\title{
Development of a Southern Vilnius Standard Photometric System
}

\author{
by \\ Murray Charles Forbes
}

A thesis

submitted to the Victoria University of Wellington in fulfilment of the requirements for the degree of Doctor of Philosophy in Physics.

(c) Victoria University of Wellington

September 1996 
ACTORIA UNIVERSITY OF WELLINCTL 


\begin{abstract}
The Vilnius Standard Photometric System is said to have several advantages over other photometric systems; reduction procedures free of systematic errors, a homogeneous set of standard stars, accurate dereddening, spectral classification and calibration of physical parameters for normal stars, and a good detection rate of abnormal stars. To investigate these, two southern, open star clusters (Omicron Velorum and Kappa Crucis) have been measured in the Vilnius system. The observations were used to derive astrophysical parameters such as age $\left((45 \pm 15) \times 10^{6}\right.$ and $(10 \pm 3) \times 10^{6}$ years respectively), composition (both solar metallicity), distance to the clusters $\left(\mathrm{m}-\mathrm{M}=5^{m} \cdot 94 \pm 0.02\right.$ and $\left.12^{m} \cdot 18 \pm 0 \cdot 05\right)$, interstellar reddening along our light of sight to the clusters $\left(E_{\mathrm{Y}-\mathrm{V}}=0^{m} \cdot 00 \pm 0.02\right.$ and $\left.0 \cdot 31 \pm 0 \cdot 09\right)$, and cluster membership probabilities for the individual stars. These compared favorably with the consensus of similar analyses made by observers using other photometric systems, with one exception being the distance to Kappa Crucis $\left(\langle\mathrm{m}-\mathrm{M}\rangle=11^{m} \cdot 59\right)$.
\end{abstract}

As no stars further south than declination $-26^{\circ}$ have been measured in the (original) Vilnius System, it was necessary to calibrate the local system to the standard system by measuring equatorial stars common to both. To alleviate this problem in the future, suitable southern stars were measured to form a southern standard system. Initially bright stars evenly spread across the sky were calibrated - this will ensure any future observing programme will have nearby standards. The next phase of the programme was to calibrate the E-region stars - this is a set of stars in common use as southern standards in other photometric systems. The final phase was to calibrate a sequence of stars near the south celestial pole - producing a set of stars so that the same standards can be observed at any time during the year. This southern standard system could not be completely established in the time available (it is approximately $50 \%$ finished), due to the larger than expected uncertainties in the measurements largely caused by the atmospheric effects of the Mount Pinatubo eruption. 


\section{Acknowledgements.}

I thank my family for their patience and support, and Tim and Hà Banks for livening up the office. A big thanks goes to the Physics Department (for providing the part-time teaching job) and staff, especially Grant Carter. My co-supervisors (Drs' Denis Sullivan and Richard Dodd) are thanked for their many constructive suggestions. I also thank the University of Canterbury for the use of their observing facilities, and the Mount John University Observatory staff for their friendly assistance (especially Mike Clark and family). Thanks also go to the Vilnius Institute of Theoretical Physics and Astronomy for providing the filters, and Dr Vytautas Straižys. The Centre de Données Astronomiques de Strasbourg is thanked for providing the Vilnius catalogues, and the use of the Set of Identifications, Measurements and Bibliography for Astronomical Data. Dr. Kurucz is thanked for providing the grid of Vilnius stellar models, and Dr. Schaerer and collaborators for the evolutionary models. I acknowledge the financial support of the New Zealand Foundation for Research, Science and Technology and the Victoria University of Wellington's Internal Grants Committee. 


\section{Contents}

$\begin{array}{ll}\text { Abstract. } & \text { iii }\end{array}$

Acknowledgements. $\quad$ v

1 Introduction to Photometric Systems. 1-1

1.1 Possible information found from Spectroscopy. . . . . . . . . . . . 1-1

1.2 Why do photometry? .................

1.3 Designing a Photometric system. . . . . . . . . . . . . . 1-13

1.4 Characteristics of photometric system. . . . . . . . . . . 1-15

1.5 Development of the UBV Photometric System. . . . . . . . . . 1-21

1.6 Development of the $u v b y$ Photometric System. . . . . . . . . . . 1-26

1.7 Development of the Vilnius Photometric System. . . . . . . . . . . . 1-32

1.8 The Q-Search Classification Method. . . . . . . . . . . . . . . 1-37

1.9 The Q-Spectra Classification Method. . . . . . . . . . . . 1-38

1.10 The Q-Isolines Classification Method. . . . . . . . . . . . . . 1-40

1.11 The QQ-diagrams Classification Method. . . . . . . . . . . . . . 1-44

1.12 Summary. . . . . . . . . . . . . . . . . . . . . . 1-51

2 Observation and Reduction Techniques. 2-1

2.1 The Equipment used. . . . . . . . . . . . . . . . . . . . 2-1

2.2 The Dark Current. . . . . . . . . . . . . . . . . . 2-3

2.3 Time Corrections. . . . . . . . . . . . . . . . 2-5

2.4 Dead-time Corrections. . . . . . . . . . . . . . . . . . . 2-5

2.5 Sky Background Correction. . . . . . . . . . . . . . . . 2-9

2.6 The Monochromatic Extinction Correction. . . . . . . . . . . . . . 2-13

2.7 The Heterochromatic Extinction Correction. . . . . . . . . . . . . 2-26

2.8 Transformation to the Standard Filter System. . . . . . . . . . . . . 2-31

2.9 Planning an Observing Session. . . . . . . . . . . . . 2-36

2.10 Summary. . . . . . . . . . . . . . . . . . . . 2-38 
3 The Southern Vilnius Standard System. 3-1

3.1 Selection of Standard star candidates. . . . . . . . . . . . . . 3-1

3.2 Error analysis. . . . . . . . . . . . . . . . . . . . . $3-2$

3.3 Selection of the Standard stars. . . . . . . . . . . . . . . 3-8

3.4 Derivation of the Southern Vilnius System's Passbands. . . . . . . . . . . 3 3-9

3.5 Transformations to the Johnson System. . . . . . . . . . . . . 3-10

3.6 Transformations to the Strömgren System. . . . . . . . . . . . . 3-19

3.7 Summary. . . . . . . . . . . . . . . . . . . . . . . . . . . . $3-24$

4 The Omicron Velorum and Kappa Crucis elusters. 4-1

4.1 Isochrones in the Vilnius System. . . . . . . . . . . . . . . . 4-1

4.2 Literature review of the Omicron Velorum cluster. . . . . . . . . . . . 4-4

4.3 Vilnius Photometry of the Omicron Velorum cluster. . . . . . . . . . 4-30

4.4 Literature review of the Kappa Crucis cluster. . . . . . . . . . . . . 4-38

4.5 Vilnius Photometry of the Kappa Crucis cluster. . . . . . . . . . . 4 4-49

4.6 Summary. . . . . . . . . . . . . . . . . . . . 4-53

5 Discussion and Conclusions.

$\begin{array}{ll}\text { A Overview of the Appendices. } & \text { A-1 }\end{array}$

B Catalogs of Candidate Primary and Secondary Standard Stars. B-1

C The Observational Log. $\quad$ C-1

$\begin{array}{ll}\text { D The Reduced Observations. } & \text { D-1 }\end{array}$

E The Mean Reduced Observations. $\quad$ E-1

F Derivation of Nikonov's Method to determine Instantaneous Extinction. F-1

G Long term changes in extinction and the effect of Mount Pinatubo. G-1

G.1 Correlations between nights. . . . . . . . . . . . . . . . G-1

G.2 The effect of the Mount Pinatubo eruption. . . . . . . . . . G-3

H The Rayleigh, Ozone and Aerosol Extinction Coefficients. H-1

H.1 Introduction. . . . . . . . . . . . . . . . . H

H.2 Rayleigh. . . . . . . . . . . . . . . . H

H.3 Ozone. . . . . . . . . . . . . . . . . . . . . . . . H

H.4 Aerosol. . . . . . . . . . . . . . . . . . . . . H H

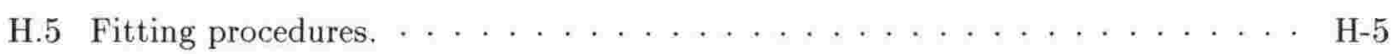


H.6 Results. . . . . . . . . . . . . . . . . . . H 6 . 6

H.7 Conclusions. . . . . . . . . . . . . . . . H-11

I Formulation of the Vilnius Filters using Schott glasses. I-1

J Tables and Appendices from Straižys book. J-1

K Glossary of Acronyms. $\quad$ K-1

L List of Publications. $\quad$ L-1

$\begin{array}{ll}\text { References. } & \text { R-1 }\end{array}$ 


\section{List of Figures}

1.1 Black Body Spectra. . . . . . . . . . . . . . . . . 1-3

1.2 Example of the hydrogen Balmer series of absorption lines. . . . . . . 1-5

1.3 Spectra of various Main Sequence stars. . . . . . . . . . . 1-11

1.4 Convolution of various Main Sequence spectra with Johnson V filter. . . . . 1-12

1.5 Convolution of various Main Sequence spectra with Vilnius V filter. . . . . 1-12

1.6 Transmission Response curves of the UBV Photometric System. . . . . . . 1-24

1.7 The Johnson System's intrinsic U-B, B-V diagram. . . . . . . . . . . 1 1-25

1.8 Transmission Response curves of the uvby Photometric System. . . . . . . 1-27

1.9 Schematic explanation of $c_{1}$ and $m_{1}$ in the uvby system. . . . . . . 1-29

1.10 The Strömgren $b-y, c_{1}$ diagram. . . . . . . . . . . . 1-31

1.11 Transmission Response curves of the Vilnius Photometric System. . . . . . 1-33

1.12 Test of the Q-Search Spectral classification method. . . . . . . . . 1-39

1.13 Pseudo-Q-Spectra of main-sequence stars. . . . . . . . . . . . 1-41

1.14 Pseudo-Q-Spectra for different luminosity classes. . . . . . . . . . . . 1-42

1.15 Examples of Q-isolines classifications. . . . . . . . . . . . . 1-43

1.16 Initial Spectral classification of QQ-diagrams method. . . . . . . . . . 1-44

1.17 Separation of M-type stars for QQ-diagrams classification method. . . . . . 1-44

1.18 Alternative separation of M-type stars. . . . . . . . . . . . . 1-46

1.19 Alternative separation of M-type stars continued. . . . . . . . . . . 1-47

1.20 Classification of normal M stars using QQ-diagrams. . . . . . . . . . 1-47

1.21 Separation of Be stars from normal B stars. . . . . . . . . . . . 1-49

1.22 Separation of subdwarves from normal stars. . . . . . . . . . . 1-49

1.23 Separation of Ap and Bp stars from normal stars. . . . . . . . . . 1-49

1.24 Separation of white dwarves from normal stars. . . . . . . . . . 1-49

1.25 Classification of normal B stars using QQ-diagrams. . . . . . . . 1-50

1.26 Separation of metallic-line, Ap and Bp stars from normal stars. . . . . . 1-51

1.27 Classification of normal A-F stars using QQ-diagrams. . . . . . . . 1-52

1.28 Classification of normal A5-7 III-V stars using QQ-diagrams. . . . . . . . 1-53

1.29 Separation of $\mathrm{CH}$, barium and R-type stars from normal stars. . . . . . 1-54 
1.30 Separation of metal-deficient giants from normal stars. . . . . . . . . . 1-54

1.31 Classification of subdwarves using QQ-diagrams. . . . . . . . 1-55

1.32 Classification of normal $\mathrm{G}$ stars using QQ-diagrams. $\ldots \ldots \ldots \ldots$ 1-56

1.33 Classification of normal K stars using QQ-diagrams. . . . . . . . 1-57

2.1 View of the buildings at the top of the Mount John University Observatory. . 2-2

2.2 The Boller and Chivens telescope at Mount John University Observatory. . . 2 2-2

2.3 Bouguer's Law plot to determine monochromatic extinction. . . . . . . . 2-16

2.4 Observational error as a function of airmass at MJUO . . . . . . . 2-17

2.5 Variable extinction in a Bouguer Law plot. . . . . . . . . 2-18

2.6 Effect on a Bouguer plot due to three possible causes of changing extinction. - 2-20

2.7 Effect on the extinction coefficient of different types of extinction change. $\cdots \quad 2-21$

2.8 Possible variation of monochromatic extinction during a night. $\ldots \ldots \ldots \ldots .22$

2.9 Possible variation of the instrumental zero-point during a night. . . . . . 2-22

2.10 Effect of extinction or zero-point changes on transformation to the standard system. . . . . . . . . . . . . . . . . . 2-23

2.11 Nikonov's Method used to determine the exact magnitude of the extinction star. 2-25

2.12 Typical atmospheric extinction across the optical region. . . . . . . . 2 26 26

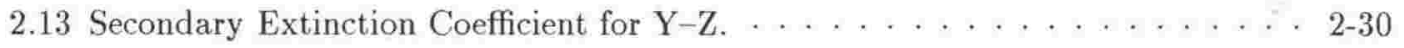

2.14 Comparison of local and Standard Vilnius filter responses. . . . . . . 2-34

2.15 Transformation from the local to the Standard Vilnius System. . . . . . 2 2-35

2.16 The airmass of $\mathrm{O}$ Vel at midnight of every night during 1993 at MJUO. . . . 2-36

2.17 Choosing the Declination as a function of HA between the extinction and control stars. . . . . . . . . . . . . . . . . . . . 2-37

2.18 The airmass of the extinction star E6_047 at MJUO during 19 May 1993 . . . 2-38

3.1 Observational error as a function of instrumental magnitude at MJUO. . . . 3-4

3.2 Histogram of residuals of the primary standards observations. . . . . . . 3-5

3.3 Histogram of residuals of the primary standard stars. . . . . . . . . 3-6

3.4 Johnson filters as a linear combination of Vilnius filters. . . . . . . . . 3-11

3.5 Transformations between Vilnius and Johnson V magnitudes. . . . . . 3-12

3.6 Transformations between Vilnius U-Y and Johnson U-B colours. . . . . . 3-14

3.7 Transformations between Vilnius colours and Johnson B-V. . . . . . . 3 3-16

3.8 Transformations between Vilnius V-S and Johnson V-R. $\ldots \ldots \ldots \ldots \ldots$ 3-17

3.9 Transformations between Vilnius V-S and Johnson V-I. . . . . . . . . 3-18

3.10 Strömgren filters as a linear combination of Vilnius filters. . . . . . . . . 3-19

3.11 Transformations between the Vilnius and Strömgren magnitudes. . . . . . 3-21 
3.12 Transformations to the Strömgren $u-b$ colour. . . . . . . . . . 3-21

3.13 Transformations to the Strömgren $v-b$ colour. . . . . . . . . . . 3-23

3.14 Transformations to the Strömgren $b-y$ colour. . . . . . . . . . . . 3-24

4.1 Grid of Vilnius V magnitudes (solar metallicity models). . . . . . . . . . 4-2

4.2 Calibration of Kurucz's models to the standard Vilnius system. . . . . . . 4 4-3

4.3 The Vilnius ZAMS 'isochrone'. . . . . . . . . . . . . . . . . . . 4-4

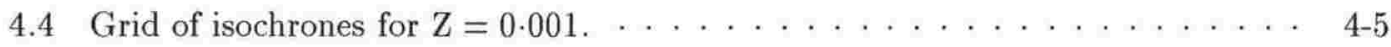

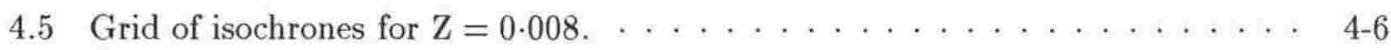

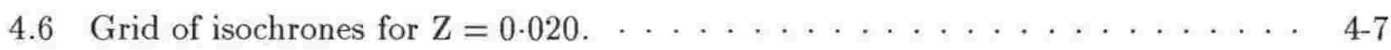

4.7 Star Chart for O Vel. . . . . . . . . . . . . . . . . . . . . 4-11

4.8 Proper Motion distribution for the $\mathrm{O}$ Vel cluster. . . . . . . . . . . 4-15

4.9 Radial Velocity distribution for the $\mathrm{O}$ Vel cluster. . . . . . . . . . . 4-17

4.10 Colour-Magnitude diagram of $\mathrm{O}$ Vel, from the UBV literature. . . . . . . . 4-23

4.11 Colour-Colour diagram of $\mathrm{O}$ Vel, from the UBV literature. . . . . . . . . . 4-24

4.12 Colour-Magnitude diagram of $\mathrm{O}$ Vel, from the $u v b y$ literature. . . . . . . . 4-27

$4.13 H_{\beta}$-Absolute Magnitude diagram of $\mathrm{O}$ Vel, from the $u v b y$ literature. . . . . . 4-29

4.14 Colour-Magnitude diagrams of $\mathrm{O}$ Vel from Vilnius photometry. . . . . . . 4-33

4.15 Vilnius isochrones for $\mathrm{O}$ Vel. . . . . . . . . . . . . . 4-35

4.16 Distance modulus and colour excess for Kappa Crucis from the literature. . . 4-40

4.17 Star Chart for Kappa Crucis. . . . . . . . . . . . . . . . . . . . . 4-40

4.18 Proper motions histograms for Kappa Crucis. . . . . . . . . . . . . . 4-43

4.19 Colour excesses $E_{B-V}$ for Kappa Crucis stars from the literature. . . . . . . 4-46

4.20 Colour-Magnitude diagram of Kappa Crucis, from the UBV literature. . . . . 4-47

4.21 Colour-Colour diagram of Kappa Crucis, from the UBV literature. . . . . . . 4-48

4.22 Colour excess and distance moduli distributions for Vilnius photometry of Kappa

Crucis. . . . . . . . . . . . . . . . . . . . 4 4-51

4.23 Spatial distribution of Vilnius colour excesses for Kappa Crucis. . . . . . . 4 4-52

4.24 Vilnius isochrones for Kappa Crucis. . . . . . . . . . . . . . . 4-53

G.1 Auto-correlation of extinction between consecutive nights at MJUO. . . . . G-2

G.2 The effect of the Mount Pinatubo eruption on extinction at MJUO. . . . . G-4

H.1 The three different aerosol size distributions and their corresponding extinction spectrum. . . . . . . . . . . . . . . . . H $\ldots$ H

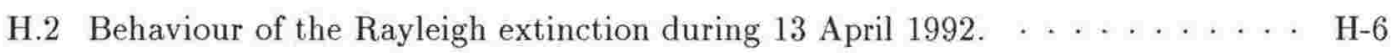

H.3 Behaviour of the ozone extinction during 13 April 1992. . . . . . . . H H

H.4 Behaviour of the aerosol extinction during 13 April 1992. . . . . . . H 
H.5 Behaviour of the aerosol size distribution parameter during 13 April 1992 . . H-9

H.6 The mean extinction fitted with Rayleigh, Ozone and aerosol components. . . H-10

I.1 Comparison of Measured and Catalog responses of Schott glasses. . . . . . . I-2

I.2 Realization of the Vilnius Photometric System using Schott glasses. . . . . . . I-4

I.3 Comparison of intermediate Schott filters with the Vilnius System. . . . . . . I-9 


\section{List of Tables}

1.1 Characteristics of the Standard UBV System Passbands. . . . . . . . . 1-23

1.2 Characteristics of the Standard uvby System Passbands. . . . . . . . . 1-27

1.3 Transmission Response curves of the Vilnius Photometric System. . . . . . 1-34

1.4 Characteristics of the Standard Vilnius System Passbands. . . . . . . 1-35

1.5 Approximate colour excess ratios. . . . . . . . . . . . . 1-51

1.6 Comparison of the UBV, uvby, and Vilnius Systems. . . . . . . . . 1-59

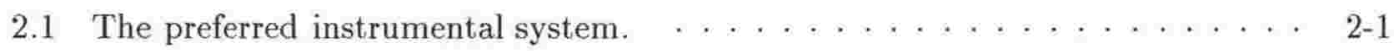

2.2 Dead-time measurements. . . . . . . . . . . . . . . . . 2-8

2.3 Vilnius Colour Extinction Coefficients. . . . . . . . . . . . . . 2-31

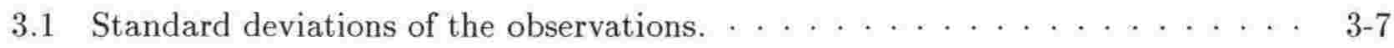

3.2 Error estimates of the Southern Vilnius system standard stars. . . . . . . 3-9

4.1 Review of main characteristics of $\mathrm{O}$ Vel. . . . . . . . . . . . 4-8

4.2 Synonyms for O Vel's stars. . . . . . . . . . . . . . . . 4-10

4.3 Spectral type for $\mathrm{O}$ Vel stars from the literature. . . . . . . . . . . . . . 4-12

4.4 Proper motion for $\mathrm{O}$ Vel stars from the literature. . . . . . . . . . . 4-14

4.5 Radial velocity for $\mathrm{O}$ Vel stars from the literature. . . . . . . . . . 4 4-16

4.6 Orbital elements of Spectroscopic binary $\mathrm{O}$ Vel 3. . . . . . . . . . . 4-17

4.7 Membership for $\mathrm{O}$ Vel stars from the literature. . . . . . . . . . . . . 4-19

4.8 Visual magnitude $\mathrm{V}$ for $\mathrm{O}$ Vel stars from the literature. . . . . . . . 4-20

$4.9 \mathrm{U}-\mathrm{B}$ and $\mathrm{B}-\mathrm{V}$ colours for $\mathrm{O}$ Vel stars from the literature. . . . . . . . . 4-22

$4.10 b-y$ colours for $\mathrm{O}$ Vel stars from the literature. . . . . . . . . . 4 $4-25$

$4.11 m_{1}$ and $c_{1}$ colours for $\mathrm{O}$ Vel stars from the literature. . . . . . . . $4-26$

$4.12 H_{\beta}$ colours for $\mathrm{O}$ Vel stars from the literature. . . . . . . . . . 4-28

4.13 Spectral Classifications of $\mathrm{O}$ Vel stars from Vilnius photometry. . . . . . . 4 4-36

4.14 Membership of $\mathrm{O}$ Vel stars from Vilnius photometry. . . . . . . . . . . 4-37

4.15 Review of main characteristics of Kappa Crucis. . . . . . . . . . . . 4-39

4.16 Synonyms for Kappa Crucis's stars. . . . . . . . . . . . . . . . . 4-41 
4.17 Spectral types for Kappa Crucis stars from the literature. . . . . . . . . . . . 4-57

4.18 Proper motion and radial velocity for Kappa Crucis stars from the literature. * 4-58

4.19 Membership for Kappa Crucis stars from the literature. . . . . . . . . . . . . 4-59

4.20 Visual magnitude V for Kappa Crucis stars from the literature. . . . . . . . 4-60

4.21 Variability of Kappa Crucis stars from the literature. . . . . . . . . . . . . . 4-61

$4.22 \mathrm{U}-\mathrm{B}$ and B-V colours for Kappa Crucis stars from the literature. . . . . . . 4-62

4.23 Colour excesses $E_{B-V}$ for Kappa Crucis stars from the literature. . . . . . 4-63

4.24 Spectral Classifications of Kappa Crucis stars from Vilnius photometry. . . . . 4-64

4.25 Dereddening of Kappa Crucis stars from Vilnius photometry. . . . . . . . . 4-65

B.1 Bright, southern Vilnius stars selected for use as primary standards. . . . . . . B-2

B.2 Bright, southern stars selected for use as secondary standards. . . . . . . . . B-11

B.3 SPS stars selected for use as secondary standards. . . . . . . . . . . B-18

C.I A summary of the observations made on each night. . . . . . . . . . C-3

C.2 List of observed stars : coordinates, star type, Johnson $V$ and Spectral class. . C-7

D.1 The individual reduced observations for the primary standard stars. . . . . D-2

D.2 The individual reduced observations for the SPS standard stars. . . . . . . D-19

D.3 The individual reduced observations for the bright, secondary standard stars. * D-21

D.4 The individual reduced observations for the E region standard stars. . . . . D-45

D.5 The individual reduced observations for the cluster O Vel. . . . . . D-71

D.6 The individual reduced observations for the cluster Kappa Crucis. . . . . . D-77

E.1 The mean reduced observations for the primary standard stars. . . . . . . E-2

E.2 The mean reduced observations for the bright, southern secondary standard stars. E-4

E.3 The mean reduced observations for the E Region standard stars. . . . . . . E 5

E.4 The mean reduced observations for the unsuccessful primary standard stars. . . E-7

E.5 The mean reduced observations for the unsuccessful SPS standard stars. . . . E-10

E.6 The mean reduced observations for the unsuccessful bright, southern secondary standard stars. . . . . . . . . . . . . . . . . E E-11

E.7 The mean reduced observations for the unsuccessful E Region standard stars. • E-15

E.8 The mean reduced observations for the cluster $\mathrm{O}$ Vel. . . . . . . . . . E-17

E.9 The mean reduced observations for the cluster Kappa Crucis. . . . . . . . E-19

H.1 Effective Rayleigh and Ozone extinction coefficients for the standard Vilnius photometric system. . . . . . . . . . . . . . H-5

H.2 The mean Vilnius extinction coefficients at MJUO. . . . . . . . . . H-10

H.3 The fitted Rayleigh, Ozone and aerosol coefficients to the mean extinction. . . H-11 
I.1 The Vilnius Filter System from Schott glasses. $\ldots \ldots \ldots \ldots$ I-3 . . . . . . .

I.2 Filters between the wavelengths of the Vilnius Filter System from Schott glasses. I-8

J.1 Ratio of extinction to colour excess. . . . . . . . . . . . . J-1

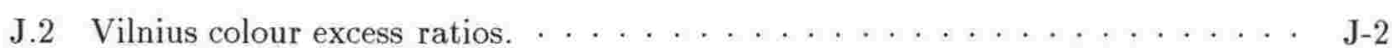

J.3 Intrinsic Vilnius colours for the luminosity $\mathrm{V}$ and the ZAMS stars. . . . . . J J-3

J.4 Intrinsic Vilnius colours for the luminosity IV stars. . . . . . . . . . J J-4

J.5 Intrinsic Vilnius colours for the luminosity III stars. . . . . . . . J J-5

J.6 Intrinsic Vilnius colours for the luminosity I stars. . . . . . . . . . J J-6

J.7 Intrinsic color indices $(\mathrm{Y}-\mathrm{V})_{0}$ as a function of $\mathrm{Sp}$ and $\mathrm{M}_{\mathrm{V}} \ldots \ldots \ldots \ldots . \quad \ldots \quad \ldots$

J.8 Calibration of spectral classes MK in absolute magnitude $\mathrm{M}_{\mathrm{V}} \ldots \ldots \ldots \ldots \ldots$ J-9

J.9 Logarithms of stellar effective temperatures and bolometric corrections. . . J J-10

J.10 Logarithms of stellar surface gravities. . . . . . . . . . . . . . . J-11

J.11 Logarithms of stellar masses in solar units. . . . . . . . . . . J J-12

J.12 Logarithms of stellar radii in solar units. . . . . . . . . . . . J-13 


\section{Chapter 1}

\section{Introduction to Photometric Systems.}

\subsection{Possible information found from Spectroscopy.}

Some possible sources of information about stars and interstellar material are electro-magnetic radiation (of all wavelengths, for example gamma rays, X-rays, ultra-violet (UV), visible light, infrared, or radio waves), cosmic rays (consisting of high energy electrons, protons, and heavier nuclei, plus neutrons and mesons, with some anti-matter), neutrinos and anti-neutrinos, and, perhaps one day, gravitational waves. Traditionally most measurements have been made of e-m radiation, with observations in the optical region having the longest history. Generally e-m observations give information only about the surface of stars, while neutrinos give complementary information about stellar cores. As this work concerned a visible light measuring system, a brief outline of the information that can be found from this region will be given next - any university undergraduate textbook on quantum mechanics or e-m radiation should be referred to for more detail.

A 'black body' is defined as any object that absorbs all incident light (at all wavelengths) and reflects none. It is also a perfect emitter of light, producing a continuous spectrum in a way that only depends on it's temperature;

$$
E(\lambda, T)=\frac{2 \pi h c^{2}}{\lambda^{5}} \frac{1}{e^{h c / \lambda k T}-1}
$$

$E$ is the energy emitted per second, per unit wavelength interval, per unit (surface) area of the black body, into a unit solid angle, $h$ is Planck's constant, $k$ is Boltzmann's constant, $c$ is the speed of light, $\lambda$ is the wavelength and $T$ is the (absolute) temperature. Black bodies do not actually exist ${ }^{1}$ but any hot, opaque solid, liquid or (compressed) gas will behave approximately like one.

Two important results for black bodies are best seen in the following equations (which can

\footnotetext{
${ }^{1}$ If Quantum black holes exist, they are expected to radiate as a black body via quantum fluctuations in the size of their event horizon
} 
be derived from equation 1.1). The first is Wien's displacement law

$$
\lambda_{\max } T=2 \cdot 9 \times 10^{7} \quad(\lambda \text { in } \AA)
$$

which states the black body emission has it's maximum intensity at the wavelength $\lambda_{\max }$, and this 'peak' wavelength shifts to shorter wavelengths as the temperature increases. The second result is Stefan's law

$$
I=\sigma T^{4}
$$

which states that the total energy emitted (i.e. over all wavelengths) per second per unit area $(I)$ increases (rapidly) with temperature, where $\sigma$ is Stefan's constant.

Thus the temperature of a black body can be measured in three ways; fitting a black body curve to the measured spectrum, measuring the maximum intensity wavelength, or measuring the total energy emitted (over all wavelengths). The latter is not generally used due to difficulties in calibrating the measurements in absolute units, measuring the radius or angular size of the object (which is difficult for stars) and covering a sufficiently large wavelength range (to approximate the complete range).

If a star is spherical (radius $R$ ) and has the same temperature over it's entire surface, then the total energy emitted by the star is

$$
L \propto I R^{2}
$$

Approximating the star as a black body with an effective surface temperature $\left(T_{e}\right)$ gives

$$
L \propto T_{e}^{4} R^{2}
$$

Normalizing by dividing with solar units (subscript $\odot$ ) gives

$$
\frac{L}{L_{\odot}}=\left(\frac{T_{e}}{T_{e_{\odot}}}\right)^{4}\left(\frac{R}{R_{\odot}}\right)^{2}
$$

This is more commonly used in logarithmic form

$$
\log \frac{L}{L_{\odot}}=4 \log \frac{T_{e}}{T_{e_{\odot}}}+2 \log \frac{R}{R_{\odot}} .
$$

An alternative form uses the surface gravity $(g)$

$$
\log \frac{L}{L_{\odot}}=4 \log \frac{T_{e}}{T_{e_{\odot}}}+\log \frac{\mathcal{M}}{\mathcal{M}_{\odot}}-\log \frac{g}{g_{\odot}}
$$

vis $g=G \mathcal{M} / R^{2}$, where $G$ is the universal gravitational constant and $\mathcal{M}$ is the mass of the star. These relationships are expected to hold when a star is in energy equilibrium (for example, on 


\section{Black Body Spectra}

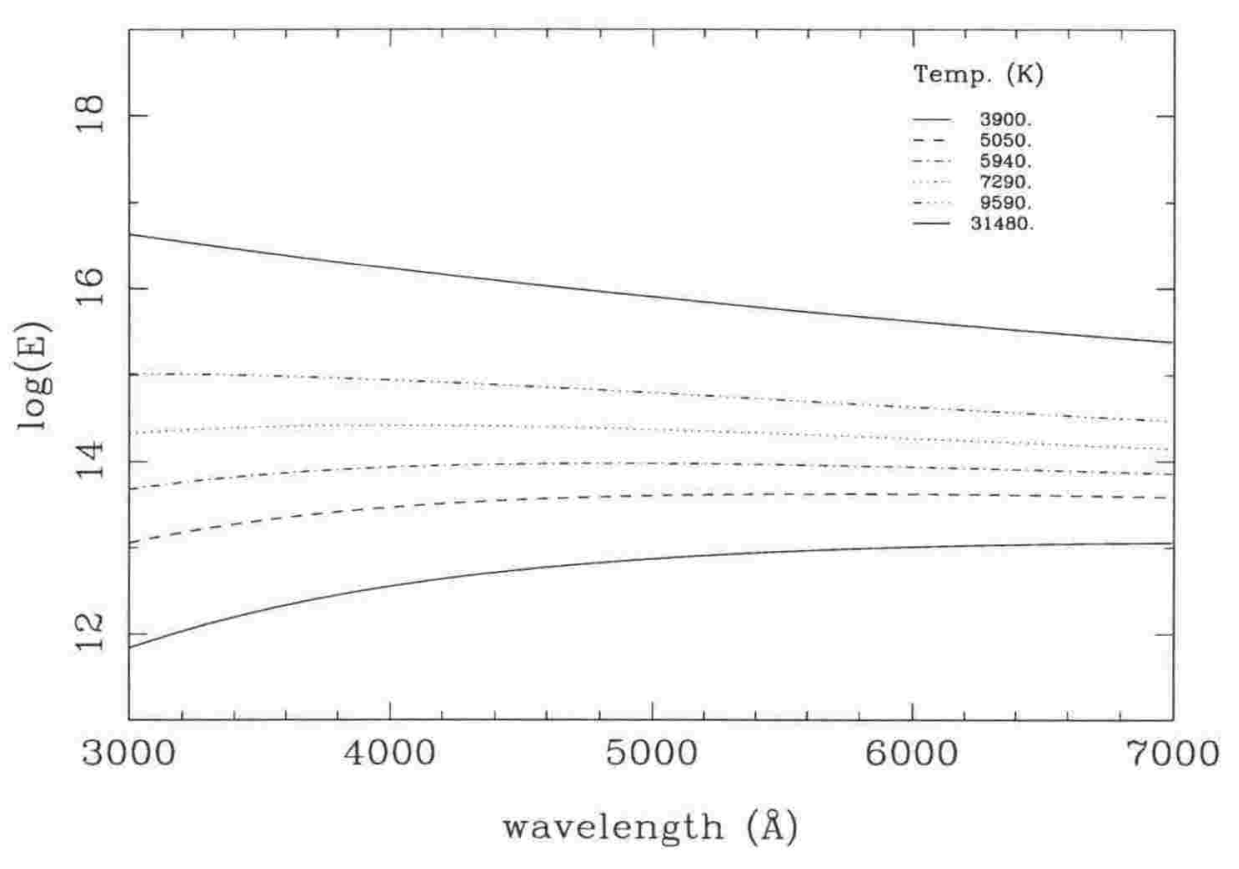

Figure 1.1: Black Body emission spectra at different temperatures over the optical wavelength region. The temperatures correspond to the effective surface temperatures of M0 V $(3900 \mathrm{~K})$, K0 V $(5050 K)$, G0 V $(5940 K)$, F0 V $(7290 K)$, A0 V $(9590 K)$, and B0 V $(31480 K)$ stars Temperatures taken from Appendix 3 of Straižys (1992). The emitted energy is plotted using the logarithm (to base 10) as the maximum energy (for each temperature) varies by a factor of $10^{4}$.

the main sequence) - if the star is non-spherical or has a non-uniform temperature distribution, then the radius or temperature may be replaced by effective parameters (averaged over the star's surface).

Light can be described by both wave and particle models, ${ }^{2}$ with the connection between the models given by

$$
E=h \nu=h c / \lambda
$$

where $E$ is the energy of the particle (called a photon), $h$ is Planck's constant, $c$ is the speed of light through the medium (usually a vacuum), and $\nu, \lambda$ are the frequency and wavelength respectively of the e-m wave. This is often used in alternate form

$$
E=\hbar \omega
$$

where $\omega$ is the angular frequency $(\omega=2 \pi \nu)$ and $\hbar=h / 2 \pi$.

\footnotetext{
${ }^{2}$ What are normally considered particles, for example electrons or atoms, can also be described by both particle and wave models.
} 
This particle/wave duality has important consequences when combined with the quantum nature of matter. The energy states of atomic and molecular systems are found to only occur in certain discrete values (i.e. they are 'quantized') rather than being free to take any value from a continuous range. To change the state of a system, it must absorb (or emit) energy corresponding to the difference in energy between it's initial and higher (or lower) final energy state. Thus a system can only absorb a photon if the photon's wavelength corresponds to a energy, via equation 1.7 , that will excite the system to a higher level from it's current state. The converse applies when an excited system decays to a lower energy level by emitting a photon; the photon's wavelength corresponds to the difference in energy levels.

However, quantum systems may have some energy states so closely spaced together that they merge to form a continuous energy band, with the system able to take on any energy level within the band. This is more common in larger systems, as, according to the correspondence principle, any quantum system behaves more like a classical system (which can have any energy from a continuous range) as it's size increases.

The energy states of concern here are the 'bound' electrons which orbit the nucleus of an atom. An electron excited to a higher energy state moves to another orbit which is, on average, further from the nucleus than the initial orbit. ${ }^{3}$ If the electron receives enough energy that it escapes from the attraction of the nuclei's electric field, the atom is said to be ionized. Such 'free' electrons are actually then part of a much larger quantum system (of all the neighboring atoms and other free electrons) which has an continuous energy band from the ionisation energy upwards. Further, the energy levels of the atom change when it becomes ionized and changes again each time it loses another electron.

The quantum states (and transition probabilities) are unique to each element, meaning the resulting pattern of absorption (or emission) lines allow that element to be identified. However the absence of an element's absorption lines (in a star) does not mean the element itself is absent as the star's surface may not be at the right temperature and pressure to maintain a large enough population of atoms excited to the right energy levels that the lines can be seen (in the wavelength region observed).

Consider the hydrogen atom; to a good approximation it's energy levels are

$$
E \propto \frac{1}{n^{2}}
$$

where $n$ is the quantum state, ranging from the ground state $(n=1)$ to the ionisation level $(n=\infty)$. Thus the wavelength of a photon arising from a transition from the $n_{i}$ initial state

\footnotetext{
${ }^{3}$ The electrons don't follow well defined orbits (in the sense of planets orbiting a star) but instead have their position given by a probability function, in keeping with their dual particle/wave nature.
} 


\section{Example of the hydrogen Balmer series}

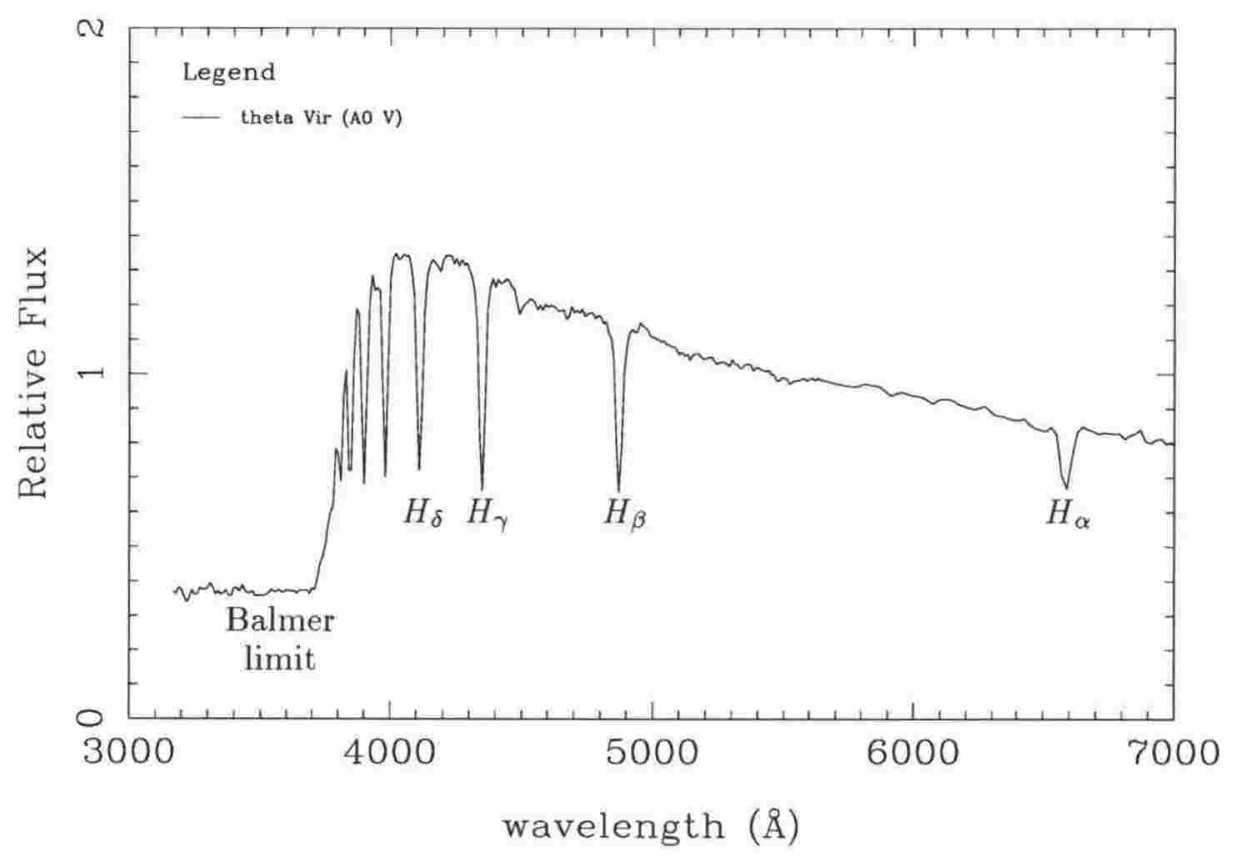

Figure 1.2: Example of the hydrogen Balmer series of absorption lines. The star is $\theta$ Vir, an A0 V type star, whose spectrum was measured by Gunn \& Stryker (1983).

to the $n_{j}$ final state is

$$
\frac{1}{\lambda} \propto \frac{1}{n_{i}^{2}}-\frac{1}{n_{j}^{2}},
$$

the photon being absorbed if $n_{i}<n_{j}$ or emitted if $n_{i}>n_{j}$. For instance, the Balmer series of lines arises when one of the quantum states is $n=2$ and the other is $n>2$. Given that the first line in the Balmer series ( $H_{\alpha}$, between the $n=2$ and $n=3$ energy levels) corresponds to $\lambda=6564 \AA$, the next lines in the series occur at $H_{\beta}=4861 \AA, H_{\gamma}=4342 \AA, H_{\delta}=4102 \AA$ etc. The lines become increasingly packed together (in wavelength) as the series increases, ultimately stopping at $\lambda=3646 \AA$ when the atom is ionized. As the electron can take on a continuum of energies above the ionisation level, this absorption 'line' spreads out over all wavelengths for $\lambda<3646 \AA$. An example of the Balmer series in a star is shown by Figure 1.2.

Clearly the Balmer series is only seen in stars which are hot enough to maintain a large population of hydrogen atoms (on the star's surface) excited to the first energy level. However, if the star is too hot then most of the hydrogen is ionized and the Balmer series is not seen. Hence the intensity of the Balmer lines indicate the temperature of the star's surface; unfortunately the strength initially increases with temperature and then decreases again with further temperature increases. The lines also sharpen, and so increase their strength, with increasing pressure (i.e. surface gravity - see later). However each series of lines shows a similar tem- 
perature dependence over a different temperature range - also each element shows a different temperature dependence. Thus the ratios of the line strengths between different elements can indicate the surface temperature of the star.

The absorbed/emitted photons for such atomic transitions generally have wavelengths in the UV and visible regions. However a molecule has additional energy states available, due to rotations of the entire molecule or vibrations of the component atoms. These energy levels are generally spaced more closely together than the atomic states, hence the corresponding photons have longer wavelengths and occur in the infrared or far-infrared regions for vibrational or rotational transitions respectively. Note that most molecules will break up before any of their component atoms can become ionized and so are only found in the cooler stars.

The conditions which give rise to a continuous spectrum, or discrete emission or absorption lines can be summarized by the following rules found empirically by Kirchoff;

1. A hot and opaque solid, liquid or highly compressed gas emits a continuous spectrum.

2. A hot, transparent gas produces an emission line spectrum.

3. If a continuous spectrum passes through a transparent gas at a lower temperature, then the gas produces absorption lines in the spectrum.

Thus the interior of a star produces a continuous spectrum, approximately described by a black body function, while the cooler, more transparent atmosphere produces absorption lines. Note that emission lines may also be seen, originating in the corona where the temperature increases again.

The Heisenberg uncertainty principle is also important for this discussion. This states that the product of the uncertainties of two complementary variables can not be less than Planck's constant, for instance energy and time $(\Delta E \Delta \tilde{S} h)$. Each transition between energy levels in a quantum system has an associated probability of occurring. If the transition has a high probability, then the average time the system will spend in the initial state before undergoing the transition is small. Hence the uncertainty in that time is also small, and so the uncertainty of the change in energy is large. Thus the frequency of an emitted (or absorbed) photon is not a precise value $\left(\omega_{0}\right)$ but described by a intensity function of the form

$$
I(\omega) \propto \frac{a}{a^{2}+\left(\omega-\omega_{0}\right)^{2}}
$$

whose intensity and line width is determined by the constant ' $a$ ' (which depends on the transition probability). Thus less probable energy state transitions give narrower, more intense absorption (or emission) lines.

This 'natural' line width is seldom seen, as a variety of processes act to broaden it. For 
instance, collisions between atoms can stimulate an energy transition, i.e. increase the transition probability and hence increase the line width. This can give a measure of the absolute temperature $(T)$ and pressure $(P)$ of the gas; the collision rate $\left(R_{c}\right)$ can be found by $R_{c}=n \bar{v} \sigma$ where $n$ is the density of atoms in the gas, $\bar{v}$ is the average velocity of the atoms, and $\sigma$ is the effective cross-sectional area of an atom. Combining this with the kinetic theory relation

$$
\frac{1}{2} m \bar{v}^{2}=\frac{3}{2} k T
$$

and the ideal gas law

$$
n=\frac{P}{k T}
$$

gives

$$
R_{c}=\frac{\sigma P}{k T}\left(\frac{3 k T}{m}\right)^{\frac{1}{2}}
$$

where $m$ is the mass of an atom and $k$ is Boltzmann's constant. In other words, as the temperature decreases or pressure increases (of the region from which this line originates), the (frequency) line width increases. Astronomers usually measure the local gravity $(g)$ rather than the pressure - these are related via the hydrostatic equilibrium equation

$$
\frac{d P}{d r}=-n g,
$$

where $r$ is the radius from the center of the star.

However, in tenuous interstellar gas clouds, collisions are rare and so these sharp 'natural width' lines may be seen provided they are not hidden by lines from the star illuminating the clouds. If a cloud is predominantly hydrogen, interstellar lines are most easily seen when the star is sufficiently hot that all it's hydrogen is ionized, and thus has no hydrogen lines of it's own. Further, the low density of the clouds means so-called 'forbidden' lines may be seen. These are actually very low probability transitions, such as simultaneous two photon emission, which require the excited atom to be left undisturbed sufficiently long that it can spontaneously decay to a lower energy level via this particular transition. These sharp lines may allow individual clouds along the observer's line of sight to be resolved by their radial velocities and temperatures via the Doppler effect (see later discussion).

Thermally re-emitted light (for example from a dense gas cloud) is un-polarized. However, if the interstellar material are metallic grains, or elongated grains aligned by some process (for example by an electric field), then the scattered light is polarized.

In the previous discussion, many of the energy levels are actually the combination of several quantum states which have the same energy - these are called 'degenerate states'. However, in the presence of an electric field (from a neighboring atom say) or magnetic field these quantum 
states will split into different energy levels, closed spaced above the degenerate energy level. This is known as the Stark effect or Zeeman splitting for electric or magnetic fields respectively. There are now many possible transitions (between these energy states) with very similar changes in energy, which can appear to blend together and increase the line width. Fortunately some of the different energy states produce photons with different polarizations, so splitting the light through a polarizer can allow the individual lines to be resolved. As these effects are proportional to the field strength (for weak fields; a quadratic term becomes important in stronger fields), it may be possible to measure the field strength if it can be untangled from the other broadening processes. For example, Jaschek \& Jaschek (1987) give the line width $(\Delta \lambda)$ due to Zeeman splitting as $\Delta \lambda \propto \lambda^{2} H$, where $H$ is the field strength.

Another important process is the Doppler effect; when an object is moving with a velocity $\mathbf{v}$ relative to an observer, any waves emitted by the object appear to the observer to be shifted in frequency compared to the frequency seen when the object is stationary. The 'relativistic' Doppler effect is

$$
\frac{\Delta \nu}{\nu} \propto \frac{v}{c} \frac{1}{\sqrt{1-\left(\frac{v}{c}\right)^{2}}},
$$

where $v$ is the magnitude of the velocity. For objects not moving at a significant fraction of the speed of light, the 'classical' Doppler effect is sufficiently accurate

$$
\frac{\Delta \nu}{\nu} \propto \frac{v_{r}}{c} \quad(v \ll c)
$$

where $v_{r}$ is the velocity component of the axis between the object and the observer (the 'radial' velocity). An object approaching the observer has a negative velocity and hence the wave is 'blue-shifted' to a lower frequency, with the converse case of a receding object being 'red-shifted' to a higher frequency.

If the object consists of a collection of smaller objects all emitting (or absorbing) waves and moving with different velocities (described by some statistical distribution), the Doppler effect results in a smearing out of the wave's frequency as what is actually seen is the combination of the individually Doppler shifted waves of all the (smaller) objects. For instance, the velocity distribution of atoms in a gas is given by the Maxwell-Boltzmann distribution (if they collide frequently) so the average radial velocity $\left(\bar{v}_{r}\right)$, which is measured from an absorption line width, is related to the temperature by $\bar{v}_{r}^{2}=k T / m$. The same line broadening effect can occur on the larger scale of micro-turbulence; consider a small cell of gas moving relative to other cells of gas (where a cell is a region in which the atoms can be described by a particular velocity distribution). Yet larger scales of turbulence can be found, right up to full-blown convection of the star.

The largest scale for which Doppler broadening can occur is in the case of a rotating star; 
a quarter of the star is approaching the observer while another quarter is receding - the remaining half is on the 'back' side of the star out of sight of the observer. This broadening is proportional to $v \sin (i)$, where $v$ is the rotation rate and $i$ is the inclination angle (the angle between the rotation axis of the star and the axis connecting the observer to the star). As the inclination angle is not usually known, statistical studies of large numbers of stars must be used to gain information about rotation rates. There is also the added difficulty that for stars with very fast rotation rates, the lines become so broad and shallow that they are difficult to resolve from the continuum. Another effect of high rotation is that the centripetal force makes the star bulge out around it's equator, and so the local gravity and temperature varies across the star's surface.

Finally, the Doppler shift of the star's lines gives the radial velocity of the star. If the star is sufficiently close to the observer that it's proper motion ${ }^{4}$ can be measured, then it's actual velocity may be found.

In a binary star system, the velocity curves of one, or both components, of the system may be found, which gives the period of the orbits and the total mass of the system. If the system's orbital plane is nearly edge on to the observer's line of sight, then measurements of the continuum during a complete orbit can give the angle of inclination of the system, the relative brightness of the component stars and their mass ratio. Combining this with the velocity curves gives a complete description of the system's orbit and the masses of the individual components. Alternately, if the star is sufficiently close to the observer that the component stars can be resolved (so it's proper motion and trigometric parallax can be measured), the orbit and individual masses may again be found.

Most of the previous discussion assumed that all the light from a star originates from the star's 'surface', which is characterized by one combination of temperature, pressure and chemical composition. However the opacity of a star's atmosphere varies with wavelength, so measurements in different wavelength regions will see to different depths and hence regions of different temperature, pressure and composition.

\subsection{Why do photometry?}

Given the information about stars and interstellar material that can be gleamed from spectroscopy, why then are so many measurements made using photometry instead? Spectroscopy, due to the low photon arrival rate per unit wavelength interval, requires very large telescopes and/or is restricted to very bright stars to get a sufficient signal in the limited time available

\footnotetext{
${ }^{4}$ The proper motion of a star is the transverse motion of the star, measured against the background of very distant (and therefore apparently motionless) stars.
} 
for an observation (at most the duration of a night ${ }^{5}$ ). Further, large telescopes are expensive and difficult to obtain observing time on, and most stars are fainter than can be measured spectroscopically. Finally, much of the spectrum (of normal stars) contains redundant information (Bessell 1993), for instance only two or three points on the continuum are needed to derive the effective temperature. Thus the first question to answer is if the planned investigation requires the wavelength resolution of spectroscopy or will photometry suffice.

Assuming photometry is adequate, the next question is which of the existing photometric systems are suitable, or will a new system need to be designed. As each photometric system is (usually) designed to investigate a particular question (which accounts for the large number of systems, for example, the UBV system (and it's clones and extensions), Washington, Geneva, DDO, Strömgren, Walraven, Borgman, Wing, and Vilnius systems to name but a few), there does not exist a single 'best' system - indeed many observers do not believe an 'ideal' system is even possible as it would have to satisfy many, often contradictory, requirements. Other observers however believe it is possible, with subsets of filters from the 'ideal' system selected to suit each investigation and having the added advantage of needing only one set of standard stars (Straižys 1966). To help explain why the Vilnius system was chosen for this study, two other photometric systems (the popular Johnson and Strömgren systems) will be described later and compared with the Vilnius system.

One important characteristic of a photometric system is the typical bandwidth of it's filters. These are usually sub-divided into the three categories given below;

$$
\begin{array}{rll}
\lambda_{1}-\lambda_{2} & <90 \AA & \text { narrow } \\
90 \AA<\lambda_{1}-\lambda_{2} & <300 \AA & \text { intermediate } \\
300 \AA<\lambda_{1}-\lambda_{2} & & \text { wide (or broad) }
\end{array}
$$

according to Strömgren $(1963,1966)$, where $\lambda_{1}$ and $\lambda_{2}$ are the minimum and maximum wavelengths through which the filter will transmit light. However it should be noted that these thresholds are only approximate. Figure 1.3 shows a selection of stellar spectra measured at spectrocopic resolution while Figures 1.4 and 1.5 show the same spectra seen at wide and intermediate bandwidth resolution respectively. Clearly broadband systems can only measure the largest features of a spectrum, such as the continuum and the Balmer discontinuity, while intermediate systems can measure additional features such as molecular absorption bands and some of the strong (single) absorption lines.

Another important characteristic of a photometric or spectroscopic system is it's purity. Consider a photometric system with $n$ passbands, so a measurement of a star can be thought of

\footnotetext{
${ }^{5}$ Some early photographic observations were made over several nights by re-exposing the same film with the same star field each night (Dodd 1994).
} 


\section{Spectra}

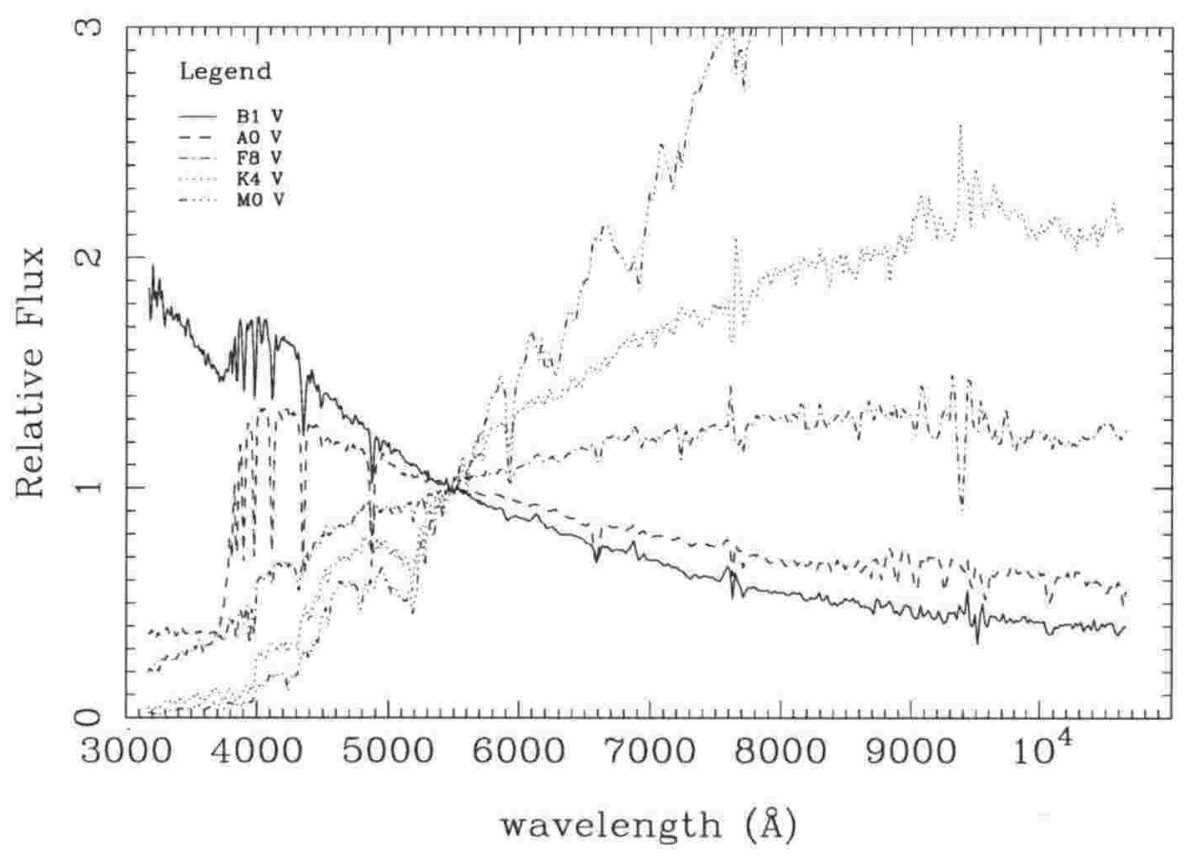

Figure 1.3: Spectra of various Main Sequence stars, taken from the Gunn \& Stryker (1983) catalog, de-reddened and normalised to the effective wavelength of the Standard Johnson V filter.

defining a point in an $n$-dimensional space. The measuring errors means a precise point is not defined, but rather a 'fuzzy' region known as a photometric box (Golay 1974, Jaschek \& Jaschek 1987). ${ }^{6}$ Thus all stars falling in the same box are identical according to that photometric system even though they may in fact have different properties. The purity of each box is defined as the number of stars in the box having the desired property divided by the total number of stars in the box (Jaschek \& Frankel 1985). The total number of stars in a box is usually increased by using dereddened colours (as a colour index cancels the inverse square law reduction of the star's intensity with distance), giving an $n-1$ dimensional space. ${ }^{7}$

A necessary preliminary to the investigation is to pre-select stars of the appropriate type, so increasing the purity of the photometric system. This is often done as a survey with a form of spectroscopy using coarser resolution but covering a wider wavelength range than discussed previously, for example the $100 \AA / \mathrm{mm}$ dispersion of the MK classification system. The survey system is not usually suitable for the investigation itself as it gives qualitative information (i.e. by grouping similar stars together), while photometric systems give quantitative information. However some photometric systems can be used for both the survey and measurement

\footnotetext{
${ }^{6}$ These are also known as stellar or Golay boxes.

${ }^{7}$ An $n$ passband system has $n ! /(n-2)$ ! possible colours but only $n-1$ independent colours.
} 
Spectra smoothed by Standard Johnson V filter

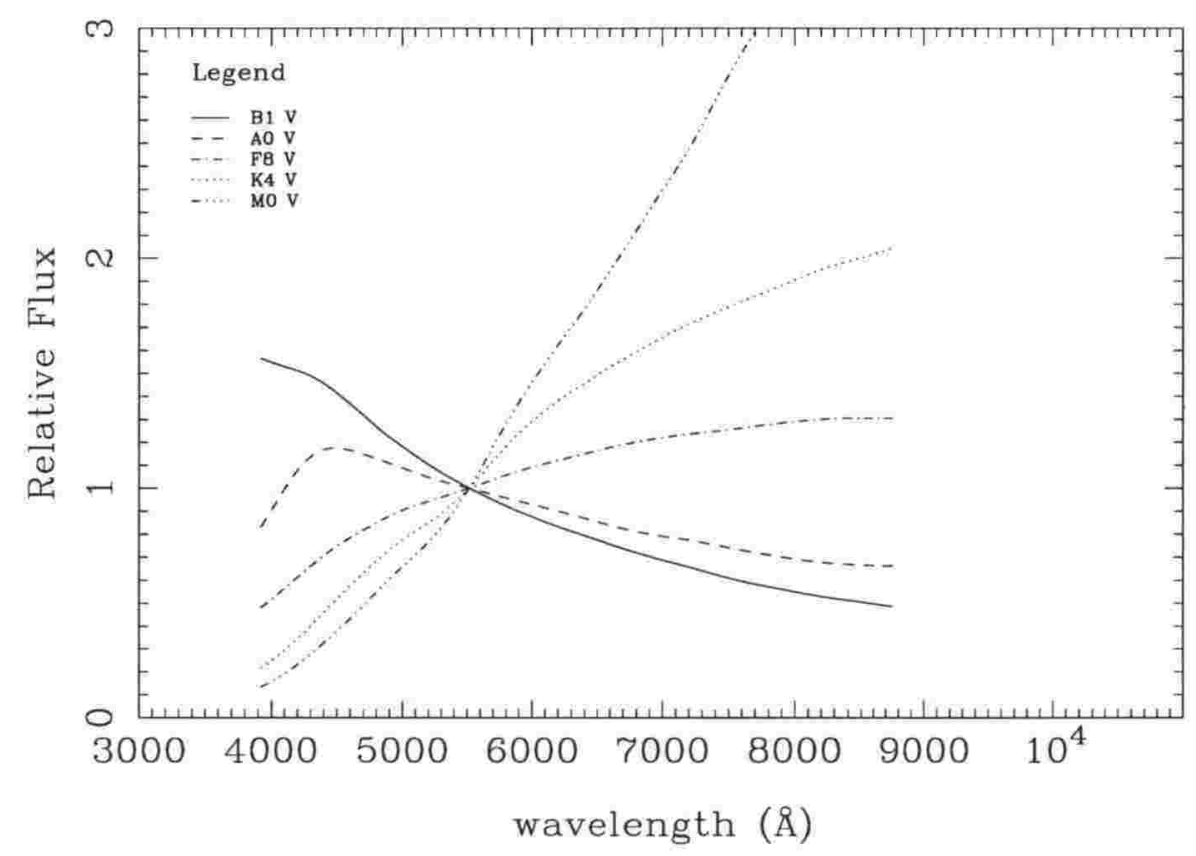

Figure 1.4: Convolution of various Main Sequence spectra with Johnson V filter. Spectra taken from the Gunn \& Stryker (1983) catalog and V filter response from Straižys (1992).

Spectra smoothed by Standard Vilnius V filter

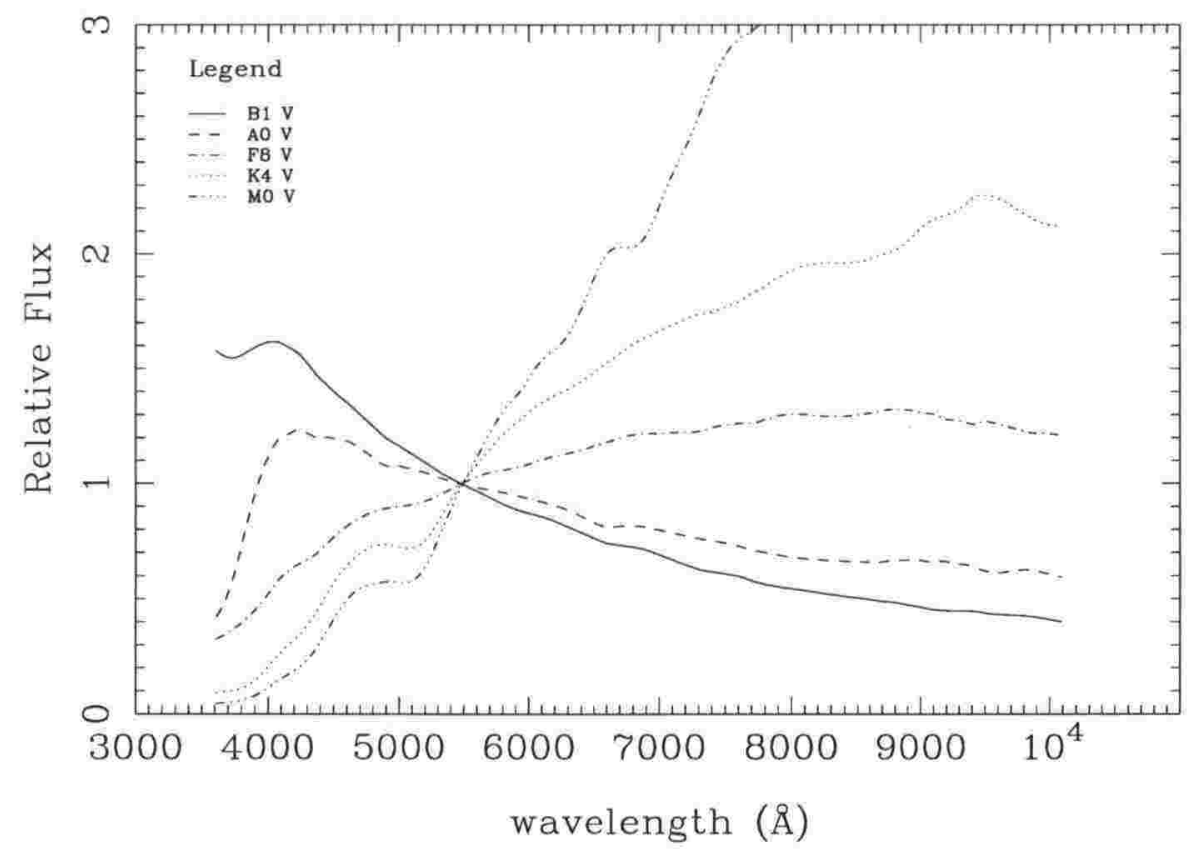

Figure 1.5: Convolution of various Main Sequence spectra with Vilnius V filter. Spectra taken from the Gunn \& Stryker (1983) catalog and V filter response from Straižys (1992). 
phases, for instance the Vilnius system can correctly classify $95 \%$ of all stars in the MK scheme (Straižys \& Philip 1994) and measure effective temperatures etc. (see section 1.7).

\subsection{Designing a Photometric system.}

A brief outline of the design of a photometric system is given next. As an example, consider a hypothetical system designed to measure the effective (surface) temperature of stars $\left(T_{e}\right)$. It would be advantageous to situate the filters in the wavelength region of the star's maximum intensity, which can be estimated from Wien's law $\left(\lambda_{\max } T_{e}=\right.$ constant $)$ or Table 4.3 of Jaschek \& Jaschek (1987). However for ground based observatories, the filters must placed in an atmospheric 'window' of low extinction and avoid regions with highly variable extinction (for example water absorption bands - see Figure 2.12).

The bandwidth of the filters (i.e. narrow, intermediate or broadband) is set as a compromise between the need to reduce bandwidth effects (for example on heterochromatic extinction - see section 2.7) and increase the limiting magnitude of the system. Note also that the placement and shape of the filter responses becomes more crucial as the bandwidth decreases - for instance the effect of excluding a spectral feature by a filter which is slightly narrower or shifted in wavelength from what was intended (i.e. the standard filter) becomes more important as the feature occupies a larger fraction of the filter's bandwidth.

The number of filters in the system is similarly a compromise between the need to maximize information about the star's spectra $(E(\lambda))$ and minimize the time required to observe a star. For instance, a minimum of two filters (placed in continuum regions well approximated by a black body function) are required to measure the effective temperature - the ratio of the intensities cancels the inverse square law reduction of the star's intensity with distance to the observer. However if the stars are not nearby, they are likely to be reddened by interstellar extinction. Therefore more filters would be required to measure this effect, perhaps with one in the red or near-infrared where the reddening is weaker.

Another consideration is the wavelength spacing between the filters relative to their bandwidth, i.e. their overlap. Young $(1988,1992 a, 1994)$ has found that it is vital the filters are spaced no further than their Full Width at Half Maximum (FWHM) apart so transformations (such as the heterochromatic extinction or from the local system to the standard system) for any star is free of systematic errors. This spacing is equivalent to satisfying the critical sampling theorem, i.e. to adequately sampling the spectrum so it can be fully described at the resolution level set by the bandwidth of the filters. As most photometric systems are designed for restricted sets of stars (for example early stars in the uvby system) which all give approximately the same systematic errors, this requirement has usually been ignored. Unfortunately most 
photometric system have been extended to sets of stars for which they were never intended (Jaschek \& Jaschek 1987), with the ensuing systematic transformation errors being different for each local system.

The reduction procedure used to convert the raw observations into measurements in the standard system (for example, see Chapter 2) must also be given. This ensures any systematic errors in the reduction procedures are the same for all observers, hopefully cancelling out when comparing results from local copies of the standard system.

In addition to the astrophysical factors discussed above, there are several practical considerations. Clearly the technology (i.e. filters, detectors) must be available and affordable. The filters need to be chosen to provide the highest transmittance possible consistent with the required effective wavelength and bandwidth.

The stability of the filters and detector is another important consideration; for instance interference filters cost more, have a smaller temperature sensitivity but are less stable (due to water vapour entering the layers), hence having a shorter lifetime, and produce narrower passbands (with high transmission) than glass filters (Young 1974). Each filter must be measured over a wide wavelength range to check for 'leaks' outside the intended passband - often these leaks can be removed by choosing a detector which is only sensitive over the intended wavelength range of the system, otherwise a blocking filter must be added. If observing near a city, or other light source, the system will need to avoid the wavelength(s) of the light and/or make frequent measurements of the sky background.

The system's properties, such as its purity, may be explored during the design stage using magnitudes and colours synthesized by convolving the passband response curves with stellar spectra (from either spectroscopic measurements or stellar atmosphere models). Further, reduction and dereddening procedures may be investigated. Synthesized magnitudes are also useful after the system has been implemented, reducing the number and variety of stars that need to be measured to calibrate the system and its reduction procedures (for example, see section 1.7 describing the Vilnius system).

A full description of the original (standard) system must be given, including the instrument (for example a $61 \mathrm{~cm}$ Aluminum coated mirror in a $\mathrm{f} 13$ Cassegrain telescope), the receiver (for example a photon counting 9558B PhotoMultiplier Tube (PMT) at $-1500 \mathrm{~V}$ and cooled to $-10^{\circ} \mathrm{C}$, with an amplifier/discriminator) and filters (for example the $\mathrm{U}$ filter consists of $1 \mathrm{~mm}$ thick Schott WG355 glass glued to $7 \mathrm{~mm}$ thick Schott UG11 glass with Canadian Balsam). This will give other observers a starting point for their own local implementations of the system; however the definitive description is given by the transmittance curves of the entire system for each filter. These are usually calculated by convolving the measured transmittance curves of each filter with the receiver's response (often taken as the typical response from the 
manufacturer's catalog) and the reflectance/transmittance curves of any optical elements in the telescope and photometer. An alternative is to measure some stars whose spectra are known (either by spectroscopic measurements or from a stellar atmosphere model). The response curve for each passband is convolved with the spectra and least squares fitted to match the measured magnitudes of the stars. To reduce the number of data points used in the fit, each response curve can be approximated by a cubic spline fitted to $4-5$ points or Fourier series of 4-5 sine functions (Jaschek \& Jaschek 1987), using the calculated passbands as a starting point. This works best if there are no strong spectral features within the passband of each filter, i.e. use O-B stars with various amounts of reddening if the filter is not situated on the continuum.

As standard light sources for calibration of photometric systems are very difficult to produce in an observatory situation (Young 1974, Straižys 1992, Bessell 1993), a set of (non-variable) 'standard' stars are normally used for this purpose. These should be well distributed across the sky, cover a large magnitude range and the spectral and luminosity classes for which the system was designed (and preferably the complete range of spectral/luminosity classes and peculiar stars). In general both reddened and unreddened stars should be included. If the receiver is subject to fatigue effects (for example dead-time in PMT's), very bright stars should be excluded. When establishing a new photometric system it is helpful to include a set of circumpolar standards for internal calibration using the same stars through-out the year (as the original filters will change with age, and PMT characteristics drift on all time scales).

Finally, the photometric system must be calibrated against stellar parameters such as the effective temperature, surface gravity, chemical composition etc. As there are few large, homogeneous samples of such calibrated stars (Jaschek \& Jaschek 1987), it is more common to calibrate using approximate quantities such as spectral type, luminosity class, $[\mathrm{Fe} / \mathrm{H}]$ ratio etc. These also have the advantage of being model-independent, so will not have to be revised every time stellar models are improved. The calibrations may be tested by deriving the distance modulus of the individual stars in a binary or cluster - these should all be the same if the calibrations are correct (assuming the stars are all at the same distance, have the same $[\mathrm{Fe} / \mathrm{H}]$ ratio, interstellar reddening and the cluster membership is known).

\subsection{Characteristics of photometric system.}

The intensity measured through a filter in a photometric system is

$$
E_{m}=\int_{\lambda_{1}}^{\lambda_{2}} S(\lambda) R(\lambda) d \lambda
$$

where $S(\lambda)$ is the spectral function of the light incident on the receiver system $R(\lambda)$, over the wavelength interval $\left[\lambda_{1}, \lambda_{2}\right]$ where the receiver's response is non-zero. The incident light 
$S(\lambda)$ includes the star's intrinsic intensity $E(\lambda)$, the inverse square law reduction in intensity due to the distance ' $r$ ' between the star and the receiver, the transmission function $I(\lambda)$ of interstellar matter along the light of sight and the transmission function $A(\lambda)$ of the Earth's atmosphere. These functions are usually grouped together (as $S(\lambda))$ because they vary from one measurement to another, while the receiver system is assumed to be stable.

If the star's spectrum $(S(\lambda))$ is continuous and the receiver's response function does not change sign in the interval $\left[\lambda_{1}, \lambda_{2}\right]$, then the mean value theorem says the measured (heterochromatic) intensity $E_{m}$ corresponds to the monochromatic intensity at one, or more, wavelengths in the interval, i.e.

$$
E_{m}=\int_{\lambda_{1}}^{\lambda_{2}} S(\lambda) R(\lambda) d \lambda=S\left(\lambda_{i}\right) \int_{\lambda_{1}}^{\lambda_{2}} R(\lambda) d \lambda,
$$

apart from a constant factor $\left(\int R(\lambda) d \lambda\right)$. This is known as the isophotal wavelength(s)(Golay 1974), and is different for each star, and even different measurements of the same star due to the atmospheric function $A(\lambda)$ changing. Unfortunately the isophotal wavelength requires knowledge of the star's spectrum (which is what we wish to measure) before it can be calculated.

If $S(\lambda)$ is continuous and it's derivatives are continuous in the interval $\left[\lambda_{1}, \lambda_{2}\right]$, then it may be expanded in a Taylor series about $\lambda_{0} \in\left[\lambda_{1}, \lambda_{2}\right]$

$$
S(\lambda)=S\left(\lambda_{0}\right)+\frac{\left(\lambda-\lambda_{0}\right)}{1 !} S^{\prime}\left(\lambda_{0}\right)+\frac{\left(\lambda-\lambda_{0}\right)^{2}}{2 !} S^{\prime \prime}\left(\lambda_{0}\right)+\ldots
$$

where the primes are differentiation with respect to wavelength. This expands Equation 1.18 to

$$
E_{m}=S\left(\lambda_{0}\right) \int_{\lambda_{1}}^{\lambda_{2}} R(\lambda) d \lambda+S^{\prime}\left(\lambda_{0}\right) \int_{\lambda_{1}}^{\lambda_{2}}\left(\lambda-\lambda_{0}\right) R(\lambda) d \lambda+\frac{1}{2 !} S^{\prime \prime}\left(\lambda_{0}\right) \int_{\lambda_{1}}^{\lambda_{2}}\left(\lambda-\lambda_{0}\right)^{2} R(\lambda) d \lambda+\ldots
$$

Rewriting the equation for brevity by dropping the integral limits, the explicit wavelength dependence and denoting the evaluation of a function at $\lambda_{0}$ by a subscript zero gives

$$
E_{m}=S_{0} \int R d \lambda+S_{0}^{\prime} \int\left(\lambda-\lambda_{0}\right) R d \lambda+\frac{1}{2 !} S_{0}^{\prime \prime} \int\left(\lambda-\lambda_{0}\right)^{2} R d \lambda+\ldots
$$

By rearranging slightly, the equation can be further abbreviated using the normalised $n^{\text {th }}$ moments

$$
\mu_{n}^{n}=\frac{\int\left(\lambda-\lambda_{0}\right)^{n} R d \lambda}{\int R d \lambda}
$$

producing

$$
E_{m}=S_{0} \int R d \lambda\left\{1+\frac{S_{0}^{\prime}}{S_{0}} \mu_{1}^{1}+\frac{1}{2 !} \frac{S_{0}^{\prime \prime}}{S_{0}} \mu_{2}^{2}+\ldots\right\}
$$


The wavelength $\lambda_{0}$ may be chosen so the first moment vanishes, i.e. $\mu_{1}^{1}=0$, which requires

$$
\lambda_{0}=\frac{\int \lambda R d \lambda}{\int R d \lambda}
$$

This is known as the effective wavelength (King 1952) (or the equivalent wavelength (Strömgren 1937), or mean wavelength (Golay 1974)). This is significant because if the second order and higher derivative terms are negligible, then $E_{m}=S_{0} \int R d \lambda$, i.e. the effective wavelength equals the isophotal wavelength. In practise, the higher order terms only converge slowly (Young $1992 b)$ and the effective wavelength accurately estimates the isophotal wavelength if the star's spectrum has a point of inflexion in the interval $\left[\lambda_{1}, \lambda_{2}\right]$ (Golay 1974).

Consider the case where the spectrum is free of strong features, so that only the second and/or third derivative terms are important say, and can be well approximated by an assumed function such as the Black Body equation. Then the following

$$
\lambda_{e}=\frac{\int \lambda S R d \lambda}{\int S R d \lambda}
$$

is considered to give a better estimate of the isophotal wavelength than the effective wavelength. ${ }^{8}$ The only parameter required for the black body equation is the temperature, being here the effective surface temperature of the star. An iterative process of fitting a black body equation to the star's intensity through two different filters to derive the temperature, ${ }^{9}$ which then gives an improved estimated of the filters isophotal wavelengths for that star using equation 1.26 can be done.

In general, a single absorption line will not have a detectable effect on a photometric measurement (consider a $1000 \AA$ passband filter giving measurements to a precision of $\pm 1 \%$ and an absorption line with an equivalent width less than $10 \AA$ ). However the cumulative effect of many absorption lines can be significant. Thus, to ensure the effective wavelength accurately gives the isophotal wavelength restricts filters to being placed on the continuum. This is difficult to satisfy for all stars, especially late-type stars which have so many absorption bands that the regions with only weak features are considered a pseudo-continuum. In these cases the spectrum is split into a smooth continuum $S^{*}(\lambda)$ and the energy removed by absorption features $s(\lambda)$. The fraction of energy removed from the continuum is

$$
\eta(\lambda)=\frac{\int_{\lambda-\varepsilon}^{\lambda+\varepsilon}\left[S^{*}-s\right] d \lambda}{\int_{\lambda-\varepsilon}^{\lambda+\varepsilon} S^{*} d \lambda}
$$

\footnotetext{
${ }^{8}$ This is also called the effective wavelength by some authors, for example King (1952). Here the term 'effective wavelength' will refer to equation 1.25 unless explicitly stated otherwise.

${ }^{9}$ An effective temperature derived by fitting a black body to a colour index is appropriately known as a 'colour' temperature.
} 
with the wavelength range of the integrals usually being $\varepsilon=5-50 \AA$ (Jaschek \& Jaschek 1987). This gives the 'blocking coefficient' as

$$
\gamma(\lambda)=1-\eta(\lambda)=\frac{\int s d \lambda}{\int S^{*} d \lambda}
$$

which increases rapidly with spectral type, luminosity but decreases with increasing wavelength (Ardeberg \& Virdefors 1975). Some photometric systems have filters designed to measure this blocking coefficient and thus the star's metal abundance. ${ }^{10}$

However, if the star is in energy equilibrium this blocked energy must be re-emitted but not necessarily at the wavelength which it was originally absorbed. This effect is known as 'backwarming' as most of the energy is re-emitted in the continuum, which increases the effective temperature of the continuum as shown next. The integrated blocking coefficient

$$
\gamma_{\text {int }}=\frac{\int_{0}^{\infty} \gamma S^{*} d \lambda}{\int_{0}^{\infty} S^{*} d \lambda}
$$

is the total energy left after blocking, hence the original effective temperature $\left(T_{e_{1}}\right)$ is related to the back-warmed effective temperature $\left(T_{e_{2}}\right)$ by $T_{e_{1}}^{4}=\gamma_{\text {int }} T_{e_{2}}^{4}$, i.e.

$$
T_{e_{2}}^{4}=\frac{1}{\gamma_{i n t}} T_{e_{1}}^{4}>T_{e_{1}}^{4}
$$

as $0<\gamma_{\text {int }}<1$. Both effects (blocking and back-warming) are together referred to by the term 'blanketing'.

A special case of blocking is when a filter is situated on a single, isolated strong line, such as $\mathrm{H}_{\beta}$. The removed energy $(s)$ is now the line profile and $\eta$ measures the equivalent width $(W)$ of the line. An additional wider passband filter centred on the line, or filters on each side of the line, is necessary to measure the continuum $S^{*}$. The first case, where the two filters have (nearly) the same isophotal wavelength has the additional advantages of having the same interstellar and atmospheric extinction, which can then be cancelled out (Jaschek \& Jaschek 1987).

In photometry it is conventional to use a magnitude scale rather than intensity. The original magnitude scale was based on the visibility of stars during evening twilight (Sterken \& Manfroid 1992a). The time between sunset and full darkness was divided into six equal intervals, with stars of the first magnitude being visible in the first interval and stars of the sixth magnitude becoming visible in the last interval. Around 1850 it was found that this approximately corresponded to a logarithmic scale with one magnitude step being a factor of 2.5 in intensity. Pogson

\footnotetext{
${ }^{10}$ Photometrists use the term 'metal abundance' to refer to all elements/molecules causing absorption features, while spectroscopists use the term 'metallicity' to refer to actual metals and stellar interior modelers use that term to refer to all elements other than hydrogen and helium (Jaschek \& Jaschek 1987).
} 
(1856) choose the value 2.512 , as this is the fifth root of 100 and so the base 10 logarithmic scale could be used.

Therefore the modern magnitude scale is defined by

$$
\begin{aligned}
\frac{E_{1}}{E_{2}} & =2.512^{m_{1}-m_{2}} \\
\Longleftrightarrow m_{1}-m_{2} & =-2.5 \log \left(\frac{E_{1}}{E_{2}}\right)
\end{aligned}
$$

where $E_{1}, E_{2}$ are the measured intensities (of either two stars through the same passband or one star through two passbands, denoted by the numeric subscripts) and $m_{1}, m_{2}$ are the corresponding magnitudes. By normalizing to some intensity (equivalently, setting some magnitude to zero), the magnitude scale of a star becomes

$$
m=-2 \cdot 5 \log (E)+C
$$

where $C$ is a constant chosen for the normalization, known as the 'zero-point'. Similarly, a 'colour' (or 'colour index') was defined as the difference in magnitudes (measured at different wavelengths) for the same star, giving

$$
c_{12}=m_{1}-m_{2}=-2 \cdot 5 \log \left(\frac{E_{1}}{E_{2}}\right) .
$$

The 'apparent' magnitude measured by the observer is fainter than the star's actual magnitude due to the inverse square law reduction of intensity with distance between the star and observer. To correct for this effect, the 'absolute' magnitude (denoted by $M$, or $m_{0}$ ) is defined as the star's magnitude measured at a distance $(\rho)$ of 10 parsecs from the star, which, assuming no other causes of light reduction, gives

$$
m-M=-2 \cdot 5 \log \left(\frac{E / \rho^{2}}{E / 10^{2}}\right)=5 \log (\rho)-5
$$

where $m-M$ is called the 'distance modulus'. Note that a colour is independent of the star's distance, i.e. $c=c_{0}$ as $c_{12}=m_{1}-m_{2}=\left(M_{1}+5 \log (\rho)-5\right)-\left(M_{2}+5 \log (\rho)-5\right)=M_{1}-M_{2}=c_{0_{12}}$.

The presence of interstellar material will also reduce the star's apparent magnitude, producing

$$
m-M=5 \log (\rho)-5+A
$$

with $A$ being the interstellar extinction as will be shown next. If $x$ is the optical thickness of the interstellar material and $k(\lambda)$ is it's absorption coefficient, then the measured intensity 
(equation 1.18) becomes

$$
E_{m}=\int E(\lambda) e^{-k(\lambda) x} R(\lambda) d \lambda
$$

where $x=0$ corresponds to no extinction and $x=1$ corresponds to complete extinction.

In the case of a narrow passband filter such that $k(\lambda) \approx$ constant, $k$ say, then

$$
E_{m}=e^{-k x} E_{m_{0}}
$$

where $E_{m_{0}}$ is the star's intrinsic intensity $\left(\int E(\lambda) R(\lambda) d \lambda\right)$. Converting to magnitudes gives

$$
\begin{aligned}
m & =-2 \cdot 5 \log \left(E_{m}\right) \\
& =m_{0}+a x, \quad \text { where } a=\frac{2 \cdot 5}{2 \cdot 3026} k \\
\text { or } & =m_{0}+A, \quad \text { where } A=a x
\end{aligned}
$$

with $m_{0}$ being the star's intrinsic magnitude and $A$ is the interstellar extinction. In terms of colours, this gives

$$
\begin{aligned}
c_{12} & =m_{1}-m_{2} \\
& =\left(m_{0_{1}}+a_{1} x\right)-\left(m_{0_{2}}+a_{2} x\right) \\
& =c_{0_{12}}+E_{12}, \quad \text { where } E_{12}=\left(a_{1}-a_{2}\right) x=A_{1}-A_{2},
\end{aligned}
$$

with $c_{0_{12}}$ being the star's intrinsic colour and $E_{12}$ is the colour excess. In general the extinction decreases as the wavelength increases, so a star's observed colour is larger (i.e. redder) than it's intrinsic colour, hence the term 'reddening'.

The extinction at one wavelength can be written in terms of that at another wavelength, i.e. $A_{1}=f A_{2}$, so

$$
\begin{aligned}
E_{12} & =A_{1}-A_{2}=f A_{2}-A_{2} \\
\Longleftrightarrow A_{2} & =\frac{1}{f-1} E_{12} \\
& =R E_{12} \text { and } A_{1}=(R+1) E_{12}
\end{aligned}
$$

where $R$ is the 'ratio of extinction to colour excess' and is only dependent on the form of the interstellar extinction law, i.e. independent of the amount of interstellar material (as $R=$ $\left.1 /(f-1)=A_{2} / E_{12}=a_{2} /\left(a_{1}-a_{2}\right)\right)$. This ratio can be used to calculate intrinsic magnitudes, for example from the UBV system $V_{0}=V-R E_{B-V}$, or reddening free magnitude parameters, for example $P=V-R(\mathrm{~B}-\mathrm{V})$ also from the UBV system. ${ }^{11}$

\footnotetext{
${ }^{11}$ It can be easily shown that $P$ is equivalent to $P=V_{0}-R(\mathrm{~B}-\mathrm{V})_{0}$, which is free of reddening as it can be
} 
These ideas can be extended to colours for systems with two or more colours, via the 'colour excess ratios'

$$
\chi_{123}=\frac{E_{12}}{E_{23}} \quad \text { or } \quad \chi_{1234}=\frac{E_{12}}{E_{34}}
$$

which are also dependent only of the form of the interstellar extinction law (as $\chi_{123}=\left(a_{1}-\right.$ $\left.\left.a_{2}\right) /\left(a_{2}-a_{3}\right)\right)$. It follows from the above definition that the intrinsic colour can be calculated by

$$
c_{0_{12}}=c_{12}-\chi_{123} E_{23}
$$

and reddening free colour parameters from

$$
Q_{123}=c_{12}-\chi_{123} c_{23} \quad \text { or } \quad Q_{1234}=c_{12}-\chi_{1234} c_{34}
$$

as the ' $Q$ parameters' (also known as ' $Q$ factors') are easily shown to be equivalent to $Q_{123}=$ $c_{0_{12}}-\chi_{123} c_{0_{23}}$ etc.

All of the above colour excesses and excess ratios assumed the monochromatic case (i.e. narrow passbands). In the general case, the colour excesses depend on the star's spectrum (i.e. spectral and luminosity class), the optical thickness of the interstellar material $(x)$, and the particular form of the extinction law followed by the interstellar material along the line of sight to the star, with the dependence increasing as the filter bandwidths increase. The colour excess ratios also depends on the star's spectrum and the form of the extinction law. Finally, these all depend on the effective wavelength of the filter so correct transformation of measurements to the wavelengths of the standard system is crucial (Manfroid 1992).

\subsection{Development of the UBV Photometric System.}

In the late 1940's, Johnson and his co-workers measured photoelectrically (using wide-band blue and green filters) stars in the North Polar Sequence (NPS) and three Kapteyn regions which were standard stars in the International System of photographic $\left(\mathrm{M}_{\mathrm{pg}}\right)$ and photovisual $\left(\mathrm{M}_{\mathrm{pv}}\right)$ magnitudes (Stebbins et al. 1950). Their intention was to investigate how to duplicate, and improve on, the International System using a photoelectric system, and develop a system closely tied to the MK spectral classification scheme. It was found that the two filters gave non-linear, multi-valued transformations to the International System. This was due to the inclusion of different amounts of the Balmer jump between the blue filter and $\mathbf{M}_{\mathrm{pg}}$, as shown by further observations with several blue filters with different UV cut-offs in their response functions. Hence, a third filter (to measure the UV) is required for linear transformations.

expressed in terms of intrinsic magnitudes and colours. 
Based on the above results, Johnson proposed the UBV photoelectric system consisting of an ultra-violet U filter (Corning 9863 glass), a blue B filter (Schott GG13 and Corning 5030 glass) and a green V filter (Corning 3384 glass) with an uncooled Antimony-Caesium RCA 1P21 PMT. The UV cut-off of the U filter is determined by the response of the PMT's glass envelope and the (steeply increasing) atmospheric extinction. The red cut-off of the $V$ filter is set by the long wavelength response of the PMT. Observations in this system (to establish standard stars etc.) were started in the winter of 1950/51 using $32 \mathrm{~cm}$ and $2 \mathrm{~m}$ Cassegrain telescopes with Aluminized mirrors and balsam cemented quartz-fluorite Fabry lens. The response functions of the system were calculated from room temperature double-beam monochromator measurements of the filters transmissions and the PMT's sensitivity - the effects of the mirrors, Fabry lens and change from room temperature to the Observatory's ambient temperature were ignored (Johnson \& Morgan 1951). Further, the response functions were never given in numerical form but only shown in graphical form.

In the summer of 1951, the Fabry lens was replaced with a crystalline quartz lens and the PMT cooled with dry ice (Johnson 1962). The resulting 'summer' system was then transformed back to the 'winter' system, which was considered the standard system (even though it no longer existed). The U and B filters were found to have red 'leaks' at $\lambda>6800 \AA$ and $\lambda>7200 \AA$ respectively, with the $B$ filter's red leak being an order of magnitude less than the $U$ filter. Johnson \& Morgan (1953) corrected the observations for the red leak of the U filter only.

The zero-point of the $\mathrm{V}$ magnitude was adjusted to correspond to the photovisual system via measurements of nine NPS stars (which became primary standards of the UBV system). The earlier observations by Stebbins et al. (1950) had already established that there was no colour equation between $\mathrm{V}$ and $\mathrm{M}_{\mathrm{pv}}$. The remaining zero-points were set by taking the average colours (U-B,B-V) of un-reddened A0 V stars (based on six stars) as zero. Further standard stars were given by Johnson \& Harris (1954), and later by Johnson (1955).

Six stars were also used to investigate the atmospheric extinction corrections (see section 2.7 ), taken as

$$
\begin{aligned}
\mathrm{V}_{0} & =\mathrm{V}-\left(k_{\mathrm{V}}^{\prime}+k_{\mathrm{V}}^{\prime \prime}(\mathrm{B}-\mathrm{V})\right) X \\
(\mathrm{U}-\mathrm{B})_{0} & =\mathrm{U}-\mathrm{B}-k_{\mathrm{U}-\mathrm{B}}^{\prime} X \\
(\mathrm{~B}-\mathrm{V})_{0} & =\mathrm{B}-\mathrm{V}-\left(k_{\mathrm{B}-\mathrm{V}}^{\prime}+k_{\mathrm{B}-\mathrm{V}}^{\prime \prime}(\mathrm{B}-\mathrm{V})\right) X
\end{aligned}
$$

where $k_{\mathrm{U}-\mathrm{B}}^{\prime \prime}$ is defined as zero. This has caused many problems as the extinction in wide-band systems (especially in the UV) strongly depends on the star's temperature and luminosity, plus the amount of interstellar and atmospheric extinction - this is a result of the higher order terms of the Taylor expansion of the star's spectrum (equation 1.22) being significant. Thus 
Table 1.1: Characteristics of the Standard UBV System Passbands.

\begin{tabular}{|ccccccc|}
\hline filter & $\begin{array}{c}\lambda_{R_{\max }} \\
(\AA)\end{array}$ & $\begin{array}{c}\text { FWHM } \\
(\AA)\end{array}$ & $\begin{array}{c}\lambda_{0} \\
(\AA)\end{array}$ & $\begin{array}{c}\mu_{2}^{2} \\
\left(\mathrm{~m}^{2}\right)\end{array}$ & $\begin{array}{c}\mu_{3}^{3} \\
\left(\mathrm{~m}^{3}\right)\end{array}$ & $\begin{array}{c}\mu_{4}^{4} \\
\left(\mathrm{~m}^{4}\right)\end{array}$ \\
\hline $\mathrm{U}$ & 3730 & 440 & 3760 & $4 \cdot 29 \times 10^{-15}$ & $1 \cdot 24 \times 10^{-21}$ & $4 \cdot 11 \times 10^{-28}$ \\
$\mathrm{~B}$ & 4280 & 1010 & 4450 & $1.32 \times 10^{-15}$ & $1.32 \times 10^{-23}$ & $4 \cdot 41 \times 10^{-30}$ \\
V & 5280 & 820 & 5500 & $1.35 \times 10^{-15}$ & $3.95 \times 10^{-23}$ & $6.86 \times 10^{-30}$ \\
\hline
\end{tabular}

setting $k_{\mathrm{U}-\mathrm{B}}^{\prime \prime}=0$ gives errors dependent on the airmass, which were seen by Johnson (1963) as declination effects (as the southern stars were always seen at large airmasses).

Therefore each standard star has it's own colour equation (or equivalently, it's own response function) dependent on the average airmass through which it was observed. This problem is made worse as part of the response functions of the $\mathrm{U}$ and $\mathrm{V}$ are determined by the PMT, which is considerably less stable than a glass filter. Further, the PMT was un-cooled, i.e. left to drift with the observatory's ambient temperature, giving seasonal variations which would probably be seen as Right Ascension (RA) effects. All of this leads to systematic errors in transforming a local system (typically with an internal precision of $\pm 0^{m} \cdot 01$ ) to the standard system of up to $\pm 0^{m} \cdot 05$ in $\mathrm{U}-\mathrm{B}$, and makes it difficult to do synthetic magnitude studies.

Several investigations have been made of the UBV response functions, for example Melbourne (1960), Arp (1961), Sharov (1962), Matthews \& Sandage (1963), Johnson (1965a), but have still ignored the effects of the mirrors, lens, temperature or red leaks. Later investigations based on matching synthesized colours to observed colours, for example Ažusienis \& Straižys (1969), Bessell (1990), should give more accurate results, but are still only correct for the average airmass through which the system was observed - see Figure 1.6. Although the filters overlap in wavelength, they do not meet the critical sampling theorem which will lead to further systematic errors in extinction and standardisation transformations. Some of the system's characteristics are given in Table $1.1 ; \lambda_{R_{\max }}$ is the wavelength giving the maximum transmittance for each filter, the FWHM was measured directly from Figure 1.6, $\lambda_{0}$ is the effective wavelength and $\mu_{2}^{2}$ etc. are the moments of the filter passbands.

The B-V colour serves as a temperature (spectral type) indicator while the U-B colour measures the size of the Balmer jump, which depends on both the temperature and surface gravity (luminosity class). Unfortunately the long wavelength response of the $\mathrm{U}$ filter extends into the Balmer jump itself so does not give an accurate measure of the intensity on the short wavelength side of the jump. The spectral sequences for different luminosity classes is shown in Figure 1.7. Notice the bends in the sequences (around A0-G0, and after M0) and the shallow angle between the reddening line and the sequences - this means heavily reddened early type stars can fall in the same position in the diagram as un-reddened later type stars (or white dwarves or sub-dwarves). Further, the sequences intersect at several points on the diagram, 


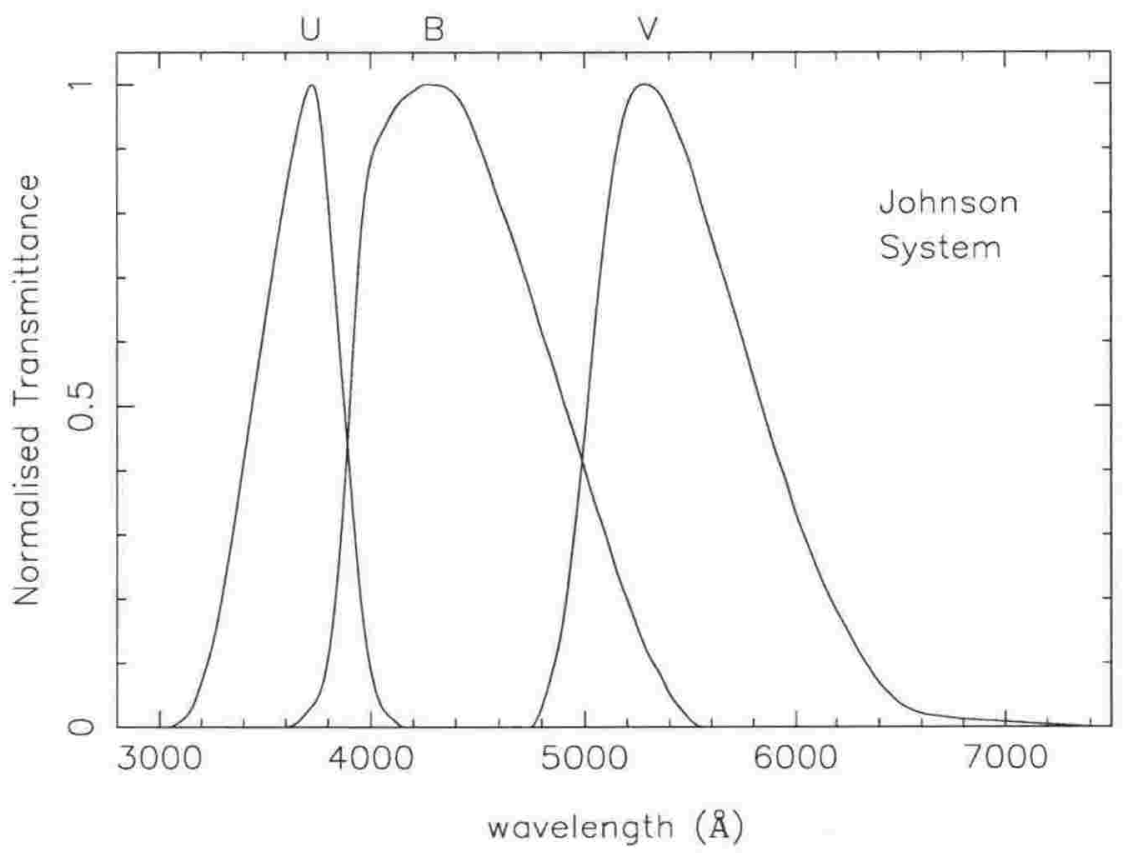

Figure 1.6: Transmission Response curves of the UBV Photometric System, normalised and spline fitted. Taken from Straižys (1992).

for example G2 I, G5 III, and K2 V stars are all in the same position, giving ambiguous results when classifying stars in the diagram.

These factors give rise to three cases when trying to classify stars in the UBV system; Firstly, if the reddening is known, or is small enough to be neglected, then the star's (de-reddened) observed colours equal it's intrinsic colours and the star may be classified using the diagram (subject to the ambiguities due to different luminosity classes). Secondly, if the luminosity class is known, then the star may be de-reddened by extrapolating it's observed colours back to it's intrinsic colours using a colour excess ratio (or equivalently, it's Q factor may be calculated). However ambiguous results are still possible, for example an apparently un-reddened G9 V star may in fact be a reddened F0 V star or a heavily reddened B7 V star. Only in the third case, when both the reddening and luminosity class of the star is known, can it's spectral class be uniquely determined. This also allows another factor, such as the star's metallicity, to be found (although this may still give ambiguous results due to other factors such as rotation or binarity).

This is further complicated by the bandwidth effect on the reddening which means the slope of the reddening line in the $\mathrm{U}-\mathrm{B}, \mathrm{B}-\mathrm{V}$ diagram (i.e. the colour excess ratio) depends on both the star's spectrum and the amount of interstellar material. According to Straižys (1992), $A_{V}$ and $A_{B}$ are approximately linear with $(\mathrm{B}-\mathrm{V})_{0}$ for $0<(\mathrm{B}-\mathrm{V})_{0}<1$ and is approximately 


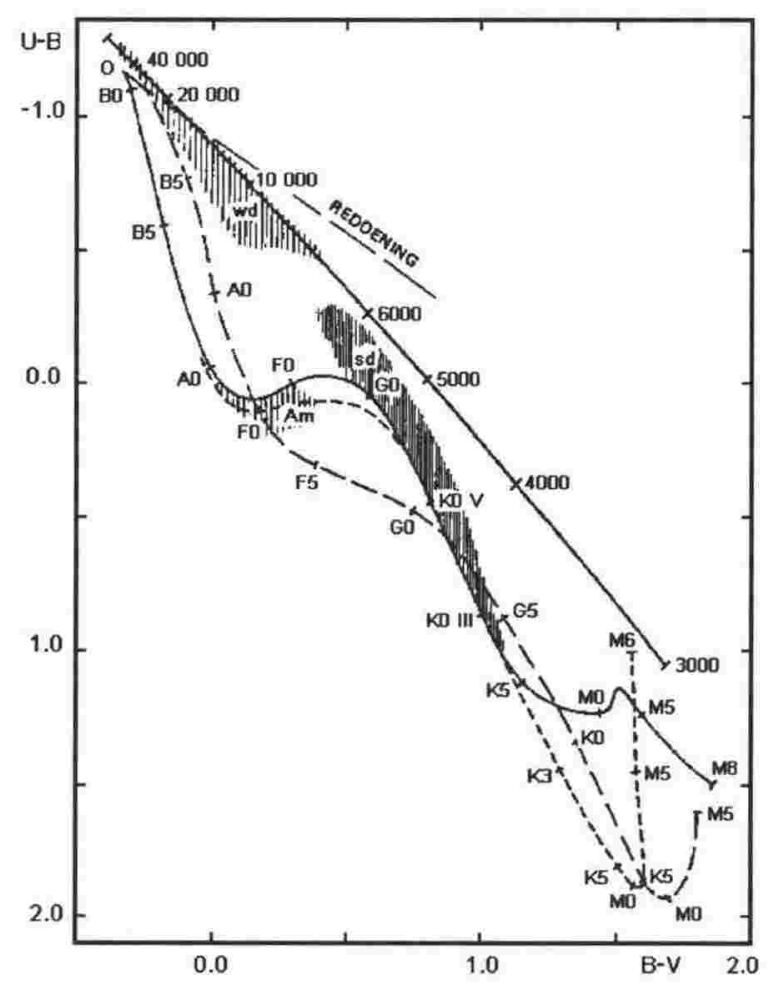

Figure 1.7: The Johnson System's intrinsic U-B, B-V sequences of luminosity V (solid line), III (short-dashed line), and I (long-dashed line) stars, the black body line, and the positions of white dwarves, metallic-line stars and sub-dwarves. Taken from Straižys (1992).

independent of luminosity. However $A_{U}$ shows a bend around $(\mathrm{B}-\mathrm{V})_{0}=0$ due to the Balmer jump and separate sequences for different luminosities. They all show a quadratic dependence on the amount of interstellar material (for $0<x<1$ ) and so the reddening line is not linear (approximately parabolic for small reddening) and it's slope depends on $(\mathrm{B}-\mathrm{V})_{0}$. Finally, the red leaks have a strong effect on the reddening line for late type stars.

Despite it's problems, the UBV system was quickly adopted and is still widely used today, with at least 90,000 stars observed to date (Mermilliod 1987a, b) - see Straižys (1992) for further references to catalogues. Possible reasons for it's popularity are the large number of standard stars spread across the (northern) sky established by Johnson, it's wide bandwidths meaning faint stars may be observed, and that the system can be easily realized for photographic sky surveys (after-all, it was intended to duplicate a photographic system).

There are also several clones or extensions of the system in use (Bessell 1986). For instance, Johnson (1965b) extended the system by adding the red and infrared filters R,I,J,H,K,L,M,N. These suffer smaller blanketing effects and interstellar reddening than the bluer UBV filters and are better temperature indicators for late type stars (as they are nearer the maximum emission of these cooler stars). Straižys (1973 a) proposed a W filter to replace the U filter, which was designed to a) reduce the long wavelength response into the Balmer jump (giving a 
better measure of the size of the jump in conjunction with the B filter), b) remove the red leak, c) determine an accurate response function and d) correct the $(W-B)$ colour for atmospheric extinction allowing for the spectral type, luminosity class and reddening of the star. A final example is the $\mathrm{U}_{C} \mathrm{BV}$ variant produced by the Cape Observatory of South Africa, generally known as the Cousins system as most of the standard stars (predominately from the Harvard $\mathrm{E}$ regions of stars evenly spaced in RA at Declination $\sim-45^{\circ}$ ) were observed by Cousins (Menzies \& Laing 1988, Menzies et al. 1989). One major difference between the systems is that $\left(\mathrm{U}_{C}-\mathrm{B}\right)$ is not (incorrectly) transformed outside the atmosphere as was done by Johnson but corrected to an airmass of $1 \cdot 1$. The resulting $(\mathrm{V})$ magnitude and colours are then corrected to the UBV system via multi-valued non-linear transformations.

\subsection{Development of the uvby Photometric System.}

In the 1960's an intermediate bandwidth photometric system was developed by Strömgren $(1963, b, 1966)$ to measure the Balmer discontinuity, temperature and metallicity of $\mathrm{B}, \mathrm{A}$ and $\mathrm{F}$ stars. It consisted of four filters, with the $u$ filter being $8 \mathrm{~mm}$ of Schott UG11 glass cemented to $1 \mathrm{~mm}$ of WG3 Schott glass, and the remaining three $(v, b$ and $y)$ made of interference filters. The $v$ magnitude was adjusted to approximately correspond to Johnson $\mathrm{V}$, while the colours were left in the instrumental system of the original photometer. The filters are spaced in wavelength so they measure approximately equal increments in interstellar extinction, hence the reddening nearly cancels when taking differences in colours (Jaschek \& Jaschek 1987).

The ultraviolet $u$ filter is placed between the atmosphere's extinction cutoff (at $\sim 3000 \AA$ ) and the beginning of the Balmer jump, overcoming the two major problems with the Johnson U filter. The violet $v$ filter measures the flux at $\sim 4000 \AA$, being centred near the $H_{\delta}$ line, with the other Balmer lines falling in the short wavelength tail of the response function and so having little effect. However in late type stars, this filter is strongly effected by blanketing. Centred at $4600 \AA$, the blue $b$ filter is situated between the $H_{\gamma}$ and $H_{\beta}$ lines with the long wavelength tail slightly overlapping the $H_{\beta}$ line. This filter also avoids the interstellar absorption band at $4430 \AA$, and is only weakly effected by blanketing. The yellow $y$ filter is essentially a narrower Johnson V filter, having nearly the same effective wavelength. It is placed in a region largely free of spectral features, and gives a good apparent magnitude measurement. For F and G stars, this filter has approximately the same blanketing as the $b$ filter.

The response functions of the filters were initially given by Crawford (1966), with slightly different functions found by Matsushima (1969), again by Crawford \& Barnes (1970), and Stokes $(1972, b)$. Olson (1974) also included the 1P21 PMT in his calculations, which has only a minor effect as the passband responses are nearly completely defined by the filter responses 


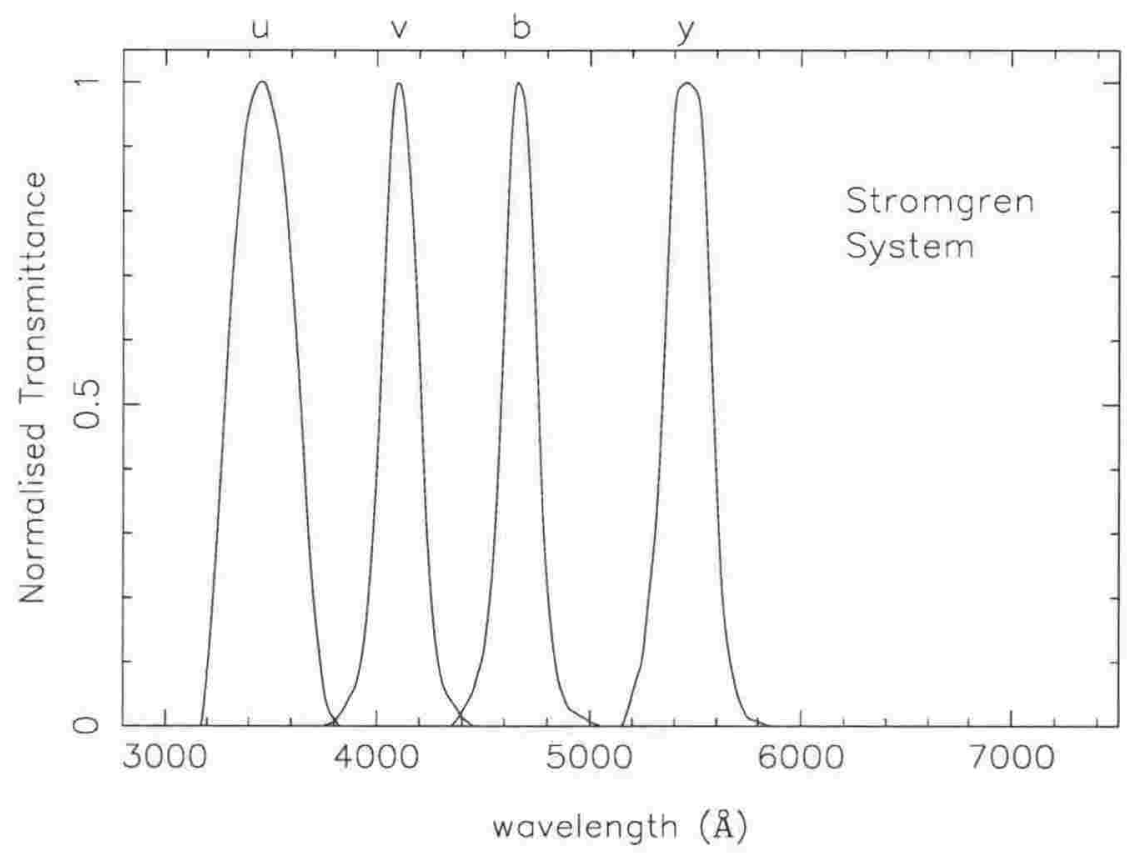

Figure 1.8: Transmission Response curves of the uvby Photometric System, normalised and spline fitted. Taken from Jaschek \& Jaschek (1987).

Table 1.2: Characteristics of the Standard uvby System Passbands.

\begin{tabular}{|ccccccc|}
\hline filter & $\begin{array}{c}\lambda_{R_{\max }} \\
(\AA)\end{array}$ & $\begin{array}{c}\text { FWHM } \\
(\AA)\end{array}$ & $\begin{array}{c}\lambda_{0} \\
(\AA)\end{array}$ & $\begin{array}{c}\mu_{2}^{2} \\
\left(\mathrm{~m}^{2}\right)\end{array}$ & $\begin{array}{c}\mu_{3}^{3} \\
\left(\mathrm{~m}^{3}\right)\end{array}$ & $\begin{array}{c}\mu_{4}^{4} \\
\left(\mathrm{~m}^{4}\right)\end{array}$ \\
\hline$u$ & 3460 & 360 & 3470 & $1 \cdot 50 \times 10^{-16}$ & $+1 \cdot 36 \times 10^{-25}$ & $5 \cdot 20 \times 10^{-32}$ \\
$v$ & 4100 & 200 & 4110 & $8 \cdot 04 \times 10^{-17}$ & $-1 \cdot 28 \times 10^{-27}$ & $2 \cdot 30 \times 10^{-32}$ \\
$b$ & 4660 & 180 & 4670 & $7 \cdot 50 \times 10^{-17}$ & $+2 \cdot 67 \times 10^{-26}$ & $2 \cdot 22 \times 10^{-32}$ \\
$y$ & 5460 & 240 & 5460 & $9 \cdot 64 \times 10^{-17}$ & $-5 \cdot 27 \times 10^{-27}$ & $2 \cdot 81 \times 10^{-32}$ \\
\hline
\end{tabular}

(Manfroid \& Sterken 1987). The passbands shown in Figure 1.8 are from Jaschek \& Jaschek (1987), and were originally taken from Lamla (1982). Some of the system's characteristics are given in Table $1.2 ; \lambda_{R_{\max }}$ is the wavelength giving the maximum transmittance for each filter, the FWHM was measured directly from Figure $1.8, \lambda_{0}$ is the effective wavelength and $\mu_{2}^{2}$ etc. are the moments of the filter passbands.

The system is often used with two extra filters; a narrow $\left(\beta_{N}\right)$ and wider filter $\left(\beta_{W}\right)$ both centred on the $H_{\beta}$ line (Crawford 1958, 1960). As these have (nearly) the same effective wavelength, the beta index (in magnitudes, $\beta=\beta_{N}-\beta_{W}$ ) is free from interstellar reddening, and, for simultaneous measurements through both filters, atmospheric extinction (Crawford \& 
Mander 1966). As the strength of the Balmer lines change in proportion to each other, ${ }^{12}$ the $\beta$ index can be used to correct for the effect of the $H_{\delta}$ line in the $v$ filter (Golay 1974). The $\beta_{N}$ and $\beta_{W}$ response functions have been given by Crawford (1966), later revised by Crawford \& Mander (1966), and also given by Stokes $(1972, b)$.

The bandwidths of the filters are sufficiently small that higher order terms in the Taylor expansion of the star's spectrum (equation 1.22) are negligible, except for the $c_{1}$ and $u-b$ which shows a complicated dependence of the star's spectrum and reddening (Straižys 1992) - see later for a definition of $c_{1}$. However it was assumed that all the colours had negligible higher order terms, so the reduction procedure used to correct for atmospheric extinction results in systematic errors for $c_{1}$ and $u-b$ colours. Further, as the filters do not overlap in wavelength, significant systematic errors when transforming measurements to the standard system are possible.

For early stars $b$ and $y$ are approximately blanketing free, while for late stars they have nearly the same blanketing (Hendon \& Kaitchuck 1990). Thus the $b-y$ colour is free of blanketing effects and serves as a temperature indicator in a similar manner to Johnson B-V. The $v$ filter is in a strong blanketing region but clear of the Balmer lines so the difference between the actual $v-b$ colour and what it would have in the absence of blanketing (estimated by $b-y$ ) gives the metallicity $m_{1}=(v-b)-(b-y)$, as can be seen in Figure 1.9. The $u$ filter's intensity is reduced by both blanketing and the Balmer jump $\left(c_{1}\right)$. As the blanketing of the $u$ filter is approximately twice that of the $v$ filter, Figure 1.9 shows that $(u-y)-c_{1}-2 m_{1}=3(b-y)$ which reduces to the Balmer jump $c_{1}=(u-v)-(v-b)$. Finally, as described in section 1.4, the $\beta$ index measures the equivalent width of the $H_{\beta}$ line (see Golay (1974) for a proof of this). For B and early A stars this width is correlated with the absolute magnitude of the star, while for late $\mathrm{A}$ and $\mathrm{F}$ stars it is a function of temperature (Straižys 1992). Hence the following parameters

$$
\begin{aligned}
b-y & \\
m_{1} & =(v-b)-(b-y) \\
c_{1} & =(u-v)-(v-b) \\
\beta & =\beta_{N}-\beta_{W}
\end{aligned}
$$

are more commonly used than the other colour indices.

These parameters still depend slightly on interstellar reddening (Strömgren 1966) so the

\footnotetext{
${ }^{12}$ The shorter wavelength lines are actually slightly more sensitive to luminosity (i.e. gravity) than the longer wavelengths, via the Stark effect (Golay 1974).
} 


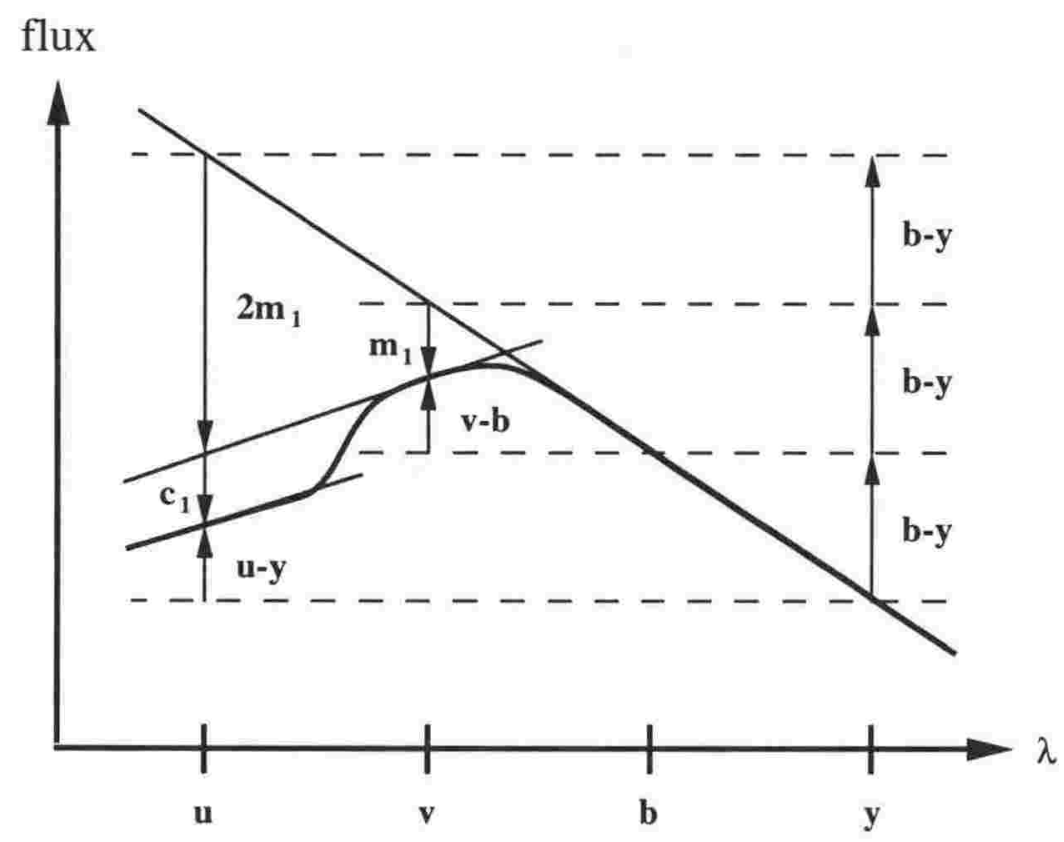

Figure 1.9: Schematic explanation of $c_{1}$ and $m_{1}$ in the $u v b y$ system. Adapted from Golay (1974) and originally given by Crawford.

following reddening free parameters are used;

$$
\begin{aligned}
{\left[c_{1}\right] } & =c_{1}-\frac{E_{c_{1}}}{E_{b-y}}(b-y) \\
{\left[m_{1}\right] } & =m_{1}-\frac{E_{m_{1}}}{E_{b-y}}(b-y) \\
\text { and sometimes, }[u-b] & =(u-b)-\frac{E_{u-b}}{E_{b-y}}(b-y)=\left[c_{1}\right]+2\left[m_{1}\right],
\end{aligned}
$$

where $[u-b]$ is useful as a temperature indicator in very hot (B type) stars (Budding 1993). The filter bandwidths are sufficiently narrow that the reddening lines are nearly straight as the extinction increases. However the slopes of the lines, and hence the value of the colour excess ratios, do depend slightly on the star's spectrum. Typical values are $E_{c_{1}} / E_{b-y}=$ $0 \cdot 19, E_{m_{1}} / E_{b-y}=-0 \cdot 33$, and $E_{u-b} / E_{b-y}=1 \cdot 53$, while synthesized ratios are given by Straižys (1992, Table 34) for different spectral and luminosity classes.

In an attempt to minimize the variations of local versions from the uvby system, Crawford of Kitt Peak Observatory (KPO) bulk ordered filters which were then supplied to different observers (Bessell 1993, Straižys 1992). Unfortunately a manufacturing error resulted in some of the $v$ filters having the wrong FWHM, being too narrow, which has caused systematic errors in $c_{1}$ and $m_{1}$ colours. Mount Stromlo and Siding Springs Observatories received two of these wrong filters (Eggen 1976), as was shown by comparison of the transmission with that of the standard filter (Eggen 1982). The transformation errors were so severe that Eggen $(1976,1977$, $1978,1982)$ decided to use their instrumental system as a new $\left(b-y, M_{1}, C_{1}, \beta\right)$ standard 
system. Eggen (1976) also extended this system for use with late type stars.

Another variant of the uvby system used a spectrophotometer, where the light was spread into a spectrum using a prism or diffraction grating, split into the required passbands by slots in a mask and the passbands measured simultaneously by four PMT's. One version used uvby filters between the slots and PMT's (Grønbech et al. 1976) but the tails of the passbands were cutoff by the slots and the passbands themselves did not closely match the standard passbands so this system did not transform well to the standard system (Manfroid 1985, Manfroid \& Sterken 1987, Young 1988). The other version did not use filters but instead defined the passbands by the slots, resulting in rectangular response curves which had severe transformation problems (Olsen 1983).

In general, observers get high internal precision in the uvby system (typically $\pm 0^{m} \cdot 005-$ $\left.0^{m} \cdot 009\right)$ but need multi-valued, non-linear transformations to the standard system and still have large systematic errors. For instance, there are differences of $\pm 0^{m} \cdot 03-0^{m} \cdot 05$ in $c_{1}$ and $m_{1}$ between catalogs of the northern and southern hemisphere (Straižys 1992). Thus there seem to be at least two uvby systems; the original system defined by northern hemisphere standard stars (Grønbech et al. 1976, Grønbech \& Olsen 1977, Olsen 1983) and the southern hemisphere E region standards measured by Cousins (1987), including $H_{\beta}$ standards (Cousins 1989), and extended to fainter stars by Kilkenny \& Laing (1992). A large number of stars (Hauck \& Mermilliod $(1980,1985)$ list approximately 40000 stars in their catalogue) have been observed in this system, which is second in popularity to only the UBV system.

Several procedures have been developed for classifying stars in this system, with a good description given by Straižys (1992), so only an outline will be given here. First it is assumed that only the stars for which the system was designed ( $\mathrm{B}, \mathrm{A}$ and $\mathrm{F}$ stars) have been observed. ${ }^{13}$ Figure 1.10 shows the intrinsic sequences in a $b-y, c_{1}$ diagram, with the sequences of different luminosities have similar shapes but being shifted down and to the right slightly. Thus reddened main sequence stars can overlap with supergiants and heavily reddened B stars can overlap with the $\mathrm{A}$ and $\mathrm{F}$ stars. Therefore a star has to be grouped in it's approximate spectral and luminosity class before this diagram can be used, with various other diagrams such as $(b-y$, $\left.m_{1}\right)$ or $\left(m_{1}, \beta\right)$ available for this.

Once separated into the approximate spectral group, the $b-y$ and $c_{1}$ colours may then be de-reddened using an iterative method. For instance, with B III-V stars the observed $c_{1}$ value gives an estimate of the intrinsic $c_{0}$ colour, which is then used to look up an improved estimate of the intrinsic $(b-y)_{0}$ colour in a table of intrinsic $(b-y)_{0}, c_{0}$ colours. This then gives the colour excess $E_{b-y}\left(=(b-y)-(b-y)_{0}\right)$, leading to the colour excess $E_{c_{1}}$ via the colour excess ratio

\footnotetext{
${ }^{13}$ Some qualitative and quantitative schemes have been developed for the restricted case of un-reddened $\mathrm{G}$ and $\mathrm{K}$ III-V stars.
} 


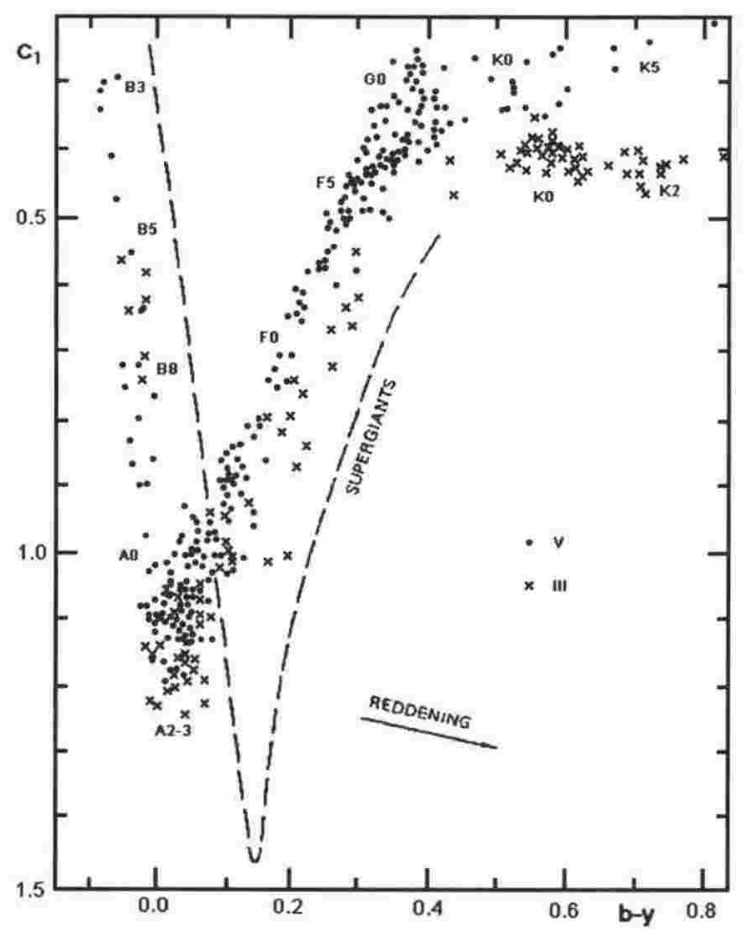

Figure 1.10: The Strömgren $b-y, c_{1}$ diagram, taken from Straižys (1992).

$E_{c_{1}} / E_{b-y}$, and resulting in an improved estimate of the intrinsic $c_{0}$ colour. The process is repeated until the values converge, usually only requiring a few iterations. Similar procedures can be used for most of the other groups, with the A stars being most difficult to classify due to their position in the turning point of the sequence.

An alternative procedure is to use the Golay box method, comparing the unknown stars with reference stars taken from a catalog and occupying the same $\left(b-y, c_{1}, m_{1}\right.$ or $\left.(b-y)_{0}, c_{0}, m_{0}\right)$ box. Either procedure results in similar accuracy, correctly classifying the stars to within $\pm 1-2$ spectral subclasses and \pm 1 luminosity classes. Calibrations to physical parameters have been found by Crawford $(1975,1978,1979)$.

Various types of peculiarities may be recognized with success rates varying from $50-75 \%$, with the lower rates due to stars with a mild form of the peculiarity occupying the same position on the diagrams as normal stars. For instance, metallic line (Am) stars can be separated with a $75 \%$ success rate using $m_{1}, b-y$ or $m_{1}, \beta$ diagrams. Some Blue Horizontal Branch (BHB) stars may be recognized, while white dwarves can be separated about half the time and both components of a binary can usually be classified. In some restricted cases, the effects of metallicities (in clusters), rotation or micro-turbulence may be detected. 


\subsection{Development of the Vilnius Photometric System.}

According to Straižys (1973 b), in the 1960's a chaotic situation existed with many intermediate and narrow bandwidth filter systems in use. These were usually very specialized in design and limited in the temperature range, luminosity and population type that could be classified (using dozens of proposed methods) and all needing extensive sets of standard stars for transformation (of local implementations of the filters to the standard system) and calibration (Straižys 1973 b). The situation was not helped as it was becoming clear that transformations (both from the local to standard system and between different standard systems) were plagued by systematic errors. The Vilnius group felt there was a need for one general system, based on astrophysical needs (rather than availability of the filters) and in particular, a system which could derive a star's spectral type, luminosity and reddening using only the photometric measurements from the system. In addition to requiring standard stars for only one system, this would allow many more stars, in particular fainter stars or stars in dusty regions, to be classified than could currently be done by spectroscopic means. Some applications of such a system could be statistical studies of stellar populations or investigations of interstellar dust and gas. Further, specialized studies could be possible by taking a subset of the general system.

The development of such a system became possible with the photoelectric measurements of energy distributions of stars of different types and the (mean) interstellar dust transmission curve in the 1960's. Synthetic colours and Q factors could be calculated and by changing the effective wavelength, FWHM and shape of the response curves, optimum passbands could be derived. A trade-off between bandwidth effects and limiting magnitude implied the use of intermediate bandwidth filters (bandwidth $\sim 400 \AA$ ).

Initially Straižys (1964) investigated existing filter systems for their ability to measure reddening and found they could only do so if the stars all belonged to the same group (for example a cluster or association) and had the same reddening. Further, due to a 'break' in the colourcolour diagrams, the reddening lines (for early stars) would intersect the sequence of (later-type) unreddened stars and stars of different luminosities formed their own sequences. Straižys (1963, 1964) found that the ideal system met three conditions; 1) there is a large angle between the sequence of unreddened stars (the intrinsic sequence) and the reddening lines, 2) the intrinsic sequence does not have large changes in slope, especially towards the reddening lines, and 3) the intrinsic sequences of different luminosities lie close together on the diagram. To achieve this requires the following three filters; X ( $\lambda_{0} \sim 4000 \AA$, bandwidth $\sim 400 \AA$ ), Y (at the breakpoint of the interstellar reddening line when plotted against $\lambda^{-1}$, i.e. $\lambda_{0} \sim 4500 \AA$, bandwidth $\sim 400 \AA)$ and Z $\left(\lambda_{0}=5000-6000 \AA\right.$, bandwidth $\left.\sim 500 \AA\right)$.

This allowed reddening to be determined for early-type stars (Zdanavičius \& Straižys 1964) but the addition of an $\mathrm{S}$ filter was required to find the reddening and classify late-type stars 


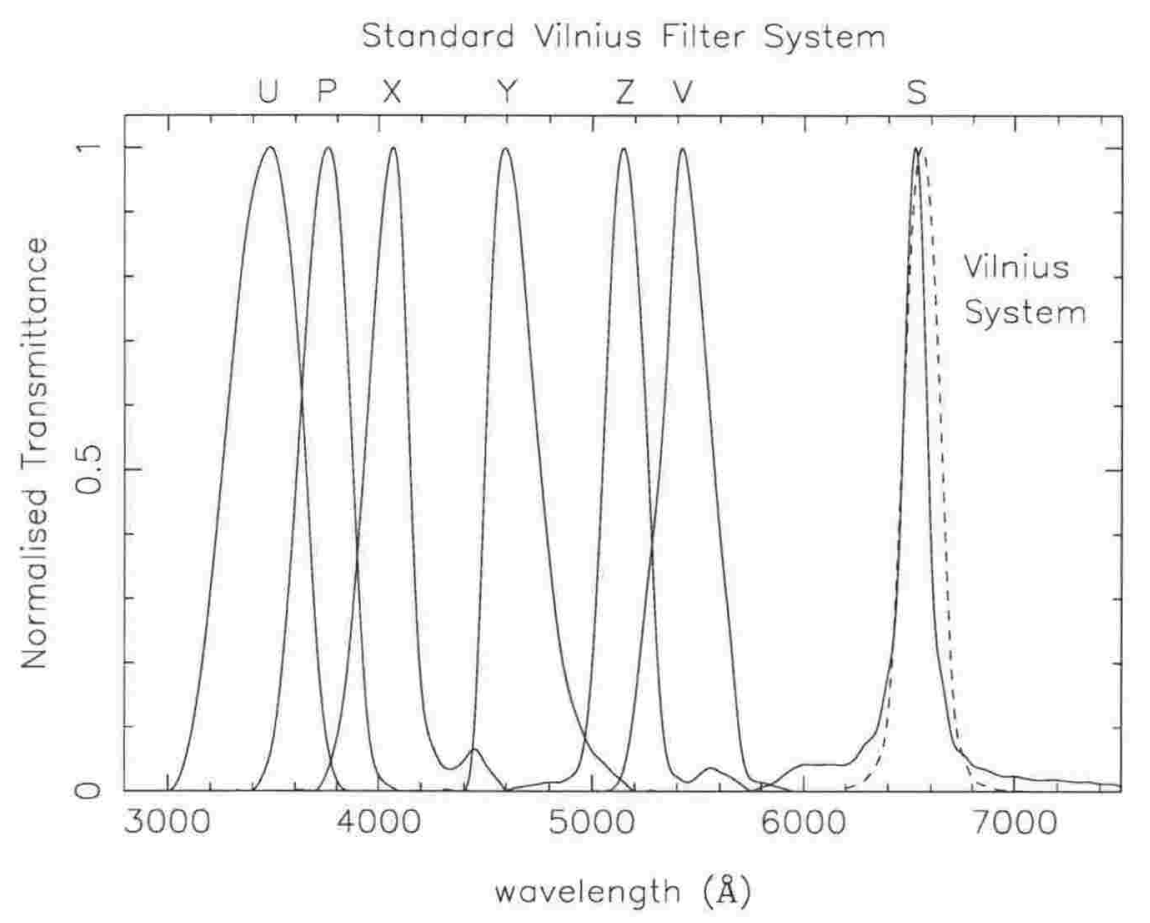

Figure 1.11: Transmission Response curves of the Vilnius Photometric System, normalised and spline fitted. Taken from Straižys \& Zdanavičius (1970) and Straižys (1992) for the new S response curve (dashed line).

and a T filter to separate the K and M stars (Straižys \& Zdanavičius 1965). Finally, the U and P filters were added to classify early-type stars (Straižys 1965).

The proposed system was tested in 1966 using interference filters and confirmed the theoretical calculations (Zdanavičius \& Nikonov 1967). However, as interference filters are not stable, are hard to manufacture to a required transmission curve and have too small a bandwidth, they were replaced (except for the $\mathrm{S}$ filter) with glass filters (Kakaras et al. 1968). The T filter was dropped (it was only required to separate the $\mathrm{K}$ and $\mathrm{M}$ stars, which can also be done using combinations of the remaining filters) and a V filter added to form the standard system. The transmission response curves of the standard system are given by Straižys \& Zdanavičius (1970), listed in Table 1.3 and shown in Figure 1.11. Some of the system's characteristics are given in Table 1.4; $R_{\max }$ is maximum transmittance for each filter (in arbitary units), $\lambda_{R_{\max }}$ is the wavelength of the maximum transmittance, the FWHM was measured directly from Figure $1.11, \lambda_{0}$ is the effective wavelength and $\mu_{2}^{2}$ etc. are the moments of the filter passbands. The response curve of the S filter was modified by Straižys (1992), and is labelled as the 'new S' filter in Table 1.3 and shown as a dashed curve in Figure 1.11. As the new S response curve became available partway through this thesis, much of the analysis uses the response curves of Straižys \& Zdanavičius (1970). 
Table 1.3: Transmission Response data of the Standard Vilnius Photometric System, with wavelength in $\AA$. Taken from Straižys \& Zdanavičius (1970) and Straižys (1992, Table 59) for the (normalised) new $\mathrm{S}$ response curve.

\begin{tabular}{|c|c|c|c|c|c|c|c|c|c|c|c|}
\hline$\lambda$ & $\mathrm{U}$ & $\mathrm{P}$ & $\mathrm{X}$ & $\lambda$ & $\mathrm{Y}$ & $\mathrm{Z}$ & $\lambda$ & V & $\lambda$ & $\mathrm{S}$ & new $\mathrm{S}$ \\
\hline 3000 & 0.0 & & & 4400 & 0.0 & & 5050 & 0.0 & 5750 & 0.0 & \\
\hline 3050 & $3 \cdot 0$ & & & 4450 & $32 \cdot 0$ & & 5100 & $1 \cdot 0$ & 5800 & 1.0 & \\
\hline 3100 & 13.0 & & & 4500 & 156.0 & & 5150 & 8.0 & 5850 & $2 \cdot 0$ & \\
\hline 3150 & $31 \cdot 0$ & & & 4550 & $263 \cdot 0$ & & 5200 & $30 \cdot 0$ & 5900 & $4 \cdot 0$ & \\
\hline 3200 & 57.0 & & & 4600 & $287 \cdot 0$ & $0 \cdot 0$ & 5250 & $65 \cdot 0$ & 5950 & $6 \cdot 0$ & \\
\hline 3250 & 88.0 & & & 4650 & $261 \cdot 0$ & $2 \cdot 0$ & 5300 & $98 \cdot 0$ & 6000 & $7 \cdot 0$ & \\
\hline 3300 & $120 \cdot 0$ & & & 4700 & 206.0 & $3 \cdot 0$ & 5350 & $146 \cdot 0$ & 6050 & $7 \cdot 0$ & \\
\hline 3350 & $149 \cdot 0$ & & & 4750 & $150 \cdot 0$ & $4 \cdot 0$ & 5400 & $208 \cdot 0$ & 6100 & $7 \cdot 0$ & \\
\hline 3400 & 168.0 & $0 \cdot 0$ & & 4800 & $103 \cdot 0$ & $5 \cdot 0$ & 5450 & $205 \cdot 0$ & 6150 & $7 \cdot 0$ & 0.0 \\
\hline 3450 & 178.0 & $6 \cdot 0$ & & 4850 & $65 \cdot 0$ & $5 \cdot 0$ & 5500 & $169 \cdot 0$ & 6200 & $7 \cdot 0$ & 0.5 \\
\hline 3500 & 179.0 & $21 \cdot 0$ & & 4900 & 42.0 & $8 \cdot 0$ & 5550 & $126 \cdot 0$ & 6250 & 9.0 & 1.0 \\
\hline 3550 & $163 \cdot 0$ & 57.0 & & 4950 & 28.0 & $19 \cdot 0$ & 5600 & $85 \cdot 0$ & 6300 & $13 \cdot 0$ & $2 \cdot 1$ \\
\hline 3600 & $135 \cdot 0$ & $110 \cdot 0$ & & 5000 & 18.0 & $72 \cdot 0$ & 5650 & $50 \cdot 0$ & 6350 & $17 \cdot 0$ & $4 \cdot 6$ \\
\hline 3650 & 92.0 & $170 \cdot 0$ & & 5050 & 13.0 & $193 \cdot 0$ & 5700 & $15 \cdot 0$ & 6400 & 31.0 & $12 \cdot 4$ \\
\hline 3700 & $44 \cdot 0$ & $218 \cdot 0$ & 0.0 & 5100 & 8.0 & $320 \cdot 0$ & 5750 & $4 \cdot 0$ & 6450 & $63 \cdot 0$ & $41 \cdot 3$ \\
\hline 3750 & $15 \cdot 0$ & 237.0 & $6 \cdot 0$ & 5150 & 4.0 & $359 \cdot 0$ & 5800 & $3 \cdot 0$ & 6500 & 151.0 & $81 \cdot 3$ \\
\hline 3800 & 3.0 & $221 \cdot 0$ & $19 \cdot 0$ & 5200 & 0.0 & $308 \cdot 0$ & 5850 & $2 \cdot 0$ & 6550 & $153 \cdot 0$ & $100 \cdot 0$ \\
\hline 3850 & 0.0 & $160 \cdot 0$ & $44 \cdot 0$ & 5250 & & $206 \cdot 0$ & 5900 & $1 \cdot 0$ & 6600 & $62 \cdot 0$ & 87.4 \\
\hline 3900 & & 82.0 & 87.0 & 5300 & & 83.0 & 5950 & 0.0 & 6650 & $30 \cdot 0$ & $52 \cdot 0$ \\
\hline 3950 & & $25 \cdot 0$ & 139.0 & 5350 & & $20 \cdot 0$ & & & 6700 & $14 \cdot 0$ & $16 \cdot 6$ \\
\hline 4000 & & $6 \cdot 0$ & 192.0 & 5400 & & 8.0 & & & 6750 & $10 \cdot 0$ & $5 \cdot 8$ \\
\hline 4050 & & $2 \cdot 0$ & $226 \cdot 0$ & 5450 & & $5 \cdot 0$ & & & 6800 & $7 \cdot 0$ & $2 \cdot 2$ \\
\hline 4100 & & 0.0 & $210 \cdot 0$ & 5500 & & 9.0 & & & 6850 & 6.0 & $1 \cdot 1$ \\
\hline 4150 & & & $112 \cdot 0$ & 5550 & & $13 \cdot 0$ & & & 6900 & $5 \cdot 0$ & 0.5 \\
\hline 4200 & & & $39 \cdot 0$ & 5600 & & $11 \cdot 0$ & & & 6950 & $4 \cdot 0$ & 0.2 \\
\hline 4250 & & & $17 \cdot 0$ & 5650 & & 8.0 & & & 7000 & $4 \cdot 0$ & $0 \cdot 1$ \\
\hline 4300 & & & $9 \cdot 0$ & 5700 & & $4 \cdot 0$ & & & 7050 & $3 \cdot 5$ & $0 \cdot 0$ \\
\hline 4350 & & & $8 \cdot 0$ & 5750 & & 0.0 & & & 7100 & $3 \cdot 0$ & \\
\hline 4400 & & & 11.0 & & & & & & 7150 & $3 \cdot 0$ & \\
\hline 4450 & & & $15 \cdot 0$ & & & & & & 7200 & $3 \cdot 0$ & \\
\hline 4500 & & & $10 \cdot 0$ & & & & & & 7300 & 2.5 & \\
\hline 4550 & & & $5 \cdot 0$ & & & & & & 7350 & 2.5 & \\
\hline \multirow[t]{7}{*}{4600} & & & $0 \cdot 0$ & & & & & & 7400 & $2 \cdot 0$ & \\
\hline & & & & & & & & & 7450 & 2.0 & \\
\hline & & & & & & & & & 7500 & 1.5 & \\
\hline & & & & & & & & & 7550 & 1.0 & \\
\hline & & & & & & & & & 7600 & 1.0 & \\
\hline & & & & & & & & & 7650 & 0.5 & \\
\hline & & & & & & & & & 7700 & 0.0 & \\
\hline
\end{tabular}


Table 1.4: Characteristics of the Standard Vilnius System Passbands.

\begin{tabular}{|cccccccc|}
\hline filter & $R_{\max }$ & $\begin{array}{c}\lambda_{R_{\max }} \\
(\AA)\end{array}$ & $\begin{array}{c}\mathrm{FWHM} \\
(\AA)\end{array}$ & $\begin{array}{c}\lambda_{0} \\
(\AA)\end{array}$ & $\begin{array}{c}\mu_{2}^{2} \\
\left(\mathrm{~m}^{2}\right)\end{array}$ & $\begin{array}{c}\mu_{3}^{3} \\
\left(\mathrm{~m}^{3}\right)\end{array}$ & $\begin{array}{c}\mu_{4}^{4} \\
\left(\mathrm{~m}^{4}\right)\end{array}$ \\
\hline $\mathrm{U}$ & 180 & 3480 & 400 & 3440 & $2 \cdot 07 \times 10^{-16}$ & $-4 \cdot 70 \times 10^{-25}$ & $1 \cdot 05 \times 10^{-31}$ \\
$\mathrm{P}$ & 238 & 3760 & 270 & 3740 & $1 \cdot 08 \times 10^{-16}$ & $-1 \cdot 28 \times 10^{-25}$ & $3.24 \times 10^{-32}$ \\
$\mathrm{X}$ & 229 & 4070 & 220 & 4050 & $1.64 \times 10^{-16}$ & $+2 \cdot 12 \times 10^{-24}$ & $1.46 \times 10^{-31}$ \\
$\mathrm{Y}$ & 288 & 4590 & 260 & 4660 & $1.64 \times 10^{-16}$ & $+1.99 \times 10^{-24}$ & $1.05 \times 10^{-31}$ \\
$\mathrm{Z}$ & 360 & 5140 & 220 & 5160 & $1.40 \times 10^{-16}$ & $+1.36 \times 10^{-24}$ & $1.41 \times 10^{-31}$ \\
$\mathrm{~V}$ & 215 & 5420 & 230 & 5440 & $1.41 \times 10^{-16}$ & $+2 \cdot 19 \times 10^{-25}$ & $5 \cdot 21 \times 10^{-32}$ \\
$\mathrm{~S}$ & 169 & 6530 & 120 & 6530 & $5 \cdot 53 \times 10^{-16}$ & $+1.24 \times 10^{-23}$ & $2.37 \times 10^{-30}$ \\
\hline
\end{tabular}

The initial calibration of the system was based on observations of normal and peculiar stars made during 1967-70(Zdanavičius et al. 1969, Straižys \& Sviderskiené 1972) although improvements have continued to the present date, for example (Sūdžius et al. 1970, Bartkevičius et al. 1973), with the current calibrations given by Straižys (1992). The method used to correct for atmospheric extinction was described by Zdanavičius (1970) and standard star sets established in the northern hemisphere (Zdanavičius et al. 1969, Straižys et al. 1970, Zdanavičius \& Cerniene 1985, Černis et al. 1989, Černis \& Jasevičius 1992). These, and all other observations, were collected together by North (1980) in a catalog available on magnetic tape from the Centre de Données Astronomiques de Strasbourg (CDS). A more up-to-date catalog (containing 7445 stars) recently became available (Straižys \& Kazlauskas 1993) and was used for the later analysis in this thesis.

In addition to being able to classify the spectral type and luminosity of normal B-M stars with any degree of reddening, the Vilnius system was found to detect stars with certain types of peculiarities; subdwarfs (Bartkevičius \& Straižys 1970a) and late-type metal deficient giants (Bartkevičius \& Metik 1969, Bartkevičius \& Straižys 1970c, Bartkevičius et al. 1992, Bartkevičius \& Lazauskaite 1994), Am, Ap and Be stars (Bartkevičius \& Straižys 1970b), white dwarfs, double stars (Kakaras \& Straižys 1969), carbon and barium stars (S̆leivytė 1992), Herbig Ae/Be stars, and T Tauri stars. It has been estimated that this system can classify 90 - $95 \%$ of the stars in any galactic field (Straižys \& Philip 1994).

A brief discussion of the rationale for the placement of each filter follows; The $U$ filter is placed on the ultraviolet side of the Balmer Jump to measure the minimum intensity of the Jump, and hence, in combination with the $\mathrm{P}$ or X filters, measure the size of the Jump which is sensitive to the temperature (or spectral type) of an early-type star. However the filter must be at a sufficiently long wavelength to avoid atmospheric extinction which increases rapidly

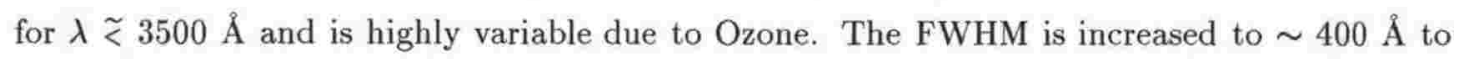
improve the limiting magnitude to which a star can be measured through the filter.

The $\mathrm{P}$ filter measures the absorption lines of the higher members of the Balmer series, 
whose depths are sensitive to the surface gravity of early stars. Thus for supergiants the $\mathrm{P}$ filter measures the maximum intensity of the Balmer Jump while for main sequence stars it measures the bottom of the Jump.

As described earlier, the $\mathrm{X}, \mathrm{Y}$ and $\mathrm{Z}$ filter measure reddening due to interstellar dust and gas. It is desirable to place the $\mathrm{X}$ and $\mathrm{Z}$ filters far away (in wavelength) from the $\mathrm{Y}$ filter to satisfy the three conditions for an ideal $\mathrm{X}-\mathrm{Y}, \mathrm{Y}-\mathrm{Z}$ diagram. However the $\mathrm{X}$ filter must avoid being on the Balmer Jump or the ultraviolet side of the Jump (as this would give a strong non-linearity in the diagrams) and hence is placed between the $\mathrm{H}_{\gamma}$ and $\mathrm{H}_{\epsilon}$ absorption lines. It also measures the maximum intensity of the Balmer Jump for early stars (especially main sequence stars) and blocking by metal lines in late-type stars.

The $\mathrm{Y}$ filter is shifted slightly from the interstellar reddening breakpoint to avoid an interstellar absorption band at $4430 \AA$.

The $\mathrm{Z}$ filter has a wide wavelength range available in which it can measure interstellar reddening and so it is placed to also measure a wide absorption feature (the $\mathrm{Mg}$ I triplet and $\mathrm{MgH}$ molecular band) in late-type stars whose depth is sensitive to surface gravity (or luminosity).

The V filter is an analog of the Johnson V filter, having nearly the same effective wavelength. There is no colour term in the transformation between the filters except for $\mathrm{K}-\mathrm{M}$ stars. This was intended to allow the Johnson V magnitudes to be adopted as the Vilnius V magnitudes and reduce the work required to set up the Vilnius system of standard stars. It is also the long wavelength side of the absorption feature measured in the $\mathrm{Z}$ filter, so $\mathrm{Z}-\mathrm{V}$ measures the depth of this feature and partly replaces the function of the T filter.

Finally, the $\mathrm{S}$ filter is centred on the $\mathrm{H}_{\alpha}$ line and can measure any emission in early stars (separating B and Be stars). For late-type stars, it measures a pseudo-continuum (actually it is the next intensity maximum of the TiO band measured by the T filter), helping to give the spectral and luminosity classes of a late star.

A variant of the standard system, the VilGen system, was made by merging the Vilnius and Geneva photometric systems as several filters have similar effective wavelengths and functions. The Geneva system has wide bandwidths, and hence fainter limiting magnitudes, so several of the Vilnius filters were widened and/or replaced by the equivalent Geneva filter. Theoretical and observational experiments were made on the proposed system by Straižys $(1977,1979)$ and North (1978). Preliminary intrinsic colours and calibrations for normal stars were measured by Straižys et al. (1982) and some peculiar stars (subdwarfs, metal deficient giants, metallic line, and Ap stars) by North et al. (1982). This system has the advantage that all VilGen colours can be easily obtained from the original Vilnius and Geneva colours (except for M-type, carbon and emission stars). A brief discussion of the system is given by Straižys (1992). 


\subsection{The Q-Search Classification Method.}

One of the methods used to classify the spectral and luminosity types of stars measured in the Vilnius system is the 'stellar box' method of Geneva astronomers (Straižys 1992) - this has also been called the 'sigma-Q' method (Smriglio et al. 1986, 1988, 1991).

The following value is calculated for each program star using a reference list of stars with known spectral and luminosity types

$$
\sigma_{\Delta Q}=\left[\sum_{i=1}^{n} \frac{\Delta Q_{i}^{2}}{n}\right]^{\frac{1}{2}}
$$

where $\Delta Q$ is the difference in the $\mathrm{Q}$ factors between the program star and a reference star, and $n$ is the number of $\mathrm{Q}$ factors used (usually five - there are at most five independent $\mathrm{Q}$ factors as there are only six Vilnius colours).

To determine the spectral class, the spectral types of the three reference stars with the smallest $\sigma_{\Delta Q}$ must agree within one class range (for example A5-F5). The assigned spectral class is the weighted mean of these three stars, with the weights being $1 / \sigma_{\Delta Q}$. Similarly, the luminosity classes of the three closest matching reference stars must lie within two consecutive luminosity classes (for example IV-V). Thus, at least two of the three reference stars have the same luminosity class and this class is assigned to the program star. Two or three iterations will be needed as the colour-excesses used to calculate the $\mathrm{Q}$ factors depend slightly on the spectral and luminosity class of the star. Initially a G0 V type star is assumed.

A variation on the general method, referred to here as the 'Q-Search' method, was used and is described next. In line with the literature, the following five $Q$ factors were used; $Q_{\mathrm{UPYV}}, Q_{\mathrm{PXYV}}, Q_{\mathrm{XYV}}, Q_{\mathrm{ZVYV}}$, and $Q_{\mathrm{VSYv}}$. However, instead of searching for the three best matching reference stars, all reference stars with $\sigma_{\Delta Q}$ less than or equal to some minimum are listed and the average spectral and luminosity classes calculated from this list. The averages are weighted by $1 /\left(\sigma_{\Delta Q}^{2}+0.001\right)$ - the 0.001 is added to avoid 'divide-by-zero' errors. At least five matching reference stars must be found before an average is calculated. This has the advantages of an adjustable and generally less stringent criteria (the minimum $\sigma_{\Delta Q}$ required was usually 0.020 to get five or more matching reference stars, although up to 0.060 was sometimes needed for $\mathrm{K}$ stars) and allows the standard deviations of the assigned spectral and luminosity classes to be calculated. The (northern) catalog of Vilnius stars (North 1980) was used for the reference list. If a star has multiple entries in the reference star list, only the first entry is considered for the search.

To test the QSearch computer program, tables of mean Vilnius intrinsic colours (Straižys 1992, Sūdžius \& Bobinas 1994) were used as program stars. It was found that the spectral type of early (O-B) and late (K-M) stars, particularly supergiants, were mis-classified with 
small systematic errors. Examination of the reference star lists used to calculate the mean spectral class revealed this was due to the spectral type of these stars (with approximately the same Q factors) varying with luminosity class. To reduce this effect, the weights for the mean spectral type were multiplied by an additional weight, the reciprocal of the absolute difference in luminosity classes between the program and reference star, i.e. $1 /(\mid \Delta$ Luminosity $\mid+1)-$ the one is added to avoid 'divide-by-zero' errors. This requires the Q-search to be iterated at least once, to calculate the luminosity class first.

The tests were repeated with the revised program, with the results shown in Figure 1.12. The additional weights did reduce the luminosity effect on the spectral classifications but not entirely eliminate it. The standard deviations give a good estimate of the possible difference between the actual and calculated spectral and luminosity classes. Typical standard deviations are \pm 1.4 spectral sub-classes and \pm 0.8 luminosity classes, with the giants and supergiants having slightly larger standard deviations $( \pm 1 \cdot 6)$ in the spectral classifications. As can be seen in Figure 1.12, M-type stars may not be accurately classified - this is due to the small selection of $\mathrm{M}$ stars in the reference list so the search criteria must be widened to find enough stars for an average. The search then also finds many $\mathrm{K}$ stars and hence the average is forced towards an earlier (in-correct) spectral class.

\subsection{The Q-Spectra Classification Method.}

Another method of spectral classification in the Vilnius system is that of the 'Q-curves' (also referred to as 'Q-Spectra' or 'Q $(\lambda)$ functions'), described by Straižys $(1974,1992)$. For monochromatic magnitudes, the following reddening free energy distribution curve is calculated

$$
Q_{m_{\lambda} m_{1} m_{2}}=\left(m_{\lambda}-m_{1}\right)-\frac{E_{m_{\lambda}-m_{1}}}{E_{m_{1}-m_{2}}}\left(m_{1}-m_{2}\right)
$$

for the program star and compared to a set of standard curves similarly calculated for different spectral types, luminosities, metallicity, peculiarities etc. According to Straižys (1974), the program stars may be classified by "simple graphical shifting of their Q-curves up to best coincidence with one of the standard curves". The method may be extended to the heterochromatic magnitudes of a photometric system by setting $\lambda$ in turn to the effective wavelength of each filter.

As the shape of the Q-curve is similar to the star's intrinsic energy curve if $m_{1}-m_{2}$ represents the colour-temperature only, i.e. the yellow-red spectral region, the Vilnius system usually uses

$$
Q_{\lambda \mathrm{VS}}=\left(m_{\lambda}-\mathrm{V}\right)-\frac{E_{m_{\lambda}-\mathrm{V}}}{E_{\mathrm{V}-\mathrm{S}}}(\mathrm{V}-\mathrm{S}) .
$$


Test of Classification methods

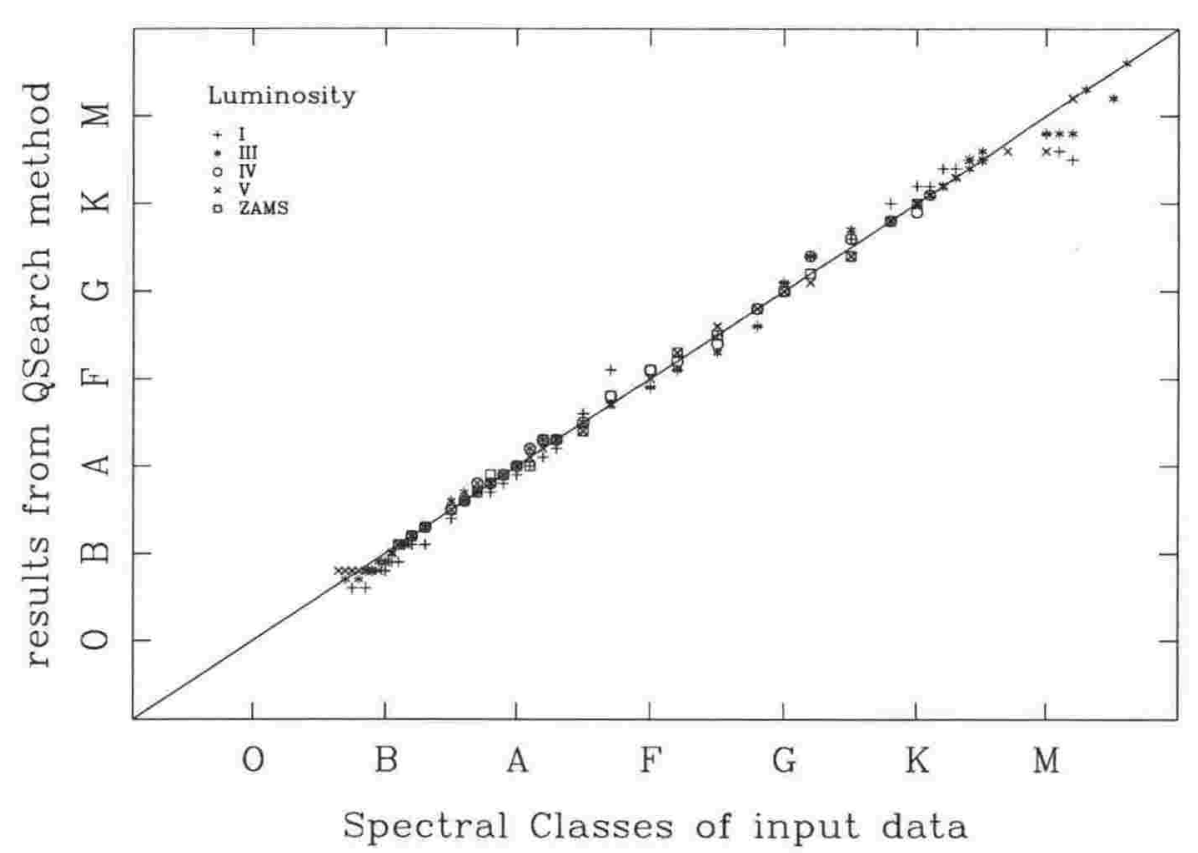

Test of Classification methods

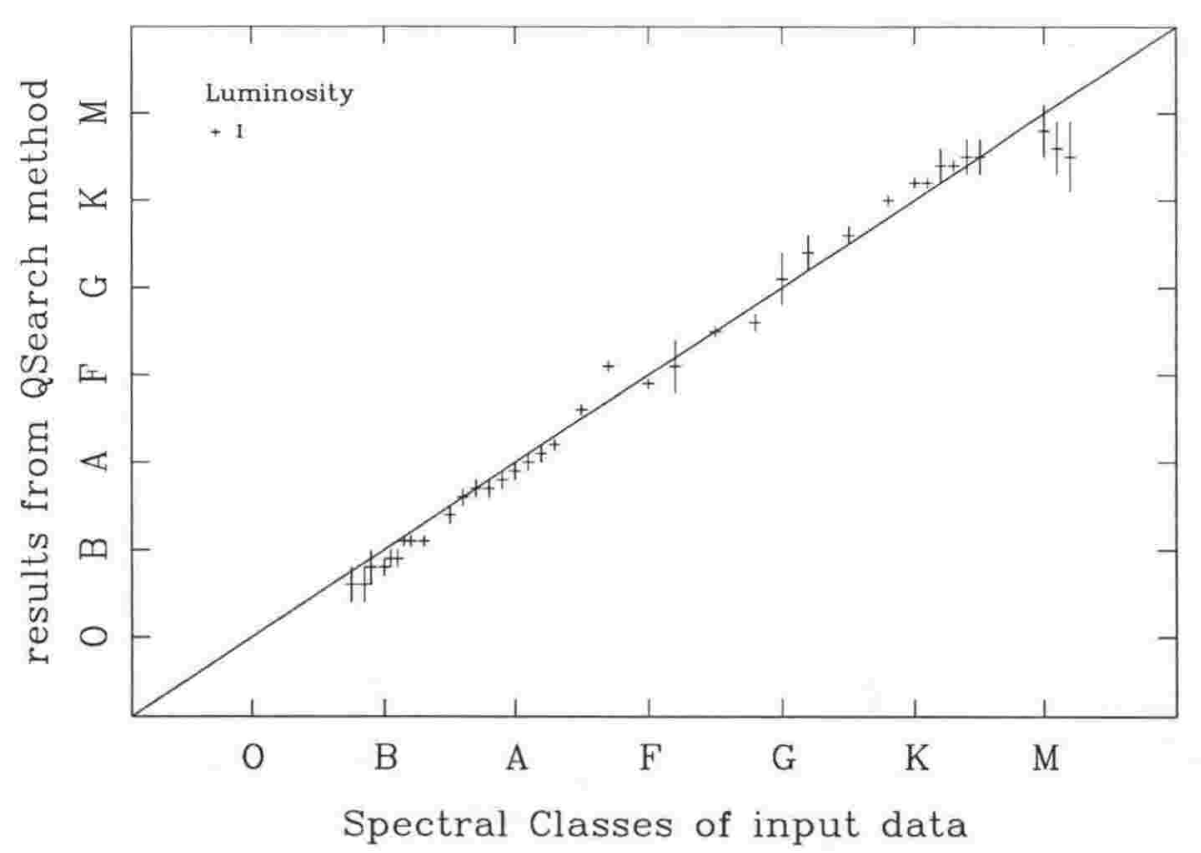

Figure 1.12: Test of the Q-Search Spectral classification method. The top figure shows the results for all luminosity classes, while the bottom figure includes only the supergiants. The error bars on the bottom figure are the standard deviations of the calculated spectral classes. 
This means seven Q-factors may be calculated ( $Q_{\mathrm{UvS}}, Q_{\mathrm{PVS}}, Q_{\mathrm{XVS}}, Q_{\mathrm{YvS}}, Q_{\mathrm{ZVS}}, Q_{\mathrm{VvS}}$, and $\left.Q_{\mathrm{SVS}}\right)$ but as $Q_{\mathrm{VvS}}=Q_{\mathrm{SvS}} \equiv 0$, only the first five can be used. As the Vilnius system defines the colours of unreddened O-type stars to be zero, the Q-Spectra for an O-type star is flat (i.e. $Q_{\lambda \mathrm{VS}} \equiv 0$ ). Examples of standard Q-Spectra are shown in Figures 1.13 and 1.14, illustrating the variation with spectral and luminosity classes.

The standard curves used here are calculated from Straižys (1992) tables of mean intrinsic colours for normal stars. The QSpectra computer program calculates the absolute difference between the program star's Q-Spectra and each of the standard Q-Spectra $\left(=\sum_{\lambda=\mathrm{U}}^{\mathrm{S}}\left|\Delta Q_{\lambda \mathrm{V} S}\right|\right)$, the standard Q-Spectra with the minimum difference giving the spectral and luminosity classes of the program star. A large absolute difference for the best matching standard Q-Spectra indicates either that there are errors in the photometry of the program star, or a standard Q-Spectra of the appropriate type has not been included. When used to classify stars in the southern Vilnius system, $\left|\Delta Q_{\text {UVS }}\right|$ and $\left|\Delta Q_{\mathrm{PVS}}\right|$ were given half the weight of the other absolute differences as the $\mathrm{U}$ and $\mathrm{P}$ measurements generally had larger errors (see Chapter 3 ).

To test the QSpectra program, the Aquila and Cygnus Standard Stars (Zdanavičius et al. 1969 ) were used as program stars. As it was not clear what Straižys meant by "simple graphical shifting of the Q-curves", both possibilities

$$
Q(\lambda)_{\text {prog }}=(1+a)\left[\left(m_{\lambda}-\mathrm{V}\right)-\frac{E_{m_{\lambda}-\mathrm{V}}}{E_{\mathrm{V}-\mathrm{S}}}(\mathrm{V}-\mathrm{S})\right]
$$

or

$$
Q(\lambda)_{\text {prog }}=\left(m_{\lambda}-\mathrm{V}\right)-(1+a) \frac{E_{m_{\lambda}-\mathrm{V}}}{E_{\mathrm{V}-\mathrm{S}}}(\mathrm{V}-\mathrm{S})
$$

were tested. The coefficient $a$ is normally zero but could be set to other (small) values to improve the fit of the program star's Q-Spectra to a standard Q-Spectra. It was found that equation 1.44 gave only slight improvements (as measured by the average difference between the calculated and actual spectral and luminosity classes of the program stars) to that of $a \equiv 0$. However equation 1.45 gives a significant improvement (nearly a factor of two) and is equivalent to correcting any random errors in $\mathrm{V}-\mathrm{S}$ (which produce systematic errors in the Q-Spectra). Based on the 57 stars in the Aquila and Cygnus regions, the Q-Spectra method allows stars to be classified to a standard deviation of \pm 1.0 sub-classes of their actual spectral type and $\pm 0 \cdot 3$ luminosity classes.

\subsection{The Q-Isolines Classification Method.}

The 'Q-isolines' method of classifying stars also uses five independent $\mathrm{Q}$ factors (usually $Q_{\mathrm{UvS}}$, $Q_{\mathrm{PVS}}, Q_{\mathrm{XVS}}, Q_{\mathrm{YVS}}$, and $\left.Q_{\mathrm{ZVS}}\right)$ and returns the effective surface temperature $\left(T_{e}\right)$ and surface 

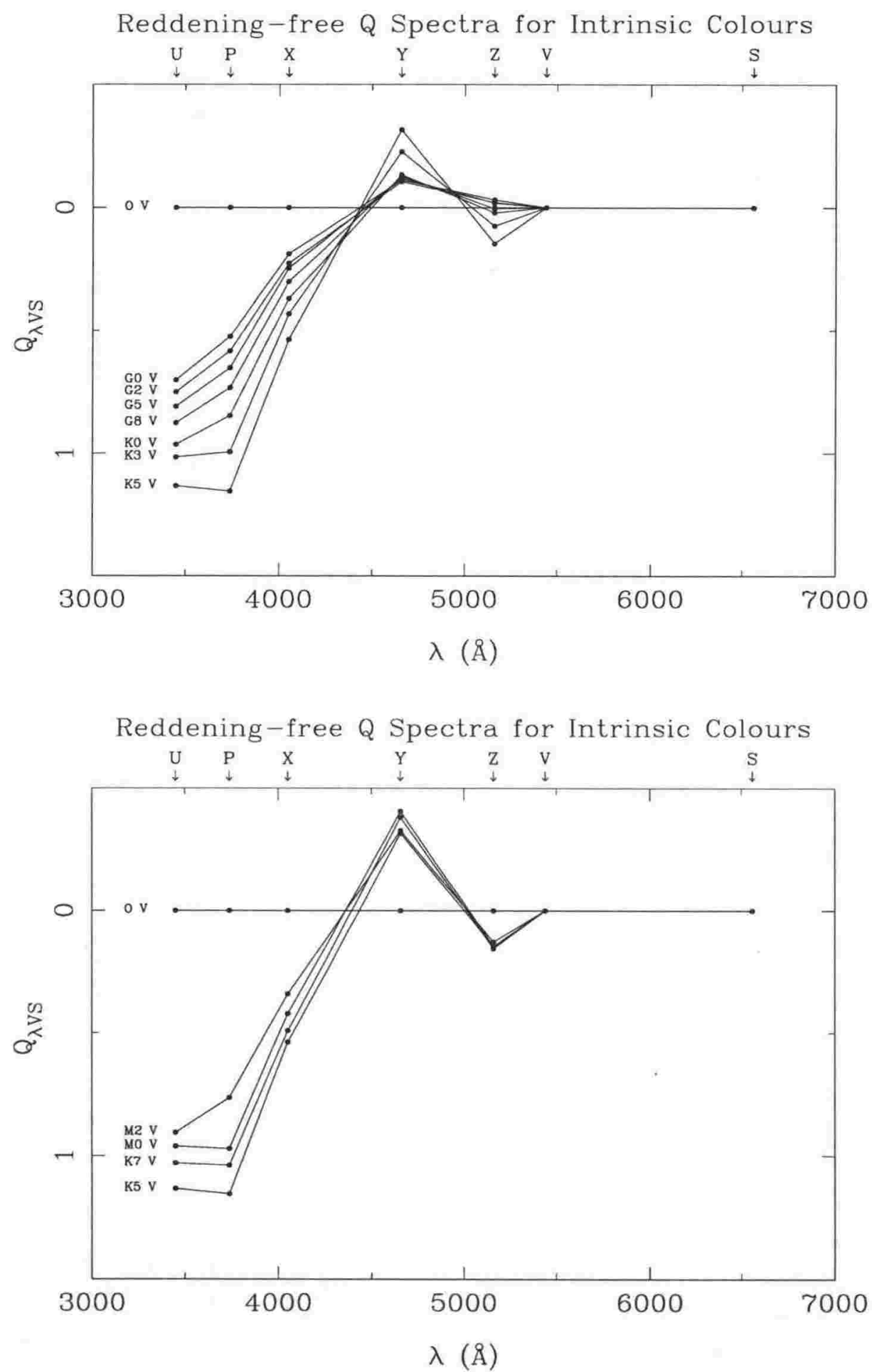

Figure 1.13: Variation of pseudo-Q-spectra with spectral class for main-sequence stars. The top figure shows the Q-Spectra for G-K stars, while the bottom figure shows K-M stars. 

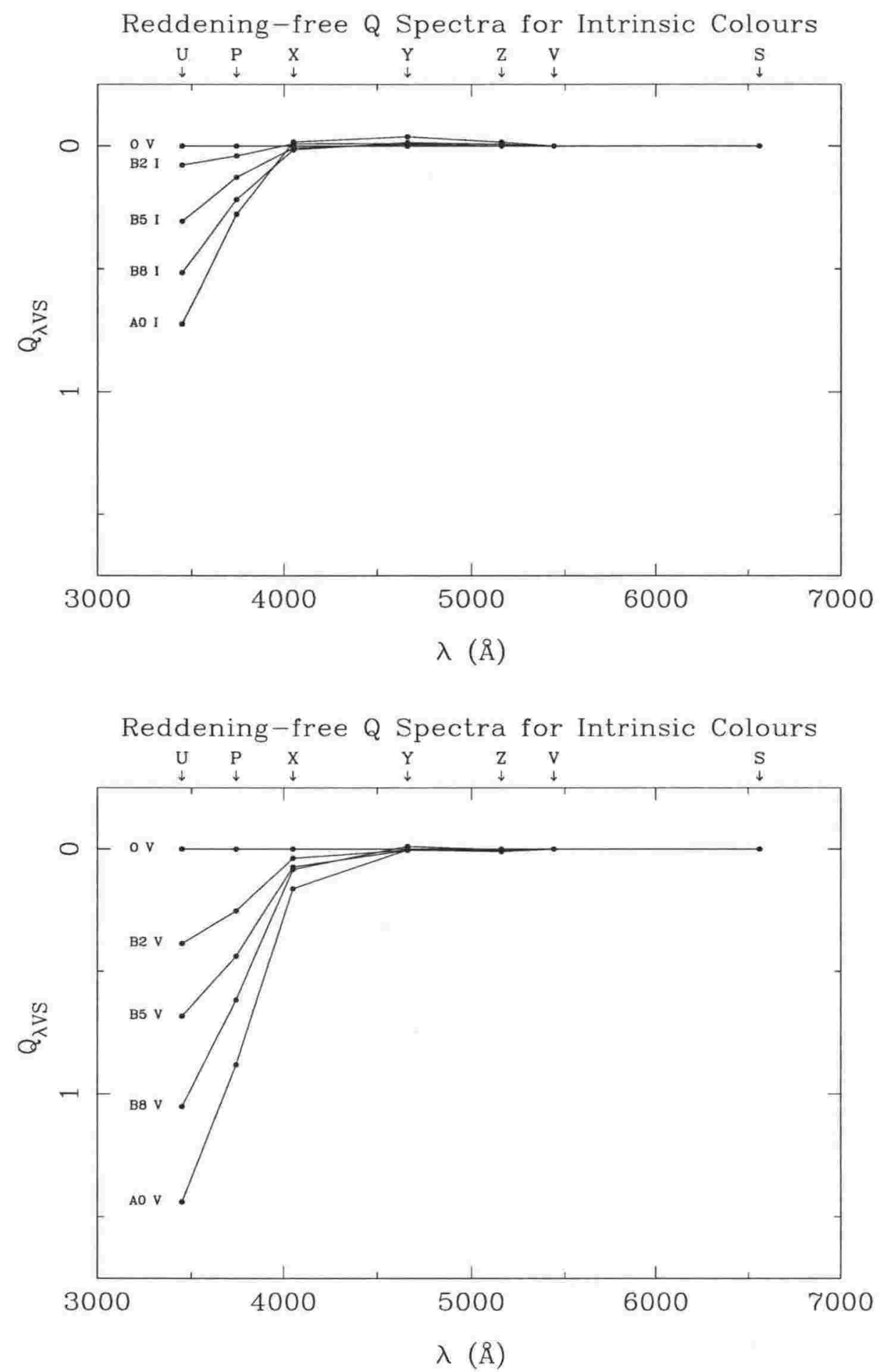

Figure 1.14: Variation of pseudo-Q-spectra with luminosity class. The top figure shows the Q-Spectra for O-A supergiants, while the bottom figure shows main-sequence stars of the same spectral classes. 

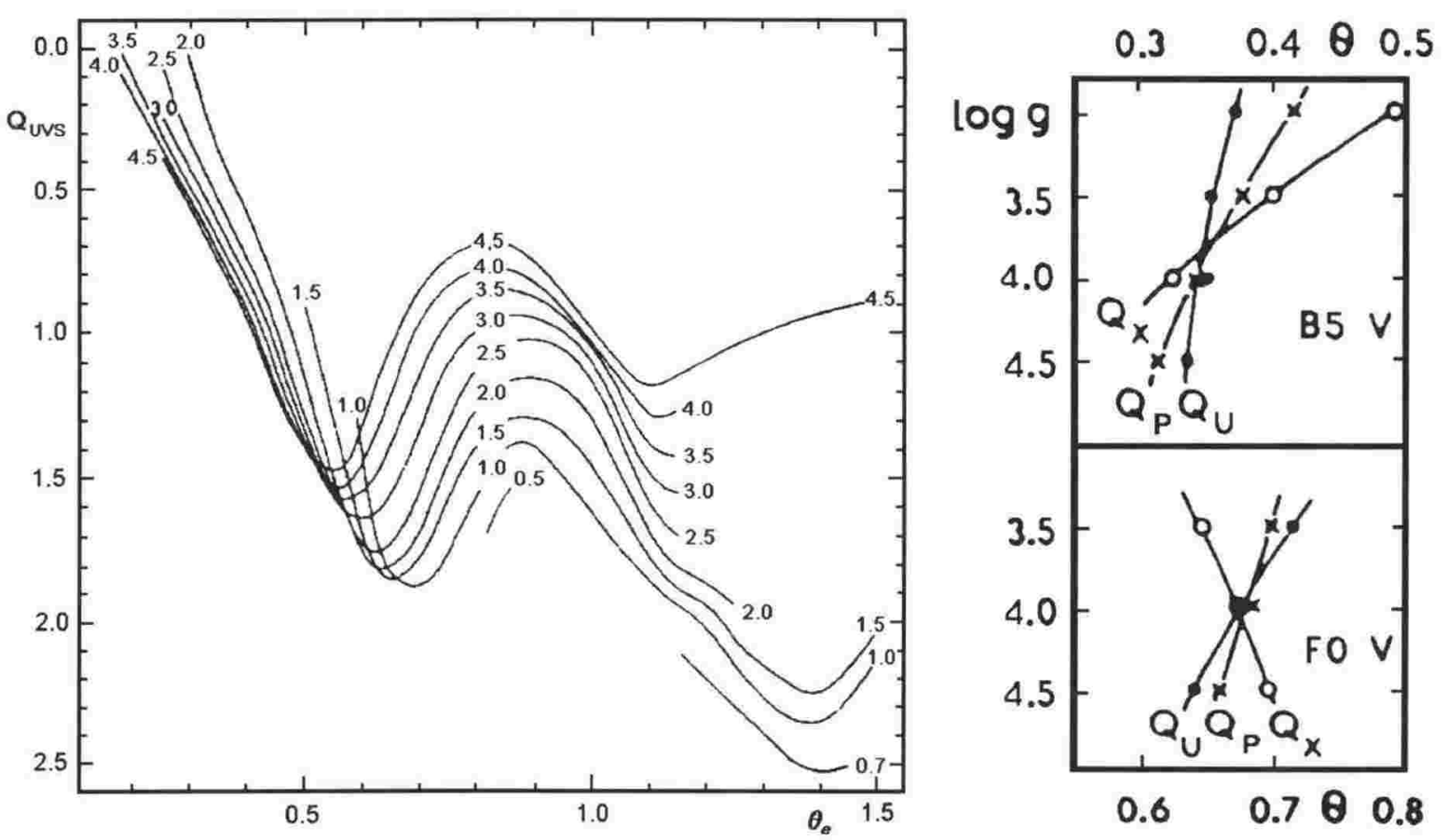

Figure 1.15: Examples of Q-isolines classifications. The left figure gives $\theta_{e}, \log (g)$ data points (for $Q_{\text {Uvs }}$ ) which are used to plot the Q-isolines in the right figure. Taken from Straižys (1978).

gravity $(g)$ of the star (Straižys 1978,1992$)$. This works by plotting in a $\theta_{e}\left(=5040 / T_{e}\right), \log (g)$ diagram five curves $\left(\mathrm{Q}\right.$-isolines) corresponding to $Q_{\mathrm{UVS}}=$ constant $_{1}, Q_{\mathrm{PVS}}=$ constant $_{2}$ etc., with the constants being the $Q$ factors of the star to be classified. Each isoline is defined by three - four $\theta_{e}, \log (g)$ points which can be read from the intersections of a $Q=$ constant line with $\log (g)=$ constant curves in diagrams such as Figure 1.15. The five isolines will intersect at a point corresponding to the $\theta_{e}$ and $\log (g)$ of the star, as shown by two examples also in Figure 1.15 .

Due to calibration and observational errors, the isolines will seldom intersect in one point, but will instead form a region whose area depends on the size of the errors. The $\theta_{e}, \log (g)$ 'point' defined by this region is given by weighted means, with the weights being the errors in each Q factor. According to Jasevičius (1986), this method agrees with the Q,Q classification method with mean square errors of \pm 0.8 spectral sub-classes and \pm 0.6 magnitudes. However, the method only works for normal stars as stars with peculiar energy distributions (for example subdwarves, metal-deficient stars, metallic-line stars, Ap stars, white dwarves or double stars) also give a larger intersection area. Fortunately a "trained investigator" can decide from the "character of deviations of different lines in the diagram" the peculiarity type of the star (Straižys 1978). 


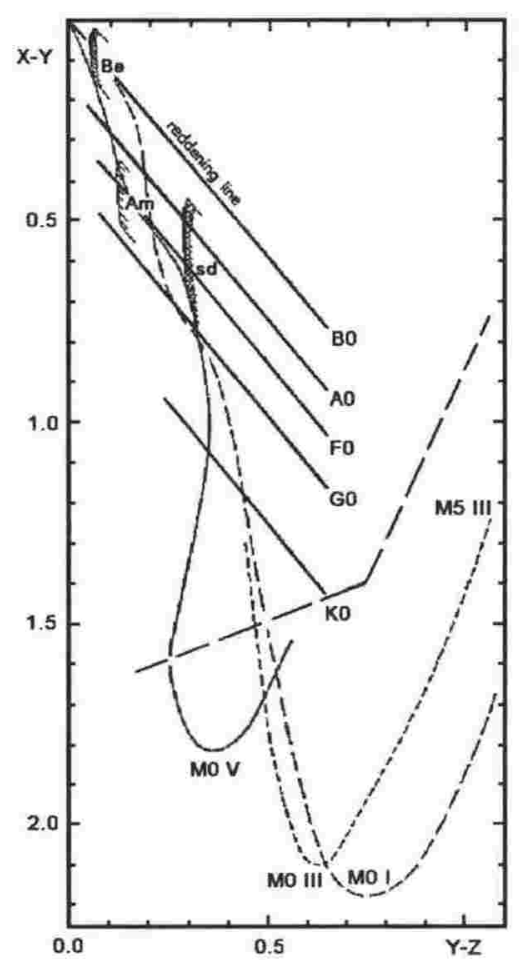

Figure 1.16: The $\mathrm{X}-\mathrm{Y}, \mathrm{Y}-\mathrm{Z}$ diagram is used to roughly classify the spectral type of a star. The curves indicate intrinsic sequences for main sequence (solid), giants (short dashes) and supergiants (long dashes). The parallel lines are reddening lines for $\mathrm{B} 0 \mathrm{~V}, \mathrm{AO} \mathrm{V}, \mathrm{F} 0 \mathrm{~V}, \mathrm{G} 0 \mathrm{~V}$ and $\mathrm{KO}$ $\mathrm{V}$ stars. The two-segment broken line separates M-type stars from earlier stars. The hatching indicates the blue limits of Beand Am-stars and subdwarfs. Taken from Straižys \& Sviderskienè (1972) (or Straižys (1992)).

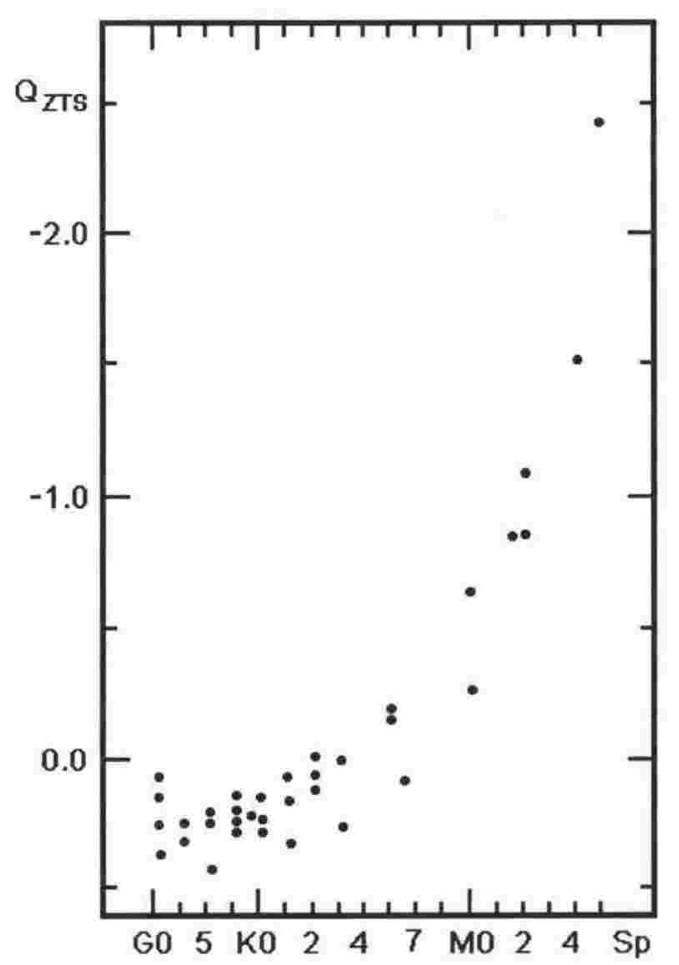

Figure 1.17: Separation of M-type stars from mixture of $\mathrm{K}$ and $\mathrm{M}$ stars found below the two-segment broken line in Figure 1.16. All M-type stars have $Q_{\text {ZTS }}<-0 \cdot 2$. Taken from Straižys \& Sviderskiene (1972).

\subsection{The QQ-diagrams Classification Method.}

The final classification method is called the 'QQ-diagrams' method as it uses calibrated diagrams of various $Q$ factors after sub-dividing into approximate spectral classes and removing peculiar stars, and is described by Straižys \& Sviderskiene (1972) and Straižys (1992).

The first step is to sub-divide the stars into approximate spectral classes using the star's (reddened) $\mathrm{X}-\mathrm{Y}$ and $\mathrm{Y}-\mathrm{Z}$ colours, as shown in Figure 1.16. The design of the Vilnius filter system ensured that the intrinsic sequences of normal stars (in this diagram) do not intersect any reddening lines, the reddening lines are at a large angle to the sequences and that $Q_{\mathrm{XYZ}}$ is (approximately) a function only of spectral class. 
The major exception to this are M-type stars, whose sequences show strong luminosity effects and bend back towards the $\mathrm{X}-\mathrm{Y}$ colours of earlier stars, and so may overlap with earlier stars that are reddened or of a different luminosity class. As M-type stars are only found below the two-segment broken line in the diagram, stars which satisfy

$$
(\mathrm{X}-\mathrm{Y})>\min \left\{\begin{array}{l}
1 \cdot 68-0.4(\mathrm{Y}-\mathrm{Z}) \\
3 \cdot 0-2 \cdot 1(\mathrm{Y}-\mathrm{Z})
\end{array}\right.
$$

(here referred to as 'possible M stars') must be checked if they are M stars before proceeding with the spectral groups classification.

The prototype Vilnius filters included a T filter, whose effective wavelength at $6250 \AA$ coincides with one of the TiO absorption bands found in M stars. Combining this with the $\mathrm{S}$ filter, which lies between two TiO bands, gives the depth of a TiO absorption band which increases with the spectral class (of M stars). To correct for reddening, a $\mathrm{Q}$ factor (using $\mathrm{T}$ and S) is needed - Straižys \& Sviderskienè (1972) use $Q_{\text {ZTS }}$ to separate M stars from earlier stars $\left(Q_{\mathrm{ZTS}}<-0 \cdot 2\right)$ in the group of 'possible M stars', and even approximately classify M stars, as shown in Figure 1.17.

However the final Vilnius system did not include the $\mathrm{T}$ filter, possibly because late $\mathrm{M}$ stars may be separated using combinations of the remaining filters. Straižys (1992) states that $Q_{\text {YSYV }}$ may be used for this, but the top diagram in Figure 1.18, using all 'possible M stars' from the Vilnius catalog (North 1980, Straižys \& Kazlauskas 1993), does not support this. However, other Q factors were found to allow separation of (late) M stars, for example $Q_{\mathrm{UXY}}, Q_{\mathrm{PXY}}$, $Q_{\mathrm{PYZ}}, Q_{\mathrm{PYS}}, Q_{\mathrm{XVS}}, Q_{\mathrm{ZVS}}$, and the bottom diagram of Figure 1.18. The discrimination between the $\mathrm{M}$ stars and others is slightly improved by combining two of the above $\mathrm{Q}$ factors, as shown by $Q_{\mathrm{ZVS}}, Q_{\mathrm{UXY}}$ in Figure 1.19 , where only $5 \%$ of the stars between $-0.55<Q_{\mathrm{ZVS}}<+0.05$ and $Q_{\mathrm{UXY}}>0.4-0.6 Q_{\mathrm{ZvS}}$ are mis-classified as M-type stars.

Once a star has been identified as a M star, it's spectral type and absolute (V) magnitude (or effective surface temperature and gravity) may be read off the calibrated $Q_{\mathrm{XYZ}}, Q_{\mathrm{Xzs}}$ diagrams shown in Figure 1.20.

After the M stars have been classified, the remaining stars may be placed in one of the four spectral groups according to

$$
\begin{array}{cr}
\text { B } & Q_{\mathrm{XYZ}} \leq 0.16 \\
\mathrm{~A}-\mathrm{F} & 0.16<Q_{\mathrm{XYZ}} \leq 0.40 \\
\mathrm{G} & 0.40<Q_{\mathrm{XYZ}} \leq 0.66 \\
\mathrm{~K} & 0.66<Q_{\mathrm{XYZ}}
\end{array}
$$

which are the reddening lines shown in Figure 1.16. 
Possible M-type stars

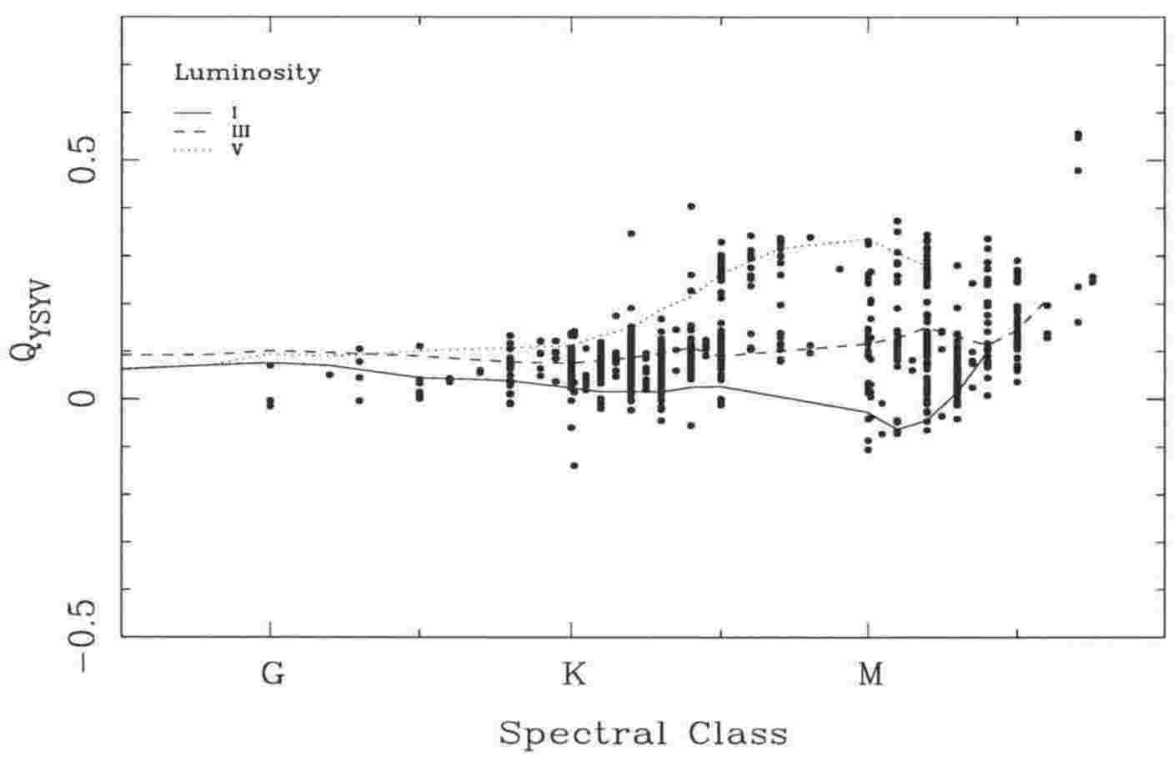

Possible M-type stars

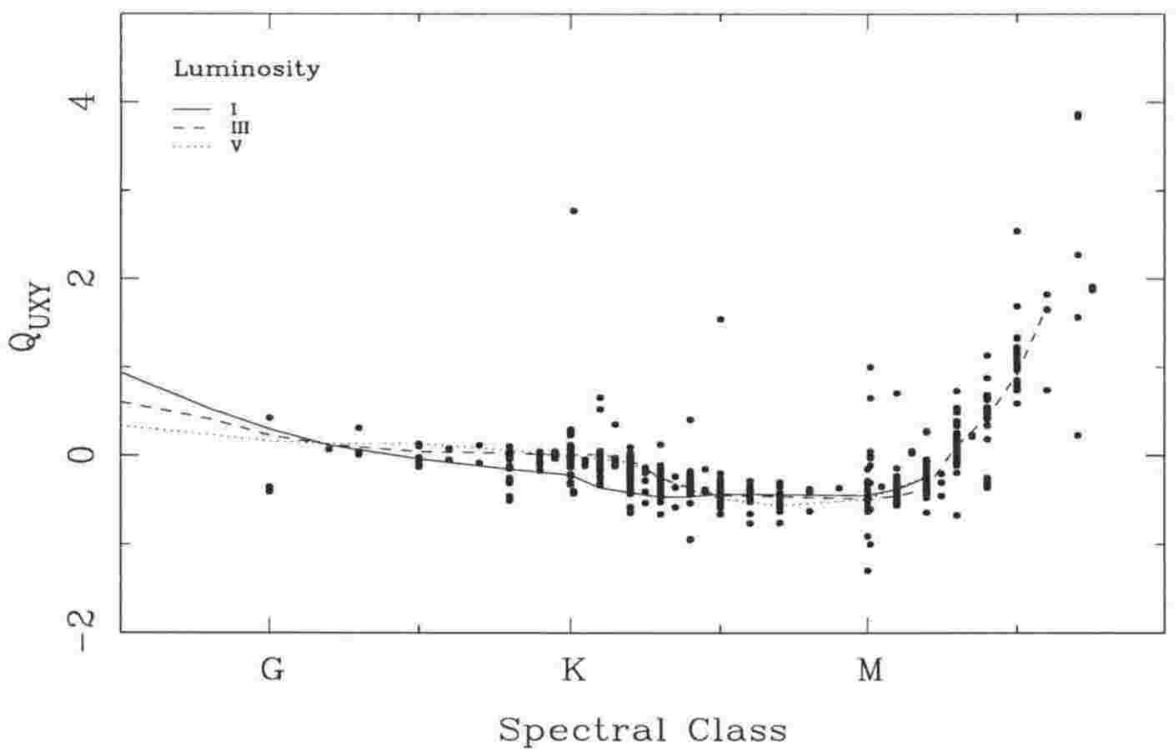

Figure 1.18: Alternative separation of M-type stars from mixture of $\mathrm{K}$ and $\mathrm{M}$ stars found below the two-segment broken line in Figure 1.16. The curves indicate intrinsic sequences for main sequence (solid), giants (long dashes) and supergiants (short dashes). Most late M-type stars have $Q_{\mathrm{UXY}} \geq 0.5$. 
Possible M-type stars

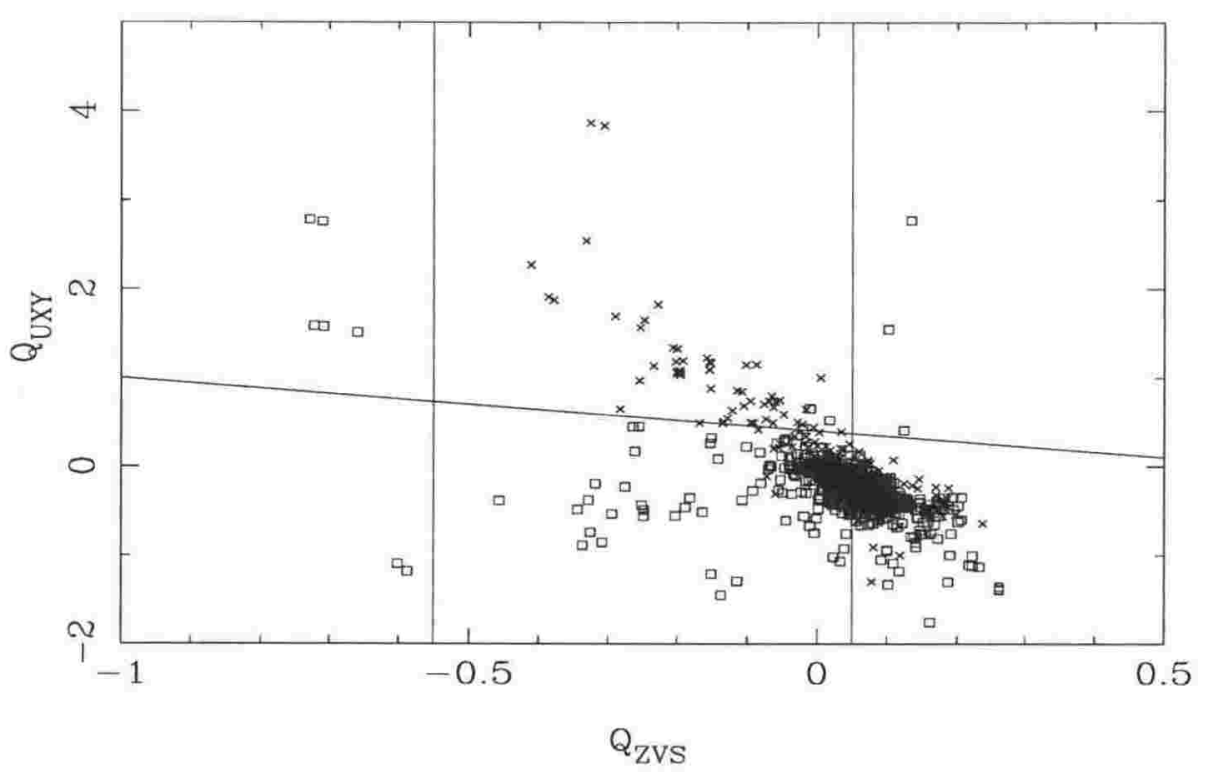

Figure 1.19: Alternative separation of M-type stars from mixture of $\mathrm{K}$ and $\mathrm{M}$ stars found below the two-segment broken line in Figure 1.16 continued. The M-type stars are crosses and the others are squares.

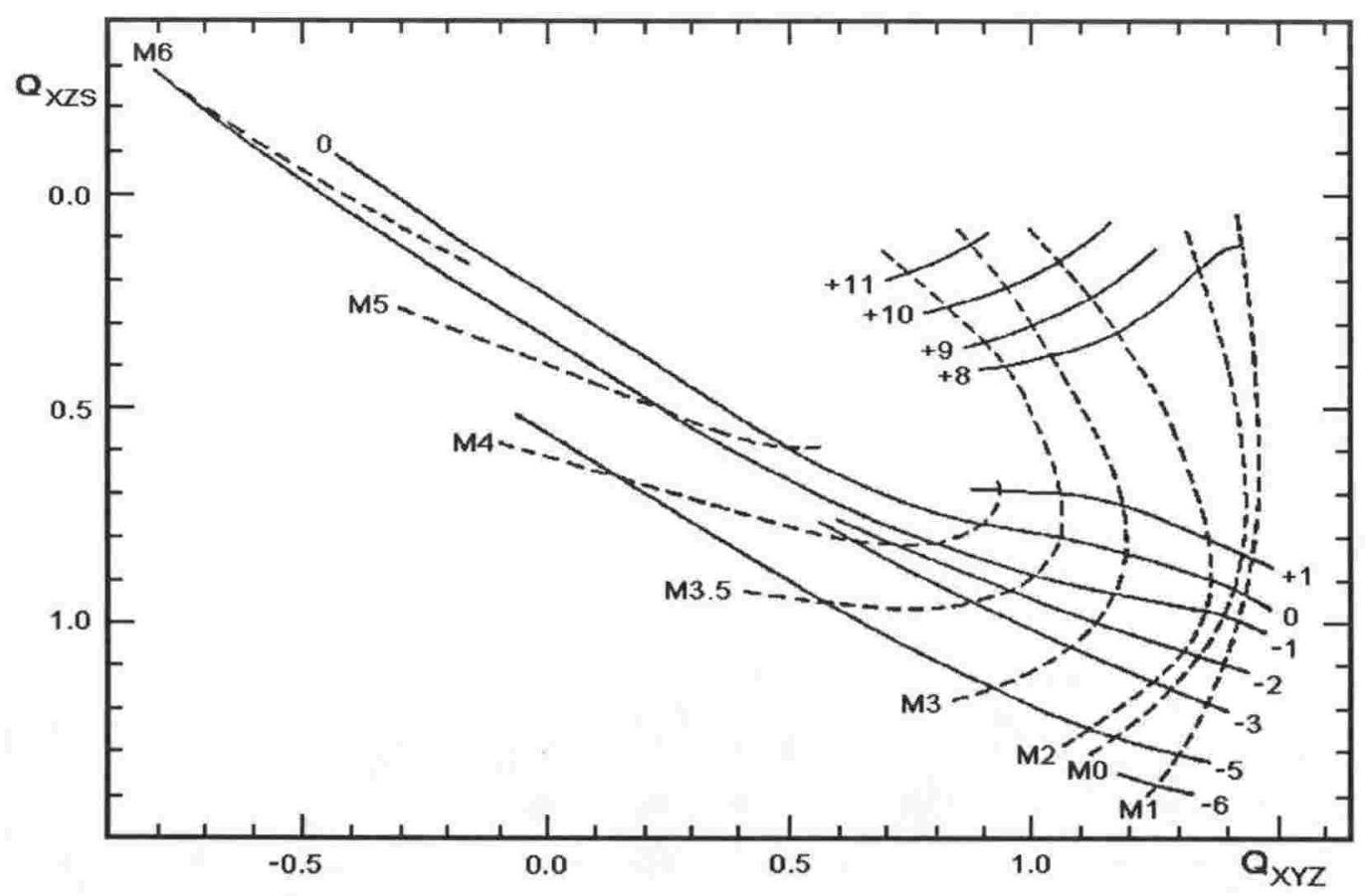

Figure 1.20: Calibrated $Q_{\mathrm{XYZ}}, Q_{\mathrm{Xzs}}$ diagrams for $\mathrm{M}$ star classifications, calibrated in spectral class and absolute magnitudes. Taken from Straižys (1992). 
The B group consists of normal O and B stars, A supergiants, Of, Be, Bp stars, OB subdwarfs, white dwarfs and extreme F subdwarfs. First the Be stars and F subdwarfs must be separated - the B group stars are divided in two groups B1 $\left(Q_{\mathrm{XYZ}} \leq 0 \cdot 10\right)$ and $\mathrm{B} 2\left(Q_{\mathrm{XYZ}} \geq 0 \cdot 10\right)$. The $\mathrm{B} 1$ group is plotted in a $Q_{\mathrm{XYZ}}, Q_{\mathrm{XzS}}$ diagram to separate the Be stars - see Figure 1.21. The B2 group is plotted in a $Q_{\mathrm{UYV}}, Q_{\mathrm{UXY}}$ diagram to separate the extreme F subdwarfs (Bartkevičius \& Straižys 1970a, Straižys 1992) - see Figure 1.22. The Bp stars (of Si type) may be separated in a Y-S, Q YZs diagram (Figure 1.23) and the white dwarfs in a Y-V, U-P diagram (Figure 1.24). The remaining stars are considered normal and are classified in calibrated $Q_{\mathrm{PYV}}, Q_{\mathrm{UPY}}$ diagrams (Figure 1.25).

The A-F group consists of normal A and F stars, Am and Ap stars, F and G subdwarfs and white dwarfs. The Am stars are recognized in a Y-V, $Q_{\mathrm{XYV}}$ diagram (Figure 1.26) and for greater reddening, in $Q_{\mathrm{XYV}}, Q_{\mathrm{UPY}}$ and X-Y,U-X diagrams (Bartkevičius \& Straižys 1970b). The Ap stars (of CrSrEu type) can be recognized in Figure 1.23. The white dwarfs and FG subdwarfs may again be separated in Figures 1.24 and 1.22. The remaining normal stars are classified in the calibrated $Q_{\mathrm{XYV}}, Q_{\mathrm{UPY}}$ diagram in Figure 1.27. As the spectral type or temperature isolines are too close together or overlapping for A5-7 III-V stars, these are classified in a $Q_{\mathrm{UPYV}}, Q_{\mathrm{UXY}}$ diagram instead (Figure 1.28).

The G group consists of normal G type stars, G and K subdwarfs, metal-deficient giants, $\mathrm{CH}$ and barium stars. The subdwarfs may be separated as before using Figure 1.22. Barium and CH stars are separated in a QXzS, QUPYV diagram (Figure 1.29). The metal-deficient giants are separated using $Q_{\mathrm{UXY}}, Q_{\mathrm{UYV}}$ and $Q_{\mathrm{PYZ}}, Q_{\mathrm{XYZ}}$ diagrams (see Figure 1.30). All the subdwarfs found earlier (in the B, A-F and G groups) may be classified in $\mathrm{T}_{\text {eff }}$ and $[\mathrm{Fe} / \mathrm{H}]$ using a calibrated $Q_{\mathrm{UXY}}, Q_{\mathrm{UYV}}$ diagram (see Figure 1.31). The remaining normal G stars are classified using calibrated $Q_{\mathrm{XZS}}, Q_{\mathrm{UPY}}$ diagrams (Figure 1.32).

The K group consists of normal K dwarfs, G8-K giants, G5-K supergiants, K subdwarfs, metal-deficient giants, and R, $\mathrm{CH}$ and barium stars. The subdwarfs, metal-deficient giants and carbon rich stars are separated using the same diagrams as before (Figures 1.22, 1.30 and 1.29 respectively). The remaining normal stars are classified in a calibrated $Q_{\mathrm{XYZ}}, Q_{\mathrm{XZS}}$ diagram (see Figure 1.33).

Initially a G0 V spectral type is assumed for each star, so the colour excesses may be found (see Table 1.5) and the $\mathrm{Q}$ factors calculated. The classification procedure is then repeated using the spectral type found in the previous iteration. More precise colour excess ratios (for the later iterations) are given in Appendix J, copied from Straižys (1992) and Kurilienè \& Sūdžius (1974).

The different Vilnius classification methods have largely complementary strengths and weaknesses, so classifications will generally be made using as many of the methods as possible and 


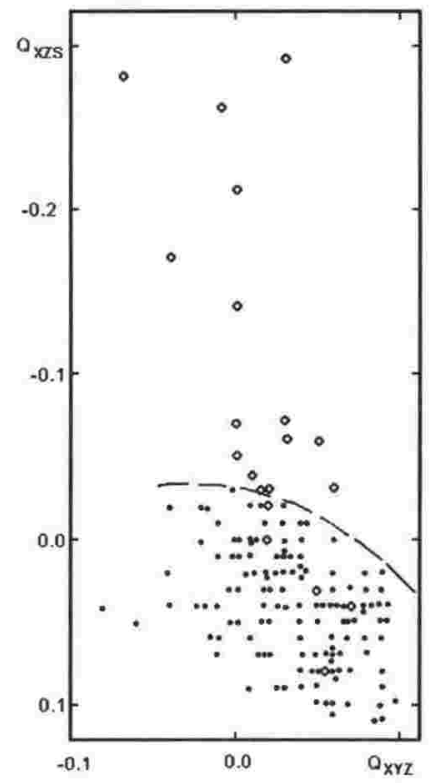

Figure 1.21: Separation of $\mathrm{Be}$ stars (circles) from normal B stars (dots) of different luminosities. The dashed line shows the limit of normal stars. Taken from Straižys \& Sviderskiene (1972) (or Straižys (1992)).

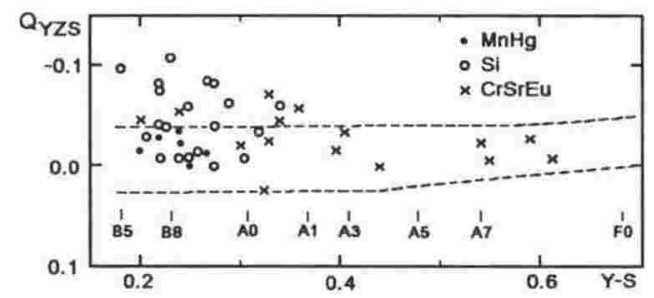

Figure 1.23: Separation of Ap and Bp stars from normal stars. Here $Q_{\mathrm{YZS}}=\mathrm{Y}-\mathrm{Z}-$ $0 \cdot 54(\mathrm{Z}-\mathrm{S})$. The normal stars of luminosities III, IV, and V are between the dashed lines, and the positions of the main sequence spectral classes are marked. Taken from Straižys (1992).

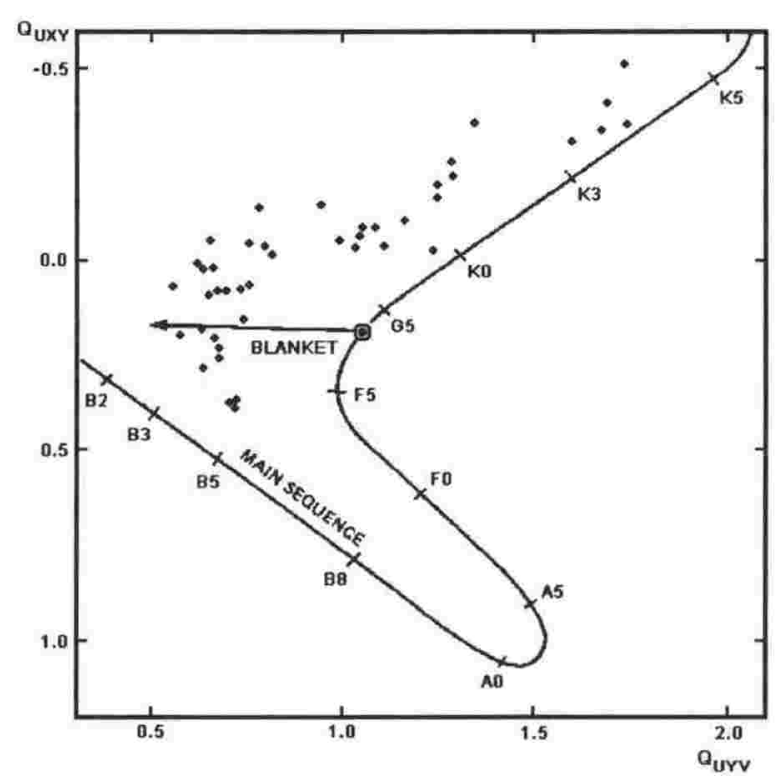

Figure 1.22: Separation of subdwarves from normal stars. The arrow is the blanketing vector for the Sun. Taken from Straižys (1992).

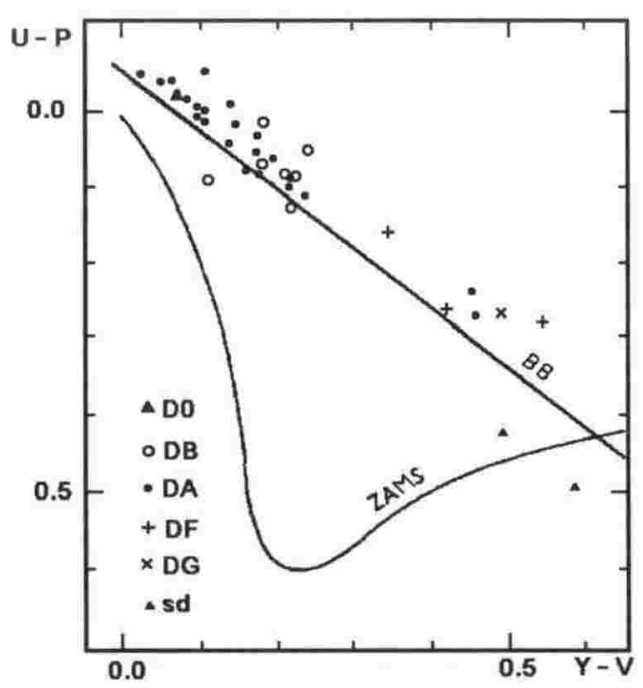

Figure 1.24: Separation of white dwarves from normal stars. The main sequence and black body curves are shown. The triangles are extreme $\mathbf{F}$ subdwarves. Taken from Straižys (1992). 

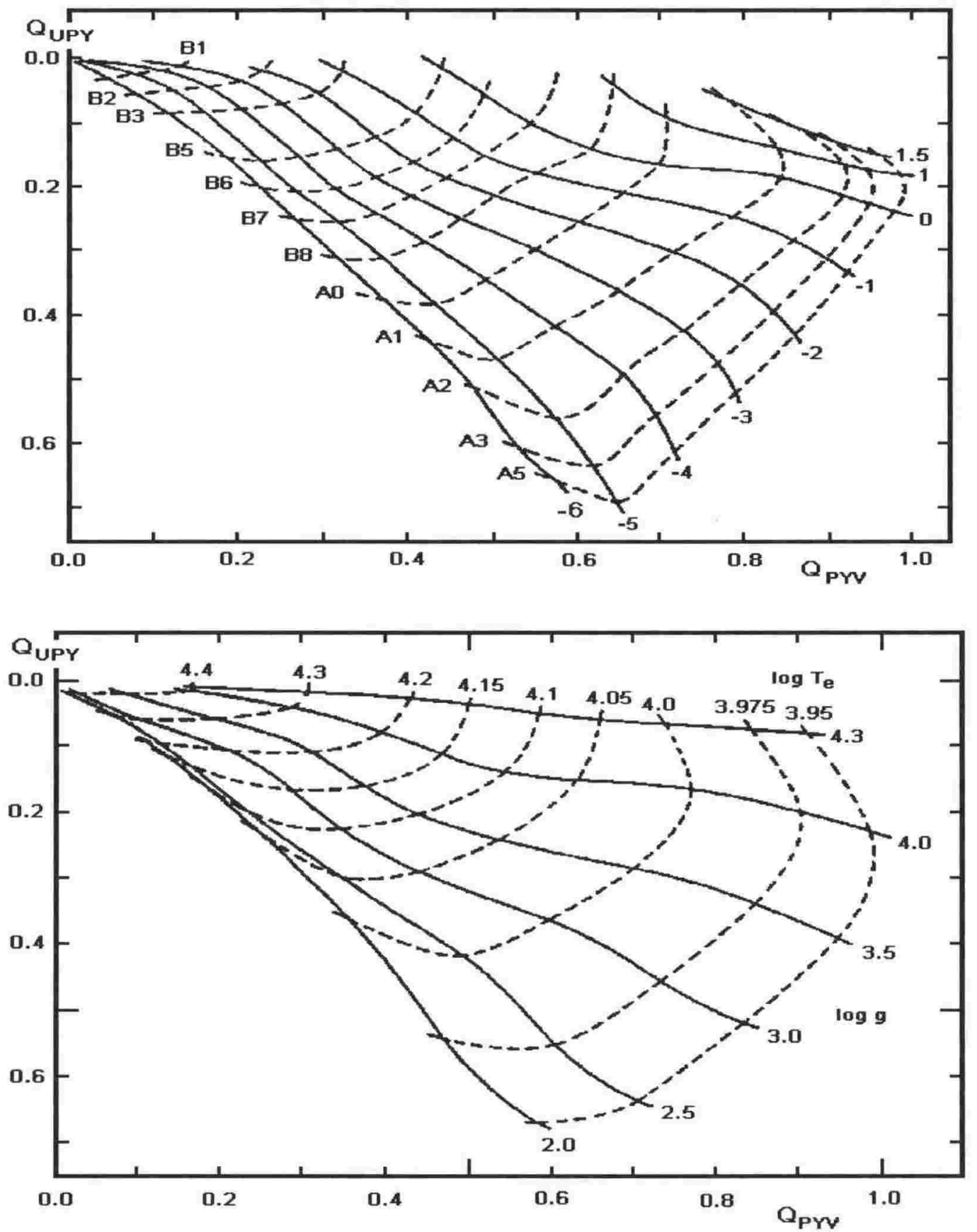

Figure 1.25: Classification of normal B stars using $Q_{\mathrm{PYV}}, Q_{\mathrm{UPY}}$ diagrams calibrated in spectral class and absolute magnitude (top figure) and effective temperature and gravity (bottom figure). Taken from Straižys (1992). 


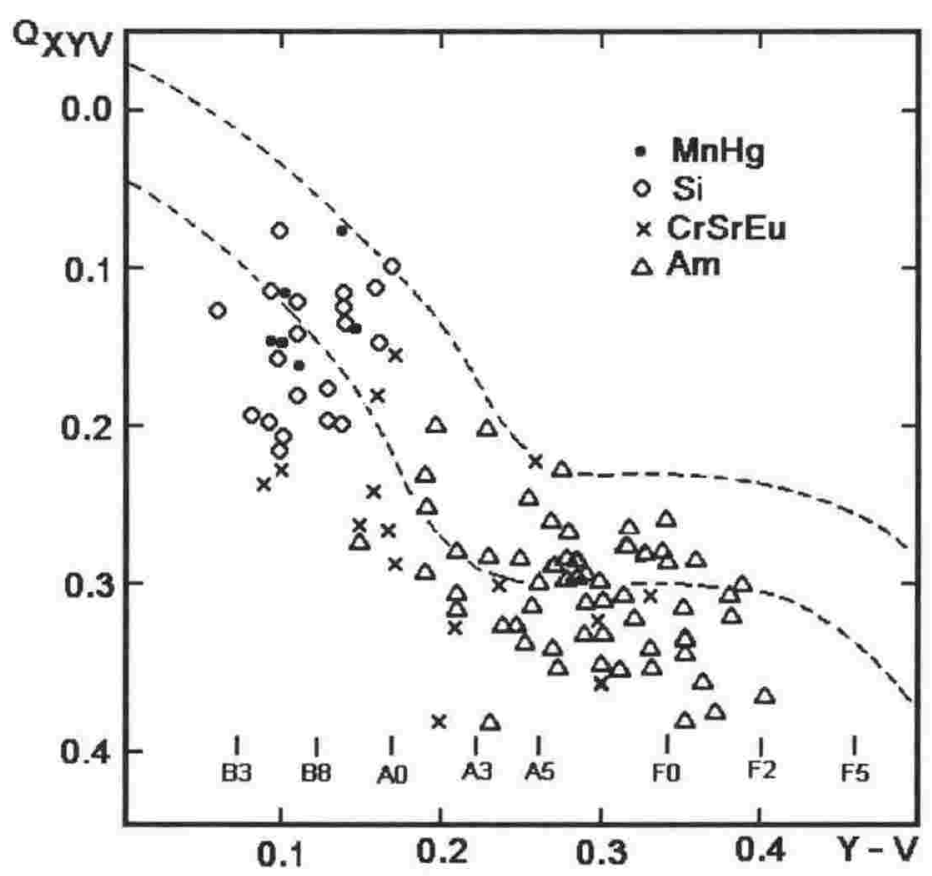

Figure 1.26: Metallic-line, Ap and Bp stars. The normal stars of luminosities III, IV, and V are between the dashed lines, and the positions of the main sequence spectral classes are marked. Taken from Straižys (1992).

an average taken for the spectral class.

\subsection{Summary.}

Much information about stellar surfaces and material along the observer's line of sight can be gained from the electro-magnetic radiation emitted by a star. Ideally the entire e-m spectrum should be measured at fine wavelength resolution (spectroscopy). In practice, only limited wavelength ranges can be measured (due to high opacity wavelength regions in the Earth's atmosphere and the need for a variety of detectors for different wavelength regions). Further,

\begin{tabular}{|c|c|c|c|c|c|c|c|c|}
\hline Sp. & $\frac{E E_{U-P}}{U_{n}}$ & $\frac{E_{\mathrm{P}-\mathrm{Y}}}{\Gamma}$ & $\frac{E_{X-Y}}{\mathrm{X}}$ & $\frac{E_{X-Y}}{X}$ & $E_{\mathrm{X}-\mathrm{z}}$ & $\frac{E_{U-X}}{n_{U}}$ & $E_{U-Y}$ & $\frac{E_{P}-Y}{P}$ \\
\hline B & $\frac{E_{P}-Y}{0.43}$ & $\frac{E_{Y}-\mathrm{V}}{1.12}$ & $\frac{E_{Y-V}}{0.75}$ & $\frac{E_{Y-Z}}{1.16}$ & $\frac{E_{\mathrm{Z}-\mathrm{s}}}{116}$ & $\frac{E X-Y}{15}$ & $\frac{E_{\mathrm{Y}}-\mathrm{V}}{160}$ & $\frac{E_{Y-z}}{173}$ \\
\hline A & 0.45 & 109 & 0.73 & 1.14 & 1.15 & 1.15 & 1.59 & 169 \\
\hline F & 0.41 & 1.11 & 0.73 & 1.14 & 1.15 & 1.15 & 1.58 & 1.74 \\
\hline G & 0.38 & 1.15 & 0.73 & 1.13 & 1.13 & 1.18 & 1.58 & 1.79 \\
\hline $\mathrm{K}$ & 0.37 & 1.15 & 0.70 & 1.11 & 1.12 & 1.23 & 1.57 & 1.79 \\
\hline M & 0.35 & 1.14 & 0.70 & 1.06 & 1.10 & 1.20 & 1.54 & 1.77 \\
\hline
\end{tabular}

Table 1.5: Approximate colour excess ratios for initial spectral classification. Taken from Straižys (1992, Table 75). 

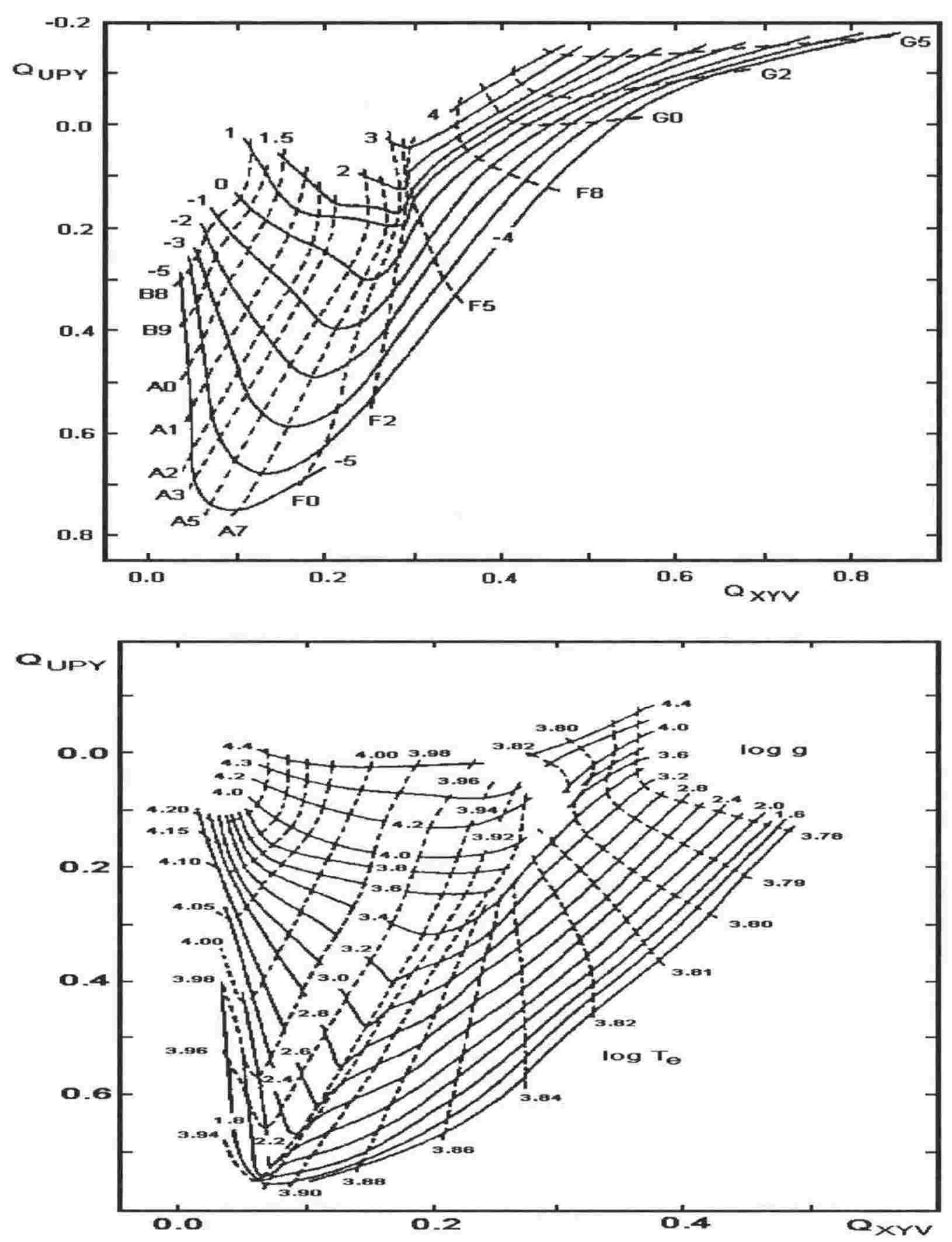

Figure 1.27: Classification of normal A-F stars using $Q_{\mathrm{XYV}}, Q_{\mathrm{UPY}}$ diagrams calibrated in spectral class and absolute magnitude (top figure) and effective temperature and gravity (bottom figure). Taken from Straižys (1992). 

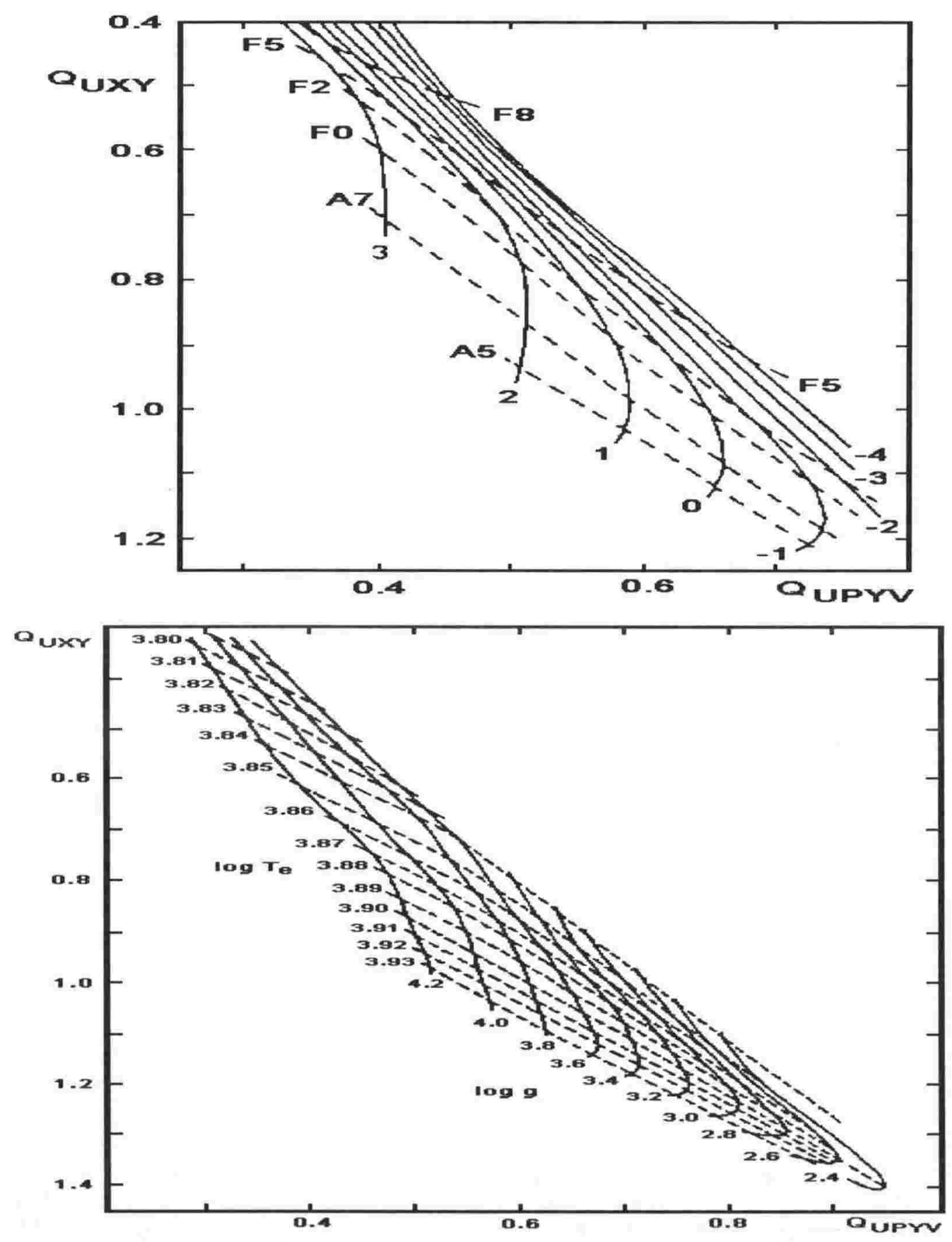

Figure 1.28: Classification of normal A5-7 III-V stars using $Q_{\mathrm{UPYV}}, Q_{\mathrm{UXY}}$ diagrams calibrated in spectral class and absolute magnitude (top figure) and effective temperature and gravity (bottom figure). Taken from Straižys (1992). 


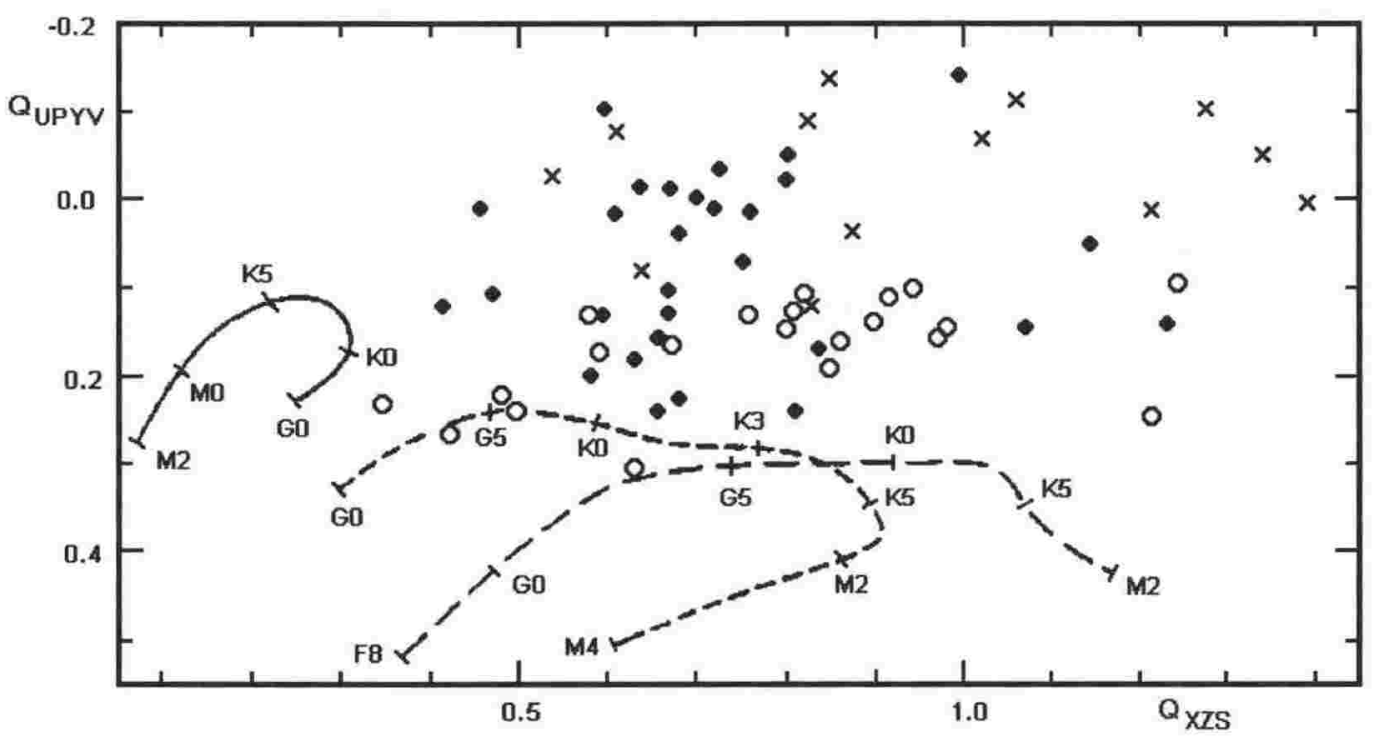

Figure 1.29: $\mathrm{CH}$ (crosses), barium (circles) and R-type (dots) stars. The curves are the main sequence (solid), giant sequence (short dashes) and supergiant sequence (long dashes). Taken from Straižys (1992).
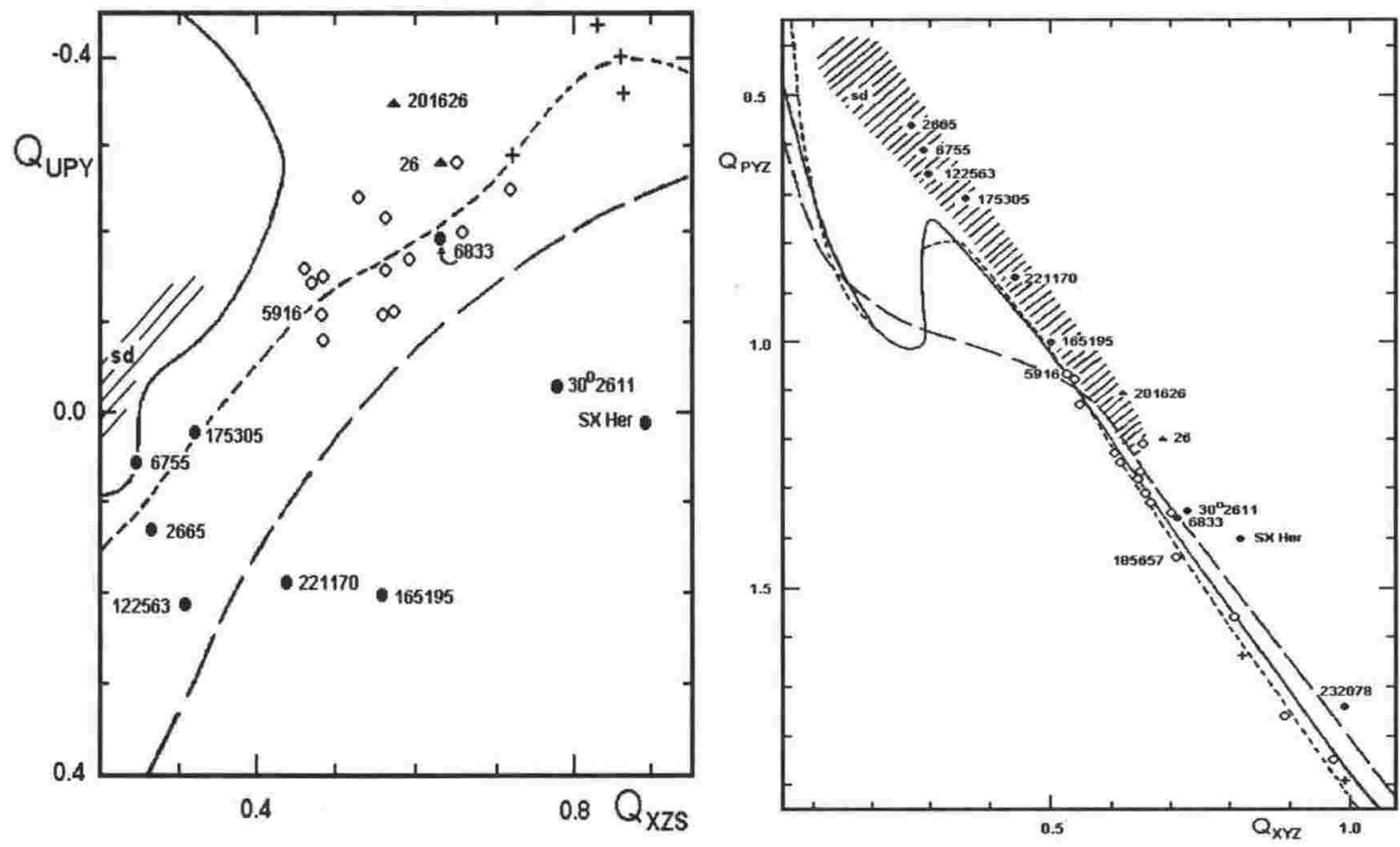

Figure 1.30: Separation of metal-deficient giants from normal stars. Pluses are CN stars, triangles $\mathrm{CH}$, circles $-0.3>[\mathrm{Fe} / \mathrm{H}]>-0.7$, and dots are $-0.7>[\mathrm{Fe} / \mathrm{H}]$. The main sequence (solid line), giant sequence (short dashes) and supergiants (long dashes) are also shown, and the shaded region are subdwarves. Taken from Bartkevičius \& Straižys $(1970 c)$. 


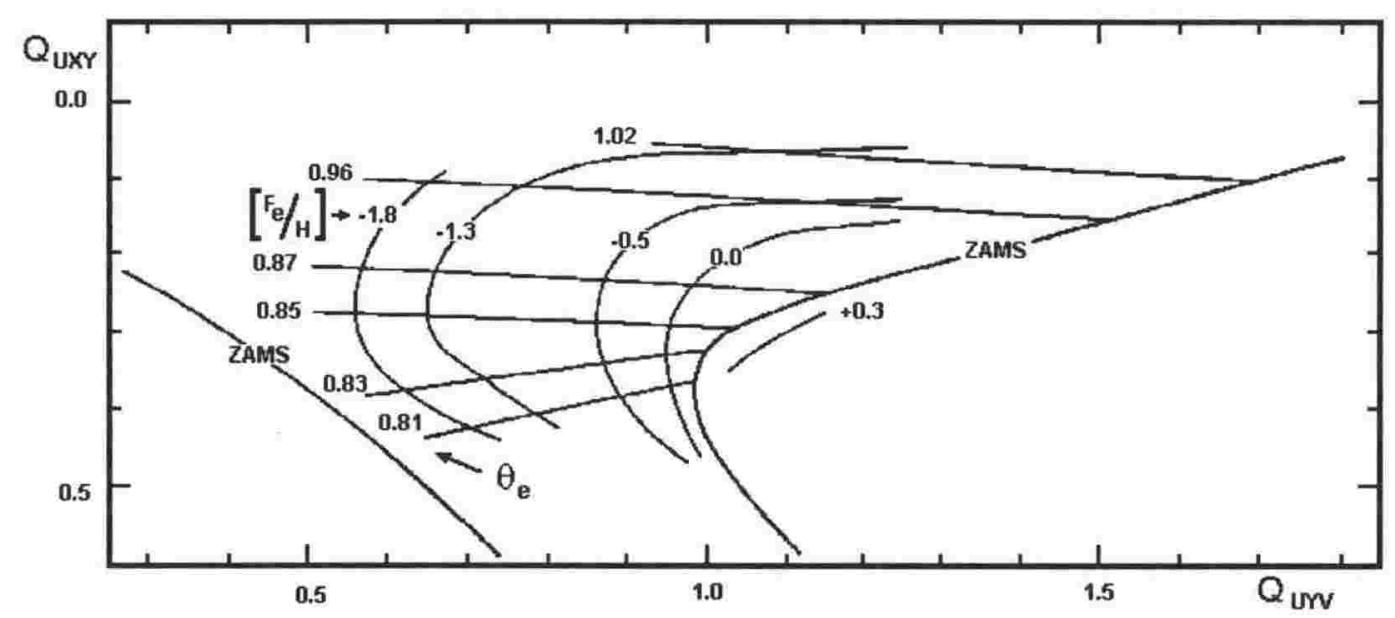

Figure 1.31: Classification of subdwarves using a $Q_{\mathrm{UXY}}, Q_{\mathrm{UYV}}$ diagram calibrated in metallicity and effective temperature. Taken from Bartkevičius \& Straižys (1970a).

to obtain adequate signal to noise in the time available only the brightest stars can be measured at fine resolution in these wavelength 'windows' and only by using large telescopes (which are expensive and difficult to obtain observing time on). Thus many observers are required to make measurements with coarse wavelength resolution (photometry) using smaller telescopes.

There are a large number of photometric systems in use, most being designed to investigate a particular question and so being restricted to use on a small subset of types of stars (if the 'purity' of the system is not good, then a survey phase will be needed to reject the stars for which the system was not intended). To allow a meaningful analysis of measurements made in any photometric system, and to enable other observers to duplicate the original system, a system must be described (numerically) by the transmission spectrum through each filter. In addition, a description of the components making up the system - telescope/mirrors size and type, filter composition (glass or interference filter type and thickness), detector type and operating conditions - is useful as a starting point to duplicate the system. Further, the reduction procedure to transform the raw measurements to those equivalent to the original system (but above the Earth's atmosphere) must be described - this will depend on the bandwidth, number of filters and wavelength spacing between the filters and requires a large number of stars accurately measured in the original system for use as 'standard' stars. Finally, measurements in the system should be calibrated against astrophysical parameters relevant to the investigation, for instance the surface temperature, gravity, chemical composition, rotation rate, and/or magnetic field strength of stars.

To illustrate these points, and to allow a comparison with the photometric system chosen for this study (the Vilnius system), two of the most commonly used systems (the Johnson and Strömgren systems) were reviewed together with the Vilnius system. The Vilnius system is 

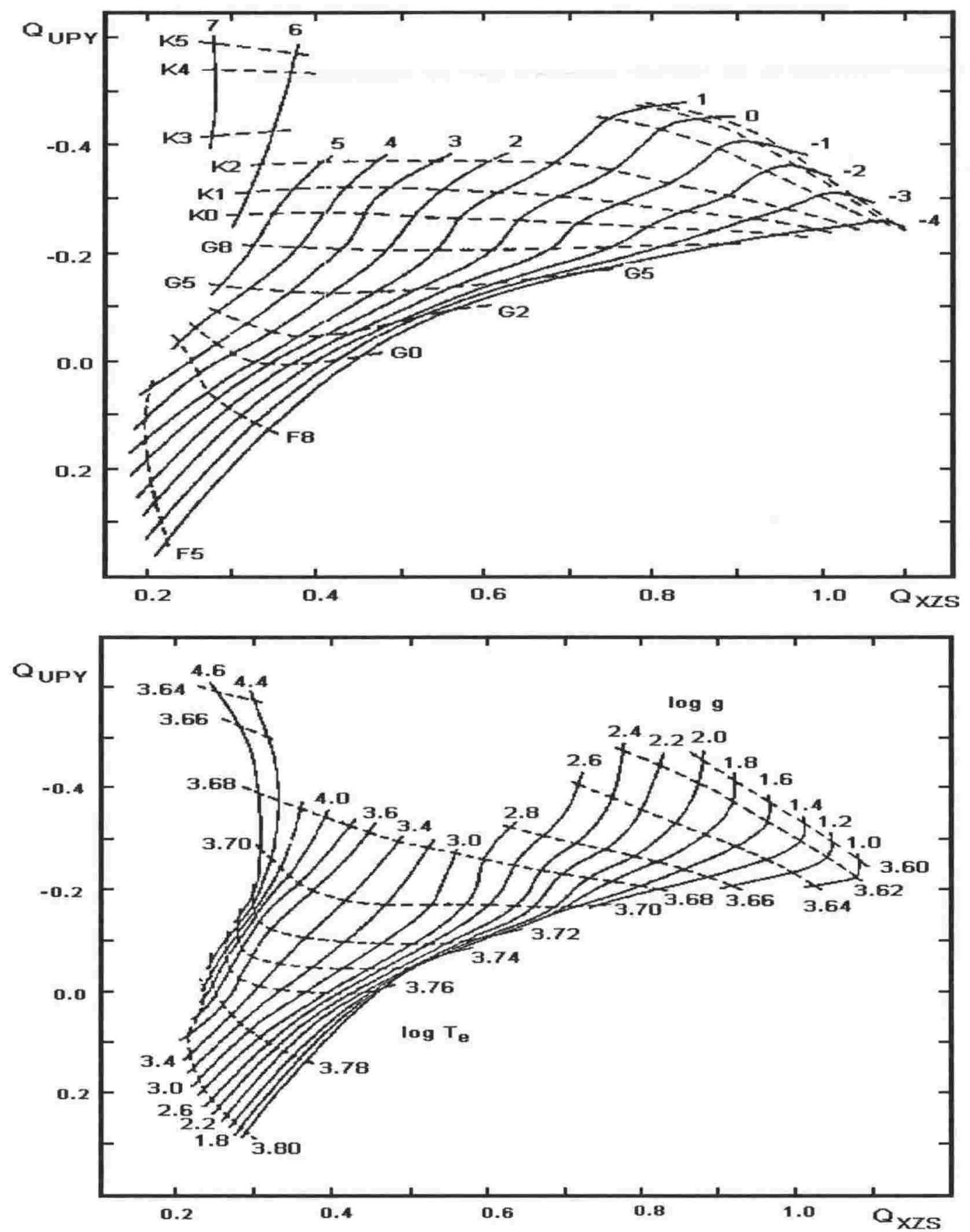

Figure 1.32: Classification of normal G stars using $Q_{\mathrm{XZS}}, Q_{\mathrm{UPY}}$ diagrams calibrated in spectral class and absolute magnitude (top figure) and effective temperature and gravity (bottom figure). Taken from Straižys (1992). 

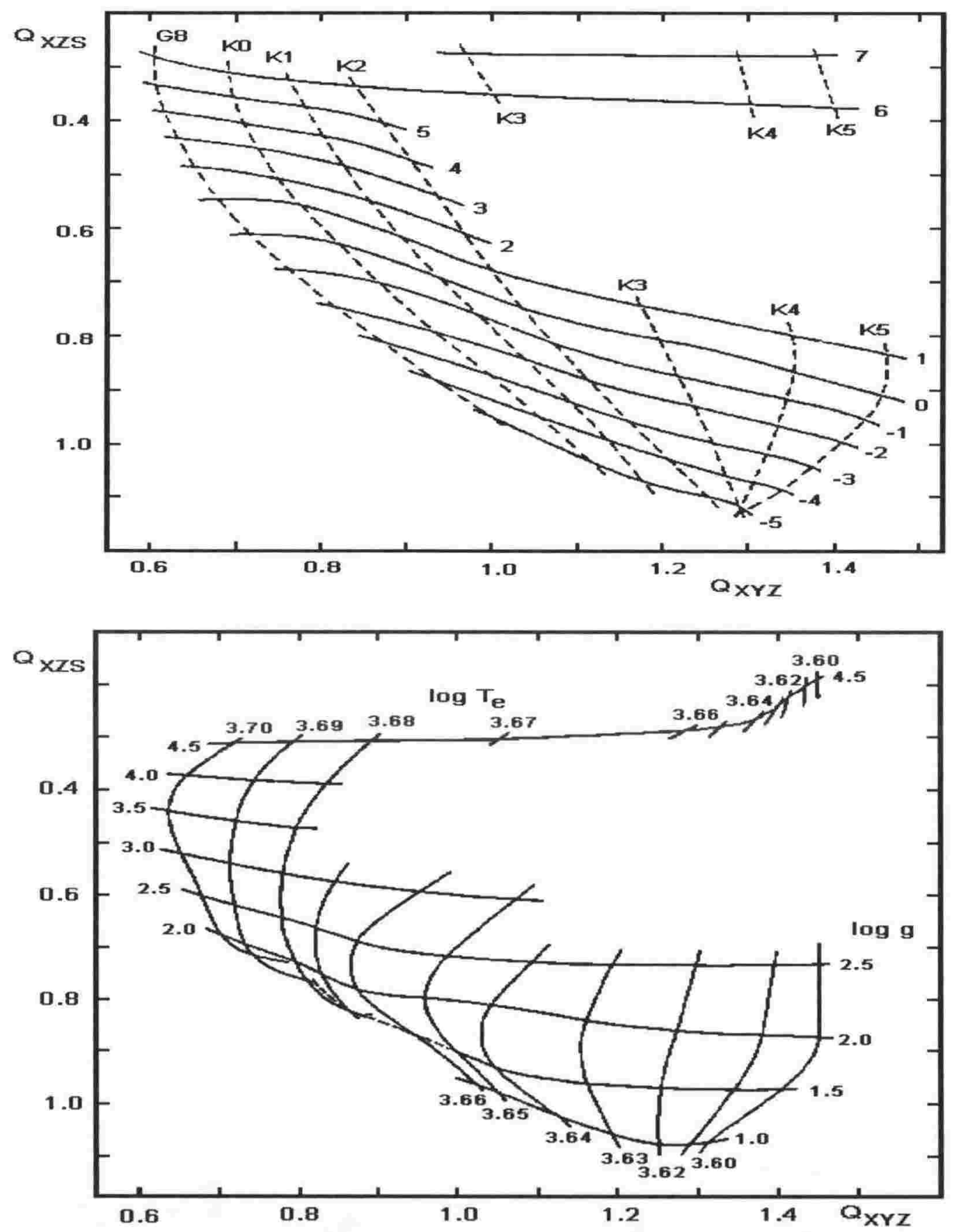

Figure 1.33: Classification of normal K stars using $Q_{\mathrm{XYZ}}, Q_{\mathrm{XZS}}$ diagrams calibrated in spectral class and absolute magnitude (top figure) and effective temperature and gravity (bottom figure). Taken from Straižys (1992). 
notable as being the first designed for MK classification and measurement of the main physical parameters of all normal stars, using the then recently available stellar spectra to synthesize magnitudes and colours to investigate the system's properties. It was also found to have a high detection rate of peculiar stars (i.e. high purity), virtually eliminating the need for a survey phase of any investigation. The MK classification can be done via several complementary methods based on the reddening-free $Q$ factors.

A summary of the differences between the three photometric systems is given in Table 1.6. The wavelength range of each system is taken as the difference in effective wavelengths of the bluest and reddest filters. Some of the comparisons are necessarily subjective, but show why the Vilnius system was chosen for use at Victoria University of Wellington (VUW) and Carter Observatory. The only major shortcoming of this system (relative to the others) is the small number of stars observed and lack of standard stars in the southern hemisphere - the latter being precisely what this study intended to remedy. Note also that there have been no critical studies of transformations of local systems to the standard Vilnius system, hence the question marks. 
Table 1.6: Comparison of the UBV, uvby, and Vilnius Systems.

\begin{tabular}{|c|c|c|c|}
\hline & UBV & $u v b y$ & Vilnius \\
\hline number of filters & 3 & 4 & 7 \\
\hline bandwidth of filters & wide & intermediate & intermediate \\
\hline wavelength range & $1600 \AA$ & $2000 \AA$ & $3100 \AA$ \\
\hline $\begin{array}{l}\text { critical sampling of } \\
\text { spectrum }\end{array}$ & nearly & under-sampled & $\begin{array}{l}\text { mixture of } \\
\text { under-sampling and } \\
\text { nearly critical }\end{array}$ \\
\hline $\begin{array}{l}\text { intended range of } \\
\text { stars }\end{array}$ & ? & normal B-A-F & all normal stars \\
\hline limiting magnitude & faint & bright & moderately bright \\
\hline $\begin{array}{l}\text { number of stars } \\
\text { observed }\end{array}$ & $\sim 90000$ & $\sim 40000$ & $\sim 7000$ \\
\hline description of system & poor & good & good \\
\hline $\begin{array}{l}\text { stability of } \\
\text { instrumental system }\end{array}$ & poor & good & good \\
\hline red leaks & major & none & none \\
\hline $\begin{array}{l}\text { clones/extensions to } \\
\text { the system }\end{array}$ & many & some & one (VilGen) \\
\hline $\begin{array}{l}\text { atmospheric } \\
\text { extinction correction } \\
\text { procedure }\end{array}$ & $\begin{array}{l}\text { systematic errors, } \\
\text { especially in U }\end{array}$ & $\begin{array}{l}\text { small systematic } \\
\text { errors in } c_{1} \text { and } u-b\end{array}$ & good \\
\hline standard star lists & $\begin{array}{l}\text { whole sky } \\
\text { distribution, } \\
\text { non-homogeneous lists }\end{array}$ & $\begin{array}{l}\text { whole sky } \\
\text { distribution, } \\
\text { non-homogeneous lists }\end{array}$ & $\begin{array}{l}\text { not in southern } \\
\text { hemisphere, } \\
\text { homogeneous (?) }\end{array}$ \\
\hline $\begin{array}{l}\text { standardisation } \\
\text { transformation }\end{array}$ & systematic errors & systematic errors & $?$ \\
\hline $\begin{array}{l}\text { dereddening } \\
\text { procedure }\end{array}$ & poor & good & excellent \\
\hline parameters measured & $\begin{array}{l}\text { Balmer jump, } \\
\text { temperature, } \\
\text { apparent magnitude }\end{array}$ & $\begin{array}{l}\text { Balmer jump, } \\
\text { temperature, } \\
\text { metallicity, } \beta \rightarrow \\
\text { absolute magnitude }\end{array}$ & $\begin{array}{l}\text { Balmer jump, } \\
\text { temperature, } \\
\text { metallicity, } \\
\text { apparent magnitude }\end{array}$ \\
\hline $\begin{array}{l}\text { purity/ability to do } \\
\text { MK classifications }\end{array}$ & bad & moderate & good \\
\hline $\begin{array}{l}\text { calibration of physical } \\
\text { parameters }\end{array}$ & poor & good & good \\
\hline $\begin{array}{l}\text { detection of peculiar } \\
\text { stars }\end{array}$ & poor & moderate & good \\
\hline
\end{tabular}




\section{Chapter 2}

\section{Observation and Reduction Techniques.}

\subsection{The Equipment used.}

The observations were made at Mount John University Observatory (MJUO) (see Figure 2.1), located by Lake Tekapo in the South Island of New Zealand and run by University of Canterbury (UoC). The observatory has three main telescopes; an Optical Craftsman (O/C) $61 \mathrm{~cm}$ Cassegrain, a Boller and Chivens (B\&C) $61 \mathrm{~cm}$ Cassegrain (see Figure 2.2), and the McLellan $1 \mathrm{~m}$ telescope (constructed by UoC). To try to increase the long term stability of the observations, the same instrumental system, as listed in Table 2.1, was used when scheduling permitted. The actual system used for each session is given in Appendix C.

The PMT was used in pulse-counting mode, with the output being amplified and re-shaped to a 'square' shape (suitable for counting) by the amplifier/discriminator circuit. The discriminator part of this circuit rejects any pulses smaller than (an adjustable) threshold, which increases the signal-to-noise ratio (with the noise here being predominately due to the dark counts of the PMT - see section 2.2). The total number of pulses in a given 'integration' period are counted and logged by a computer (initially a PET computer, and then a PC after April 1992).

For bright stars ( $\mathrm{V}$ brighter than $6^{m} \cdot 0$, say), five second integrations (10 seconds for the $\mathrm{U}$ and $\mathrm{S}$ filters) give sufficient accuracy by ensuring the count is larger than 10,000 (see section 2.5). At least three such integrations were done for each filter, allowing the mean $(\bar{x})$ and variance $\left(s^{2}\right)$ of the observation to be calculated. The data logging program uses these to give a real-time

Table 2.1: The preferred instrumental system.

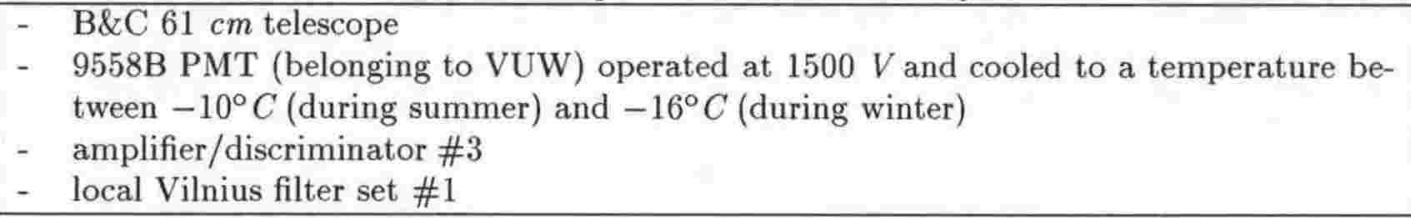




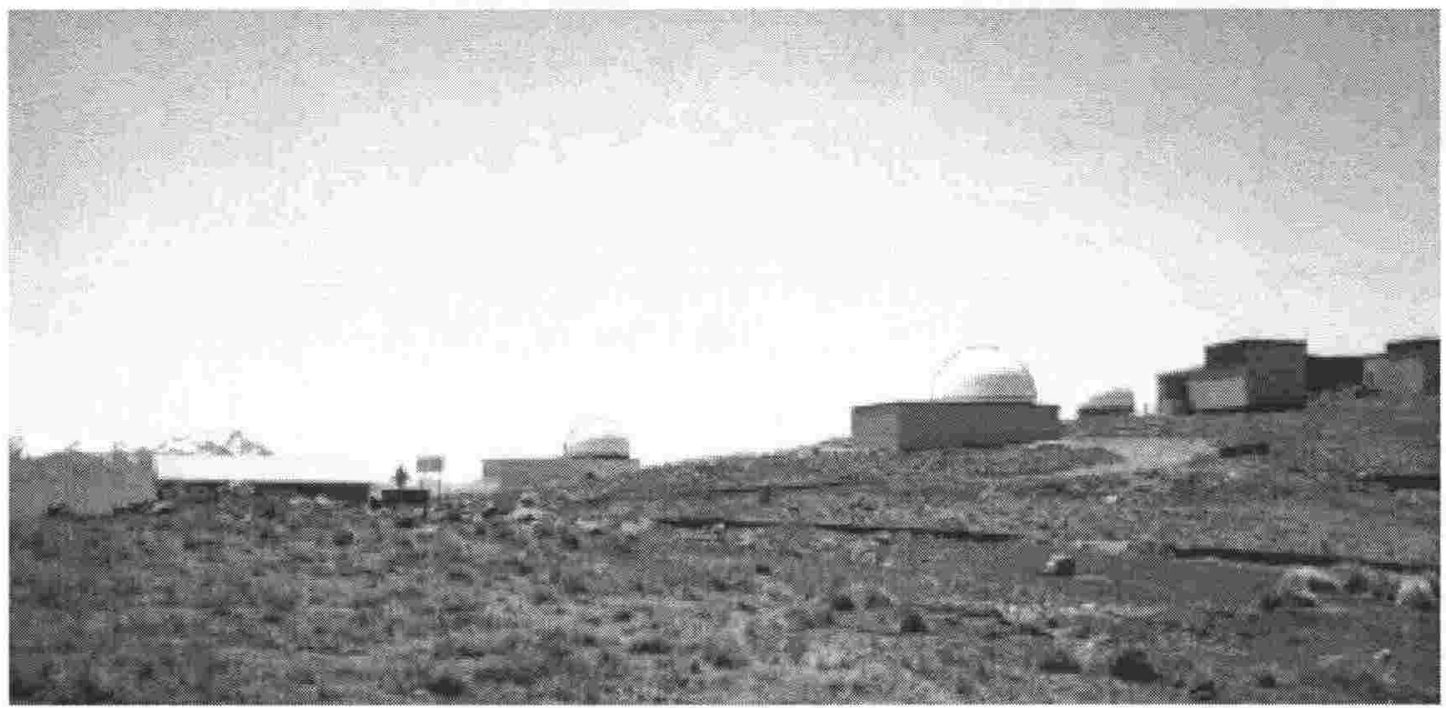

Figure 2.1: View of the buildings at the top of the Mount John University Observatory, located at longitude $+170^{\circ} 27 \cdot 9^{\prime}$ East, latitude $-43^{\circ} 59 \cdot 2^{\prime}$, and $1027 \mathrm{~m}$ above sea-level.

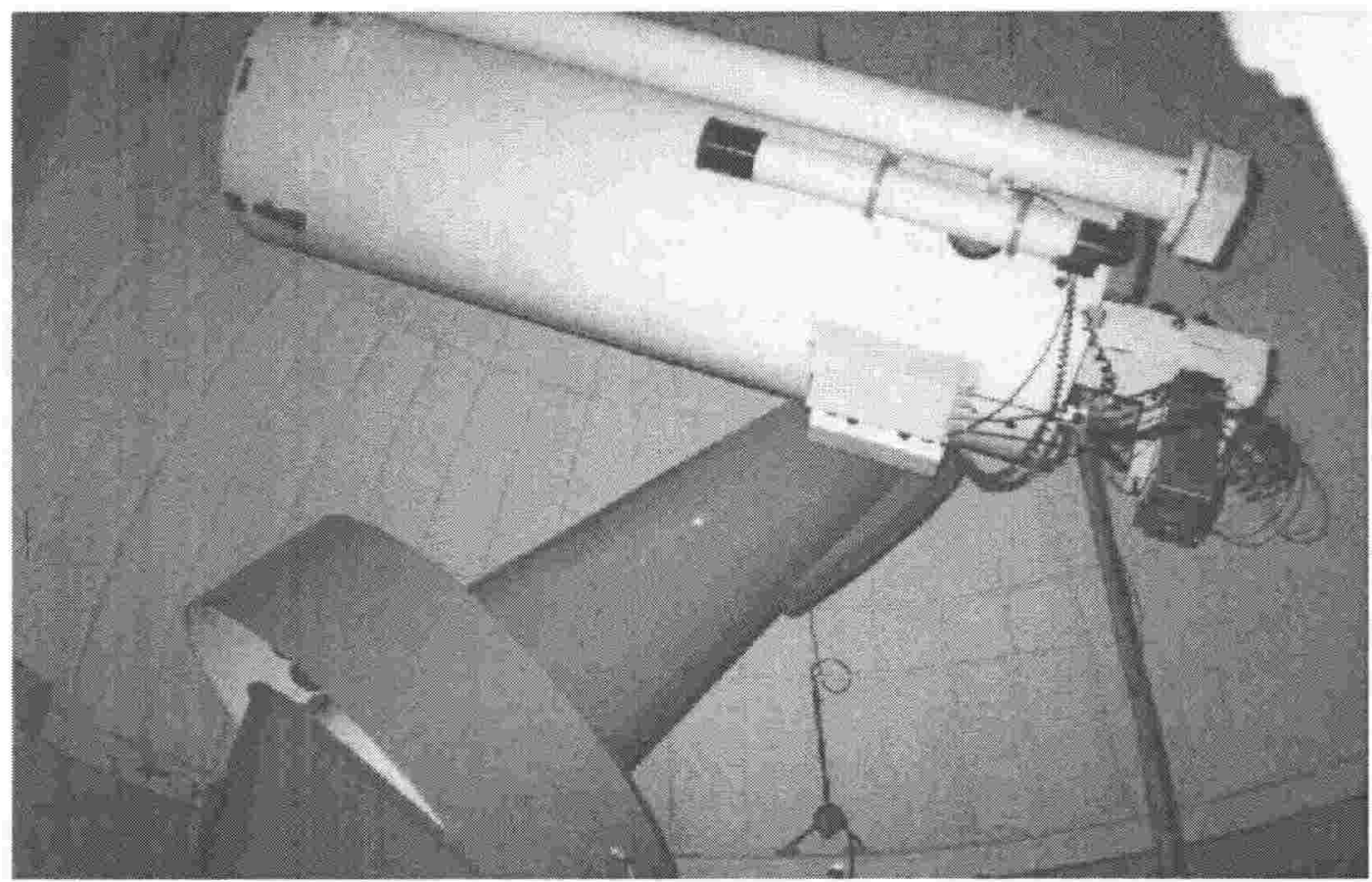

Figure 2.2: The Boller and Chivens telescope at Mount John University Observatory. 
indication of the quality of the observations

$$
\mathrm{QF}=\frac{s^{2}}{\bar{x}}\left\{\begin{array}{l}
\sim 1 \Rightarrow \text { 'good' observation } \\
>1 \Longrightarrow \text { 'bad' observation }
\end{array}\right.
$$

assuming the 'noise' follows a Poisson distribution. The reduction program calculates the errors in the reduced observations by propagating the variance of the integrations in the standard manner using

$$
s_{y}^{2}=\frac{1}{y^{2}} \sum_{i=1}^{n} s_{x,}^{2}\left(\frac{\partial y}{\partial x_{i}}\right)^{2}
$$

for any function $y=f\left(x_{i}\right)$ with independent variables $x_{i}, i=1 \ldots n$.

Two filter sets were supplied by the Vilnius Institute of Theoretical Physics and Astronomy. The first set is $15 \mathrm{~mm}$ in diameter and up to $12.5 \mathrm{~mm}$ thick, and fit in a filter-wheel which can be used on either the $\mathrm{B} \& \mathrm{C}$ or $\mathrm{O} / \mathrm{C}$ telescopes. The second set are $28 \mathrm{~mm}$ diameter, and were designed to fit in the Charge Coupled Device (CCD) photometer for use on the $1 \mathrm{~m}$ telescope in addition to PMT photometers on the $\mathrm{B} \& \mathrm{C}$ and $\mathrm{O} / \mathrm{C}$ telescopes (by using the appropriate filter-wheel). A history of the two filter sets is given below;

Oct 1987 First trial of filter set \#1. No Z observations were made as this filter would not fit in the filter-wheel.

June 1988 (Thinner) replacement Z filter used in set \#1.

Aug 1992 Filter set \#2 arrives. The P filter was found to be cracked and the Y filter was 'cloudy' near one edge.

Nov 1992 Replacement P filter for set \#2 arrives.

Dec 1992 Set \#2 first used (using the clear region of the $\mathrm{Y}$ filter).

June 1993 Replacement Y filter arrives

Sept 1993 Set \#2 padded to a common physical thickness ( $\approx$ optical depth) using FK5 or BG270 glass and Dow-Corning Q2-3068 'glue'. This ensures the CCD system will have the same focal point through all Vilnius filters (of set \#2).

\subsection{The Dark Current.}

A PMT will produce a (small) signal even in the total absence of any light. In Direct Current (DC) mode, this is called the dark current while in Pulse Counting (PC) mode, it is called the dark count. The mean dark count can be simply subtracted from a star's signal, however fluctuations in the dark count add to the total noise in the signal. As this dark count noise scales with the mean dark count, it is desirable to reduce the dark count.

The main sources of the dark count are

- cosmic rays 
- radioactivity

- after pulses

- electrical leakage

- thermally ejected electrons

Cosmic rays are detected by the Cherenkov radiation they produce on striking the PMT's glass window. The amount of cosmic rays increase with the observatory's height above sea-level, being $\sim 1 /$ minute $/ \mathrm{cm}^{2}$ at sea-level (Young 1974), and depend on the area of the photocathode. The Cherenkov radiation is roughly white and strong in the ultra-violet so it's contribution also depends on the spectral transmittance of the PMT window and the spectral response of the photocathode. The pulse produced is usually larger than a signal pulse so can be removed if the amplifier/discriminator circuit has maximum voltage threshold.

Naturally occuring radioactive isotopes are present in small quantities in all PMT window and envelope materials. The decay of these isotopes can produce spurious signals via Cherenkov radiation or ejecting electrons from the photocathode. Fortunately the count rate is generally low $(\sim 2 /$ minute $)$ and slowly reduces with time.

After pulses are extra pulses which may follow any signal (or dark count) pulse. These are due to ionisation by the initial pulse of any residual gas in the PMT. The positively charged ion is then accelerated towards and collides with the photocathode, causing electrons to be ejected and produce the after pulse. The standard vacuum bake out technique can not be used to reduce the amount of residual gas as it would destroy the photocathode. Cooling the PMT reduces the after pulse rate as the 'gas' is more likely to stick to a cold surface.

The connecting pins to the cathode, dynodes and anode all pass through the glass envelope at the back of the PMT, in close proximity to each other. With the large voltages between the cathode, dynodes and anode and the small currents which form the signal, the resistivity of this envelope has to be large to prevent any leakage current between the pins. Cooling the PMT may decrease the resistivity of the envelope and so increase the leakage current.

The major source of dark counts is usually the electrons thermally ejected from the photocathode or the dynodes. Around the region of $0^{\circ} \mathrm{C}$, the dark count is an exponential function of absolute temperature, so cooling the PMT will dramatically reduce the dark count. However excessive cooling may cause other problems in addition to the electrical leakage mentioned above. For instance, the conductivity of the photocathode decreases with decreasing temperature and also differential contraction between different materials in the PMT may lead to the vacuum seal being broken. For this reason, the MJUO PMT is permanently cooled to a set temperature, between $-10^{\circ}$ and $-16^{\circ} .^{1}$ This gives an additional benefit of thermal stability, as, for example, the spectral response of the PMT changes with temperature.

\footnotetext{
${ }^{1}$ The cooling unit is limited by the maximum temperature difference it can maintain between the PMT and
} 
The thermal dark count also depends on the work function of the photocathode material and the area of the photocathode. The choice of the photocathode material is determined by the required spectral range, but the star image (via the fabry lens) should cover the entire photocathode area so none of the photocathode contributes to the dark count while not contributing to the signal.

The dark count can be measured by closing a shutter which is mounted between the PMT and the rest of the photometer. These measurements were done at the beginning of each night before starting the observations and again at the end of the night. As this was usually done during twilight, the dome was closed and all interior lights turned off to reduce any light leakage into the PMT. This provided a crude check of the correct operation of the PMT as experience has shown that the dark count (of the PMT used here) is normally $\sim 30 \mathrm{~Hz}$.

\subsection{Time Corrections.}

The time of the beginning of each integration measurement is recorded by the data logging program, using the computer's internal clock. This clock is nominally set to Universal Time (UT) but sometimes has a slow drift away from the true time, on the order of seconds per night. For this reason, a radio tunable to one of the time standard services (WWV - the American service or VNG - the Australian service) is provided in the $\mathrm{B} \& \mathrm{C}$ and $\mathrm{O} / \mathrm{C}$ domes.

At the beginning of each night, provided the radio reception is suitable, the computer clock is compared with the standard's time pips. Any discrepancy is then either recorded (for later use in the reduction program) or the computer clock is reset to the correct time. This should ensure the recorded time is accurate at the beginning of the night (to approximately one third of a second) and within a few seconds throughout the entire night. As the observing program consists of (nominally non-variable) standard stars, this level of accuracy should be sufficient.

\subsection{Dead-time Corrections.}

In a pulse counting system, when a photon is detected the system is un-responsive to further photons for a certain time interval, known as the dead-time. For instance, this could be due to the finite duration of the output pulse from the amplifier/discriminator circuit or the ability of the frequency counter to resolve two close pulses as two, rather than only one pulse. It is assumed that the photons arriving during during a dead-time interval do not contribute their own dead-time to the original interval, i.e. the system is non-paralyzable. It is particularly important to correct for these un-detected photons when measuring bright stars.

the ambient temperature. Thus the PMT is usually kept at a higher temperature during summer than during winter. 
As photons are Bosons, they obey Bose-Einstein population statistics where they 'bunch' together more than would be expected from a uniform random distribution. The probability density function of two photons being separated by an interval $t$ is

$$
p(t)=f e^{-f t}
$$

where $f$ is the arrival frequency of the photons (Poretti 1992). The number of photons arriving in an interval $\tau$ (the dead-time) is

$$
n_{\tau}=N \int_{0}^{\tau} p(t) d t=N \int_{0}^{\tau} f e^{-f t} d t
$$

where $N$ is the number of photons arriving during the total measurement interval. Setting this measurement interval to one second gives $N=f$ and

$$
n_{\tau}=N \int_{0}^{\tau} N e^{-N t} d t=N\left[1-e^{-N \tau}\right] .
$$

However the number of measured photons is

$$
n=N-n_{\tau}=\frac{N}{e^{N \tau}}
$$

on substituting for $n_{\tau}$. This is an exact relation between the measured and actual number of photons, but it can not be analytically solved for $N$. However, if $N \tau$ is small, then a MacLaurin expansion on the exponential term to first order gives

$$
\begin{aligned}
n & \approx \frac{N}{1+N \tau} \\
\Longleftrightarrow N & \approx \frac{n}{1-n \tau}
\end{aligned}
$$

which approximation is used to correct the measured photon count rate for the dead-time.

Poretti (1992) pointed out in a correction to his earlier paper that Bose-Einstein statistics "is not valid since to observe the effects of Bose-Einstein clumping it is necessary to use much faster equipment and much more monochromatic light than we have in ordinary photometry". Instead the arrival of photons should be treated as independent events and described by Poisson statistics (Evans 1955) where the probability of the interval between consecutive photon arrivals being between $t$ and $t+d t$ is

$$
d P_{t}=f e^{-f t} d t
$$

Therefore the number of intervals between $t=0$ and $t=\tau$ (i.e. the number of photons arriving 
during the dead-time) is

$$
n_{\tau}=N \int_{0}^{\tau} d P_{t}=N \int_{0}^{\tau} f e^{-f t} d t
$$

which the same as equation 2.4 and hence the same results follow.

The approximation to the dead-time correction (equation 2.6) is equivalent to assuming the photons arrive at a uniform rate, as will be shown next. During a one second interval, each measured photon produces a dead-time $\tau$ so the total dead-time is $n \tau$. If the photon arrivals are uniformly distributed throughout the interval, then $N n \tau$ arrive during the total dead-time. However the number of undetected photons is also $N-n$, hence

$$
\begin{aligned}
N-n & =N n \tau \\
\Longleftrightarrow N & =\frac{n}{1-n \tau}
\end{aligned}
$$

which is the same result as the approximation formula 2.6 .

A variety of methods have been developed to measure the dead-time, see for example Hall \& Genet (1982), Cooper \& Walker (1989), Morris \& Naftilan (1994), Sterken \& Manfroid (1992a), and Hendon \& Kaitchuck (1990). The method used here is given by Wadsworth (1988) and is based on the following;

A stable, diffuse light source is used to illuminate the telescope and measurements through two different sized photometer diaphragms are made giving

$$
N_{S}=\frac{n_{S}}{1-n_{S} \tau} \quad \text { and } \quad N_{L}=\frac{n_{L}}{1-n_{L} \tau}
$$

where the subscripts denote the Small and Large diaphragms respectively. Dividing the smaller diaphragm measurement by the larger gives

$$
\begin{aligned}
\frac{N_{S}}{N_{L}} & =\frac{n_{S}}{n_{L}} \frac{1-n_{L} \tau}{1-n_{S} \tau} \\
\Longleftrightarrow \frac{n_{L}}{n_{S}} & =\frac{N_{L}}{N_{S}}\left(1-n_{L} \tau\right)\left(1-n_{S} \tau\right)^{-1}
\end{aligned}
$$

Provided $n_{S} \tau$ is small, a binomial expansion may be used

$$
\frac{n_{L}}{n_{S}} \approx \frac{N_{L}}{N_{S}}\left(1-n_{L} \tau\right)\left(1+n_{S} \tau\right)=\frac{N_{L}}{N_{S}}\left[1-\left(n_{L}-n_{S}\right) \tau\right]
$$

to first order. By making a series of measurements with the light source adjusted to different intensities, a straight line may be least squares fitted to $\left(n_{L} / n_{S}\right) v s\left(n_{L}-n_{S}\right)$, giving a slope of $-\frac{N_{L}}{N_{S}} \tau$ and intercept $\frac{N_{L}}{N_{S}}$ and thus $\tau=-\frac{\text { slope }}{\text { intercept }}$.

The light source used at MJUO consists of differently coloured Light Emitting Diodes 
(LEDs) arranged in rings behind 'clouded' glass (to diffuse the light) in a 'tophat' and powered by an adjustable, constant current supply run from a rechargable battery (for stability).

The LED 'tophat' can be mounted in the primary mirror baffle of either the B\&C or the O/C telescopes. It is recommended that dead-time measurements be made on a cloudy night with all the dome lights off (to reduce any light leakage into the PMT) and the following sequence used;

1. Choose a suitable combination of LED (colour), filter, small/large diaphragms and LED intensity range so that measurements of at least ten different intensities may be made from $n \sim 10^{4} \rightarrow 10^{6} \mathrm{~Hz}$.

2. Set the LED to some intensity and allow time to stabilize (for example five minutes). Measure the dark current while waiting.

3. Make a quick series of measurements alternating between the small and large diaphragms (for example small, large, small, ..., large, small) at uniform time intervals (for example ten second measurements with five seconds between each measurement) so any instrumental drift may be corrected to first order by interpolating the small diaphragm measurements to the time of the large diaphragm measurements.

4. Measure the dark current again.

5. Change the LED intensity and go back to step 2 .

The interpolated dark current count rates should be subtracted from each set of measurements to give $n_{S}$ and $n_{L}$.

From the EMI PMT cat-

Table 2.2: Dead-time measurements on the B\&C telescope using the VUW PMT at $1500 \mathrm{~V}$.

\begin{tabular}{|c|c|c|c|c|c|}
\hline Date & $\begin{array}{c}\text { Ampl } \\
\#\end{array}$ & LED & Filter & $\begin{array}{c}\tau \\
(n s)\end{array}$ & $\begin{array}{c}<\tau> \\
(n s)\end{array}$ \\
\hline 27 Feb 91 & 1 & Green & $\mathrm{V}$ & $370 \pm 50$ & $370 \pm 50$ \\
\hline 14 May 91 & 3 & Red & $\mathrm{S}$ & 330 & \\
07 Feb 92 & 3 & Green & $\mathrm{Z}$ & 270 & \\
17 Apr 92 & 3 & Red & $\mathrm{S}$ & 299 & \\
26 Jun 92 & 3 & Red & $\mathrm{S}$ & 288 & $297 \pm 25$ \\
\hline
\end{tabular}

alog, the rise time $\left(t_{r}\right)$ of the output pulse in response to a detected photon for a $9558 \mathrm{~B}$ PMT is $10 n s$, so $3 t_{r}$ should be a good estimate of the pulse duration and hence the deadtime of the PMT. The same catalog gives the dead-time of some amplifier/discriminator circuits as being between $10-100 \mathrm{~ns}$. As the dead-time of a system is due to the longest dead-time of any component of the system, dead-times (due to the amplifier/discriminator circuit) of the order $10-100$ ns could be expected. This is also borne out in results reported in the literature, for example Morris \& Naftilan (1994), Poretti (1992), and Fernie (1976). 
The results of dead-time measurements made at MJUO are shown in Table 2.2, with the average values being used in the data reductions. These are somewhat larger than the expected values, but clearly depend on which amplifier/ discriminator is used.

Fernie (1976) investigated the effect of very bright stars and found that the dead-time (approximate) correction (equation 2.6) fails for stars brighter than $\sim 4^{m} \cdot 0$, using the same sized telescope as those at MJUO $(61 \mathrm{~cm})$. The dead-time appeared to increase as the count rate increased $\left(\tau^{\prime}=\tau n^{b}\right)$, leading to a power law correction

$$
N=\frac{n}{1-\tau n^{1+b}}
$$

with $b=0.052$. The Vilnius filters have narrower bandwidths and lower peak transmittance than those used by Fernie so at MJUO this effect could be expected to occur from a brighter magnitude, above $\sim 2^{m} \cdot 0$ say. Although only a few $(\sim 8)$ stars brighter than this were measured, the MJUO dead-time measurements were also fitted with this correction. This yielded a slightly better fit in some cases, but $b$ was found to be very sensitive to measurement errors and $\tau$ was strongly correlated with $b$, so this power law correction was not used.

\subsection{Sky Background Correction.}

When measuring the intensity of any star, a background contribution from the night sky is always included and must be subtracted to obtain the star's correct intensity. Listed below are the natural sources of the night sky's background light (Hendon \& Kaitchuck 1990);

- integrated light from distant galaxies

- integrated star light from within our own galaxy

- twilight emission lines

- zodiacal light

- Moon light

- airglow

- aurora

Galactic and extra-galactic background light is probably the limiting factor when trying to minimize the 'sky' background contribution. This background light can vary significantly over an angular distance of a few arc-minutes so the correct background subtraction may not be possible. However, the background is 'static' in that it is always the same for the same position (in equatorial or galactic coordinates).

Twilight emission lines are caused by the Sun illuminating certain layers (of Sodium, emitting the Sodium D lines at $5892 \AA$ and Oxygen, emitting red lines at 6300 and $6364 \AA$ ) in 
the Earth's upper atmosphere. This can only be seen when the Sun is at the correct angle below the horizon, called the solar dispersion angle. This is between $7^{\circ}$ and $10^{\circ}$ and occurs for approximately one hour (during nautical twilight ${ }^{2}$ ), and ends at astronomical twilight. ${ }^{3}$ Only the Oxygen red lines occur within the Vilnius filters response curves, at the short wavelength 'tail' of the S filter, so the twilight emission lines should make little contribution to the sky background. Inspection of the sky background at dawn twilight on several nights during the observing program show that the largest increase occurs in the $\mathrm{Z}$ filter, followed by the $\mathrm{Y}$, $\mathrm{X}$, and $\mathrm{P}$ filters. The remaining filters (including the $\mathrm{S}$ filter) show approximately the same increase, therefore it is confirmed that the twilight emission lines make a negligible contribution to the sky background.

The zodiacal light is sunlight reflected or scattered off dust in the plane of the solar system, and so is confined to the ecliptic plane. It's intensity increases towards the Sun, but is approximately constant on the scale of arc-minutes. The light is highly polarized and shows a solar spectra.

The intensity of Moon light depends on the phase of the Moon and angular distance from the Moon.

The night airglow is a major contributor to the total sky background. It is due to ionized atoms and molecules recombining (and thus emitting light) following collisions with high energy particles and radiation, which are mainly from the Sun. This mostly occurs in a layer approximately $100 \mathrm{~km}$ above the Earth. It's spectra consists of a pseudo-continuum plus some emission lines, the major lines being atomic Oxygen ( $5577 \AA$ ), Sodium (5892 $\AA$ ), molecular Oxygen $(7617,8645 \AA)$ and hydroxyl $\left(\mathrm{OH}^{-}\right.$, in the infrared). The intensity increases towards the horizon but is generally variable, depending on the latitude, season, local time, solar activity and sky conditions.

The aurora is essentially the same as the airglow but occurs when high energy charged particles (usually from the solar wind or flares) are trapped by the Earth's magnetic field and forced to spiral in towards the magnetic poles. As the particles are concentrated in an irregular oval around each pole, the aurora can be several hundred times brighter than the airglow. The major emission lines are due to atomic Oxygen (5577, 6300, 6364 $\AA$ ), Hydrogen (6563 $\AA$ ) and molecular Nitrogen (red), giving colours ranging from a white-green to deep red. It's intensity varies with latitude, solar cycle and flare activity and season (peaking in March and October). The irregular and highly variable nature of aurora mean that accurate sky measurements are extremely difficult to make and it is recommended that observations not be made during an

\footnotetext{
${ }^{2}$ Nautical twilight is defined by the Astronomical Almanac as the interval during which the central point of the Sun's disk is between $6^{\circ}$ and $12^{\circ}$ below the horizon

${ }^{3}$ Astronomical twilight is defined by the Astronomical Almanac as the interval during which the central point of the Sun's disk is between $12^{\circ}$ and $18^{\circ}$ below the horizon
} 
aurora (Hendon \& Kaitchuck 1990).

Given that the aim of the observing program was to measure stars rather than the sky, it is worth investigating the optimum (minimum) time to spend measuring the sky. It is usual to observe the star until the shot noise ${ }^{4}$ (assumed to be the dominant error here) achieves the required accuracy level. Denoting the total integrated count as $n$, the integration time as $t$ and the count rate as $\dot{n}(=n / t)$, the shot noise is $n^{\frac{1}{2}}$. Aiming for a $1 \%$ accuracy level gives

$$
\frac{\text { shot noise }}{\text { signal }}=\frac{n_{\text {star }+ \text { sky }}^{\frac{1}{2}}}{n_{\text {star }+ \text { sky }}}<0.01 \Rightarrow n_{\text {star }+ \text { sky }}>10000
$$

and an (absolute) error in the count rate of

$$
\begin{aligned}
\varepsilon_{\text {star }+s k y} & =\frac{\text { shot noise }}{\text { integ. time }} \\
& =\left(\frac{\dot{n}_{\text {star }+ \text { sky }}}{t_{\text {star }+ \text { sky }}}\right)^{\frac{1}{2}}
\end{aligned}
$$

A simple way to minimize the time spent measuring the sky while retaining the required accuracy is to measure the sky until it's error is less than that of the star, i.e.

$$
\begin{aligned}
& \varepsilon_{s k y}<\varepsilon_{s t a r+s k y} \\
& \Longleftrightarrow\left(\frac{\dot{n}_{s k y}}{t_{s k y}}\right)^{\frac{1}{2}}<\left(\frac{\dot{n}_{\text {star }+s k y}}{t_{\text {star }+s k y}}\right)^{\frac{1}{2}} \\
& \Longleftrightarrow \frac{t_{s k y}}{t_{\text {star }+s k y}}>\frac{\dot{n}_{s k y}}{\dot{n}_{\text {star }+ \text { sky } y}}
\end{aligned}
$$

with the total error being

$$
\begin{aligned}
\varepsilon_{s t a r}^{2} & =\varepsilon_{s t a r+s k y}^{2}+\varepsilon_{s k y}^{2} \\
& =\frac{\dot{n}_{s t a r+s k y}}{t_{s t a r+s k y}}+\frac{\dot{n}_{s k y}}{t_{s k y}}
\end{aligned}
$$

In the limiting case of a faint star (equal to the sky) and equal errors in the star and sky, then $t_{s k y}=t_{\text {star }+s k y}$ and in general $t_{s k y} \leq t_{\text {star+sky }}$.

Hendon \& Kaitchuck (1990) and Sterken \& Manfroid (1992a) both derive the same expression for the optimum fraction of the total integration time to be spent observing the star $\left(p=t_{\text {star }+s k y} / t_{\text {total }}\right)$ as

$$
\frac{1-p}{p}=\left(\frac{\dot{n}_{s k y}}{\dot{n}_{s t a r+s k y}}\right)^{\frac{1}{2}}
$$

This follows from minimizing the total error with respect to $p$.

\footnotetext{
${ }^{4}$ Shot noise is also called photon noise.
} 
Given that the peak transmittance of each filter is different and that the count rates for each star will also be different, an optimum sky integration time would need to be calculated for each filter and star combination. It was considered that the time saved by using the optimum time for the sky measurements would be comparable with the time required to do the calculations (in the dome, after observing the star) and reset the integrations times. Therefore it was decided to use the same integration times for both the star and the sky - that way the sky's error contribution should always be less than that of the star.

Another consideration when measuring the sky background level is the diaphragm size. As the sky appears as an extended source (rather than the point source of a star), increasing the size of the diaphragm increases the sky signal and increases the limiting intensity of the faintest star that may be measured. This means the diaphragm size should be the minimum necessary (to satisfy the other conditions on the diaphragm size) when observing faint stars. The most commonly used diaphragm in this program gave, on a Moon-less night, a sky background of $\sim 50 \mathrm{~Hz}$. As this includes the $\sim 30 \mathrm{~Hz}$ contribution from the dark count, using a smaller diaphragm would mean the background level would be set by the dark count rather than the sky background.

The observing sequence between star and sky was adapted to suit each night's conditions and intended program, with some examples listed below;

- In general, the sky was measured before and after the measurement of each star. However, if the star was bright and the sky conditions were stable, the sky was measured only once (after the star measurement, say).

- Choose a location for the sky measurement which appears clear of faint stars and is approximately five diaphragm diameters away from the star. However, if the star has a companion star whose magnitude and proximity are sufficient to contribute significantly to the sky background (yet not close enough that they have to be measured together through the same diaphragm), choose the location of the sky measurement so that it matches the star's background. For instance, choose a location on an arc which is centred on the companion star and runs through the observed star.

- If observing during twilight or Moon rise/set, only bright stars were measured and the sky was measured immediately before and after the star measurement.

- If observing a sequence of 2-4 bright stars which are close together in the sky, then the sky would be measured before and after the sequence rather than with each individual star.

- If re-measuring a previously observed star, use the same location as before for the sky measurement. This is particularly useful for time series or differential photometry (for example 
variable, comparison and check stars).

The reduction program was written to allow flexibility in the star/sky sequence used, as the program could be instructed by the user to

- automatically assign all sky measurements to the closest preceding star measurement

- automatically assign all sky measurements to the closest following star measurement

- automatically interpolate the closest sky measurements surrounding the star measurement

- least squares fit a polynomial (order specified by the user) to the sky measurements

- allow the user to over-ride any individual star/sky assignment

while warning the user if any star has not yet had a sky measurement assigned to it.

\subsection{The Monochromatic Extinction Correction.}

When star light passes through the Earth's atmosphere, some of the photons will be scattered or absorbed. However, as far as the observer is concerned the star appears to be dimmed, hence the term 'extinction' is used for both processes. The main sources of extinction are; Rayleigh scattering by molecules, scattering off aerosols (dust particles and small droplets), and molecular absorption - see Appendix $\mathrm{H}$ for more details.

Consider a monochromatic ray of light (wavelength $\lambda$, intensity $I$ ) passing through a small element of absorbing material with length $\delta h$ and unit cross-section, at a height $h$ above the observer. The intensity loss $\delta I$ is

$$
\delta I=-I \kappa(\lambda, h) \rho(h) d h
$$

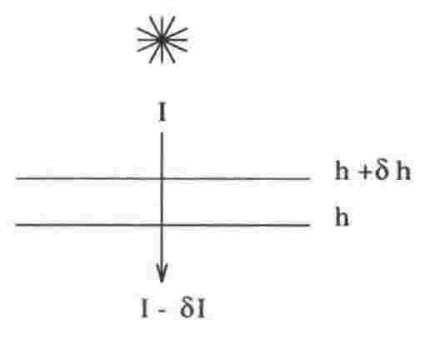

where $\kappa(\lambda, h)$ is the absorption coefficient per unit mass and $\rho(h)$ is the mass density of the air in the element.

In general, the star is not at the zenith so $d h$ is replaced by $\sec (z) d h$

$$
\delta I=-I \kappa(\lambda, h) \rho(h) \sec (z) d h
$$

where $z$ is the $z$ enith distance. Integrating gives

$$
I\left(h_{0}\right)=I\left(h_{1}\right) e^{-\int_{h_{0}}^{h_{1}} \kappa(\lambda, h) \rho(h) \sec (z) d h}
$$


where $h_{0}$ is the height of the observer and $h_{1}$ is the top of the atmosphere.

However, this assumes the ray travels in a straight line

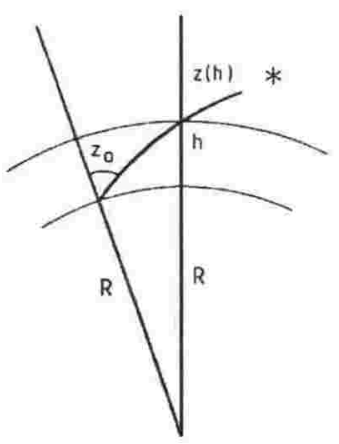
through the atmosphere. In fact the angle $z$ varies with height because of the Earth's curvature and refraction of the ray, i.e. $z=z(h)$. Note that $z_{0}$ is the apparent zenith distance of the star as seen by the observer.

Assuming the absorption coefficient is constant throughout the atmosphere, i.e. $\kappa=\kappa(\lambda)$, then

$$
I\left(h_{0}\right)=I\left(h_{1}\right) e^{-\kappa(\lambda) \int_{h_{0}}^{h_{1}} \rho(h) \sec (z(h)) d h} .
$$

The integral represents the mass of air in a column along the ray's path. Defining the air mass as

$$
X=\frac{\int_{h_{0}}^{h_{1}} \rho(h) \sec (z(h)) d h}{\int_{h_{0}}^{h_{1}} \rho(h) d h},
$$

which is the amount of air in the column relative to that at the zenith. Hence

$$
\begin{aligned}
I\left(h_{0}\right) & =I\left(h_{1}\right) e^{-\kappa(\lambda) X(z(h)) \int_{h_{0}}^{h_{1}} \rho(h) d h} \\
& =I\left(h_{1}\right) e^{-\kappa(\lambda) X(z(h)) \alpha}
\end{aligned}
$$

with $\alpha\left(=\int_{h_{0}}^{h_{1}} \rho(h) d h\right)$ being the total mass of air in a vertical column (of unit cross-section) above the observer.

Converting to magnitudes gives Bouguer's Law

$$
\begin{aligned}
m\left(h_{0}\right) & =m\left(h_{1}\right)+2 \cdot 5 \log _{10}(e) \kappa(\lambda) X(z(h)) \alpha \\
& =m\left(h_{1}\right)+k(\lambda) X(z(h)),
\end{aligned}
$$

with the extinction coefficient $k(\lambda)=2.5 \log _{10}(e) \kappa(\lambda) \alpha$.

Bemporad (1904) calculated $X$ as the function of the apparent zenith distance for a standard atmosphere (i.e. at a particular sea level temperature and pressure and having a certain composition and structure). Hardie (1962) shows that $X=\sec (z)$ is a reasonable approximation near the zenith, giving an error of 0.005 airmasses at $z=60^{\circ}$. Hardie also fitted a polynomial to Bemporad's data

$$
X=\sec \left(z_{0}\right)-0.0018167\left(\sec \left(z_{0}\right)-1\right)-0.002875\left(\sec \left(z_{0}\right)-1\right)^{2}-0.0008083\left(\sec \left(z_{0}\right)-1\right)^{3}
$$

but this uses the apparent zenith distance (which is difficult to calculate). Young \& Irvine 
(1967) derive a fit to Bemporad's tables

$$
X=\sec (z)\left(1-0.0012\left(\sec ^{2}(z)-1\right)\right)
$$

which uses the zenith distance.

Equation 2.16 was used here to calculate the airmass as the zenith distance may be calculated using spherical trigonometry on coordinate transformations,

$$
\sec (z)=(\sin (\phi) \sin (\delta)+\cos (\phi) \cos (\delta) \cos (H A))^{-1}
$$

where $\phi$ is the observer's latitude, $\delta$ is the star's declination and $H A$ is the star's Hour Angle. The hour angle is found from $H A=L S T-\alpha$, where $L S T$ is the Local Sidereal Time and $\alpha$ is the star's Right Ascension.

The local sidereal time is calculated in turn from the Universal Time as follows; the fractional Julian Century is

$$
T_{u}=\frac{J D-2451545}{36525}
$$

as the Julian Date at the beginning of the century is 2451545 and there are 36525 days in this century. The Julian Date must be calculated from the calendar date, using a BASIC subroutine given by Duffett-Smith (1985) and re-written in FORTRAN.

$T_{u}$ allows the sidereal time for Greenwich at $0^{h}$ UT to be found;

$$
G S T_{0}=24110 \cdot 54841+8640184 \cdot 812866 T_{u}+0 \cdot 093104 T_{u}^{2}-6 \cdot 2 \times 10^{-6} T_{u}^{3}(\text { seconds })
$$

and hence the Greenwich sidereal time at the time of the observation

$$
G S T=G S T_{0}+\frac{366 \cdot 2422}{365 \cdot 2422} U T \text { (hours) }
$$

where $366 \cdot 2422$ is the number of sidereal days in a year while $365 \cdot 2422$ is the number of (mean) solar days in a year. Now the local sidereal time can be found

$$
L S T=G S T+\lambda
$$

where $\lambda$ is the observer's longitude (East of Greenwich, in hours).

Recall that Bouguer's Law was derived assuming the absorption coefficient per unit mass $(\kappa)$ was constant, i.e. the components making up the atmosphere are well mixed. This is true for the major atmospheric constituents but not for ozone or dust and water droplet aerosols (Sterken \& Manfroid 1992a). Ozone is a major component in a layer at $\sim 30 \mathrm{~km}$ height 


\section{2- 6-1989 extn. star HD122066 P magnitude}

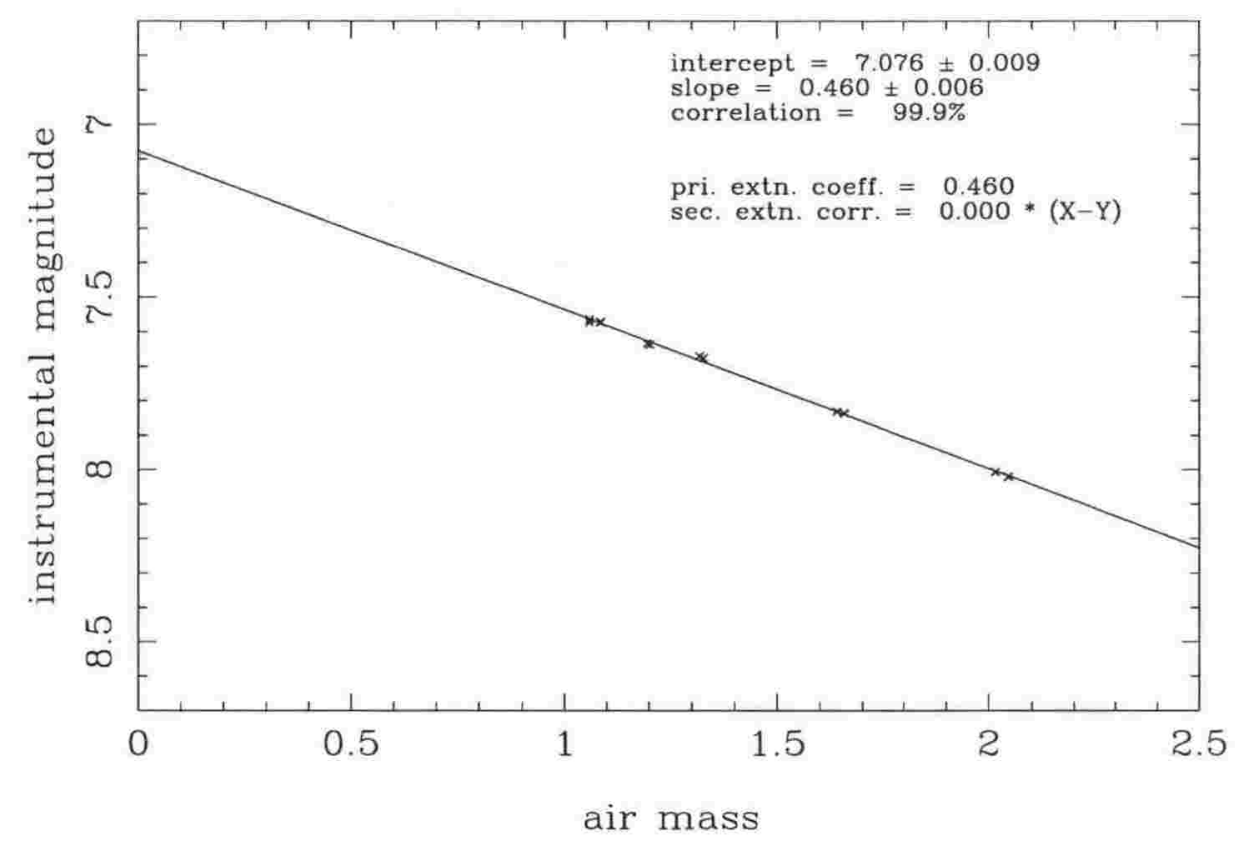

Figure 2.3: Bouguer plot to determine monochromatic extinction (for the $\mathrm{P}$ filter). Observations were made of the extinction star HD122066 during 22 June 1989, yielding $\left\langle k_{P}\right\rangle=0 \cdot 460 \pm 0 \cdot 006$.

while the aerosols are largely found just above the ground, with a scale height of $\sim 1 \mathrm{~km}$. Hence, at some wavelengths where the ozone or aerosol extinction is strong, Bemporad's tables (and equation 2.16) may not give the correct values for the airmass. However, Young (1974) has shown that the ozone and aerosols require corrections in opposite directions (i.e. partially cancel out) and are not significant for $X \lesssim 2 \cdot 3$.

All that remains is to find the extinction coefficient $k(\lambda)$. Assuming the extinction is uniform across the sky and constant during the night, then observing a star through a range of airmasses gives $k(\lambda)$ by least squares fitting a straight line to the instrumental magnitude $\left(m\left(h_{1}\right)\right)$ versus the airmass. The slope of the line gives the extinction coefficient while the intercept gives the extra-terrestrial instrumental magnitude $\left(m\left(h_{0}\right)\right)$. As example can be seen in Figure 2.3, and is known as a Bouguer plot.

Measuring the star at larger airmasses should increase the precision of $k(\lambda)$. However, "it is a common experience that the error [of a measurement of the star] is an increasing function of airmass" according to Young (1974). This was investigated for MJUO in Figure 2.4, showing a possible quadratic relationship - but the scarcity of observations at large airmasses mean this relationship can not be definitely confirmed. Assuming however that the accuracy does decrease with increasing airmass, then there will be an optimum airmass $\left(X_{\text {opt }}\right)$ beyond which the benefits 
Relationship between the raw magnitude errors and air mass

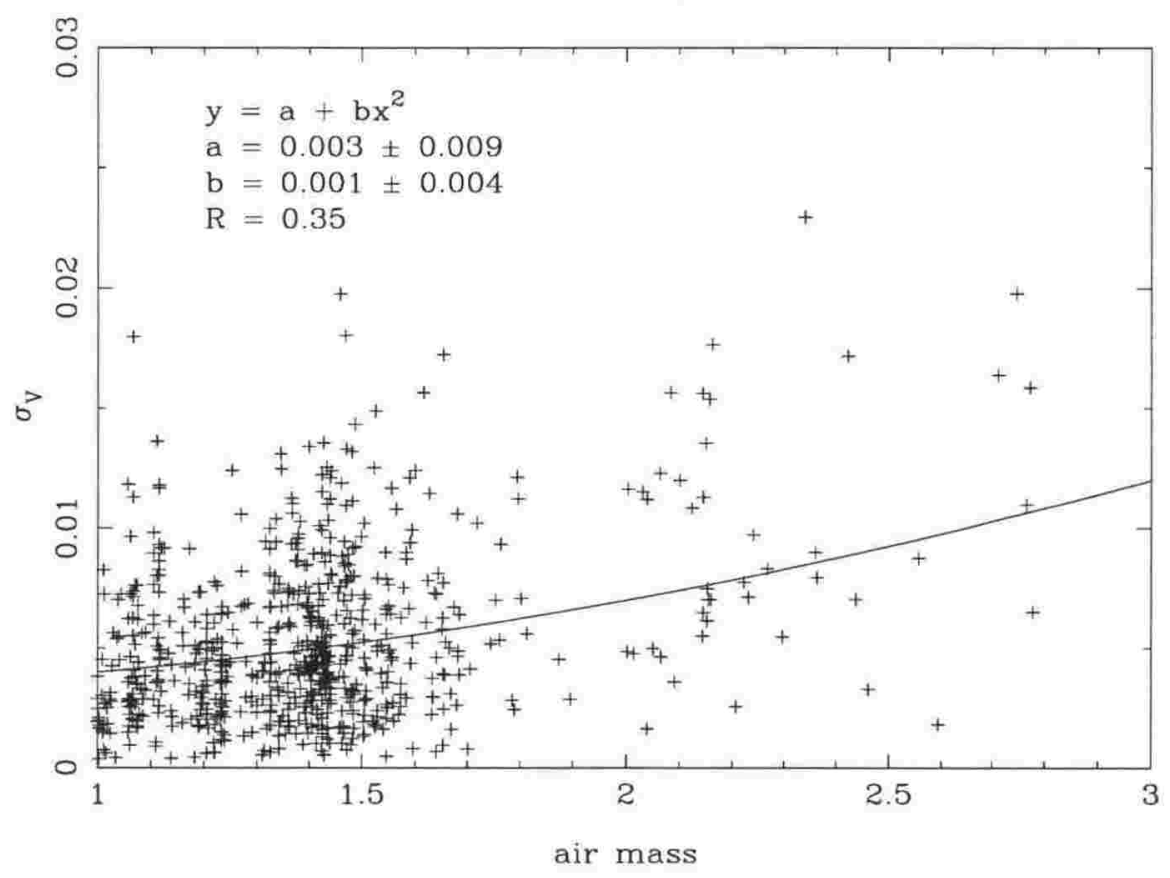

Figure 2.4: Observational error as a function of airmass at MJUO. The standard deviation of the $\mathrm{V}$ filter measurements are plotted against the airmass of the observation.

of increasing $X$ will be offset by the increasing error. Using a power law relationship, Young (1974) finds $X_{\text {opt }} \sim 2$ so observations of extinction stars at MJUO were generally restricted to $X \lesssim 2$. Young (1974) also shows that observing the extinction star twice as often at the larger airmasses than at the smaller airmasses gives the correct weighting to the least squares fit this was usually done at MJUO by observing the star as it rises and again as it sets.

Unfortunately the previous assumption that the extinction remains constant across the sky and throughout the night is seldom true. Figure 2.5 shows a Bouguer plot on a night with an apparently slowly changing extinction - as the star sets it does not re-trace the path it took in the plot while rising.

A standard lamp could be used to measure any change in the zero-point, and hence indicate if an instrumental drift is responsible for the changing 'extinction' in Figure 2.5. An ideal standard lamp would be stable for at least the duration of the night, would illuminate the telescope in the same manner as a star and emit light over the spectral range covered by the filter set used.

Unfortunately, such a standard lamp is not yet available at MJUO as the LED Tophat used to measure the dead-time requires too long to install in the primary mirror baffle (of the B\&C telescope) to be practical for frequent measurements. Alternatively, the $\beta$-light source can be 


\section{3- 4-1992 extn. star HD114613 P magnitude}

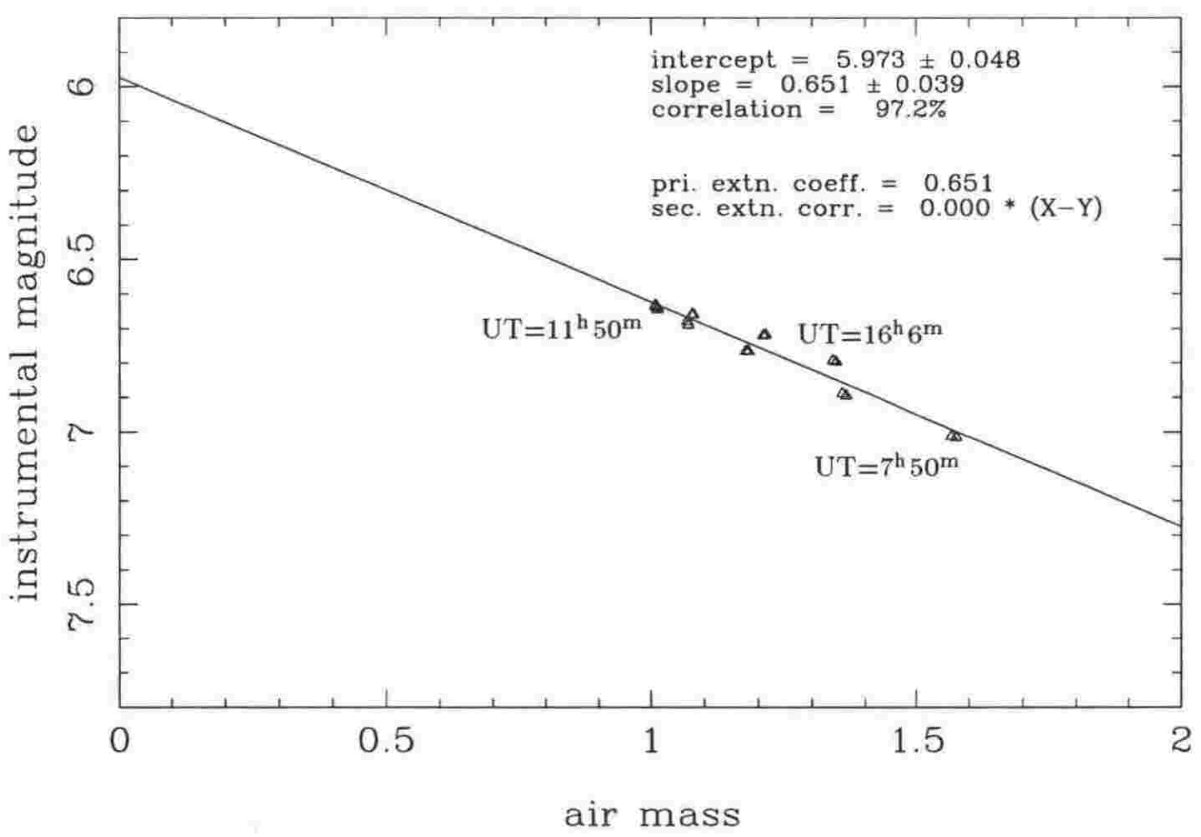

Figure 2.5: Variable extinction in a Bouguer plot. Observations were made of the extinction star HD114613 during 13 April 1992, giving an incorrect average extinction of $k_{P}=0.651 \pm 0.039$ (which is too high). The times of the first, middle and last pair of observations are shown.

mounted in a filter-wheel. This is a radioactive isotope, with an $\sim 12$ year half-life, which emits $\beta$-particles (electrons) that excites a phosphor into glowing. However, it's intensity is strongly temperature dependent (as phonon production is a competing process also excited by the $\beta$ particles) and so would need to be thermally regulated, or at least the temperature recorded. Further, it emits light in a narrow spectral range and does not illuminate the filters or telescope mirrors. Finally, as it does not have a shutter, it would be an additional source of background light whose intensity depended on the position of the filter-wheel. This means the cause of the variable extinction in Figure 2.5 must be found from measurements of extinction stars only.

Young (1974) investigated the shapes of Bouguer plots with slowly changing extinction due to different causes - a horizontal extinction gradient, a time-varying extinction coefficient, or an instrumental zero-point drift. Assuming an extinction coefficient which varies linearly with horizontal distance (at some height above the observer), then $m=m_{0}+\left(k_{0}+k_{1} \tan (z)\right) \sec (z)$ in a flat-earth approximation, which shows two convex branches meeting in a common tangent at the minimum airmass in a Bouguer plot. Alternately, a linearly time varying extinction coefficient gives $m=m_{0}+\left(k_{0}+k_{1} t\right) \sec (z)$, which shows two nearly straight branches meeting at the minimum airmass. Finally, a linearly time varying instrumental zero-point gives $m=$ $m_{0}+k_{0} \sec (z)+k_{1} t$, showing two nearly straight, parallel lines with a rounded end at minimum 
airmass. These can be seen in Figure 2.6, which were adjusted by eye to fit the extinction data from 13 April 1992.

The cause of the variable extinction on 13 April 1992 is difficult to decide on the basis of Figure 2.6, as the difference in the shape of the curves is only readily apparent at larger airmasses. However two other stars were also used to measure the extinction during the same night. As these stars are $\sim 3^{\text {h }}$ apart (in RA) from each other, and so are at different airmasses when observed (nearly) simultaneously, a plot of the extinction coefficient versus time should behave in the different manner for each star depending on the source of the extinction variations.

This is shown in Figure 2.7, calculating the extinction coefficient for each observation using $k(t)=\left(m(t)-m_{0}\right) / X$ (the extra-terrestrial magnitude $\left(m_{0}\right)$ for each star was accurately determined using Nikonov's Method - see later). For each possible cause of extinction change, a curve was fitted to HD114613 and the derived coefficients used to draw curves for the other two stars. As the correct source of the extinction change should fit all three stars equally well, the horizontal gradient in extinction can be immediately ruled out. Both the time dependent extinction and zero-point changes appear to match the data well, with the latter being a slightly better fit.

As the changes may not be linear, the extinction coefficients (or zero-point shifts) for all three stars were plotted against time in Figures 2.8 and 2.9 and a polynomial fitted so the required adjustment may be found for the time of any observation. Using these adjustments, the transformation to the standard system was fitted in Figure 2.10. Despite the fact that the standard stars were observed at larger airmasses where the two possible adjustments $(\Delta m=$ $k_{1} t X$ or $\Delta m=k_{1} t$ ) should show the greatest difference, no significant difference was found.

As the program stars are usually observed at their minimum airmass, where the adjustments are nearly equal, it was assumed that the changing Bouguer plot was due to a slowly decreasing extinction coefficient. Almost all nights which had a changing extinction showed a slowly deceasing extinction, which was approximately linear with time. According to Young (1974), this is due to a slow fall-out of aerosols in the atmosphere during the night as the convection (stirring up the aerosols) driven by solar heating ceases.

Recall that the calculation of the instantaneous extinction coefficient using $k=\left(m-m_{0}\right) / X$ required an accurate value for $m_{0}$. One method for finding this was devised by Nikonov (1953), and a variation on the technique was used here. Other methods to find $m_{0}$, or otherwise determine better values of the extinction coefficient than from a Bouguer plot, can be found in the literature, for example Hardie (1962), Harris, FitzGerald \& Reed (1981), Acker \& Jaschek (1986), Sterken \& Manfroid (1992a), Straižys (1992), Forbes (1992), and Poretti \& Zerbi (1993).

In Nikonov's method, two extinction stars are used; an 'extinction' star and a 'control' star. They are chosen such that when the extinction star culminates (i.e. at it's minimum airmass so 
time-varying extinction coefficient

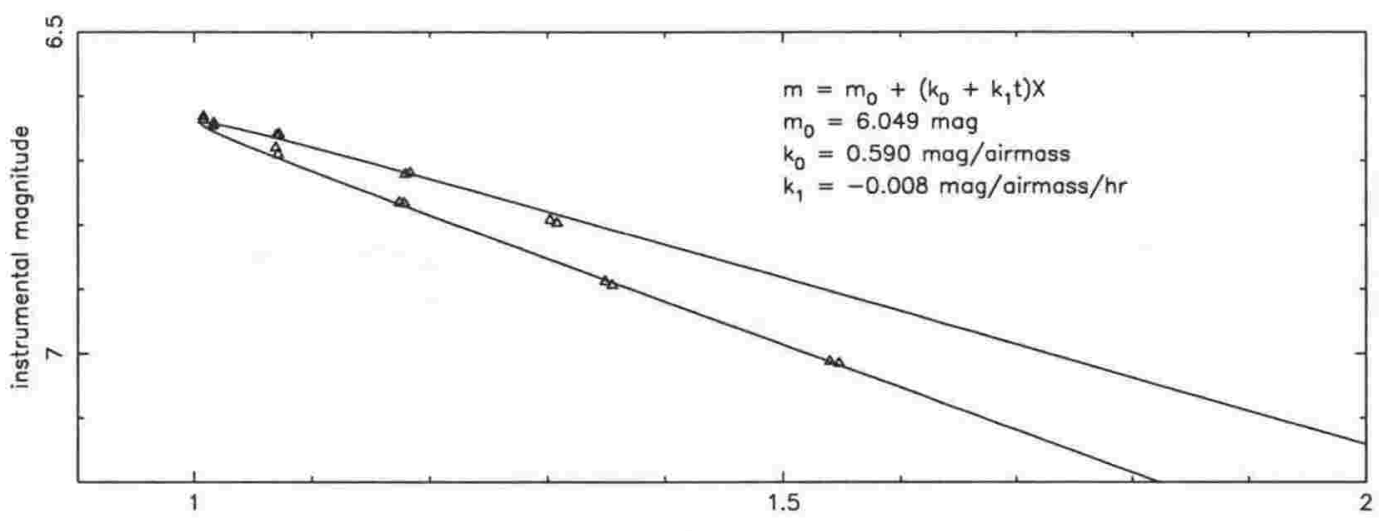

air mass

horizontal gradient in extinction

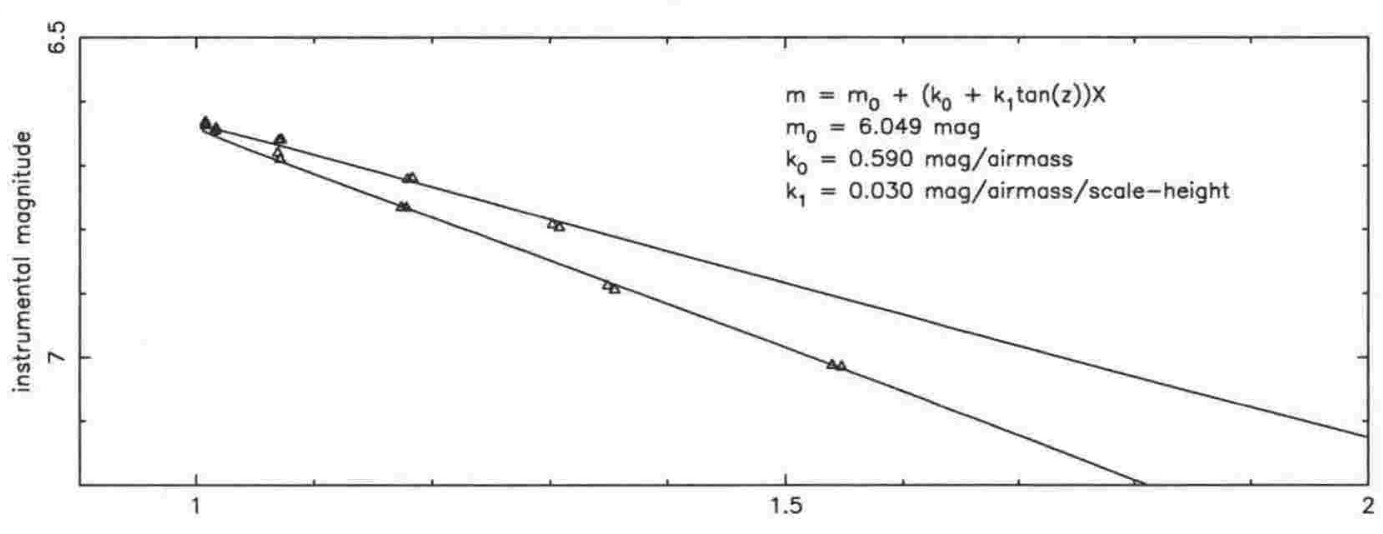

oir mass

time-varying instrumental zero-point

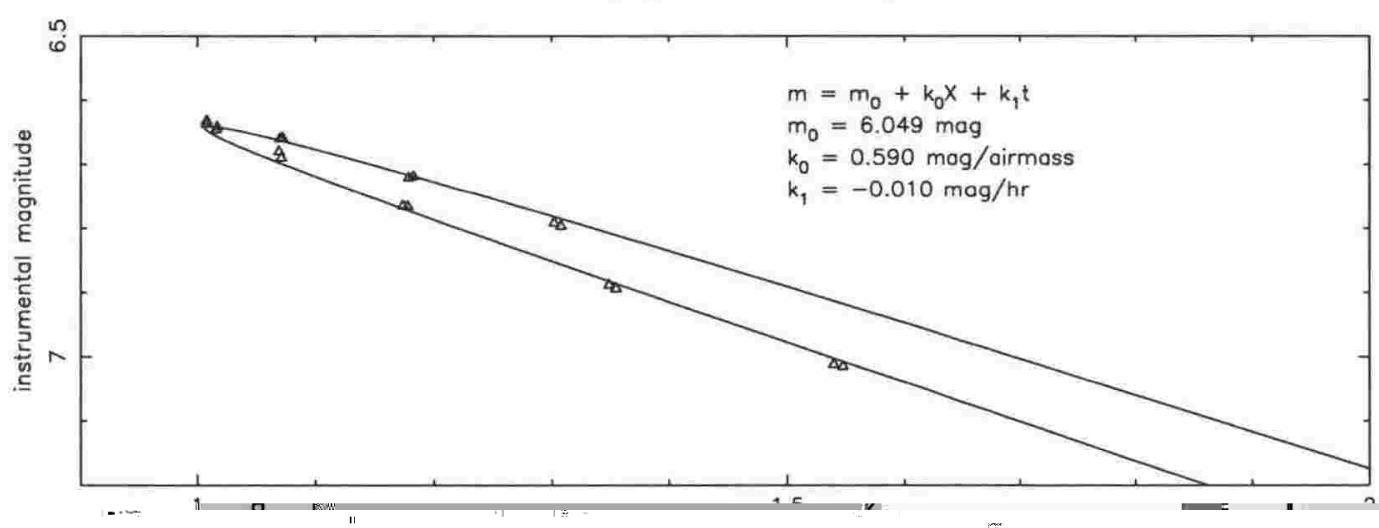

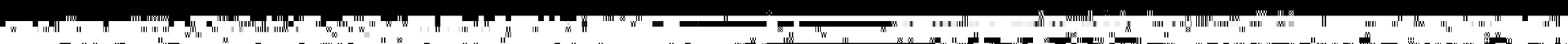
年 m

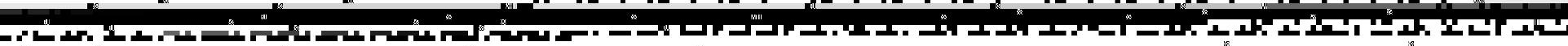

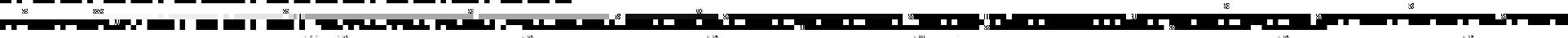
"

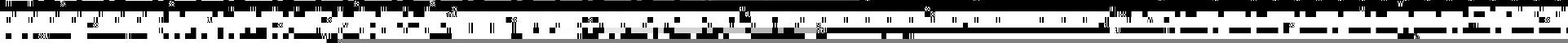


time-varying extinction coefficient
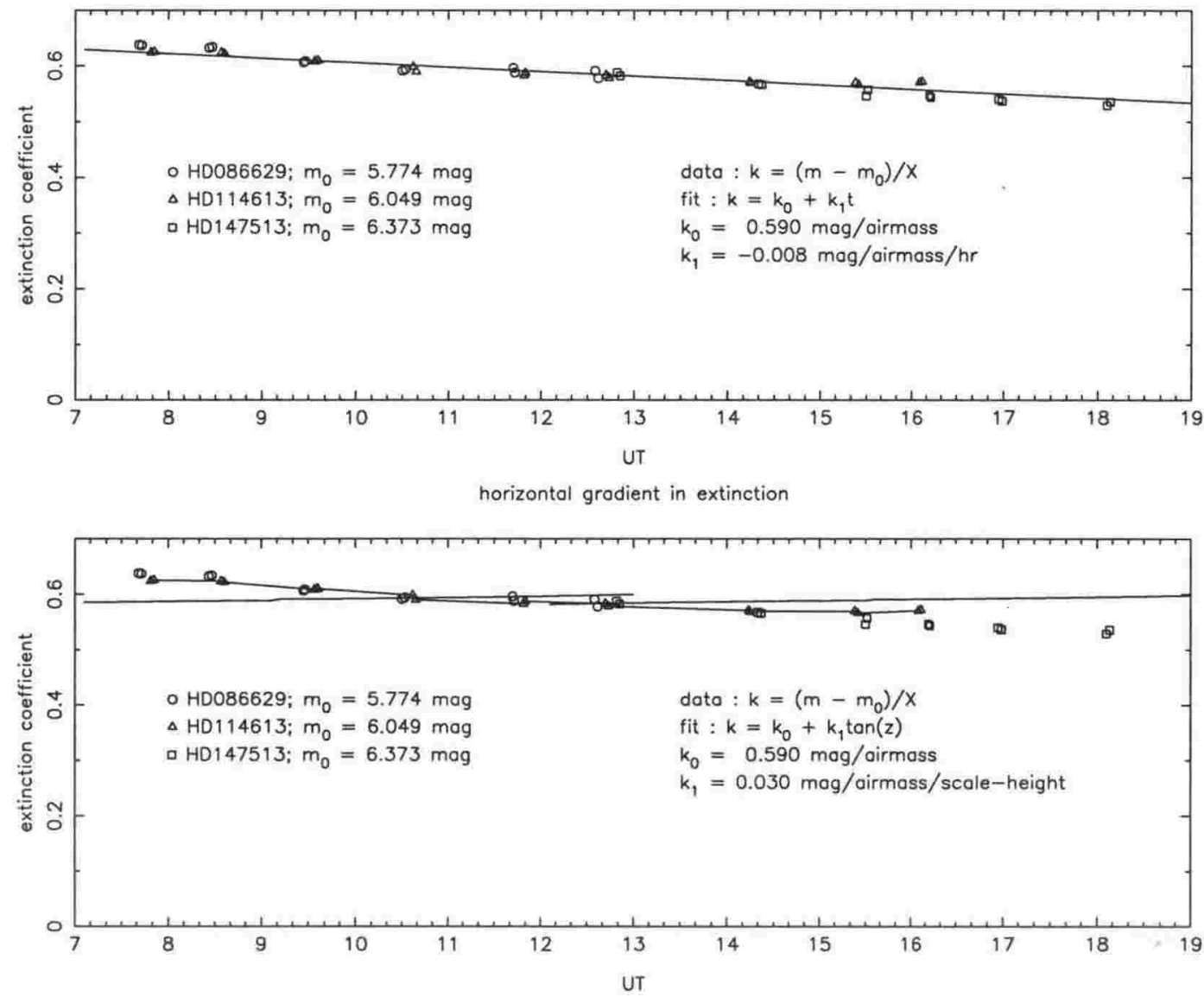

time-varying instrumental zero-point

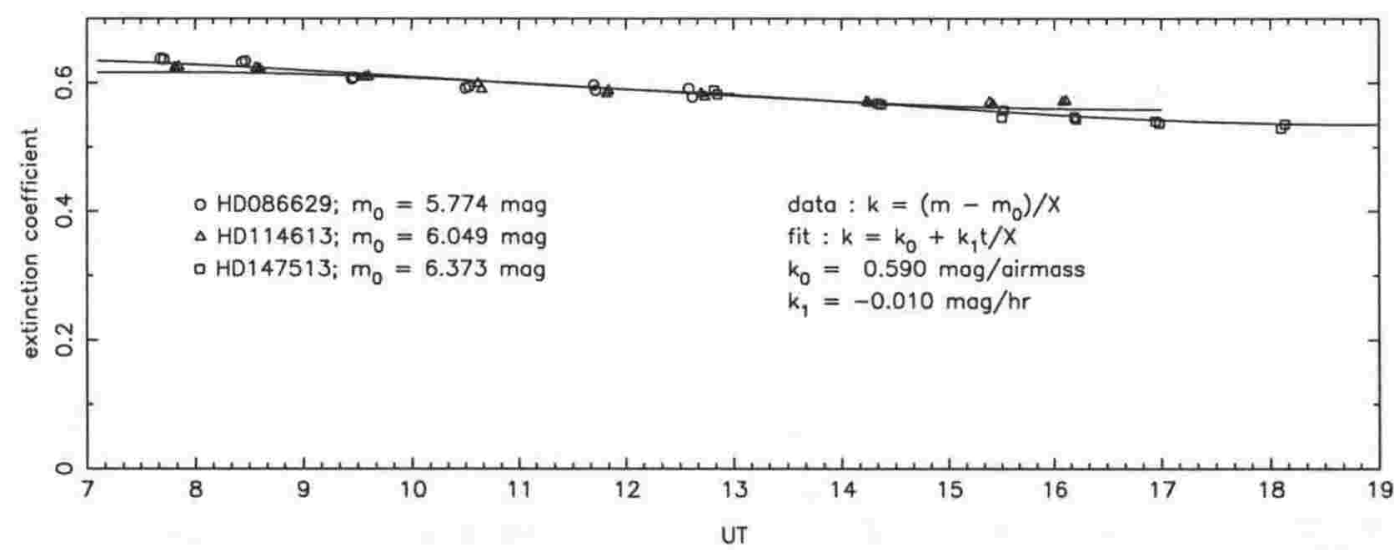

Figure 2.7: Effect on the P filter extinction coefficient of different types of extinction change. During 13 April 1992, three extinction stars were used to find the cause of apparently changing extinction, assuming linearly changing extinction, instrumental zero-point or a horizontal gradient in the extinction. 


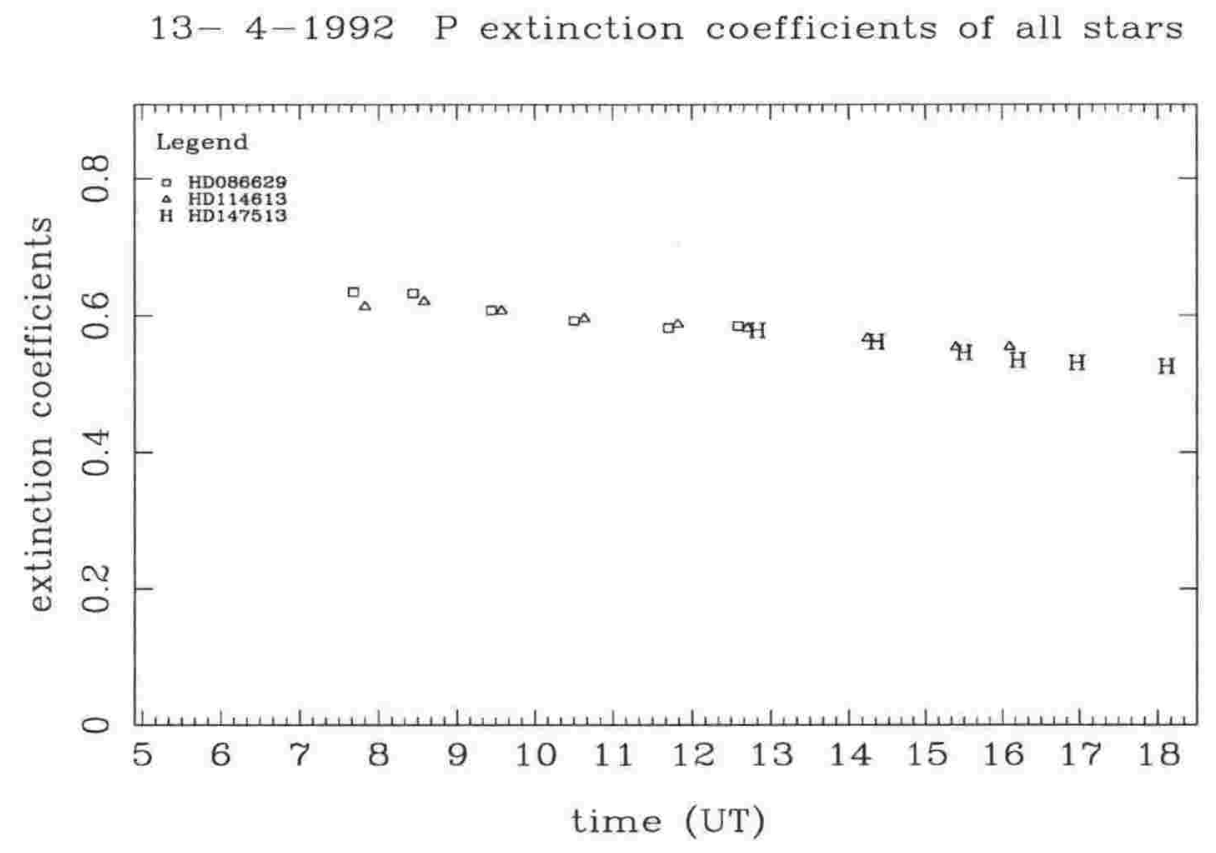

Figure 2.8: Possible variation of monochromatic extinction during a night. Using the exact magnitudes of HD086629, HD114613 and HD147513, which were determined by Nikonov's Method, the extinction at the time of each observation during 13 April 1992 can be determined.

$$
\text { 13-4-1992 P zero-point shift }
$$

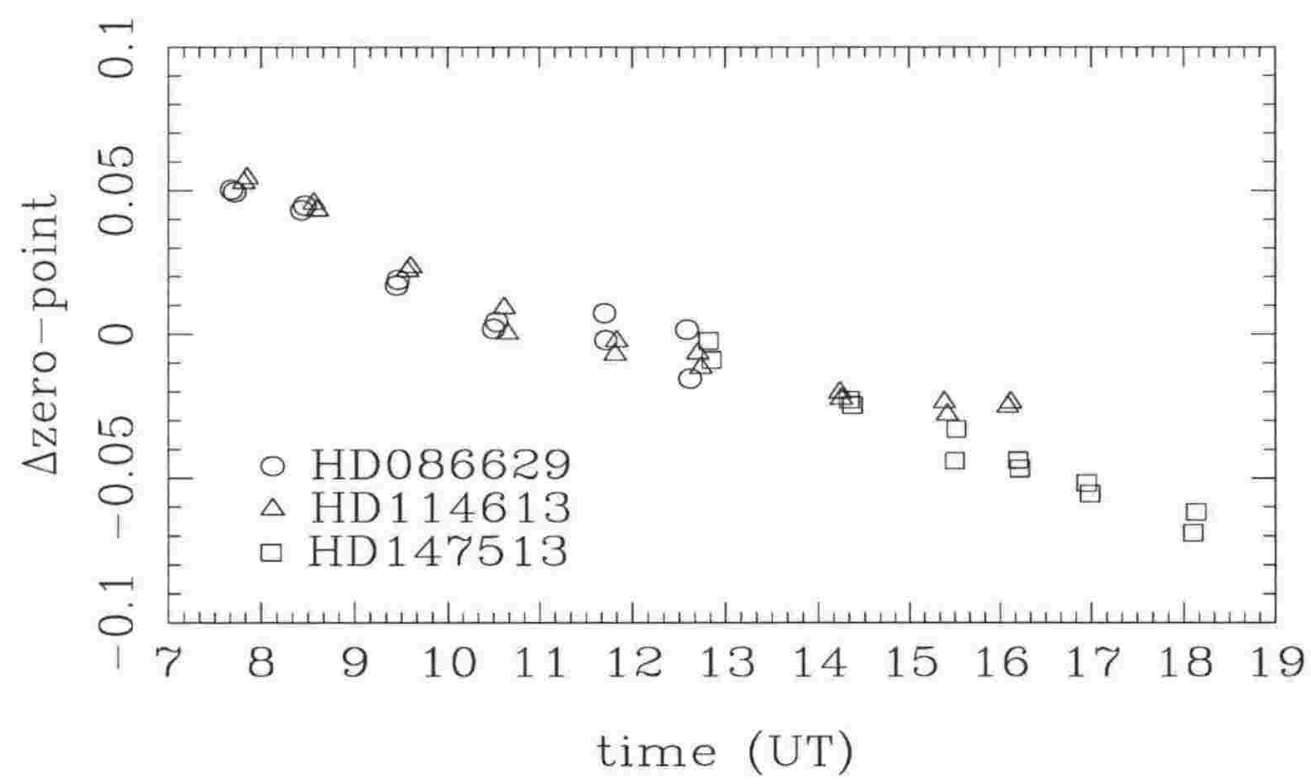

Figure 2.9: Possible variation of the instrumental zero-point during a night. Using the exact magnitudes of HD086629, HD114613 and HD147513, which were determined by Nikonov's Method, the zero-point at the time of each observation during 13 April 1992 can be determined. 
time-varying extinction coefficient

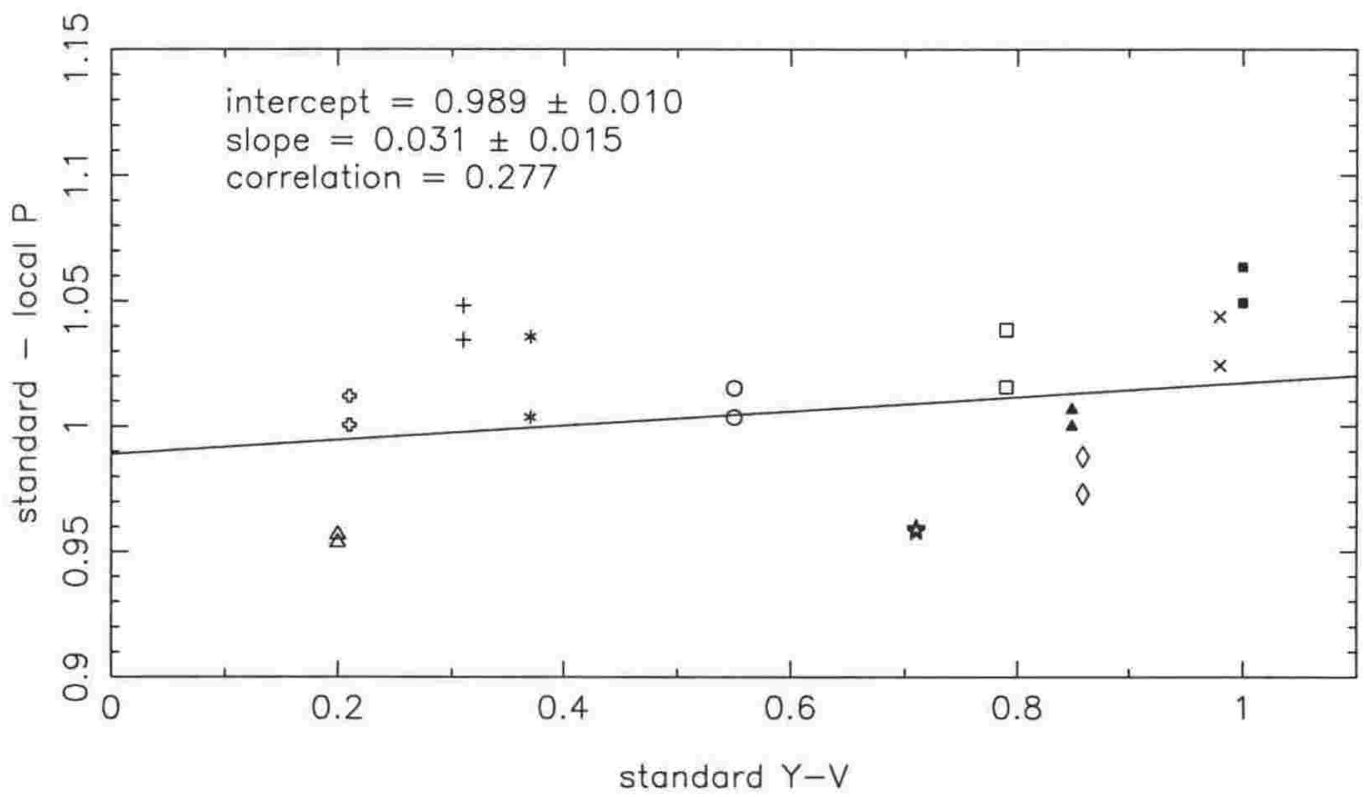

time-varying instrumental zero-point

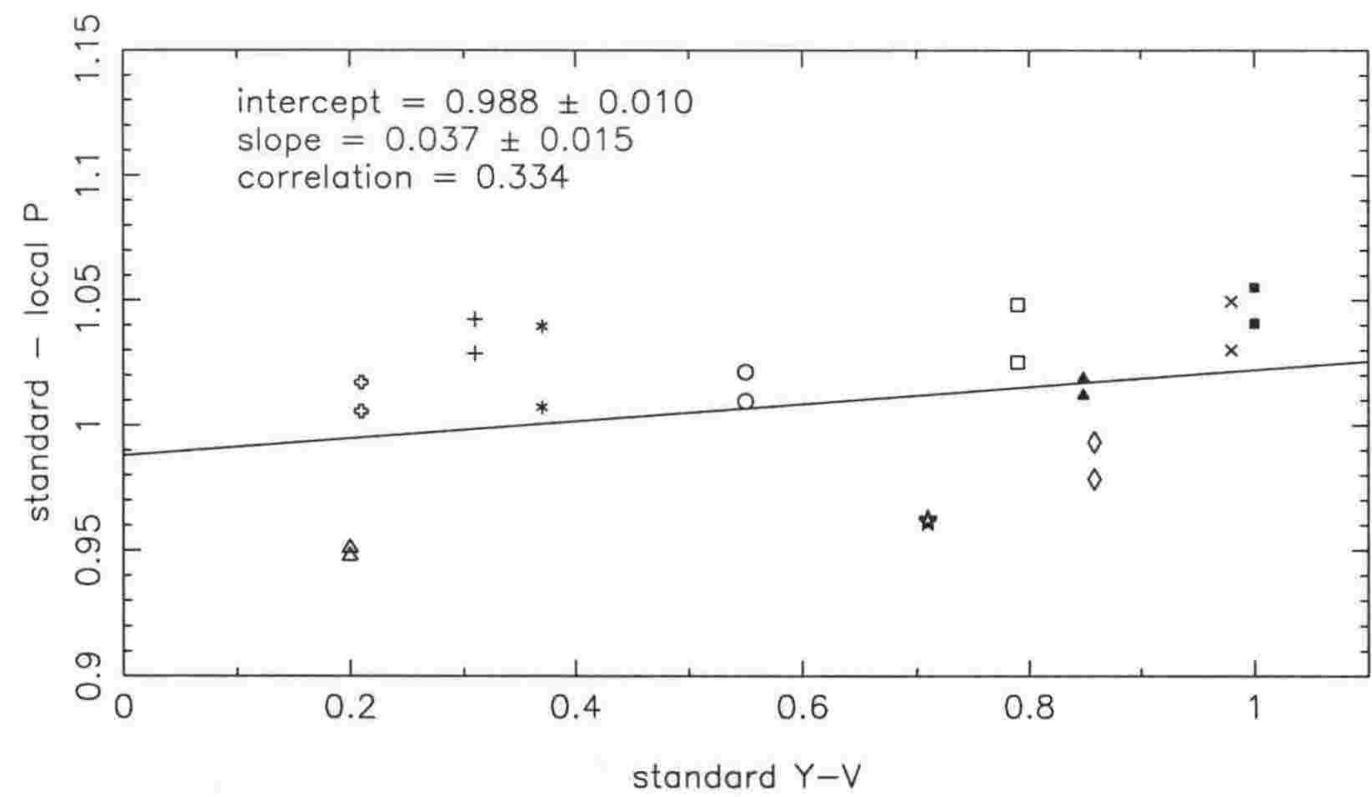

Figure 2.10: Effect of extinction or zero-point changes on transformation to the standard system for 13 April 1992. The symbols in the plots each represent a star; from left to right, the triangle is HD163245, the hollow plus is HD172850, the plus is HD150366, the asterisk is HD150451, the circle is HD157089, the pentagon is HD167768, the square is HD160823, the solid triangle is HD171767, the diamond is HD165462, the $\mathrm{x}$ is HD157415, and the solid square is HD175190. 
$X \sim 1$ ), the control star is rising in the East (i.e. starting at a large airmass, $X \sim 2$ say). The control star is only measured twice - once at the beginning of the night (when the extinction star culminates) and again at the end of the night (when the control star culminates and the extinction star sets). Measurements of the extinction star are made (nearly) simultaneously with these observations. Further measurements of the extinction star are made during the night at appropriate intervals, for instance hourly. In the variation of Nikonov's method used here, the control star is measured simultaneously with every extinction star observation rather than only at the beginning and end of the night.

The following criterion, in addition to those above, are used to select the control and extinction stars;

- non-variable

- appropriate (and equal) spectral types

- appropriate (and equal) magnitudes

To try to ensure the stars are non-variable, variable star catalogs (such as the General Catalog of Variable Stars (GCVS)) are checked for their absence. Preference is given to a star that has been observed as part of a standard stars system, for example from the E region Johnson standards. The spectral types should be the same so any heterochromatic extinction correction (see Section 2.7) is the same for both stars. They should also be of the appropriate spectral type for the filter system used so the heterochromatic extinction correction is (nearly) zero, for example use stars in the range F5-G2 V for the Vilnius system. Finally, both stars should be the same magnitude so any non-linearities in the measuring system cancel out. The magnitude should also be suitable for the size of the telescope, for example on the $\mathrm{B} \& \mathrm{C} 61 \mathrm{~cm}$ telescope, control and extinction stars in the range $m_{V} \sim 5.0 \rightarrow 7.0$ are acceptable. This range is bright enough to give good signal-to-noise, minimizing the importance of the dark count or sky background, the length of time required to make a measurement and not too bright to make the effect of the dead-time correction important.

The reduction algorithm is;

- Calculate the approximate magnitude of the extinction star $\left(\widetilde{m}_{o, e x t n}\right)$ from a Bouguer plot.

- Improve the accuracy of the approximate magnitude by taking the average over each photometric night of the observing run, giving $\left\langle\tilde{m}_{o, \text { extn }}\right\rangle$.

- For each simultaneous pair of control and extinction star observations (subscripted i), 


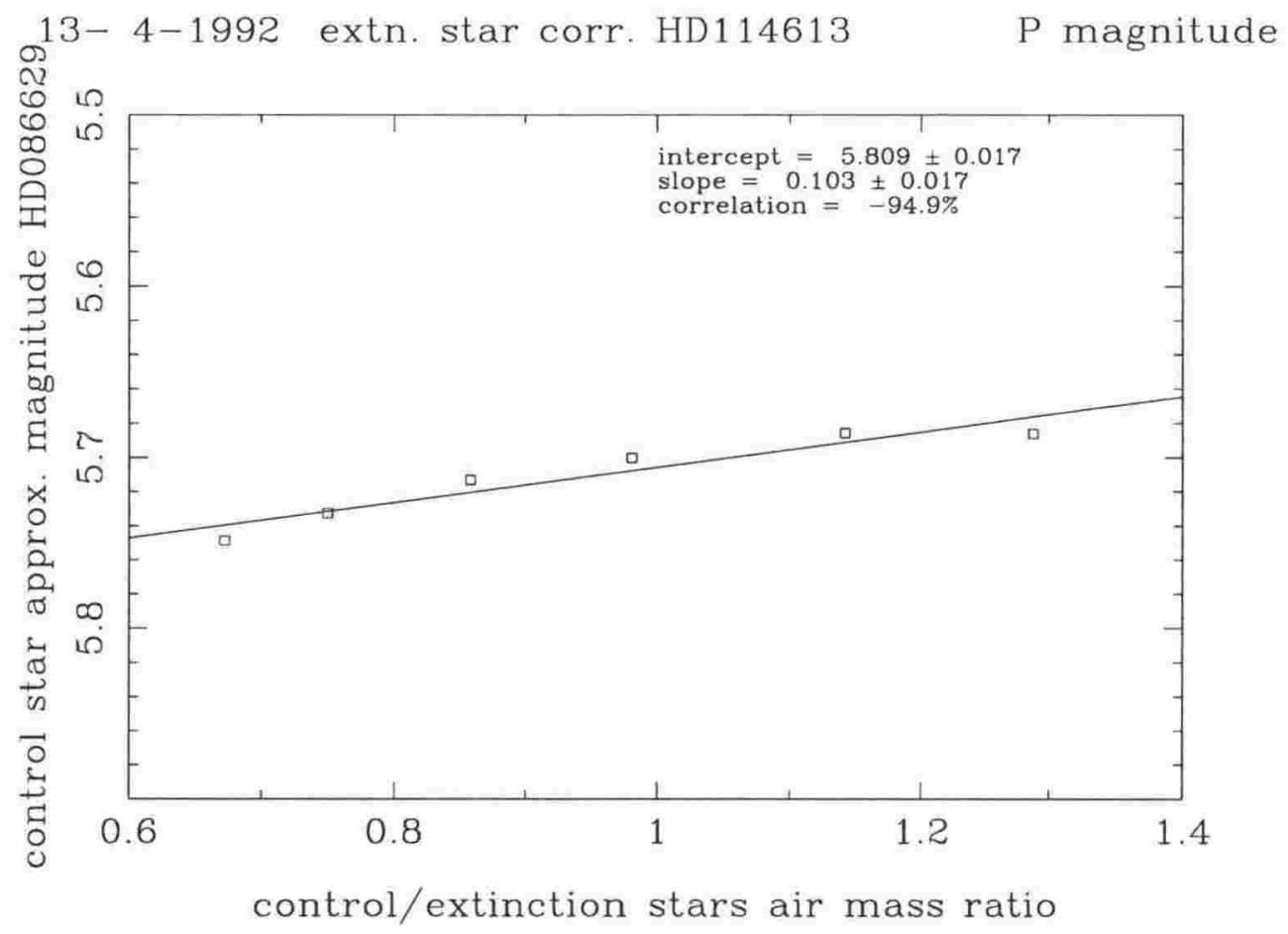

Figure 2.11: Nikonov's Method used to determine the exact $(\mathrm{P})$ magnitude of the extinction star. During 13 April 1992, the extinction star was HD114613, and the control star HD086629, yielding $m_{\text {exact }}=m_{\text {approx }}+(0.103 \pm 0.017)$.

calculate the approximate magnitude of the control star using

$$
\tilde{m}_{o, c n t l_{i}}=m_{c n t l_{i}}-\left(m_{e x t n_{i}}-<\tilde{m}_{o, e x t n_{i}}>\right) \frac{X_{c n t l_{i}}}{X_{e x t n_{i}}} .
$$

- Least squares fit $X_{\text {entl }_{i}} / X_{e x t n_{i}}$ (as the independent variable) versus $\widetilde{m}_{o, \text { entl }}$. The intercept gives the 'exact' mag of the control star $\left(m_{o, c n t l}\right)$ and the slope gives $\delta_{o, e x t n}$, which is the difference between the approximate and 'exact' magnitude of the extinction star $\left(m_{o, e x t n}=<\tilde{m}_{o, e x t n}>+\delta_{o, e x t n}\right)$.

- Improve the accuracy of the 'exact' magnitude of the control and extinction stars by averaging.

An example of the least squares fitting is shown in Figure 2,11. The derivation of this method is given in Appendix F, with the key assumptions being that the extinction coefficient is uniform across the sky at any one moment and that the observations of the control and extinction star are made simultaneously. The assumption about the uniform extinction is more likely to hold true than those made for a Bouguer plot. 
Typical extinction function for the Earth's atmosphere

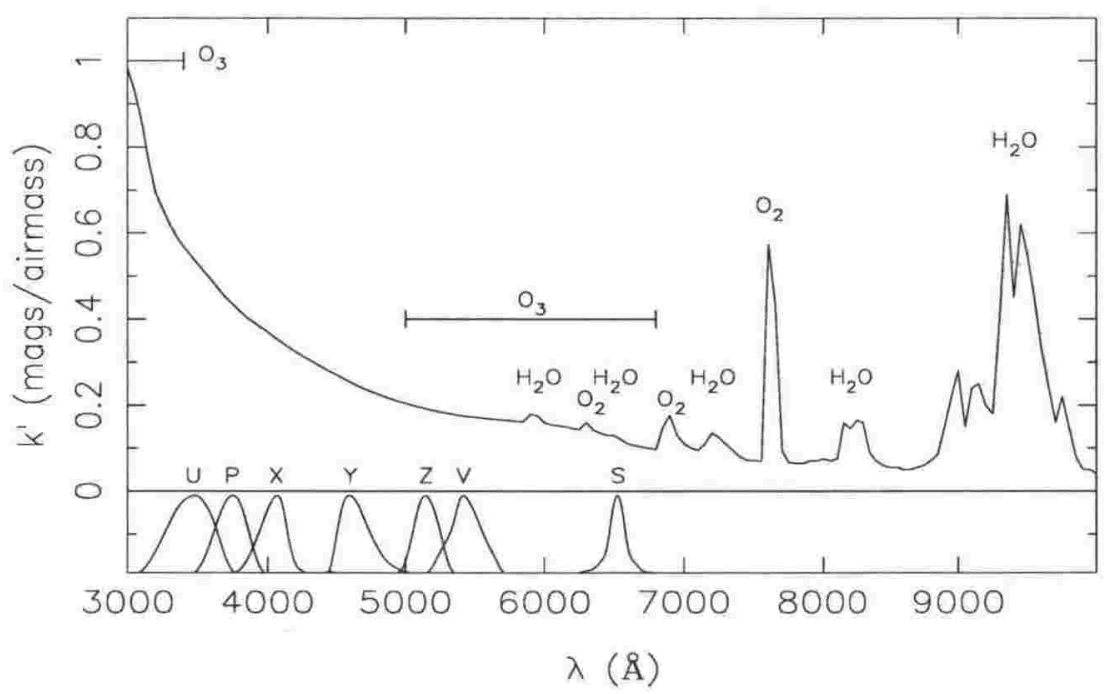

Figure 2.12: Typical atmospheric extinction across the optical region, assuming the equivalent of $10 \mathrm{~mm}$ of water vapour and $3 \mathrm{~mm}$ of Ozone. Taken from Table 7 of Straižys (1992). The Vilnius System passbands are included for comparison.

\subsection{The Heterochromatic Extinction Correction.}

The previous discussion about extinction assumed a monochromatic ray of light passing through the Earth's atmosphere. However each observation is made over a wavelength range defined by the particular filter used (or more strictly, the combined responses of the filter, PMT, any lenses in the photometer and the telescope mirrors). If the extinction coefficient was uniform across this wavelength range, then the same situation as for the monochromatic extinction would be obtained. However, as Figure 2.12 shows, this is seldom the case.

Generally the extinction is larger on the short wavelength side of a filter passband, hence the colour of a star appears reddened, with blue stars being affected more than red stars.

This reddening also increases with larger airmass; for example if the extinction on the short wavelength (blue) side of the filter was twice that of the long wavelength side at some airmass, then doubling the airmass increases the blue extinction by a factor of four over the red. This is equivalent to reducing the bandwidth of the filter, so at larger airmasses any (non-linear) effects of heterochromatic extinction are reduced and the Bouguer plot becomes a straight line - this is known as the Forbes effect, from Forbes (1842). Unless this effect is accounted for, an extrapolation of a straight line fitted from observations at large airmass will give an incorrect extra-terrestrial magnitude.

Thus the measured extinction coefficient depends on the star's spectral class (and luminos- 
ity, interstellar reddening etc.) in addition to the wavelength dependence of the atmospheric extinction and airmass of the observation. To obtain the extinction coefficient for another star, the measured coefficient must be 'corrected' for the difference in spectral class and airmass between the stars.

Recall the measured heterochromatic intensity is

$$
E_{m}=\int_{\lambda_{1}}^{\lambda_{2}} S(\lambda) R(\lambda) d \lambda
$$

where $S(\lambda)$ is the spectral function of the light incident on the receiver system (with response function $R(\lambda)$ ). A Taylor expansion is made on $S(\lambda)$ about $\lambda_{0}$, where $\lambda_{0}$ is chosen so that the first moment cancels, i.e. $\lambda_{0}=\int_{\lambda_{1}}^{\lambda_{2}} \lambda R(\lambda) d \lambda / \int_{\lambda_{1}}^{\lambda_{2}} R(\lambda) d \lambda$ and the other moments are denoted by $\mu_{n}^{n}\left(=\int_{\lambda_{1}}^{\lambda_{2}}\left(\lambda-\lambda_{0}\right)^{n} R(\lambda) d \lambda / \int_{\lambda_{1}}^{\lambda_{2}} R(\lambda) d \lambda\right)$;

$$
E_{m}=S\left(\lambda_{0}\right) \int_{\lambda_{1}}^{\lambda_{2}} R(\lambda) d \lambda+\frac{1}{2 !} \mu_{2}^{2} S^{\prime \prime}\left(\lambda_{0}\right) \int_{\lambda_{1}}^{\lambda_{2}} R(\lambda) d \lambda+\frac{1}{3 !} \mu_{3}^{3} S^{\prime \prime \prime}\left(\lambda_{0}\right) \int_{\lambda_{1}}^{\lambda_{2}} R(\lambda) d \lambda+\ldots
$$

where the primes indicate differentiation with respect to $\lambda$. Dropping the integral limits $\lambda_{1}$ and $\lambda_{2}$, the explicit $\lambda$ dependence and denoting $\lambda_{0}$ by a zero subscript on the function for brevity;

$$
E_{m}=S_{0} \int R d \lambda\left\{1+\frac{1}{2 !} \mu_{2}^{2} \frac{S_{0}^{\prime \prime}}{S_{0}}+\frac{1}{3 !} \mu_{3}^{3} \frac{S_{0}^{\prime \prime \prime}}{S_{0}}+\ldots\right\}
$$

The measured magnitude $m\left(h_{0}\right)$ has $S=E(\lambda) A(\lambda)$, with $E$ being the star's irradiance and $A$ being the Earth's atmospheric transmission function, so

$$
\begin{aligned}
m\left(h_{0}\right)= & z-2 \cdot 5 \log \left[E_{0} A_{0} \int R d \lambda\left\{1+\frac{1}{2 !} \mu_{2}^{2} \frac{\left(E_{0} A_{0}\right)^{\prime \prime}}{E_{0} A_{0}}+\frac{1}{3 !} \mu_{3}^{3} \frac{\left(E_{0} A_{0}\right)^{\prime \prime \prime}}{E_{0} A_{0}}+\ldots\right\}\right] \\
= & z-2 \cdot 5 \log \left\{E_{0}\right\}-2 \cdot 5 \log \left\{A_{0}\right\}-2 \cdot 5 \log \left\{\int R d \lambda\right\}- \\
& 2 \cdot 5 \log \left\{1+\frac{1}{2 !} \mu_{2}^{2} \frac{\left(E_{0} A_{0}\right)^{\prime \prime}}{E_{0} A_{0}}+\frac{1}{3 !} \mu_{3}^{3} \frac{\left(E_{0} A_{0}\right)^{\prime \prime \prime}}{E_{0} A_{0}}+\ldots\right\} .
\end{aligned}
$$

Similarly, the extra-terrestrial magnitude $m\left(h_{1}\right)$ has $S(\lambda)=E(\lambda)$, so

$$
\begin{gathered}
m\left(h_{1}\right)=z-2 \cdot 5 \log \left[E_{0} \int R d \lambda\left\{1+\frac{1}{2 !} \mu_{2}^{2} \frac{E_{0}^{\prime \prime}}{E_{0}}+\frac{1}{3 !} \mu_{3}^{3} \frac{E_{0}^{\prime \prime \prime}}{E_{0}}+\ldots\right\}\right] \\
=z-2 \cdot 5 \log \left\{E_{0}\right\}-2 \cdot 5 \log \left\{\int R d \lambda\right\}- \\
\quad 2 \cdot 5 \log \left\{1+\frac{1}{2 !} \mu_{2}^{2} \frac{E_{0}^{\prime \prime}}{E_{0}}+\frac{1}{3 !} \mu_{3}^{3} \frac{E_{0}^{\prime \prime \prime}}{E_{0}}+\ldots\right\} .
\end{gathered}
$$

The required transformation (which is usually called a 'correction') is

$$
\Delta m=m\left(h_{1}\right)-m\left(h_{0}\right)
$$




$$
\begin{gathered}
=2 \cdot 5 \log \left\{A_{0}\right\}-2 \cdot 5 \log \left\{1+\frac{1}{2 !} \mu_{2}^{2} \frac{E_{0}^{\prime \prime}}{E_{0}}+\frac{1}{3 !} \mu_{3}^{3} \frac{E_{0}^{\prime \prime \prime}}{E_{0}}+\ldots\right\}+ \\
2.5 \log \left\{1+\frac{1}{2 !} \mu_{2}^{2} \frac{\left(E_{0} A_{0}\right)^{\prime \prime}}{E_{0} A_{0}}+\frac{1}{3 !} \mu_{3}^{3} \frac{\left(E_{0} A_{0}\right)^{\prime \prime \prime}}{E_{0} A_{0}}+\ldots\right\} .
\end{gathered}
$$

If the higher order terms in the $\log$ functions are small, then the approximation $\ln (1+x) \approx x$ may be used

$$
\begin{array}{r}
\Delta m \approx 2 \cdot 5 \log \left\{A_{0}\right\}-2 \cdot 5 \log (e)\left\{\frac{1}{2 !} \mu_{2}^{2} \frac{E_{0}^{\prime \prime}}{E_{0}}+\frac{1}{3 !} \mu_{3}^{3} \frac{E_{0}^{\prime \prime \prime}}{E_{0}}+\ldots\right\}+ \\
2 \cdot 5 \log (e)\left\{\frac{1}{2 !} \mu_{2}^{2} \frac{\left(E_{0} A_{0}\right)^{\prime \prime}}{E_{0} A_{0}}+\frac{1}{3 !} \mu_{3}^{3} \frac{\left(E_{0} A_{0}\right)^{\prime \prime \prime}}{E_{0} A_{0}}+\ldots\right\}
\end{array}
$$

with the $\log (e)$ factors coming from the conversion of base 10 logarithms to natural logarithms.

Now the derivatives of $E_{0} A_{0}$ expand in a binomial series, for example $\left(E_{0} A_{0}\right)^{\prime \prime}=E_{0}^{\prime \prime} A_{0}+$ $2 E_{0}^{\prime} A_{0}^{\prime}+E_{0} A_{0}^{\prime \prime}$, which gives

$$
\begin{gathered}
\Delta m \approx 2 \cdot 5 \log \left\{A_{0}\right\}+2 \cdot 5 \log (e)\left\{\frac{1}{2 !} \mu_{2}^{2}\left[2 \frac{E_{0}^{\prime}}{E_{0}} \frac{A_{0}^{\prime}}{A_{0}}+\frac{A_{0}^{\prime \prime}}{A_{0}}\right]+\right. \\
\left.\frac{1}{3 !} \mu_{3}^{3}\left[3 \frac{E_{0}^{\prime \prime}}{E_{0}} \frac{A_{0}^{\prime}}{A_{0}}+3 \frac{E_{0}^{\prime}}{E_{0}} \frac{A_{0}^{\prime \prime}}{A_{0}}+\frac{A_{0}^{\prime \prime \prime}}{A_{0}}\right]+\ldots\right\}
\end{gathered}
$$

The 'normalised' derivatives are derivatives of the quantity in magnitudes as $x^{\prime} / x=\{\ln (x)\}^{\prime}=$ $\ln (10)\{\log (x)\}^{\prime}$ and the higher order derivatives can be written in similar terms using the earlier derivatives, for example $x^{\prime \prime} / x=\left(x^{\prime} / x\right)^{2}+\left(x^{\prime} / x\right)^{\prime}$. Therefore, (assuming the airmass $X$ does not have any wavelength dependence)

$$
\begin{aligned}
2.5 \log \left\{A_{0}\right\} & =-k_{0} X \\
\frac{A_{0}^{\prime}}{A_{0}} & =-\frac{\ln (10)}{2 \cdot 5} k_{0}^{\prime} X \text { and } \\
\frac{A_{0}^{\prime \prime}}{A_{0}} & =\left[-\frac{\ln (10)}{2 \cdot 5} k_{0}^{\prime} X\right]^{2}-\frac{\ln (10)}{2 \cdot 5} k_{0}^{\prime \prime} X \quad \text { etc. }
\end{aligned}
$$

with the other extinction terms involving further derivatives of the monochromatic extinction at $\lambda_{0}$ and powers of the airmass $X$. Similar results are also found for the extra-terrestrial monochromatic magnitude terms.

In principle, the extinction at $\lambda_{0}$ and it's derivatives can be found by measuring the extinction at several wavelengths closely spaced about $\lambda_{0}$. To first order, the extinction follows a power law $k(\lambda)=a \lambda^{-n}$, where $n \approx 4$ as Rayleigh scattering is usually the dominant cause of extinction. In particular, this is true for the Vilnius System as the $\mathrm{H}_{2} \mathrm{O}$ and $\mathrm{O}_{2}$ absorption bands do not coincide with the filter passbands, or are very weak if they are included. As the extinction is thus a fairly smooth function, the spacing of the filters is not critical, and the monochromatic extinction values from the filter set in use is adequate to derive the significant 
terms.

Unfortunately a star's spectra is not smooth, consisting of absorption or emission lines superimposed on a black body curve. In general, the derivatives of $E_{0}$ do not diminish very fast. Young (1988) has shown that the first and second derivatives can be found easily by splitting $E$ into a smooth (parabolic) function $E_{s}$ and a remainder function $E_{r}$. The coefficients of the parabola are found by requiring $\int E_{r} A R d \lambda=0$ so the equations above (to second order) hold for the smooth function. A Taylor expansion of this condition about the extinction $A_{0}$ gives

$$
A_{0} \int E_{r} R d \lambda+A_{0}^{\prime} \int\left(\lambda-\lambda_{0}\right) E_{r} R d \lambda+A_{0}^{\prime \prime} \int\left(\lambda-\lambda_{0}\right)^{2} E_{r} R d \lambda=0
$$

As $A_{0}, A_{0}^{\prime}$, and $A_{0}^{\prime \prime}$ depend on the airmass through which a star is measured, to ensure the condition is always true requires each integral to vanish. Young (1988) finds this requires the filters to spaced less than half of the FWHM apart in wavelength to get good results.

This condition is (nearly) satisfied for only the U,P,X and the $\mathrm{Z}$ and $\mathrm{V}$ filters of the Vilnius System. Hence the expansion can only be carried to the second moment (and also dropping $\left.A_{0}^{\prime \prime} / A_{0}\right)$

$$
\begin{aligned}
\Delta m & \approx 2 \cdot 5 \log \left\{A_{0}\right\}+2 \cdot 5 \log (e) \frac{2}{2 !} \mu_{2}^{2} \frac{E_{0}^{\prime}}{E_{0}} \frac{A_{0}^{\prime}}{A_{0}} \\
& \approx-k_{0} X+c k_{0}^{\prime} X\left\{m\left(h_{1}\right)_{0}\right\}^{\prime}
\end{aligned}
$$

where $c$ absorbs the various constant terms. The derivative of the extra-terrestrial magnitude about $\lambda_{0}$ is found from some colour index, for instance in the Vilnius System $\mathrm{X}-\mathrm{Y}$ is used. The usual form of this equation is

$$
\Delta m \approx-\left(k^{\prime}+k^{\prime \prime} \text { Colour }\right) X
$$

where $k^{\prime}$ and $k^{\prime \prime}$ absorb the various constant factors and are the primary (or monochromatic) and secondary (or heterochromatic or colour) extinction coefficients.

A better approximation can be found by retaining the $A_{0}^{\prime \prime} / A_{0}$ term. This is done by not expanding $\left(E_{0} A_{0}\right)^{\prime \prime}$ but instead adding the second moment terms (in equation 2.20) together

$$
\ldots \mu_{2}^{2}\left[\frac{E_{0}^{\prime \prime}}{E_{0}}+\frac{\left(E_{0} A_{0}\right)^{\prime \prime}}{E_{0} A_{0}}\right] \ldots
$$

i.e. using the average of the measured and extra-terrestrial colour (Young \& Irvine 1967, Young 1974).

As most filter systems are not spaced close enough together in wavelength to correct for heterochromatic extinction more accurately than the second derivatives, according to Sterken 
$21-5-1993$ Y-Z colour sec. extn. coeff. fit

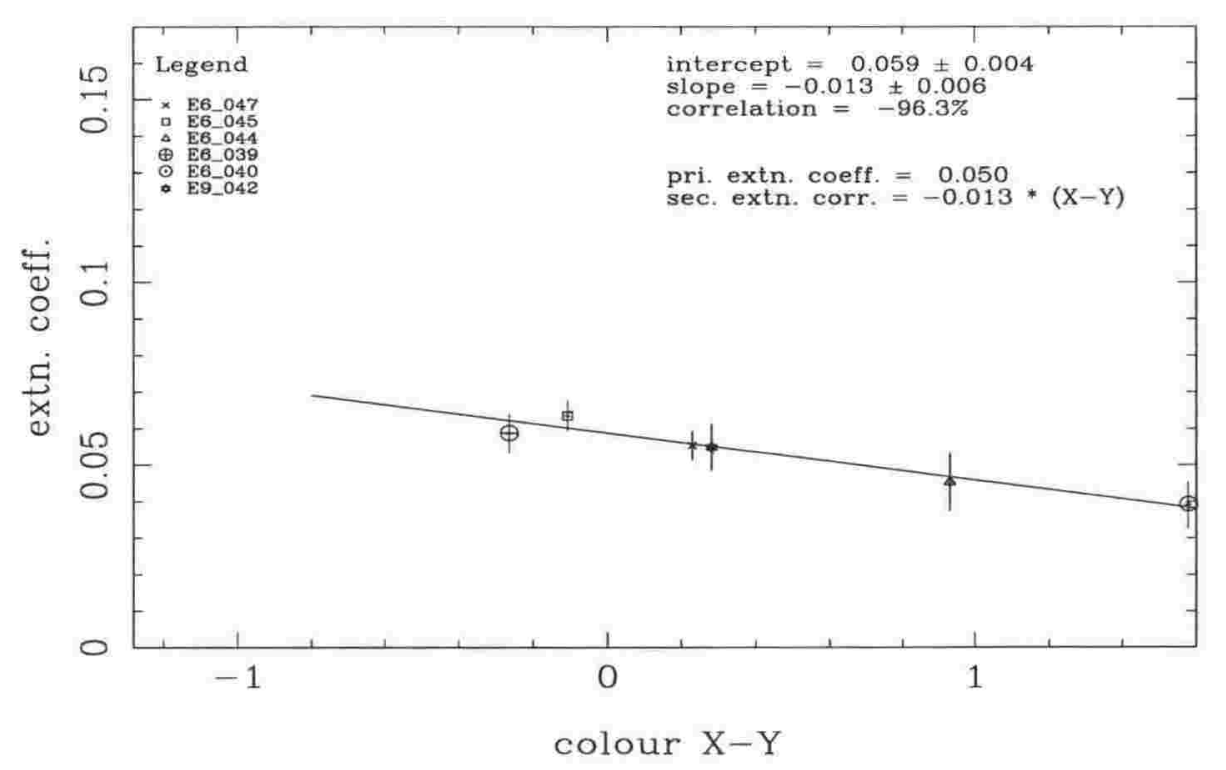

Figure 2.13: Secondary Extinction Coefficient for $\mathrm{Y}-\mathrm{Z}$ of Vilnius filter set \#2, determined from the slope of the heterochromatic extinction coefficients of six stars versus their $\mathrm{X}-\mathrm{Y}$ colours. The colour $\mathrm{X}-\mathrm{Y}$ is the extra-terrestrial colour in the instrumental system.

\& Manfroid (1992a) it is better that everyone use the same reduction methods (even if they are not strictly correct). Thus everyone will have the same systematic errors, which will at least allow better comparisons between different observers. For this reason, equation 2.24 (with the extra-terrestrial colour) was used here, following the procedures from the originators of the Vilnius System.

The colour extinction coefficient was determined for each filter set by measuring the heterochromatic extinction $\left(k=k^{\prime}+k^{\prime \prime}\right.$ Colour $)$ for several extinction stars of different spectral types on a photometric night. To minimize the effect of any errors in measuring the extinction, (for instance, due to variations in $k$ across the sky or during the night) the stars should be close together in the sky and be measured simultaneously. The extinction $k$ is calculated for each star in a Bouguer plot and these then plotted against the extra-terrestrial colour of each star, as shown in Figure 2.13.

The only terms that can vary in the secondary coefficient $k_{0}^{\prime}$ (see equation 2.23) are the derivative of the extinction spectral function $\left(A_{0}^{\prime} / A_{0}\right)$ and the second moment of the filter passbands $\left(\mu_{2}^{2}\right)$. Neither of these terms are considered to change very quickly, so estimates for the required measurement frequency of the secondary coefficients vary from monthly to yearly (Young 1974, Hendon \& Kaitchuck 1990).

The results for the two filter sets is shown in Table 2.3 , with most magnitudes and colours 
not having a significant coefficient, i.e. the coefficient was not larger than the measuring errors. The colour term is set to zero for an arbitrary spectral class, being G0 V for the Vilnius System - in the table, this is the $(\mathrm{X}-\mathrm{Y})_{\text {offset }}$ in the local (instrumental) system. The coefficients were measured on 21 Feb 1991 for filter set \#1 and on 25 May 1993 for set \#2.

The theoretical results for the standard Vilnius System are also listed in the table. The

Table 2.3: Vilnius Colour Extinction Coefficients, measured on $21 \mathrm{Feb} 1991$ (filter set \#1) and 25 May 1993 (set \#2)

Standard System coefficients wer
obtained by convolving the sys-
tem passbands with a typical
extinction spectral curve at dif-
ferent airmasses and for different stars with measured spectra and colours (Straižys 1992,

\begin{tabular}{|c|c|c|c|}
\hline colour & \multicolumn{3}{|c|}{ filter set } \\
& standard & $15 \mathrm{~mm}$ & $28 \mathrm{~mm}$ \\
\hline$(\mathrm{X}-\mathrm{Y})_{\text {offset }}$ & +0.78 & $-0.03 \pm 0.01$ & $+0.25 \pm 0.01$ \\
$\mathrm{U}$ & - & - & $-0.016 \pm 0.007$ \\
$\mathrm{~S}$ & - & - & $+0.012 \pm 0.005$ \\
$\mathrm{Y}-\mathrm{V}$ & - & - & $-0.012 \pm 0.010$ \\
$\mathrm{U}-\mathrm{P}$ & -0.027 & $-0.020 \pm 0.006$ & $-0.028 \pm 0.009$ \\
$\mathrm{P}-\mathrm{X}$ & +0.013 & $+0.017 \pm 0.006$ & $+0.018 \pm 0.007$ \\
$\mathrm{X}-\mathrm{Y}$ & -0.011 & $-0.013 \pm 0.006$ & - \\
$\mathrm{Y}-\mathrm{Z}$ & - & - & $-0.013 \pm 0.006$ \\
\hline
\end{tabular}
Zdanavičius 1970). The agreement between the different filter sets and the Standard System is reasonably close. The values are also quite small, as the error in measuring the primary extinction coefficient is often a similar size (especially for the nights where the extinction was changing).

\subsection{Transformation to the Standard Filter System.}

Now the extra-terrestrial magnitude $m\left(h_{1}\right)$, which was measured in the local instrumental system (also called the natural or internal instrumental system or simply the instrumental system), has to be transformed to the equivalent measurement in the standard system (which is defined by the original instrumental system of the photometric system).

In general, the corresponding filters in each system will not have the same effective wavelength. Hence, in a Taylor expansion about $\lambda_{0}$ (choosing $\lambda_{0}$ between the effective wavelengths of each filter, say) the first moments will be non-zero. Denoting the local system by the subscript $A$ and the standard system by $B$,

$$
\begin{aligned}
m\left(h_{1}\right)_{A}=z_{A}-2 \cdot 5 \log \left\{E_{0}\right\}-2 \cdot 5 \log \left\{\int R_{A} d \lambda\right\}- & \\
& 2 \cdot 5 \log \left\{1+\mu_{1, A} \frac{E_{0}^{\prime}}{E_{0}}+\frac{1}{2 !} \mu_{2, A}^{2} \frac{E_{0}^{\prime \prime}}{E_{0}}+\frac{1}{3 !} \mu_{3, A}^{3} \frac{E_{0}^{\prime \prime \prime}}{E_{0}}+\ldots\right\}, \\
m\left(h_{1}\right)_{B}=z_{B}-2 \cdot 5 \log \left\{E_{0}\right\}-2 \cdot 5 \log \left\{\int R_{B} d \lambda\right\}- & \\
& 2 \cdot 5 \log \left\{1+\mu_{1, B} \frac{E_{0}^{\prime}}{E_{0}}+\frac{1}{2 !} \mu_{2, B}^{2} \frac{E_{0}^{\prime \prime}}{E_{0}}+\frac{1}{3 !} \mu_{3, B}^{3} \frac{E_{0}^{\prime \prime \prime}}{E_{0}}+\ldots\right\} .
\end{aligned}
$$


Absorbing $\int R_{A} d \lambda$ into the zero-point $z_{A}$ etc. and combining the zero-points as $z_{A B}$, the transformation between systems $A$ and $B$ is

$$
\begin{aligned}
m\left(h_{1}\right)_{A}-m\left(h_{1}\right)_{B}=z_{A B} & -2 \cdot 5 \log \left\{1+\mu_{1, A} \frac{E_{0}^{\prime}}{E_{0}}+\frac{1}{2 !} \mu_{2, A}^{2} \frac{E_{0}^{\prime \prime}}{E_{0}}+\frac{1}{3 !} \mu_{3, A}^{3} \frac{E_{0}^{\prime \prime \prime}}{E_{0}}+\ldots\right\} \\
& +2.5 \log \left\{1+\mu_{1, B} \frac{E_{0}^{\prime}}{E_{0}}+\frac{1}{2 !} \mu_{2, B}^{2} \frac{E_{0}^{\prime \prime}}{E_{0}}+\frac{1}{3 !} \mu_{3, B}^{3} \frac{E_{0}^{\prime \prime \prime}}{E_{0}}+\ldots\right\} \\
=z_{A B} & -2 \cdot 5 \log \left\{1+\frac{\mu_{1, A}}{\lambda_{0}} \frac{\lambda_{0} E_{0}^{\prime}}{E_{0}}+\frac{1}{2 !}\left(\frac{\mu_{2, A}}{\lambda_{0}}\right)^{2} \frac{\lambda_{0}^{2} E_{0}^{\prime \prime}}{E_{0}}+\ldots\right\} \\
& +2 \cdot 5 \log \left\{1+\frac{\mu_{1, B}}{\lambda_{0}} \frac{\lambda_{0} E_{0}^{\prime}}{E_{0}}+\frac{1}{2 !}\left(\frac{\mu_{2, B}}{\lambda_{0}}\right)^{2} \frac{\lambda_{0}^{2} E_{0}^{\prime \prime}}{E_{0}}+\ldots\right\}\{2
\end{aligned}
$$

The latter form allows the derivatives of $E_{0}$ to be replaced by derivatives of $\ln (E)$ with respect to $\ln (\lambda)\left(\right.$ at $\left.\lambda_{0}\right)$

$$
\begin{aligned}
\frac{\lambda E^{\prime}}{E} & =\frac{\lambda}{E} \frac{d E}{d \lambda}=\frac{d(\ln E)}{d(\ln \lambda)} \\
\frac{\lambda^{2} E^{\prime \prime}}{E} & =\frac{\lambda^{2}}{E} \frac{d^{2} E}{d \lambda^{2}}=\frac{d^{2}(\ln E)}{d(\ln \lambda)^{2}}+\left[\frac{d(\ln E)}{d(\ln \lambda)}\right]^{2}-\frac{d(\ln E)}{d(\ln \lambda)}
\end{aligned}
$$

going from the compact prime notation to the explicit $d y / d x$ notation to show the change of variables. As before, a star's spectra can be split into a smooth approximation and a remainder $\left(E=E_{s}+E_{r}\right)$ and $E_{s}$ chosen such that the above equations hold for $E_{s}$ instead of $E$. Hence, the logarithmic derivatives of the smoothed spectra can be estimated by a colour index (for $d(\ln E) / d(\ln \lambda))$ and difference between adjacent colour indices (for $\left.d^{2}(\ln E) / d(\ln \lambda)^{2}\right)$. Young (1988) has found that the colours (and differences) give good estimates of the first and second derivatives if the filters (in a system) are spaced less than half of the FWHM apart in wavelength.

It is usually assumed that the higher order terms are all small, so the logarithmic approximation $\ln (1+x) \approx x$ for $x \ll 1$ may be used

$$
\begin{aligned}
& m\left(h_{1}\right)_{A}-m\left(h_{1}\right)_{B} \approx z_{A B}-2 \cdot 5 \log (e)\left\{\frac{\mu_{1, A}}{\lambda_{0}} \frac{\lambda_{0} E_{0}^{\prime}}{E_{0}}+\frac{1}{2 !}\left(\frac{\mu_{2, A}}{\lambda_{0}}\right)^{2} \frac{\lambda_{0}^{2} E_{0}^{\prime \prime}}{E_{0}}+\ldots\right\} \\
&+2 \cdot 5 \log (e)\left\{\frac{\mu_{1, B}}{\lambda_{0}} \frac{\lambda_{0} E_{0}^{\prime}}{E_{0}}+\frac{1}{2 !}\left(\frac{\mu_{2, B}}{\lambda_{0}}\right)^{2} \frac{\lambda_{0}^{2} E_{0}^{\prime \prime}}{E_{0}}+\ldots\right\} \\
& \approx z_{A B}++2 \cdot 5 \log (e)\left\{\frac{\mu_{1, B}-\mu_{1, A}}{\lambda_{0}} \frac{\lambda_{0} E_{0}^{\prime}}{E_{0}}+\right. \\
&\left.\frac{1}{2 !} \frac{\mu_{2, B}^{2}-\mu_{2, A}^{2}}{\lambda_{0}^{2}} \frac{\lambda_{0}^{2} E_{0}^{\prime \prime}}{E_{0}}+\ldots\right\}
\end{aligned}
$$

with the $\log (e)$ factors coming from the conversion of base 10 logarithms to natural logarithms. 
Denoting the effective wavelengths of the systems as $\lambda_{A}$ and $\lambda_{B}$, then

$$
\mu_{1, A}=\frac{\int\left(\lambda-\lambda_{0}\right) R_{A} d \lambda}{\int R_{A} d \lambda}=\frac{\int \lambda R_{A} d \lambda}{\int R_{A} d \lambda}-\lambda_{0} \frac{\int R_{A} d \lambda}{\int R_{A} d \lambda}=\lambda_{A}-\lambda_{0}
$$

and similarly, $\mu_{1, B}=\lambda_{B}-\lambda_{0}$. This gives

$$
\mu_{1, B}-\mu_{1, A}=\lambda_{B}-\lambda_{A} .
$$

If the higher order moments of each system are equal, i.e. the filters have (nearly) identical shapes but are shifted in wavelength, then

$$
\begin{aligned}
m\left(h_{1}\right)_{A}-m\left(h_{1}\right)_{B} & \approx z_{A B}-2 \cdot 5 \log (e)\left(\frac{\lambda_{B}-\lambda_{A}}{\lambda_{0}}\right)\left[\frac{d(\ln E)}{d(\ln \lambda)}\right]_{\lambda_{0}} \\
& \approx z_{A B}+\gamma(\text { colour })_{\lambda_{0}}
\end{aligned}
$$

which is the linear transformation equation commonly used.

The transmittance of the local Vilnius filter sets were measured from $\lambda=1900-8200 \AA$ in $20 \AA$ steps using a double-beam spectrometer, courtesy of the Chemistry Department, VUW. These are compared with the response curves of the Standard Vilnius system (Straižys 1992) in Figure 2.14. The local filters generally match the standard filters' shapes but are shifted slightly in wavelength, so the equation 2.28 is expected to be a good transformation. However the different shapes of the $\mathrm{Z}$ and (interference) $\mathrm{S}$ filter, which shows a double peak, may cause some problems.

Convolving the 1990 measurements of filter set \#1 and the Standard filter responses with stellar spectra (Gunn \& Stryker 1983) to synthesize magnitudes, various transformations between the local and Standard systems were plotted. It was found that using $(\mathrm{Y}-\mathrm{V})_{0}$ as the colour in equation 2.28, single-valued (but often non-linear) transformations were required the zero subscript now indicates a measurement in the standard system. Using (standard) colours closer in wavelength to the magnitude (or colour) being transformed, for example $\mathrm{V}-\mathrm{V}_{0}=a+b(\mathrm{Z}-\mathrm{V})_{0}$, sometimes gave a multi-valued (and non-linear) transformation. The errors from assuming a linear transformation were $\sim 0^{m} \cdot 02$, which is approximately the same size as the uncertainties in the observations of real (rather than synthesized) standard stars and much less than the scatter in the (real) transformation diagrams (see Figure 2.15).

As the observations could not resolve between linear and non-linear transformations, nor between transformations using $(\mathrm{Y}-\mathrm{V})_{0}$ or a 'closer' colour as an estimate of the derivative of $E_{s}$, the following linear transformations were used for all observations;

$$
(\mathrm{Y}-\mathrm{V})_{0}=a_{0}+a_{1}(\mathrm{Y}-\mathrm{V})
$$


Comparison of local and standard Vilnius systems

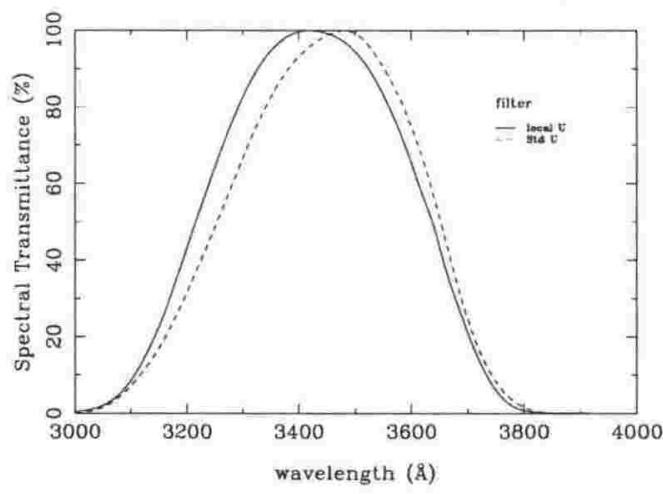

Comparison of local and standard Vilnius systems

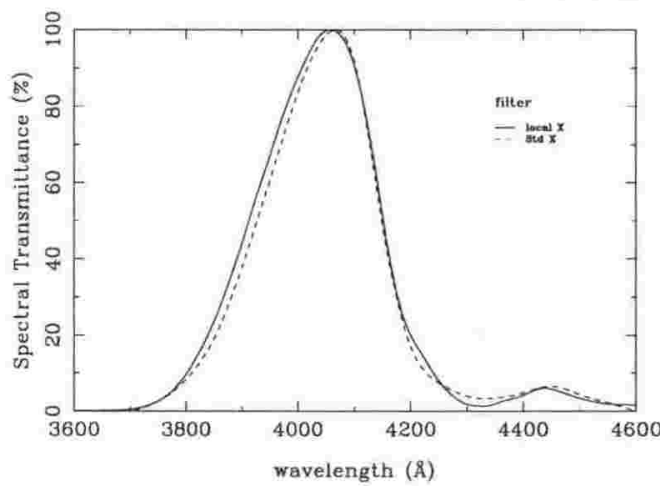

Comparison of local and standard Vilnius systems

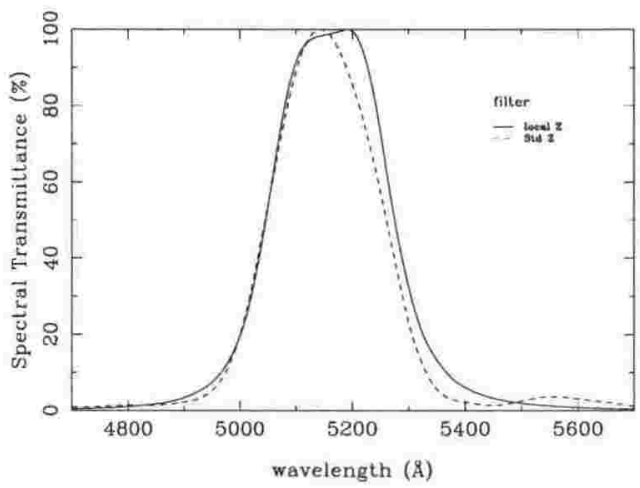

Comparison of local and standard Vilnius systems

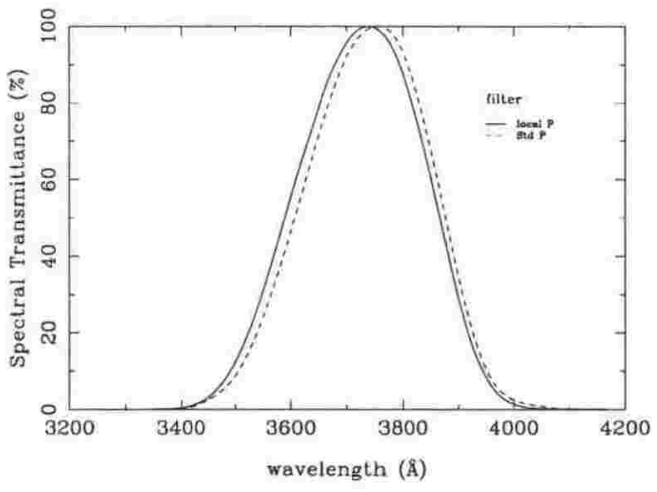

Comparison of local and standard Vilnius systems

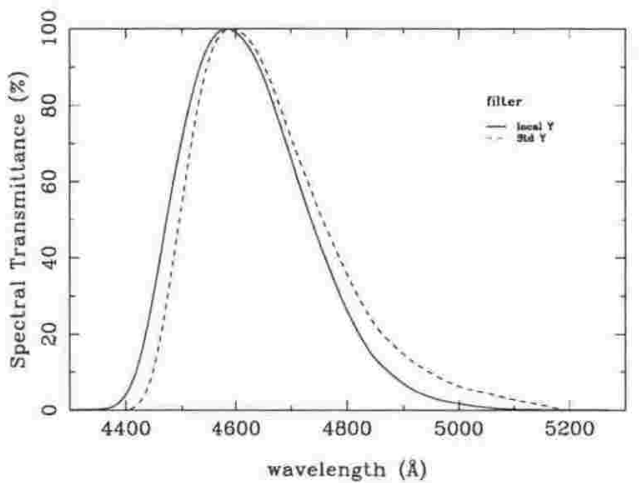

Comparison of local and standard Vilnius systems

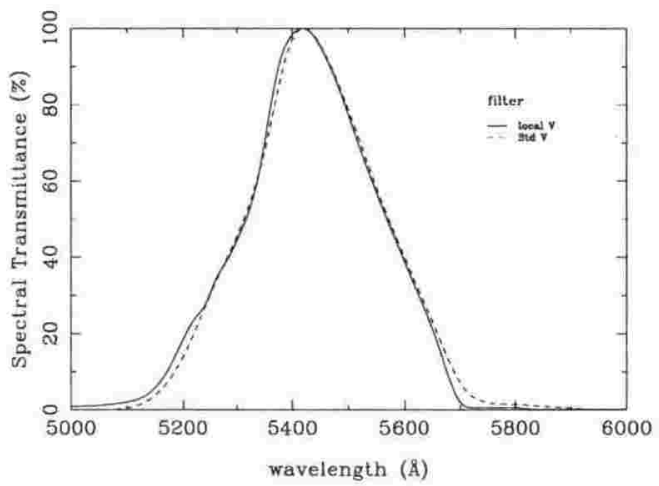

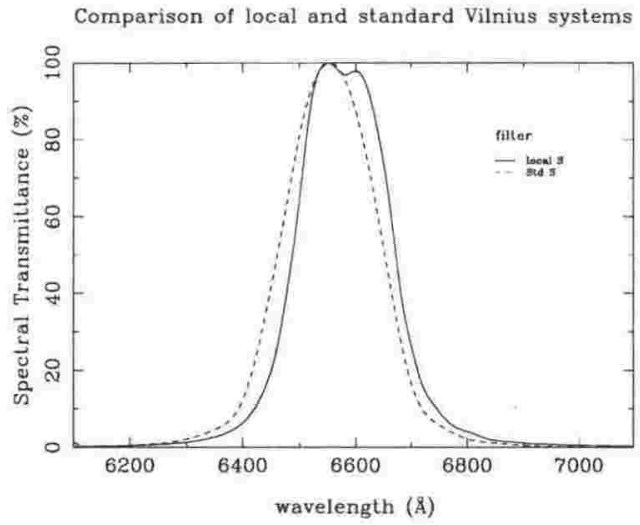

Figure 2.14: Comparison of local and Standard Vilnius filter responses, normalised to $100 \%$ transmittance. The local filters are Set \#1, measured in 1990, and the standard filters are from Straižys (1992). 


\section{3-7-1993 observed vrs standard magnitudes}

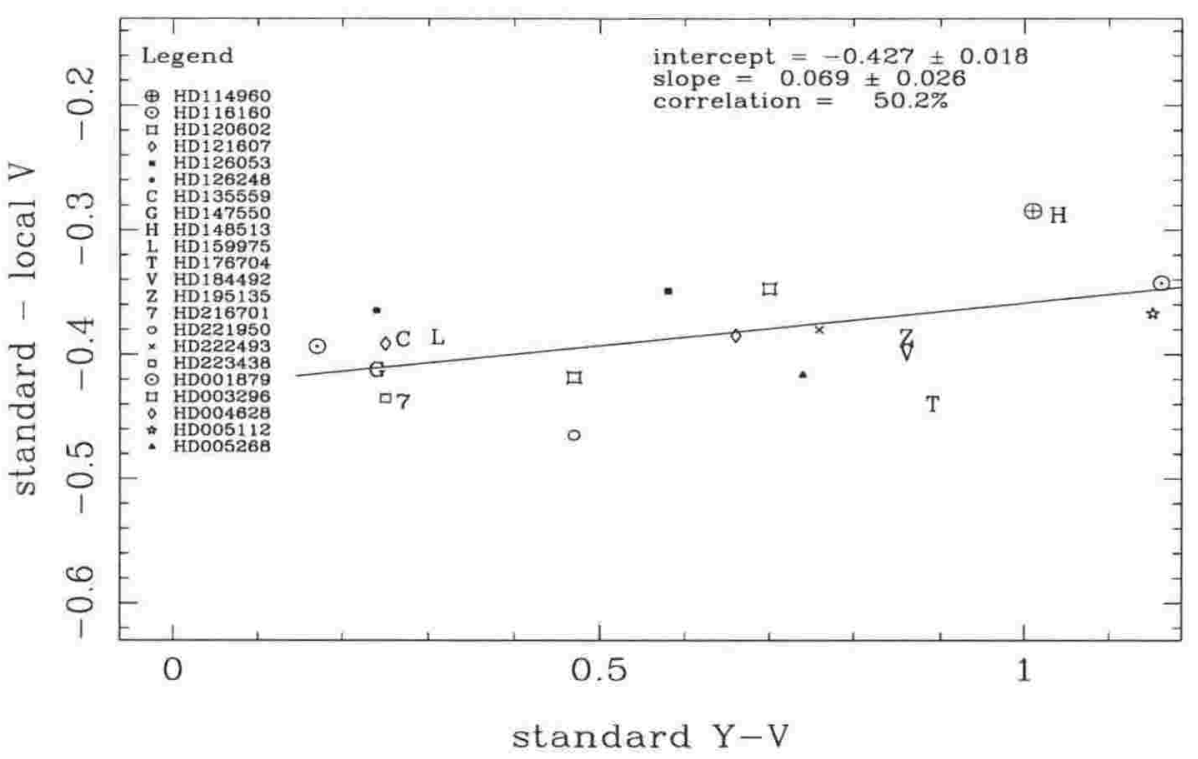

Figure 2.15: Transformation from the local to the Standard Vilnius System for 3 July 1993 for the $\mathrm{V}$ filter.

$$
\begin{aligned}
\mathrm{V}-\mathrm{V}_{0} & =b_{0}+b_{1}(\mathrm{Y}-\mathrm{V})_{0} \\
(\mathrm{U}-\mathrm{P})-(\mathrm{U}-\mathrm{P})_{0} & =c_{0}+c_{1}(\mathrm{Y}-\mathrm{V})_{0} \\
(\mathrm{P}-\mathrm{X})-(\mathrm{P}-\mathrm{X})_{0} & =d_{0}+d_{1}(\mathrm{Y}-\mathrm{V})_{0} \\
(\mathrm{X}-\mathrm{Y})-(\mathrm{X}-\mathrm{Y})_{0} & =e_{0}+e_{1}(\mathrm{Y}-\mathrm{V})_{0} \\
(\mathrm{Y}-\mathrm{Z})-(\mathrm{Y}-\mathrm{Z})_{0} & =f_{0}+f_{1}(\mathrm{Y}-\mathrm{V})_{0} \\
(\mathrm{Z}-\mathrm{V})-(\mathrm{Z}-\mathrm{V})_{0} & =g_{0}+g_{1}(\mathrm{Y}-\mathrm{V})_{0} \\
(\mathrm{~V}-\mathrm{S})-(\mathrm{V}-\mathrm{S})_{0} & =h_{0}+h_{1}(\mathrm{Y}-\mathrm{V})_{0}
\end{aligned}
$$

On some nights, insufficient standards were observed to get an accurate transformation. For such nights, the possibility of using an average transformation determined from all the observations of standard stars over the entire observing run was investigated. It was found that the fitted coefficients for average transformations are of similar accuracy to the fitted coefficients for individual nights, i.e. the instrumental system is stable over the entire observing run. This means an average transformation may be used for nights with few standards - this was particularly useful for observations made during summer. 
O Velorum $(\mathrm{RA}=8 \mathrm{~h} 40 \mathrm{~m}$ Os Dec $=-53 \mathrm{~d} \quad 2 \mathrm{~m} \quad 0 \mathrm{~s})$

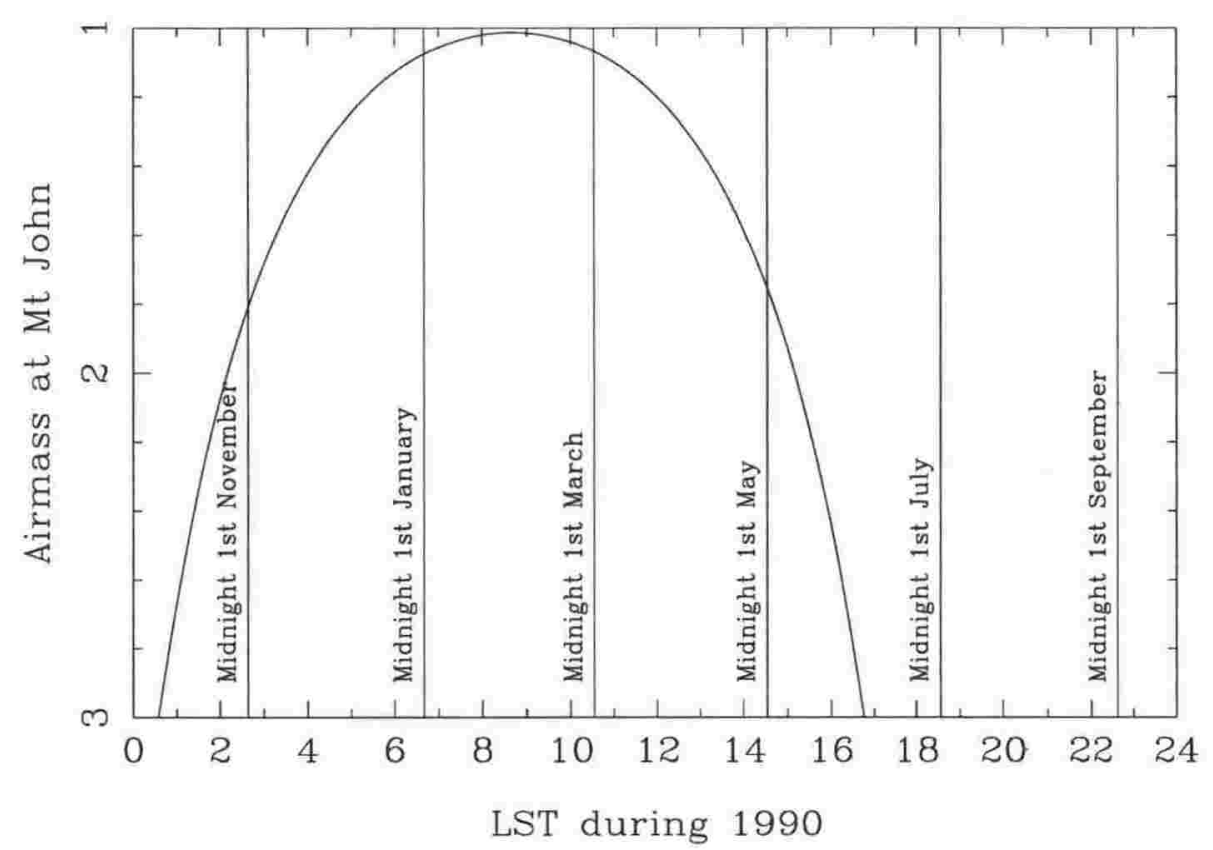

Figure 2.16: The airmass of $\mathrm{O}$ Vel at midnight of every night during 1993 at MJUO.

\subsection{Planning an Observing Session.}

To make optimum use of photometric conditions during an observing run, it is essential to decide on the observing programme beforehand. Some examples of this pre-planning are discussed below.

Program stars are in general best placed for observing at certain times of the year. An example using the O Vel cluster is shown in Figure 2.16 - this shows that O Vel cluster stars can be measured (at small airmass) after midnight during a December observing run, throughout the night from January to April, and before midnight (during the longer winter nights) until the beginning of June.

Similarly, the extinction and control stars need to be chosen to fulfill the conditions discussed in section 2.6(page 2-19). The declination of the extinction and control stars can be chosen with the aid of Figure 2.17. This shows the relation (for MJUO) between the declination of the control and extinction stars and the Hour Angle (HA) of the control star, fixing the control star at two airmasses while the extinction star is at its minimum airmass (so its HA is zero).

For instance, bright stars such as the extinction and control stars can be observed to approximately halfway between twilight and sunrise (or sunset). In May 1993, this was approximately 13 hours which is also the required difference in HA between the extinction and control stars. This is clearly not practical in this case, so for longer winter nights two control stars would be 


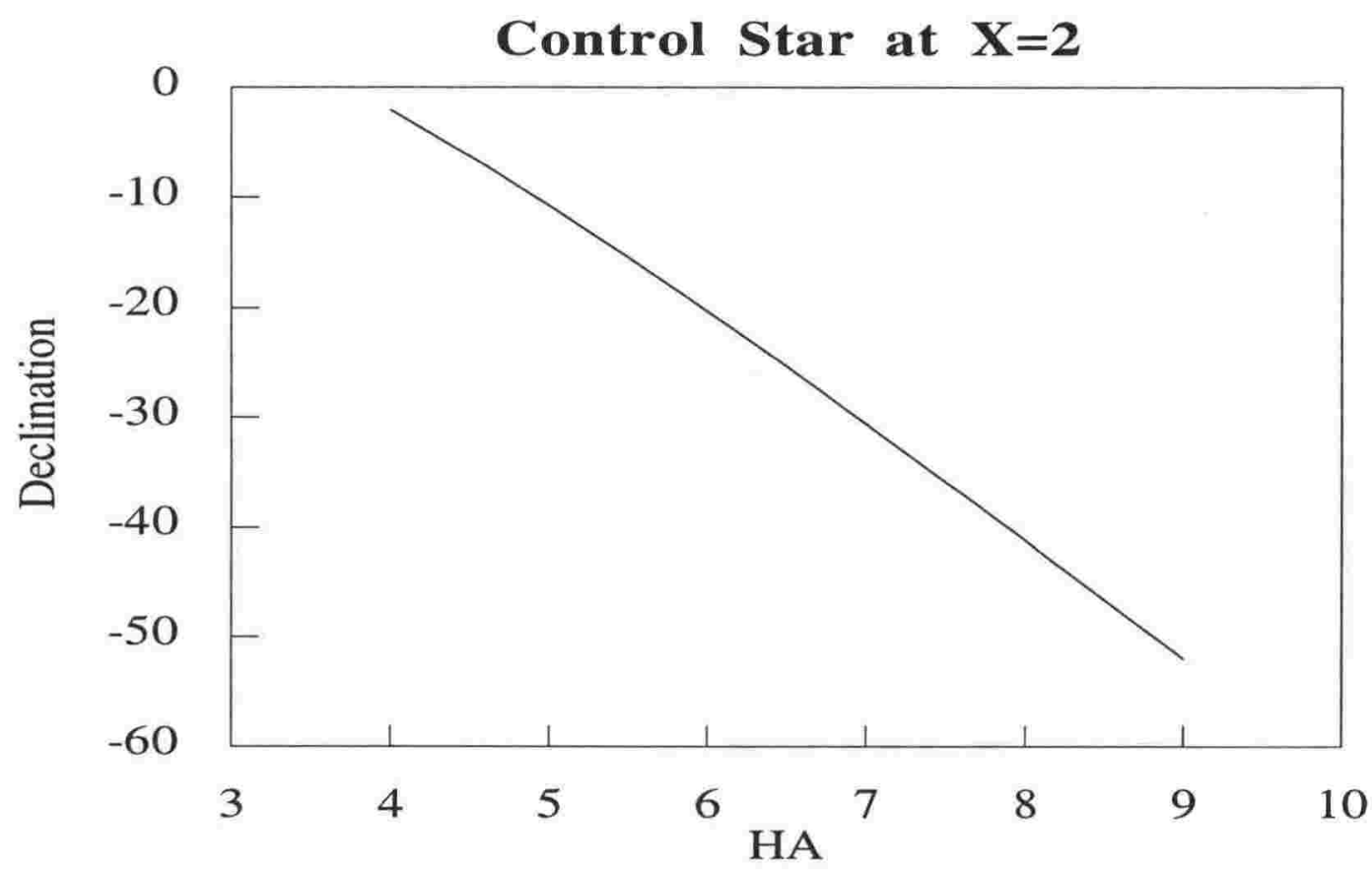

Figure 2.17: Choosing the Declination as a function of the control star's HA, when the control star is at two airmasses and the extinction star is at its minimum airmass (i.e. its HA is zero). This allows the stars to be picked to match the duration of the night, for example six hours in summer or two six hours segments in winter.

chosen by dividing the night into two segments. There are now three stars, labeled $\operatorname{star}_{1}, \operatorname{star}_{2}$, and $\operatorname{star}_{3}$ in order of increasing RA. At the beginning of the night, star ${ }_{1}$ is the extinction star and star $_{2}$ is the control star. Halfway through the night, star $_{1}$ is setting and star $_{2}$ is culminating while star $_{3}$ is rising so star $_{2}$ becomes the extinction star and star 3 the control.

By dividing the night in half, the required difference in HA between the three stars is now 6.5 hours, and Dec $\sim-25^{\circ}$. In practice, the extinction and control stars were chosen from the $\mathrm{E}$ region standard stars, with $\mathrm{Dec} \sim-45^{\circ}$ and hence a difference in HA of approximately 8.5 hours - this has the advantages that near the middle of the night all three stars may be observed together (which gives a check on the extinction determinations) and that program stars (i.e. candidates for the Southern Vilnius Standard System) are being used to determine the extinction.

The RA of the extinction star (i.e. star 2 during winter), and hence of the control star(s), may be chosen from diagrams such as Figure 2.18. This shows that the $\mathrm{E}$ region standard star E6_047 was chosen as the extinction star for the May 1993 observing run as it has an airmass of $\sim 2$ at the beginning and end of astronomical twilight and is at its minimum airmass near the middle of the night (in addition to being non-variable etc.).

Finally, star charts need to be checked to ensure there is no possibility of confusion over finding the correct star. If there was another star of similar, or brighter, intensity within $\frac{1}{2}^{\circ}$ 
E6_047 (RA $=14 \mathrm{~h} 59 \mathrm{~m} 45 \mathrm{~s}$ Dec $=-43 \mathrm{~d} 48 \mathrm{~m} 33 \mathrm{~s})$

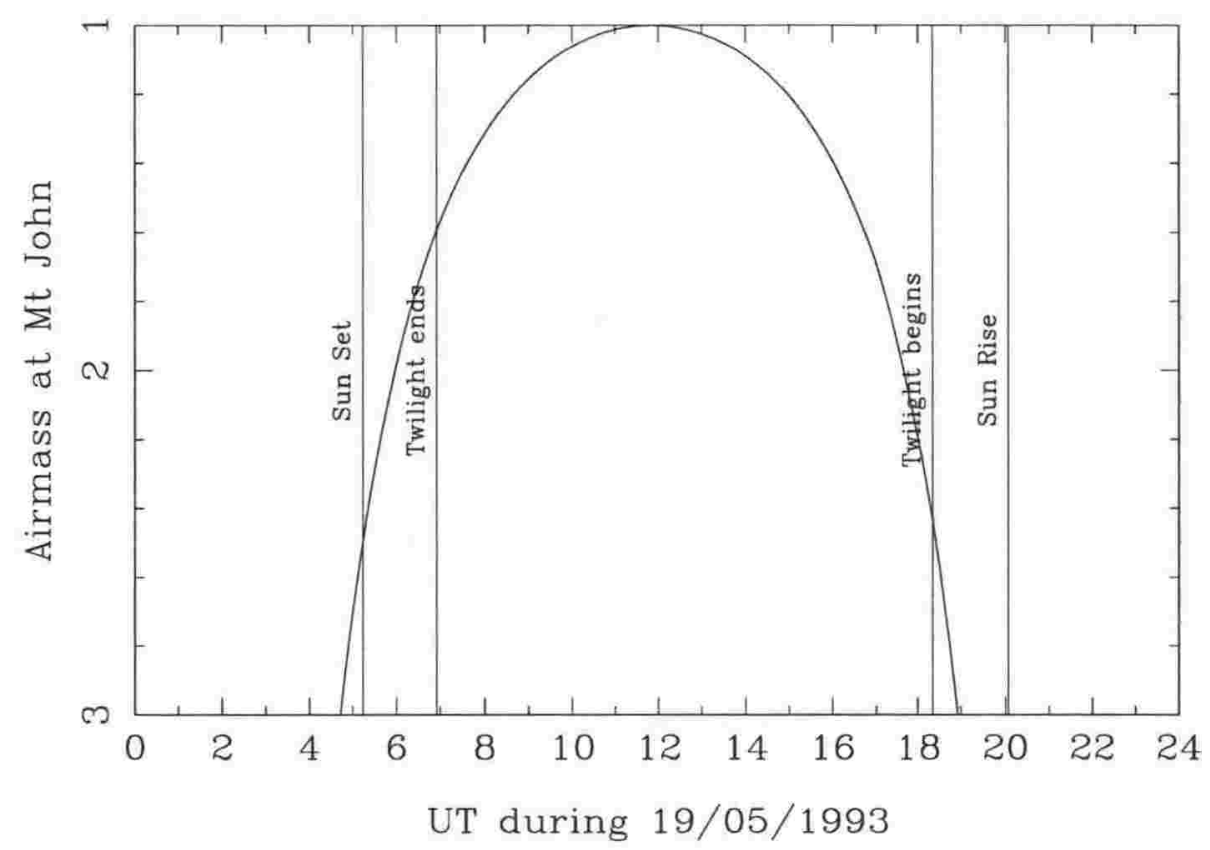

Figure 2.18: The airmass of the extinction star E6_047 at MJUO during 19 May 1993.

say, a finding chart was prepared (using the "starchart" program which reads the Hubble Space Telescope (HST) Guide Star Catalog from Compact Disk Read Only Memory (CDROM)'s). The orientation and scale of the chart should match the view seen through the finder telescope or the pre-diaphragm viewer on the main telescope so the charts are usually printed on transparencies with appropriate scale indications. An example (the finding chart for O Vel) can be seen in Figure 4.7.

\subsection{Summary.}

The observations for this study were made at Mount John University Observatory, in the South Island of New Zealand. The preferred system was the B\&C $61 \mathrm{~cm}$ Cassegrain telescope using the VUW 9558B PMT in pulse counting mode and the Vilnius filter set \#1. A brief background on the observation and reduction procedures for photoelectric photometry is given, together with a detailed description of the methods used in this study. These were all 'standard' methods, except for the extinction correction described next.

The (monochromatic) extinction correction used was Nikonov's Method, which only assumes the extinction is uniform across the sky at the moment of measurement. This allows any changes in extinction during the night to be measured, unlike the more commonly used Bouguer's 
method which required the extinction to be uniform both across the sky and throughout the entire night. During the reductions it was found that apparent extinction variations during a night were common at MJUO- an investigation concluded that this effect is due to either time dependent drifting in the instrument's zero-point or time dependent changes in the extinction. Due to the particular observing program used, there were no detectable differences between the two possible causes of 'extinction' variations on the reduced measurements so the effect was assumed to be from the latter cause.

The final section of the chapter discusses planning for observing sessions, i.e. choosing which extinction, standard and program stars are to observed depending on the time of year and time of night, together with preparation of finding charts. 


\section{Chapter 3}

\section{The Southern Vilnius Standard System.}

An extensive set of standard stars was established around the North Celestial Pole (Černis et al. 1989), the Kapteyn areas (Černis \& Jasevičius 1992), the Cygnus Standard Region (Zdanavičius et al. 1969, Zdanavičius \& Cerniene 1985) and Aquila Standard Region (Zdanavičius et al. 1969). As none of these standard regions is south of the celestial equator it became necessary to establish first a set of southern standard stars before any observations in the southern hemisphere could be made. A set of stars that are observable from the southern hemisphere was selected from those previously measured in the Vilnius system (North 1980) to be used as 'primary standards'. The selection was made by accepting only stars brighter than $7^{m} \cdot 00$ and further south than declination $+10^{\circ}$. Initially any stars in the catalog which had Johnson $\mathrm{V}$ magnitudes rather than Vilnius V magnitudes was rejected. Similarly any known or suspected variable stars were also rejected. However these last two criteria had to be relaxed when it was found that they resulted in too few standard stars (especially during summer observations). The final list is given in appendix B.

As the so-called 'primary standards' are not in fact standard stars, the scatter in transformation diagrams (such as Figure 2.15 on page 2-35) was larger than normal and so required more stars to be observed than usual. In addition, as it is likely that measurements made here would be more accurate than the original observations in the northern system (as, in general, more observations per star were made) it was decided to include these stars as part of the Southern Vilnius Standard System.

\subsection{Selection of Standard star candidates.}

Lists of possible southern standard stars were prepared (see appendix B) which, if measured to sufficient precision and accuracy, will become 'secondary standards' of the Vilnius photometric system. However, the inaccurate transformations inherent in under-sampled filter systems (Young 1988) mean that a new photometric system (the Southern Vilnius System) will actually be established which is on average the same as the original system. 
The candidate southern standard stars were selected in three groups; a South Polar Sequence (the 'SPS' standards), a group of bright stars spread across the southern sky (the 'all-sky' standards, or sometimes simply the 'secondary standards'), and a group of standard stars from other photometric systems (the 'E region' standards).

The South Polar Sequence (SPS) stars were selected using the Set of Identifications, Measurements and Bibliography for Astronomical Data (SIMBAD) database with the following search criterion; non-variable, $\mathrm{V} \leq 7^{m} \cdot 00$, and $\delta \leq-80^{\circ}$. The advantage of a polar sequence over other standard star regions is that being circumpolar, the same set of stars may be observed at any time of the year, for example allowing long term instrumental (zero-point) drifts to be monitored. Further, polar sequence stars move through a smaller range in airmass, reducing the effects of inexact extinction corrections on transformations to the standard system. If establishing a completely new photometric system, such a sequence is essential and should be observed first, before the system can change (due to 'aging' of the filters, long-term drifts in the PMT etc.).

The bright, all-sky group of stars were also selected using SIMBAD with the search criterion being; non-variable, $\mathrm{V} \leq 6^{m} \cdot 00$, and $-70^{\circ} \leq \delta \leq-30^{\circ}$ (although some stars were observed outside this declination range). Choosing stars spread in an approximately uniform manner across the sky ensures there will always be some standard stars near in the sky to any program star (for example as comparison and check stars of a variable star program). Similarly, there should always be some standard stars suitable as both standard and extinction stars for use in a variety of observing methods (see references given in section 2.6, page 2-19).

The final group of candidate standard stars is taken from a set of standard stars already established for the Johnson photometric system by Menzies et al. (1989). The group consists of nine $\mathrm{E}$ regions which contain approximately sixty stars each, ranging from $\mathrm{V}=3^{\mathrm{m}} \cdot 0 \rightarrow 12^{\mathrm{m}} \cdot 0$, centred near $\delta \approx-45^{\circ}$ and spaced $2-3$ hours apart in RA. The initial program is to observe all bright stars ( $\mathrm{V} \leq 7$, say) from an $\mathrm{E}$ region and then move to progressively fainter stars in the region. In general, the stars denoted as variable, or with optical companions that may not be consistently included (or excluded, following Menzies's notes) in the photometer diaphragm, were not observed. The $\mathrm{E}$ region stars are also suitable for a variety of extinction and/or standardisation observing methods, in a complimentary manner to the all-sky group.

\subsection{Error analysis.}

An error analysis was performed, to check the reliability of the standard deviations (see section 2.1, page 2-1) as an indicator of the uncertainty in the data, and to check for any problems in the instrumental system. A list of all the observed stars is given in appendix $\mathrm{C}$, while 
the magnitudes and standard deviations of all the 'good' individual observations are given in appendix D - the definition of 'good' observations is given later on page 3-3.

Some other terms used in the analysis are explained below; The term 'internal errors' refers to the standard deviations of the extinction corrected observations, i.e. the observations are still in the local, or internal, photometric system. Similarly, after transforming to the standard ('external') system, the standard deviations are referred to as the 'external errors'. For the transformed primary standards, a third type of error can be calculated - the 'residual error'. This is the difference between the magnitudes (or colours) from the original (northern) system and the (transformed) local system.

A quick review of the terms 'precision' and 'accuracy' is also appropriate here. Precision refers to the number of significant figures in a measurement and is usually associated with the random error of a measurement, for example a measured $\mathrm{V}$ magnitude of $6^{m} \cdot 75$ may be known to a precision of $\pm 0^{m} \cdot 01$. Accuracy refers to how close the measured value is to the true value and is usually associated with systematic errors; in the example above, the accuracy would be $0^{m} \cdot 05$ if the true $\mathrm{V}$ magnitude was $6^{m} \cdot 70$. The internal errors are assumed to be predominately random errors, the external errors a mixture of random and systematic and the residuals predominately systematic errors.

First, correlations between the internal V errors and the other filters' errors were investigated. It was found that the other errors show slight to definite correlations with the $\mathrm{V}$ errors, hence the V errors could be taken as 'typical' and so further analysis will be described for the V filter only.

Plots of the internal errors against instrumental colour of the star, RA of the star, and the seasonal phase (of the year) of the observation showed no trends, and hence there are no indications of any instrumental problems. However, the errors do show a slight dependence on the airmass through which the observation was made (see Figure 2.4 on page 2-17). There was also a dependence of the errors on the instrumental magnitude of the star (see Figure 3.1). This was plotted from observations made between 1.0 to 1.4 airmasses, where the airmass dependence is small. Hence, the errors increase (as expected) with decreasing brightness and/or increasing airmass of the observations.

A histogram of the internal $\mathrm{V}$ errors $\left(s_{V}\right)$ shows the main distribution is between $s_{V}=$ $0^{m} \cdot 00 \rightarrow 0^{m} \cdot 05$, followed by a small number of 'outliers' with $s_{V}>0^{m} \cdot 05$. All the other filters were also found to have similar distributions with outliers from $0^{m} \cdot 05$, except for $U$ (whose outliers occurred from $s_{U}>0 \cdot 09$ ). These outliers were considered 'bad' observations and rejected from the data set.

The catalogs in Appendix D list the 'good' individual observations of the stars - the individual observations of each star, rather than just the mean values, were included so later 


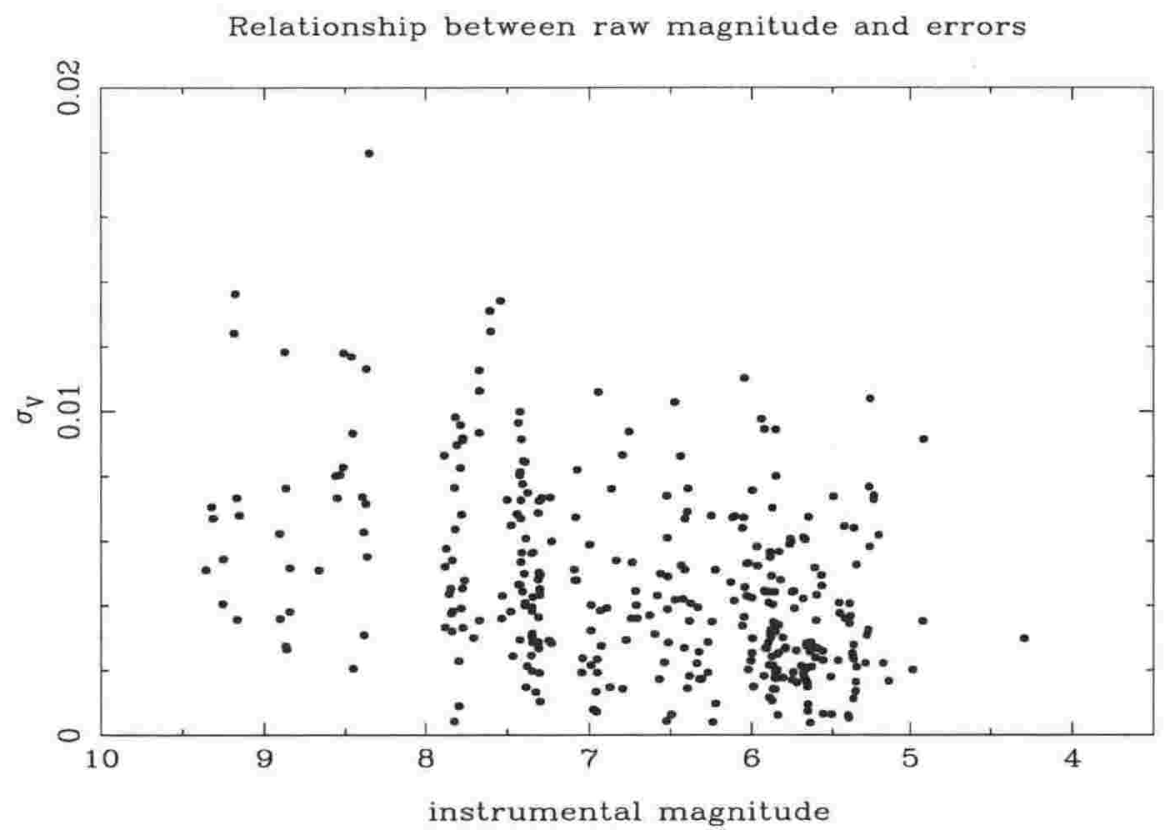

Figure 3.1: Observational error as a function of instrumental magnitude at MJUO. The internal standard deviation of the $\mathrm{V}$ filter measurements are plotted against the instrumental magnitude of the observation.

observations may be added to the data set and new mean values calculated, and investigations of possible variability of any star may be made later.

Initially two consecutive observations of each star were made during a night. It was found that the internal errors also indicates the size of the difference between these consecutive observations, so some of the later measurements took advantage of this by making only one observation of each star during a night (except extinction stars, which were measured hourly).

It was hoped that the external errors would correlate with the residual errors of the primary standards, and so indicate how accurately the secondary standards were transformed to the northern Vilnius system. Unfortunately no significant correlation was found but the errors both vary over approximately the same range of values (for example most of the external $\mathrm{V}$ errors are found between $0^{m} \cdot 01 \rightarrow 0^{m} \cdot 05$ while the residuals are between $0^{m} \cdot 00 \rightarrow 0^{m} \cdot 06$ )

A histogram of the $\mathrm{V}$ residuals (of the individual observations) is shown in Figure 3.2, together with a fitted exponential function ${ }^{1}$

$$
\text { frequency }=N \Delta x \frac{1}{\sigma e^{\bar{x} / \sigma}} e^{-\left|\frac{x-\bar{x}}{\sigma}\right|}=a e^{-\frac{\left|\mathrm{v}_{\text {residual }}\right|}{b}},
$$

assuming the mean residual $(\bar{x})$ is zero. To improve the fit, two discrepant data bins were

\footnotetext{
${ }^{1}$ The 'absolute value of the residuals' function was used, rather than the normal function, simply because it gave a better fit to the data.
} 
distribution of residuals of primary Vilnius standards (indiv. obs.)

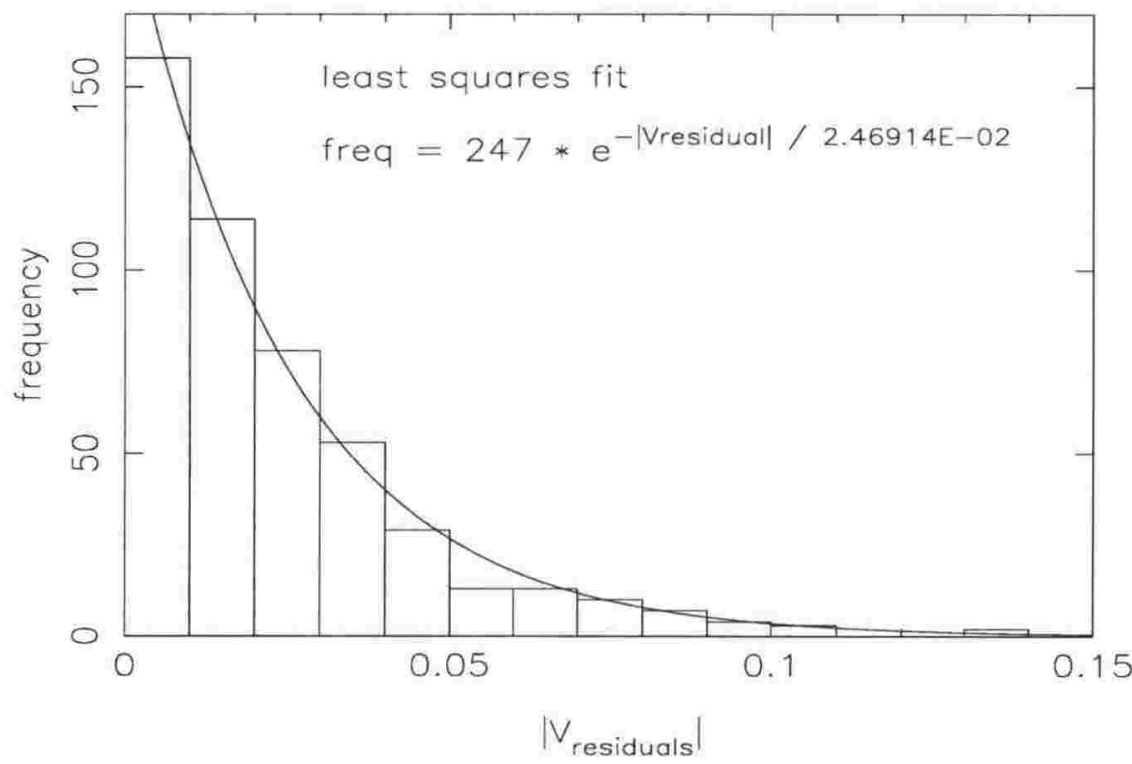

Figure 3.2: Histogram of residuals from all the 'good' observations of primary standard stars, together with a least squares fitted exponential curve.

rejected $\left(0.05 \leq\left|V_{\text {residual }}\right|<0.06,0.13 \leq\left|V_{\text {residual }}\right|<0.14\right)$, removing 13 observations to leave a total of 471 . The fitted terms ( $a$ and $b$ ) can be interpreted analogously to a normal distribution

$$
\text { frequency }=N \Delta x \frac{1}{\sqrt{2 \pi} \sigma} e^{-\frac{1}{2}\left(\frac{x-\not{x}}{\sigma}\right)^{2}}
$$

where $N$ is the number of observations, $x$ is a residual, with $\bar{x}$ and $\sigma$ being the mean and standard deviation of the residuals. The mean residual was then shifted by the histogram bin width $(\Delta x)$ to align the data with the fitted curve. The fitted term $(a=247)$ then gives $N=436$, which agrees reasonably well with the actual number of observations (471). The $b$ factor $(=0.025)$ should be close to the standard deviation of the residuals, which for the full 484 observations is 0.022 .

Next the average value for each primary standard star from the individual observations was calculated and the residuals plotted against the number of observations $(N)$ made of each star see Figure 3.3. The lower curve drawn in the histogram shows the expected $N^{-1 / 2}$ dependence of the average residual - recall this is 0.025 for a single (average) observation. The upper curve is the expected standard deviation of the residual added to the lower curve. This is calculated assuming a Poisson distribution for observations of a star, with the standard deviation being $\sqrt{0 \cdot 025} \approx 0 \cdot 16$. The number in the exponential decay term is $1 /\langle N\rangle(=1 / 4 \cdot 7 \approx 0 \cdot 21)$, where $\langle N\rangle$ is the average number of observations per star. 


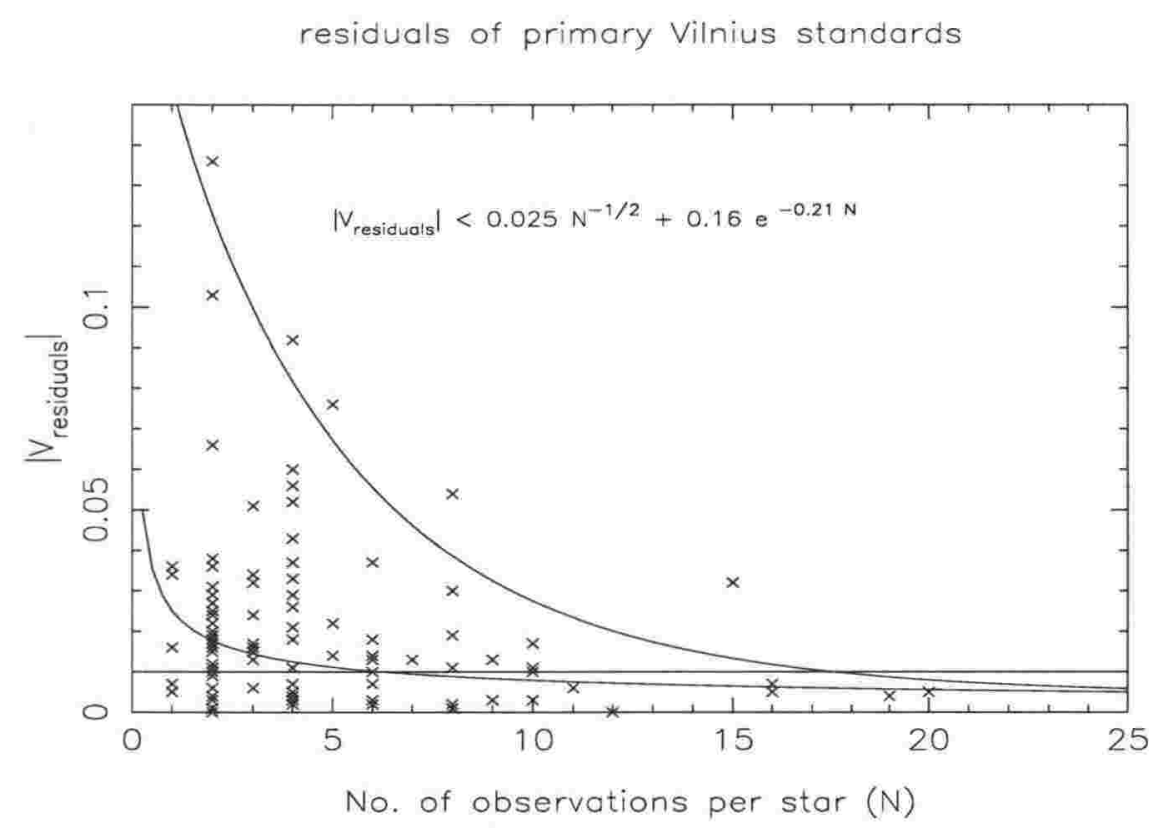

Figure 3.3: Histogram of residuals of the primary standard stars (averaged) observations.

The horizontal line indicates the desired $0^{m} \cdot 01$ accuracy and precision - the lower curve shows six observations of each star are required to reduce the random errors (in the observations and transformations) so that the average residual is $\sim 0^{m} \cdot 01$. The upper curve indicates that up to 18 observations per star might be required to reduce the random (transformation) errors so all 'good' standard stars are measured to better than $0^{m} \cdot 01$ accuracy. Conversely, any star above the upper curve would be a 'bad' standard star; it may not transform correctly with the simple (linear) transformations used here, the value in the standard system may be inaccurate, the star may be variable, or (for $N<6$, say) not enough observations have been made of the star (in the local system). However, the upper curve should only be used as a guide, especially the 18 observations per star requirement, as very few stars have had a large number of observations made. Note that if the precision and accuracy goal is relaxed to $0^{m} \cdot 02$, the number of observations per star becomes 1.5 and 12 respectively.

The overall precision and accuracy of the observations can be gauged from the standard deviations shown in Table 3.1. The internal errors show that $0^{m} \cdot 01 \leftrightarrow 0^{m} \cdot 02$ precision is being achieved in the local system, and the errors increase by less than a factor of two when being transformed to the standard system. The standard deviations were also calculated separately for the primary standard stars and the three groups of secondary standard stars. However the results for the SPS stars are probably not reliable due to the small number of observations made.

The internal errors of each group of stars generally decrease as the average airmass through 
Table 3.1: Standard deviations from all the $(N)$ individual observations, calculated for the internal, external and (primary standard stars) residual errors. The observations are also split into the three secondary standard groups and primary standards discussed in the text.

\begin{tabular}{|c|c|c|c|c|c|c|c|c|c|}
\hline observations & $\mathrm{N}$ & errors & V & $\mathrm{U}-\mathrm{P}$ & $\mathrm{P}-\mathrm{X}$ & $\mathrm{X}-\mathrm{Y}$ & $\mathrm{Y}-\mathrm{Z}$ & $\mathrm{Z}-\mathrm{V}$ & $\mathrm{V}-\mathrm{S}$ \\
\hline \multirow[t]{2}{*}{ SPS standards } & 33 & internal & 0.013 & 0.010 & 0.011 & 0.011 & 0.013 & $0 \cdot 012$ & 0.008 \\
\hline & & external & 0.048 & 0.030 & 0.023 & 0.021 & 0.018 & 0.014 & 0.015 \\
\hline \multirow[t]{2}{*}{ all-sky standards } & 681 & internal & 0.023 & 0.020 & 0.012 & 0.012 & $0 \cdot 014$ & 0.016 & 0.012 \\
\hline & & external & 0.045 & 0.037 & 0.027 & 0.023 & 0.022 & 0.022 & 0.023 \\
\hline \multirow[t]{2}{*}{ E region standards } & 764 & internal & 0.017 & 0.018 & $0 \cdot 010$ & 0.009 & $0 \cdot 009$ & 0.009 & 0.011 \\
\hline & & external & 0.027 & 0.030 & 0.017 & 0.014 & 0.015 & 0.013 & 0.017 \\
\hline \multirow[t]{3}{*}{ primary standards } & 484 & internal & 0.020 & 0.023 & 0.015 & $0 \cdot 013$ & 0.014 & 0.015 & $0 \cdot 012$ \\
\hline & & external & 0.032 & 0.034 & 0.023 & 0.020 & 0.020 & 0.019 & 0.021 \\
\hline & & residuals & 0.022 & 0.022 & 0.015 & 0.012 & $0 \cdot 012$ & 0.011 & 0.016 \\
\hline \multirow[t]{2}{*}{ all observations } & 1962 & internal & 0.020 & $0 \cdot 020$ & $0 \cdot 012$ & 0.011 & $0 \cdot 012$ & 0.013 & 0.012 \\
\hline & & external & 0.035 & 0.034 & 0.022 & 0.019 & $0 \cdot 019$ & 0.018 & 0.020 \\
\hline
\end{tabular}

which they were observed decreases (in decreasing order; primary standards, all-sky, E region secondary standards). The consistently larger U-P error is probably due to the integration time for the $\mathrm{U}$ filter being too short (despite already being twice as long as the other filters) it is recommended that the $\mathrm{U}$ filter integration time be 3-4 times longer than the other filters (i.e. 15-20 seconds for bright stars). The $\mathrm{V}$ magnitude error is also consistently larger than the colours - this is probably because reducing the data as a colour removes to first order any instrumental drifts and/or any extinction variations having a large 'grey' component.

The external errors do not show the same trends between the groups of stars as seen in the internal errors. The airmass dependence is probably responsible for the primary standards' errors being larger than the $\mathrm{E}$ region standards. However the all-sky standards were (unfortunately) almost exclusively observed during the period immediately following the Mount Pinatubo eruption, unlike the other groups of standards (which were observed both before and during the eruption and after it's effects had started to decrease). This probably accounts for the larger standard deviations of the all-sky standards, and is more clearly seen by plotting the mean internal error of each photometric night against the Julian Date - this shows the mean errors significantly increase after the eruption's aerosol clouds arrived over MJUO, which is due to the increased variation in the atmospheric extinction (see Appendix G).

The sum (of the squares) of the primary standard stars' internal and residual errors show a good correlation with the external errors, while slightly under-estimating them. Therefore it was concluded that the external errors give an excellent estimate of the average precision and accuracy of the observations of the Southern Vilnius System. 


\subsection{Selection of the Standard stars.}

Using the 'good' observations discussed earlier, the weighted mean and standard deviation of the mean was calculated for each candidate southern standard star. To select those stars that have been measured to sufficient precision and accuracy to become standards, the following criteria were applied;

- for the (northern) primary 'standards', the star must not be a known or suspected variable (the secondary standards have already been checked for this).

- more than $N$ observations have been made of a star.

- the standard deviation of the mean value for a star must be less than some cut-off value for each filter.

- the star's spectral classification based on it's mean Vilnius colours agrees within two sub-classes of the spectral type given in the literature and within one luminosity class.

Setting the required number of observations per star $(N)$ to six (to achieve an average accuracy of $0^{m} \cdot 01$, as shown in Figure 3.3) results in very few stars being accepted as standards. For this reason, it was relaxed to $0^{m} \cdot 02$, which meant two observations of a star should be sufficient - in fact $N$ was set to three so the standard deviation (of the mean) of each star could be calculated.

For the $\mathrm{E}$ region stars, the required standard deviation cut-off was set to $0^{\mathrm{m}} \cdot 02$ (except for U-P, which was relaxed slightly to $0^{m} \cdot 025$ ). To achieve a reasonable number of stars acceptable as standards in the remaining groups of stars (primary standards, all-sky and SPS secondary standard candidates), which were generally measured through a larger airmass, the error cutoffs were increased to $0^{m} \cdot 04$ for the $\mathrm{V}$ magnitude and $0^{m} \cdot 03$ for the colours. Both the Vilnius 'Q-Search' and 'Q-Spectra' classification methods were used to derive the star's spectral type, with a star being acceptable if successfully matched by both methods. A star was considered marginally acceptable if matched by one method, and nearly matched by the other - this will allow for any shortcomings in the reference lists used by each method. The resulting lists of standard stars for the Southern Vilnius System are given in Appendix E. As the majority of unacceptable stars were rejected due to an insufficient number of observations, these were also included (in separate tables) at the back of the appendix for the benefit of later observers continuing to develop the Southern Vilnius System.

The overall accuracy and precision of the standard stars (rather than that of an individual observation) may be estimated from

$$
\varepsilon=\frac{\sigma}{\sqrt{\frac{\sum n_{i}}{N}}}
$$


Table 3.2: Error estimates of the Southern Vilnius system standard stars. The observations are also split into the two secondary standard groups and primary standards discussed in the text.

\begin{tabular}{|lrrrrccccc|}
\hline stars & $\sum n_{i}$ & $N$ & $\mathrm{~V}$ & $\mathrm{U}-\mathrm{P}$ & $\mathrm{P}-\mathrm{X}$ & $\mathrm{X}-\mathrm{Y}$ & $\mathrm{Y}-\mathrm{Z}$ & $\mathrm{Z}-\mathrm{V}$ & $\mathrm{V}-\mathrm{S}$ \\
\hline primary standards & 338 & 49 & 0.012 & 0.013 & 0.009 & 0.008 & 0.008 & 0.007 & 0.008 \\
all-sky standards & 677 & 31 & 0.010 & 0.008 & 0.006 & 0.005 & 0.005 & 0.005 & 0.005 \\
E region standards & 764 & 60 & 0.008 & 0.008 & 0.005 & 0.004 & 0.004 & 0.004 & 0.005 \\
\hline all standard stars & 1779 & 140 & 0.010 & 0.010 & 0.006 & 0.005 & 0.005 & 0.005 & 0.006 \\
\hline
\end{tabular}

where $\sigma$ is the standard deviation of a single observation (taken from the external errors in Table 3.1), $n_{i}$ is the number of observations of the $i^{\text {th }}$ star and $N$ is the total number of stars measured. The results are given in Table 3.2 , and were also calculated separately for the primary standard stars and two groups of secondary standard stars (none of the SPS stars was accepted as standards, due to small number of observations per star).

\subsection{Derivation of the Southern Vilnius System's Pass-} bands.

Following the general method described by Bessell (1990), it was intended to derive the passbands of the Southern Vilnius System (relative to the passbands of the Northern Vilnius system).

First all the stars in the Northern Vilnius Catalog (North 1980) that are also in the Gunn \& Stryker (1983) catalog of stellar spectra were collated, giving a data set of 39 stars. As the Vilnius colours are not dereddened but the G-S spectra are (assuming a particular reddening law), the spectra were restored to their original reddened state. Convolving the spectra $(S(\lambda))$ with the standard passbands of the Northern Vilnius system (Straižys \& Zdanavičius 1970) gives synthesized colours

$$
\mathrm{Col}_{A B}=\frac{\int_{\lambda_{1}}^{\lambda_{2}} S(\lambda) R_{A} d \lambda}{\int_{\lambda_{3}}^{\lambda_{4}} S(\lambda) R_{B} d \lambda}
$$

where $R_{A}, R_{B}$ are the passbands of the particular Vilnius colour. The G-S spectra have been normalised at the effective wavelength of the Johnson $\mathrm{V}$ filter - unfortunately the original intensity at that wavelength was not recorded in the catalog so only colours, but not magnitudes, may be synthesized.

These synthesized colours were then compared with the measured colours. For each colour, all the spectra were multiplied by a polynomial whose coefficients were adjusted until the synthesized colours matched the actual colours. In all cases, a polynomial to first order $(f(\lambda)=$ $\left.a_{0}+a_{1} \lambda\right)$ was sufficient.

Next all G-S stars that have also been measured in the Southern Vilnius system were collated. Using the same polynomial found for the northern system, the adjusted spectra were convolved 
with the southern system passbands and the synthesized colours compared with the measured colours. The filter passbands would then be adjusted so that the synthesized colours match the actual colours, using the northern passbands as the initial guess. Unfortunately only 5 stars were found to be common to the G-S catalog and the Southern Vilnius system, which was insufficient to see if there was any difference between the northern and southern passbands.

\subsection{Transformations to the Johnson System.}

To gauge the accuracy of transforming measurements in the Vilnius system to the Johnson system, Vilnius standard passbands were 'added' together in various linear combinations to match each Johnson filter (using the standard passbands given by Straižys (1992)). The best combinations (in the least squares sense) were found to be;

$$
\begin{aligned}
\mathrm{U}_{j} & =a_{1} \mathrm{U}_{v}+a_{2} \mathrm{P}_{v}+a_{3} \mathrm{X}_{v} \\
\mathrm{~B}_{j} & =a_{1} \mathrm{P}_{v}+a_{2} \mathrm{X}_{v}+a_{3} \mathrm{Y}_{v}+a_{4} \mathrm{Z}_{v}+a_{5} \mathrm{~V}_{v} \\
\mathrm{~V}_{j} & =a_{1} \mathrm{Z}_{v}+a_{2} \mathrm{~V}_{v}+a_{3} \mathrm{~S}_{v}
\end{aligned}
$$

with the ' $j$ ' and ' $v$ ' subscripts indicating Johnson or Vilnius filters respectively. The actual value of the coefficients $a_{1}$ etc. is irrelevant; what is important is how well the best linear combination matches the shape of the corresponding Johnson passband, which can be seen in Figure 3.4.

The Johnson U filter is closely duplicated by the combination of Vilnius filters given above and so accurate transformations (using the same transformation coefficients) should be possible for any star. However the Johnson B and V filters are poorly matched, so separate transformations will be needed for different subsets of stars, for example late type stars have an absorption feature at $\sim 6000 \AA$ which is not measured by the Vilnius combination and hence will not have the same transformation as early type stars (which do not have this feature).

Some actual transformations between the Vilnius and Johnson systems may be found from the E region's observations, ${ }^{2}$ with the Johnson magnitudes and colours taken from Menzies et al. (1989). Transformations using fluxes (Young 1994) were made in addition to those using magnitudes but gave no improvement in the precision of the least squares fits and so are not presented here. Several transformations to each Johnson colour are included for two reasons; first, the stars may have only been measured using a subset of the Vilnius filter set (for example Banks et al. (1994) CCD photometry), and secondly, if all the transformations give

\footnotetext{
${ }^{2}$ E3_087 produced a data point lying a significant 'distance' from the relations found in all the following transformations. This is due to it's companion star being included in Menzies et al. (1989) measurements of the star but not in the Vilnius observations, and so E3_087 was excluded from the transformations.
} 


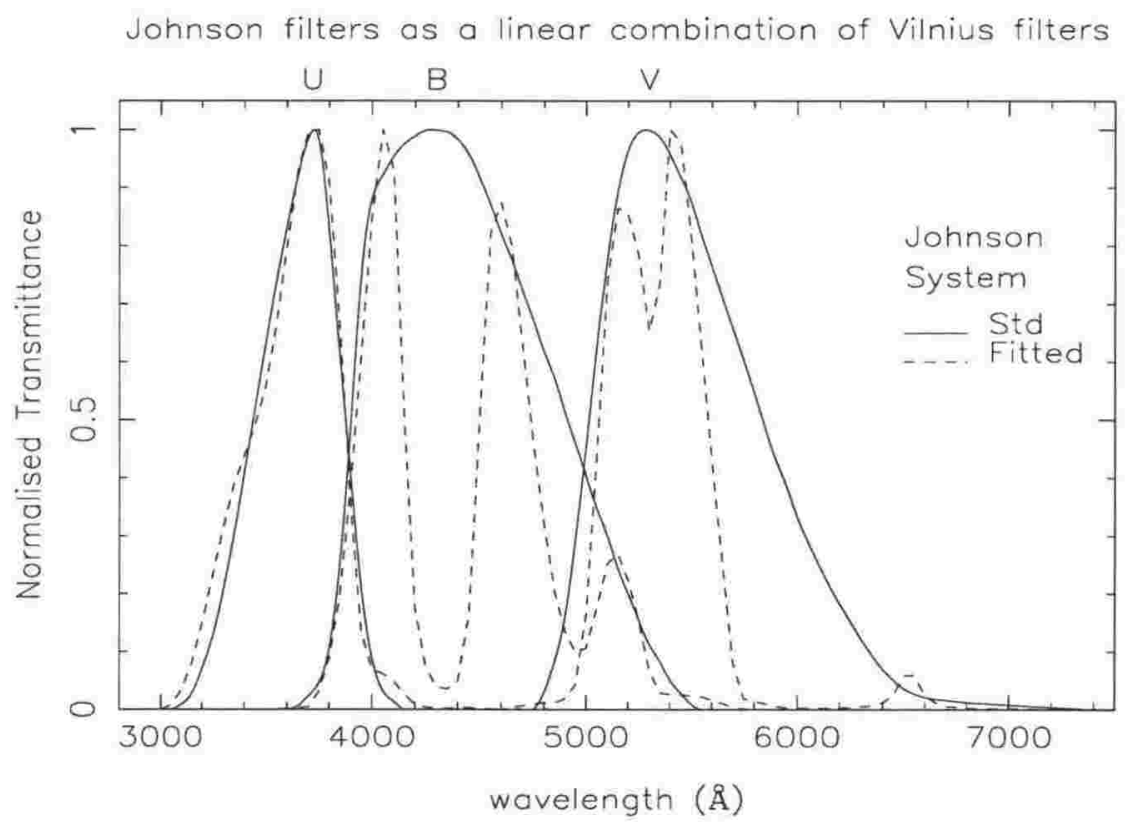

Figure 3.4: Least squared fitted linear combination of Vilnius filters to match the Johnson filters.

(approximately) the same Johnson colour then the transformations can be considered correct for that particular star.

The first transformation is between the Vilnius and Johnson V magnitudes; ${ }^{3}$

$$
V_{j}=a_{0}+a_{1} V_{v} \begin{cases}a_{0}=-0.029 \pm 0.028 & \\ a_{1}=+0.999 \pm 0.004 & \mathrm{R}=0.9994 \\ \sigma=0.029 & \mathrm{R}=0.9994\end{cases}
$$

where $R$ are the linear correlation coefficients for each independent variable and $\sigma$ is the standard deviation of the residuals between the actual and transformed Johnson $\mathrm{V}$ magnitudes (the $R$ next to this is the overall correlation coefficient for the fit). This linear transformation is included so transformations may be made in either direction, i.e. from or to the Johnson system. This shows close agreement with the zero-point difference $(-0.03 \pm 0.02)$ found by Dzérvítis \& Paupers (1994).

\footnotetext{
${ }^{3}$ All the coefficients ( $a_{0}$ etc.) are given to milli-magnitudes regardless of the size of the associated standard deviation. This is because the large linear correlation coefficients indicate the standard deviations probably over-estimate the actual uncertainties, due to the correlations between the coefficients.
} 
Transformation between Johnson and Vilnius systems for the E Regions

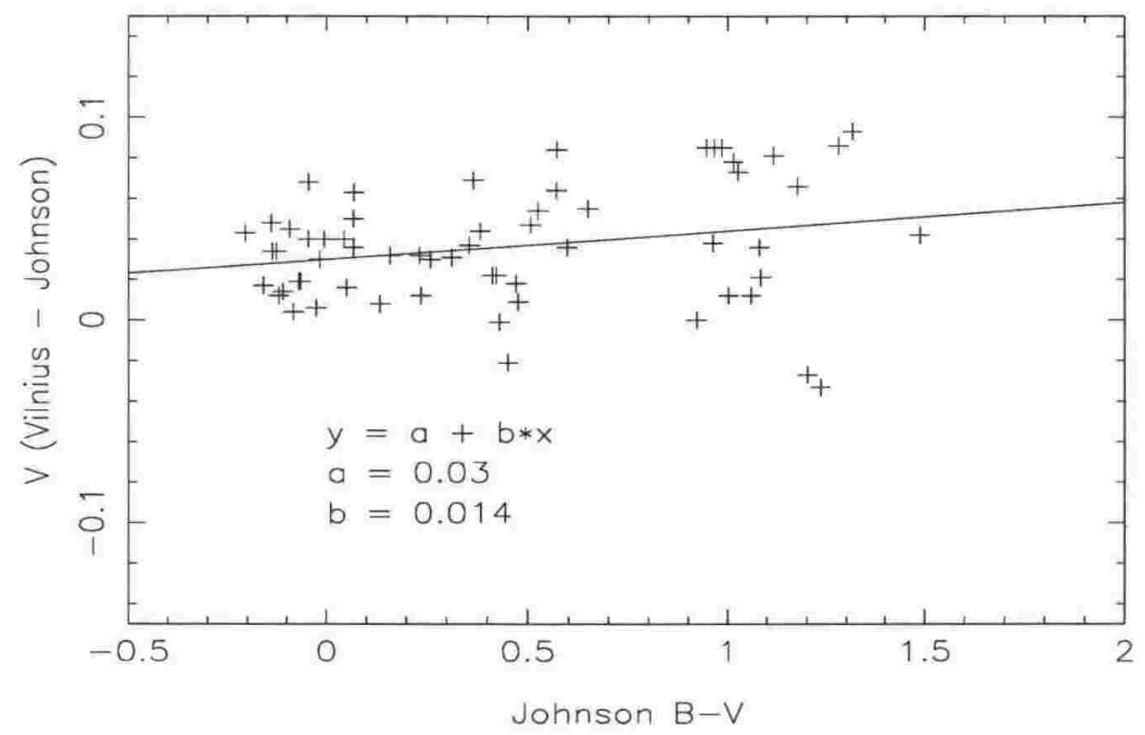

Figure 3.5: Transformations between Vilnius and Johnson V magnitudes, based on E region stars measurements.

The next transformation between the $\mathrm{V}$ magnitudes requires the Johnson $\mathrm{B}-\mathrm{V}$ colour

$$
V_{v}-V_{j}=a_{0}+a_{1}(\mathrm{~B}-\mathrm{V})_{j}\left\{\begin{array}{rlrl}
a_{0} & =+0.030 \pm 0.005 & & \\
a_{1} & =+0.014 \pm 0.007 & \mathrm{R} & =0.228 \\
\sigma & =0.028 & \mathrm{R} & =0.228
\end{array}\right.
$$

and is shown in Figure 3.5. This can be compared with Sviderskiene \& Straižys (1970) 'theoretical' transformation ( $\left.a_{0} \equiv 0, a_{1}=0.01\right)$ which is based on synthesized magnitudes and colours. The difference in zero-points is probably due to the 'standard' passbands used to synthesize the Johnson magnitudes not corresponding exactly to the actual passbands (for instance, see (Bessell 1990) and (Menzies et al. 1991)), and so not reproducing what appears to be a genuine difference (of 0.03) between the Johnson and Vilnius V magnitudes. The colour terms show good agreement (differing by only 0.004 ) and so the $\mathrm{E}$ region measurements confirm the theoretical V transformation.

According to Sviderskiené \& Straižys (1970), interstellar extinction (reddening) moves a star's position in the transformation diagram (Figure 3.5 ) to the right approximately parallel to the data line, except for late-type stars. Most of the other Vilnius-Johnson transformations (see later Figures 3.6 etc.) also have reddening lines nearly parallel to the intrinsic sequence(s). A histogram of the $E_{\mathrm{Y}-\mathrm{V}}$ colour excesses (calculated from $E_{\mathrm{Y}-\mathrm{V}}=(\mathrm{Y}-\mathrm{V})_{0}-\mathrm{Y}-\mathrm{V}$ and 'looking up' it's intrinsic $(\mathrm{Y}-\mathrm{V})_{0}$ colour from Tables J.7 and J.8, given the star's spectral classification) 
shows some of the stars have significant reddening $\left(E_{\mathrm{Y}-\mathrm{V}} \sim 0.5-1.0\right)$. However there is no strong correlation between the amount of reddening and the star's deviation from the intrinsic sequence, confirming the reddening lines do run nearly parallel to the intrinsic sequences.

An alternative transformation using the Vilnius $\mathrm{Y}-\mathrm{V}$ colour is

$$
V_{v}-V_{j}=a_{0}+a_{1}(\mathrm{Y}-\mathrm{V})_{v} \begin{cases}a_{0}=+0.026 \pm 0.007 & \\ a_{1}=+0.023 \pm 0.013 & \mathrm{R}=0.224 \\ \sigma=0.029 & \mathrm{R}=0.224\end{cases}
$$

The final $\mathrm{V}$ transformation is

$$
V_{j}=a_{0}+a_{1} V_{v}+a_{2} Z_{v}+a_{3} S_{v} \quad \begin{cases}a_{0}=-0.002 \pm 0.003 & \\ a_{1}=+0.436 \pm 0.3 & \mathrm{R}=0.9994 \\ a_{2}=+0.369 \pm 0.2 & \mathrm{R}=0.9882 \\ a_{3}=+0.192 \pm 0.1 & \mathrm{R}=0.9573 \\ \sigma=0.028 & \mathrm{R}=0.9995\end{cases}
$$

Sviderskiene \& Straižys (1970) found that relations between Vilnius colours and Johnson U-B were non-linear, with strong luminosity effects and reddening lines non-parallel to the intrinsic sequence, and generally not suitable for transformations. The best relation was between the Vilnius $\mathrm{U}-\mathrm{Y}$ and Johnson $\mathrm{U}-\mathrm{B}$, as can be seen in Figure 3.6 and the following piece-wise equation

$$
(\mathrm{U}-\mathrm{B})_{j}=a_{0}+a_{1}(\mathrm{U}-\mathrm{Y})_{v}\left\{\begin{array}{l}
\mathrm{U}-\mathrm{B}<0.5 \\
\mathrm{U}-\mathrm{B}>0.5 \begin{cases}a_{0}=-1.089 \pm 0.02 \\
a_{1}=+0.632 \pm 0.01 & \mathrm{R}=0.998 \\
\sigma=0.017 & \mathrm{R}=0.998\end{cases} \\
\begin{array}{ll}
a_{0}=-1.353 \pm 0.05 \\
a_{1}=+0.765 \pm 0.02 & \mathrm{R}=0.999 \\
\sigma=0.011 & \mathrm{R}=0.999
\end{array}
\end{array}\right.
$$

Sviderskiené \& Straižys (1970) do not give any equations for the (U-Y) - (U-B) relations but their plotted data agrees well with Figure 3.6. 


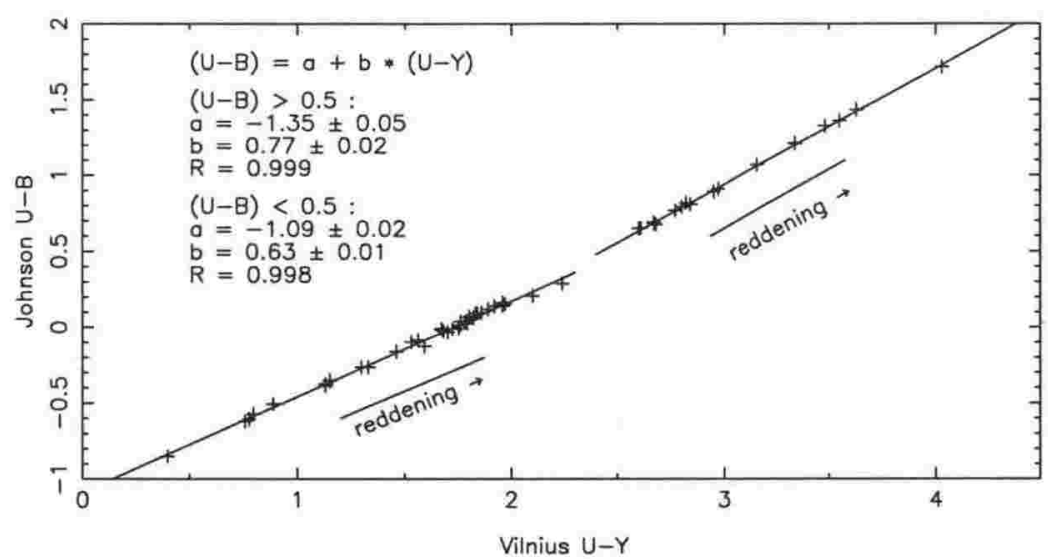

Figure 3.6: Transformations between Vilnius U-Y and Johnson U-B colours, based on E region stars measurements.

Another relation with the U-B colour was found

$$
(\mathrm{U}-\mathrm{B})_{j}=a_{0}+a_{1}(\mathrm{P}-\mathrm{X})_{v}+a_{2}(\mathrm{X}-\mathrm{Y})_{v}+a_{3}(\mathrm{Y}-\mathrm{Z})_{v} \quad \begin{cases}a_{0}=-1.101 \pm 0.01 & \\ a_{1}=+1.152 \pm 0.02 & \mathrm{R}=0.9469 \\ a_{2}=+0.582 \pm 0.04 & \mathrm{R}=0.9632 \\ a_{3}=+0.188 \pm 0.09 & \mathrm{R}=0.9203 \\ \sigma=0.020 & \mathrm{R}=0.9994\end{cases}
$$

using the $\mathrm{E}$ region measurements.

The following piece-wise transformation was found between the Vilnius $\mathrm{X}-\mathrm{V}$ and the Johnson B-V colour

$$
(\mathrm{B}-\mathrm{V})_{j}=a_{0}+a_{1}(\mathrm{X}-\mathrm{V})_{v}\left\{\begin{array}{l}
\mathrm{B}-\mathrm{V}<0.8 \\
\mathrm{~B}-\mathrm{V}>0.8 \begin{cases}a_{0}=-0.329 \pm 0.007 \\
a_{1}=+0.708 \pm 0.009 \\
\sigma=0.011 & \mathrm{R}=0.999\end{cases} \\
\begin{array}{ll}
a_{0}=+0.05 \pm 0.03 \\
a_{1}=+0.48 \pm 0.02 \\
\sigma=0.008 & \mathrm{R}=0.999
\end{array}
\end{array}\right.
$$

and is also plotted in Figure 3.7 for comparison with Sviderskienè \& Straižys (1970) graph. Similarly, a nearly linear transformation between Vilnius $\mathrm{Y}-\mathrm{V}$ and Johnson B-V was compared,

$$
(\mathrm{B}-\mathrm{V})_{j}=a_{0}+a_{1}(\mathrm{Y}-\mathrm{V})_{v}\left\{\begin{array}{rlrl}
a_{0} & =-0.312 \pm 0.004 & & \\
a_{1}=+1.708 \pm 0.007 & \mathrm{R} & =0.9992 \\
\sigma & =0.020 & \mathrm{R} & =0.9992
\end{array}\right.
$$


confirming the theoretical relations. Both these relations have reddening lines nearly parallel to the intrinsic sequences and small luminosity effects (being largest at $\mathrm{B}-\mathrm{V} \widetilde{\sim} 1.0$ ).

Sviderskiene \& Straižys (1970) give $\mathrm{B}-\mathrm{V}=-0.33+0 \cdot 50[(\mathrm{X}-\mathrm{V})+(\mathrm{Y}-\mathrm{V})]$ for $\mathrm{B}-\mathrm{V} \leq 0.80$ as the best relation between the Vilnius system and Johnson B-V. The E region measurements confirm this relation;

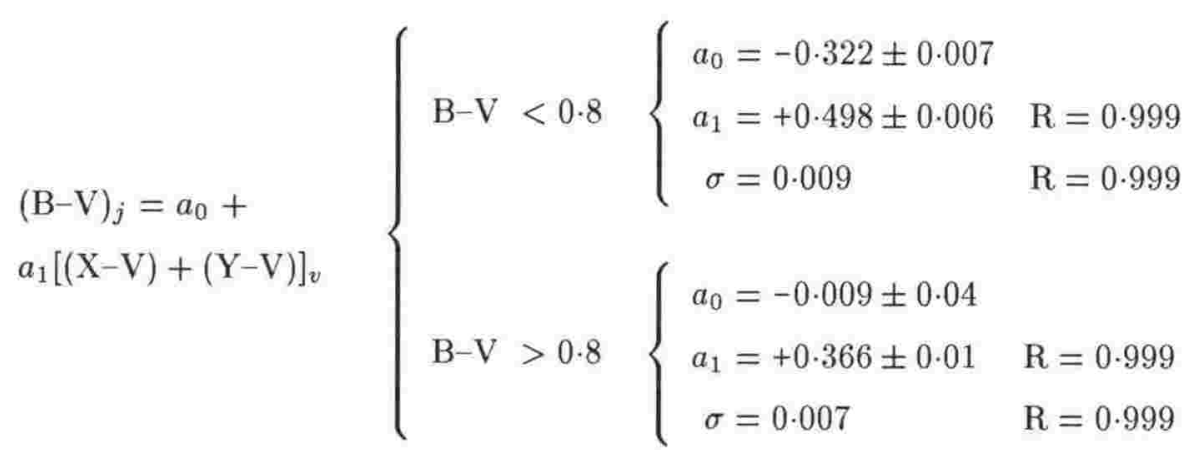

and extend it piece-wise for $\mathrm{B}-\mathrm{V}>0 \cdot 80$.

Finally, a new transformation to Johnson B-V was found

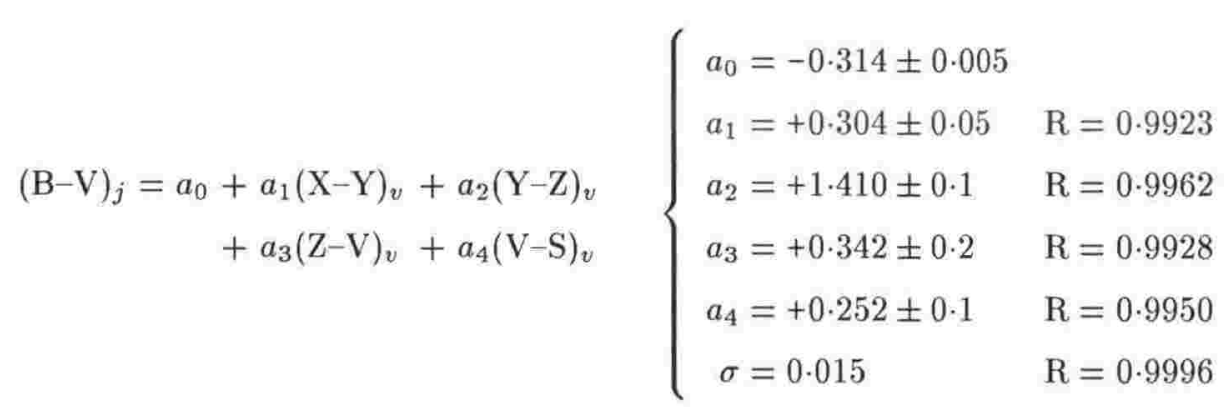

Although the Vilnius filters do not cover the same wavelength range as the extended Johnson filter system (R,I,J,K,L,M,M, and Q), Menzies et al. (1989) E region measurements include V-R and V-I colours, which are based on Cousins version of the $\mathrm{R}$ and I filters. The Vilnius V-S colour gives good transformations to both $\mathrm{V}-\mathrm{R}$ and $\mathrm{V}-\mathrm{I}$, as shown by the following equations and Figures 3.8 and 3.9 .

$$
(\mathrm{V}-\mathrm{R})_{j}=a_{0}+a_{1}(\mathrm{~V}-\mathrm{S})_{v} \quad \begin{cases}a_{0}=-0.133 \pm 0.003 & \\ a_{1}=+0.898 \pm 0.06 & \mathrm{R}=0.9987 \\ \sigma=0.012 & \mathrm{R}=0.9987\end{cases}
$$



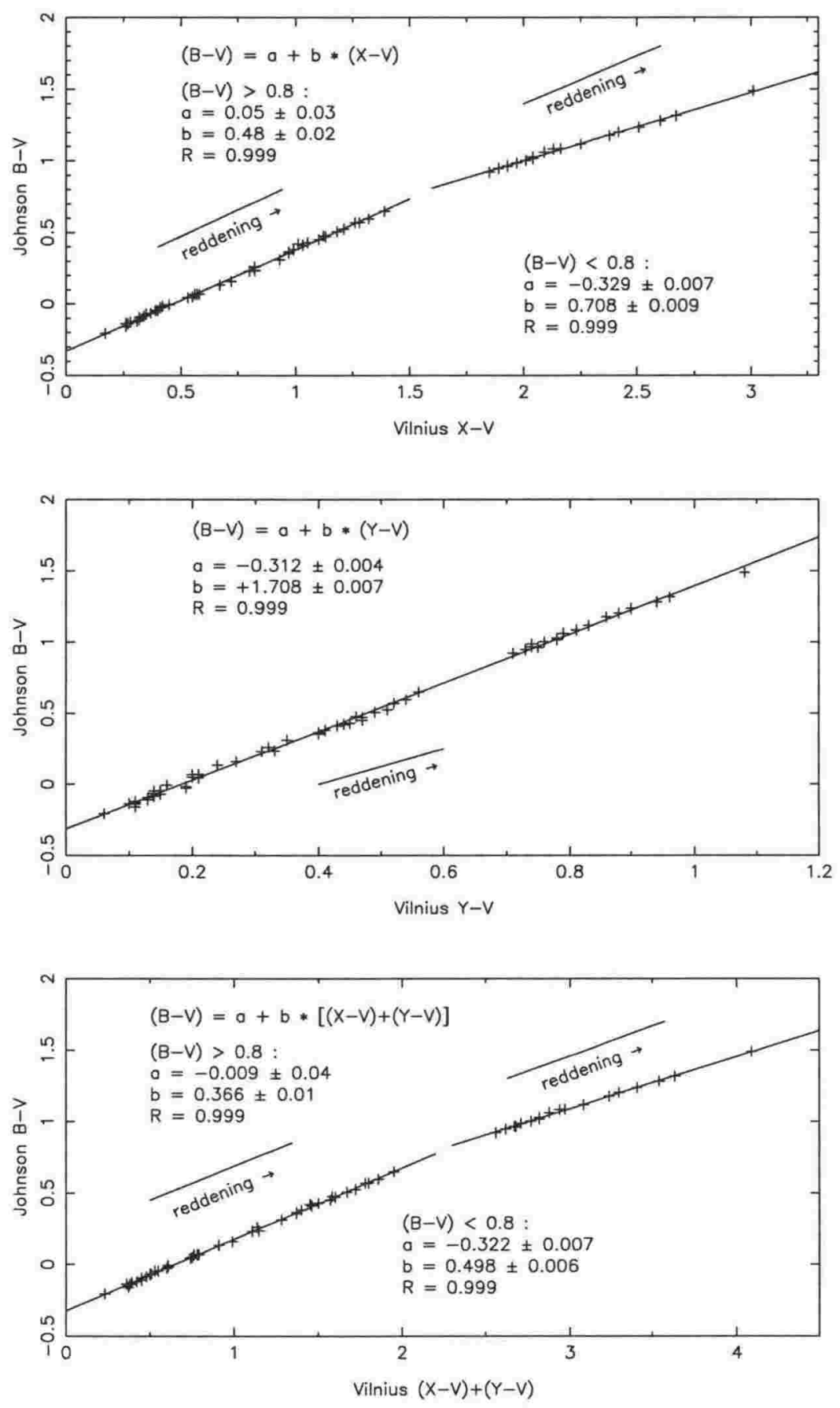

Figure 3.7: Transformations between Vilnius colours and Johnson B-V, based on E region stars measurements. 
Transformation between Johnson and Vilnius systems for the E Regions

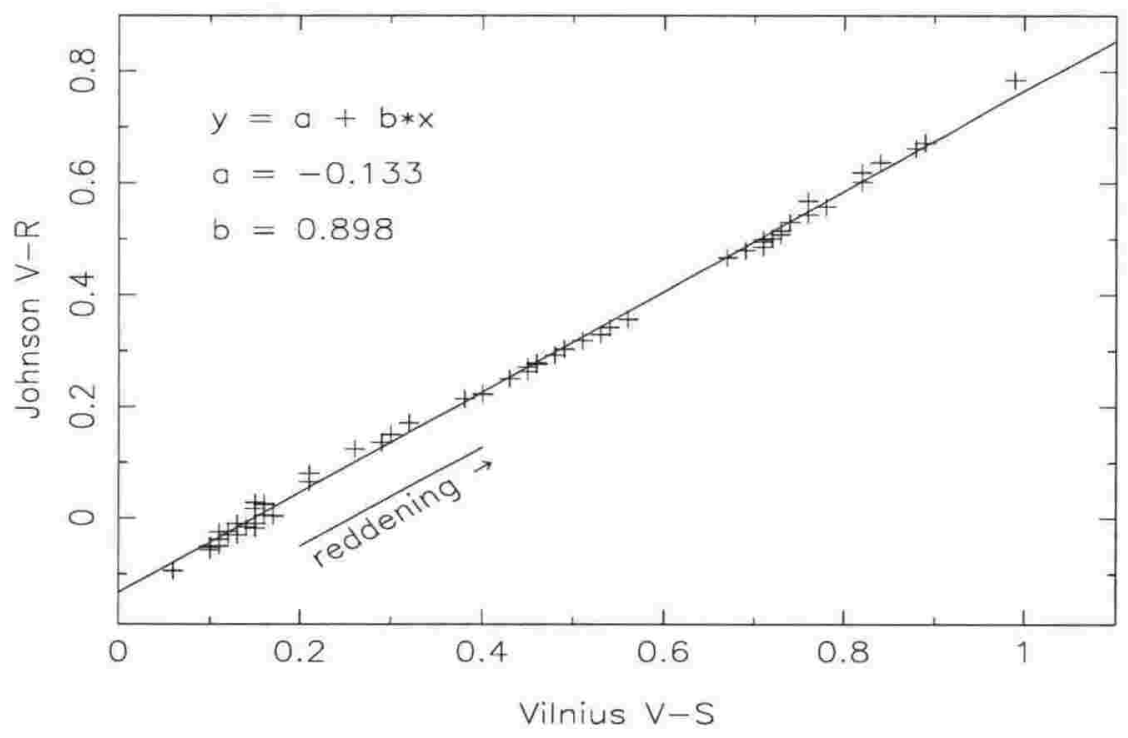

Figure 3.8: Transformations between Vilnius $\mathrm{V}-\mathrm{S}$ and Johnson $\mathrm{V}-\mathrm{R}$, based on $\mathrm{E}$ region stars measurements.

$$
(\mathrm{V}-\mathrm{I})_{j}=a_{0}+a_{1}(\mathrm{~V}-\mathrm{S})_{v} \quad\left\{\begin{array}{rlrl}
a_{0} & =-0.255 \pm 0.007 & & \\
a_{1}=+1.720 \pm 0.01 & \mathrm{R} & =0.9978 \\
\sigma & =0.031 & \mathrm{R} & =0.9978
\end{array}\right.
$$

It was found that adding progressively bluer colour terms (i.e. $\mathrm{Z}-\mathrm{V}$, then $\mathrm{Z}-\mathrm{V}$ and $\mathrm{Y}-\mathrm{Z}$ etc.) to $\mathrm{V}-\mathrm{S}$ improved the transformations (except for $\mathrm{U}-\mathrm{P}$ ). Only the equations for the most precise transformations (using all the colours $\mathrm{P}-\mathrm{X}$ to $\mathrm{V}-\mathrm{S}$ ) are given below;

$$
\begin{aligned}
(\mathrm{V}-\mathrm{R})_{j}=a_{0}+a_{1}(\mathrm{P}-\mathrm{X})_{v}+a_{2}(\mathrm{X}-\mathrm{Y})_{v} \\
+a_{3}(\mathrm{Y}-\mathrm{Z})_{v}+a_{4}(\mathrm{Z}-\mathrm{V})_{v} \\
+a_{5}(\mathrm{~V}-\mathrm{S})_{v}
\end{aligned} \quad \begin{cases}a_{0}=-0.150 \pm 0.005 & \\
a_{1}=+0.034 \pm 0.1 & \mathrm{R}=0.7625 \\
a_{2}=-0.089 \pm 0.02 & \mathrm{R}=0.9855 \\
a_{3}=+0.311 \pm 0.07 & \mathrm{R}=0.9940 \\
a_{4}=+0.726 \pm 0.1 & \mathrm{R}=0.9937 \\
a_{5}=+0.523 \pm 0.06 & \mathrm{R}=0.9987 \\
\sigma=0.007 & \mathrm{R}=0.9996\end{cases}
$$


Transformation between Johnson and Vilnius systems for the E Regions

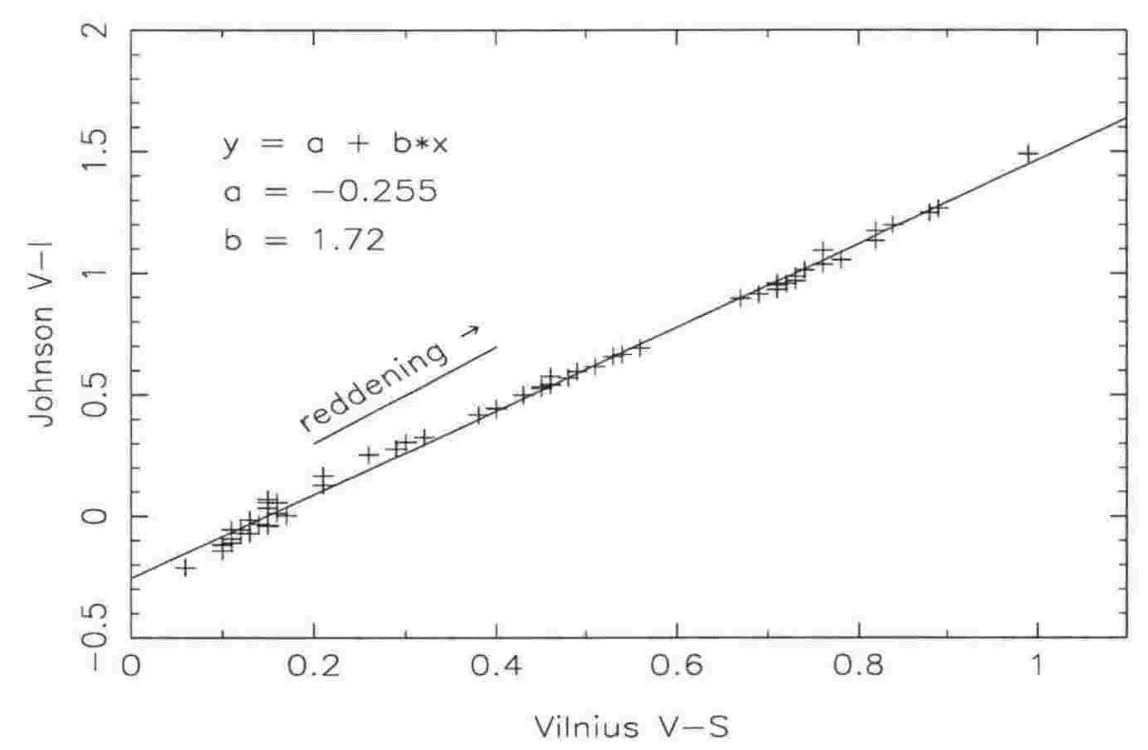

Figure 3.9: Transformations between Vilnius V-S and Johnson V-I, based on E region stars measurements.

$$
\begin{gathered}
(\mathrm{V}-\mathrm{I})_{j}=a_{0}+a_{1}(\mathrm{P}-\mathrm{X})_{v}+a_{2}(\mathrm{X}-\mathrm{Y})_{v} \\
+a_{3}(\mathrm{Y}-\mathrm{Z})_{v}+a_{4}(\mathrm{Z}-\mathrm{V})_{v} \\
+a_{5}(\mathrm{~V}-\mathrm{S})_{v}
\end{gathered} \quad\left\{\begin{array}{cc}
a_{0}=-0.336 \pm 0.02 \\
a_{1}=+0.142 \pm 0.03 & \mathrm{R}=0.7604 \\
a_{2}=-0.438 \pm 0.07 & \mathrm{R}=0.9811 \\
a_{3}=+0.968 \pm 0.2 & \mathrm{R}=0.9938 \\
a_{4}=+1.808 \pm 0.4 & \mathrm{R}=0.9909 \\
a_{5}=+1.018 \pm 0.2 & \mathrm{R}=0.9978 \\
\sigma=0.021 & \mathrm{R}=0.9991
\end{array}\right.
$$

In all the colour transformation diagrams (Figures 3.6, 3.7, 3.8, and 3.9), a 'gap' in the sequence of data points can be seen (at $\mathrm{U}-\mathrm{B} \sim 0.5, \mathrm{~B}-\mathrm{V} \sim 0.8, \mathrm{~V}-\mathrm{R} \sim 0.4$, and $\mathrm{V}-\mathrm{I} \sim 0.8$ ). This same gap can be seen in Sviderskienè \& Straižys (1970) diagrams as a reduced density of data points and is due to the following selection and luminosity effects. Consider the $(\mathrm{Y}-\mathrm{V})-$ $(\mathrm{B}-\mathrm{V})$ relation in Figure 3.7 as an example. The early type stars $(\mathrm{B}-\mathrm{V}<0.8)$ are predominately main-sequence stars, but the late type stars are exclusively brighter luminosity classes (I-III). This is because late type main-sequence stars are intrinsically fainter than early type mainsequence stars (at the effective wavelength of the $\mathrm{V}$ filter) and so have not yet been observed from the $\mathrm{E}$ region star lists (the selection effect). For early type stars, $\mathrm{B}-\mathrm{V}$ is approximately independent of luminosity class but for later type stars ( $\sim \mathrm{G}$ class onwards), B-V increases with 


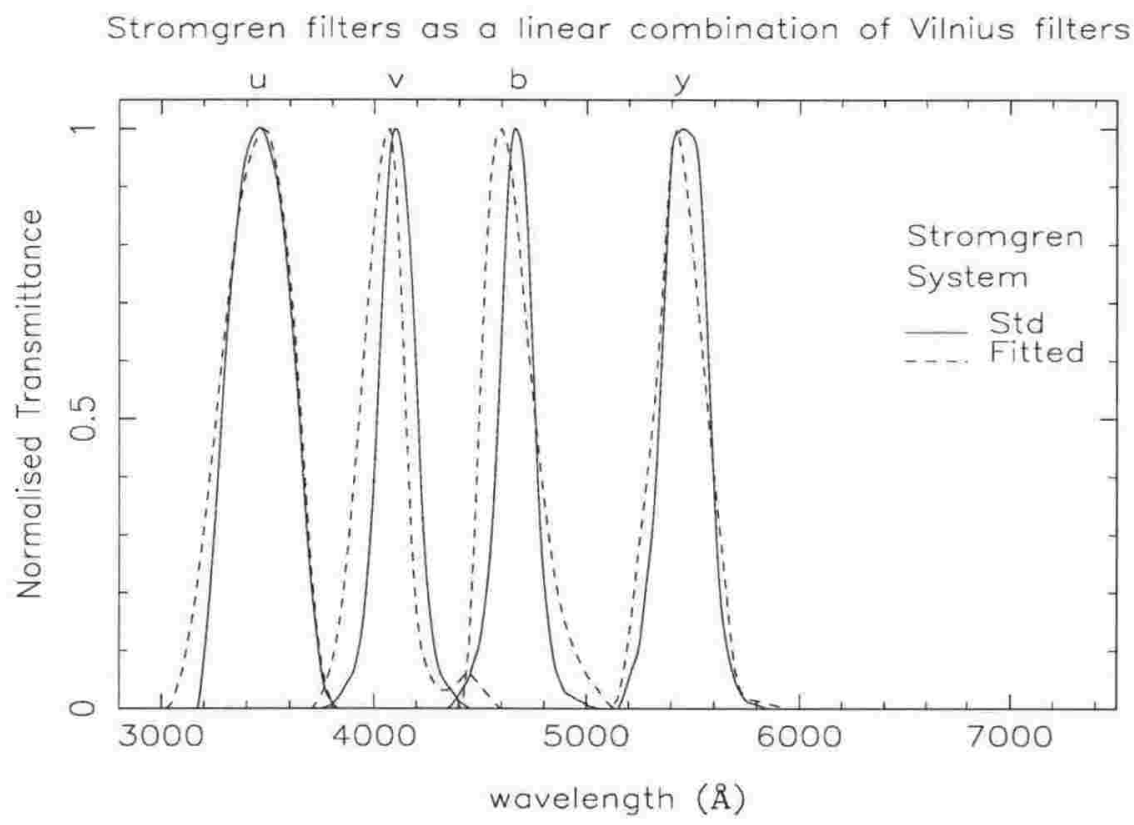

Figure 3.10: Least squared fitted linear combination of Vilnius filters to match the Strömgren filters.

luminosity for a given spectral class. However $\mathrm{Y}-\mathrm{V}$ is approximately independent of luminosity for all spectral classes (except M, which wasn't observed here). Hence the selection effect (which meant no main-sequence stars later than G4 were observed) combined with the luminosity effect (which starts at $\sim$ G class) produces the gap in the diagrams.

\subsection{Transformations to the Strömgren System.}

Transformations from the Vilnius to the Strömgren systems were also investigated. As for the Johnson filters, Vilnius standard passbands were added in linear combinations to match each Strömgren filter. The best combinations were;

$$
\begin{aligned}
u & =a_{1} \mathrm{U} \\
v & =a_{1} \mathrm{X} \\
b & =a_{1} \mathrm{Y}+a_{2} \mathrm{Z} \\
y & =a_{1} \mathrm{Z}+a_{2} \mathrm{~V}
\end{aligned}
$$

with lower-case or upper-case indicating Strömgren or Vilnius filters respectively - see also Figure 3.10 .

The Strömgren $u$ filter is closely matched by the Vilnius U filter, apart from the short 
wavelength tail. However the atmospheric extinction is large at these wavelengths and so the difference between the filters should have negligible effect. The remaining Strömgren filters are reasonably well matched by the Vilnius filter combinations, however in general the fitted filter has a shorter effective wavelength than the standard filter.

The Strömgren $y$ filter has nearly the same effective wavelength as the Vilnius $\mathrm{V}$ filter so a simple linear fit between the two magnitudes should give good results;

$$
y=a_{0}+a_{1} V\left\{\begin{array}{rlrl}
a_{0} & =-0.061 \pm 0.04 & & \\
a_{1}=+1.001 \pm 0.07 & \mathrm{R} & =0.9990 \\
\sigma & =0.034 & \mathrm{R} & =0.9990
\end{array}\right.
$$

A slight colour term remains, which is fit to approximately the same precision by the following colours;

$$
\mathrm{V}-y=a_{0}+a_{1}(\mathrm{Col}) \quad\left\{\begin{array}{ccccc}
\mathrm{Col} & a_{0} & a_{1} & \sigma & \mathrm{R} \\
\mathrm{X}-\mathrm{Y} & 0.023 \pm 0.007 & 0.043 \pm 0.009 & 0.027 & 0.608 \\
\mathrm{Y}-\mathrm{Z} & 0.017 \pm 0.007 & 0.133 \pm 0.02 & 0.027 & 0.624 \\
\mathrm{Z}-\mathrm{V} & 0.024 \pm 0.007 & 0.164 \pm 0.03 & 0.028 & 0.591 \\
\mathrm{~V}-\mathrm{S} & 0.020 \pm 0.007 & 0.078 \pm 0.02 & 0.027 & 0.611 \\
v-b & 0.028 \pm 0.006 & 0.046 \pm 0.009 & 0.027 & 0.596 \\
b-y & 0.033 \pm 0.005 & 0.073 \pm 0.01 & 0.027 & 0.613
\end{array}\right.
$$

The best fit is with the $\mathrm{Y}-\mathrm{Z}$ colour, which is shown in Figure 3.11 .

All the 'usual' Vilnius colours give non-linear and multi-valued transformations to the Strömgren $u-b$ colour. However, on the basis of the linear combinations of filters, the U-Y colour is expected to give a good fit. As Figure 3.12 shows, the transformation is linear and single-valued, with the following coefficients;

$$
u-b=a_{0}+a_{1}(\mathrm{U}-\mathrm{Y}) \begin{cases}a_{0}=-0.293 \pm 0.007 & \\ a_{1}=+0.968 \pm 0.004 & \mathrm{R}=0.9997 \\ \sigma=0.018 & \mathrm{R}=0.9997\end{cases}
$$

The reddening line for this, and all the following colour transformations, lie nearly parallel to the intrinsic sequence and hence the transformations should be nearly independent of the reddening. 
Transformation between Stromgren and Vilnius systems for the E Regions

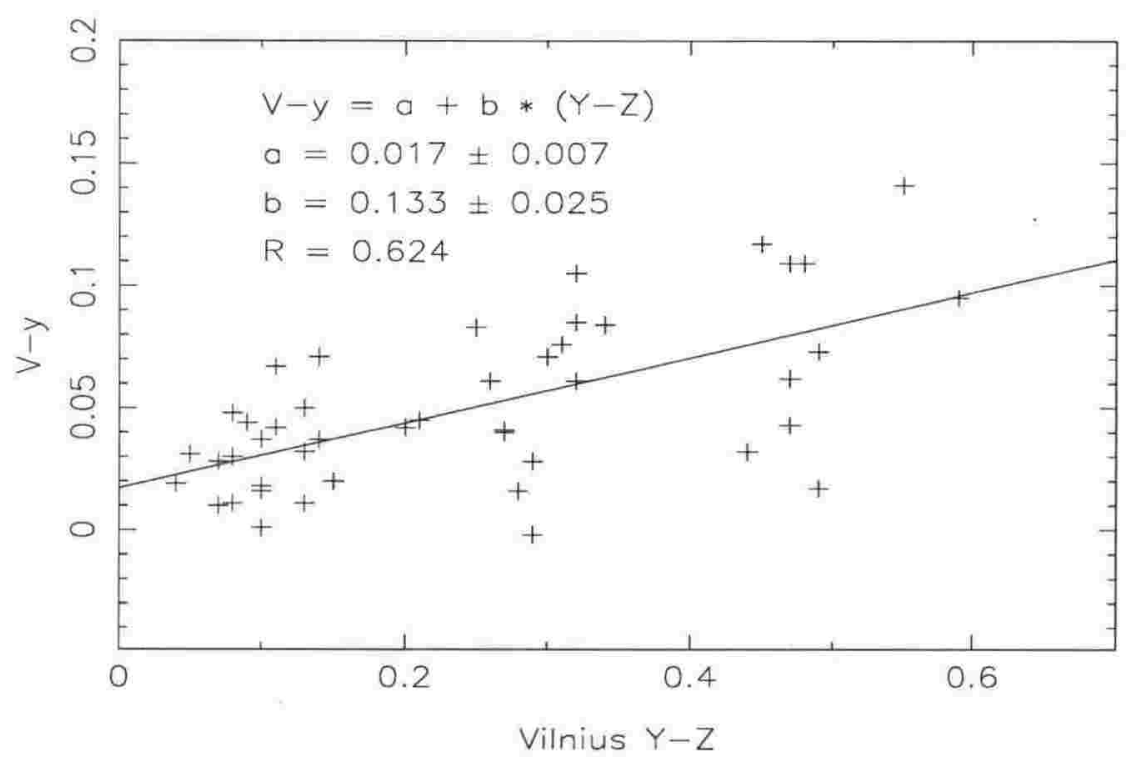

Figure 3.11: Transformations between the Vilnius and Strömgren magnitudes, based on E region stars measurements.

Transformation between Stromgren and Vilnius systems for the E Regions

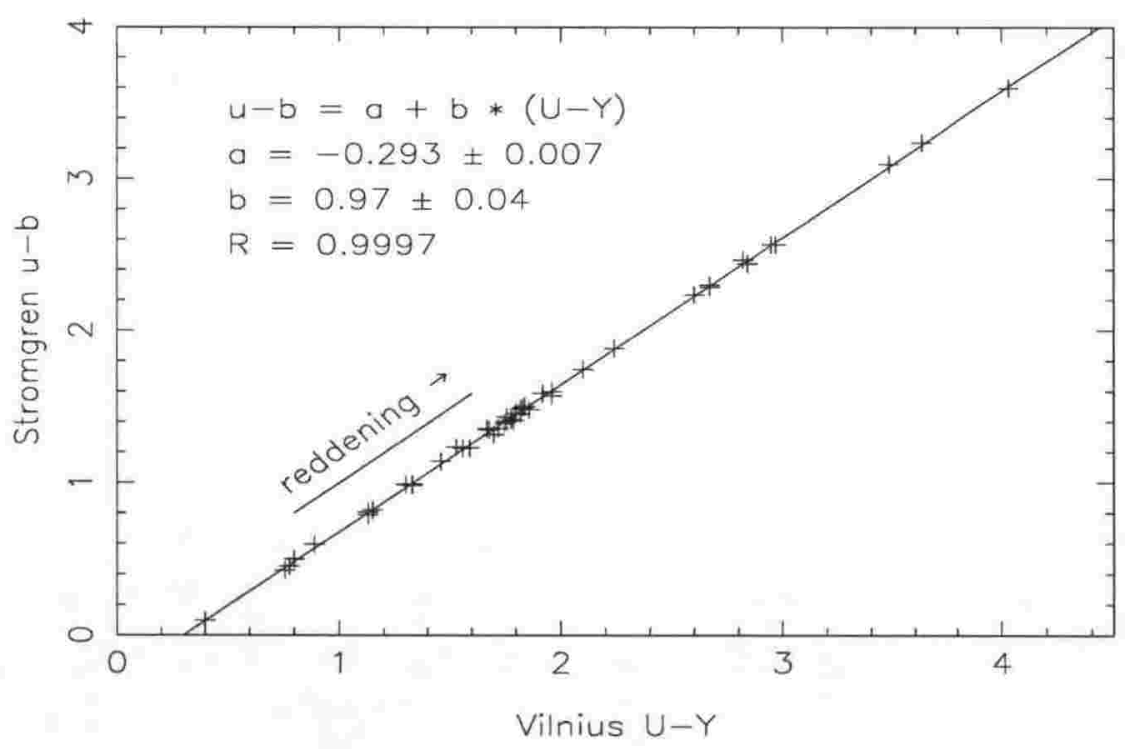

Figure 3.12: Transformations between the Vilnius U-Y and Strömgren $u-b$ colours, based on E region stars measurements. 
As with the $y$ magnitude, a slight improvement is made by the addition of the $\mathrm{Z}$ filter;

$$
u-b=a_{0}+a_{1}(\mathrm{U}-\mathrm{Y})+a_{2}(\mathrm{Y}-\mathrm{Z}) \quad \begin{cases}a_{0}=-0.308 \pm 0.008 & \\ a_{1}=+0.996 \pm 0.008 & \mathrm{R}=0.9997 \\ a_{2}=-0.148 \pm 0.04 & \mathrm{R}=0.9143 \\ \sigma=0.016 & \mathrm{R}=0.9998\end{cases}
$$

or the Strömgren $b-y$ colour

$$
u-b=a_{0}+a_{1}(\mathrm{U}-\mathrm{Y})+a_{2}(b-y) \begin{cases}a_{0}=-0.327 \pm 0.01 & \\ a_{1}=+0.997 \pm 0.009 & \mathrm{R}=0.9997 \\ a_{2}=-0.086 \pm 0.02 & \mathrm{R}=0.9216 \\ \sigma=0.016 & \mathrm{R}=0.9998\end{cases}
$$

Straižys (1973 a) gives a similar transformation

$$
(u-b)-(\mathrm{U}-\mathrm{Y})=0 \cdot 024(b-y)_{0}+c E_{b-y}
$$

where $c \approx 0.005$ for early stars, increases to $c \approx 0.016$ for F0 I stars and decreases to $c \approx 0.0$ for late type stars. As most of the $\mathrm{E}$ region stars have little reddening, only a crude check of Straižys' transformation could be made using $b-y \approx(b-y)_{0}$

$$
(u-b)-(\mathrm{U}-\mathrm{Y})=a_{0}+a_{1}(b-y) \begin{cases}a_{0}=-0.330 \pm 0.003 & \\ a_{1}=-0.093 \pm 0.008 & \mathrm{R}=-0.850 \\ \sigma=0.016 & \mathrm{R}=-0.850\end{cases}
$$

which shows little agreement.

Again on the basis of the linear combinations of the filters, the Vilnius $\mathrm{X}-\mathrm{Y}$ colour is expected to give a good transformation to $v-b$. However, according to Straižys $(1973 a)$, this transformation is expected to be non-linear and multi-valued for stars of different luminosity and reddening. This is due to the position of the $v$ and $\mathrm{X}$ passbands - for example early type stars have a strong $\mathrm{H}_{\delta}$ absorption line near the effective wavelength of the $v$ filter but on the edge of the $\mathrm{X}$ filter. Nevertheless, the $(v-b)-(\mathrm{X}-\mathrm{Y})$ transformation based on the $\mathrm{E}$ region stars is approximately linear, with small 'oscillations' about the line (see Figure 3.13 ) and the following coefficients

$$
v-b=a_{0}+a_{1}(\mathrm{X}-\mathrm{Y}) \begin{cases}a_{0}=-0.113 \pm 0.004 & \\ a_{1}=+0.917 \pm 0.005 & \mathrm{R}=0.9992 \\ \sigma=0.017 & \mathrm{R}=0.9992\end{cases}
$$


Transformation between Stromgren and Vilnius systems for the E Regions

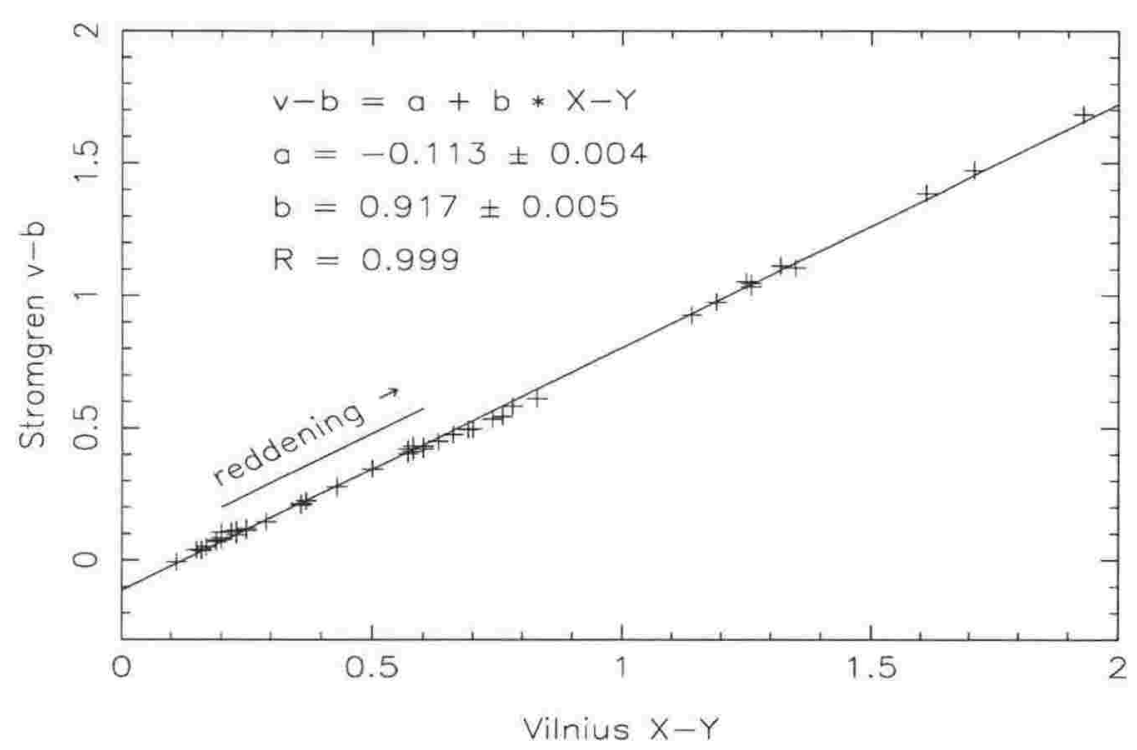

Figure 3.13: Transformations between the Vilnius $\mathrm{X}-\mathrm{Y}$ and Strömgren $v-b$ colours, based on $\mathrm{E}$ region stars measurements.

Slight improvements to the precision of the fit are possible using an additional colour;

$$
v-b=a_{0}+a_{1}(\mathrm{X}-\mathrm{Y})+a_{2}(\mathrm{Y}-\mathrm{Z}) \quad \begin{cases}a_{0}=-0.101 \pm 0.004 & \\ a_{1}=+1.020 \pm 0.02 & \mathrm{R}=0.9992 \\ a_{2}=-0.316 \pm 0.06 & \mathrm{R}=0.9733 \\ \sigma=0.014 & \mathrm{R}=0.9995\end{cases}
$$

or

$$
v-b=a_{0}+a_{1}(\mathrm{X}-\mathrm{Y})+a_{2}(b-y) \quad\left\{\begin{array}{lll}
a_{0}=-0.145 \pm 0.007 & \\
a_{1}=+1.058 \pm 0.03 & \mathrm{R}=0.9992 \\
a_{2}=-0.240 \pm 0.05 & \mathrm{R}=0.9840 \\
\sigma=0.014 & \mathrm{R}=0.9995
\end{array}\right.
$$

The final Strömgren colour, $b-y$, is best fitted by the Vilnius $\mathrm{Y}-\mathrm{V}$ colour, as implied by the linear combination of filters. This is shown in Figure 3.14 and the following equation

$$
b-y=a_{0}+a_{1}(\mathrm{Y}-\mathrm{V}) \begin{cases}a_{0}=-0.170 \pm 0.002 & \\ a_{1}=+1.014 \pm 0.004 & \mathrm{R}=0.9995 \\ \sigma=0.009 & \mathrm{R}=0.9995\end{cases}
$$


Transformation between Stromgren and Vilnius systems for the E Regions

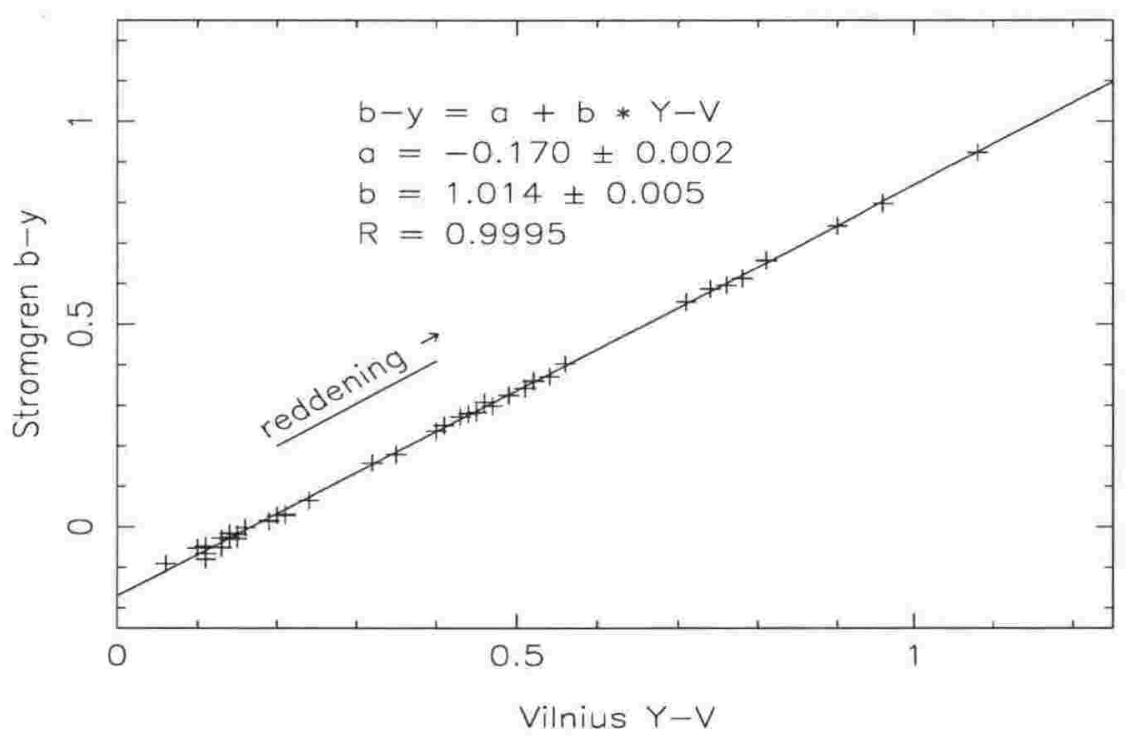

Figure 3.14: Transformations between the Vilnius $\mathrm{Y}-\mathrm{V}$ and Strömgren $b-y$ colours, based on E region stars measurements.

A slight improvement may be made by the addition of the $\mathrm{Z}$ filter

$$
b-y=a_{0}+a_{1}(\mathrm{Y}-\mathrm{V})+a_{2}(\mathrm{Z}-\mathrm{V}) \quad\left\{\begin{array}{lll}
a_{0}=-0.173 \pm 0.003 & \\
a_{1}=+1.080 \pm 0.04 & \mathrm{R}=0.9995 \\
a_{2}=-0.152 \pm 0.1 & \mathrm{R}=0.9928 \\
\sigma=0.009 & \mathrm{R}=0.9996
\end{array}\right.
$$

or the Strömgren $b-y$ colour (with the equation in the form used by Straižys)

$$
(b-y)-(\mathrm{Y}-\mathrm{V})=a_{0}+a_{1}(b-y) \quad \begin{cases}a_{0}=-0.168 \pm 0.002 & \\ a_{1}=+0.014 \pm 0.005 & \mathrm{R}=0.429 \\ \sigma=0.009 & \mathrm{R}=0.429\end{cases}
$$

where Straižys (1973a) gives $a_{0} \equiv 0$ and $a_{1}=0.021$ or later $a_{0}=-0.16$ and $a_{1}=0.01$ (Straižys 1992), with the latter agreeing well with the results from the E region measurements.

\subsection{Summary.}

At the time this study started there were no Vilnius standard star regions observable from MJUO, so one had to be established before any research using the Vilnius system could be 
started. As the stars (from the Vilnius catalog) used to tie the new standards back to the original system were not themselves standards, there was a larger than average scatter in the transformation diagrams, so requiring more stars to be observed than usual. Note that due to under-sampling of the wavelength region measured by the Vilnius filters, the southern standards will actually form a new (Southern) Vilnius system, which on average equals the northern system.

The southern standards were divided into three groups; the South Polar Sequence (SPS), the all-sky group and the E Regions. The SPS is a set of bright, circumpolar stars chosen so seasonal effects (for example due to changes in the average temperature between summer and winter) may be eliminated. The all-sky set of stars consists of bright stars evenly scattered around the sky so an observer should always be able to find a standard near any program star or for use as extinction stars. The $\mathrm{E}$ regions extend to a fainter magnitude range than the other standard sets, and are spaced in approximately two hour intervals in Right Ascension and centred about $-45^{\circ}$ in declination. These stars are already used as standard stars in the Johnson and Strömgren systems so are so less likely to include variable stars.

An error analysis was carried out on the observations of the proposed standard stars. A comparison between the different filters revealed that the $\mathrm{V}$ errors were typical of the other filters, so further analysis was done only on the $\mathrm{V}$ measurements. The only trends found in the internal errors (i.e. before transforming to the standard system) are with the airmass of the observation and brightness of the star (which are to be expected) so there were no signs of instrumental problems. Outliers $\left(s_{\mathrm{v}}>0^{m} \cdot 05\right)$ from main distribution of internal errors were considered 'bad' observations and rejected. Similar thresholds were found for the other filters.

The original intention was to achieve $0^{m} \cdot 01$ accuracy and precision in the new standard system. However the accuracy and precision achieved was significantly affected by the Mount Pinatubo eruption, which increased the mean value and scatter of the extinction, so it was necessary to relax the precision and accuracy goal slightly to $0^{m} \cdot 02$. For instance, based on the residuals (differences between the measurements of stars observed in both the southern and northern systems), it was found that for $50 \%$ of the measured stars to meet the original $0^{m} \cdot 01$ goal requires six observations per star, while eighteen observations per star are needed for $99 \%$ of the stars. The revised $0^{m} \cdot 02$ goal shifts these to 1.5 and 12 observations per star, while the actual average number of observations per star is $4 \cdot 7$.

Another measure of the precision of the observations are the standard deviations of the external (i.e. after transformation to the standard system) measurements. All filters, except $\mathrm{V}\left(0^{m} \cdot 035\right)$ and U-P $\left(0^{m} \cdot 034\right)$, had a standard deviation of approximately $0^{m} \cdot 020$. Thus it is recommended that the integration times for $\mathrm{U}$ filter be increased to 3-4 times longer than the other filters (it is already twice that of the other filters). 
Thus the following thresholds were developed to accept or reject a measured star as a standard; more than three observations must have been made of the star, the standard deviation of the mean value of the star for each filter must be less than some value (for example $0^{m} \cdot 02$ for the E Regions stars, except for U-P which is $0^{m} \cdot 025$ ), and the star's spectral classification based on mean Vilnius colours agrees within two sub-classes of the spectral type given in the literature and within one luminosity class. An obvious precondition is that the star must not be listed in any of the catalogues of known or suspected variables.

After this selection process, the overall accuracy and precision of the southern Vilnius standard system was estimated (using equation 3.3 ) as approximately $0^{m} \cdot 005$ in each of the magnitudes and colours (except for $\mathrm{V}$ and $\mathrm{U}-\mathrm{P}$, which are about $0^{m} \cdot 01$ ). Note that this is the accuracy and precision achievable by using all of the southern standard stars to transform a program star to the Vilnius system.

As the E Region stars are already standard stars in the Johnson and Strömgren systems, the opportunity arose to investigate transformations to/from the Vilnius system. From trying to reproduce the Johnson or Strömgren filter passbands as linear combinations of the Vilnius passbands, it was expected that good transformations should be possible between the $\mathrm{U}$ (and $u$ ) filters but not the others. However it was found several (often piece-wise) transformations to each Johnson or Strömgren filter was possible, to a precision of about $0^{m} \cdot 02-0{ }^{m} \cdot 03$. Some of these transformations had previously been investigated in the literature, with similar results to this study. It should be remembered however that these are only valid for same type of star used to derive the transformation, which here were predominately normal main sequence stars. 


\section{Chapter 4}

\section{The Omicron Velorum and Kappa Crucis clusters.}

\subsection{Isochrones in the Vilnius System.}

There is a large body of literature available describing stellar models, which, for example, can be used for population synthesis studies, or fitting isochrones to star clusters. These models usually give results in both 'theoretical' units (such as effective surface temperature $T_{e}$ and solar luminosities $L / L_{\odot}$ ) and observational units (such as absolute visual magnitude $\mathrm{M}_{\mathrm{V}}$ and Johnson colour B-V). Unfortunately none have yet been published in the colours of the Vilnius system, apart from the limited case of B-type stars given recently by Straižys et al. (1993). However one of the authors of that paper (Kurucz 1995) provided Vilnius magnitudes and colours for a more complete grid of temperatures, gravities and metallicities - see Figure 4.1 for an example.

Kurucz's Vilnius magnitudes and colours are in a natural system and need to be transformed to the standard system (see section 2.8). As they were calculated by convolving model stellar spectra with the passbands of the standard filters, a simple zero-point shift was expected to suffice. However Kurucz's magnitudes are actually the magnitude per unit surface area $(-2.5 \log (I))$, and so need to be integrated over the star's surface first - this is not needed for the colours as the surface area terms cancel. Rearrangement of equation 1.4 leads to a more convenient form

$$
-2.5 \log (L)=-2.5 \log (I)-5 \log (R)
$$

in terms of magnitudes.

To calibrate the natural magnitudes and colours, the intrinsic colours and absolute magnitudes $\left(M_{V}\right)$ for main sequence stars were taken from Straižys (1992). The effective temperature, gravity and radii for the corresponding spectral types were also taken from Straižys (1992), and Kurucz's magnitudes (after correction for stellar radii) and colours interpolated to these values, assuming solar metallicity $([\mathrm{M}]=0 \cdot 0)$. This was repeated for giant and super-giant sequences for the $\mathrm{V}$ magnitude, and is shown in Figure 4.2. 


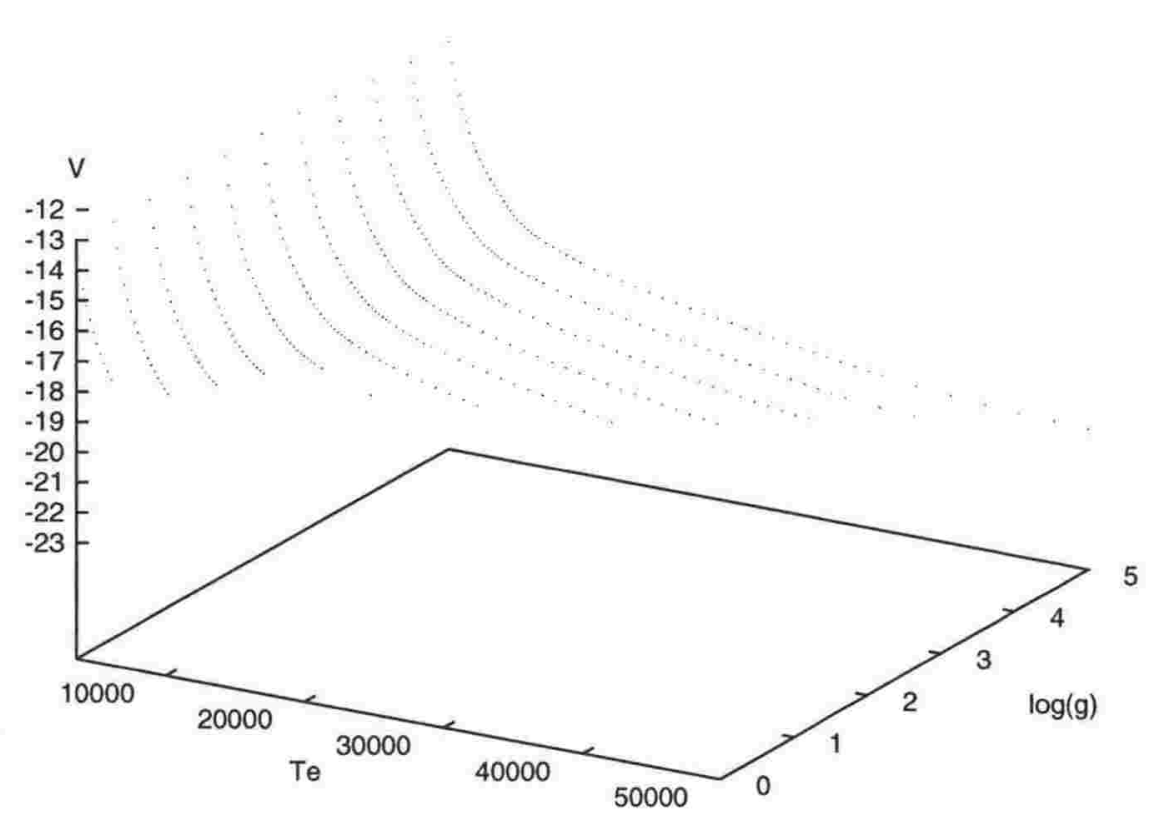

Figure 4.1: Grid of Vilnius V magnitudes (per unit surface area) from Kurucz's solar metallicity models, not yet converted to the standard system. Other metallicities available range from $[\mathrm{M}]=-5 \cdot 00$ to $+1 \cdot 00$.

The V calibration curve can be closely fitted by a straight line having a slope of 1.0, i.e. a zero-point shift, with the three luminosity classes all falling on the same line. However Kurucz's late-type Ia stars appear systematically too faint, not overlapping the earlier stars when they loop back in the sequence. This doesn't occur for late-type stars in the other luminosity classes, i.e. in the same range of temperatures and gravities in Kurucz's grid, therefore the 'error' is not due to Kurucz's models and was ignored. The Y-V calibration curve can also be fit by a zero-point shift, but there appear to be some systematic deviations from a straight line with Kurucz's Y-V being too blue for early-type stars.

The required theoretical data to be input to Kurucz's grid was provided by a grid of evolutionary models computed by Schaerer and collaborators (Schaller et al. 1992, Schaerer et al. 1993). These cover an initial mass range of 0.8 to $120 \mathcal{M}_{\odot}$, for three metallicities $Z=0.001$, 0.008 , and $0.020 .{ }^{1}$ A program provided by Dr. Schaerer interpolated between this grid of models to produce an isochrone for a given metallicity and age. To test the results, a Zero Age Main Sequence (ZAMS) was constructed by joining piece-wise segments of various isochrones (with $Z=0.020$ ) and comparing to the Vilnius ZAMS in Figure 4.3. Two different calibration

\footnotetext{
${ }^{1}$ Kurucz used solar metallicities $[\mathrm{M}]$, which is related to the metallicity used by Schaerer via $[\mathrm{M}]=\log \left(Z / Z_{\odot}\right)$, where $Z_{\odot}$ is the Sun's metallicity (taken here as 0.020 ).
} 

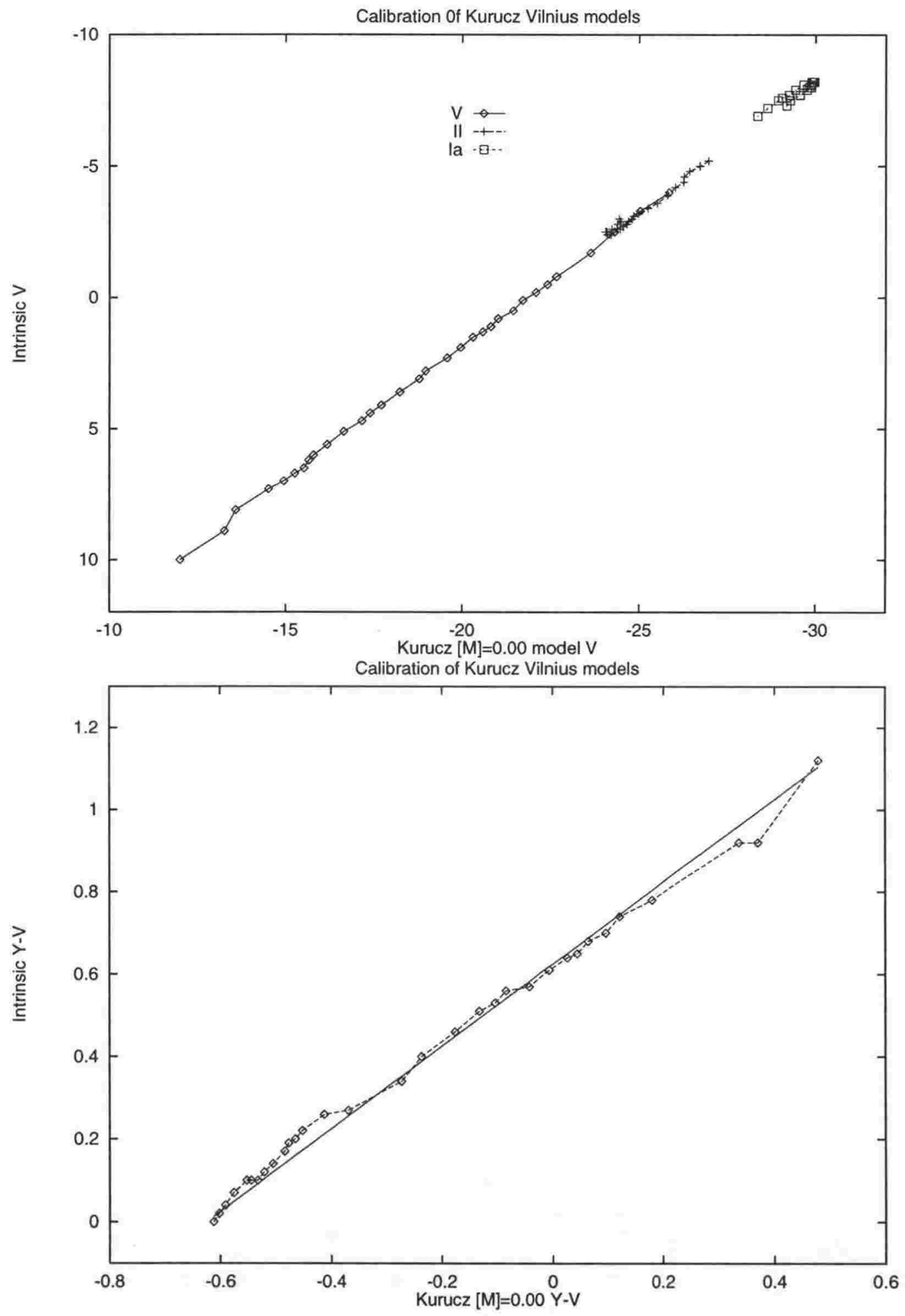

Figure 4.2: Calibration of Kurucz's models to the standard Vilnius system, for $\mathrm{V}\left(=\mathrm{M}_{\mathrm{V}}\right)$ and $\mathrm{Y}-\mathrm{V}$. The top diagram includes luminosity classes Ia, III, and V, while the bottom diagram includes only the main sequence, with a straight line corresponding to a zero-point shift drawn through it. 


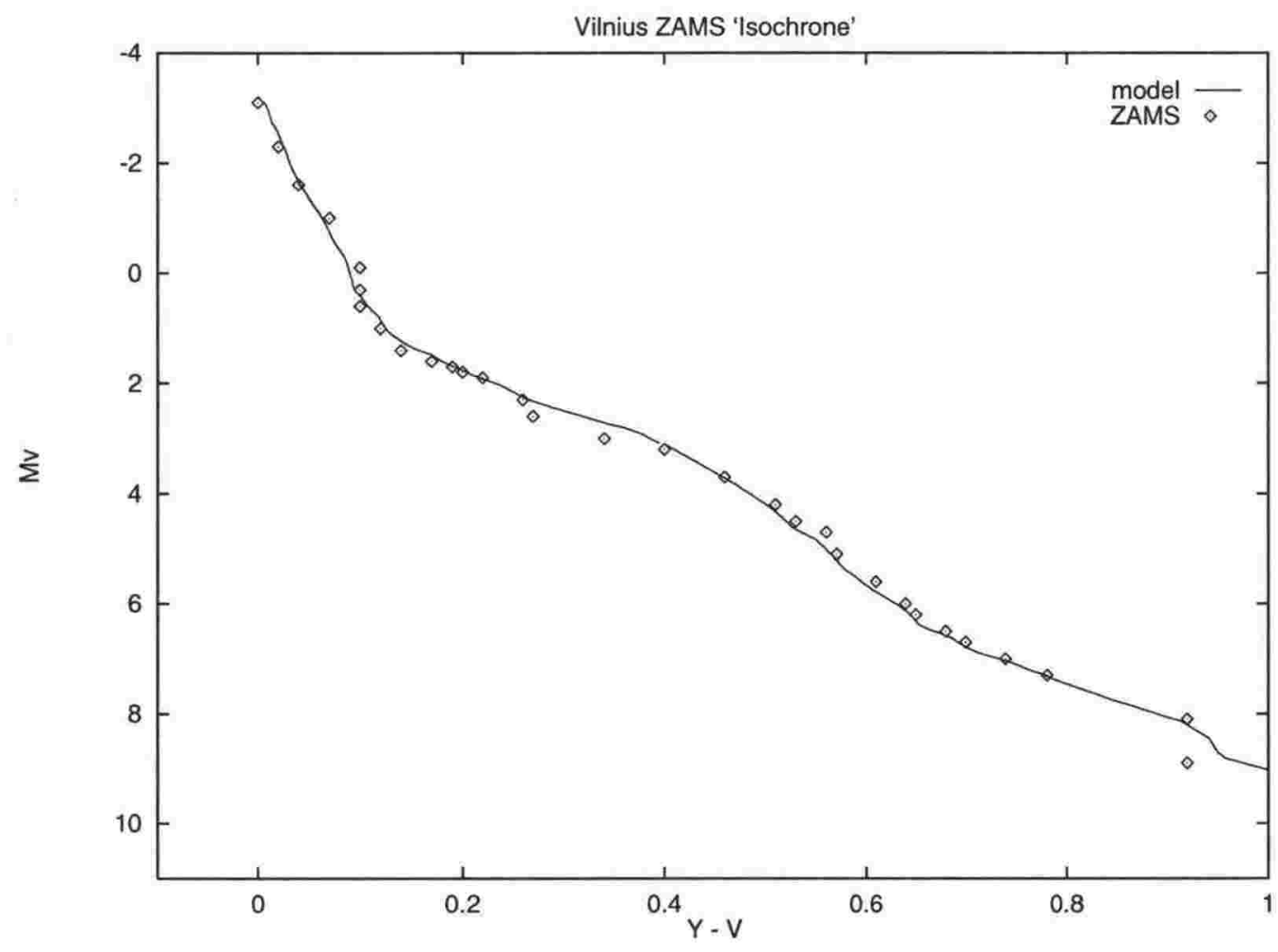

Figure 4.3: Comparison of the ZAMS with the model ZAMS (constructed piece-wise from various age isochrones).

methods (of Kurucz's natural magnitudes and colours) were tested; one using zero-point shifts and the other using Figure 4.2 as calibration curves. The best results were found by taking the average value between the two calibration methods, which is shown in the figure.

This enabled a series of isochrones for three metallicities to be calculated, shown in Figures 4.4 to 4.6 . These range from $\log ($ age $)=6.5$ to 10.1 in 0.1 increments, with the age in years. As there is essentially a linear transformation between the Johnson B-V colour and the Vilnius $\mathrm{Y}-\mathrm{V}$ (see section 3.5), the isochrones show the same features as found in the literature for the UBV system (for example, see Meynet et al. (1993)).

\subsection{Literature review of the Omicron Velorum cluster.}

The first photoelectric study of the open star cluster 'Omicron Velorum'2 appears to have been done by Lyngå (1959), hence Lyngå's numbering scheme was adopted here. The cluster is located at $\mathrm{RA}=8^{\mathrm{h}} 38^{\mathrm{m}} 8$, Declination $(\mathrm{Dec})=-52^{\circ} 53^{\prime}(1950)$ or galactic coordinates $\mathrm{l}=270 \cdot 4^{\circ}$, $\mathrm{b}=-6 \cdot 9^{\circ}$, with an angular diameter of $\sim 45^{\prime}$.

\footnotetext{
${ }^{2}$ So named due to the bright star Omicron Velorum in the middle of the cluster. The $O$ Vel cluster is also known as IC 2391 from the Index Catalogue or C0838-528 using it's International Astronomical Union (IAU) designation.
} 


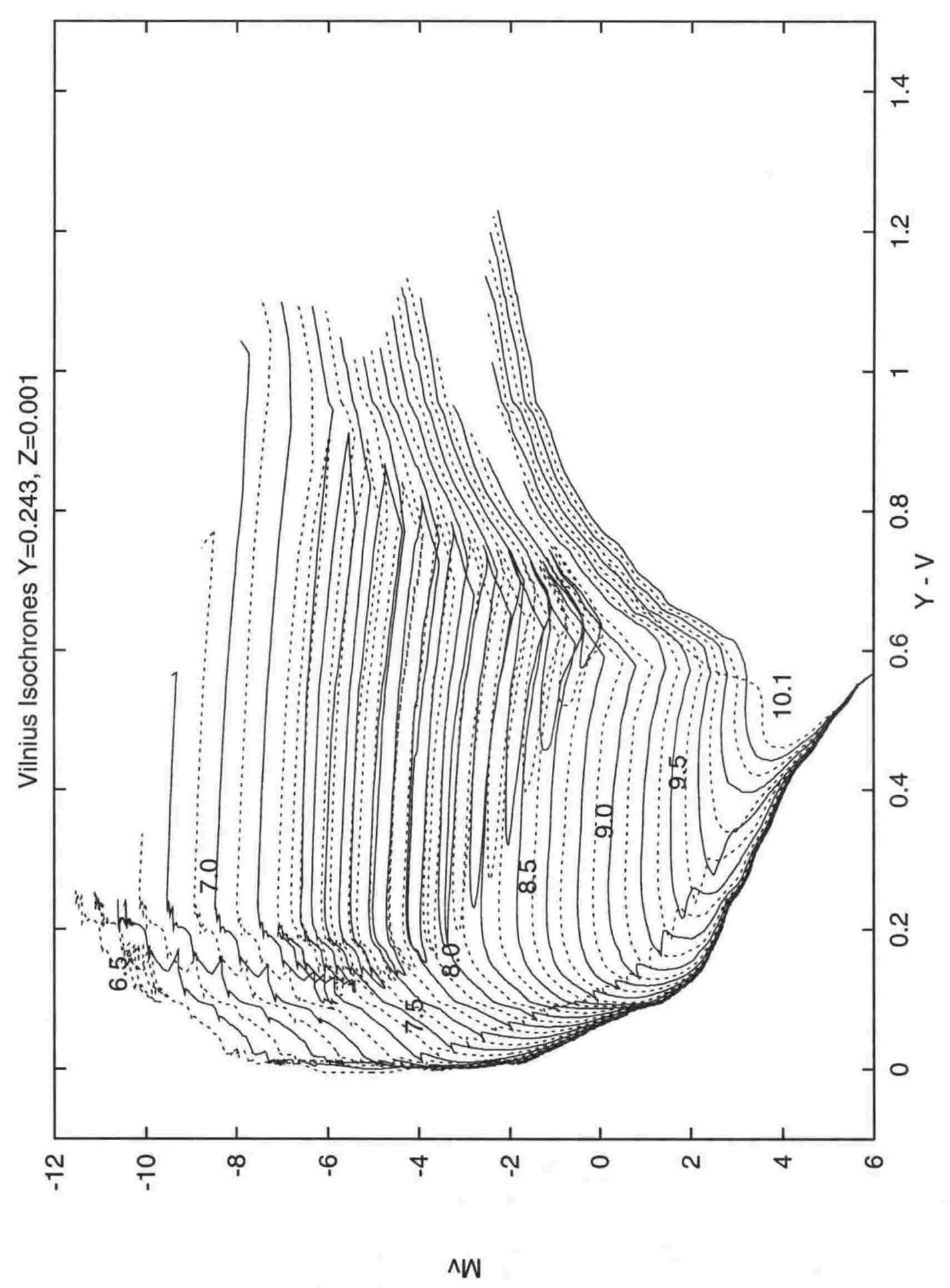

Figure 4.4: Grid of isochrones for $\mathrm{Z}=0.001$, between $\log ($ age $)=6.5$ to 10.1 in 0.1 increments (age in years). 


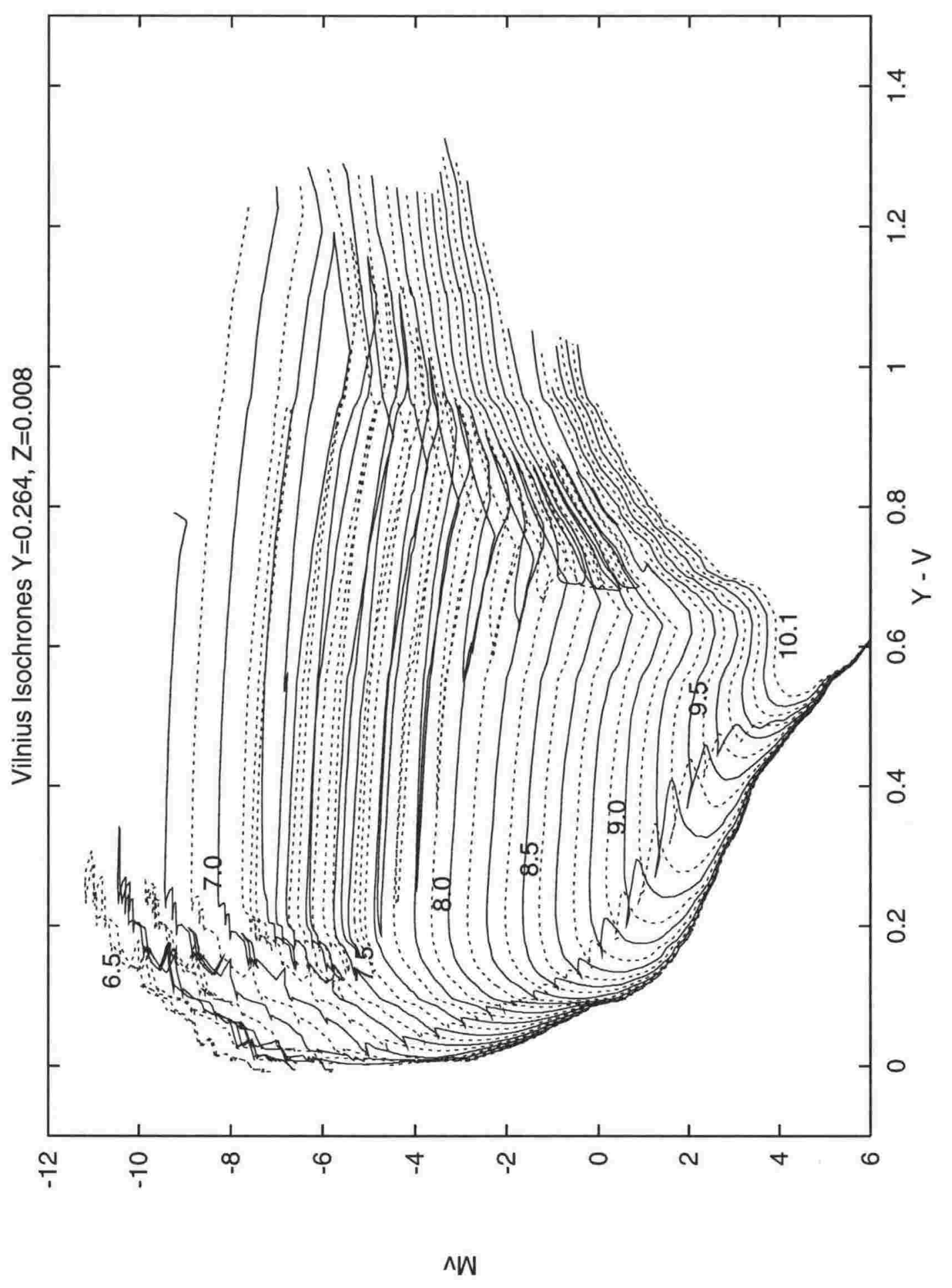

Figure 4.5: Grid of isochrones for $\mathrm{Z}=0.008$, between $\log ($ age $)=6.5$ to 10.1 in 0.1 increments (age in years). 


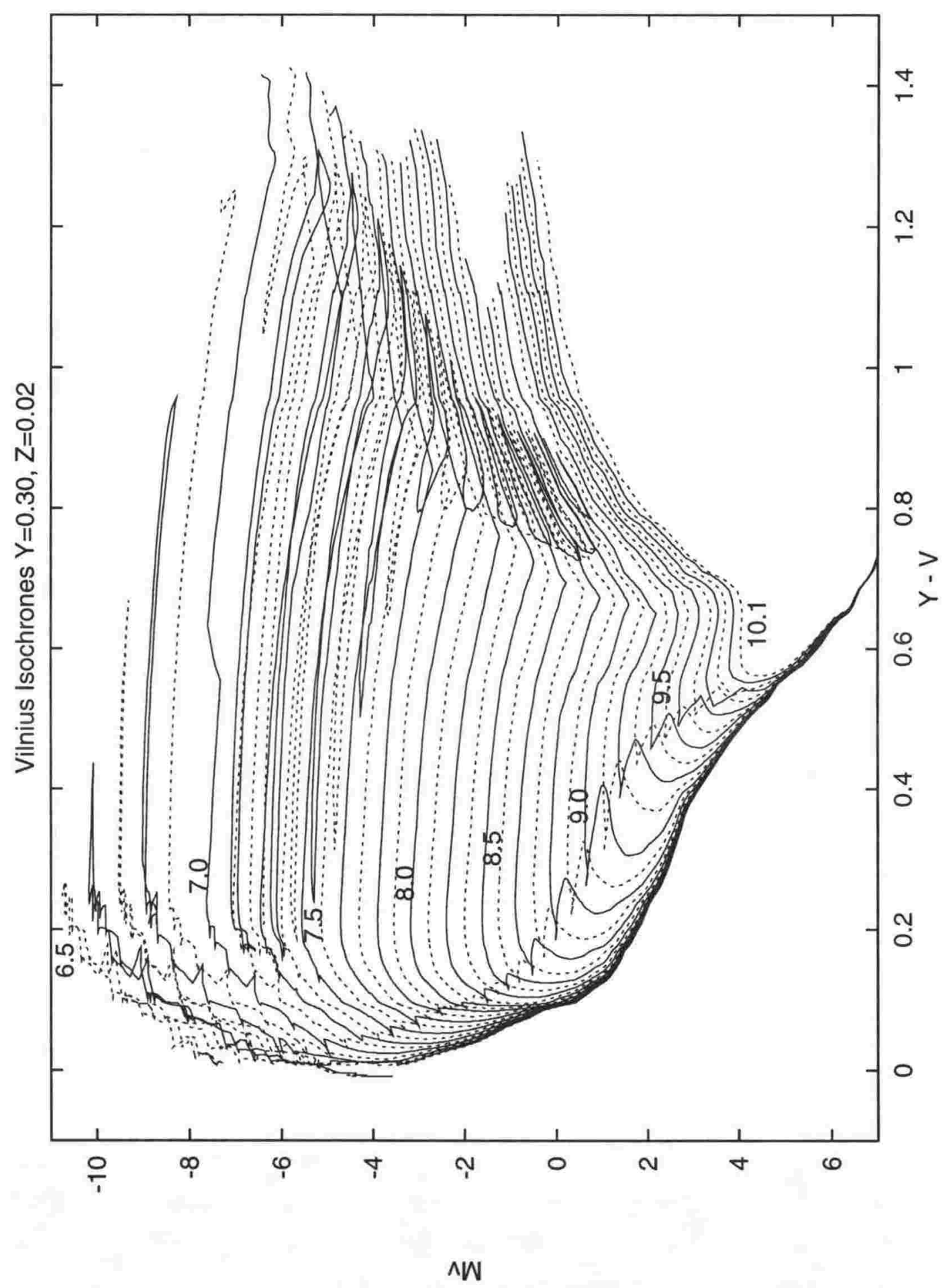

Figure 4.6: Grid of isochrones for $Z=0.020$, between $\log ($ age $)=6.5$ to 10.1 in 0.1 increments (age in years). 
Table 4.1: Review of main characteristics of O Vel.

\begin{tabular}{|llll|}
\hline author & \multicolumn{1}{c|}{$\begin{array}{c}\text { distance } \\
(p c)\end{array}$} & \multicolumn{1}{c|}{$\begin{array}{c}\text { reddening } \\
\left(E_{B-V}\right)\end{array}$} & \multicolumn{1}{c|}{$\begin{array}{c}\text { age } \\
(\text { years })\end{array}$} \\
\hline Hogg & $153 \pm 3(210,120,260)$ & $0.004 \pm 0.003$ & $25-53 \times 10^{6}\left(19 \times 10^{6}\right)$ \\
Lynga & 160 & 0.017 & $40 \times 10^{6}\left(<20 \times 10^{6}\right)$ \\
Buscombe & $210 \pm 10$ & & \\
Graham & $226 \pm 40$ & 0.000 & \\
P\&H & $148 \pm 4$ (uvby $158 \pm 4)$ & $0.00 \pm 0 \cdot 01$ & $30 \times 10^{6}\left(>30 \times 10^{6}\right)$ \\
Eggen (UBV) & 151 & 0.02 & \\
Eggen (uvby) & $166 \pm 22$ & $0.001 \pm 0.007$ & $30 \times 10^{6}$ \\
L\&W & $140 \pm 11$ & $0.1 \pm 0.1$ & $36 \times 10^{6}$ \\
B\&S & 167 & $0.022 \pm 0.011$ & \\
L\&M & $150 \pm 6$ & 0.02 & \\
K\&R & & & $57-80 \times 10^{6}$ \\
M\&C & $154 \pm 6$ & 0.000 & \\
\hline median & 154 & 0.010 & $30 \times 10^{6}$ \\
\hline
\end{tabular}

A summary of the cluster's characteristics found by different authors is given in Table 4.1. Hogg (1960) also lists distance determinations from three other authors (shown in parenthesis) and gives ages from the main sequence turn-off (the nuclear age) and the pre-main-sequence turn-on (the gravitational contraction age, shown in parenthesis). Lynga (1961) fits the nuclear age using two methods; that of Sandage, and von Hoerner (parenthesis). Buscombe (1965) has a note added in proof that the $(0 \cdot 06)$ discrepancy between his distance (modulus) and Lyngå and Hogg appears to be due to the difference between absolute magnitude calibrations of field stars near A0 V and the ZAMS. Graham (1967) assumes zero reddening and used a preliminary $H_{\beta}$-absolute magnitude calibration, which seems to have the same discrepancy found by Buscombe. Perry \& Hill (1969) give distances based separately on their UBV and $u v b y$ photometry, and the nuclear and contraction (parenthesis) ages. Eggen $(1972,1983)$ measured the cluster first using the UBV system and then the $u v b y$ system - here $E_{b-y}$ has been converted to $E_{B-V}$. Balona \& Shobbrook (1984) find the cluster distance assuming the Pleiades is 126 pc. Levato \& Malaroda (1984) recalculated the cluster's mean reddening using the UBV intrinsic colours based on their spectral classifications and Perry \& Hill's (reddened) colours. Koester \& Reimers (1985) fit isochrones to a colour-magnitude diagram from previous authors observations. Maitzen \& Catalano (1986) conclude the difference in reddening between Perry \& Hill and Lyngå \& Wramdemark (1984) is due to the zero-point difference (in $b-y$ ), and the reddening is actually zero. The final row of the table lists the median values ${ }^{3}$ - note that the actual $E_{B-V}$ may be smaller than the median as negative $E_{B-V}$ 's are not allowed.

Different authors have used a variety of other naming systems for the stars in the cluster, which have been summarized in Table 4.2. The RA and Dec (in hours, minutes, seconds and

\footnotetext{
${ }^{3}$ The median was used here to avoid the difficulties in calculating a mean with data (age) involving inequalities.
} 
degrees, minutes respectively) are taken from SIMBAD and are to the Epoch 2000. The third column gives the numbers used by Hogg (1960) while the fourth column is from volume 19 of the Cape Photographic Catalogue (CPC). The next column (23910000+CDS) are those used by Mermilliod in the SIMBAD database. The ninth column $(236000+\mathrm{SAO})$ is from the Smithsonian Astrophysical Observatory (SAO) catalog. The remaining catalogues are sufficiently well known not to need further explanation. Star CPD -521579 has a question mark as it appears to be a (much fainter) visual companion to $\mathrm{O}$ Vel 3 , while a CPD number for $\mathrm{O}$ Vel 3 itself could not be found.

A star chart of $\mathrm{O}$ Vel suitable for viewing through a finder scope was prepared using Lyngå's numbers and is shown in Figure 4.7. The occasional concentric white ring inside a black circle (for example see $\mathrm{O}$ Vel 13) is due to slight discrepancies in the visual magnitude as recorded in the two catalogues (the Yale HR and the SAO) used to prepare the chart.

Table 4.3 gives all the spectral classifications found in the literature for $\mathrm{O}$ Vel. These classifications are derived from spectroscopic measurements unless noted otherwise below. The author(s) are listed at the top of each column; Lynga (1961) - the first column is from the HD catalog, the second from Lyngå's UBV results using the $Q$ method. Feins. = Feinstein (1961), while Busc. = Buscombe (1965), P\&H = Perry \& Hill (1969), and P\&B = Perry \& Bond (1969). Craw. = Crawford, Barnes \& Golson (1971), with the spectral classes taken from Hoffleit (1964), Lesh (1969) or Hiltner et al. (1969). Eggen = Eggen (1983), with the spectral classes taken from Houk (1978). L\&M = Levato \& Malaroda (1984), and finally Renson (1988). Most of the stars appear to be on the main sequence, the exceptions being O Vel 1 (B3 III-IV), O Vel 2 (B3 IV-V), O Vel 3 (B5 IV-V), and possibly O Vel 26 (K0-3 III-V), O Vel 27 (F0-2 IV-V), and O Vel 29 (F2 IV-V). The spectral classes generally show good agreement between different authors, apart from the metallic-line or peculiar stars $(5,10,15,20,21,23$, and 25). Maitzen \& Catalano (1986) investigated O Vel for chemically peculiar stars, and confirmed that stars 5 and 10 are peculiar while the status of stars 20 and 38 are unclear. They also note that it has become well known the luminosity classification for hot chemically peculiar stars is often wrong (so $\mathrm{O}$ Vel 5 and 10 may actually be on the main sequence).

Table 4.4 lists the proper motions found in the literature. The RA proper motion has been converted into arc-seconds, via the conversion factor ' $15 \cos (D e c)$ ', from the usual units for RA (time, in seconds). As the values often show little agreement, even allowing for the probable errors of $0.005-0.010^{\prime \prime} / y r$, the median was calculated in the final columns. Hogg's (1960) data was copied from the Cape Annals Vol 19, so have been supplemented here with further values from the Cape Annals for stars not observed by Hogg. The data given by Lynga (1961) is the weighted mean of Lyngå's values and the Cape Annals Vol 19. As it was not possible to recover Lyngå's own values, it was assumed that where Lyngå's values are identical to the Cape's values 
Table 4.2: Synonyms for O Vel's stars.

\begin{tabular}{|c|c|c|c|c|c|c|c|c|c|}
\hline Lynga & RA & Dec & Hogg & CPC & CDS & HD & HR & $\mathrm{SAO}$ & CPD \\
\hline 01 & 084018 & $-5255 \cdot 3$ & 00 & 3070 & 20 & 74195 & 3447 & 164 & $-52 \quad 1583$ \\
\hline 02 & 084225 & -5306.9 & 03 & 3092 & 34 & 74560 & 3467 & 205 & -521607 \\
\hline 03 & 083958 & $-5303 \cdot 3$ & 02 & 3065 & 16 & 74146 & 3442 & 157 & $-521579 ?$ \\
\hline 04 & $\begin{array}{lll}08 & 39 & 22\end{array}$ & $-5326 \cdot 2$ & 12 & 3062 & 13 & 74071 & 3440 & 151 & -531796 \\
\hline 05 & 084219 & $-5306 \cdot 0$ & 04 & 3090 & 31 & 74535 & 3466 & 202 & -521605 \\
\hline 06 & 084018 & $-5300 \cdot 9$ & 01 & 3071 & 21 & 74196 & 3448 & 165 & -521584 \\
\hline 07 & 083845 & $-5305 \cdot 4$ & 13 & 3055 & 08 & 73952 & 3435 & 142 & -521565 \\
\hline 08 & 083825 & $-5343 \cdot 5$ & & 3054 & 06 & 73904 & & 140 & -531789 \\
\hline 09 & 083510 & $-5412 \cdot 4$ & & 3034 & 01 & 73287 & & 100 & -531760 \\
\hline 10 & 084002 & $-5316 \cdot 3$ & 11 & 3067 & 18 & 74169 & & 160 & -521581 \\
\hline 11 & 084050 & $-5248 \cdot 2$ & 06 & 3075 & 23 & 74275 & & 177 & -521587 \\
\hline 12 & 084003 & $-5351 \cdot 1$ & & 3069 & 19 & 74182 & & 162 & -531804 \\
\hline 13 & $0842 \quad 13$ & $-5258 \cdot 2$ & 08 & 3086 & 29 & 74516 & & 198 & -521601 \\
\hline 14 & 084450 & $\begin{array}{lll}-53 & 05 \cdot 1\end{array}$ & & 3105 & 41 & 74955 & & 233 & -521634 \\
\hline 15 & 084149 & $\begin{array}{lll}-53 & 04.2\end{array}$ & 09 & 3081 & 27 & 74438 & & 190 & -521598 \\
\hline 16 & 084305 & $-5304 \cdot 6$ & 15 & 3099 & 39 & 74678 & & 217 & -521622 \\
\hline 17 & $0843 \quad 36$ & -5331.9 & & 3102 & 40 & 74762 & & 223 & -531842 \\
\hline 18 & 084208 & $-5245 \cdot 2$ & 07 & 3084 & 28 & 74497 & & 196 & -521599 \\
\hline 19 & 083711 & $-5315 \cdot 6$ & 33 & 3045 & 03 & 73681 & & 125 & -521552 \\
\hline 20 & 084224 & -5320.7 & 32 & 3088 & 32 & 74536 & & 201 & -521603 \\
\hline 21 & 084251 & -5344.8 & & 3098 & 38 & 74665 & & 214 & -531834 \\
\hline 22 & $\begin{array}{lll}08 & 36 & 24\end{array}$ & $\begin{array}{ll}-53 & 02 \cdot 2\end{array}$ & & 3041 & 02 & 73503 & & 118 & -521545 \\
\hline 23 & 084000 & $-5242 \cdot 6$ & & 3068 & 15 & 74145 & & 161 & -521580 \\
\hline 24 & 084208 & -5309.6 & 10 & 3085 & 30 & 74517 & & 197 & -521600 \\
\hline 25 & 084211 & -5409.5 & & 3089 & 33 & 74537 & & & -531825 \\
\hline 26 & 084019 & $-5326 \cdot 2$ & & 3072 & 22 & 74212 & & 168 & -531808 \\
\hline 27 & 083738 & -5411.0 & & 3049 & 05 & 73778 & & 131 & -531784 \\
\hline 28 & 083730 & $-5239 \cdot 1$ & & 3047 & 04 & 73722 & & 129 & -521554 \\
\hline 29 & 083943 & $-5257 \cdot 7$ & 25 & 3064 & 14 & 74117 & & 155 & -521578 \\
\hline 30 & 084130 & $-5259 \cdot 7$ & 31 & & 26 & 74387 & & & -521597 \\
\hline 31 & 083918 & $-53 \quad 17 \cdot 1$ & & 3061 & 12 & 74056 & & 150 & -521574 \\
\hline 32 & 084218 & $-5354 \cdot 2$ & & 3091 & 35 & 74561 & & 203 & -531826 \\
\hline 33 & $\begin{array}{lll}08 & 42 & 12\end{array}$ & $-5306 \cdot 1$ & 05 & 3087 & & 74535B & & & $\begin{array}{lll}-52 & 1602\end{array}$ \\
\hline 34 & 083900 & -5301.5 & 27 & & 72 & & & & -521568 \\
\hline 35 & 083922 & -5301.5 & 26 & & 71 & & & & -521575 \\
\hline 36 & 084109 & -5253.0 & & & 24 & 74340 & & & -521592 \\
\hline 37 & 083939 & $-5310 \cdot 4$ & & & & & & & \\
\hline 38 & 084125 & $-53 \quad 17.9$ & & & & 74373 & & & \\
\hline 39 & 083932 & $-53 \quad 19 \cdot 3$ & & & & & & & \\
\hline 40 & 083856 & $-5258 \cdot 2$ & 28 & & 73 & & & & -521567 \\
\hline 41 & 0840.59 & -5230.9 & & & & & & & \\
\hline 42 & 083932 & $-53 \quad 19 \cdot 3$ & & & & & & & \\
\hline 43 & 083918 & $-53 \quad 17 \cdot 1$ & & & & & & & \\
\hline
\end{tabular}


$z \ddot{n}$

$\ddot{\ddot{m}}$

三乙

三

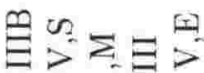

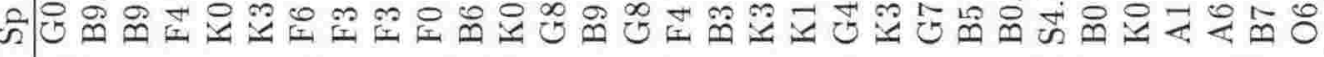

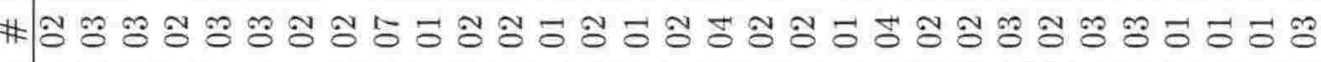

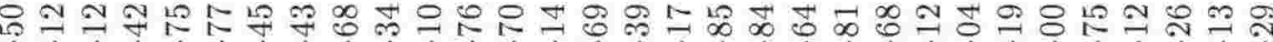

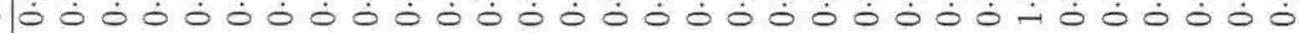

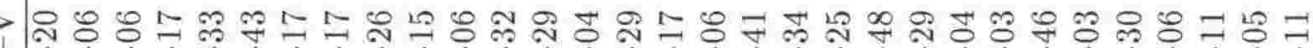

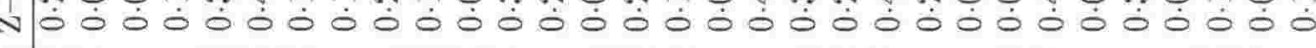

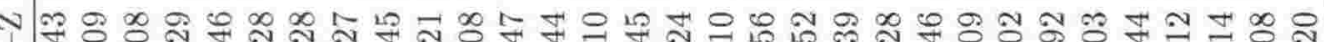

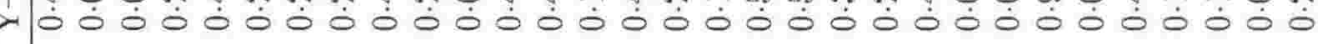

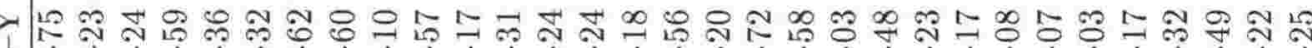

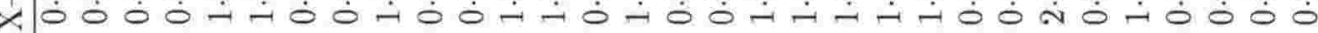

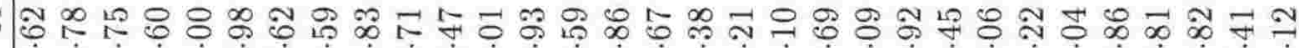

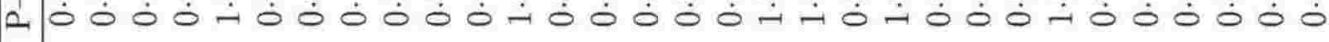

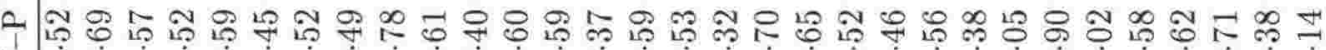

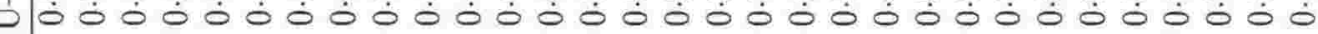

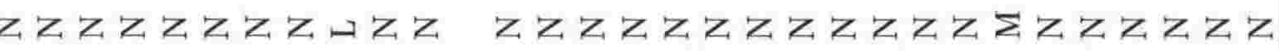
ஜㅇㅇㅇ L F F F

m+

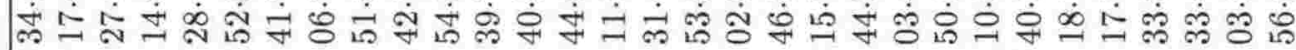

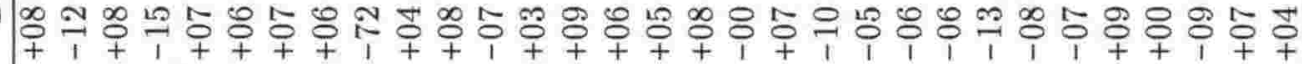

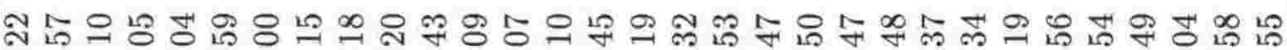

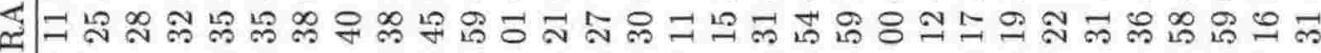

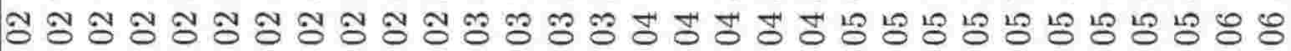
๓ \# 코웅

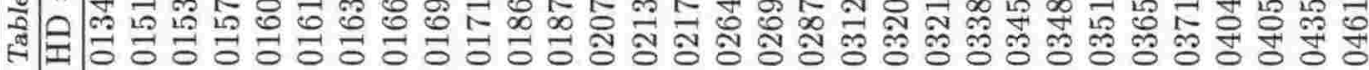




\begin{tabular}{|c|c|}
\hline$\Leftrightarrow$ & 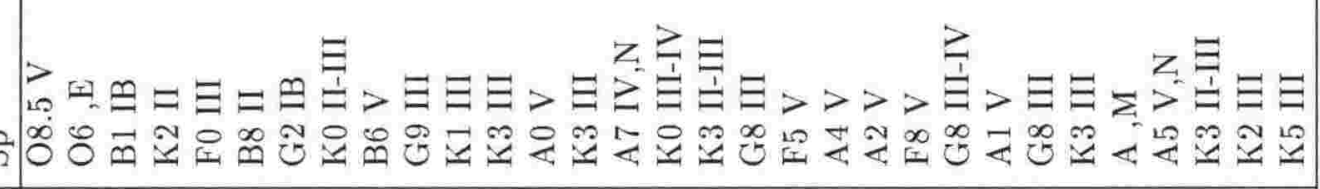 \\
\hline$\neq$ & \# 농 응 \\
\hline$v$ & 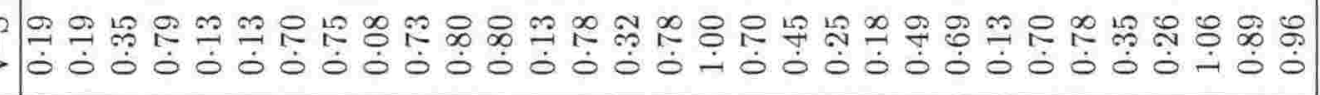 \\
\hline & 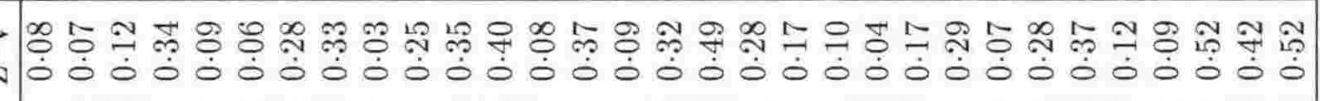 \\
\hline & 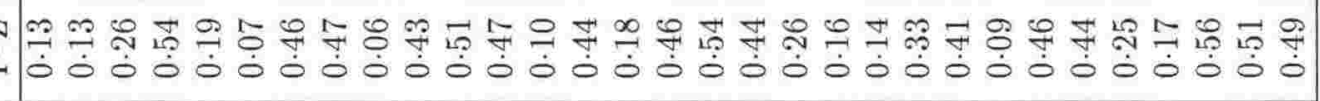 \\
\hline & 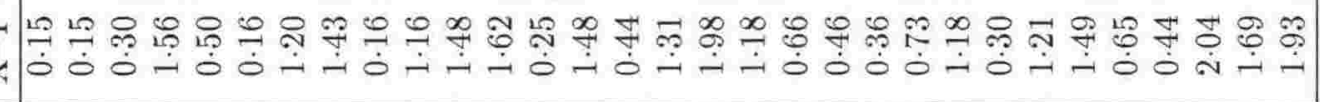 \\
\hline & 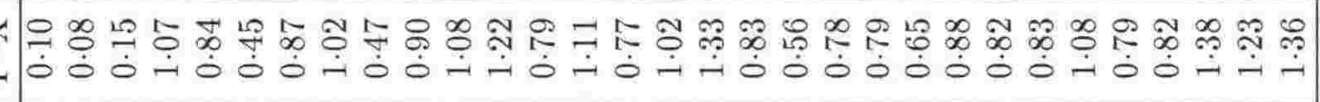 \\
\hline & 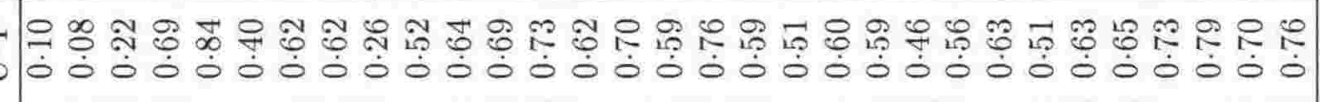 \\
\hline 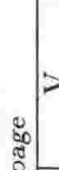 & 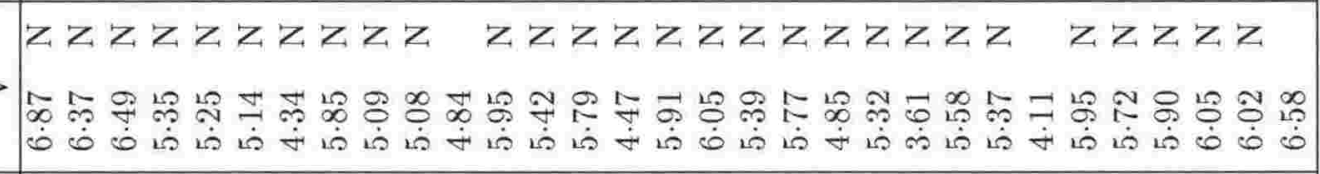 \\
\hline 至 & 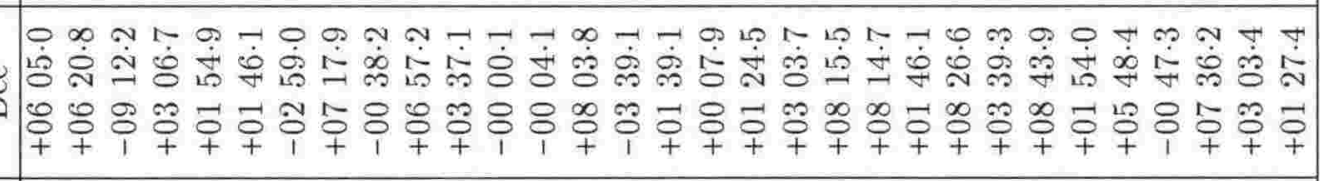 \\
\hline & 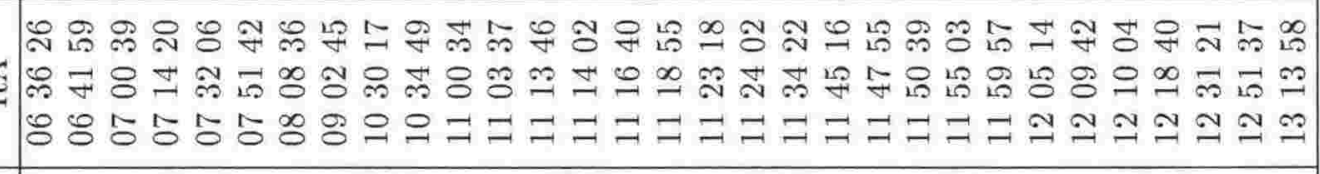 \\
\hline & 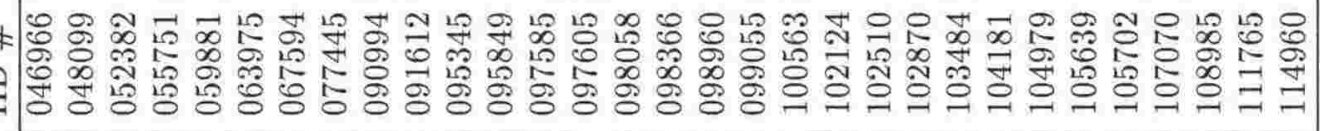 \\
\hline
\end{tabular}




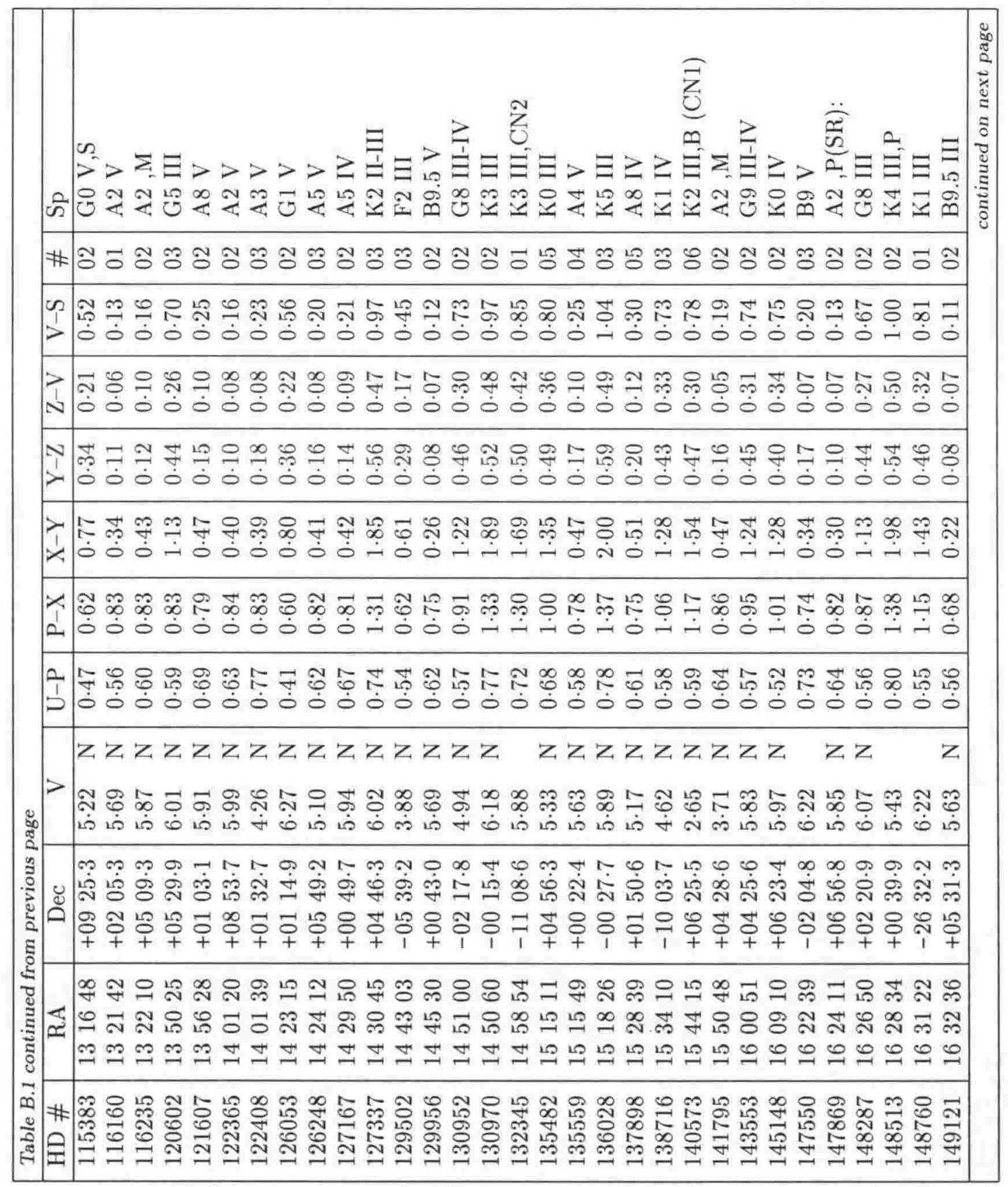




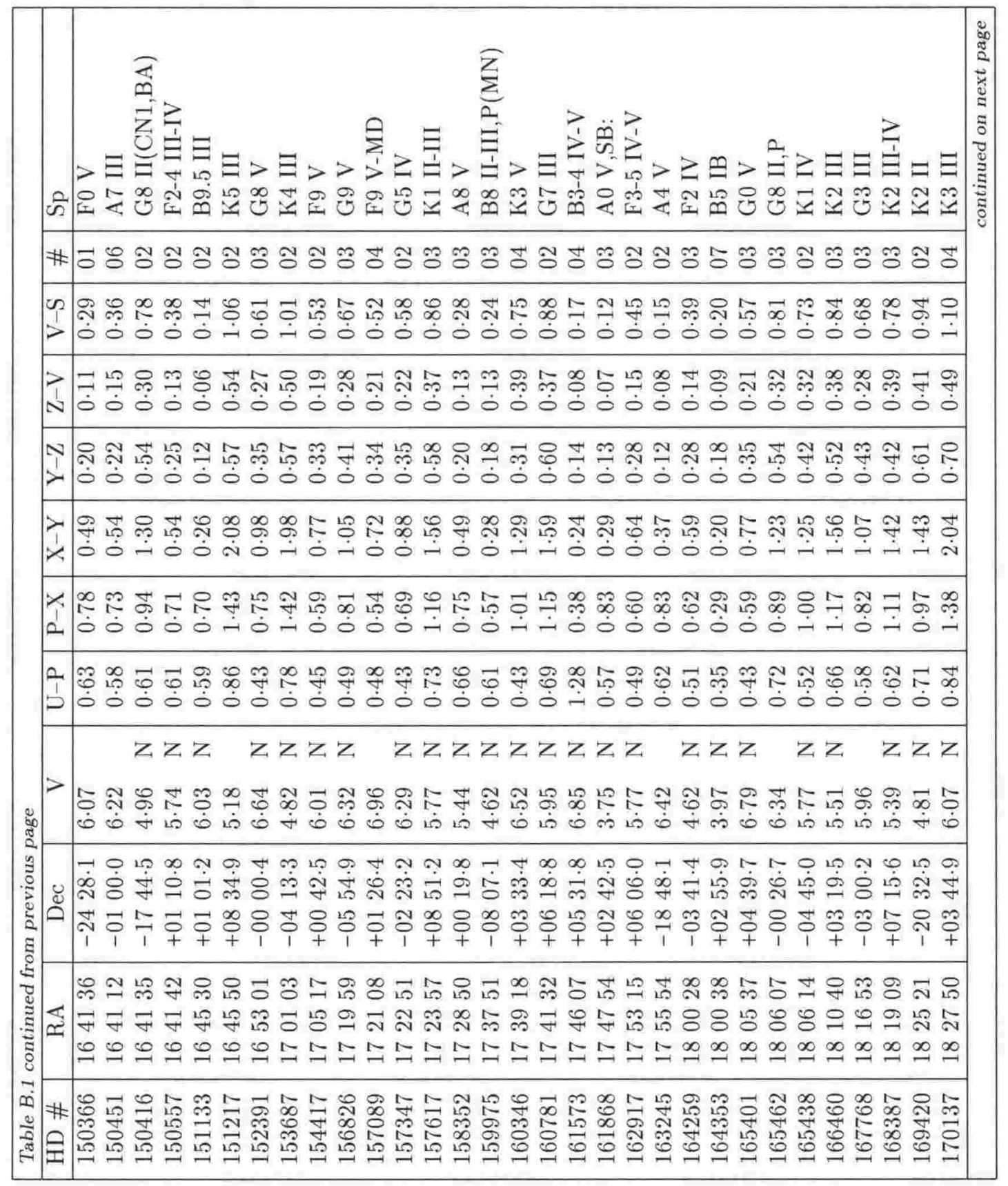




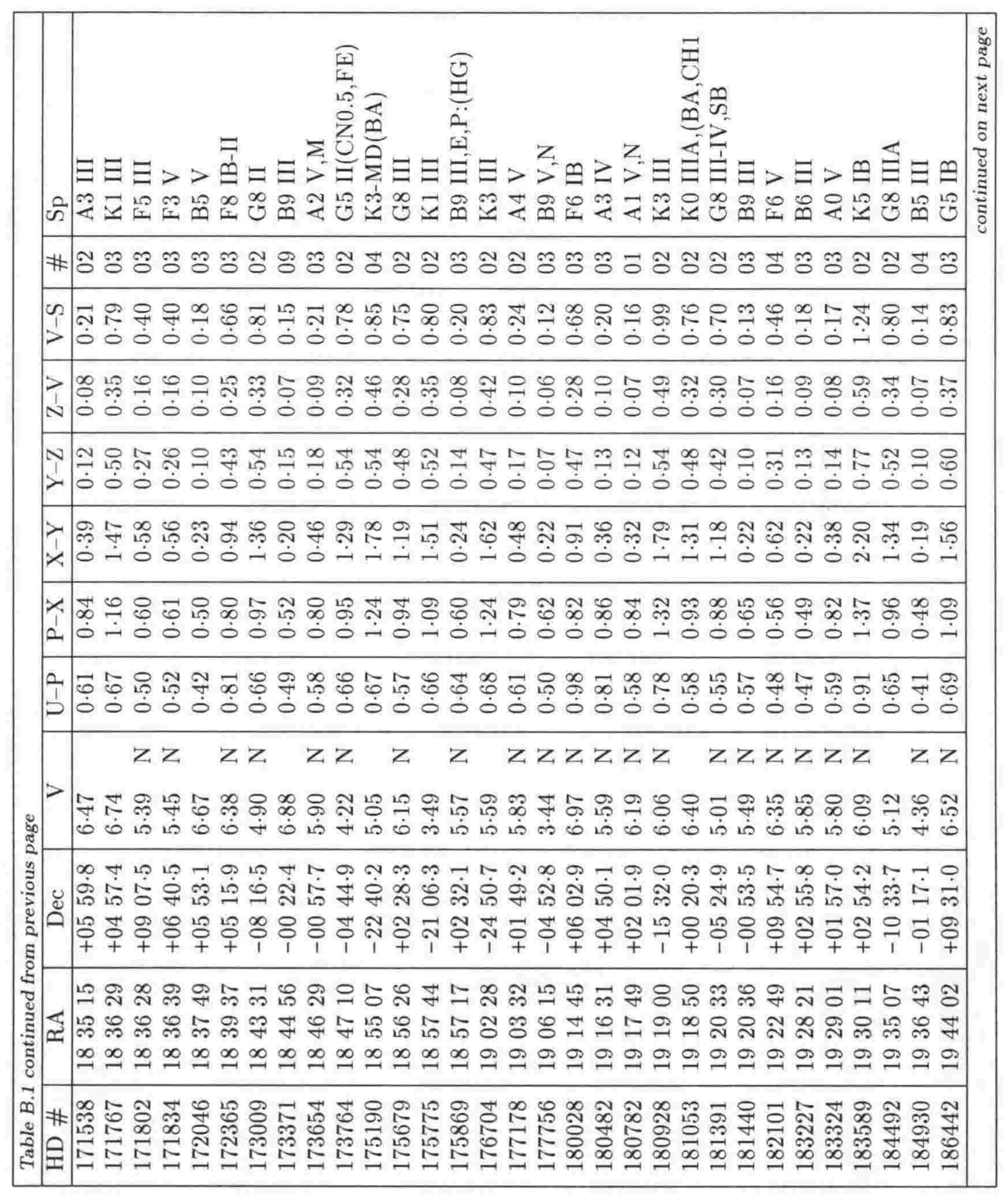




\begin{tabular}{|c|c|}
\hline & 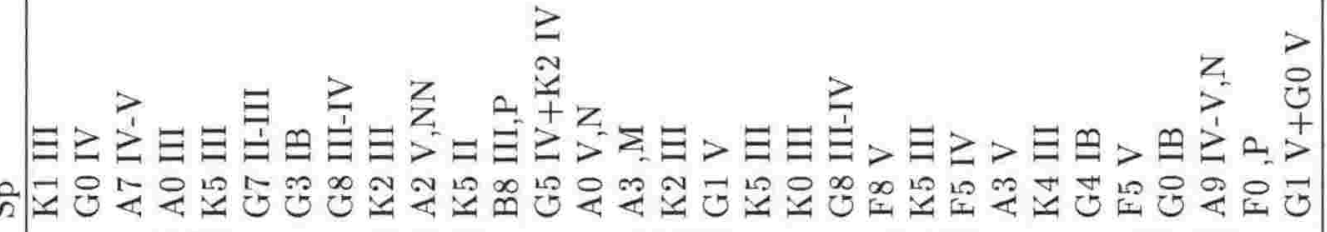 \\
\hline & 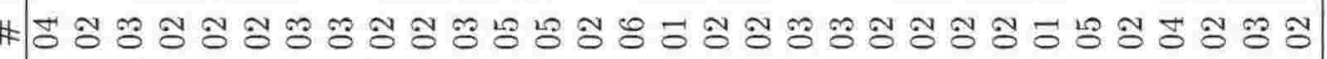 \\
\hline & 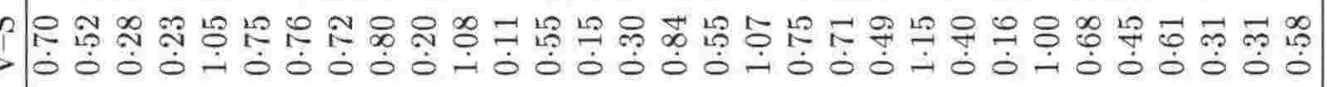 \\
\hline & 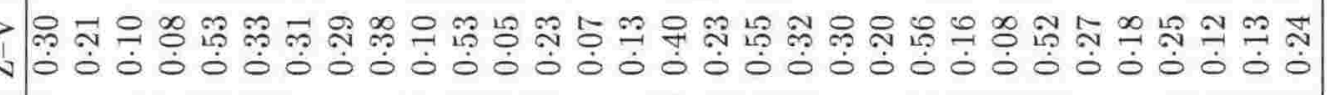 \\
\hline & 삼 \\
\hline & 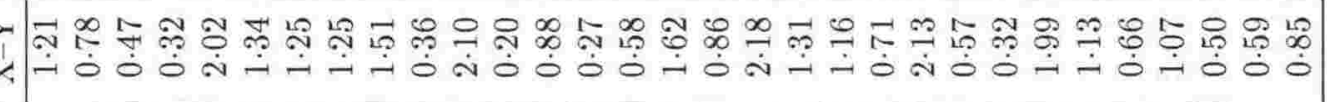 \\
\hline & L \\
\hline & 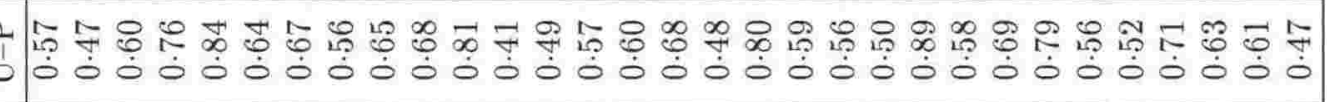 \\
\hline$\because$ & 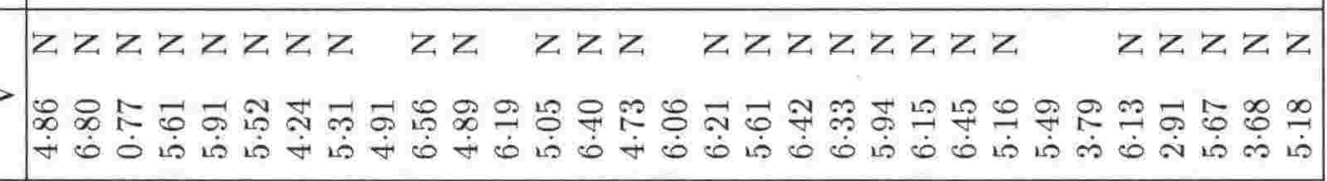 \\
\hline 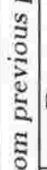 & 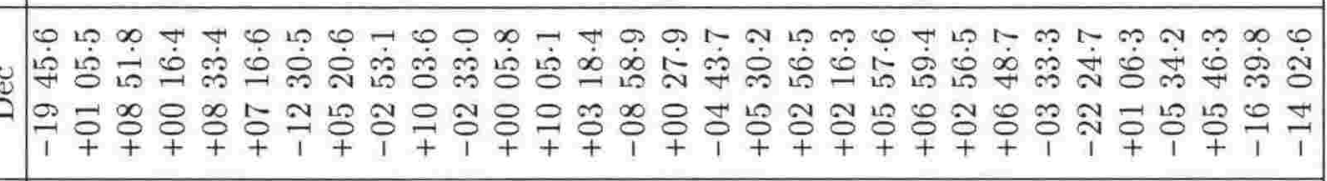 \\
\hline 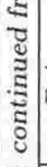 & 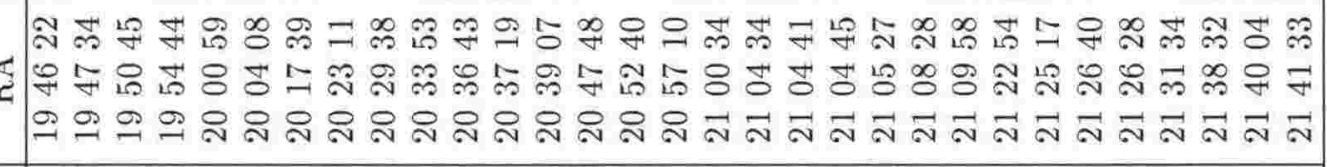 \\
\hline : & 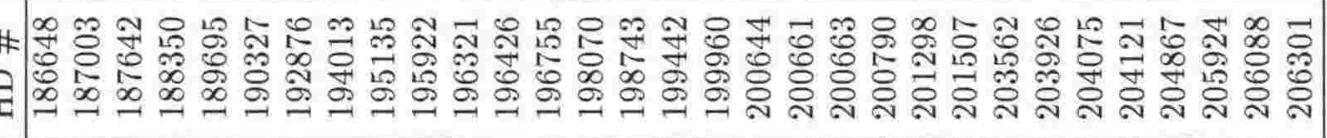 \\
\hline
\end{tabular}




\begin{tabular}{|c|c|}
\hline की & 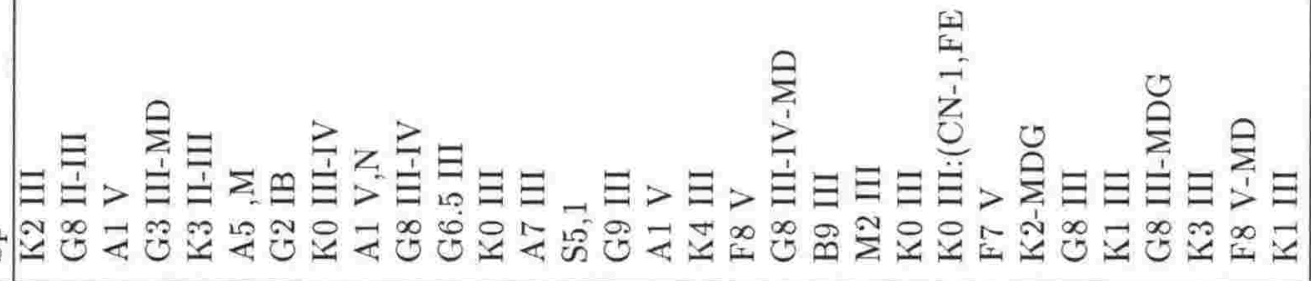 \\
\hline \# & 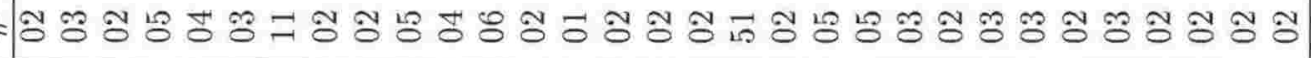 \\
\hline & 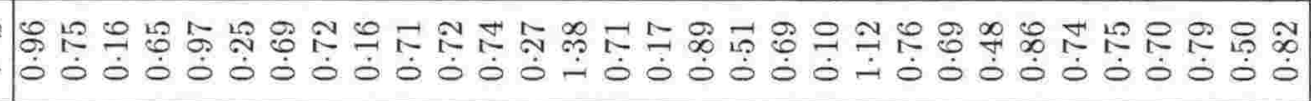 \\
\hline 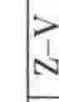 & 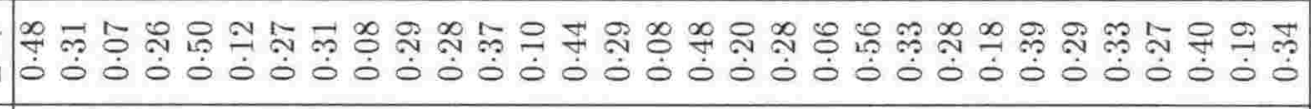 \\
\hline & 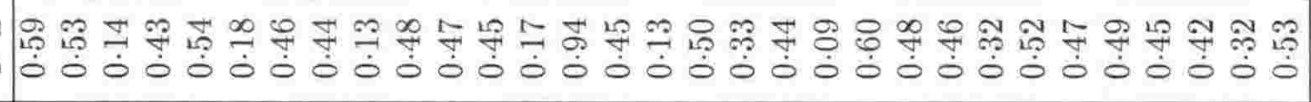 \\
\hline & 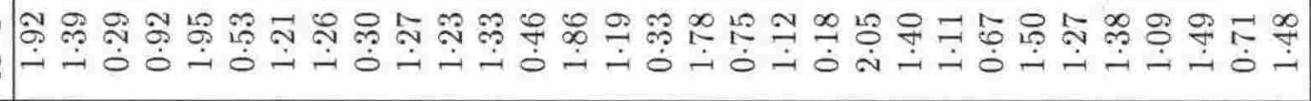 \\
\hline & 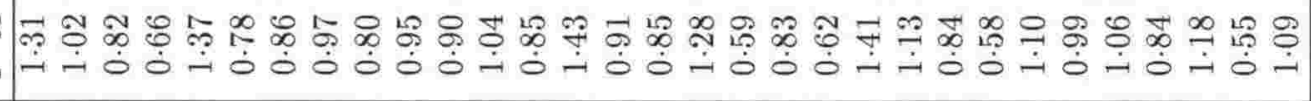 \\
\hline F & 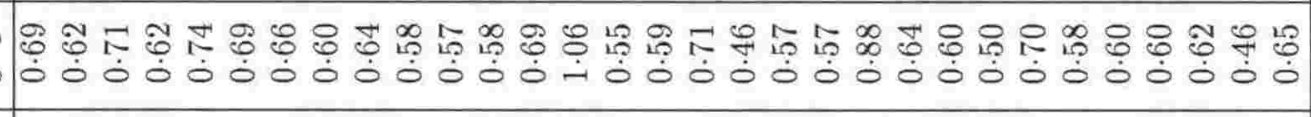 \\
\hline 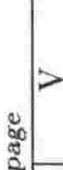 & 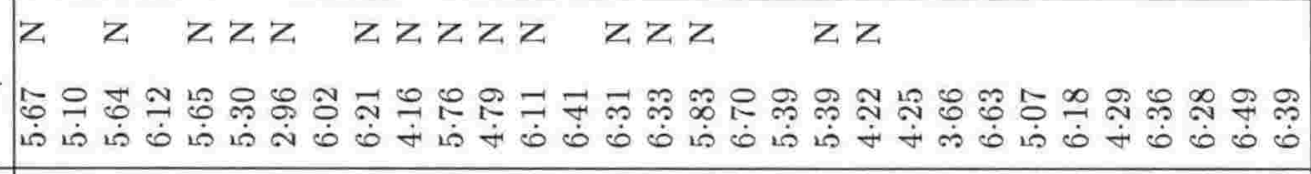 \\
\hline 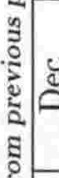 & 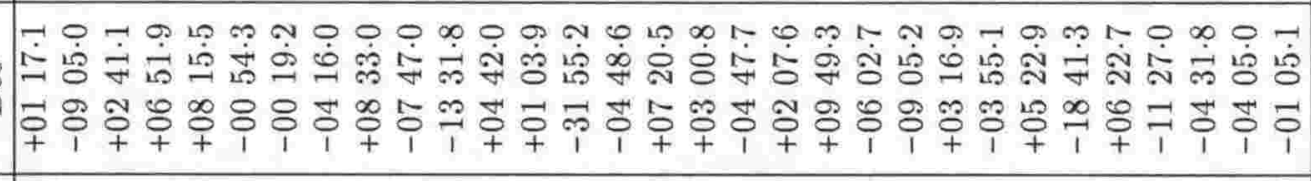 \\
\hline (ֶ) & 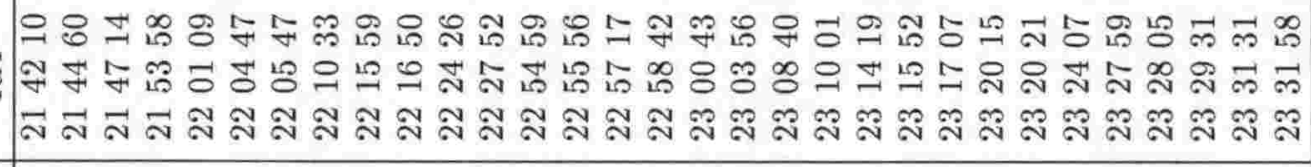 \\
\hline & 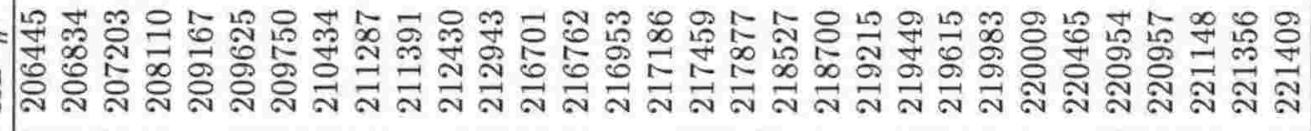 \\
\hline
\end{tabular}


$\sum_{1} \sum_{i=1}^{\theta}$

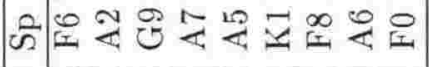

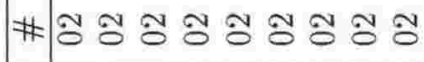

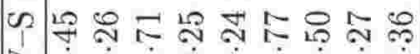

$>000000000$

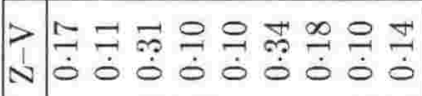

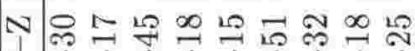

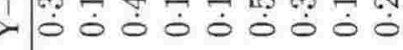

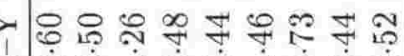

ব人் $\dot{0} \dot{0} \dot{0} \dot{0} 0 \dot{0}$

4

ح 0 00 $00 \dot{0} 00$

雨年

乙 乙 乙 乙

|

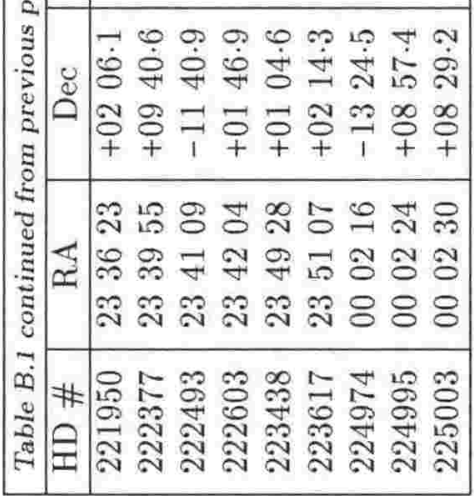


B-11

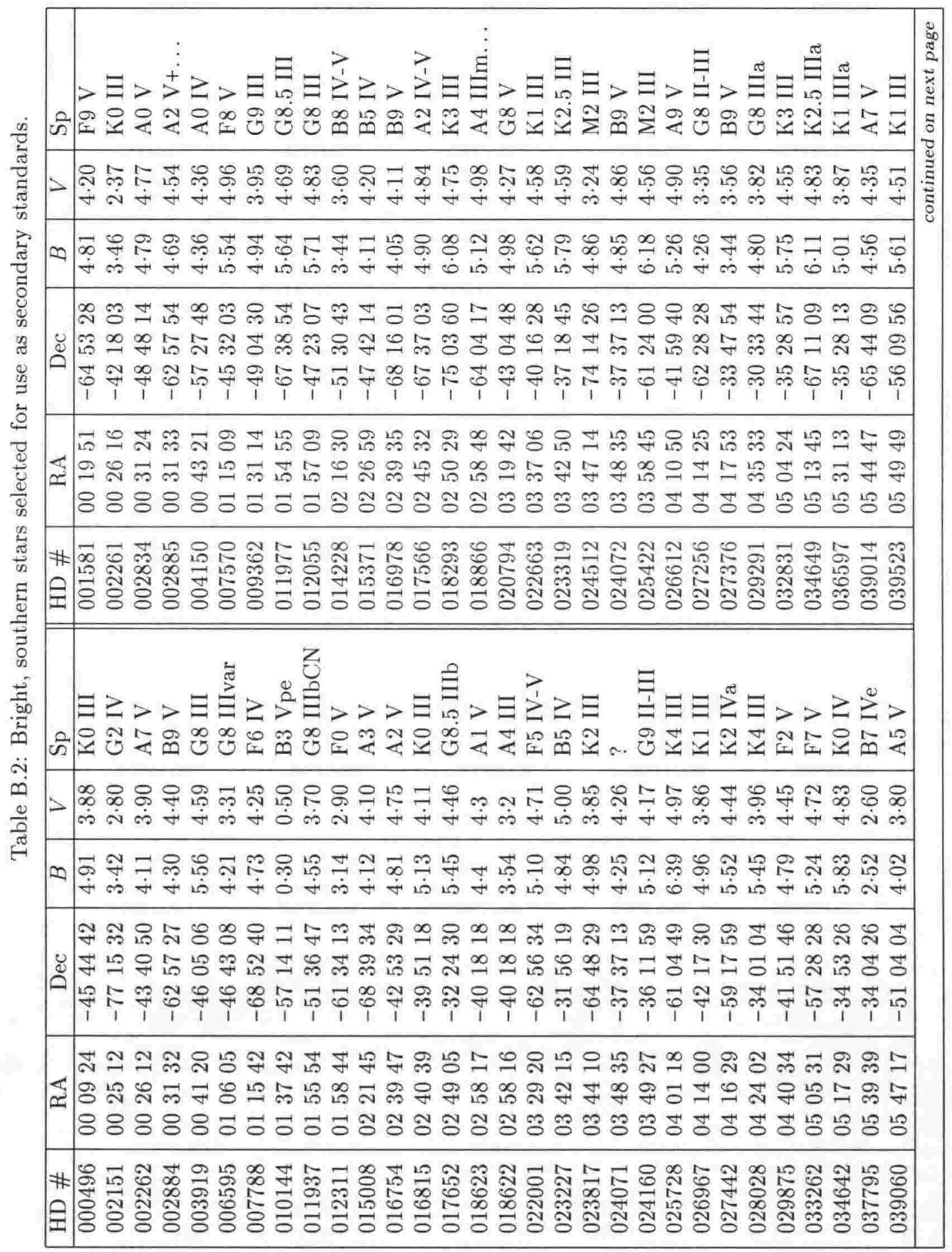




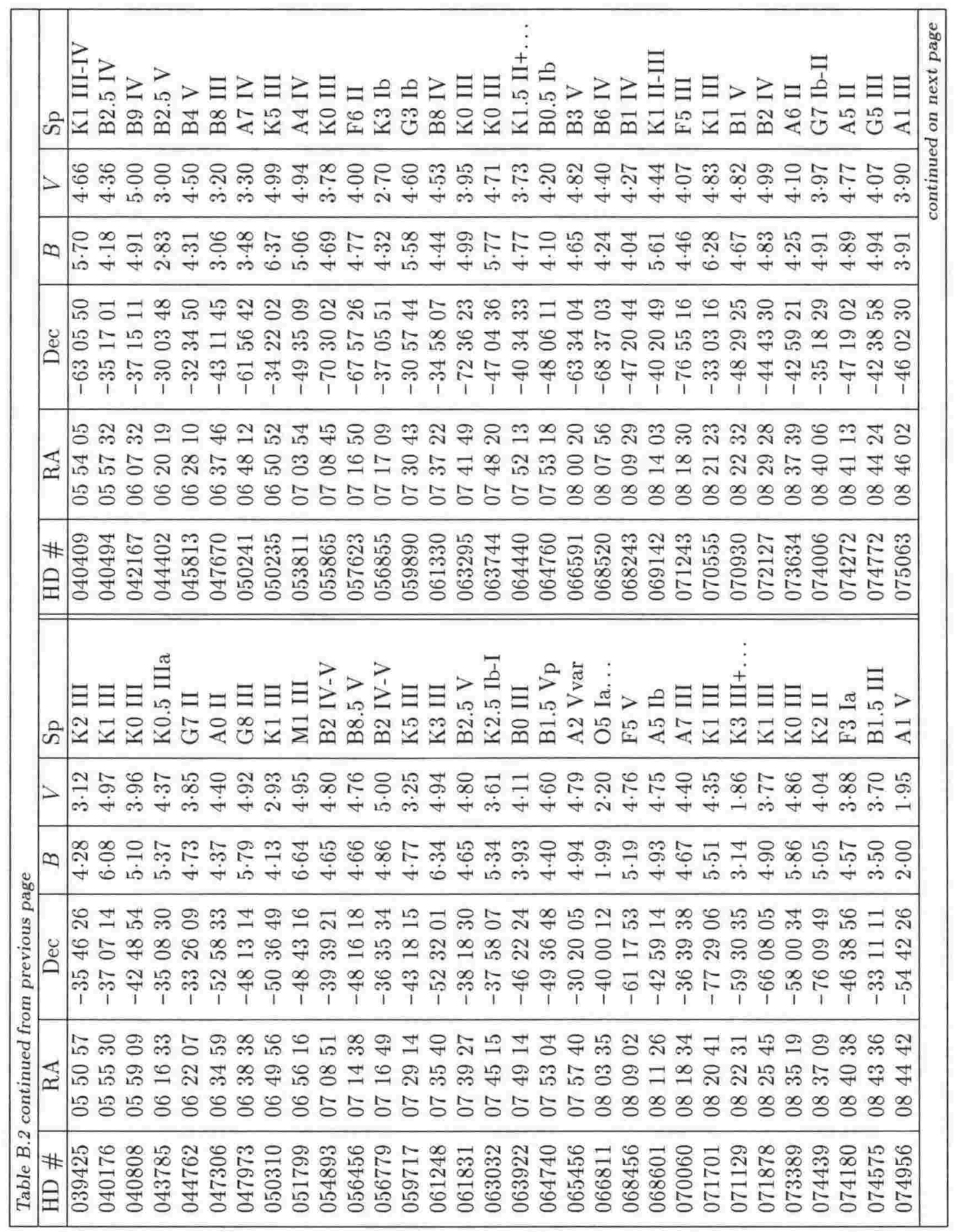




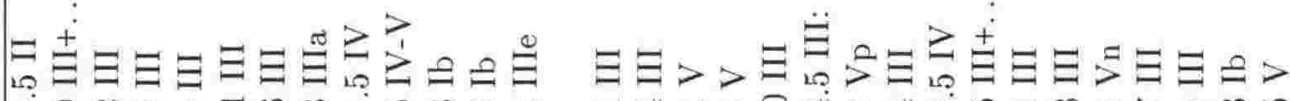

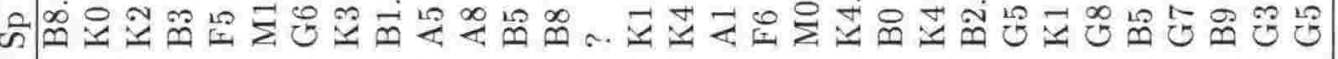

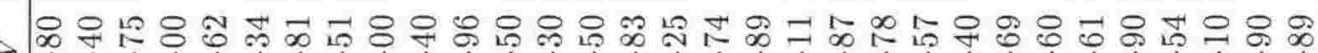

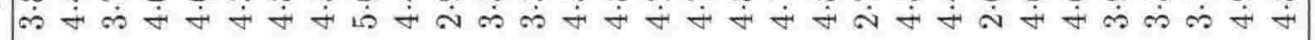

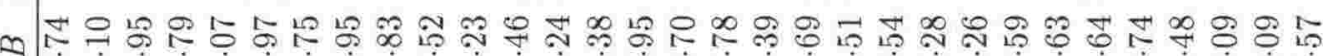
的

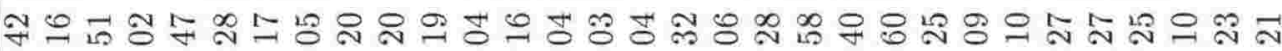

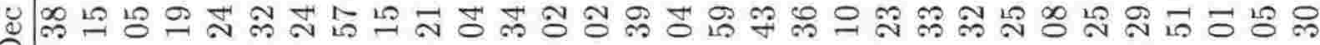

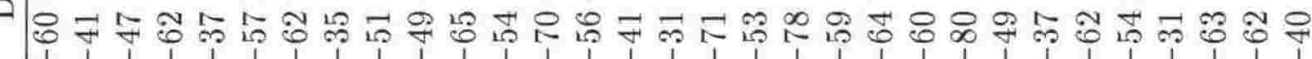

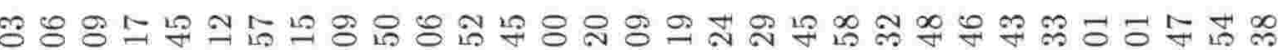
这绍8

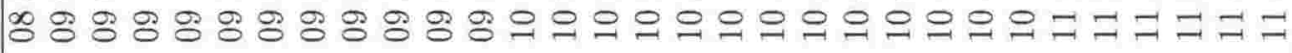

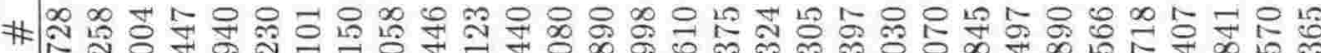

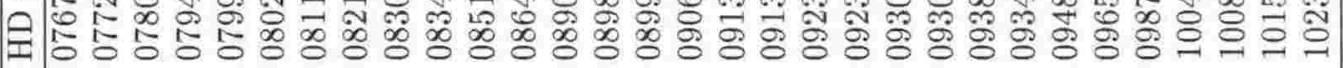

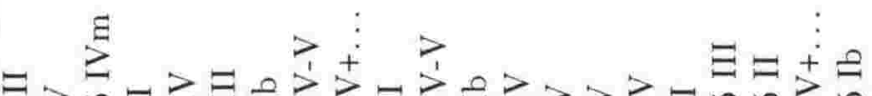

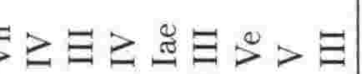

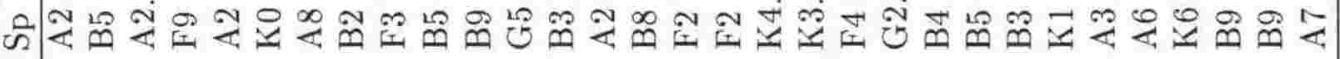

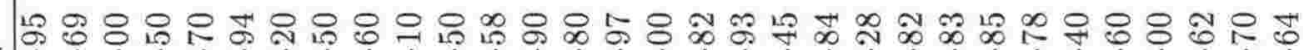

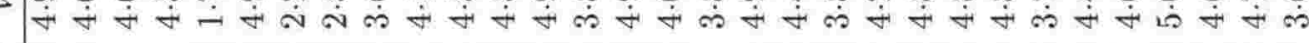

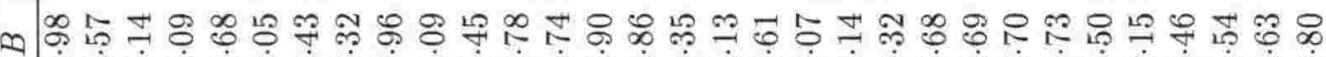

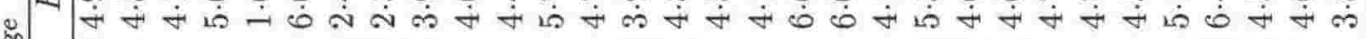

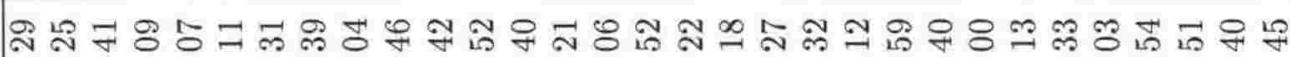

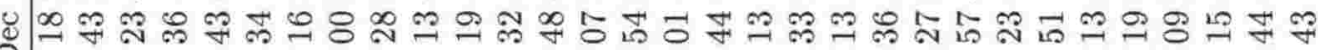

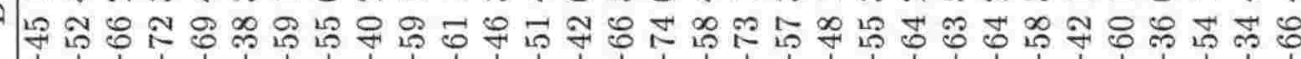

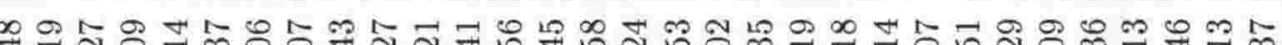
A ת ת m

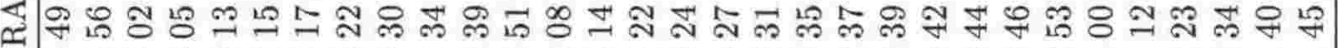

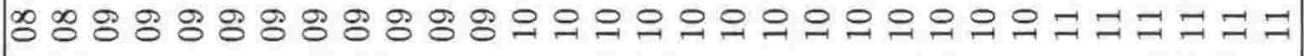
๓

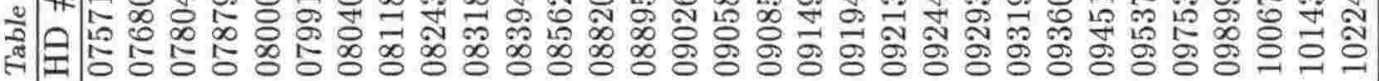




\begin{tabular}{|c|c|c|}
\hline & 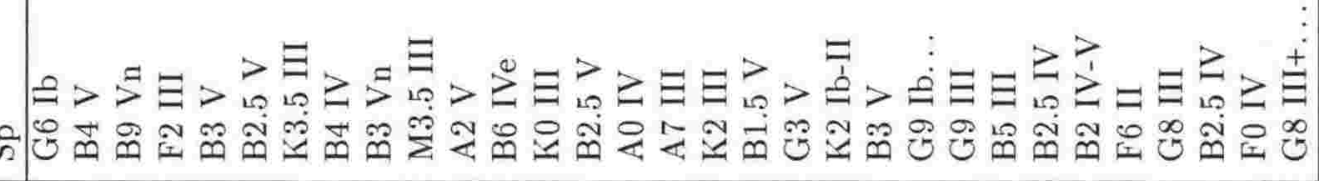 & 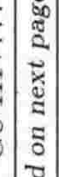 \\
\hline & 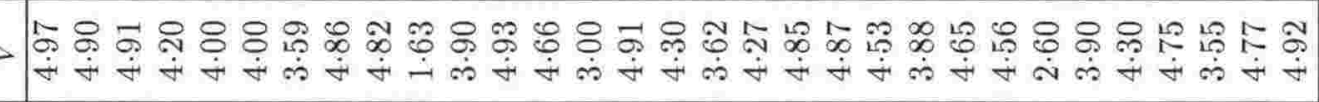 & \\
\hline & 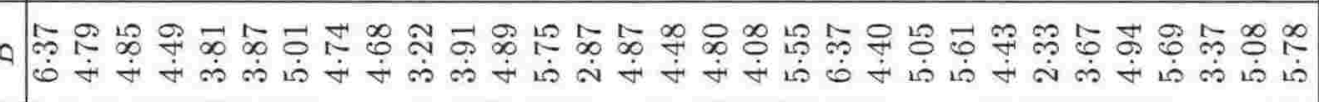 & \\
\hline & 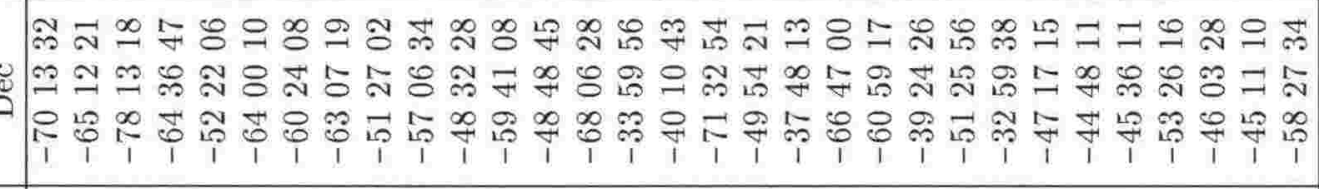 & \\
\hline & 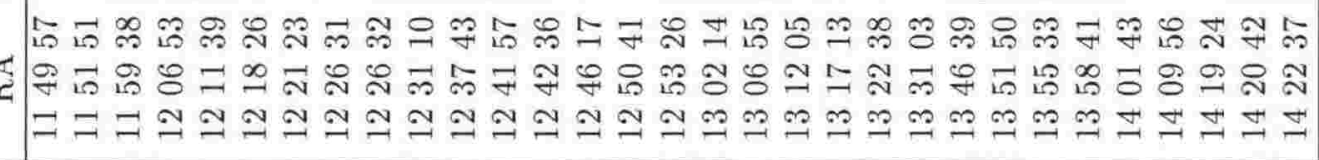 & \\
\hline & 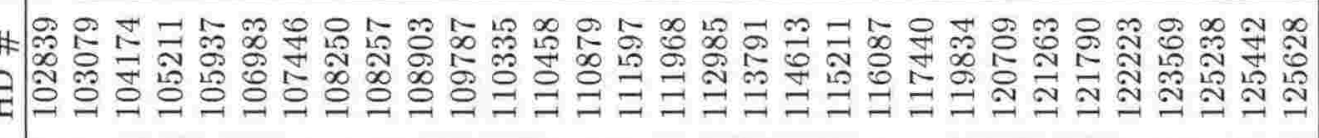 & \\
\hline & 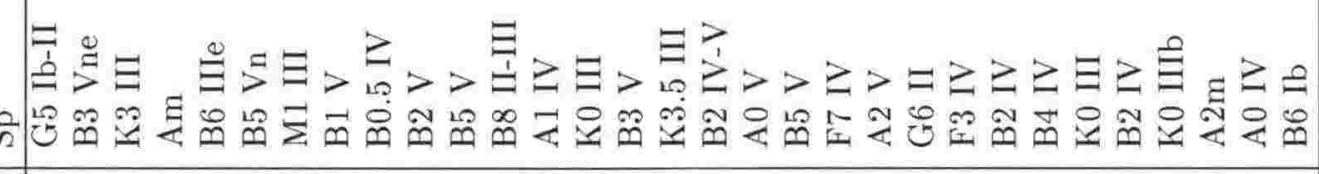 & \\
\hline & 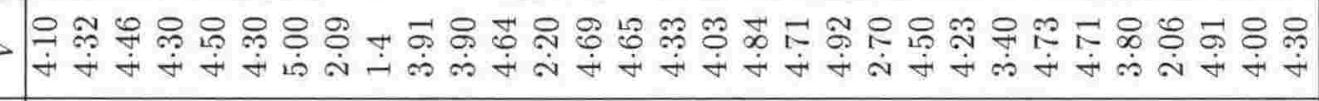 & \\
\hline & b & \\
\hline 至 & 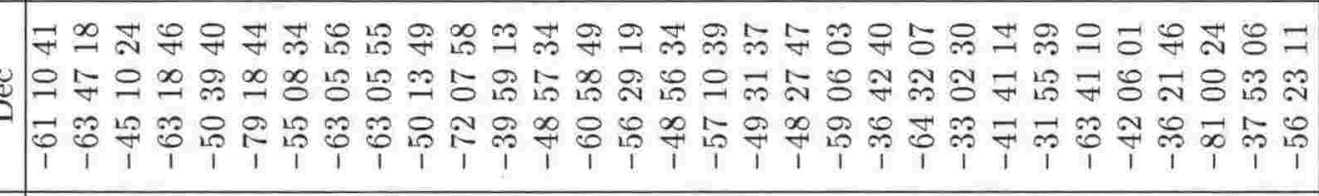 & \\
\hline . & 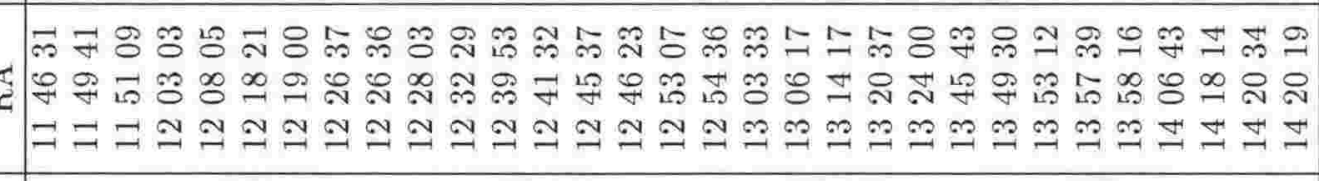 & \\
\hline & 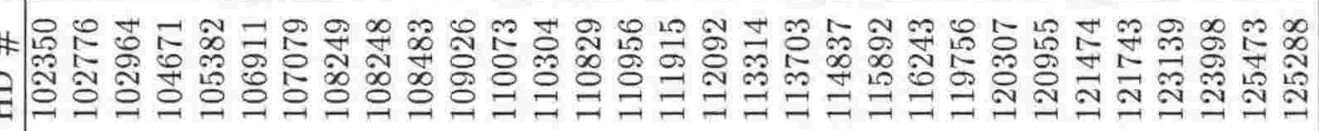 & \\
\hline
\end{tabular}




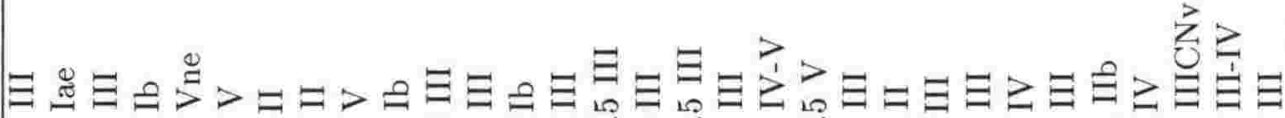

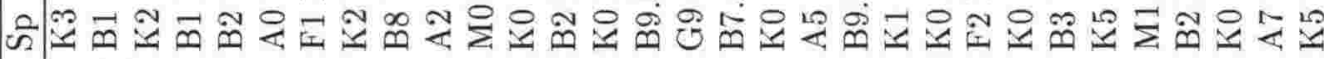

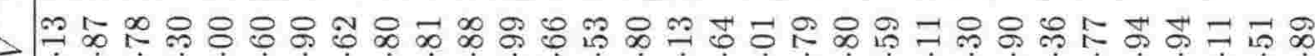

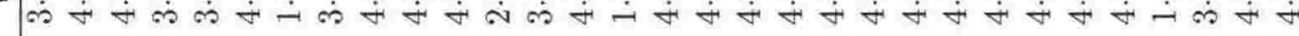

๓ $\dot{\gamma}$ ம

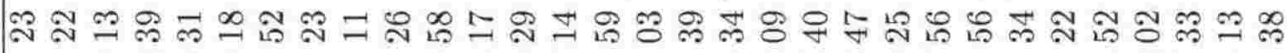

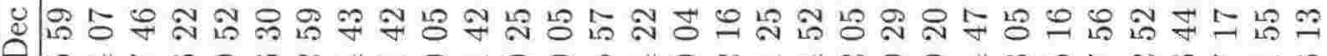

ผ

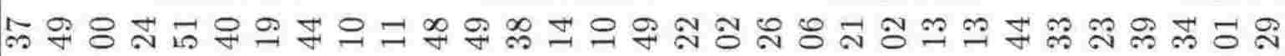

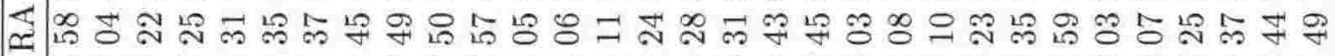

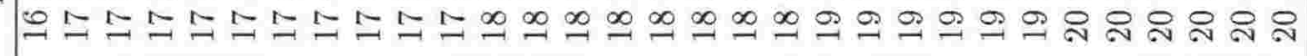

\#

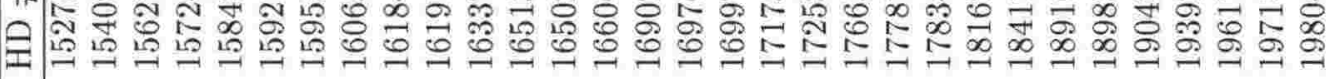

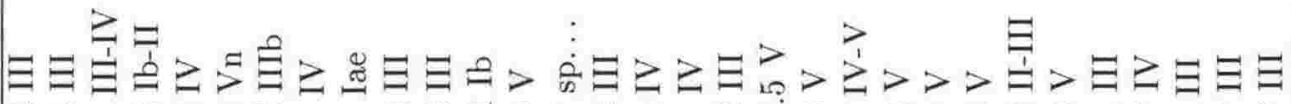
ค月せ

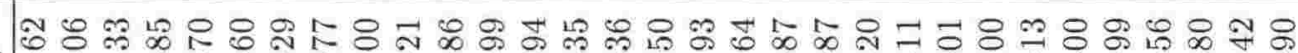

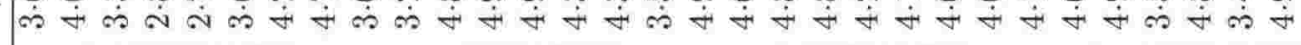

๓ - मे ம

옹ㅇำ

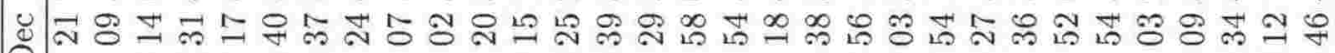
華

요 용 弪

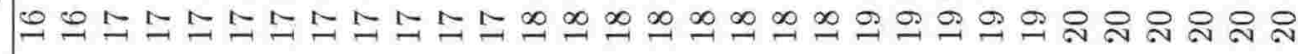
๓ \#

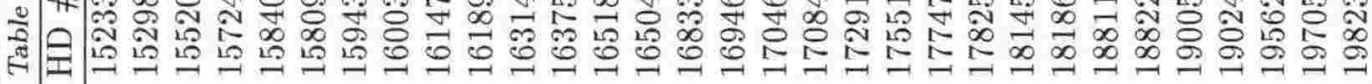




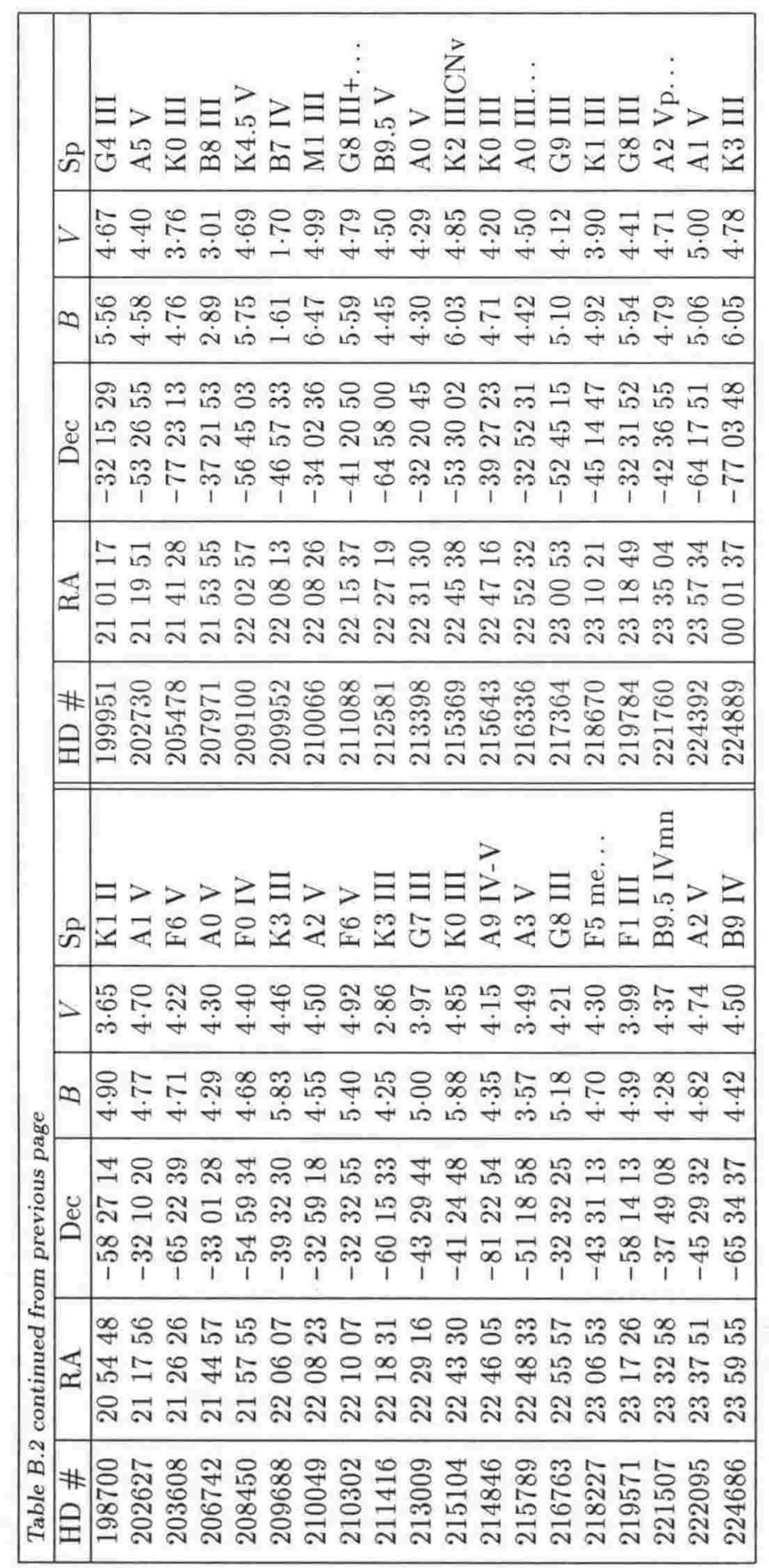




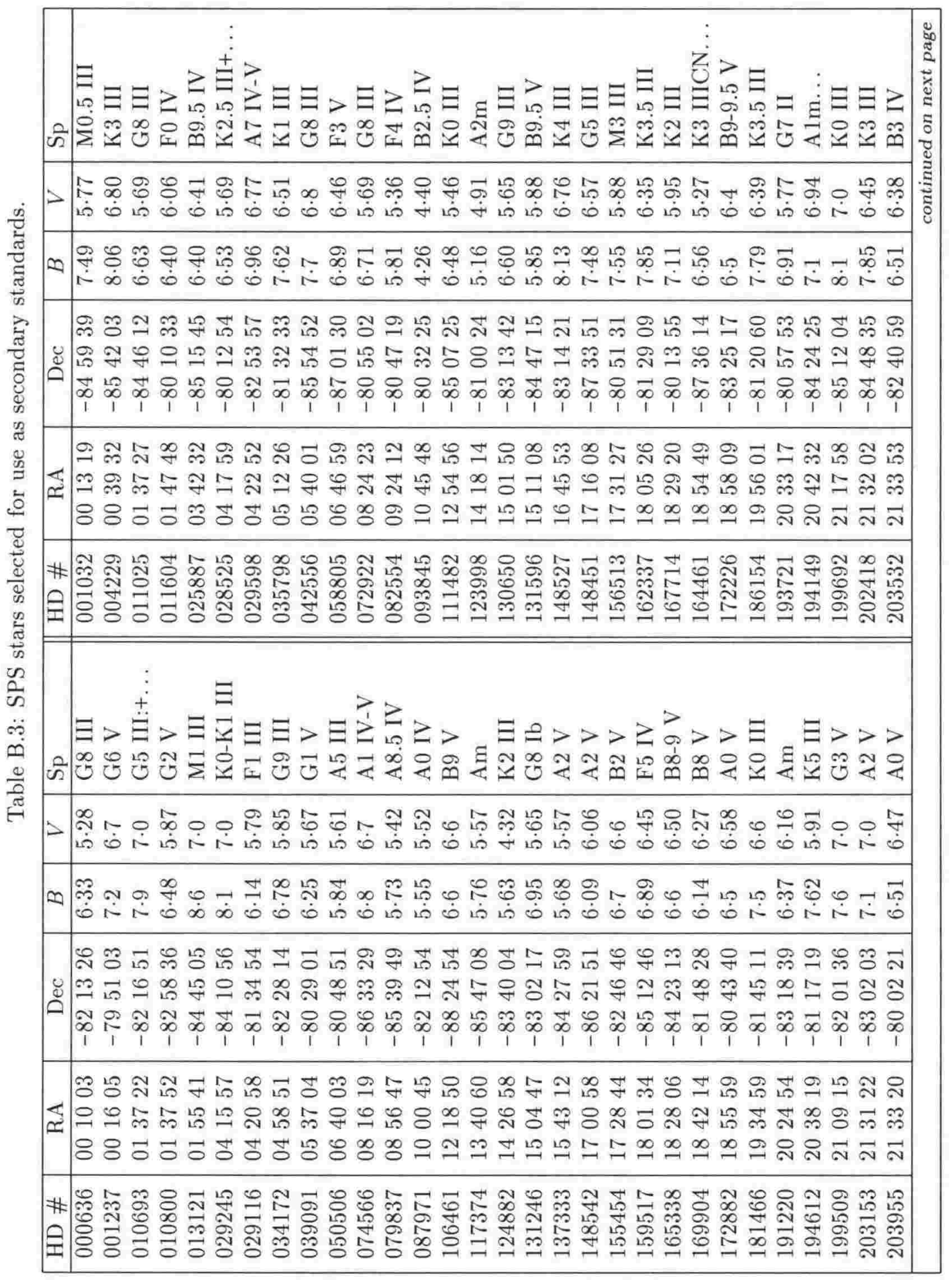




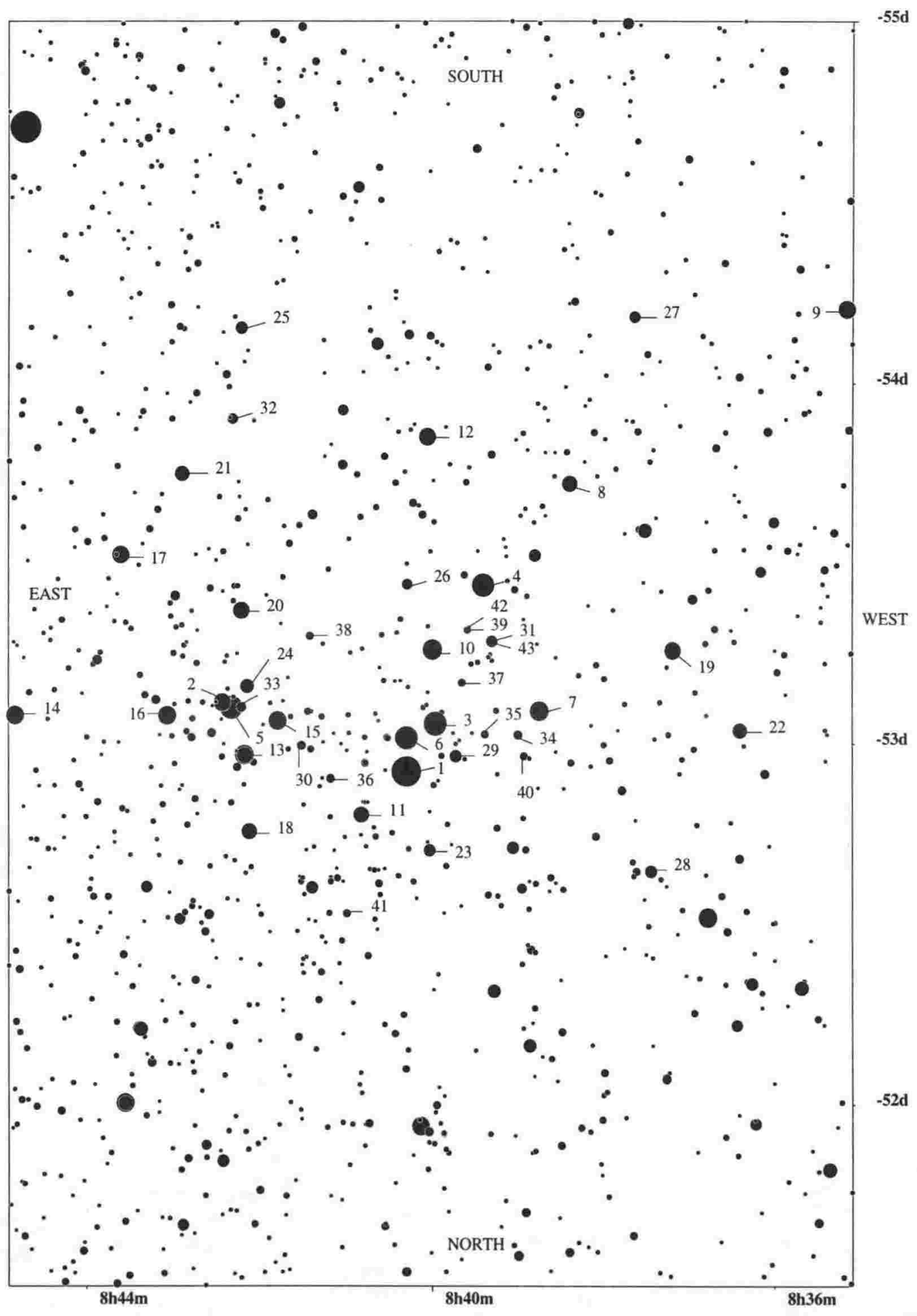

Figure 4.7: Star Chart for O Vel. 
Table 4.3: Spectral type for O Vel stars from the literature.

\begin{tabular}{|c|c|c|c|c|c|c|c|c|c|c|}
\hline \# & Ly & & Feins. & Busc. & P\&H & $\mathrm{P} \& \mathrm{~B}$ & Craw. & Eggen & L\&M & Renson \\
\hline 01 & B3 & B3 & B3 IV & B3 III & B3 III & & & $\mathrm{B} 3 / 5(\mathrm{~V})$ & B2.5 IV & \\
\hline 02 & B5 & B3 & B3 V & B4 IV & B3 V & & B3 IV & B3 IV & B3 V & \\
\hline 03 & B5 & B5 & B5 V & A8 V & B5 V & & B4 IV & B5 IV & B5 V & \\
\hline 04 & B5 & B5 & $\mathrm{B} 6 \mathrm{~V}$ & B5 V & $\mathrm{B} 6 \mathrm{~V}$ & & B5 V & B3 V & B6 V & \\
\hline 05 & B9 & B5 & A0p & B8 Si & B9.5 III & & & Ap & B 8.5 IVp & \\
\hline 06 & B5 & B5 & B5 ? & B8 $\mathrm{V}$ & B5 V & & B8 IV & $\mathrm{B} 9 / \mathrm{A} 0$ & B6 Vn & B7 He \\
\hline 07 & B9 & B8 & B9 V & B8 V & & & & B8 V & B8.5 Vn & \\
\hline 08 & A0 & B9 & & A1 V & & & & A2 $\mathrm{V}$ & A1 Vn & \\
\hline 09 & B9 & B7 & & B7 V & & & & & B7 V & \\
\hline 10 & A0 & B9 & $\mathrm{A} 0 \mathrm{~m}$ & A0 $\mathrm{m}$ & A0 IV:p & & & & $\begin{array}{l}\mathrm{A} 2 \mathrm{p}(\mathrm{Si}-\mathrm{Cr}- \\
\mathrm{Sr})\end{array}$ & $\begin{array}{l}\mathrm{A} 1 \mathrm{Cr}- \\
\mathrm{Eu}-\mathrm{Sr}\end{array}$ \\
\hline 11 & A0 & B9 & AO V & A0 V & $\mathrm{A} 0 \mathrm{~V}$ & & & $\mathrm{~A} 0 \mathrm{~V}$ & $\mathrm{AO} \mathrm{Vb}$ & \\
\hline 12 & A3 & & & A5 IV & & & & & & \\
\hline 13 & A0 & B9 & $\mathrm{A} 1 \mathrm{~V}$ & $\mathrm{~A} 1 \mathrm{~V}$ & $\mathrm{~A} 0 \mathrm{~V}$ & & & Al V & $\mathrm{A} 0 \mathrm{~V}$ & \\
\hline 14 & A. 0 & B9 & & A $1 \mathrm{~V}$ & & & & Al V & A2 $\mathrm{V}$ & \\
\hline 15 & A3 & & A2 $\mathrm{V}$ & A3 $\mathrm{V}$ & & & & & & A $2-8 m$ \\
\hline 16 & A2 & & A $1 \mathrm{~V}$ & A1 V & & & & A1 V & $\mathrm{A} 1 \mathrm{~V}$ & \\
\hline 17 & A2 & & & A5 V & & & & & & \\
\hline 18 & G5 & & & G3 V & & & & & & \\
\hline 19 & A0 & B9 & $\mathrm{A} 1 \mathrm{~V}$ & A3 V & $\mathrm{A} 2 \mathrm{~V}$ & & & A1 V & $\mathrm{A} 2 \mathrm{~V}$ & \\
\hline 20 & B8 & B5 & B7 V: & B5 $V_{p}$ & & & & & B6:IV:pec & B6p? \\
\hline 21 & A3 & & & $\mathrm{A} 3 \mathrm{~m}$ & & & & A3 V & $\mathrm{A} 7: \mathrm{Vnn}$ & \\
\hline 22 & A0 & B9 & & $\mathrm{A} 1 \mathrm{~V}$ & & & & & $\mathrm{~A} 0 \mathrm{~V}$ & \\
\hline 23 & A5 & & A5 V & A7 $\mathrm{V}$ & & & & $\mathrm{Am}$ & $\operatorname{Am}(\mathrm{K} / \mathrm{H} / \mathrm{M}$ & $\mathrm{A} 6 \mathrm{~m}$ ? \\
\hline 24 & A0 & A0 & & A3 V & & & & & /АI/AY/FII & \\
\hline 25 & A2 & & & A5 V & & & & & $\operatorname{Am}(\mathrm{K} / \mathrm{H} / \mathrm{M}$ & \\
\hline & & & & & & & & & /A4/A7/A9) & \\
\hline 26 & Ko & & & K3 V & & & & & Ko IIIa & \\
\hline 27 & F0 & & & F2 IV & & & & Fo V & Fo V & \\
\hline 28 & F5 & & & F5 V & & $\mathrm{F} 4 \mathrm{~V}$ & & & F3 V & \\
\hline 29 & F2 & & & $\mathrm{F}_{2} \mathrm{~V}$ & & & & F2 IV/V & F2 IV & \\
\hline 30 & KO & & & & & & & & & \\
\hline 31 & A2 2 & & & $\mathrm{~A} 2 \mathrm{~V}$ & & & & & & \\
\hline 32 & F2 & & & F5 V & & & & & F3 V & \\
\hline 33 & & & & F5 V & & & & & F6 V & \\
\hline 34 & & & & F5 V & & & & & F6 V & \\
\hline 35 & & & & K5 V & & & & & & \\
\hline 36 & G5 & & & $\mathrm{F} 6 \mathrm{~V}$ & & & & F5 V & & \\
\hline 37 & & & & & & & & & & \\
\hline 38 & A0 & B9 & & A2 V & & & & & & \\
\hline 39 & & B9 & & & & & & & & \\
\hline 40 & & & & & & & & & & \\
\hline 41 & & & & & & & & & & \\
\hline $\begin{array}{l}42 \\
43\end{array}$ & & & & & & & & & & \\
\hline
\end{tabular}


(marked with a colon in the table) they are actually the Cape values and were ignored when calculating the median values. The data given by Perry \& Bond (1969) is originally from the SAO Catalog, while Eggen's (1983) values are from either the CPC Catalog or Lesh (1972). Again, the table was supplemented from the original catalogues for stars not observed by the authors. Lyngå and Hogg used the FK3 system, while Perry \& Bond and Eggen used it's successor, the FK4 system. Comparisons between the different authors revealed that several stars common to Hogg and Eggen differ by exactly $0.006^{\prime \prime} / y r$ in Dec (marked by a colon under Eggen) - these were assumed to be values copied from the FK3 catalogues and corrected for systematic errors when transformed to the FK4 system and so were ignored when calculating the median values.

Figure 4.8 show histograms of the median values of the proper motions. The RA shows a bimodal distribution, probably due to the separation of field and cluster stars, while the Dec histogram hints at a similar bimodal distribution. As the field star contamination can be expected to increase with the fainter stars, following Lyngå (1961) the data was split into two groups (brighter and fainter than $V=8 \cdot 0$, see Table 4.8) and the median found for each. The brighter stars have (median) proper motions of $\mathrm{RA}=-0.023 \pm 0.011^{\prime \prime} / y \mathrm{r}$ and Dec $=+0.015 \pm 0.015^{\prime \prime} / y r$ while the fainter stars have RA $=-0.006 \pm 0.019^{\prime \prime} / y r$ and Dec $=+0.008 \pm 0.018^{\prime \prime} / y r$. According to Lyngå (1961), field stars at the distance of the cluster should have parallactic motions of -0.015 and $+0.006^{\prime \prime} / \mathrm{yr}$ respectively and smaller values with increasing distance. Hence the results are consistent with the fainter stars being predominantly field stars.

Table 4.5 gives the available radial velocities $(\mathrm{km} / \mathrm{s})$ for $\mathrm{O}$ Vel from the literature. The authors are Feinstein (1961), Buscombe (1965), Perry \& Bond (1969), Gieseking (1981), Eggen (1983), and Levato et al. (1988). Buscombe's data are the mean of his results plus measurements from the Lick, Cordoba and Stromolo observatories, while Eggen's are taken from Wilson (1953). Comparisons of the radial velocities between the different authors revealed a scatter generally consistent with the individual errors and showing no systematic differences, so the median for each star was calculated in the final column of the table.

A histogram of the (median) radial velocities is given in Figure 4.9, giving an average radial velocity of $13 \pm 9 \mathrm{~km} / \mathrm{s}$ (the uncertainty of the mean is \pm 1.5 and the median is $13.5 \mathrm{~km} / \mathrm{s}$ ) this compares well with Feinstein's mean (15 $\pm 1 \cdot 8)$, Buscombe's (14 $\pm 0 \cdot 7)$, and Levato (13 $\pm 1 \cdot 6$, which is given as a typographical error of -13) while Perry \& Bond and Eggen do not have sufficient values to allow useful mean values. This can also be compared with the theoretically expected variation in radial velocity for cluster members of $\sim 0.8 \mathrm{~km} / \mathrm{s}$ (Buscombe 1965).

Several of the stars are listed as a known or suspected Spectroscopic Binary (SB) (usually on the basis of double-lined spectra) or showing variable radial velocities. As the stars were 
Table 4.4: Proper motion for $\mathrm{O}$ Vel stars from the literature.

\begin{tabular}{|c|c|c|c|c|c|c|c|c|c|c|}
\hline \multirow[t]{2}{*}{$\#$} & \multicolumn{5}{|c|}{$\mathrm{RA}\left(0 \cdot 001^{\prime \prime} / y r\right)$} & \multicolumn{5}{|c|}{$\operatorname{Dec}\left(0.001^{\prime \prime} / y r\right)$} \\
\hline & Hogg & Lynga & P\&B & Eggen & median & Hogg & Lynga & P\&B & Eggen & median \\
\hline 01 & -17 & $-17:$ & -20 & -16 & -17 & +24 & $+24:$ & +19 & +20 & +20 \\
\hline 02 & -17 & -07 & -18 & $-19 \cdot 3$ & -17 & +11 & +11 & +21 & $+19 \cdot 8$ & +15 \\
\hline 03 & -25 & $-25:$ & -23 & $-16 \cdot 9$ & -23 & +03 & $+03:$ & +08 & $+19 \cdot 4$ & +08 \\
\hline 04 & -21 & -25 & -33 & $-25 \cdot 4$ & -25 & +24 & +25 & +18 & $+08 \cdot 2$ & +21 \\
\hline 05 & -17 & $-17:$ & -32 & & -25 & +17 & $+17:$ & +22 & & +20 \\
\hline 06 & -21 & $-21:$ & -32 & -16 & -21 & +23 & $+23:$ & +29 & $+21 \cdot 2$ & +23 \\
\hline 07 & -32 & $-32:$ & -12 & $-30:$ & -22 & +18 & $+18:$ & +12 & $+24:$ & +15 \\
\hline 08 & -21 & $-21:$ & -19 & $-19:$ & -20 & +43 & $+43:$ & +40 & $+49:$ & +41 \\
\hline 09 & -15 & $-15:$ & -23 & & -19 & +05 & $+05:$ & +17 & & +11 \\
\hline 10 & -30 & -32 & -27 & & -30 & +15 & +15 & +12 & & +15 \\
\hline 11 & -25 & -34 & -22 & $-22:$ & -25 & +27 & +27 & +24 & $+33:$ & +27 \\
\hline 12 & -16 & $-16:$ & +04 & & -06 & -24 & $-24:$ & -23 & & -23 \\
\hline 13 & -29 & -30 & -46 & $-27:$ & -30 & +10 & +10 & +19 & $+16:$ & +10 \\
\hline 14 & -05 & $-05:$ & -57 & $-02:$ & -32 & +21 & $+21:$ & +04 & $+27:$ & +12 \\
\hline 15 & -30 & -29 & -65 & & -30 & +03 & +05 & +07 & & +05 \\
\hline 16 & -44 & -41 & -35 & $-42:$ & -41 & +17 & +17 & -08 & $+23:$ & +17 \\
\hline 17 & -01 & $-01:$ & -46 & & -23 & -17 & $-17:$ & -31 & & -24 \\
\hline 18 & -27 & -24 & -24 & & -24 & +24 & +20 & +21 & & +21 \\
\hline 19 & -41 & -37 & -39 & $-39:$ & -39 & +33 & +27 & +30 & $+39:$ & +30 \\
\hline 20 & +13 & -11 & +15 & & +13 & +02 & +15 & -01 & & +02 \\
\hline 21 & -15 & $-15:$ & -24 & $-13:$ & -20 & +10 & $+10:$ & +00 & $+16:$ & +05 \\
\hline 22 & -09 & -12 & -06 & & -09 & +43 & +38 & +40 & & +40 \\
\hline 23 & -29 & -29 & -26 & -27 & -28 & +19 & +18 & +16 & +26 & +18 \\
\hline 24 & -17 & -23 & -14 & & -17 & -05 & +20 & -08 & & -05 \\
\hline 25 & -06 & $-06:$ & & & -06 & +02 & $+02:$ & & & +02 \\
\hline 26 & +32 & +15 & +35 & & +32 & -21 & -15 & -24 & & -21 \\
\hline 27 & -43 & $-43:$ & -42 & $-40:$ & -42 & +46 & $+46:$ & +43 & $+52:$ & +44 \\
\hline 28 & -03 & -13 & +00 & & -03 & +19 & +24 & +17 & & +19 \\
\hline 29 & -46 & -32 & -43 & $-44:$ & -43 & +18 & +18 & +15 & $+24:$ & +18 \\
\hline 30 & & +30 & & & +30 & & +18 & & & +18 \\
\hline 31 & -03 & -06 & +00 & & -03 & -27 & -16 & -30 & & -27 \\
\hline 32 & -25 & $-25:$ & -23 & & -24 & +13 & $+13:$ & +10 & & +11 \\
\hline 33 & -16 & -11 & & & -13 & +16 & +11 & & & +13 \\
\hline 34 & & -02 & & & -02 & & +10 & & & +10 \\
\hline 35 & & -11 & & & -11 & & +08 & & & +08 \\
\hline 36 & & -02 & & & -02 & & +06 & & & +06 \\
\hline 37 & & +02 & & & +02 & & -06 & & & -06 \\
\hline 38 & & -06 & & & -06 & & +17 & & & +17 \\
\hline 39 & & -17 & & & -17 & & -01 & & & -01 \\
\hline 40 & & +01 & & & +01 & & +08 & & & +08 \\
\hline 41 & & -34 & & & -34 & & +37 & & & +37 \\
\hline 42 & & +02 & & & +02 & & -17 & & & -17 \\
\hline 43 & & +01 & & & +01 & & -02 & & & -02 \\
\hline
\end{tabular}



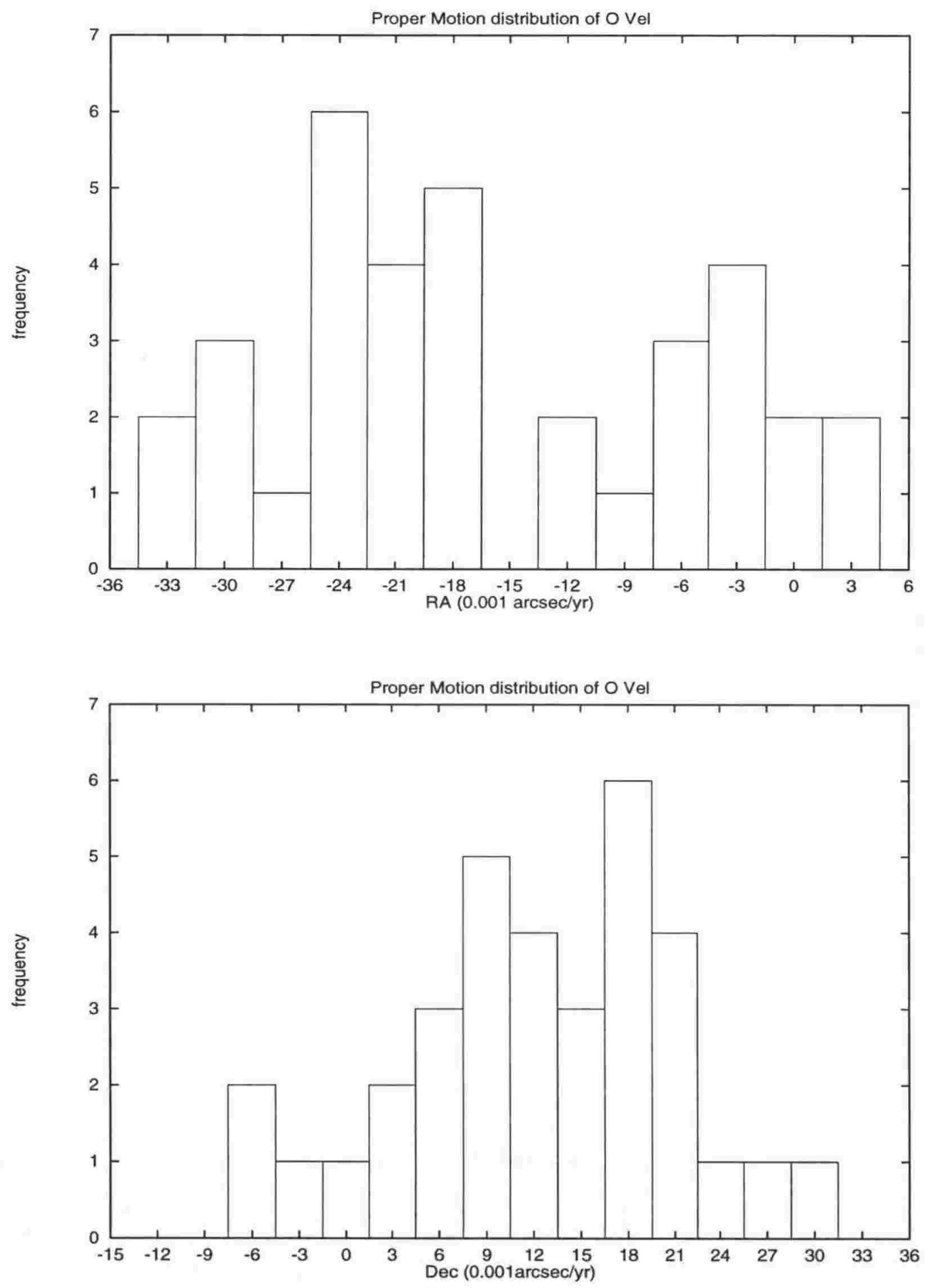

Figure 4.8: Proper Motion distribution for the O Vel cluster. 
Table 4.5: Radial velocity $(\mathrm{km} / \mathrm{s})$ for O Vel stars from the literature.

\begin{tabular}{|c|c|c|c|c|c|c|c|c|c|c|c|}
\hline \# & \multicolumn{2}{|c|}{ Feinstein } & \multicolumn{2}{|c|}{ Buscombe } & \multirow{2}{*}{$\begin{array}{l}\mathrm{P} \& \mathrm{~B} \\
+15\end{array}$} & \multirow[t]{2}{*}{ Gieseking } & \multicolumn{2}{|c|}{ Eggen } & \multicolumn{2}{|c|}{ Levato } & median \\
\hline 01 & $+15 \cdot 4$ & & +16 & & & & $+17 \cdot 1$ & & +14 & & +15 \\
\hline 02 & +19.7 & Var & +19 & SB & & & +21.6 & & +12 & & +19 \\
\hline 03 & $+12 \cdot 0$ & SB & +12 & SB & & & & SB & +16 & SB & +12 \\
\hline 04 & $+15 \cdot 2$ & & +15 & & & & +9 & Var & +10 & & +13 \\
\hline 05 & $+12 \cdot 7$ & SB? & +15 & & & & +19.8 & & +13 & & +14 \\
\hline 06 & +24.9 & & +16 & SB & & & & SB & +05 & Var & +16 \\
\hline 07 & $+04 \cdot 2$ & & +15 & & & & & & +13 & & +13 \\
\hline 08 & & & $+21:$ & SB & & & & & +09 & & +15 \\
\hline 09 & & & +10 & & & & & & +08 & Var & +09 \\
\hline 10 & $+12 \cdot 0$ & & +06 & SB & & & & & +19 & & +12 \\
\hline 11 & +15 & SB? & +15 & SB & & & & & +12 & & +15 \\
\hline 12 & & & +06 & & & & & & & & +06 \\
\hline 13 & +09.0 & & -02 & & & & & & +13 & & +09 \\
\hline 14 & & & +10 & SB & & & & & +06 & & +08 \\
\hline 15 & +12.5 & & +21 & & & & & & & & +17 \\
\hline 16 & & & +09 & SB & & & & & +02 & SB & +06 \\
\hline 17 & & & -03 & SB & & & & & & & -03 \\
\hline 18 & & & +15 & & & & & & & & +15 \\
\hline 19 & $+13 \cdot 2$ & & $+06:$ & & & & & & +08 & & +08 \\
\hline 20 & +31.6 & & +23 & & & $+8.0 \mathrm{SB} ?$ & & & +16 & & +20 \\
\hline 21 & & & +18 & SB & & & & & +10 & SB & +14 \\
\hline 22 & & & $+22:$ & & & & & & +08 & & +15 \\
\hline 23 & $+09 \cdot 3$ & & +16 & & & & & & +15 & & +15 \\
\hline 24 & & & +03 & SB & & & & & & & +03 \\
\hline 25 & & & +38 & & & & & & +31 & & +34 \\
\hline 26 & & & +03 & SB & & & & & +50 & & +26 \\
\hline 27 & & & $-08:$ & & & & & & -09 & & -08 \\
\hline 28 & & & +15 & & +02 & & & & +03 & & +03 \\
\hline 29 & & & +19 & & & & & & +15 & Var & +17 \\
\hline 30 & & & & & & & & & & & \\
\hline 31 & & & +16 & & & & & & & & +16 \\
\hline 32 & & & +12 & & & & & & +14 & & +13 \\
\hline 33 & & & +16 & & & & & & +01 & & +08 \\
\hline 34 & & & $+52:$ & & & & & & +12 & & +32 \\
\hline 35 & & & +29: & & & & & & & & +29 \\
\hline 36 & & & +13 & Var & Var? & & & & & & +13 \\
\hline 37 & & & & & & & & & & & \\
\hline 38 & & & +06 & & -15 & & & & & & -04 \\
\hline 39 & & & & & & & & & & & \\
\hline 40 & & & & & & & & & & & \\
\hline 41 & & & & & & & & & & & \\
\hline 42 & & & & & & & & & & & \\
\hline 43 & & & & & & & & & & & \\
\hline
\end{tabular}




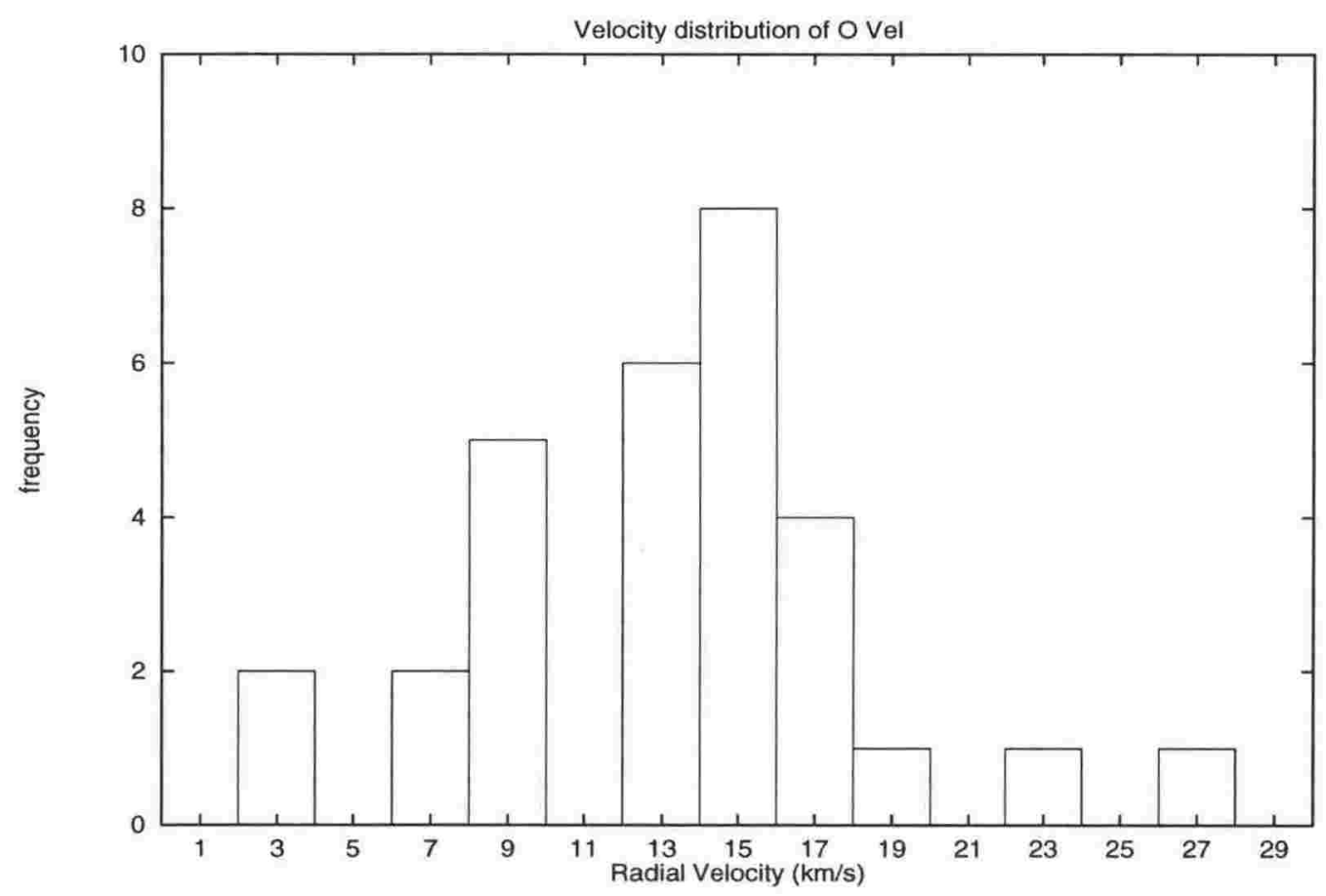

Figure 4.9: Radial Velocity distribution for the O Vel cluster.

not generally observed over a sufficient time to find their centre-of-mass radial velocity, this is also reflected in the larger variation in radial velocities between different authors found for these stars. However, O Vel 3 was observed by Feinstein long enough to derive its orbital elements. Levato later re-observed this star and gave the following orbital elements based on all observations found in the literature (see Table 4.6).

Table 4.7 contains the membership assessments from the literature. Hogg (1960) and Lyngå (1961) based their membership on proper motions, while Feinstein (1961), Perry \& Bond (1969), and Lyngå \& Wramdemark (1984) used photometric results (colour excesses and distance modulus). Buscombe (1965) used radial velocities and spectroscopic classifications, while Levato

Table 4.6: Orbital elements of Spectroscopic binary O Vel 3.

$$
\begin{aligned}
\text { Period } & =3 \cdot 192276 \pm 0.000008 \text { days } \\
T_{o} & =2419729.49 \pm 0.05 \text { Julian days } \\
e & =0.05 \pm 0.03 \\
V_{o} & =14.5 \pm 1.2 \mathrm{~km} / \mathrm{s} \\
\Omega & =288 \pm 31 \text { degrees } \\
K & =57 \pm 1.6 \mathrm{~km} / \mathrm{s} \\
a \sin (i) & =(2.4 \pm 0.085) \times 10^{6} \mathrm{~km}
\end{aligned}
$$


et al. (1988) used radial velocities, proper motions and spectroscopic classifications. Finally, Stauffer et al. (1989) used proper motions and spectroscopic classifications. The last column gives the median membership value (i.e. majority of the membership assessments). The median values in parenthesis are for those stars with no assessments in the literature and result from fitting normal curves to the proper motion and radial velocity histograms using the median and standard deviations for the cluster, finding the probabilities that a star will have its particular values, multiplying these probabilities together and assigning yes/maybe/no values to the 70 and 40 percentage thresholds. To gauge the 'accuracy' of this simple assessment, these probabilities were calculated for all the stars and compared with the median values, indicating the probability assessment agrees about $50 \%$ of the time, and is within one assessment class (for example the median gives yes while the probability indicates maybe) about $70 \%$ of the time.

Table 4.8 lists all the visual magnitudes in the literature - these are from the UBV system unless stated otherwise. The earliest observations are from Lyngå (1959) (with a fuller report given later (Lyngå 1961)) and Hogg (1960). Perry \& Hill (1969) made measurements in both the UBV and uvby systems, as did Eggen $(1972,1983)$, with the $y$ magnitude taken as the visual magnitude. Note the typographical error by Eggen (1972); O Vel 36 (HD74340) should be V = $9 \cdot 84$, not $7 \cdot 84$. Rufener (1981) lists measurements of $\mathrm{O}$ Vel stars from the Geneva photometric system's catalog, with their $M_{V}$ corresponding to a visual magnitude. The final authors are Lyngå \& Wramdemark (1984). Comparisons between the different authors revealed colour dependent systematic errors in the $\mathrm{V}$ magnitude, probably due to incorrect transformations to the standard UBV system. As it was not possible to tell which system is closest to the standard, the median was calculated in the hope that both the systematic and random errors would be reduced.

Several of the brighter stars in the cluster have been investigated for variability. However the results are complicated by the fact that the comparison stars used in several investigations were found to be variable by others, as described next.

O Vel 1 (Omicron Velorum itself) was found to be a radial velocity and photometric variable by Van Hoof (1972) with a change in $\mathrm{V}$ of $0^{m} \cdot 03-0^{m} \cdot 04$ and a period just over 3 hours, and confirmed by later observations (Van Hoof 1973). However Balona (1977) and Jakate (1979) found it to be constant (within $\pm 0^{m} \cdot 02$ ) over the order of nights. The Geneva catalog has a larger than usual dispersion and notes that it is listed in the GCVS(Rufener 1981). Later observations by Waelkens \& Rufener (1983) in the Geneva system confirm a variability of $\pm 0^{m} \cdot 012$ in $\mathrm{V}$ with a period of $\sim 2.7$ days. Finally, Eggen (1983) finds it to vary over $\Delta \mathrm{V} \approx 0.1$ and in $H_{\beta}$.

$\mathrm{O}$ Vel 2 was found to be variable $( \pm 0.015)$ with a period of 0.0284 days. Note however that stars 1 and 5 were used as comparison stars and found to be stable. Balona (1982) also found 
Table 4.7: Membership for O Vel stars from the literature.

\begin{tabular}{|c|c|c|c|c|c|c|c|c|c|}
\hline \# & Hogg & Fein. & Lynga & Busc. & P\&B & L\&W & Levato & Stauffer & median \\
\hline 01 & yes & yes & yes & yes & yes & & yes & & yes \\
\hline 02 & yes & yes & yes & yes & yes & & yes & & yes \\
\hline 03 & yes & yes & yes & yes & yes & yes & yes & & yes \\
\hline 04 & yes & yes & yes & yes & yes & & yes & & yes \\
\hline 05 & yes & yes & yes & yes & no & yes & yes & & yes \\
\hline 06 & yes & yes & yes & yes & yes & yes & yes & & yes \\
\hline 07 & yes & yes & yes & yes & yes & & yes & & yes \\
\hline 08 & & & & yes & & & yes & & yes \\
\hline 09 & & & & yes & & & no & & maybe \\
\hline 10 & yes & yes & yes & yes & no & & yes & & yes \\
\hline 11 & yes & yes & yes & yes & yes & & yes & & yes \\
\hline 12 & & & & yes & no & & & & maybe \\
\hline 13 & yes & yes & yes & yes & yes & yes & yes & & yes \\
\hline 14 & & & & & yes & & yes & & yes \\
\hline 15 & doubtful & no & & yes & no & & & & maybe \\
\hline 16 & no & yes & & yes & yes & yes & & & yes \\
\hline 17 & & & & no & no & & yes & & no \\
\hline 18 & yes & & yes & no & no & no & & & no \\
\hline 19 & & yes & & yes & yes & & yes & & yes \\
\hline 20 & no & no & yes & no & no & no & no & & no \\
\hline 21 & & & & yes & yes & & yes & & yes \\
\hline 22 & & & & yes & & & no & & maybe \\
\hline 23 & & yes & yes & yes & yes & yes & yes & & yes \\
\hline 24 & yes & & yes & yes & no & & & & yes \\
\hline 25 & & & & yes & & & no & & maybe \\
\hline 26 & & & & no & & & no & & no \\
\hline 27 & & & & yes & & & no & & maybe \\
\hline 28 & & & yes & yes & no & & no & & maybe \\
\hline $\begin{array}{l}29 \\
30\end{array}$ & yes & & yes & yes & yes & yes & yes & & $\begin{array}{c}\text { yes } \\
\text { (no) }\end{array}$ \\
\hline 31 & & & & yes & no & & & & maybe \\
\hline 32 & & & yes & yes & & & yes & & yes \\
\hline 33 & yes & & & yes & & & yes & & yes \\
\hline 34 & & & & yes & & yes & yes & & yes \\
\hline 35 & & & & no & & & & & no \\
\hline 36 & & & & yes & yes & yes & & & yes \\
\hline 37 & & & & & & & & & (no) \\
\hline 38 & & & & no & no & & & & no \\
\hline 39 & & & & & & & & & (maybe) \\
\hline 40 & & & & & & yes & & yes & yes \\
\hline 41 & & & & & & & & & (no) \\
\hline 42 & & & & & & & & & (no) \\
\hline 43 & & & & & & & & & (no) \\
\hline
\end{tabular}


Table 4.8: Visual magnitude $\mathrm{V}$ for $\mathrm{O}$ Vel stars from the literature.

\begin{tabular}{|c|c|c|c|c|c|c|c|c|c|}
\hline \# & Lynga & Hogg & $\begin{array}{l}{ }_{(V)}{ }^{\mathrm{P}} \\
\text { ( }\end{array}$ & $\begin{array}{l}\mathrm{H} \\
\text { (uvby) }\end{array}$ & $\begin{array}{c}\text { Eggen } \\
\text { (UBV) }\end{array}$ & $\begin{array}{r}\text { Rufener } \\
(\mathrm{Mv})\end{array}$ & $\begin{array}{l}\text { Eggen } \\
\text { (uvby) }\end{array}$ & L\&W & median \\
\hline 01 & $3 \cdot 61$ & 3.67 & 3.64 & 3.63 & & 3.606 & $3 \cdot 60$ & & $3 \cdot 62$ \\
\hline 02 & 4.84 & 4.90 & 4.89 & 4.88 & 4.78 & 4.804 & 4.85 & & $4 \cdot 85$ \\
\hline 03 & $5 \cdot 19$ & $5 \cdot 27$ & $5 \cdot 21$ & $5 \cdot 23$ & & $5 \cdot 176$ & $5 \cdot 21$ & $5 \cdot 20$ & $5 \cdot 21$ \\
\hline 04 & $5 \cdot 48$ & $5 \cdot 52$ & $5 \cdot 50$ & $5 \cdot 50$ & & $5 \cdot 441$ & 5.48 & & $5 \cdot 49$ \\
\hline 05 & $5 \cdot 52$ & $5 \cdot 56$ & & $5 \cdot 54$ & 5.47 & $5 \cdot 487$ & $5 \cdot 49$ & $5 \cdot 52$ & $5 \cdot 52$ \\
\hline 06 & 5.57 & $5 \cdot 62$ & 5.60 & $5 \cdot 59$ & & $5 \cdot 548$ & 5.58 & $5 \cdot 56$ & 5.58 \\
\hline 07 & 6.46 & $6 \cdot 48$ & $6 \cdot 47$ & 6.48 & & $6 \cdot 429$ & $6 \cdot 47$ & & 6.47 \\
\hline 08 & $7 \cdot 67$ & & & & & $7 \cdot 647$ & 7.67 & & $7 \cdot 67$ \\
\hline 09 & 7.07 & & & & & & & & $7 \cdot 07$ \\
\hline 10 & $7 \cdot 23$ & $7 \cdot 27$ & & $7 \cdot 27$ & $7 \cdot 27$ & $7 \cdot 215$ & & & $7 \cdot 27$ \\
\hline 11 & $7 \cdot 29$ & $7 \cdot 31$ & & $7 \cdot 31$ & $7 \cdot 28$ & $7 \cdot 275$ & $7 \cdot 31$ & & $7 \cdot 30$ \\
\hline 12 & $7 \cdot 36$ & & $7 \cdot 40$ & $7 \cdot 38$ & & & & & $7 \cdot 38$ \\
\hline 13 & $7 \cdot 39$ & $7 \cdot 41$ & & $7 \cdot 41$ & 7.38 & $7 \cdot 362$ & $7 \cdot 38$ & $7 \cdot 38$ & $7 \cdot 38$ \\
\hline 14 & 7.58 & & $7 \cdot 60$ & $7 \cdot 59$ & $7 \cdot 55$ & $7 \cdot 541$ & $7 \cdot 56$ & & $7 \cdot 57$ \\
\hline 15 & 7.59 & $7 \cdot 60$ & & $7 \cdot 60$ & 7.54 & $7 \cdot 548$ & & & 7.59 \\
\hline 16 & $7 \cdot 68$ & $7 \cdot 69$ & $7 \cdot 70$ & $7 \cdot 70$ & $7 \cdot 66$ & $7 \cdot 655$ & $7 \cdot 68$ & $7 \cdot 68$ & $7 \cdot 68$ \\
\hline 17 & $7 \cdot 79$ & & $7 \cdot 79$ & $7 \cdot 77$ & $7 \cdot 78$ & 7.729 & & & $7 \cdot 78$ \\
\hline 18 & $7 \cdot 81$ & 7.88 & & $7 \cdot 85$ & & & & $7 \cdot 83$ & $7 \cdot 84$ \\
\hline 19 & $7 \cdot 85$ & $7 \cdot 89$ & & $7 \cdot 86$ & 7.83 & $7 \cdot 817$ & $7 \cdot 86$ & & 7.86 \\
\hline 20 & 7.96 & 7.98 & & 7.96 & & & & 7.92 & 7.96 \\
\hline 21 & $8 \cdot 17$ & & $8 \cdot 17$ & $8 \cdot 14$ & 8.14 & $8 \cdot 113$ & $8 \cdot 12$ & & $8 \cdot 14$ \\
\hline 22 & 8.35 & & & & & & & & $8 \cdot 35$ \\
\hline 23 & 8.55 & & $8 \cdot 57$ & $8 \cdot 56$ & $8 \cdot 52$ & 8.526 & 8.55 & 8.53 & 8.55 \\
\hline 24 & $8 \cdot 60$ & $8 \cdot 63$ & & 8.63 & & & & & $8 \cdot 63$ \\
\hline 25 & 8.68 & & & & & 8.659 & & & 8.67 \\
\hline 26 & 8.68 & & & & & & & & $8 \cdot 68$ \\
\hline 27 & 8.77 & & & & & $8 \cdot 753$ & 8.78 & & 8.77 \\
\hline 28 & 8.88 & & & 8.93 & 8.91 & 8.885 & & & 8.90 \\
\hline 29 & 9.09 & $9 \cdot 12$ & $9 \cdot 10$ & $9 \cdot 10$ & $9 \cdot 09$ & 9.073 & 9.08 & $9 \cdot 11$ & $9 \cdot 10$ \\
\hline 30 & $9 \cdot 21$ & $9 \cdot 32$ & & & & & & & $9 \cdot 25$ \\
\hline 31 & $9 \cdot 38$ & & & $9 \cdot 42$ & & & & & $9 \cdot 40$ \\
\hline 32 & $9 \cdot 38$ & & & & & $9 \cdot 357$ & & & $9 \cdot 37$ \\
\hline 33 & 9.59 & $9 \cdot 69$ & & & $9 \cdot 59$ & & & & $9 \cdot 59$ \\
\hline 34 & $9 \cdot 62$ & $9 \cdot 66$ & & & & $9 \cdot 606$ & & $9 \cdot 62$ & $9 \cdot 62$ \\
\hline 35 & $9 \cdot 66$ & $9 \cdot 74$ & & & & & & & $9 \cdot 70$ \\
\hline 36 & $9 \cdot 83$ & & $9 \cdot 88$ & $9 \cdot 87$ & $9 \cdot 84$ & $9 \cdot 824$ & 9.86 & $9 \cdot 84$ & $9 \cdot 84$ \\
\hline 37 & 9.95 & & & & & & & & 9.95 \\
\hline 38 & $10 \cdot 15$ & & $10 \cdot 18$ & $10 \cdot 17$ & & & & & $10 \cdot 17$ \\
\hline 39 & $10 \cdot 16$ & & & & & & & & $10 \cdot 16$ \\
\hline 40 & $10 \cdot 33$ & $10 \cdot 36$ & & & & $10 \cdot 288$ & & $10 \cdot 31$ & $10 \cdot 32$ \\
\hline 41 & $10 \cdot 63$ & & & & & & & & $10 \cdot 63$ \\
\hline 42 & 11.48 & & & & & & & & 11.48 \\
\hline 43 & 11.57 & & & & & & & & 11.57 \\
\hline
\end{tabular}


O Vel 2 to be stable but Waelkens \& Rufener (1983) find variations of \pm 0.015 in $\mathrm{V}$ with a period of 1.553 days and Eggen (1983) notes it as a possible variable.

O Vel 3 is listed as appearing variable by Van Hoof (1973) but no other authors have noted any variability. Recall however that it was found to be a spectroscopic binary by Feinstein (1961) and Levato et al. (1988). O Vel 4 was used as a comparison star by Balona (1977) and found to be stable but Eggen (1983) states it varies by $\Delta \mathrm{V}=0.02$ with a period of 0.26 days.

Thus, it seems that the four brightest stars in the cluster are irregularly variable at the $0^{m} \cdot 01$ level with various periods ranging from hours to days. A possible explanation for the observations during which the stars appeared constant could be the presence of two, or more, frequencies (perhaps due to intrinsic and orbital variations) whose 'waves' approximately cancel out during certain (short) intervals.

There have been only four investigations using the UBV system, by Lyngå (1959), Hogg (1960), Perry \& Hill (1969), and Eggen (1972). The results are shown in Table 4.9, with comparisons between the authors again revealing systematic differences so the median values will be taken as best representing the standard system.

Colour-Magnitude and Colour-Colour diagrams are shown in Figures 4.10 and 4.11. These show all candidate stars for the cluster and probable members (based on Table 4.7). The position of O Vel 1 in the Colour-Magnitude Diagram (CMD) and spectral type (B3 III-V) indicates it is probably just leaving the main sequence, consistent with a cluster age of $30 \times 10^{6}$ years. O Vel 24 , although given as a probable member in Table 4.7, appears further off the main sequence than could be expected from observational errors and may not be a member. O Vel 40 is the faintest probable member, and is considered by Stauffer et al. (1989) to be a pre-main sequence star, again consistent with a cluster age of $30 \times 10^{6}$ years.

There have been only four investigations of O Vel in the uvby system; that of Perry \& Hill (1969), Crawford et al. (1971), Eggen (1983), and Lyngå \& Wramdemark (1984). These are summarized in Tables 4.10 and 4.11 and CMDs plotted in Figure 4.12 using the median values. O Vel 1 and O Vel 40 show the same behaviour seen in the UBV CMD, but the 'blue excess' stars do not (instead they generally lie on the main sequence). O Vel 24 still lies slightly to the left of the main sequence but is within the observational error.

Finally, measurements of the $H_{\beta}$ index (using the extension to the uvby system) are summarized in Table 4.12. The authors are Graham (1967), Perry \& Hill (1969), Crawford et al. (1971), Grønbech \& Olsen (1977), Eggen (1983), and Lyngå \& Wramdemark (1984). The median values are compared with the $H_{\beta}$-absolute magnitude calibration in Figure 4.13. O Vel 1 is again brighter than the ZAMS, but no conclusion can be drawn for O Vel 40 as it has not yet been observed in the $H_{\beta}$ system.

Comparison between the CMDs and the absolute magnitude calibration of all candidate 
Table 4.9: U-B and B-V colours for O Vel stars from the literature.

\begin{tabular}{|c|c|c|c|c|c|c|c|c|c|c|}
\hline \multirow[t]{2}{*}{ \# } & \multicolumn{5}{|c|}{ U-B } & \multicolumn{5}{|c|}{$\mathrm{B}-\mathrm{V}$} \\
\hline & Lynga & Hogg & P\&H & Eggen & median & Lynga & Hogg & P\&H & Eggen & median \\
\hline 01 & -0.67 & -0.66 & -0.66 & & -0.66 & -0.18 & -0.18 & -0.18 & & -0.18 \\
\hline 02 & -0.67 & -0.66 & -0.66 & -0.67 & -0.66 & $-0 \cdot 17$ & -0.18 & $-0 \cdot 17$ & -0.16 & $-0 \cdot 17$ \\
\hline 03 & -0.60 & -0.57 & -0.58 & & -0.58 & $-0 \cdot 14$ & -0.15 & -0.15 & & -0.15 \\
\hline 04 & -0.59 & -0.55 & -0.58 & & -0.58 & $-0 \cdot 15$ & $-0 \cdot 16$ & -0.15 & & -0.15 \\
\hline 05 & -0.57 & -0.54 & & -0.54 & -0.54 & $-0 \cdot 15$ & -0.17 & & -0.13 & -0.15 \\
\hline 06 & -0.54 & -0.50 & -0.50 & & -0.50 & $-0 \cdot 13$ & -0.14 & $-0 \cdot 14$ & & -0.14 \\
\hline 07 & -0.33 & -0.37 & -0.32 & & $-0 \cdot 33$ & -0.09 & -0.11 & $-0 \cdot 10$ & & $-0 \cdot 10$ \\
\hline 08 & -0.04 & & & & -0.04 & +0.08 & & & & +0.08 \\
\hline 09 & -0.46 & & & & $-0 \cdot 46$ & $-0 \cdot 11$ & & & & -0.11 \\
\hline 10 & -0.17 & -0.04 & & -0.11 & $-0 \cdot 11$ & -0.01 & -0.06 & & -0.05 & -0.05 \\
\hline 11 & -0.11 & $+0 \cdot 00$ & & -0.025 & -0.03 & +0.01 & -0.03 & & -0.00 & -0.00 \\
\hline 12 & -0.03 & & +0.07 & & +0.02 & +0.29 & & +0.26 & & +0.27 \\
\hline 13 & -0.08 & +0.01 & & +0.025 & +0.01 & +0.02 & -0.02 & & +0.03 & +0.02 \\
\hline 14 & -0.01 & & +0.06 & +0.08 & +0.06 & +0.08 & & +0.08 & +0.08 & +0.08 \\
\hline 15 & -0.01 & +0.02 & & +0.08 & +0.02 & +0.27 & +0.22 & & +0.27 & +0.27 \\
\hline 16 & -0.03 & +0.02 & +0.05 & +0.065 & +0.03 & $+0 \cdot 10$ & +0.04 & +0.07 & +0.09 & +0.08 \\
\hline 17 & +0.02 & & +0.13 & $+0 \cdot 12$ & +0.12 & $+0 \cdot 20$ & & +0.19 & $+0 \cdot 19$ & +0.19 \\
\hline 18 & +0.20 & $+0 \cdot 16$ & & & +0.18 & +0.66 & +0.65 & & & +0.65 \\
\hline 19 & -0.01 & +0.02 & & +0.06 & +0.02 & +0.11 & +0.05 & & $+0 \cdot 10$ & $+0 \cdot 10$ \\
\hline 20 & -0.50 & -0.40 & & & -0.45 & +0.02 & +0.02 & & & +0.02 \\
\hline 21 & +0.00 & & +0.11 & +0.07 & +0.07 & +0.21 & & +0.19 & $+0 \cdot 21$ & +0.21 \\
\hline 22 & -0.11 & & & & -0.11 & +0.04 & & & & +0.04 \\
\hline 23 & +0.02 & & +0.06 & +0.06 & +0.06 & +0.20 & & +0.24 & +0.24 & +0.24 \\
\hline 24 & +0.06 & +0.03 & & & +0.04 & $+0 \cdot 15$ & +0.11 & & & +0.13 \\
\hline 25 & +0.04 & & & & +0.04 & +0.22 & & & & +0.22 \\
\hline 26 & +0.82 & & & & +0.82 & +1.06 & & & & +1.06 \\
\hline 27 & -0.07 & & & & -0.07 & +0.36 & & & & +0.36 \\
\hline 28 & -0.04 & & & -0.06 & -0.05 & +0.44 & & & +0.43 & +0.43 \\
\hline 29 & -0.07 & +0.02 & -0.02 & -0.055 & -0.04 & +0.35 & +0.37 & +0.36 & +0.36 & +0.36 \\
\hline 30 & +0.75 & +0.76 & & & +0.75 & +1.05 & +1.05 & & & +1.05 \\
\hline 31 & +0.03 & & & & +0.03 & $+0 \cdot 12$ & & & & +0.12 \\
\hline 32 & -0.08 & & & & -0.08 & +0.44 & & & & +0.44 \\
\hline 33 & -0.15 & +0.02 & & -0.08 & -0.08 & $+0 \cdot 44$ & $+0 \cdot 42$ & & $+0 \cdot 50$ & +0.44 \\
\hline 34 & -0.08 & +0.02 & & & -0.03 & $+0 \cdot 46$ & $+0 \cdot 46$ & & & +0.46 \\
\hline 35 & +1.00 & +1.14 & & & +1.07 & $+1 \cdot 20$ & +1.21 & & & +1.20 \\
\hline 36 & -0.06 & & -0.04 & -0.055 & -0.05 & $+0 \cdot 49$ & & +0.46 & $+0 \cdot 45$ & +0.46 \\
\hline 37 & +0.12 & & & & $+0 \cdot 12$ & +0.64 & & & & +0.64 \\
\hline 38 & +0.05 & & $+0 \cdot 14$ & & +0.10 & $+0 \cdot 18$ & & +0.15 & & $+0 \cdot 17$ \\
\hline 39 & +0.03 & & & & +0.03 & $+0 \cdot 19$ & & & & +0.19 \\
\hline 40 & $+0 \cdot 18$ & $+0 \cdot 17$ & & & +0.17 & $+0 \cdot 68$ & +0.68 & & & +0.68 \\
\hline 41 & $-0 \cdot 36$ & & & & $-0 \cdot 36$ & $+0 \cdot 15$ & & & & +0.15 \\
\hline 42 & & & & & & +1.82 & & & & +1.82 \\
\hline 43 & -0.01 & & & & -0.01 & +0.35 & & & & +0.35 \\
\hline
\end{tabular}



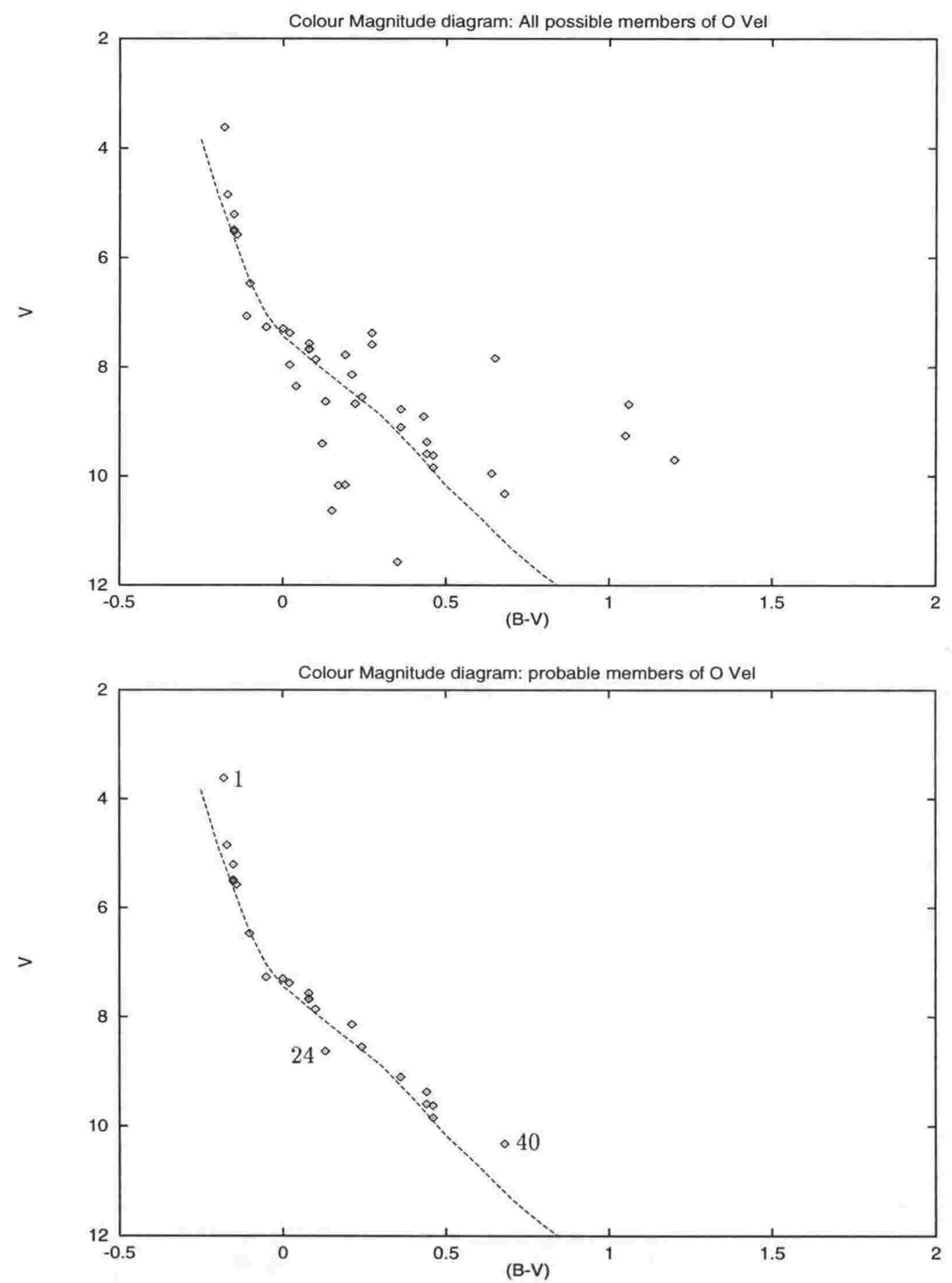

Figure 4.10: Colour-Magnitude diagram of $\mathrm{O}$ Vel, using the median values from the UBV literature. The line is the ZAMS, taken from Johnson (1963, page 216, Table 4) and assuming a distance modulus of $5.94(=154 \mathrm{pc})$. The top diagram is for all stars from Lyngå's list while the bottom diagram are the probable members according to the median column of Table 4.7. 

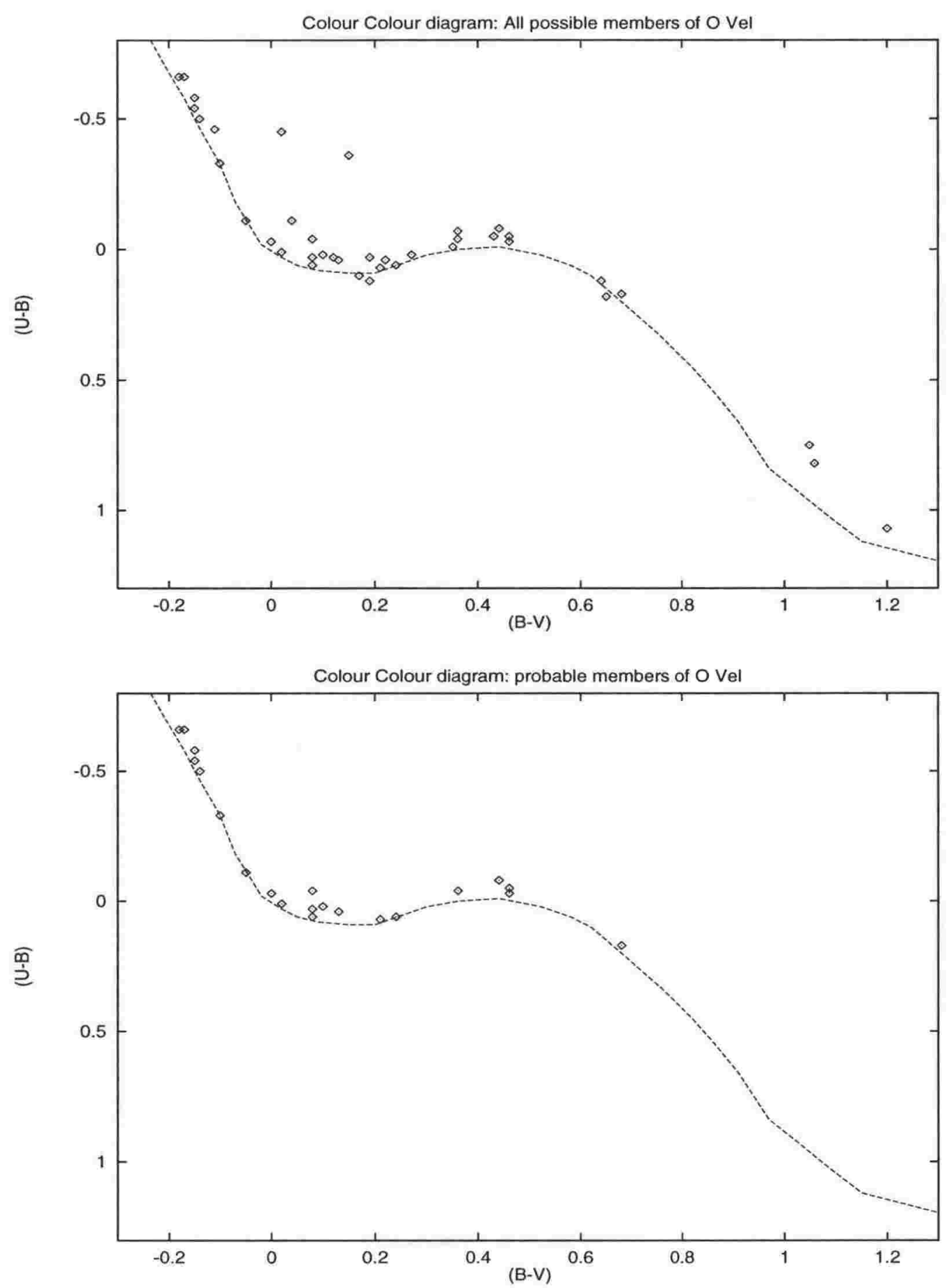

Figure 4.11: Colour-Colour diagram of $\mathrm{O}$ Vel, using the median values from the UBV literature. The line is the main sequence, taken from Tables 14 and 15 of Straižys (1992). The top diagram is for all stars from Lyngå's list while the bottom diagram are the probable members according to the median column of Table 4.7 . 
Table 4.10: $b-y$ colours for O Vel stars from the literature.

\begin{tabular}{|c|c|c|c|c|c|}
\hline \# & $\mathrm{P} \& \mathrm{H}$ & Crawford & Eggen & $\mathrm{L} \& \mathrm{~W}$ & median \\
\hline 01 & -0.075 & & -0.080 & & -0.077 \\
\hline 02 & -0.071 & -0.078 & -0.075 & & -0.075 \\
\hline 03 & -0.073 & -0.098 & -0.070 & -0.061 & -0.072 \\
\hline 04 & -0.070 & -0.079 & -0.070 & & -0.070 \\
\hline 05 & -0.085 & & -0.078 & -0.073 & -0.078 \\
\hline 06 & -0.054 & -0.058 & -0.060 & -0.053 & -0.056 \\
\hline 07 & $-0 \cdot 040$ & & $-0 \cdot 040$ & & $-0 \cdot 040$ \\
\hline 08 & & & +0.029 & & +0.029 \\
\hline \multicolumn{6}{|l|}{09} \\
\hline 10 & -0.042 & & & & -0.042 \\
\hline 11 & -0.010 & & -0.011 & & -0.010 \\
\hline 12 & $+0 \cdot 163$ & & & & +0.163 \\
\hline 13 & -0.005 & & -0.005 & +0.008 & -0.005 \\
\hline 14 & +0.029 & & +0.031 & & +0.030 \\
\hline 15 & +0.134 & & & & +0.134 \\
\hline 16 & +0.028 & & +0.025 & +0.045 & +0.028 \\
\hline 17 & +0.099 & & & & +0.099 \\
\hline 18 & +0.419 & & & +0.428 & +0.423 \\
\hline 19 & +0.024 & & +0.025 & & +0.024 \\
\hline 20 & +0.029 & & & +0.054 & +0.026 \\
\hline 21 & +0.123 & & $+0 \cdot 110$ & & +0.016 \\
\hline \multicolumn{6}{|l|}{22} \\
\hline 23 & $+0 \cdot 140$ & & +0.140 & +0.154 & $+0 \cdot 140$ \\
\hline 24 & +0.074 & & & & +0.074 \\
\hline \multicolumn{6}{|l|}{25} \\
\hline \multicolumn{6}{|l|}{26} \\
\hline 27 & & & $+0 \cdot 244$ & & $+0 \cdot 244$ \\
\hline 28 & +0.288 & & & & +0.288 \\
\hline 29 & +0.242 & & $+0 \cdot 240$ & +0.255 & $+0 \cdot 242$ \\
\hline 30 & & & & & \\
\hline 31 & +0.052 & & & & +0.052 \\
\hline \multirow{2}{*}{\multicolumn{6}{|c|}{$\begin{array}{l}32 \\
33\end{array}$}} \\
\hline & & & & & \\
\hline \multirow{2}{*}{\multicolumn{6}{|c|}{$\begin{array}{l}54 \\
35\end{array}$}} \\
\hline & & & & & \\
\hline 36 & $+0 \cdot 320$ & & $+0 \cdot 310$ & +0.327 & +0.320 \\
\hline \multicolumn{6}{|l|}{37} \\
\hline 38 & +0.106 & & & & +0.106 \\
\hline \multicolumn{6}{|l|}{39} \\
\hline 40 & & & & +0.435 & +0.435 \\
\hline \multicolumn{6}{|l|}{41} \\
\hline 42 & & & & & \\
\hline 43 & & & & & \\
\hline
\end{tabular}


Table 4.11: $m_{1}$ and $c_{1}$ colours for $\mathrm{O}$ Vel stars from the literature.

\begin{tabular}{|c|c|c|c|c|c|c|c|c|c|c|}
\hline \multirow[t]{2}{*}{ \# } & \multicolumn{5}{|c|}{$m_{1}$} & \multicolumn{5}{|c|}{$c_{1}$} \\
\hline & P\&H & Craw. & Eggen & L\&W & median & P\&H & Craw. & Eggen & L\&W & median \\
\hline 01 & 0.093 & & 0.090 & & 0.091 & 0.344 & & 0.350 & & 0.347 \\
\hline 02 & 0.093 & $0 \cdot 105$ & $0 \cdot 100$ & & 0.100 & 0.337 & $0 \cdot 310$ & 0.330 & & $0 \cdot 330$ \\
\hline 03 & $0 \cdot 122$ & 0.131 & 0.115 & $0 \cdot 106$ & 0.118 & $0 \cdot 396$ & 0.412 & 0.400 & $0 \cdot 398$ & $0 \cdot 399$ \\
\hline 04 & $0 \cdot 112$ & 0.116 & $0 \cdot 110$ & & 0.112 & 0.427 & 0.419 & 0.428 & & 0.427 \\
\hline 05 & 0.143 & & $0 \cdot 135$ & 0.124 & $0 \cdot 135$ & 0.428 & & 0.435 & 0.436 & 0.435 \\
\hline 06 & $0 \cdot 104$ & $0 \cdot 102$ & $0 \cdot 108$ & $0 \cdot 101$ & $0 \cdot 103$ & 0.515 & 0.516 & 0.520 & 0.514 & 0.516 \\
\hline 07 & 0.124 & & 0.125 & & 0.124 & 0.698 & & 0.700 & & $0 \cdot 699$ \\
\hline 08 & & & $0 \cdot 191$ & & $0 \cdot 191$ & & & 1.038 & & 1.038 \\
\hline 09 & & & & & & & & & & \\
\hline 10 & 0.212 & & & & 0.212 & $0 \cdot 830$ & & & & $0 \cdot 830$ \\
\hline 11 & $0 \cdot 166$ & & 0.162 & & $0 \cdot 164$ & 0.931 & & 0.940 & & 0.935 \\
\hline 12 & $0 \cdot 181$ & & & & $0 \cdot 181$ & 0.822 & & & & 0.822 \\
\hline 13 & $0 \cdot 184$ & & $0 \cdot 182$ & $0 \cdot 154$ & 0.182 & 0.986 & & 0.998 & 0.991 & 0.991 \\
\hline 14 & 0.211 & & $0 \cdot 196$ & & 0.204 & 0.958 & & 1.002 & & 0.980 \\
\hline 15 & 0.220 & & & & $0 \cdot 220$ & 0.767 & & & & 0.767 \\
\hline 16 & $0 \cdot 196$ & & $0 \cdot 195$ & $0 \cdot 159$ & $0 \cdot 195$ & 0.984 & & 0.997 & 1.012 & 0.997 \\
\hline 17 & 0.213 & & & & $0 \cdot 213$ & 0.937 & & & & 0.937 \\
\hline 18 & 0.204 & & & $0 \cdot 221$ & 0.212 & $0 \cdot 380$ & & & $0 \cdot 315$ & $0 \cdot 347$ \\
\hline 19 & 0.219 & & $0 \cdot 209$ & & 0.214 & 0.960 & & 0.969 & & 0.964 \\
\hline 20 & $0 \cdot 101$ & & & 0.069 & 0.085 & 0.479 & & & 0.487 & $0 \cdot 483$ \\
\hline 21 & $0 \cdot 185$ & & $0 \cdot 185$ & & 0.185 & 0.924 & & 0.950 & & 0.937 \\
\hline 22 & & & & & & & & & & \\
\hline 23 & 0.208 & & 0.197 & 0.182 & 0.197 & 0.772 & & 0.795 & 0.782 & 0.782 \\
\hline 24 & $0 \cdot 196$ & & & & 0.196 & 1.026 & & & & 1.026 \\
\hline 25 & & & & & & & & & & \\
\hline 26 & & & & & & & & & & \\
\hline 27 & & & $0 \cdot 136$ & & 0.136 & & & 0.573 & & 0.573 \\
\hline 28 & $0 \cdot 149$ & & & & 0.149 & 0.477 & & & & 0.477 \\
\hline 29 & $0 \cdot 164$ & & $0 \cdot 150$ & $0 \cdot 150$ & $0 \cdot 150$ & 0.561 & & 0.570 & 0.541 & 0.561 \\
\hline 30 & & & & & & & & & & \\
\hline 31 & $0 \cdot 184$ & & & & 0.184 & 1.018 & & & & 1.018 \\
\hline 32 & & & & & & & & & & \\
\hline 33 & & & & & & & & & & \\
\hline 34 & & & & $0 \cdot 140$ & 0.140 & & & & 0.424 & 0.424 \\
\hline 35 & & & & & & & & & & \\
\hline $\begin{array}{l}36 \\
37\end{array}$ & $0 \cdot 143$ & & $0 \cdot 147$ & 0.144 & $0 \cdot 144$ & $0 \cdot 379$ & & 0.443 & $0 \cdot 393$ & $0 \cdot 393$ \\
\hline 38 & 0.129 & & & & 0.129 & 1.165 & & & & 1.165 \\
\hline 39 & & & & & & & & & & \\
\hline 40 & & & & 0.219 & $0 \cdot 219$ & & & & 0.318 & 0.318 \\
\hline 41 & & & & & & & & & & \\
\hline 42 & & & & & & & & & & \\
\hline 43 & & & & & & & & & & \\
\hline
\end{tabular}



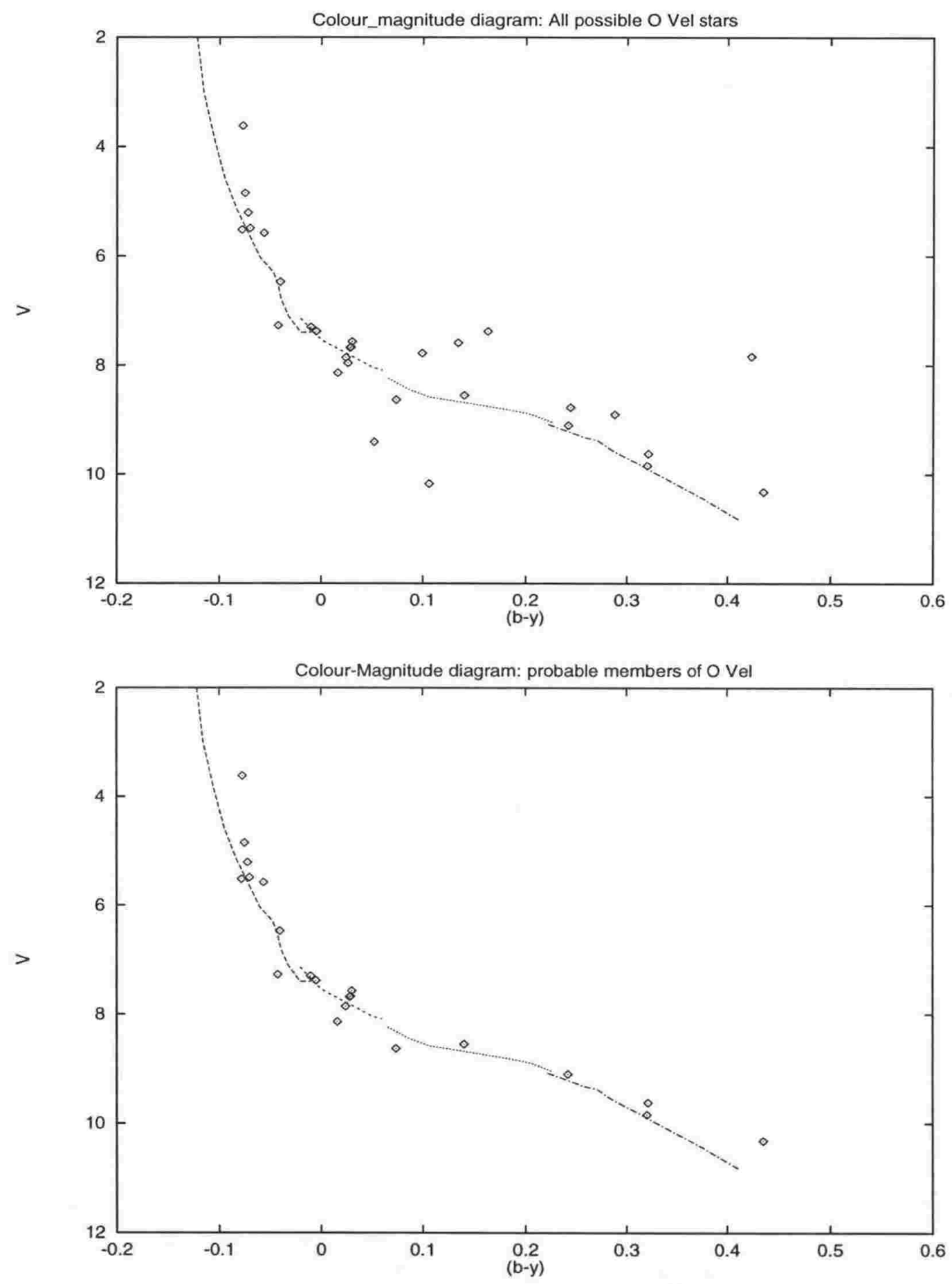

Figure 4.12: Colour-Magnitude diagram of $\mathrm{O}$ Vel, using the median values from the $u v b y$ literature. The line segments are the main sequence, taken from Tables 35, 39, 40, 42 and 43 of Straižys (1992) and assuming a distance modulus of $5.94(=154 \mathrm{pc})$. The top diagram is for all stars from Lyngå's list while the bottom diagram are the probable members according to the median column of Table 4.7. 
Table 4.12: $H_{\beta}$ colours for $\mathrm{O}$ Vel stars from the literature.

\begin{tabular}{|c|c|c|c|c|c|c|c|}
\hline \# & Gra. & $\mathrm{P} \& \mathrm{H}$ & Craw. & Gron. & Eggen & L\&W & median \\
\hline 01 & $2 \cdot 66$ & $2 \cdot 666$ & & & $2 \cdot 66 \mathrm{~V}$ & 2.674 & 2.663 \\
\hline 02 & $2 \cdot 68$ & $2 \cdot 688$ & 2.674 & $2 \cdot 689$ & 2.695 & $2 \cdot 689$ & $2 \cdot 689$ \\
\hline 03 & $2 \cdot 68$ & $2 \cdot 713$ & $2 \cdot 692$ & 2.760 & $2 \cdot 710$ & & $2 \cdot 710$ \\
\hline 04 & $2 \cdot 70$ & $2 \cdot 717$ & $2 \cdot 693$ & 2.712 & $2 \cdot 710$ & & $2 \cdot 710$ \\
\hline 05 & $2 \cdot 73$ & 2.723 & & 2.732 & $2 \cdot 730$ & $2 \cdot 728$ & $2 \cdot 730$ \\
\hline 06 & $2 \cdot 69$ & $2 \cdot 710$ & $2 \cdot 681$ & $2 \cdot 712$ & $2 \cdot 710$ & 2.712 & $2 \cdot 710$ \\
\hline 07 & $2 \cdot 76$ & 2.778 & & $2 \cdot 790$ & $2 \cdot 785$ & & 2.782 \\
\hline 08 & & & & & 2.879 & & 2.879 \\
\hline \multicolumn{8}{|l|}{09} \\
\hline 10 & $2 \cdot 85$ & $2 \cdot 855$ & & & & & $2 \cdot 852$ \\
\hline 11 & $2 \cdot 85$ & $2 \cdot 896$ & & & $2 \cdot 892$ & & $2 \cdot 892$ \\
\hline 12 & & $2 \cdot 788$ & & & & & 2.788 \\
\hline 13 & $2 \cdot 85$ & 2.911 & & & $2 \cdot 916$ & 2.920 & 2.914 \\
\hline 14 & & 2.900 & & & $2 \cdot 894$ & & 2.897 \\
\hline 15 & & $2 \cdot 831$ & & & & & $2 \cdot 831$ \\
\hline 16 & & $2 \cdot 887$ & & & $2 \cdot 899$ & & $2 \cdot 893$ \\
\hline 17 & & $2 \cdot 857$ & & & & & 2.857 \\
\hline 18 & & 2.598 & & & & 2.586 & $2 \cdot 592$ \\
\hline 19 & & 2.918 & & & $2 \cdot 903$ & & 2.910 \\
\hline 20 & & $2 \cdot 705$ & & & & 2.705 & $2 \cdot 705$ \\
\hline 21 & & $2 \cdot 834$ & & & $2 \cdot 830$ & & $2 \cdot 832$ \\
\hline \multicolumn{8}{|l|}{22} \\
\hline 23 & & $2 \cdot 801$ & & & $2 \cdot 800$ & 2.801 & $2 \cdot 801$ \\
\hline 24 & & $2 \cdot 860$ & & & & & $2 \cdot 860$ \\
\hline \multicolumn{8}{|l|}{25} \\
\hline \multicolumn{8}{|l|}{26} \\
\hline 27 & & & & & $2 \cdot 681$ & & $2 \cdot 681$ \\
\hline 28 & & $2 \cdot 663$ & & & & & $2 \cdot 663$ \\
\hline 29 & & $2 \cdot 703$ & & & $2 \cdot 700$ & 2.699 & 2.700 \\
\hline \multicolumn{8}{|l|}{30} \\
\hline 31 & & $2 \cdot 864$ & & & & & $2 \cdot 864$ \\
\hline \multicolumn{8}{|l|}{32} \\
\hline \multicolumn{8}{|l|}{33} \\
\hline \multicolumn{8}{|l|}{34} \\
\hline \multicolumn{8}{|l|}{35} \\
\hline 36 & & $2 \cdot 635$ & & & $2 \cdot 637$ & & $2 \cdot 636$ \\
\hline \multicolumn{8}{|l|}{37} \\
\hline 38 & & $2 \cdot 833$ & & & & & $2 \cdot 833$ \\
\hline \multicolumn{8}{|l|}{39} \\
\hline \multicolumn{8}{|l|}{40} \\
\hline \multicolumn{8}{|l|}{41} \\
\hline 42 & & & & & & & \\
\hline 43 & & & & & & & \\
\hline
\end{tabular}



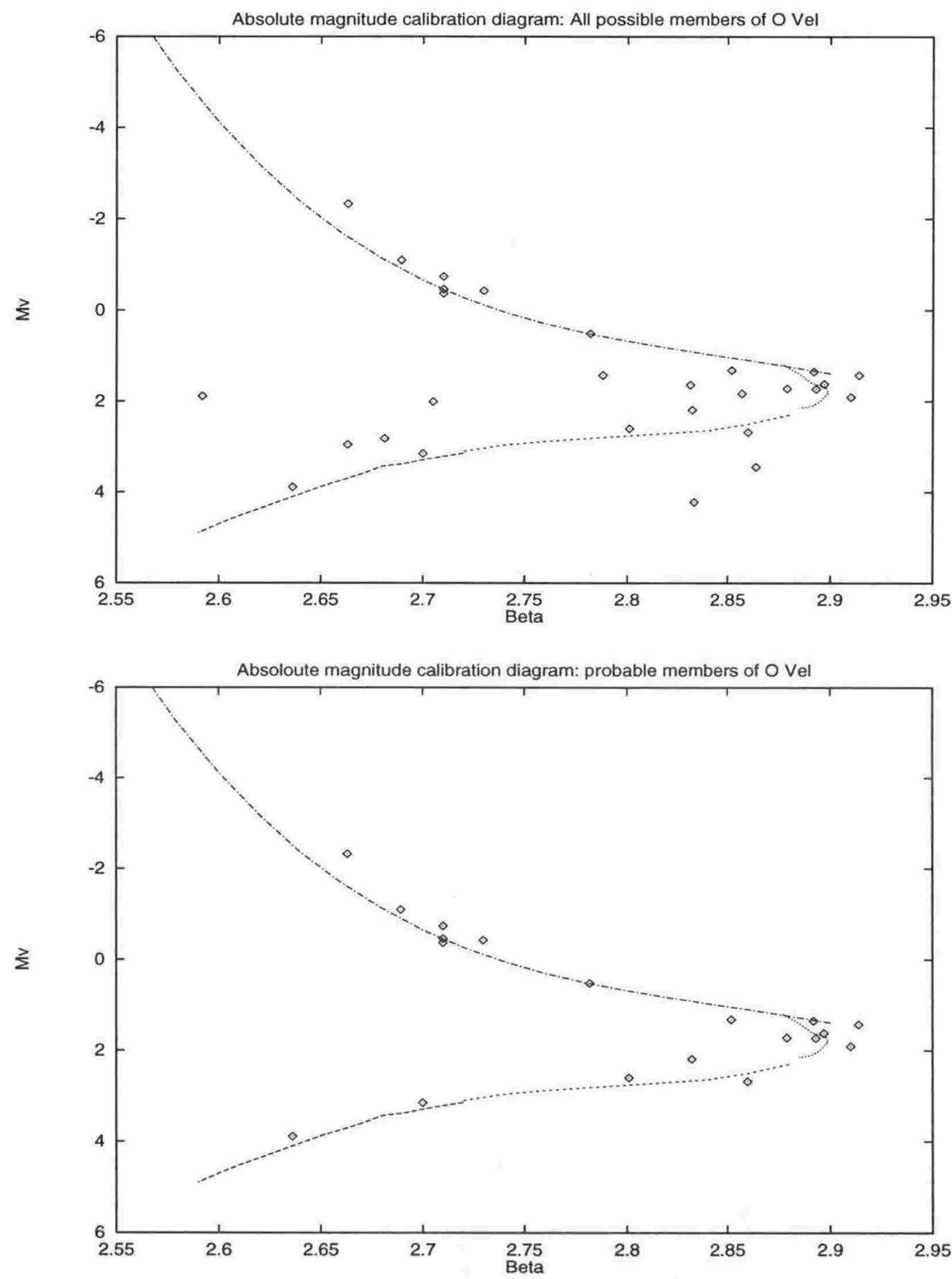

Figure 4.13: $H_{\beta}$-Absolute Magnitude diagram of $\mathrm{O}$ Vel, using the median values from the $u v b y$ literature. The lines are the $H_{\beta}$-absolute magnitude calibration for the ZAMS, taken from Tables 39, 40, 42, and 43 of Straižys (1992). The top diagram is for all stars from Lyngå's list while the bottom diagram are the probable members according to the median column of Table 4.7 and assuming a distance modulus of $5.94(=154 p c)$. 
stars and probable members of the $\mathrm{O}$ Vel cluster imply the membership assessments given in Table 4.7 are reliable in the sense that few, or none, of the stars are incorrectly assessed as members (i.e. false-positive) - the most likely false-positive star being O Vel 24. Similarly, there appear to be no stars incorrectly assessed as non-members (i.e. false-negative) - as the few non-members which appear near the main sequence in the UBV CMD are not near the uvby main sequence and vice versa.

\subsection{Vilnius Photometry of the Omicron Velorum cluster.}

Recall the purpose of the observations of the O Vel cluster was to compare the analysis from Vilnius photometry alone with those found by other photometric and spectroscopic systems. For this reason, none of the prior knowledge available from the literature was assumed when analyzing the results from the Vilnius observations.

The individual observations are given in Table D.5, while the mean results for each star is given in Table E.8. At the precision level achieved by this study $\left(0^{\mathrm{m}} \cdot 02\right.$ for a single observation), none of the observed stars were found to be variable. As a further check, the standard deviation of the mean for each star was compared with the expected deviation for a star with $n$ observations, using the formula found for primary standards in Figure 3.3 - no stars were indicated as variable using this criteria. To investigate possible variability to higher precision would require dedicated programs of differential photometry, which may be undertaken at a later date. The Vilnius mean $\mathrm{V}$ magnitudes compare well with the visual magnitudes found in the literature (see Table 4.8 ); $\sim 50 \%$ agree within $\pm 0^{m} \cdot 01$ of the median value, $\sim 90 \%$ within $\pm 0^{m} \cdot 02$ and all but two stars lie within the range covered in the literature.

The next stage in the analysis is to derive the spectral classification of each star, in this case using the Vilnius Q-Search, Q-Spectra and QQ-diagrams methods described in Chapter 1 (sections 1.8, 1.9, and 1.11 respectively), with the results given in Table 4.13. The Q-Search results list the spectral types one standard deviation above and below the mean value, and note in parenthesis any peculiarities of the matching reference stars. The Q-Spectra notes the 'graphical shifting' factor as $1+a$, with 1.00 meaning no shifting was required to achieve a good match. If the match of the closest reference spectra appeared to be worse than would be expected from the observational errors, then '(p)' follows the spectral type. Recall the QQ-diagrams are calibrated in absolute magnitudes rather than luminosity class, and may also indicate possible spectral peculiarities. Finally, an 'average' spectral type is assigned in the last column. These were compared with the spectral types from the literature (see Table 4.3); $\sim 55 \%$ agree within \pm 1.0 spectral sub-classes, which is the expected standard deviation of the classification methods (see Chapter 1 , sections 1.8 and 1.9), and all agree within \pm 3.0 spectral 
sub-classes. Three stars have been classified for the first time; O Vel 37 (G2 V), O Vel 40 (G1 V) and O Vel 41 (B6 V).

Given the spectral type of each star (the 'average' column of Table 4.13), the average reddening and distance modulus can be found for the cluster as follows;

1. Using the spectral and luminosity class, look up the intrinsic magnitude $\mathrm{V}_{0}$ and colour $(\mathrm{Y}-\mathrm{V})_{0}$ from Appendix 1 and Table 73 of Straižys (1992) for each star. ${ }^{4}$

2. Calculate the colour excess for each star from the intrinsic and apparent (reddened) colour using $E_{\mathrm{Y}-\mathrm{V}}=(\mathrm{Y}-\mathrm{V})-(\mathrm{Y}-\mathrm{V})_{0}$.

3. Calculate the distance modulus for each star, using the 'extinction to colour excess' ratio $(R)$ from Table 74 of Straižys (1992) and $\mathrm{m}-\mathrm{M}=\mathrm{V}-\mathrm{V}_{0}-R E_{\mathrm{Y}-\mathrm{V} \cdot{ }^{4}}$

4. Examine the distribution of distance moduli and calculate the mean $(<\mathrm{m}-\mathrm{M}>)$ from the probable members. ${ }^{5}$ Alternatively, use the median value.

5. Re-calculate the intrinsic magnitude for each star using the mean distance modulus $\left(V_{0}=\right.$ $\left.V-<\mathrm{m}-\mathrm{M}>-R E_{\mathrm{Y}-\mathrm{v}}\right)$.

6. Lookup the intrinsic colour $(\mathrm{Y}-\mathrm{V})_{0}$ using the spectral type and intrinsic magnitude with Table 73 .

7. Return to step 2 and repeat until the iterated mean distance modulus converges. Note that the distance moduli and colour excess of the individual stars will only converge for cluster members.

This gives the cluster's distance moduli as $5.94 \pm 0.02^{6}$ and a distribution about the mean measured by a standard deviation of 0.03 . Similarly, the mean colour excess is $0.00 \pm 0.02^{7}$ with a distribution of 0.03 (which is strongly correlated with the distance moduli). Assuming the cluster is spherical, its angular diameter $\left(\sim 45^{\prime}\right)$ at the mean distance corresponds to a spread in the distance moduli for individual cluster members of \pm 0.03 . Thus it seems reasonable that the entire spread in values is due to the differences in distance moduli of individual cluster stars and that there is no interstellar material causing reddening along the line of sight to the cluster.

Once the cluster's distance modulus and colour excess have been determined, then the distance and excess of individual stars may be used to decide on the star's cluster membership.

\footnotetext{
${ }^{4}$ See Appendix J for a copy of these tables.

${ }^{5}$ Convert the distance moduli into linear units, for example parsecs via $\rho=10^{(\mathrm{m}-\mathrm{M}-5) / 5}$, before calculating the mean.

${ }^{6}$ This is the standard deviation of the mean.

${ }^{7}$ Although negative colour excesses have no physical meaning, they can arise due to observational and calibration errors in the case of weak, or zero, reddening and should be retained for the purpose of calculating means.
} 
A histogram of the distance moduli (for a fixed, zero colour excess) was examined, with stars outside the cluster's distribution considered non-members and stars in the tails of the distribution considered 'maybe' members. The results are shown in Table 4.14; note that the 'partial' disagreement for O Vel 40 may be due to an incorrect spectral classification from the Vilnius photometry, as it is probably a pre-main sequence star (for which the Vilnius system is not calibrated). Only two stars show complete disagreement; O Vel 37 was assessed from the radial velocity and proper motions studies rather than directly by the authors, while O Vel 17 is considered a cluster member by one (of the three) authors in the literature so a 'maybe' membership assessment could be more accurate - note how the Vilnius assessment is revised to a 'maybe' by examination of the spectral sequence in the CMD (see later).

Figure 4.14 shows the CMD after correcting for the cluster's mean distance modulus and reddening. The error bars in the top diagram indicate more observations need to be made of the fainter stars $\left(\mathrm{V}_{0}>2 \Longleftrightarrow \mathrm{V}>8\right.$ ) before analysis of the (fainter) probable cluster members can be done with confidence. The bottom diagram show the probable members; the various sequences indicate that O Vel 1 appears to be B4 III rather than B4 IV, the stars O Vel 2-7 may have moved off the ZAMS to the main sequence, O Vel 24 again appears too far to the left of the ZAMS to be a member, and that O Vel 37 and 40 are pre-main sequence stars. The, 
Colour Magnitude diagram: All possible members of O Vel

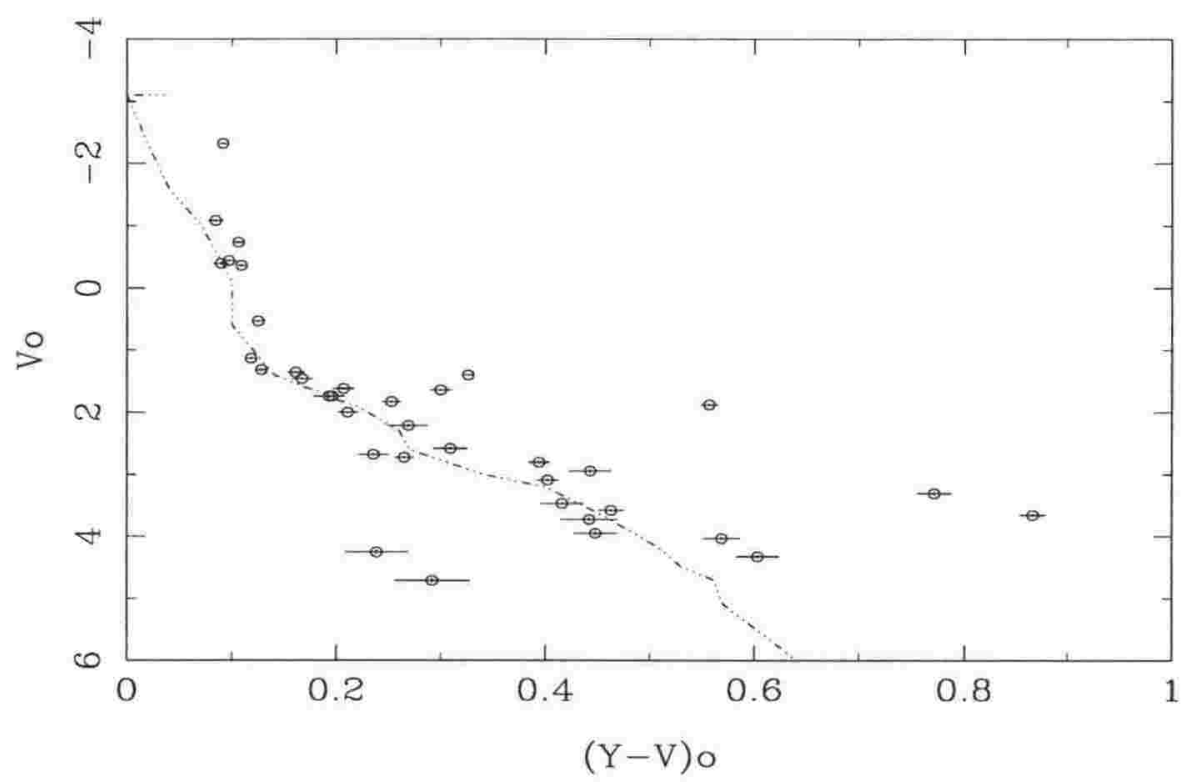

Colour Magnitude diagram: probable members of $\mathrm{O}$ Vel

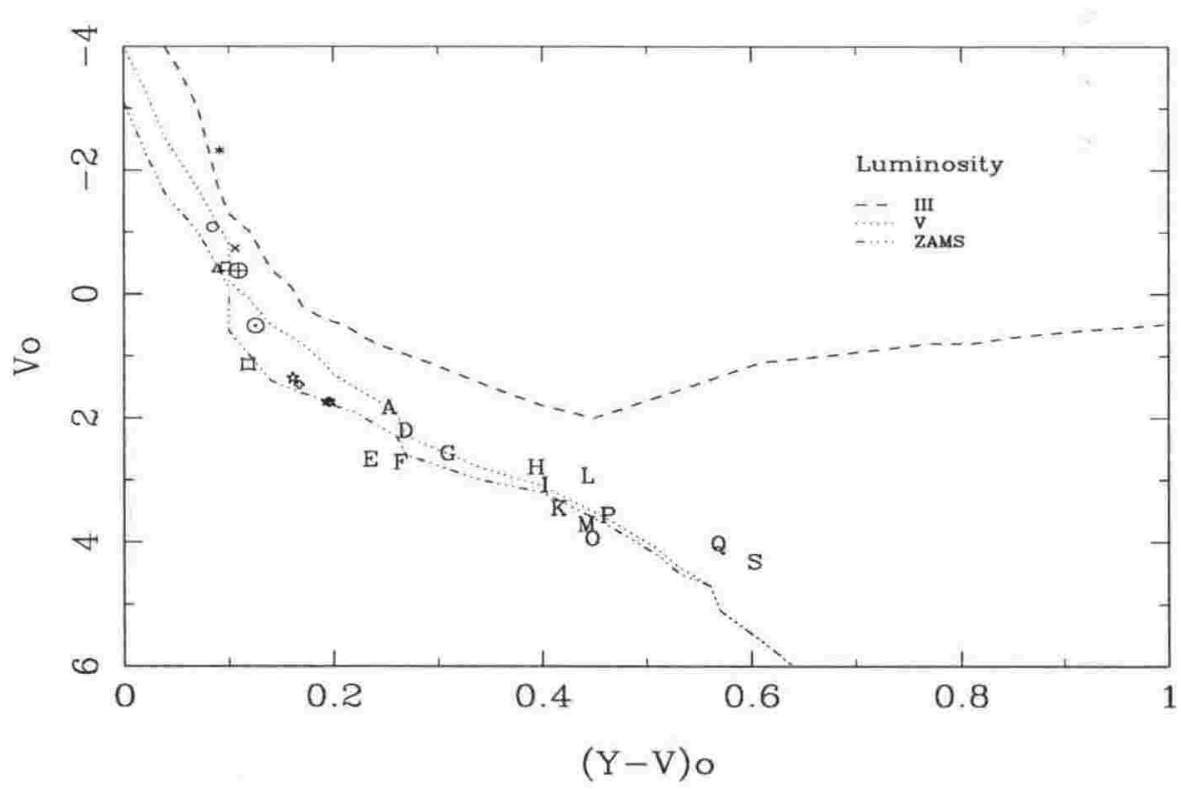

Legend

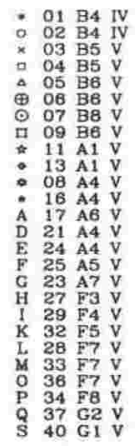

Figure 4.14: Colour-Magnitude diagrams of $\mathrm{O}$ Vel from Vilnius photometry. The top diagram, with all candidate members of the cluster, has error bars indicating the size of the standard deviation of the mean magnitudes and colours for each star, and the ZAMS taken from Straižys (1992) (shown by a dashed line). The bottom diagram shows the probable cluster members (according to median column of Table 4.14) and the ZAMS, main, and giant sequences. 
the median age from the literature $\left(30 \times 10^{6}\right.$ years, see Table 4.1$)$. 

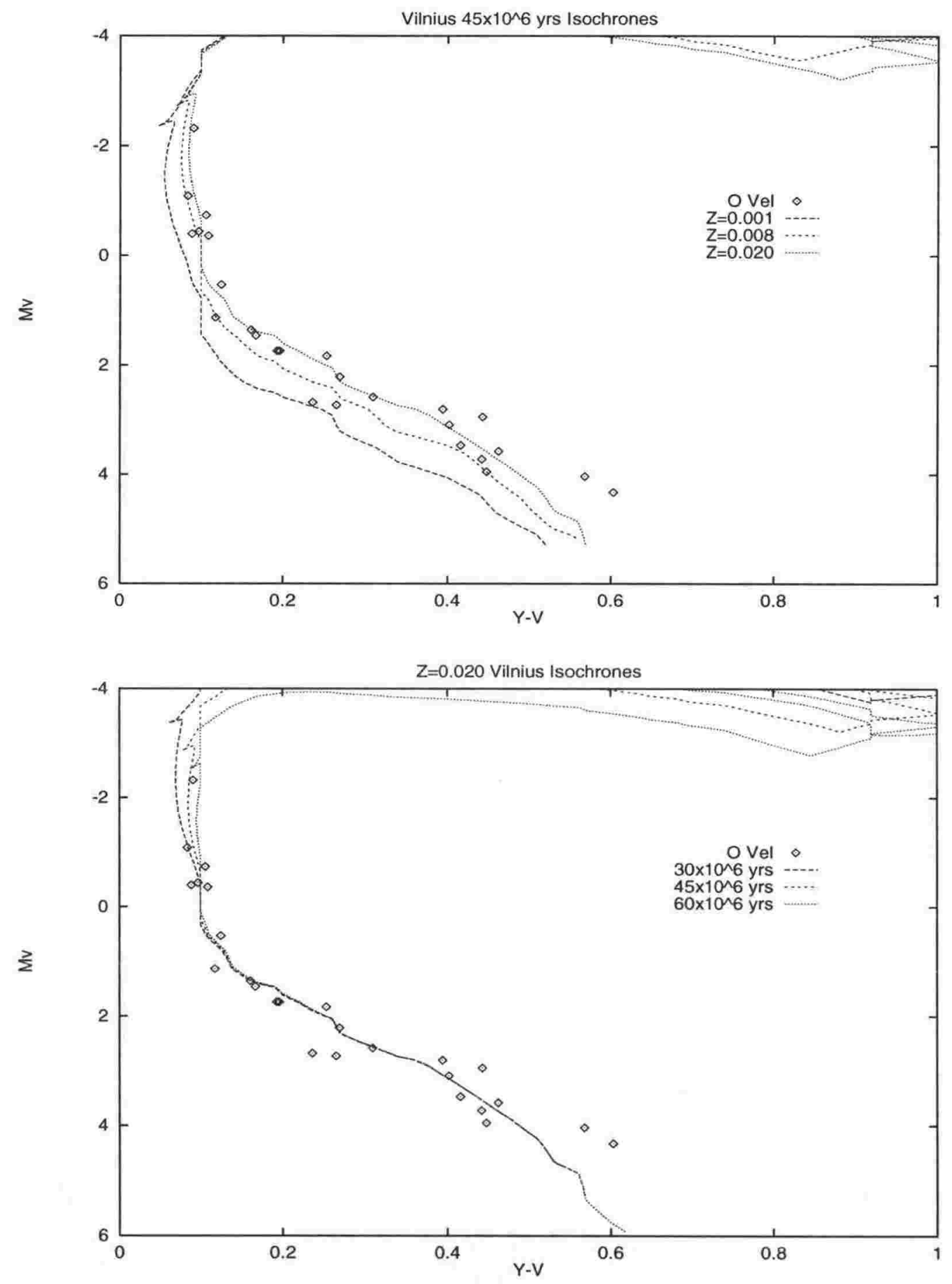

Figure 4.15: Vilnius isochrones for $\mathrm{O}$ Vel. The top diagram compares different metallicities (for an approximate age) to the probable members of $\mathrm{O}$ Vel, while the bottom diagram compares different ages for the best fitting metallicity. 
Table 4.13: Spectral Classifications of O Vel stars from Vilnius photometry.

\begin{tabular}{|c|c|c|c|c|c|c|c|}
\hline \# & Q-Search & \multicolumn{2}{|c|}{ Q-Spectra } & \multicolumn{3}{|c|}{ QQ-diagrams } & average \\
\hline 1 & B3-5 IV-V & B3-4 IV & 1.00 & $\mathrm{~B} 3 \cdot 5$ & -1 & & B4 IV \\
\hline 2 & B3-5 IV-V & B5 V (p) & $1 \cdot 00$ & B4 & $-0 \cdot 2$ & dble? & B4 IV \\
\hline 3 & $\mathrm{~B} 6 \mathrm{~V}(\mathrm{SB})$ & B5 $\mathrm{V}$ & 1.00 & B5 & +0.05 & & B5 V \\
\hline 4 & B4-6 V (SB) & B5 V & 1.50 & B $5 \cdot 3$ & $+0 \cdot 2$ & & B5 V \\
\hline 5 & B5-7 IV-V (SB) & B6 V & $1 \cdot 20$ & B6 & +0.5 & dble? & B6 V \\
\hline 6 & B5-7 III-V & B5 III & $1 \cdot 00$ & B6 & +0.05 & & B6 V \\
\hline 7 & B9 V & B8 IV-V & $1 \cdot 00$ & B $8 \cdot 1$ & +1.05 & & B8 $\mathrm{V}$ \\
\hline 8 & A1-5 V & A5 V & $1 \cdot 00$ & A3 & $+2 \cdot 1$ & & $\mathrm{~A} 4 \mathrm{~V}$ \\
\hline 9 & B7-9 III-V & $\mathrm{B} 6 \mathrm{~V}$ & $1 \cdot 40$ & B 6.5 & +0.5 & & $\mathrm{~B} 6 \mathrm{~V}$ \\
\hline 10 & A1 IV-V (SB?) & A1 V & $0 \cdot 65$ & A1 & +2 & $\mathrm{Bp}(\mathrm{Si})$ ? or $\mathrm{Ap}$ ? & A $1 \mathrm{~V}$ \\
\hline 11 & A0-2 V & A1 V & $1 \cdot 00$ & A1 & +2 & & A1 V \\
\hline 12 & A6-F1 III-V & A7 V & 1.00 & A7 & +2.8 & Ap? & A7 V \\
\hline 13 & A0-2 V & A2 V & $1 \cdot 00$ & A1 & $+2 \cdot 5$ & & A $1 \mathrm{~V}$ \\
\hline 14 & A0-4 V & $A 3 V(p)$ & 1.00 & A3 & $+2 \cdot 4$ & & A3 V \\
\hline 15 & A4-F1 IV-V (m?) & F0 IV & 1.00 & A 6.5 & $+3 \cdot 1$ & & $\mathrm{~A} 6 \mathrm{~V}$ \\
\hline 16 & A0-4 V & A5 V & $1 \cdot 00$ & A3 & $+2 \cdot 6$ & & A $4 \mathrm{~V}$ \\
\hline 17 & A4-8 V (m) & A7 V & 1.00 & A6 & $+2 \cdot 4$ & Am? & $\mathrm{A} 6 \mathrm{~V}$ \\
\hline 18 & G4-8 V & G2 V & $1 \cdot 00$ & G5? & $+6-7$ & sd? & G4 V \\
\hline 20 & B3-7 IV-V & $\mathrm{B} 6 \mathrm{~V}$ & 0.89 & B5. 3 & $-0 \cdot 3$ & reddened? & B5 V \\
\hline 21 & A3-9 V & $\mathrm{A} 3 \mathrm{~V}$ & 0.85 & $\mathrm{~A} 6$ & $+2 \cdot 2$ & & $\mathrm{~A} 4 \mathrm{~V}$ \\
\hline 23 & A3-F0 V (m) & A7 V & $1 \cdot 10$ & A7 & $+3 \cdot 1$ & Ap? & A7 V \\
\hline 24 & A1-7 V & A3 IV & $1 \cdot 00$ & A4 & $+2 \cdot 0$ & & A $4 \mathrm{~V}$ \\
\hline 25 & A3-7 V & A5 V & 1.00 & A6 & $+2 \cdot 7$ & Am? & A5 V \\
\hline 27 & F1-5 IV-V & $\mathrm{F} 2 \mathrm{~V}$ & $1 \cdot 00$ & F4 & $+3 \cdot 5$ & & F3 V \\
\hline 28 & F6-8 V & F8 V (p) & $1 \cdot 00$ & $\mathrm{~F} 6$ & +4 & & F7 V \\
\hline 29 & F2-6 III-V & F5 V & $1 \cdot 00$ & F4 & $+3 \cdot 5$ & & F4 V \\
\hline 30 & G8-K0 III & K0 IV & $1 \cdot 00$ & G9-K0 & +1.8 & & K0 IV \\
\hline 32 & F5-7 V & F5 V & $0 \cdot 91$ & F6 & +4 & Am? & F5 V \\
\hline 33 & $\mathrm{~F} 6-8 \mathrm{~V}$ & $\mathrm{~F} 8 \mathrm{~V}$ & 0.93 & F6 & +5 & & F7 V \\
\hline 34 & F7-9 V & F8 V (p) & 0.90 & $\mathrm{~F} 2$ ? & $+5 ?$ & & $\mathrm{~F} 8 \mathrm{~V}$ \\
\hline 35 & K2 V & K3 V (p) & 1.05 & K2 & $+4 \cdot 2$ & sd? or carbon? & $\mathrm{K} 2 \mathrm{~V}$ \\
\hline 36 & F6-8 V & F8 V (p) & 0.88 & F5-6 & $+4-5$ & & F7 V \\
\hline 37 & G1-7 V & G2 V (p) & $1 \cdot 00$ & G5? & $+6-7$ & sd? & G2 V \\
\hline 38 & A2-6 IV-V & A2 III & $0 \cdot 83$ & A4 & $+1 \cdot 7$ & & A3 V \\
\hline 40 & G0-4 IV-V & G0 V (p) & 1.06 & G9 & $+7-8$ & sd & G1 V \\
\hline 41 & B6-8 III-V & B6 V (p) & 1.09 & $\mathrm{~B} 6 \cdot 7$ & +0.6 & $\mathrm{Be}$ ? or reddened? & $\mathrm{B} 6 \mathrm{~V}$ \\
\hline
\end{tabular}


Table 4.14: Membership of O Vel stars from Vilnius photometry. The second column is based on the distance modulus and colour excess analysis from the Vilnius photometry. The third column is the revised memberships (showing only the changes from the previous column), based on the spectral classes sequence in the CMD. The fourth column is copied from the median column of Table 4.7 for comparison.

\begin{tabular}{|c|c|c|c|c|c|c|c|}
\hline \# & \multicolumn{3}{|c|}{ member? } & \multirow[t]{3}{*}{ \# } & \multicolumn{3}{|c|}{ member? } \\
\hline \# & \multicolumn{2}{|c|}{ Vilnius } & \multirow{2}{*}{$\begin{array}{l}\text { Literature } \\
\text { (median) }\end{array}$} & & \multicolumn{2}{|c|}{ Vilnius } & \multirow{2}{*}{$\begin{array}{c}\text { Literature } \\
\text { (median) }\end{array}$} \\
\hline & $\mathrm{m}-\mathrm{M}$ & Sp & & & $\mathrm{m}-\mathrm{M}$ & $\mathrm{Sp}$ & \\
\hline 1 & yes & & yes & 20 & no & & no \\
\hline 2 & yes & & yes & 21 & yes & & yes \\
\hline 3 & yes & & yes & 23 & yes & & yes \\
\hline 4 & yes & & yes & 24 & yes & & yes \\
\hline 5 & yes & & yes & 25 & yes & & maybe \\
\hline 6 & yes & & yes & 27 & yes & & maybe \\
\hline 7 & yes & & yes & 28 & yes & & maybe \\
\hline 8 & yes & & yes & 29 & yes & & yes \\
\hline 9 & yes & maybe & maybe & 30 & no & & (no) \\
\hline 10 & maybe & & yes & 32 & yes & & yes \\
\hline 11 & yes & & yes & 33 & yes & & yes \\
\hline 12 & maybe & & maybe & 34 & yes & & yes \\
\hline 13 & yes & & yes & 35 & no & & no \\
\hline 14 & maybe & & yes & 36 & yes & & yes \\
\hline 15 & maybe & & maybe & 37 & yes & & (no) \\
\hline 16 & yes & & yes & 38 & no & & no \\
\hline 17 & yes & maybe & no & 40 & maybe & & yes \\
\hline 18 & no & & no & 41 & no & & (no) \\
\hline
\end{tabular}




\subsection{Literature review of the Kappa Crucis cluster.}

The $\kappa$ Crucis cluster ${ }^{8}$ is located at RA $=12^{\mathrm{h}} 53^{\mathrm{m}}$, Dec $=-60^{\circ} 17^{\prime}$ or galactic coordinates $1=303^{\circ}, \mathrm{b}=+2^{\circ}$, with an angular diameter of $\sim 5 \cdot 1^{\prime}$ (Dachs \& Kaiser 1984). The numbering scheme of Dachs \& Kaiser was adopted, which is based on the first photoelectric study of the cluster by Arp \& Van Sant (1958) with the quadrant number becoming the hundred's digit, for example II-06 becomes 206 .

A summary of the cluster's characteristics found by different authors is given in Table 4.15. Arp \& Van Sant (1958) find the cluster age between that of the $h+\chi$ Persei and Pleiades clusters, with star A assumed to be at the Schonberg-Chandrasekhar limit giving an age of $3 \times 10^{6}$ years. Assuming $E_{B-V}=0.44$, Hernández (1960) calculated the cluster's distance via an absolute magnitude/spectral type calibration. The other authors used a variety of similar calibrations involving intrinsic colours or reddening-free parameters, for example Graham (1967) used a preliminary $H_{\beta}$ /absolute magnitude calibration and a UBV intrinsic colour/spectral type calibration to derive reddening and distance of the cluster. Shobbrook (1984) converted $E_{b-y}=0.44$ to $E_{B-V}$ using the colour excess ratio $E_{b-y} / E_{B-V}=0.74$, while the mean reddening $E_{b-y}=0.278$ found by Balona \& Koen (1994) was converted using the colour excess ratio appropriate to B-type stars $\left(E_{b-y} / E_{B-V}=0.77\right.$, from Straižys (1992, Table 34)). Dachs \& Kaiser (1984) find two overlapping ages for the cluster, based on age calibrations of the intrinsic colours of the bluest stars in a cluster and the bolometric magnitudes of cluster blue supergiants, while Balona \& Koen fit isochrones to the CMD.

As the calculated intrinsic magnitude is a function of both the apparent magnitude and colour excess $\left(V_{0}=V-R E_{B-V}\right.$, see section 1.4), it follows that the derived cluster distance and colour excess will be correlated. Hence, to compare results from the different authors, the colour excesses and distance modulus were plotted in Figure 4.16. This shows that all except Arp \& Van Sant (1958), Becker (1961, 1963), and Mermilliod \& Maeder (1986) fall about the straight line defined by the median value with a slope equal to the ratio of extinction to colour excess ( $R$, taken as 3.0). However the values from Mermilliod \& Maeder are "critically evaluated" averages from a literature survey - as their distance modulus is significantly above the main trend, this implies there must be other results (with greater distance moduli) that are not included in Table 4.15.

Different authors have used a variety of other naming systems for the stars in the cluster, which have been summarized in Table 4.16. The RA and Dec (in hours, minutes, seconds and degrees, minutes, seconds respectively) are taken from King (1980) and are to the Epoch 1950. Coordinates marked with an asterisk were calculated by calibrating the $\mathrm{CCD}(\mathrm{x}, \mathrm{y})$ coordinates

\footnotetext{
${ }^{8}$ So named due to the bright star $\kappa$ Crucis (star B using the numbering system adopted here) near the middle of the cluster. The $\kappa$ Crucis cluster is also known as NGC 4755 or C1250-600 using it's IAU designation.
} 
Table 4.15: Review of main characteristics of Kappa Crucis.

\begin{tabular}{|c|c|c|c|}
\hline author & $\begin{array}{c}\text { distance } \\
(p c)\end{array}$ & $\begin{array}{c}\text { reddening } \\
\left(E_{B-V}\right)\end{array}$ & $\begin{array}{c}\text { age } \\
\text { (years) }\end{array}$ \\
\hline Arp \& Van Sant (1958) & $830 \pm 230$ & $0.31 \pm 0.03$ & $3 \times 10^{6}$ \\
\hline Wallenquist (1959) & $1445 \pm 200$ & & \\
\hline Hernández (1959) & 2630 & & \\
\hline Hernández (1960) & 2100 & 0.44 (assumed) & \\
\hline Becker (1961) & 1585 & 0.44 & \\
\hline Becker (1963) & 1050 & 0.31 & \\
\hline Buscombe (1963) & 2290 & 0.40 & \\
\hline Feast (1963) & $2355 \pm 150$ & $0.48 \pm 0.01$ & \\
\hline Kennedy (1966) & 2400 & 0.46 & \\
\hline Graham (1967) & $2250 \pm 1000$ & 0.40 & \\
\hline Schild $(1970)$ & 2090 & 0.42 & \\
\hline Becker \& Fenkart (1970) & 1035 & 0.31 & \\
\hline Perry et al. (1976) & $2290 \pm 210$ & $0.40 \pm 0.01$ & \\
\hline Kaiser (1980) & 2290 & 0.44 & \\
\hline Mermilliod (1981) & 1845 & $0 \cdot 35$ & \\
\hline Shobbrook (1984) & 1900 & $0.38 \pm 0.03$ & \\
\hline de Waard et al. (1984) & $1780 \pm 200$ & $0.36 \pm 0.04$ & \\
\hline Dachs \& Kaiser (1984) & $2310 \pm 70$ & $0.44 \pm 0.04$ & $\begin{array}{l}8 \cdot 3-11.5 \times 10^{6} \\
7 \cdot 8-10 \cdot 4 \times 10^{6}\end{array}$ \\
\hline Brown et al. (1986) & $1910 \pm 330$ & $0.39 \pm 0.04$ & \\
\hline Mermilliod \& Maeder (1986) & 3090 & 0.35 & $11 \times 10^{6}$ \\
\hline Balona \& Koen (1994) & $2040 \pm 47$ & $0.36 \pm 0.05$ & $(10 \pm 2) \times 10^{6}$ \\
\hline median & 2075 & 0.40 & $10 \times 10^{6}$ \\
\hline
\end{tabular}

of Balona \& Koen (1994) with King's coordinates, and using the CCD coordinates to fill in the equatorial coordinates missing from King's list. The two coordinates marked with ':' were interpolated from known coordinates of other stars using the finding chart (Figure 4.17). The $\mathrm{CD}, \mathrm{CPD}$ and $\mathrm{CPC}$ numbers are for declination $-59^{\circ},-59^{\circ}$ and zone 20.1 respectively. The question marks indicate there is some disagreement between authors regarding the catalog number, for example CPD $-59^{\circ} 4553$ is listed as star 103 by several authors but given as star $\mathrm{R}$ by the SIMBAD database. Where two numbers are given, the first is from the SIMBAD database while the second is from Perry et al. (1976) and later authors.

A star chart of $\kappa$ Crucis suitable for viewing through a finder scope was prepared using the numbering scheme of Dachs \& Kaiser (1984) and is shown in Figure 4.17.

Table 4.17 gives the spectral classifications found in the literature for $\kappa$ Crucis, which are all based on spectroscopic observations. The authors are, in left to right order, Bidelman (1954), Arp \& Van Sant (1958), Hernández (1960), Feast (1963), Schild (1970), Sowell (1987), and Slettebak (1985). The results of Arp \& Van Sant are from the unpublished (1958) observations of Gratton and Hernandez. They also quote Bidelman's spectral classes, but mix up stars A and C according to Hernández (1960). The most complete list is from Feast, with the other 
Correlation between distance modulus and reddening for $\mathrm{K}$ Cru

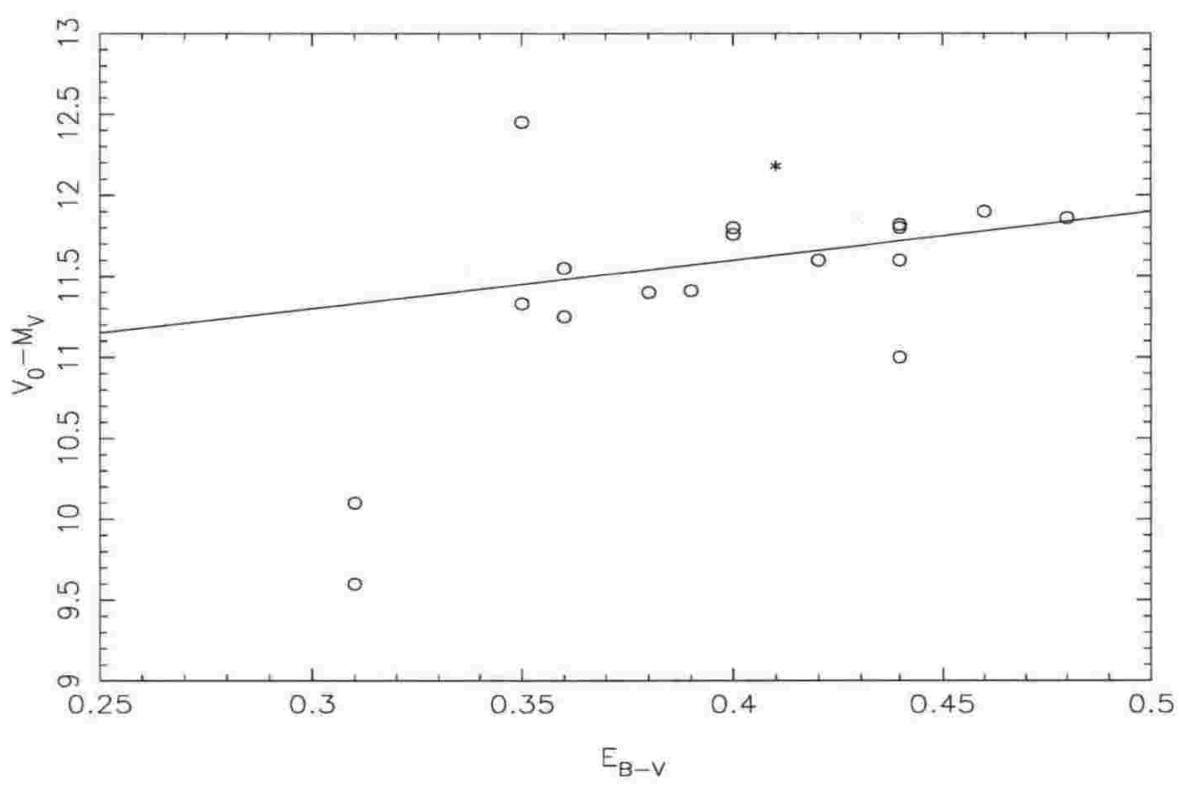

Figure 4.16: Distance modulus and colour excess for Kappa Crucis from the literature. The straight line passes through the median value (see Table 4.15) with a slope $R$ (the ratio of extinction to colour excess, taken as 3.0 ). The asterisk symbol is the value from this study, after converting from the Vilnius to Johnson systems.

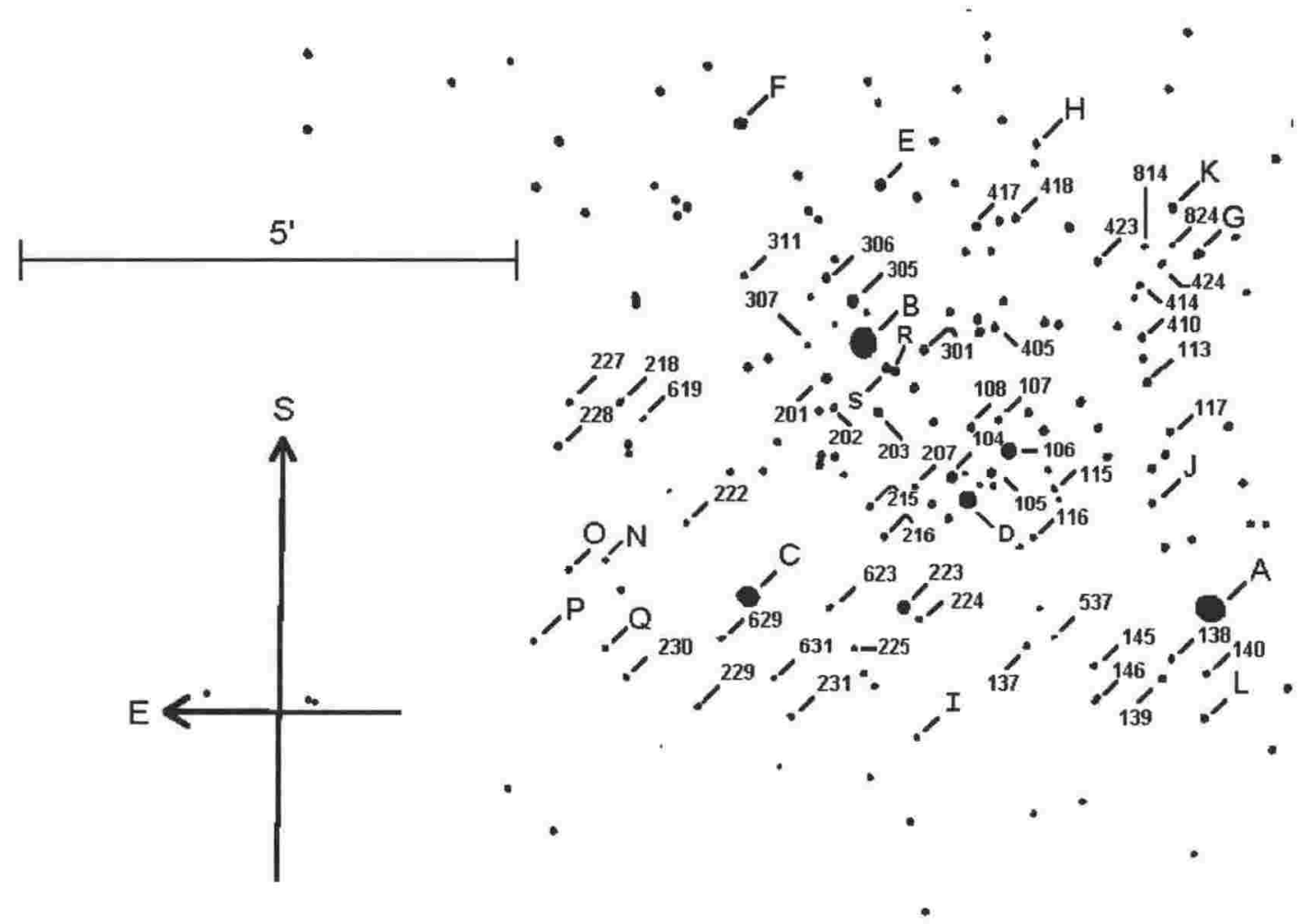

Figure 4.17: Star Chart for Kappa Crucis. The cluster is approximately centered around $\mathrm{RA}=12^{\mathrm{h}} 53^{\mathrm{m}}$, Dec $=-60^{\circ} 17^{\prime}$. 
Table 4.16: Synonyms for Kappa Crucis's stars.

\begin{tabular}{|c|c|c|c|c|c|c|c|c|}
\hline D\&K & RA & Dec & HD & $\mathrm{SAO}$ & $\mathrm{HR}$ & CD & CPD & $\mathrm{CPC}$ \\
\hline A & 125022 & -600325 & 111904 & 252069 & 4887 & 4455 & 4529 & 3732 \\
\hline B & 125049 & $\begin{array}{lll}-60 & 06 & 17\end{array}$ & 111973 & 252077 & 4890 & 4460 & 4555 & 3740 \\
\hline C & 125100 & -600351 & 111990 & 252080 & & 4461 & 4566 & 3743 \\
\hline D & 125041 & -600441 & & 252073 & & 4459 & 4547 & 3736 \\
\hline $\mathrm{E}$ & 125046 & -600755 & & 252074 & & & 4552 & 3737 \\
\hline $\mathrm{F}$ & 125057 & $\begin{array}{llll}-60 & 08 & 41\end{array}$ & & 252078 & & & 4564 & 3741 \\
\hline G & 125021 & -600659 & & & & 4454 & 4528 & \\
\hline $\mathrm{H}$ & 125033 & -600816 & & & & & 4540 & \\
\hline I & 125047 & -600219 & & & & & 4550 & \\
\hline $\mathbf{J}$ & 125026 & -600431 & & & & & 4537 & \\
\hline $\mathrm{K}$ & 125023 & -600730 & & & & & 4530 & \\
\hline $\mathrm{L}$ & *12 5024 & -600219 & & & & & & \\
\hline M & *12 5019 & -600233 & & & & & & \\
\hline 104 & *12 5042 & -600456 & & & & & & \\
\hline 105 & 125047 & $\begin{array}{lll}-60 & 06 & 02\end{array}$ & & & & & $4553 ?$ & \\
\hline 106 & 125038 & -600508 & 111934 & 252070 & & 4458 & 4543 & 3734 \\
\hline 107 & *12 5038 & -600527 & & & & & & \\
\hline 108 & *12 5036 & -600530 & & & & & & \\
\hline 113 & 125026 & -600543 & & & & & 4532 & \\
\hline 115 & 125034 & -600443 & & & & & 4541 & \\
\hline 117 & 125024 & -600514 & & & & & 4531 & \\
\hline 137 & *12 5038 & $\begin{array}{lll}-60 & 0310\end{array}$ & & & & & & \\
\hline 138 & 125027 & -600243 & & & & & 4536 & \\
\hline 139 & *125027 & -600243 & & & & & & \\
\hline 201 & 125052 & -600559 & & & & & $4558 ?$ & \\
\hline 202 & 125053 & -600514 & & & & & $4562 / 58 ?$ & \\
\hline 203 & 125049 & -600646 & & & & & $4557 / 66 ?$ & \\
\hline 207 & ${ }^{*} 125045$ & -600451 & & & & & & \\
\hline 215 & *12 5049 & -600441 & & & & & & \\
\hline 216 & $: 125048$ & -600425 & & & & & & \\
\hline 218 & :125108 & -600547 & & & & & & \\
\hline 223 & 125047 & -600338 & & 252075 & & & 4551 & 3738 \\
\hline 224 & *12 5046 & $\begin{array}{llll}-60 & 03 & 31\end{array}$ & & & & & & \\
\hline 228 & 125114 & -600531 & & & & & 4571 & \\
\hline 305 & 125051 & -600700 & & & & & $4559 / 57 ?$ & \\
\hline 306 & 125052 & -600737 & & & & & $4561 / 59 ?$ & \\
\hline 311 & 125058 & -600706 & & & & & 4565 & \\
\hline 405 & 125038 & $\begin{array}{lll}-60 & 06 & 22\end{array}$ & & & & & 4544 & \\
\hline 410 & *12 5026 & -600609 & & & & & 4533 & \\
\hline 414 & 125026 & -600642 & & & & & 4535 & \\
\hline 417 & 125039 & -600727 & & & & & 4546 & \\
\hline 418 & 125035 & -600730 & & & & & 4542 & \\
\hline
\end{tabular}


authors generally showing good agreement - the major exceptions are stars D, F and G from Arp \& Van Sant and star A from Sowell. However Sowell has found that he obtained latertype classifications for O-F type stars when comparing his results with others. Comparison with the apparent V magnitude (see Table 4.20) shows the luminosity classes scale with the magnitude, with stars brighter than $8^{m} \cdot 5$ being luminosity I, fainter than $\sim 9^{m} \cdot 5$ are luminosity $\mathrm{V}$ and in-between are luminosity III.

Only one proper motion study of $\kappa$ Crucis could be found in the literature, that of King (1980) - this paucity is probably due to the difficulty in measuring proper motions due to the cluster's large distance $(2075 \mathrm{pc}$ ). King's results for the stars observed in this study are given in Table 4.18, while the proper motion histograms for all of King's data is plotted in Figure 4.18. These show approximately normal distributions, but with an excessive tail centred around $\mathrm{RA}=-100 \mathrm{x} 0 \cdot 01^{\prime \prime} / 100 \mathrm{yr}$. Splitting the data above and below the median value of King's photographic magnitudes $\left(11^{m} \cdot 3\right)$ shows a greater proportion of the fainter stars have proper motions in this tail region than the brighter stars, suggesting this corresponds to the proper motion of non-members. King also assigned membership probabilities on the basis of the star's proper motion (see Table 4.19) and using a 50\% threshold, concluded that 89 stars were likely members and 75 stars were likely non-members.

Table 4.18 also contains the radial velocities found in the literature, in left to right order, from Hernández (1960), Feast (1963), Marraco \& Orsatti (1982), and Sowell (1987), with Feast giving the most complete set of radial velocities. As nearly $50 \%$ of the stars do not have radial velocity measurements, no further analysis was performed other than to note possible variables.

There are only three studies that explicitly assess cluster membership of the stars; that of King (1980), Dachs \& Kaiser (1984), and Shobbrook (1984). Shobbrook lists several stars as possible foreground objects, but none of these were observed here so only the results from King and Dachs \& Kaiser are given in Table 4.19. King's are based on proper motions and are given as a percentage. Dachs \& Kaiser derive their memberships based on the differences from the mean distance modulus and reddening, using categories 1-4 denoting 'certain', 'probable', 'possible' and 'impossible' membership respectively. To calculate the average membership, the categories were converted to percentages (the thresholds were $75 \%, 50 \%$, and $25 \%$ ) and the resulting average re-categorized. This results in only one star being considered a non-member (star 104).

Table 4.20 lists all the visual magnitudes in the literature, from Arp \& Van Sant (1958), Graham (1967), Schild (1970), Perry et al. (1976), Grønbech \& Olsen (1976), McGregor \& Hyland (1984), de Waard et al. (1984), Dachs \& Kaiser (1984), Shobbrook (1984), and Balona \& Koen (1994). Arp \& Van Sant observed stars A to Q photoelectrically, with the remaining stars measured photographically. Graham quotes some UBV results as unpublished by the 

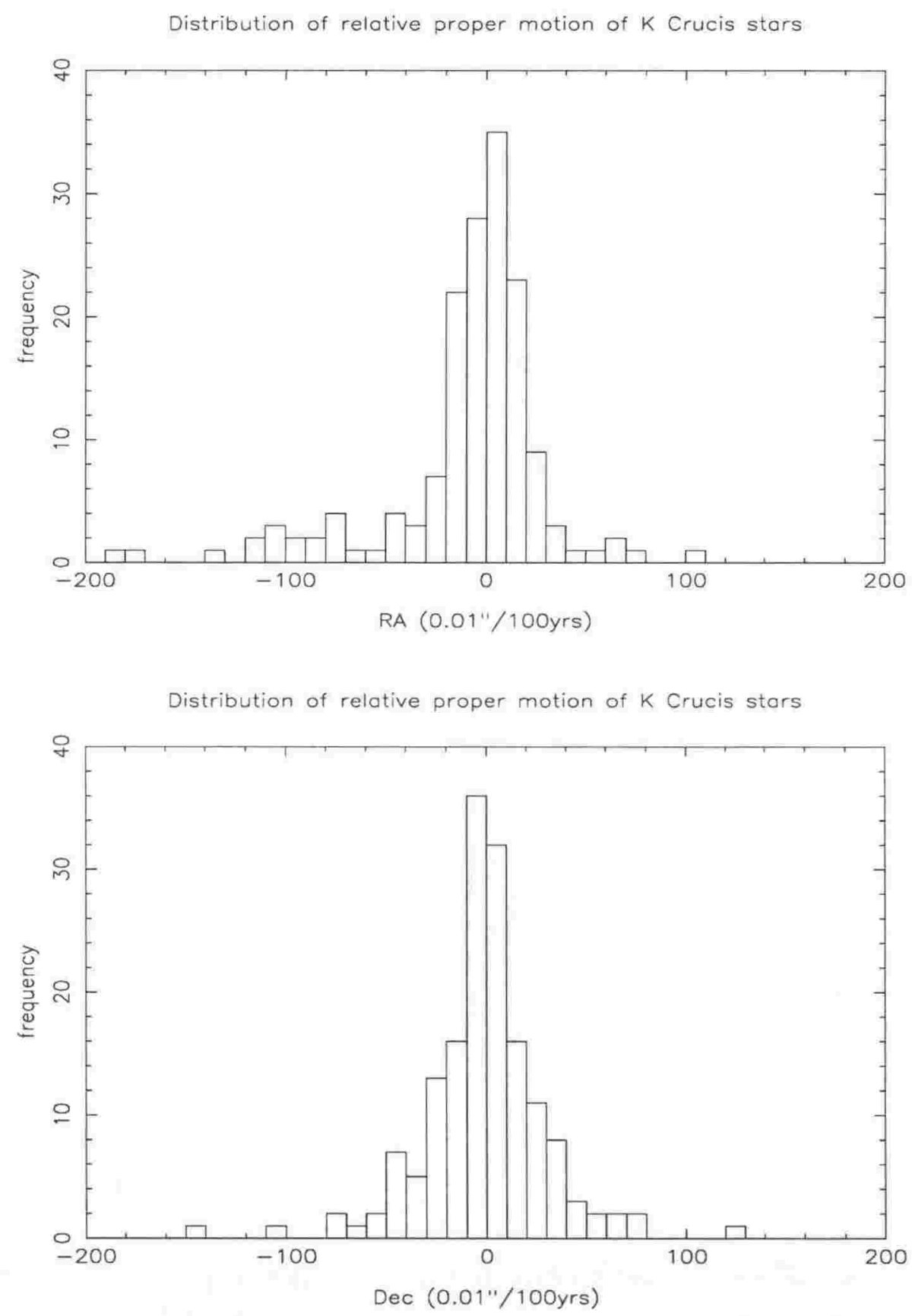

Figure 4.18: Proper motions histograms for Kappa Crucis, using all the data from King (1980) rather than just from Table 4.18. 
observer (A. R. Hogg), but they had in fact being published by Hogg (1958) according to Marraco \& Orsatti (1982). Perry et al. made observations in both the UBV and uvby systems, combining the Johnson V and Strömgren $y$ to form the visual magnitude. Grønbech \& Olsen observed one $\kappa$ Crucis cluster star (B) as part of their southern bright star uvby catalog, while McGregor \& Hyland used a red-ward extension of the UBV system (BVHJK) to examine latetype cluster supergiants (measuring star D in $\kappa$ Crucis). de Waard et al. observed in the Walraven 5 channel system and converted their results to the UBV system. Dachs \& Kaiser measured 86 stars photoelectrically, and a total of 533 stars photographically in the UBV system, with only the photoelectric results being given in Table 4.20. Shobbrook and Balona \& Koen both observed in the uvby system, quoting $y$ as a visual magnitude. As only two stars are not B-type, comparisons between the different authors were not expected to allow any colour dependent systematic errors in the $\mathrm{V}$ magnitude to be found (and none were seen). One magnitude dependent systematic error was found; Arp \& Van Sant consistently measure the faintest stars (below $12^{m} \cdot 0$ ) as too bright compared to all other authors - however the brighter stars show no systematic deviations. Another possible source of error is due to the crowded nature of the cluster; most stars have faint companion stars, or could be affected by scattered light from the bright cluster stars, either of which being included by differing amounts in the photometry of each author. Therefore a median (rather than a mean, which would be strongly affected by the systematic differences of Arp \& Van Sant) was calculated to reduce both the systematic and random errors.

Several authors have conducted investigations which included specific searches for variable stars in the cluster. Their findings are summarized in Table 4.21, with the authors being Jakate (1978), Shobbrook (1984), Dachs \& Kaiser (1984), Koen (1993), and Balona \& Koen (1994). Jakate searched for $\beta$ Cephei stars, selecting as candidates stars having B0.5-2 III-V spectral classifications. While Shobbrook did not specifically search for variables, his results confirmed the variability of star $\mathrm{F}$, found an additional variable ( $\operatorname{star} \mathrm{H}$ ) but found star 418 to be constant, contradicting the results of Jakate (however Shobbrook considered the possibility that star 418 was multi-periodic and had been observed during a low amplitude phase). However as the other two authors who find star 418 to be constant can easily reach the precision required to detect the apparent variability found by Jakate, and as it is unlikely that the star, if multiperiodic, would be observed all three times during a low amplitude phase, then it is probable that star 418 is actually constant. Dachs \& Kaiser conclude star D was variable by comparing their results with Arp \& Van Sant (1958) and Schild (1970) - however as star D is the only late-type star in the cluster, it seems more likely the differences are due to discrepancies in the standardisation transformations. They also find star 223 to be variable, contradicting Jakate who found it constant. However Dachs \& Kaiser monitored this star over several days (finding 
a period of $3 \cdot 24$ days) while Jakate only measured the star for six hours. Koen conducted a further search for $\beta$ Cephei stars, confirming most of Jakate's results. Finally, Balona \& Koen conducted a special CCD search for variable stars, following up with photoelectric photometry to refine the periods and confirming most of the variables found earlier by Jakate or Koen. The apparent contradictions between different authors (for stars 113, 223, 306, and 417) can be easily explained by differences in the precision and time-scale of the measurements as all these stars have low amplitude variations and/or long periods.

There have been seven investigations of $\kappa$ Crucis in the UBV system, by Arp \& Van Sant (1958), Graham (1967), Schild (1970), Perry et al. (1976), McGregor \& Hyland (1984), de Waard et al. (1984), and Dachs \& Kaiser (1984). These are listed in Table 4.22, with the exception of McGregor \& Hyland who observed only one star in common with this study ( star $\mathrm{D}, \mathrm{B}-\mathrm{V}=2 \cdot 20$ ). Arp \& Van Sant do not have any U-B colours as they used a very early U-type filter (giving a colour index $\mathrm{Cu}$ ) which could not be transformed to the Johnson system. The other authors have been previously discussed in the visual magnitudes section. Some of the earlier investigations (Arp \& Van Sant, Graham, Schild) show systematic differences compared to the later measurements so the median values will be taken as best representing the standard system.

Table 4.23 lists the $E_{B-V}$ reddening found in the literature; the authors are Arp \& Van Sant (1958), Graham (1967), Schild (1970), Perry et al. (1976), Shobbrook (1984), de Waard et al. (1984), and Dachs \& Kaiser (1984). Arp \& Van Sant use two methods; the first based on spectral classes given by Bidelman (1954) or Gratton \& Hernández (unpublished), the second method used a colour-colour diagram. Graham used a preliminary $\beta /$ absolute magnitude calibration, while Schild used a colour-spectral type calibration. Perry et al. used several methods, all based on either intrinsic colours and/or spectral type calibrations. Shobbrook gives the reddening as $E_{\mathrm{b}-\mathrm{y}}$, which are converted to $E_{\mathrm{B}-\mathrm{v}}$ using the colour excess ratio appropriate for B-type stars. de Waard et al. converted their results from the Walraven system, while Dachs \& Kaiser did both photoelectric and photographic observations in the UBV system. In addition, Sowell (1987) found star D to have a large infrared excess, suggesting the presence of a thick dust cloud but doesn't give an $E_{\mathrm{B}-\mathrm{V}}$ value.

The distribution of the median reddening from the literature is plotted in Figure 4.19. This gives a median reddening for the cluster of $E_{\mathrm{B}-\mathrm{V}}=0.40$ and a mean of $0.41 \pm 0.04$. The small standard deviation implies a constant reddening for the cluster, while the literature gives mixed results. For instance, Arp \& Van Sant (1958) implicitly assume constant reddening, Balona \& Koen (1994) finds uniform reddening as does Mermilliod (1981) (based on a literature survey) and Shobbrook (1984). Further, Shobbrook concludes all the absorption is in front of the cluster while Feast (1963) finds most of the absorption is not associated with the cluster 
Distribution of reddening of $K$ Crucis stars

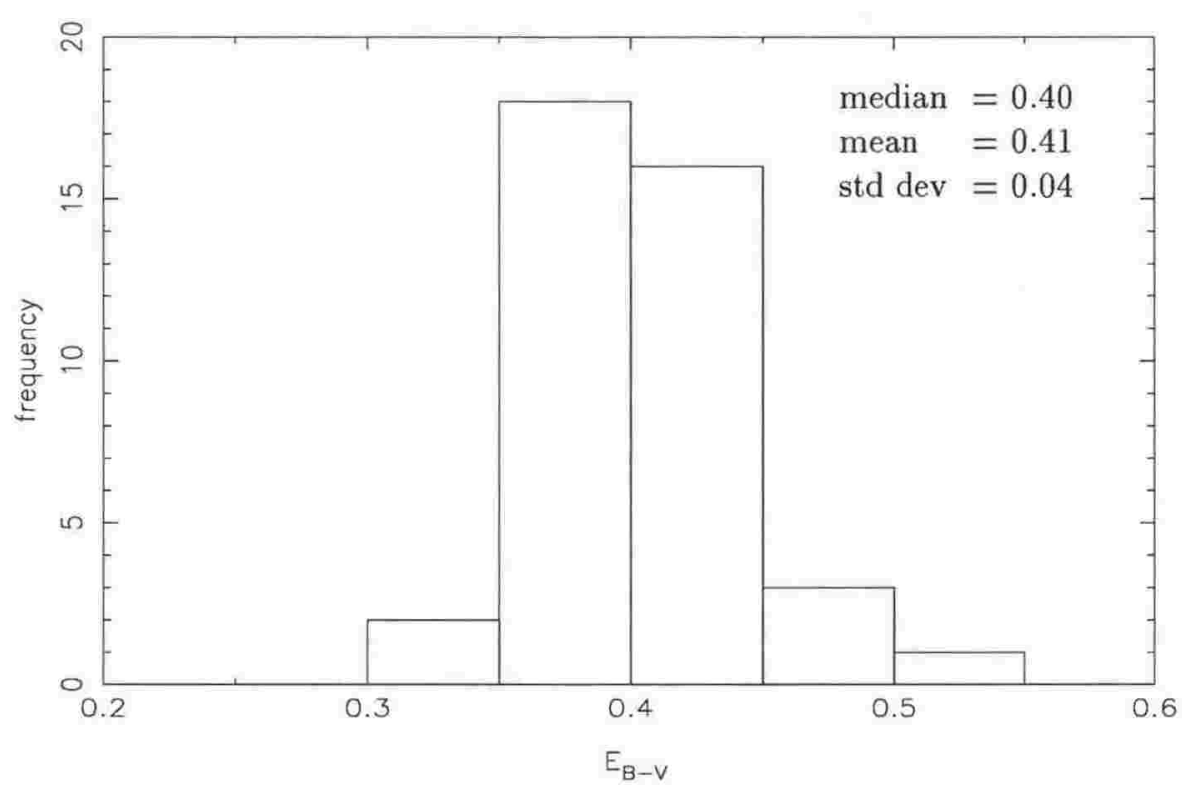

Figure 4.19: Colour excesses $E_{B-V}$ for Kappa Crucis stars from the literature (see the median values from Table 4.23).

(i.e. in front). However de Waard et al. (1984), Dachs \& Kaiser (1984), and Sagar (1987) find differential reddening across the cluster, with Dachs \& Kaiser stating the absorption increases with galactic latitude and angular distance from the Coal Sack but the other authors not finding any systematic variations.

The dereddened colours and magnitudes are plotted in Figures 4.20 and 4.21. The median distance modulus from Table 4.15 and both the median colour excess for the cluster (Table 4.15) and individual stars (Table 4.23) are used to calculate the intrinsic colours and magnitudes. ${ }^{9}$ The possible $\beta$ Cephei and other variable stars (Table 4.21) are indicated with different symbols; on the CMD most of the variables lie in an instability strip devoid of constant stars. Clearly star 104 is not a cluster member, while star D could be a late-type supergiant member of the cluster and all other stars are probably cluster members (which is consistent with membership assessments found in the literature, see Table 4.19). Except for the faintest stars, the scatter in the CMD seems reduced when using the individual colour excesses (rather than the median for the cluster). This effect is seen more clearly in the colour-colour diagram, and is to be expected as the calculated excess simply absorbs any measurement errors to force each star back onto the appropriate sequence. A solar metallicity isochrone is super-imposed on the CMD, showing the median age (Table 4.15 ) from the literature agrees well with the data.

\footnotetext{
${ }^{9}$ Note that as the U-B colour is not available for star 108 and the individual colour excess for star 104 are not available from the literature, these stars are not present in all the diagrams.
} 

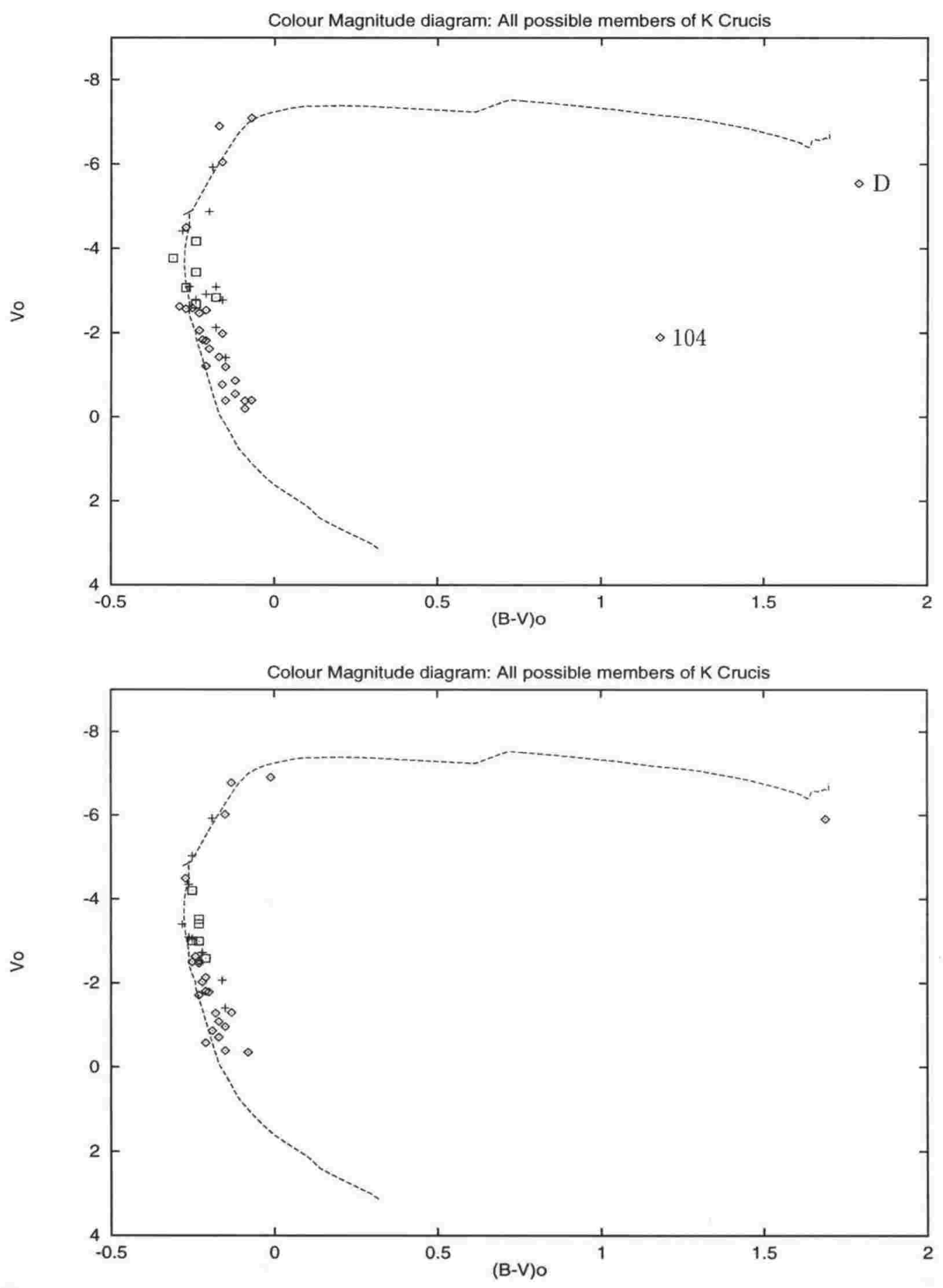

Figure 4.20: Colour-Magnitude diagram of Kappa Crucis, using the median values from the UBV literature (see Tables 4.20 and 4.22), assuming a distance modulus of $11.59(=2075 p c)$. The line is a solar metallicity isochrone of $10 \times 10^{6}$ years. The top diagram is dereddened using the median colour excess for the cluster (see table 4.15) while the bottom diagram is dereddened using the median colour excesses for the individual stars (see Table 4.23). The box and plus symbols indicate possible $\beta$ Cephei and other variable stars respectively (see Table 4.21). 

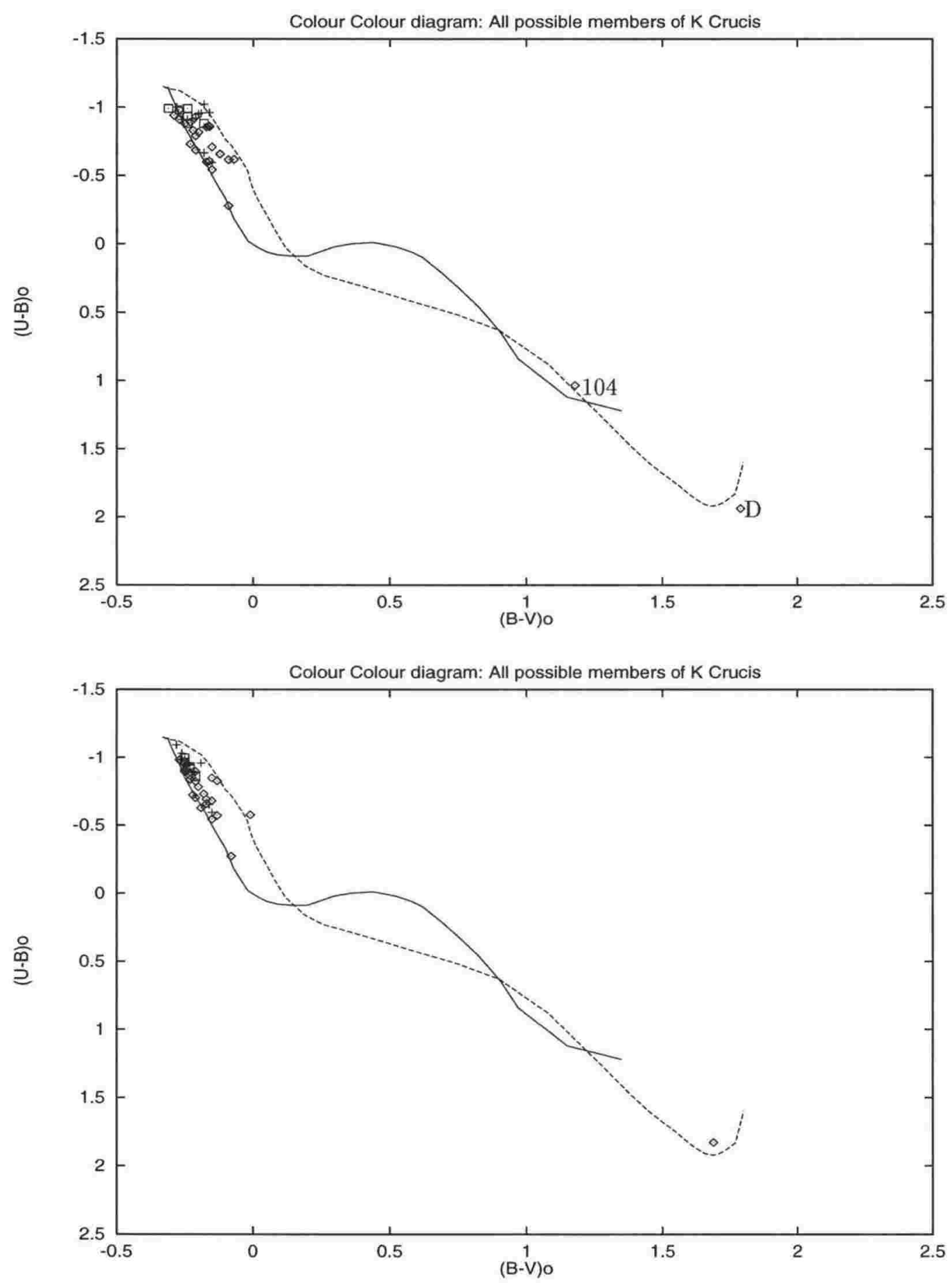

Figure 4.21: Colour-Colour diagram of Kappa Crucis, using the median values from the UBV literature (see Table 4.22). The main and luminosity Ia sequences are shown as solid and dashed lines, taken from Tables 14 and 15 of Straižys (1992). The top diagram is dereddened using the median colour excess for the cluster (see Table 4.15) while the bottom diagram is dereddened using the median colour excesses for the individual stars (see Table 4.23). The box and plus symbols indicate possible $\beta$ Cephei and other variable stars respectively (see Table 4.21). 


\subsection{Vilnius Photometry of the Kappa Crucis cluster.}

The individual observations of the $\kappa$ Crucis stars are given in Table D.6, while the mean values are in Table E.9. The precision level achievable by this study $\left(0^{\mathrm{m}} \cdot 02\right.$ for a single observation) is not met by several stars but it should be noted that these are all fainter than $10^{m} \cdot 0$. Further, when the standard deviation of the mean for each star was compared with the expected deviation for a star with $n$ observations, using the formula found for primary standards in Figure 3.3, only two stars were indicated as possible variables (137 and 218). To investigate possible variability to higher precision would require dedicated programs of differential photometry, which may be undertaken at a later date.

The Vilnius mean V magnitudes do not compare well with the visual magnitudes found in the literature (see Table 4.20); $\sim 20 \%$ agree within $\pm 0^{m} \cdot 01$ of the median value, $\sim 36 \%$ within $\pm 0^{m} \cdot 02$. However as $\sim 70 \%$ lie within the range covered in the literature, these results are as much due to the large disagreements in the literature as to errors in the Vilnius photometry.

The next stage in the analysis is to derive the spectral classification of each star, in this case using the Vilnius Q-Search, Q-Spectra and QQ-diagrams methods described in Chapter 1 (sections 1.8, 1.9, and 1.11 respectively), with the results given in Table 4.24. The Q-Search results list the spectral types one standard deviation above and below the mean value, and note in parenthesis any peculiarities of the matching reference stars. The Q-Spectra notes the 'graphical shifting' factor as $1+a$, with 1.00 meaning no shifting was required to achieve a good match. If the match of the closest reference spectra appeared to be worse than would be expected from the observational errors, then ' $(\mathrm{p})$ ' follows the spectral type. In some cases, two or three reference spectra which matched equally well are listed. Recall the QQ-diagrams are calibrated in absolute magnitudes rather than luminosity class, and may also indicate possible spectral peculiarities. Note however that the luminosity classes have larger errors than usual due to poor separation in luminosity for early (O and B-type) stars. Finally, an 'average' spectral type is assigned in the last column.

These averages were compared with the spectral types from the literature (see Table 4.17); 24 (out of 30 assigned spectral classes in the literature) lie within the range given by the literature, or within \pm 1.0 spectral sub-classes if only one spectral class is given in the literature. The remaining 12 stars have been assigned spectral classes for the first time. Note that star 104 is identified as an R-type star, for which the Vilnius system is not calibrated. Further, this (R-type) classification has since been superceded by a C-type in the classification scheme but the reference catalogues used have not been updated to reflect this.

Given the spectral type of each star, the iterative procedure described in section 4.3 was used to deredden the measurements. The results are given in Table 4.25, with the distance moduli and colour excess distributions plotted in Figure 4.22. The cluster's average distance 
modulus and reddening are found to be $12 \cdot 18 \pm 0.05$ and $0.31 \pm 0.09$. This colour excess was converted to the UBV system using the colour excess ratio appropriate to B-type stars (see Straižys (1992, Table 65)) and plotted in Figure 4.16 - it has the same excess as the median UBV value but gives a slightly larger distance modulus than found by most other authors.

Assuming the cluster is spherical, its angular diameter $\left(\sim 5 \cdot 1^{\prime}\right)$ at the average distance corresponds to a spread in the distance moduli for individual cluster members of $\pm 0{ }^{m} \cdot 003$. Hence the spread in individual moduli is not due to a spread in the distances for individual stars but to observational and calibration errors and so gives an approximately normal distribution. Further, this doesn't allow discrimination between cluster members and field stars at similar distance, and the histogram shows there are no field stars at distances significantly different from that of the cluster.

The colour excess histogram shows a normal distribution about $E_{\mathrm{Y}-\mathrm{V}}=0.31$ but also indicates significant variations, due to 'patchy' distribution of clouds either within or in front of the cluster. If these outliers are disregarded, then the standard deviation becomes $\sim 0.05$ which, after allowing for observational and calibrational uncertainties in the error budget, means there is little variation in the reddening. One extreme outlier (star M) has a negative colour excess - inspection of the mean magnitudes and colours (Table E.9) shows large uncertainties in all of its colours so this anomalous result is due to observational error (this is confirmed by the UBV colour excess for the star which lies in the middle of the $E_{B-V}$ distribution). The spatial distribution of the colour excesses were plotted in Figure 4.23 (not including star M). There are no obvious trends but the spatial distribution indicates a small, denser cloud near star D and a clearer 'window' near star B. However there are large areas within the cluster in which no stars were observed by this study - this needs to be remedied before the contours in Figure 4.23 can be confidently believed. Thus it is concluded that the cluster has a generally uniform reddening of $E_{\mathrm{Y}-\mathrm{V}}=0.31$, but some areas have significant variations from this mean so all stars need to be individually dereddened. One possibility is that the patchy reddening is due to small clouds within the cluster while the uniform reddening is due to a large foreground cloud.

The dereddened data is plotted with the three (solar metallicity) isochrones which best fit the data in Figure 4.24, implying the cluster is $(10 \pm 3) \times 10^{6}$ years old (which agrees with the median age found by other authors, see Table 4.15). As most of the stars lie on the red side of the isochrones, either isochrones with larger $\mathrm{Z}$ values are needed (which are not currently available for the models used in this study), or most observations of stars included a faint visual companion, are binaries, or Be stars. ${ }^{10}$ Three stars do not fall on the sequence; stars D, M, and 104. Star D is consistent with being a late-type giant member of the cluster, while star M

\footnotetext{
${ }^{10}$ Mermilliod (1981) made an atlas of composite open cluster CMD's and found Be stars are a strong characteristic of open clusters with Kappa Crucis's age, and that Be stars lie red-ward of the main sequence in V,B-V diagrams.
} 
Distance Modulus distribution of $\mathrm{K}$ Crucis stors from Vilnius photometry

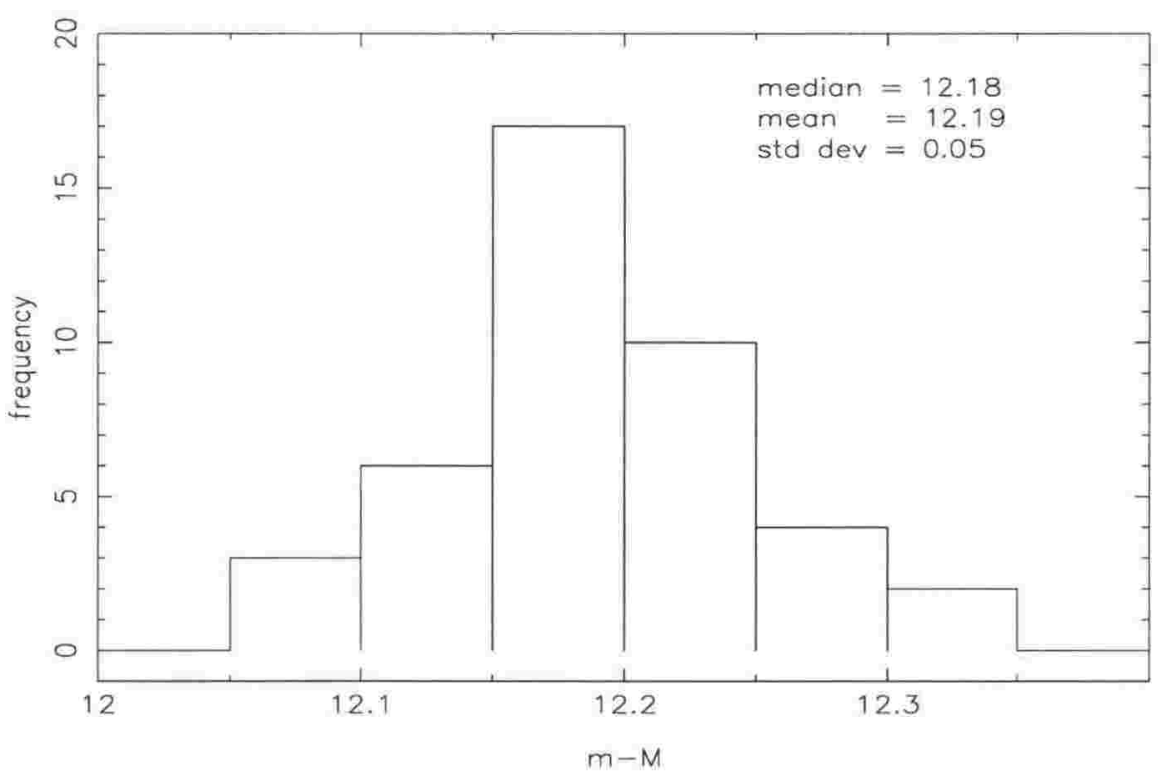

Colour Excess distribution of $K$ Crucis stors from Vilnius photometry

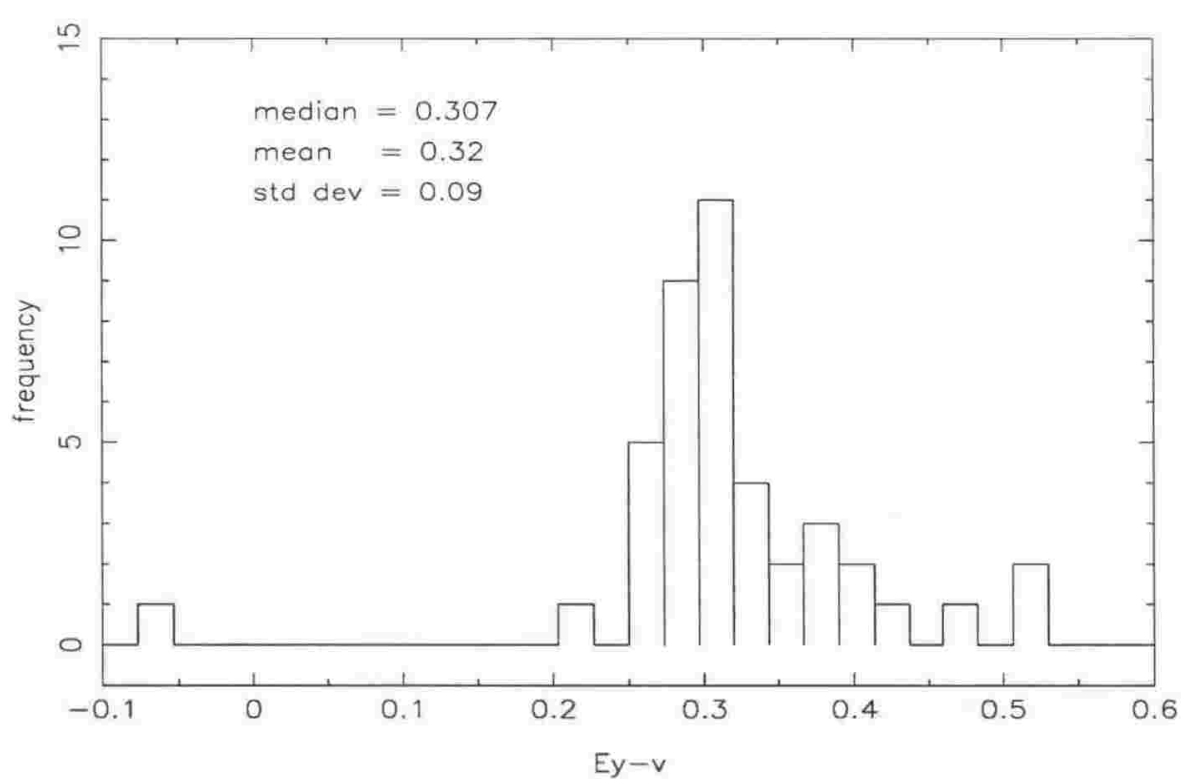

Figure 4.22: Colour excess and distance moduli distributions for Vilnius photometry of Kappa Crucis, from Table 4.25 . 

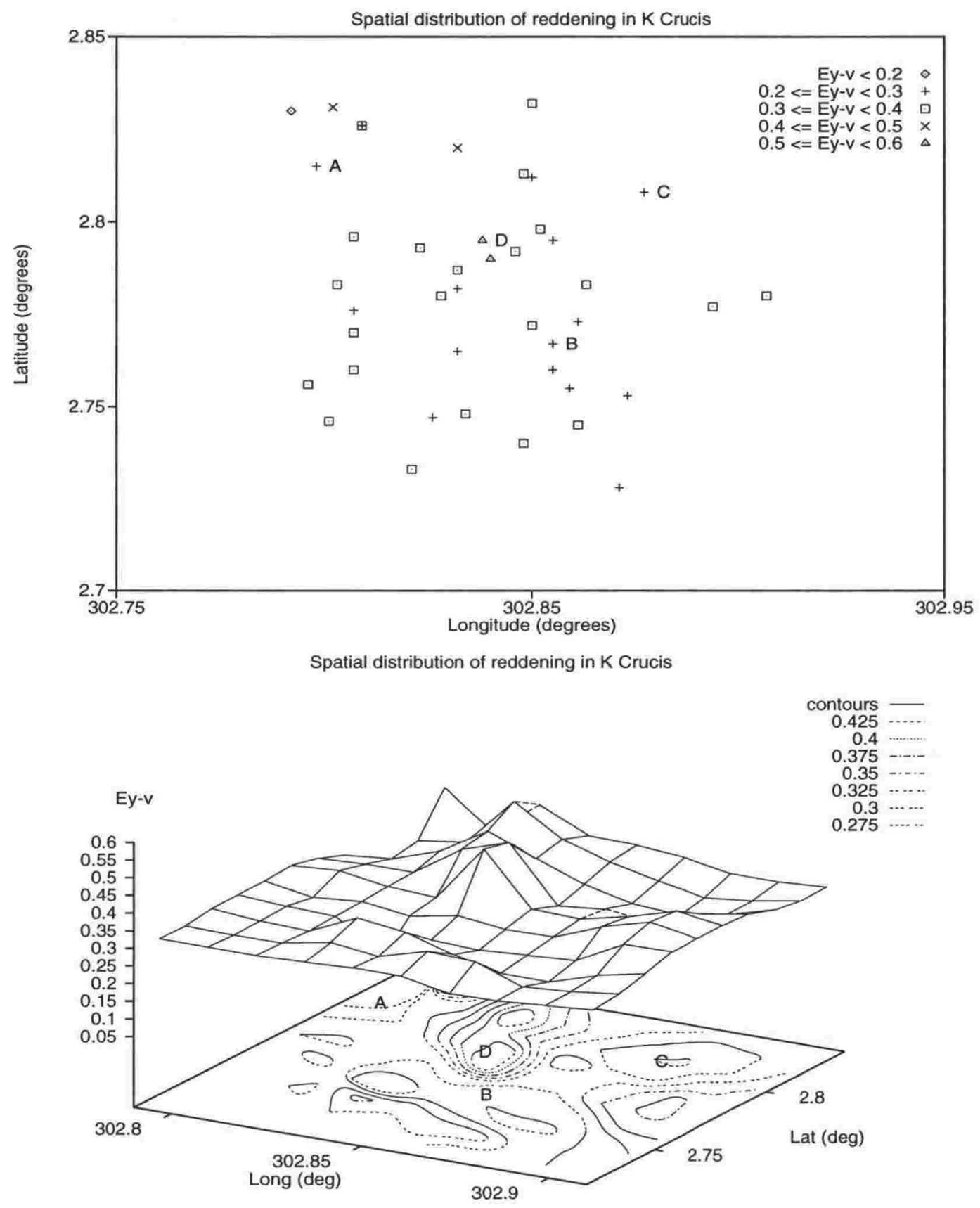

Figure 4.23: Spatial distribution (in Galactic coordinates) of Vilnius colour excesses for Kappa Crucis. Stars A to D are indicated by labels just to the right of each star. 


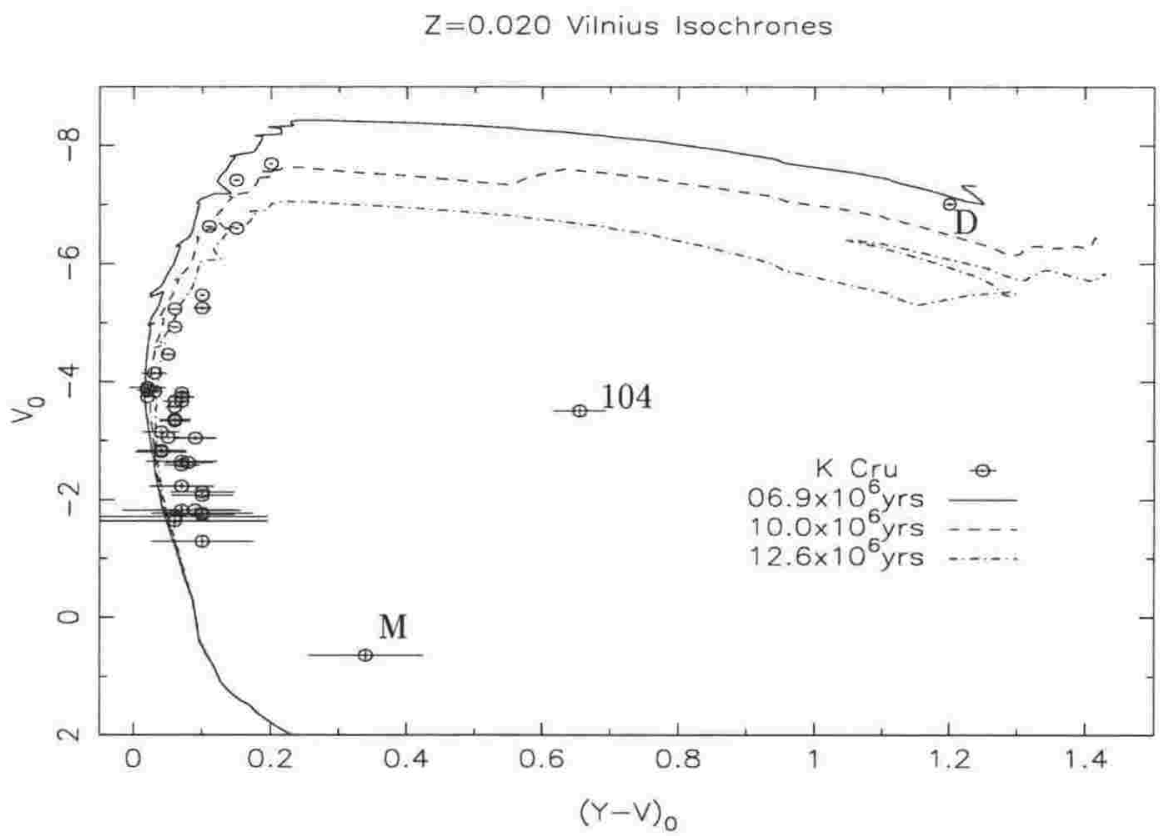

Figure 4.24: Vilnius isochrones for Kappa Crucis, using a distance modulus of 12.18 and dereddening from the individual colour excesses (see Table 4.25). The error bars indicate one standard deviation, as from Table E.9 (i.e. without allowing for the errors in the dereddening procedure).

may be a pre-main sequence member but star 104 is definitely a non-member. However star M appears to have a negative colour excess as was discussed earlier - if the observations are reasonably accurate, this anomaly could be due to the fact that main sequence calibrations of the Vilnius system were used (pre-main sequence calibrations are not available). However, assuming the median distance modulus and colour excess for star $\mathrm{M}$ gives $\left((\mathrm{Y}-\mathrm{V})_{0}, \mathrm{~V}_{0}\right)=$ $(-0.04,-1.05)$ which places it back close to the bottom of the observed sequence. In view of the UBV results, the latter case is considered more likely and emphasize the need for more observations per star for the fainter stars.

\subsection{Summary.}

Two well studied, young, open clusters were observed to compare the astrophysical information that could be derived from the Vilnius system in comparison with other photometric systems. However no stellar models are available yet in the Vilnius system, except for the limited case of B-type stars. To overcome this, Kurucz's grid of (non-standard) magnitudes and colours was calibrated with standard magnitudes from Straižys (1992) and Schaerer's grid of evolutionary models used to produce isochrones. It was found that the $\mathrm{Y}-\mathrm{V}, \mathrm{V}$ isochrones are qualitatively similar to B-V, V isochrones in the Johnson system. 
A literature review of the first cluster (Omicron Velorum) found it to be a close (154 pc), young $\left(30 \times 10^{6}\right.$ years $)$ open cluster with little reddening $\left(E_{\mathrm{B}-\mathrm{V}}=0 \cdot 01\right)$. Individual studies used a wide variety of catalogues to identify the cluster stars, with Lyngå's system being adopted here. Spectral classifications generally show good agreement between different authors, with the cluster (to the limiting magnitude of this study) consisting of B-F type stars. Four proper motion studies have been made, showing a bimodal distribution due to the cluster members and the field stars (which are more common among the fainter stars). Six radial velocity studies were found in the literature, showing the spread about the mean is consistent with the expected variation within the cluster. Several stars are known, or suspected to be, spectroscopic binaries but only $\mathrm{O}$ Vel 3 has be measured long enough to determine its orbital elements. Assessments of cluster membership have been made by eight authors, generally showing good agreement with each other, and implying just over half of the stars observed in this study ( 23 out of 43 ) are members.

Systematic differences between the $\mathrm{V}$ magnitudes in the literature were found. As it is not possible to tell which system is closest to the true standard system, the medium values (for this and all later magnitudes and colours) were used in an attempt to reduce both the systematic and random errors. Differential photometry was used in several studies to investigate variability of individual cluster stars. It appears that the four brightest stars are variable at the $0{ }^{m} \cdot 01-$ $0^{m} \cdot 02$ level but the variability may be irregular (or non-existent) as results from different authors often contradict each other.

Only four studies have been made using the Johnson system. Colour-magnitude and colourcolour diagrams of the probable member stars are consistent with the cluster characteristics given earlier, with the brightest star ( $\mathrm{O}$ Vel 1$)$ just leaving the main sequence and the faintest (O Vel 40) being a pre-main sequence star. The Strömgren system studies show similar results, while the $H_{\beta}$ measurements show reasonable agreement with a ZAMS $H_{\beta}$-absolute magnitude calibration.

To compare the Vilnius system with the most commonly used photometric systems (Johnson and Strömgren), no prior knowledge of the cluster (from the literature) was assumed during the analysis (but extensive comparisons made afterwards). At the precision level achieved by this study $(0 \cdot 02)$, no stars were found to be variable and $90 \%$ of the stars agree with the medium values from the literature. Spectral types and luminosity classes from the Vilnius photometry all agree with the literature values (within the expected accuracy of the classification methods).

Using the 'Vilnius' spectral types, the reddening and distance modulus of the individual stars were found iteratively and closely agree with the medium results of the literature. As the cluster is 'close', a simple error analysis suggests the 'errors' are largely due to differences in the distance of individual stars and that the cluster is unreddened. A histogram of the distance 
moduli was used to assign cluster membership for individual stars, agreeing almost completely with the literature.

The CMD indicates that the brightest star ( $\mathrm{O}$ Vel 1$)$ has moved off the main sequence, the next brighter stars (O Vel $2-7$ ) have moved from the ZAMS to the main sequence and that the two faintest stars (O Vel 37 and 40) are pre-main sequence stars. More observations are needed for the fainter stars to reduce the uncertainties in the colours (in particular $\mathrm{Y}-\mathrm{V}$ which is used in the CMD). The order of the stars along the CMD sequence suggests some minor changes to the Vilnius spectral classification (or downgrading of membership probabilities) are needed for two stars (O Vel 9 and 17). Fitting (by eye) a grid of isochrones to the CMD indicates that cluster has solar metallicity $(\mathrm{Z}=0.020)$, with an age of $(45 \pm 15) \times 10^{6}$ years.

A literature review of Kappa Crucis finds it to be $2075 p c$ away, and reddened $\left(E_{\mathrm{B}-\mathrm{V}}=0.40\right)$ but a quite young $\left(10 \times 10^{6}\right.$ years $)$ cluster. The variation in $\mathrm{M}_{\mathrm{V}}$ and $E_{\mathrm{B}-\mathrm{V}}$ between different authors is reduced when the correlation between those variables is considered. The numbering system used by Dachs \& Kaiser was adopted as the other catalogues do not provide complete coverage of the cluster. All spectral classifications are from spectroscopic observations; there is general agreement between different authors with Feast (1963) giving most complete coverage.

Only one proper motion study (by King (1980)) was found in the literature; an excessive tail in the distribution curve appear to be from non-members of the cluster. There have been only four radial velocity studies, with Feast (1963) providing the best coverage. The two studies of membership (for the stars observed here) show only one star considered a non-member (star 104) and one not assessed (star 108).

There have been ten studies of V magnitudes, with Arp \& Van Sant (1958)'s fainter stars being consistently too bright compared to all the other authors. Several stars were investigated for variability with somewhat contradictory results between authors, probably due to the low amplitude and long periods of the proposed variability.

Seven investigations using the Johnson system have been made, showing systematic differences between the studies. The same number of reddening $\left(E_{\mathrm{B}-\mathrm{V}}\right)$ studies were done using a variety of methods, but resulting in no agreement on the uniformity of the reddening across the cluster, although the statistics of the medium values suggest the reddening is constant. Colourmagnitude and colour-colour diagrams of the medium values show that most of the variables lie in an instability strip devoid of constant stars, star 104 is not a member and star D could be a late-type supergiant member.

Again, to compare the Vilnius system with the Johnson system, no prior knowledge of the cluster (from the literature) was assumed during the analysis. Several of the faintest stars do not meet the precision level normally achievable by this study. Two stars (137 and 218) are suggested as variable based on the standard deviations about their mean magnitudes, how- 
ever a dedicated program of differential photometry would be required to investigate possible variability at the levels found in the literature. Vilnius $\mathrm{V}$ magnitudes do not agree well with the medium values of the literature, but the large (and systematic) difference in the literature suggest that the Vilnius photometry is not solely to blame.

Most (24 out of 30 ) stars' spectral types and luminosities agree with the literature values and 12 stars are classified for the first time. Using the 'Vilnius' spectral types, the reddening and distance modulus of the individual stars were found iteratively - the reddening (after converting to $E_{\mathrm{B}-\mathrm{V}}$ ) agrees with the medium of the literature but the distance modulus is slightly larger. The reddening is generally uniform, but some areas show significant variations so the dereddening needs to be done individually for each star.

The Vilnius colour-magnitude diagram is best fitted by a $(10 \pm 3) \times 10^{6}$ years isochrone of solar metallicity. Most of the stars are on the red side of the isochrone, implying either isochrones with larger Z (metallicity) values are needed (which are not currently available), most of the stars are binaries, Be stars or the observations included faint companion stars. Three stars do not lie on the sequence; Star D is probably a late-type member of the cluster, star M may be a pre-main sequence star (but is probably a main sequence star whose anomalous results are due to an observational error) while star 104 is definitely a non-member. 
Table 4.17: Spectral types for Kappa Crucis stars from the literature.

\begin{tabular}{|c|c|c|c|c|c|c|c|}
\hline \# & Bidelman & AvS & Hernandez & Feast & Schild & Sowell & Slettebak \\
\hline A & B9 Ia & B8-9 Ib & B9 Ia-ab & B9 Iab & B9 Ia & A2 Iab & \\
\hline B & B3 Iab & B5 II or Ib & B3 Iab & B3 Ia & B5 Ia & & \\
\hline $\mathrm{C}$ & & B3-4 II & B3 Ib & B2 Ib & B2 lb & & \\
\hline D & & K5 giant & M2-3 Iab & M2 Ib & M2 lab & & \\
\hline $\mathrm{E}$ & & B5 II-III & B0.5-1 & B1 III & B1 III & & \\
\hline $\mathrm{F}$ & & B6 III: & B0.5-1 II-III & B2 III & B2 III & & \\
\hline G & & B6 V: & & B0. $5 \mathrm{~V}$ & & & \\
\hline $\mathrm{H}$ & & & & & & & \\
\hline I & & & & B1 Vn & & & \\
\hline $\mathrm{J}$ & & & & B1 V & & & \\
\hline K & & & & B8 V & & & \\
\hline $\mathrm{L}$ & & & & B8: V & & & \\
\hline M & & & & A0: $V$ & & & \\
\hline 104 & & & & & & & \\
\hline 105 & & & B1-2 II-III & B0.5 III(n) & B1 III & & \\
\hline 106 & & & B2 Ib: & $\mathrm{B} 1.5 \mathrm{Ib}$ & B1.5 Ib & & \\
\hline 107 & & & & & & & \\
\hline 108 & & & & & & & \\
\hline 113 & & & & B1 V & & & \\
\hline 115 & & & & $\mathrm{~B} 1 \mathrm{~V}$ & & & \\
\hline 117 & & & & Bnn & & & \\
\hline 137 & & & & & & & \\
\hline 138 & & & & & & & \\
\hline 139 & & & & & & & \\
\hline 201 & & & & B1 V & B0.5 V & & \\
\hline 202 & & & & B1 V & & & \\
\hline 203 & & & & B1 V & & & \\
\hline 207 & & & & & & & \\
\hline 215 & & & & & & & \\
\hline 216 & & & & & & & \\
\hline 218 & & & & & & & \\
\hline 223 & & & & B0 V? & B0.5 Vn & & \\
\hline 224 & & & & B0 V & & & \\
\hline 228 & & & & & & & \\
\hline 305 & & & B2:: & B0.5 V & $\mathrm{B} 0.5 \mathrm{IVn}$ & & \\
\hline 306 & & & & Bnne & B2 IVne & & B1 V \\
\hline 311 & & & & $\mathrm{~B} 2: \mathrm{V}$ & & & \\
\hline 405 & & & & B2 V & & & \\
\hline 410 & & & & B3:V: & & & \\
\hline 414 & & & & B1 V & & & \\
\hline 417 & & & & Bnne & B2 IVne & & B1 V \\
\hline 418 & & & & $\mathrm{~B} 2: \mathrm{V}$ & & & \\
\hline
\end{tabular}


Table 4.18: Proper motion $\left(0 \cdot 001^{\prime \prime} / \mathrm{yr}\right)$ and radial velocity $(\mathrm{km} / \mathrm{s})$ for Kappa Crucis stars from the literature.

\begin{tabular}{|c|c|c|c|c|c|c|}
\hline \multirow{2}{*}{$\begin{array}{l}\text { star } \\
\#\end{array}$} & \multirow{2}{*}{$\begin{array}{r}\text { RA } \\
\text { King }\end{array}$} & \multirow{2}{*}{$\begin{array}{r}\text { Dec } \\
\text { King }\end{array}$} & \multicolumn{4}{|c|}{ Radial Velocity } \\
\hline & & & Hernandez & Feast & $\mathrm{M} \& \mathrm{O}$ & Sowell \\
\hline A & 13 & 35 & $-20 \cdot 4$ & $-16(-15)$ & $(-23)$ & \\
\hline B & -27 & -7 & $-14 \cdot 2$ & $-12(-1 \cdot 3)$ & & \\
\hline $\mathrm{C}$ & 9 & 5 & $-29 \cdot 2$ & -11 & $(-6)$ & \\
\hline D & 11 & 17 & -22 & -17 & & $(-24)$ \\
\hline $\mathrm{E}$ & -2 & 12 & $-21 \cdot 2$ & -23 & & \\
\hline $\mathrm{F}$ & 0 & -6 & $-5,-40,+7, \mathrm{SB} ?$ & -22 & & \\
\hline G & -8 & 1 & -27 & -23 & & \\
\hline $\mathrm{H}$ & -8 & -2 & & & & \\
\hline I & 7 & -4 & & -32 var? & & \\
\hline $\mathbf{J}$ & 14 & -2 & & -34 & & \\
\hline K & 28 & 8 & & & & \\
\hline $\mathrm{L}$ & & & & & & \\
\hline M & & & & & & \\
\hline 104 & & & & & & \\
\hline 105 & -15 & 14 & $-26 \cdot 2$ & -23 & & \\
\hline 106 & 0 & -4 & -18.5 & -20 & $(-17)$ & \\
\hline 107 & & & & & & \\
\hline 108 & & & & & & \\
\hline 113 & 12 & 0 & & -19 & & \\
\hline 115 & 8 & -2 & & -22 & & \\
\hline 117 & 9 & -10 & & & & \\
\hline 137 & & & & & & \\
\hline 138 & -12 & -4 & & & & \\
\hline 139 & & & & & & \\
\hline 201 & -7 & -1 & & -16 & & \\
\hline 202 & 0 & 13 & & -33 var? & & \\
\hline 203 & 2 & 9 & & -22 & & \\
\hline 207 & & & & & & \\
\hline 215 & & & & & & \\
\hline 216 & & & & & & \\
\hline 218 & & & & & & \\
\hline 223 & 14 & -2 & $-95,+12,-98$, SB? & VAR & & \\
\hline 224 & & & & -10 & & \\
\hline 228 & -49 & -1 & & & & \\
\hline 305 & -10 & 0 & $46,30,-76,-13$, SB? & VAR & & \\
\hline 306 & 15 & 1 & & & & \\
\hline 311 & 13 & 5 & & VAR & & \\
\hline 405 & 11 & 5 & & -18 & & \\
\hline 410 & & & & -24 & & \\
\hline 414 & 4 & -9 & & -19 & & \\
\hline 417 & 0 & 3 & & & & \\
\hline 418 & 3 & -3 & & VAR & & \\
\hline
\end{tabular}


Table 4.19: Membership for Kappa Crucis stars from the literature.

\begin{tabular}{|c|c|c|c|c|c|c|c|}
\hline$\#$ & King & D\&K & average & \# & King & D\&K & average \\
\hline $\mathrm{A}$ & 22 & probable & possible & 137 & & possible & possible \\
\hline B & 54 & certain & probable & 138 & 88 & certain & certain \\
\hline $\mathrm{C}$ & 91 & probable & probable & 139 & & certain & certain \\
\hline D & 81 & certain & certain & 201 & 92 & probable & probable \\
\hline $\mathrm{E}$ & 90 & certain & certain & 202 & 89 & certain & certain \\
\hline $\mathrm{F}$ & 92 & probable & probable & 203 & 91 & certain & certain \\
\hline G & 91 & certain & certain & 207 & & certain & certain \\
\hline $\mathrm{H}$ & 91 & certain & certain & 215 & & certain & certain \\
\hline I & 92 & certain & certain & 216 & & certain & certain \\
\hline $\mathrm{J}$ & 89 & certain & certain & 218 & & probable & probable \\
\hline $\mathrm{K}$ & 56 & probable & probable & 223 & 89 & certain & certain \\
\hline $\mathrm{L}$ & & certain & certain & 224 & & probable & probable \\
\hline M & & probable & probable & 228 & 1 & certain & possible \\
\hline 104 & & impossible & impossible & 305 & 90 & probable & probable \\
\hline 105 & 78 & certain & certain & 306 & 88 & certain & certain \\
\hline 106 & 93 & certain & certain & 311 & 89 & probable & probable \\
\hline 107 & & probable & probable & 405 & 90 & certain & certain \\
\hline 108 & & & & 410 & & probable & probable \\
\hline 113 & 90 & certain & certain & 414 & 91 & certain & certain \\
\hline 115 & 92 & certain & certain & 417 & 93 & certain & certain \\
\hline 117 & 90 & probable & probable & 418 & 93 & certain & certain \\
\hline
\end{tabular}


Table 4.20: Visual magnitude V for Kappa Crucis stars from the literature.

\begin{tabular}{|c|c|c|c|c|c|c|c|c|c|c|c|}
\hline$\#$ & AvS & Gra. & Schild & Perry & $\mathrm{G} \& \mathrm{O}$ & Mc. & Waard & D\&K & Shob. & B\&K & med. \\
\hline A & $5 \cdot 75$ & $5 \cdot 80$ & & 5.76 & & & 5.75 & $5 \cdot 77$ & $5 \cdot 732$ & $5 \cdot 728$ & $5 \cdot 75$ \\
\hline B & 5.94 & 6.00 & & 5.94 & 5.913 & & $5 \cdot 92$ & 5.98 & $5 \cdot 933$ & 5.971 & 5.94 \\
\hline $\mathrm{C}$ & 6.80 & 6.84 & & $6 \cdot 77$ & & & $6 \cdot 76$ & 6.80 & 6.786 & & 6.79 \\
\hline D & $7 \cdot 58$ & & $7 \cdot 66$ & $7 \cdot 42$ & & $7 \cdot 7$ & $7 \cdot 47$ & $7 \cdot 45$ & & $7 \cdot 500$ & 7.50 \\
\hline $\mathrm{E}$ & $8 \cdot 35$ & & $8 \cdot 3$ & 8.32 & & & $8 \cdot 34$ & 8.37 & $8 \cdot 360$ & $8 \cdot 369$ & 8.35 \\
\hline F & 9.09 & & $9 \cdot 07$ & 9.03 & & & 9.08 & $9 \cdot 10$ & 9.060 & $9 \cdot 099$ & 9.08 \\
\hline G & $9 \cdot 79$ & & & $9 \cdot 75$ & & & $9 \cdot 79$ & $9 \cdot 83$ & $9 \cdot 764$ & $9 \cdot 790$ & 9.78 \\
\hline $\mathrm{H}$ & 9.93 & & & 9.58 & & & 9.94 & 9.90 & $9.9 \mathrm{~V}$ & & 9.93 \\
\hline I & $10 \cdot 01$ & & & $10 \cdot 00$ & & & $10 \cdot 04$ & $10 \cdot 02$ & $10 \cdot 005$ & 9.986 & $10 \cdot 01$ \\
\hline $\mathrm{J}$ & $10 \cdot 58$ & & & 10.99 & & & 11.02 & 11.01 & & 11.015 & 11.01 \\
\hline K & $11 \cdot 42$ & & & $11 \cdot 42$ & & & $11 \cdot 47$ & $11 \cdot 40$ & 11.436 & 11.434 & 11.43 \\
\hline L & 11.88 & & & 11.99 & & & $11 \cdot 85$ & 12.07: & & $12 \cdot 258$ & 11.99 \\
\hline M & $12 \cdot 40$ & & & $12 \cdot 48$ & & & $12 \cdot 45$ & 12.70: & & $12 \cdot 658$ & 12.48 \\
\hline 104 & $10 \cdot 70$ & & & & & & & $11 \cdot 03$ & & $11 \cdot 110$ & 11.03 \\
\hline 105 & 8.59 & & & 8.61 & & & $8 \cdot 70$ & 8.69 & 8.659 & $8 \cdot 690$ & 8.68 \\
\hline 106 & 7.00 & 6.99 & & $6 \cdot 86$ & & & 6.91 & 6.92 & $6 \cdot 891$ & 6.889 & $6 \cdot 91$ \\
\hline 107 & $11 \cdot 34$ & & & 11.18 & & & $11 \cdot 44$ & 11.45 & & 11.437 & 11.44 \\
\hline 108 & $12 \cdot 27$ & & & & & & & & & $12 \cdot 620$ & $12 \cdot 45$ \\
\hline 113 & $10 \cdot 23$ & & & $10 \cdot 17$ & & & $10 \cdot 19$ & $10 \cdot 17$ & $10 \cdot 160$ & $10 \cdot 168$ & $10 \cdot 17$ \\
\hline 115 & $10 \cdot 39$ & & & $10 \cdot 24$ & & & & $10 \cdot 40$ & & $10 \cdot 377$ & $10 \cdot 38$ \\
\hline 117 & $10 \cdot 87$ & & & $10 \cdot 82$ & & & $10 \cdot 76$ & $10 \cdot 85$ & & $10 \cdot 873$ & $10 \cdot 85$ \\
\hline 137 & $12 \cdot 48$ & & & & & & & 12.65 & & 12.663 & 12.65 \\
\hline 138 & 11.18 & & & & & & $11 \cdot 24$ & $11 \cdot 25$ & & $11 \cdot 222$ & $11 \cdot 23$ \\
\hline 139 & 11.57 & & & & & & $11 \cdot 73$ & 11.68 & & $11 \cdot 636$ & 11.66 \\
\hline 201 & $9 \cdot 37$ & & & $9 \cdot 41$ & & & $9 \cdot 42$ & $9 \cdot 40$ & & $9 \cdot 438$ & $9 \cdot 41$ \\
\hline 202 & 10.06 & & & $10 \cdot 04$ & & & 10.08 & $10 \cdot 06$ & & $10 \cdot 085$ & 10.06 \\
\hline 203 & $10 \cdot 22$ & & & $10 \cdot 26$ & & & $10 \cdot 30$ & $10 \cdot 29$ & $10 \cdot 277$ & $10 \cdot 287$ & $10 \cdot 28$ \\
\hline 207 & 11.91 & & & 12.03 & & & $12 \cdot 04$ & $12 \cdot 13$ & $12 \cdot 15$ & $12 \cdot 149$ & 12.08 \\
\hline 215 & 11.49 & & & 11.64 & & & 11.64 & 11.72 & $11 \cdot 640$ & 11.635 & 11.64 \\
\hline 216 & $12 \cdot 22$ & & & & & & $12 \cdot 45$ & $12 \cdot 48$ & & & $12 \cdot 45$ \\
\hline 218 & $12 \cdot 07$ & & & & & & $12 \cdot 35$ & $12 \cdot 30$ & & & $12 \cdot 30$ \\
\hline 223 & $7 \cdot 93$ & 8.01 & & 7.90 & & & 7.97 & $7 \cdot 97$ & $7 \cdot 97:$ & 8.012 & 7.97 \\
\hline 224 & $10 \cdot 18$ & & & $10 \cdot 31$ & & & $10 \cdot 22$ & $10 \cdot 30$ & $10 \cdot 343$ & $10 \cdot 343$ & $10 \cdot 31$ \\
\hline 228 & 10.97 & & & 11.01 & & & & 11.05 & 11.040 & & 11.03 \\
\hline 305 & $8 \cdot 43$ & & & 8.46 & & & $8 \cdot 39$ & $8 \cdot 58$ & & 8.398 & 8.43 \\
\hline 306 & $9 \cdot 98$ & & $10 \cdot 19$ & $9 \cdot 88$ & & & $10 \cdot 09$ & $10 \cdot 05$ & & $10 \cdot 093$ & 10.07 \\
\hline 311 & 10.78 & & & $10 \cdot 78$ & & & $10 \cdot 85$ & $10 \cdot 78$ & $10 \cdot 806$ & $10 \cdot 798$ & 10.79 \\
\hline 405 & $10 \cdot 27$ & & & $10 \cdot 20$ & & & $10 \cdot 20$ & $10 \cdot 22$ & & $10 \cdot 217$ & $10 \cdot 22$ \\
\hline 410 & $10 \cdot 72$ & & & 10.71 & & & $10 \cdot 76$ & 10.72 & & $10 \cdot 734$ & $10 \cdot 72$ \\
\hline 414 & $10 \cdot 26$ & & & $10 \cdot 27$ & & & $10 \cdot 27$ & $10 \cdot 24$ & & $10 \cdot 243$ & $10 \cdot 26$ \\
\hline 417 & $9 \cdot 76$ & & $9 \cdot 76$ & $9 \cdot 73$ & & & $9 \cdot 77$ & $9 \cdot 96$ & & 9.832 & $9 \cdot 76$ \\
\hline 418 & 9.86 & & & $9 \cdot 65$ & & & $9 \cdot 76$ & 9.83 & $9 \cdot 742$ & 9.727 & 9.75 \\
\hline
\end{tabular}


Table 4.21: Variability of Kappa Crucis stars from the literature.

\begin{tabular}{|c|c|c|c|c|c|}
\hline$\#$ & $\begin{array}{l}\text { Jakate } \\
\text { (Strom. b) } \\
\end{array}$ & $\begin{array}{l}\text { Shobbrook } \\
\text { (Strom. y) }\end{array}$ & $\begin{array}{l}\text { D\&K } \\
\text { (John. V) }\end{array}$ & $\begin{array}{l}\text { Koen } \\
\text { (John. B) }\end{array}$ & $\begin{array}{l}\text { B\&K } \\
\text { (Strom. y) }\end{array}$ \\
\hline $\mathrm{C}$ & const & & & & \\
\hline $\mathrm{D}$ & & & $\begin{array}{l}\text { var, } \\
\Delta=0.2\end{array}$ & & const? \\
\hline $\mathrm{E}$ & const & & & const & \\
\hline $\mathrm{F}$ & $\begin{array}{l}\beta \text { Cep, } \\
\Delta=0 \cdot 036, P=4 \cdot ? h\end{array}$ & $\begin{array}{l}\text { var, } \\
\Delta=0.03 \\
P=0.2 d\end{array}$ & & $\begin{array}{l}\beta \text { Cep, } \\
\Delta=0.007, \mathrm{f}=4.89 / \mathrm{d} \\
\Delta=0.006, \mathrm{f}=6 \cdot 16 / \mathrm{d}\end{array}$ & $\begin{array}{l}\beta \text { Cep, } \\
\Delta=0.006, \mathrm{f}=4.9 / \mathrm{d} \\
\Delta=0.005, \mathrm{f}=4.5 / \mathrm{d} \\
\Delta=0.003, \mathrm{f}=3.0 / \mathrm{d}\end{array}$ \\
\hline $\mathrm{G}$ & $\begin{array}{l}\text { var, } \\
\Delta=0.033, P=6.6 \mathrm{~h}\end{array}$ & & & $\begin{array}{l}\beta \text { Cep, } \\
\Delta=0.005, \mathrm{f}=6.62 / \mathrm{d} \\
\Delta=0.006, \mathrm{f}=6.63 / \mathrm{d} \\
\Delta=0.003, \mathrm{f}=0.21 / \mathrm{d}\end{array}$ & $\begin{array}{l}\beta \text { Cep, } \\
\Delta=0.004, \mathrm{f}=6.4 / \mathrm{d}\end{array}$ \\
\hline $\mathrm{H}$ & & $\begin{array}{l}\text { var, } \\
\Delta>0.2\end{array}$ & & & \\
\hline I & & & & $\begin{array}{l}\beta \text { Cep, } \\
\Delta=0.007, \mathrm{f}=5.59 / \mathrm{d}\end{array}$ & $\begin{array}{l}\beta \text { Cep, } \\
\Delta=0.007, \mathrm{f}=5 \cdot 6 / \mathrm{d} \\
\Delta=0.005, \mathrm{f}=0.2 / \mathrm{d}\end{array}$ \\
\hline 105 & $\begin{array}{l}\beta \text { Cер, } \\
\Delta=0.01, P=4 \mathrm{~h}\end{array}$ & & & var? & $\begin{array}{l}\text { var, } \\
\Delta=0.03, P=1.005 \mathrm{~d}\end{array}$ \\
\hline 106 & el. var? & & & & const? \\
\hline 107 & & & & & $\begin{array}{l}\text { var, } \\
\Delta=0.01, P=0.75 \mathrm{~d}\end{array}$ \\
\hline 113 & & & & const & $\begin{array}{l}\beta \text { Cep, } \\
\Delta=0.002, \mathrm{f}=4.3 / \mathrm{d}\end{array}$ \\
\hline 201 & & & & & $\begin{array}{l}\beta \text { Cep, } \\
\Delta=0.008, \mathrm{f}=5.5 / \mathrm{d}\end{array}$ \\
\hline 202 & & & & & $\begin{array}{l}\text { binary? } \\
P=6 \cdot 4 d\end{array}$ \\
\hline 223 & const & & $\begin{array}{l}\text { var? } \\
\Delta=0.08 \\
P=3 \cdot 24 \mathrm{~d}\end{array}$ & ell. var? & const? \\
\hline 305 & $\begin{array}{l}\text { var, } \\
\Delta>0.06, P>7 \mathrm{~h}\end{array}$ & & & $\begin{array}{l}\text { binary, } \\
\mathrm{f}=0.51 / \mathrm{d}\end{array}$ & $\begin{array}{l}\text { var, } \\
\Delta=0.12, \mathrm{P}=1.96 \mathrm{~d}\end{array}$ \\
\hline 306 & const & & & & $\begin{array}{l}\text { var, } \\
\Delta=0.08, P=0.79 d\end{array}$ \\
\hline 410 & & & & & $\begin{array}{l}\text { var, } \\
\Delta=0.07\end{array}$ \\
\hline 417 & const & & & & $\begin{array}{l}\text { var, } \\
\Delta=0.03, P=2.21 d\end{array}$ \\
\hline 418 & $\begin{array}{l}\beta \text { Cер, } \\
\Delta=0.032, P=3.2 \mathrm{~h}\end{array}$ & const? & & const? & const? \\
\hline
\end{tabular}


Table 4.22: U-B and B-V colours for Kappa Crucis stars from the literature.

\begin{tabular}{|c|c|c|c|c|c|c|c|c|c|c|c|c|}
\hline \multirow[t]{2}{*}{$\#$} & \multicolumn{5}{|c|}{ U-B } & \multicolumn{7}{|c|}{$B-V$} \\
\hline & Gm. & Sd. & Py. & DK. & mn. & AvS & Gm. & Sd. & Py. & Wd. & DK. & $\mathrm{mn}$. \\
\hline A & -0.30 & & $-0 \cdot 34$ & -0.34 & -0.34 & 0.325 & 0.39 & & 0.33 & 0.33 & 0.32 & 0.33 \\
\hline B & -0.54 & & -0.57 & -0.58 & -0.57 & 0.224 & $0 \cdot 27$ & & $0 \cdot 24$ & 0.23 & 0.22 & 0.23 \\
\hline $\mathrm{C}$ & -0.54 & & -0.57 & -0.58 & -0.57 & 0.243 & $0 \cdot 30$ & & 0.24 & 0.24 & 0.24 & 0.24 \\
\hline D & & $+2 \cdot 20$ & +2.38 & +2.57 & $+2 \cdot 38$ & $(2 \cdot 07)$ & & $2 \cdot 16$ & $2 \cdot 19$ & $2 \cdot 22$ & $2 \cdot 22$ & $2 \cdot 19$ \\
\hline $\mathrm{E}$ & & -0.69 & -0.69 & -0.72 & -0.69 & 0.118 & & 0.13 & $0 \cdot 13$ & 0.14 & 0.14 & 0.13 \\
\hline $\mathrm{F}$ & & -0.70 & -0.70 & -0.70 & -0.70 & 0.153 & & 0.08 & 0.09 & 0.09 & 0.07 & 0.09 \\
\hline G & & & -0.67 & -0.70 & -0.69 & 0.195 & & & 0.13 & 0.14 & 0.11 & 0.13 \\
\hline $\mathrm{H}$ & & & -0.59 & -0.67 & -0.63 & 0.194 & & & $0 \cdot 22$ & 0.19 & 0.18 & 0.19 \\
\hline I & & & -0.57 & -0.60 & -0.59 & 0.336 & & & 0.20 & 0.22 & 0.22 & 0.22 \\
\hline $\mathrm{J}$ & & & -0.52 & -0.56 & -0.54 & 0.151 & & & $0 \cdot 18$ & 0.18 & 0.22 & 0.18 \\
\hline K & & & -0.32 & -0.32 & -0.32 & 0.321 & & & 0.21 & 0.20 & 0.26 & 0.23 \\
\hline $\mathrm{L}$ & & & -0.37 & -0.39 & -0.38 & 0.302 & & & $0 \cdot 25$ & 0.29 & 0.28 & 0.28 \\
\hline M & & & -0.02 & +0.01 & -0.00 & 0.384 & & & 0.30 & 0.31 & 0.32 & 0.31 \\
\hline 104 & & & & +1.39 & +1.39 & 1.59 & & & & & 1.57 & 1.58 \\
\hline 105 & & & -0.70 & -0.71 & -0.70 & 0.21 & & & $0 \cdot 16$ & 0.15 & 0.15 & 0.16 \\
\hline 106 & -0.66 & & -0.67 & -0.67 & -0.67 & $0 \cdot 18$ & 0.26 & & $0 \cdot 22$ & 0.21 & 0.20 & 0.21 \\
\hline 107 & & & -0.32 & -0.30 & -0.31 & 0.26 & & & 0.25 & $0 \cdot 21$ & 0.25 & 0.25 \\
\hline 108 & & & & & & 0.33 & & & & & & 0.33 \\
\hline 113 & & & -0.58 & -0.60 & -0.59 & 0.22 & & & $0 \cdot 13$ & 0.14 & 0.18 & 0.16 \\
\hline 115 & & & -0.58 & -0.61 & -0.60 & $0 \cdot 17$ & & & $0 \cdot 14$ & & 0.18 & 0.17 \\
\hline 117 & & & -0.56 & -0.60 & -0.58 & 0.24 & & & $0 \cdot 18$ & 0.24 & 0.22 & 0.24 \\
\hline 137 & & & & -0.33 & -0.33 & 0.32 & & & & & 0.31 & 0.31 \\
\hline 138 & & & & -0.53 & -0.53 & 0.24 & & & & 0.20 & 0.20 & 0.20 \\
\hline 139 & & & & -0.42 & -0.42 & $0 \cdot 25$ & & & & 0.22 & 0.25 & 0.25 \\
\hline 201 & & & -0.63 & -0.65 & -0.64 & 0.18 & & & 0.13 & 0.14 & 0.17 & 0.16 \\
\hline 202 & & & -0.61 & -0.62 & -0.62 & $0 \cdot 19$ & & & 0.13 & 0.13 & 0.18 & 0.16 \\
\hline 203 & & & $-0 \cdot 60$ & -0.64 & -0.62 & 0.22 & & & 0.12 & 0.12 & 0.15 & 0.13 \\
\hline 207 & & & -0.31 & -0.34 & -0.32 & 0.28 & & & $0 \cdot 22$ & 0.24 & 0.25 & 0.24 \\
\hline 215 & & & $-0 \cdot 38$ & -0.41 & -0.40 & 0.27 & & & $0 \cdot 18$ & 0.21 & 0.16 & 0.19 \\
\hline 216 & & & & -0.26 & -0.26 & 0.28 & & & & 0.21 & 0.25 & 0.25 \\
\hline 218 & & & & -0.37 & -0.37 & 0.32 & & & & 0.21 & 0.28 & 0.28 \\
\hline 223 & -0.62 & & -0.66 & -0.67 & -0.66 & 0.20 & $0 \cdot 24$ & & $0 \cdot 20$ & 0.19 & 0.20 & 0.20 \\
\hline 224 & & & -0.62 & -0.66 & -0.64 & 0.27 & & & $0 \cdot 16$ & 0.18 & 0.20 & 0.19 \\
\hline 228 & & & -0.49 & -0.50 & -0.50 & 0.22 & & & $0 \cdot 18$ & 0.17 & 0.20 & 0.19 \\
\hline 305 & & & -0.71 & -0.72 & -0.71 & $0 \cdot 18$ & & & 0.12 & 0.12 & 0.11 & 0.12 \\
\hline 306 & & -0.67 & -0.65 & -0.67 & -0.67 & 0.36 & & $0 \cdot 24$ & $0 \cdot 22$ & 0.24 & 0.28 & 0.24 \\
\hline 311 & & & $-0 \cdot 43$ & -0.45 & -0.44 & 0.23 & & & 0.15 & 0.14 & $0 \cdot 20$ & 0.17 \\
\hline 405 & & & -0.64 & -0.66 & -0.65 & 0.24 & & & 0.09 & 0.11 & 0.11 & 0.11 \\
\hline 410 & & & -0.38 & -0.39 & -0.38 & 0.27 & & & $0 \cdot 19$ & 0.18 & 0.24 & 0.22 \\
\hline 414 & & & -0.57 & -0.60 & -0.59 & 0.21 & & & $0 \cdot 13$ & 0.14 & $0 \cdot 16$ & 0.15 \\
\hline 417 & & -0.73 & -0.72 & -0.78 & -0.73 & 0.24 & & 0.26 & $0 \cdot 22$ & $0 \cdot 20$ & 0.16 & 0.22 \\
\hline 418 & & & -0.60 & -0.65 & -0.62 & $0 \cdot 24$ & & & 0.15 & 0.14 & 0.12 & 0.14 \\
\hline
\end{tabular}


Table 4.23: Colour excesses $E_{B-V}$ for Kappa Crucis stars from the literature.

\begin{tabular}{|c|c|c|c|c|c|c|c|c|}
\hline \# & AvS & Graham & Schild & Perry & Shob. & Waard & D\&K & median \\
\hline A & $0.30 \quad 0.34 \quad 0.34$ & 0.38 & 0.33 & $0 \cdot 34$ & $0 \cdot 40$ & $0 \cdot 36:$ & $0 \cdot 32$ & $0 \cdot 34$ \\
\hline B & $\begin{array}{llll}0.25 & 0.33 & 0.26\end{array}$ & $0 \cdot 40$ & $0 \cdot 31$ & $0 \cdot 38$ & $0 \cdot 37$ & $0 \cdot 36$ : & $0 \cdot 36$ & $0 \cdot 36$ \\
\hline $\mathrm{C}$ & $0.34 \quad 0.38 \quad 0.35$ & 0.40 & 0.41 & 0.41 & 0.39 & 0.37 : & $0 \cdot 40$ & 0.39 \\
\hline D & & & 0.42 & 0.55 & & & $0 \cdot 50$ & 0.50 \\
\hline $\mathrm{E}$ & $0 \cdot 28$ & & $0 \cdot 39$ & 0.43 & $0 \cdot 40$ & 0.40 & 0.41 & 0.40 \\
\hline F & $0 \cdot 29$ & & $0 \cdot 32$ & 0.36 & 0.33 & 0.32 & $0 \cdot 33$ & 0.32 \\
\hline G & 0.34 & & & $0 \cdot 42$ & $0 \cdot 38$ & 0.37 & $0 \cdot 39$ & 0.38 \\
\hline $\mathrm{H}$ & & & & $0 \cdot 50$ & $0 \cdot 42$ & 0.41 & $0 \cdot 46$ & 0.44 \\
\hline I & & & & 0.48 & 0.43 & 0.42 & 0.49 & 0.45 \\
\hline $\mathrm{J}$ & & & & 0.42 & $0 \cdot 35$ & $0 \cdot 36$ & 0.48 & $0 \cdot 39$ \\
\hline $\mathrm{K}$ & & & & 0.36 & $0 \cdot 33$ & $0 \cdot 35$ & $0 \cdot 46$ & 0.36 \\
\hline $\mathrm{L}$ & & & & 0.43 & 0.41 & 0.43 & $0 \cdot 49$ & 0.43 \\
\hline M & & & & $0 \cdot 36$ & & 0.39 & 0.40 & $0 \cdot 39$ \\
\hline 104 & & & & & & & & \\
\hline 105 & 0.34 & & 0.47 & 0.38 & 0.42 & 0.41 & 0.44 & 0.41 \\
\hline 106 & $0 \cdot 32$ & $0 \cdot 40$ & & 0.46 & 0.39 & 0.40: & $0 \cdot 37$ & $0 \cdot 40$ \\
\hline 107 & & & & 0.40 & & 0.39 & 0.44 & 0.40 \\
\hline 108 & & & & & & & & \\
\hline 113 & & & & 0.38 & 0.36 & 0.36 & 0.44 & $0 \cdot 37$ \\
\hline 115 & & & & 0.40 & 0.36 & & $0 \cdot 43$ & 0.40 \\
\hline 117 & & & & 0.45 & 0.41 & & 0.49 & 0.45 \\
\hline 137 & & & & & & & 0.52 & 0.52 \\
\hline 138 & & & & & & 0.42 & $0 \cdot 44$ & 0.43 \\
\hline 139 & & & & & & 0.40 & $0 \cdot 47$ & 0.43 \\
\hline 201 & & & 0.46 & 0.39 & 0.34 & 0.37 & $0 \cdot 44$ & $0 \cdot 39$ \\
\hline 202 & & & & $0 \cdot 39$ & 0.33 & 0.36 & 0.45 & 0.38 \\
\hline 203 & & & & $0 \cdot 38$ & $0 \cdot 38$ & 0.35 & 0.42 & $0 \cdot 38$ \\
\hline 207 & & & & 0.41 & 0.36 & 0.44 & 0.45 & 0.43 \\
\hline 215 & & & & $0 \cdot 38$ & 0.37 & 0.35 & $0 \cdot 36$ & 0.36 \\
\hline 216 & & & & & & 0.36 & 0.43 & 0.40 \\
\hline 218 & & & & & & 0.38 & 0.49 & 0.45 \\
\hline 223 & 0.32 & 0.40 & 0.48 & 0.50 & 0.45 & 0.42 & $0.47:$ & 0.45 \\
\hline 224 & & & & 0.46 & 0.41 & 0.41 & 0.47 & 0.43 \\
\hline 228 & & & & 0.40 & 0.36 & 0.37 & 0.44 & 0.39 \\
\hline 305 & 0.31 & & 0.46 & 0.41 & 0.37 & 0.37 & 0.39 & 0.38 \\
\hline 306 & & & 0.48 & 0.52 & 0.48 & & 0.60: & 0.50 \\
\hline 311 & & & & 0.38 & 0.41 & 0.33 & 0.42 & 0.39 \\
\hline 405 & & & & $0 \cdot 36$ & $0 \cdot 33$ & 0.36 & 0.36 & $0 \cdot 36$ \\
\hline 410 & & & & $0 \cdot 39$ & $0 \cdot 35$ & $0 \cdot 37$ & 0.45 & $0 \cdot 38$ \\
\hline 414 & & & & $0 \cdot 39$ & 0.35 & 0.37 & 0.41 & 0.38 \\
\hline 417 & & & 0.50 & 0.52 & 0.47 & & $0.50:$ & 0.50 \\
\hline 418 & & & & 0.41 & 0.37 & 0.40 & 0.38 & $0 \cdot 39$ \\
\hline
\end{tabular}


Table 4.24: Spectral Classifications of Kappa Crucis stars from Vilnius photometry.

\begin{tabular}{|c|c|c|c|c|c|c|}
\hline \# & Q-Search & Q-Spectra & \multicolumn{3}{|c|}{ QQ-diagrams } & average \\
\hline A & B5-8 I-II & B8 I 0.90 & $\mathrm{~B} 6 \cdot 5$ & $-6 \cdot 5$ & $\overline{\mathrm{Be}}$ ? & B7 I \\
\hline B & O9-B1 III-IV & B5 I 0.92 & B3-4 & -6.5 & & B3 I \\
\hline $\mathrm{C}$ & BO III & B3 I 0.95 & B3 & $-6 \cdot 3$ & & B3 I \\
\hline $\mathrm{D}$ & K2-6 III & K3 I (p) 0.95 & Ko & $-5 \cdot 0$ & & K2 I \\
\hline $\mathrm{E}$ & BO-2 III-IV & B3 I 1.00 or B1 III 0.98 & B1 & $-4 \cdot 5$ & & B1 III \\
\hline F & BO-2 III-IV & B1 VI 1.03 or B3 I 1.12 & B1 & $-2 \cdot 5$ & & B1 IV \\
\hline G & BO II-IV & B1 VI 0.90 or B3 I 1.00 or B1 V 0.93 & $\mathrm{~B} 1$ & $-3 \cdot 0$ & & B1 IV \\
\hline $\mathrm{H}$ & B1-3 III-IV & $\mathrm{B} 2 \mathrm{~V} 0.75$ or B2 III 0.80 & $\mathrm{~B} 2$ & $-2 \cdot 0$ & $\mathrm{Be}$ & B2 IV \\
\hline I & B0-2 III-V & B1 VI 1.05 or B3 I 1.13 & $\mathrm{~B} 2$ & $-4 \cdot 7$ & & B2 III \\
\hline $\mathrm{J}$ & B1-5 IV-V & B2 IV $1 \cdot 12$ & $\mathrm{~B} 2$ & -3.0 & & B2 IV \\
\hline K & B5 IV-V (SB) & B5 VI 1.15 or B5 V 1.13 & B4-5 & -0.5 & & B5 V \\
\hline $\mathrm{L}$ & B2-6 III-V & B3 III 1.65 & B3-4 & $-3 \cdot 2$ & & B3 III \\
\hline M & F0-6 II-III & F0 III (p) 0.58 & $\mathrm{~F} 2-3$ & $-1 \cdot 7$ & & F1 III \\
\hline 104 & Ro & G8 IV (p) 1.00 & $\mathrm{R}$ & & & $\mathrm{R}$ \\
\hline 105 & B0-2 I-V & B1 III 0.98 or B2 I 1.03 & $\mathrm{~B} 1$ & $-6 \cdot 0$ & & B1 I \\
\hline 106 & B0-2 II-IV & B3 I 0.98 & $\mathrm{~B} 2$ & $-7 \cdot 0$ & & B2 I \\
\hline 107 & $\mathrm{~B} 4-\mathrm{A} 2 \mathrm{~V}(\mathrm{SB})$ & B5 VI (p) 1.00 & B5-6 & $+1 \cdot 0$ & SB & B5 V \\
\hline 108 & B5-7 IV-V (P,SB) & B5 V (p) 1.23 & B6 & +0.0 & SB & B5 V \\
\hline 113 & B0-4 IV-V & B2 III 1.18 & B2 & -3.0 & & B2 IV \\
\hline 115 & B2 IV-V & B2 VI 1.00 or B2 III-IV 1.03 & B1-2 & $-2 \cdot 0$ & & B2 IV \\
\hline 117 & B1-5 IV-V & $\mathrm{B} 2 \mathrm{~V} 1.08$ & $\mathrm{~B} 2-3$ & $-3 \cdot 0$ & & B2 IV \\
\hline 137 & B2-8 II-V & B3 III (p) 1.83 & B4 & $-6 \cdot 0$ & & B3 II \\
\hline 138 & B3 III-IV & B3 III 1.00 & $\mathrm{~B} 2.5$ & $-3 \cdot 0$ & & B3 III \\
\hline 139 & B2-8 III-V & B5 V (p) 0.95 & B5 & $-2 \cdot 0$ & ? & B5 IV \\
\hline 201 & O9 V & B0 I 0.98 or $\mathrm{B} 0 \mathrm{~V} 0.93$ & $\mathrm{~B} 2$ & $-3 \cdot 5$ & $\mathrm{Be}$ ? & B0 IV \\
\hline 202 & B2 IV-V & B2 III $1 \cdot 25$ & $\mathrm{~B} 2$ & $-4 \cdot 0$ & & B2 III \\
\hline 203 & B0-2 III-V & B2 III 1.00 & $\mathrm{~B} 2$ & $-4 \cdot 5$ & & B2 III \\
\hline 207 & B3 II-V & B3 V $0.83(p)$ & B4 & -3.5 & $\mathrm{Be}$ & B3 III \\
\hline 215 & B3-5 IV-V & B3 IV 1.13 & B4 & $-0 \cdot 5$ & & B4 IV \\
\hline 216 & B5-9 I-II & B9 I 1.10 or B5 V 1.00 & B5-6 & $-3 \cdot 0$ & & B5 III \\
\hline 218 & B2-6 III-V & B3 V (p) 2.05 & B4 & $-2 \cdot 0$ & & B3 IV \\
\hline 223 & B0-1 II-IV & B3 I 0.95 & $\mathrm{~B} 1-2$ & $-5 \cdot 5$ & & B2 II \\
\hline 224 & B0-2 III-V & B1 V (p) 0.88 & B1 & $-4 \cdot 0$ & $\mathrm{Be}$ & B1 III \\
\hline 228 & B1-7 IV-V & $\mathrm{B} 2 \mathrm{~V} 1.28$ & $\mathrm{~B} 2$ & -3.5 & & B2 IV \\
\hline 305 & B1-3 II-IV & B3 I 1.20 & $\mathrm{~B} 1-2$ & $-6 \cdot 0$ & ? & B2 II \\
\hline 306 & O7-B1 I-II & $?$ & B0 & $-3 \cdot 0$ & & BO III \\
\hline 311 & B3 III-V & B3 V 0.87 or B3 IV 0.88 & B3 & -1.5 & & B3 V \\
\hline 405 & B0-2 II-V & B2 IV 0.78 & $\mathrm{~B} 2$ & $-2 \cdot 0$ & $\mathrm{Be}$ ? & B2 IV \\
\hline 410 & B2-7 IV-V & B3 III 1.55 & B3 & $-2 \cdot 5$ & & B3 IV \\
\hline 414 & B1-5 III-V & B2 IV 1.23 & B2 & $-3 \cdot 5$ & & B2 IV \\
\hline 417 & $\mathrm{O} 7-\mathrm{B} 2 \mathrm{~V}, \mathrm{E}$ & $?$ & B0 & -3 & $?$ & Bo V \\
\hline 418 & B0-2 III-V & B1 VI 1.03 or B3 I 1.10 & $\mathrm{~B} 1-2$ & $-3 \cdot 5$ & & B2 III \\
\hline
\end{tabular}


Table 4.25: Dereddening of Kappa Crucis stars from Vilnius photometry. $V_{0}$ was calculated using the median of the distance moduli.

\begin{tabular}{|c|c|c|c|c|c|c|c|}
\hline \# & $\mathrm{V}$ & $\mathrm{Y}-\mathrm{V}$ & $E_{\mathrm{Y}-\mathrm{V}}$ & $(\mathrm{Y}-\mathrm{V})_{0}$ & $\mathrm{R}$ & $\mathrm{m}-\mathrm{M}$ & $\mathrm{V}_{0}$ \\
\hline $\mathrm{A}$ & $5 \cdot 747$ & 0.481 & +0.281 & 0.20 & 4.48 & $12 \cdot 18$ & -7.69 \\
\hline B & $5 \cdot 965$ & $0 \cdot 417$ & +0.267 & $0 \cdot 15$ & 4.48 & $12 \cdot 22$ & $-7 \cdot 41$ \\
\hline C & $6 \cdot 792$ & $0 \cdot 420$ & $+0 \cdot 270$ & $0 \cdot 15$ & $4 \cdot 48$ & $12 \cdot 22$ & $-6 \cdot 60$ \\
\hline D & $7 \cdot 480$ & 1.709 & +0.509 & $1 \cdot 20$ & 4.54 & $12 \cdot 18$ & -7.01 \\
\hline $\mathrm{E}$ & 324 & $0 \cdot 368$ & +0.308 & 0.06 & 4.48 & $12 \cdot 27$ & $-5 \cdot 24$ \\
\hline $\mathrm{F}$ & 009 & & +0.288 & 0.05 & .48 & 12.27 & $-4 \cdot 46$ \\
\hline G & $9 \cdot 770$ & 347 & $+0 \cdot 317$ & 0.03 & 48 & $12 \cdot 18$ & $-3 \cdot 83$ \\
\hline $\mathrm{H}$ & $9 \cdot 874$ & 0.373 & +0.303 & 0.07 & $4 \cdot 48$ & $12 \cdot 27$ & $-3 \cdot 66$ \\
\hline & 9.989 & 0.416 & $+0 \cdot 346$ & 0.07 & $4 \cdot 48$ & $12 \cdot 18$ & $-3 \cdot 74$ \\
\hline J & 10.952 & 0.393 & $+0 \cdot 313$ & 0.08 & 4.48 & $12 \cdot 31$ & -2.63 \\
\hline K & ·391 & $0 \cdot 400$ & $+0 \cdot 300$ & $0 \cdot 10$ & 4.48 & $12 \cdot 18$ & $-2 \cdot 13$ \\
\hline L & .854 & 0.495 & $+0 \cdot 425$ & 0.07 & $4 \cdot 48$ & $12 \cdot 14$ & $-2 \cdot 23$ \\
\hline M & 518 & $0 \cdot 272$ & -0.068 & 0.34 & 4.48 & $12 \cdot 09$ & +0.64 \\
\hline 104 & 10.9 & 1.172 & +0.517 & 66 & $4 \cdot 48$ & $12 \cdot 16$ & $-3 \cdot 51$ \\
\hline 105 & 663 & $0 \cdot 375$ & $+0 \cdot 315$ & 0.06 & $4 \cdot 48$ & $12 \cdot 09$ & -4.93 \\
\hline 106 & 6.914 & 0.414 & +0.304 & 0.11 & 4.48 & $12 \cdot 22$ & $-6 \cdot 63$ \\
\hline 107 & .304 & 0.368 & +0.268 & $0 \cdot 10$ & $4 \cdot 48$ & $12 \cdot 18$ & $-2 \cdot 08$ \\
\hline 108 & & 402 & +0.3 & $0 \cdot 10$ & $4 \cdot 48$ & $12 \cdot 18$ & -1.77 \\
\hline 113 & .113 & & +0.2 & 0.06 & 4.48 & $12 \cdot 22$ & $-3 \cdot 36$ \\
\hline 115 & & $0 \cdot 370$ & $+0 \cdot 3$ & 0.06 & 4.48 & $12 \cdot 22$ & $-3 \cdot 33$ \\
\hline 117 & & & $+0 \cdot 3$ & 0.04 & $4 \cdot 48$ & $12 \cdot 14$ & $-2 \cdot 83$ \\
\hline 137 & & 0.527 & +0.4 & 0.065 & $4 \cdot 48$ & $12 \cdot 20$ & -1.71 \\
\hline 138 & .057 & 0.416 & $+0 \cdot 341$ & 0.075 & 4.48 & 12.18 & $-2 \cdot 65$ \\
\hline 139 & $11 \cdot 641$ & $0 \cdot 370$ & +0.270 & $0 \cdot 10$ & $4 \cdot 48$ & 18 & -1.75 \\
\hline 201 & 93 & $0 \cdot 313$ & +0.293 & 0.0 & $4 \cdot 48$ & $12 \cdot 18$ & $-3 \cdot 90$ \\
\hline 202 & 906 & 71 & +0.311 & & 4.48 & $12 \cdot 14$ & -3.67 \\
\hline 203 & 270 & $0 \cdot 321$ & $+0 \cdot 2$ & 0.045 & $4 \cdot 48$ & 07 & $-3 \cdot 15$ \\
\hline 207 & .064 & $0 \cdot 451$ & $+0 \cdot 3$ & 0.07 & $4 \cdot 48$ & $12 \cdot 18$ & -1.82 \\
\hline 215 & & & +0.2 & 0.09 & $4 \cdot 48$ & $12 \cdot 18$ & -1.82 \\
\hline 216 & & & & & 4.48 & .18 & -1.29 \\
\hline 218 & & & & & $4 \cdot 48$ & & $-1 \cdot 64$ \\
\hline 223 & & & +0.2 & $0 \cdot 1$ & $4 \cdot 48$ & $12 \cdot 20$ & -5.47 \\
\hline 224 & $10 \cdot 202$ & $0 \cdot 419$ & +0.394 & 0.025 & $4 \cdot 48$ & $12 \cdot 11$ & $-3 \cdot 74$ \\
\hline 228 & $10 \cdot 990$ & $0 \cdot 402$ & +0.362 & 0.04 & 4.48 & $12 \cdot 14$ & -2.81 \\
\hline 305 & & $0 \cdot 396$ & +0.291 & 0 . & 4.48 & $12 \cdot 20$ & $-5 \cdot 25$ \\
\hline 306 & $10 \cdot 047$ & $0 \cdot 405$ & +0.385 & 0.02 & $4 \cdot 48$ & $12 \cdot 18$ & $-3 \cdot 86$ \\
\hline 311 & $10 \cdot 766$ & 0.337 & $+0 \cdot 262$ & 0.075 & $4 \cdot 48$ & $12 \cdot 25$ & -2.59 \\
\hline 405 & $10 \cdot 093$ & 0.266 & $+0 \cdot 216$ & 0.05 & $4 \cdot 48$ & $12 \cdot 18$ & -3.05 \\
\hline 410 & $10 \cdot 642$ & 0.426 & +0.336 & 0.09 & 4.48 & $12 \cdot 27$ & -3.04 \\
\hline 414 & 9.981 & $0 \cdot 366$ & +0.306 & 0.06 & $4 \cdot 48$ & $12 \cdot 22$ & -3.57 \\
\hline 417 & $9 \cdot 790$ & & +0.391 & 0.03 & 4.48 & $12 \cdot 31$ & $-4 \cdot 14$ \\
\hline 418 & $9 \cdot 660$ & $0 \cdot 357$ & +0.287 & 0.07 & $4 \cdot 48$ & $12 \cdot 18$ & $-3 \cdot 81$ \\
\hline
\end{tabular}




\section{Chapter 5}

\section{Discussion and Conclusions.}

Given that photometry is a practical necessity for most observations (as spectroscopy is limited to the brightest stars using large telescopes), the question arises of which photometric system should be used. Most photometric systems have been designed to investigate a particular astrophysical question, so would be a logical choice for further work in that area. However this must be balanced against the availability of standard stars in that system and the ability to compare your results with those of other observers made in different systems. This has led some observers to consider the idea of one 'perfect all-purpose' photometric system used by everyone, with subsets of the filters chosen to suit any particular investigation. However others do not believe such a system is possible, stating that the differing needs of each investigation would lead to conflicting requirements of the filters.

The Vilnius system was carefully designed to classify all normal stars (regardless of reddening) in the MK system, without recourse to MK spectroscopy. It also turned out to be able to detect many types of peculiar stars, so is also ideally suited for the survey phase necessary before a photometric system designed for that type of peculiar star is used (in many cases the Vilnius system itself will adequately measure the peculiar star). Thus the Vilnius system is arguably the closest any photometric system has come to being the 'perfect all-purpose' system.

Regardless of personal philosophy concerning specialized verses all-purpose systems, the Vilnius system satisfies most of the requirements of a 'good' photometric system. For instance, a full description of the system is given via the transmission passbands of each filter and the system is quite stable, having only one alteration made (to the $\mathrm{S}$ filter) since it was established. The choice of glass for the filters (rather than interference filters) means each local filter set itself is stable, and the glasses were picked to ensure there are no red leaks. The reduction procedures (for extinction, standardization and dereddening) are fully described, and have the advantage of measuring the extinction at intervals throughout the night rather than using a single, average value for the entire night. The medium bandwidth of the filters is a compromise between the need for broad bandwidths to measure fainter stars verses the need for narrow bandwidths to measure specific spectral features and reduce the complications in the reduction 
procedures arising from non-monochromatic measurements. From the astrophysical point of view, the system has high purity, has several complementary classification methods based on reddening-free Q factors, and is well calibrated in both the MK system and physical parameters such as effective temperature and gravity.

The one major flaw of the Vilnius system (which apparently all photometric systems suffer from) is that it doesn't provide critical sampling of the spectrum at the filters bandwidth resolution, i.e. the filters should be spaced no further apart in wavelength than half their FWHM. The importance of this was not appreciated at the time the system was designed, but insufficient sampling has since been shown to lead to systematic errors in any reduction procedure. As shown in Appendix I, the addition of some extra filters situated between the existing filters should reduce the systematic errors to an acceptable level - however the experimental proof of this has to be left to a future observer.

Note that critical sampling at the medium FWHM of the Vilnius filters ( $230 \AA$ ) over the wavelength range covered $(3100 \AA)$ would require $\sim 27$ filters and would give all information available from a stellar spectrum at that wavelength resolution. Even with the improved filter and detector technology now available this would take too long to measure a star to be practical. However widening the FWHM of filters measuring wavelengths with little astrophysical information (such as the continuum) would significantly reduce the number of filters, suggesting a method to design the 'perfect all-purpose' photometric system.

The use of Nikonov's method to determine the extinction at MJUO has revealed apparent variations throughout the night, which were probably not detected before as the other observing programs at MJUO involve differential photometry using mean extinction values. This has also highlighted the need for a standard lamp at MJUO to check and/or correct for drifts in the instrumental zero-points. It would also be useful for future observations with the Vilnius system to measure the outside air temperature and pressure throughout the night. This may lead to night-time measures of the ozone level and aerosol distribution that would compliment those currently made during the day by other New Zealand researchers (for example the National Institute of Water and Atmospheric Research), in addition to any astrophysical benefits from improved extinction corrections.

The filters supplied by the Vilnius Institute of Theoretical Physics and Astronomy show a close match to the passbands of the standard filters, so should allow transformations to the standard system at least as good as those between local and standard filters of any photometric system (notwithstanding systematic errors due to non-critical sampling). Although the $0^{m} \cdot 01$ precision and accuracy goal was not achieved (reaching $0^{m} \cdot 02$ instead), this was largely due to the effects of the Mount Pinatubo eruption on the atmospheric extinction at MJUO. Further, the largest uncertainties were generally found in the U filter measurements - these appear to 
be due to the lower signal because of the larger extinction at the U filter's effective wavelength and its corresponding greater sensitivity to changes in the extinction, and the smaller signal from the star itself after the Balmer jump - and should be easily remedied by making longer measurements through the $\mathrm{U}$ filter than the others (at least 4-5 times longer, as they were already observed for 2-3 times longer in this study). Another important factor concerning the $0^{m} \cdot 02$ results is that the stars used as Vilnius standards for the transformations are not actually standards (an equatorial region of standards only became available after the observational phase of this study was completed). Thus the failure to achieve the original goal should not be taken as a reflection on the quality of the Vilnius system or the MJUO facilities.

At the time this observing program was planned, the difficulty of establishing a standard star system in the New Zealand maritime climate (hindsight has reduced the original estimate of photometric nights from $\sim 30 \%$ down to $\sim 10 \%$ ) was balanced against the difficulty (and expense) of obtaining observing time at overseas observatories for such a program. The generous scheduling at MJUO allowed this problem to be overcome by making a large number of observations per star. Having a insufficient number of observations per star accounts for most of the stars that were not accepted as standards, so with relatively little effort a future observer could approximately double the number of standard stars in the southern Vilnius system by completing these measurements.

The possibility of more crowded regions of fainter stars suitable for CCD photometry standards should also be considered. Note that as PMT's are generally used to measure standards (they are still more precise than CCD's), the standards must not be too crowded. Open clusters, the 'outskirts' of globular clusters, and regions near the galactic equator should provide a adequate number of standard stars which can be observed together in one CCD frame (or an acceptably small number of frames) with a wide range in magnitude and colour. It is recommended that the CCD standard regions of other photometric systems be considered, as was done with the $\mathrm{E}$ regions.

Two young, open clusters (Omicron Velorum and Kappa Crucis) were observed in this study to demonstrate some of the advantages the Vilnius system has over other photometric systems. As the Vilnius photometry gives the same results (membership, distance modulus, reddening, age and metallicity) as the other photometric systems (without the additional input from spectroscopy and proper motion studies that the other systems need), I believe this has been achieved. However more work needs to be done on isochrones in the Vilnius system, as other CMD's or colour-colour diagrams (than the Y-V, V CMD used here) may have differing sensitivities to reddening, metallicity, age etc. In addition, simultaneous isochrone fitting of different CMD's should increase the precision and accuracy of the results.

As the literature does not agree on the variability (or otherwise) of the brighter members 
of each cluster, a dedicated program of differential photometry would be needed to settle this question. More measurements are also needed of the faintest stars observed in this study to decrease their uncertainties in $\mathrm{Y}-\mathrm{V}$. Among other things, this would suggest eclipsing binary candidates (based on either their variability or position in the CMD relative to the main sequence). Measurements of eclipsing binaries would provide a valuable independent check on the Vilnius system calibrations (of temperature, gravity etc.) and isochrones as light curves of eclipsing binaries, in conjunction with radial velocity curves, yield orbital and astrophysical parameters (for example mass, gravity, luminosity, distance).

Once the brighter cluster members are accurately known, they can be used as a zero-point tie to Vilnius CCD photometry which could rapidly complete observation of all cluster members to a given limiting magnitude (which could be much fainter than that achieved here given a larger telescope, such as the $\mathrm{M}^{\mathrm{c}}$ Lellan one metre at MJUO). This would also allow a better investigation of reddening variations along the line of sight and within the cluster itself. It would also allow the possibility of detecting a 'pre-main sequence' sequence, which has been hinted at by the results from this study.

In conclusion, the basis of a standard star network for the southern Vilnius photometric system has been established, and the utility of the Vilnius system for astrophysical measurements has been demonstrated. Building on these results, the size of the southern standard star system could be doubled with relatively little extra observations (which time did not permit in this study). Similarly, some further measurements of the cluster stars, plus some CCD observations to complete the coverage of cluster members and extend the limiting magnitude, would yield extra astrophysical information. 


\section{Appendix A}

\section{Overview of the Appendices.}

A brief description of the appendices is given here - note that for the appendices containing tables, a description of the tables are given at the front of the appendix.

Appendix B lists the stars selected as potential standard stars, either for transforming the local system to the northern standard system (the 'primary' standards) or as standards of the southern system (the 'secondary' standards). These have been included for the benefit of any future observers who wish to continue the development of the southern Vilnius standard system.

Appendix C has two tables; the first is a summarized form of the Observational Log of this study while the second lists all stars observed in this study with relevant information for an observer (for example coordinates, visual magnitude, spectral type).

Appendix D gives the individual observations of the primary and secondary standard stars and the two clusters observed ( $\kappa$ Crucis and O Velorum). These have been included so future observers may add further observations to each star and recalculate their means, and also investigate possible variability of a star. Appendix E lists the mean values for each star, splitting the tables into those acceptable as standards and those that currently do not qualify.

Appendix F gives the derivation of Nikonov's method for calculating monochromatic extinction at any time during observations. Appendix G examines the longer term variations in extinction at MJUO, to see if a running average over several nights (or seasonally) can be used for a night for which the extinction was not measured. It was concluded that a running average is only useful for a photometric night during which the uncertainty in the measured extinction exceeds 0.03 , i.e. the large uncertainty is due to a lack of extinction star observations rather a poor quality night. Further, the variation in the night-to-night extinction was of the same size as the change in the mean extinction during different seasons, so mean values for the extinction should not be used at MJUO. The aerosols and gases ejected into the atmosphere by the June 1991 Mount Pinatubo eruption were found to significantly increase both the mean value and variation in the extinction at MJUO.

Appendix $\mathrm{H}$ continues examining extinction, this time using data from the seven Vilnius filters to investigate the various components making up atmospheric extinction over the optical 
region (Rayleigh scattering, aerosol scattering and molecular absorption). As four parameters have to be fit, the results had such a large scatter that no trends could be found. However, fixing the Rayleigh parameter reduced the scatter in the remaining parameters sufficiently that trends throughout a night in the aerosol scattering and ozone absorption could be seen. The effect of the Mount Pinatubo eruption on the individual components could also be seen; on average the aerosol extinction was increased by a factor of four while the ozone absorption was halved.

Appendix I looks at the possibility of making Vilnius filters out of Schott glasses. Only three of the Schott glass combinations match the standard filters as well as the local filters provided by the Vilnius Institute of Theoretical Physics and Astronomy, so Schott glasses should not be used to implement a Vilnius filter system. However Schott glasses might be used to construct additional filters centred at wavelengths between that of the Vilnius filters so critical sampling of the wavelength region is achieved. This should result in better transformations to the standard system (compared to using the local Vilnius filters alone), but still awaits experimental confirmation.

Appendix $\mathrm{J}$ lists several tables from the literature that were used in this study. They have been included here as most are from a book by Straižys (1992), which may be difficult to obtain.

Appendix $\mathrm{K}$ gives a glossary of the acronyms used in this study. The meaning of the acronym is also given the first time it is used in the thesis. Finally, in Appendix L a list of papers relevant to the project published by this author and coworkers is given. There are also other papers planned for publication after the completion of this thesis, which can probably be found in later volumes of Baltic Astronomy. 


\section{Appendix B}

\section{Catalogs of Candidate Primary and Secondary Standard Stars.}

The following tables list the stars selected as candidates for primary or secondary standard stars.

The first table are stars selected from the Vilnius catalog (North 1980) to be used to transform the local photometric system to the standard Vilnius system. The first column is the star name, with the next two being the equatorial coordinates (for the Epoch 2000). The following seven columns are the Vilnius V magnitude and colours. An ' $\mathrm{N}$ ' in the V magnitude column indicates that it is actually a Johnson V magnitude taken from the catalogue of Nicolet (1978). Similarly, an 'M' indicates Johnson V from the catalogues of Mermilliod (1987a, b), and 'L' indicates Johnson $\mathrm{V}$ from other sources. The second to last column gives the number of measurements made of the star in the standard Vilnius system, and the last column lists the star's spectral type.

The following two tables are stars selected as secondary standards. The first three columns are again the star's name and coordinates. The next two columns are the Johnson B and V magnitudes, followed by the star's spectral type. The E region secondary standard stars are not listed here as they can be found in the literature, for example from Menzies et al. (1989). 


\begin{tabular}{|c|c|}
\hline की & 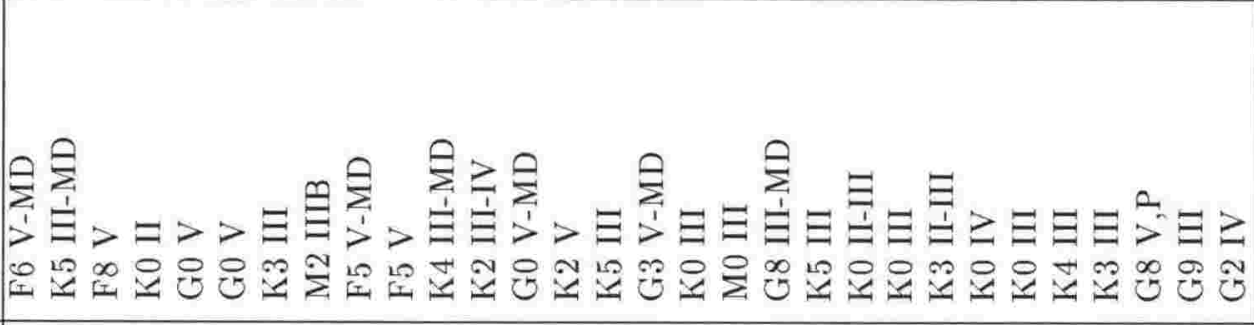 \\
\hline \# & |ேீலல \\
\hline $\begin{array}{l}\infty \\
1 \\
>\end{array}$ & 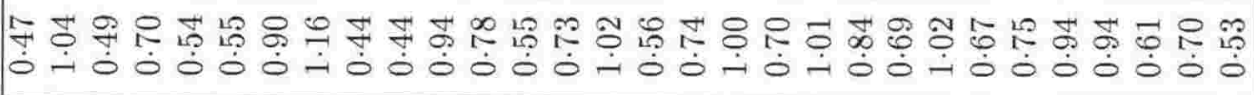 \\
\hline$\vec{N}$ & 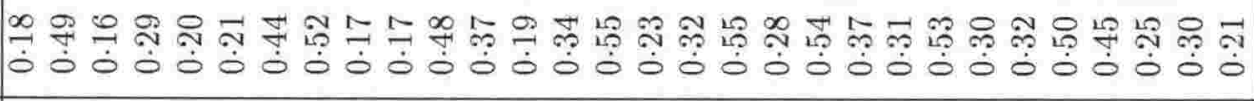 \\
\hline $\mid \begin{array}{lll}N \\
1 \\
>\end{array}$ & 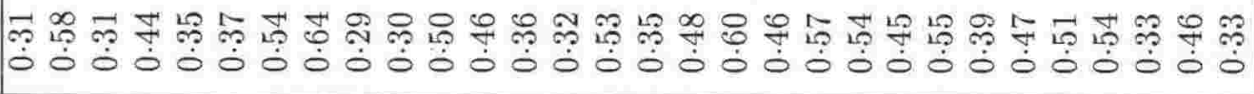 \\
\hline$\frac{7}{1}$ & 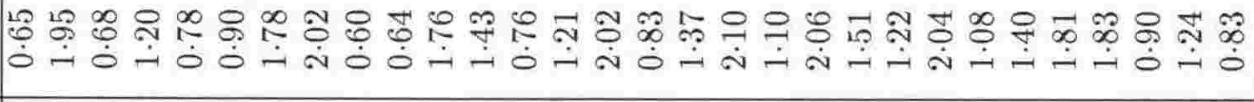 \\
\hline $\begin{array}{l}x \\
1 \\
2\end{array}$ & 总 \\
\hline$\underline{T}$ & 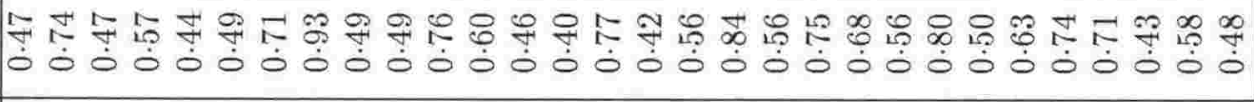 \\
\hline$>$ & 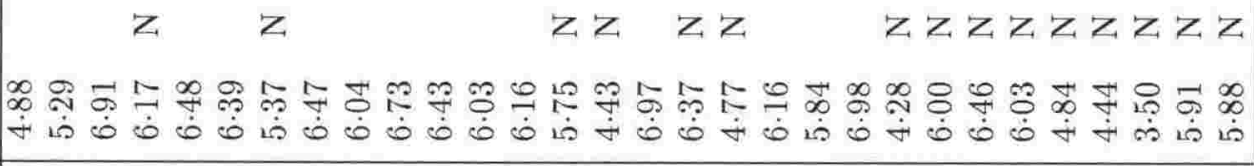 \\
\hline$\stackrel{\cup}{\square}$ & 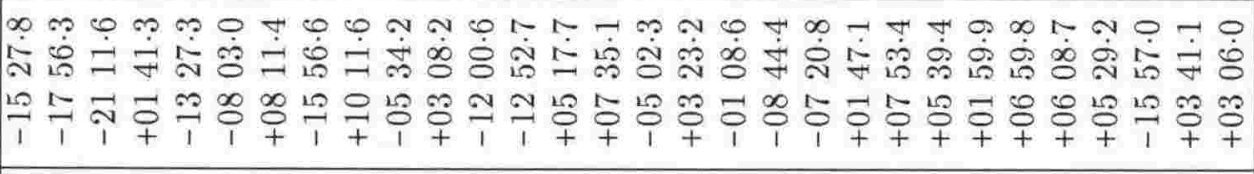 \\
\hline$\underset{2}{2}$ & 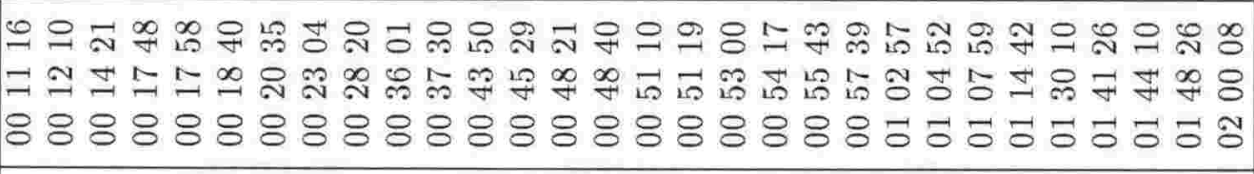 \\
\hline $\begin{array}{l}\# \\
\text { \# }\end{array}$ & 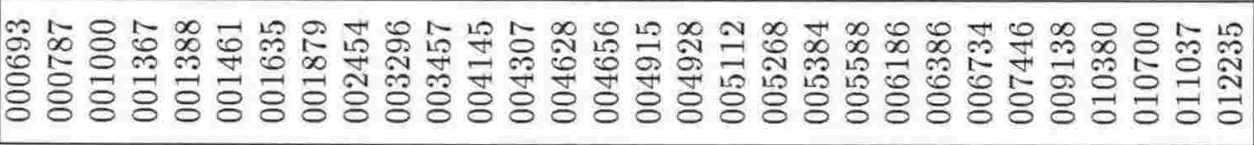 \\
\hline
\end{tabular}




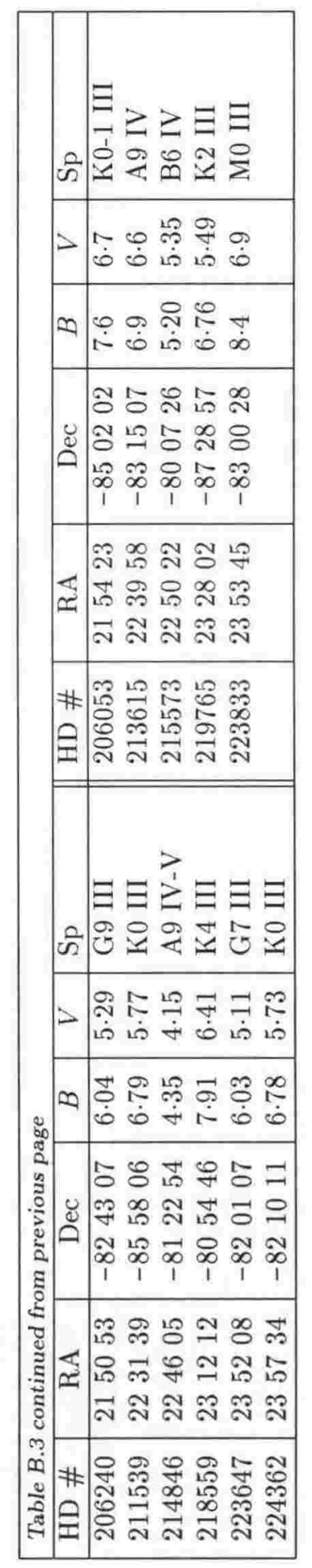




\section{Appendix C}

\section{The Observational Log.}

This appendix contains two tables describing the observations.

The first (Table C.1) summarizes the observations made each night. Column one shows the calendar date (at the start of the night), with an asterisk indicating a note at the end of the table. The second column gives the Julian Date (minus 2440000.5 days). The next column lists the observers; RJD = Richard Dodd, MF = Murray Forbes. The instrumental setup is described in the fourth column;

telescopes :

O/C - Optical Craftsman $61 \mathrm{~cm}$

B\&C - Boller and Chivens $61 \mathrm{~cm}$

$1 \mathrm{~m}-\mathrm{M}^{\mathrm{c}}$ Lellan $1 \mathrm{~m}$ with either the $\mathrm{f} 13$ or $\mathrm{f} 8$ secondary mirror

data loggers :

PET - the PET computer

$\mathrm{PC}$ - a PC computer

detectors (and settings) :

VUW PMT - Victoria University of Wellington PhotoMultiplier Tube EMI 9558B

CCD - Thomson CSF TH7882 CDA Charge Coupled Device

Ampl \# - the amplifier/discriminator used with the PMT

filters :

Vil 1 - Vilnius Set 1, $15 \mathrm{~mm}$ diameter filters

Vil 2 - Vilnius Set 2, $28 \mathrm{~mm}$ diameter filters

An assessment of the photometric quality of the night is given in the fifth column. The last column summarizes the number of stars (of each type) observed that night;

std - Vilnius primary standard stars

SPS - South Polar Sequence Vilnius secondary standard candidate stars

sec std - bright, southern secondary standard candidate stars

E? - E region secondary standard candidate stars 
R Arae - differential photometry of the eclipsing binary R Arae and a comparison and check star

drift-scans - attempts to measure the components of an optical double by drifting the stars across the diaphragm

std lamp - investigation of beta light source

$\kappa$ Crucis - stars in the $\kappa$ Crucis open cluster

O Vel - stars in the O Vel open cluster

M41 - stars in the M41 open cluster

NGC1818 - a Large Magellanic Cloud (LMC) "globular cluster" observed for T. Banks

sec extn - stars used to calibrate the secondary extinction coefficients

dead-time - dead-time calibration measurements (using LEDs)

The second (Table C.2) lists all the stars observed, sorted alphabetically by star name. The first column gives the star name (or cluster name). Any symbols (in parenthesis) following the name denote;

(v) - variable star, if primary standard then only used if no others are observable at the time

$(\mathrm{v}$ ?) - possibly variable star, if primary standard then only used if no others are observable at the time

$(\infty)$ - optical double star (may not be accepted as standard star due to possible errors if both stars not consistently included in later measurements)

$(\ddagger)$ - nearby star of similar intensity, so not accepted as standard star due to possible errors in identifying correct star

The second column gives the Epoch (years) of the star's coordinates, which are given in the next two columns. These are equatorial coordinates Right Ascension (hours, minutes, seconds) and Declination (degrees, minutes). The next column lists the star's 'type';

PriStd - Vilnius northern Primary Standard stars

SPS - South Polar Sequence Vilnius secondary standard candidate stars

SecStd - bright, southern Vilnius Secondary Standard candidate stars

ESecStd - E region Vilnius Secondary Standard candidate stars

PriExtn - southern secondary standard star used to measure the Primary Extinction

SecExtn - southern secondary standard star used to measure the Secondary Extinction

R Arae - an eclipsing binary star

Comp - southern secondary standard star used as the Comparison star for differential photometry of R Arae

Check - southern secondary standard star used as the Check star for differential photometry of R Arae

Program - all remaining stars (not intended as standards)

The last two columns give the Johnson $V$ magnitude and spectral class, taken from either the Vilnius catalog (for the primary standards) or SIMBAD (all other stars). 
Table C.1: A summary of the observations made on each night.

\begin{tabular}{|c|c|c|c|c|c|}
\hline date & $\begin{array}{l}\text { julian } \\
\text { date }\end{array}$ & $\begin{array}{l}\text { obser- } \\
\text { ver(s) }\end{array}$ & equipment & $\begin{array}{l}\text { photometric } \\
\text { quality }\end{array}$ & observations \\
\hline 15 Oct $87^{*}$ & 7083 & RJD & O/C scope & \multicolumn{2}{|c|}{ no reductions made } \\
\hline 21 Jun $88^{*}$ & 7333 & \multirow[t]{4}{*}{ R.JD } & \multirow[t]{4}{*}{ O/C scope } & cloudy & 8 SPS \\
\hline 22 Jun 88 & 7334 & & & $100 \%$ cloud & \\
\hline 23 Jun 88 & 7335 & & & $100 \%$ cloud & \\
\hline 24 Jun 88 & 7336 & & & photometric & 5 std, 8 SPS, R Arae \\
\hline 27 May 89 & 7673 & R.JD & O/C scope & clear 3 hours & \\
\hline 04 Jun $89^{*}$ & 7681 & \multirow[t]{9}{*}{ RJD } & \multirow[t]{9}{*}{ O/C scope } & marginal & \\
\hline 05 Jun 89 & 7682 & & & photometric & 3 std, 3 SPS, $8 \kappa$ Crucis \\
\hline 06 Jun 89 & 7683 & & & $100 \%$ cloud & \\
\hline 07 Jun 89 & 7684 & & & $100 \%$ cloud & \\
\hline 08 Jun 89 & 7685 & & & photometric & 9 std, $5 \kappa$ Crucis \\
\hline 09 Jun 89 & 7686 & & & $100 \%$ cloud & \\
\hline 1 & 1 & & & 1 & \\
\hline 21 Jun 89 & 7698 & & & $100 \%$ cloud & \\
\hline 22 Jun 89 & 7699 & & & photometric & 4 std, 9 SPS \\
\hline 05 Nov 90 & 8200 & \multirow[t]{4}{*}{ RJD } & \multirow{15}{*}{$\begin{array}{l}\text { O/C scope, } \\
\text { PET logger, } \\
\text { Vil \#1 }\end{array}$} & clear 3 hours & \\
\hline 06 Nov 90 & 8201 & & & $100 \%$ cloud & \\
\hline 07 Nov 90 & 8202 & & & good & $1 \mathrm{std}, 3 \mathrm{sec}$ extn \\
\hline 08 Nov 90 & 8203 & & & $100 \%$ cloud & \\
\hline 09 Nov 90 & 8204 & \multirow{2}{*}{$\begin{array}{l}\text { RJD } \\
\& \text { MF }\end{array}$} & & marginal & 6 std \\
\hline 10 Nov 90 & 8205 & & & $100 \%$ cloud & \\
\hline 11 Nov 90 & 8206 & \multirow[t]{9}{*}{ MF } & & $100 \%$ cloud & \\
\hline 1 & 1 & & & 1 & \\
\hline 21 Nov 90 & 8216 & & & $100 \%$ cloud & \\
\hline $22 \mathrm{Nov} 90$ & 8217 & & & clear 3 hours & \\
\hline 23 Nov 90 & 8218 & & & $100 \%$ cloud & \\
\hline 24 Nov 90 & 8219 & & & cloudy & \\
\hline 25 Nov 90 & 8220 & & & clear 2 hours & 4 std \\
\hline 26 Nov 90 & 8221 & & & $100 \%$ cloud & \\
\hline 27 Nov 90 & 8222 & & & $100 \%$ cloud & \\
\hline $17 \mathrm{Feb} 91$ & 8304 & \multirow[t]{10}{*}{ MF } & \multirow{10}{*}{$\begin{array}{l}\text { B\&C scope, } \\
\text { PET logger, } \\
\text { Ampl \#3, Vil \#1 }\end{array}$} & $100 \%$ cloud & \\
\hline 18 Feb 91 & 8305 & & & $100 \%$ cloud & \\
\hline $19 \mathrm{Feb} 91$ & 8306 & & & cloudy & . \\
\hline $20 \mathrm{Feb} 91$ & 8307 & & & cloudy & \\
\hline $21 \mathrm{Feb} 91$ & 8308 & & & good & $7 \mathrm{sec}$ extn, $70 \mathrm{Vel}$ \\
\hline $22 \mathrm{Feb} 91$ & 8309 & & & cloudy & \\
\hline 1 & 1 & & & 1 & \\
\hline $26 \mathrm{Feb} 91$ & 8313 & & & cloudy & \\
\hline $27 \mathrm{Feb} 91^{*}$ & 8314 & & & clear 4 hours & $5 \mathrm{std}, 10 \mathrm{O} \mathrm{Vel}$, dead-time \\
\hline $28 \mathrm{Feb} 91$ & 8215 & & & photometric & $6 \mathrm{std}, 10 \mathrm{O} \mathrm{Vel}$ \\
\hline & & & & & continued on next page \\
\hline
\end{tabular}




\begin{tabular}{|c|c|c|c|c|c|}
\hline \multicolumn{6}{|c|}{ Table C.1 continued from previous page } \\
\hline date & $\begin{array}{l}\text { julian } \\
\text { date }\end{array}$ & $\begin{array}{l}\text { obser- } \\
\operatorname{ver}(s)\end{array}$ & equipment & $\begin{array}{l}\text { photometric } \\
\text { quality }\end{array}$ & observations \\
\hline 11 Apr 91 & 8357 & \multirow[t]{4}{*}{ RJD } & \multirow{4}{*}{$\begin{array}{l}\text { O/C scope, } \\
\text { PET logger; } \\
\text { Ampl \#1, Vil \#1 }\end{array}$} & cloudy & \\
\hline $12 \mathrm{Apr} 91$ & 8358 & & & $100 \%$ cloud & \\
\hline $13 \mathrm{Apr} 91$ & 8359 & & & photometric & 1 std, 210 Vel \\
\hline $14 \mathrm{Apr} 91$ & 8360 & & & photometric & 6 std, 90 Vel \\
\hline 11 May 91 & 8387 & \multirow[t]{13}{*}{ MF } & \multirow{13}{*}{$\begin{array}{l}\text { B\&C scope, } \\
\text { PET logger, } \\
\text { VUW PMT at } \\
1500 \mathrm{~V} \&-10^{\circ} \mathrm{C}, \\
\text { Ampl \#3, Vil \#1 }\end{array}$} & photometric & 8 std, $3 \kappa$ Crucis, 60 Vel \\
\hline 12 May 91 & 8388 & & & cloudy & \\
\hline 13 May 91 & 8389 & & & clear 3 hours & 5 std \\
\hline 14 May 91 & 8390 & & & clear 5 hours & $5 \kappa$ Crucis, $7 \mathrm{O}$ Vel, dead-time \\
\hline 15 May 91 & 8391 & & & $100 \%$ cloud & \\
\hline 16 May 91 & 8392 & & & $100 \%$ cloud & \\
\hline 17 May 91 & 8393 & & & clear 7 hours & 8 std, $10 \kappa$ Crucis \\
\hline 18 May 91 & 8394 & & & cloudy & \\
\hline 1 & 1 & & & 1 & \\
\hline 20 May 91 & 8396 & & & cloudy & \\
\hline 21 May 91 & 8397 & & & marginal & 9 std, $11 \kappa$ Crucis, 7 O Vel \\
\hline 22 May 91 & 8398 & & & cloudy & \\
\hline 23 May 91 & 8399 & & & clear 3 hours & 4 std, $6 \kappa$ Crucis \\
\hline $\begin{array}{c}29 \text { Nov } 91 \\
\text { । } \\
02 \text { Dec } 91\end{array}$ & $\begin{array}{c}8589 \\
1 \\
8592 \\
\end{array}$ & \multirow[t]{4}{*}{ MF } & \multirow{3}{*}{$\begin{array}{l}\text { B\&C scope, } \\
\text { PET logger, } \\
\text { VUW PMT at } \\
1500 \mathrm{~V} \&-10^{\circ} \mathrm{C} \text {, } \\
\text { Ampl \#3, Vil \#1 }\end{array}$} & $\begin{array}{l}100 \% \text { cloud } \\
1 \\
100 \% \text { cloud }\end{array}$ & \\
\hline 03 Dec 91 & 8593 & & & good & $1 \mathrm{std}, 4 \mathrm{sec}$ std, $6 \mathrm{M} 41$ \\
\hline $04 \mathrm{Dec} 91$ & 8594 & & & good & 4 std, $4 \mathrm{sec}$ std, 2 SPS, 6 M41 \\
\hline $\begin{array}{c}05 \operatorname{Dec} 91 \\
1 \\
12 \operatorname{Dec} 91 \\
\end{array}$ & $\begin{array}{c}8595 \\
1 \\
8602 \\
\end{array}$ & & $\begin{array}{l}1 \mathrm{~m} \text { scope at } \mathrm{f} 13, \\
\mathrm{CCD} \\
\text { Vil \#1 }\end{array}$ & $\begin{array}{l}100 \% \text { cloud } \\
1 \\
100 \% \text { cloud }\end{array}$ & \\
\hline $01 \mathrm{Feb} 92$ & 8653 & \multirow[t]{10}{*}{ MF } & \multirow{5}{*}{$\begin{array}{l}1 \mathrm{~m} \text { scope at } \mathrm{f} 13, \\
\mathrm{CCD} \\
\text { Vil \#1 }\end{array}$} & cloudy & $30 \mathrm{Vel}$ \\
\hline $02 \mathrm{Feb} \quad 92$ & 8654 & & & cloudy & $1 \kappa$ Crucis \\
\hline $03 \mathrm{Feb} 92$ & 8655 & & & $100 \%$ cloud & \\
\hline $04 \mathrm{Feb} 92$ & 8656 & & & $100 \%$ cloud & \\
\hline $05 \mathrm{Feb} 92$ & 8657 & & & cloudy & NGC1818, R Arae \\
\hline $06 \mathrm{Feb} 92$ & 8658 & & \multirow{5}{*}{$\begin{array}{l}\text { B\&C scope, } \\
\text { PET logger, } \\
\text { VUW PMT at } \\
1500 \mathrm{~V} \&-12^{\circ} \mathrm{C}, \\
\text { Ampl \#3, Vil \#1 }\end{array}$} & photometric & 8 std, 8 O Vel, 3 M41 \\
\hline $07 \mathrm{Feb} 92$ & 8659 & & & $100 \%$ cloud & dead-time \\
\hline $08 \mathrm{Feb} 92$ & 8660 & & & good & 2 std, $3 \kappa$ Crucis, $130 \mathrm{Vel}$ \\
\hline $09 \mathrm{Feb} 92$ & 8661 & & & good & $9 \mathrm{std}, 130 \mathrm{Vel}$ \\
\hline $10 \mathrm{Feb} 92$ & 8662 & & & good & 11 std, 110 Vel \\
\hline 11 Apr 92 & 8723 & \multirow[t]{7}{*}{ MF } & \multirow{5}{*}{$\begin{array}{l}\text { B\&C scope, } \\
\text { PC logger, } \\
\text { VUW PMT at } \\
1500 \text { V \& }-16^{\circ} \mathrm{C}, \\
\text { Ampl \#3, Vil \#1 }\end{array}$} & photometric & 6 std, $17 \kappa$ Crucis, $\mathrm{R}$ Arae drift-scans \\
\hline 12 Apr 92 & 8724 & & & good & 9 std, 6 sec std, $1 \mathrm{E} 7,15 \kappa$ Crucis \\
\hline 13 Apr 92 & 8725 & & & good & $11 \mathrm{std}, 6 \mathrm{sec}$ std, $21 \kappa$ Crucis \\
\hline $14 \mathrm{Apr} 92$ & 8726 & & & good & 9 std, 20 sec std, $13 \kappa$ Crucis \\
\hline $15 \mathrm{Apr} 92$ & 8727 & & & good & 11 std, 30 sec std, 4 SPS \\
\hline 16 Apr 92 & 8728 & & $1 \mathrm{~m}$ scope at $\mathrm{f} 13$ & cloudy & $10 \kappa$ Crucis \\
\hline 17 Apr 92 & 8729 & & B\&C scope & $100 \%$ cloud & dead-time \\
\hline
\end{tabular}




\begin{tabular}{|c|c|c|c|c|c|}
\hline \multicolumn{6}{|c|}{ Table C.1 continued from previous page } \\
\hline date & $\begin{array}{l}\text { julian } \\
\text { date }\end{array}$ & $\begin{array}{l}\text { obser- } \\
\operatorname{ver}(\mathbf{s})\end{array}$ & equipment & $\begin{array}{l}\text { photometric } \\
\text { quality }\end{array}$ & observations \\
\hline $18 \mathrm{Apr} 92$ & 8730 & & \multirow{4}{*}{$\begin{array}{l}1 \mathrm{~m} \text { scope at } \mathrm{f} 13, \\
\mathrm{CCD} \\
\text { Vil \#1 }\end{array}$} & $100 \%$ cloud & \\
\hline 19 Apr 92 & 8731 & & & good & 2 std, $6 \kappa$ Crucis, $\mathrm{R}$ Arae \\
\hline $20 \mathrm{Apr} 92$ & 8732 & & & cloudy & \\
\hline $21 \mathrm{Apr} 92$ & 8733 & & & good & 5 std, $3 \kappa$ Crucis, $\mathrm{R}$ Arae \\
\hline 23 Jun $92^{*}$ & 8796 & \multirow[t]{8}{*}{ MF } & O/C scope & cloudy & \\
\hline 24 Jun $92^{*}$ & 8797 & & \multirow{7}{*}{$\begin{array}{l}\text { B\&C scope, } \\
\text { PC logger, } \\
\text { VUW PMT at } \\
1500 \mathrm{~V} \&-16^{\circ} \mathrm{C}, \\
\text { Ampl \#3, Vil \#1 }\end{array}$} & good & 12 std, 11 sec std, 1 SPS \\
\hline 25 Jun $92^{*}$ & 8798 & & & good & $13 \mathrm{std}, 27 \mathrm{sec}$ std, $1 \mathrm{SPS}, 1 \mathrm{E} 7,1 \mathrm{E} 8, \mathrm{R}$ Arae \\
\hline 26 Jun 92 & $8799^{\prime}$ & & & $100 \%$ cloud & dead-time \\
\hline 27 Jun $92^{*}$ & 8800 & & & marginal & $13 \mathrm{std}, 34 \mathrm{sec}$ std, 4 SPS, 1 E8, 1 E9, $R$ Arae \\
\hline 28 Jun 92 & 8801 & & & good & 12 SPS, R Arae eclipse \\
\hline 29 Jun 92 & 8802 & & & $100 \%$ cloud & \\
\hline 30 Jun 92 & 8803 & & & cloudy & 9 std, 14 sec extn, 5 sec std \\
\hline $\begin{array}{c}17 \text { Dec } 92 \\
1 \\
19 \operatorname{Dec} 92 \\
\end{array}$ & $\begin{array}{c}8973 \\
1 \\
8975 \\
\end{array}$ & \multirow[t]{7}{*}{ MF } & \multirow[t]{2}{*}{$\begin{array}{l}1 \mathrm{~m} \text { scope at } \mathrm{f} 13, \\
\mathrm{CCD}, \\
\text { Vil \#2 }\end{array}$} & $\begin{array}{l}100 \% \text { cloud } \\
1 \\
100 \% \text { cloud } \\
\end{array}$ & \\
\hline 20 Dec 92 & 8976 & & & cloudy & $2 \mathrm{M} 41$ \\
\hline 21 Dec 92 & 8977 & & \multirow{5}{*}{$\begin{array}{l}\text { B\&C scope, } \\
\text { PC logger, } \\
\text { VUW PMT at } \\
1500 \mathrm{~V} \&-15^{\circ} \mathrm{C}, \\
\text { Ampl \#3, Vil \#2 }\end{array}$} & $100 \%$ cloud & \\
\hline 1 & 1 & & & 1 & \\
\hline 26 Dec 92 & 8982 & & & $100 \%$ cloud & \\
\hline 27 Dec 92 & 8983 & & & photometric & 6 std, 4 E2, 9 E3, 4 M41 \\
\hline 28 Dec 92 & 8984 & & & cloudy & \\
\hline $12 \mathrm{Feb} 93$ & 9030 & \multirow[t]{7}{*}{ MF } & \multirow{7}{*}{$\begin{array}{l}\text { B\&C scope, } \\
\text { PC logger, } \\
\text { VUW PMT at } \\
1500 \mathrm{~V} \&-16^{\circ} \mathrm{C}, \\
\text { Ampl \#3, Vil \#2 }\end{array}$} & $100 \%$ cloud & \\
\hline $13 \mathrm{Feb} 93$ & 9031 & & & $100 \%$ cloud & \\
\hline $14 \mathrm{Feb} 93^{*}$ & 9032 & & & marginal & 3 std, $8 \mathrm{E} 3$ \\
\hline $15 \mathrm{Feb} 93^{*}$ & 9033 & & & photometric & 4 std, $5 \mathrm{E} 3,16 \mathrm{E} 4$ \\
\hline 16 Feb $93^{*}$ & 9034 & & & good & 11 std, $2 \mathrm{E} 3,16 \mathrm{E} 4$ \\
\hline $17 \mathrm{Feb} 93^{*}$ & 9035 & & & good & 4 std, $12 \mathrm{E} 4$ \\
\hline $18 \mathrm{Feb} 93^{*}$ & 9036 & & & good & 13 std, 15 E4, $9 \kappa$ Crucis \\
\hline $\begin{array}{c}14 \text { May } 93 \\
\text { । } \\
17 \text { May } 93\end{array}$ & $\begin{array}{c}9121 \\
1 \\
9124 \\
\end{array}$ & \multirow[t]{7}{*}{ MF } & \multirow{7}{*}{$\begin{array}{l}\text { B\&C scope, } \\
\text { PC logger, } \\
\text { VUW PMT at } \\
1500 \mathrm{~V} \&-12^{\circ} \mathrm{C}, \\
\text { Ampl \#3, Vil \#2 }\end{array}$} & $\begin{array}{l}100 \% \text { cloud } \\
\quad \\
1 \\
100 \% \text { cloud }\end{array}$ & \\
\hline 18 May 93 & 9125 & & & cloudy & \\
\hline 19 May 93 & 9126 & & & $100 \%$ cloud & \\
\hline 20 May 93 & 9127 & & & photometric & 16 std, 1 E4, 11 E6, 1 E9, R Arae \\
\hline 21 May 93 & 9128 & & & clear 7 hours & $\begin{array}{l}8 \mathrm{std}, 6 \mathrm{sec} \text { extn ( } 5 \mathrm{E} 6,1 \mathrm{E} 9), \mathrm{R} \text { Arae, std } \\
\text { lamp }\end{array}$ \\
\hline 22 May 93 & 9129 & & & cloudy & 10 std, 1 E4, 5 E6, $10 \mathrm{E} 8$, R Arae \\
\hline 23 May 93 & 9130 & & & good & $\begin{array}{l}11 \text { std, } 1 \text { E4, } 14 \text { E6, } 8 \text { E8, } 1 \text { E9, } 4 \kappa \text { Crucis, } \\
\text { R Arae }\end{array}$ \\
\hline 29 Jun 93 & 9167 & \multirow[t]{3}{*}{ MF } & \multirow{3}{*}{$\begin{array}{l}\text { B\&C scope, } \\
\text { PC logger, } \\
\text { VUW PMT at } \\
1500 \mathrm{~V} \&-12^{\circ} \mathrm{C},\end{array}$} & clear 9 hours & 13 std, $1 \mathrm{E} 1,2 \mathrm{E} 7,12 \mathrm{E} 9$, R Arae \\
\hline 30 Jun 93 & 9168 & & & cloudy & \\
\hline $01 \mathrm{Jul} 93$ & 9169 & & & good & $\begin{array}{l}14 \text { std, } 1 \text { E1, } 1 \text { E5, } 12 \text { E6, } 2 \text { E7, } 11 \text { E9, } \\
\text { R Arae }\end{array}$ \\
\hline
\end{tabular}




\begin{tabular}{|c|c|c|c|c|c|}
\hline \multicolumn{6}{|c|}{ Table C.1 continued from previous page } \\
\hline date & $\begin{array}{l}\text { julian } \\
\text { date }\end{array}$ & $\begin{array}{l}\text { obser- } \\
\text { ver(s) }\end{array}$ & equipment & $\begin{array}{l}\text { photometric } \\
\text { quality }\end{array}$ & observations \\
\hline 02 Jul 93 & 9170 & & Ampl \#3, & clear 6 hours & 10 std, 1 E5, 11 E6, 2 E7, R Arae \\
\hline $03 \mathrm{Jul} \quad 93$ & 9171 & & Vil \#2+ new $\mathrm{Y}$ & good & $\begin{array}{l}22 \mathrm{std}, 1 \mathrm{E} 1,1 \mathrm{E} 5,17 \mathrm{E} 6,2 \mathrm{E} 7,18 \mathrm{E} 9 \text {, } \\
\mathrm{R} \text { Arae }\end{array}$ \\
\hline $04 \mathrm{Jul} \quad 93$ & 9172 & & & clear 6 hours & $5 \mathrm{std}, 1 \mathrm{E} 5,17 \mathrm{E} 6,2 \mathrm{E} 7$ \\
\hline $05 \mathrm{Jul}$ & 9173 & & & $100 \%$ cloud & \\
\hline $06 \mathrm{Jul} \quad 93$ & 9174 & & & good & 7 std, 1 E1, 1 E5, 9 E6, 2 E7, 8 E9, R Arae \\
\hline $07 \mathrm{Jul} \quad 93$ & 9175 & & & good & $\begin{array}{l}18 \mathrm{std}, 1 \mathrm{E} 1,1 \mathrm{E} 5,14 \mathrm{E} 6,2 \mathrm{E} 7,14 \mathrm{E} 9 \text {, } \\
\text { R Arae }\end{array}$ \\
\hline $08 \mathrm{Jul} \quad 93$ & 9176 & & & good & 12 std, 1 E5, $2 \mathrm{E} 7,7 \kappa$ Crucis, $\mathrm{R}$ Arae \\
\hline
\end{tabular}

Notes :

\section{Oct $87 \quad Z$ filter missing}

21 Jun 88 the extinction star (HD122066) differs by $\sim 1^{m} \cdot 5$ from 24 Jun 88 , probably observed wrong star (BS5250 is nearby and similar $V$ magnitude).

04 Jun 89 the extinction star (HD122066) changed by $\sim 1^{m} \cdot 0$ during the night, ditto above note for 21 Jun 88.

27 Feb 91 the PET disk drive was not working, data entered from the printout

23 Jun 92 dark-counts vary according to scope's position (electrical-noise from tracking motors?)

24 Jun 92 moved to the B\&C scope due to noise problems found on 23 Jun 92

25-27 Jun 92 used wrong check star for R Arae (HD150898 instead of HD150745)

14 Feb 93 the dome shutter power socket shorted and destroyed the computer

14-18 Feb 93 strange results from the U filter 
Table C.2: List of observed stars : coordinates, star type, Johnson $V$ and Spectral class.

\begin{tabular}{|c|c|c|c|c|c|c|c|}
\hline Name & Ep & RA & Dec & Type & $V$ & \multicolumn{2}{|l|}{ Sp } \\
\hline CPD-80 0962 & 2000 & 203622 & $-8031 \cdot 0$ & SPS & & K0 & \\
\hline E1_036 & 2000 & 011507 & $-4532 \cdot 0$ & ESecStd & $4 \cdot 97$ & G0 V & \\
\hline E2_008 & 2000 & 035102 & $-4422 \cdot 0$ & ESecStd & $8 \cdot 01$ & F2 III & \\
\hline E2_014 & 2000 & 035617 & $-4337 \cdot 0$ & ESecStd & $8 \cdot 13$ & F5-6 V & \\
\hline E2_015 & 2000 & 040108 & $-4427 \cdot 0$ & ESecStd & 8.46 & F6 V & \\
\hline E2_052 & 2000 & 034949 & $-4244 \cdot 0$ & ESecStd & $7 \cdot 35$ & A5-6 V & \\
\hline E3_002 & 2000 & 063730 & $-4536 \cdot 0$ & ESecStd & $8 \cdot 28$ & B8-9 V & \\
\hline E3_005 & 2000 & 063313 & $-4523 \cdot 0$ & ESecStd & 8.05 & A1 IV & \\
\hline E3_006 & 2000 & 063829 & $-4522 \cdot 0$ & ESecStd & $8 \cdot 17$ & A2 III & \\
\hline E3_007 & 2000 & 063841 & $-4610 \cdot 0$ & ESecStd & $8 \cdot 38$ & A $0 \mathrm{~V}$ & \\
\hline E3_018 & 2000 & 063439 & $-4631 \cdot 0$ & ESecStd & $8 \cdot 58$ & $\mathrm{~F} 0 \mathrm{~V}$ & \\
\hline E3_020 & 2000 & 063815 & $-45 \quad 17 \cdot 0$ & ESecStd & $8 \cdot 28$ & $\mathrm{~F} 2 \mathrm{~V}$ & \\
\hline E3_036( $\infty)$ & 2000 & 064957 & $-4527 \cdot 0$ & ESecStd & $6 \cdot 54$ & K5-M0 III & \\
\hline E3_037 & 2000 & 063219 & $-4519 \cdot 0$ & ESecStd & $7 \cdot 17$ & K0 III & \\
\hline E3_041 & 2000 & 063327 & $-4504 \cdot 0$ & ESecStd & $8 \cdot 68$ & K0 III & \\
\hline E3_045( $\infty)$ & 2000 & 062543 & $-4811 \cdot 0$ & ESecStd & $5 \cdot 76$ & B9 V & \\
\hline E3_047 & 2000 & 064954 & $-4637 \cdot 0$ & ESecStd & $5 \cdot 14$ & F5 V & \\
\hline E3_050 & 2000 & 064047 & $-4737 \cdot 0$ & ESecStd & $6 \cdot 65$ & B3-5 V & \\
\hline E3_051 & 2000 & 065800 & $-4606 \cdot 0$ & ESecStd & $6 \cdot 83$ & A0 IV & \\
\hline E3_052 & 2000 & 065841 & $-4546 \cdot 0$ & ESecStd & $6 \cdot 22$ & A0 V & \\
\hline E3_072 & 2000 & 062859 & $-4522 \cdot 0$ & ESecStd & $10 \cdot 75$ & & \\
\hline E3_087 $(\infty)$ & 2000 & 063837 & $-4813 \cdot 0$ & ESecStd & 4.94 & G8 III & \\
\hline E3_089 & 2000 & $0645 \quad 10$ & $-4713 \cdot 0$ & ESecStd & $7 \cdot 22$ & B2 III & \\
\hline E4_028 & 2000 & 093033 & $-4534 \cdot 0$ & ESecStd & $6 \cdot 63$ & K2-3 III & \\
\hline E4_029 & 2000 & 092015 & $-4510 \cdot 0$ & ESecStd & $6 \cdot 70$ & K2 III & \\
\hline E4_030( $\infty)$ & 2000 & 091851 & $-4500 \cdot 0$ & ESecStd & $7 \cdot 20$ & K0 III & \\
\hline E4_039 & 2000 & 091214 & $-4550 \cdot 0$ & ESecStd & $6 \cdot 66$ & F3-5 V & \\
\hline E4_040( $\infty)$ & 2000 & 091230 & $-4337 \cdot 0$ & ESecStd & $5 \cdot 56$ & B8 V(Psi) & \\
\hline E4_041( $\infty)$ & 2000 & 091514 & $-4533 \cdot 0$ & ESecStd & $6 \cdot 25$ & B8 V & \\
\hline E4_044 & 2000 & 092224 & $-4603 \cdot 0$ & ESecStd & $5 \cdot 74$ & G6-8 III & \\
\hline E4_045 & 2000 & 092922 & $-4311 \cdot 0$ & ESecStd & $6 \cdot 60$ & F5 IV & \\
\hline E4_046 & 2000 & 093119 & $-4757 \cdot 0$ & ESecStd & $6 \cdot 53$ & A9 IV-V & \\
\hline E4_047 & 2000 & 093801 & $-4311 \cdot 0$ & ESecStd & $5 \cdot 50$ & G8 II & \\
\hline E4_078 & 2000 & 091408 & $-4409 \cdot 0$ & ESecStd & $5 \cdot 85$ & B5 V & \\
\hline E4_079( $\infty)$ & 2000 & 091424 & $-4314 \cdot 0$ & ESecStd & $5 \cdot 24$ & B3-5 V & \\
\hline E4_093 & 2000 & 091133 & $-4635 \cdot 0$ & ESecStd & $5 \cdot 78$ & B2 III-IV & \\
\hline E4_094 & 2000 & 091334 & $-4720 \cdot 0$ & ESecStd & $5 \cdot 91$ & B9 V & \\
\hline E4_096 & 2000 & 091658 & $-4501 \cdot 0$ & ESecStd & $6 \cdot 74$ & $\mathrm{~A} 0 \mathrm{~V}$ & \\
\hline E4_097 & 2000 & 092325 & $-4817 \cdot 0$ & ESecStd & $6 \cdot 27$ & B3 IV & \\
\hline E4_098 & 2000 & 092339 & $-4655 \cdot 0$ & ESecStd & $6 \cdot 21$ & B7 III & \\
\hline E5_040 & 2000 & 120338 & $-4225 \cdot 9$ & ESecStd & $5 \cdot 15$ & F5 V & \\
\hline
\end{tabular}




\begin{tabular}{|c|c|c|c|c|c|c|}
\hline \multicolumn{7}{|c|}{ Table C.2 continued from previous page } \\
\hline Name & Ep & $\mathrm{RA}$ & Dec & Type & V & Sp \\
\hline E6_012 & 2000 & 145614 & $-4405 \cdot 4$ & ESecStd & $7 \cdot 89$ & A3 IV \\
\hline E6_013 & 2000 & 145525 & $-4420 \cdot 0$ & ESecStd & 7.99 & $\mathrm{~A} 4 \mathrm{~V}$ \\
\hline E6_014 & 2000 & 145304 & $-4343 \cdot 6$ & ESecStd & $8 \cdot 15$ & A5 V \\
\hline E6_033 & 2000 & 143812 & $-4552 \cdot 2$ & ESecStd & 6.83 & K2 III \\
\hline E6_039( $\infty)$ & 2000 & 143008 & $-4519 \cdot 2$ & ESecStd & $5 \cdot 51$ & B8 V \\
\hline E6_040 & 2000 & 143618 & -4614.7 & ESecStd & $5 \cdot 54$ & K3 III \\
\hline E6_041 & 2000 & 143911 & $-4634 \cdot 8$ & ESecStd & $6 \cdot 06$ & F6 V \\
\hline E6_042( $\infty)$ & 2000 & 144557 & $-4452 \cdot 0$ & ESecStd & $6 \cdot 91$ & $\mathrm{~A} 0 \mathrm{~V}$ \\
\hline E6_043 & 2000 & 144628 & $-4726 \cdot 4$ & ESecStd & $5 \cdot 73$ & A1 V \\
\hline E6_044 & 2000 & 144731 & $-4333 \cdot 3$ & ESecStd & $6 \cdot 30$ & G8 III \\
\hline E6_045 & 2000 & 145624 & $-4442 \cdot 2$ & ESecStd & $6 \cdot 75$ & $\mathrm{~A} 1 \mathrm{~V}$ \\
\hline E6_046( $\infty)$ & 2000 & 145918 & $-4328 \cdot 2$ & ESecStd & $7 \cdot 04$ & F2 III \\
\hline E6_047 & 2000 & 145945 & $-4348 \cdot 5$ & ESecStd & $6 \cdot 58$ & F7 IV-V \\
\hline E6_078(v) & 2000 & 142607 & $-4513 \cdot 2$ & ESecStd & $4 \cdot 6$ & B2 IV \\
\hline E6_079( $\infty)$ & 2000 & 142610 & $-4522 \cdot 7$ & ESecStd & $4 \cdot 36$ & F7 \\
\hline E6_080 & 2000 & 142712 & $-4607 \cdot 9$ & ESecStd & $5 \cdot 82$ & $\mathrm{~A} 1 \mathrm{~mA} 5 / 7-\mathrm{F} 2$ \\
\hline E6_081( $\infty)$ & 2000 & 142851 & $-4759 \cdot 4$ & ESecStd & $6 \cdot 40$ & $\mathrm{ApSi}$ \\
\hline E6_082 & 2000 & 143509 & $-4627 \cdot 6$ & ESecStd & $6 \cdot 89$ & B9 V \\
\hline E6_084 & 2000 & 143549 & $-4648 \cdot 9$ & ESecStd & 6.78 & G8 III \\
\hline E6_087 & 2000 & 143754 & $-4801 \cdot 3$ & ESecStd & $6 \cdot 65$ & B8 V \\
\hline E6_088( $\infty)$ & 2000 & 144018 & $-4547 \cdot 5$ & ESecStd & $6 \cdot 63$ & $\mathrm{ApSi}$ \\
\hline E6_090 & 2000 & 145058 & $-4249 \cdot 3$ & ESecStd & 6.83 & A2 V \\
\hline E6_091( $\infty)$ & 2000 & 145137 & $-4334 \cdot 4$ & ESecStd & $4 \cdot 32$ & B5 IV \\
\hline E6_092( $\infty)$ & 2000 & 145631 & $-4752 \cdot 6$ & ESecStd & $5 \cdot 63$ & B9 V \\
\hline E6_095 & 2000 & 145926 & $-4309 \cdot 5$ & ESecStd & $6 \cdot 10$ & F7 II \\
\hline E6_100 & 2000 & 145400 & $-4335 \cdot 8$ & ESecStd & $8 \cdot 22$ & G8 II \\
\hline E6_101 & 2000 & 145547 & $-4441 \cdot 7$ & ESecStd & 7.94 & K3 III \\
\hline E7_048 & 2000 & 172443 & $-4500 \cdot 5$ & ESecStd & $6 \cdot 66$ & F3 V \\
\hline E7_061 & 2000 & 171209 & $-4314 \cdot 0$ & ESecStd & $3 \cdot 33$ & F3 III-IVp \\
\hline E7_065 & 2000 & 173753 & $-4233 \cdot 7$ & ESecStd & $7 \cdot 17$ & G2-3 V \\
\hline E8_026 & 2000 & $2011 \quad 16$ & $-4339 \cdot 6$ & ESecStd & $6 \cdot 54$ & G5-6 III \\
\hline E8_028 & 2000 & 200236 & $-4511 \cdot 7$ & ESecStd & $6 \cdot 57$ & K1 III \\
\hline E8_029 & 2000 & 201005 & -4353.5 & ESecStd & 6.93 & K0 III \\
\hline E8_030 & 2000 & 200508 & $-4605 \cdot 7$ & ESecStd & $6 \cdot 95$ & K3-4 III \\
\hline E8_042 & 2000 & 200048 & $-4506 \cdot 7$ & ESecStd & $5 \cdot 79$ & F0-2 IV \\
\hline 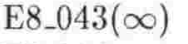 & 2000 & 200125 & $-4723 \cdot 7$ & ESecStd & $6 \cdot 95$ & F2 IV \\
\hline E8_044 & 2000 & 201917 & $-4734 \cdot 6$ & ESecStd & $6 \cdot 12$ & F5 V \\
\hline E8_045 & 2000 & 202227 & $-4202 \cdot 8$ & ESecStd & $5 \cdot 58$ & A0 V \\
\hline E8_069 & 2000 & 195515 & $-4152 \cdot 0$ & ESecStd & $4 \cdot 12$ & K0 II-III \\
\hline E8_071( $\infty)$ & 2000 & 202353 & $-4225 \cdot 3$ & ESecStd & $5 \cdot 64$ & A5 V \\
\hline E8_072 & 2000 & 202604 & -4639.5 & ESecStd & $6 \cdot 73$ & A9 III-IV \\
\hline E9_024 & 2000 & 223211 & $-4315 \cdot 7$ & ESecStd & $6 \cdot 91$ & K0 III \\
\hline E9_025 & 2000 & 224830 & $-4326 \cdot 7$ & ESecStd & $7 \cdot 06$ & G8 III \\
\hline
\end{tabular}




\begin{tabular}{|c|c|c|c|c|c|c|}
\hline \multicolumn{7}{|c|}{ Table C.2 continued from previous page } \\
\hline Name & Ep & RA & Dec & Type & $V$ & Sp \\
\hline E9_029 & 2000 & 223354 & $-4441 \cdot 4$ & ESecStd & $6 \cdot 79$ & G8 III \\
\hline E9_030 & 2000 & 225315 & $-4508 \cdot 8$ & ESecStd & 6.85 & KO III \\
\hline E9_031 & 2000 & 225226 & $-4559 \cdot 4$ & ESecStd & $7 \cdot 25$ & K4-5 III \\
\hline E9_040 & 2000 & 223043 & $-4405 \cdot 6$ & ESecStd & 6.94 & A0-1 V \\
\hline E9_042 & 2000 & 223641 & $-4328 \cdot 2$ & ESecStd & $6 \cdot 76$ & F8 V \\
\hline E9_043 & 2000 & 224243 & $-4414 \cdot 9$ & ESecStd & 6.07 & K0 III \\
\hline E9_044 & 2000 & 224541 & $-4632 \cdot 8$ & ESecStd & $5 \cdot 51$ & K2 III \\
\hline E9_048 & 2000 & 223015 & $-4217 \cdot 4$ & ESecStd & $6 \cdot 92$ & B9.5 V \\
\hline E9_049 & 2000 & 224709 & -4141.5 & ESecStd & $6 \cdot 83$ & K2 III \\
\hline E9_063( $\infty)$ & 2000 & 224237 & $-4712 \cdot 3$ & ESecStd & $5 \cdot 99$ & G0 V \\
\hline E9_071 & 2000 & 221535 & $-4427 \cdot 1$ & ESecStd & $6 \cdot 10$ & G8-K0 III \\
\hline E9_072 & 2000 & 222307 & $-4555 \cdot 6$ & ESecStd & $5 \cdot 62$ & F0 V \\
\hline E9_073 & 2000 & 222325 & $-4640 \cdot 1$ & ESecStd & $6 \cdot 91$ & A $1 \mathrm{~V}$ \\
\hline E9_074 & 2000 & 222916 & $-4329 \cdot 7$ & ESecStd & 3.97 & G6-8 III \\
\hline E9_079 & 2000 & 224330 & $-4124 \cdot 8$ & ESecStd & 4.84 & K0 III \\
\hline E9_080( $\infty)$ & 2000 & 224643 & $-4656 \cdot 4$ & ESecStd & 6.56 & A9 IIIm \\
\hline HD000496 & 2000 & 000924 & $-4545 \cdot 0$ & SecStd & $3 \cdot 88$ & K0 III \\
\hline HD000636 & 2000 & 001003 & $-8213 \cdot 0$ & SPS & $5 \cdot 28$ & G8 III \\
\hline HD000693 & 2000 & 001116 & $-1527 \cdot 8$ & PriStd & 4.88 & F6 V-MD \\
\hline HD000787 & 2000 & 001210 & $-1756 \cdot 3$ & PriStd & $5 \cdot 29$ & K5 III-MD \\
\hline HD001032 & 2000 & $\begin{array}{llll}00 & 13 & 19\end{array}$ & $-8500 \cdot 0$ & SPS & $5 \cdot 77$ & M0.5 III \\
\hline HD 001237 & 2000 & 001605 & $-7951 \cdot 0$ & SPS & $6 \cdot 7$ & G6 V \\
\hline HD001581 & 2000 & 001951 & -6453.0 & SecStd & $4 \cdot 20$ & F9 V \\
\hline HD001879 & 2000 & 002304 & $-1556 \cdot 6$ & PriStd & $6 \cdot 47$ & M2 IIIB \\
\hline HD002151 & 2000 & 002512 & $-77 \quad 16 \cdot 0$ & SPS & $2 \cdot 80$ & G2 IV \\
\hline HD002261 & 2000 & 002616 & $-4218 \cdot 0$ & SecStd & $2 \cdot 37$ & K0 III \\
\hline HD 002262 & & $00 \quad 2612$ & $-4341 \cdot 0$ & SecStd & $3 \cdot 90$ & $\mathrm{~A} 7 \mathrm{~V}$ \\
\hline HD002834 & 2000 & 003124 & $-4848 \cdot 0$ & SecStd & $4 \cdot 77$ & $\mathrm{~A} 0 \mathrm{~V}$ \\
\hline HD003296 & 2000 & 003601 & $-0534 \cdot 2$ & PriStd & 6.73 & F5 V \\
\hline HD004229 & 2000 & 003932 & $-8542 \cdot 0$ & SPS & $6 \cdot 80$ & K3 III \\
\hline HD 004628 & 2000 & 004821 & $+0517 \cdot 7$ & PriStd & $5 \cdot 75$ & $\mathrm{~K} 2 \mathrm{~V}$ \\
\hline HD005112 & 2000 & 005300 & $-0108 \cdot 6$ & PriStd & 4.77 & M0 III \\
\hline HD005268 & 2000 & 005417 & $-0844 \cdot 4$ & PriStd & $6 \cdot 16$ & G8 III-MD \\
\hline HD005384 & 2000 & 005543 & -0720.8 & PriStd & $5 \cdot 84$ & K5 III \\
\hline HD006186 & 2000 & 010257 & +0753.4 & PriStd & $4 \cdot 28$ & G9 III \\
\hline HD010800 & 2000 & 013753 & $-8259 \cdot 0$ & SPS & 5.87 & G2 V \\
\hline HD011025 & 2000 & 013727 & $-8446 \cdot 0$ & SPS & $5 \cdot 69$ & G8 III \\
\hline HD011171 & & 014936 & $-1041 \cdot 0$ & SecStd & 4.77 & F3 III \\
\hline HD011604 & 2000 & 014748 & $-8011 \cdot 0$ & SPS & 6.06 & F0 IV \\
\hline HD012311 & & 015844 & $-6134 \cdot 0$ & SecStd & $2 \cdot 90$ & F0 V \\
\hline HD012872(v) & 2000 & $02 \quad 06 \quad 13$ & +0814.9 & PriStd & $6 \cdot 31$ & M4 \\
\hline HD014802 & 2000 & 022232 & $-2349 \cdot 0$ & PriExtn & $5 \cdot 37$ & $\mathrm{G} 2 \mathrm{~V}$ \\
\hline HD014882 & & 022315 & -2952.0 & PriExtn & $6 \cdot 94$ & G1 V \\
\hline
\end{tabular}




\begin{tabular}{|c|c|c|c|c|c|c|}
\hline \multicolumn{7}{|c|}{ Table C.2 continued from previous page } \\
\hline Name & Ep & RA & Dec & Type & $V$ & Sp \\
\hline HD015048 & 2000 & 022426 & $-3722 \cdot 0$ & PriExtn & 6.98 & F5 V \\
\hline HD015182 & 2000 & 022545 & $-3745 \cdot 0$ & SecStd & $9 \cdot 0$ & G8 III \\
\hline HD016399 & 2000 & 023800 & $+0741 \cdot 7$ & PriStd & $6 \cdot 39$ & F5 \\
\hline HD017081 & & 024407 & $-1352 \cdot 0$ & SecStd & $4 \cdot 39$ & B7 IV \\
\hline HD018331 & & 025637 & $-0343 \cdot 0$ & SecStd & $5 \cdot 27$ & $\mathrm{~A} 1 \mathrm{Vn}$ \\
\hline HD018784 & 2000 & 030109 & $-0739 \cdot 7$ & PriStd & $5 \cdot 78$ & K0 II \\
\hline HD020630 & 2000 & 031921 & $+0322 \cdot 0$ & SecStd & 4.96 & G5 Vvar \\
\hline HD020791 & 2000 & 032107 & $+0340 \cdot 5$ & PriStd & $5 \cdot 69$ & G8.5 III \\
\hline HD022049 & & 033259 & $-0927 \cdot 0$ & SecStd & $3 \cdot 81$ & K2 V \\
\hline HD023246 & 2000 & 034426 & $+2423 \cdot 7$ & PriStd & $8 \cdot 17$ & A $8 \mathrm{~V}$ \\
\hline HD023249(v?) & 2000 & 034315 & $-0946 \cdot 5$ & PriStd & $3 \cdot 54$ & K0 IV \\
\hline HD023288 & 2000 & 034449 & $+2417 \cdot 4$ & PriStd & $5 \cdot 46$ & B7 IV \\
\hline HD023338 & 2000 & 034513 & $+2428 \cdot 1$ & PriStd & $4 \cdot 30$ & B6 IV \\
\hline HD023608 & 2000 & 034717 & +2407.7 & PriStd & $8 \cdot 69$ & F $3 \mathrm{~V}$ \\
\hline HD023850 & 2000 & 034910 & $+2403 \cdot 0$ & SecStd & $3 \cdot 80$ & B8 III \\
\hline HD025422 & & 035845 & $-6124 \cdot 0$ & SecStd & 4.56 & M2 III \\
\hline HD026912 & 2000 & 041532 & +0853.5 & PriStd & $4 \cdot 29$ & B3 IV \\
\hline HD028525 & & 042118 & $-8020 \cdot 0$ & SPS & $5 \cdot 69$ & K2.5 III+ \\
\hline HD028556 & 2000 & 043037 & $+1343 \cdot 0$ & PriStd & $5 \cdot 43$ & F0 V \\
\hline HD028873 & & 043050 & $-4457 \cdot 0$ & SecStd & $5 \cdot 16$ & B2 IV-V \\
\hline HD029116 & & 042519 & $-8142 \cdot 0$ & SPS & $5 \cdot 79$ & F1 III \\
\hline HD029712 & & 043646 & $-6205 \cdot 0$ & SecStd & & M8 IIIe \\
\hline HD029875 & & 044034 & $-4152 \cdot 0$ & PriExtn & $4 \cdot 45$ & $\mathrm{~F} 2 \mathrm{~V}$ \\
\hline HD030652 & 2000 & 044949 & +0658.0 & SecStd & $3 \cdot 31$ & F6 V \\
\hline HD031139(v?) & 2000 & 045323 & $+0230 \cdot 5$ & PriStd & $5 \cdot 33$ & M1 III \\
\hline HD031237(v) & 2000 & 045416 & $+0226 \cdot 4$ & PriStd & 3.72 & $\mathrm{~B} 3 \mathrm{III}+\mathrm{B} 0 \mathrm{~V}$ \\
\hline HD 031283 & 2000 & 045447 & $+1125 \cdot 4$ & PriStd & $5 \cdot 19$ & A3 V \\
\hline HD033111 & & 050751 & $-0505 \cdot 0$ & SecStd & 2.92 & A3 III \\
\hline HD033544 & 2000 & 051138 & +1538.5 & PriStd & $5 \cdot 20$ & K5 III \\
\hline HD034484 & & 051624 & $-3231 \cdot 0$ & PriExtn & $7 \cdot 06$ & F6-F7 V \\
\hline HD035299 & & 052342 & $-0010 \cdot 0$ & SecStd & $5 \cdot 70$ & $\mathrm{~B} 1.5 \mathrm{~V}$ \\
\hline HD036512 & & 053156 & $-0718 \cdot 0$ & SecStd & $4 \cdot 63$ & B0 V \\
\hline HD037224 & 2000 & 053527 & $-3316 \cdot 0$ & PriExtn & $6 \cdot 69$ & $\mathrm{~F} 6 \mathrm{~V}$ \\
\hline HD037495 & 2000 & 053745 & $-2841 \cdot 0$ & PriExtn & $5 \cdot 31$ & F4 V \\
\hline HD038558 & 2000 & 054726 & $+1743 \cdot 7$ & PriStd & $5 \cdot 49$ & F0 III \\
\hline HD042301 & & 060858 & $-2226 \cdot 0$ & SecStd & $5 \cdot 46$ & $\mathrm{~A} 0 \mathrm{~V}$ \\
\hline HD045416 & 2000 & 062644 & +00 $18 \cdot 0$ & SecStd & $5 \cdot 20$ & \\
\hline HD046150 & 2000 & 063155 & $+0456 \cdot 6$ & PriStd & $6 \cdot 73$ & $\mathrm{O} 6 \mathrm{~V}, \mathrm{E}$ \\
\hline HD050082 & 2000 & 065154 & $+0636 \cdot 0$ & PriStd & $7 \cdot 40$ & G0 (BA) \\
\hline HD051199 & 2000 & 065537 & $-2008 \cdot 0$ & SecExtn & $4 \cdot 62$ & F2 IV-V \\
\hline HD051208 & 2000 & 065427 & $-4222 \cdot 0$ & SecExtn & $6 \cdot 00$ & C II... \\
\hline HD052382 & 2000 & 070039 & $-0912 \cdot 2$ & PriStd & $6 \cdot 49$ & B1 IB \\
\hline HD052877(‡) & 2000 & 070144 & $-2756 \cdot 0$ & SecStd & $3 \cdot 46$ & M0 Iab \\
\hline
\end{tabular}




\begin{tabular}{|c|c|c|c|c|c|c|}
\hline \multicolumn{7}{|c|}{ Table C.2 continued from previous page } \\
\hline Name & Ep & RA & Dec & Type & $V$ & Sp \\
\hline HD053704 & 2000 & 070403 & $-4220 \cdot 0$ & SecExtn & $5 \cdot 26$ & $\mathrm{Am}$ \\
\hline HD054118 & 2000 & 070418 & $-5645 \cdot 0$ & SecExtn & $5 \cdot 30$ & A0p... \\
\hline HD054439 & 2000 & 070823 & $-1151 \cdot 0$ & PriStd & $7 \cdot 68$ & B2 III,N \\
\hline HD055751 & 2000 & 071420 & $+0306 \cdot 7$ & PriStd & $5 \cdot 35$ & K2 II \\
\hline HD055892 & 2000 & 071234 & $-4646 \cdot 0$ & SecExtn & $4 \cdot 47$ & F0 IV \\
\hline HD056855 & 2000 & 071709 & $-3706 \cdot 0$ & SecExtn & $2 \cdot 74$ & K3 Ib \\
\hline HD057150 & 2000 & $0718 \quad 19$ & -3644.0 & SecStd & 4.68 & $B 2 V+\ldots$ \\
\hline HD057623 & 2000 & 071650 & $-6757 \cdot 0$ & SecExtn & $4 \cdot 02$ & F6 II \\
\hline HD059881 & 2000 & 073206 & +0154.9 & PriStd & $5 \cdot 25$ & F0 III \\
\hline HD061831 & 2000 & 073927 & -3818.0 & SecExtn & $4 \cdot 91$ & B2.5 V \\
\hline HD062058 & 2000 & 074053 & $-3140 \cdot 0$ & SecExtn & $6 \cdot 64$ & F8-G0 Ia \\
\hline HD062345( $\infty)$ & 2000 & 074427 & $+2424 \cdot 0$ & PriStd & $3 \cdot 68$ & G8 IIIa \\
\hline HD063032 & 2000 & 074515 & -3758.0 & SecExtn & $3 \cdot 72$ & K2.5 Ib-II \\
\hline HD063077 & & 074536 & -3412.0 & PriExtn & $5 \cdot 39$ & G0 V \\
\hline HD063975 & 2000 & 075142 & $+0146 \cdot 1$ & PriStd & $5 \cdot 14$ & B8 II \\
\hline HD064379 & 2000 & $0752 \quad 17$ & $-3443 \cdot 0$ & PriExtn & $5 \cdot 01$ & F5 V \\
\hline HD066811 & & 080311 & $-3958 \cdot 0$ & SecStd & $2 \cdot 20$ & O5 If \\
\hline HD067594 & 2000 & 080836 & $-0259 \cdot 0$ & PriStd & $4 \cdot 34$ & G2 Ib \\
\hline HD069142 & 2000 & 081403 & $-4021 \cdot 0$ & SecStd & $4 \cdot 44$ & K1 II-III \\
\hline HD070569 & 2000 & 082322 & $+1820 \cdot 0$ & PriStd & $5 \cdot 95$ & A9 V \\
\hline HD072127( $\infty)$ & 2000 & 082928 & $-4443 \cdot 0$ & SecStd & 4.99 & B2 IV \\
\hline HD073389( & 2000 & 083519 & -5801.0 & SecStd & $4 \cdot 86$ & K0 III \\
\hline HD073390 & 2000 & 083516 & -5813.0 & SecStd & $5 \cdot 40$ & B3 $V+\ldots$ \\
\hline HD074006 & 2000 & 084006 & $-3518 \cdot 0$ & SecStd & $3 \cdot 97$ & G7 Ib-II \\
\hline HD074180 & 2000 & 084038 & $-4639 \cdot 0$ & SecStd & $3 \cdot 88$ & F3 Ia \\
\hline HD 074272 & 2000 & $0841 \quad 13$ & $-4719 \cdot 0$ & SecStd & $4 \cdot 77$ & A5 II \\
\hline HD074521(v) & 2000 & 084445 & $+1004 \cdot 9$ & PriStd & $5 \cdot 66$ & $\mathrm{~A} 1, \mathrm{P}(\mathrm{EU}-\mathrm{CR})$ \\
\hline HD075063(‡) & 2000 & 084602 & $-4602 \cdot 0$ & SecStd & $3 \cdot 90$ & A1 III \\
\hline HD075710 & 2000 & 084948 & $-4518 \cdot 0$ & SecStd & 4.95 & A2 III \\
\hline HD076728 & 2000 & 085503 & $-6039 \cdot 0$ & SecStd & $3 \cdot 80$ & B8.5 II \\
\hline HD076805 & 2000 & 085619 & $-5243 \cdot 0$ & SecStd & $4 \cdot 69$ & B5 V \\
\hline HD077258( $\infty)$ & 2000 & 090006 & $-4115 \cdot 0$ & SecStd & $4 \cdot 40$ & K0 III $+\ldots$ \\
\hline HD078004 & 2000 & 090410 & $-4706 \cdot 0$ & SecStd & $3 \cdot 75$ & K2 III \\
\hline HD078045 & 2000 & 090227 & $-6624 \cdot 0$ & SecStd & $4 \cdot 00$ & $\mathrm{~A} 2.5 \mathrm{IVm}$ \\
\hline HD079447 & 2000 & $0911 \quad 17$ & $-6219 \cdot 0$ & SecStd & $4 \cdot 00$ & B3 III \\
\hline HD079940 & 2000 & 091545 & $-3725 \cdot 0$ & SecStd & $4 \cdot 62$ & F5 III \\
\hline HD082434 & 2000 & 093043 & $-4028 \cdot 0$ & SecStd & $3 \cdot 60$ & F3 IV $+\ldots$ \\
\hline HD083058 & 2000 & 093409 & $-5115 \cdot 0$ & SecStd & $5 \cdot 00$ & B1.5 IV \\
\hline HD083183 & 2000 & 093427 & $-5914 \cdot 0$ & SecStd & $4 \cdot 10$ & B5 II \\
\hline HD083446 & 2000 & 093650 & $-4921 \cdot 0$ & SecStd & $4 \cdot 40$ & A5 IV-V \\
\hline HD083944 & 2000 & 093921 & $-6120 \cdot 0$ & SecStd & $4 \cdot 50$ & B9 IV-V \\
\hline HD085123( $\infty)$ & 2000 & 094706 & $-6504 \cdot 0$ & SecStd & $2 \cdot 96$ & A8 Ib \\
\hline HD 085622 & 2000 & 095141 & $-4633 \cdot 0$ & SecStd & 4.58 & G5 Ib \\
\hline
\end{tabular}




\begin{tabular}{|c|c|c|c|c|c|c|}
\hline \multicolumn{7}{|c|}{ Table C. 2 continued from previous page } \\
\hline Name & Ep & RA & Dec & Type & $V$ & Sp \\
\hline HD086440 & 2000 & 095652 & $-5434 \cdot 0$ & SecStd & $3 \cdot 50$ & B5 Ib \\
\hline HD086629 & 2000 & 095853 & $-3553 \cdot 0$ & PriExtn & $5 \cdot 23$ & F1 III-IV \\
\hline HD087901(v?) & 2000 & 100824 & $+1158 \cdot 0$ & PriStd & $1 \cdot 35$ & B7 V \\
\hline HD088206 & 2000 & 100856 & $-5149 \cdot 0$ & SecStd & 4.90 & B3 IV \\
\hline HD088742 & 2000 & 101326 & $-3302 \cdot 0$ & PriExtn & $6 \cdot 37$ & G1 V \\
\hline HD088955 & 2000 & 101445 & $-4207 \cdot 0$ & SecStd & $3 \cdot 80$ & A2 $\mathrm{V}$ \\
\hline HD089080 & 2000 & 101345 & $-7002 \cdot 0$ & SPS & $3 \cdot 30$ & B8 IIIe \\
\hline HD090994 & 2000 & 103017 & $-0038 \cdot 2$ & PriStd & $5 \cdot 09$ & B6 V \\
\hline HD091316(v) & 2000 & 103249 & +09 $18 \cdot 4$ & PriStd & $3 \cdot 85$ & B1 IB,SB \\
\hline HD091612 & 2000 & 103449 & +0657.2 & PriStd & $5 \cdot 08$ & G9 III \\
\hline HD093779(‡) & 2000 & 104517 & $-8028 \cdot 0$ & SPS & $5 \cdot 48$ & K0 III \\
\hline HD093845(‡) & 2000 & 104548 & $-8032 \cdot 0$ & SPS & $4 \cdot 40$ & B2.5 IV \\
\hline HD095345 & 2000 & 110034 & $+0337 \cdot 1$ & PriStd & $4 \cdot 84$ & K1 III \\
\hline HD095370 & 2000 & 110009 & $-4214 \cdot 0$ & SecStd & $4 \cdot 40$ & A3 IV \\
\hline HD095456 & 2000 & 110041 & $-3150 \cdot 0$ & PriExtn & $6 \cdot 06$ & F8 V \\
\hline HD095849 & 2000 & 110337 & $-0000 \cdot 1$ & PriStd & $5 \cdot 95$ & K3 III \\
\hline HD096566 & 2000 & 110633 & $-6225 \cdot 0$ & SecStd & $4 \cdot 61$ & G8 III \\
\hline HD097585 & 2000 & 111346 & $-0004 \cdot 1$ & PriStd & $5 \cdot 42$ & A0 V \\
\hline HD098058 & 2000 & 111640 & $-0339 \cdot 1$ & PriStd & $4 \cdot 47$ & A7 IV,N \\
\hline HD098718 & 2000 & 112101 & $-5429 \cdot 0$ & SecStd & $3 \cdot 90$ & B5 Vn \\
\hline HD100286 & & 113216 & $-2916 \cdot 0$ & PriExtn & $5 \cdot 86$ & F8 V \\
\hline HD100563 & 2000 & 113422 & $+0303 \cdot 7$ & PriStd & $5 \cdot 77$ & F5 V \\
\hline HD100953 & 2000 & 113701 & $-3259 \cdot 0$ & PriExtn & $6 \cdot 28$ & F6 III-IV \\
\hline HD101431 & 2000 & 114013 & $-3445 \cdot 0$ & SecStd & $4 \cdot 70$ & B9 V \\
\hline HD102124 & 2000 & 114516 & +0815.5 & PriStd & 4.85 & $\mathrm{~A} 4 \mathrm{~V}$ \\
\hline HD102510 & 2000 & 114755 & +0814.7 & PriStd & $5 \cdot 32$ & A2 V \\
\hline HD102647(v?) & 2000 & 114906 & +1434.4 & PriStd & $2 \cdot 14$ & A3 V \\
\hline HD102776 & 2000 & 114941 & $-6347 \cdot 0$ & SecStd & $4 \cdot 32$ & B3 Vne \\
\hline HD102870 & 2000 & 115039 & $+0146 \cdot 1$ & PriStd & $3 \cdot 61$ & F8 V \\
\hline HD102964 & 2000 & 115109 & $-4510 \cdot 0$ & SecStd & $4 \cdot 46$ & K3 III \\
\hline HD103026 & 2000 & 115142 & $-3050 \cdot 0$ & PriExtn & $5 \cdot 85$ & F7 V \\
\hline HD103484 & 2000 & 115503 & $+0826 \cdot 6$ & PriStd & $5 \cdot 58$ & G8 III-IV \\
\hline HD104181 & 2000 & 115957 & $+0339 \cdot 3$ & PriStd & $5 \cdot 37$ & A1 V \\
\hline HD104979 & 2000 & 120514 & +0843.9 & PriStd & $4 \cdot 11$ & G8 III \\
\hline HD105211 & 2000 & 120653 & -6437.0 & SecStd & $4 \cdot 20$ & F2 III \\
\hline HD105452 & 2000 & 120825 & $-2444 \cdot 0$ & SecStd & $4 \cdot 02$ & F0 IV-V \\
\hline HD105639 & 2000 & 120942 & +0154.0 & PriStd & $5 \cdot 95$ & K3 III \\
\hline HD105937 & 2000 & 121139 & $-5222 \cdot 0$ & SecStd & $4 \cdot 00$ & B3 V \\
\hline HD107070 & 2000 & 121840 & $-0047 \cdot 3$ & PriStd & $5 \cdot 90$ & A5 V,N \\
\hline HD107079 & 2000 & 121900 & $-5509 \cdot 0$ & SecStd & $5 \cdot 00$ & M1 III \\
\hline HD107328(v?) & 2000 & 122023 & +0318.9 & PriStd & 4.96 & K0 III \\
\hline HD108483 & 2000 & 122803 & $-5014 \cdot 0$ & SecStd & $3 \cdot 91$ & B2 V \\
\hline HD110423(v?) & 2000 & 124157 & $+0648 \cdot 4$ & PriStd & $5 \cdot 59$ & A2 V \\
\hline
\end{tabular}




\begin{tabular}{|c|c|c|c|c|c|c|}
\hline \multicolumn{7}{|c|}{ Table C.2 continued from previous page } \\
\hline Name & Ep & RA & Dec & Type & $V$ & Sp \\
\hline HD111721 & 2000 & 125126 & $-1329 \cdot 0$ & PriStd & 7.98 & G-MDSGE \\
\hline HD 111765 & 2000 & 125137 & +0303.4 & PriStd & $6 \cdot 02$ & K2 III \\
\hline HD111915 & 2000 & 125307 & $-4857 \cdot 0$ & SecStd & $4 \cdot 33$ & K3.5 III \\
\hline HD111968 & 2000 & 125326 & $-4011 \cdot 0$ & SecStd & $4 \cdot 30$ & A7 III \\
\hline HD112300(v?) & 2000 & 125538 & +0323.9 & PriStd & $3 \cdot 38$ & M3 III \\
\hline HD112985 & 2000 & 130214 & $-7133 \cdot 0$ & SPS & $3 \cdot 62$ & K2 III \\
\hline HD113703 & 2000 & 130617 & $-4828 \cdot 0$ & SecStd & 4.71 & B5 V \\
\hline HD114435 & 2000 & 131109 & $-4214 \cdot 0$ & PriExtn & $5 \cdot 79$ & F7 IV \\
\hline HD114613 & 2000 & 131205 & $-3748 \cdot 0$ & PriExtn & 4.85 & G3 V \\
\hline HD114837 & 2000 & 131417 & $-5906 \cdot 0$ & SecStd & 4.92 & F7 IV \\
\hline HD114960 & 2000 & 131358 & $+0127 \cdot 4$ & PriStd & 6.58 & K5 III \\
\hline HD116160 & 2000 & 132142 & $+0205 \cdot 3$ & PriStd & $5 \cdot 69$ & A2 V \\
\hline HD116235 & 2000 & 132210 & $+0509 \cdot 3$ & PriStd & $5 \cdot 87$ & $\mathrm{~A} 2, \mathrm{M}$ \\
\hline HD118022(v) & 2000 & 133408 & +0339.5 & PriStd & 4.94 & A1, $\mathrm{P}$ (SR-CR-EU) \\
\hline HD119756 & 2000 & 134543 & $-3303 \cdot 0$ & PriExtn & $4 \cdot 23$ & F3 IV \\
\hline HD120602 & 2000 & 135025 & +0529.9 & PriStd & $6 \cdot 01$ & G5 III \\
\hline HD120955(œ) & 2000 & 135312 & $-3156 \cdot 0$ & SecStd & $4 \cdot 73$ & B4 IV \\
\hline HD120987 & 2000 & 135333 & $-3540 \cdot 0$ & PriExtn & $5 \cdot 54$ & F4 V \\
\hline HD121263 & 2000 & 135533 & $-4717 \cdot 0$ & SecStd & $2 \cdot 60$ & B2.5 IV \\
\hline HD121447 & 2000 & 135547 & $-1815 \cdot 0$ & PriStd & 7.90 & $\mathrm{M}, \mathrm{P}$ \\
\hline HD121607 & 2000 & 135628 & $+0103 \cdot 1$ & PriStd & 5.91 & A $8 \mathrm{~V}$ \\
\hline HD 122066 & 2000 & 140001 & $-2501 \cdot 0$ & PriExtn & $5 \cdot 80$ & F6 V \\
\hline HD122408 & 2000 & 140139 & +0132.7 & PriStd & $4 \cdot 26$ & A3 V \\
\hline HD123139 & 2000 & 140643 & $-3622 \cdot 0$ & SecStd & $2 \cdot 06$ & K0 IIIb \\
\hline HD 123998 & 2000 & 141814 & $-8100 \cdot 0$ & SPS & 4.91 & A2 m \\
\hline HD124639 & 2000 & 142423 & $-8251 \cdot 0$ & SPS & $6 \cdot 37$ & $\mathrm{~B} 8 \mathrm{Ve}$ \\
\hline HD124780 & 2000 & 141618 & $-3314 \cdot 0$ & PriExtn & $6 \cdot 56$ & F0 V \\
\hline HD124850(v?) & 2000 & 141601 & $-0559 \cdot 7$ & PriStd & $4 \cdot 08$ & F6 III \\
\hline HD124882 & & 142658 & $-8340 \cdot 0$ & SPS & $4 \cdot 32$ & K2 III \\
\hline HD126053 & 2000 & 142315 & $+0114 \cdot 9$ & PriStd & $6 \cdot 27$ & G1 V \\
\hline HD126248 & 2000 & 142412 & $+0549 \cdot 2$ & PriStd & $5 \cdot 10$ & A5 V \\
\hline HD126868(v?) & 2000 & 142812 & $-0213 \cdot 7$ & PriStd & $4 \cdot 81$ & G2 IV \\
\hline HD129116 & 2000 & 144158 & $-3748 \cdot 0$ & SecStd & $4 \cdot 00$ & B3 V \\
\hline HD129956 & 2000 & 144530 & $+0043 \cdot 0$ & PriStd & $5 \cdot 69$ & B9.5 V \\
\hline HD130650 & 2000 & 150150 & $-8314 \cdot 0$ & SPS & $5 \cdot 65$ & G9 III \\
\hline HD131246 & 2000 & 150447 & $-8302 \cdot 0$ & SPS & $5 \cdot 65$ & G8 Ib \\
\hline HD132345 & 2000 & 145854 & $-1108 \cdot 6$ & PriStd & $5 \cdot 88$ & K3 III,CN2 \\
\hline HD133955 & 2000 & 150851 & $-4517 \cdot 0$ & SecStd & $4 \cdot 05$ & B3 V \\
\hline HD134169 & 2000 & 150818 & +0356.0 & PriStd & $7 \cdot 68$ & F8-SD \\
\hline HD135559 & 2000 & 151549 & $+0022 \cdot 4$ & PriStd & $5 \cdot 63$ & A4 V \\
\hline HD136014 & 2000 & 151932 & $-3706 \cdot 0$ & PriExtn & $6 \cdot 19$ & G6 III-IV \\
\hline HD136028 & 2000 & 151826 & $-0027 \cdot 7$ & PriStd & $5 \cdot 89$ & K5 III \\
\hline HD136422 & 2000 & 152149 & $-3616 \cdot 0$ & SecStd & $3 \cdot 56$ & K5 III \\
\hline
\end{tabular}




\begin{tabular}{|c|c|c|c|c|c|c|}
\hline \multicolumn{7}{|c|}{ Table C.2 continued from previous page } \\
\hline Name & Ep & RA & Dec & Type & $V$ & Sp \\
\hline HD137058 & 2000 & 152520 & $-3844 \cdot 0$ & SecStd & $4 \cdot 60$ & A0 IV \\
\hline HD 137613 & 2000 & 152748 & -2510.0 & PriStd & 7.63 & $\mathrm{R} 3$ \\
\hline HD138716 & 2000 & 153410 & $-1003 \cdot 7$ & PriStd & $4 \cdot 64$ & K1 IV \\
\hline HD139409 & 2000 & 153842 & $-1740 \cdot 0$ & PriStd & $7 \cdot 19$ & K0 (BA) \\
\hline HD140008 & 2000 & 154241 & $-3443 \cdot 0$ & SecStd & 4.75 & B5 V \\
\hline HD 140573 & 2000 & 154415 & +0625.5 & PriStd & 2.65 & K2 IIIb \\
\hline HD141556 & 2000 & 155057 & $-3338 \cdot 0$ & SecStd & $3 \cdot 95$ & B9 IV \\
\hline HD143118( $\infty)$ & 2000 & 160007 & $-3824 \cdot 0$ & SecStd & $3 \cdot 41$ & B2.5 IV \\
\hline HD143474( $\infty)$ & 2000 & 160333 & $-5746 \cdot 0$ & SecStd & $4 \cdot 63$ & A7 IV \\
\hline HD143553 & 2000 & 160051 & $+0425 \cdot 6$ & PriStd & $5 \cdot 83$ & G9 III-IV \\
\hline HD144470 & 2000 & 160649 & $-2040 \cdot 0$ & SecStd & 3.96 & B1 V \\
\hline HD145366 & & 162021 & $-7842 \cdot 0$ & SPS & $4 \cdot 78$ & M5 IIIb \\
\hline HD146836 & 2000 & 161932 & $-3054 \cdot 0$ & PriExtn & $5 \cdot 49$ & F6 III \\
\hline HD147196 & 2000 & 162119 & $-2342 \cdot 0$ & PriStd & 7.03 & B8 V,NN,P \\
\hline HD147432 & 2000 & 162252 & -2307.0 & PriStd & $7 \cdot 50$ & A2 V \\
\hline HD147513 & 2000 & 162401 & $-3912 \cdot 0$ & PriExtn & $5 \cdot 40$ & G5 V \\
\hline HD 147550 & 2000 & 162239 & $-0204 \cdot 8$ & PriStd & $6 \cdot 22$ & B9 V \\
\hline HD147675 & & 163329 & -7854.0 & SPS & $3 \cdot 89$ & G9 III \\
\hline HD 147977 & 2000 & 162815 & $-5836 \cdot 0$ & Comp & $5 \cdot 78$ & B9 II-III \\
\hline HD148513 & 2000 & 162834 & $+0039 \cdot 9$ & PriStd & $5 \cdot 43$ & K4 III,P \\
\hline HD148542 & 2000 & 170057 & $-8622 \cdot 0$ & SPS & $6 \cdot 06$ & A2 V \\
\hline HD148605 & 2000 & $\begin{array}{llll}16 & 30 & 13\end{array}$ & -2507.0 & SecStd & $4 \cdot 78$ & B3 V \\
\hline HD148703 & 2000 & 163123 & $-3442 \cdot 0$ & SecStd & $4 \cdot 23$ & B2 III-IV \\
\hline HD148760 & 2000 & 163122 & $-2632 \cdot 2$ & PriStd & $6 \cdot 22$ & K1 III \\
\hline HD149324 & & 164309 & $-7731 \cdot 0$ & SPS & $4 \cdot 24$ & K0 III \\
\hline HD149447 & 2000 & 163622 & $-3515 \cdot 0$ & SecStd & $4 \cdot 16$ & K6 III \\
\hline HD149730 & 2000 & 163915 & $-5659 \cdot 0$ & R Arae & & B9 IV-V \\
\hline HD150366 & 2000 & 164136 & $-2428 \cdot 1$ & PriStd & $6 \cdot 07$ & FO V \\
\hline HD150416 & 2000 & 164135 & $-1744 \cdot 5$ & PriStd & 4.96 & $\mathrm{G} 8 \mathrm{II}(\mathrm{CN} 1, \mathrm{BA})$ \\
\hline HD150451 & 2000 & 164112 & $-0100 \cdot 0$ & PriStd & $6 \cdot 22$ & A7 III \\
\hline HD150745 & 2000 & 164621 & $-5828 \cdot 0$ & Check & $5 \cdot 94$ & B3 \\
\hline HD150898 & 2000 & 164720 & $-5820 \cdot 0$ & SecStd & $5 \cdot 76$ & B0 \\
\hline HD151046 & 2000 & 164459 & $-0037 \cdot 0$ & PriStd & $7 \cdot 68$ & K5 III \\
\hline HD151431(v?) & 2000 & 164710 & +0203.9 & PriStd & $6 \cdot 10$ & A $3 \mathrm{~V}$ \\
\hline HD152980 & 2000 & 165935 & $-5310 \cdot 0$ & SecStd & $4 \cdot 06$ & K4 III \\
\hline HD153210(v) & & 165518 & +0927.0 & PriStd & $3 \cdot 20$ & K2 III \\
\hline HD153687 & 2000 & 170103 & $-04 \quad 13 \cdot 3$ & PriStd & $4 \cdot 82$ & K4 III \\
\hline HD154090 & 2000 & 170449 & $-3407 \cdot 0$ & SecStd & $4 \cdot 87$ & B1 Iae \\
\hline HD154417 & 2000 & 170517 & +0042.5 & PriStd & $6 \cdot 01$ & F9 V \\
\hline HD156190 & & 172206 & $\begin{array}{lll}-70 & 07.0\end{array}$ & SPS & $5 \cdot 60$ & B9 $V+\ldots$ \\
\hline HD156277 & 2000 & 172160 & $-6746 \cdot 0$ & SecStd & $4 \cdot 78$ & K2 III \\
\hline HD156513 & 2000 & 173127 & $-8052 \cdot 0$ & SPS & $5 \cdot 88$ & M3 III \\
\hline HD156826 & 2000 & 171959 & $-0554 \cdot 9$ & PriStd & $6 \cdot 32$ & G9 V \\
\hline
\end{tabular}




\begin{tabular}{|c|c|c|c|c|c|c|}
\hline \multicolumn{7}{|c|}{ Table C.2 continued from previous page } \\
\hline Name & Ep & RA & Dec & Type & $V$ & Sp \\
\hline HD157089 & 2000 & 172108 & $+0126 \cdot 4$ & PriStd & $6 \cdot 96$ & F9 V-MD \\
\hline HD157244 & 2000 & 172518 & -5532.0 & SecStd & 2.85 & K3 Ib-II \\
\hline HD157246 & 2000 & 172524 & $-5623 \cdot 0$ & SecStd & $3 \cdot 30$ & B1 Ib \\
\hline HD 157415 & 2000 & 172302 & $+0050 \cdot 0$ & PriStd & $7 \cdot 78$ & K0 III-MD \\
\hline HD158094 & 2000 & 173106 & $-6041 \cdot 0$ & SecStd & 3.60 & $\mathrm{~B} 8 \mathrm{Vn}$ \\
\hline HD 158352 & 2000 & 172850 & $+0019 \cdot 8$ & PriStd & $5 \cdot 44$ & A $8 \mathrm{~V}$ \\
\hline HD 158427 & 2000 & 173151 & $-4952 \cdot 0$ & SecStd & 3.00 & B2 Vne \\
\hline HD158926 & 2000 & 173338 & $-3706 \cdot 0$ & SecStd & 1.63 & B1.5 IV \\
\hline HD 159217 & 2000 & 173540 & $-4630 \cdot 0$ & SecStd & 4.60 & $\mathrm{~A} 0 \mathrm{~V}$ \\
\hline HD 159433 & 2000 & 173633 & $-3838 \cdot 0$ & SecStd & $4 \cdot 29$ & K0 IIIb \\
\hline HD 159517 & 2000 & 180134 & $-8513 \cdot 0$ & SPS & $6 \cdot 45$ & F5 IV \\
\hline HD 159532 & 2000 & 173719 & $-4300 \cdot 0$ & SecStd & 1.90 & F1 II \\
\hline HD159975 & 2000 & 173751 & $-0807 \cdot 1$ & PriStd & $4 \cdot 62$ & B8 II-III,P(MN) \\
\hline HD 160032 & 2000 & 174023 & $-4925 \cdot 0$ & SecStd & 4.77 & F3 IV \\
\hline HD160346 & 2000 & 173918 & $+0333 \cdot 4$ & PriStd & $6 \cdot 52$ & K3 V \\
\hline HD160635 & 2000 & 174544 & $-6443 \cdot 0$ & SecStd & $3 \cdot 62$ & K2 II \\
\hline HD160781 & 2000 & 174132 & $+0618 \cdot 8$ & PriStd & $5 \cdot 95$ & G7 III \\
\hline HD160823 & 2000 & 174156 & +0422.0 & PriStd & 7.01 & G2 II-MD \\
\hline HD161868 & 2000 & 174754 & +0242.5 & PriStd & 3.75 & A0 V,SB: \\
\hline HD161912 & 2000 & 175011 & $-4005 \cdot 0$ & SecStd & 4.81 & A2 Ib \\
\hline HD162917 & 2000 & 175315 & $+0606 \cdot 0$ & PriStd & $5 \cdot 77$ & F3-5 IV-V \\
\hline HD163245 & 2000 & 175554 & -1848.1 & PriStd & $6 \cdot 42$ & A4 V \\
\hline HD 163376 & 2000 & 175748 & $-4143 \cdot 0$ & SecExtn & $4 \cdot 88$ & M0 III \\
\hline HD164259 & 2000 & 180028 & $-0341 \cdot 4$ & PriStd & 4.62 & F2 IV \\
\hline HD164353 & 2000 & 180039 & $+0256 \cdot 0$ & PriStd & 3.97 & B5 Ib \\
\hline HD164461 & 2000 & 185449 & $-8736 \cdot 0$ & SPS & $5 \cdot 27$ & K3 IIICN... \\
\hline HD165174(v) & 2000 & 180438 & $+0155 \cdot 1$ & PriStd & $6 \cdot 14$ & B0 IIIn \\
\hline HD 165189 & 2000 & 180650 & $-4325 \cdot 0$ & SecExtn & 4.94 & A5 V \\
\hline HD165462 & 2000 & 180607 & $-0026 \cdot 7$ & PriStd & $6 \cdot 34$ & G8 II,P \\
\hline HD166063 & 2000 & 181114 & $-4557 \cdot 0$ & SecExtn & 4.53 & K0 III \\
\hline HD166114 & 2000 & 181105 & $-4122 \cdot 0$ & PriExtn & $5 \cdot 90$ & F2 V \\
\hline HD166460 & 2000 & 181040 & +0319.5 & PriStd & $5 \cdot 51$ & K2 III \\
\hline HD 167768 & 2000 & 181653 & $-0300 \cdot 2$ & PriStd & $5 \cdot 96$ & G3 III \\
\hline HD169111 & 2000 & 182236 & +1201.8 & PriStd & $5 \cdot 90$ & $\mathrm{~A} 2 \mathrm{~V}$ \\
\hline HD169191 & 2000 & 182249 & +1749.5 & PriStd & $5 \cdot 25$ & K3 III \\
\hline HD169467 & 2000 & 182658 & $-4558 \cdot 0$ & SecStd & $3 \cdot 50$ & B3 IV \\
\hline HD169767 & 2000 & 182849 & $-4904 \cdot 0$ & SecStd & $4 \cdot 13$ & G9 III \\
\hline HD169904 & 2000 & 184214 & $-8148 \cdot 0$ & SPS & $6 \cdot 27$ & B8 V \\
\hline HD170465(‡) & 2000 & 183145 & $-4555 \cdot 0$ & SecStd & 4.93 & B6 IV \\
\hline HD170521 & 2000 & 183156 & $-4330 \cdot 0$ & SecStd & $5 \cdot 71$ & K2 III \\
\hline HD170845 & 2000 & 183330 & $-4219 \cdot 0$ & SecExtn & 4.64 & G8 III \\
\hline HD171538 & 2000 & 183515 & $+0559 \cdot 8$ & PriStd & $6 \cdot 47$ & A3 III \\
\hline HD171767 & 2000 & 183629 & +0457.4 & PriStd & 6.74 & K1 III \\
\hline
\end{tabular}




\begin{tabular}{|c|c|c|c|c|c|c|}
\hline \multicolumn{7}{|c|}{ Table C.2 continued from previous page } \\
\hline Name & Ep & RA & Dec & Type & $V$ & $\mathrm{Sp}$ \\
\hline HD172046 & 2000 & 183749 & $+0553 \cdot 1$ & PriStd & $6 \cdot 67$ & B5 V \\
\hline HD172365 & 2000 & 183937 & $+0515 \cdot 9$ & PriStd & $6 \cdot 38$ & F8 Ib-II \\
\hline HD 172850 & 2000 & 184230 & $-0118 \cdot 0$ & PriStd & $7 \cdot 78$ & B8 V \\
\hline HD172910 & 2000 & 184419 & $-3539 \cdot 0$ & SecExtn & $4 \cdot 87$ & $\mathrm{~B} 2.5 \mathrm{~V}$ \\
\hline HD173009 & 2000 & 184331 & $-0816 \cdot 5$ & PriStd & 4.90 & G8 II \\
\hline HD173371 & 2000 & 184456 & $-0022 \cdot 4$ & PriStd & $6 \cdot 88$ & B9 III \\
\hline HD175190 & 2000 & 185507 & $-2240 \cdot 2$ & PriStd & 4.97 & $\mathrm{~K} 3-\mathrm{MD}(\mathrm{BA})$ \\
\hline HD175679 & 2000 & 185626 & $+0228 \cdot 3$ & PriStd & $6 \cdot 15$ & G8 III \\
\hline HD176638 & 2000 & 190306 & $-4206 \cdot 0$ & SecExtn & $4 \cdot 80$ & B9.5 V \\
\hline HD176704 & 2000 & 190228 & $-2450 \cdot 7$ & PriStd & 5.59 & K3 III \\
\hline HD176723 & 2000 & 190318 & $-3815 \cdot 0$ & PriExtn & $5 \cdot 73$ & F2 III-IV \\
\hline HD177474 & 2000 & 190625 & $-3704 \cdot 0$ & SecExtn & $4 \cdot 20$ & F7 IV-V \\
\hline HD177482 & 2000 & 210840 & $-8857 \cdot 0$ & SPS & $5 \cdot 46$ & F0 III \\
\hline HD 177756 & 2000 & 190615 & -0452.8 & PriStd & 3.44 & B9 V,N \\
\hline HD177873 & 2000 & 190821 & $-4030 \cdot 0$ & SecExtn & 4.59 & K1 III \\
\hline HD178125(v) & 2000 & 190658 & $+1104 \cdot 3$ & PriStd & $5 \cdot 09$ & B8 III \\
\hline HD178253 & 2000 & 190928 & $-3754 \cdot 0$ & SecExtn & $4 \cdot 11$ & $\mathrm{~A} 2 \mathrm{~V}$ \\
\hline HD179406(v?) & 2000 & 191241 & $-0756 \cdot 4$ & PriStd & $5 \cdot 34$ & B3 V \\
\hline HD181383 & 2000 & 191953 & $+1132 \cdot 1$ & PriStd & $6 \cdot 02$ & $\mathrm{~A} 2 \mathrm{~V}$ \\
\hline HD181391 & 2000 & 192033 & -0524.9 & PriStd & $5 \cdot 01$ & G8 III-IV,SB \\
\hline HD182101 & 2000 & 192249 & $+0954 \cdot 7$ & PriStd & $6 \cdot 35$ & F6 V \\
\hline HD182296 & 2000 & 192338 & $+0839 \cdot 6$ & PriStd & 7.07 & G3 Ib \\
\hline HD182640 & 2000 & 192529 & +0307.0 & SecStd & $3 \cdot 36$ & F0 IV \\
\hline HD183324 & 2000 & 192901 & $+0157 \cdot 0$ & PriStd & $5 \cdot 80$ & A0 V \\
\hline HD183630(v?) & 2000 & 193040 & $-0247 \cdot 3$ & PriStd & $5 \cdot 03$ & M1 III \\
\hline HD183936 & 2000 & 193143 & $+0546 \cdot 1$ & PriStd & $7 \cdot 20$ & F2 III \\
\hline HD184127 & 2000 & 193513 & $-4806 \cdot 0$ & SecStd & 4.90 & KO III \\
\hline HD184313(v) & 2000 & 193346 & $+0528 \cdot 0$ & PriStd & $6 \cdot 33$ & M8 V \\
\hline HD184406(v?) & 2000 & 193405 & +0722.9 & PriStd & 4.45 & K3 III,B \\
\hline HD184492 & 2000 & 193507 & $-1033 \cdot 7$ & PriStd & $5 \cdot 12$ & G8 IIIA \\
\hline HD184915 & 2000 & 193653 & -0702.0 & SecStd & 4.95 & B0.5 III \\
\hline HD184930 & 2000 & 193643 & $-0117 \cdot 1$ & PriStd & $4 \cdot 36$ & B5 III \\
\hline HD186154 & 2000 & 195601 & $-8121 \cdot 0$ & SPS & $6 \cdot 39$ & K3.5 III \\
\hline HD186648 & 2000 & 194622 & $-1945 \cdot 6$ & PriStd & $4 \cdot 86$ & K1 III \\
\hline HD187923(v?) & 2000 & 195205 & $+1138 \cdot 0$ & PriStd & $6 \cdot 13$ & G0 V \\
\hline HD188228 & & 200035 & -7255.0 & SPS & $4 \cdot 00$ & A0 V \\
\hline HD188350 & 2000 & 195444 & $+0016 \cdot 4$ & PriStd & $5 \cdot 61$ & A0 III \\
\hline HD189103 & 2000 & 195944 & $-3517 \cdot 0$ & SecStd & $4 \cdot 36$ & B3 IV \\
\hline HD189695 & 2000 & 200059 & +0833.4 & PriStd & $5 \cdot 91$ & K5 III \\
\hline HD190248 & 2000 & 200833 & $\begin{array}{ll}-66 & 10 \cdot 0\end{array}$ & SecStd & $3 \cdot 56$ & G7 IV \\
\hline HD190421 & 2000 & 200723 & $-5253 \cdot 0$ & SecStd & 4.94 & M1 IIb \\
\hline HD191220 & 2000 & 202454 & $\begin{array}{ll}-83 & 19 \cdot 0\end{array}$ & SPS & $6 \cdot 16$ & $\mathrm{Am}$ \\
\hline HD192876(‡) & 2000 & 201739 & $-1230 \cdot 5$ & PriStd & $4 \cdot 24$ & G3 IB \\
\hline
\end{tabular}




\begin{tabular}{|c|c|c|c|c|c|c|}
\hline & & & & & & \\
\hline Name & Ep & $\mathrm{RA}$ & Dec & Type & $V$ & Sp \\
\hline HD194612 & 2000 & 203819 & $-8117 \cdot 0$ & SPS & 5.91 & K5 III \\
\hline HD195135 & 2000 & 202938 & -0253.1 & PriStd & 4.91 & K2 III \\
\hline HD195627 & 2000 & 203534 & $-6035 \cdot 0$ & SecStd & $4 \cdot 80$ & F1 III \\
\hline HD195810(v?) & 2000 & 203313 & $+1118 \cdot 0$ & PriStd & 4.03 & B6 III \\
\hline HD196171 & 2000 & 203734 & $-4718 \cdot 0$ & SecStd & $3 \cdot 11$ & K0 IIICNv \\
\hline HD196426 & 2000 & $2037 \quad 19$ & +0005.8 & PriStd & $6 \cdot 19$ & B8 III,P \\
\hline HD198070 & 2000 & 204748 & +0318.4 & PriStd & $6 \cdot 40$ & $\mathrm{~A} 0 \mathrm{~V}, \mathrm{~N}$ \\
\hline HD198743 & 2000 & 205240 & -0858.9 & PriStd & $4 \cdot 73$ & $\mathrm{~A} 3, \mathrm{M}$ \\
\hline HD199442 & 2000 & $2057 \quad 10$ & +0027.9 & PriStd & 6.06 & K2 III \\
\hline HD199951 & 2000 & 210117 & $-3215 \cdot 0$ & SecStd & $4 \cdot 67$ & G4 III \\
\hline HD199960 & 2000 & 210034 & $-0443 \cdot 7$ & PriStd & $6 \cdot 21$ & G1 V \\
\hline HD200163 & 2000 & 210258 & $-3837 \cdot 0$ & PriExtn & $5 \cdot 35$ & F3 V \\
\hline HD200644 & 2000 & 210434 & +0530.2 & PriStd & $5 \cdot 61$ & K5 III \\
\hline HD202418 & 2000 & 213202 & $-8449 \cdot 0$ & SPS & $6 \cdot 45$ & K3 III \\
\hline HD 202627 & 2000 & 211756 & $-3210 \cdot 0$ & SecStd & $4 \cdot 70$ & A1 V \\
\hline HD203532 & 2000 & 213354 & $-8241 \cdot 0$ & SPS & $6 \cdot 38$ & B3 IV \\
\hline HD203608 & 2000 & 212626 & -6523.0 & SecStd & $4 \cdot 22$ & F6 V \\
\hline HD203926 & 2000 & 212517 & $-0333 \cdot 3$ & PriStd & $5 \cdot 49$ & K4 III \\
\hline HD203955 & 2000 & 21. 3320 & $\begin{array}{lll}-80 & 02 \cdot 0\end{array}$ & SPS & $6 \cdot 47$ & A0 V \\
\hline HD204075 & 2000 & 212640 & $-2224 \cdot 7$ & PriStd & $3 \cdot 76$ & G4 IB \\
\hline HD204904 & 2000 & 213855 & -7927.0 & SPS & $6 \cdot 17$ & F4 IV \\
\hline HD205478 & 2000 & 214128 & $-7723 \cdot 0$ & SPS & $3 \cdot 76$ & K0 III \\
\hline HD205924 & 2000 & 213832 & $+0546 \cdot 3$ & PriStd & $5 \cdot 67$ & A9 IV-V,N \\
\hline HD 206742 & 2000 & 214457 & -3301.0 & SecStd & $4 \cdot 30$ & A0 V \\
\hline HD206834 & 2000 & 214460 & $-0905 \cdot 0$ & PriStd & $5 \cdot 10$ & G8 II-III \\
\hline HD208450 & 2000 & 215755 & $-5500 \cdot 0$ & SecStd & $4 \cdot 40$ & F0 IV \\
\hline HD209100 & 2000 & 220257 & $-5645 \cdot 0$ & SecStd & $4 \cdot 69$ & $\mathrm{~K} 4.5 \mathrm{~V}$ \\
\hline HD209750 & 2000 & 220547 & $-0019 \cdot 2$ & PriStd & 2.96 & G2 Ib \\
\hline HD210302 & 2000 & 221008 & $-3233 \cdot 0$ & PriExtn & 4.92 & $\mathrm{~F} 6 \mathrm{~V}$ \\
\hline HD210434 & 2000 & 221033 & $-0416 \cdot 0$ & PriStd & 6.02 & K0 III-IV \\
\hline HD210967 & 2000 & 222001 & $-8026 \cdot 0$ & SPS & $5 \cdot 10$ & M5 III \\
\hline HD211088 & 2000 & 221537 & $-4121 \cdot 0$ & SecStd & $4 \cdot 79$ & $\mathrm{G} 8 \mathrm{III}+\ldots$ \\
\hline HD211416 & 2000 & 221831 & $\begin{array}{lll}-60 & 16 \cdot 0\end{array}$ & SecStd & $2 \cdot 86$ & K3 III \\
\hline HD212581( $\infty)$ & 2000 & 222719 & $-6458 \cdot 0$ & SecStd & $4 \cdot 50$ & B9.5 V \\
\hline HD213009 & 2000 & 222916 & $-4330 \cdot 0$ & SecStd & 3.97 & G7 III \\
\hline HD213398 & 2000 & 223130 & $-3221 \cdot 0$ & SecStd & $4 \cdot 29$ & $\mathrm{~A} 0 \mathrm{~V}$ \\
\hline HD214298 & 2000 & 223705 & $+1234 \cdot 7$ & PriStd & $6 \cdot 28$ & K5 \\
\hline HD214846 & 2000 & 224605 & $-8123 \cdot 0$ & SPS & $4 \cdot 15$ & A9 IV-V \\
\hline HD215369 & 2000 & 224538 & $-5330 \cdot 0$ & SecStd & $4 \cdot 85$ & $\mathrm{~K} 2 \mathrm{IIICNv}$ \\
\hline HD216143 & 2000 & 225031 & $-0655 \cdot 0$ & PriStd & $7 \cdot 85$ & G8-MDGE \\
\hline HD216701 & 2000 & 225459 & $+0103 \cdot 9$ & PriStd & $6 \cdot 11$ & A7 III \\
\hline HD216762 & 2000 & 225556 & $-3155 \cdot 2$ & PriStd & $6 \cdot 41$ & S5,1 \\
\hline HD217364 & 2000 & 230053 & $-5245 \cdot 0$ & SecStd & $4 \cdot 12$ & G9 III \\
\hline
\end{tabular}




\begin{tabular}{|l|c|c|c|l|l|l|}
\hline \multicolumn{7}{|l|}{ Table C.2 continued from previous page } \\
\hline Name & Ep & RA & Dec & Type & $V$ & Sp \\
\hline HD217792 & & 230330 & $-3445 \cdot 0$ & PriExtn & $5 \cdot 13$ & F0 V+... \\
HD217877 & 2000 & 230356 & $-0447 \cdot 7$ & PriStd & $6 \cdot 70$ & F8 V \\
HD218527 & 2000 & 230840 & $+0207 \cdot 6$ & PriStd & $5 \cdot 39$ & G8 III-IV-MD \\
HD218559 & 2000 & 231212 & $-8055 \cdot 0$ & SPS & $6 \cdot 41$ & K4 III \\
HD218670 & 2000 & 231021 & $-4515 \cdot 0$ & SecStd & $3 \cdot 90$ & K1 III \\
HD219449 & 2000 & 231552 & $-0905 \cdot 2$ & PriStd & $4 \cdot 25$ & K0 III \\
HD219571 & 2000 & 231726 & $-5814 \cdot 0$ & SecStd & $3 \cdot 99$ & F1 III \\
HD219765 & 2000 & 232802 & $-8729 \cdot 0$ & SPS & $5 \cdot 49$ & K2 III \\
HD219784 & 2000 & 231849 & $-3232 \cdot 0$ & SecStd & $4 \cdot 41$ & G8 III \\
HD221507 & 2000 & 233258 & $-3749 \cdot 0$ & SecStd & $4 \cdot 37$ & B9.5 IVmn \\
HD221760 & 2000 & 233504 & $-4237 \cdot 0$ & SecStd & $4 \cdot 71$ & A2 Vp... \\
HD221950 & 2000 & 233623 & $+0206 \cdot 1$ & PriStd & $5 \cdot 68$ & F6 V-MD \\
HD222368(v?) & 2000 & 233956 & $+0537 \cdot 9$ & PriStd & $4 \cdot 13$ & F7 V \\
HD222493 & 2000 & 234109 & $-1140 \cdot 9$ & PriStd & $5 \cdot 92$ & G9 III \\
HD222603 & 2000 & 234204 & $+0146 \cdot 9$ & PriStd & $4 \cdot 50$ & A7 V \\
HD223438 & 2000 & 234928 & $+0104 \cdot 6$ & PriStd & $5 \cdot 77$ & A5 , M \\
HD223647 & 2000 & 235208 & $-8201 \cdot 0$ & SPS & $5 \cdot 11$ & G7 III \\
HD223833 & 2000 & 235345 & $-8300 \cdot 0$ & SPS & $6 \cdot 9$ & M0 III \\
HD224362 & 2000 & 235734 & $-8210 \cdot 0$ & SPS & $5 \cdot 73$ & K0 III \\
HD224392 & 2000 & 235734 & $-6418 \cdot 0$ & SecStd & $5 \cdot 00$ & A1 V \\
HD224686 & 2000 & 235955 & $-6535 \cdot 0$ & SecStd & $4 \cdot 50$ & B9 IV \\
HD224889 & 2000 & 000137 & $-7704 \cdot 0$ & SPS & $4 \cdot 78$ & K3 III \\
HD224935(v) & 2000 & 000158 & $-0600 \cdot 8$ & PriStd & $4 \cdot 41$ & M3 III \\
$\kappa$ Crucis & & 125254 & $-6017 \cdot 0$ & Program & & \\
O Velorum & 2000 & 084018 & $-5255 \cdot 3$ & Program & & \\
\hline
\end{tabular}




\section{Appendix D}

\section{The Reduced Observations.}

The following tables give the reduced magnitudes from the individual observations of the primary standard stars, secondary standard stars, and the clusters $\kappa$ Crucis and O Vel. They are sorted by star name, and for the same star, further sorted by date and time of observation. These tables have been included so later observers may add further observations to each star and recalculate the mean values, and also investigate possible variability of a star.

The first column in each table is the star's name. The next column is the observation's calendar date. The third column is the time (UT) of the mid-point of the observation, in hours and minutes. Immediately after this is the index number $n$ of the observation, which indicates this was the $n^{\text {th }}$ observation on that night - this is useful for easy reference back to the original data files and observation log books. The remaining seven columns are the reduced V magnitude and colours, with their standard deviations. These values are all given to milli-magnitudes. 


\begin{tabular}{|c|c|c|c|c|c|c|c|c|c|c|c|c|}
\hline & 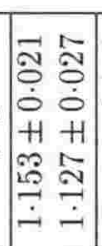 & 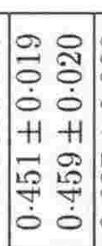 & 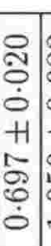 & 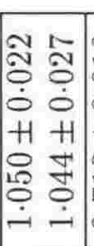 & $\left|\begin{array}{cc}\infty & -1 \\
0 & 0 \\
0 & 0 \\
+1 & +1 \\
0 & 0 \\
1 & 0 \\
0 & 0\end{array}\right|$ & 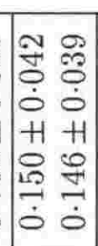 & \begin{tabular}{ll}
1 & $\infty$ \\
0 & 0 \\
0 & 0 \\
$\dot{1}$ & +1 \\
0 & 0 \\
0 & 0 \\
\hdashline & 0 \\
0 & 0
\end{tabular} & 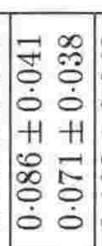 & 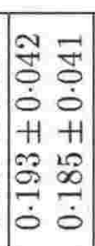 & 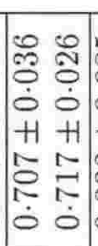 & 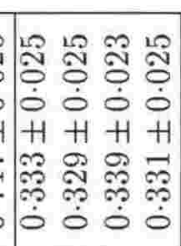 & 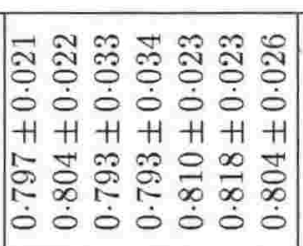 \\
\hline$>$ & 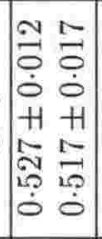 & $\mid$\begin{tabular}{cc}
2 & 0 \\
0 & 0 \\
0 & 0 \\
0 & 0 \\
$H$ & +1 \\
0 & 0 \\
\hdashline & 0 \\
0 & 0 \\
0
\end{tabular} & $\mid$\begin{tabular}{c}
20 \\
0 \\
0 \\
0 \\
+1 \\
0 \\
0 \\
\hdashline \\
0 \\
0
\end{tabular} & 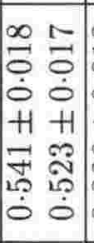 & 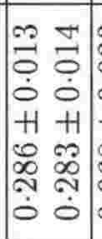 & 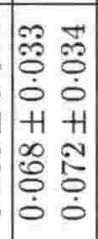 & 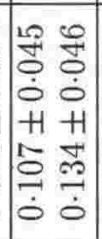 & 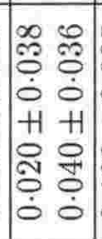 & $\mid$\begin{tabular}{cc}
1 & 2 \\
\hdashline & 0 \\
0 & 0 \\
0 & 0 \\
$H$ & +1 \\
0 & 8 \\
0 & 8 \\
0 & 0 \\
0 & 0
\end{tabular} & 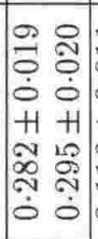 & 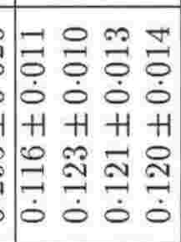 & 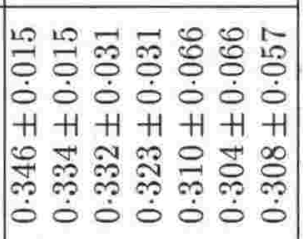 \\
\hline & 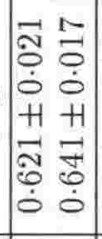 & 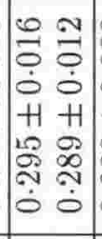 & 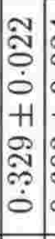 & 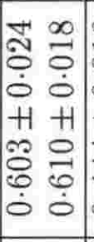 & 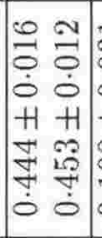 & 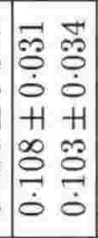 & 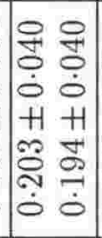 & 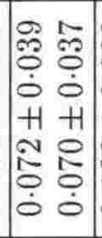 & 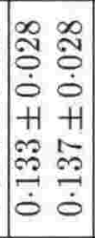 & 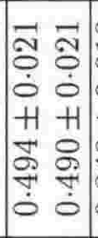 & 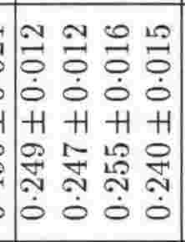 & 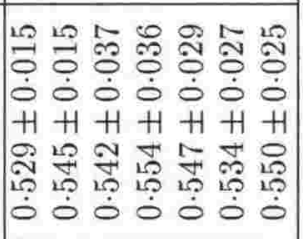 \\
\hline & 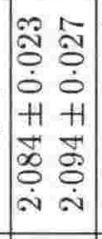 & $\left|\begin{array}{ll}0 & 0 \\
0 & 0 \\
0 & 0 \\
0 & 0 \\
1 & 1 \\
10 & 1 \\
0 & 10 \\
0 & 0 \\
0 & 0 \\
0 & 0\end{array}\right|$ & 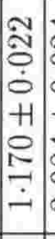 & 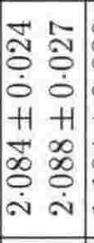 & 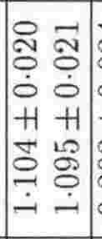 & 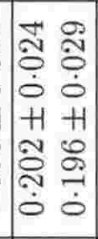 & 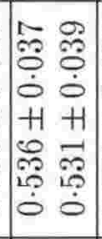 & 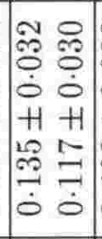 & 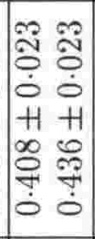 & 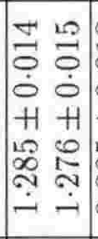 & 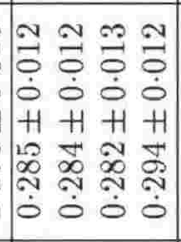 & 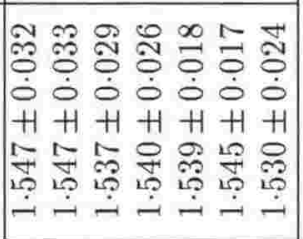 \\
\hline . & 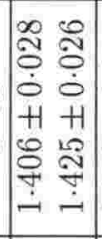 & 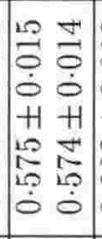 & $\begin{array}{c}0 \\
\\
0 \\
\dot{0} \\
+1 \\
\mathscr{2} \\
\mathscr{0} \\
0 \\
0\end{array}$ & 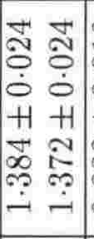 & 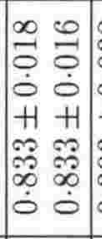 & 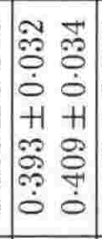 & 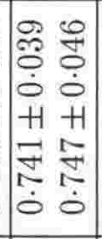 & 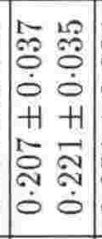 & 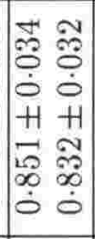 & $\left|\begin{array}{cc}0 & 0 \\
0 & 0 \\
0 & 0 \\
0 & 0 \\
1 & 1 \\
1 & 0 \\
\hdashline & 0 \\
0 & 0 \\
0 & 0\end{array}\right|$ & $\mid$\begin{tabular}{cccc}
1 & 1 & - & 0 \\
0 & 0 & 0 & 0 \\
0 & 0 & 0 & 0 \\
$H$ & $H$ & $H$ & $H$ \\
0 & $\infty$ & 2 & 0 \\
0 & 1 & $\infty$ & 0 \\
\hdashline & $\overrightarrow{0}$ & 0 & 0 \\
0 & 0 & 0 & 0
\end{tabular} & 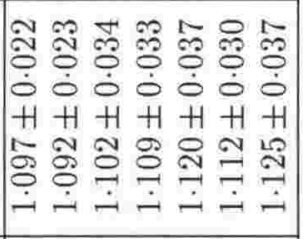 \\
\hline 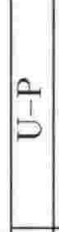 & 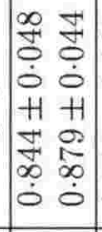 & 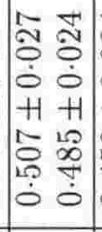 & 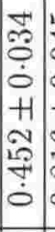 & 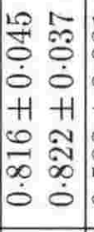 & 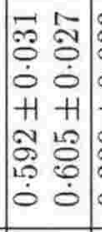 & 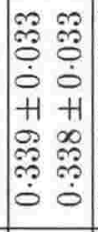 & 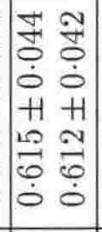 & $\mid$\begin{tabular}{cc|}
0 & 0 \\
0 & 0 \\
0 & 0 \\
0 & 0 \\
+1 & +1 \\
0 & $\mathbb{1}$ \\
0 & $\mathbb{1}$ \\
0 & 0
\end{tabular} & 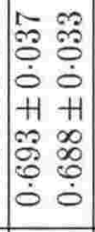 & 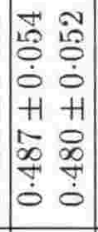 & 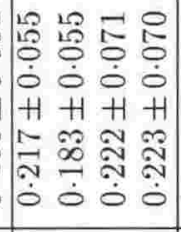 & 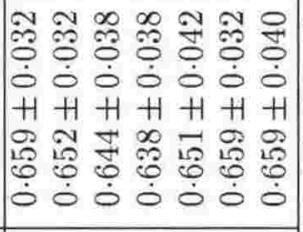 \\
\hline$>$ & 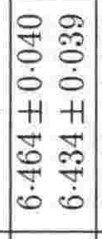 & 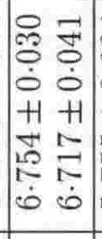 & \begin{tabular}{|l|}
5 \\
0 \\
0 \\
0 \\
+1 \\
10 \\
10 \\
10 \\
10
\end{tabular} & 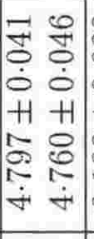 & 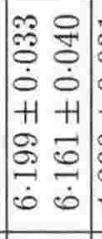 & 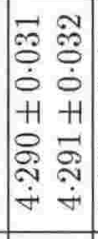 & 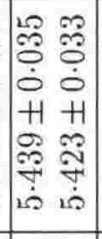 & 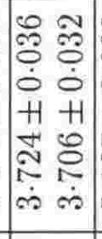 & 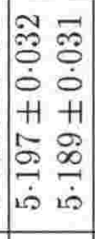 & & 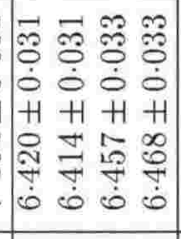 & 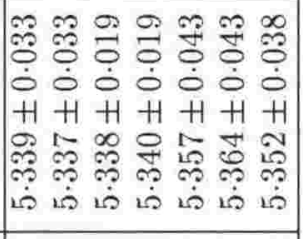 \\
\hline \# & $=\stackrel{\Xi}{\Xi}$ & $\stackrel{\infty}{\cong} \underset{\Xi}{\Xi}$ & 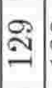 & $\stackrel{\odot}{\stackrel{\Xi}{二}}$ & 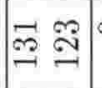 & os 0 & $\infty \stackrel{9}{-1}$ & on & $\approx \approx$ & 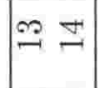 & $\dddot{⿻} \because$ & 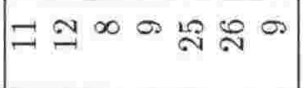 \\
\hline S & 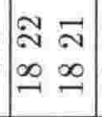 & $\left|\begin{array}{ll|}-1 & \infty \\
\infty & \infty \\
- & \sim\end{array}\right|$ & $\begin{array}{l}-\infty \\
\infty \\
-\end{array}$ & $\left|\begin{array}{ll}\forall & 2 \\
\infty & \infty \\
-1 & \infty\end{array}\right|$ & $\left|\begin{array}{cc}0 & 7 \\
\infty & 7 \\
\infty & \infty \\
-1 & -7\end{array}\right|$ & 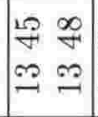 & 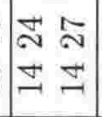 & 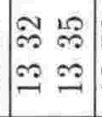 & $\left|\begin{array}{ll}\infty & -1 \\
0 & 0 \\
\hdashline & + \\
\hdashline & -1\end{array}\right|$ & $\mid \begin{array}{ll}8 & 9 \\
0 & 0 \\
- & 0\end{array}$ & 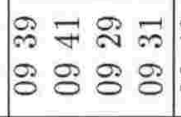 & 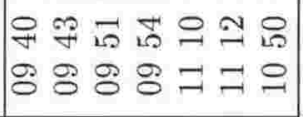 \\
\hline 量 & 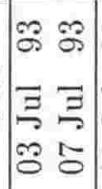 & $\left|\begin{array}{cc}\approx & \Re \\
\vdots & \Xi \\
\hdashline & 3 \\
\hdashline & 5\end{array}\right|$ & $\approx$ & ஜ & $\mid$\begin{tabular}{ll}
$\Xi$ & $\Xi$ \\
\hdashline & $\vdots$ \\
\hdashline & 5
\end{tabular} & $\left|\begin{array}{ll}3 & 3 \\
z & z \\
2 & z \\
2 & 2 \\
\end{array}\right|$ & $\mid \begin{array}{cc}8 & 8 \\
3 & 3 \\
3 & 2 \\
20 & 2 \\
& 2\end{array}$ & 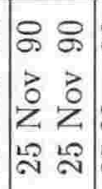 & 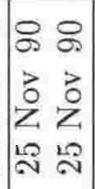 & 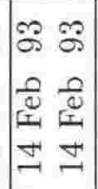 & 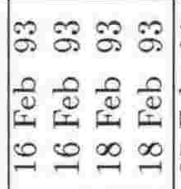 & 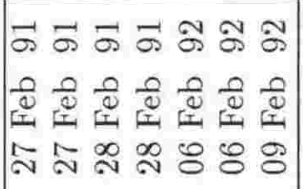 \\
\hline \# & $=\mid \begin{array}{l}0 \\
0 \\
0 \\
8 \\
\end{array}$ & 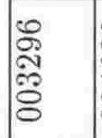 & & & 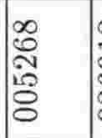 & 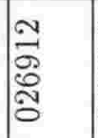 & \begin{tabular}{l}
$\infty$ \\
20 \\
10 \\
$\infty$ \\
\hdashline \\
0
\end{tabular} & 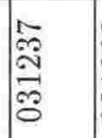 & 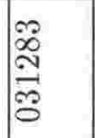 & $\begin{array}{c}\tilde{\infty} \\
\infty \\
0 \\
0 \\
0 \\
0\end{array}$ & 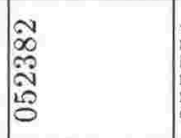 & 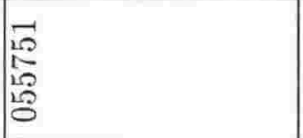 \\
\hline
\end{tabular}




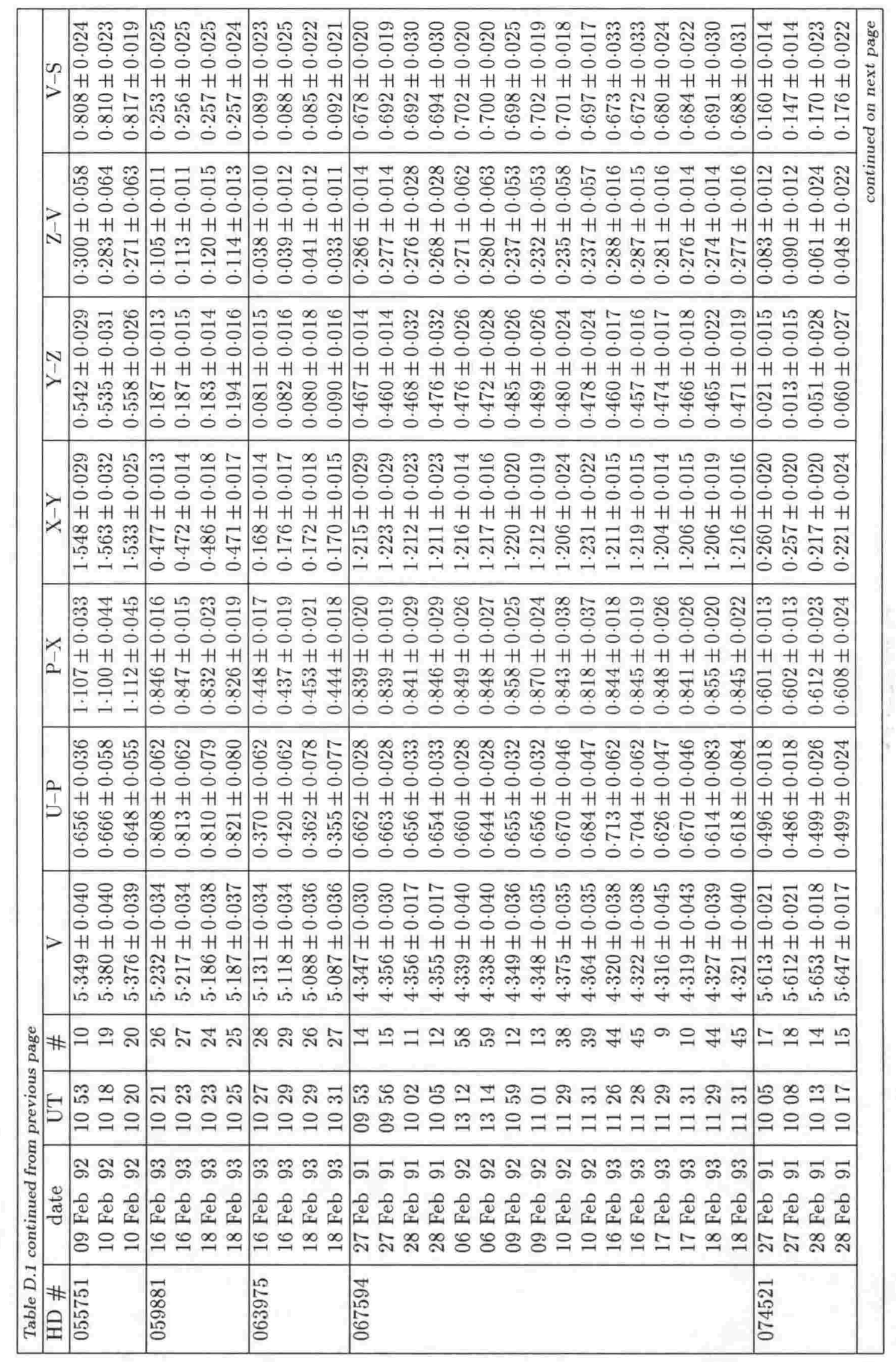




\begin{tabular}{|c|c|c|c|c|}
\hline & 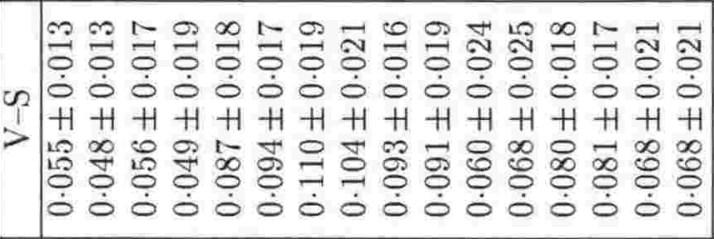 & 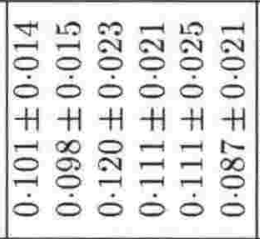 & 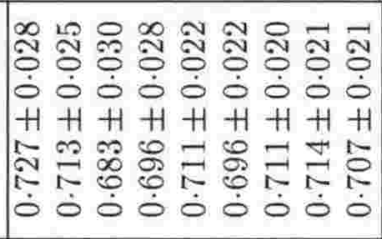 & 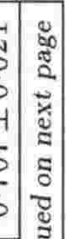 \\
\hline & 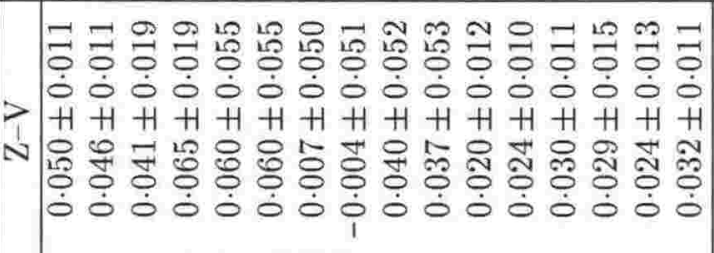 & 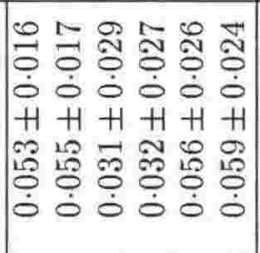 & 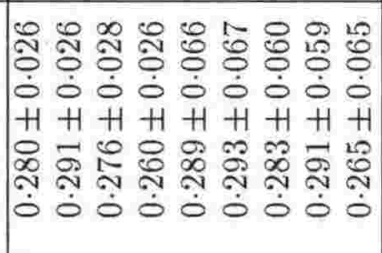 & $\begin{array}{l}5 \\
5 \\
b \\
4 \\
3 \\
\vdots \\
b\end{array}$ \\
\hline & 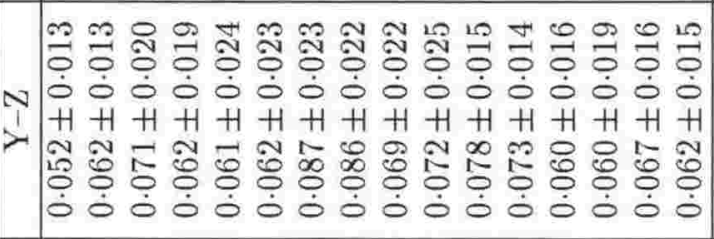 & 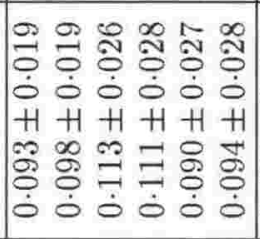 & 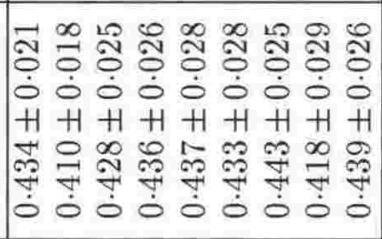 & \\
\hline & 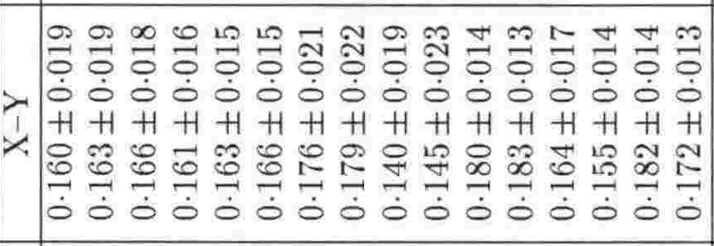 & 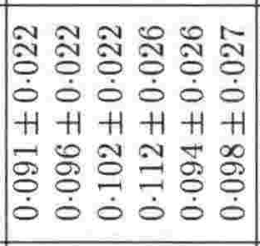 & 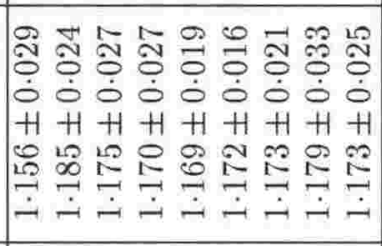 & \\
\hline & 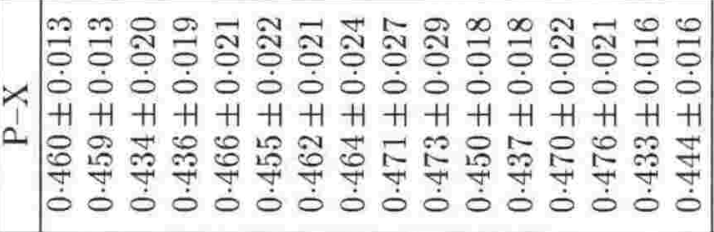 & 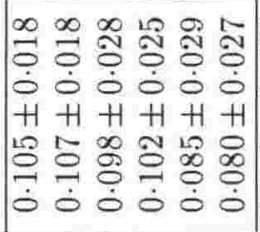 & 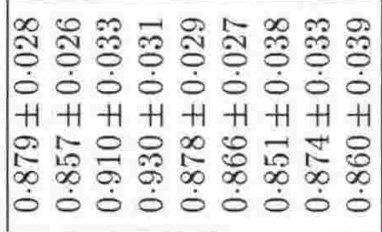 & \\
\hline & 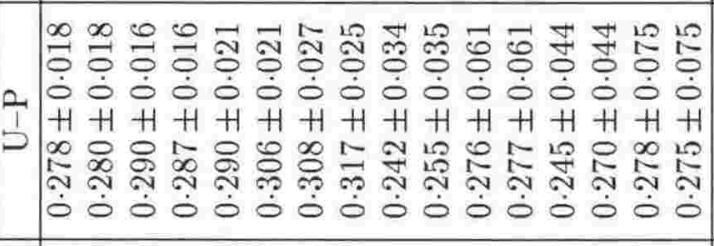 & 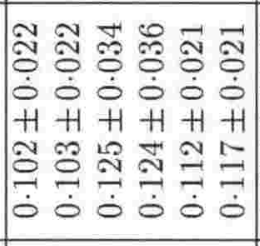 & 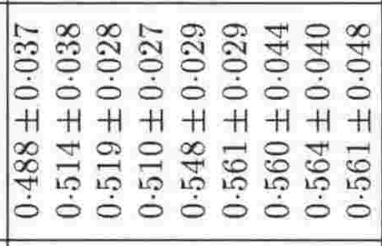 & \\
\hline & 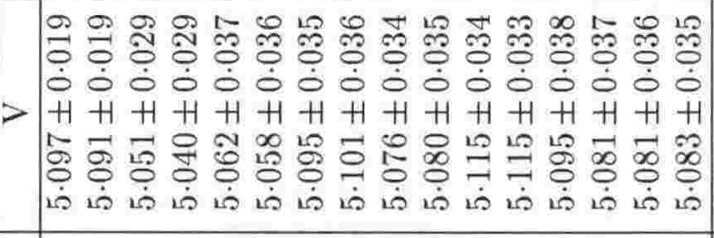 & 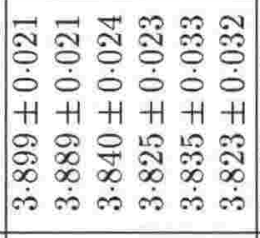 & 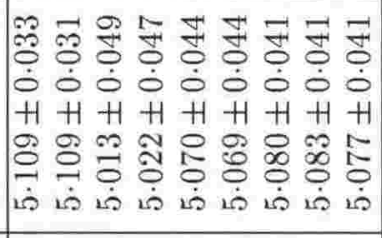 & \\
\hline & \# & $\infty 8 \neg \sim ⿻ \infty$ & 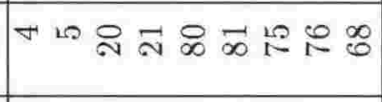 & \\
\hline & 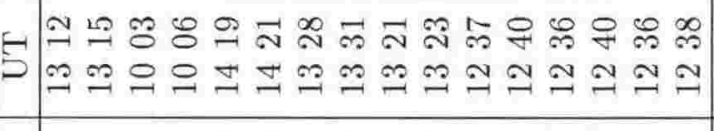 & 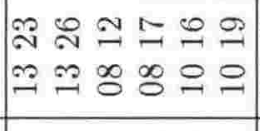 & 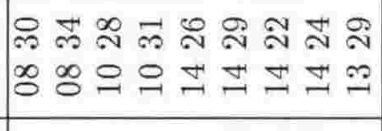 & \\
\hline & 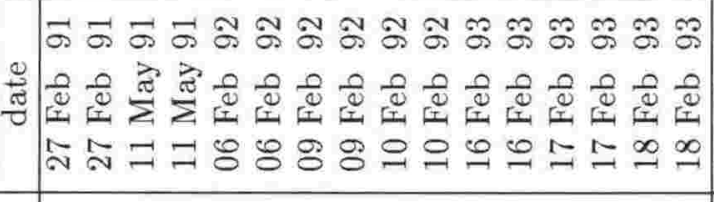 & 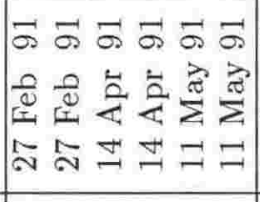 & 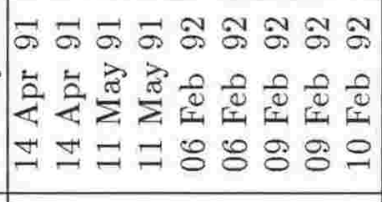 & \\
\hline & 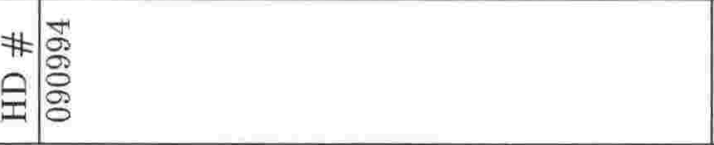 & $\frac{\infty}{20}$ & $\frac{\pi}{0}$ & \\
\hline
\end{tabular}




\begin{tabular}{|c|c|c|c|c|c|c|c|}
\hline & & $\begin{array}{lll}\infty & \overrightarrow{1} & -1 \\
0 & 0 & 0 \\
0 & 0 \\
0 & 0 & 0 \\
H & H & +1 \\
1 & 10 & 0 \\
0 & 0 & 0 \\
0 & 0 & 0 \\
0 & 0\end{array}$ & 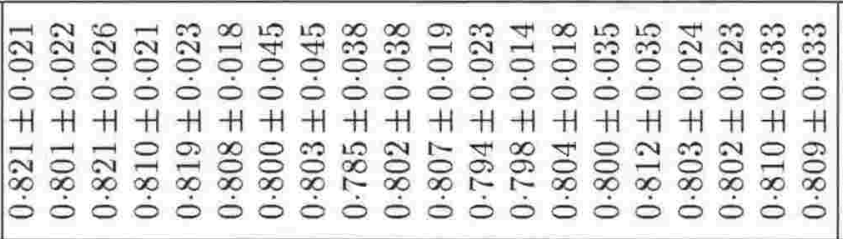 & 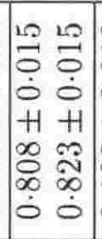 & 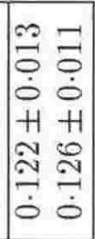 & 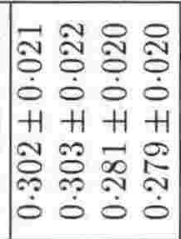 & \\
\hline & & 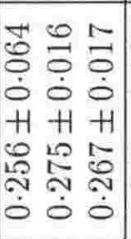 & 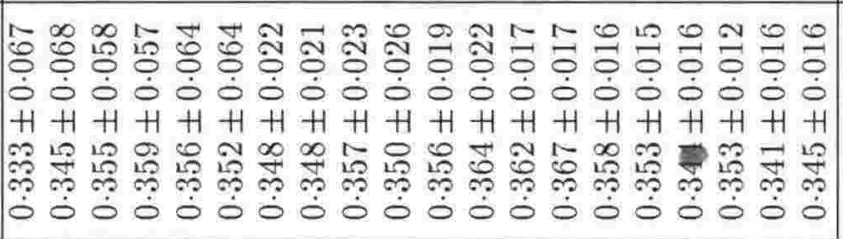 & $\mid$\begin{tabular}{cc|}
0 & 0 \\
0 & 0 \\
0 & 0 \\
0 & 0 \\
$H$ & +1 \\
0 & 0 \\
0 & 0 \\
0 & 0 \\
0 & 0
\end{tabular} & 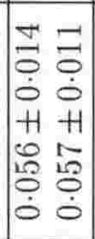 & 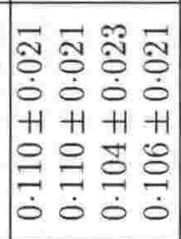 & \\
\hline & & 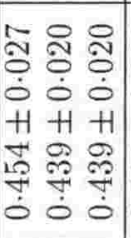 & 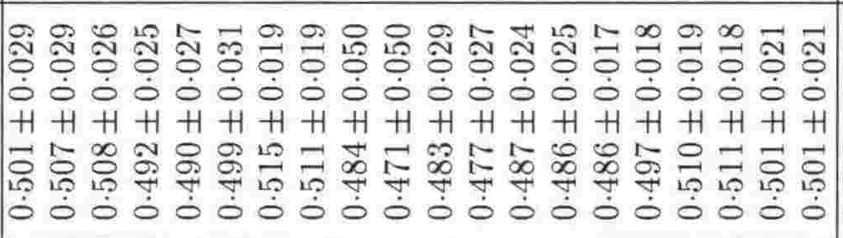 & 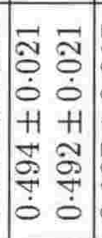 & 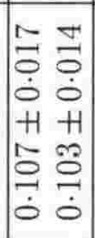 & 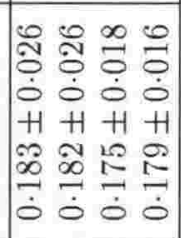 & \\
\hline & & 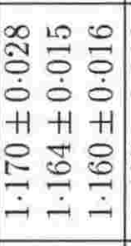 & 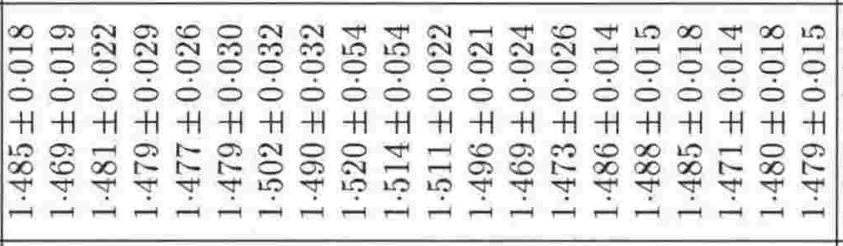 & 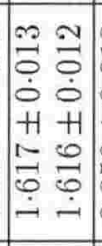 & 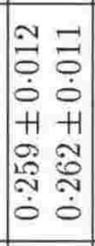 & 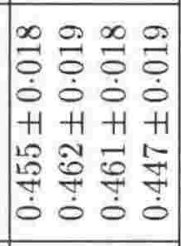 & \\
\hline & & 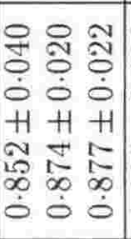 & 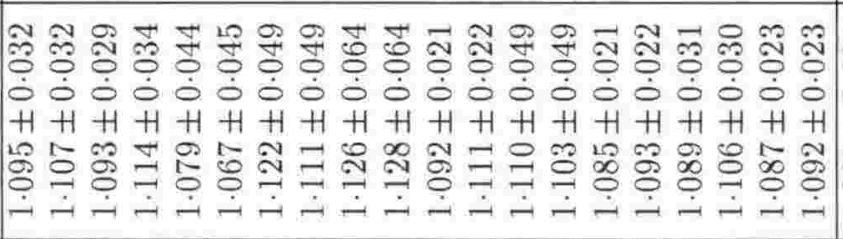 & 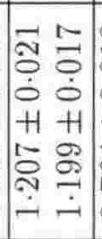 & $\begin{array}{ll}m & 2 \\
0 & 0 \\
0 & 0 \\
0 & 0 \\
1 & +1 \\
0 & 0 \\
0 & 0 \\
0 & 0 \\
0 & 0\end{array}$ & 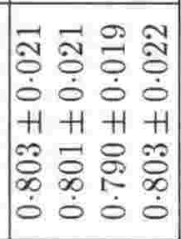 & \\
\hline & & 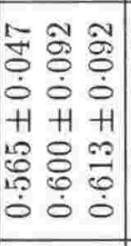 & 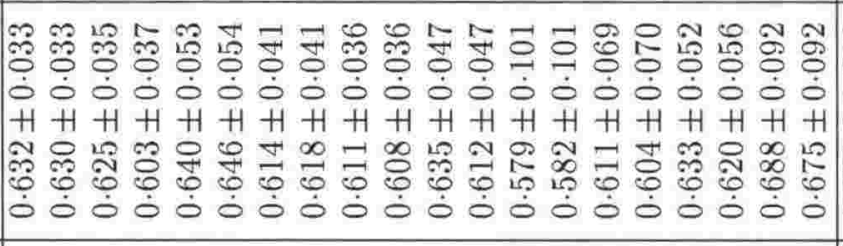 & $\left|\begin{array}{cc|}\infty & 2 \\
0 & 2 \\
0 & 0 \\
0 & 0 \\
+1 & 1 \\
0 & 1 \\
0 & 1 \\
0 & 0 \\
0 & 0 \\
0 & 0\end{array}\right|$ & $\mid$\begin{tabular}{ll|}
0 & 0 \\
0 & 0 \\
0 & 0 \\
0 & 0 \\
$H$ & $H$ \\
0 & 1 \\
$\infty$ & 0 \\
0 & 0 \\
0 & 0 \\
0 & 0
\end{tabular} & 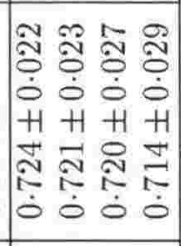 & \\
\hline & & 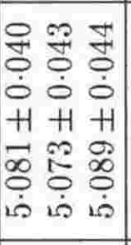 & 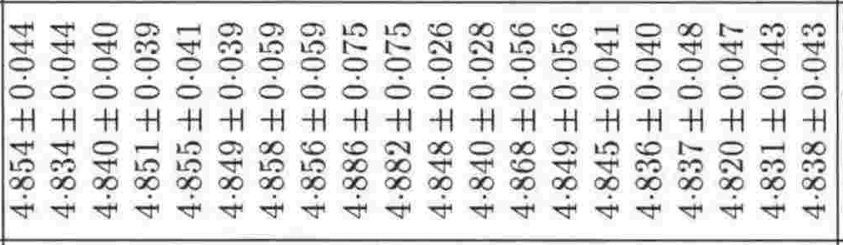 & 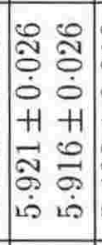 & 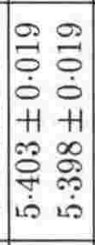 & 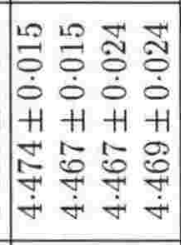 & \\
\hline & & Bกฺ & 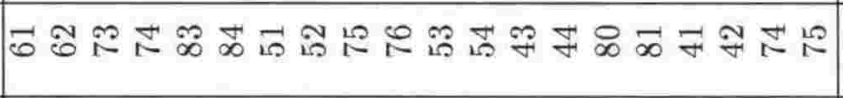 & $\cong \cong$ & $\exists \stackrel{20}{-1}$ & $18 \& 1 \infty$ & \\
\hline & & 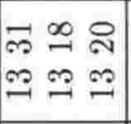 & 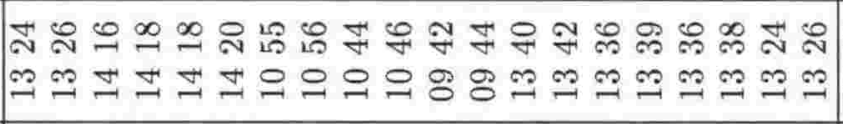 & $\left|\begin{array}{ll}\infty & \overrightarrow{5} \\
+ & 15 \\
5 & 5 \\
0 & 5\end{array}\right|$ & 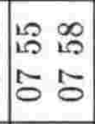 & 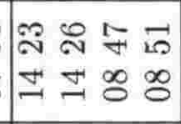 & \\
\hline : & & 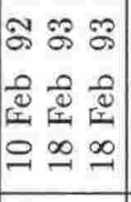 & 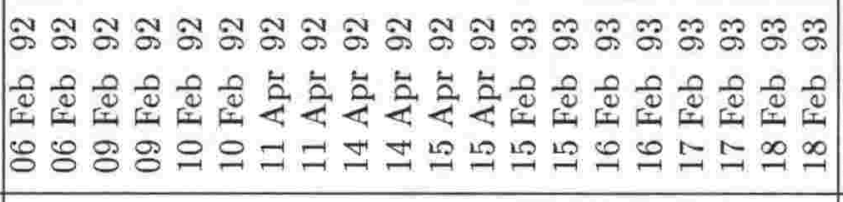 & 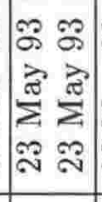 & 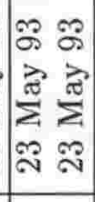 & 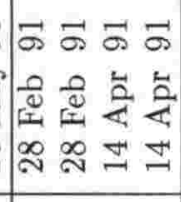 & \\
\hline & & & 量 & 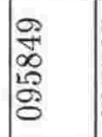 & 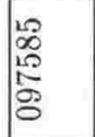 & $\begin{array}{l}\infty \\
i \\
\infty \\
\infty \\
8 \\
0\end{array}$ & \\
\hline
\end{tabular}




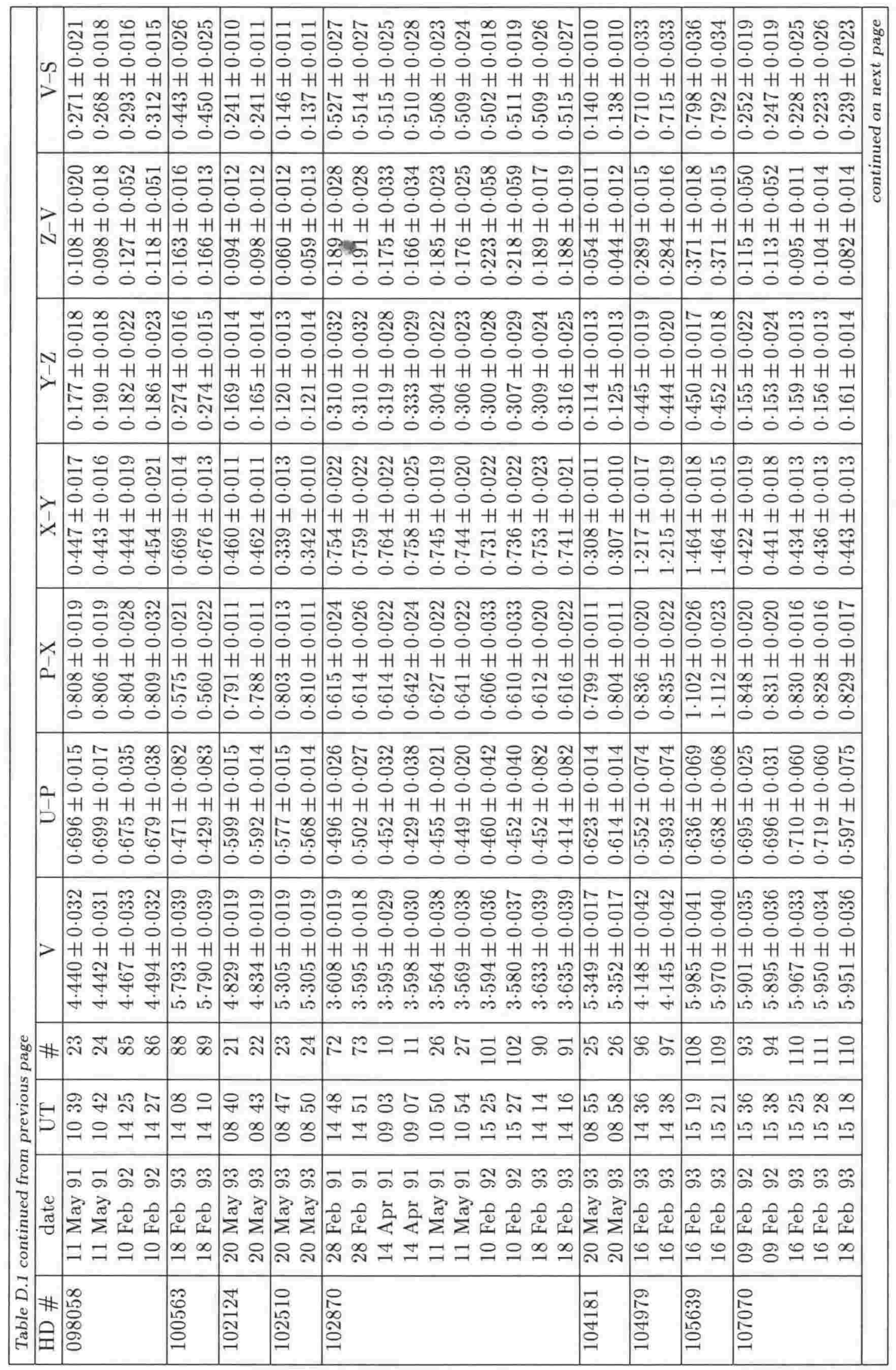




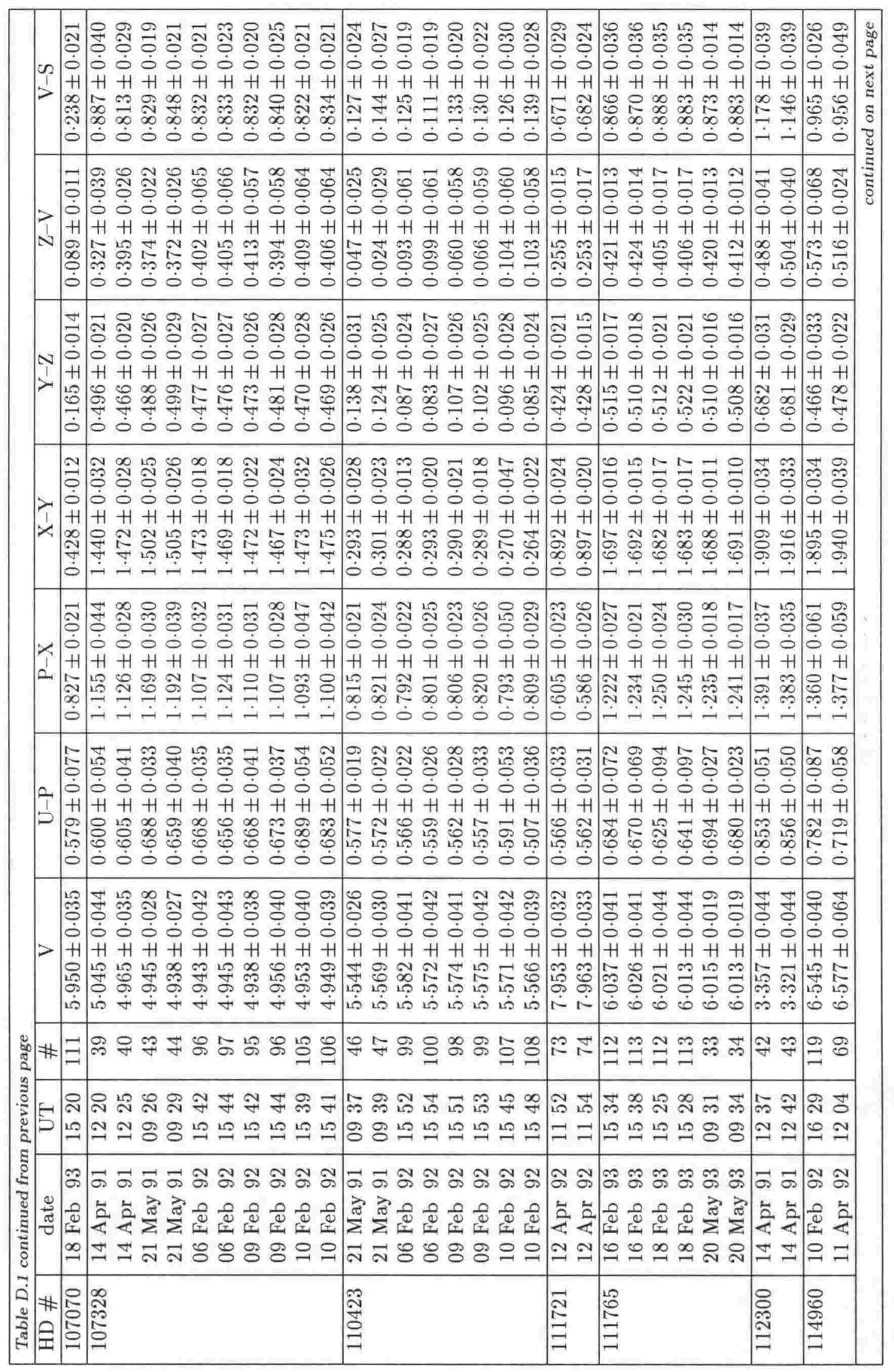




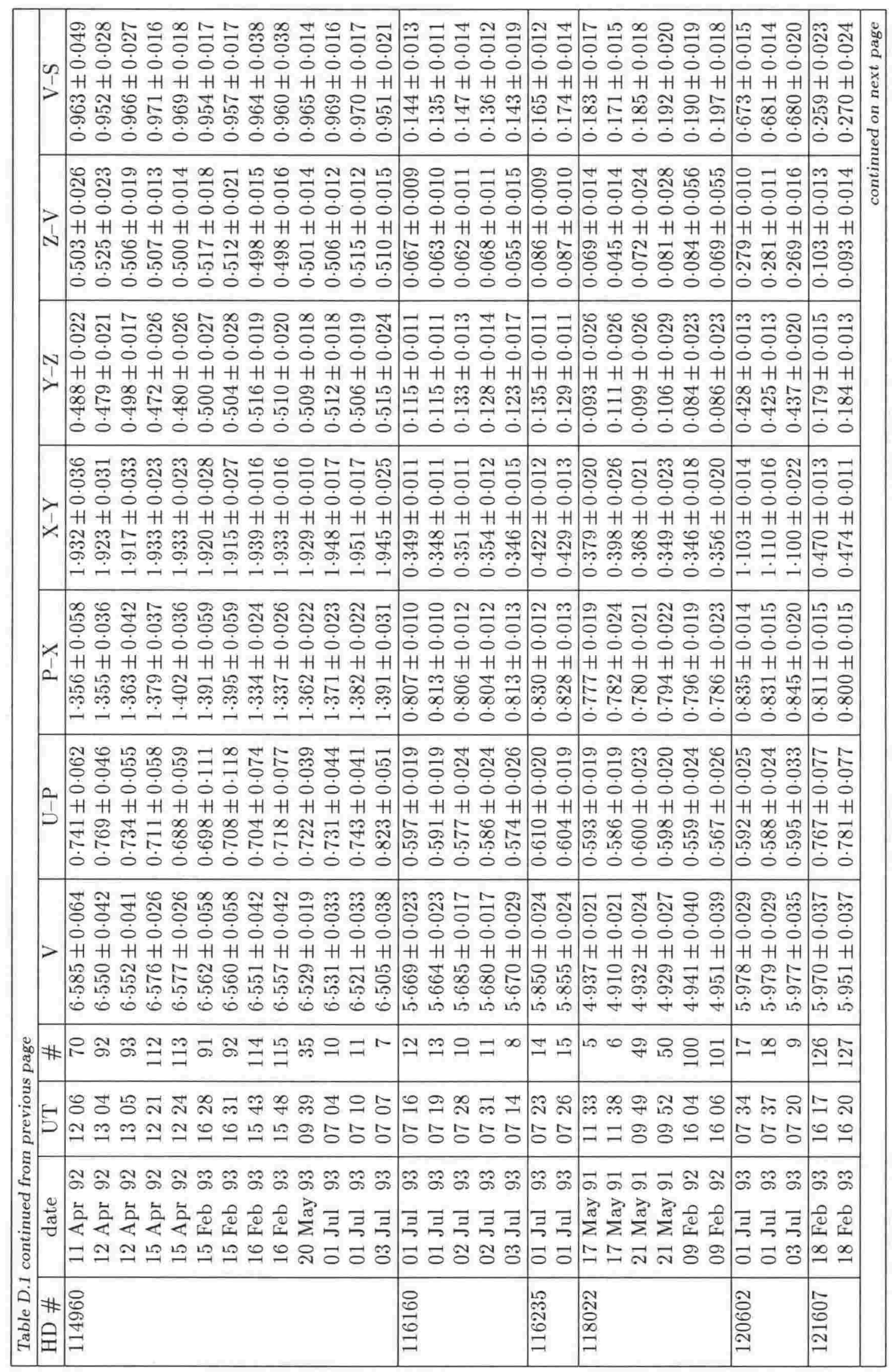




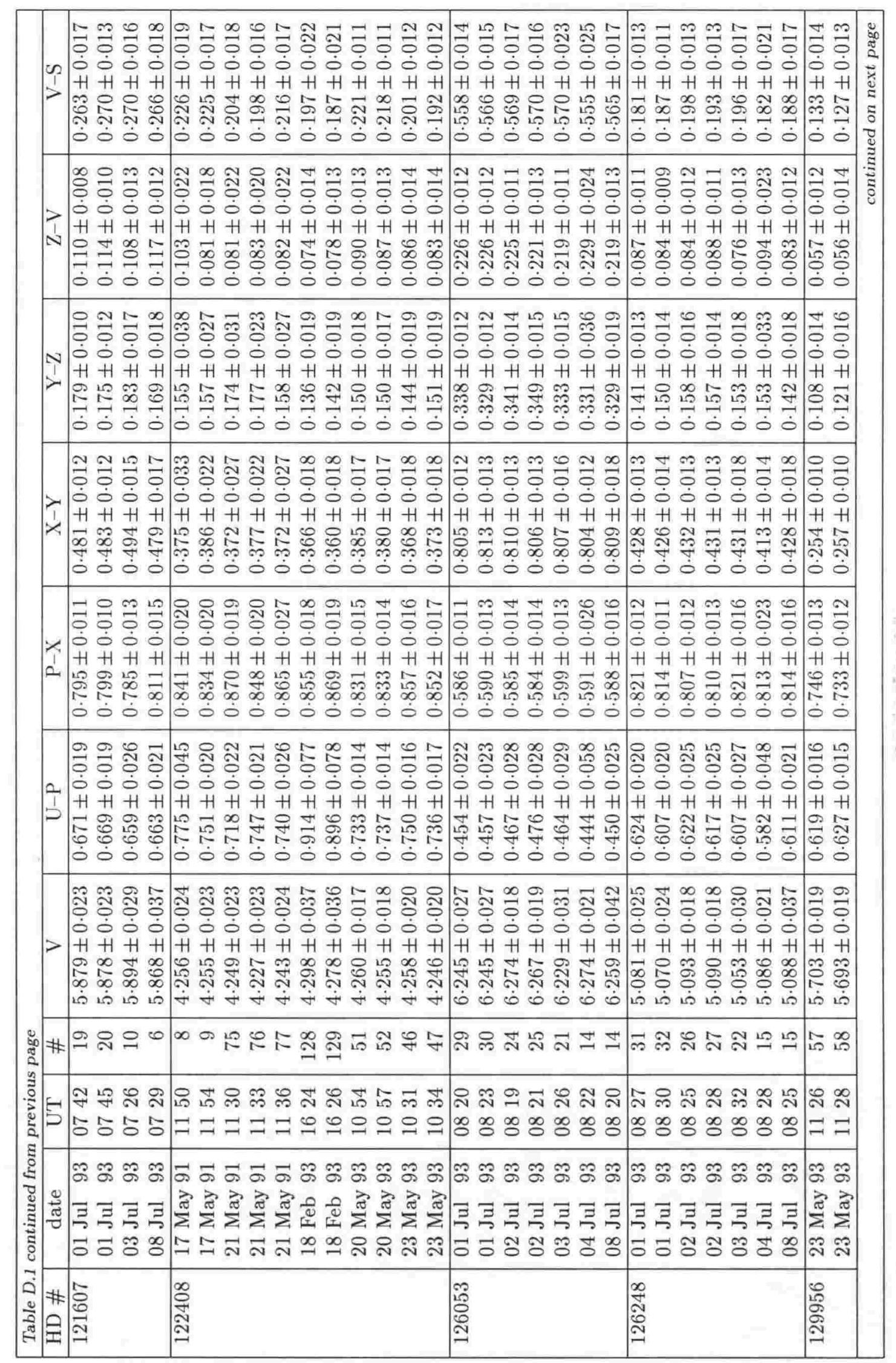




\begin{tabular}{|c|c|c|c|c|c|c|c|}
\hline & & 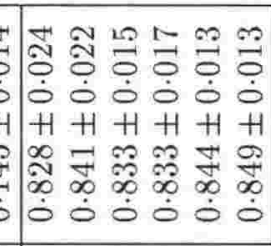 & 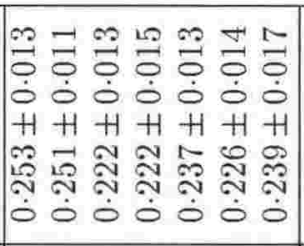 & 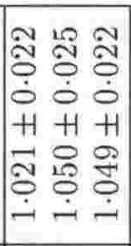 & 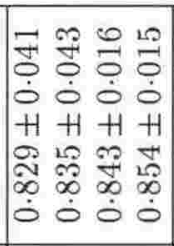 & 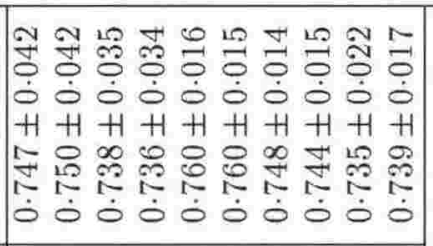 & 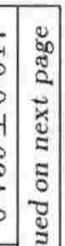 \\
\hline & & 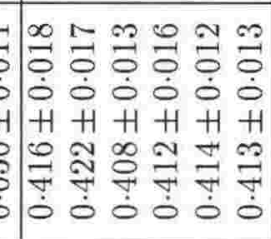 & 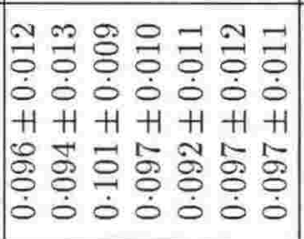 & 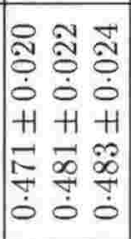 & 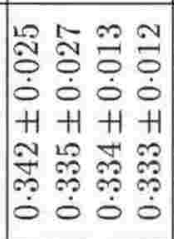 & 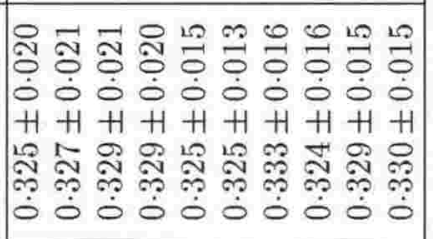 & : \\
\hline & & 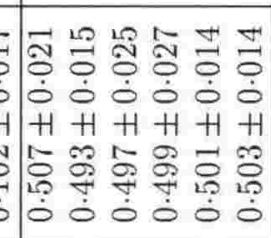 & 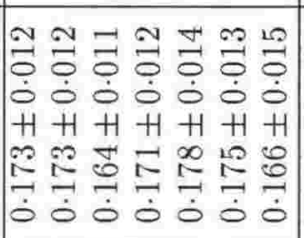 & 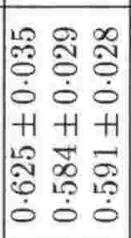 & 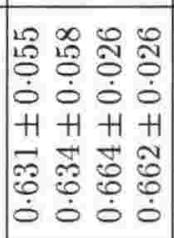 & 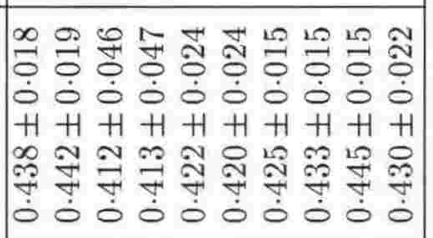 & \\
\hline & & 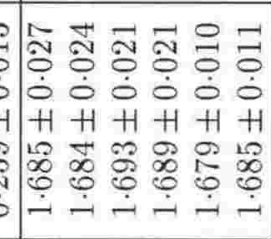 & 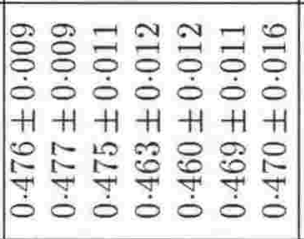 & 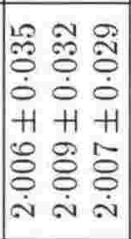 & 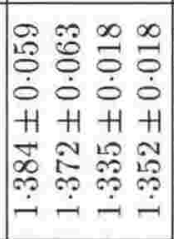 & 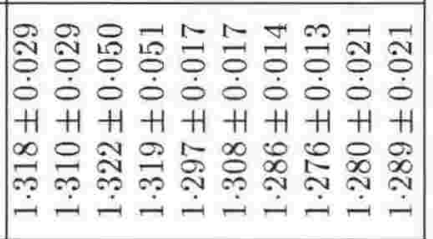 & \\
\hline & & 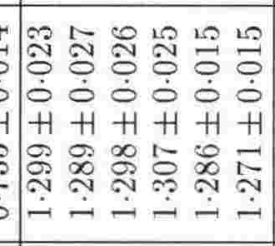 & 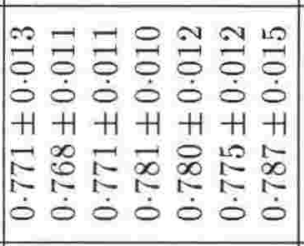 & 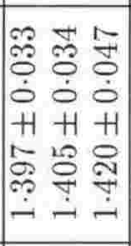 & 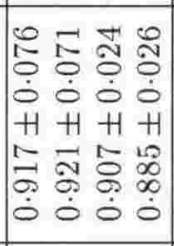 & 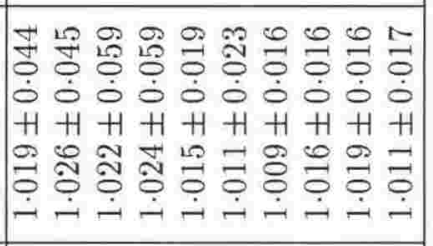 & \\
\hline & & 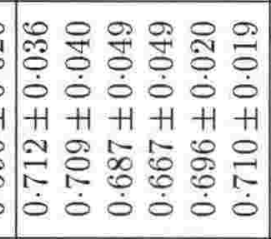 & 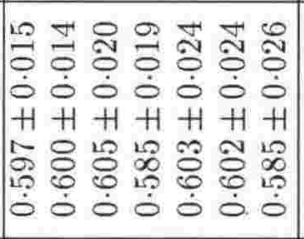 & 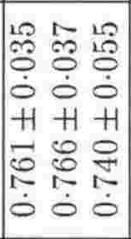 & 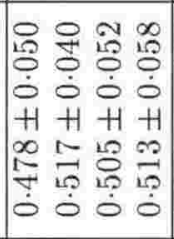 & 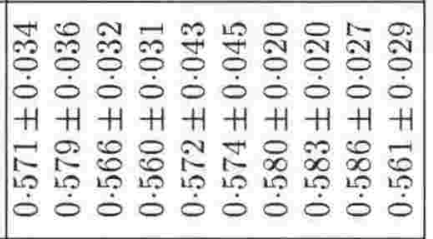 & \\
\hline & & 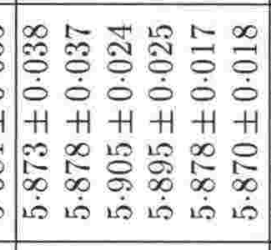 & 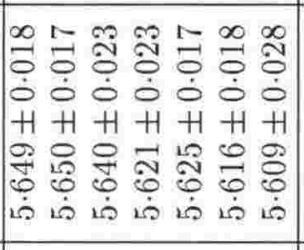 & 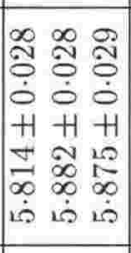 & 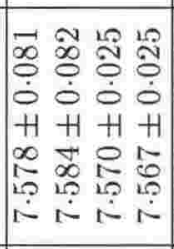 & 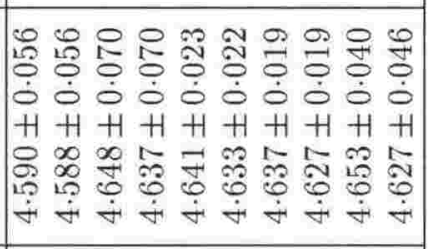 & \\
\hline$=$ & & 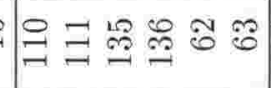 & 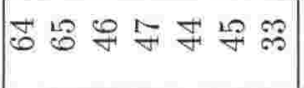 & $\because \approx$ & 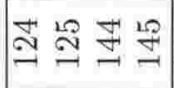 & 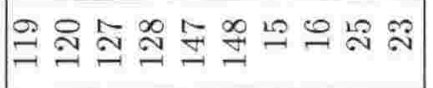 & \\
\hline & & 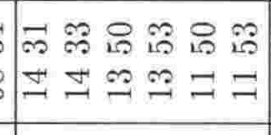 & 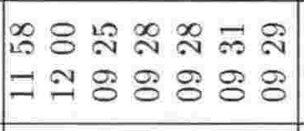 & 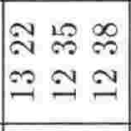 & & 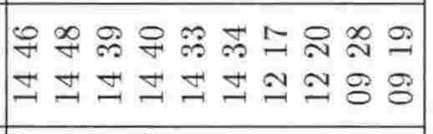 & \\
\hline & 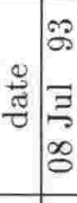 & 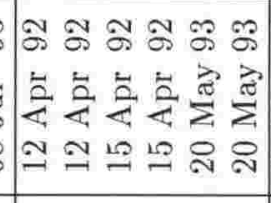 & 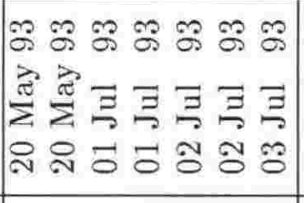 & 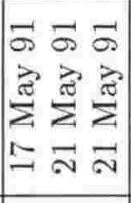 & 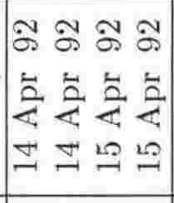 & 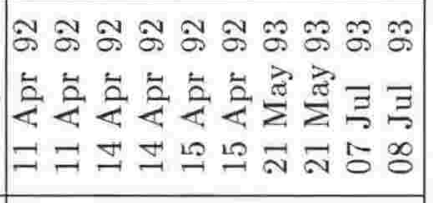 & \\
\hline & & $\frac{1}{\pi}$ & 总 & 赵 & 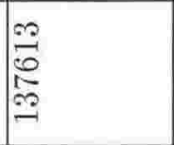 & 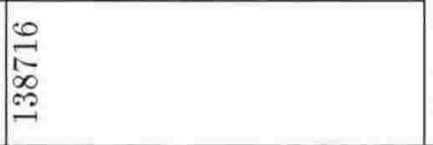 & \\
\hline
\end{tabular}




\begin{tabular}{|c|c|c|c|c|c|c|c|}
\hline & & 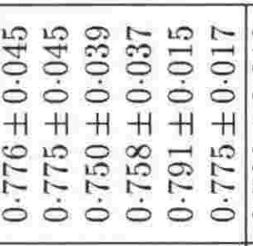 & 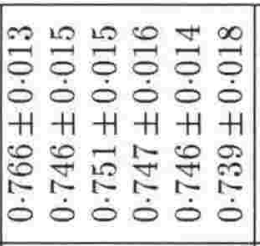 & 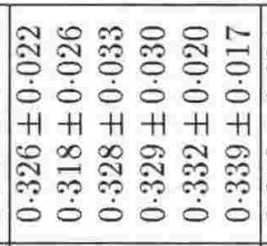 & 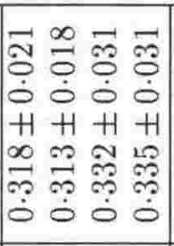 & 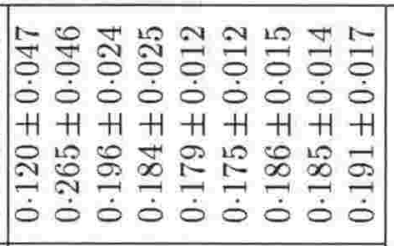 & 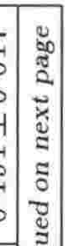 \\
\hline & & 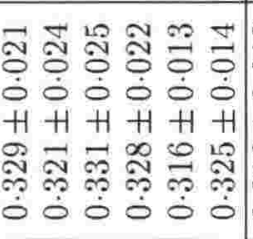 & 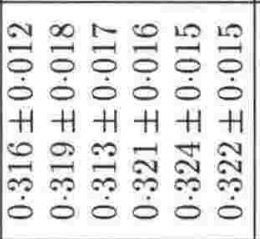 & 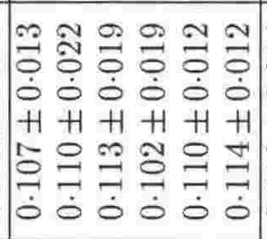 & 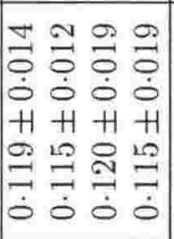 & 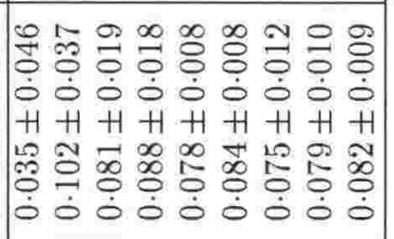 & : \\
\hline & & 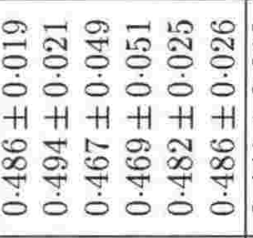 & 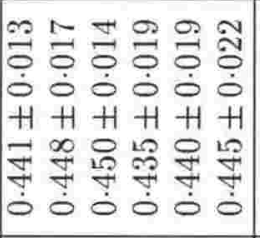 & 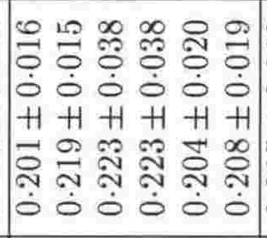 & 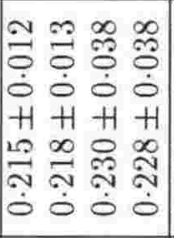 & 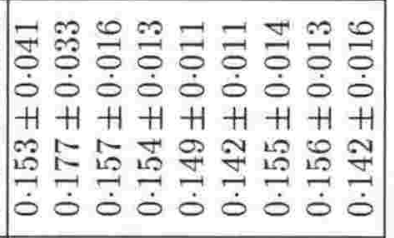 & \\
\hline & & 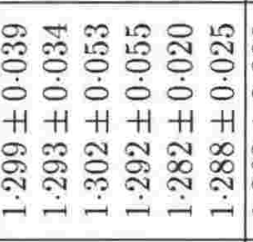 & 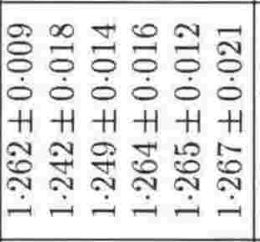 & 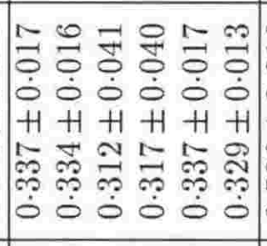 & 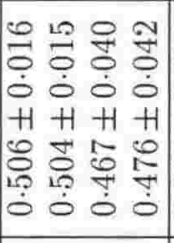 & 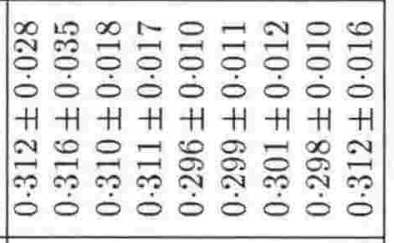 & \\
\hline & & 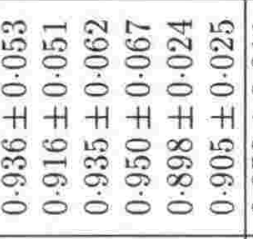 & 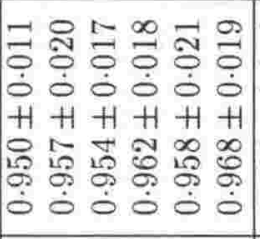 & 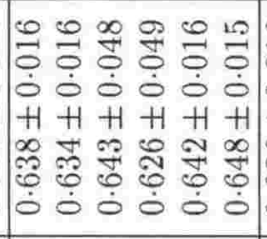 & 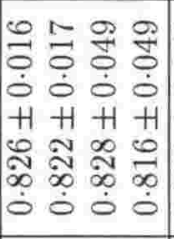 & 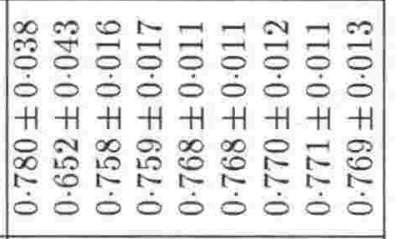 & \\
\hline & & 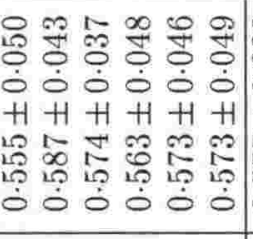 & 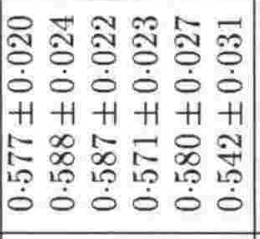 & 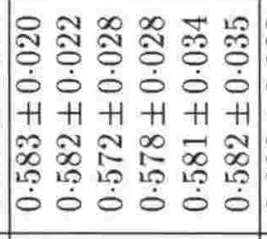 & 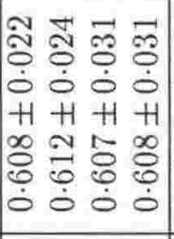 & 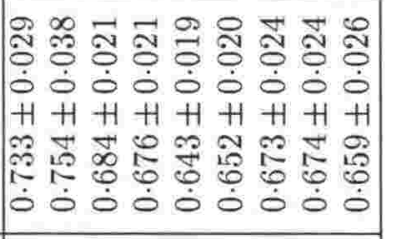 & \\
\hline & & 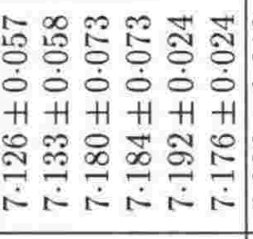 & 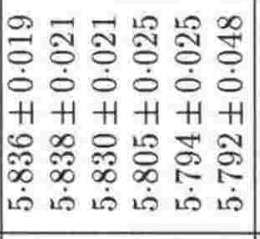 & 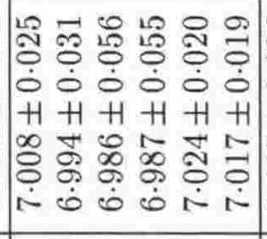 & 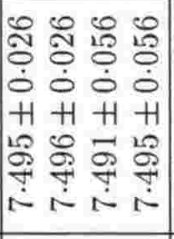 & 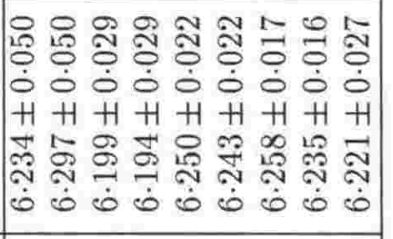 & \\
\hline & & 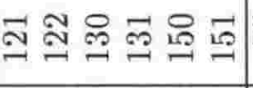 & ハニポーが & $\stackrel{ }{\cong}$ 电 & 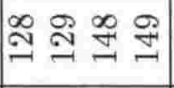 & 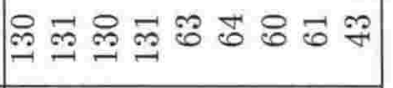 & \\
\hline & & 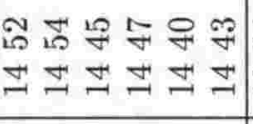 & 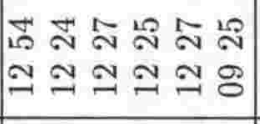 & 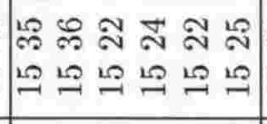 & 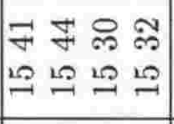 & 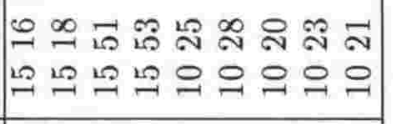 & \\
\hline . & & 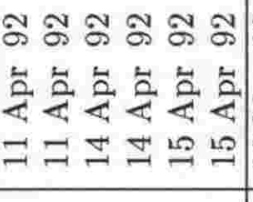 & 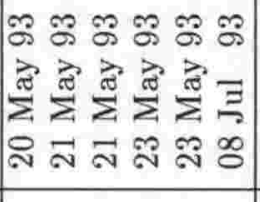 & 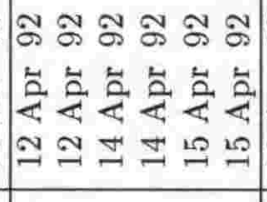 & 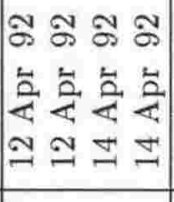 & 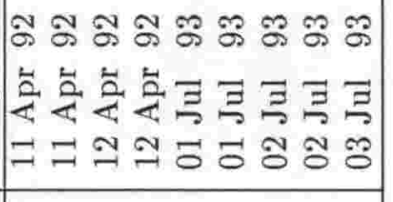 & \\
\hline & & & 旅 & 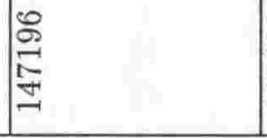 & 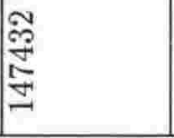 & 路 & \\
\hline
\end{tabular}




\begin{tabular}{|c|c|c|c|c|c|c|c|c|c|c|c|}
\hline & 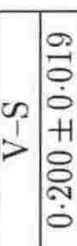 & 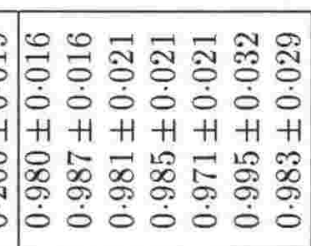 & 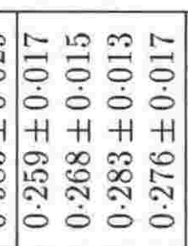 & $\left|\begin{array}{lll}\infty & 0 & \multirow{1}{0}{} \\
0 & 0 & 0 \\
0 & 0 & 0 \\
H & H & +1 \\
- & 5 & 0 \\
0 & 0 & \infty \\
0 & \infty & \leftarrow \\
0 & 0 & 0\end{array}\right|$ & $\left|\begin{array}{cc}0 & \infty \\
0 & 0 \\
0 & 0 \\
0 & 0 \\
+1 & +1 \\
10 & 0 \\
1 & 0 \\
0 & 0 \\
0 & 0\end{array}\right|$ & 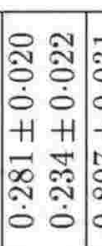 & $\mid$\begin{tabular}{cc|}
-1 & - \\
0 & 0 \\
0 & 0 \\
0 & $\dot{0}$ \\
+1 & +1 \\
& 5 \\
0 & 0 \\
0 & $\infty$ \\
0 & 0
\end{tabular} & 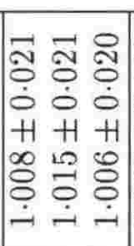 & 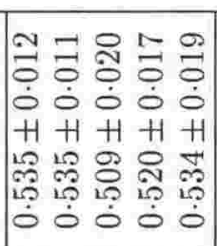 & 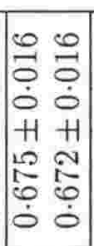 & 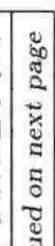 \\
\hline & 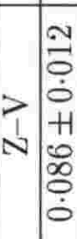 & 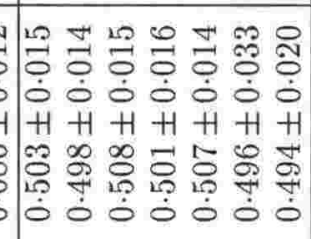 & 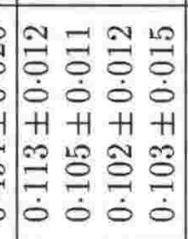 & 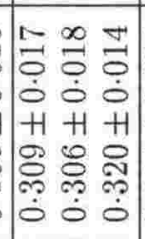 & 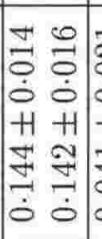 & 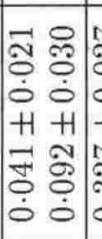 & 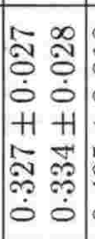 & 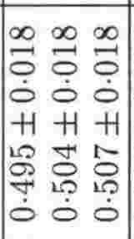 & 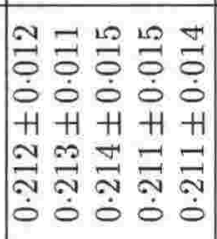 & 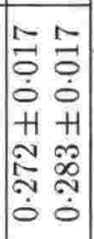 & \\
\hline & 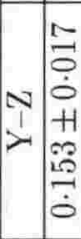 & 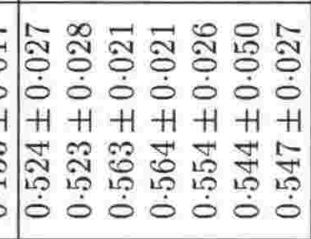 & 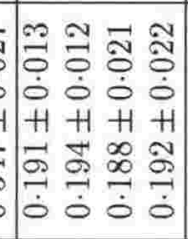 & $\mid \begin{array}{ccc}1 & - & -1 \\
0 & 0 & 0 \\
0 & 0 & 0 \\
0 & 0 & 0 \\
H & H & H \\
0 & 0 & -1 \\
0 & 0 & 0 \\
i & 0 & 0 \\
0 & 0 & 0 \\
0 & 0 & 0\end{array}$ & 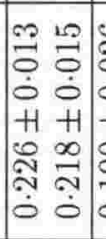 & 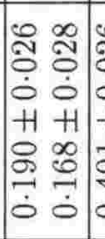 & 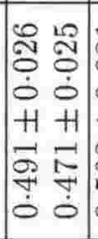 & 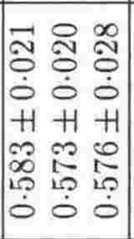 & 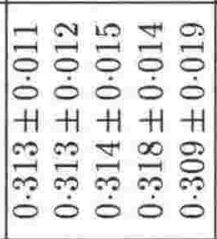 & 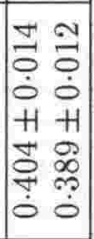 & \\
\hline & 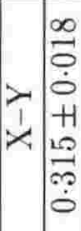 & 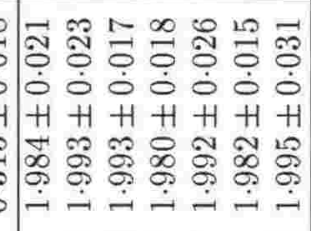 & 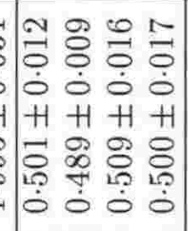 & 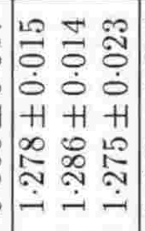 & 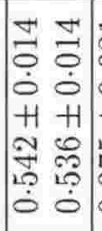 & 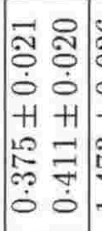 & 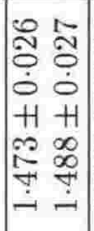 & 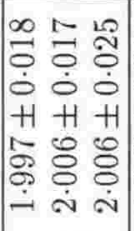 & 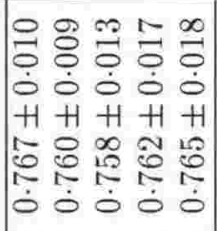 & 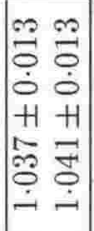 & \\
\hline & 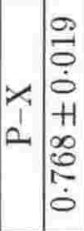 & 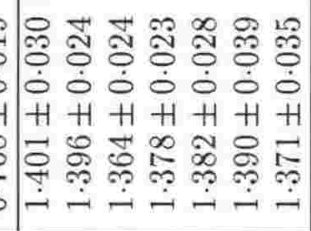 & $\begin{array}{llll}\forall & 02 & \infty & 0 \\
0 & 0 & 0 & 0 \\
0 & 0 & 0 & 0 \\
0 & 0 & 0 \\
H & H & H & H \\
10 & 0 & 0 & 0 \\
10 & 0 & 0 & 0 \\
1 & 1 & 0 & 0 \\
0 & 0 & 0 & 0\end{array}$ & $\left|\begin{array}{lll}\infty & \infty & 1 \\
0 & 0 & 0 \\
0 & 0 & 0 \\
H & H & H \\
0 & 0 & 0 \\
0 & 0 & 0 \\
0 & 0 & 0 \\
0 & 0 & 0\end{array}\right|$ & 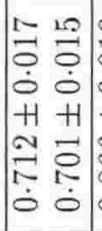 & 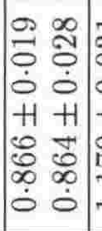 & 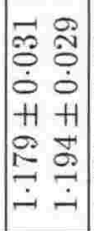 & 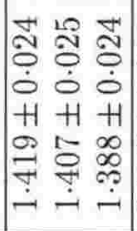 & $\begin{array}{lllll}\exists & 9 & 0 & 0 & 0 \\
0 & 0 & 0 & 0 & 0 \\
0 & 0 & 0 & 0 \\
0 & 0 & 0 & 0 & 0 \\
H & H & H & H & H \\
0 & 0 & 0 & 1 & 1 \\
0 & 0 & 0 & \infty & 0 \\
10 & 0 & 0 & 0 & 0 \\
0 & 0 & 0 & 0 & 0\end{array}$ & 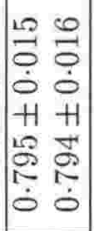 & \\
\hline & 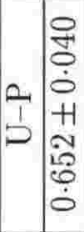 & 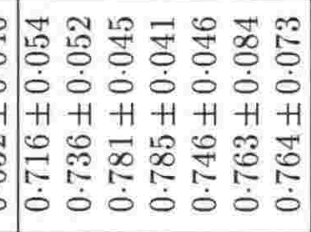 & 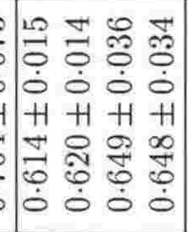 & 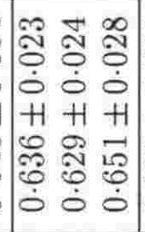 & 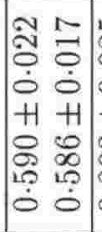 & 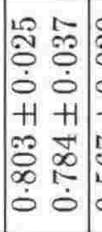 & 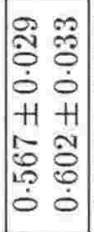 & 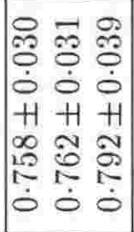 & 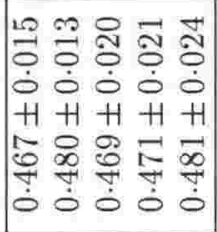 & 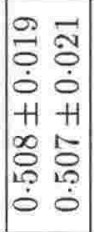 & \\
\hline & 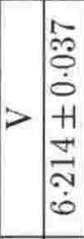 & 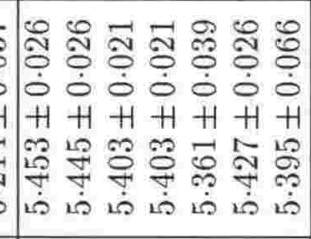 & 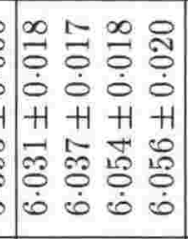 & 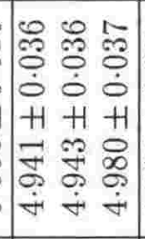 & 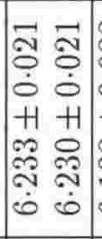 & 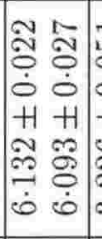 & 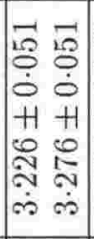 & 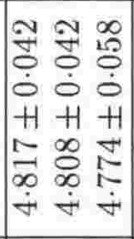 & 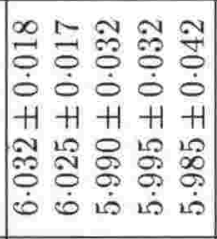 & 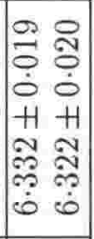 & \\
\hline & $\# \approx$ & : & 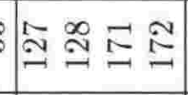 & $\infty \begin{array}{lll}\infty & 0 & 0\end{array}$ & $\stackrel{9}{\oplus}$ & $\stackrel{8}{\circ} \stackrel{-}{=}$ & $\vec{\infty} \infty$ & $=$ & 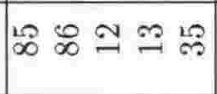 & $\infty$ & \\
\hline & 5 S & 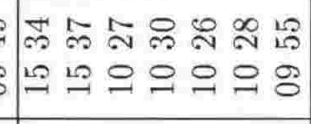 & 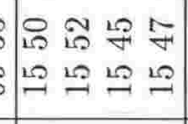 & 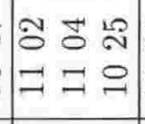 & $\left|\begin{array}{ll}\infty & 8 \\
12 & 0 \\
20 & 20 \\
-1 & -1\end{array}\right|$ & $\left|\begin{array}{ll}\text { in } & \text { in } \\
\hdashline & 90\end{array}\right|$ & 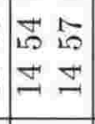 & 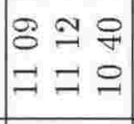 & 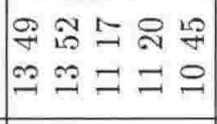 & 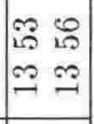 & \\
\hline & 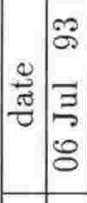 & 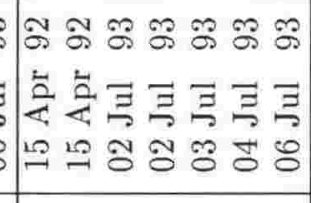 & 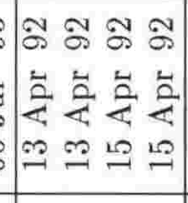 & 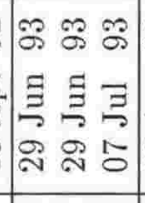 & 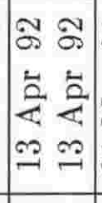 & 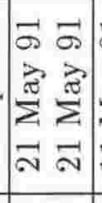 & $\begin{array}{ll}\sigma & \sigma \\
\vec{\pi} & \vec{\pi} \\
\sum^{\Sigma} & \sum \\
= & =\end{array}$ & 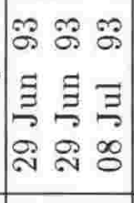 & 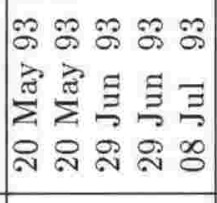 & 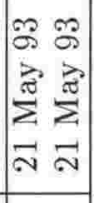 & \\
\hline & 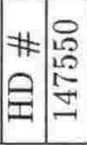 & 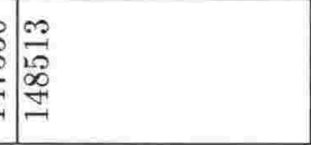 & 蒫 & 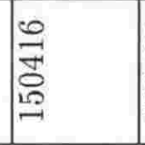 & 突 & 祃 & 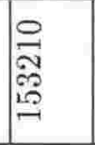 & 萬 & 部 & $\begin{array}{l}0 \\
0 \\
0 \\
5\end{array}$ & \\
\hline
\end{tabular}




\begin{tabular}{|c|c|c|c|c|c|c|c|c|}
\hline & & 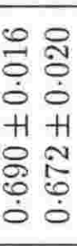 & 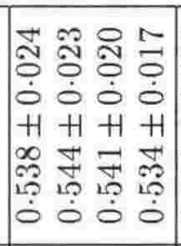 & 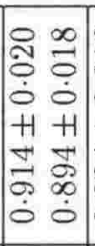 & 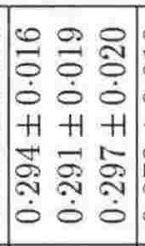 & 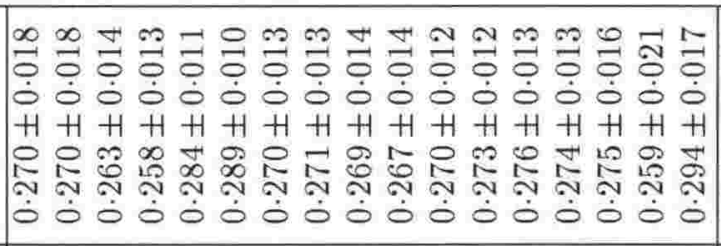 & 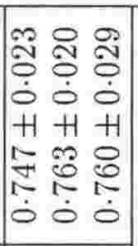 & 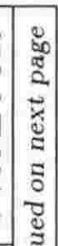 \\
\hline & & 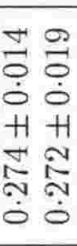 & 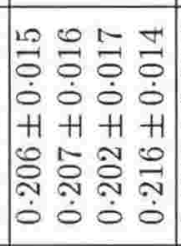 & 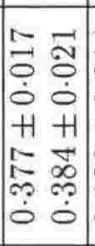 & 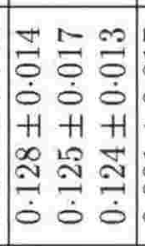 & 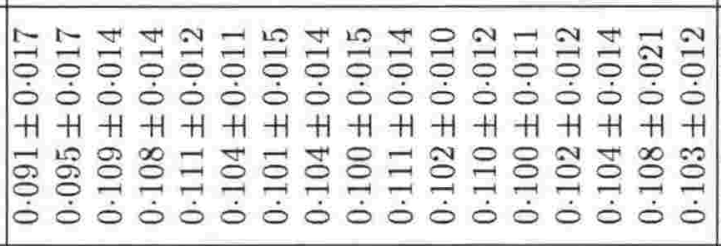 & 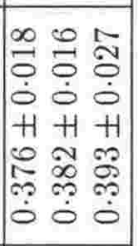 & \\
\hline & & 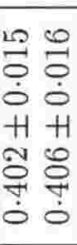 & 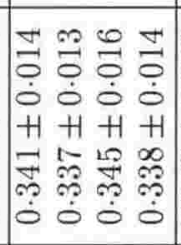 & 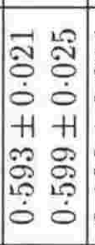 & 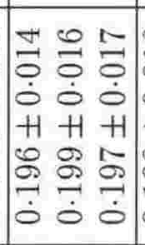 & 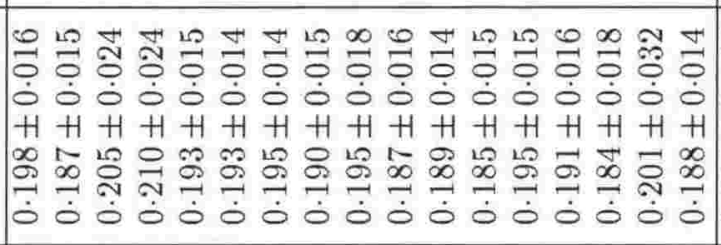 & 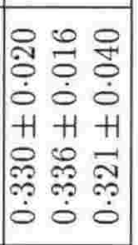 & \\
\hline & & 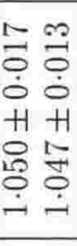 & 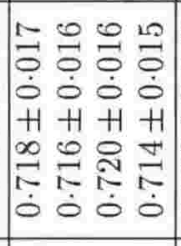 & 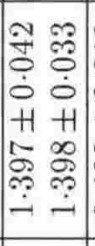 & 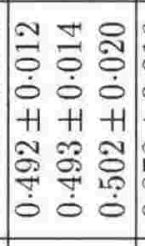 & 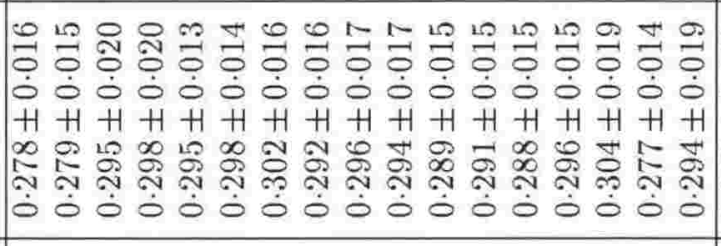 & 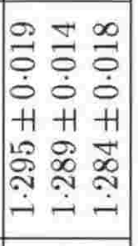 & \\
\hline & & 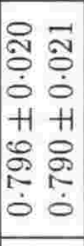 & 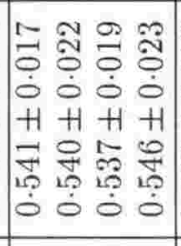 & 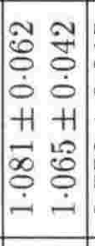 & $\left|\begin{array}{lll}\infty & \infty & - \\
0 & 0 & 0 \\
0 & 0 & 0 \\
0 & H & H \\
0 & 0 & 0 \\
0 & 0 & 0 \\
0 & 0 & 0 \\
0 & 0 & 0\end{array}\right|$ & 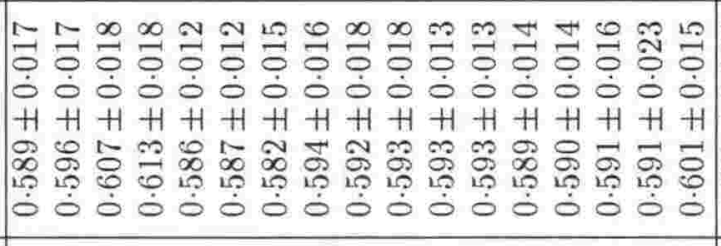 & 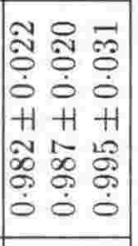 & \\
\hline & & 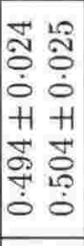 & 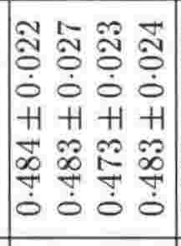 & 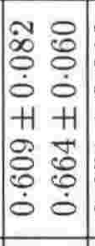 & 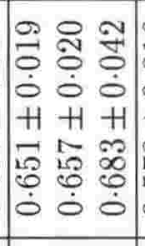 & 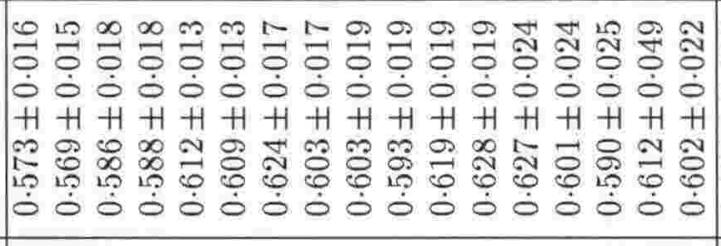 & 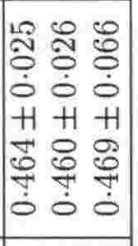 & \\
\hline & & 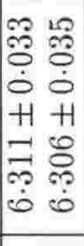 & 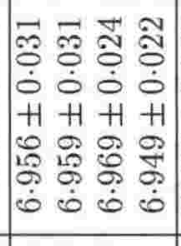 & 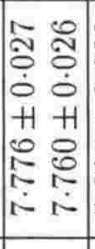 & 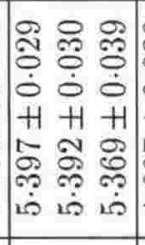 & 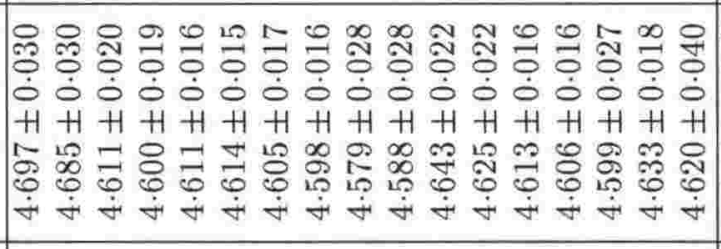 & 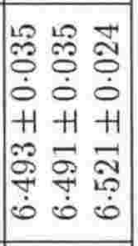 & \\
\hline & & $\exists \stackrel{20}{2}$ & 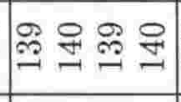 & $\underset{\exists}{\Xi}$ & $\because$ 드 & 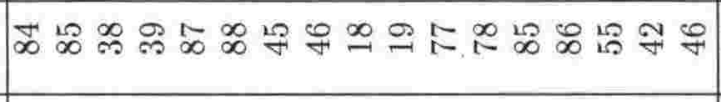 & จิ & \\
\hline & & $\begin{array}{l}\stackrel{2}{N} \\
\exists=\end{array}$ & $\begin{array}{llll}\simeq & \cap & \mathbb{N} & \Re \\
0 & 0 & 0 & 0 \\
- & - & \ddots\end{array}$ & $\left|\begin{array}{cc}\infty & -1 \\
- & \infty \\
0 & 0 \\
- & 0\end{array}\right|$ & $\because \begin{array}{lll}\because & \cdots & \approx \\
\exists & \beth & =\end{array}$ & 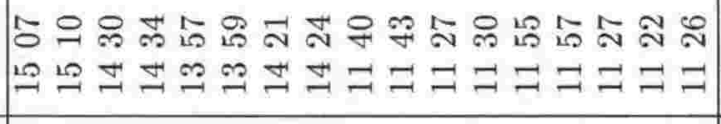 & 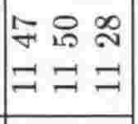 & \\
\hline |. & & 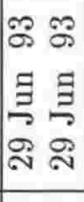 & 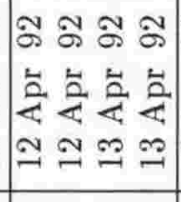 & 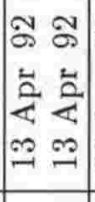 & 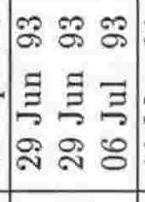 & 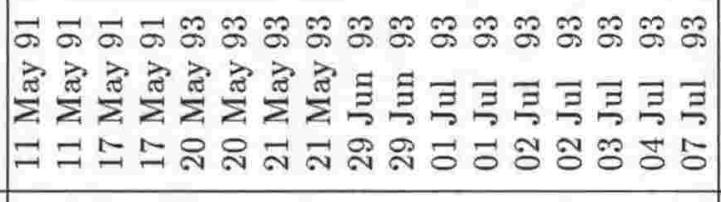 & 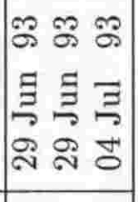 & \\
\hline & & & 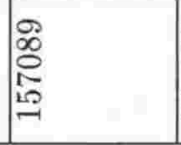 & $\underset{\substack{12 \\
\frac{12}{2}}}{=12}$ & 旅 & 量 & 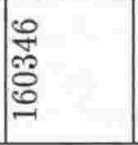 & \\
\hline
\end{tabular}




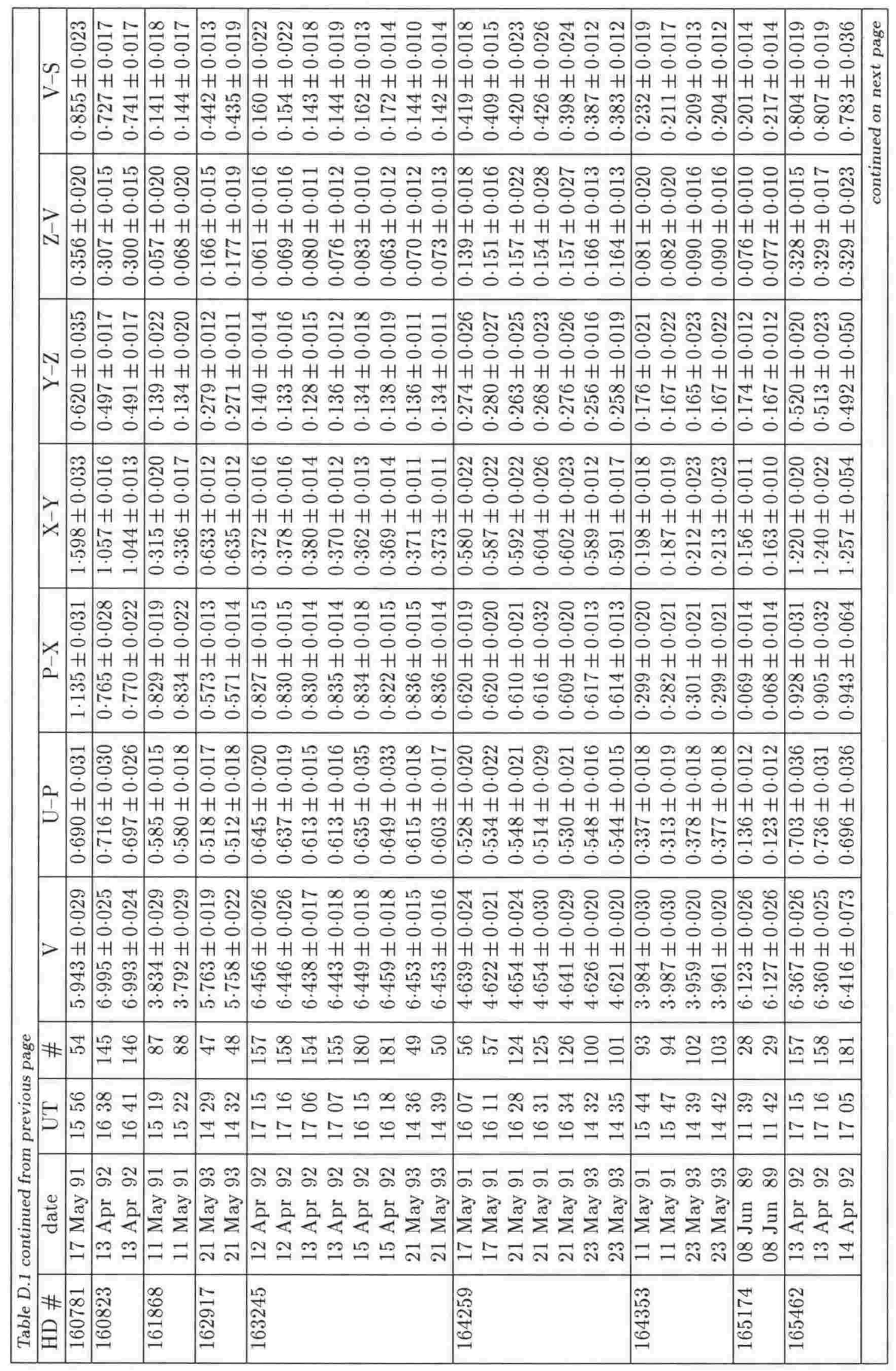




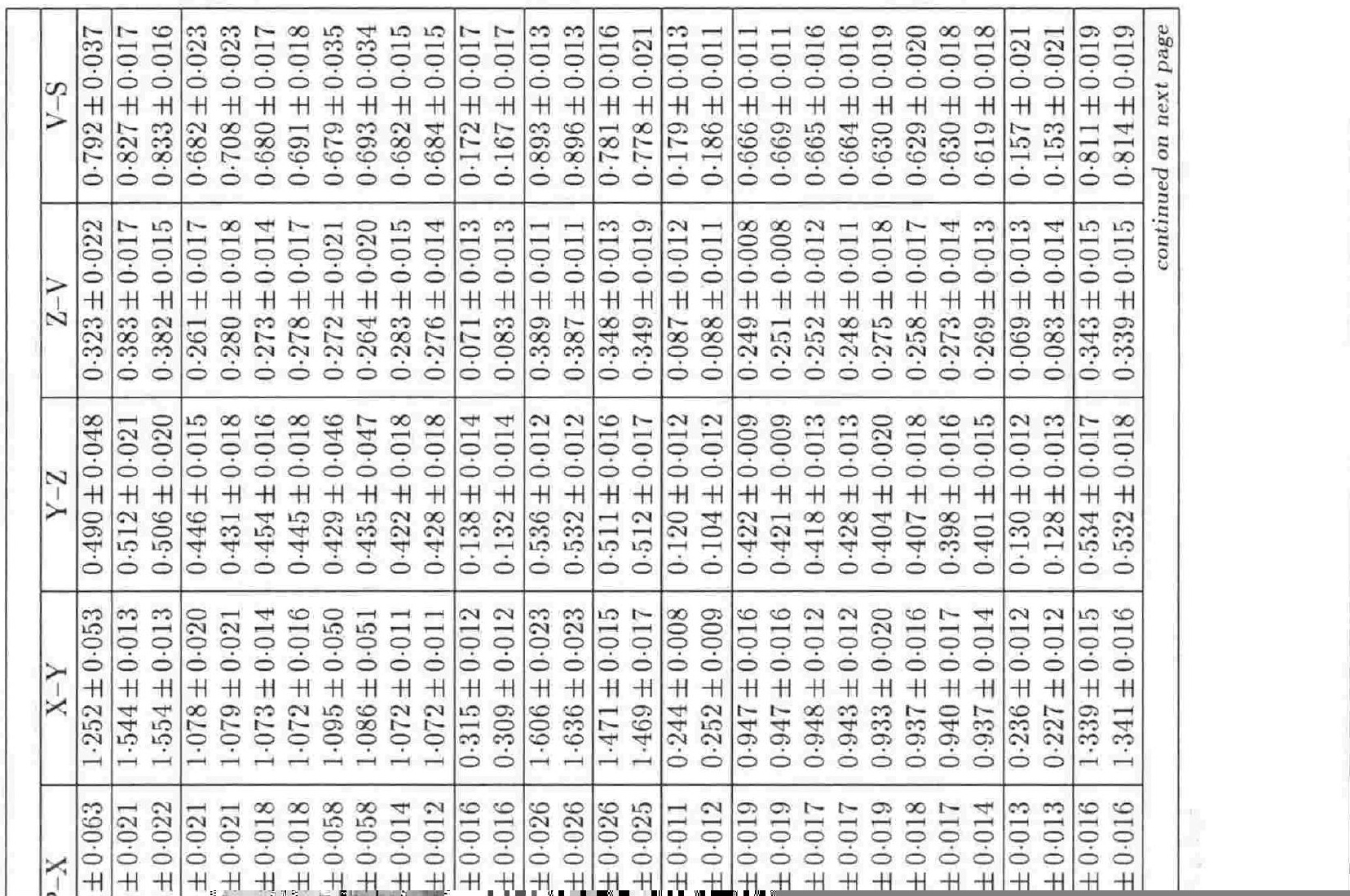




\begin{tabular}{|c|c|c|c|c|c|c|c|c|c|c|c|c|c|}
\hline & 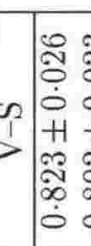 & 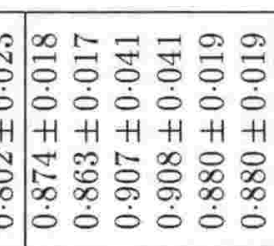 & 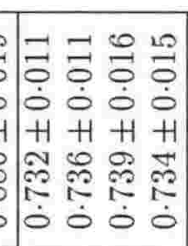 & 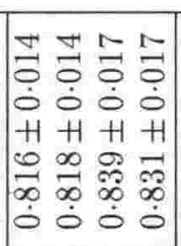 & $\begin{array}{c}0 \\
0 \\
0 \\
0 \\
+1 \\
-1 \\
0 \\
0 \\
0 \\
0\end{array}$ & 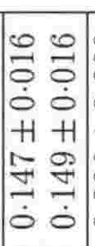 & 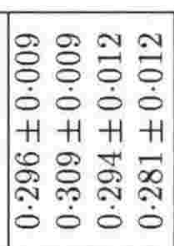 & 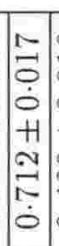 & \begin{tabular}{ll}
0 & 0 \\
0 & 0 \\
0 & 0 \\
0 & 0 \\
0 \\
$H$ & +1 \\
0 & $\mathbb{1}$ \\
0 & 1 \\
\hdashline & + \\
0 & 0
\end{tabular} & 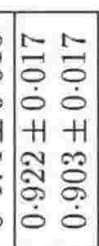 & $\mid \begin{array}{c}0 \\
0 \\
0 \\
0 \\
+1 \\
\approx 0 \\
0 \\
0 \\
0\end{array}$ & 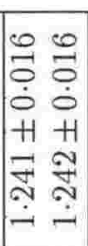 & 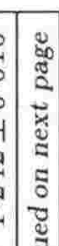 \\
\hline & 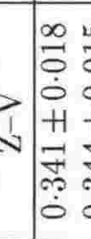 & 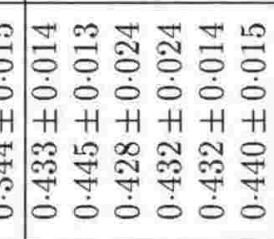 & 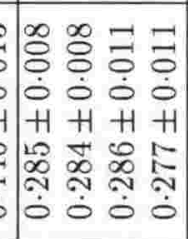 & 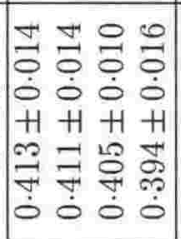 & & 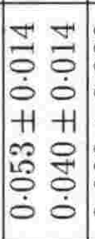 & $\begin{array}{llll}9 & 9 & 0 & 0 \\
0 & 0 & 0 & 0 \\
0 & 0 & 0 & 0 \\
0 & 0 & 0 & 0 \\
H & H & H & H \\
0 & \infty & \infty & 0 \\
\infty & \infty & \infty & 0 \\
0 & 0 & 0 & 0 \\
0 & 0 & 0 & 1 \\
0 & 0 & 0 & 0\end{array}$ & 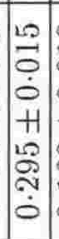 & 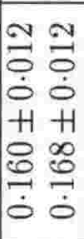 & 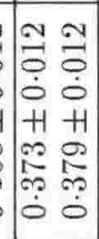 & $\mid$\begin{tabular}{c}
1 \\
\hdashline \\
0 \\
0 \\
+1 \\
10 \\
10 \\
0 \\
0 \\
0
\end{tabular} & $\begin{array}{ll}0 & 0 \\
0 & 0 \\
0 & 0 \\
0 & 0 \\
H & H \\
1 & 1 \\
0 & 0 \\
0 & 1 \\
10 & 0 \\
0 & 0 \\
0 & 0\end{array}$ & \\
\hline & 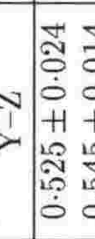 & 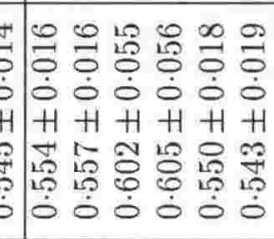 & 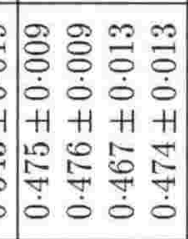 & 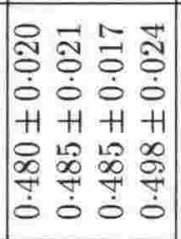 & \begin{tabular}{l} 
\\
\hdashline \\
0 \\
0 \\
+1 \\
0 \\
0 \\
0 \\
0 \\
0
\end{tabular} & 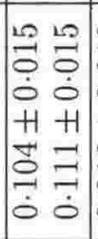 & 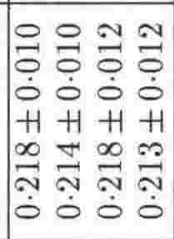 & 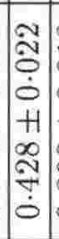 & 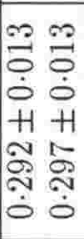 & 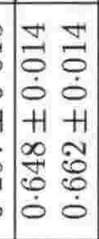 & 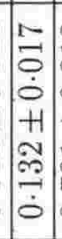 & 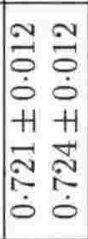 & \\
\hline & 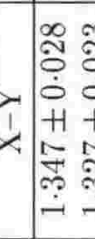 & 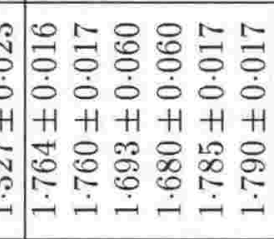 & 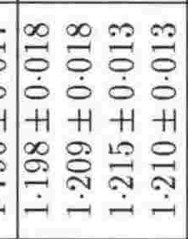 & 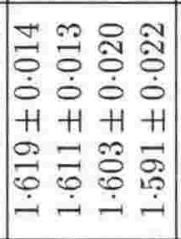 & 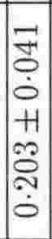 & 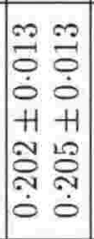 & 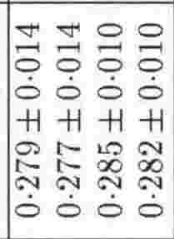 & $\begin{array}{c}\stackrel{0}{0} \\
0 \\
0 \\
+1 \\
0 \\
\\
\\
-1\end{array}$ & $\begin{array}{ll}\exists & \ddots 1 \\
& 0 \\
0 & 0 \\
0 & 0 \\
H & H \\
0 & 0 \\
0 & 0 \\
0 & 0 \\
0 & 0\end{array}$ & 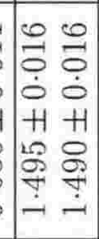 & $\begin{array}{c}0 \\
0 \\
0 \\
\dot{0} \\
+ \\
+ \\
0 \\
0 \\
? \\
0\end{array}$ & 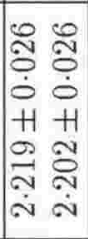 & \\
\hline & 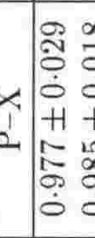 & 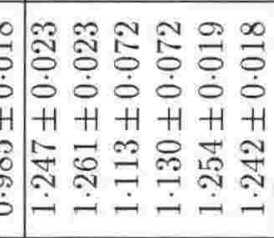 & 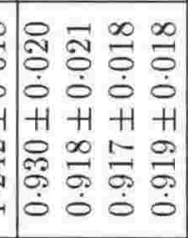 & 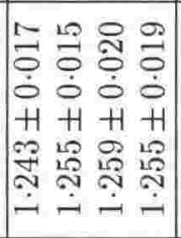 & 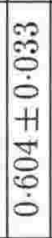 & 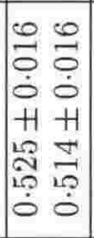 & 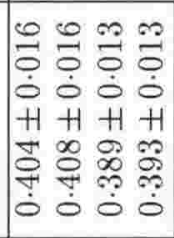 & 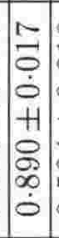 & 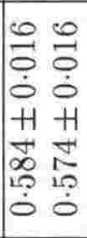 & 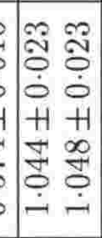 & $\begin{array}{l}- \\
\vdots \\
0 \\
0 \\
+1 \\
0 \\
0 \\
0 \\
0 \\
0\end{array}$ & 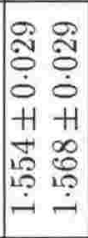 & \\
\hline & 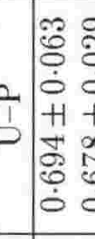 & 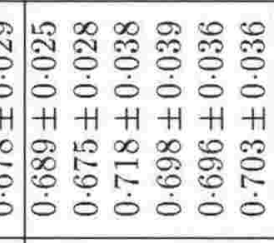 & 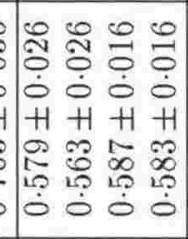 & 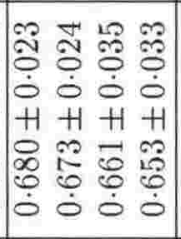 & & & 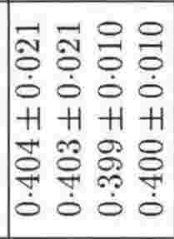 & 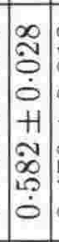 & & 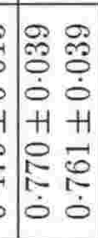 & 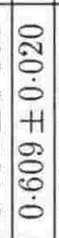 & 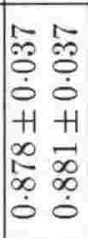 & \\
\hline & 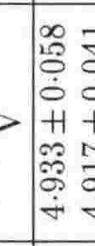 & 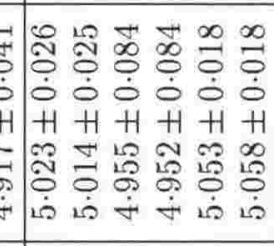 & 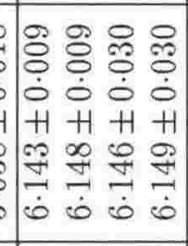 & 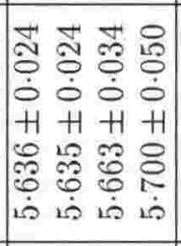 & & 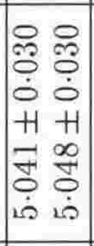 & 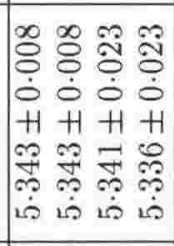 & $\begin{array}{l}0 \\
0 \\
0 \\
\dot{1} \\
+1 \\
\stackrel{2}{0} \\
0 \\
\dot{\omega}\end{array}$ & \begin{tabular}{ll}
0 & 0 \\
0 & 0 \\
0 & 0 \\
0 & 0 \\
+1 & +1 \\
9 & 9 \\
\hdashline & 0 \\
0 & 0 \\
0 & 0
\end{tabular} & 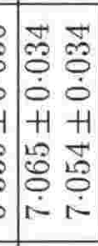 & 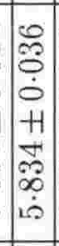 & 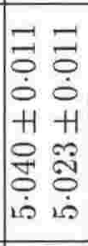 & \\
\hline 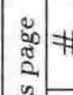 & \# 60 ir & 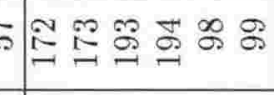 & in is 5 & 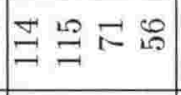 & is & $\Re$ & 궁워국 & $\begin{array}{l}\infty \\
\infty \\
10\end{array}$ & 용 & 공 & 80 & 约 & \\
\hline & 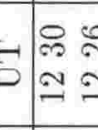 & 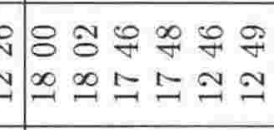 & 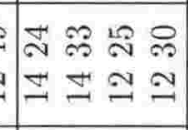 & 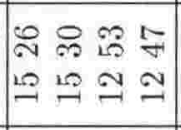 & & 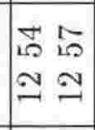 & 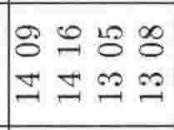 & \begin{tabular}{l}
9 \\
$i 0$ \\
\hdashline \\
\hdashline
\end{tabular} & $\stackrel{0}{\because} \stackrel{9}{9}$ & 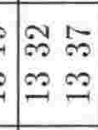 & $\begin{array}{l}\square \\
\infty \\
\infty \\
\infty\end{array}$ & $\begin{array}{ll}0 & -1 \\
10 & 0 \\
\pm & 10\end{array}$ & \\
\hline 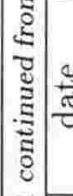 & 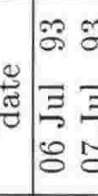 & 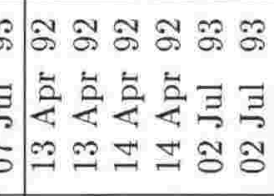 & 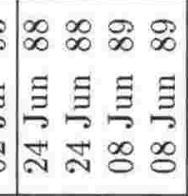 & 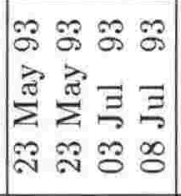 & $\approx 3$ & $\mid$\begin{tabular}{cc}
$\infty$ & 0 \\
$\infty$ & $\infty$ \\
$\Xi$ & $\Xi$ \\
\hdashline & $\vdots$ \\
0 & $\infty$
\end{tabular} & 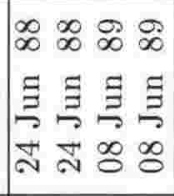 & $\begin{array}{c}\infty \\
\vdots \\
\Xi \\
\infty \\
0\end{array}$ & $\mid \begin{array}{ll}\infty & 2 \\
\infty & \infty \\
\Xi & \vdots \\
\vdots & \vdots \\
\infty & \infty \\
0 & 0\end{array}$ & {$\left[\begin{array}{ll}\infty & 2 \\
\infty & \infty \\
\Xi & \Xi \\
\vdots & \Xi \\
\infty & \infty\end{array}\right.$} & 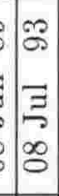 & 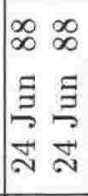 & \\
\hline 究 & \#茥| & $\frac{\sqrt{2}}{2}$ & 年 & 点 & & & 童 & 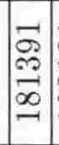 & & 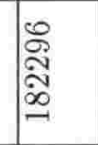 & & 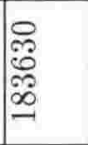 & \\
\hline
\end{tabular}




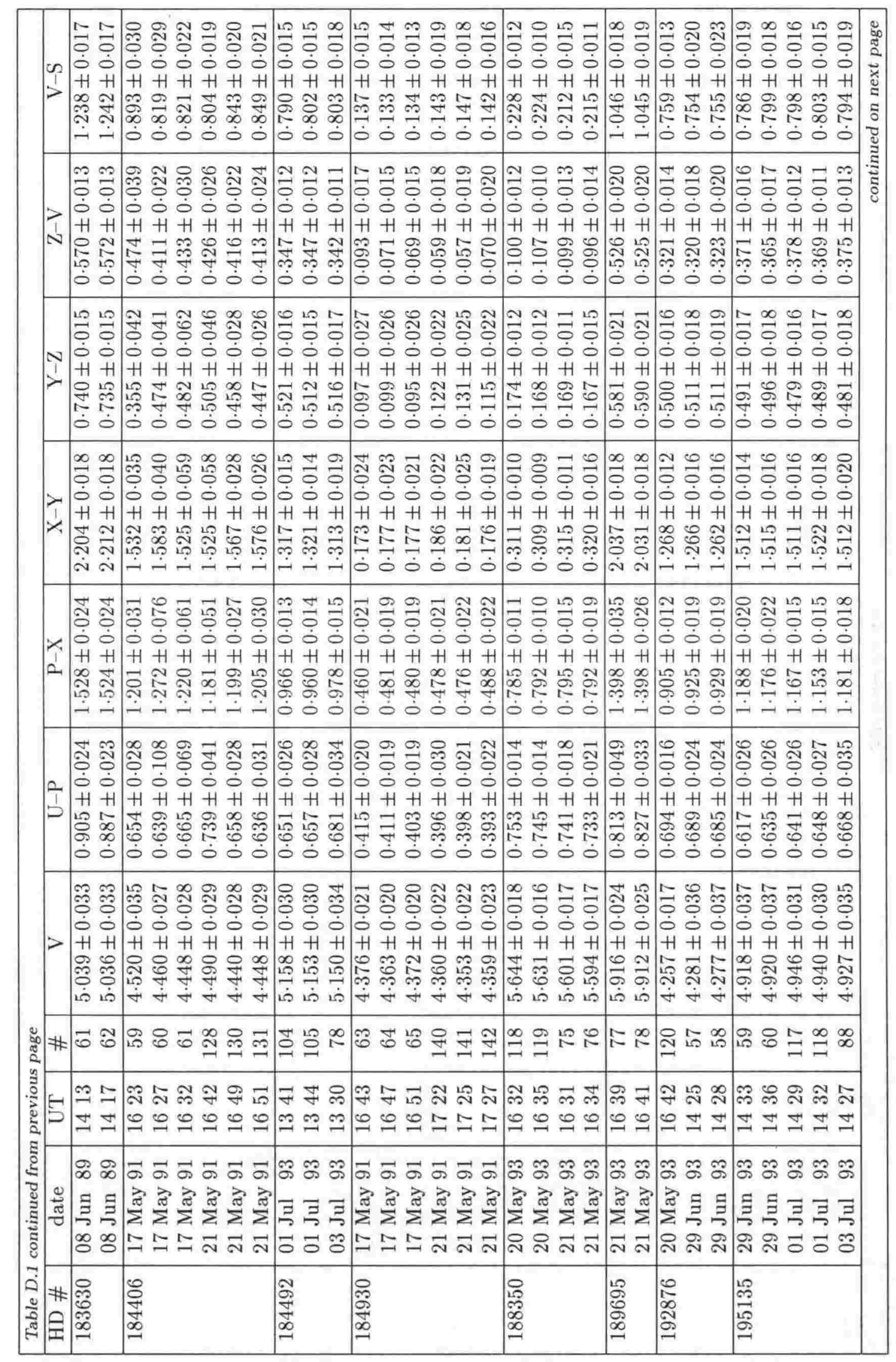




\begin{tabular}{|c|c|c|c|c|c|c|c|c|c|c|}
\hline & 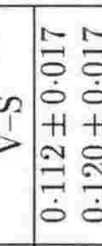 & & 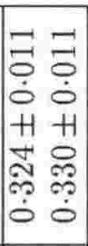 & 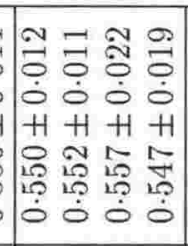 & $\begin{array}{ll}0 & 0 \\
0 & 0 \\
0 & 0 \\
0 & 0 \\
+1 & +1 \\
0 & 0 \\
0 & 0 \\
0 & 0 \\
-1 & -\end{array}$ & $\begin{array}{lll}\vec{H} & 0 & 0 \\
0 & 0 & 0 \\
0 & 0 & 0 \\
\dot{0} & \dot{0} & 0 \\
H & H & H \\
0 & 0 & 0 \\
0 & 0 & 0 \\
0 & 0 & 0 \\
0 & 0 & 0\end{array}$ & 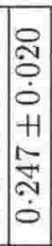 & 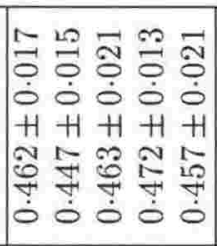 & 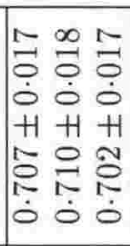 & 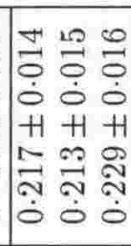 \\
\hline & 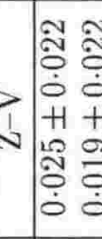 & & 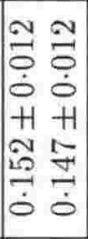 & 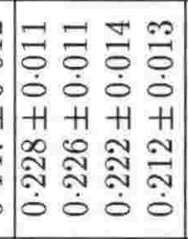 & 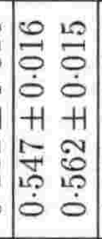 & 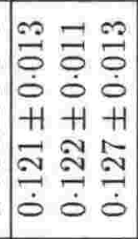 & 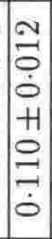 & 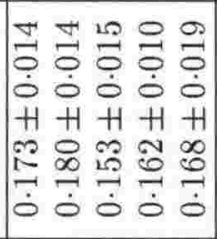 & 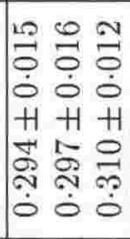 & 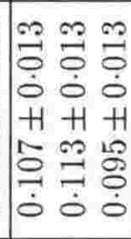 \\
\hline & 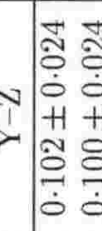 & & 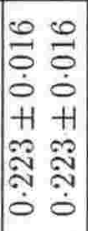 & 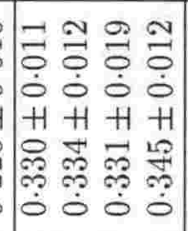 & 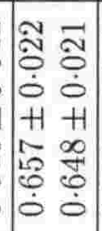 & 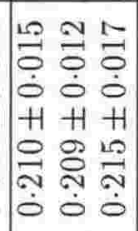 & $\begin{array}{c}0 \\
0 \\
0 \\
0 \\
0 \\
H \\
0 \\
0 \\
\\
\dot{0}\end{array}$ & 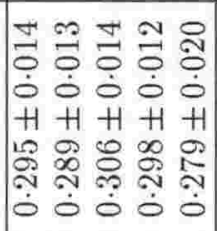 & & 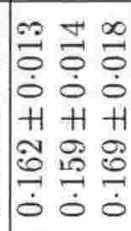 \\
\hline & 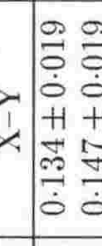 & & 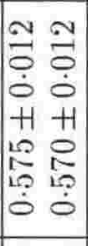 & 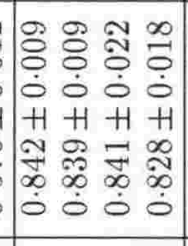 & 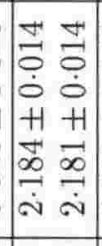 & 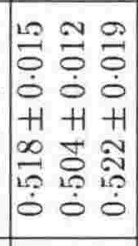 & 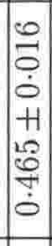 & 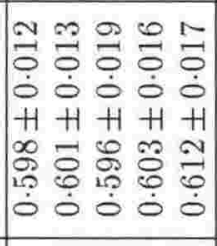 & 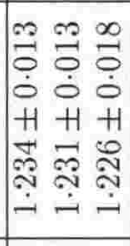 & 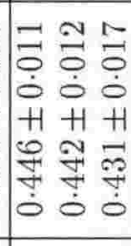 \\
\hline & 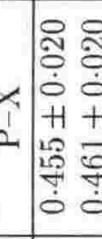 & & 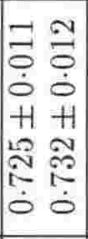 & 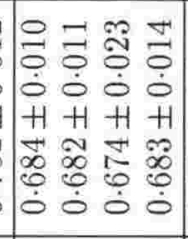 & 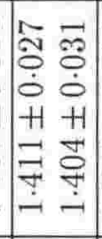 & 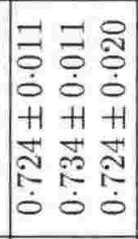 & $\begin{array}{c}12 \\
0 \\
0 \\
0 \\
1 \\
\infty \\
\infty \\
-0 \\
0 \\
0 \\
0\end{array}$ & 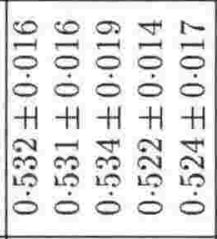 & \begin{tabular}{lll}
0 & \multicolumn{1}{c}{} & 0 \\
0 & 0 & 0 \\
0 & 0 \\
0 & 0 & 0 \\
$H$ & $H$ & $H$ \\
1 & 1 & 0 \\
10 & 10 & 0 \\
0 & 0 & 0 \\
0 & 0 & 0
\end{tabular} & $\mid \begin{array}{lll}2 & 2 & \\
0 & 0 & 0 \\
0 & 0 \\
0 & \dot{0} & 0 \\
H & H & H \\
0 & 0 & 0 \\
0 & 0 & 0 \\
0 & 0 & 0 \\
0 & 0 & 0\end{array}$ \\
\hline & 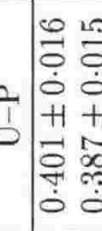 & 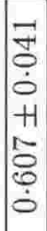 & 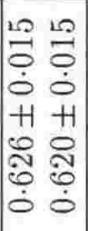 & 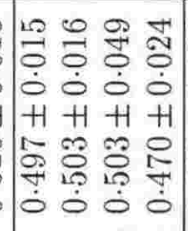 & $\mid \begin{array}{cc}2 & \infty \\
0 & 0 \\
0 & 0 \\
0 & 0 \\
1 & +1 \\
1 & 0 \\
0 & 0 \\
0 & 0 \\
0 & 0 \\
0 & 0\end{array}$ & 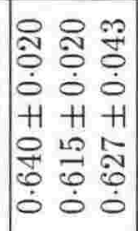 & 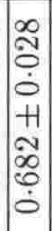 & 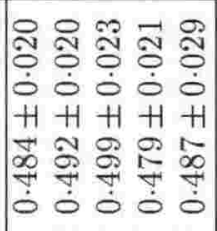 & 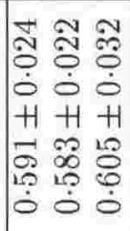 & 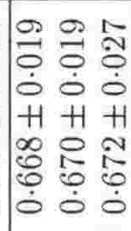 \\
\hline & 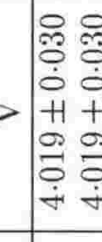 & 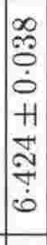 & 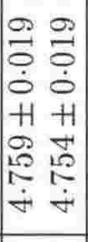 & 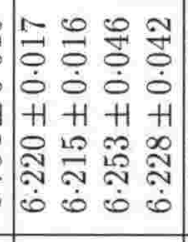 & 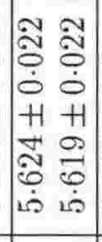 & 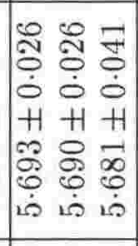 & 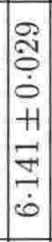 & 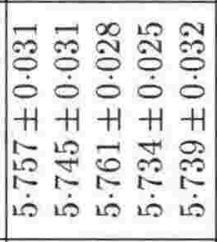 & 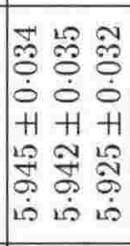 & 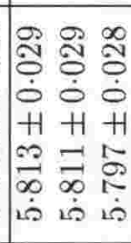 \\
\hline 要 & $\# 50$ & 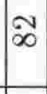 & 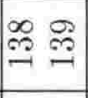 & 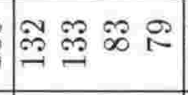 & 票骂 & $\approx$ & 8 & 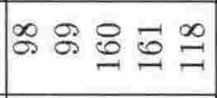 & 으을 & \%ి용 \\
\hline 造 & 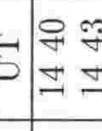 & $\stackrel{N}{a}$ & 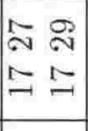 & 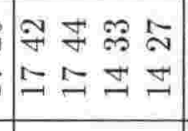 & 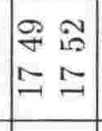 & 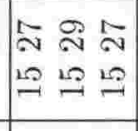 & $\begin{array}{l}\infty \\
\infty \\
0 \\
-1\end{array}$ & 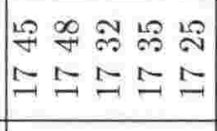 & 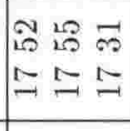 & 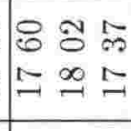 \\
\hline 这 & 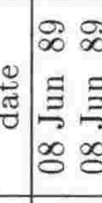 & $\because$ & 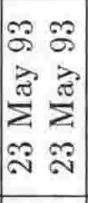 & 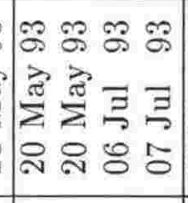 & 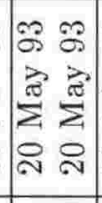 & $\mid \begin{array}{ccc}\because & 9 & 8 \\
\Xi & \Xi & \Xi \\
\vdots & 5 & 8\end{array}$ & $\mathscr{\%}$ & 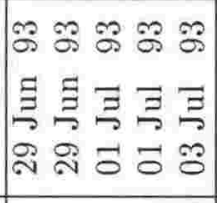 & 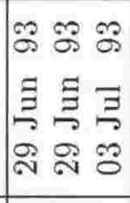 & 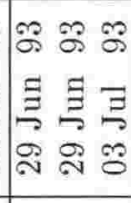 \\
\hline 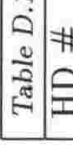 & 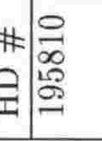 & & & 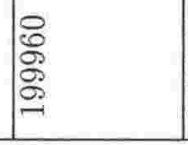 & 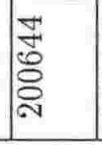 & 芯 & & & 离 & 苚 \\
\hline
\end{tabular}




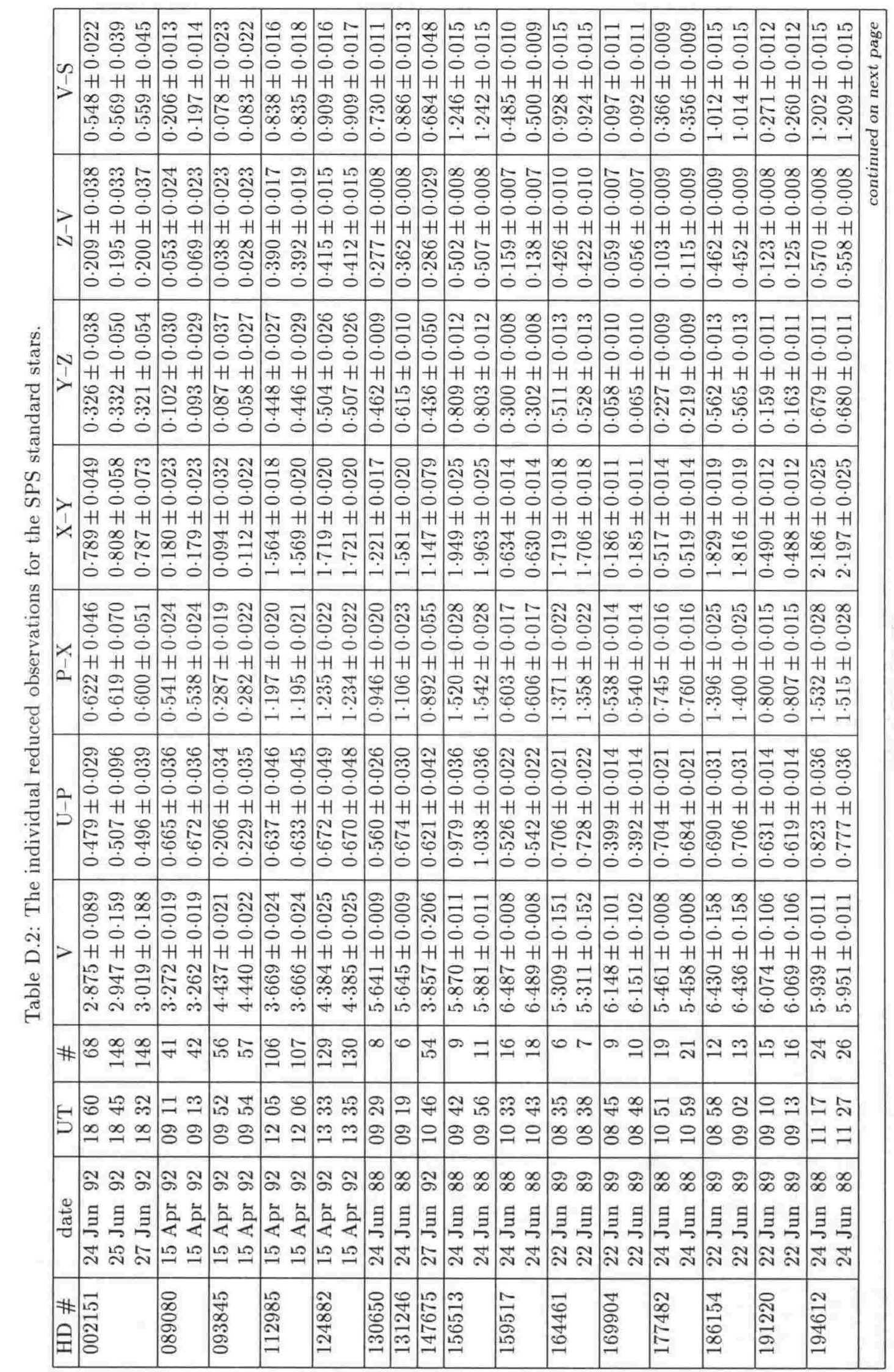




\begin{tabular}{|c|c|c|c|c|c|c|c|c|c|}
\hline & 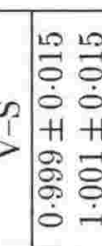 & 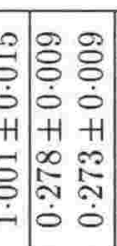 & \begin{tabular}{ll}
8 & 0 \\
0 & 0 \\
0 & 0 \\
$H$ & +1 \\
0 & 0 \\
0 & 0 \\
\hdashline & 2 \\
0 & 0 \\
0 & 0
\end{tabular} & 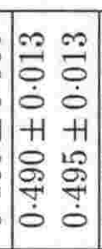 & 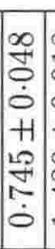 & 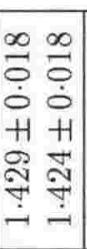 & 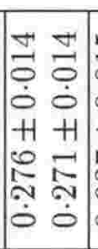 & 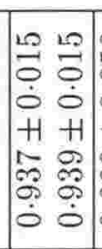 & 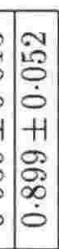 \\
\hline & 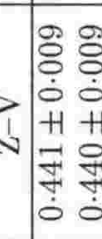 & 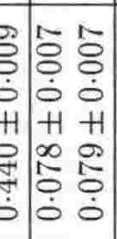 & $\mid \begin{array}{ll}8 & 8 \\
0 & 0 \\
0 & 0 \\
1 & +1 \\
0 & 0 \\
0 & 0 \\
0 & 0 \\
0 & 0\end{array}$ & $\begin{array}{l}8 \\
0 \\
0 \\
+1 \\
1 \\
0 \\
0 \\
0 \\
0\end{array}$ & & 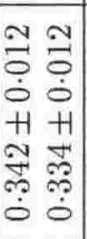 & 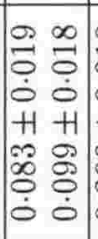 & 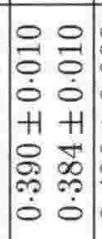 & 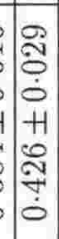 \\
\hline & 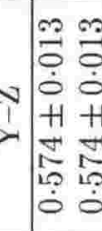 & 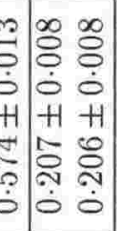 & 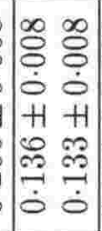 & $\mid \begin{array}{ll}0 & - \\
0 & \dot{0} \\
+ & +1 \\
0 & -1 \\
0 & - \\
0 & 0 \\
0 & 0\end{array}$ & \begin{tabular}{l}
0 \\
0 \\
+1 \\
$\infty$ \\
$\propto$ \\
\hdashline \\
$\dot{0}$
\end{tabular} & 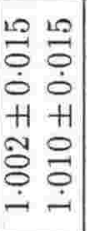 & 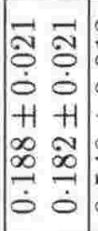 & 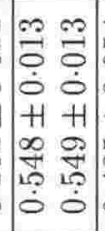 & 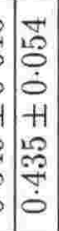 \\
\hline & 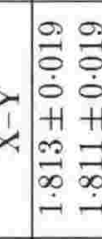 & 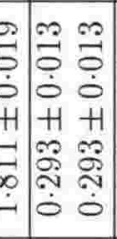 & $\mid$\begin{tabular}{ll}
0 & 0 \\
0 & 0 \\
0 & 0 \\
$H$ & +1 \\
2 & 0 \\
\hdashline & 0 \\
\hdashline & 0 \\
0 & 0
\end{tabular} & 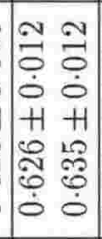 & 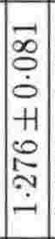 & 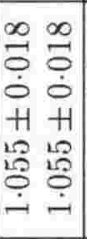 & 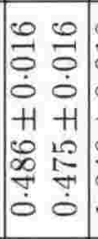 & 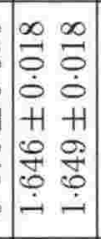 & $\begin{array}{l}+ \\
- \\
0 \\
0 \\
0\end{array}$ \\
\hline & 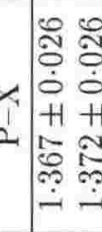 & 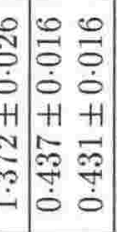 & $\mid \begin{array}{ll}0 & 0 \\
0 & 0 \\
0 & 0 \\
+1 & +1 \\
0 & 0 \\
0 & 0 \\
0 & 0 \\
0 & 0 \\
0 & 0\end{array}$ & $\mid$\begin{tabular}{ll}
0 & - \\
0 & 0 \\
0 & 0 \\
$H$ & +1 \\
\hdashline & 8 \\
\hdashline & 0 \\
0 & 0 \\
0 & 0
\end{tabular} & 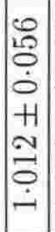 & 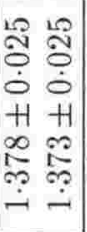 & 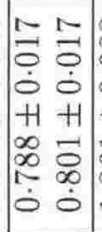 & 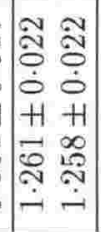 & ? \\
\hline & 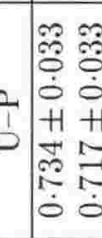 & 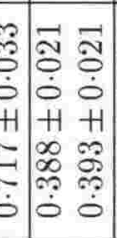 & 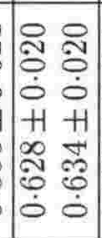 & 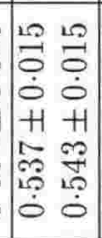 & 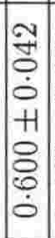 & 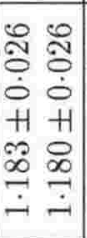 & 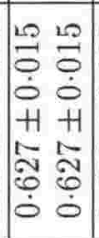 & 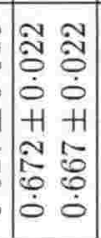 & 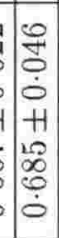 \\
\hline & 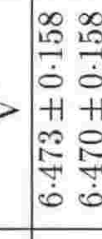 & 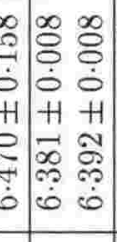 & 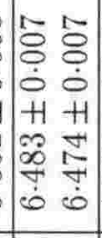 & 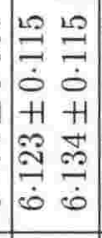 & 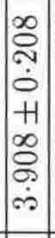 & $\begin{array}{l}\dot{0} \\
H \\
\infty \\
\infty \\
\infty \\
\dot{0} .\end{array}$ & 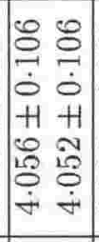 & 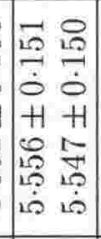 & 15 \\
\hline 究 & & & & J & $\stackrel{0}{=}$ & $\stackrel{\infty}{\sim}$ & $\vec{\curvearrowright} \vec{\infty}$ & & \\
\hline & & & & $\mid \begin{array}{ll}1 & 10 \\
0 & 4 \\
8 & 8 \\
0 & 8\end{array}$ & 20 & & $\begin{array}{l}\vec{N} \\
\varrho\end{array}$ & & \\
\hline 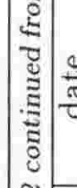 & จ & 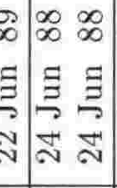 & 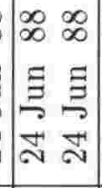 & 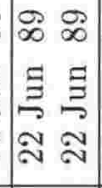 & $\tilde{\delta}$ & $\begin{array}{cc}\infty & \infty \\
\infty & \\
\Xi & \Xi\end{array}$ & & & \\
\hline 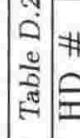 & 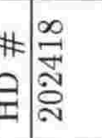 & in & 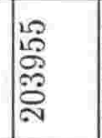 & क्ष & & & & & \\
\hline
\end{tabular}




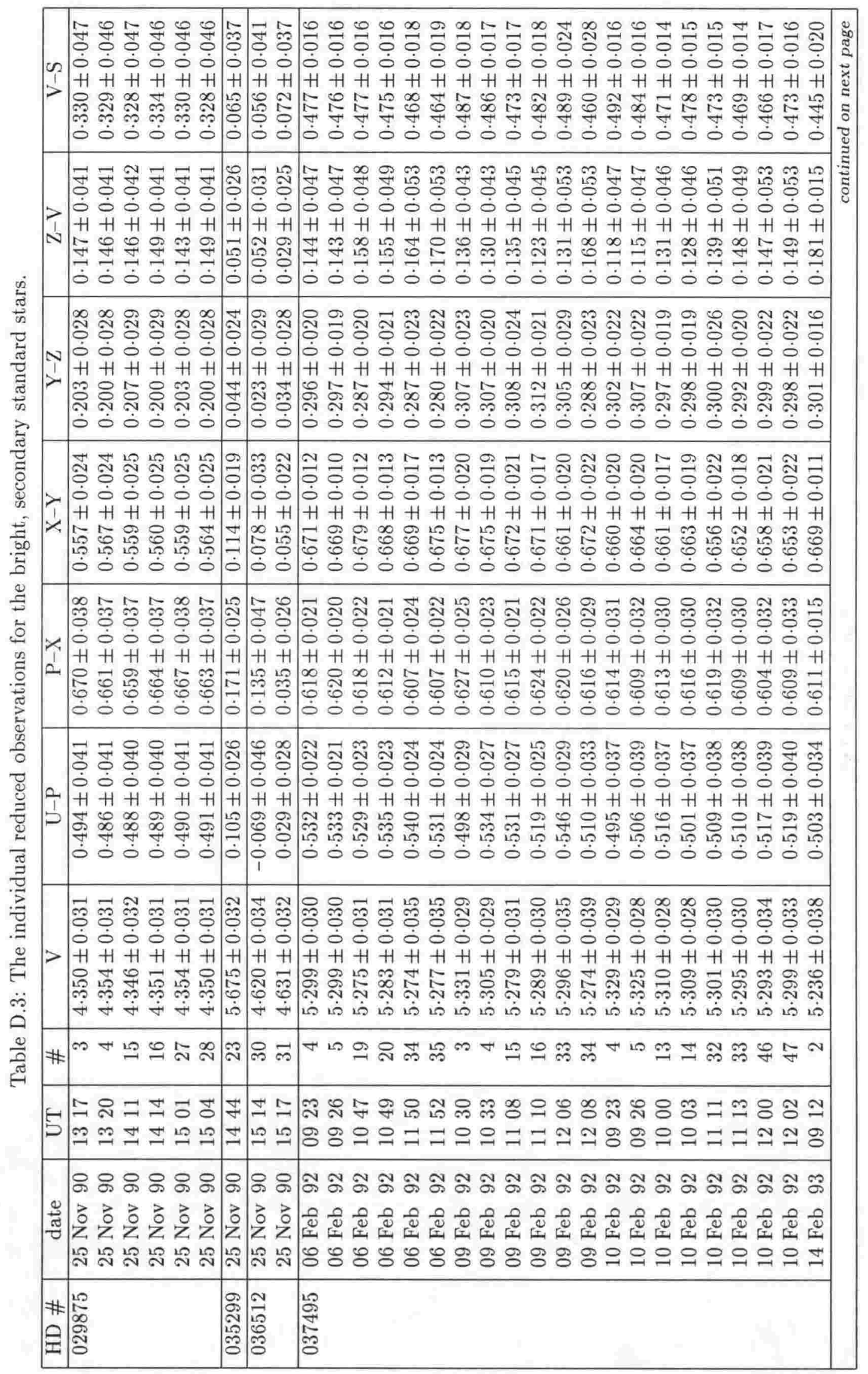




\begin{tabular}{|c|c|}
\hline & 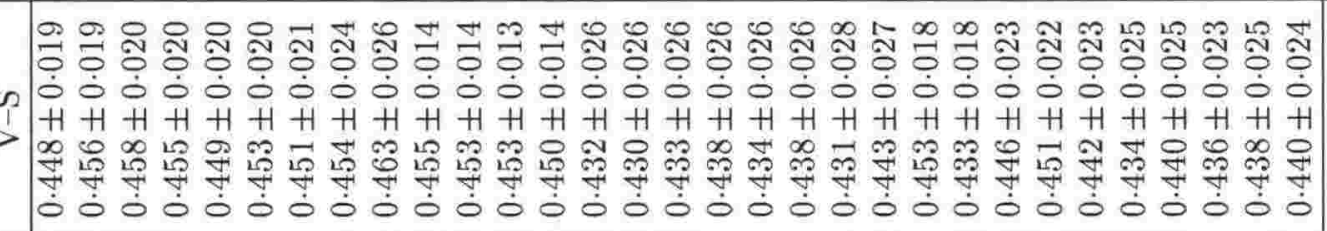 \\
\hline & 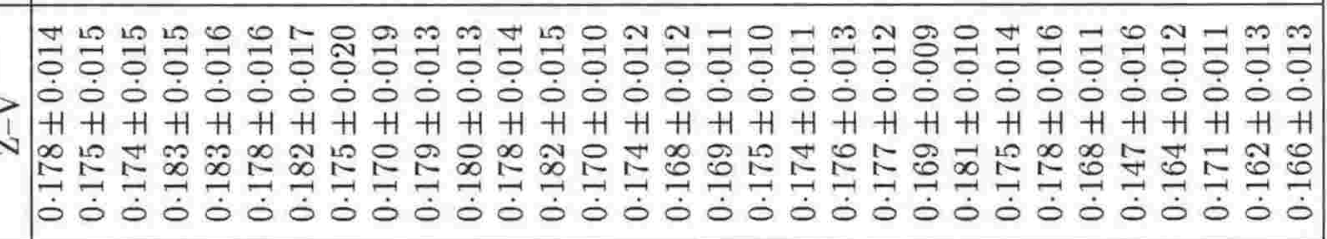 \\
\hline & 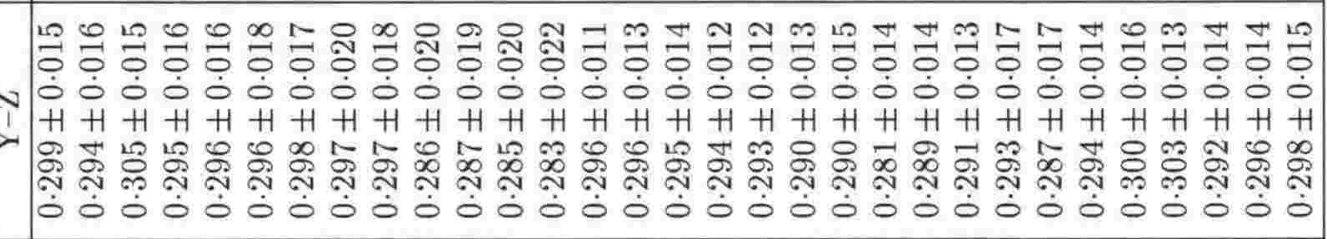 \\
\hline & 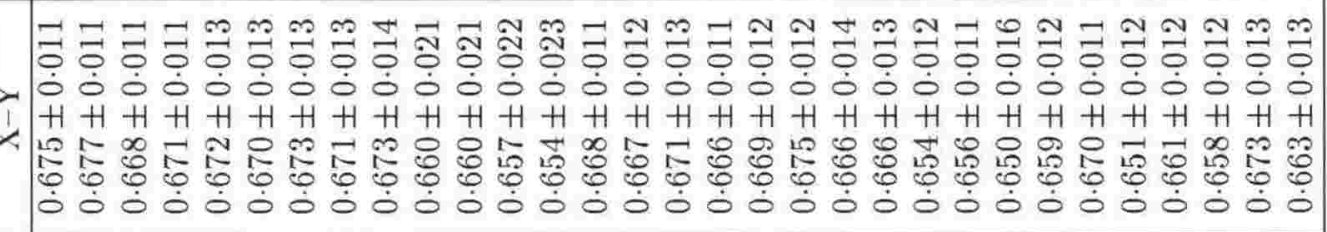 \\
\hline & 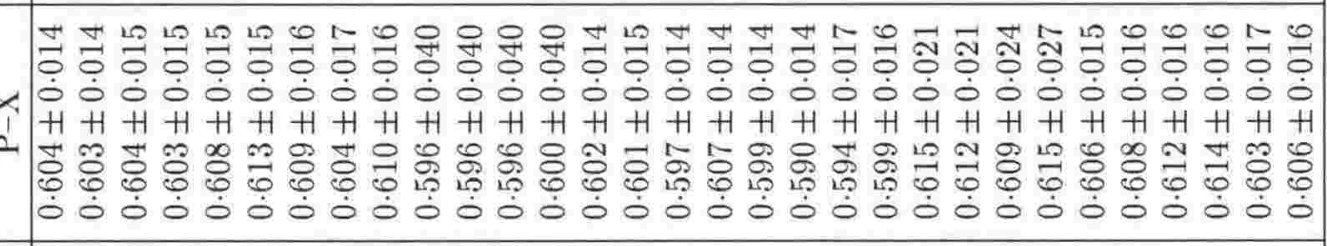 \\
\hline & 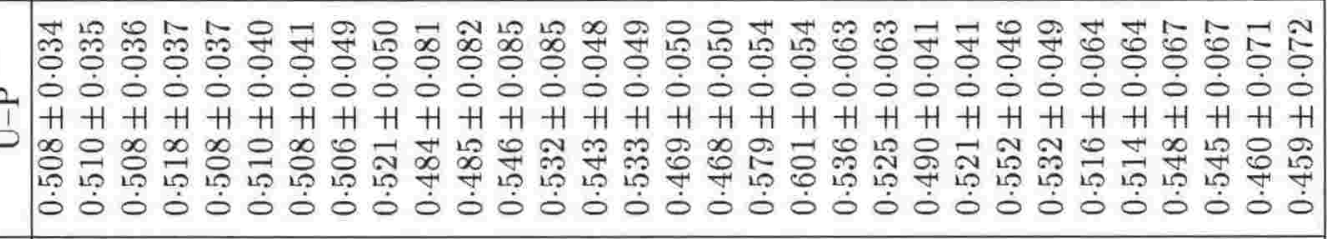 \\
\hline & 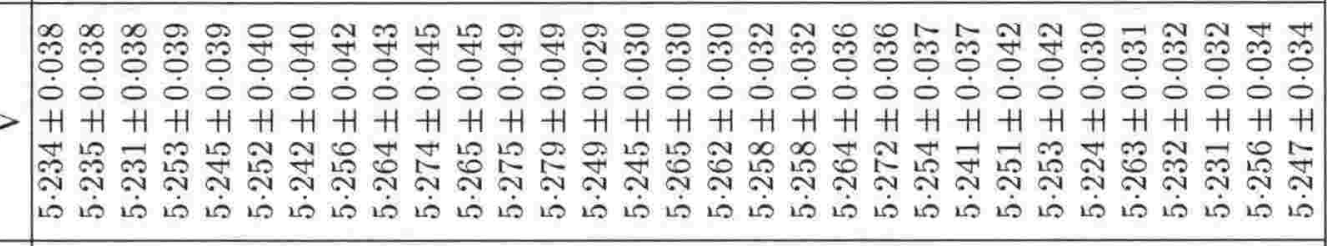 \\
\hline & \# \\
\hline & 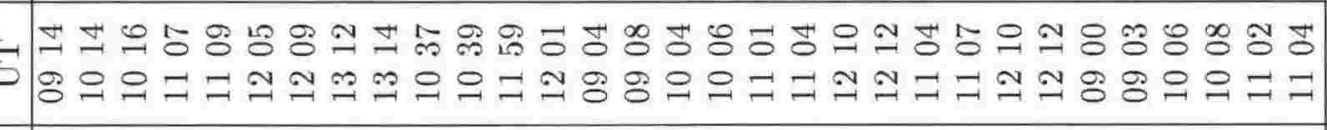 \\
\hline & 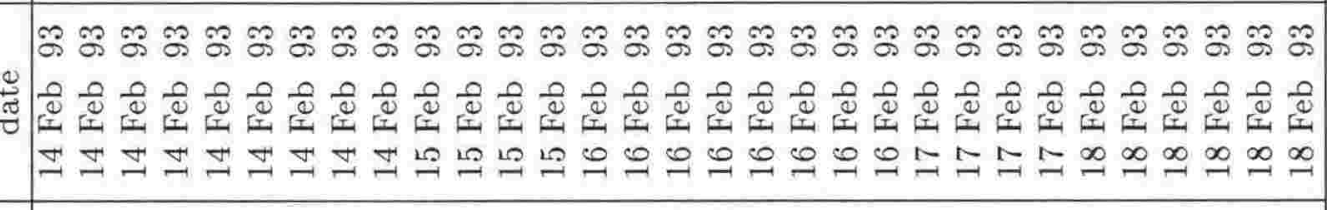 \\
\hline & \#| \\
\hline
\end{tabular}




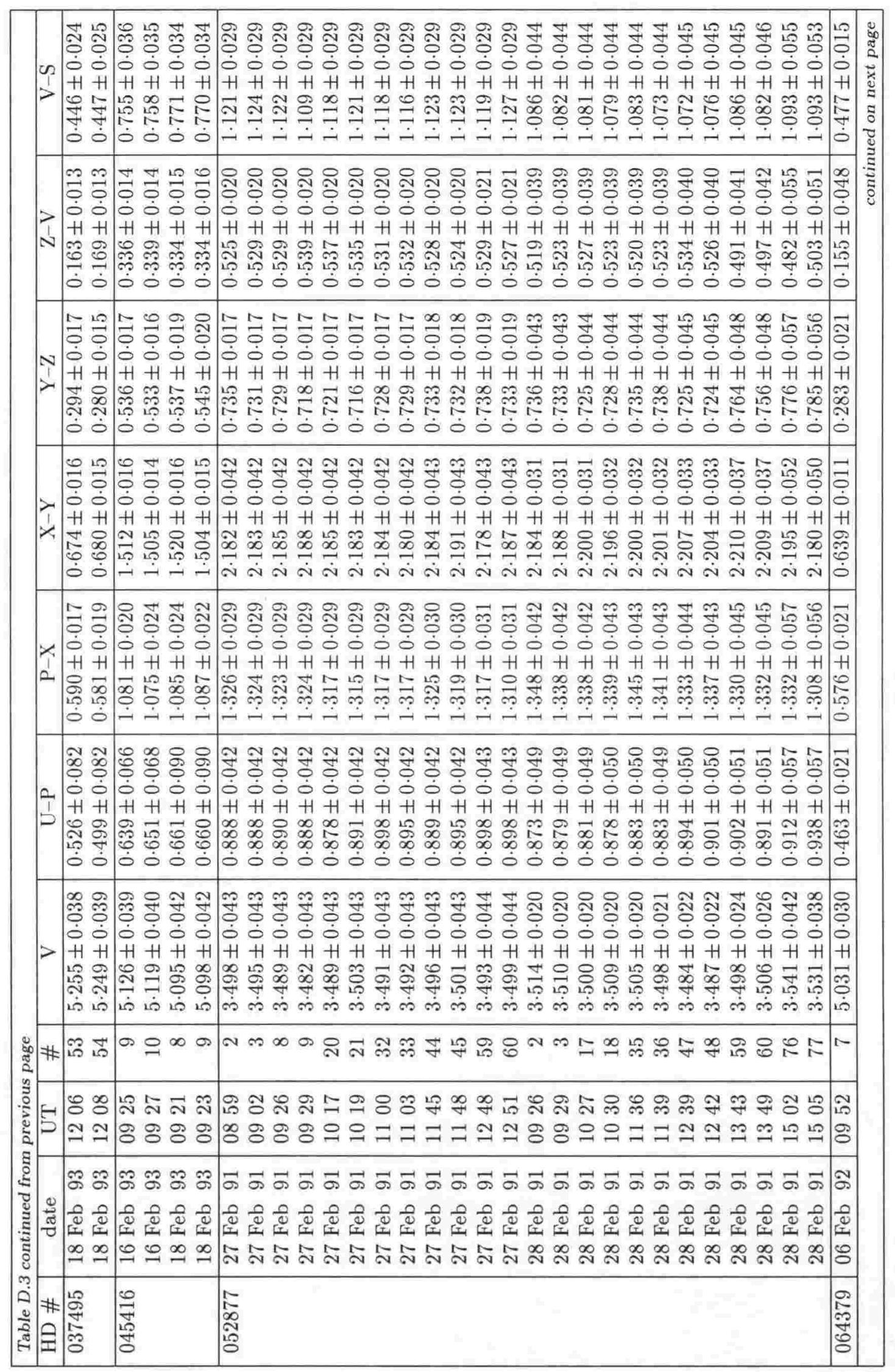




\begin{tabular}{|c|c|}
\hline & 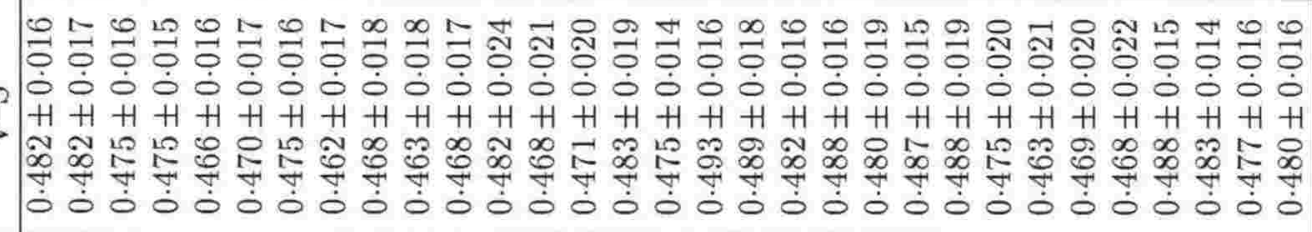 \\
\hline & 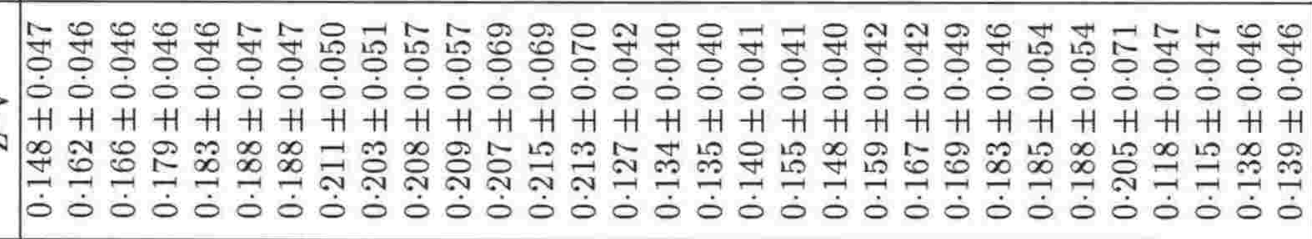 \\
\hline & 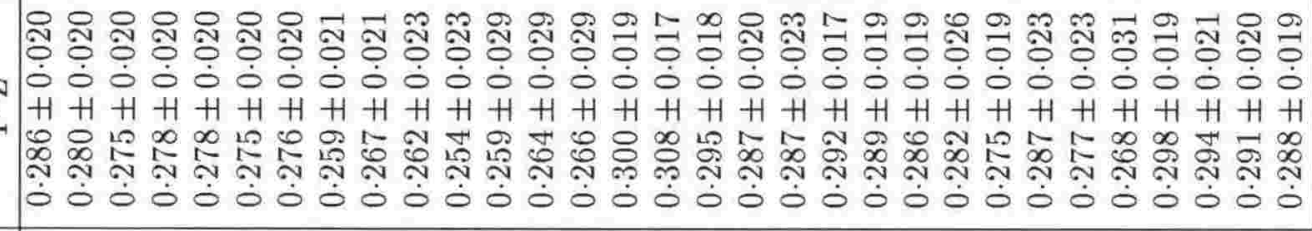 \\
\hline & 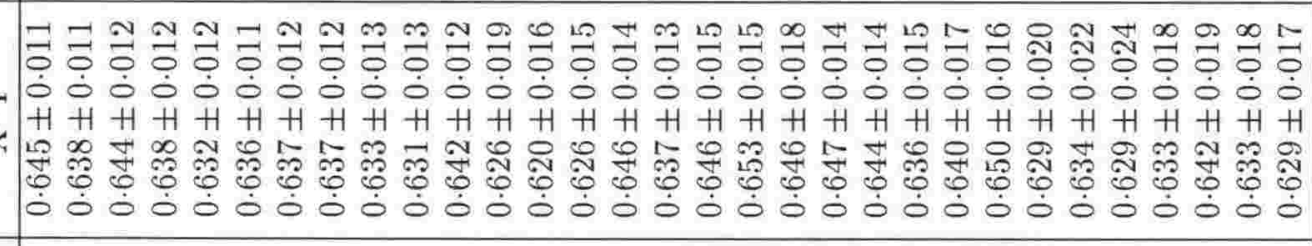 \\
\hline & 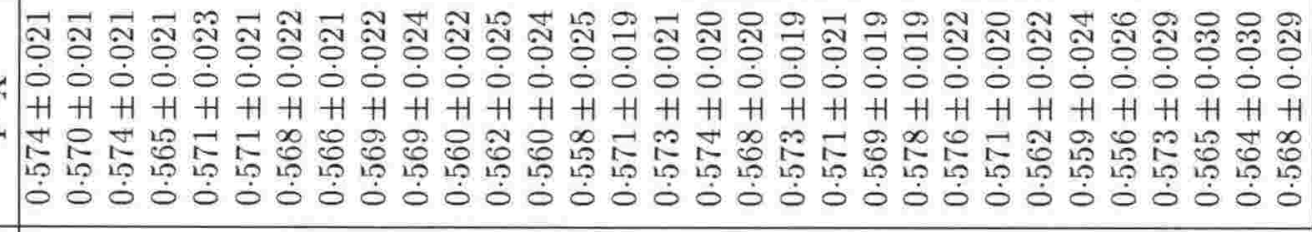 \\
\hline & 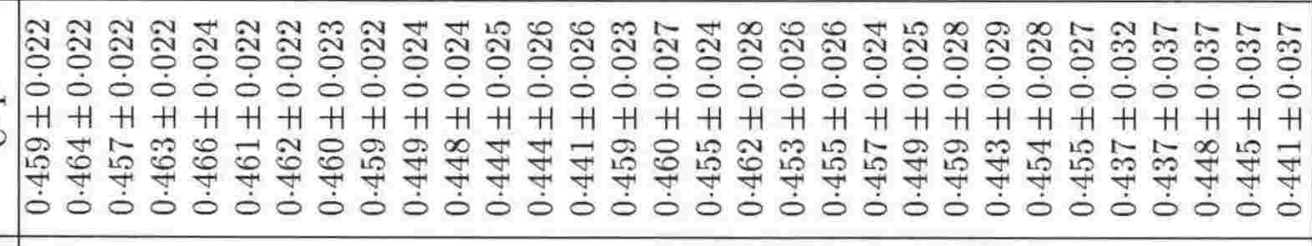 \\
\hline & 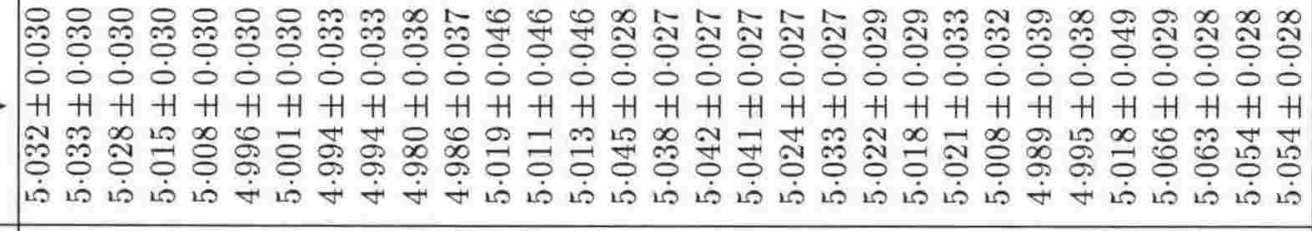 \\
\hline & $+\infty$ స \\
\hline & 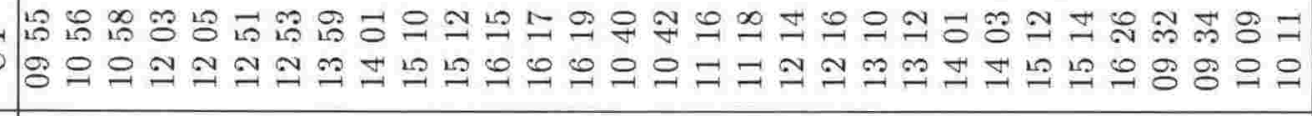 \\
\hline & 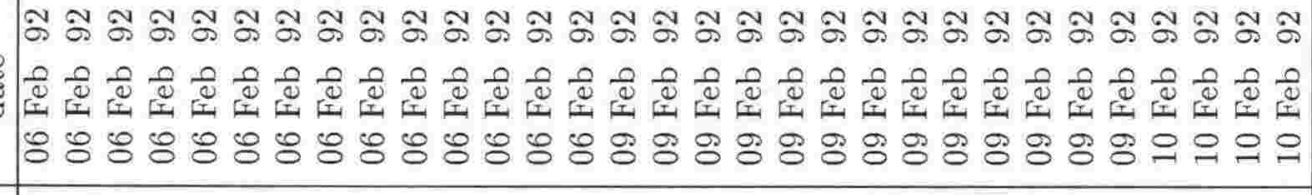 \\
\hline & $=\frac{8}{8}$ \\
\hline
\end{tabular}




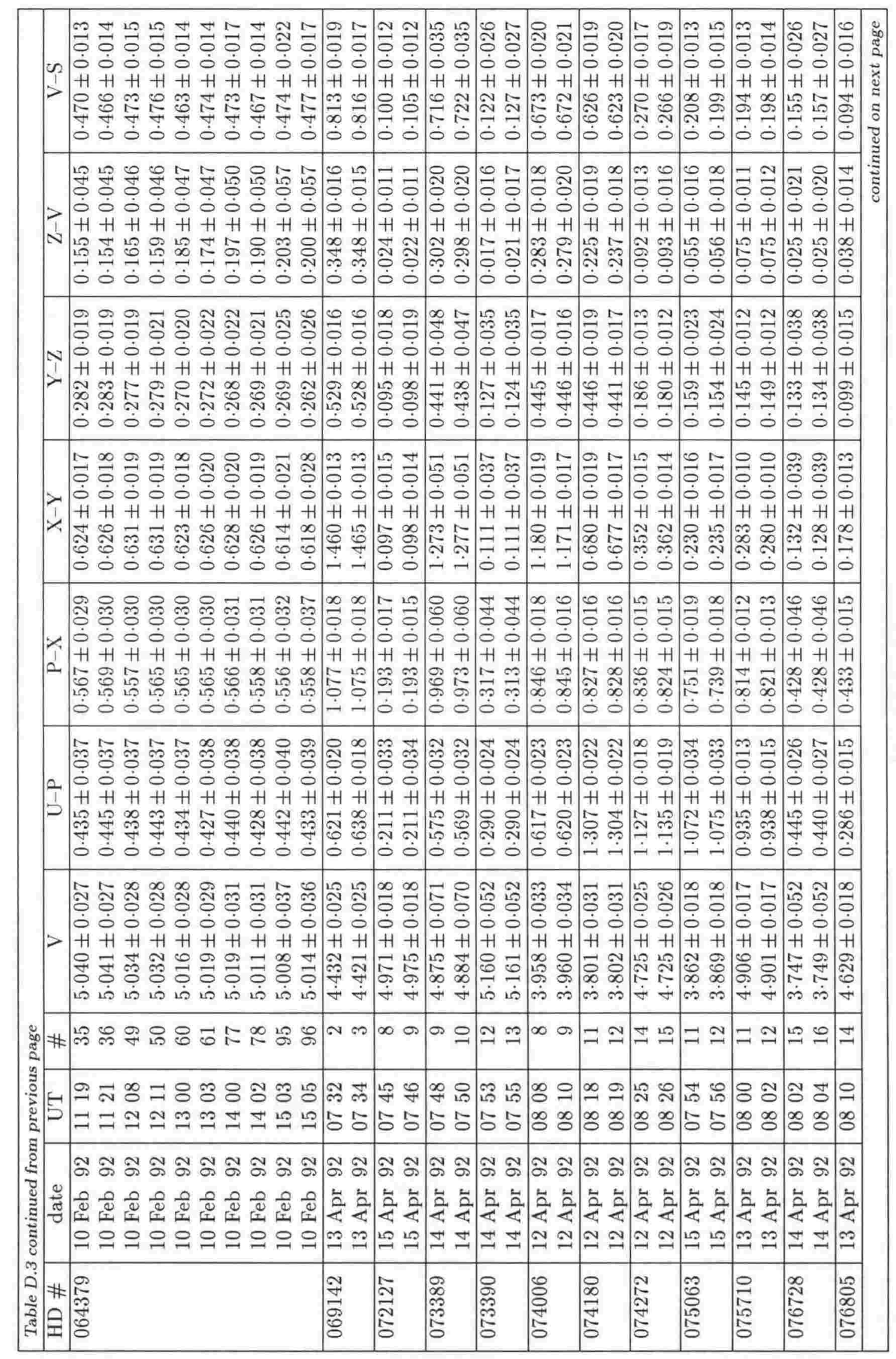




\begin{tabular}{|c|c|c|c|c|c|c|c|c|c|c|c|c|c|c|c|}
\hline & 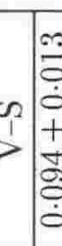 & 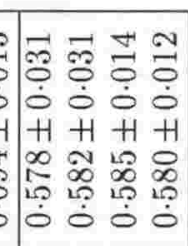 & $\begin{array}{ll}0 & 0 \\
0 & 0 \\
0 & 0 \\
0 & 0 \\
\dot{0} & \\
H & H \\
0 & 0 \\
0 & 0 \\
0 & 0 \\
0 & 0\end{array}$ & 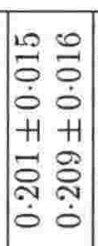 & $\begin{array}{ll}0 & 0 \\
0 & 0 \\
0 & 0 \\
0 & 0 \\
+1 \\
0 \\
0 & 0 \\
0 & 0 \\
0 & 0 \\
0 & 0\end{array}$ & 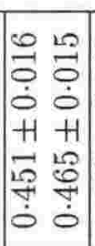 & $\mid$\begin{tabular}{cc}
0 & $\infty$ \\
0 & 0 \\
0 & 0 \\
$\dot{0}$ & $\dot{0}$ \\
$H$ & +1 \\
0 & 0 \\
0 & 0 \\
\hdashline & 0 \\
0 & 0 \\
0
\end{tabular} & $\mid \begin{array}{ll}0 & \infty \\
0 & 0 \\
0 & 0 \\
0 & 0 \\
+1 & +1 \\
0 & 0 \\
\infty & 0 \\
0 & 0 \\
0 & 0\end{array}$ & 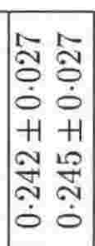 & 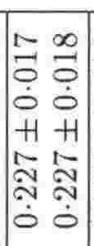 & 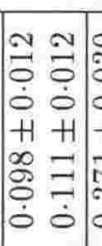 & 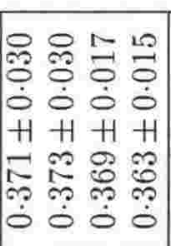 & $\begin{array}{l}0 \\
0 \\
0 \\
0 \\
+1 \\
0 \\
0 \\
1 \\
1 \\
0\end{array}$ & 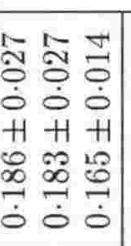 & 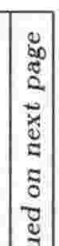 \\
\hline & & 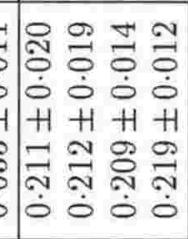 & $\begin{array}{ll}20 & 1 \\
0 & 0 \\
0 & 0 \\
0 & 0 \\
H & +1 \\
0 & 0 \\
0 & 0 \\
0 & 0 \\
0 & 0 \\
0 & 0\end{array}$ & $\begin{array}{ll}0 & 0 \\
0 & 0 \\
0 & 0 \\
0 & 0 \\
+1 & +1 \\
0 & 0 \\
0 & 0 \\
0 & 0 \\
0 & 0\end{array}$ & 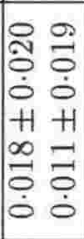 & 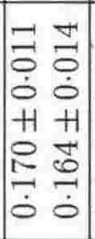 & 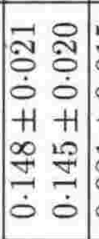 & 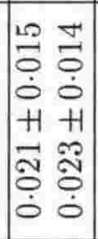 & $\begin{array}{ll}0 & 0 \\
0 & 0 \\
0 & 0 \\
\dot{0} & \dot{0} \\
+1 & H \\
0 & 0 \\
0 & 0 \\
0 & 0 \\
0 & 0\end{array}$ & 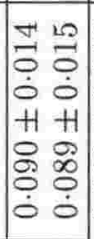 & 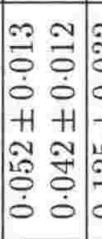 & 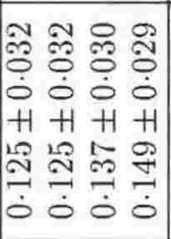 & 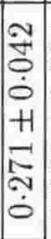 & 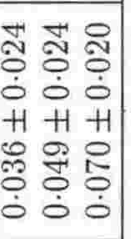 & हี \\
\hline & 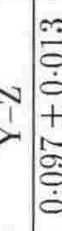 & 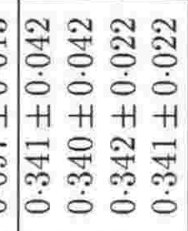 & 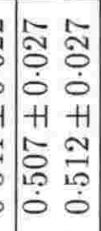 & 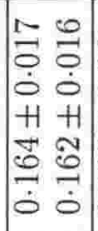 & 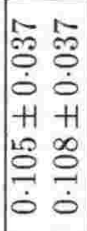 & 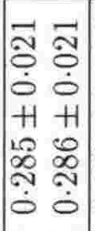 & 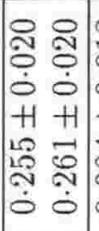 & 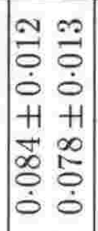 & \begin{tabular}{cc}
$\infty$ & 0 \\
\hdashline & 0 \\
0 & 0 \\
0 & 0 \\
+1 & +1 \\
0 & 0 \\
$\infty$ & 0 \\
0 & 0 \\
0 & 0
\end{tabular} & 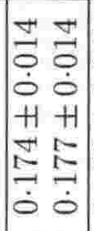 & 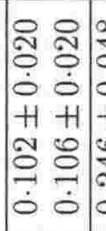 & 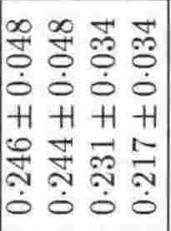 & $\begin{array}{l}0 \\
+1 \\
0 \\
10 \\
10 \\
0 \\
0\end{array}$ & 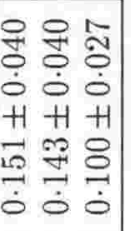 & \\
\hline & & 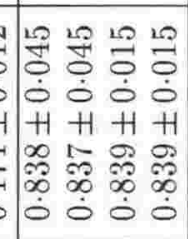 & 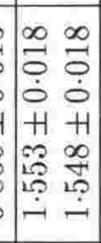 & 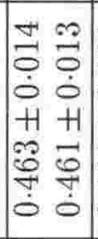 & 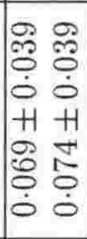 & 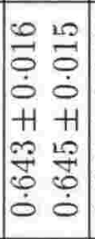 & 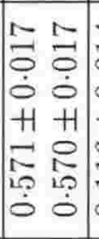 & 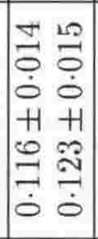 & 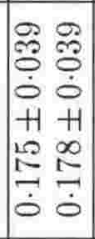 & 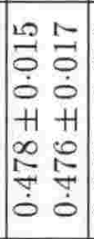 & 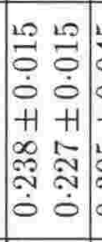 & 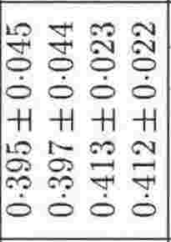 & $\begin{array}{l}+ \\
+ \\
\infty \\
\stackrel{\infty}{-} \\
\stackrel{-}{-}\end{array}$ & 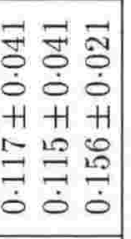 & \\
\hline & & 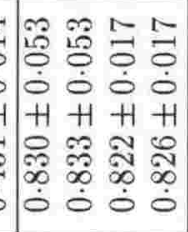 & 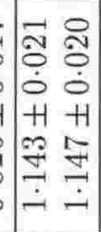 & $\mid \begin{array}{cc}1 & 0 \\
0 & 0 \\
0 & 0 \\
0 & 0 \\
1 & +1 \\
5 & 0 \\
0 & 0 \\
0 & 0 \\
0 & 0\end{array}$ & 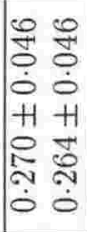 & 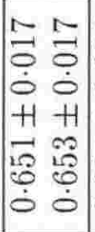 & 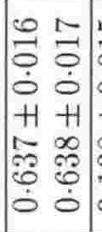 & $\mid$\begin{tabular}{cc}
20 & 0 \\
0 & 0 \\
0 & 0 \\
0 & 0 \\
$H$ & +1 \\
0 & 0 \\
\hdashline & 0 \\
\hdashline & 0 \\
0 & 0
\end{tabular} & 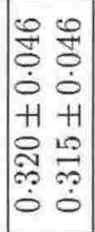 & 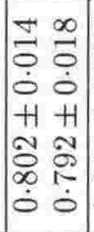 & 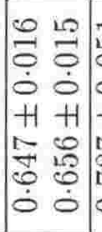 & 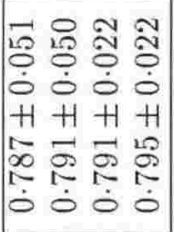 & $\begin{array}{l}\dot{0} \\
+1 \\
\dot{5} \\
\dot{S} \\
\dot{0}\end{array}$ & 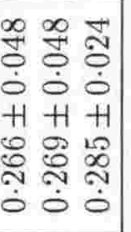 & \\
\hline & & 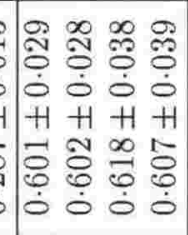 & $\begin{array}{ll}0 & 0 \\
0 & 0 \\
0 & 0 \\
0 & 0 \\
0 & 0 \\
H & +1 \\
0 & 1 \\
0 & 1 \\
0 & 0 \\
0 & 0 \\
0 & 0\end{array}$ & $\mid \begin{array}{ll}0 & 0 \\
0 & 0 \\
0 & 0 \\
0 & 0 \\
H & +1 \\
0 & 0 \\
0 & 0 \\
0 & 0 \\
0 & 0\end{array}$ & 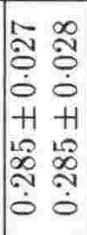 & $\mid$\begin{tabular}{cc}
1 & 0 \\
\hdashline & 0 \\
0 & 0 \\
0 & 0 \\
$H$ & +1 \\
0 & 10 \\
0 & 20 \\
0 & 0 \\
0 & 0 \\
0
\end{tabular} & 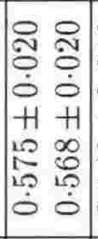 & 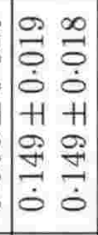 & 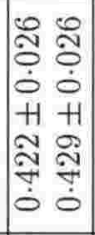 & \begin{tabular}{ll}
1 & 0 \\
0 & 0 \\
0 & 0 \\
0 & 0 \\
$H$ & 1 \\
20 & 0 \\
\hdashline & 0 \\
0 & 0 \\
0 & 0 \\
0
\end{tabular} & 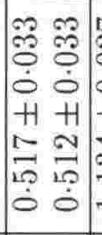 & & & 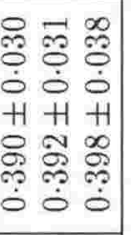 & \\
\hline & 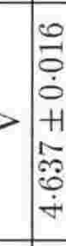 & 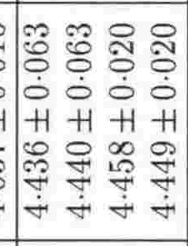 & 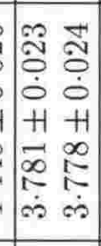 & 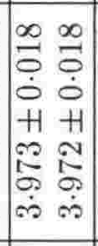 & 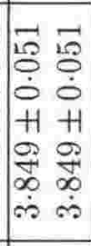 & 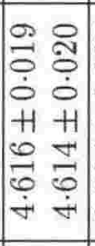 & 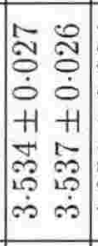 & 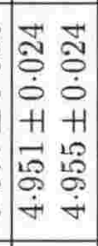 & 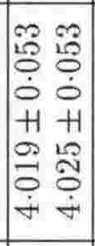 & 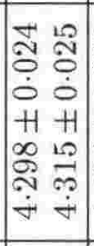 & 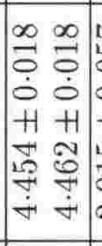 & 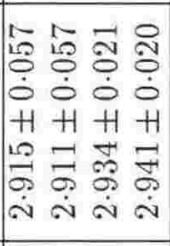 & 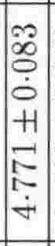 & 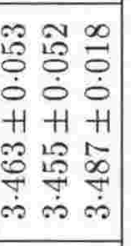 & \\
\hline 急 & $\# \stackrel{20}{2}$ & $\stackrel{\infty}{\rightarrow} \stackrel{2}{\sim}$ & $\approx \infty$ & $=\infty$ & $\vec{\curvearrowright} \lesssim$ & $\stackrel{\sim}{\sim}$ & 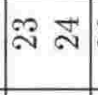 & $\because \approx$ & $\therefore$ & 尺ి & शి : & ๓ & $\because$ & 옹워 용 & \\
\hline 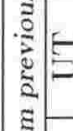 & $=$ & 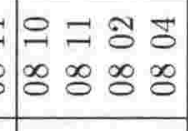 & 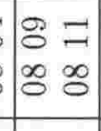 & $\left|\begin{array}{ll}\infty & 8 \\
0 & 0 \\
\infty & \infty \\
0 & 0\end{array}\right|$ & $\stackrel{\Omega}{-\infty}$ & $\mid$\begin{tabular}{ll}
1 & $\infty$ \\
\hdashline & $\infty$ \\
$\infty$ & $\infty$ \\
0 & $\infty$
\end{tabular} & $\left|\begin{array}{ll}10 & \text { iิ } \\
0 & 0 \\
0 & 0 \\
0\end{array}\right|$ & 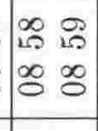 & $\mid \begin{array}{ll}\dddot{2} & \stackrel{10}{7} \\
\infty & 0 \\
0 & 0\end{array}$ & $\left|\begin{array}{ll}1 & 1 \\
0 & 5 \\
8 & 8 \\
0 & 8\end{array}\right|$ & & $\begin{array}{llll}\overrightarrow{10} & \text { in } & \text { in } & \text { in } \\
\infty & \infty & \infty & 0 \\
0 & 0 & 0 & 0\end{array}$ & & $\begin{array}{l}80 \text { is } \\
890 \\
80\end{array}$ & \\
\hline 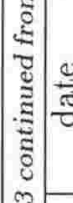 & 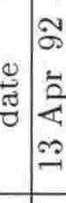 & 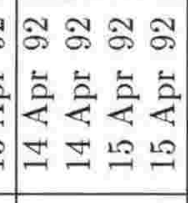 & 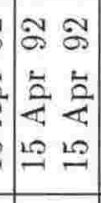 & 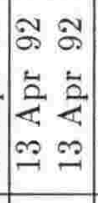 & 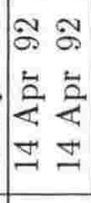 & 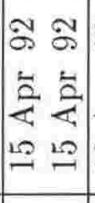 & 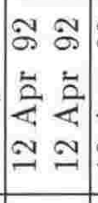 & 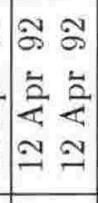 & 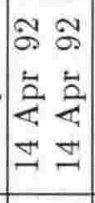 & 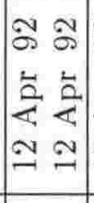 & 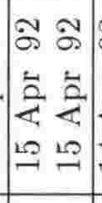 & 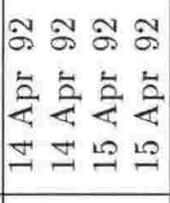 & 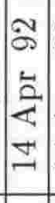 & 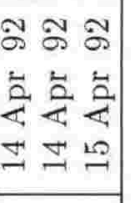 & \\
\hline 吾 & $\begin{array}{lll}* & 18 \\
0 & 0 \\
0 & 0 \\
0 & 0 \\
5\end{array}$ & 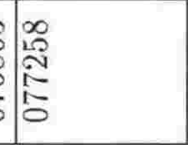 & 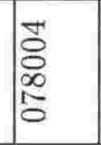 & 章 & 箱 & 융 & 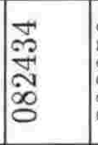 & 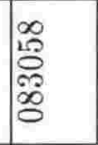 & 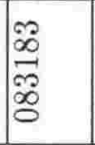 & 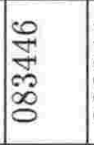 & 莸 & & & & \\
\hline
\end{tabular}




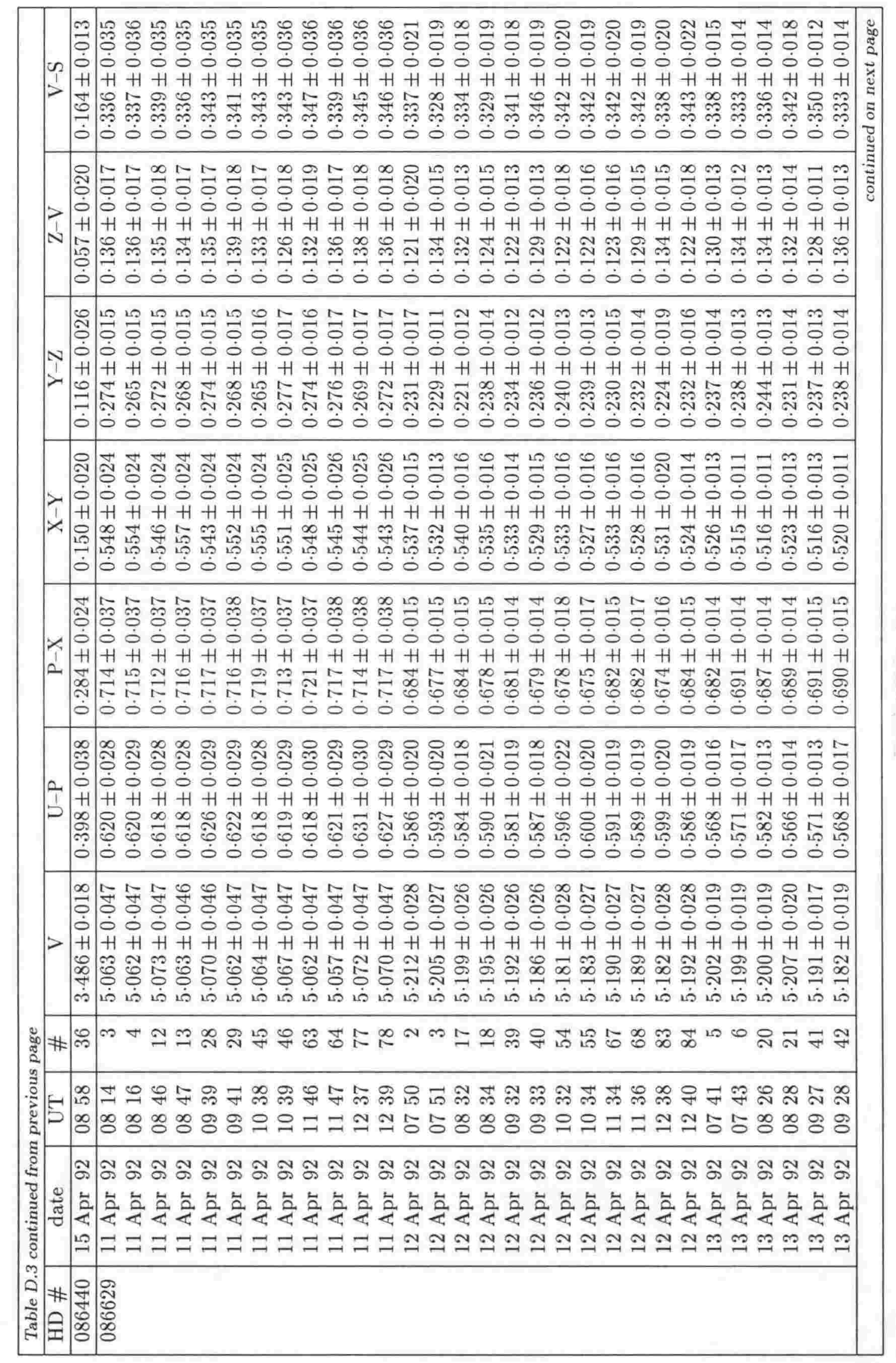




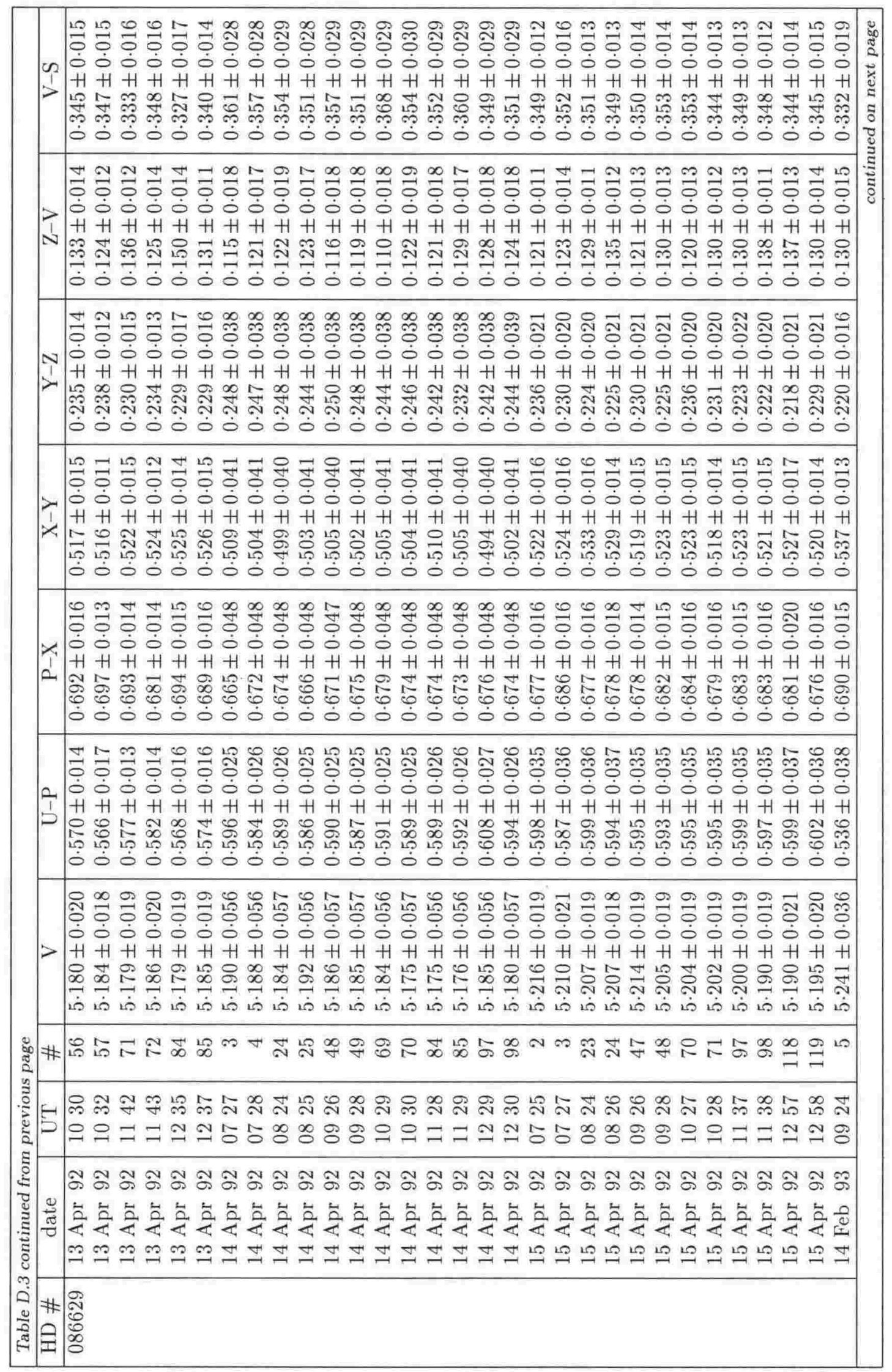




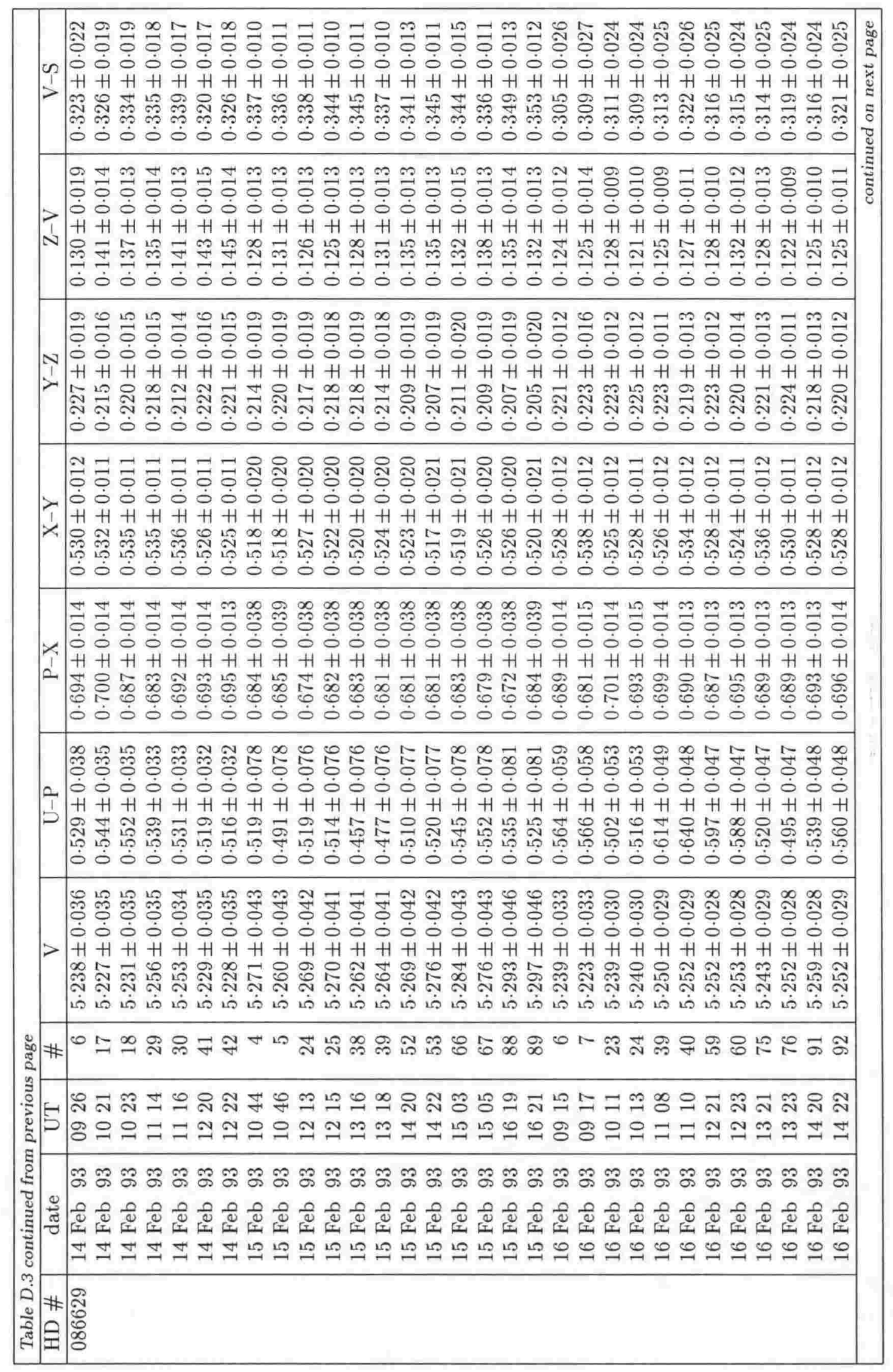




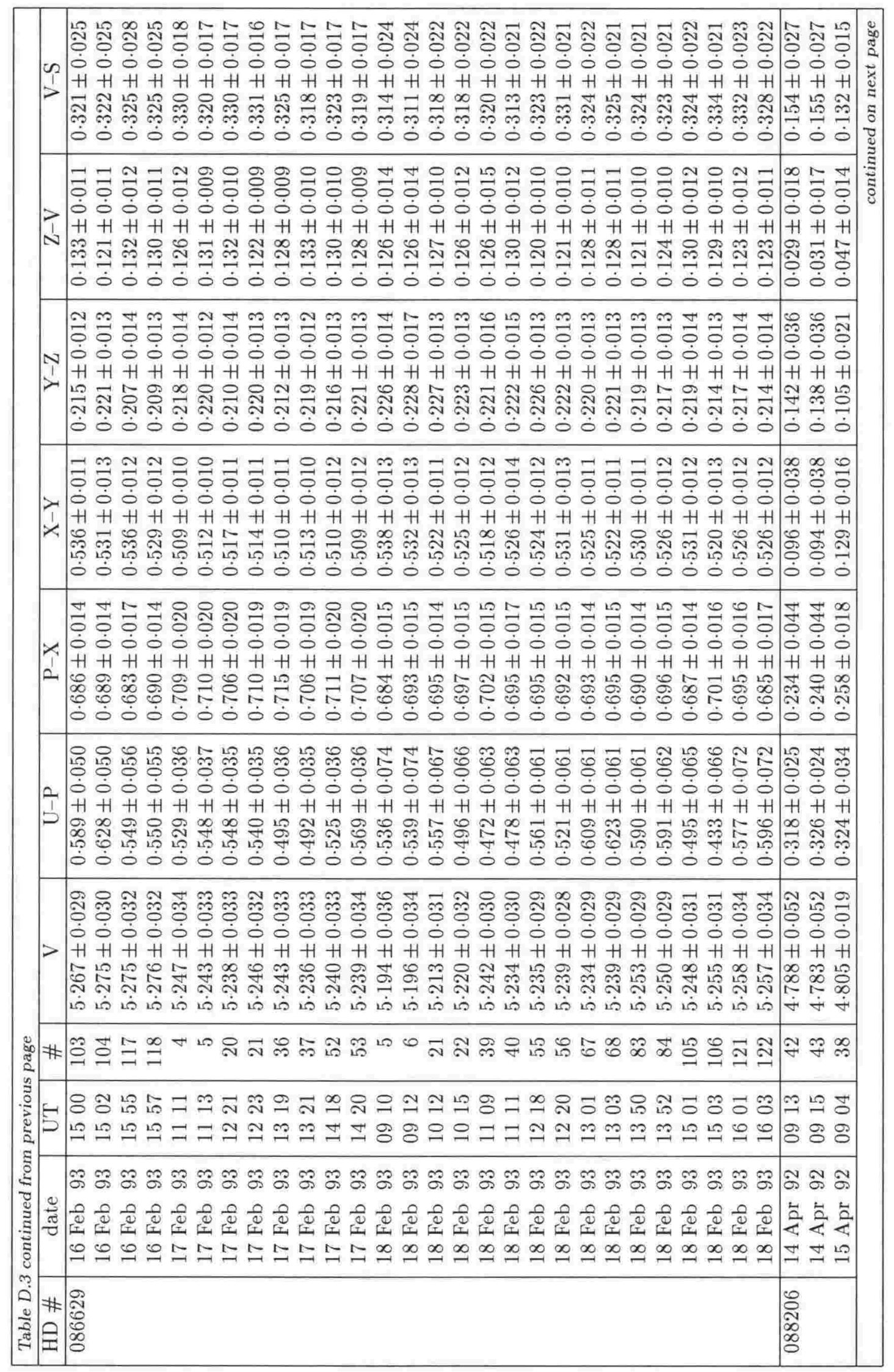




\begin{tabular}{|c|c|c|c|c|c|c|}
\hline & & 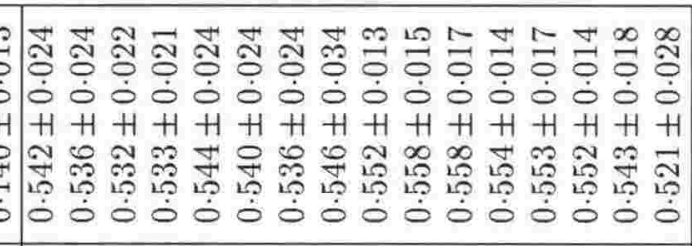 & 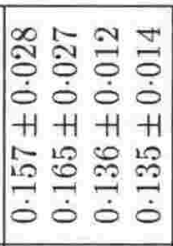 & 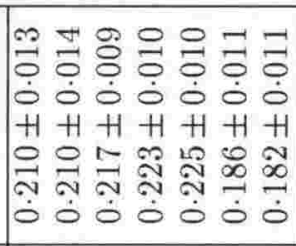 & 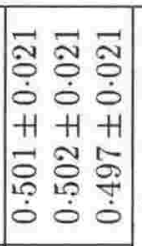 & 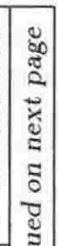 \\
\hline & & 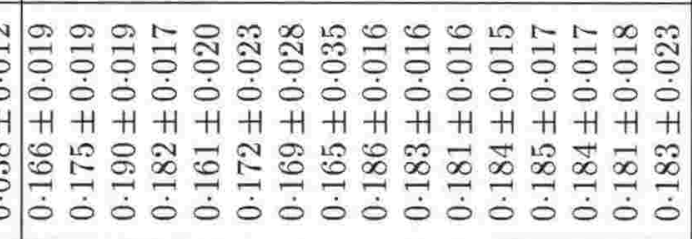 & 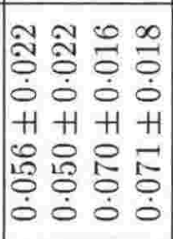 & 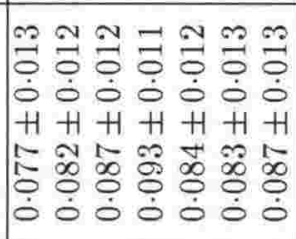 & 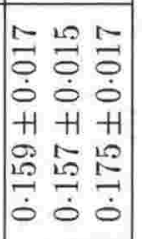 & \\
\hline & & 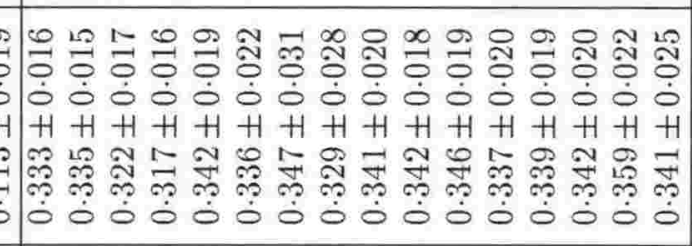 & 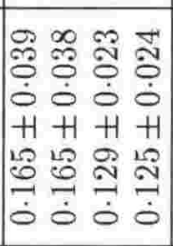 & 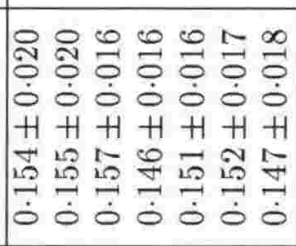 & 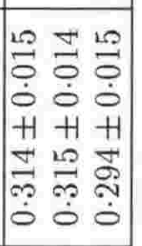 & \\
\hline & & 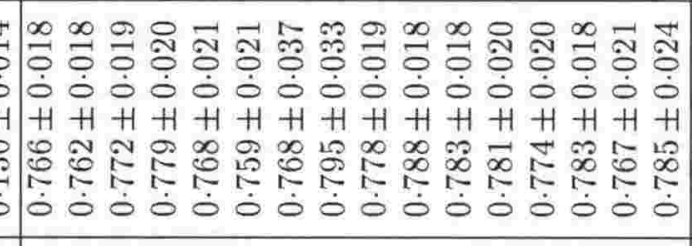 & 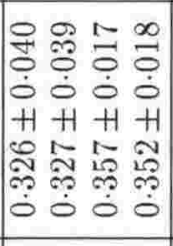 & 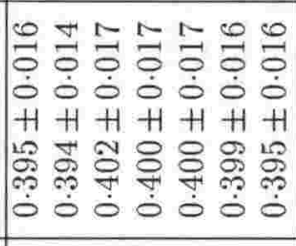 & 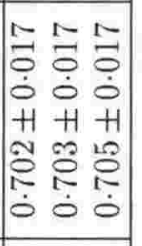 & \\
\hline & & 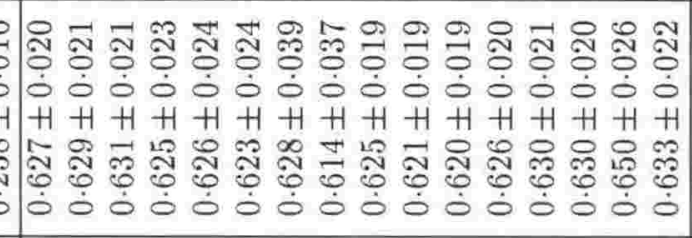 & 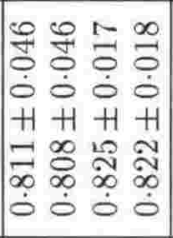 & 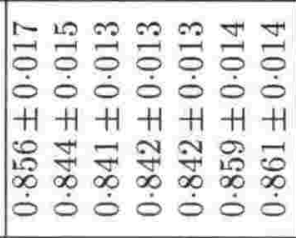 & 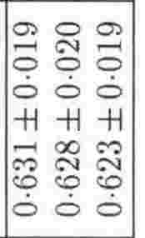 & \\
\hline & & 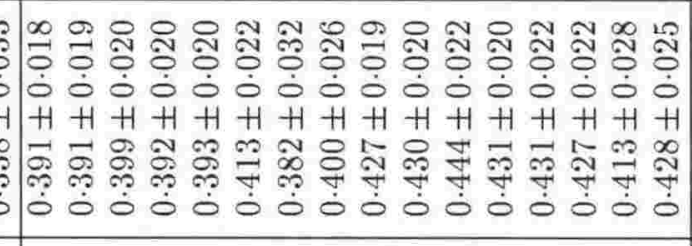 & 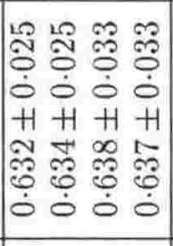 & 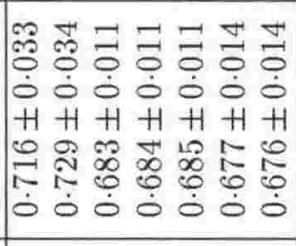 & 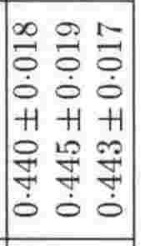 & \\
\hline & & 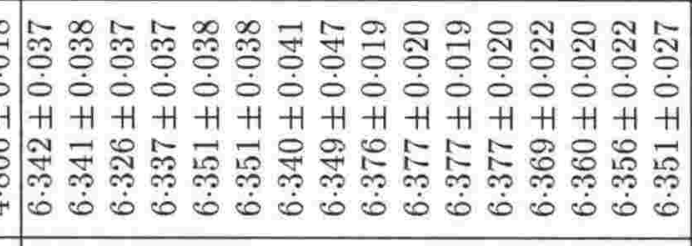 & 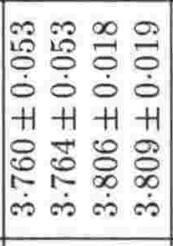 & 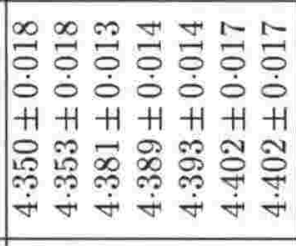 & 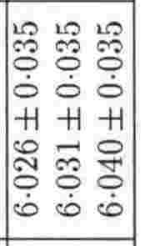 & \\
\hline & & : & 尔東 过尔 & $\overrightarrow{0}$ 웅윽 $\cong$ 윽 & $\infty \begin{array}{lll}\infty & 0 & 2\end{array}$ & \\
\hline & & 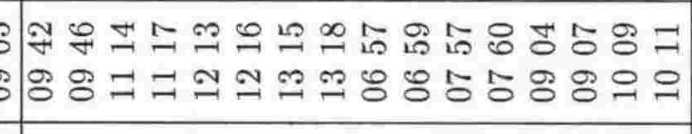 & 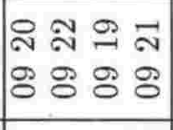 & 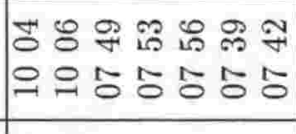 & 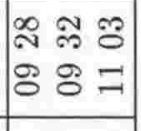 & \\
\hline & & 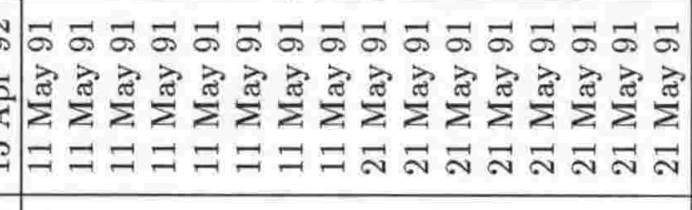 & 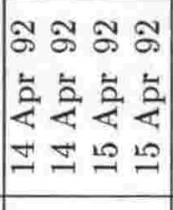 & 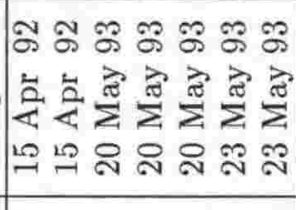 & 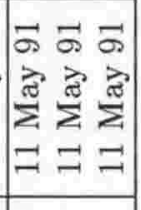 & \\
\hline & & 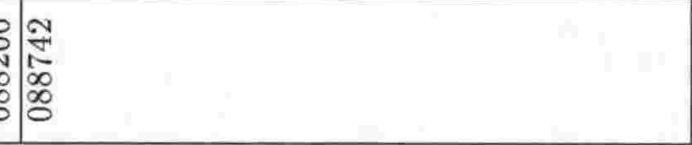 & $\begin{array}{l}2 \\
12 \\
2 \\
2 \\
0 \\
0\end{array}$ & 蕞 & $\begin{array}{l}10 \\
19 \\
18 \\
8 \\
8\end{array}$ & \\
\hline
\end{tabular}




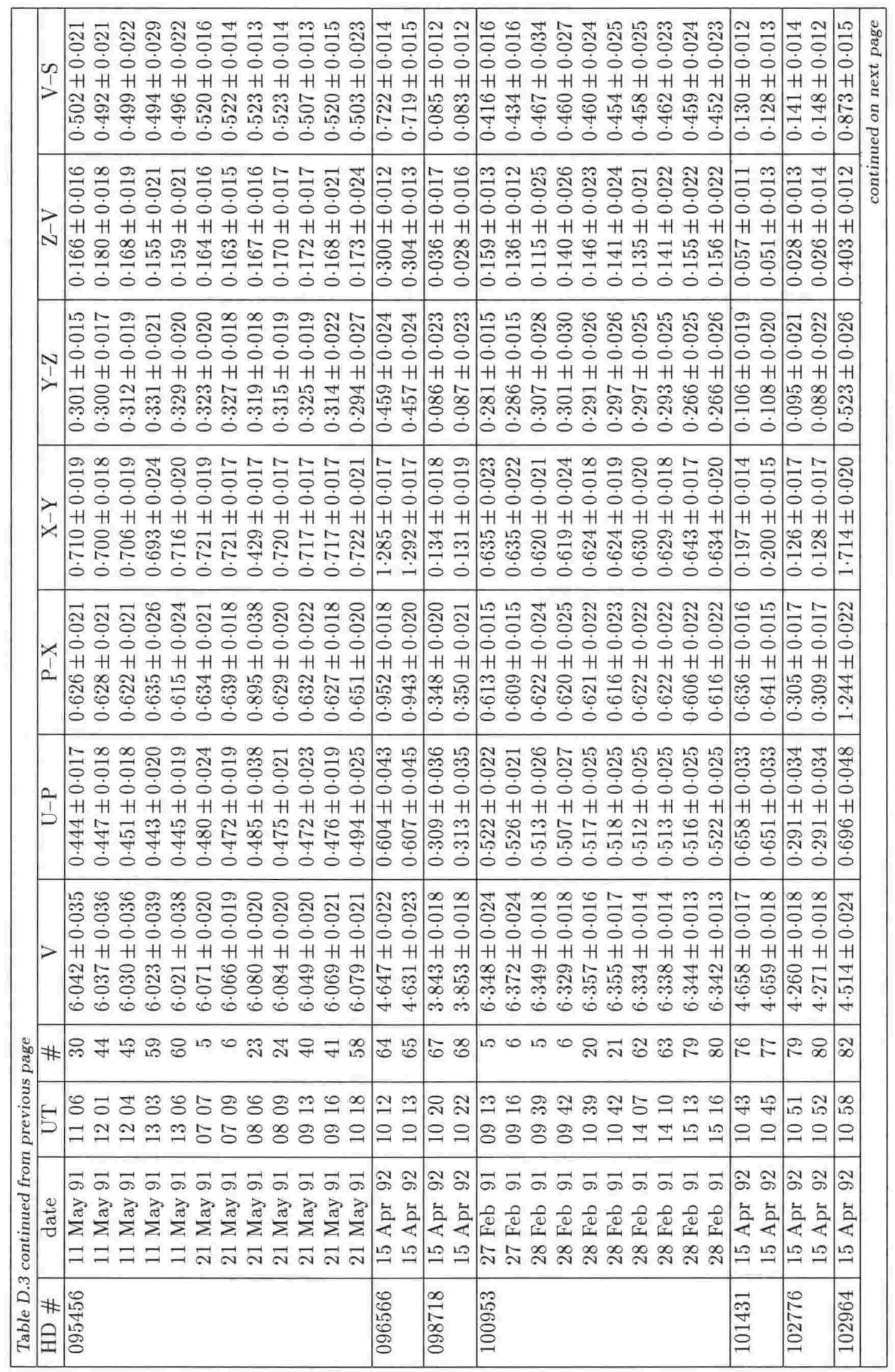




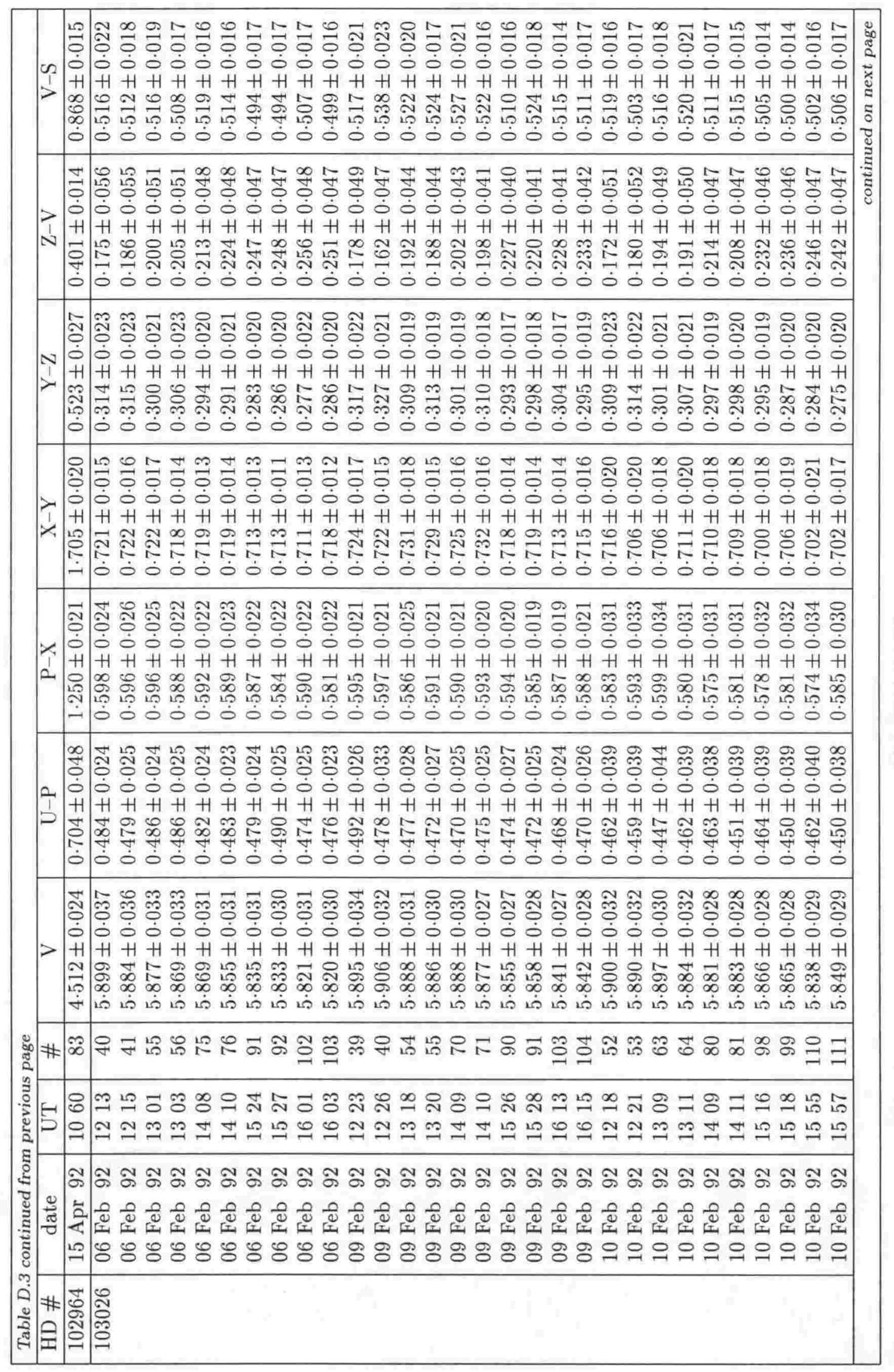




\begin{tabular}{|c|c|c|c|c|c|c|c|c|c|c|c|c|}
\hline & 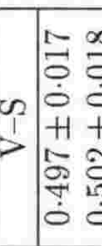 & 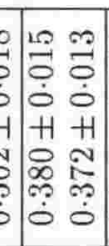 & 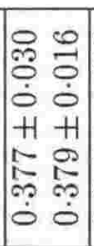 & 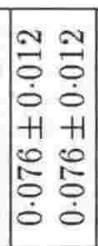 & 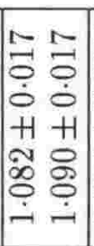 & 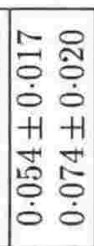 & 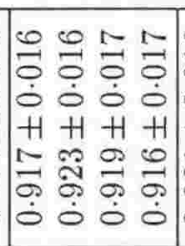 & 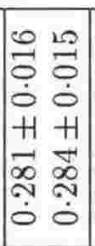 & $\left|\begin{array}{cc}0 & 2 \\
0 & 0 \\
0 & 0 \\
0 & 0 \\
H & +1 \\
0 & 0 \\
0 & 0 \\
0 & 0 \\
0 & 0\end{array}\right|$ & 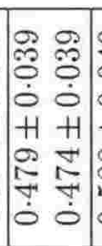 & 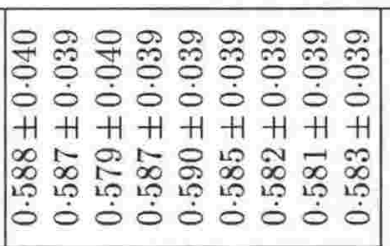 & \\
\hline & 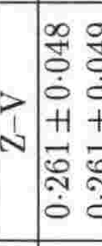 & 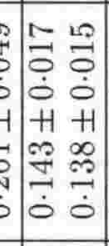 & 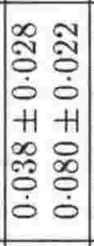 & $\mid \begin{array}{cc}20 & 0 \\
0 & 0 \\
0 & 0 \\
0 & 0 \\
H & +1 \\
0 & +4 \\
0 & 0 \\
0 & 0 \\
0 & 0\end{array}$ & 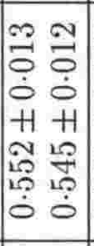 & 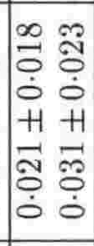 & 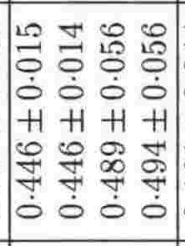 & 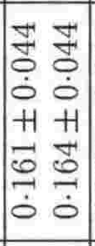 & 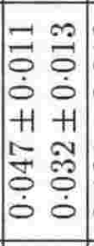 & $\mid$\begin{tabular}{cc|}
$\infty$ & $\infty$ \\
0 & 0 \\
$\dot{0}$ & 0 \\
$H$ & + \\
0 & 0 \\
\hdashline & $\infty$ \\
0 & 0 \\
0
\end{tabular} & 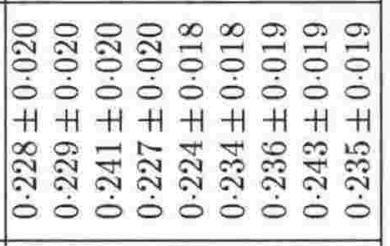 & \\
\hline & 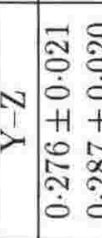 & 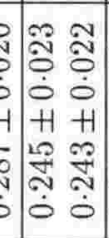 & 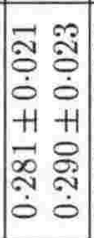 & 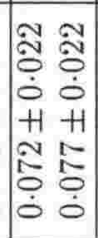 & 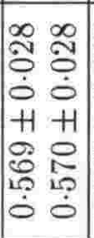 & $\mid \begin{array}{cc}\approx & 0 \\
0 & 0 \\
0 & 0 \\
\dot{1} & \dot{0} \\
1 & 1 \\
20 & 10 \\
0 & 0 \\
0 & 0 \\
0 & 0\end{array}$ & 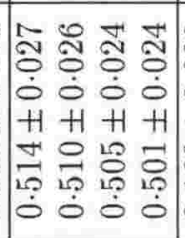 & 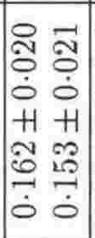 & $\mid \begin{array}{ll}0 & 0 \\
0 & 0 \\
0 & 0 \\
0 & 0 \\
H 1 & +1 \\
0 & -1 \\
0 & 0 \\
0 & 0 \\
0 & 0\end{array}$ & 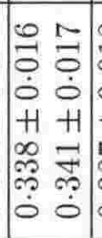 & 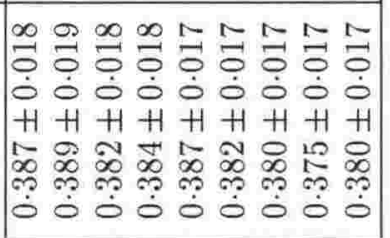 & \\
\hline & 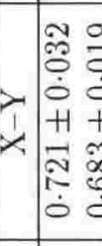 & 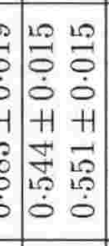 & 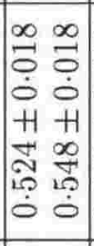 & 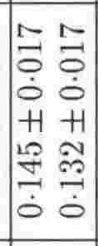 & 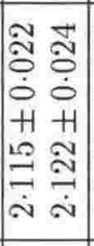 & $\mid \begin{array}{cc}\infty & 0 \\
0 & 0 \\
0 & 0 \\
0 & 0 \\
+ & +1 \\
\approx 0 & 0 \\
0 & 0 \\
0 & 0 \\
0 & 0\end{array}$ & 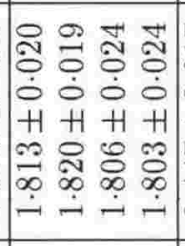 & 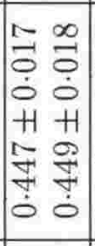 & 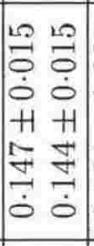 & 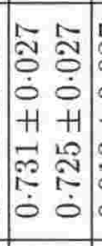 & 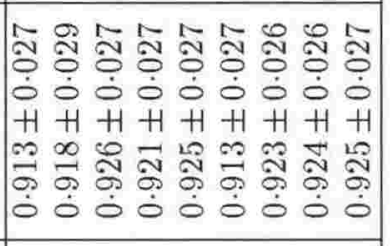 & \\
\hline & 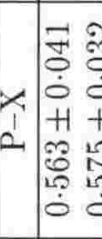 & 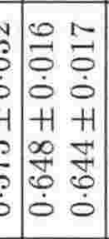 & 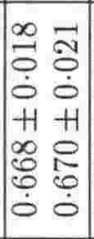 & $\left|\begin{array}{cc}0 & 0 \\
0 & 0 \\
0 & 0 \\
+1 & +1 \\
\infty & 0 \\
0 & 0 \\
\hdashline & 0 \\
0 & 0\end{array}\right|$ & 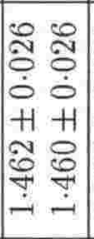 & 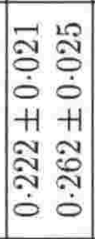 & 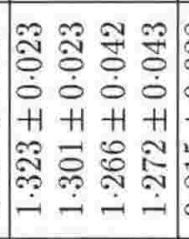 & 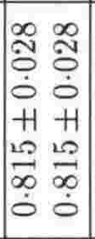 & 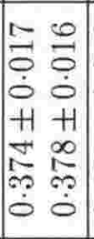 & $\left|\begin{array}{cc}0 & 0 \\
0 & 0 \\
0 & 0 \\
0 & 0 \\
1 & 1 \\
1 & 1 \\
0 & 0 \\
0 & 0 \\
0 & 0 \\
0 & 0\end{array}\right|$ & 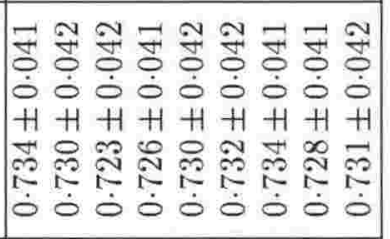 & \\
\hline & 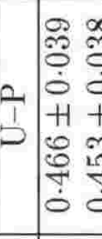 & 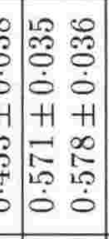 & 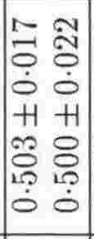 & 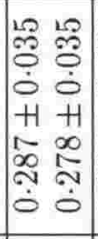 & 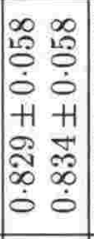 & 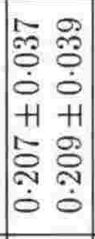 & 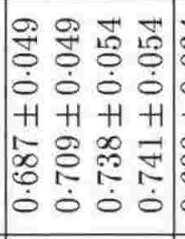 & 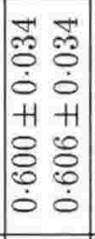 & 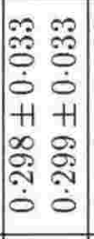 & 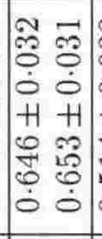 & 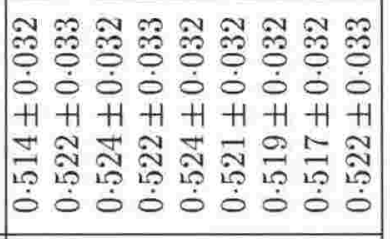 & \\
\hline & 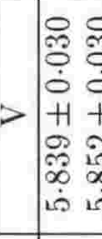 & 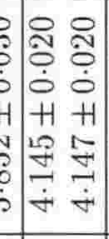 & 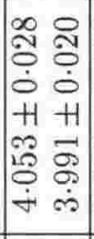 & 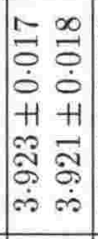 & 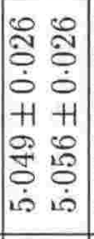 & 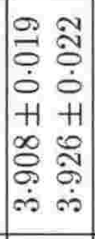 & & 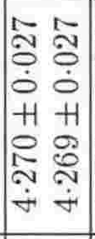 & 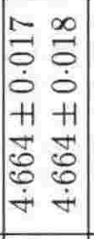 & 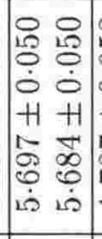 & 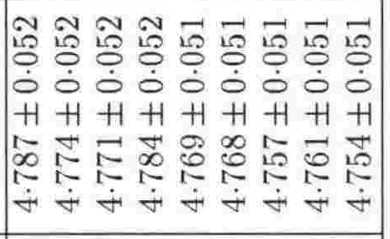 & \\
\hline & $\# \vec{\Xi}$ ฐ & $\begin{array}{lll}y & 10 & 0 \\
y & \infty & \infty\end{array}$ & iิ : & $\infty \infty_{\infty} \infty$ & $\bar{\sigma}:$ & 5 & $\because \underset{\Xi}{\Xi} \Xi$ & $\stackrel{20}{=} \cong$ & 80 & $0 N$ & 응 & \\
\hline & {$\left[\begin{array}{ll}\infty & 5 \\
\infty & 5 \\
0 & 0\end{array}\right.$} & $\begin{array}{ll}f & 0 \\
0 & 0 \\
= & =\end{array} \mid$ & $\left|\begin{array}{ll}8 & 9 \\
8 & 0 \\
8 & 0\end{array}\right|$ & & $\begin{array}{ll}\exists & \stackrel{9}{\sim} \\
\exists & \ddots\end{array}$ & $\mid \begin{array}{ll}\stackrel{2}{*} & \oplus \\
\exists & \ddots 1\end{array}$ & & & 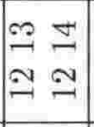 & $\left|\begin{array}{cc}\infty & -1 \\
\infty & \infty \\
\infty & 0 \\
0 & 0\end{array}\right|$ & 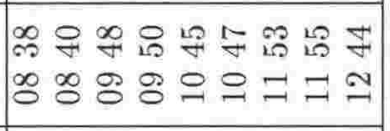 & \\
\hline 苟 & 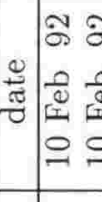 & 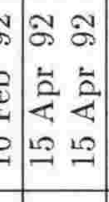 & 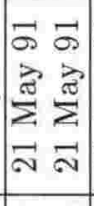 & 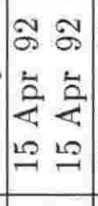 & 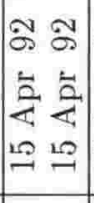 & 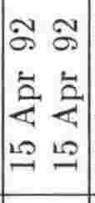 & 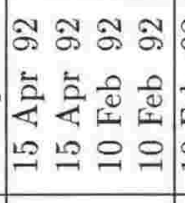 & 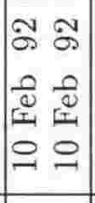 & 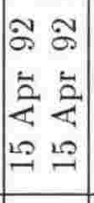 & 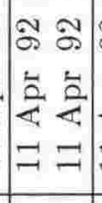 & 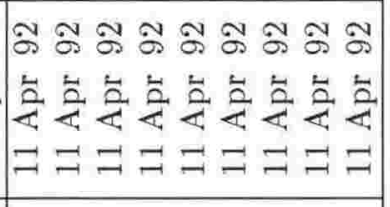 & \\
\hline & $\begin{array}{l}\# \\
\text { \#空 } \\
\text { 至 }\end{array}$ & 音 & 离 & 眥 & $\mid \begin{array}{l}0 \\
0 \\
0 \\
0 \\
0 \\
0\end{array}$ & 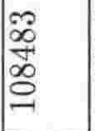 & $\stackrel{20}{\frac{2}{9}}$ & & 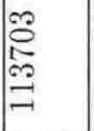 & 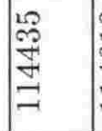 & 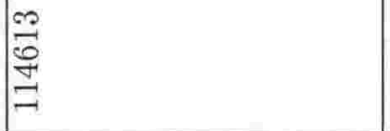 & \\
\hline
\end{tabular}




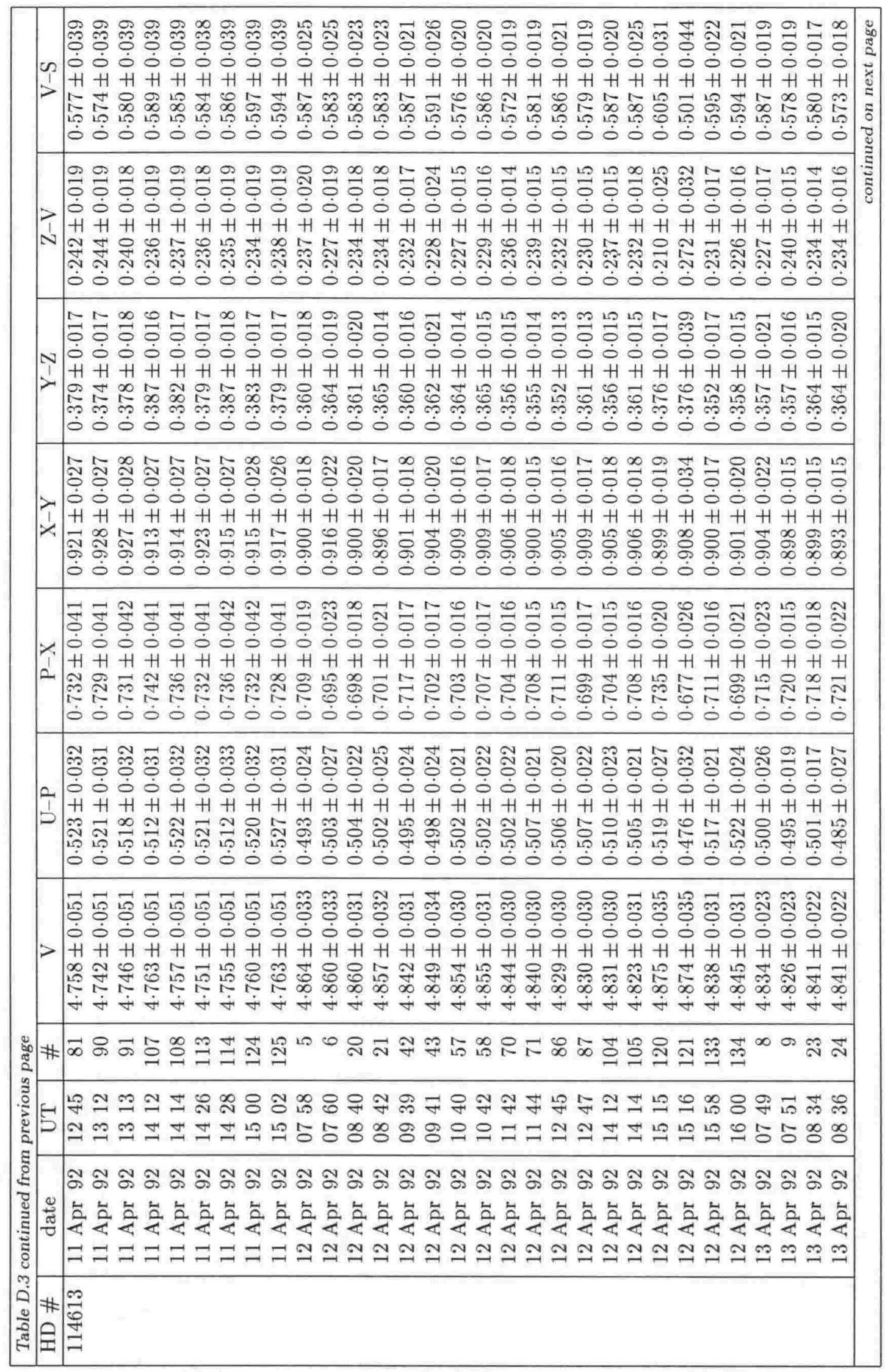




\begin{tabular}{|c|c|}
\hline & 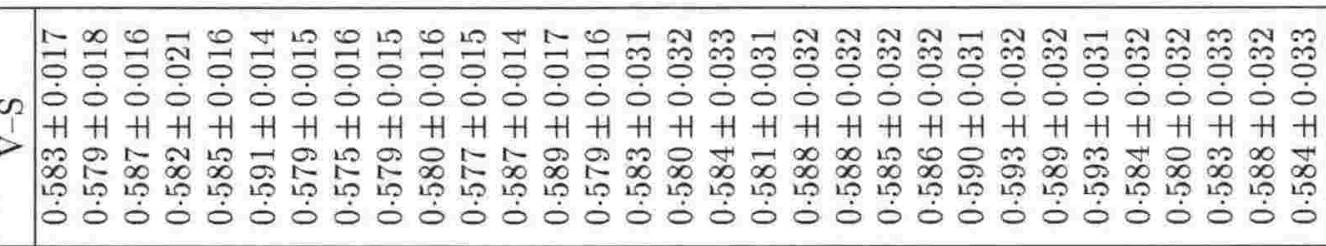 \\
\hline & 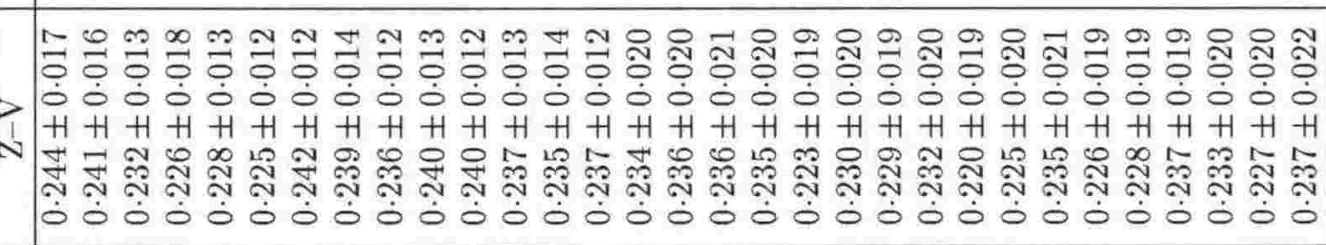 \\
\hline & 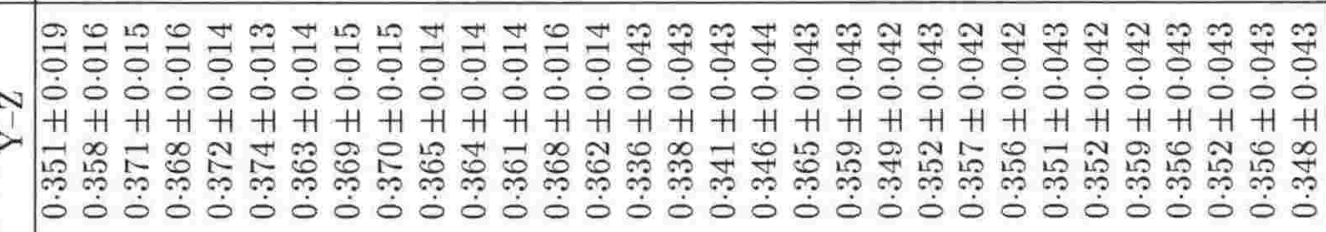 \\
\hline & 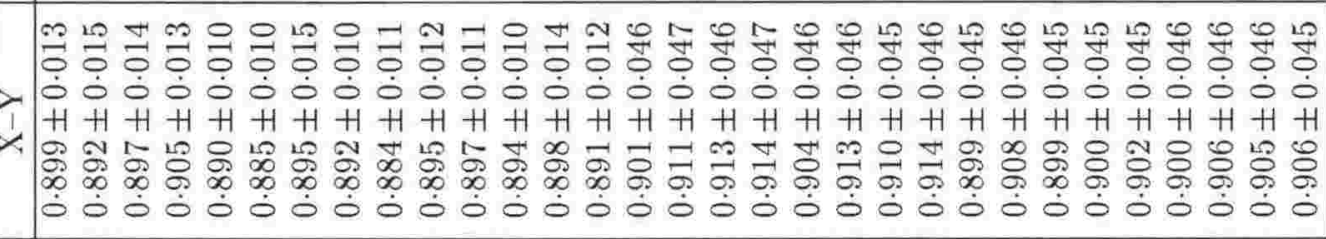 \\
\hline & 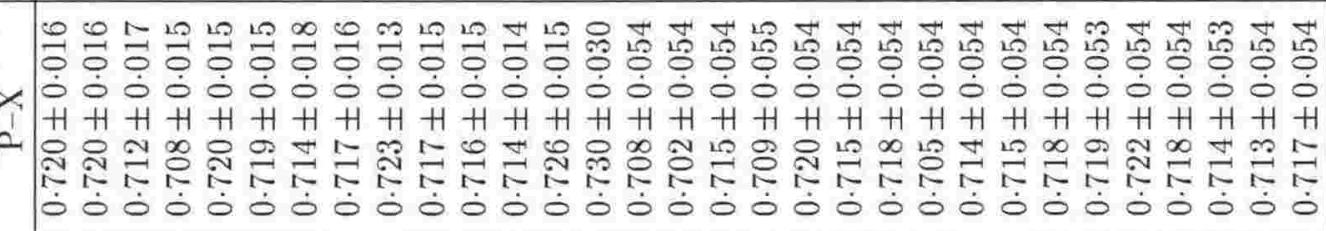 \\
\hline & 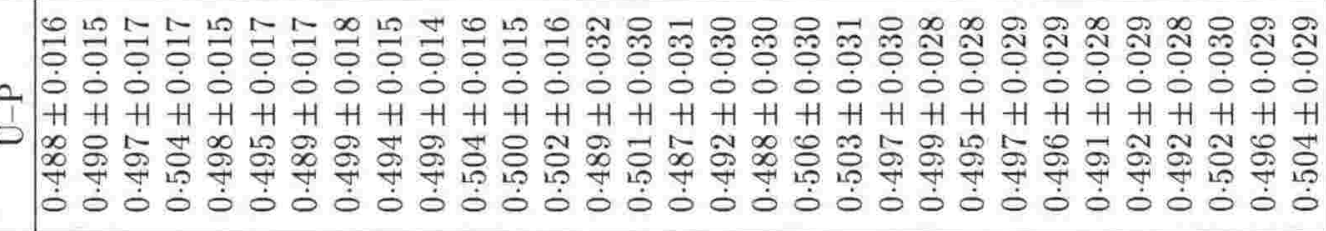 \\
\hline & 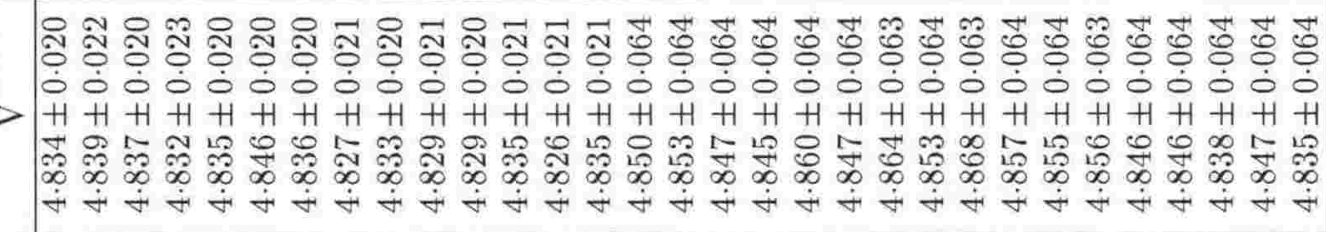 \\
\hline & 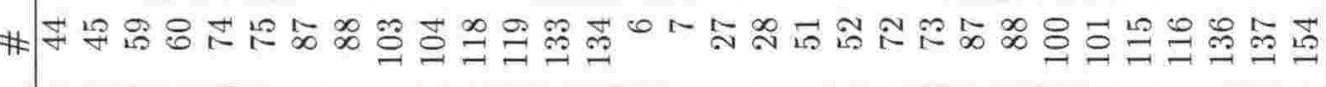 \\
\hline & 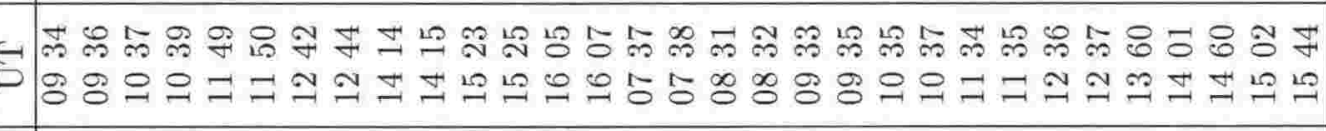 \\
\hline & 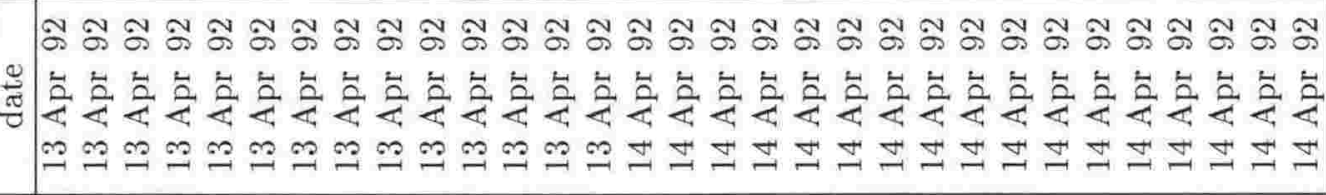 \\
\hline & 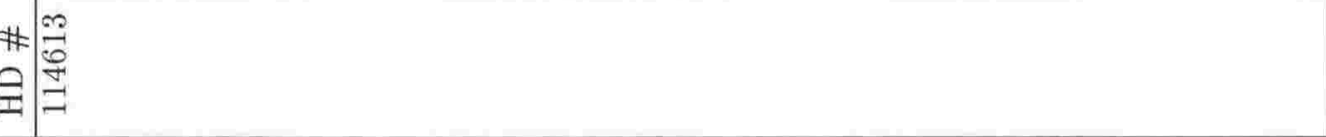 \\
\hline
\end{tabular}




\begin{tabular}{|c|c|c|c|}
\hline & 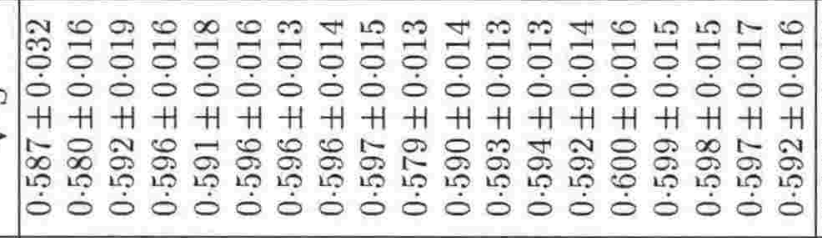 & 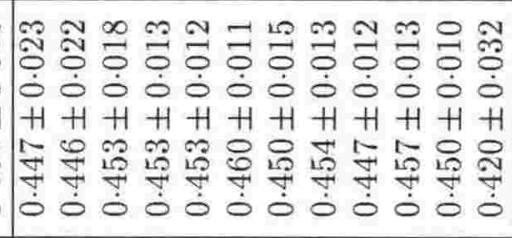 & \\
\hline & 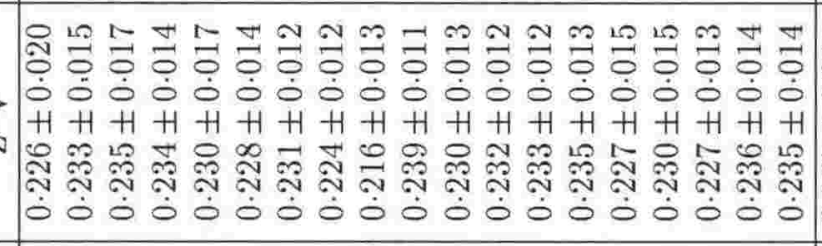 & 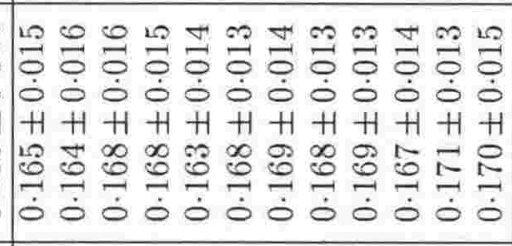 & \\
\hline & 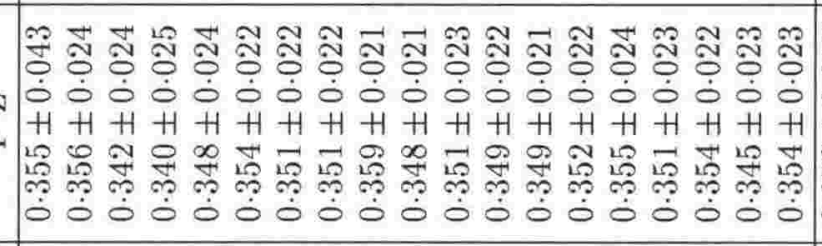 & 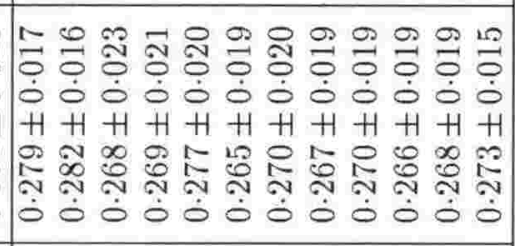 & \\
\hline & 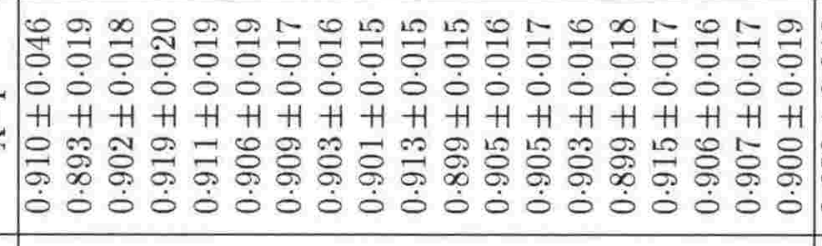 & 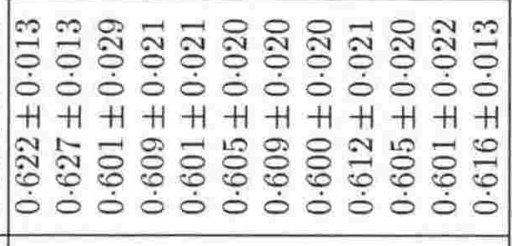 & \\
\hline & 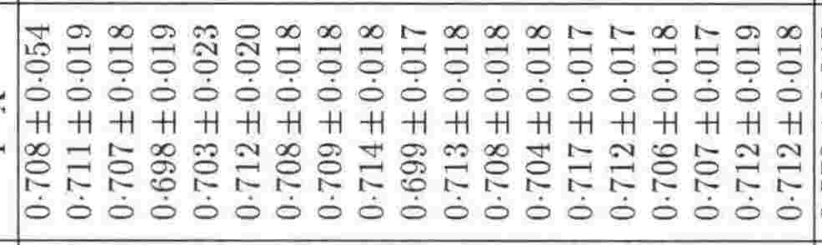 & 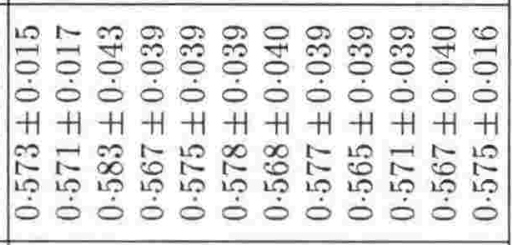 & \\
\hline & 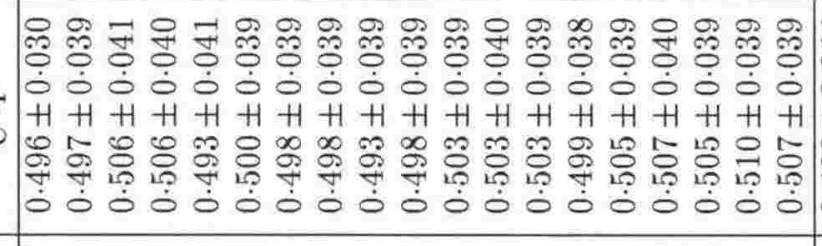 & 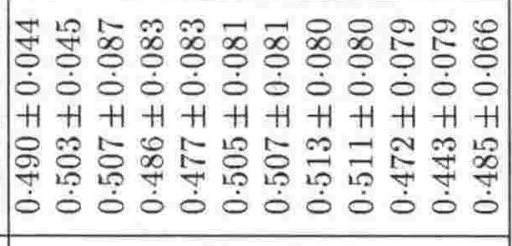 & \\
\hline & 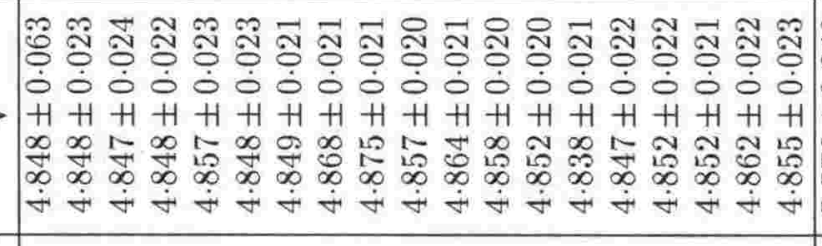 & 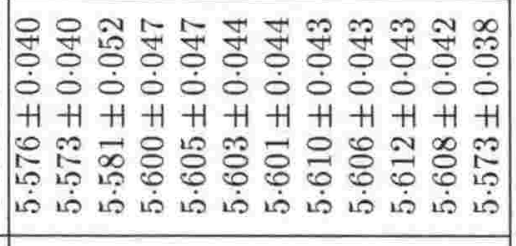 & \\
\hline & 嵒 & 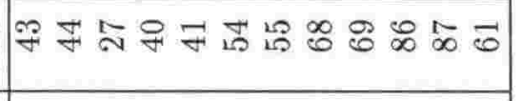 & \\
\hline & 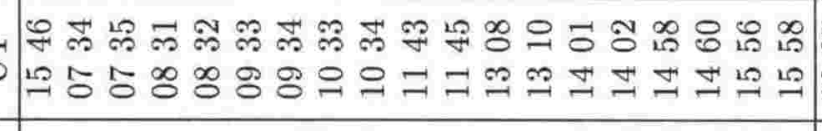 & 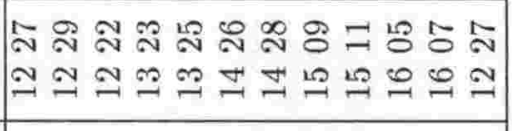 & \\
\hline & 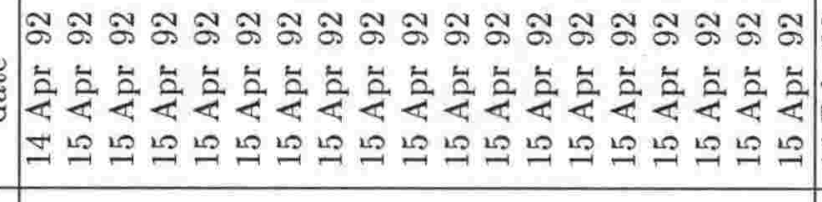 & 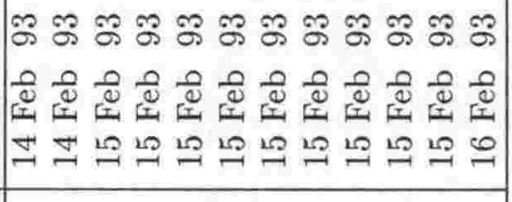 & \\
\hline & $\mid \frac{m}{0}$ & 位 & \\
\hline
\end{tabular}




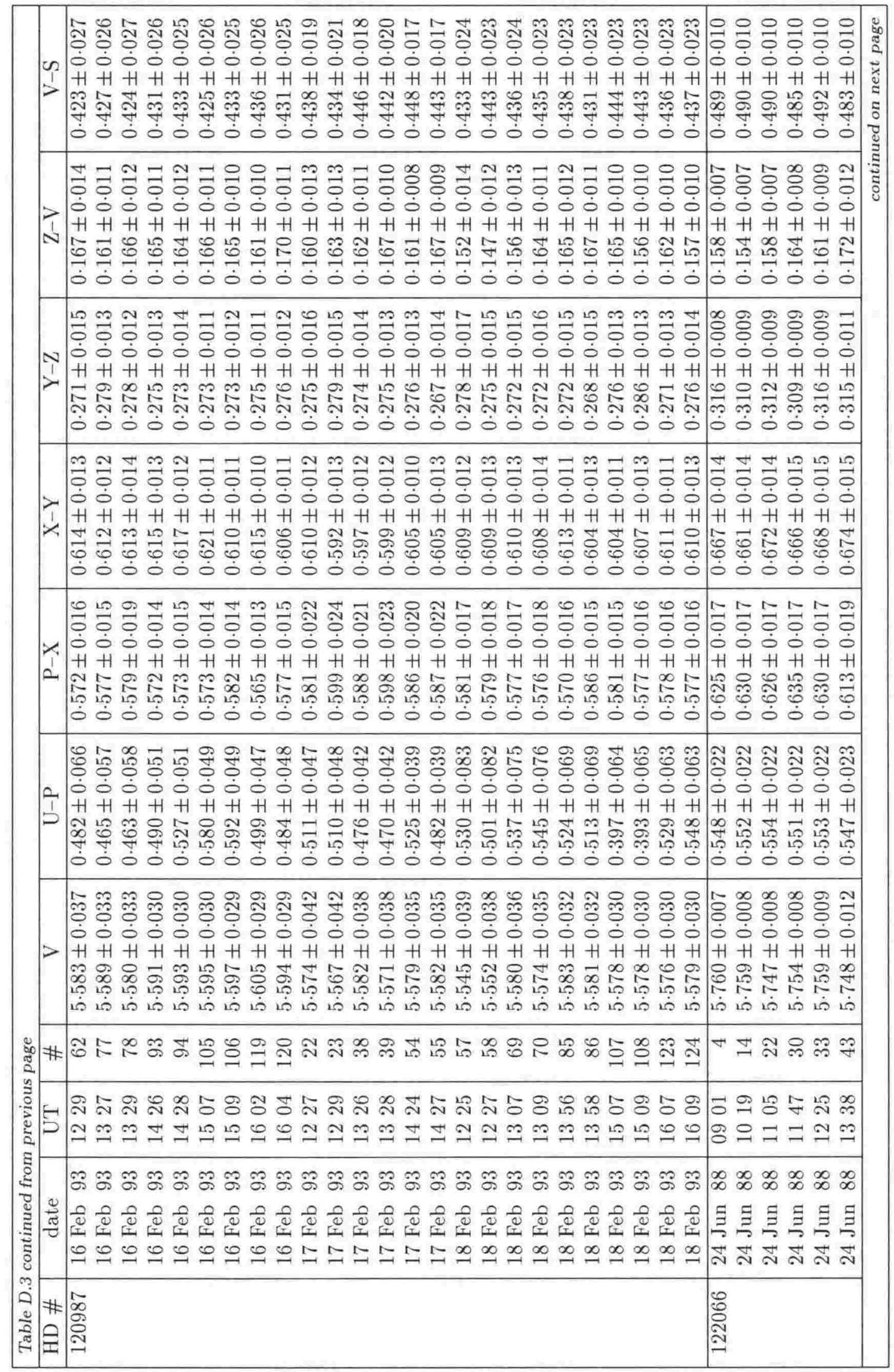




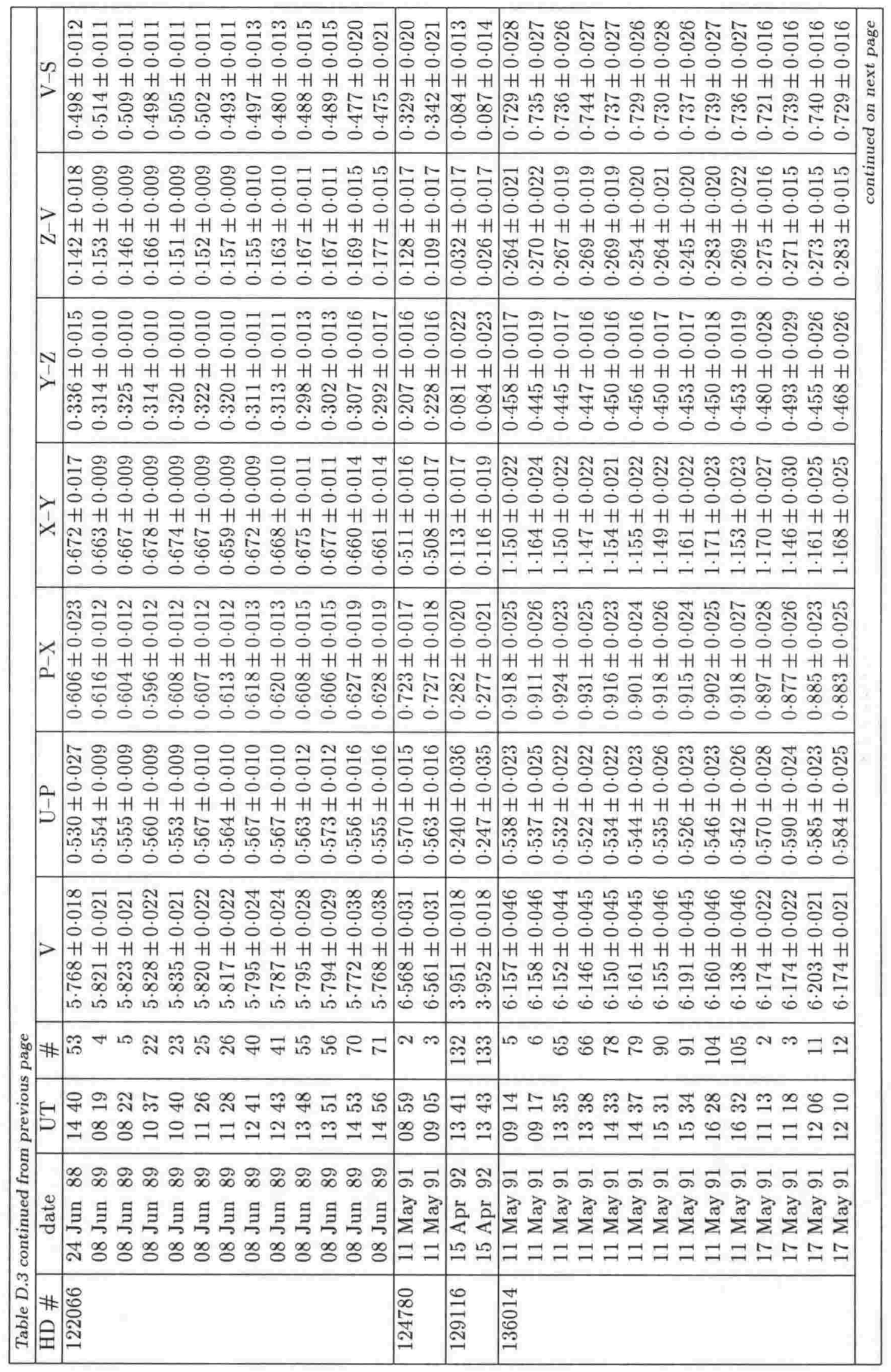




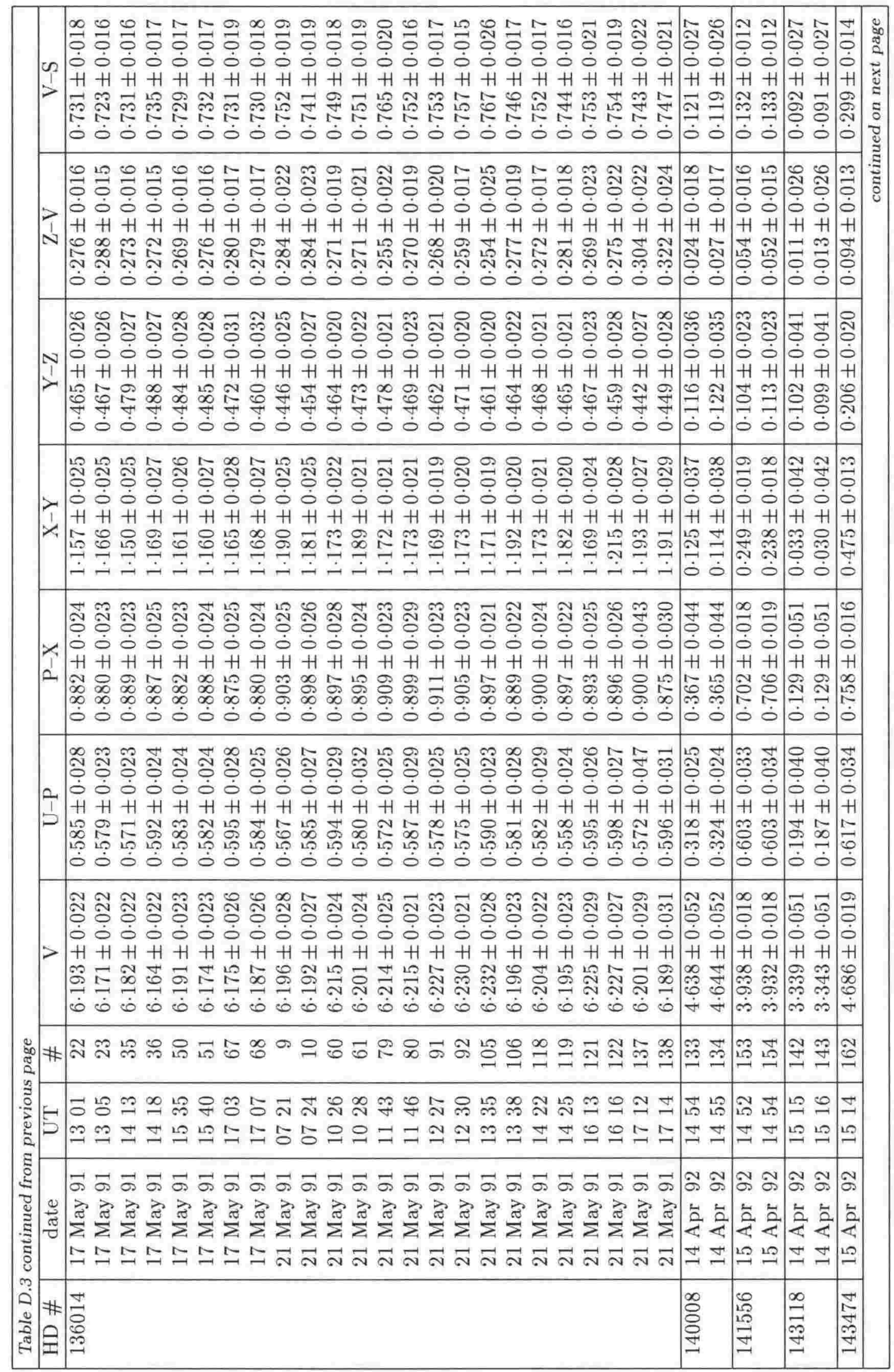




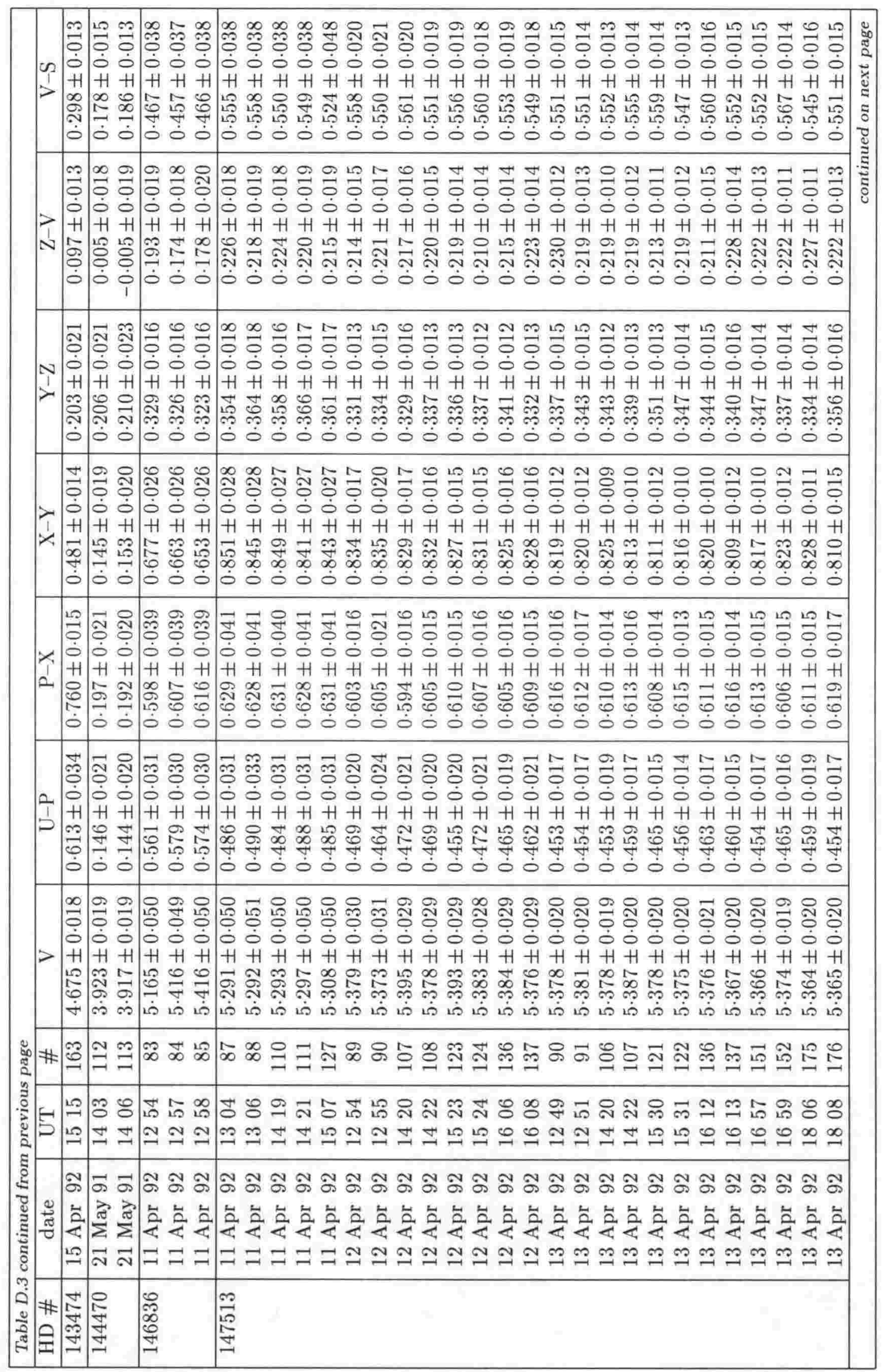




\begin{tabular}{|c|c|c|c|c|c|c|}
\hline & 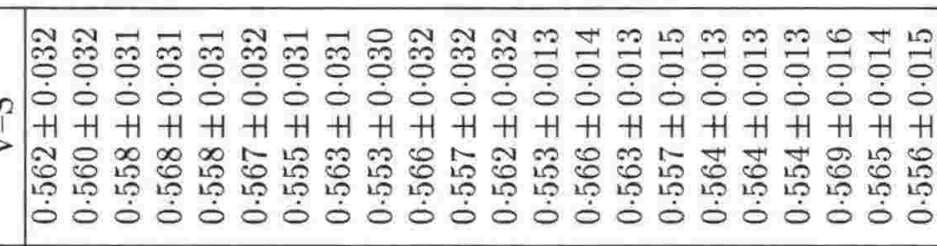 & 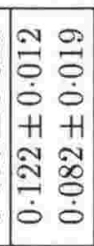 & 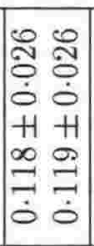 & 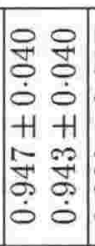 & \begin{tabular}{ll|}
1 & $\infty$ \\
0 & 0 \\
0 & 0 \\
0 & 0 \\
+1 & +1 \\
0 & 0 \\
$\infty$ & 0 \\
0 & 0 \\
0 & 0
\end{tabular} & 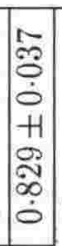 \\
\hline & 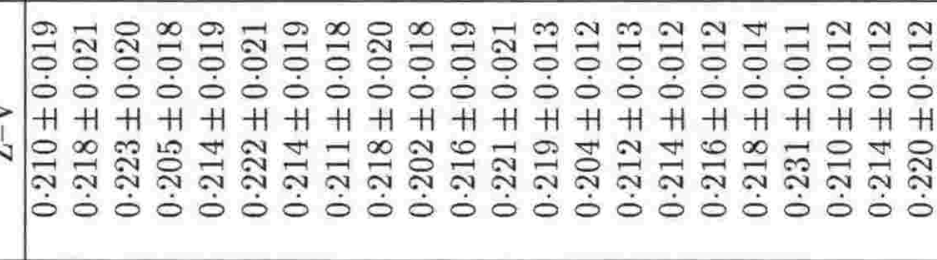 & $\begin{array}{ll}0 & -1 \\
0 & 0 \\
0 & \dot{0} \\
0 & 0 \\
H & H \\
0 & 1 \\
0 & 0 \\
0 & 0 \\
0 & 0 \\
1 & 0\end{array}$ & 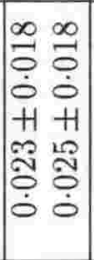 & 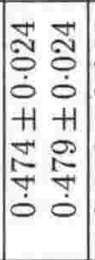 & 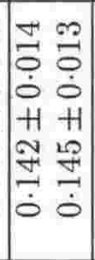 & 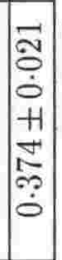 \\
\hline & 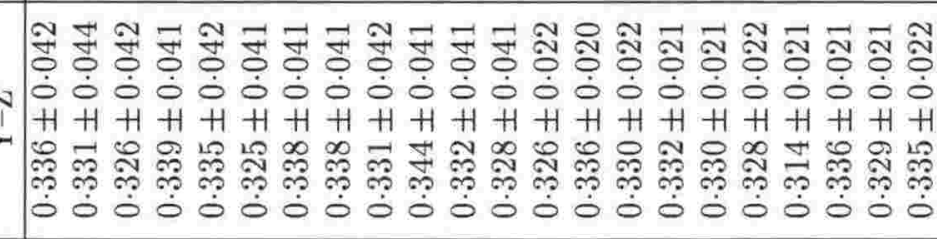 & 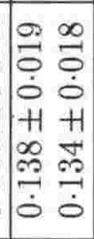 & & & & 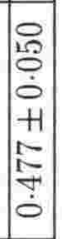 \\
\hline & 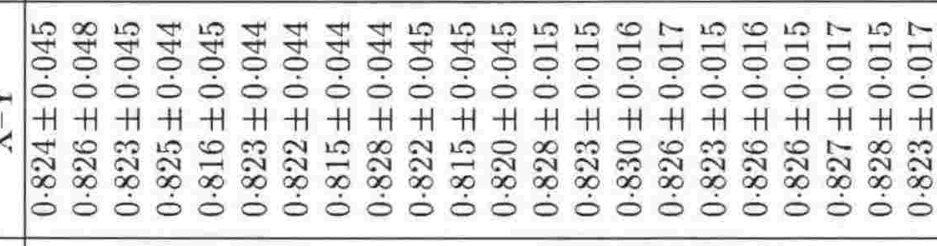 & 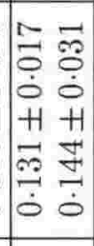 & $\begin{array}{ll}\infty & 0 \\
\approx & 0 \\
0 & 0 \\
0 & 0 \\
+1 & +1 \\
1 & 0 \\
1 & 0 \\
0 & 0 \\
0 & 0 \\
0 & 0\end{array}$ & & 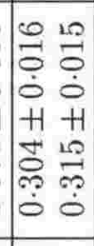 & 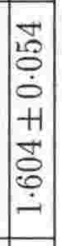 \\
\hline & 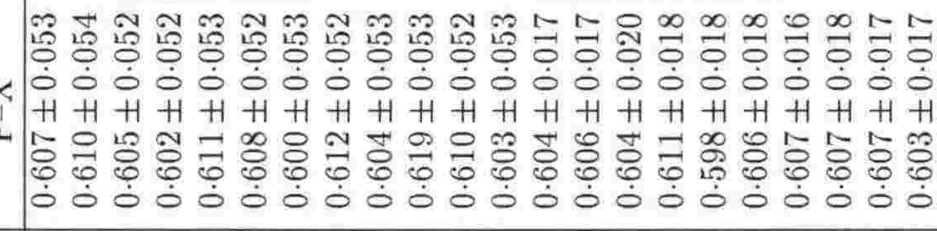 & & & & $\mid \begin{array}{ll}0 & 10 \\
0 & 0 \\
0 & 0 \\
0 & 0 \\
1 & 0 \\
0 & 1 \\
0 & 0 \\
1 & 0 \\
-1 & 0 \\
0 & 0\end{array}$ & 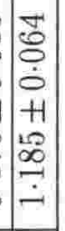 \\
\hline & 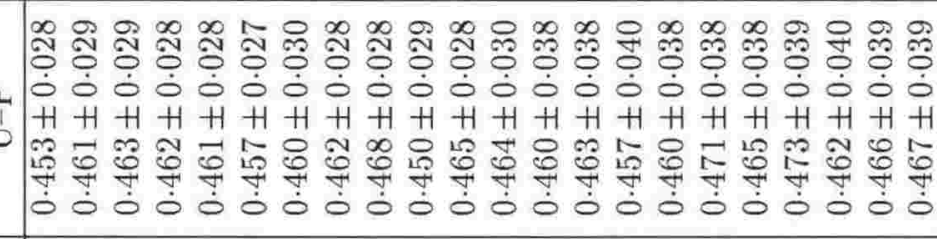 & 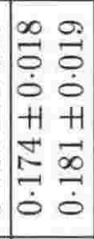 & & 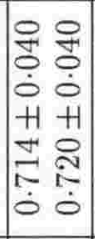 & 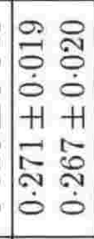 & 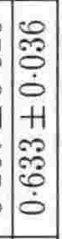 \\
\hline & 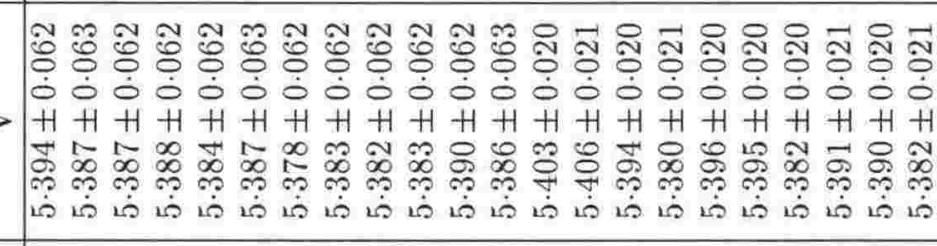 & & & & 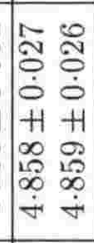 & 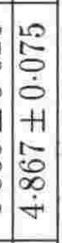 \\
\hline & 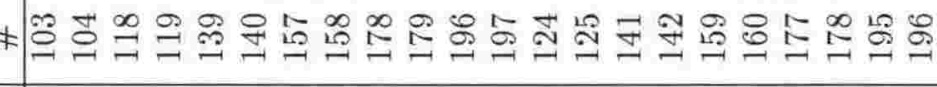 & & & $\ddot{0}$ & $\stackrel{\mathfrak{I}}{\mathfrak{I}}$ & $\because$ \\
\hline & 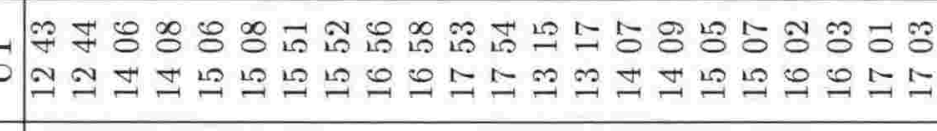 & & & & $\begin{array}{ll}2 & -1 \\
0 & 0 \\
- & 0\end{array}$ & 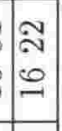 \\
\hline & 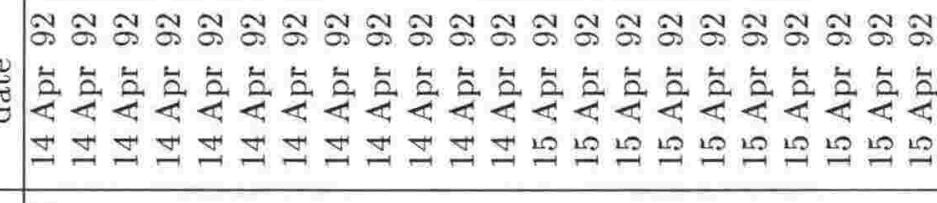 & & 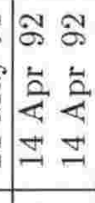 & 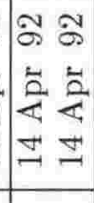 & 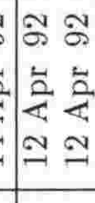 & 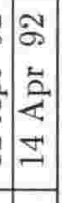 \\
\hline & 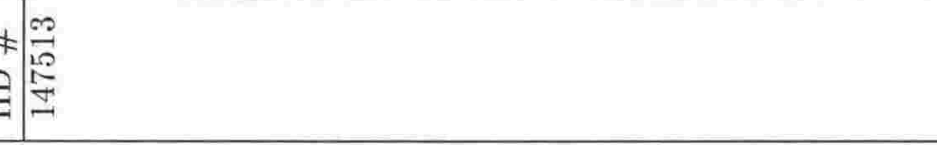 & 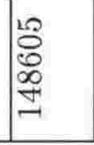 & 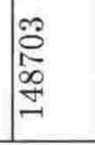 & 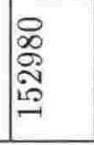 & 疍 & 点 \\
\hline
\end{tabular}




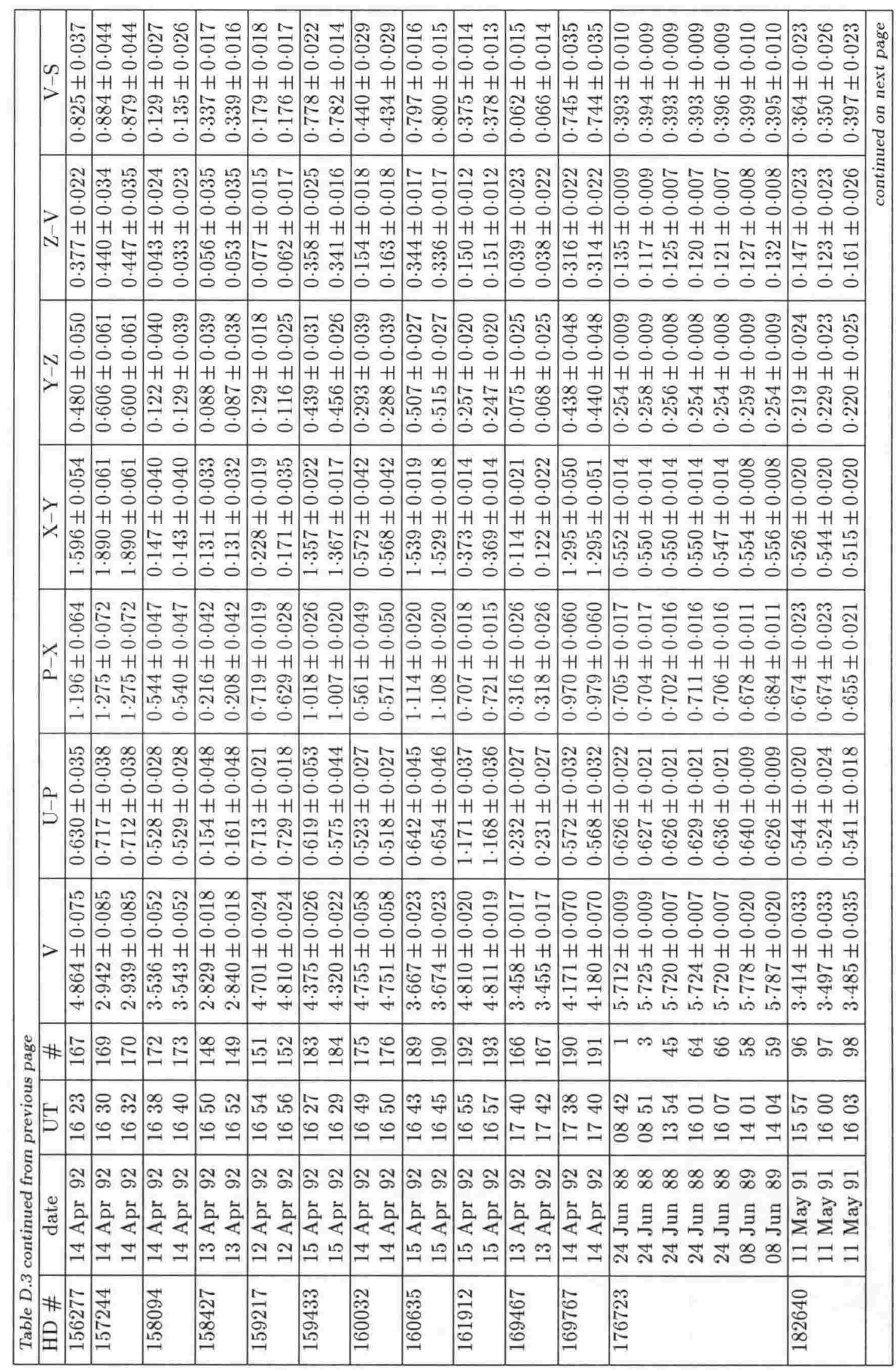




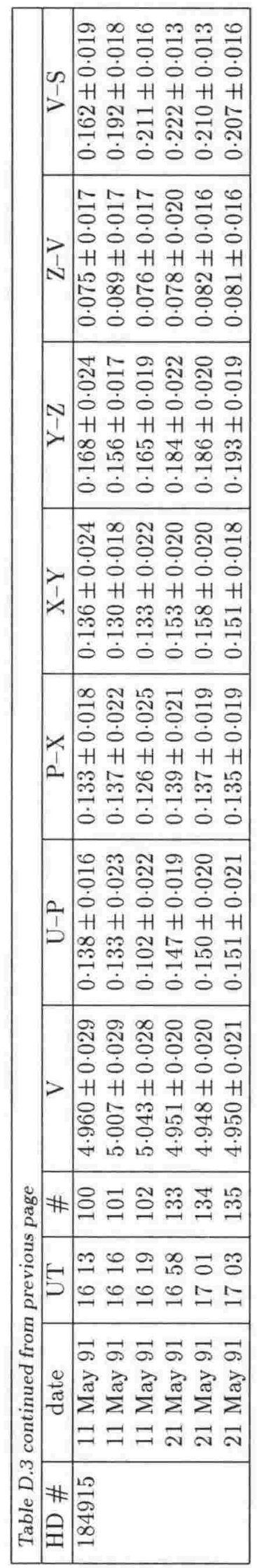




\begin{tabular}{|c|c|}
\hline & 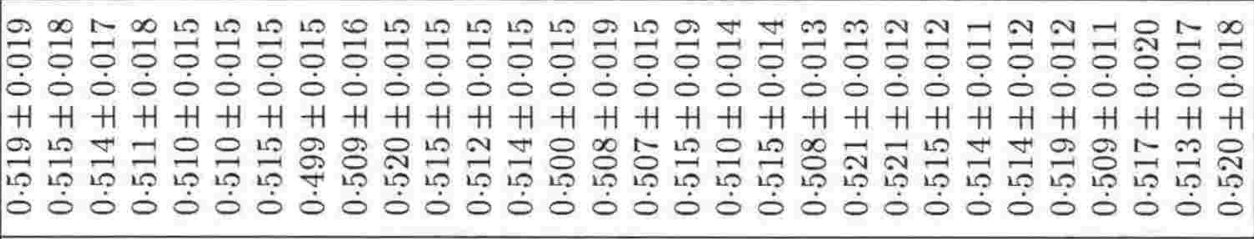 \\
\hline & 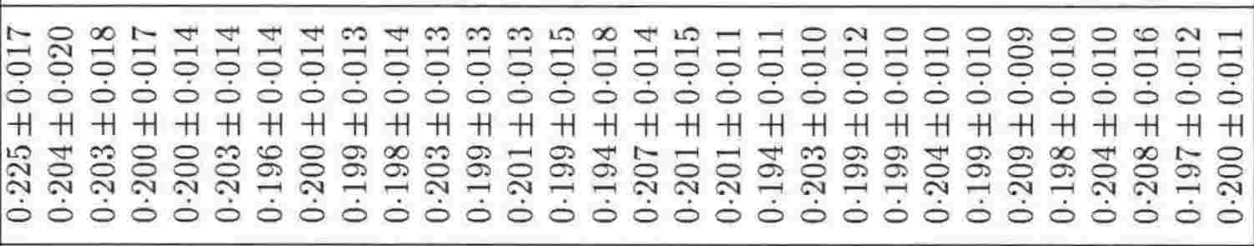 \\
\hline & 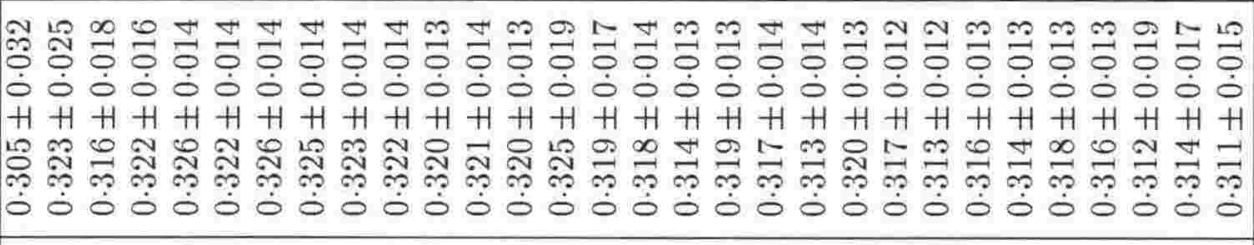 \\
\hline & 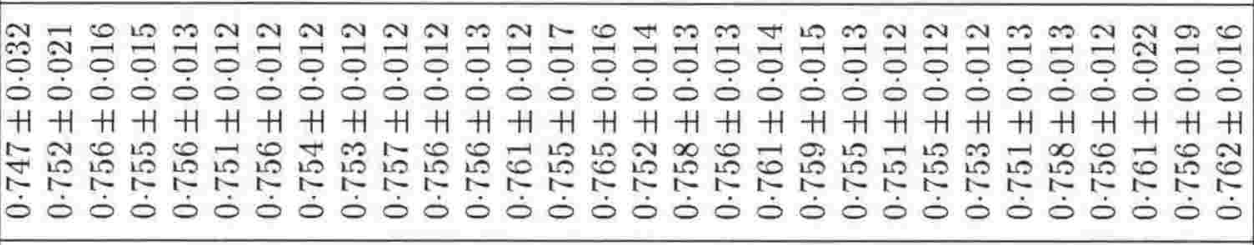 \\
\hline & 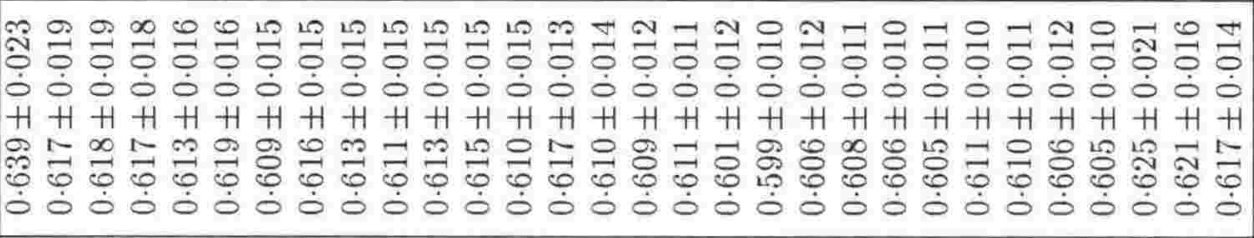 \\
\hline & 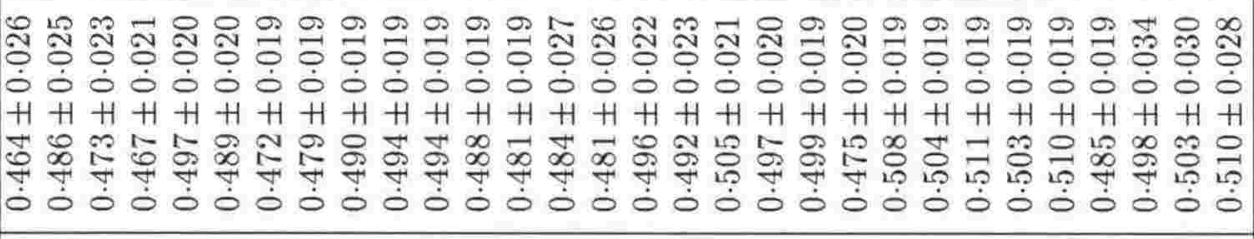 \\
\hline & 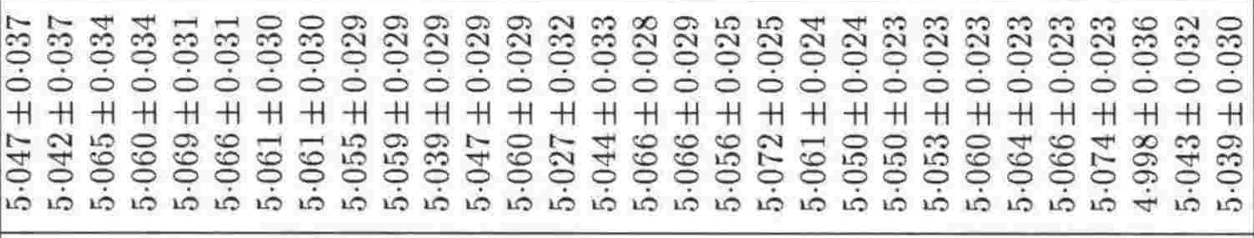 \\
\hline \# & 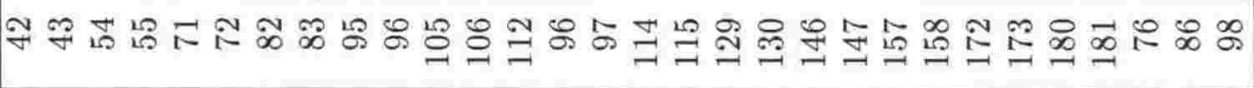 \\
\hline & 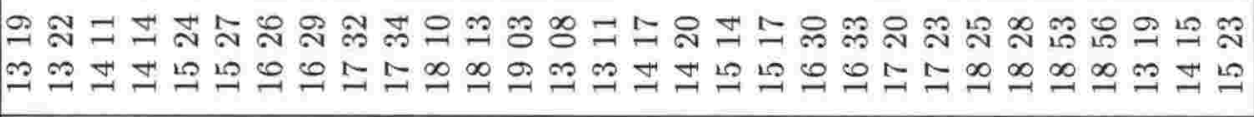 \\
\hline & 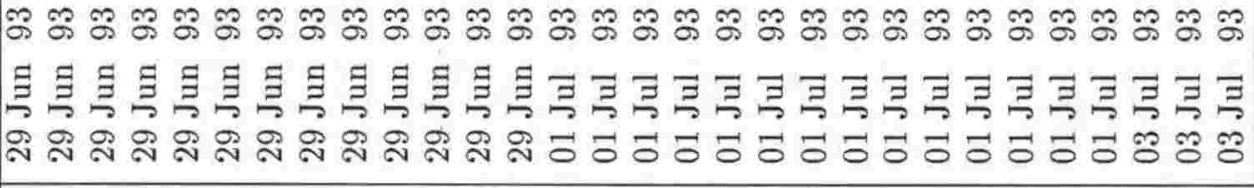 \\
\hline & \\
\hline
\end{tabular}




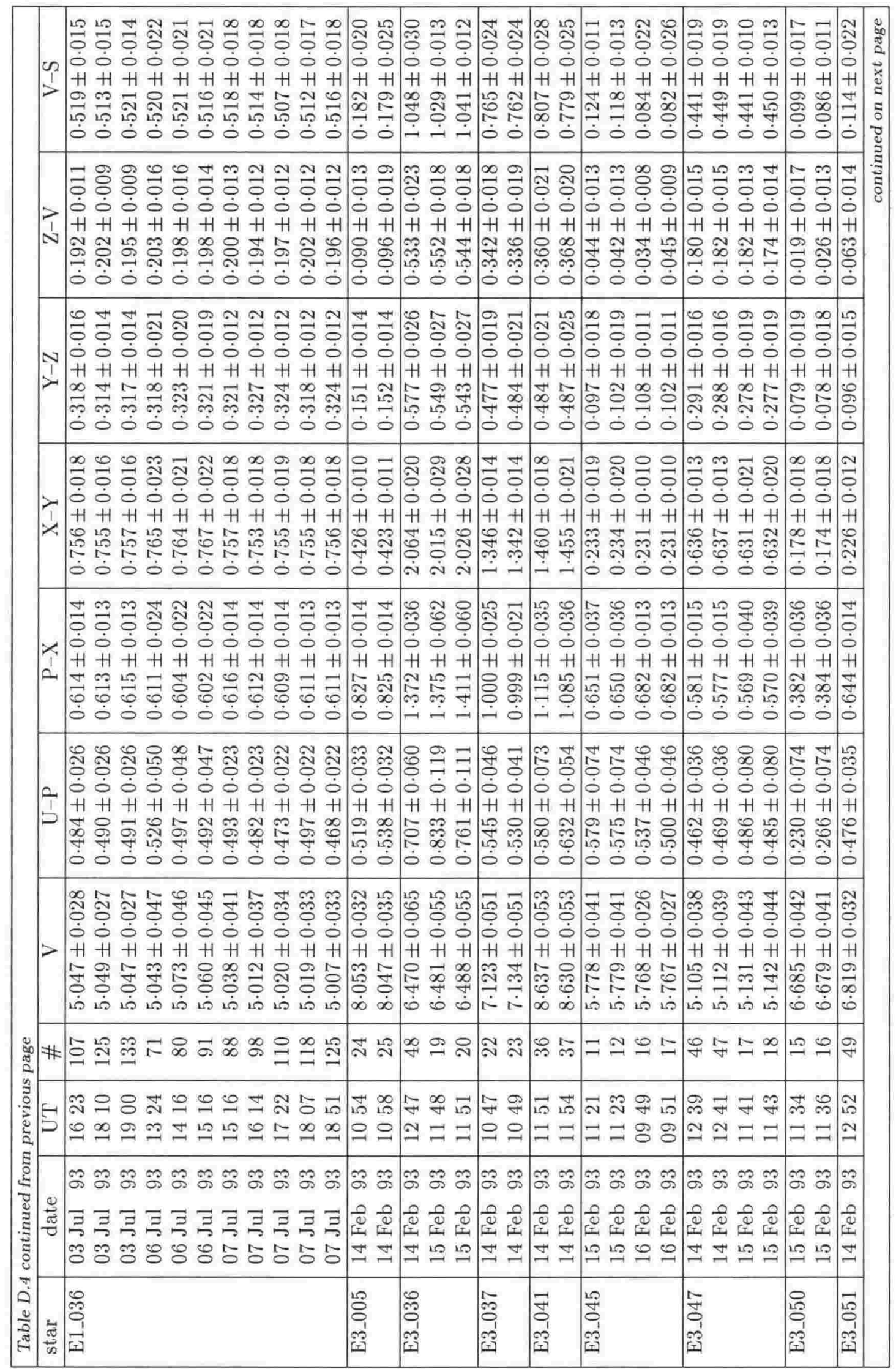




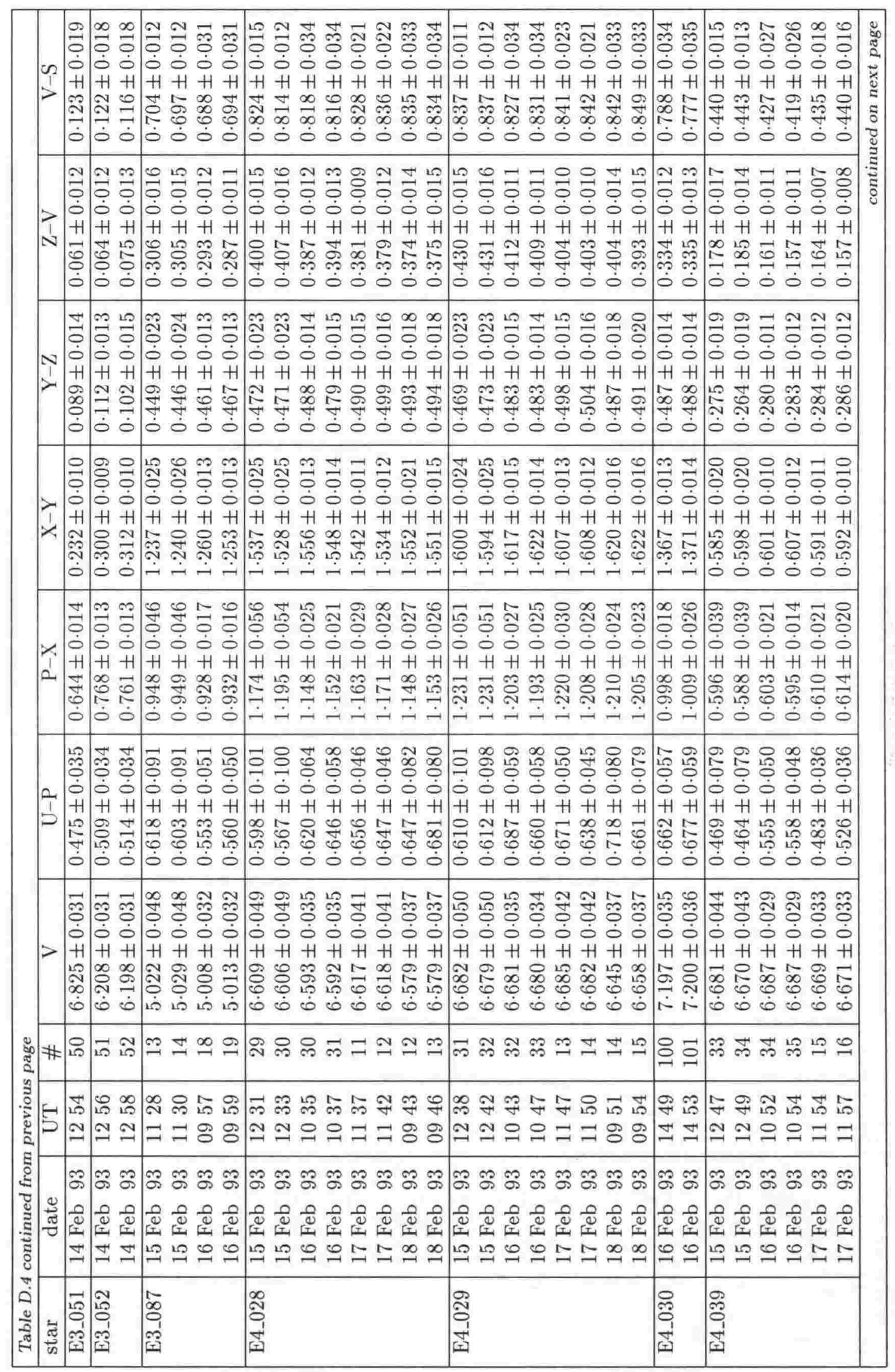




\begin{tabular}{|c|c|c|c|}
\hline & 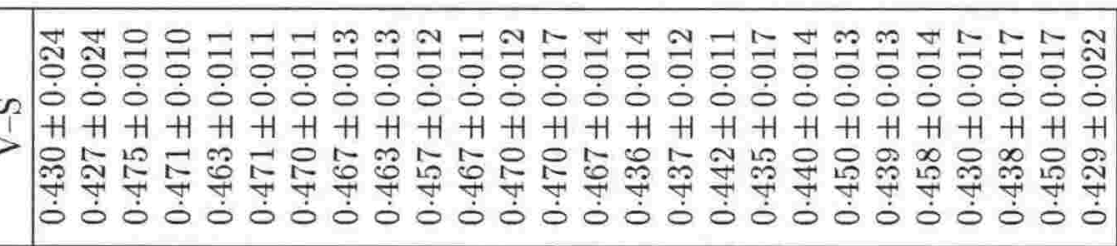 & \begin{tabular}{ll}
0 & 0 \\
0 & 0 \\
0 & 0 \\
0 & 0 \\
1 & +1 \\
0 & 0 \\
\hdashline & $\stackrel{9}{7}$ \\
$\dot{0}$ & 0 \\
\end{tabular} & 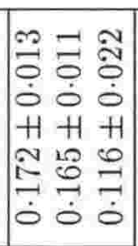 \\
\hline & 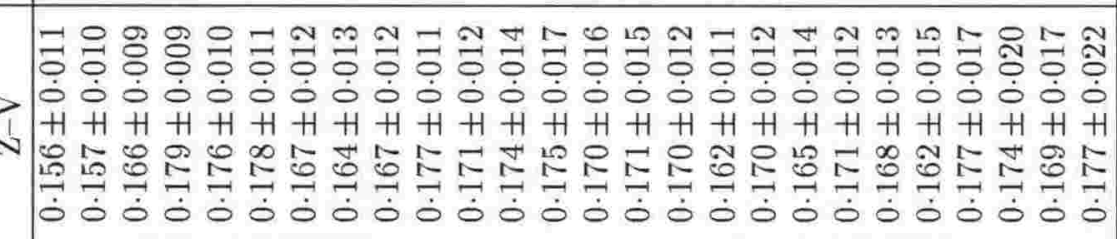 & 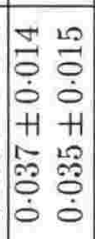 & 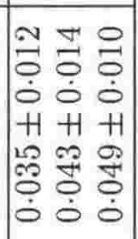 \\
\hline & 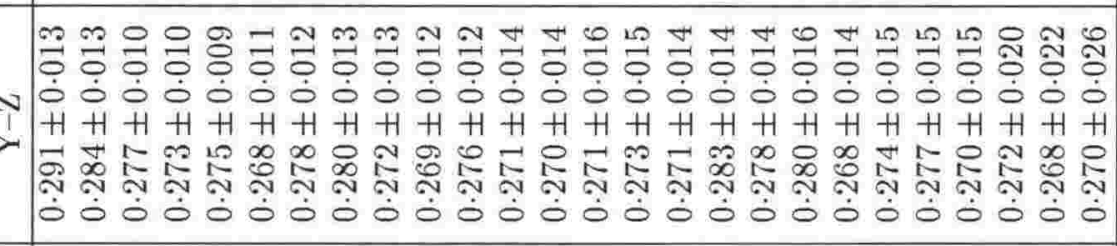 & 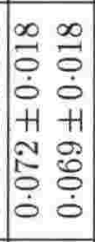 & 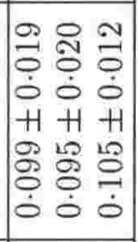 \\
\hline & 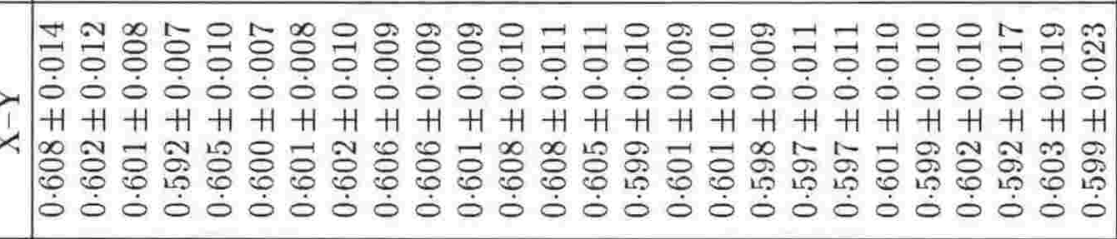 & & 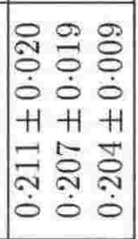 \\
\hline & 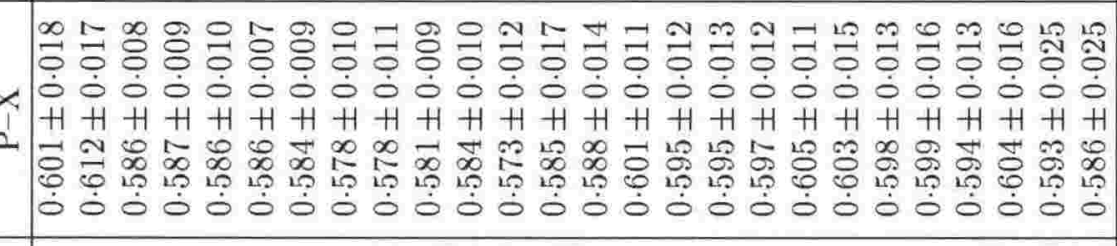 & & 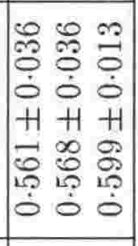 \\
\hline & 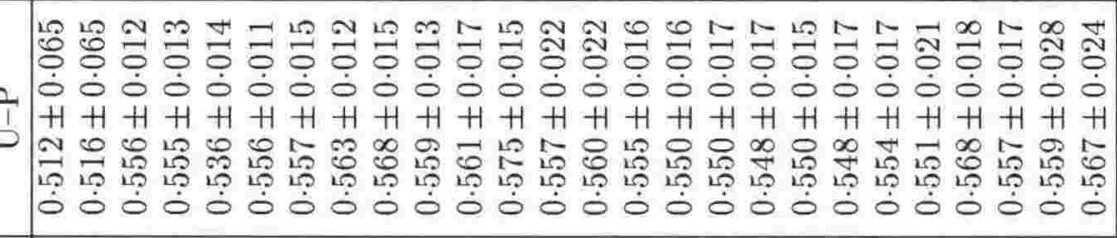 & & 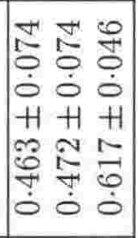 \\
\hline & 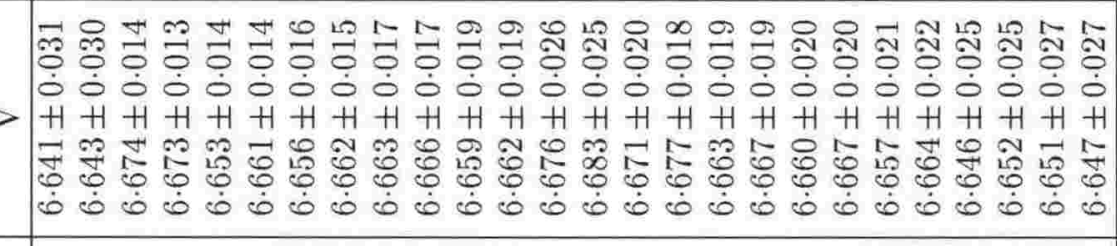 & & 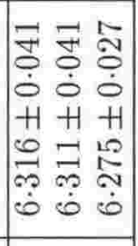 \\
\hline & 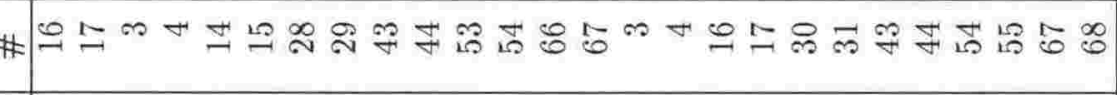 & $20 \%$ & F $\stackrel{\infty}{+}$ \\
\hline & 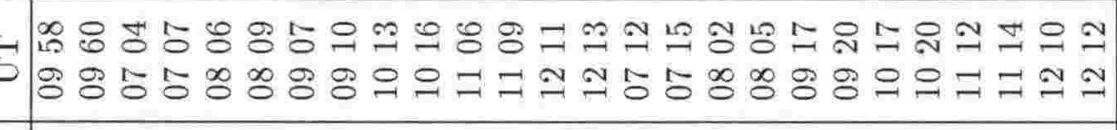 & & 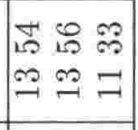 \\
\hline & 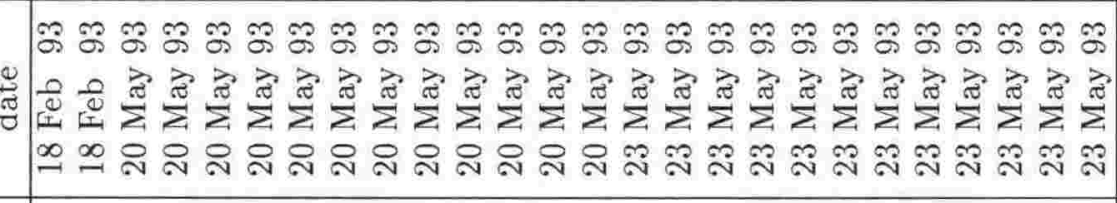 & 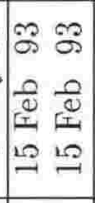 & 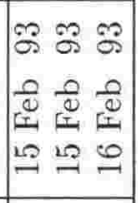 \\
\hline & 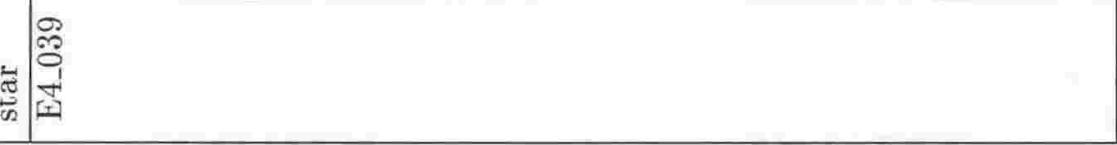 & & 素 \\
\hline
\end{tabular}




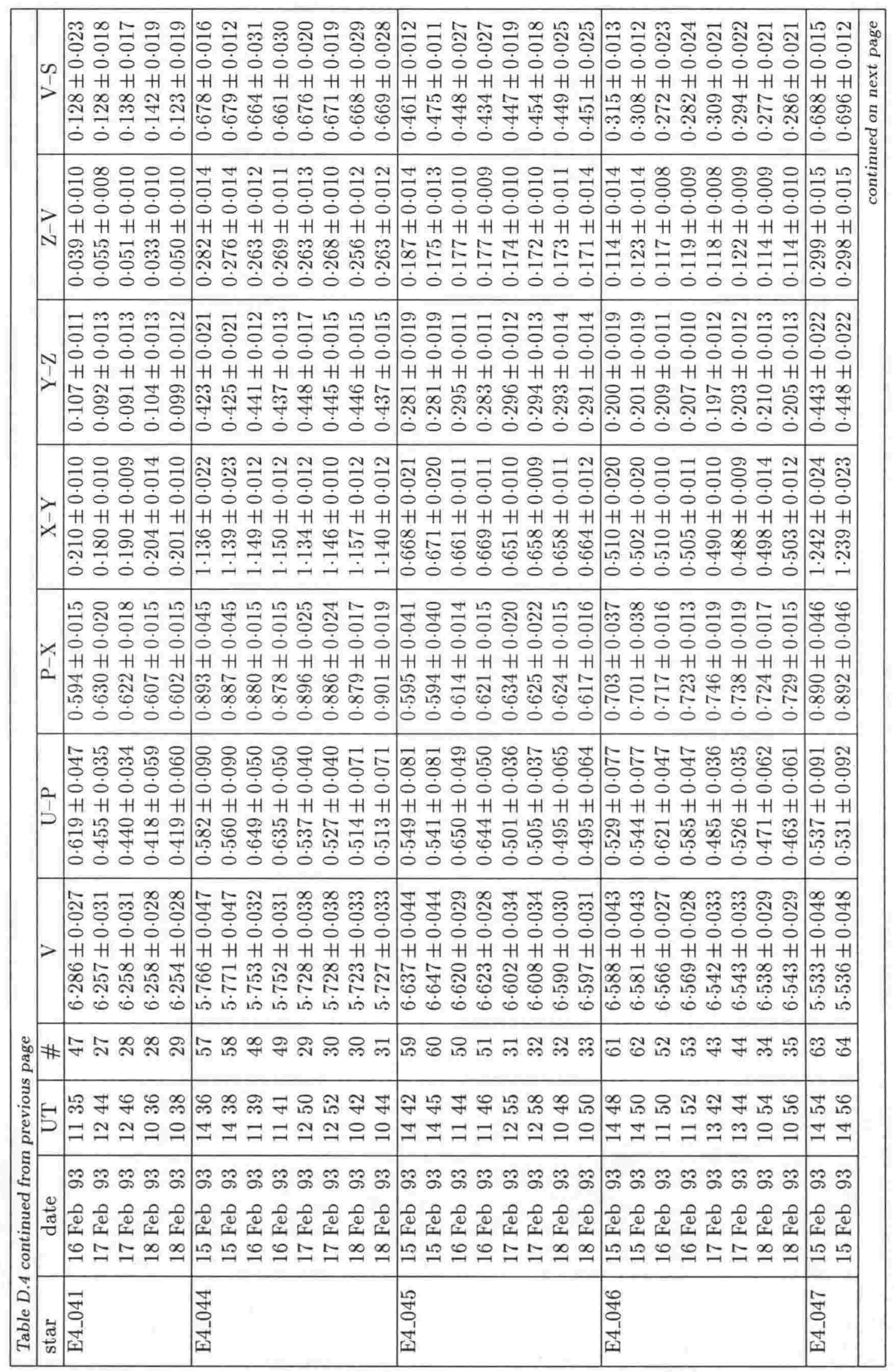




\begin{tabular}{|c|c|c|c|c|c|c|}
\hline & & 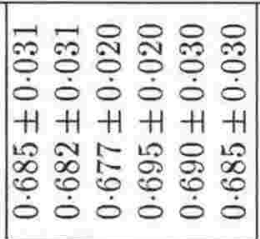 & 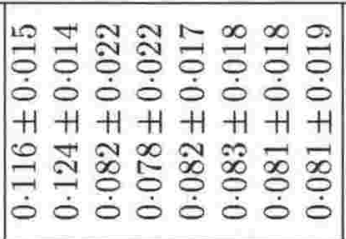 & 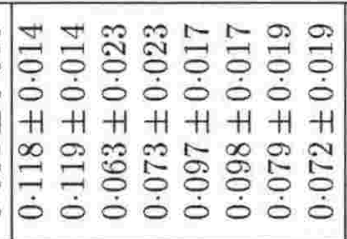 & 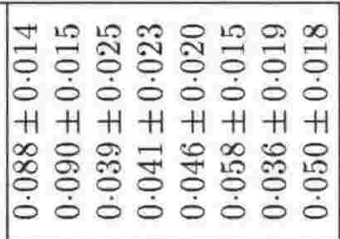 & $\begin{array}{c}- \\
\\
0 \\
0 \\
+ \\
0 \\
0 \\
0 \\
0 \\
0\end{array}$ \\
\hline & & 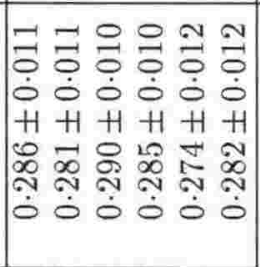 & 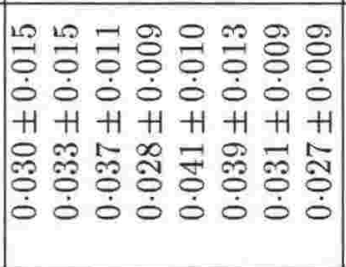 & 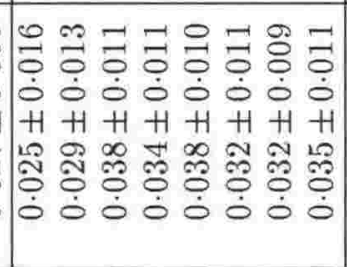 & 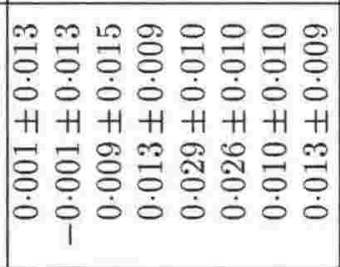 & 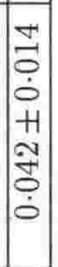 \\
\hline & & 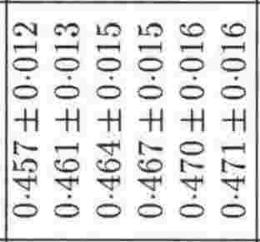 & 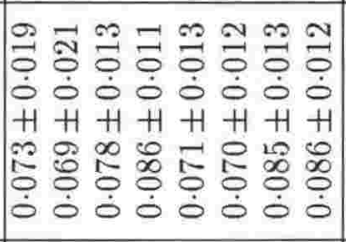 & 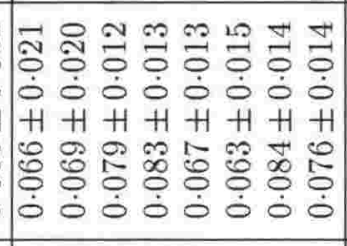 & 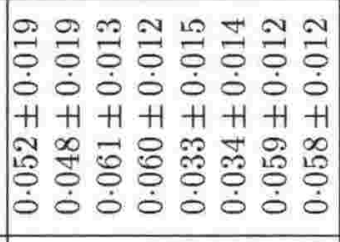 & 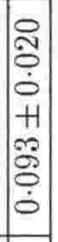 \\
\hline & & 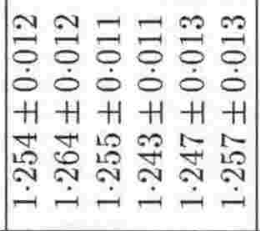 & 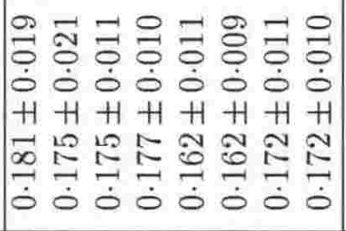 & 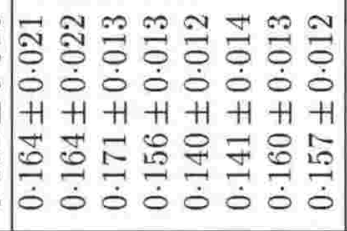 & 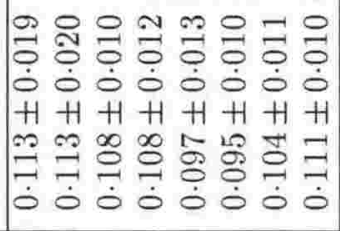 & 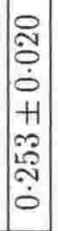 \\
\hline & & 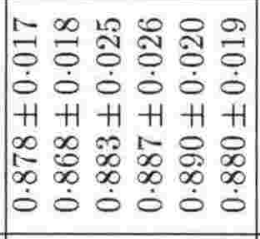 & 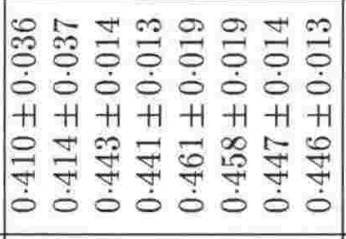 & 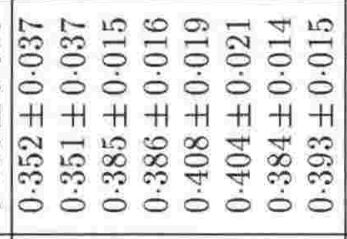 & 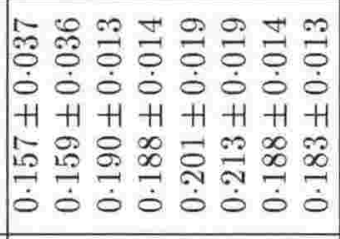 & $\begin{array}{c}1 \\
2 \\
0 \\
0 \\
0 \\
1 \\
1 \\
2 \\
0 \\
0 \\
0 \\
0\end{array}$ \\
\hline & & 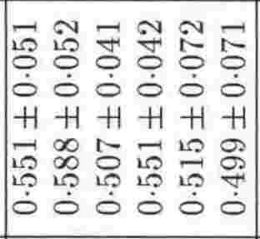 & 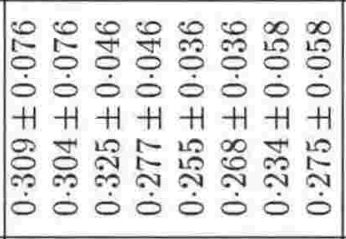 & 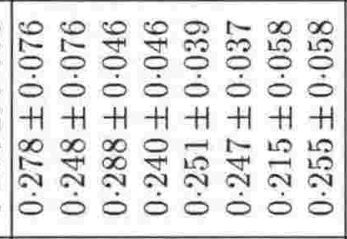 & 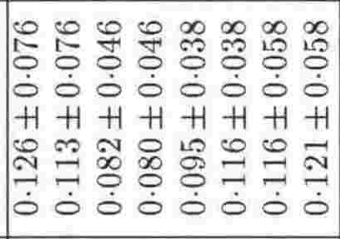 & $\begin{array}{l}1 \\
5 \\
0 \\
0 \\
+1 \\
-1 \\
2 \\
2 \\
0 \\
0\end{array}$ \\
\hline & & 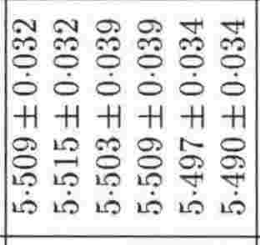 & 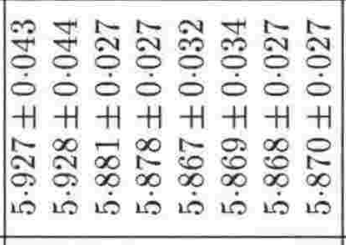 & 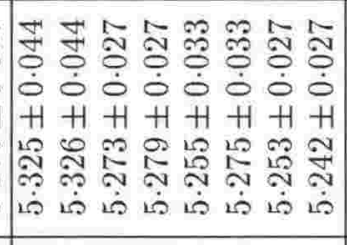 & 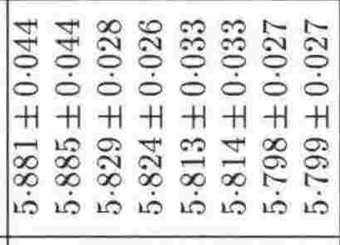 & 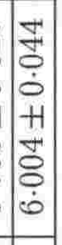 \\
\hline & & 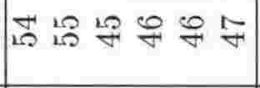 & 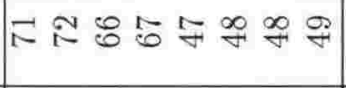 & 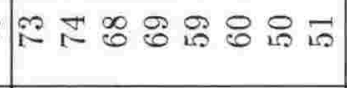 & 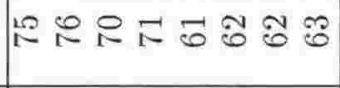 & 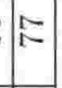 \\
\hline & & 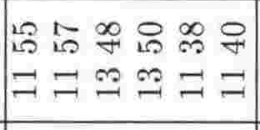 & 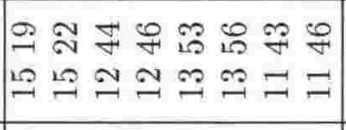 & 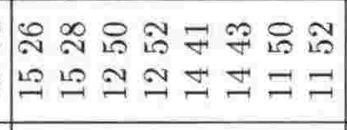 & 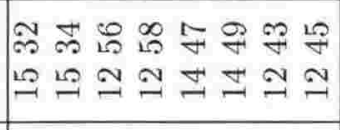 & $\begin{array}{l}\infty \\
\infty \\
20 \\
2 \\
-1\end{array}$ \\
\hline & & 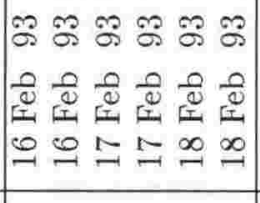 & 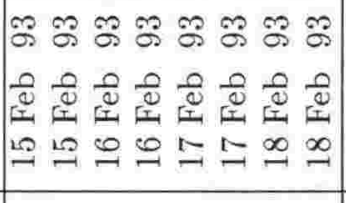 & 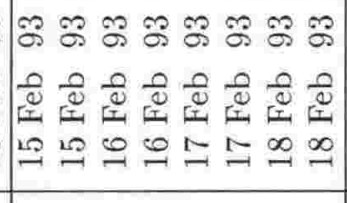 & 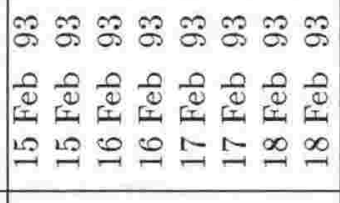 & $\begin{array}{l}2 \\
0 \\
\frac{2}{20} \\
12 \\
-12\end{array}$ \\
\hline 完. & & & 站 & $\mid \begin{array}{l}0 \\
0 \\
0 \\
7 \\
1 \\
1\end{array}$ & 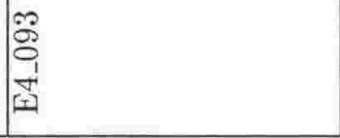 & 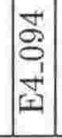 \\
\hline
\end{tabular}




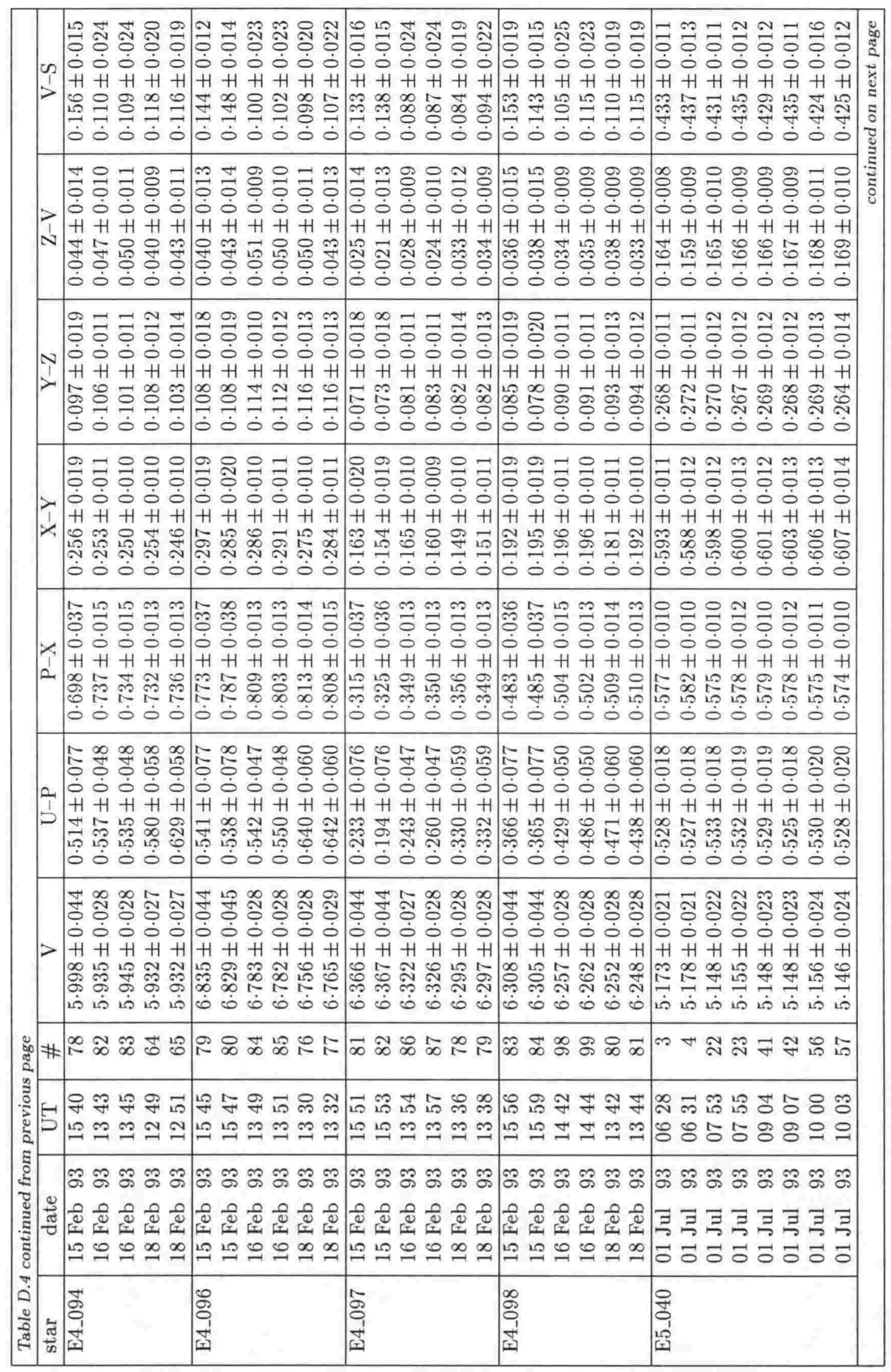




\begin{tabular}{|c|c|}
\hline & 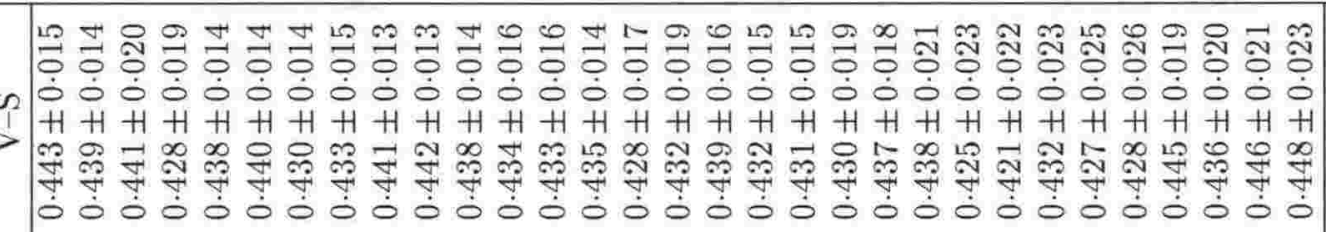 \\
\hline & 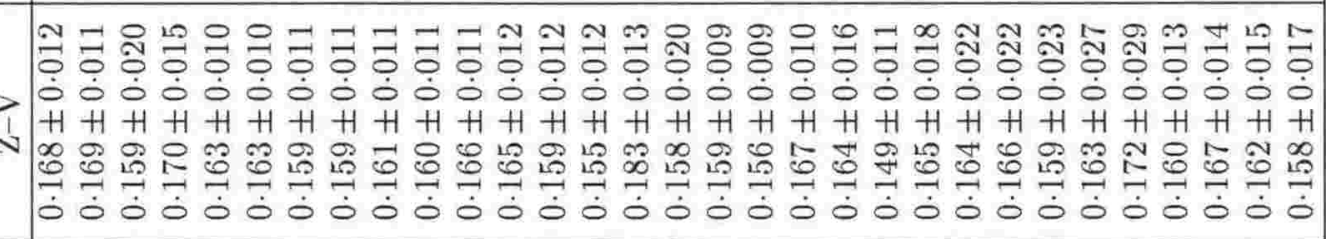 \\
\hline & 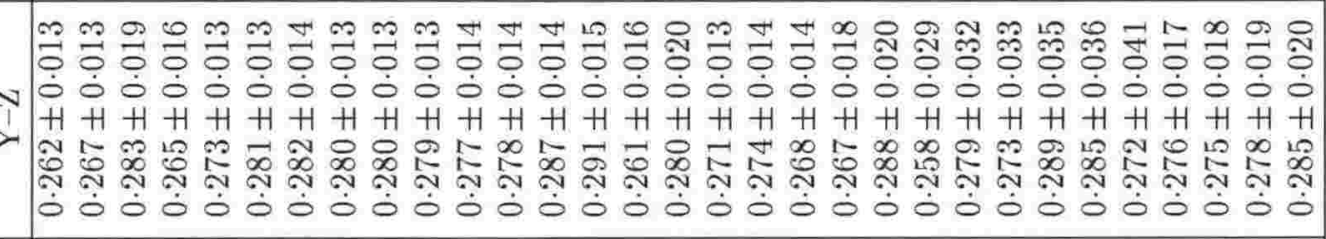 \\
\hline & 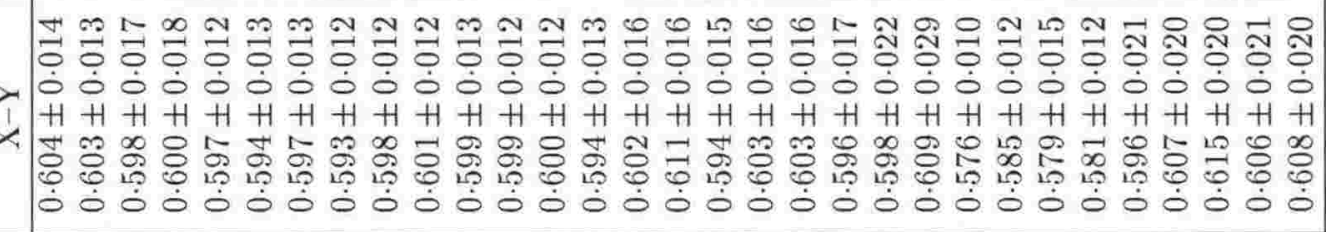 \\
\hline & 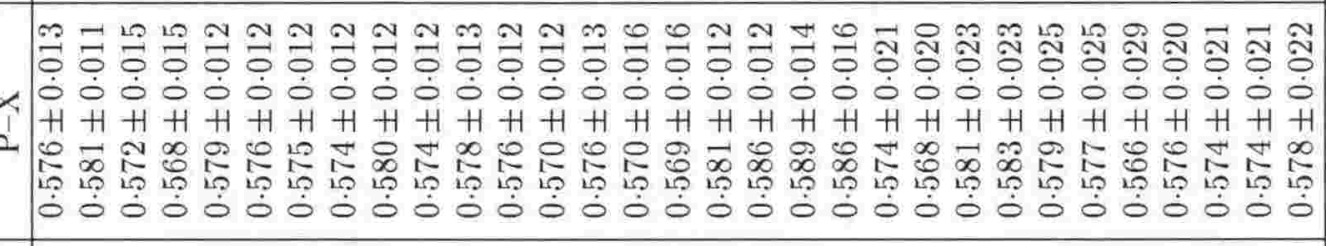 \\
\hline & 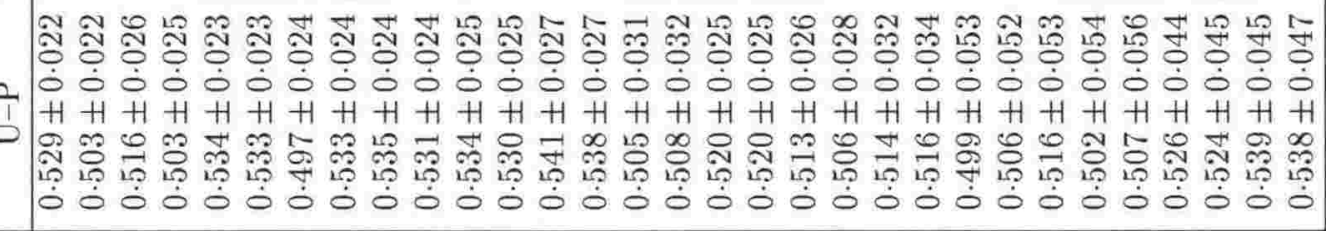 \\
\hline & 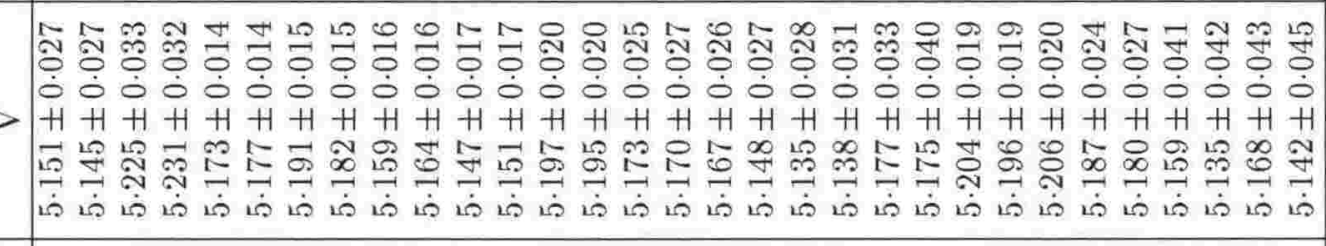 \\
\hline & 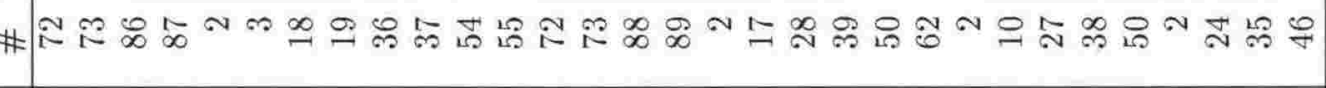 \\
\hline & 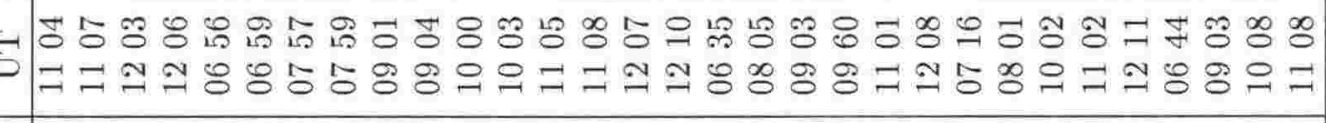 \\
\hline & 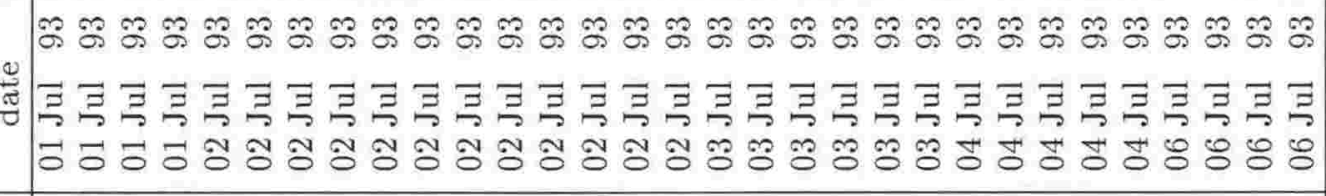 \\
\hline & 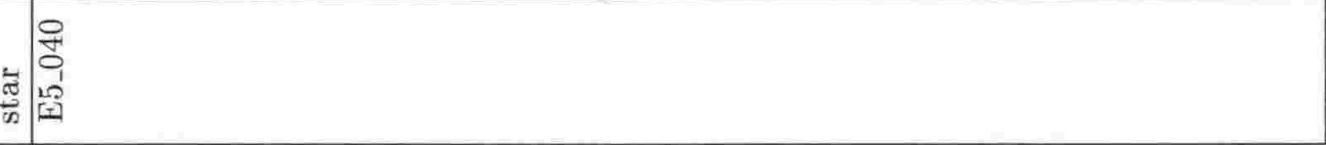 \\
\hline
\end{tabular}




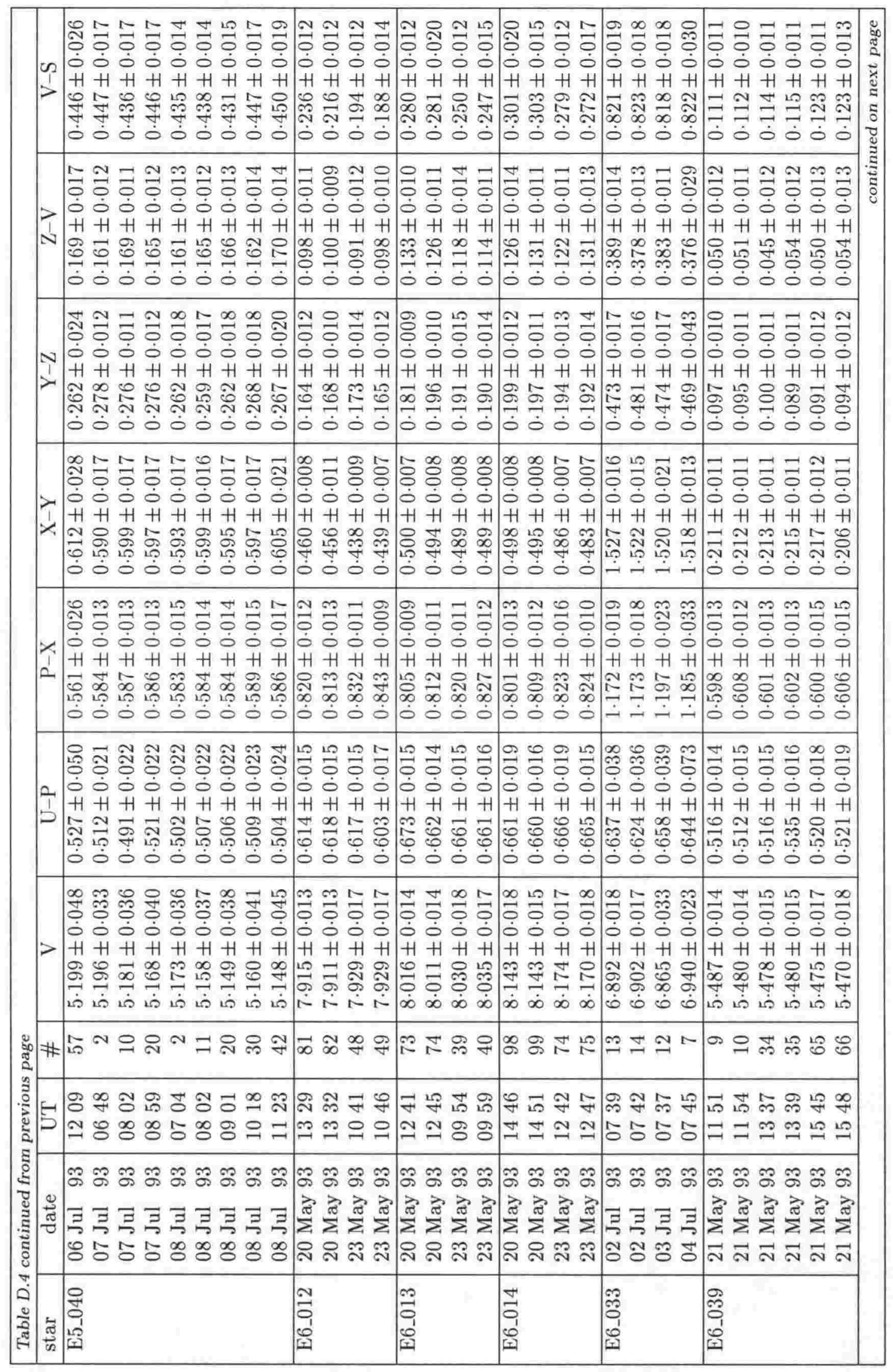




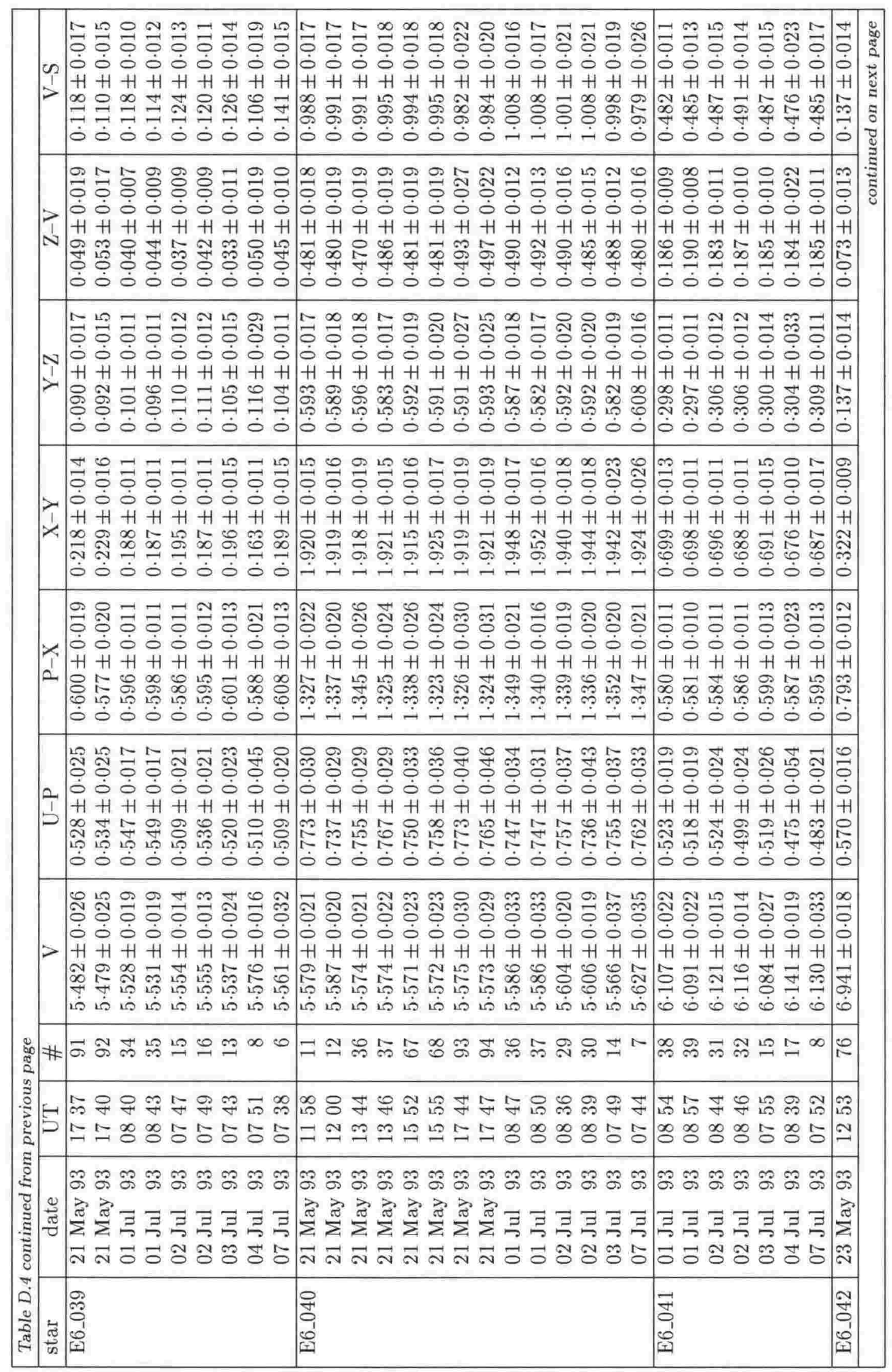




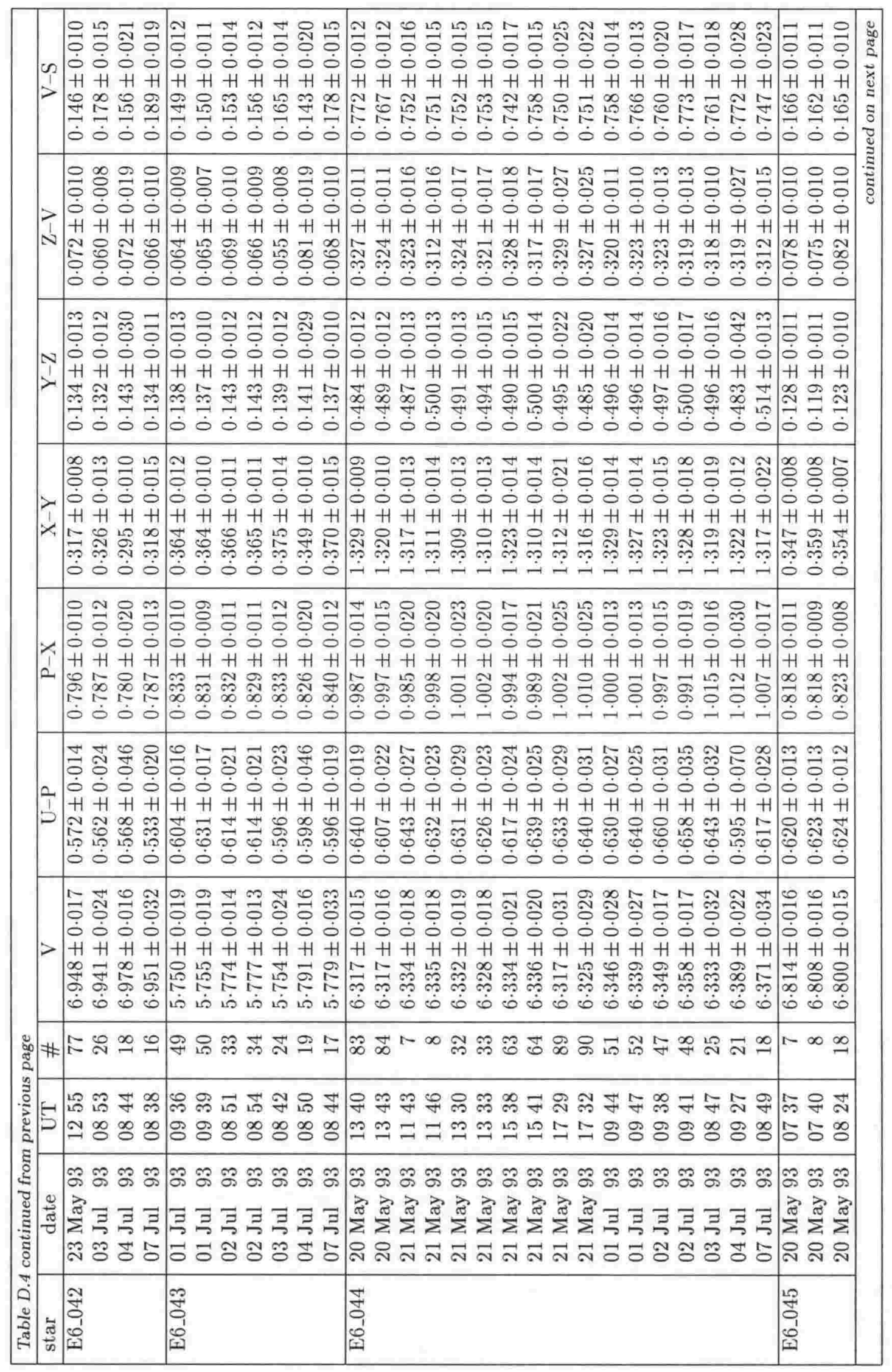




\begin{tabular}{|c|c|}
\hline & 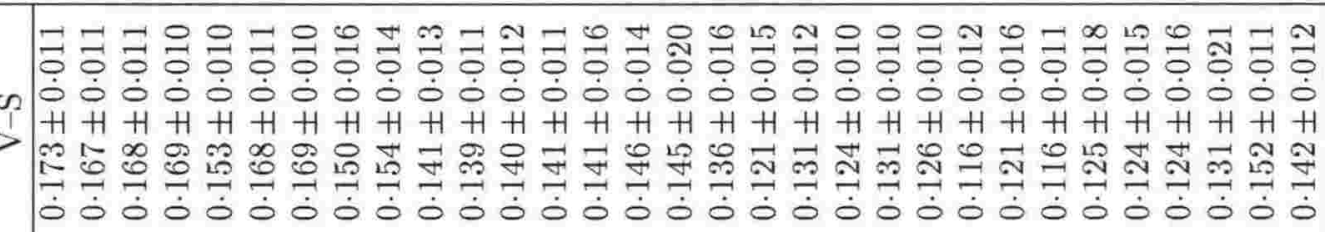 \\
\hline & 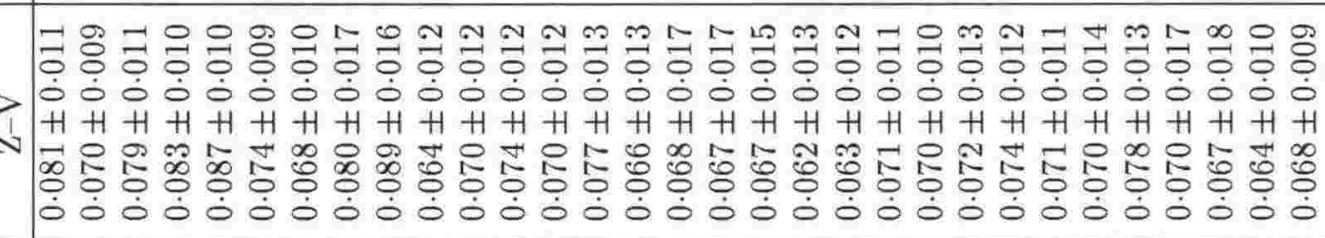 \\
\hline & 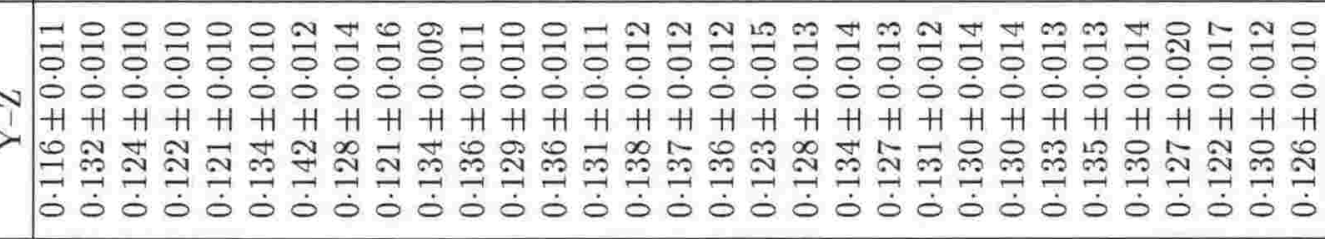 \\
\hline & 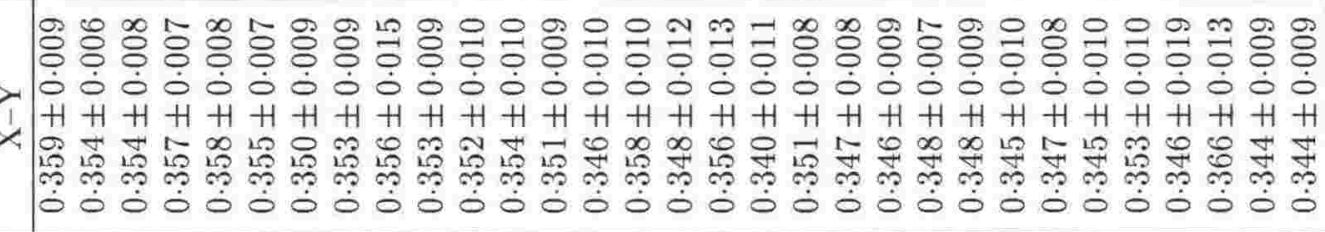 \\
\hline & 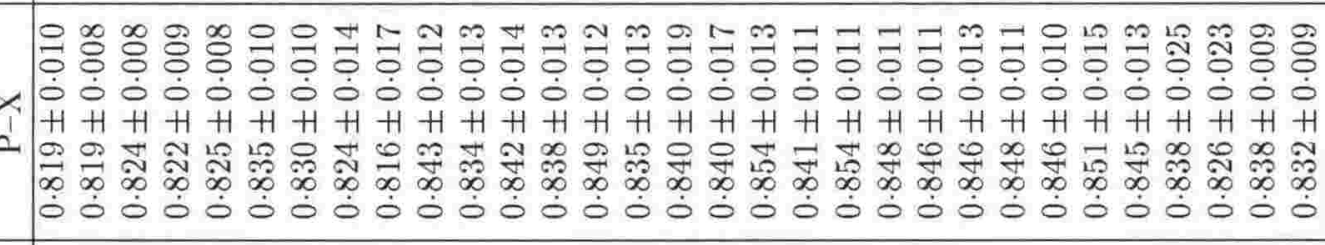 \\
\hline & 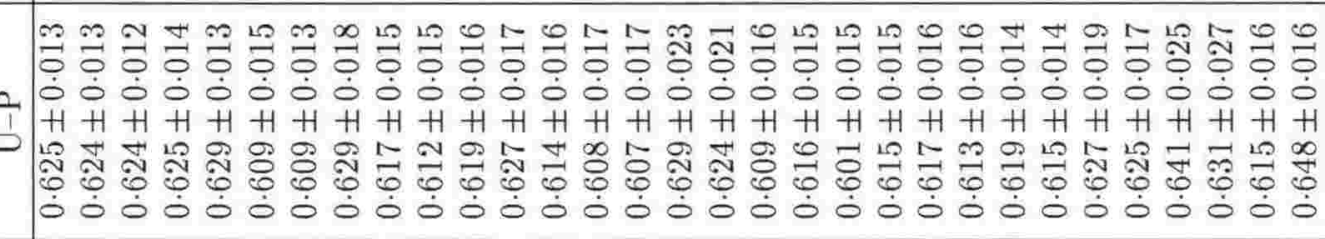 \\
\hline & 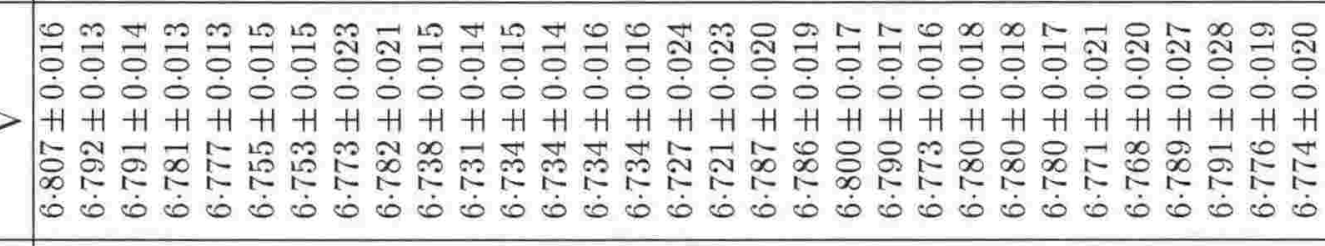 \\
\hline & 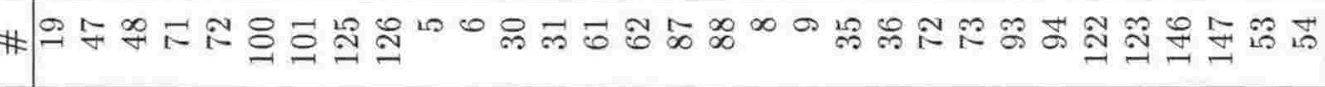 \\
\hline & 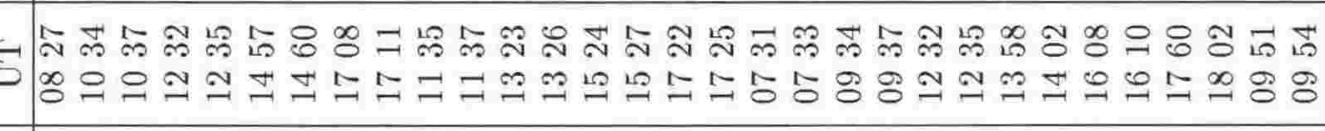 \\
\hline & 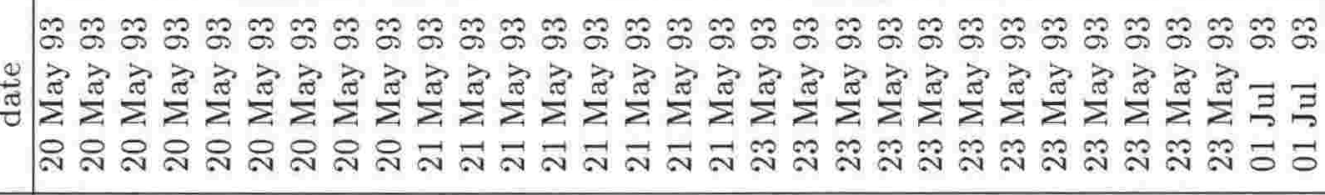 \\
\hline & 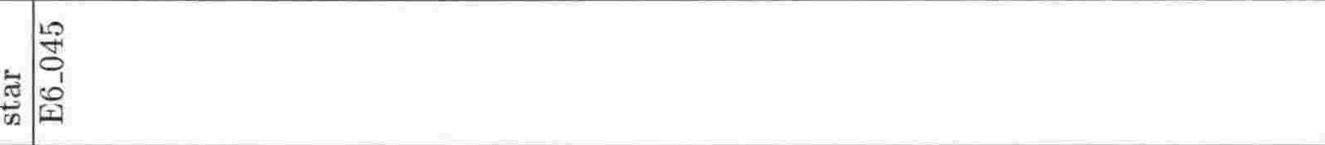 \\
\hline
\end{tabular}




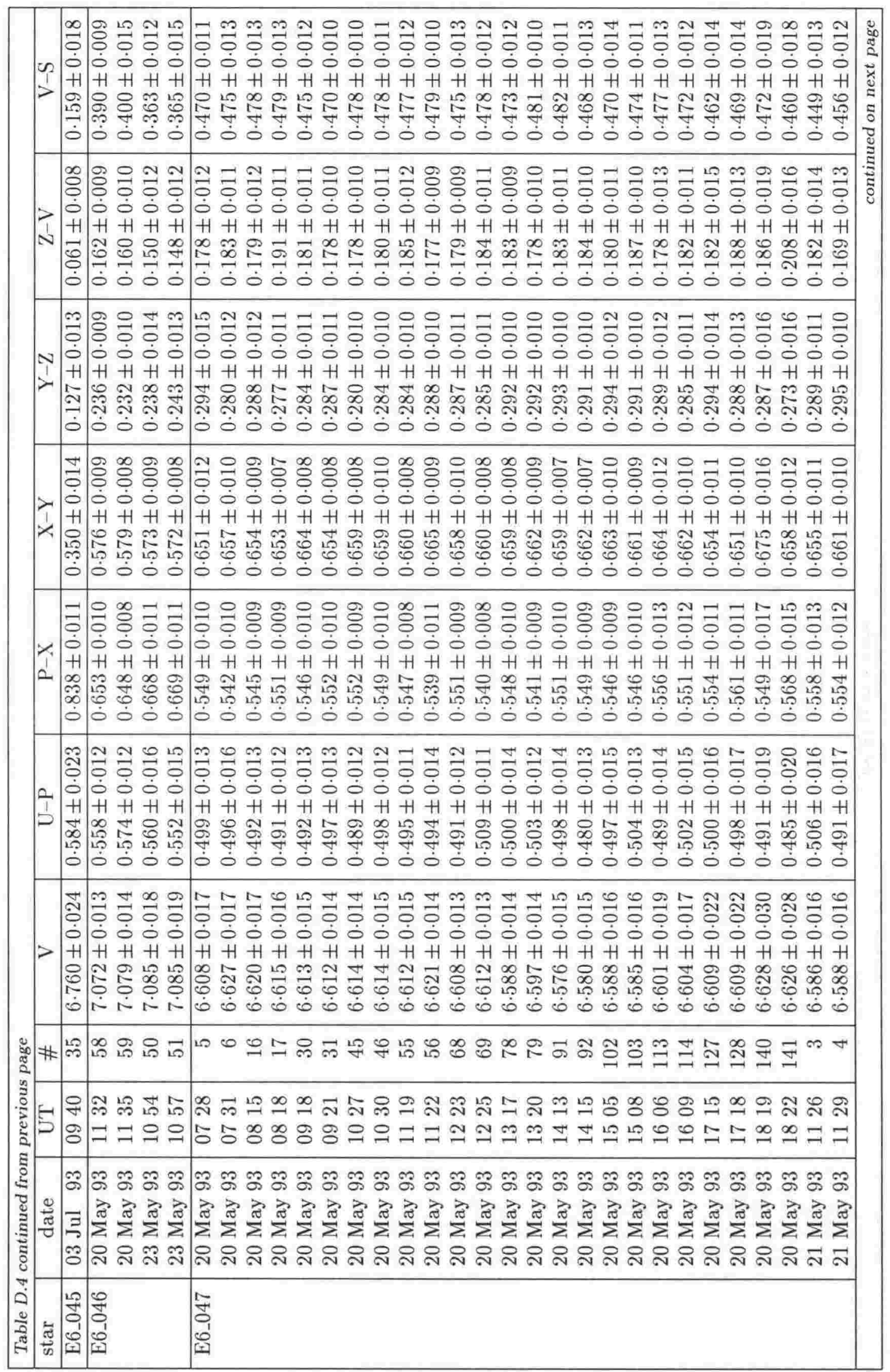




\begin{tabular}{|c|c|}
\hline & 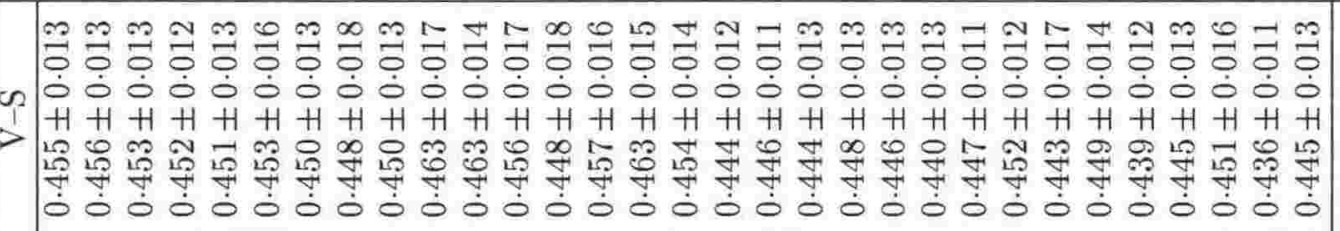 \\
\hline & 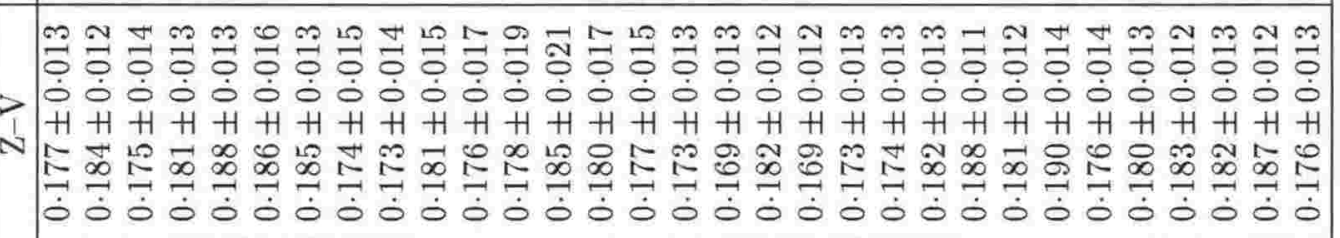 \\
\hline & 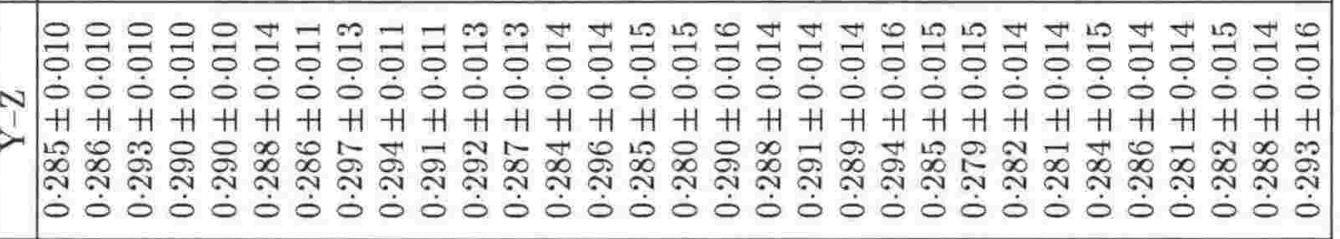 \\
\hline & 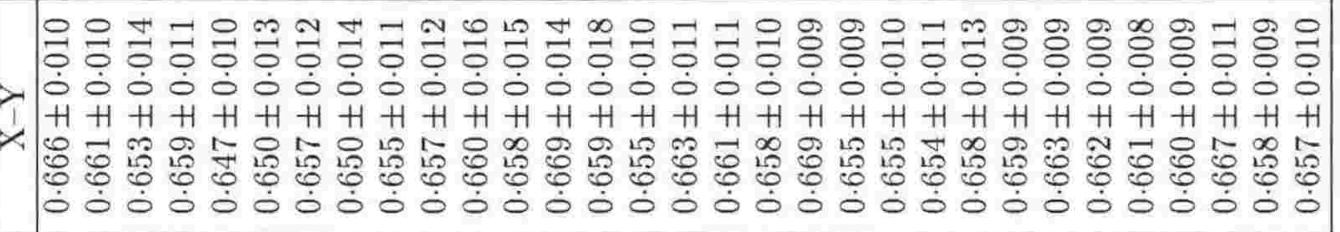 \\
\hline & 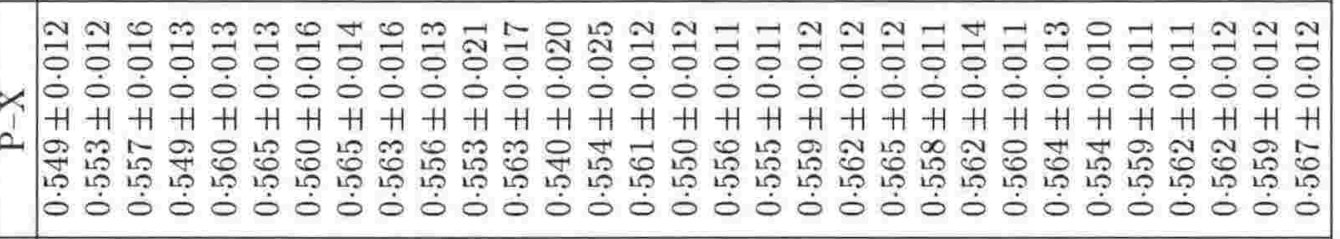 \\
\hline & 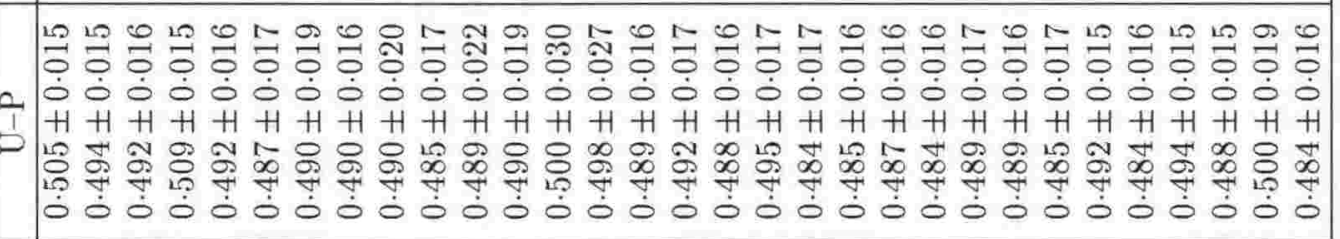 \\
\hline & 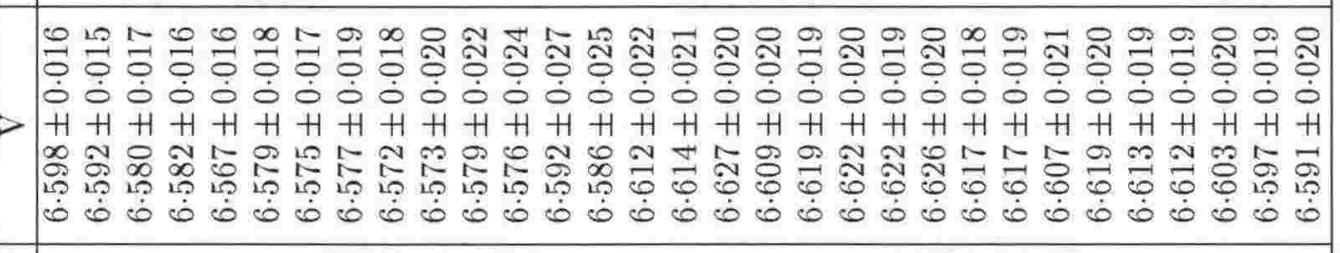 \\
\hline & \# \\
\hline & 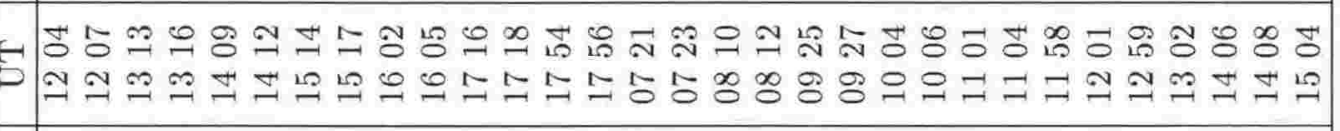 \\
\hline & 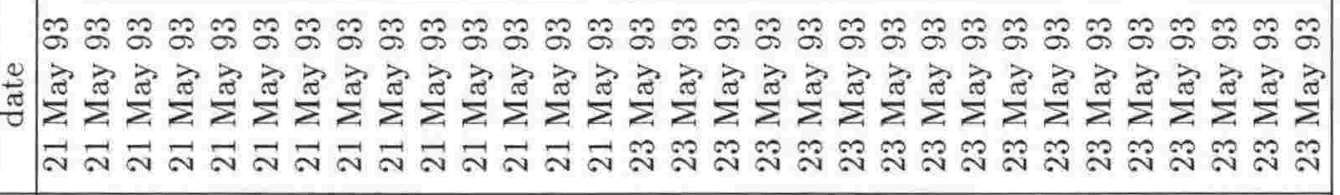 \\
\hline & 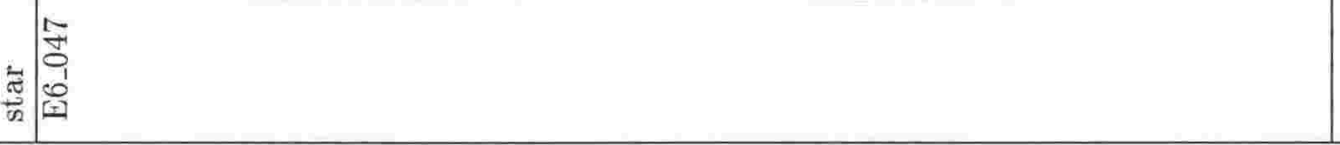 \\
\hline
\end{tabular}




\begin{tabular}{|c|c|c|c|c|c|}
\hline & 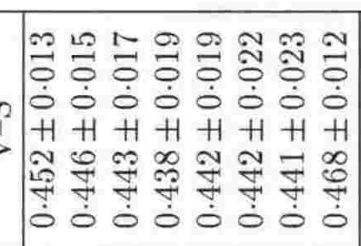 & $\mid \begin{array}{ll}\exists & -1 \\
0 & \ddots \\
0 & 0 \\
1 & 0 \\
0 & 1 \\
0 & 0 \\
0 & 0 \\
0 & 0 \\
0 & 0\end{array}$ & 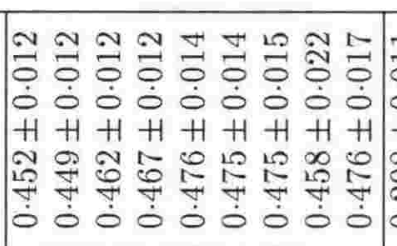 & 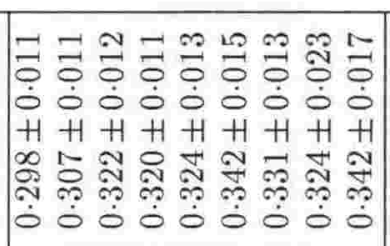 & 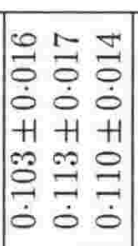 \\
\hline & 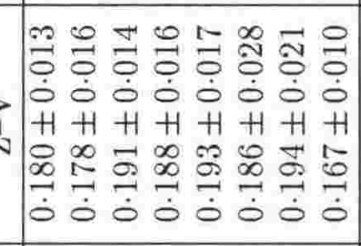 & 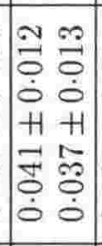 & 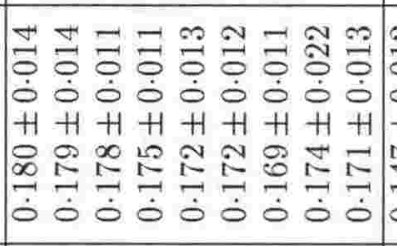 & 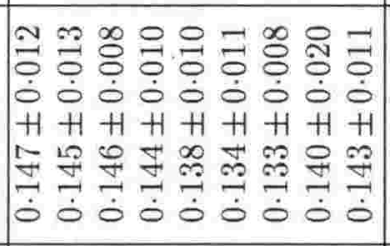 & 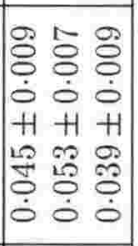 \\
\hline & 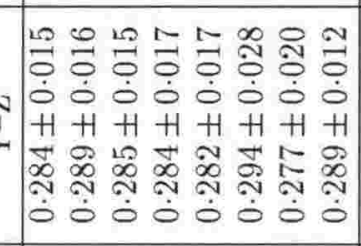 & $\begin{array}{|ll|}\infty & 1 \\
0 & 0 \\
0 & 0 \\
0 & 0 \\
H & +1 \\
0 & 0 \\
0 & 8 \\
0 & 0 \\
\dot{0} & 0 \\
& 0\end{array}$ & 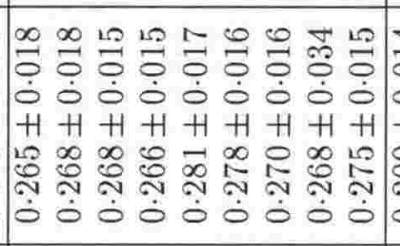 & 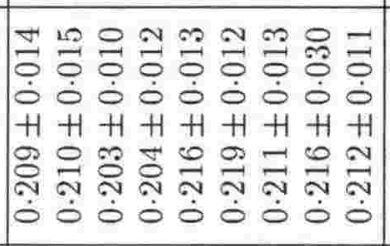 & 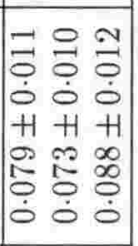 \\
\hline & 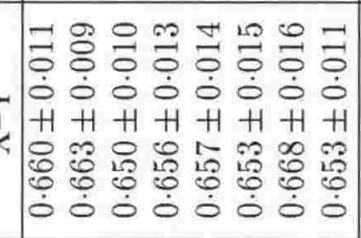 & 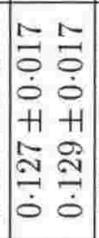 & 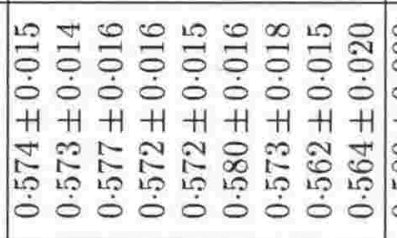 & 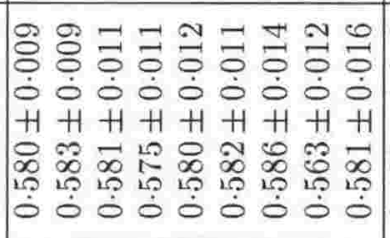 & 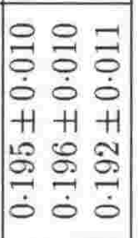 \\
\hline & 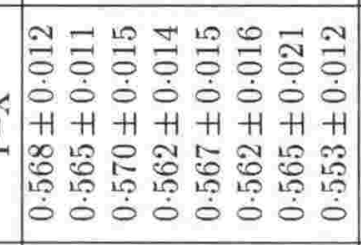 & $\begin{array}{|cc|}1 & -1 \\
0 & 0 \\
0 & 0 \\
0 & 0 \\
H & +1 \\
0 & 0 \\
0 & 0 \\
0 & 0 \\
0 & 0\end{array}$ & 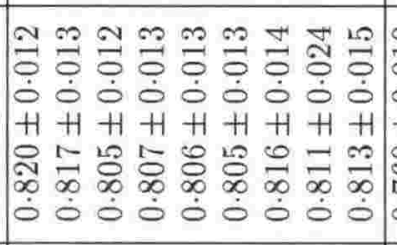 & 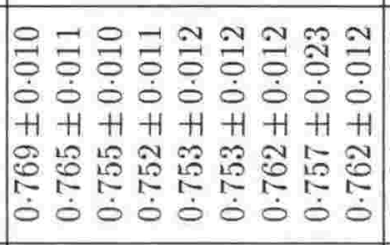 & 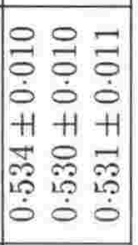 \\
\hline & 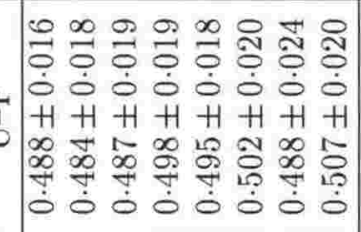 & 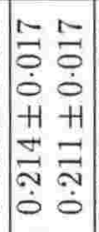 & 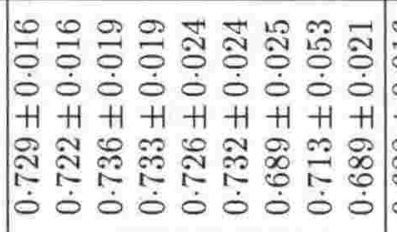 & 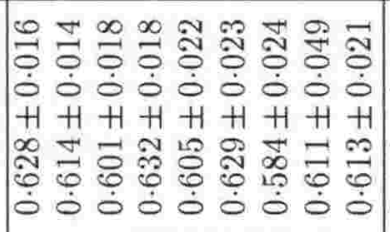 & 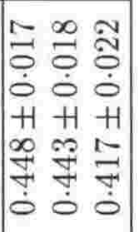 \\
\hline & 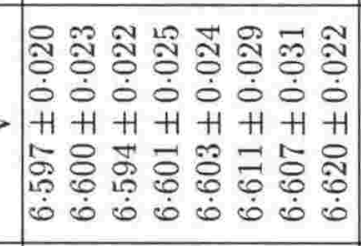 & 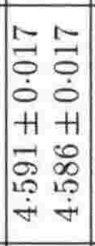 & 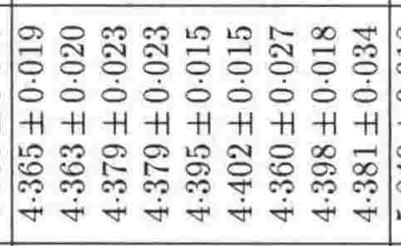 & 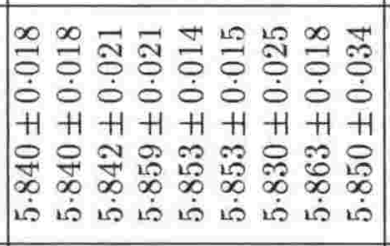 & 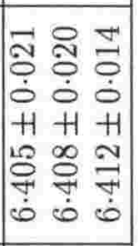 \\
\hline & 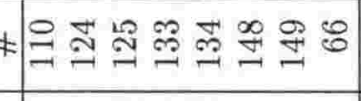 & $\infty 8$ & 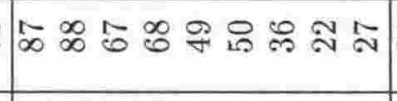 & 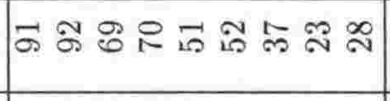 & 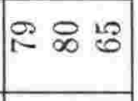 \\
\hline & 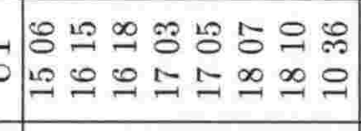 & 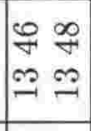 & 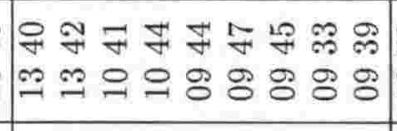 & 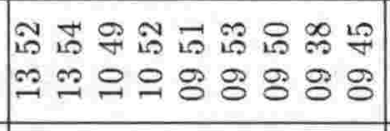 & \begin{tabular}{ccc}
$\infty$ & $\infty$ & \multicolumn{2}{c}{} \\
\hdashline & $\ddots$ & 0 \\
$=$ & $=$ & 0
\end{tabular} \\
\hline & 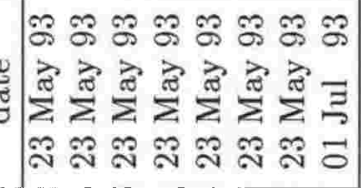 & 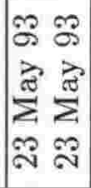 & 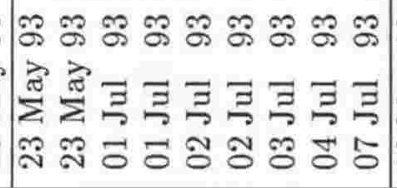 & 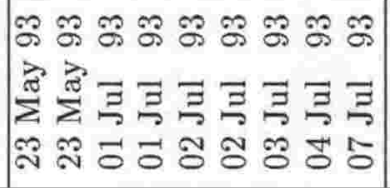 & 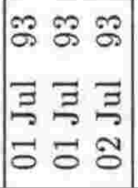 \\
\hline
\end{tabular}




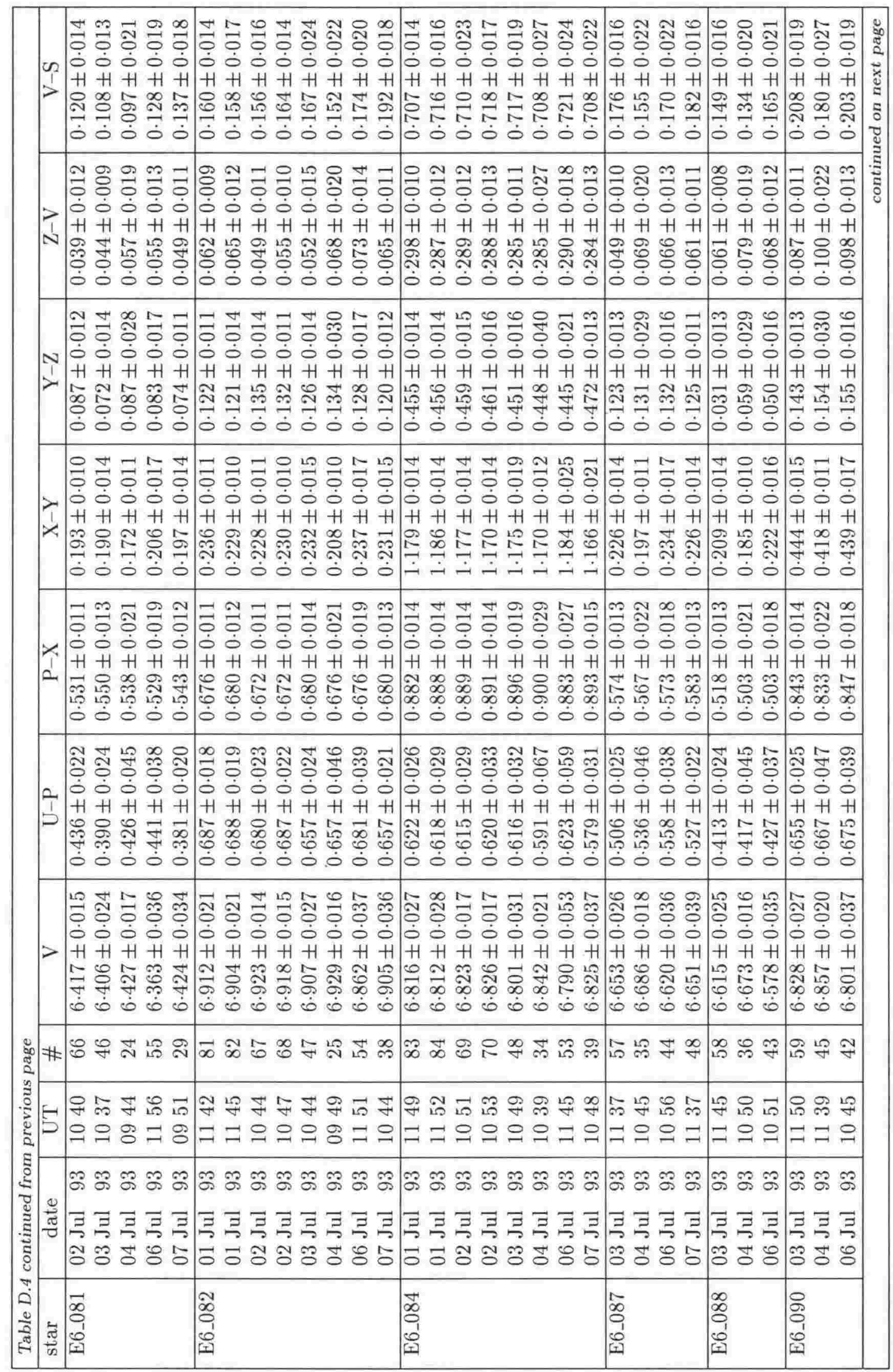




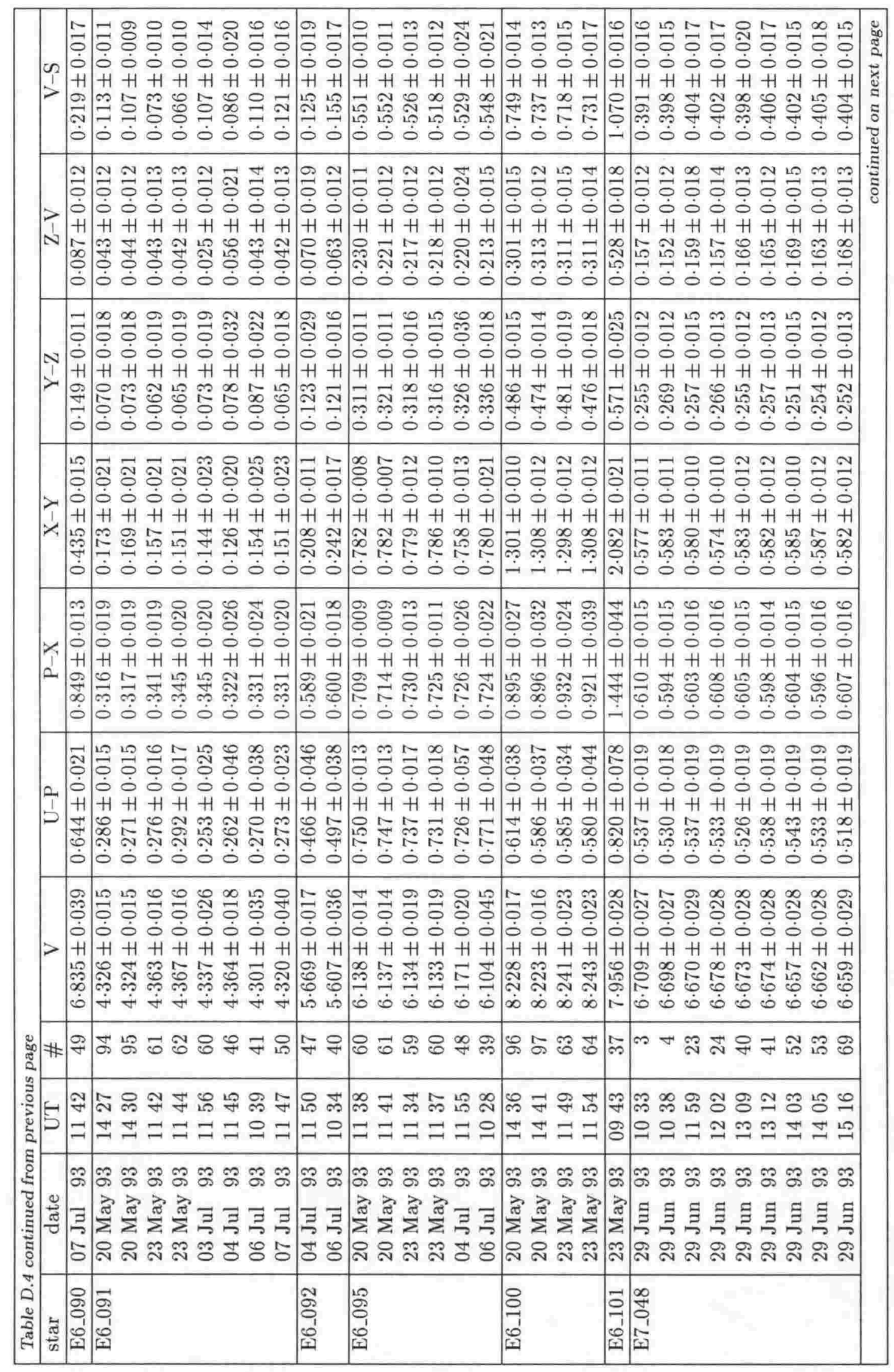




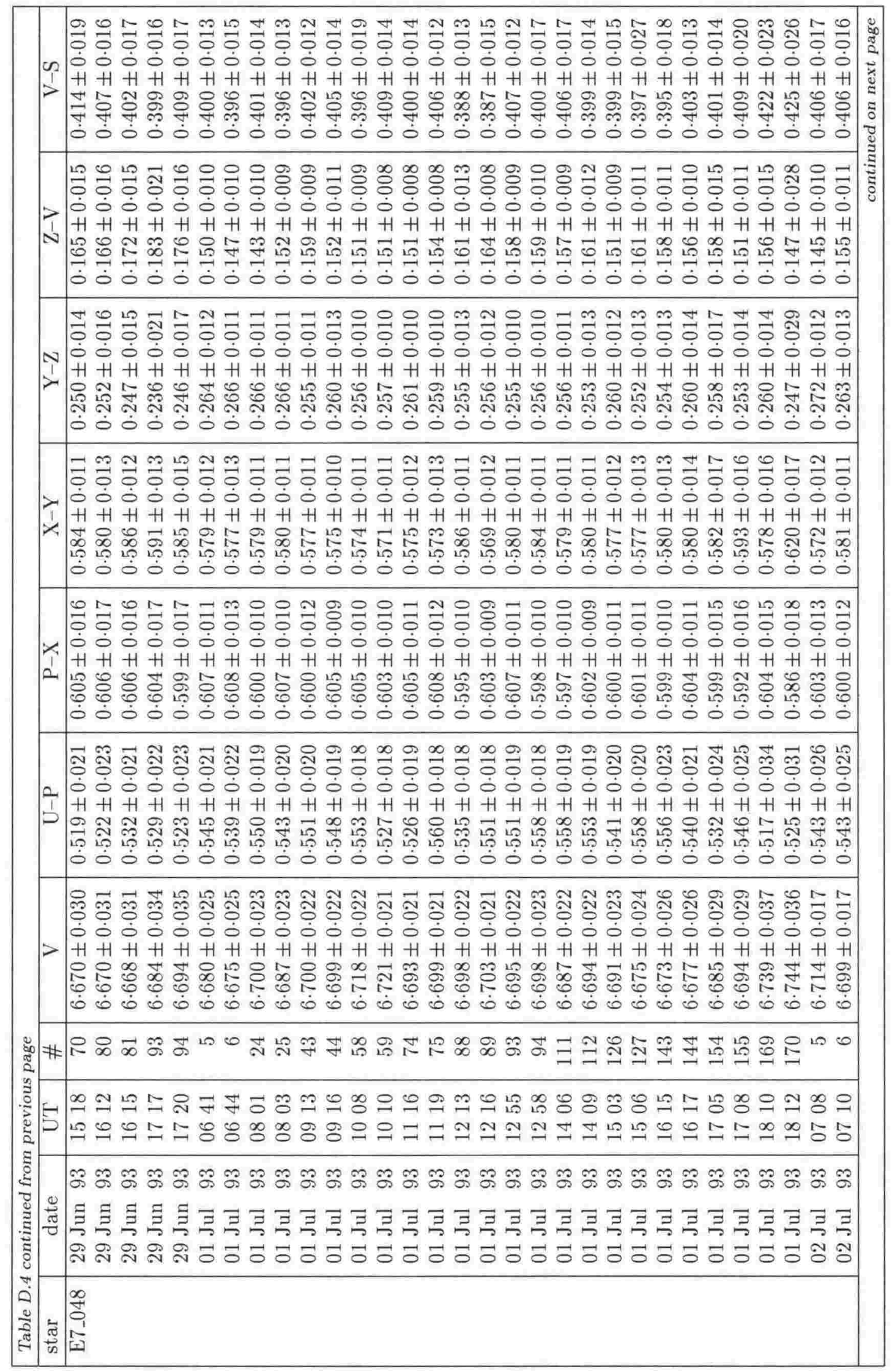




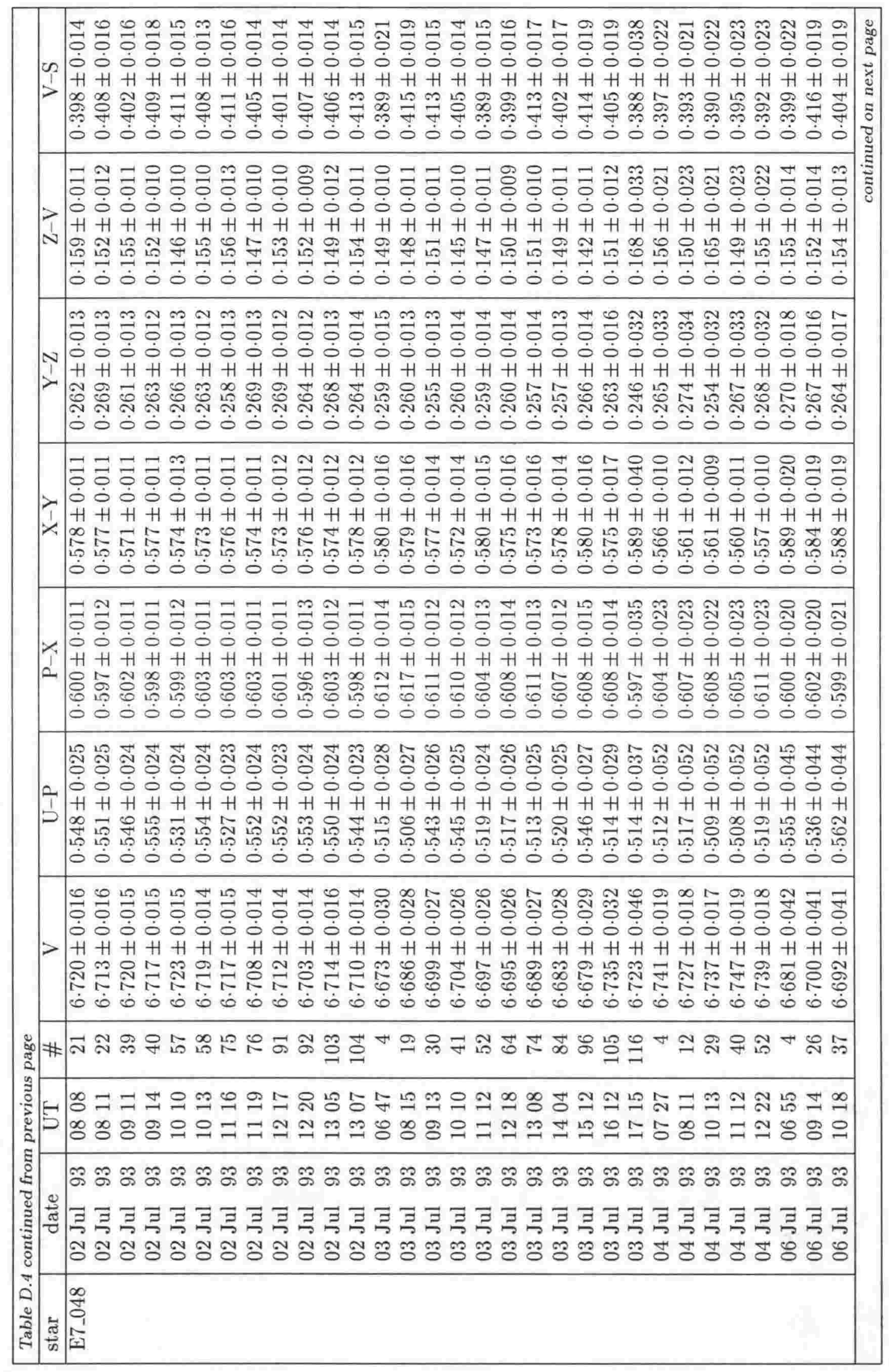




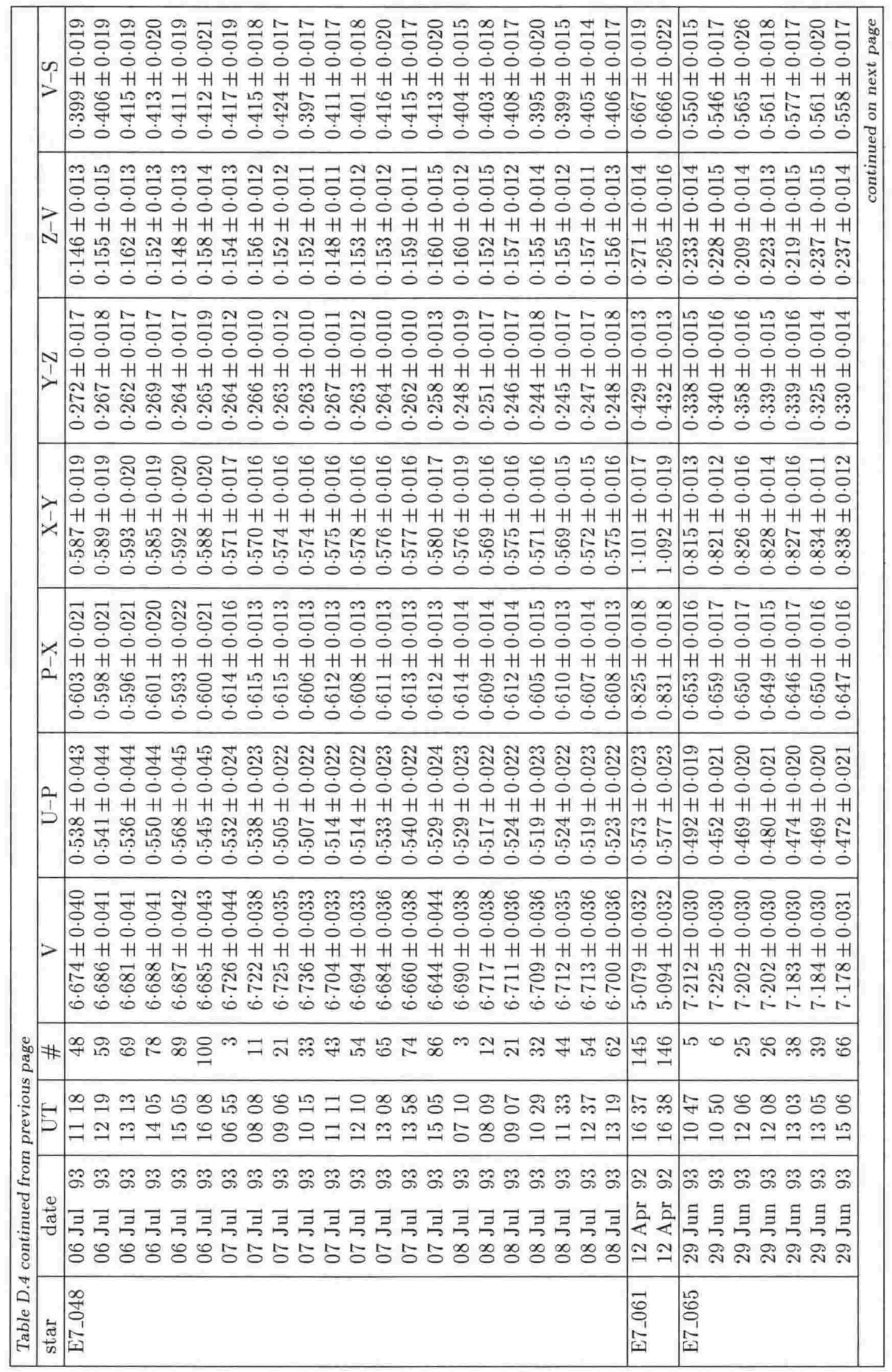




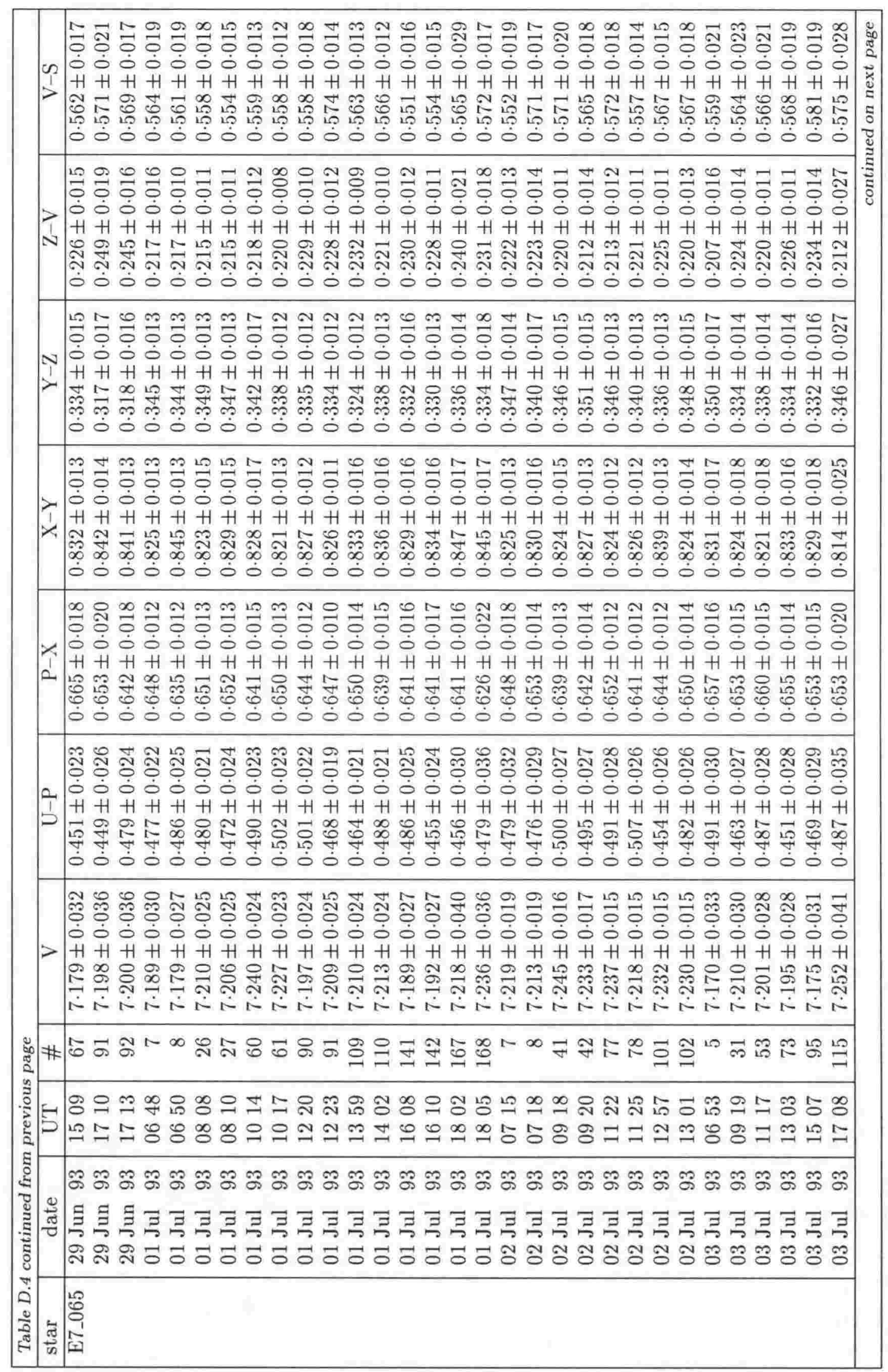




\begin{tabular}{|c|c|c|c|c|c|c|c|c|c|c|c|}
\hline & & 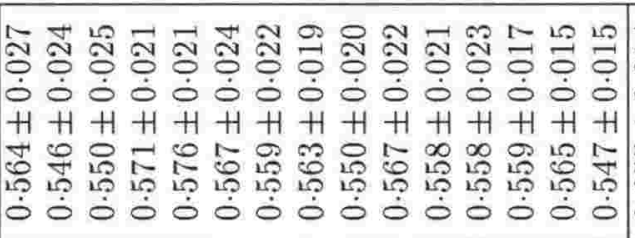 & 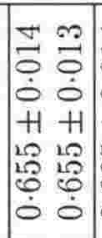 & \begin{tabular}{ll|}
$\forall$ & $\mathbb{1}$ \\
0 & 0 \\
0 & 0 \\
0 & 0 \\
$H$ & $H$ \\
1 & $\infty$ \\
0 & $\infty$ \\
0 & 0 \\
0 & 0 \\
0 & 0
\end{tabular} & 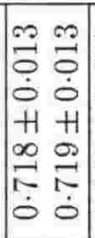 & 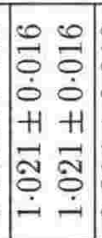 & 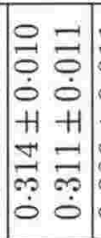 & \begin{tabular}{ll|}
- & 0 \\
0 & 0 \\
0 & 0 \\
0 & 0 \\
$H$ & 1 \\
0 & -1 \\
0 & 0 \\
0 & 0 \\
0 & 0
\end{tabular} & $\begin{array}{ll}\forall & 20 \\
0 & 0 \\
0 & 0 \\
0 & 0 \\
H & H \\
-1 & 0 \\
0 & 0 \\
0 & 0 \\
0 & 0\end{array}$ & 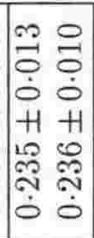 & 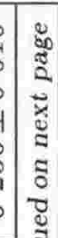 \\
\hline & & 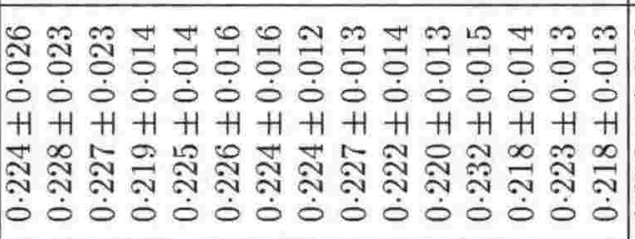 & 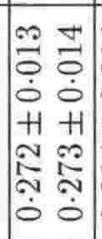 & 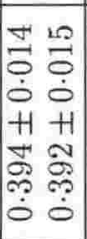 & 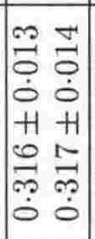 & 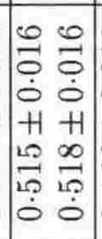 & 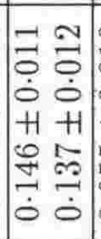 & $\mid$\begin{tabular}{ll|}
0 & 0 \\
0 & 0 \\
$\dot{0}$ & 0 \\
+1 & +1 \\
1 & 1 \\
12 & -1 \\
0 & 0 \\
0 & 0 \\
0 & 0
\end{tabular} & 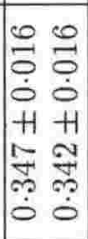 & 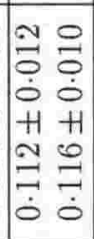 & \\
\hline & & 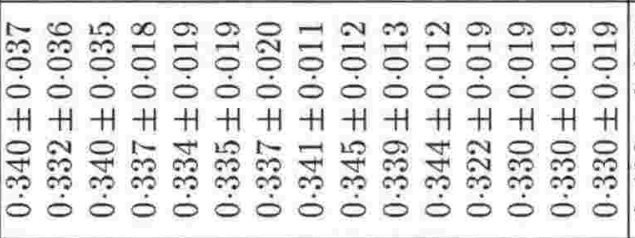 & 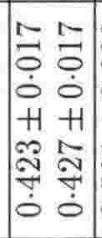 & $\mid \begin{array}{ll}0 & 0 \\
0 & 0 \\
0 & 0 \\
0 & 0 \\
+1 & +1 \\
-1 & 0 \\
0 \\
10 \\
0 & 1 \\
0 & 0 \\
\end{array}$ & 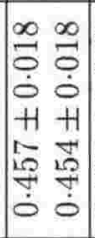 & 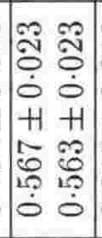 & 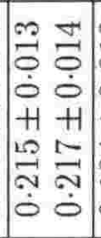 & 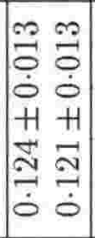 & 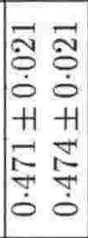 & 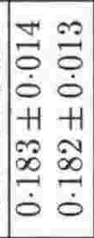 & \\
\hline & & 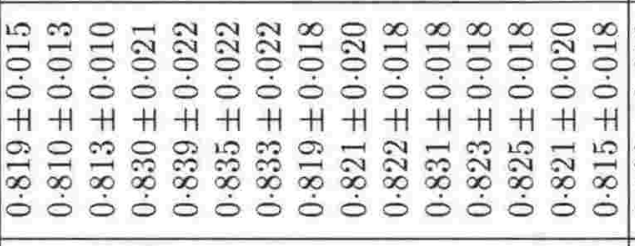 & $\begin{array}{ll}0 & 0 \\
0 & 0 \\
0 & 0 \\
0 & 0 \\
+1 & 1 \\
0 & 8 \\
0 & 0 \\
-1 & -1\end{array}$ & 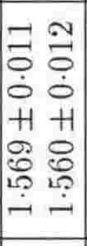 & 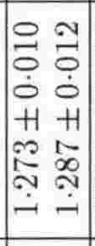 & 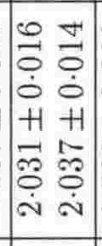 & 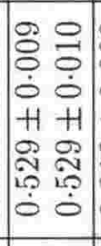 & 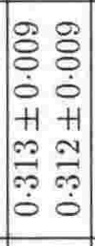 & 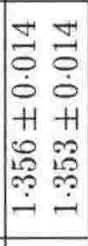 & \begin{tabular}{ll}
0 & 9 \\
0 & 0 \\
0 & 0 \\
0 & 0 \\
+1 & +1 \\
0 & 0 \\
0 & 0 \\
\hdashline & 0 \\
0 & 0
\end{tabular} & \\
\hline & & 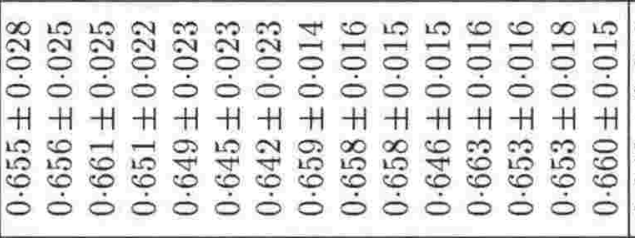 & 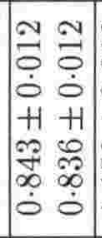 & 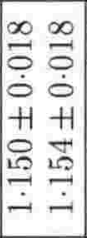 & $\mid$\begin{tabular}{cc|}
$\infty$ & $\infty$ \\
0 & 0 \\
0 & 0 \\
0 & 0 \\
1 & +1 \\
0 & 0 \\
0 & 0 \\
0 & 0 \\
0 & 0 \\
& 0
\end{tabular} & 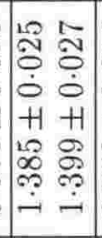 & 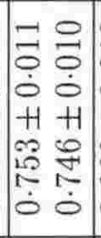 & 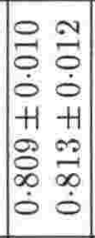 & $\begin{array}{ll}12 & 20 \\
0 & 0 \\
0 & 0 \\
0 & 0 \\
1 & 11 \\
2 & 0 \\
0 & 0 \\
0 & 0 \\
- & 0\end{array}$ & \begin{tabular}{ll}
0 & 0 \\
0 & 0 \\
0 & 0 \\
0 & 0 \\
$H$ & $H$ \\
\hdashline & $\infty$ \\
0 & 0 \\
1 & 0 \\
0 & 0
\end{tabular} & \\
\hline & & 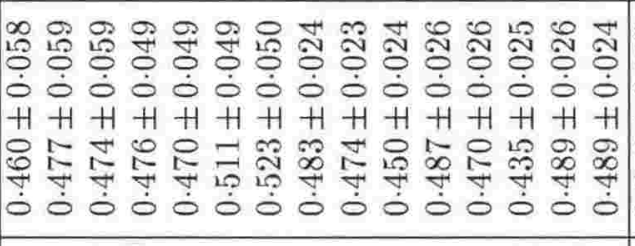 & 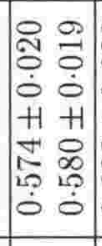 & 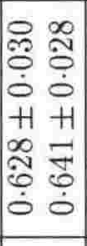 & 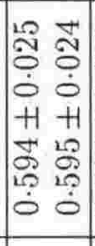 & 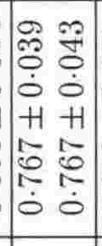 & 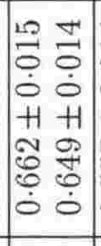 & 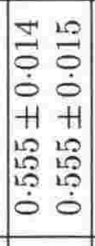 & $\begin{array}{ll}\bar{N} & \overline{1} \\
0 & 0 \\
0 & 0 \\
H & H \\
0 & \text { in } \\
0 & 0 \\
0 & 0 \\
0 & 0\end{array}$ & 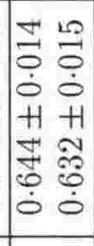 & \\
\hline & 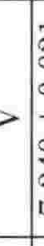 & 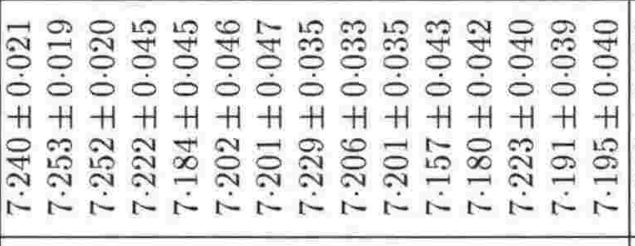 & 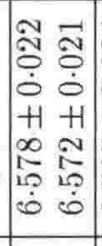 & 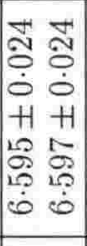 & 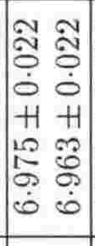 & 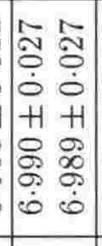 & 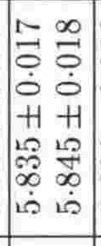 & 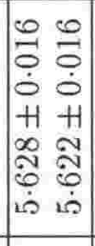 & 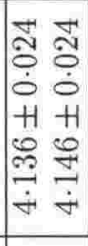 & 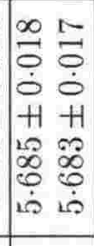 & \\
\hline & $\#$ & 以 & $\stackrel{2}{二}$ & $\stackrel{\infty}{=} \underset{=}{=}$ & $\stackrel{\Xi}{\cong}$ & : & 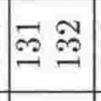 & 욤 & $\underset{\Xi}{\mathscr{I}}$ & İ $\stackrel{20}{\square}$ & \\
\hline & -5 & 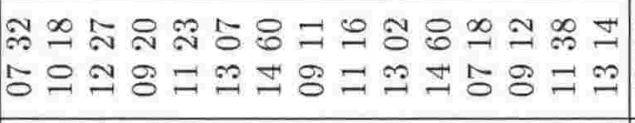 & & {$\left[\begin{array}{ll}9 & 0 \\
\hdashline & 10 \\
\hdashline & 10\end{array}\right.$} & & 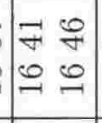 & $\left|\begin{array}{ll}1 & -1 \\
1 & 1 \\
0 & 0 \\
-1 & -1\end{array}\right|$ & 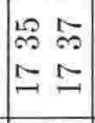 & 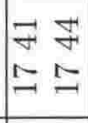 & 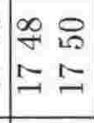 & \\
\hline & 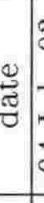 & 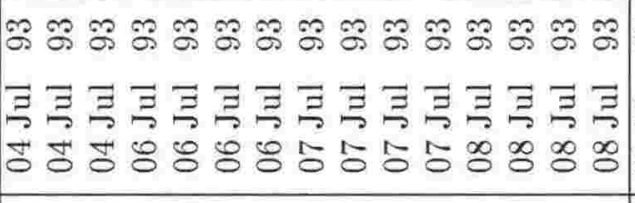 & 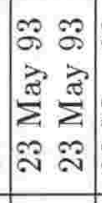 & 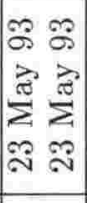 & 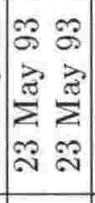 & 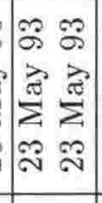 & 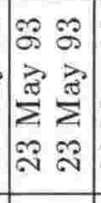 & 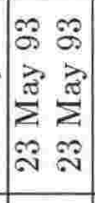 & 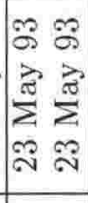 & 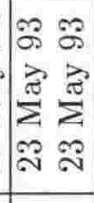 & \\
\hline & 密: & 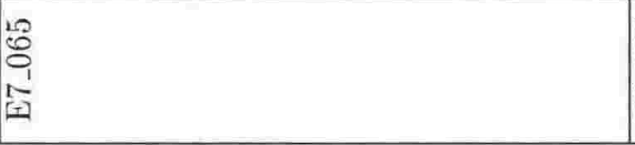 & 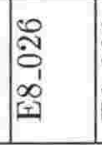 & 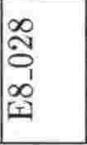 & 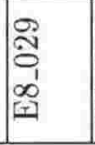 & 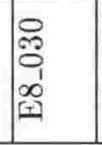 & 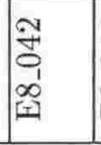 & 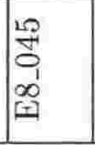 & 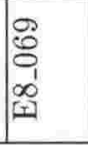 & 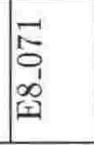 & \\
\hline
\end{tabular}




\begin{tabular}{|c|c|c|c|c|c|c|c|c|c|}
\hline & & 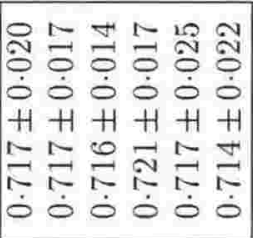 & & 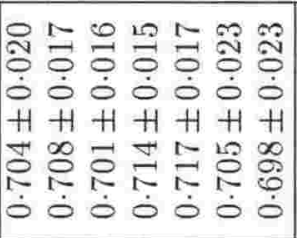 & 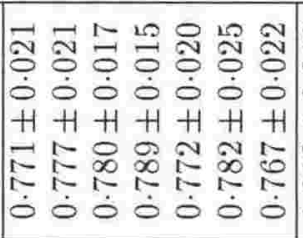 & & 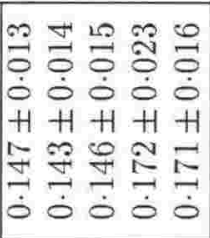 & 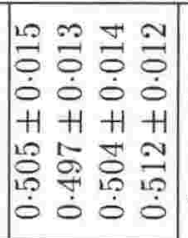 & 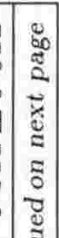 \\
\hline & & 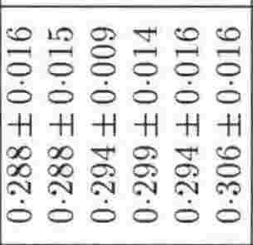 & & 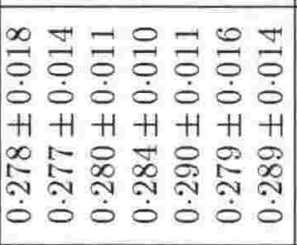 & 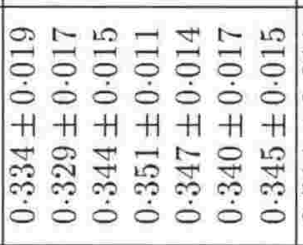 & $\begin{array}{l}\exists \\
\\
0 \\
0 \\
+1 \\
\vdots \\
0 \\
10 \\
0 \\
0\end{array}$ & 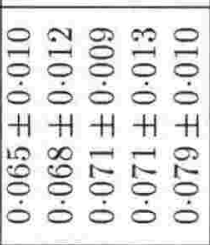 & 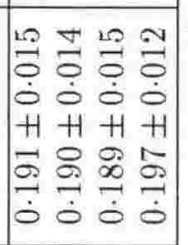 & \\
\hline & & 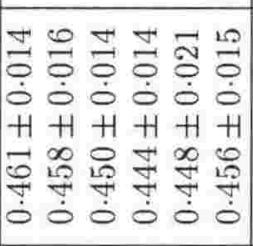 & & 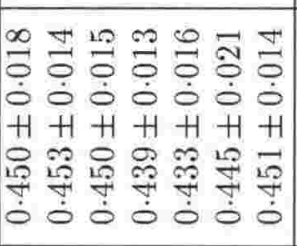 & 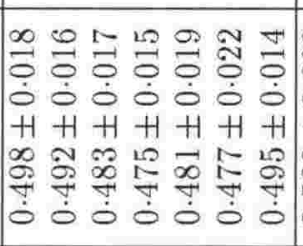 & $\begin{array}{l}\stackrel{0}{1} \\
0 \\
0 \\
0 \\
+1 \\
9 \\
0 \\
i \\
0 \\
0\end{array}$ & 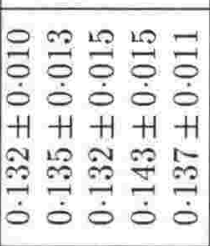 & 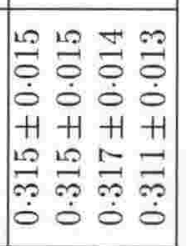 & \\
\hline & & 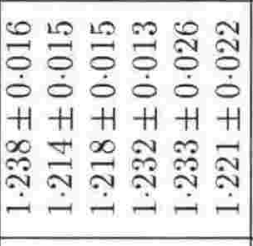 & & 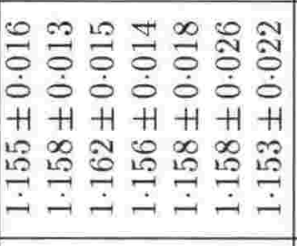 & 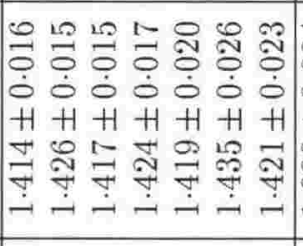 & 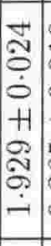 & 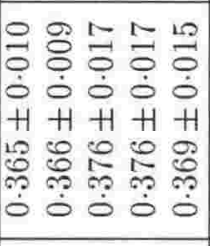 & 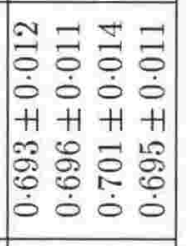 & \\
\hline & & 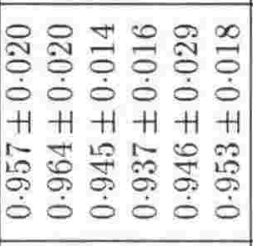 & & 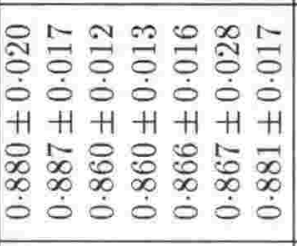 & 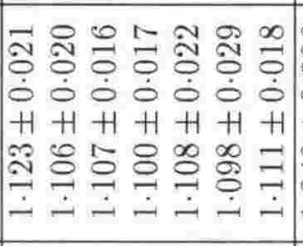 & 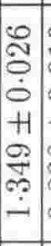 & 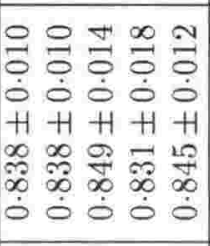 & 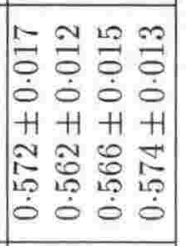 & \\
\hline & & 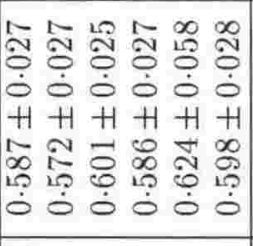 & & 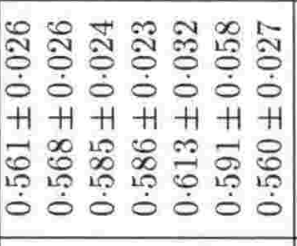 & 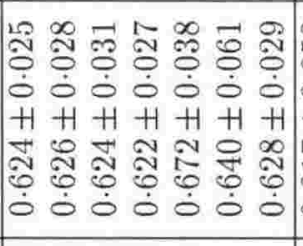 & \begin{tabular}{c}
2 \\
1 \\
0 \\
0 \\
+1 \\
1 \\
\\
\hdashline \\
0 \\
0
\end{tabular} & 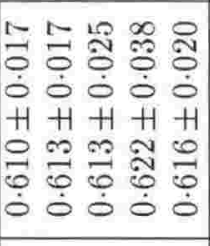 & 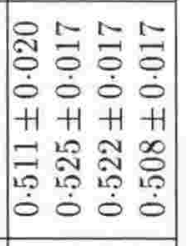 & \\
\hline & & 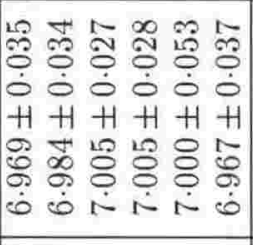 & & 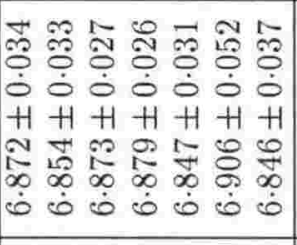 & 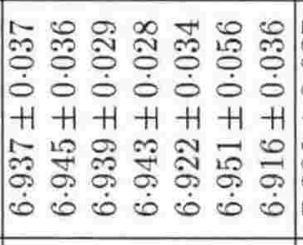 & 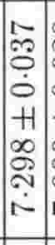 & 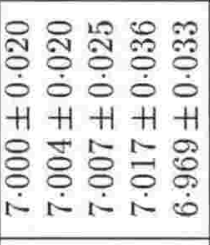 & 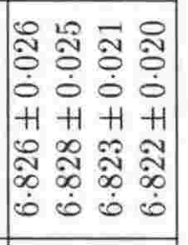 & \\
\hline & & 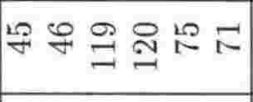 & $\underset{\Xi}{-}$ & 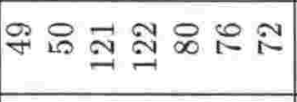 & 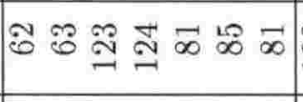 & $\stackrel{2}{\cong}$ & 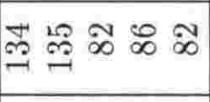 & 요 \& & \\
\hline & & 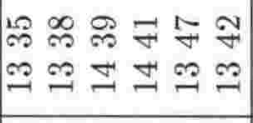 & $\infty$ & 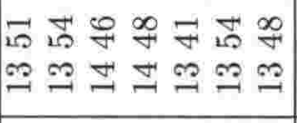 & 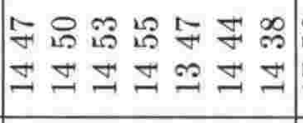 & $\mid \begin{array}{l}0 \\
20 \\
1 \\
-1\end{array}$ & 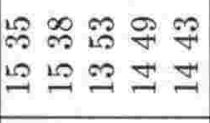 & $\left|\begin{array}{cccc}\infty & = & 2 & \infty \\
0 & 0 & 0 \\
\hdashline & 9 & \mathbb{1} & \pm\end{array}\right|$ & \\
\hline 苟 & & 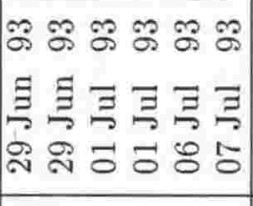 & $\mid$\begin{tabular}{l}
$\Xi$ \\
\hdashline \\
\hdashline
\end{tabular} & 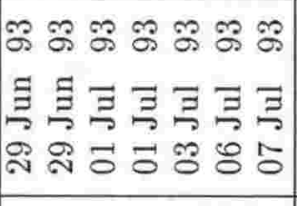 & 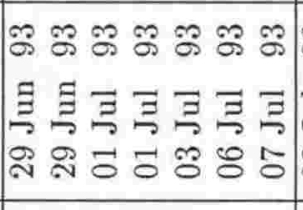 & $\approx$ & 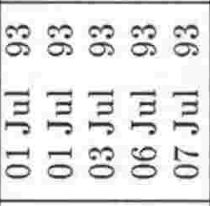 & 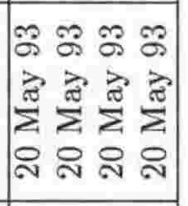 & \\
\hline 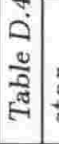 & & & & & \begin{tabular}{l}
$\%$ \\
\hdashline \\
0 \\
0 \\
0 \\
9
\end{tabular} & & & 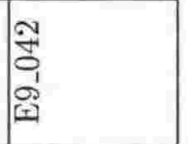 & \\
\hline
\end{tabular}




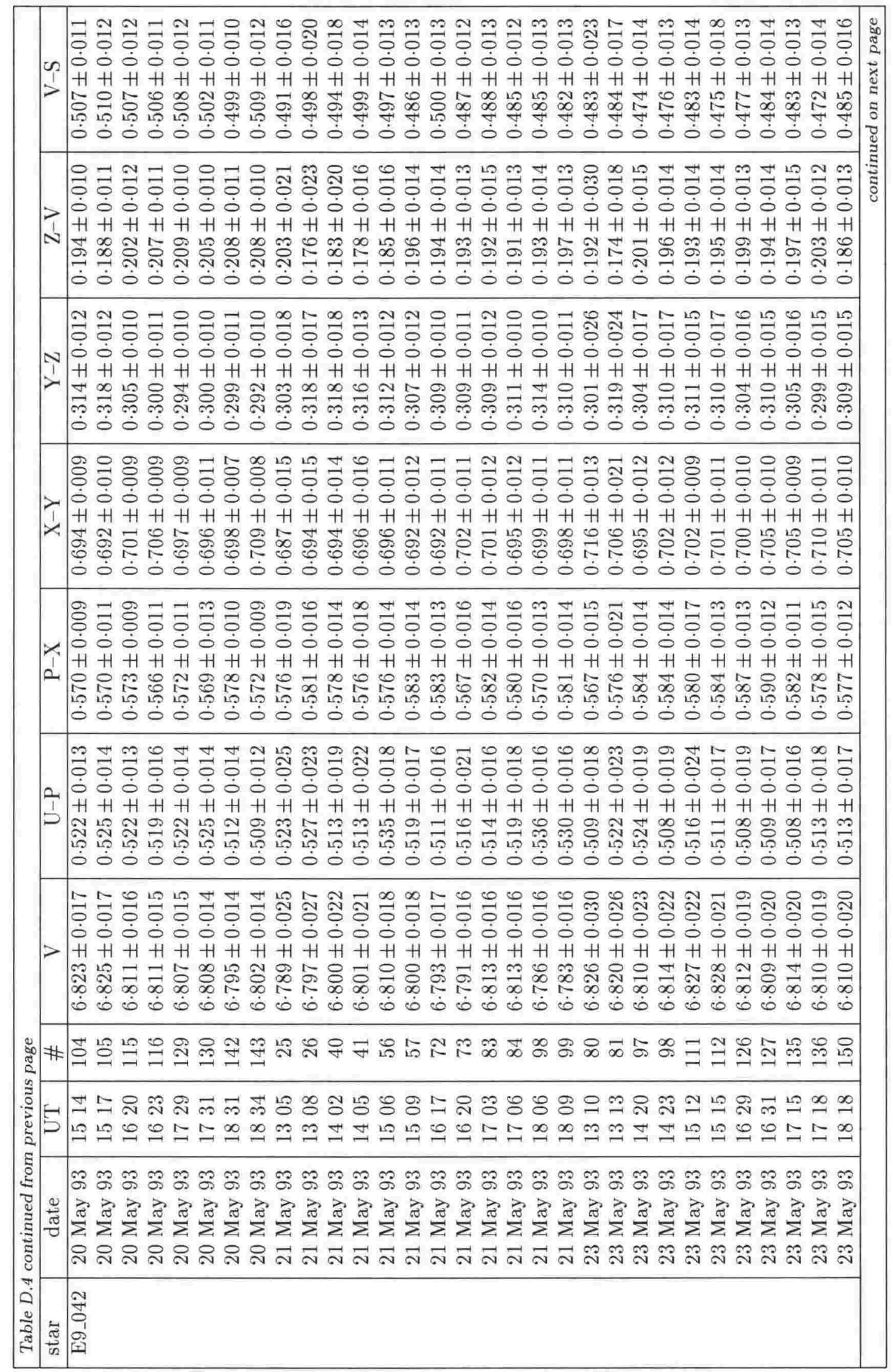




\begin{tabular}{|c|c|c|c|c|c|c|c|c|}
\hline & & 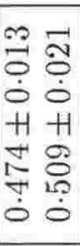 & 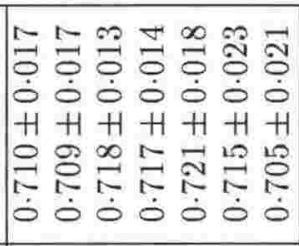 & 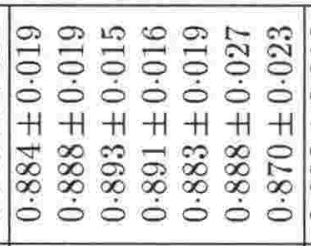 & 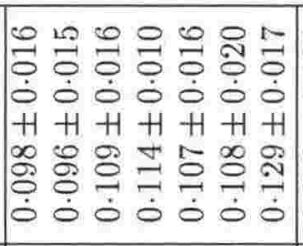 & 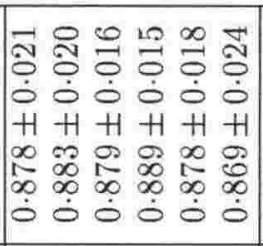 & 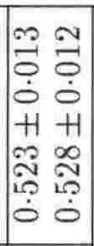 & 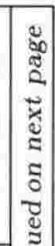 \\
\hline & & 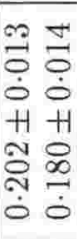 & 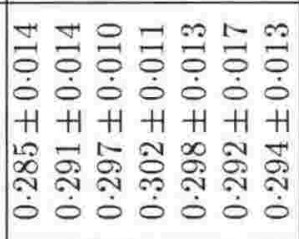 & 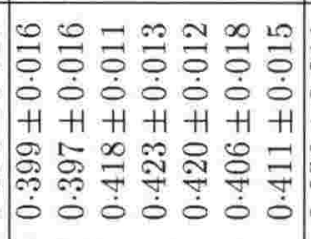 & 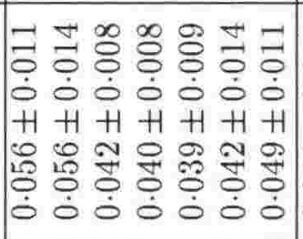 & 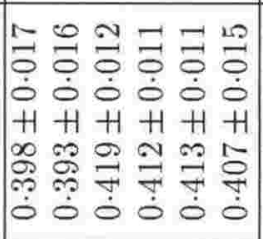 & 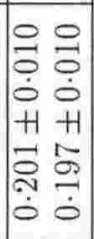 & \\
\hline & & $\begin{array}{ll}12 & \infty \\
0 & 0 \\
0 & 0 \\
0 & \\
H & H \\
0 & 0 \\
0 & 0 \\
0 & 0 \\
0 & 0\end{array}$ & 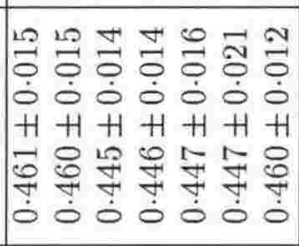 & 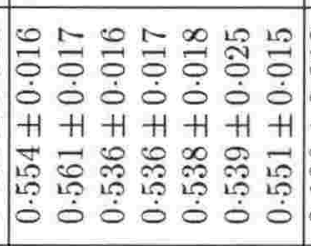 & 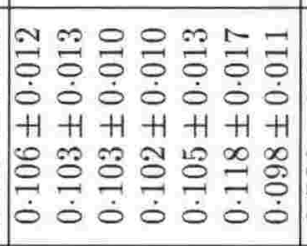 & 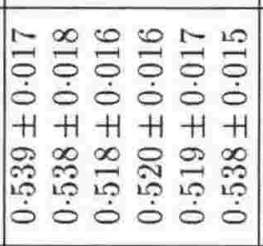 & 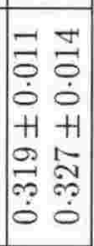 & \\
\hline & & $\begin{array}{ll}\infty & 0 \\
0 & 0 \\
0 & 0 \\
0 & 0 \\
H & +1 \\
1 & 0 \\
0 & 0 \\
0 & 0 \\
0\end{array}$ & 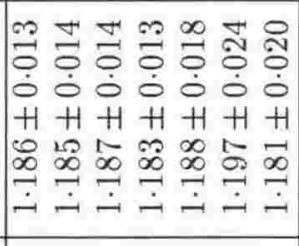 & 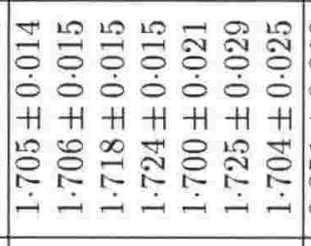 & 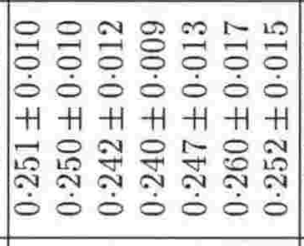 & 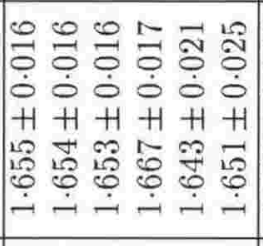 & 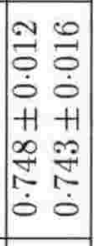 & \\
\hline & & $\begin{array}{ll}\exists & \overline{1} \\
0 & 0 \\
0 & 0 \\
0 & 0 \\
H & H \\
1 & 1 \\
0 & 5 \\
0 & 0 \\
0 & 1 \\
0 & 0 \\
0 & 0\end{array}$ & 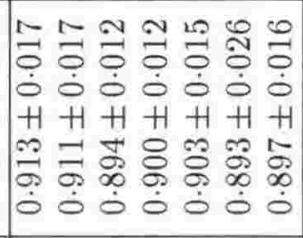 & 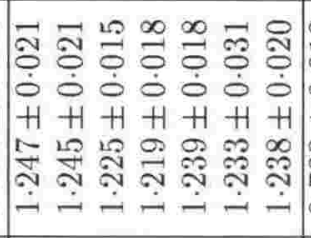 & 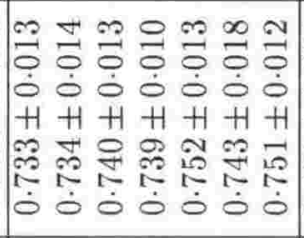 & 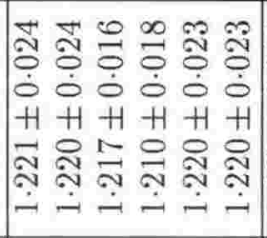 & 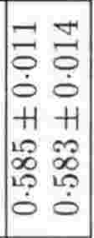 & \\
\hline & & 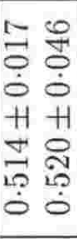 & 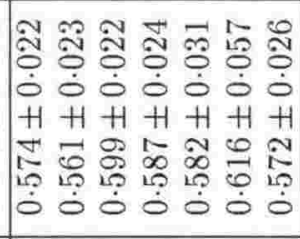 & 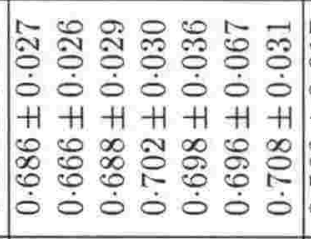 & 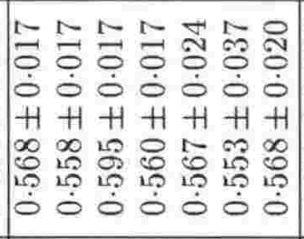 & 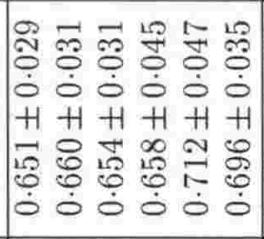 & $\begin{array}{|cc|}0 & 0 \\
0 & 0 \\
0 & 0 \\
0 & 0 \\
+1 & +1 \\
0 & 20 \\
0 & 0 \\
0 & 1 \\
0 & 0 \\
0 & 0\end{array}$ & \\
\hline & & 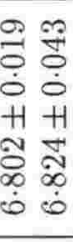 & 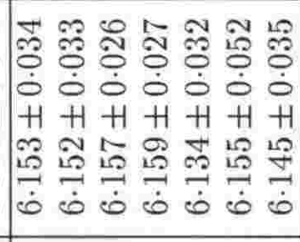 & 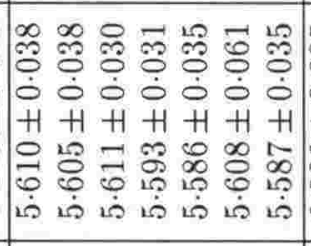 & 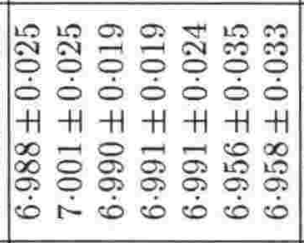 & 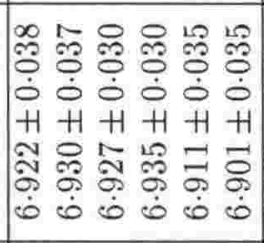 & 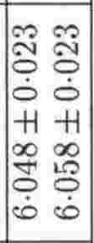 & \\
\hline & & 1025 & 묭 ஊ & 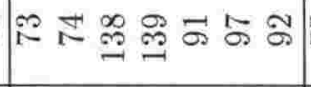 & 용 용 & トか正瓷 & $\stackrel{0}{0}$ & \\
\hline & & $\begin{array}{ll}\mathbb{N} & \infty \\
\infty & \infty \\
-1 & 10\end{array}$ & 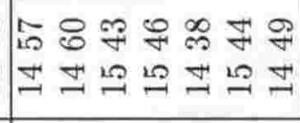 & 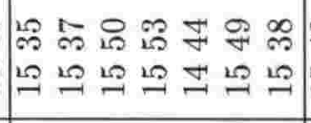 & 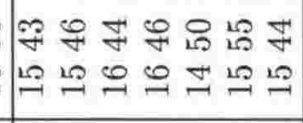 & 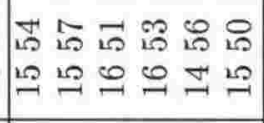 & 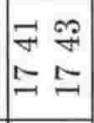 & \\
\hline & & 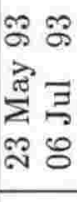 & 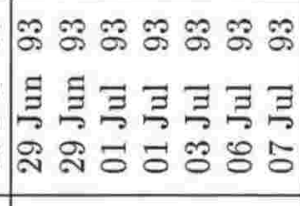 & 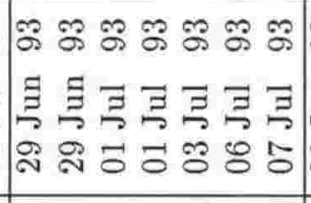 & 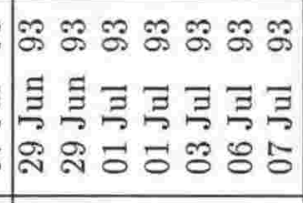 & 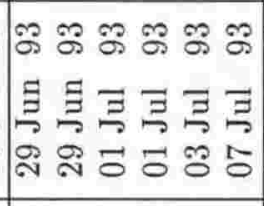 & $\begin{array}{l}\Xi \Xi \\
\Xi \Xi \\
\end{array}$ & \\
\hline & & 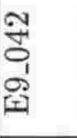 & 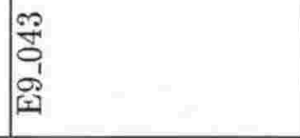 & 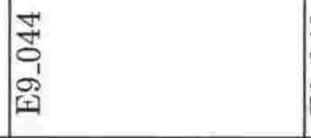 & $\begin{array}{l}\infty \\
0 \\
0 \\
0 \\
0 \\
\vdots \\
1 \\
\end{array}$ & 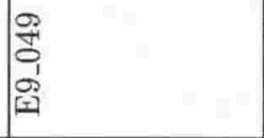 & 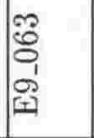 & \\
\hline
\end{tabular}




\begin{tabular}{|c|c|c|c|c|c|c|c|c|}
\hline & & 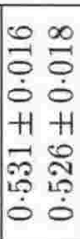 & 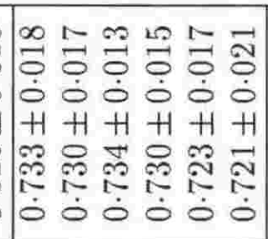 & 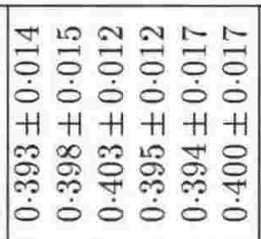 & 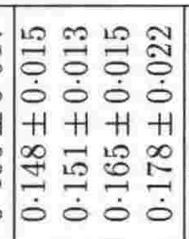 & 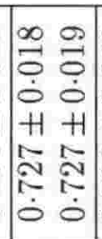 & 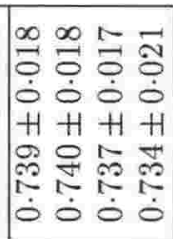 & 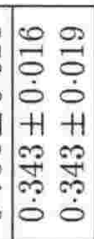 \\
\hline & & $\begin{array}{ll}0 & 0 \\
0 & 0 \\
0 & 0 \\
0 & 0 \\
H & +1 \\
0 & \mathbb{1} \\
0 & 0 \\
0 & 0 \\
0 & 0\end{array}$ & 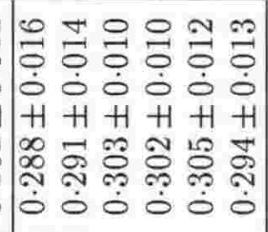 & 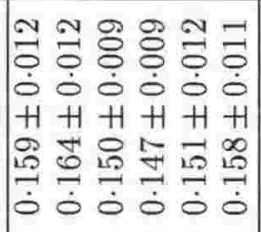 & 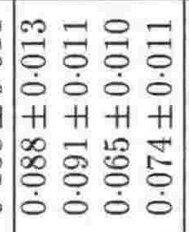 & 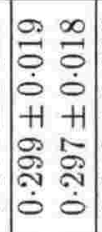 & 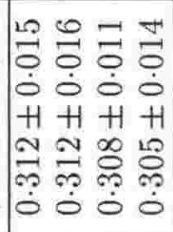 & 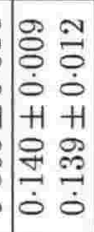 \\
\hline & & 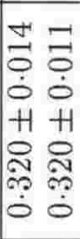 & 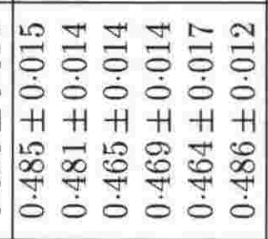 & 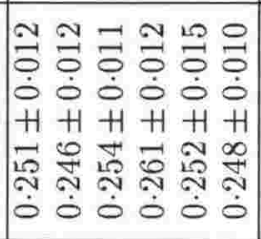 & 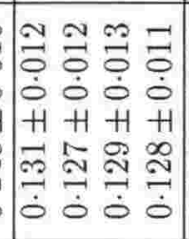 & 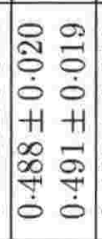 & $\begin{array}{llll}20 & 10 & 0 & 0 \\
0 & 0 & 0 & 0 \\
0 & 0 & 0 \\
0 & 0 & 0 & 0 \\
H & H & H & H \\
1 & 0 & 0 & 0 \\
0 & 0 & 0 & 0 \\
0 & 0 & 0 & 0 \\
0 & 0 & 4 & 0 \\
0 & 0 & 0 & 0\end{array}$ & 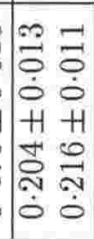 \\
\hline & & 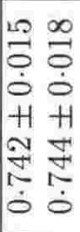 & 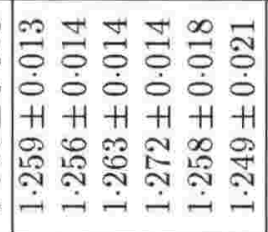 & 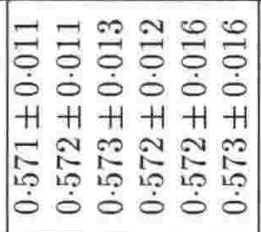 & $\mid$\begin{tabular}{cccc}
0 & 0 & $\cdots$ & 10 \\
0 & 0 & 0 & 0 \\
0 & 0 & 0 & 0 \\
$\dot{H}$ & $H$ & $H$ & $H$ \\
\hdashline & 0 & 0 & 9 \\
0 & 0 & 0 \\
0 & 0 & 0 & 0 \\
0 & 0 & 0 & 0
\end{tabular} & 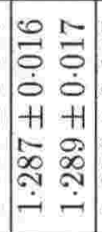 & 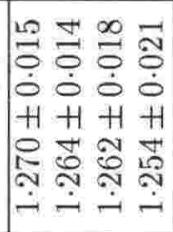 & $\begin{array}{ll}4 & 0 \\
0 & 0 \\
0 & 0 \\
0 & 0 \\
H & 11 \\
0 & 10 \\
0 & 10 \\
0 & 10 \\
0 & 0\end{array}$ \\
\hline & & 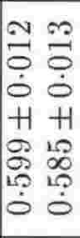 & 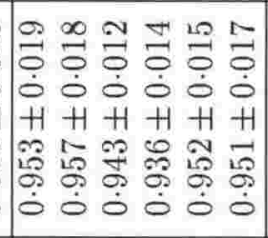 & 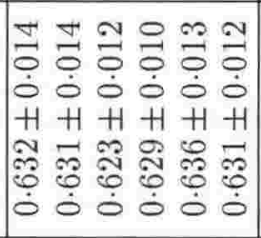 & 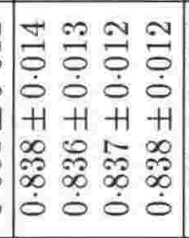 & 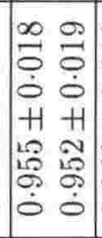 & $\begin{array}{llll}\infty & \infty & 10 & 0 \\
0 & 0 & 0 & 0 \\
0 & 0 \\
0 & 0 & 0 & 0 \\
H & H & H & H \\
0 & 0 & 0 \\
0 & 0 & 0 & 0 \\
0 & 5 & 0 & 0 \\
0 & 0 & 0 & 0\end{array}$ & \begin{tabular}{ll}
$\exists$ & 0 \\
\hdashline & 0 \\
0 & 0 \\
0 & 0 \\
$H$ & 4 \\
0 & 0 \\
0 & 0 \\
0 & 0 \\
0 & 0
\end{tabular} \\
\hline & & 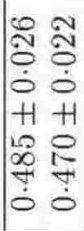 & 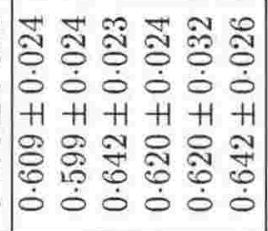 & 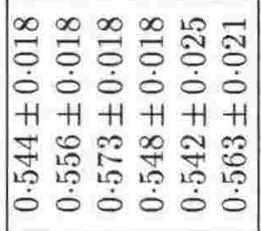 & 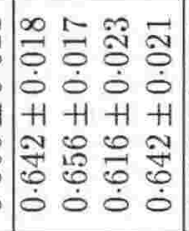 & $\mid \begin{array}{ll}\mathbb{2} & 0 \\
0 & 0 \\
0 & 0 \\
0 & 0 \\
H & H \\
0 & 0 \\
0 & 0 \\
0 & 0 \\
0 & 0\end{array}$ & 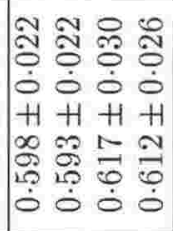 & 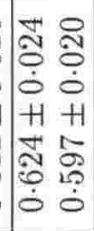 \\
\hline & & 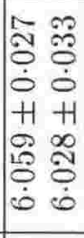 & 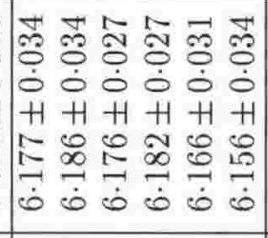 & 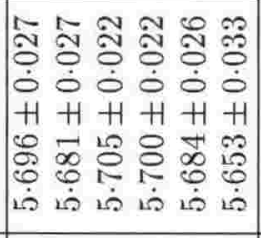 & 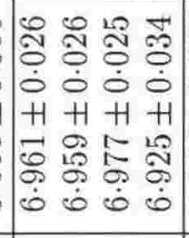 & 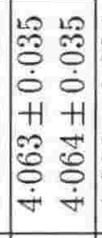 & 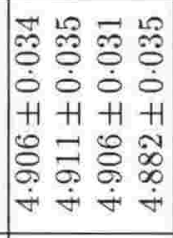 & 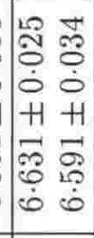 \\
\hline & & $8 \stackrel{0}{0}$ & 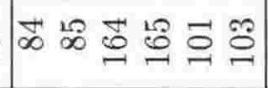 & 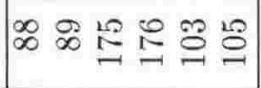 & $\stackrel{\infty}{\circ} \Xi \pm$ & F的 & 응으쿄 & $\stackrel{9}{=}$ \\
\hline & & & 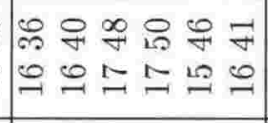 & 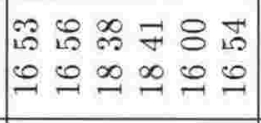 & 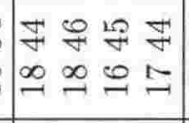 & 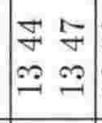 & 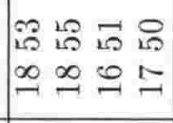 & $\begin{cases}0 & 1 \\
1 & 15 \\
0 & 15 \\
-1 & 1\end{cases}$ \\
\hline 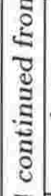 & & 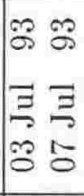 & 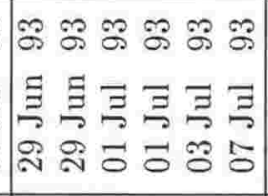 & 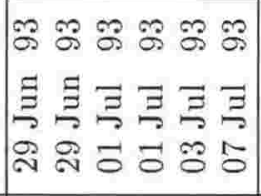 & 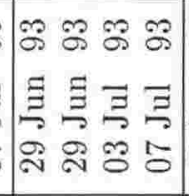 & 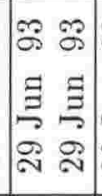 & 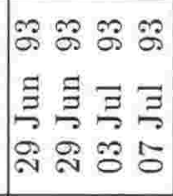 & 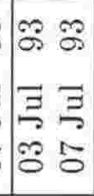 \\
\hline 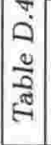 & 焉 & 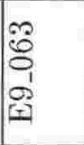 & 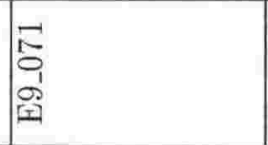 & 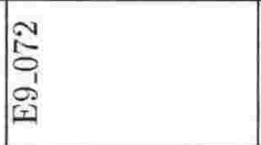 & 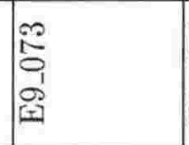 & $\begin{array}{l}\mathbb{1} \\
0 \\
0 \\
0 \\
01 \\
1\end{array}$ & $\begin{array}{l}0 \\
0 \\
0 \\
0 \\
0 \\
1 \\
1\end{array}$ & $\begin{array}{l}0 \\
0 \\
0 \\
0 \\
0 \\
0 \\
1\end{array}$ \\
\hline
\end{tabular}




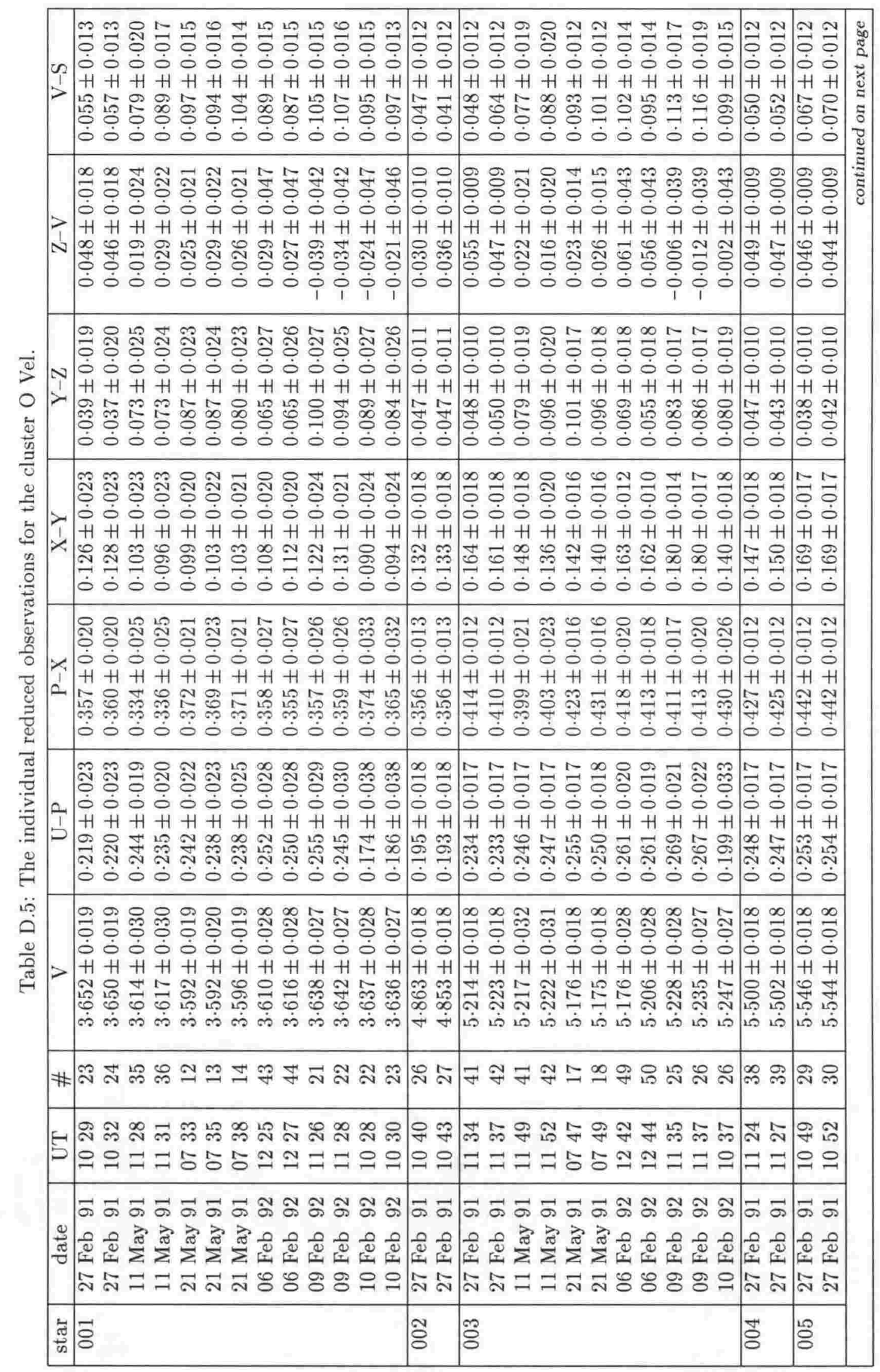




\begin{tabular}{|c|c|c|c|c|}
\hline & & 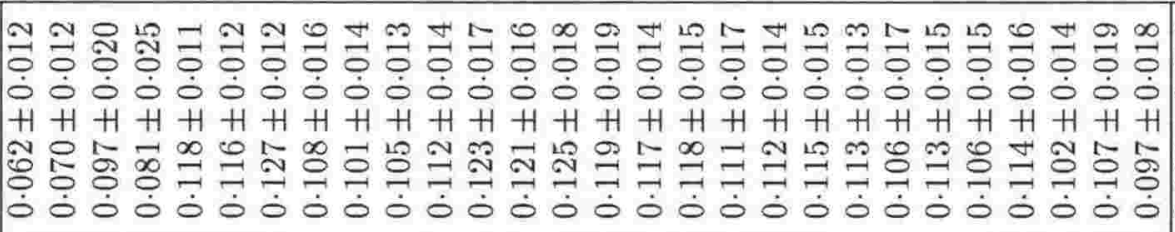 & 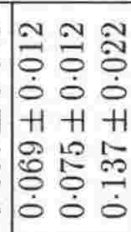 & 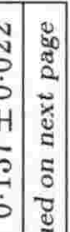 \\
\hline & & 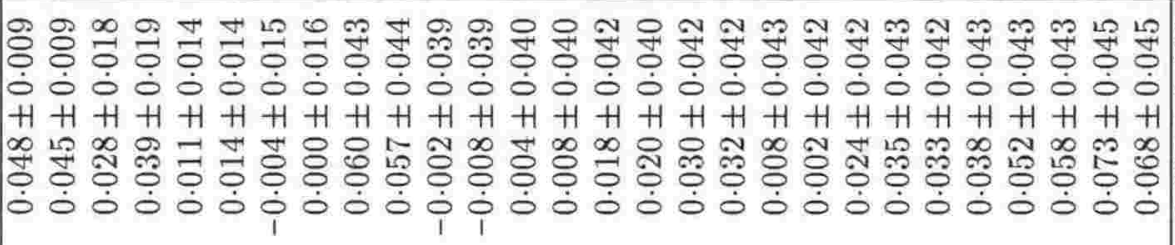 & $\begin{array}{lll}8 & 0 \\
0 & 8 & 0 \\
0 & 0 & 0 \\
0 & 0 & 0 \\
H & H \\
H & H & H \\
10 & 0 & 0 \\
0 & 0 & 0 \\
0 & 0 & 0 \\
0 & 0 & 0\end{array}$ & \\
\hline & & 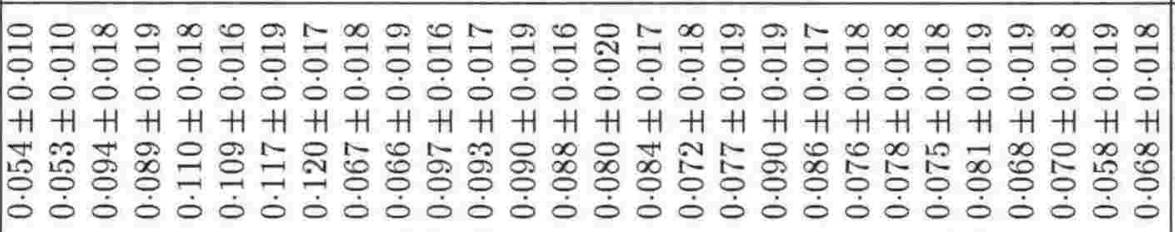 & $\begin{array}{lll}0 & 0 & -1 \\
0 & 0 & 0 \\
0 & 0 & 0 \\
0 & 0 \\
H & H & +1 \\
0 & 0 & 0 \\
0 & 0 & 0 \\
0 & 0 & 0 \\
0 & 0 & 0\end{array}$ & \\
\hline & & 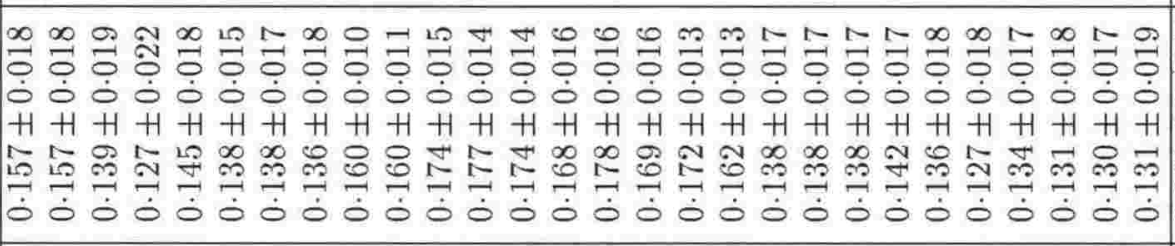 & 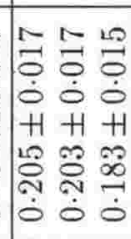 & \\
\hline & & 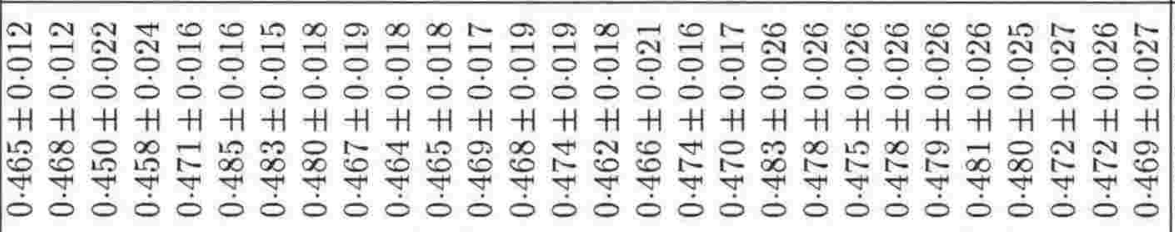 & 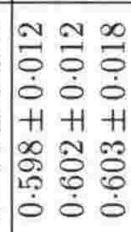 & \\
\hline & & 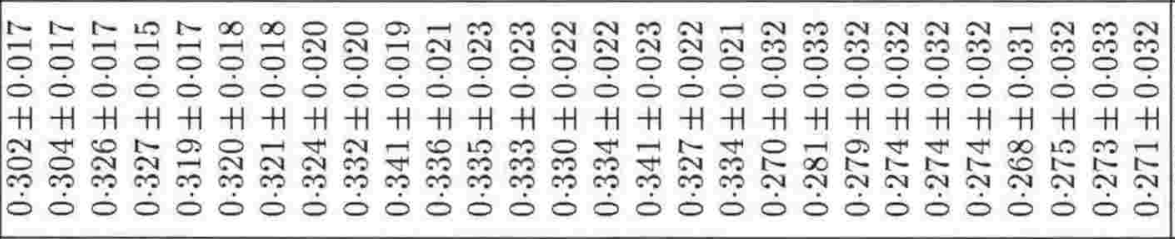 & 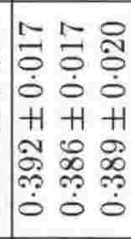 & \\
\hline & & 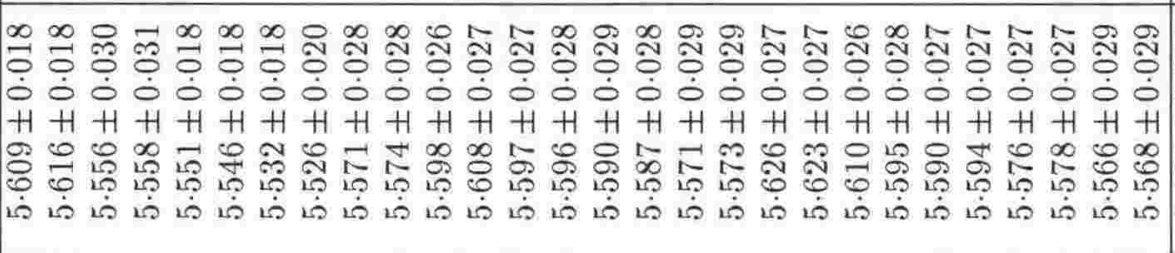 & 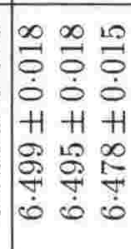 & \\
\hline & & 용 & 年 市 : & \\
\hline & & 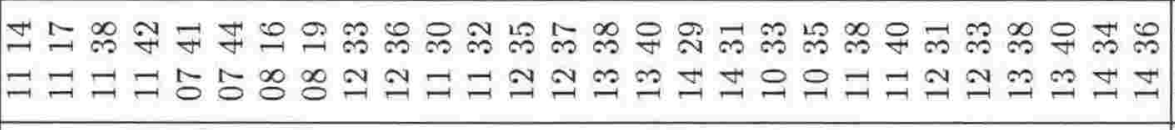 & 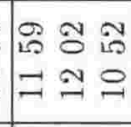 & \\
\hline & & 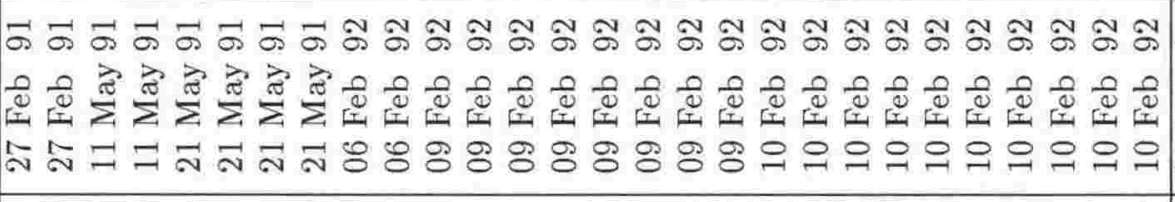 & 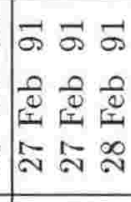 & \\
\hline & & & to & \\
\hline
\end{tabular}




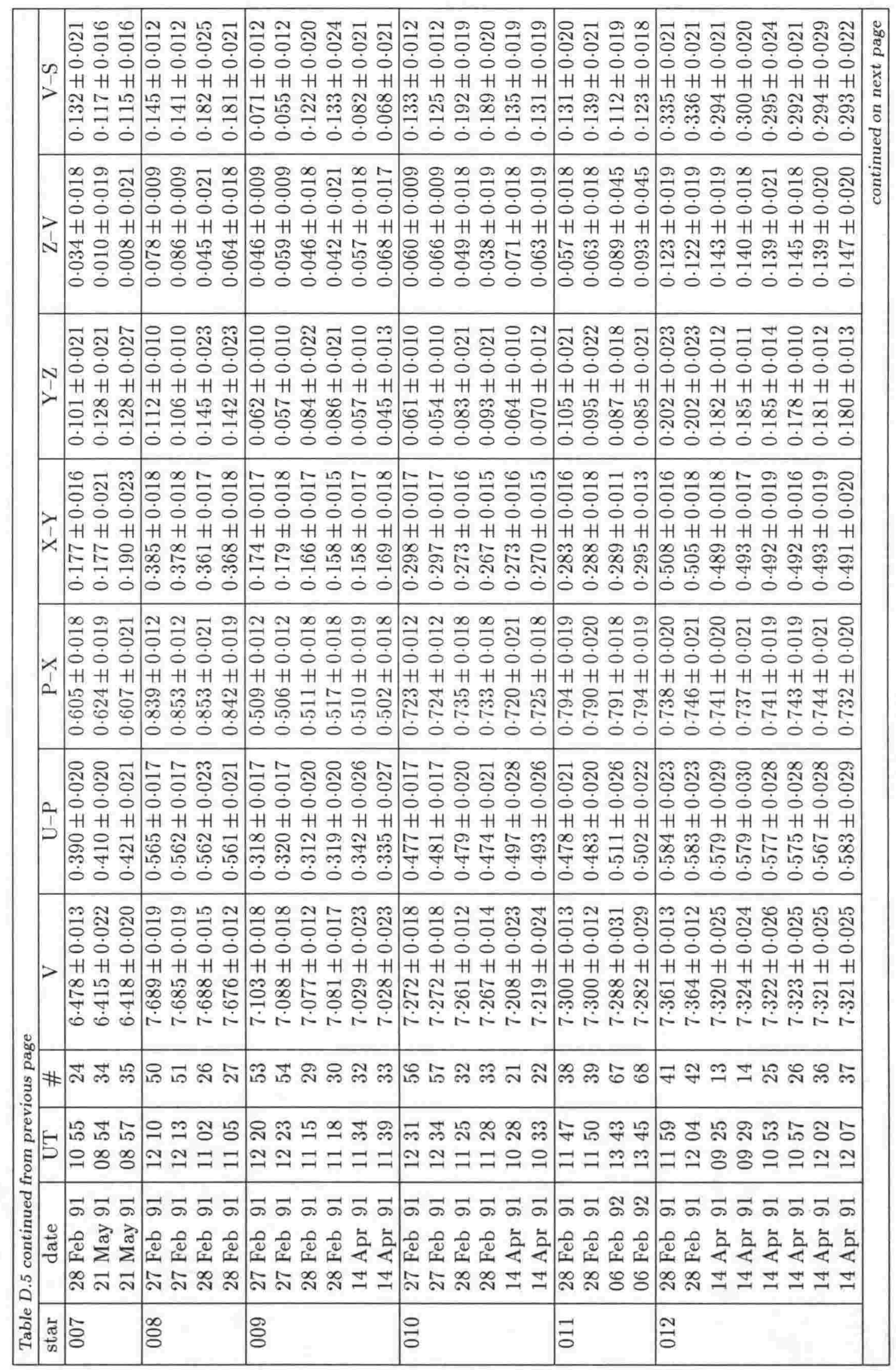




\begin{tabular}{|c|c|c|c|c|c|c|c|c|c|c|c|c|c|c|}
\hline & & 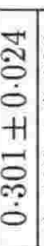 & $\begin{array}{ll}2 & ? \\
0 & 0 \\
0 & 0 \\
0 & 0 \\
H & H \\
0 & 0 \\
0 & 0 \\
& 0 \\
0 & 0\end{array}$ & 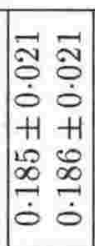 & 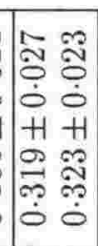 & $\begin{array}{ll}\infty & 9 \\
0 & 0 \\
0 & 0 \\
0 & 0 \\
H & H \\
0 & 1 \\
0 & 0 \\
0 & 0 \\
0 & 0\end{array}$ & 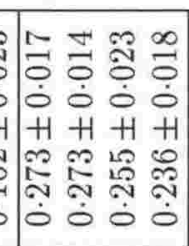 & 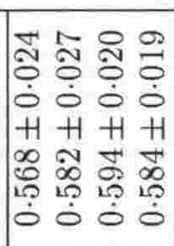 & 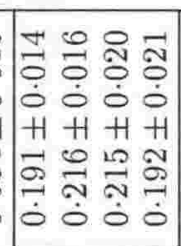 & 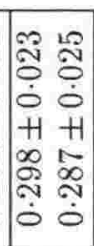 & 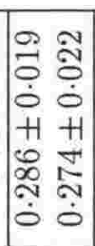 & 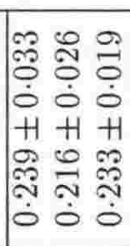 & 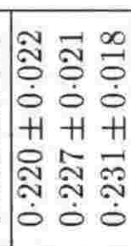 & 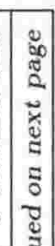 \\
\hline & & 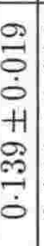 & 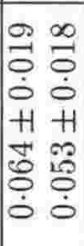 & 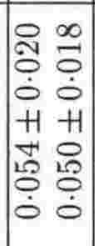 & 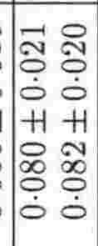 & $\begin{array}{ll}20 & 12 \\
0 & 0 \\
0 & 0 \\
0 & 0 \\
+1 & H \\
0 & +1 \\
0 & 0 \\
0 & 0\end{array}$ & 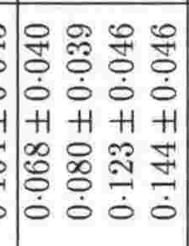 & 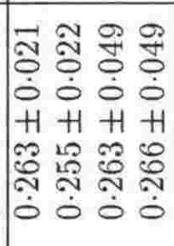 & 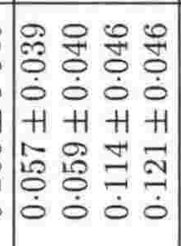 & 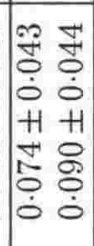 & \begin{tabular}{ll}
1 & $\infty$ \\
\multirow{5}{*}{} & 0 \\
0 & 0 \\
0 & 0 \\
+1 & +1 \\
0 & 0 \\
0 & 0 \\
\hdashline & 0 \\
0 & 0
\end{tabular} & 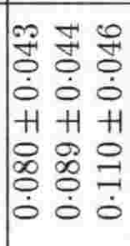 & 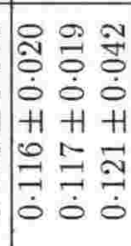 & 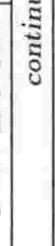 \\
\hline & & 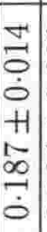 & 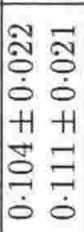 & 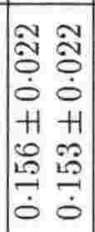 & 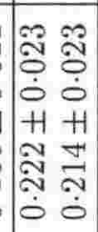 & 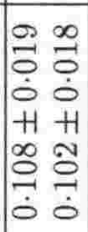 & 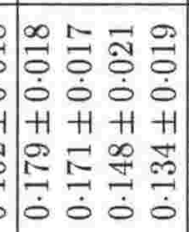 & 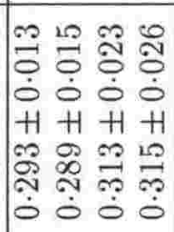 & 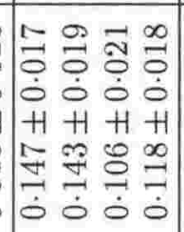 & $\begin{array}{ll}\vec{\sim} & 0 \\
0 & 0 \\
0 & 0 \\
0 & 0 \\
H & +1 \\
\dot{0} & 8 \\
0 & 0 \\
0 & 0 \\
0 & 0\end{array}$ & 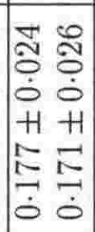 & 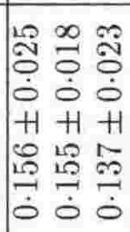 & 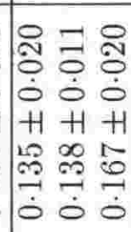 & \\
\hline & & 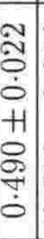 & $\begin{array}{ll}0 & 10 \\
0 & 0 \\
0 & 0 \\
0 & 0 \\
+1 & H \\
0 & 0 \\
0 & 0 \\
0 & 0 \\
0 & 0\end{array}$ & 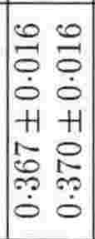 & 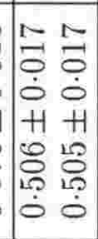 & 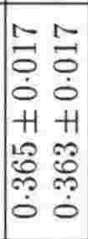 & 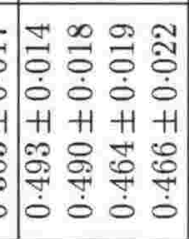 & 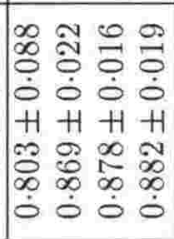 & 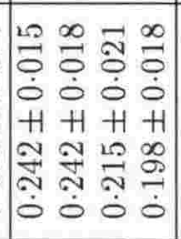 & $\begin{array}{ll}1 & 10 \\
0 & 0 \\
0 & 0 \\
0 & 0 \\
1 & +1 \\
1 & 2 \\
0 & 0 \\
0 & 0 \\
0 & 0 \\
0 & 0\end{array}$ & 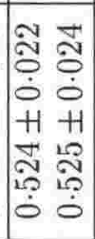 & & 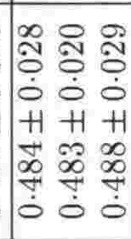 & \\
\hline & & 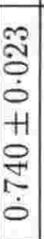 & 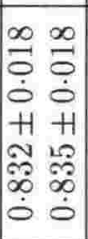 & $\mid \begin{array}{cc}0 & 0 \\
0 & 2 \\
\dot{0} & \dot{0} \\
+1 & +1 \\
\infty & 10 \\
0 & 0 \\
0 & 0 \\
0 & 0 \\
0 & 0\end{array}$ & 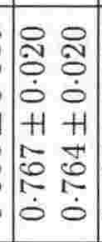 & 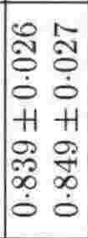 & 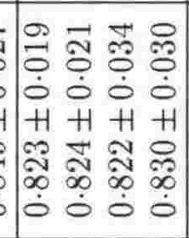 & 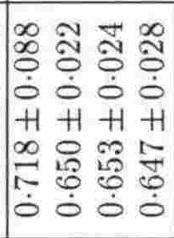 & 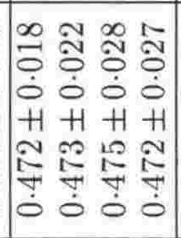 & 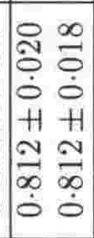 & 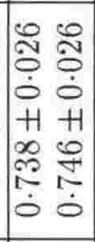 & 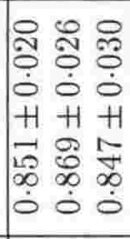 & 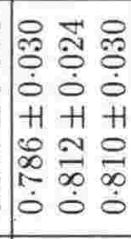 & \\
\hline & & $\begin{array}{c}2 \\
\stackrel{2}{0} \\
\dot{0} \\
+1 \\
1 \\
0 \\
1 \\
0 \\
0\end{array}$ & 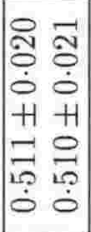 & 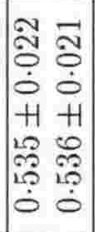 & 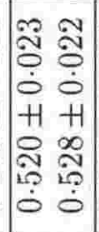 & 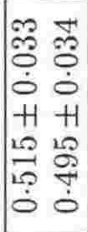 & 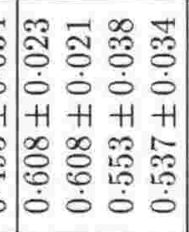 & 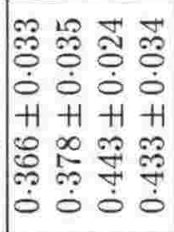 & 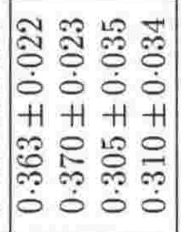 & 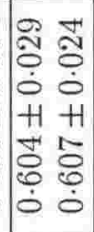 & 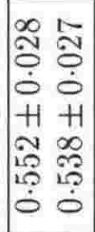 & 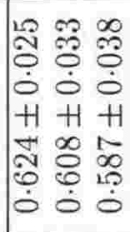 & 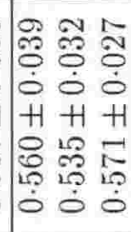 & \\
\hline & & 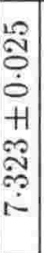 & 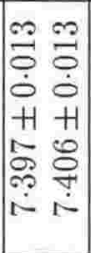 & 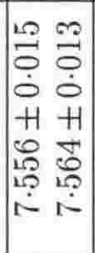 & 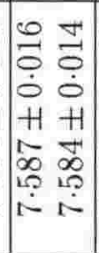 & 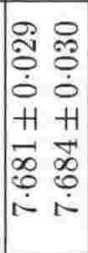 & 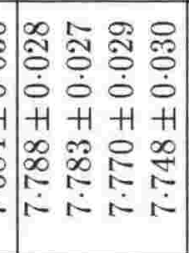 & 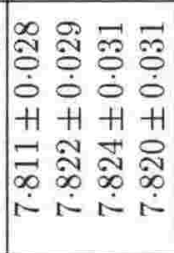 & 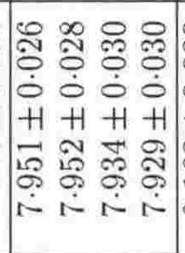 & 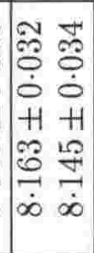 & 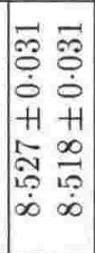 & 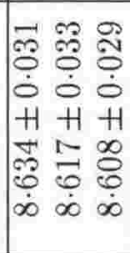 & 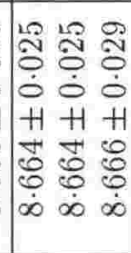 & \\
\hline & & $\stackrel{10}{8}$ & F' & 을 & 象 & 注 & च 녕 8 & 花 & ๓ & 앙 年 & $\approx \infty$ & 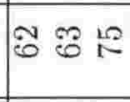 & 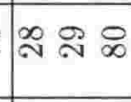 & \\
\hline & & $\begin{array}{l}\sim \\
10 \\
\sim \\
\simeq\end{array}$ & $\left|\begin{array}{ll}0 & \vec{N} \\
\stackrel{\sim}{N} & \stackrel{\sim}{\mid}\end{array}\right|$ & 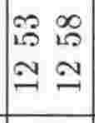 & $\begin{array}{ll}8 & 10 \\
0 & 9 \\
9 & -9\end{array}$ & 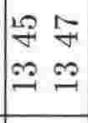 & 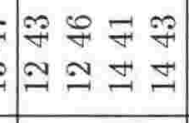 & 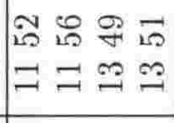 & 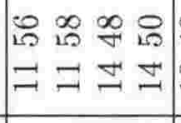 & & $\begin{array}{ll}\infty & \exists \\
\Xi & \Xi\end{array}$ & 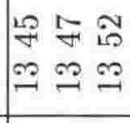 & $\begin{array}{l}O= \pm \\
= \pm \\
=\end{array}$ & \\
\hline & 总 & \begin{tabular}{c|}
$\vec{\sigma}$ \\
$\vec{a}$ \\
$\vec{a}$ \\
$\overrightarrow{1}$
\end{tabular} & 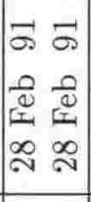 & $\left|\begin{array}{ll}\vec{\sigma} & \bar{\sigma} \\
0 & 0 \\
0 & 0 \\
0 & 0 \\
\infty & \infty \\
\sim & \infty\end{array}\right|$ & $\left|\begin{array}{ll|}\vec{\sigma} & \overrightarrow{0} \\
0 & 0 \\
0 & 0 \\
0 & 0 \\
0 & \infty\end{array}\right|$ & 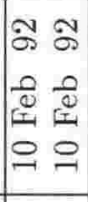 & $\begin{array}{llll}\delta & \delta & \delta & \delta \\
0 & 0 & 0 & 0 \\
0 & 0 & 0 & 0 \\
8 & 8 & 0 & 0\end{array}$ & 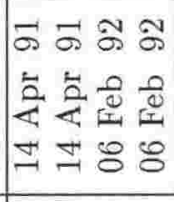 & 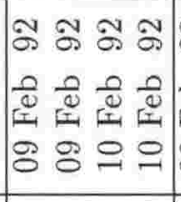 & 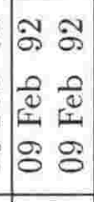 & 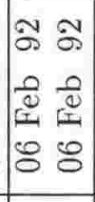 & 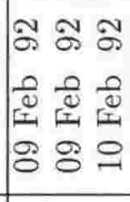 & 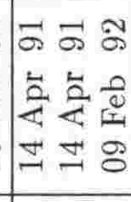 & \\
\hline & $\frac{\tilde{\pi}}{\omega}$ & & & $\underset{0}{\Xi}$ & $\frac{12}{6}$ & $\stackrel{0}{0}$ & $\stackrel{-}{0}$ & $\frac{\infty}{6}$ & 尺ి & & & & $\stackrel{\leftrightarrow}{\delta}$ & \\
\hline
\end{tabular}




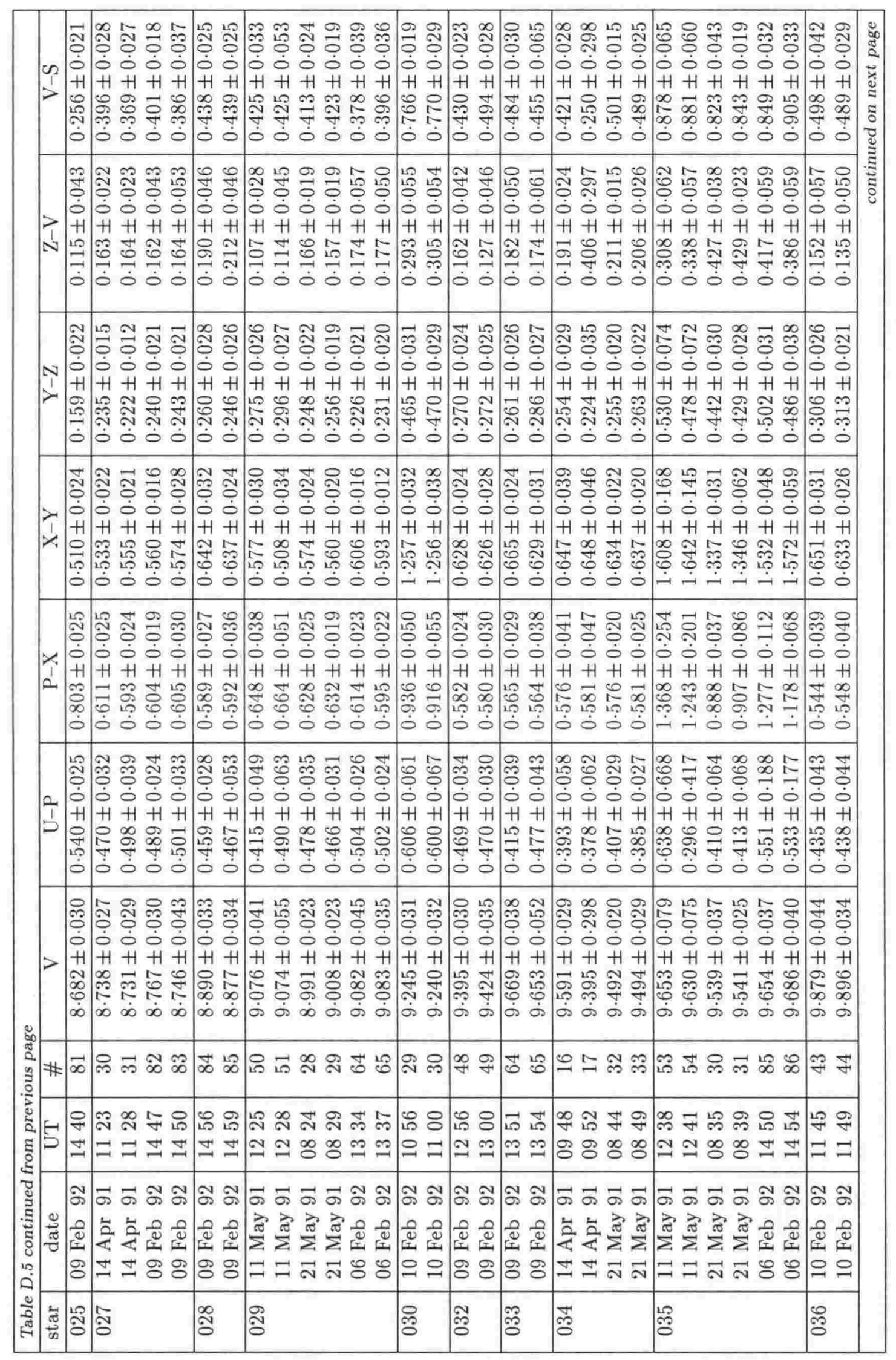




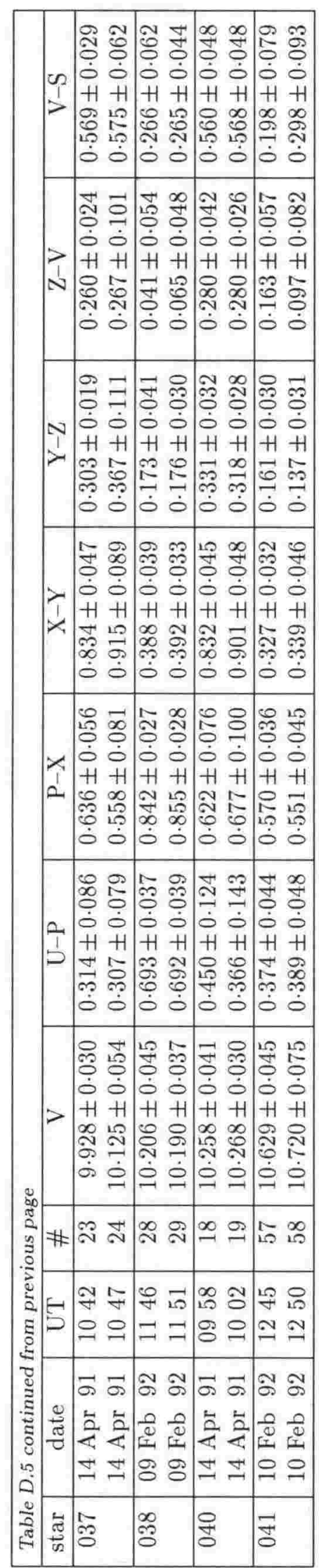




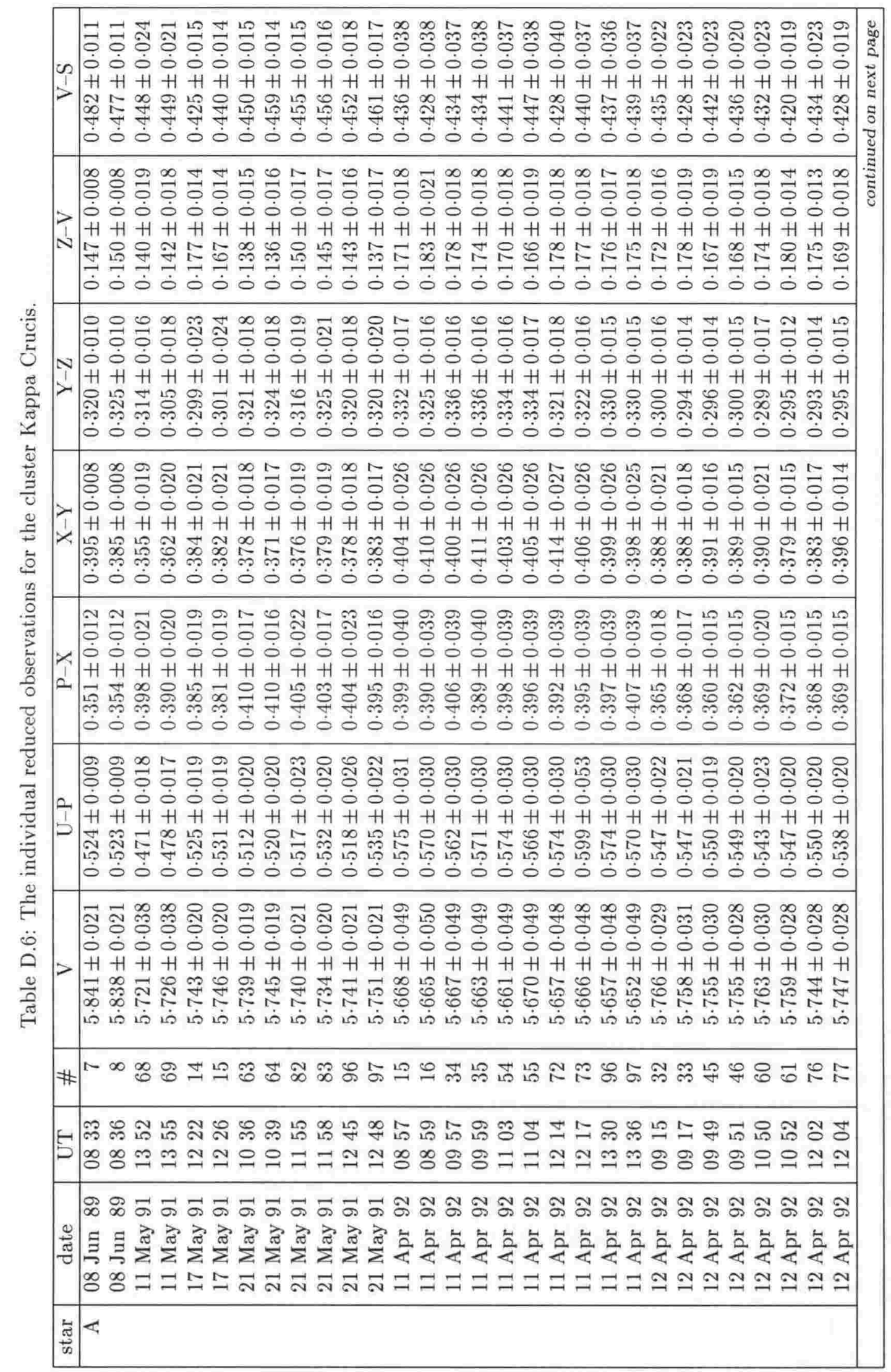




\begin{tabular}{|c|c|c|c|c|}
\hline & & 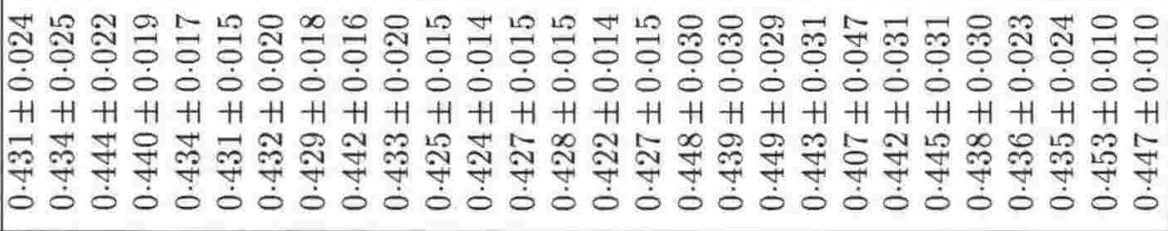 & 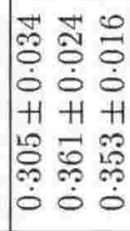 & 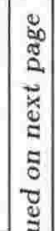 \\
\hline & & 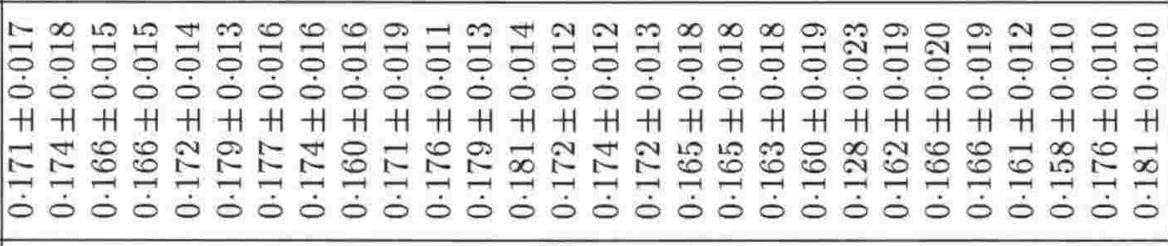 & 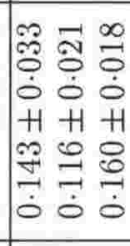 & 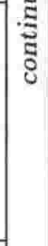 \\
\hline & & 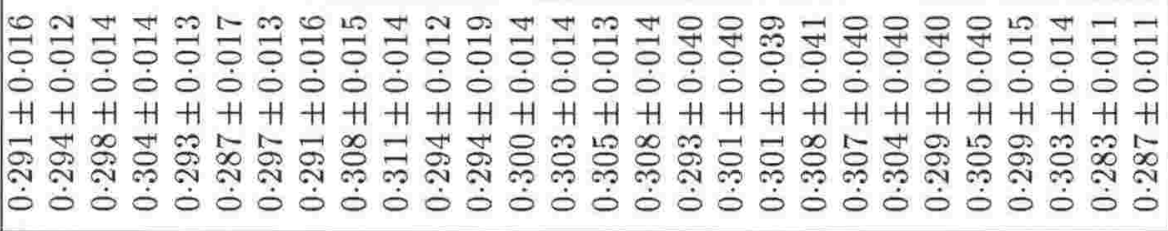 & 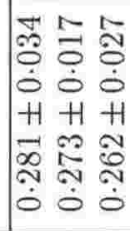 & \\
\hline & 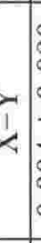 & 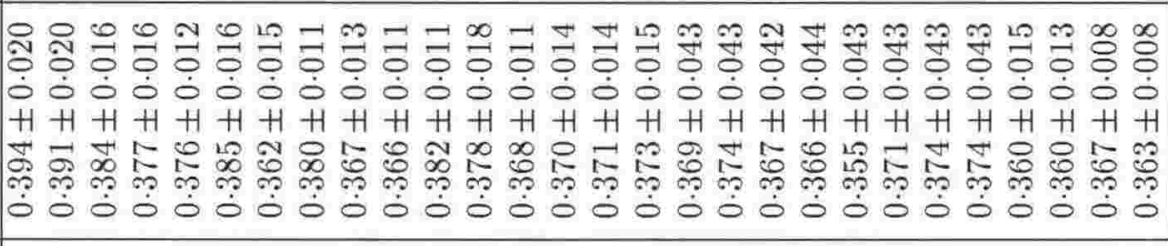 & 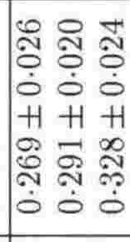 & \\
\hline & & 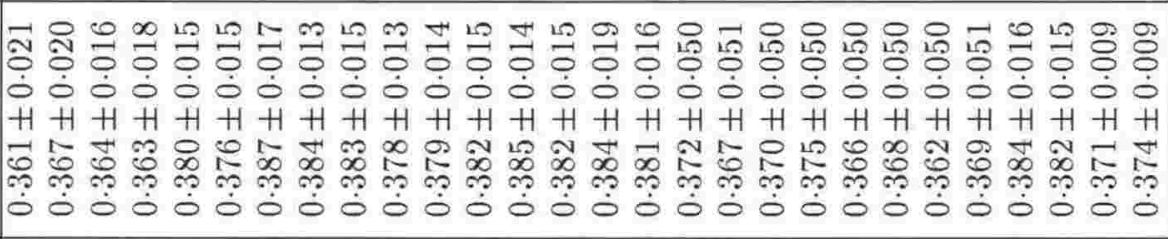 & 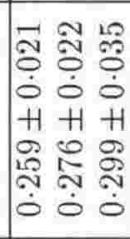 & \\
\hline & 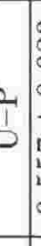 & 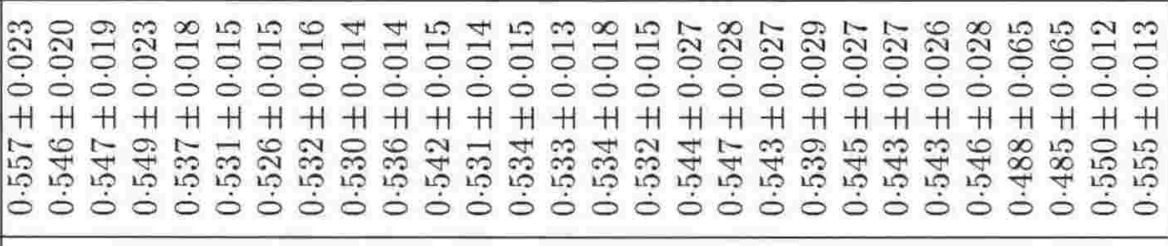 & 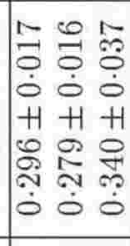 & \\
\hline & & 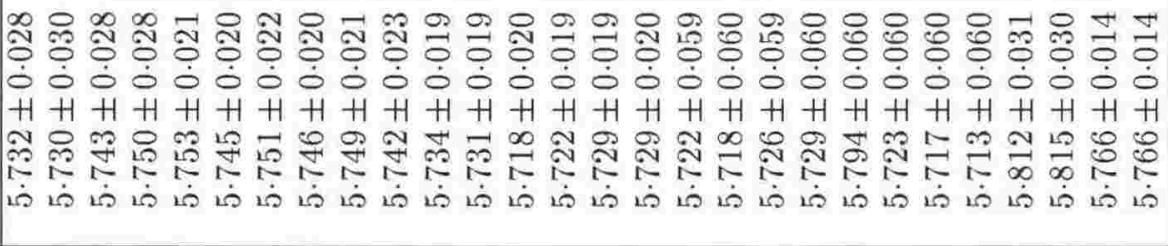 & 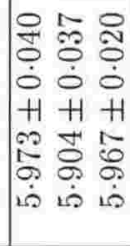 & \\
\hline & $\#$ & 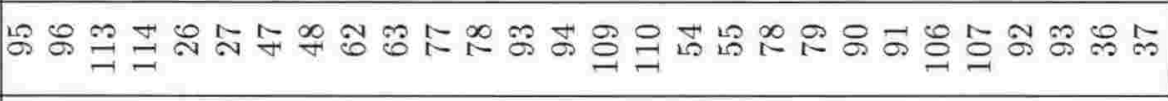 & I & \\
\hline & & 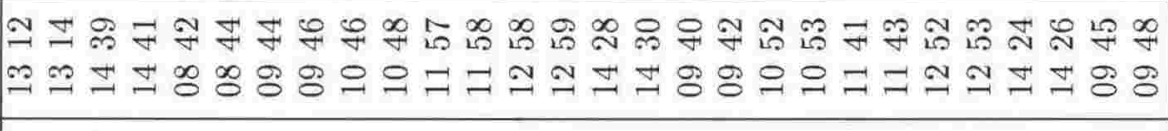 & 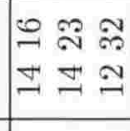 & \\
\hline & & 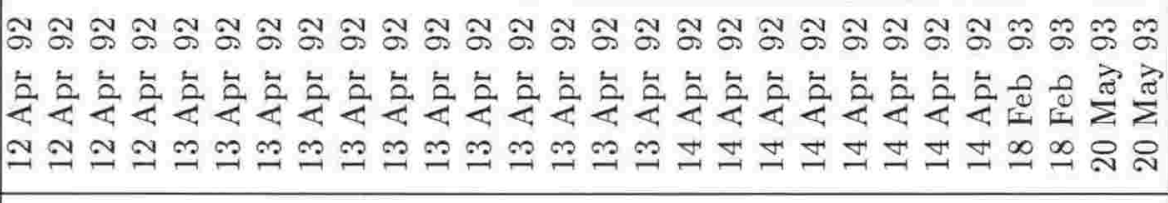 & 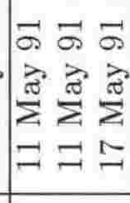 & \\
\hline & 密 & $\varangle$ & $m$ & \\
\hline
\end{tabular}




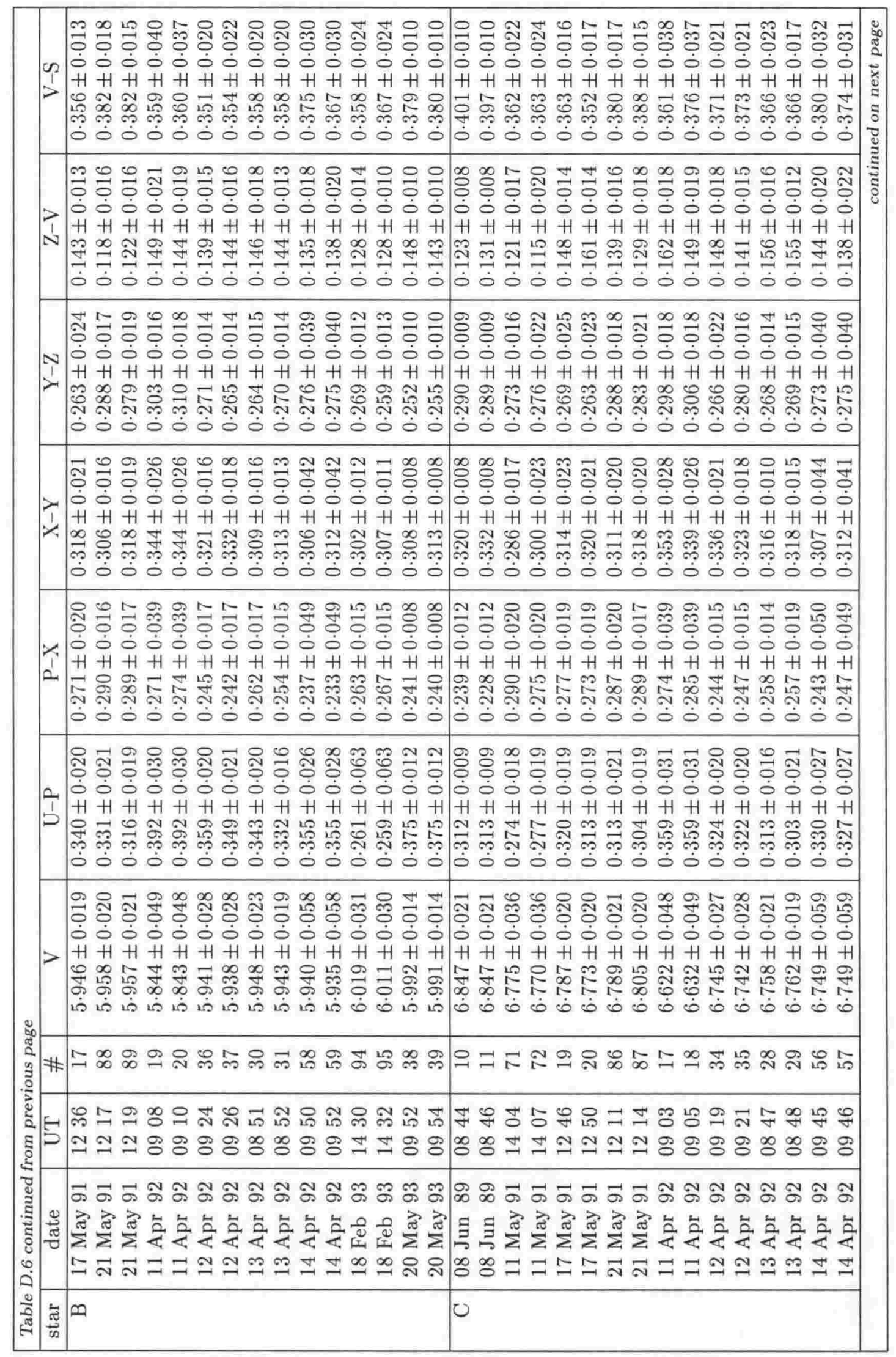




\begin{tabular}{|c|c|c|c|c|c|c|}
\hline & & 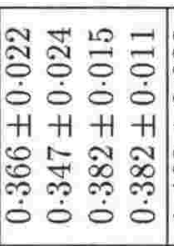 & 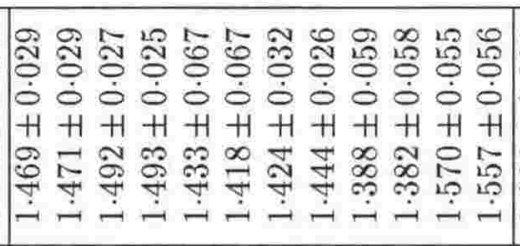 & 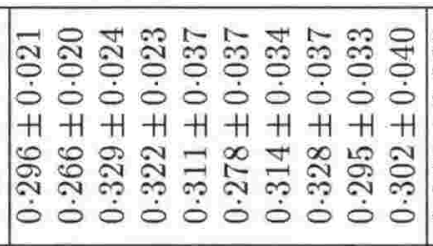 & 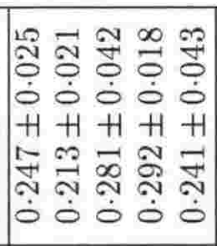 & 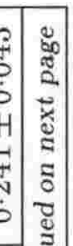 \\
\hline & & 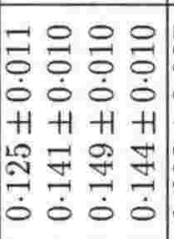 & 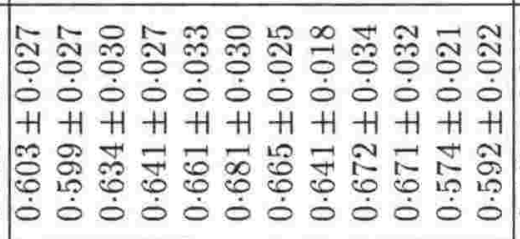 & 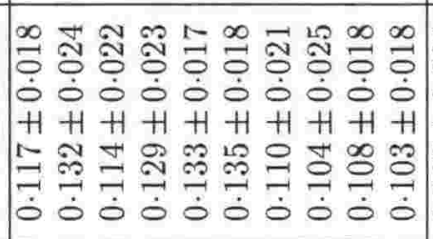 & 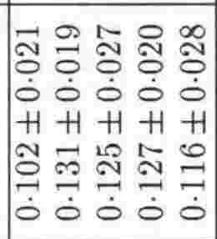 & 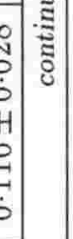 \\
\hline & & 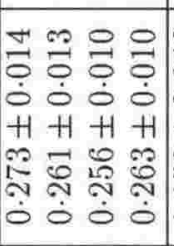 & 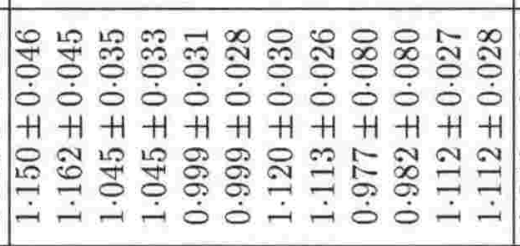 & 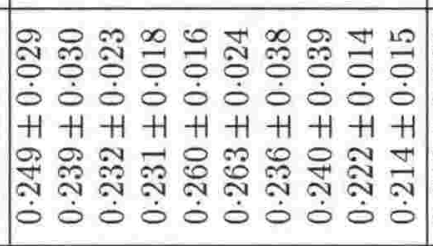 & 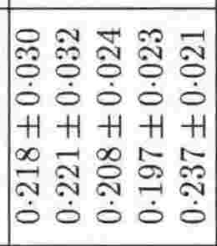 & \\
\hline & & 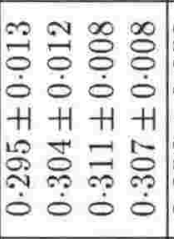 & 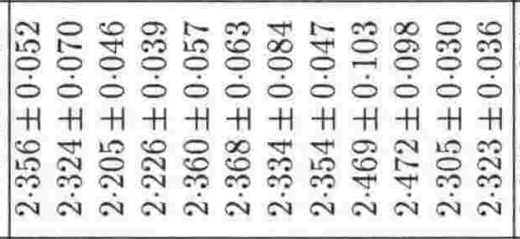 & 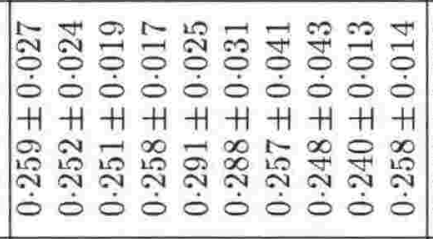 & 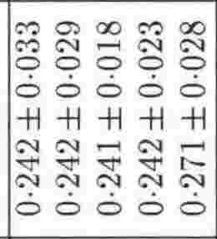 & \\
\hline & & 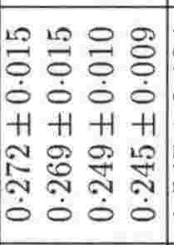 & 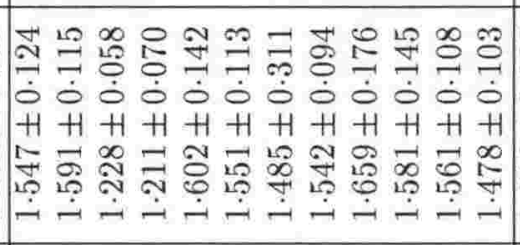 & 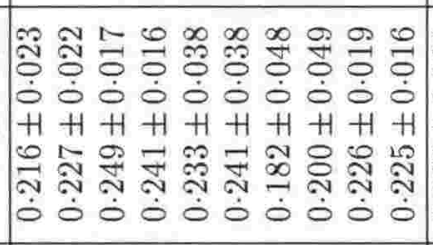 & 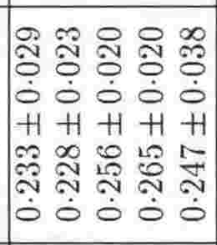 & \\
\hline & & 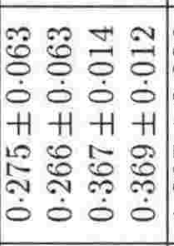 & 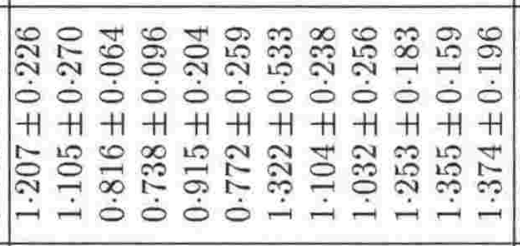 & 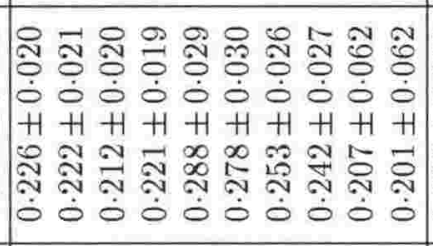 & 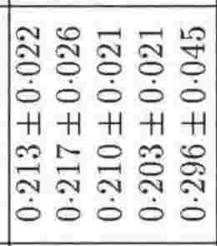 & \\
\hline & & 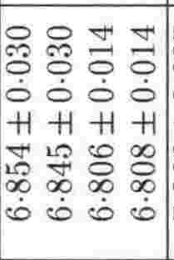 & 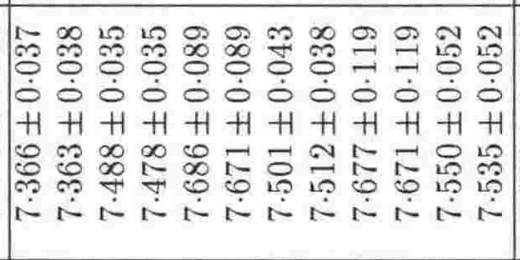 & 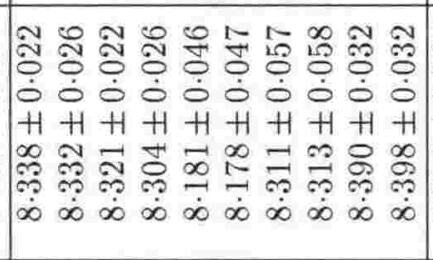 & 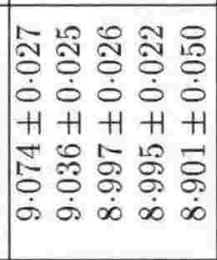 & \\
\hline & & 옹육ㄱ & 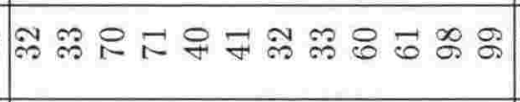 & 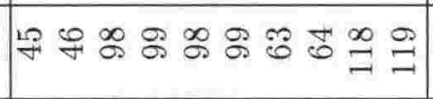 & 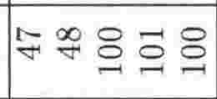 & \\
\hline & & 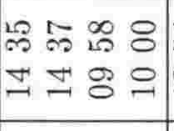 & 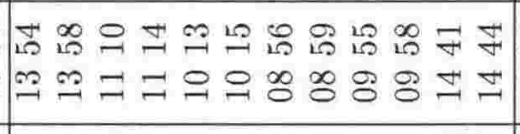 & 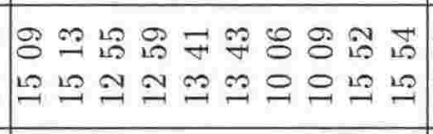 & 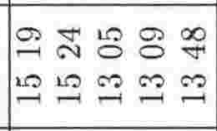 & \\
\hline & & 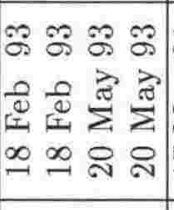 & 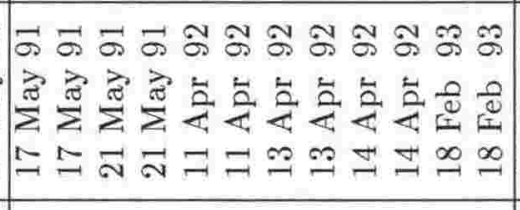 & 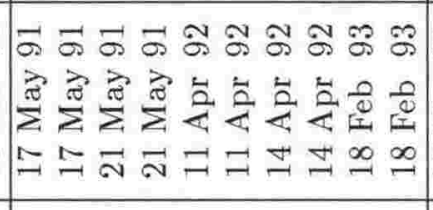 & 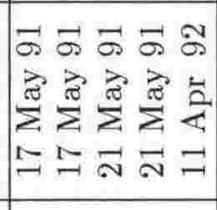 & \\
\hline & & 0 & $A$ & 뙤 & Ex & \\
\hline
\end{tabular}




\begin{tabular}{|c|c|c|c|c|c|c|c|c|c|}
\hline & & 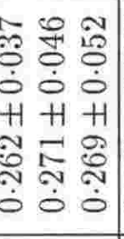 & 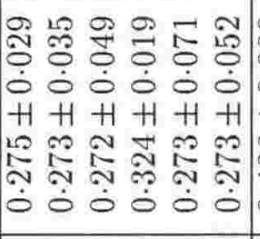 & 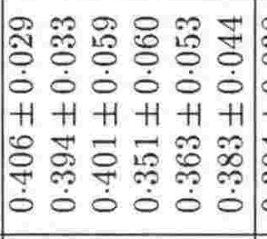 & 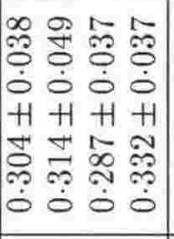 & 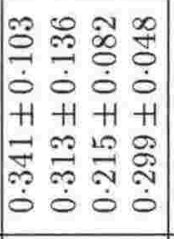 & 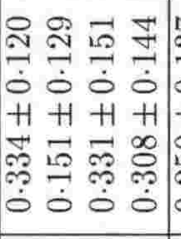 & 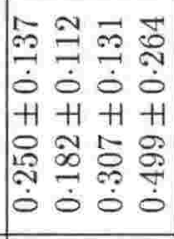 & 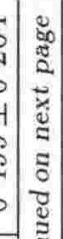 \\
\hline & & \begin{tabular}{lll}
$\infty$ & 0 & \\
0 & 0 & 0 \\
0 & 0 \\
0 & 0 & 0 \\
$H$ & $H$ & +1 \\
0 & 0 & 0 \\
\hdashline & 0 & 0 \\
0 & 0 & 0
\end{tabular} & 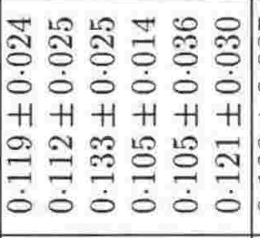 & 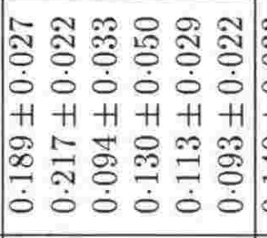 & 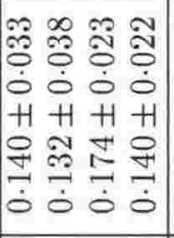 & 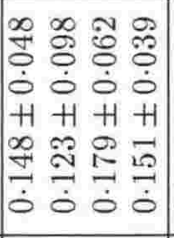 & 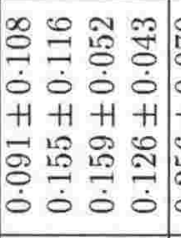 & 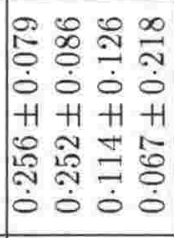 & . \\
\hline & & 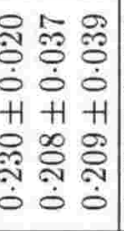 & 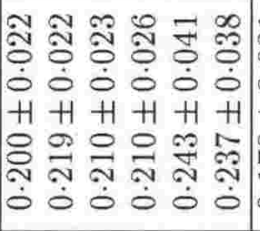 & 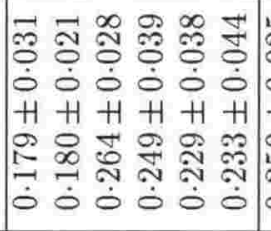 & 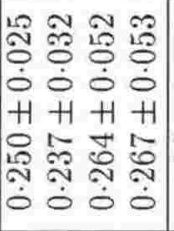 & 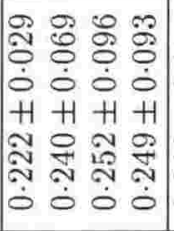 & 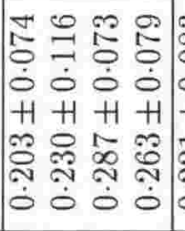 & 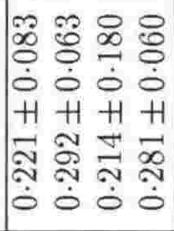 & \\
\hline & & 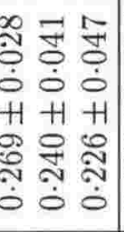 & 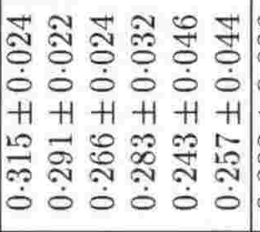 & 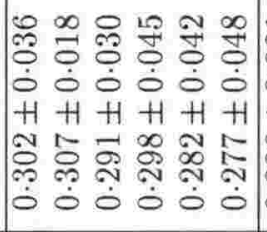 & 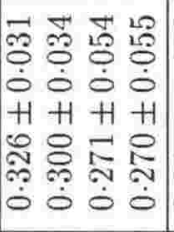 & 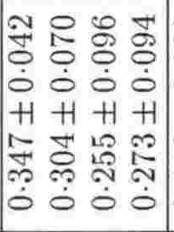 & 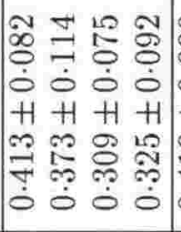 & 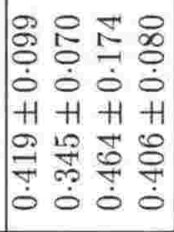 & \\
\hline & & 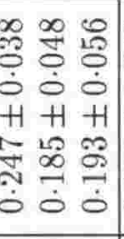 & 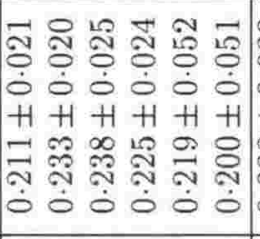 & 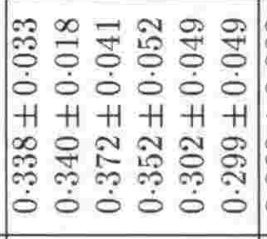 & 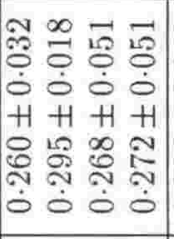 & 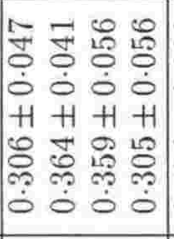 & 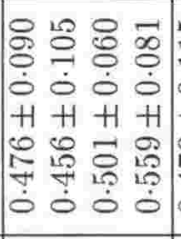 & 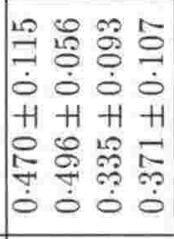 & \\
\hline & & 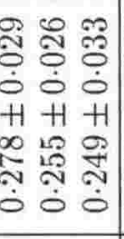 & 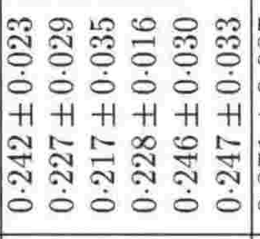 & 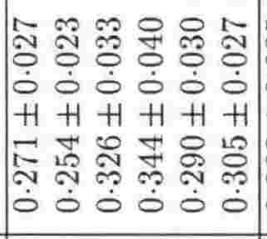 & 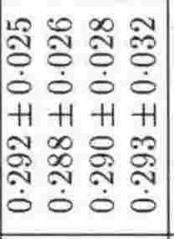 & 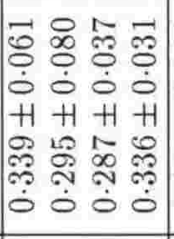 & 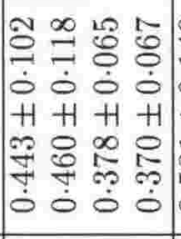 & 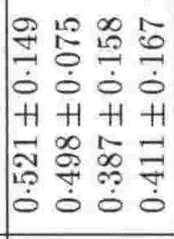 & \\
\hline 8 & & 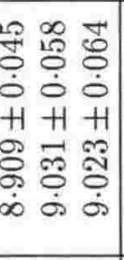 & 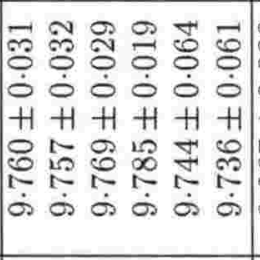 & 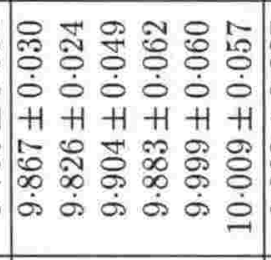 & 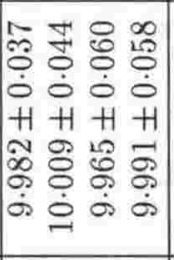 & 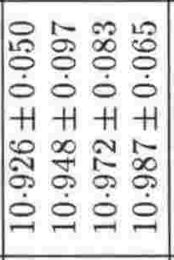 & 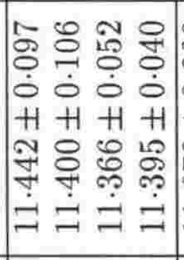 & 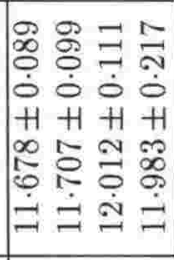 & \\
\hline & $H$ & 5.69 & $\infty$ 事 & 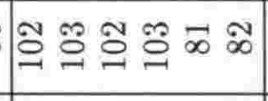 & เి & 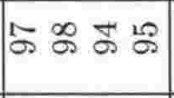 & $\infty \vec{\infty}$ เึ is & 绐合年舟 & \\
\hline & & $\begin{array}{lll}8 & \cong & 10 \\
0 & 0 & 0 \\
-1 & 0 & 0\end{array}$ & 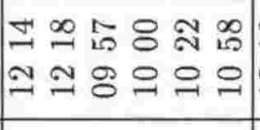 & 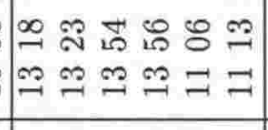 & 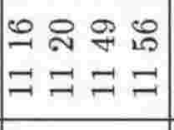 & 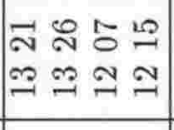 & 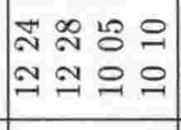 & 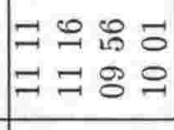 & \\
\hline & & 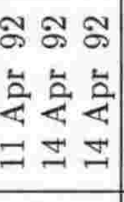 & 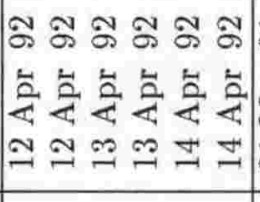 & 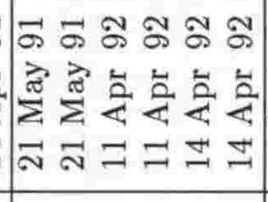 & 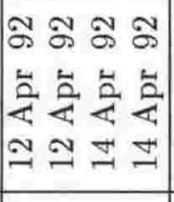 & 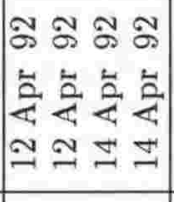 & 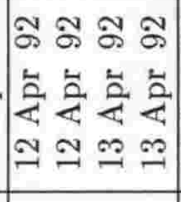 & 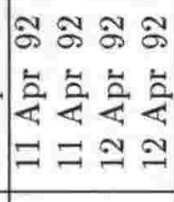 & \\
\hline ๘. & 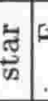 & & 0 & $I$ & - & $\rightarrow$ & $\simeq$ & 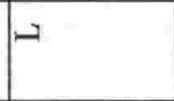 & \\
\hline
\end{tabular}




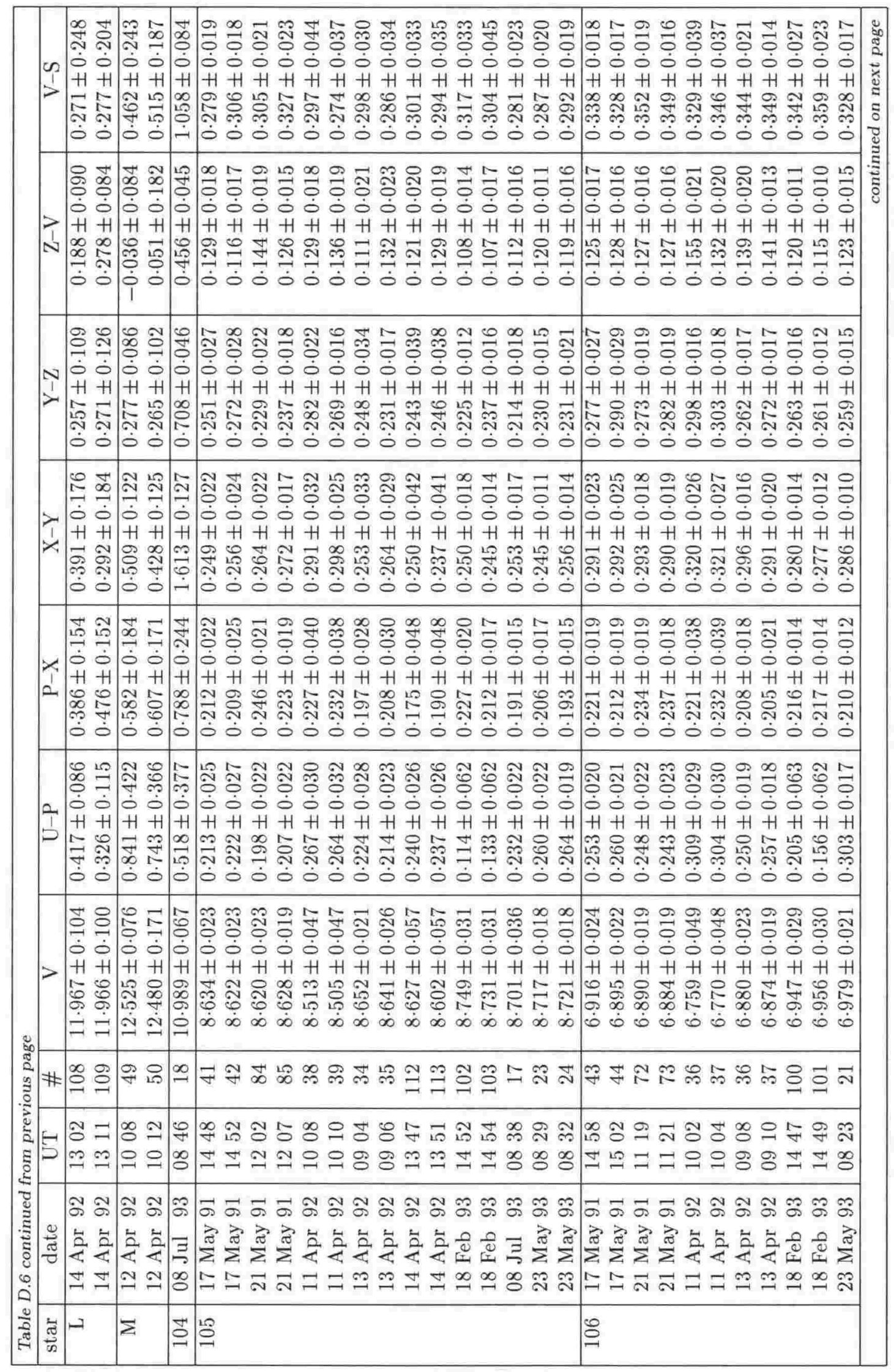




\begin{tabular}{|c|c|c|c|c|c|c|c|c|c|c|c|c|c|c|c|c|}
\hline & & & 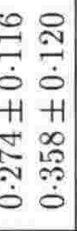 & 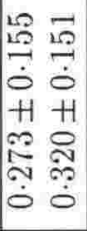 & 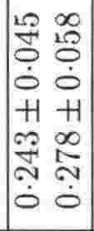 & 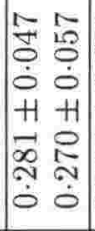 & 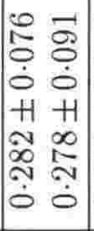 & 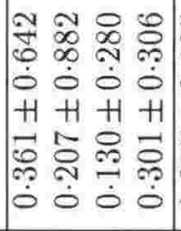 & 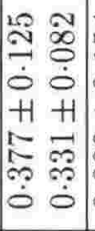 & 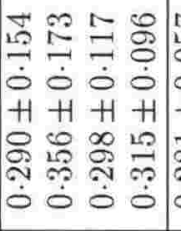 & & 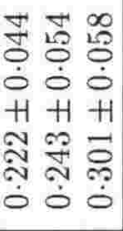 & $\begin{array}{c}\infty \\
0 \\
0 \\
\dot{0} \\
+1 \\
0 \\
0 \\
0 \\
0 \\
0\end{array}$ & 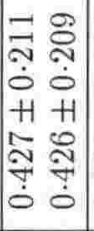 & 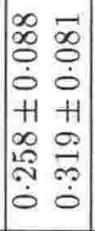 & 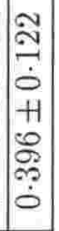 \\
\hline & & & 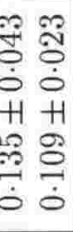 & 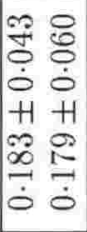 & 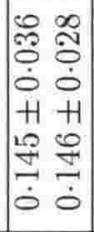 & 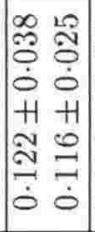 & $\mid$\begin{tabular}{ll}
1 & $\infty$ \\
10 & 0 \\
0 & 0 \\
0 & 0 \\
+1 & +1 \\
0 & -1 \\
0 & 0 \\
\hdashline & 0 \\
0 & 0
\end{tabular} & 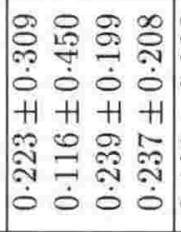 & 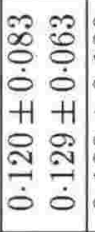 & 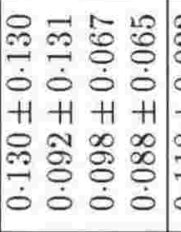 & 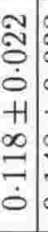 & 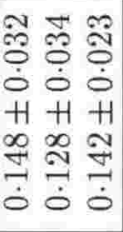 & \begin{tabular}{c}
0 \\
$\vdots$ \\
0 \\
$\dot{0}$ \\
+1 \\
0 \\
\hdashline \\
0 \\
0
\end{tabular} & 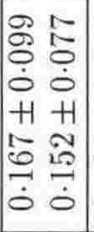 & 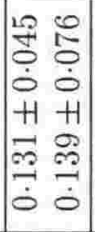 & $\begin{array}{l}\vec{\delta} \\
\dot{0} \\
0 \\
+1 \\
0 \\
\stackrel{्}{9} \\
\dot{0}\end{array}$ \\
\hline & & & 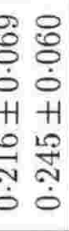 & 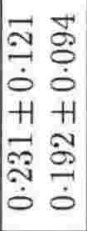 & $\mid \begin{array}{ll}2 & \infty \\
0 & 0 \\
0 & 0 \\
0 & 0 \\
+1 & 1 \\
0 & 8 \\
0 & 0 \\
0 & 0 \\
0 & 0\end{array}$ & 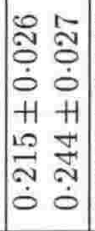 & 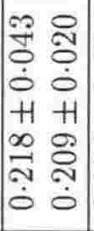 & 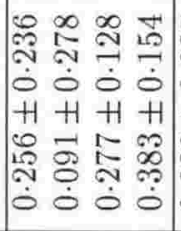 & 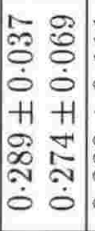 & 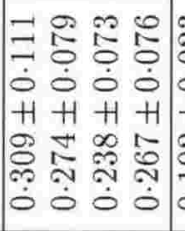 & 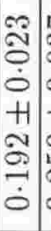 & 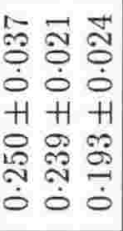 & 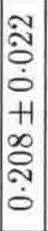 & 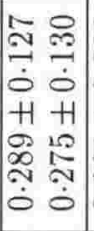 & 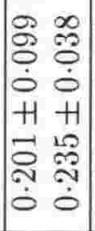 & 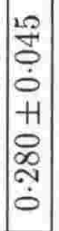 \\
\hline & & & 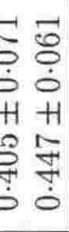 & 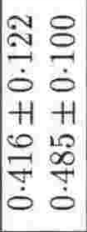 & 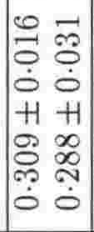 & 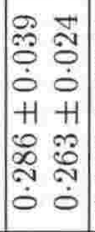 & 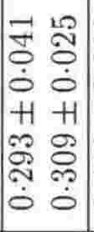 & 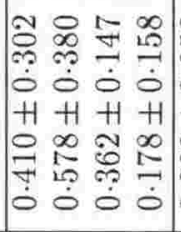 & 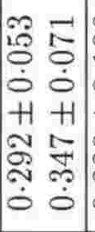 & 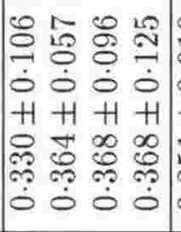 & $\begin{array}{l}0 \\
\dot{0} \\
\dot{0} \\
+ \\
H \\
\overrightarrow{2} \\
\stackrel{2}{2} \\
\dot{0}\end{array}$ & 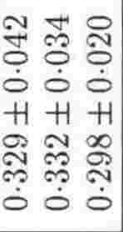 & 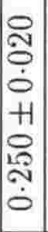 & 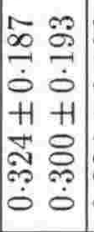 & 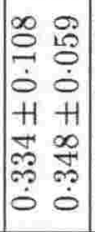 & $\begin{array}{c}\dddot{2} \\
\infty \\
0 \\
0 \\
0 \\
+1 \\
\tilde{N} \\
\infty \\
\infty \\
0 \\
0\end{array}$ \\
\hline & & & 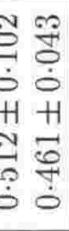 & 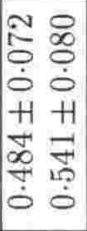 & 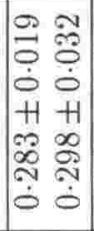 & 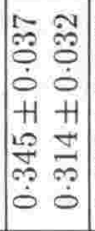 & $\mid \begin{array}{ll}2 & 4 \\
0 & 0 \\
0 & 0 \\
0 & 0 \\
H & +1 \\
0 & \infty \\
0 & 0 \\
0 & 0\end{array}$ & 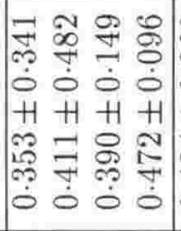 & 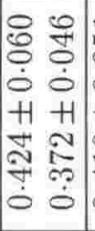 & 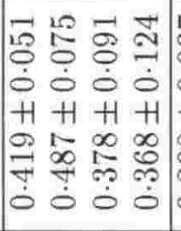 & $\begin{array}{ll}1 & \\
0 & \\
0 & \\
0 & \\
+1 & \\
\infty & \\
0 & \end{array}$ & 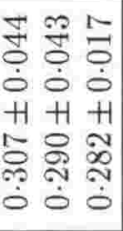 & 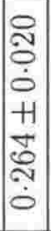 & 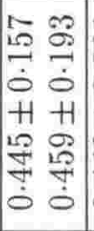 & 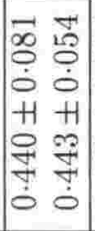 & $\begin{array}{l}\infty \\
8 \\
0 \\
0 \\
+1 \\
01 \\
0 \\
0 \\
0 \\
0\end{array}$ \\
\hline & & & 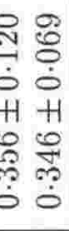 & 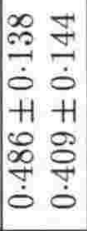 & 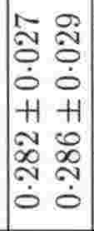 & 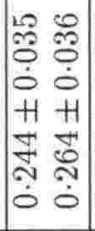 & 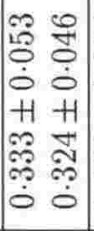 & 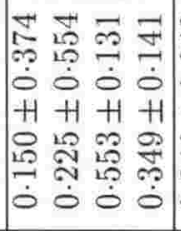 & 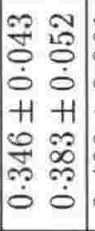 & 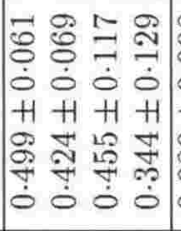 & 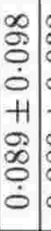 & 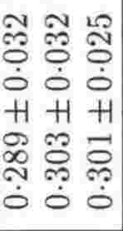 & $\begin{array}{c}\infty \\
\stackrel{0}{0} \\
\stackrel{0}{0} \\
+1 \\
0 \\
\stackrel{0}{0} \\
0\end{array}$ & 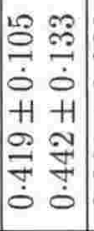 & 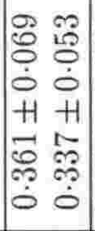 & $\begin{array}{l}2 \\
8 \\
0 \\
0 \\
0 \\
+1 \\
10 \\
10 \\
10 \\
0\end{array}$ \\
\hline & & & 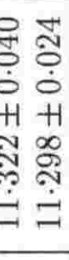 & 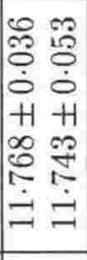 & $\begin{array}{ll}F & 0 \\
0 & 0 \\
0 & 0 \\
0 & 0 \\
+1 & +1 \\
0 & 10 \\
\exists & = \\
0 & 0 \\
0 & 0\end{array}$ & 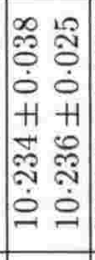 & 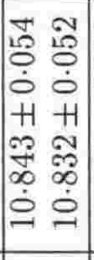 & 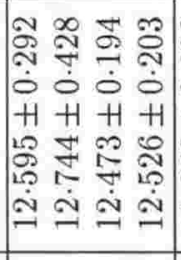 & 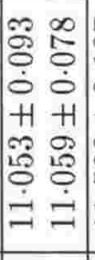 & 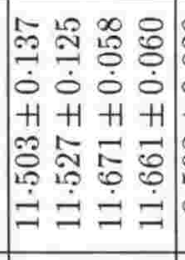 & 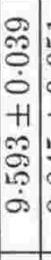 & 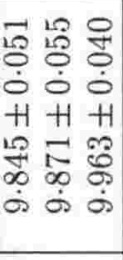 & 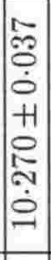 & 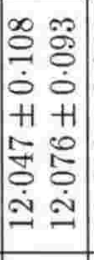 & 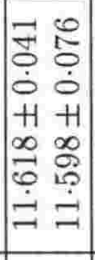 & 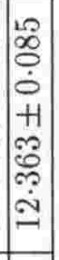 \\
\hline & $\# \approx$ & $N$ & 86 & $\infty 8$ & 흥 & $\infty$ & \& 8 & ๑ : ని : & 80 & 䑰 & $\stackrel{\infty}{\sim}$ & $\stackrel{*}{*}$ ล & $\approx$ & $\triangleq \Xi$ & $\curvearrowright \infty$ & $\vec{\infty}$ \\
\hline & $5:$ & & $\begin{array}{l}\text { 의을 } \\
\Rightarrow=\end{array}$ & 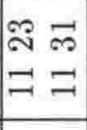 & $\mid$\begin{tabular}{ll}
10 & 9 \\
10 & 8 \\
\hdashline & \multirow{Z}{*}{}
\end{tabular} & \begin{tabular}{ll}
$\Re$ & $=$ \\
\hdashline & 8 \\
8 & 8
\end{tabular} & $\left|\begin{array}{ll}\exists & \infty \\
\hdashline & \ddots \\
\hdashline & -9\end{array}\right|$ & 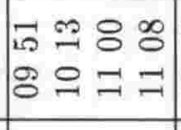 & $\begin{array}{ll}\approx & \infty \\
\approx & \ddots \\
= & =\end{array}$ & 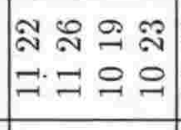 & 5 & 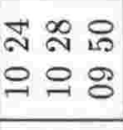 & $\begin{array}{l}7 \\
8 \\
8\end{array}$ & 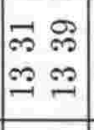 & 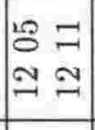 & $\begin{array}{l}\stackrel{\Omega}{2} \\
\approx \\
\simeq\end{array}$ \\
\hline 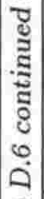 & 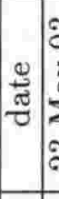 & & 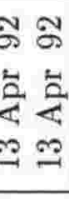 & 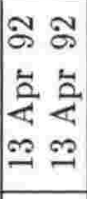 & 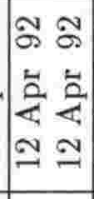 & 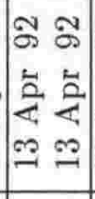 & 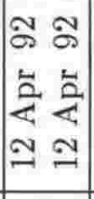 & 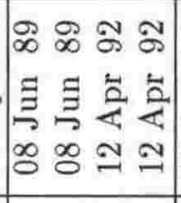 & 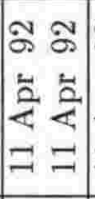 & 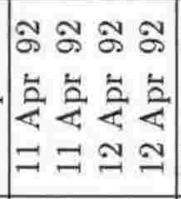 & 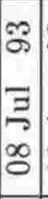 & 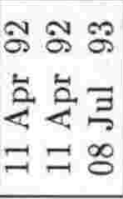 & $\begin{array}{l}\infty \\
\Xi \\
\Xi \\
\infty\end{array}$ & 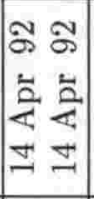 & 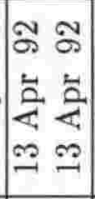 & 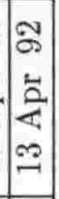 \\
\hline & 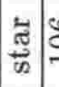 & & & $\stackrel{\infty}{0}$ & $\dddot{\nexists}$ & $\stackrel{29}{=}$ & $\cong$ & & & & & & & & जे & \\
\hline
\end{tabular}




\begin{tabular}{|c|c|c|c|c|c|c|c|}
\hline & & & 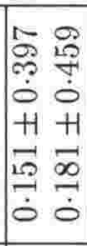 & 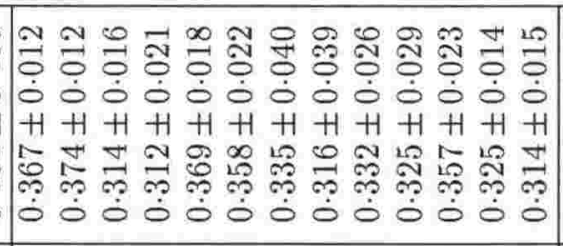 & 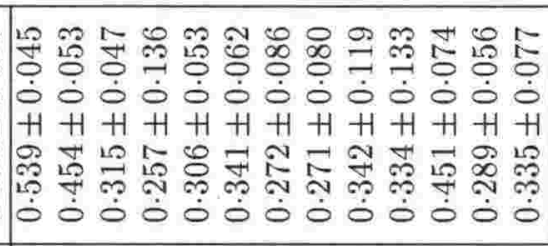 & 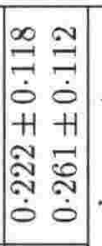 & 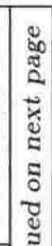 \\
\hline & & & 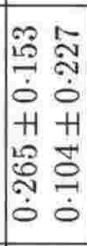 & 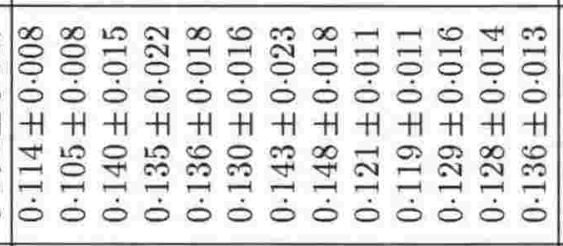 & 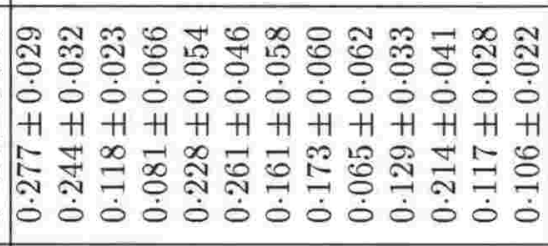 & 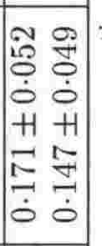 & \\
\hline & & $\begin{array}{l}5 \\
\stackrel{1}{2} \\
\dot{0} \\
+1 \\
+1\end{array}$ & 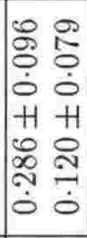 & 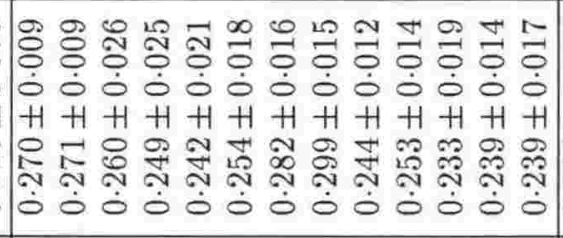 & 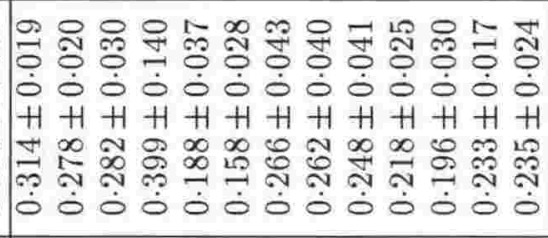 & 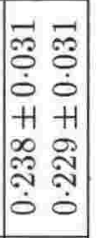 & \\
\hline & & & \begin{tabular}{ll}
2 & 0 \\
0 & 0 \\
\hdashline & 0 \\
0 & 0 \\
+ & +1 \\
$\infty$ & 0 \\
0 & 0 \\
0 & $\stackrel{1}{+}$ \\
0 & 0
\end{tabular} & 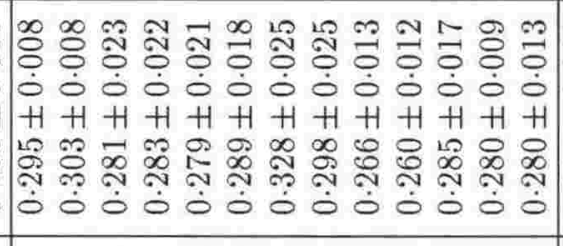 & 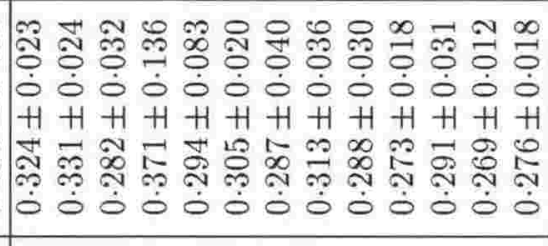 & 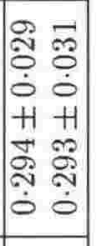 & \\
\hline & & 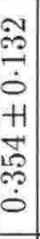 & 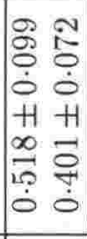 & 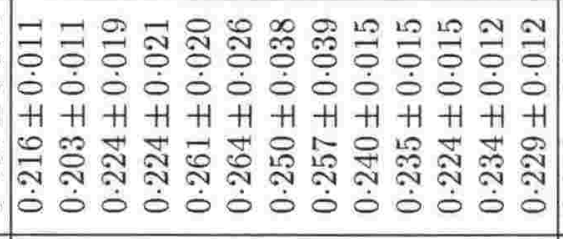 & 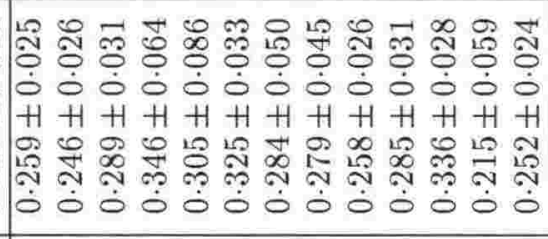 & 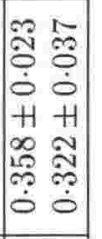 & \\
\hline & & & \begin{tabular}{ll}
$\infty$ & 8 \\
$\exists$ & 0 \\
0 & 0 \\
0 & 0 \\
+1 & $H$ \\
0 & 1 \\
0 & 10 \\
\hdashline & $f$ \\
0 & 0 \\
0
\end{tabular} & 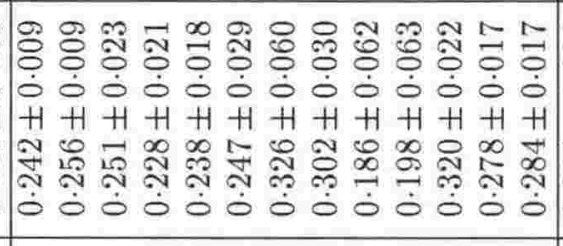 & 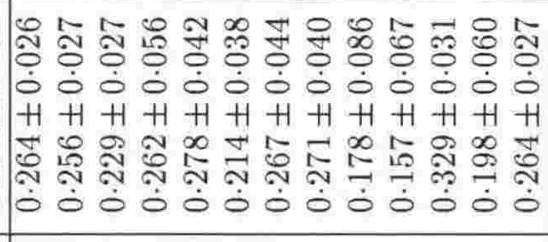 & 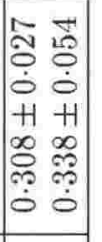 & \\
\hline & & & 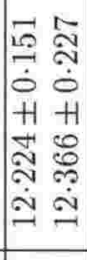 & 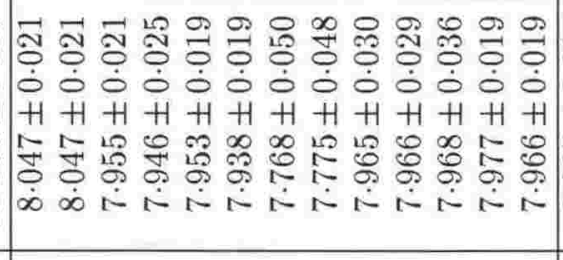 & 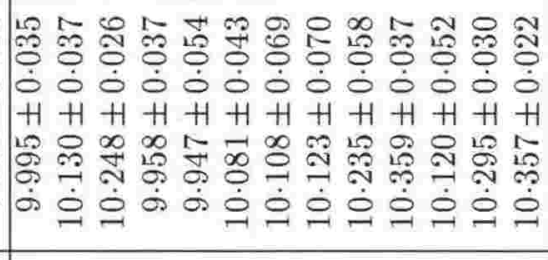 & 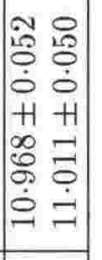 & \\
\hline & & $\infty$ & 18 & 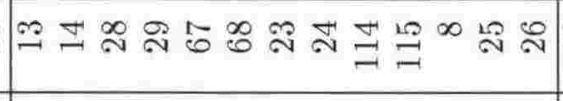 & $\because$ 듬 & $5 \infty$ & \\
\hline & & $\begin{array}{l}\stackrel{P}{2} \\
\stackrel{1}{\sim}\end{array}$ & 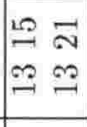 & 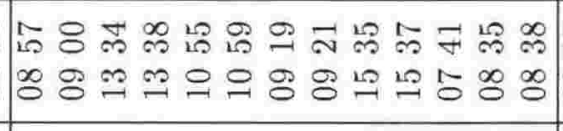 & 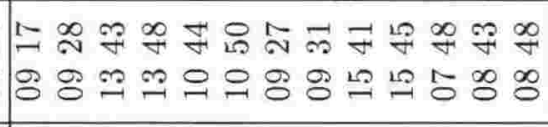 & $\left|\begin{array}{cc}0 & \infty \\
\hdashline & \ddots \\
\hdashline & \ddots-1\end{array}\right|$ & \\
\hline & & 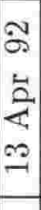 & 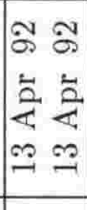 & 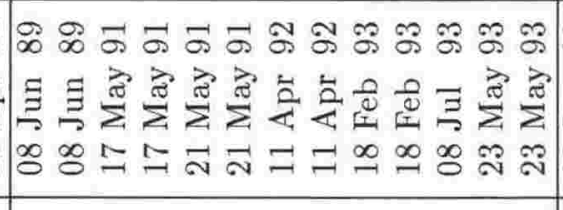 & 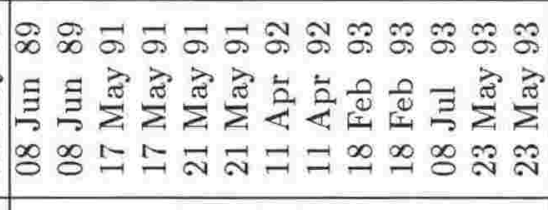 & 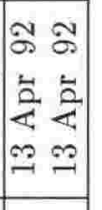 & \\
\hline & & & & $\approx \underset{\AA}{\AA}$ & ্ָন & $\stackrel{\infty}{\sim}$ & \\
\hline
\end{tabular}




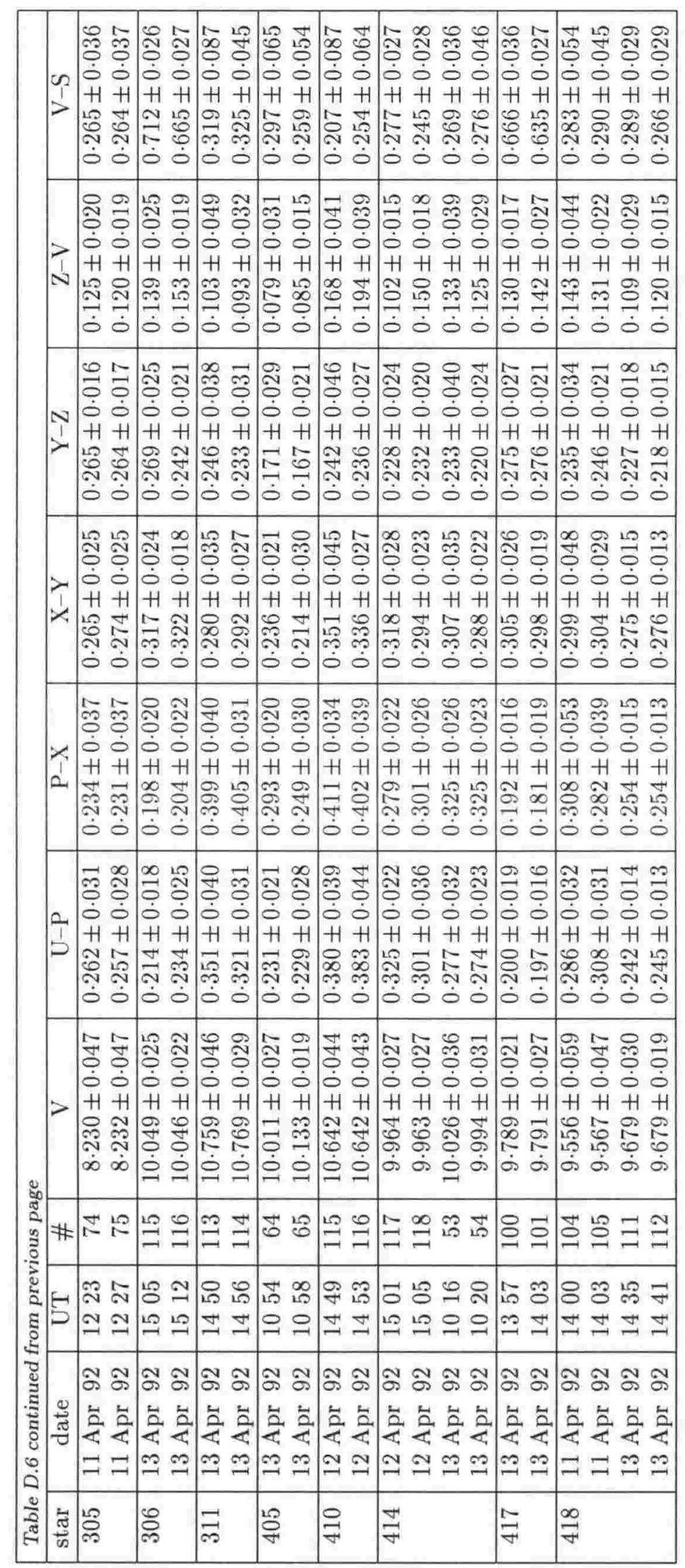




\section{Appendix E}

\section{The Mean Reduced Observations.}

The following tables give the mean reduced magnitudes from the observations of the primary standard stars, secondary standard stars, the clusters $\kappa$ Crucis and $\mathrm{O}$ Vel.

The first column in each table is the star name, being either an HD number, the E Region number used by Menzies et al. (1989), or the open cluster number (as given by Dachs \& Kaiser (1984) for $\kappa$ Crucis and Lynga (1959) for O Vel). See the description of Table C.2 for the meaning of any symbols (in parenthesis) following the name. The next eight columns contain Vilnius V magnitude and colours, including the $\mathrm{Y}-\mathrm{V}$ colour. Each column has the weighted mean (weighted by the standard deviation of the individual observations) and the standard deviation of the mean. The final column is the number of observations made of the star.

The primary standard stars are not in fact standard stars of the (northern) Vilnius system but the stars that were observable from MJUO, and so were considered as candidate secondary standard stars (for the Southern Vilnius system). The secondary standard stars are listed in two tables, the first being bright stars spread across the southern sky and the other is stars from the Harvard E Regions. Four extra tables follows the secondary standard star tables these list the remaining candidate secondary standard stars that currently do not qualify as standards, usually because insufficient observations have been made of the star.

Not all of the stars listed as observed in Table C.2 are given in these tables as some observations were rejected as bad measurements (usually due to cloud). 


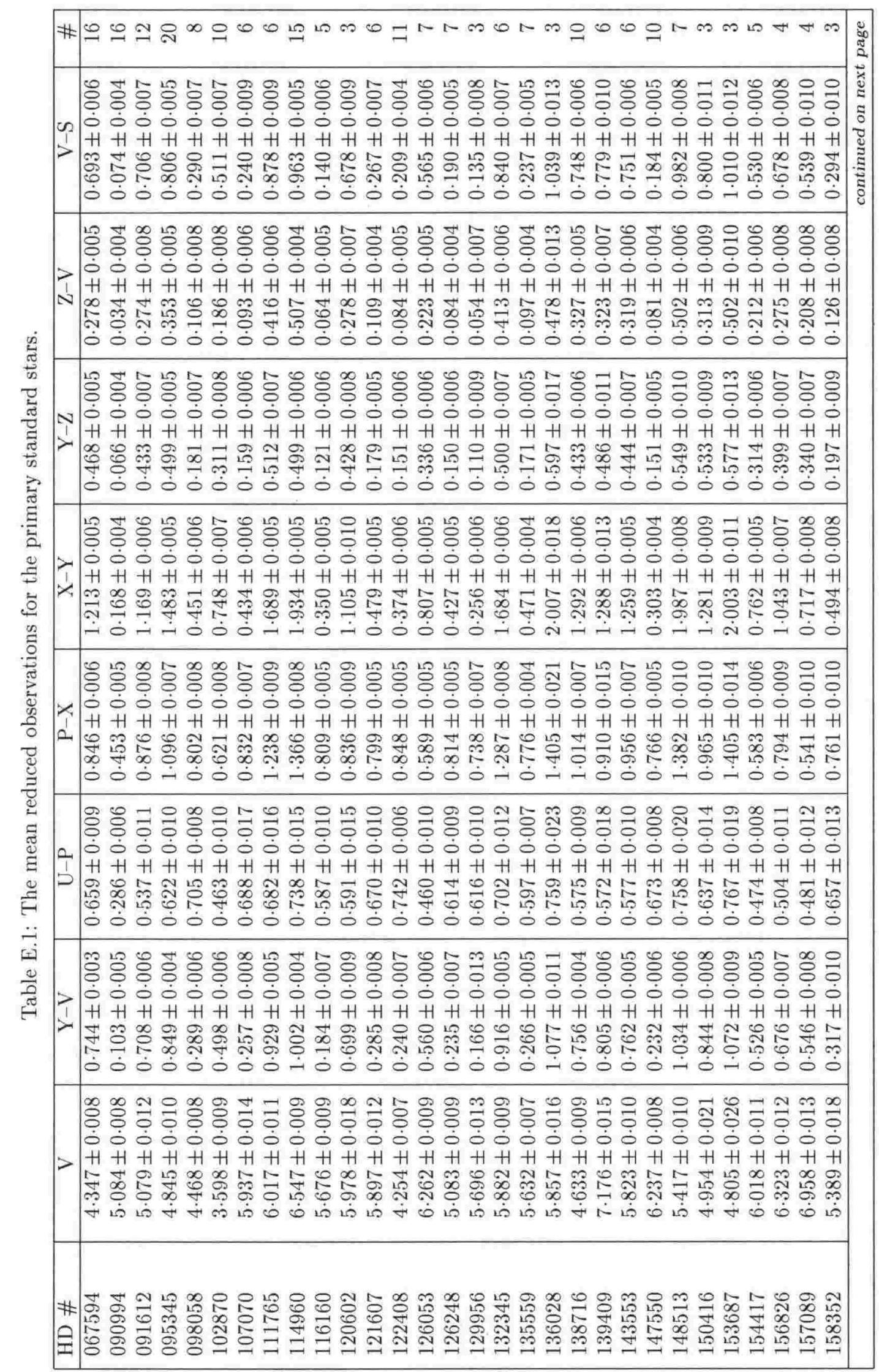




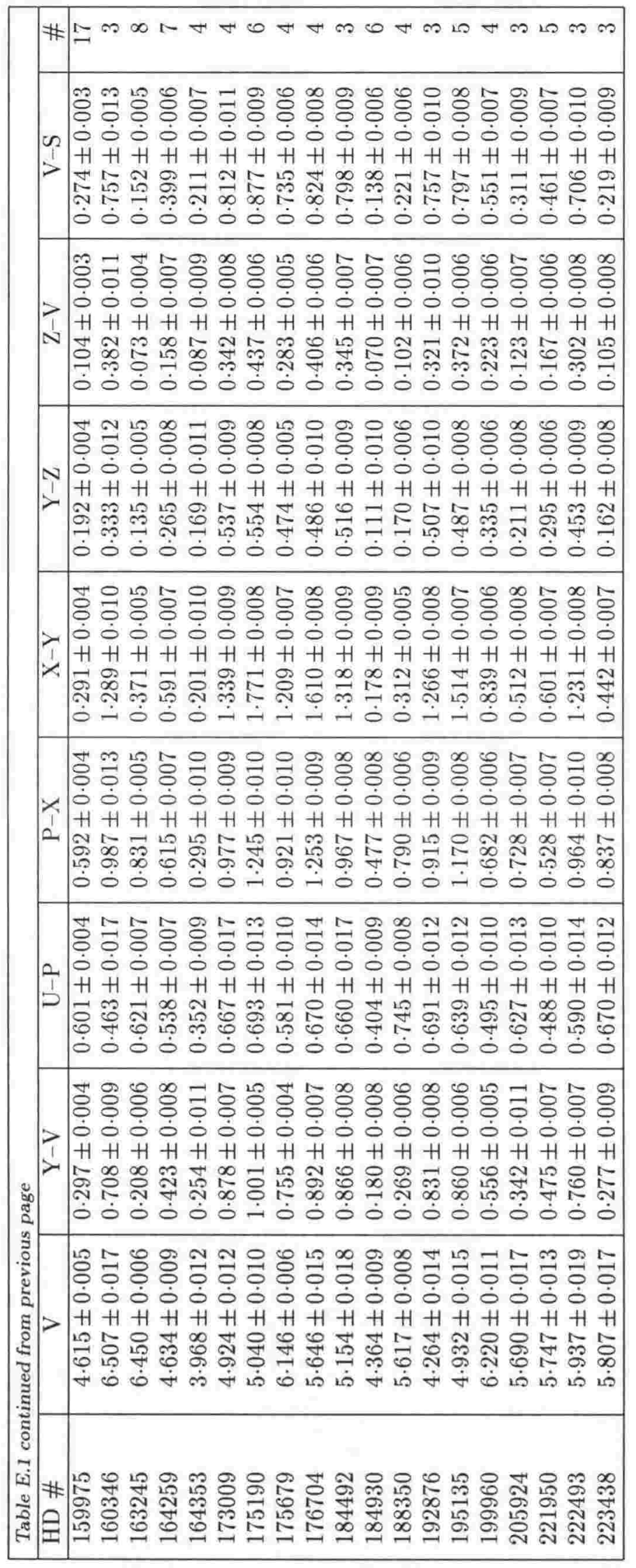




\begin{tabular}{|c|c|}
\hline & $\cong$ \\
\hline & 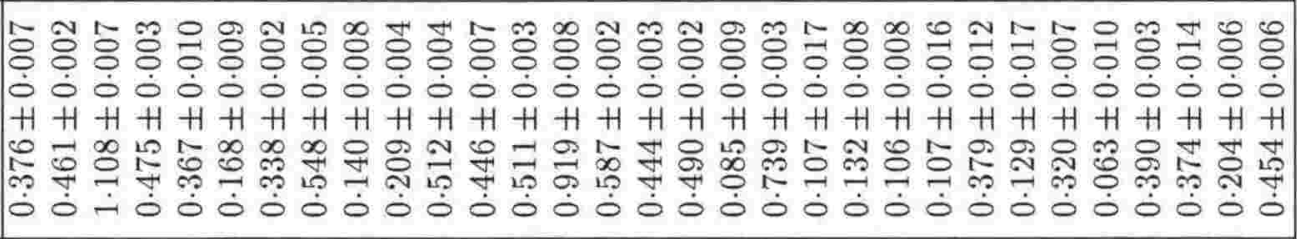 \\
\hline & 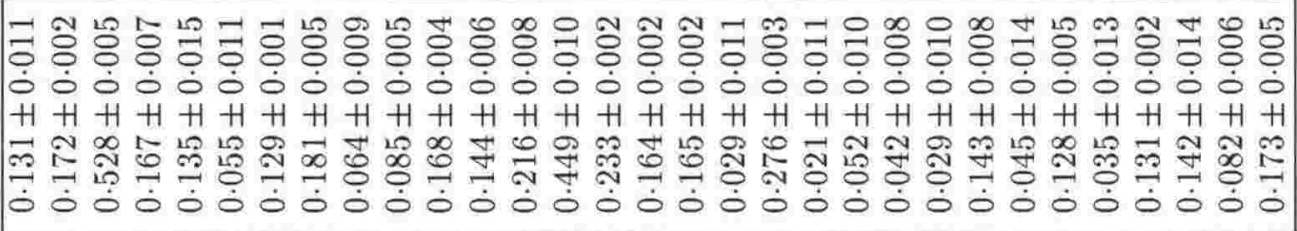 \\
\hline & 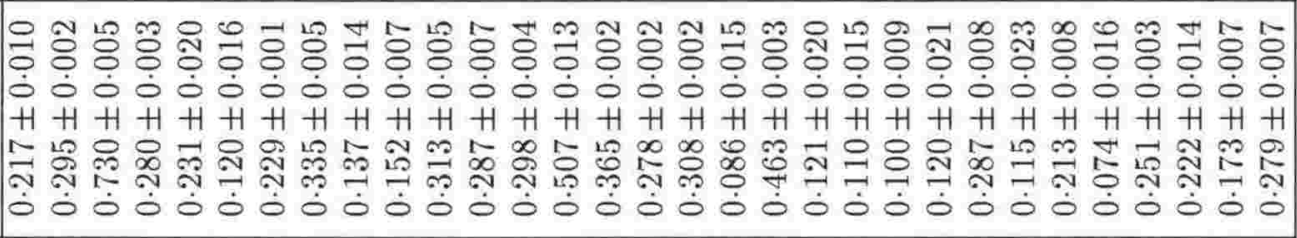 \\
\hline & 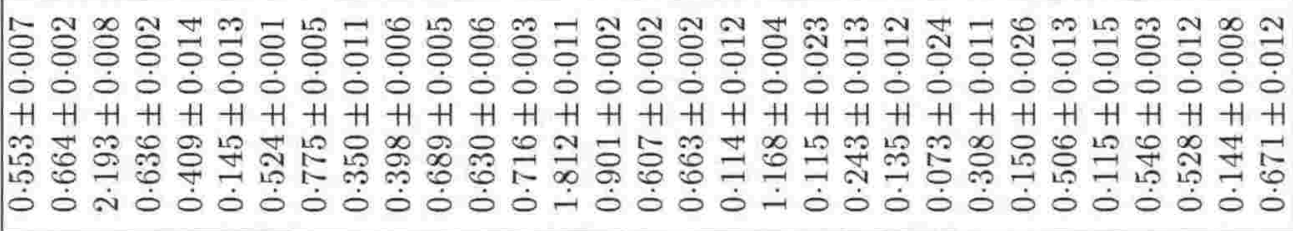 \\
\hline & 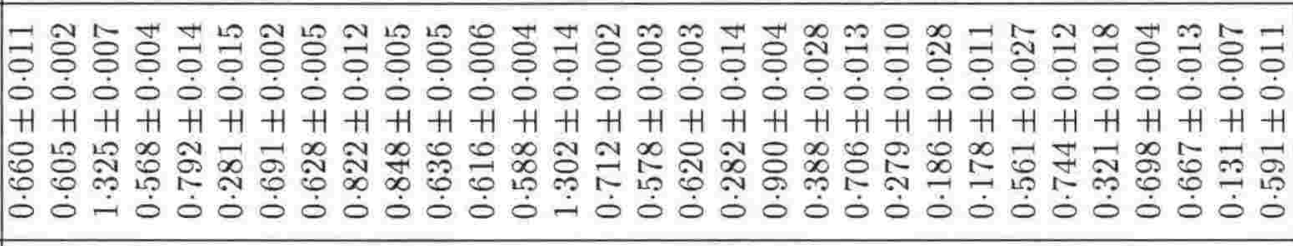 \\
\hline & 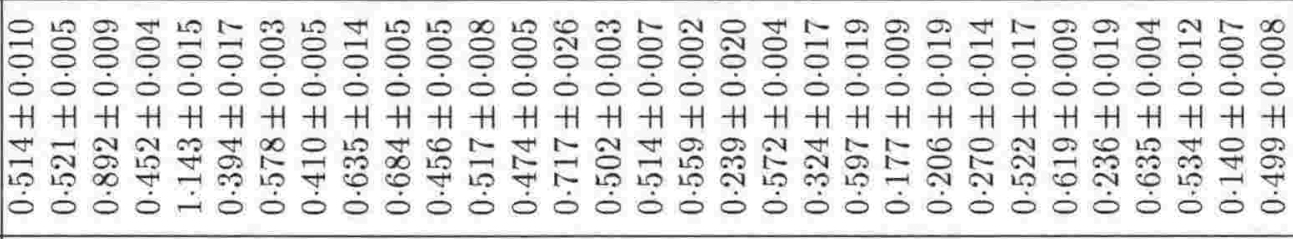 \\
\hline & 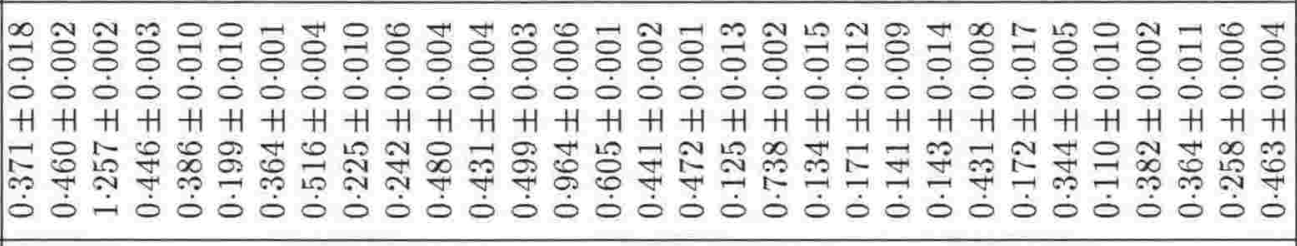 \\
\hline & 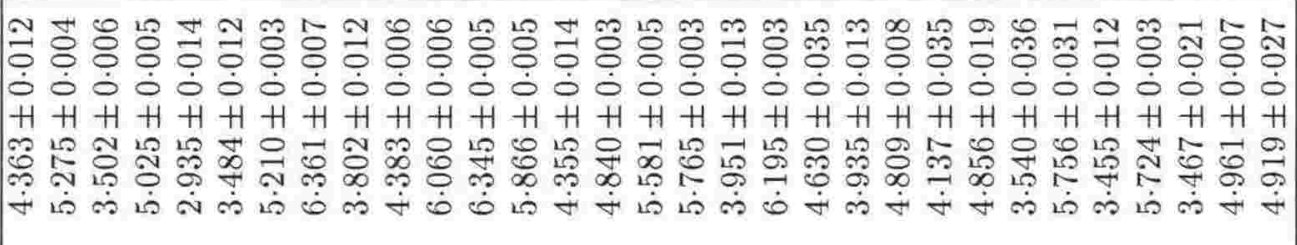 \\
\hline & 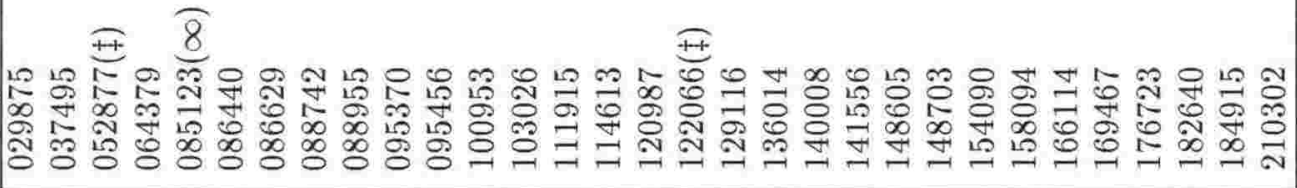 \\
\hline
\end{tabular}




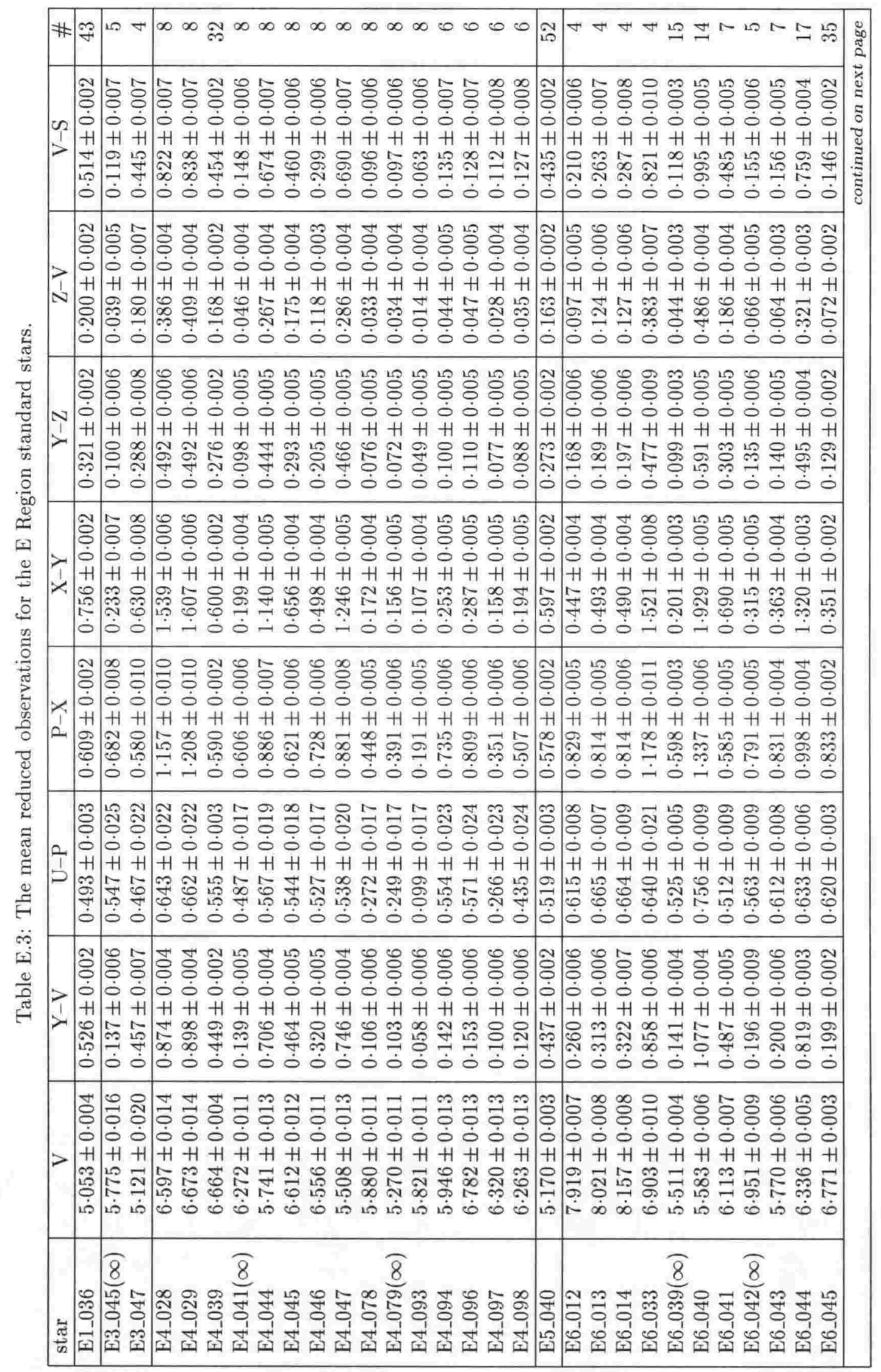




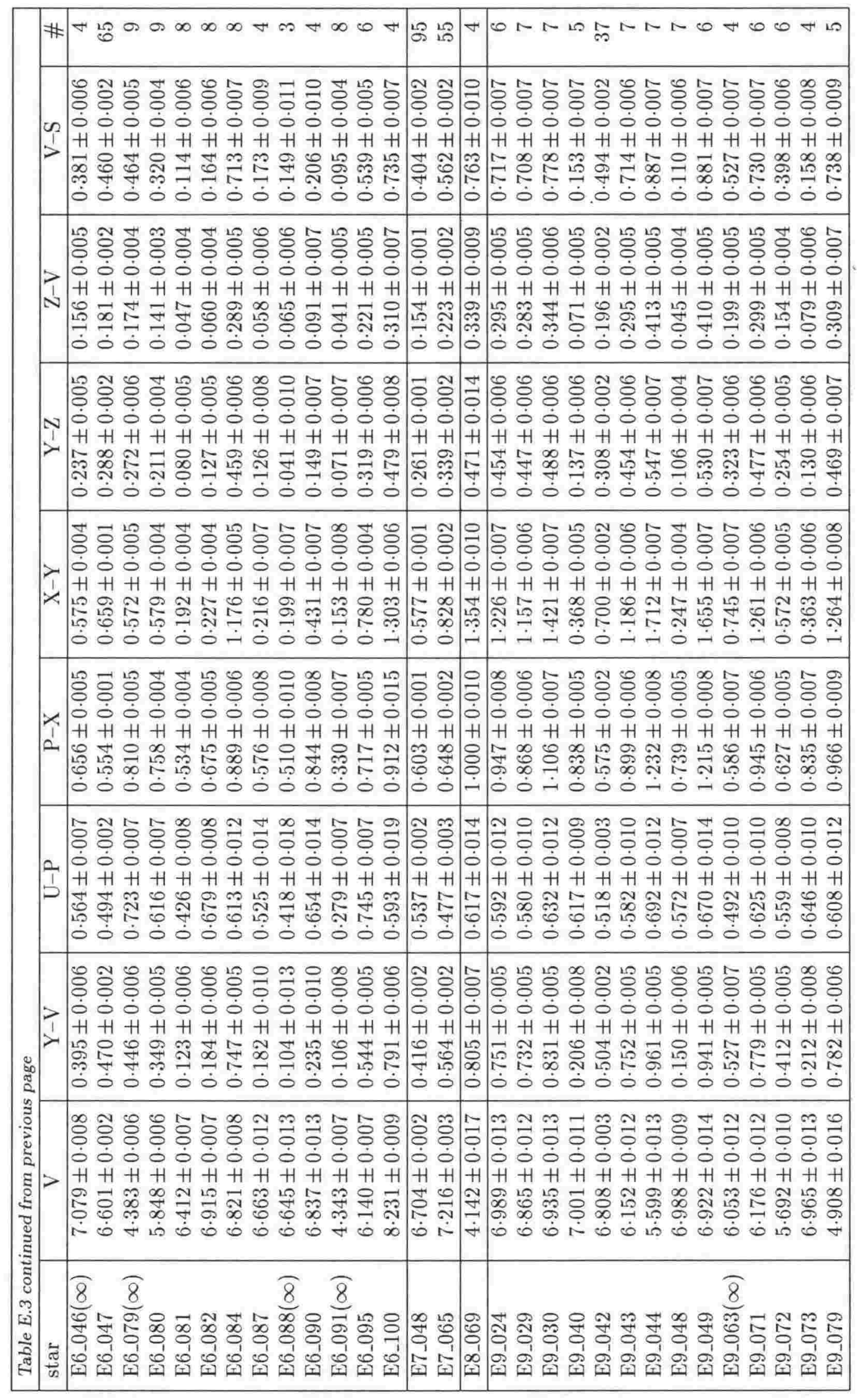




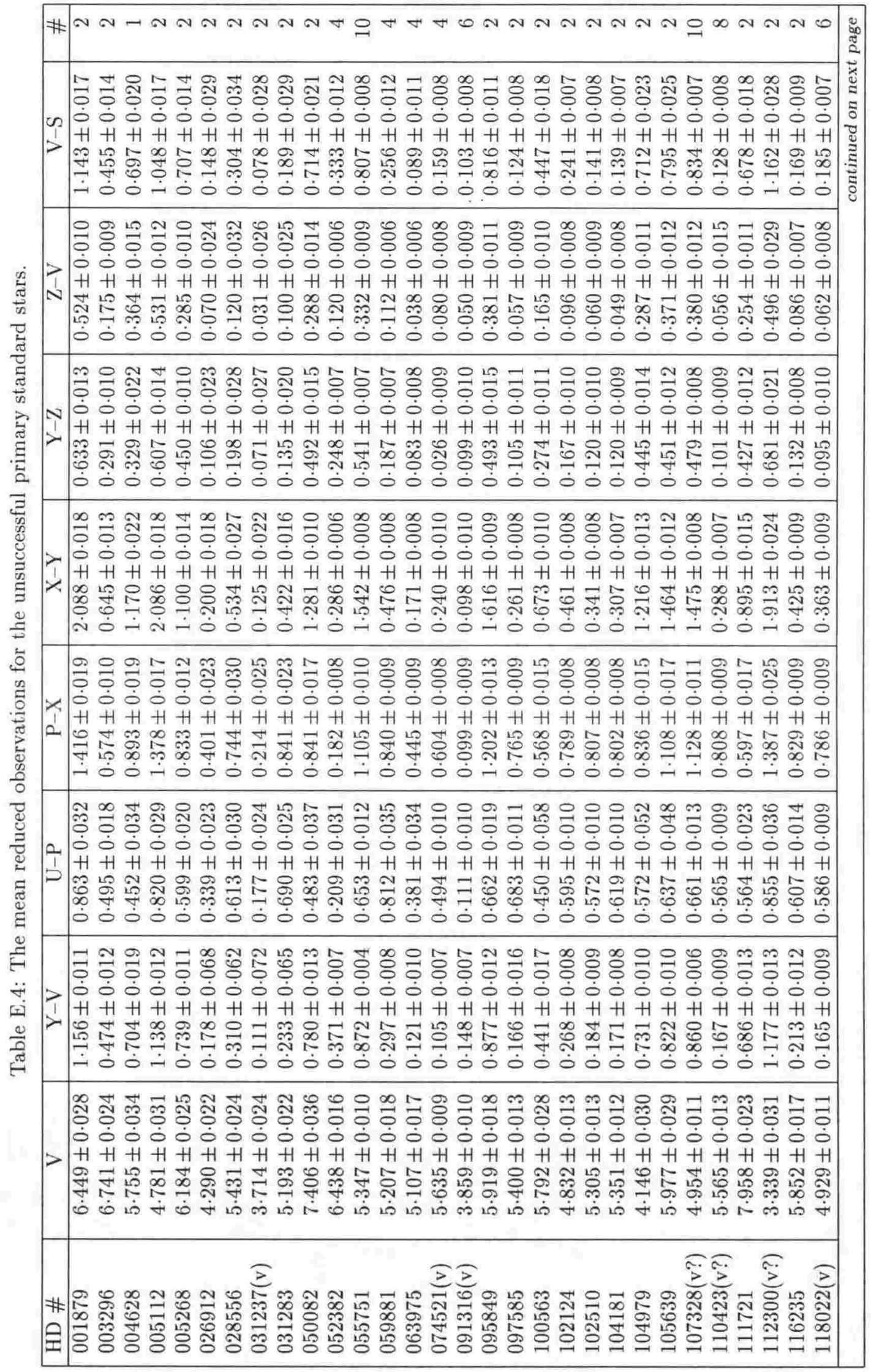




\begin{tabular}{|c|c|c|}
\hline & 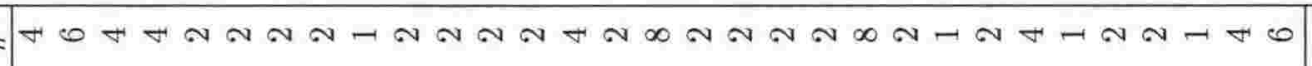 & \\
\hline & 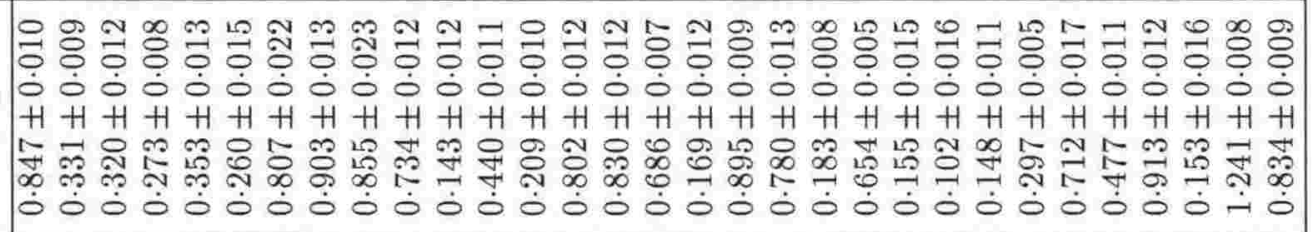 & \\
\hline & 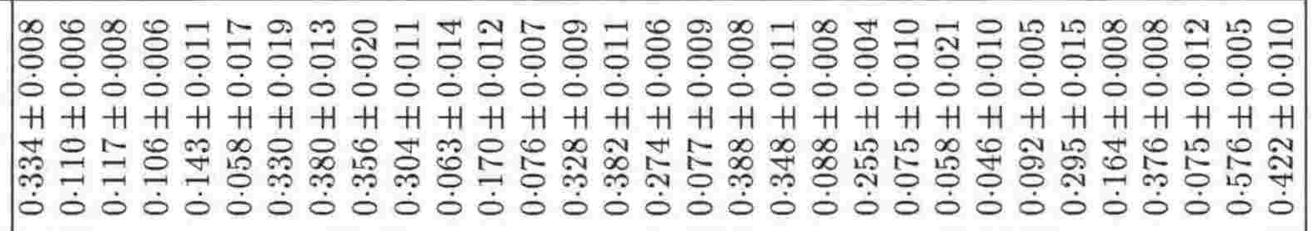 & \\
\hline & 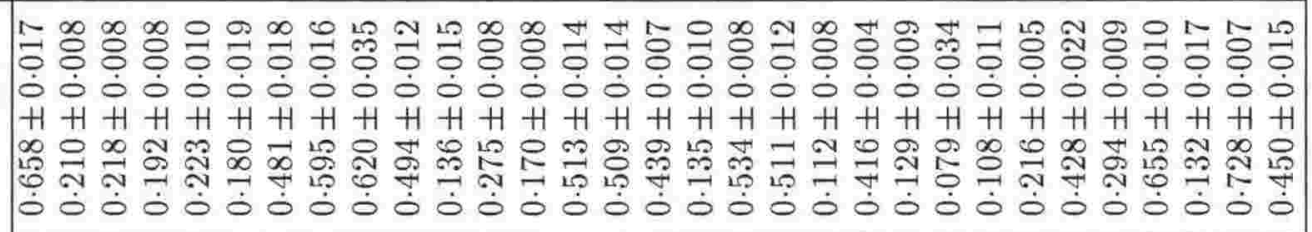 & \\
\hline & 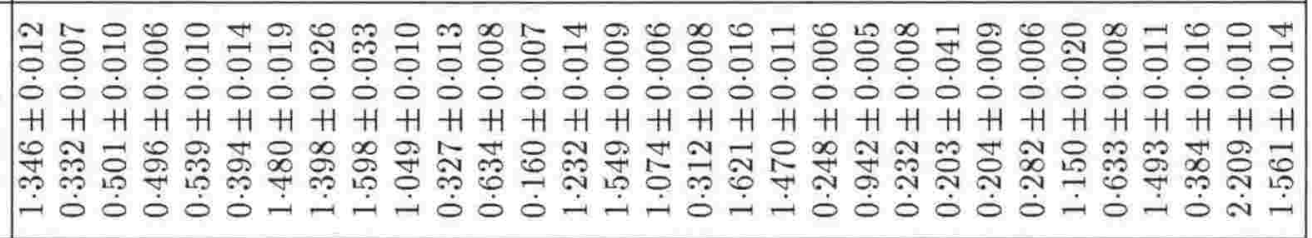 & \\
\hline & 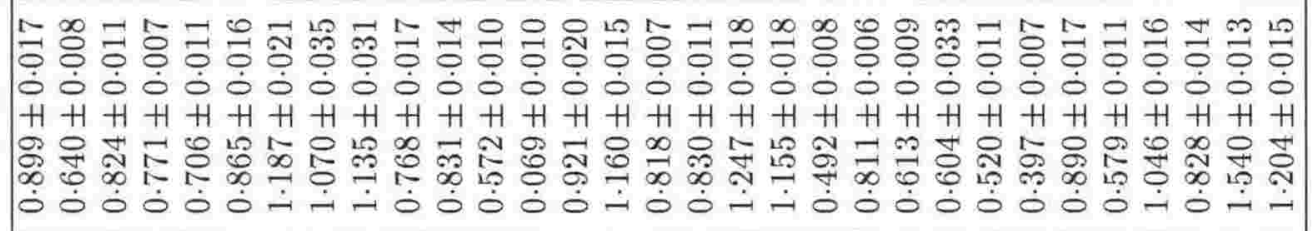 & \\
\hline$D_{2}$ & 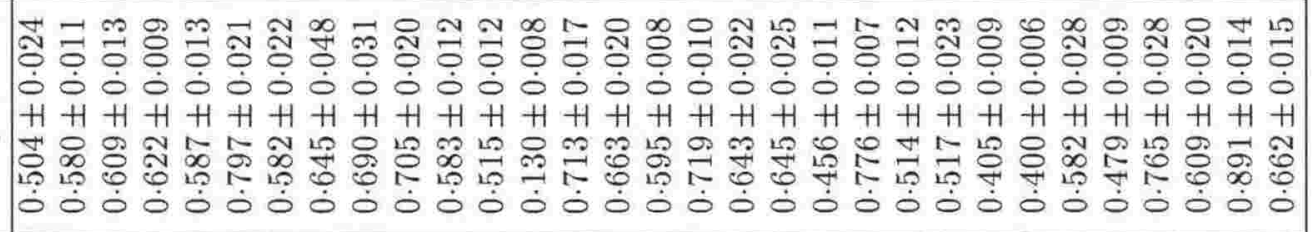 & \\
\hline & 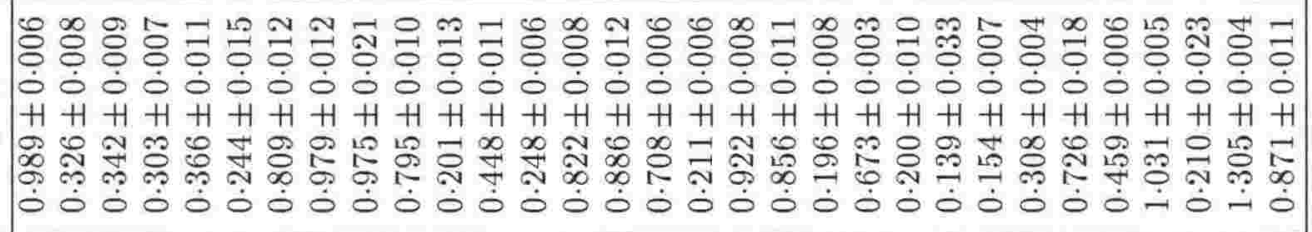 & \\
\hline & 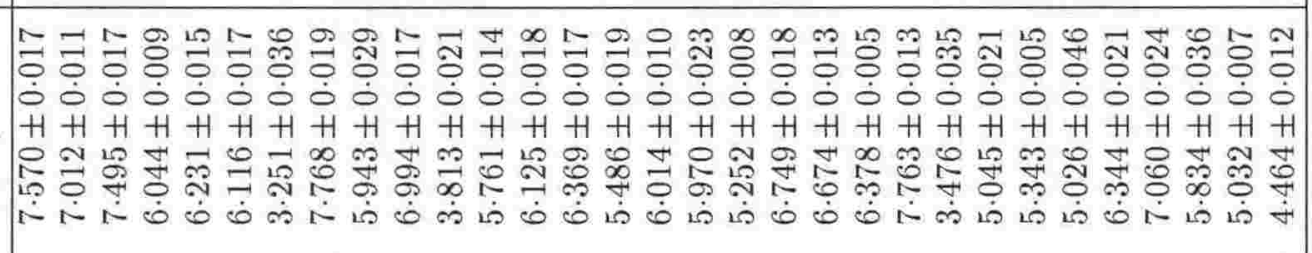 & \\
\hline & 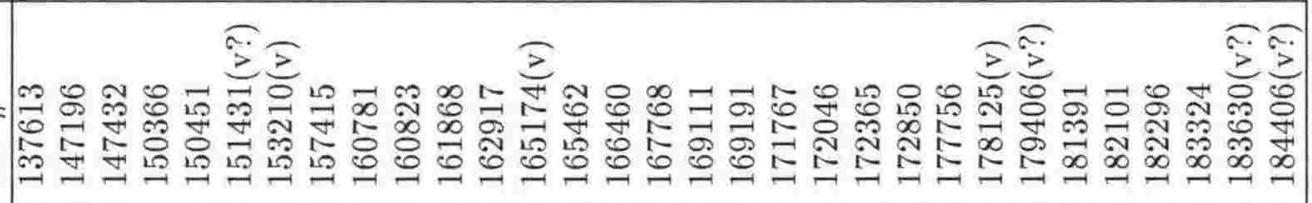 & \\
\hline
\end{tabular}




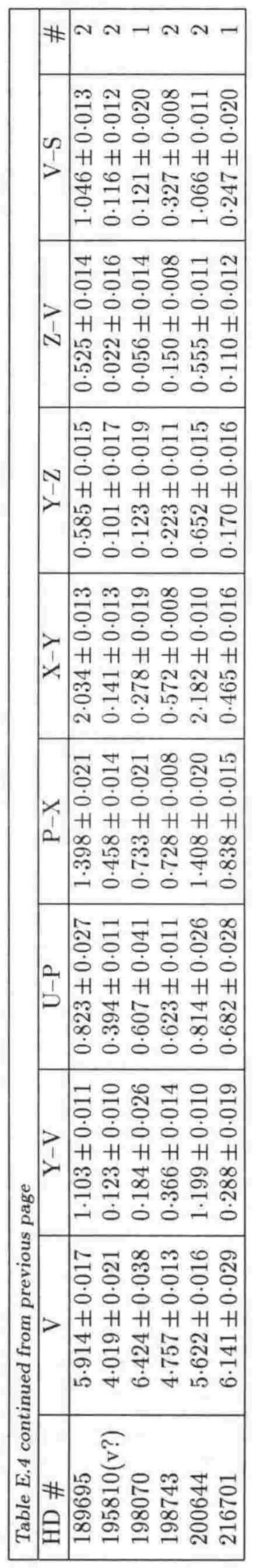




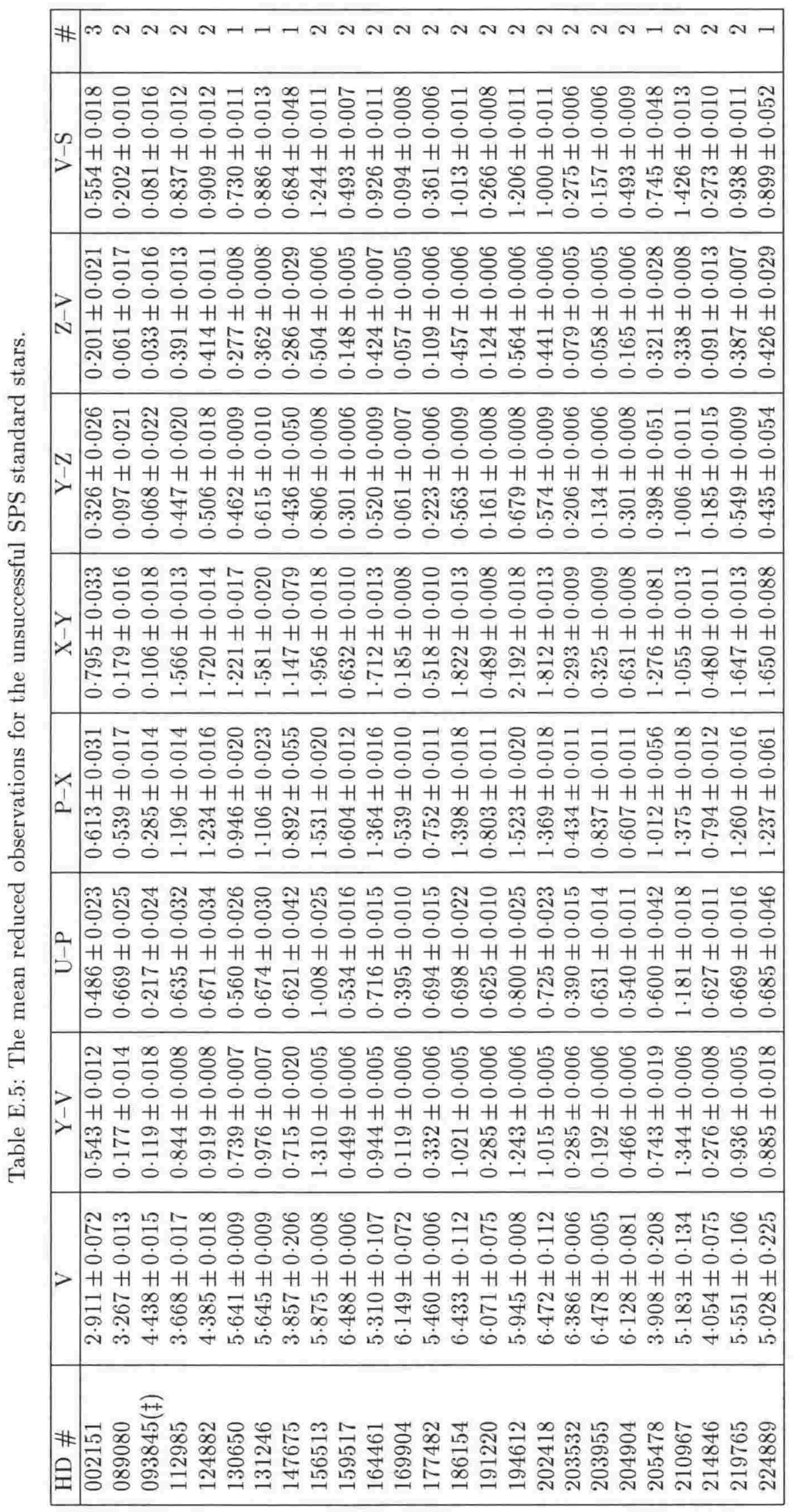




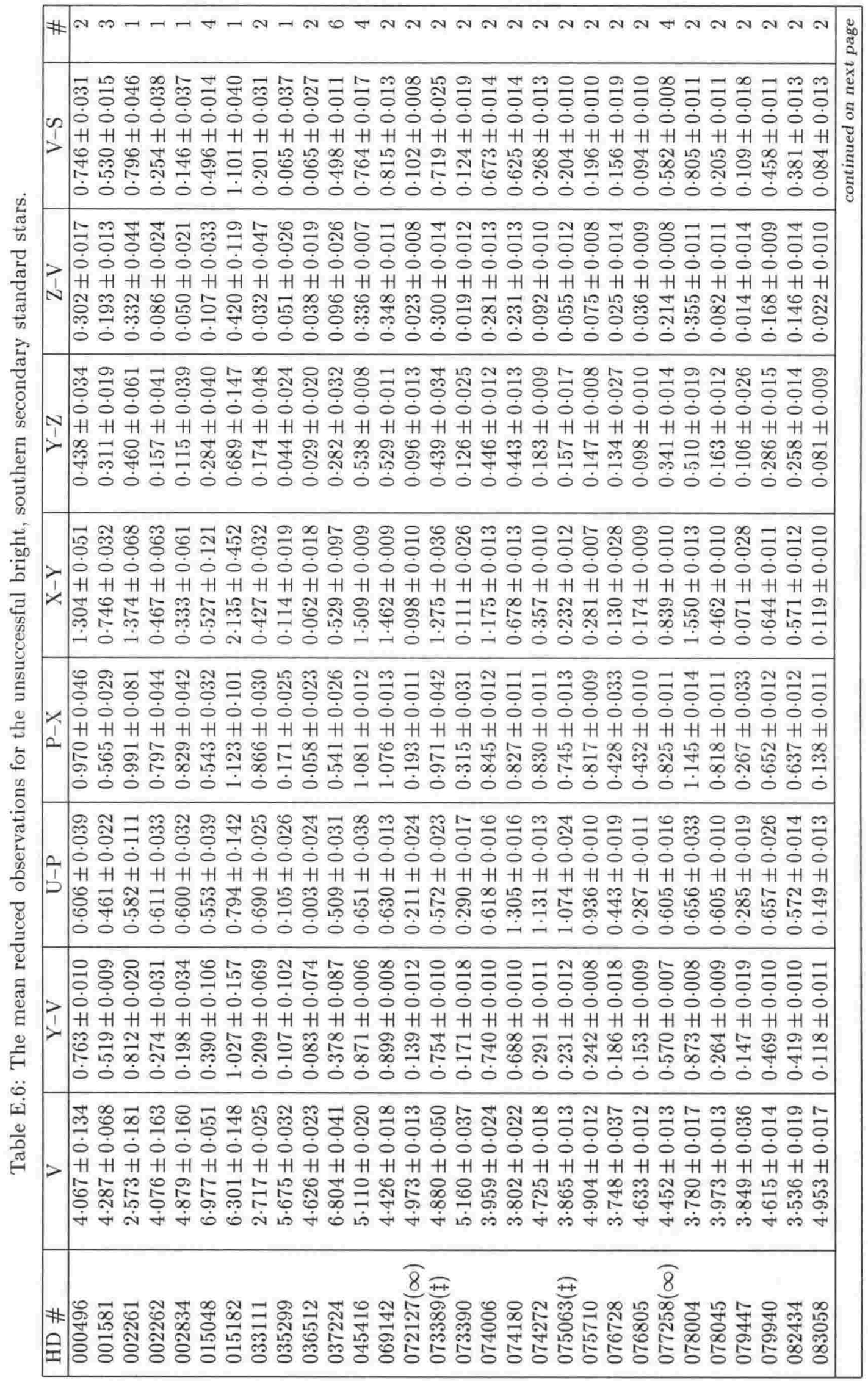




\begin{tabular}{|c|c|c|}
\hline \# & 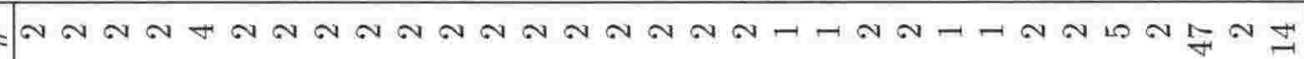 & \\
\hline$\sum_{1}^{2}$ & 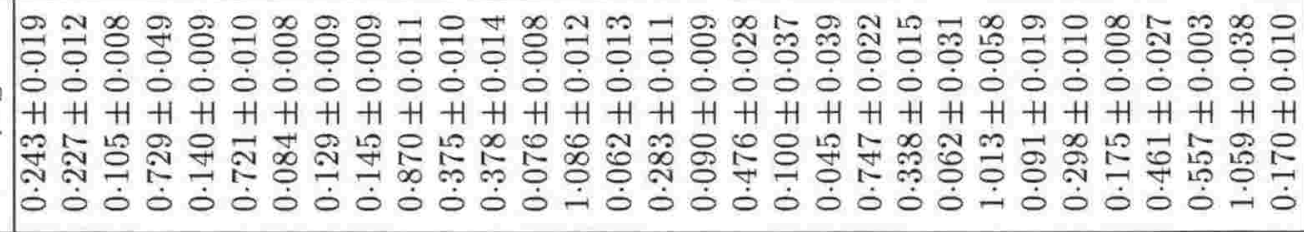 & 至 \\
\hline$\stackrel{b}{b}$ & 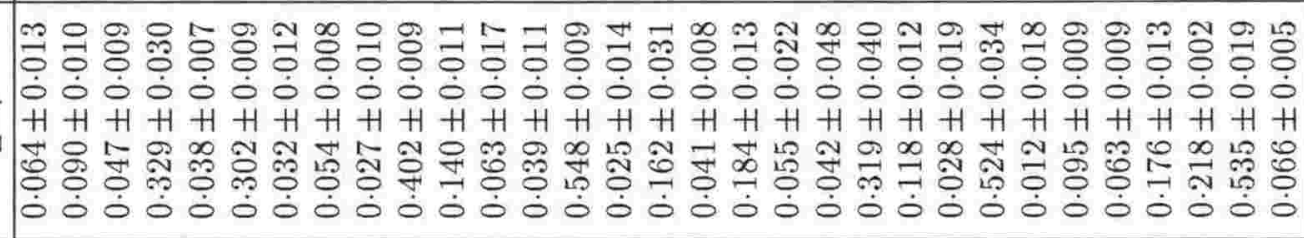 & \\
\hline i & 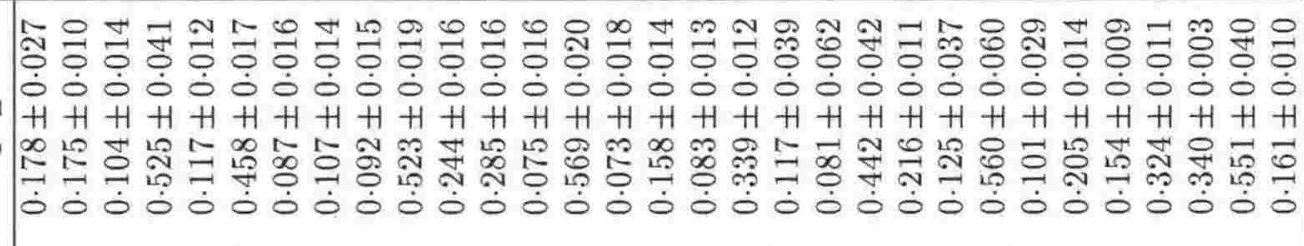 & \\
\hline & 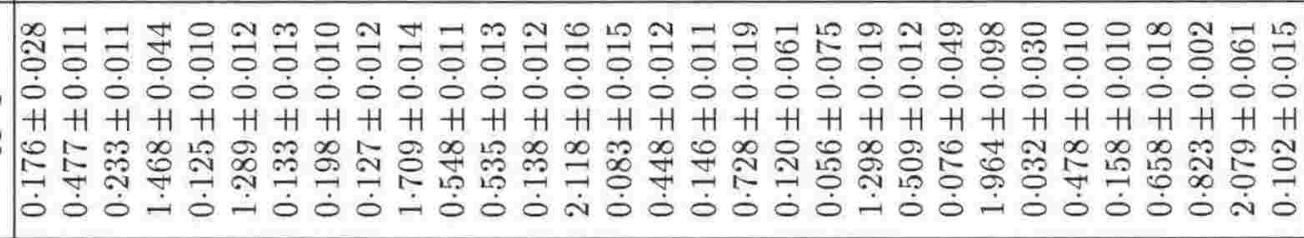 & \\
\hline $\begin{array}{l}x \\
1 \\
2\end{array}$ & 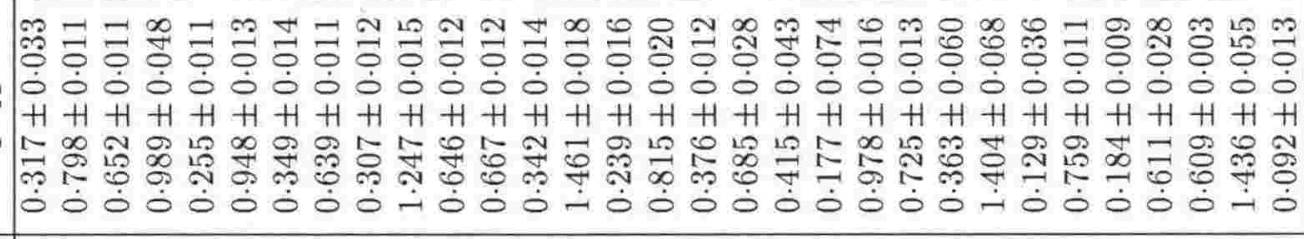 & \\
\hline$\frac{0}{1}$ & 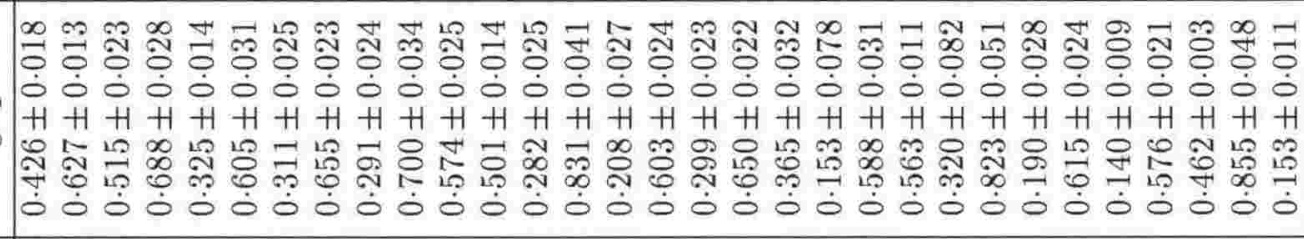 & \\
\hline & 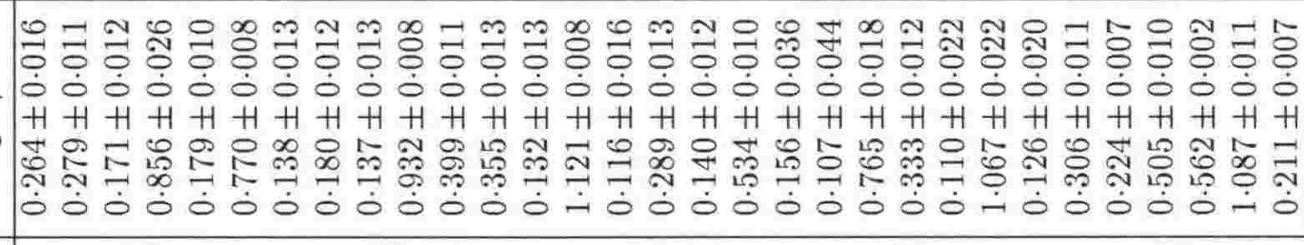 & \\
\hline & 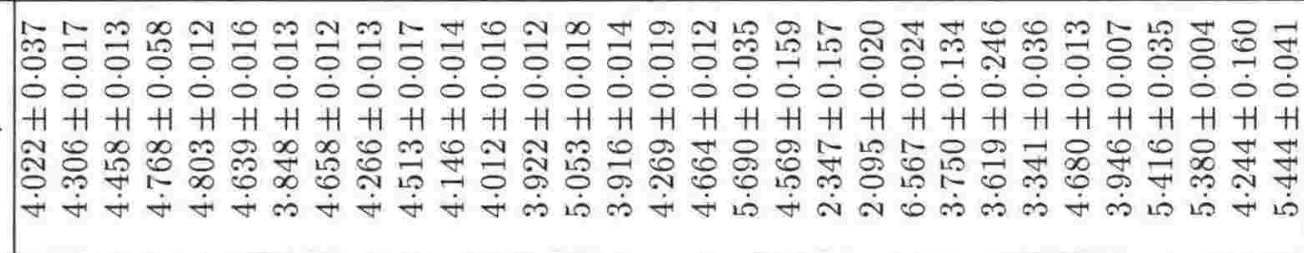 & \\
\hline & 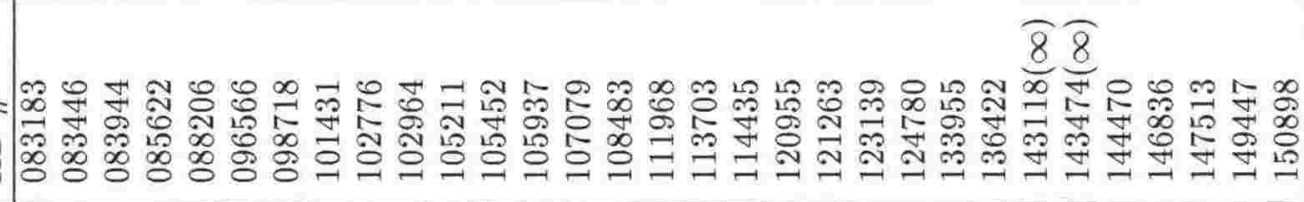 & \\
\hline
\end{tabular}




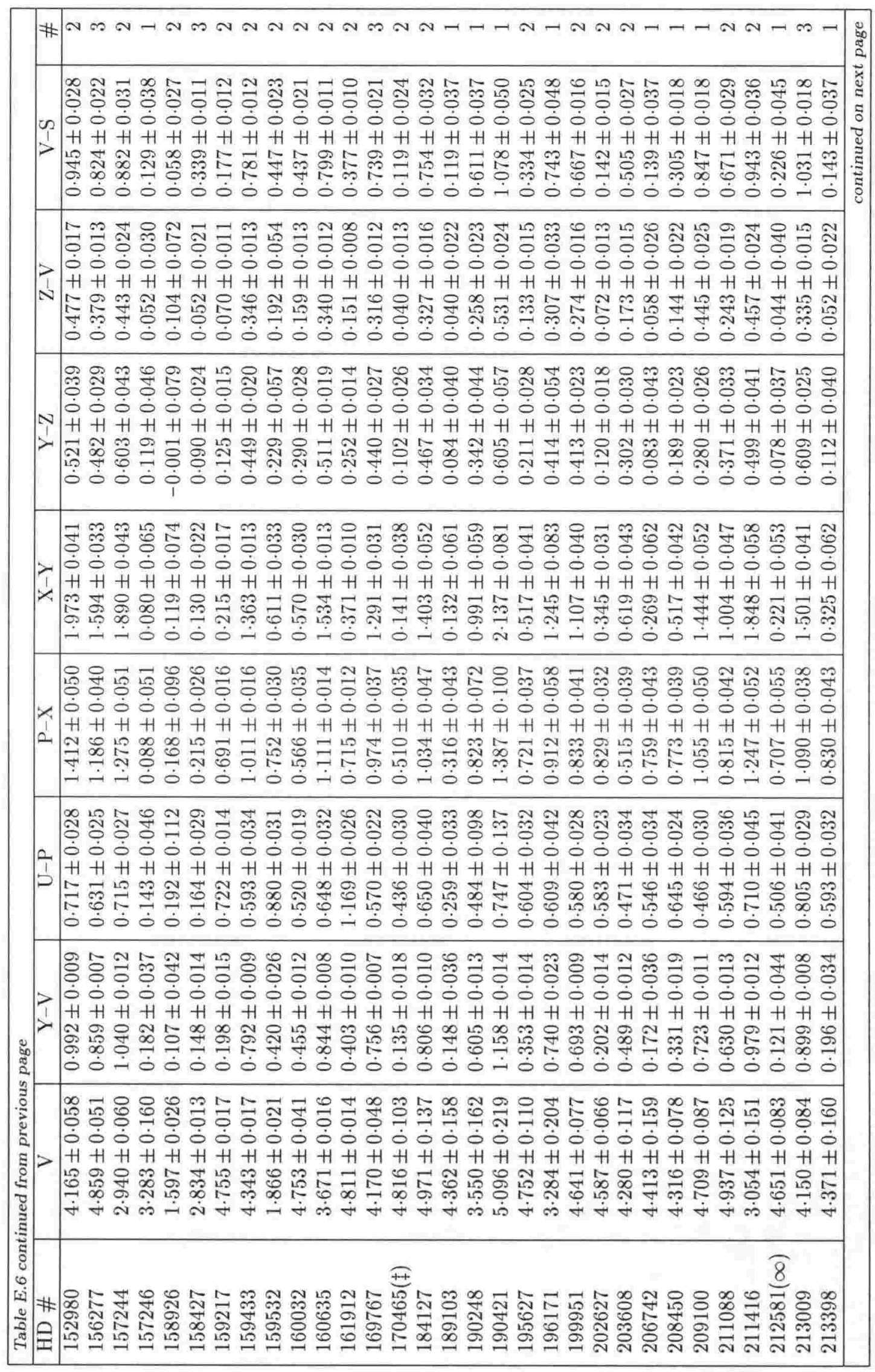




\begin{tabular}{|c|c|}
\hline \# & $H \rightarrow \infty \rightarrow \infty N \rightarrow-\infty N$ \\
\hline & 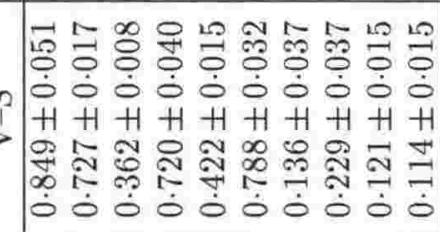 \\
\hline & 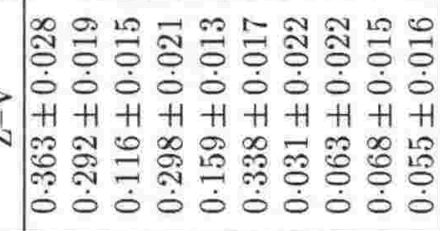 \\
\hline & 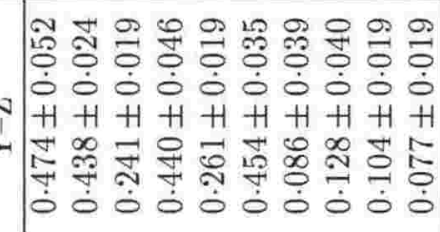 \\
\hline & 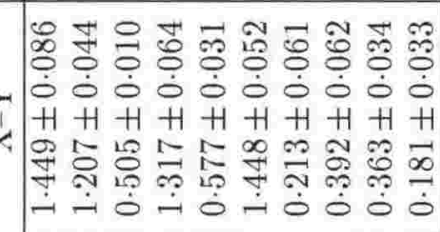 \\
\hline & 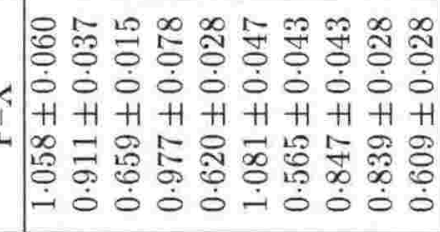 \\
\hline & 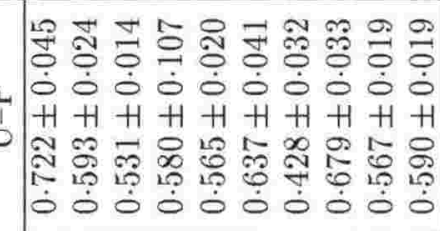 \\
\hline & 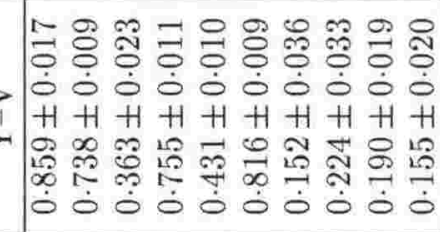 \\
\hline 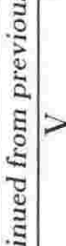 & 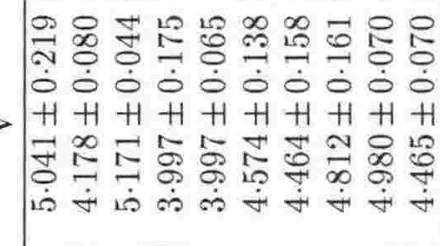 \\
\hline 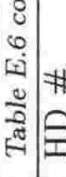 & 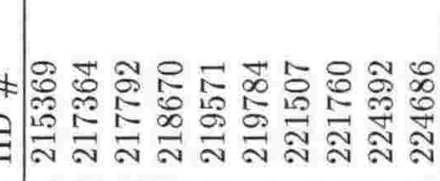 \\
\hline
\end{tabular}




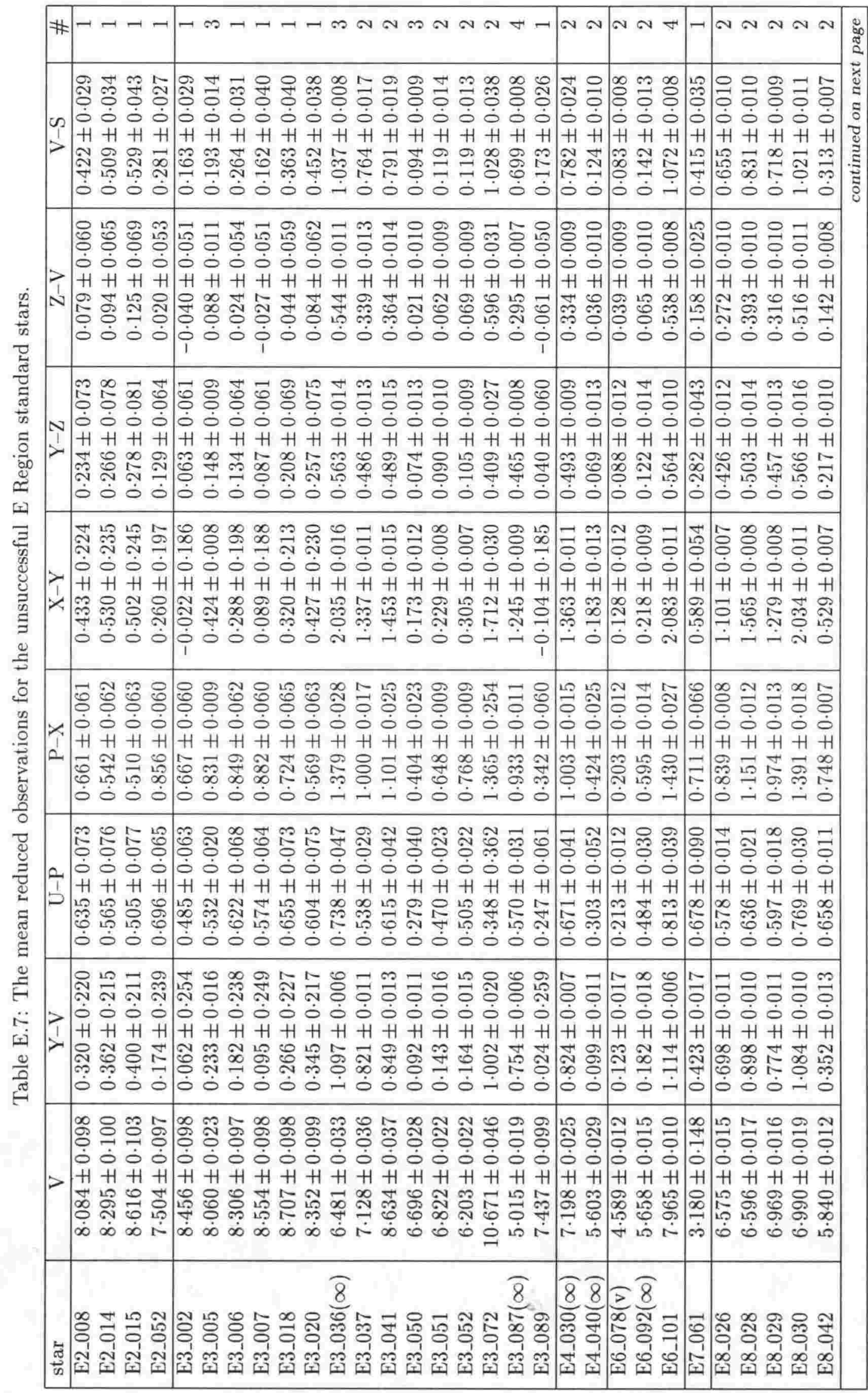




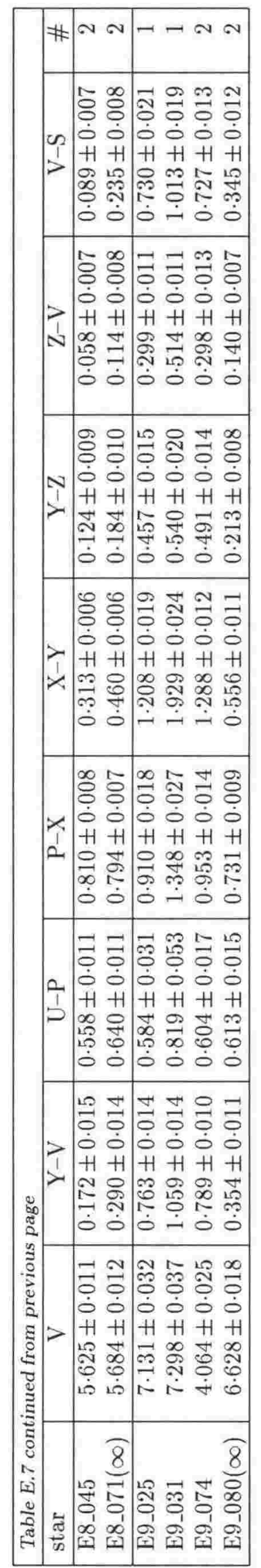




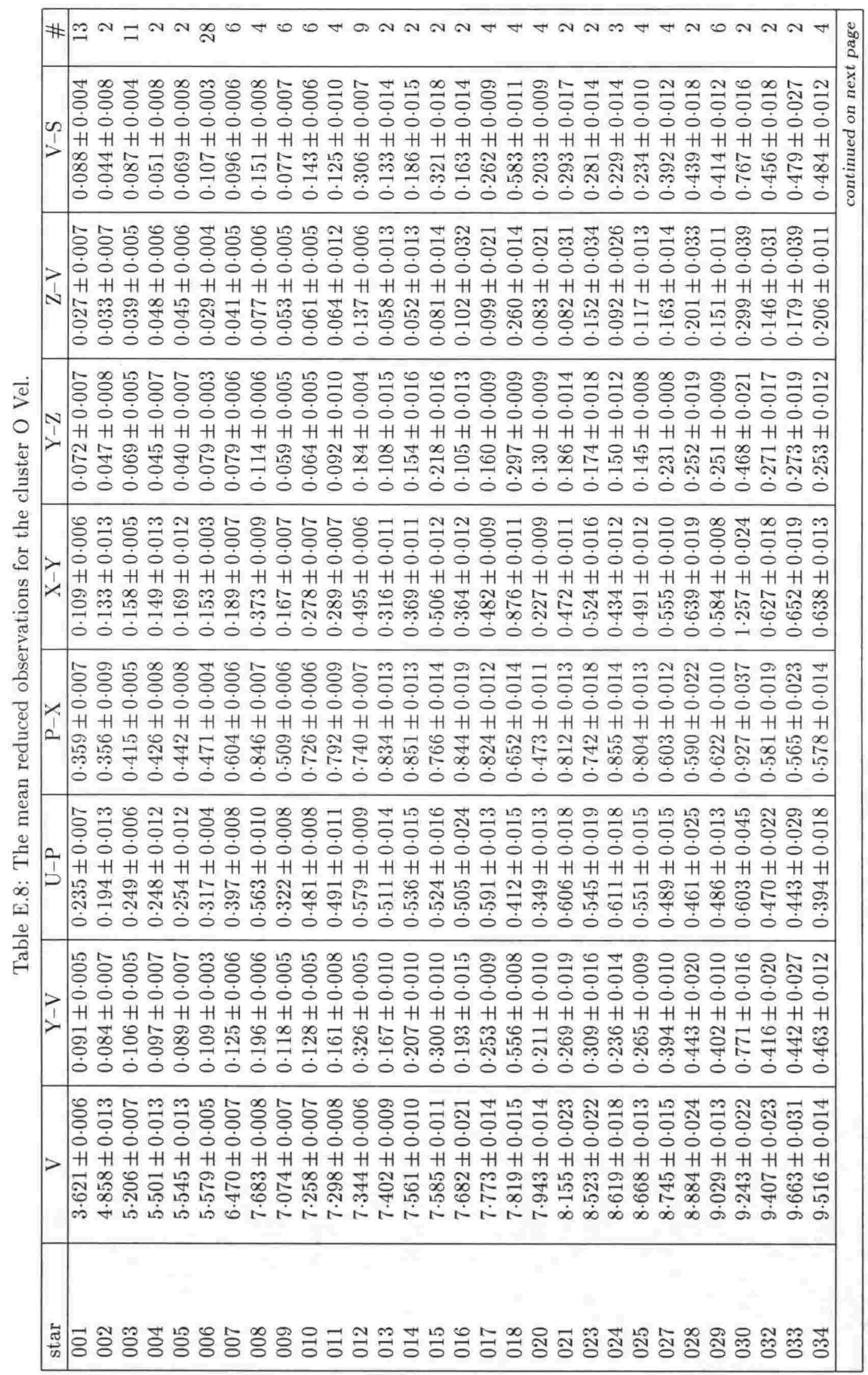




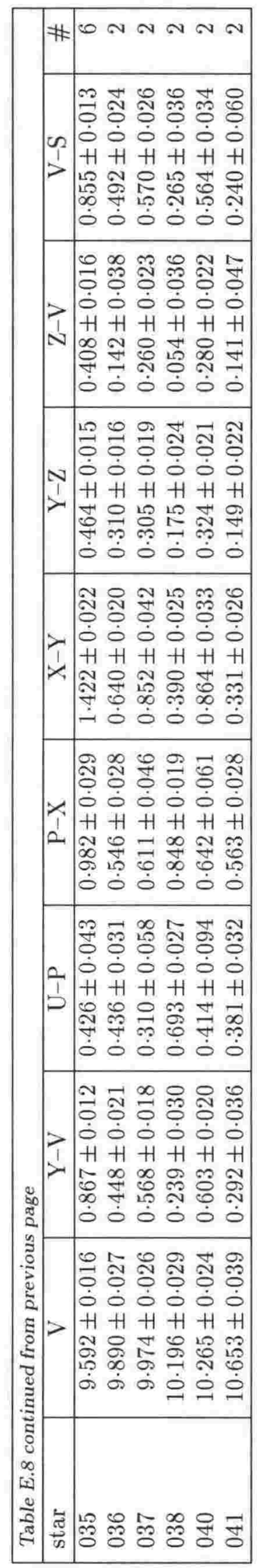




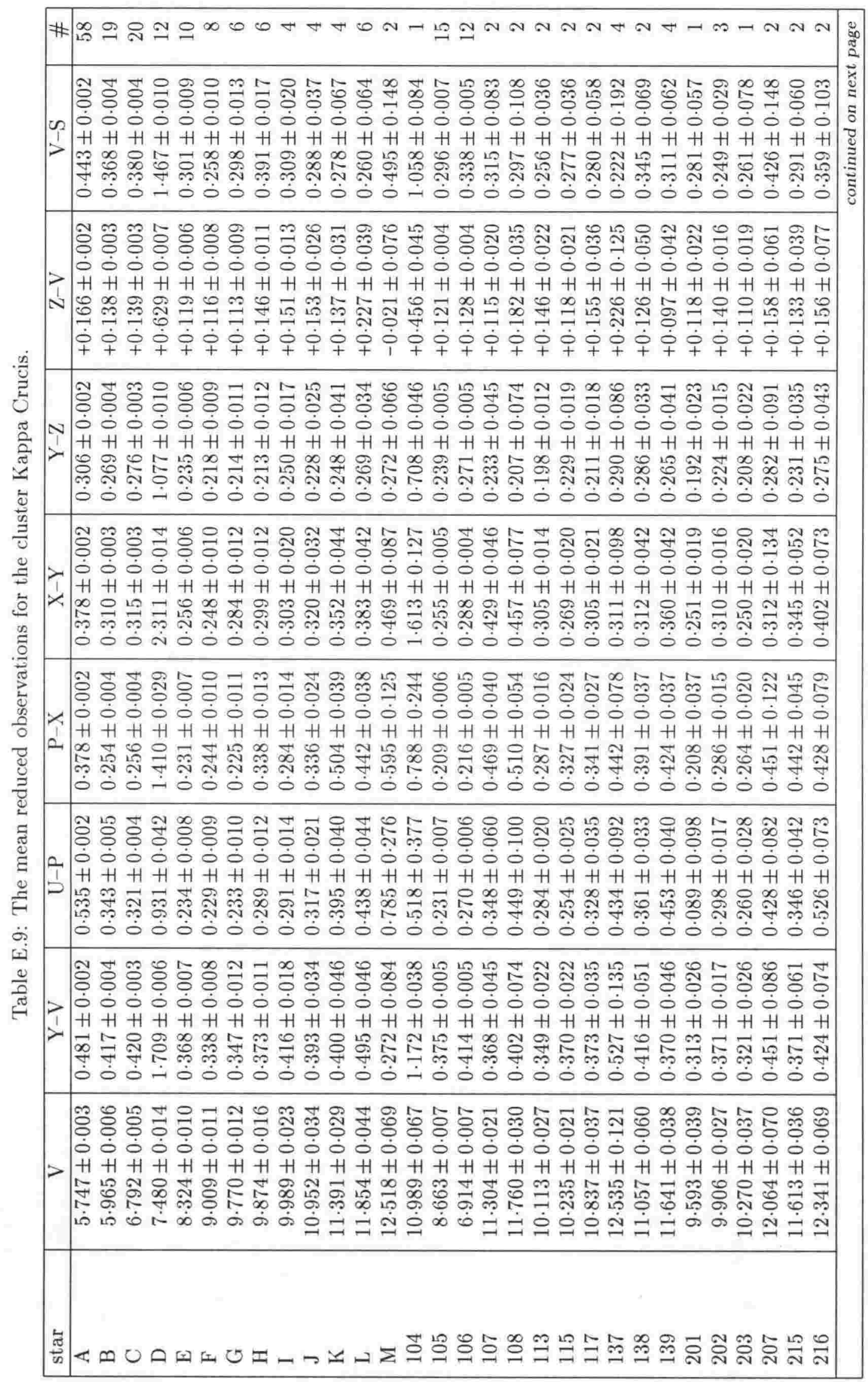




\begin{tabular}{|c|c|}
\hline & $A \cong \cong \sim \sim \sim \sim \sim ⿻$ \\
\hline & 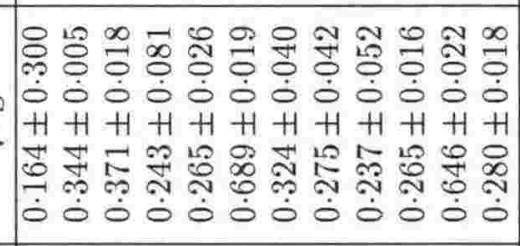 \\
\hline & 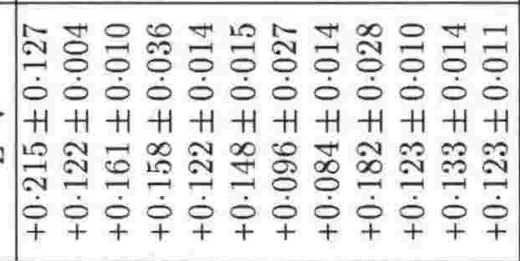 \\
\hline & 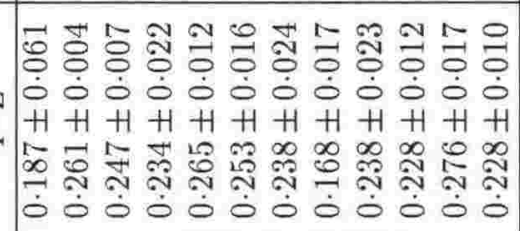 \\
\hline & 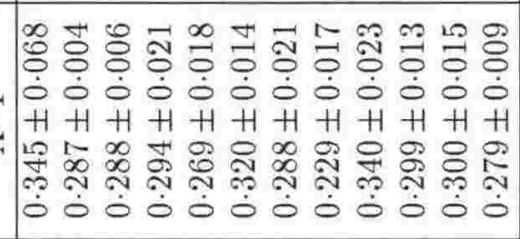 \\
\hline & 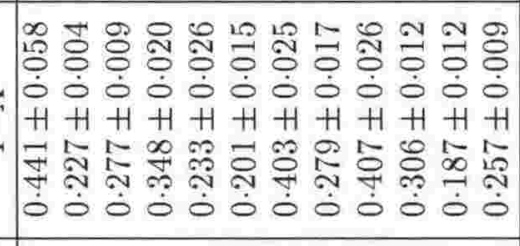 \\
\hline & 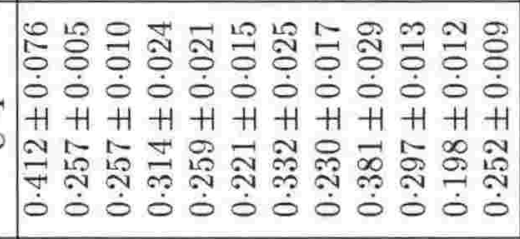 \\
\hline & 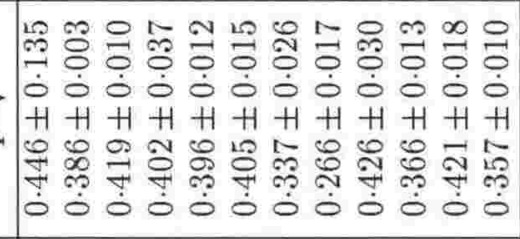 \\
\hline & 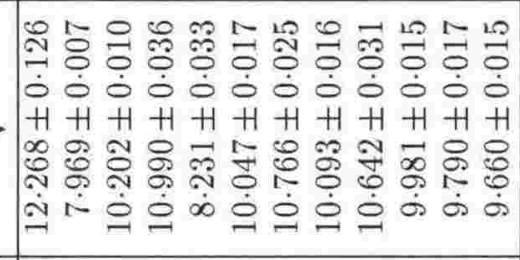 \\
\hline & 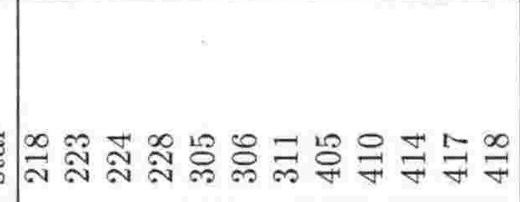 \\
\hline
\end{tabular}




\section{Appendix F}

\section{Derivation of Nikonov's Method to determine Instantaneous Extinction.}

This appendix shows how the method devised by Nikonov (1953) to allow the monochromatic extinction to be measured at any time is derived. The observational technique and reduction algorithm are described in Section 2.6.

Consider an approximate apparent magnitude of the extinction star $\left(\widetilde{m}_{o, e x t n}\right)$, which can be derived from a least squares fit of the extinction star's magnitudes verses airmasses. This differs from the exact apparent magnitude $\left(m_{o, e x t n}\right)$ by a small amount $\delta_{o, e x t n}$, ie.

$$
\tilde{m}_{o, e x t n}=m_{o, e x t n}-\delta_{o, e x t n} .
$$

The extinction star has been chosen so it has no colour dependent term in the extinction correction, ie. $m_{o}=m-k^{\prime} X$, so an approximate extinction coefficient $\left(\tilde{k}^{\prime}\right)$ can be calculated from

$$
\tilde{k}^{\prime}=\frac{m_{e x t n}-\tilde{m}_{o, e x t n}}{X_{e x t n}} .
$$

After substitution of equation F.1, this becomes

$$
\begin{aligned}
\tilde{k}^{\prime} & =\frac{m_{e x t n}-\left(m_{o, e x t n}-\delta_{o, e x t n}\right)}{X_{e x t n}} \\
& =\frac{m_{e x t n}-m_{o, e x t n}}{X_{e x t n}}+\frac{\delta_{o, e x t n}}{X_{e x t n}} \\
& =k^{\prime}+\frac{\delta_{o, e x t n}}{X_{e x t n}}
\end{aligned}
$$

where $k^{\prime}$ is the exact extinction coefficient.

Applying the approximate extinction coefficient to a control star measured simultaneously with the extinction star gives

$$
\tilde{m}_{o, c n t l}=m_{c n t l}-\tilde{k}^{\prime} X_{c n t l}
$$


Substituting $\widetilde{k^{\prime}}$ from equation F.3 produces

$$
\begin{aligned}
\tilde{m}_{o, c n t l} & =m_{c n t l}-\left(k^{\prime}+\frac{\delta_{o, e x t n}}{X_{e x t n}}\right) X_{c n t l} \\
& =\left(m_{c n t l}-k^{\prime} X_{c n t l}\right)-\delta_{o, e x t n} \frac{X_{c n t l}}{X_{e x t n}} \\
& =m_{o, c n t l}-\delta_{o, e x t n} \frac{X_{c n t l}}{X_{e x t n}}
\end{aligned}
$$

This requires a minimum of two observations to solve. The first observation is usually made when the extinction star is cumulating, the second when the control star is cumulating. Denoting the observations by the subscripts 1 and 2 ;

$$
\begin{aligned}
& \tilde{m}_{o, c n t l_{1}}=m_{o, c n t l}-\delta_{o, e x t n} \frac{X_{\text {cntl }_{1}}}{X_{\text {extn }}} \\
& \tilde{m}_{o, \text { cntl }}=m_{o, \text { cntl }}-\delta_{o, \text { extn }} \frac{X_{\text {cntl }_{2}}}{X_{\text {extn }_{2}}} .
\end{aligned}
$$

Subtracting the second observation from the first yields

$$
\widetilde{m}_{o, c n t l_{1}}-\widetilde{m}_{o, c n t l_{2}}=-\delta_{o, e x t n}\left(\frac{X_{c n t l_{1}}}{X_{e x t n_{1}}}-\frac{X_{c n t l_{2}}}{X_{e x t n_{2}}}\right) .
$$

Rearranging and substituting for the approximate apparent magnitudes of the control star gives

$$
\delta_{o, e x t n}=\frac{\left(m_{c n t l_{1}}-\tilde{k}_{1}^{\prime} X_{c n t l_{1}}\right)-\left(m_{c n t l_{2}}-\tilde{k}_{2}^{\prime} X_{c n t l_{2}}\right)}{\left(X_{c n t l_{1}} / X_{e x t n_{1}}-X_{c n t l_{2}} / X_{e x t n_{2}}\right)}
$$

where the approximate extinction coefficients $\left(\widetilde{k}_{1}^{\prime}, \widetilde{k}_{2}^{\prime}\right)$ are calculated from

$$
\tilde{k}_{i}^{\prime}=\frac{m_{e x t n_{i}}-\tilde{m}_{o, e x t n}}{X_{e x t n_{i}}} \quad(i=1,2)
$$

Alternately, if $N$ observations (more than two) of the extinction and control star have been made, a linear least squares fit may be done to calculate $\delta_{o, e x t n}$. Here the independent and dependent variables $\left(x_{i}, y_{i}\right)$ are $X_{c n t l_{i}} / X_{e x t n_{i}}$ and $\tilde{m}_{o, c n t l_{i}}$, the intercept is $m_{o, \text { cntl }}$ and slope is $\delta_{o, e x t n}$. 


\section{Appendix G}

\section{Long term changes in extinction and the effect of Mount Pinatubo.}

Short term variations of extinction (i.e. on an hourly scale) have already been discussed in Chapter 2. Longer term variations will now be investigated, motivated by the possibility of using averaged extinction coefficients for any nights that have badly determined, or completely unknown, coefficients.

\section{G.1 Correlations between nights.}

The following form of auto-correlation was used (Sterken \& Manfroid 1992b)

$$
\alpha_{\lambda}(\delta n)=<\left|k_{\lambda}^{\prime}(n+\delta n)-k_{\lambda}^{\prime}(n)\right|>
$$

where $k_{\lambda}^{\prime}(n)$ is the monochromatic extinction (averaged for each night from the hourly measurements of Nikonov's method) for the Julian Date ' $n$ ' and $k_{\lambda}^{\prime}(n+\delta n)$ is the extinction $\delta n$ days later. Plots of $\alpha$ versus $\delta n$ for each filter all have similar shapes, so, in an attempt to reduce the 'noise', $\alpha$ was averaged across all the filters for each $\delta n$ - see the top diagram in Figure G.1. This shows a monotonically increasing difference in the extinction between observations made $\delta n$ nights apart, until $\delta n \widetilde{>} 5$ days. However the apparently good correlations for $\delta n \geq 5$ are not reliable as they are based on a small frequency of observations (that have been made $\delta n$ days apart), as shown by the lower diagram in Figure G.1. This is also shown in the top diagram by the error bars, which were calculated as $\left(0^{m} \cdot 02+0^{m} \cdot 02\right) /$ frequency $(\delta n)$, where $0^{m} \cdot 02$ is the standard deviation of the extinction coefficients averaged over all nights.

Therefore, it was concluded that a running average (from $\frac{1}{2} \delta n$ nights before and after the night in question) is only useful if the standard deviation of the extinction coefficient (from that night) exceeds $0^{m} \cdot 03$ and this large uncertainty is due to a lack of suitable extinction star measurements rather than poor photometric quality of the night. 
auto-correlation of extinction at Mt. John from 1988-93

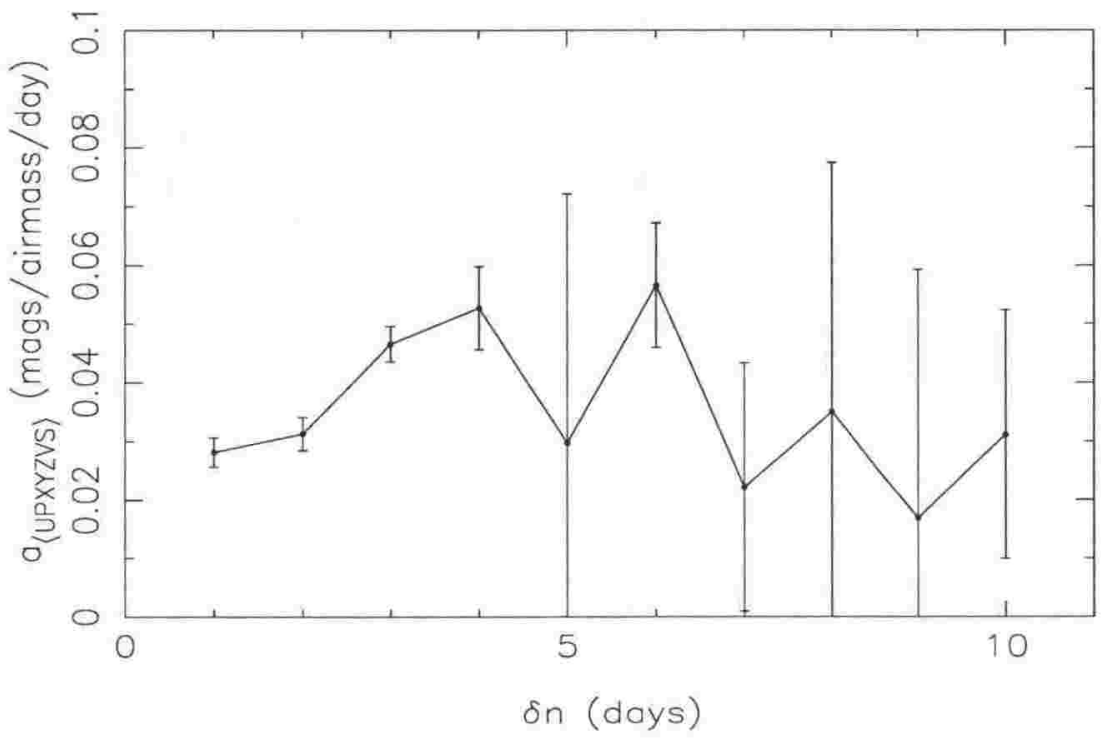

auto-correlation of extinction at Mt. John from 1988-93

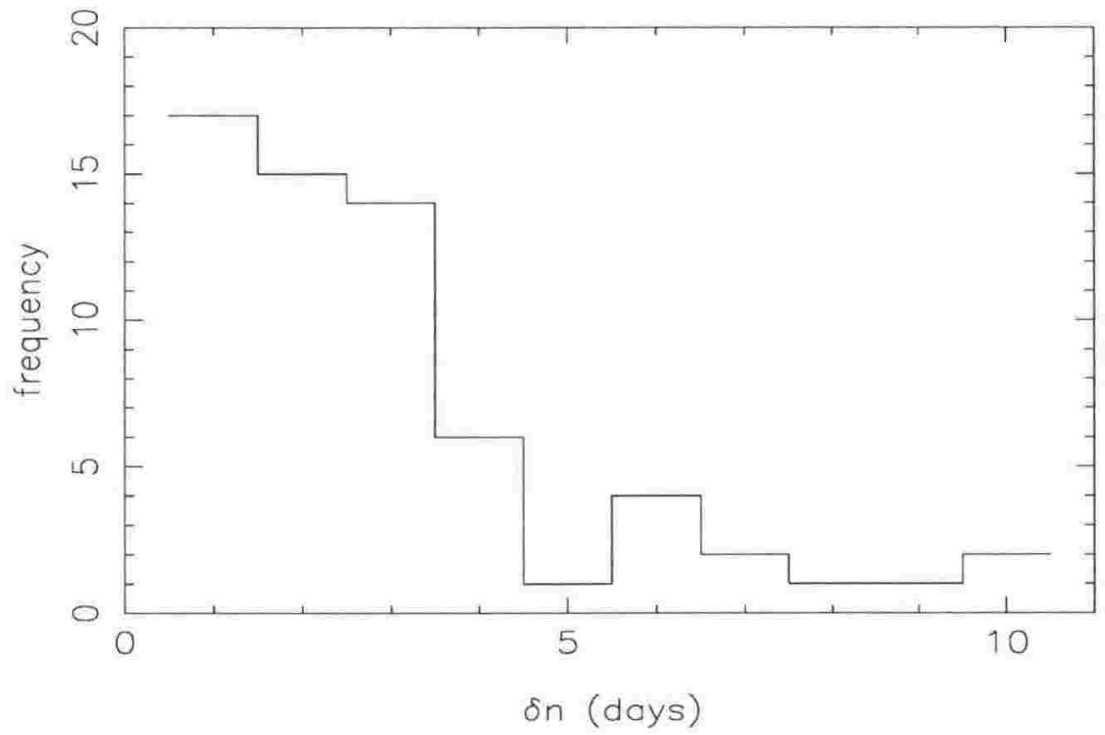

Figure G.1: Auto-correlation of extinction at MJUO that were measured $\delta n$ nights apart. The top diagram shows the auto-correlation averaged across the filters while the bottom diagram shows the frequency of observations (that were separated by $\delta n$ nights). 


\section{G.2 The effect of the Mount Pinatubo eruption.}

Another possibility is that the mean extinction (averaged over all observations, or perhaps on a seasonal basis) could be used for nights with uncertain extinction. To investigate this, a plot of the extinction coefficient (averaged for each night from the hourly measurements as before) versus the Julian Date was examined for each filter. The extinction from the different filters again closely resembled each other, so $k_{V}^{\prime}$ was taken as being typical. As the effective wavelengths of the Vilnius and Johnson V filters are nearly the same, it might be possible to increase the data set by combining the $\mathrm{V}$ extinction from other observers using a Johnson system at MJUO.

There were three other such systems in use during the period concerned (late 1987 to early 1994); The first system is a Johnson V filter on either the B\&C or the O/C telescopes, with the extinction (Gilmore \& Kilmartin 1994) derived from a Bouguer plot using the programs of Hendon \& Kaitchuck (1989).

The second system is a Johnson filter set on the $\mathbf{M}^{\mathrm{c}}$ Lellan one metre telescope using a Thomson CCD with a Photometrics control system (Tobin 1991). The extinction was calculated (Banks 1995) from observations of standard stars over a wide range of airmass, simultaneously fitting the standardisation transformation and extinction coefficients as described by Harris et al. (1981) and implemented in Image Reduction and Analysis Facility (IRAF)(Tody 1986).

The final system is a two channel photomultiplier instrument on the one metre telescope using a Johnson V filter (Sullivan 1989). The extinction data (Sullivan 1994) were derived from custom written software based on a Bouguer plot.

The extinction values found by the different systems were compared for common nights and agree within 0.03 mags/airmass. As another check, the average extinction values (before and after the Mount Pinatubo eruption) of each system were calculated and again all found to agree within 0.03 mags/airmass. This is sufficiently small compared to the long term variations in extinction that the different systems may be combined with the Vilnius system with reasonable accuracy.

The atmospheric extinction in the various V passbands measured at MJUO from late 1987 to early 1994 is shown in Figure G.2, with the solid line being a running average (smoothed over three data points before and after the current data point). The effect of the 15 June 1991 Mount Pinatubo eruption can clearly be seen, with the dashed lines representing the average extinction before and after the eruption. Both the mean extinction level and the scatter about the mean have increased, and some observers had also noted a 'ripple' pattern of haze was frequently visible during sunset and sunrise (for example this author and Banks (1994) at MJUO). The increase in average $\mathrm{V}$ extinction (0.09 mags/airmass) is similar to that found at La Silla (0.078 mags/airmass), SAAO (0.06 mags/airmass) and Kitt Peak 


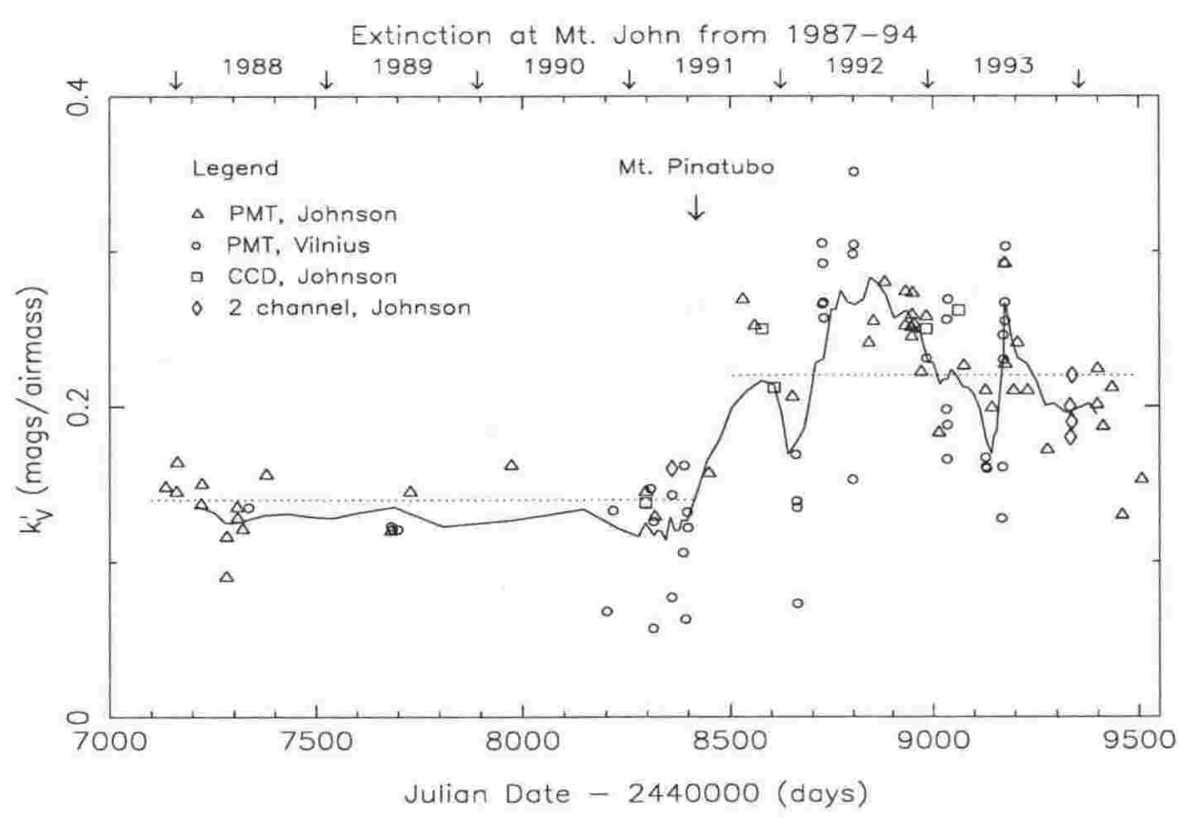

Figure G.2: The effect of the Mount Pinatubo eruption on extinction at MJUO. The V extinction coefficients from various photometric systems at MJUO are combined to increase the data set. The solid line is a running average and the dotted lines are the Pre- and Post-Pinatubo mean extinction levels.

(0.08 mags/airmass)(Grothues \& Gochermann 1992) - see Table H.2 for the other mean Vilnius extinction coefficients and changes.

It took approximately 80 days for the aerosol cloud to reach New Zealand (Wadsworth \& Cole 1994), with the extinction starting to increase around JD 2448500, and reaching an initial maximum about 1 October 1991 (JD 2448530). As of June 1994, the extinction has not yet returned to pre-eruption levels. Further, the night-to-night variations hide any exponential decay back towards the Mount Pinatubo pre-eruption levels, as also found at La Silla (Grothues \& Gochermann 1992).

The data were also investigated for seasonal effects, as there appeared to be an increase in the extinction every July since the eruption. A sinusoid was fitted (Rufener 1986) separately to the Pre-Pinatubo data, the Post-Pinatubo data and the combined data (after adjusting the Post-Pinatubo data to the same mean level as the Pre-Pinatubo data) but none were found to be statistically more significant than a single (mean) extinction value. Hence the night-to-night variations in extinction are greater than any seasonal changes, emphasizing that extinction should be determined for each night rather than using monthly averages. 


\section{Appendix $\mathbf{H}$}

\section{The Rayleigh, Ozone and Aerosol Extinction Coefficients.}

\section{H.1 Introduction.}

The total atmospheric extinction is usually considered to be comprised of three different sources of extinction; scattering off molecules (Rayleigh scattering), scattering off particles of dust or droplets of any size (aerosol scattering) and absorption by molecules (van de Hulst 1981, Hayes \& Latham 1975). In the optical region, the main molecular absorption bands are weak Ozone bands (the Huggins band from $320-340 \mathrm{~nm}$ and the weaker Chappius band centred at $575 \mathrm{~nm}$ ) (Sterken \& Manfroid 1992b) and some water absorption bands at 710, 809, 970 and $1080 \mathrm{~nm}$ (Hayes \& Latham 1975). The water absorption is highly variable and, at a good observatory site, usually very weak and can be ignored on most nights. This is particularly true for the Vilnius filter set, as can be seen in Figure 2.12. Therefore the total extinction can be written as

$$
k_{\text {total }}=k_{\text {Rayleigh }}+k_{\text {aerosol }}+k_{\text {ozone }}
$$

where the prime (indicating primary extinction) has been dropped.

Determining the total extinction coefficients at the seven different wavelengths of the Vilnius system opens the possibility of deriving these individual components and following their evolution on various time scales (hourly if determined by Nikonov's(1953) method or daily if determined by Bouguer's method (Hardie 1962)).

\section{H.2 Rayleigh.}

The Rayleigh scattering can, in principle, be calculated at Standard Temperature and Pressure (STP) from the following formula (Hayes \& Latham 1975)

$$
k_{\text {Rayleigh }_{S T P}}=9.4977 \times 10^{-3} e^{-\left(\frac{h}{7.996}\right)}\left[\frac{(n-1)_{\lambda}}{(n-1)_{\lambda=1}}\right]^{2}\left(\frac{1}{\lambda}\right)^{4}
$$


with the wavelength $\lambda$ in $\mu m$, which was adapted from Penndorf (1957). The formula has been normalised to $\lambda=1 \mu m$, with $n$ being the refractive index and the coefficient $9.4977 \times 10^{-3}$ corresponding to the optical thickness of the atmosphere at $\lambda=1 \mu \mathrm{m}$. This is adjusted for the height $h$ (in $\mathrm{km}$ ) of the observatory above sea-level with the density scale-height for the lower troposphere assumed to be $7.996 \mathrm{~km}$.

The normalised index of refraction term is given by

$$
\frac{(n-1)_{\lambda}}{(n-1)_{\lambda=1}}=0 \cdot 23465+\frac{1 \cdot 076 \times 10^{2}}{146-\left(\frac{1}{\lambda}\right)^{2}}+\frac{0 \cdot 93161}{41-\left(\frac{1}{\lambda}\right)^{2}} .
$$

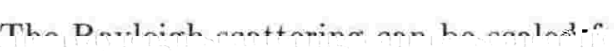


1981, King et al. 1978) gives

$$
k_{\text {aerosol }}=\int_{0}^{\infty} \pi r^{2} Q_{e x t}(r, \lambda, n) N_{c}(r) d r
$$

where $Q_{e x t}$ is the extinction efficiency factor from Mie theory and $N_{c}$ is the aerosol number density in a vertical column (cross-section $1 \mathrm{~cm}^{2}$ ) in the radius interval $r \rightarrow r+d r$.

Equation H.6 can be inverted to obtain $N_{c}$, as was done by King et al. (1978) for observations of fifty-seven days in Tucson. Figure H.1 shows schematically the (log-log) size distributions and corresponding $k_{\text {aer osol }}$ 'spectrum' for the three different classes found.

The first class is approximately a linear Junge (1963) size distribution and was found for $\sim 30 \%$ of the observations. This can be characterized in a $k_{\text {aerosol }}$ diagram by a straight line (occasionally with a slight curvature) with a large negative slope $(\alpha \sim+1 \cdot 5$, see below).

A Junge size distribution is a power-law

$$
N_{c}(r)=c r^{-\left(\nu^{*}-1\right)}
$$

for $r=0 \rightarrow \infty$, giving

$$
k_{\text {aerosol }}=\beta \lambda^{-\nu^{*}+2}
$$

which is the empirical formula found by Angström (1929)

$$
k_{\text {aerosol }}=\beta \lambda^{-\alpha}
$$

with $\alpha=\nu^{*}-2$. Assuming the size distribution can be characterized by particles with just one (effective) radius $r_{e}$, then the parameter $\alpha$ is related to this radius (Gutiérrez-Moreno et al. 1982); $\alpha \sim 1 \Rightarrow r_{e} \sim \lambda, \alpha \sim 2 \Rightarrow r_{e}<\lambda$, and $\alpha<1 \Rightarrow r_{e} \gg \lambda$. This empirical formula (equation H.9) has been used in many aerosol extinction studies, for example Hayes \& Latham (1975), Gutiérrez-Moreno et al. (1982), Rufener (1986), Reimann et al. (1992), and Sterken \& Manfroid (1992b). Correction of the unrealistic assumption of infinite spread in radius or slight deviations from the Junge distribution leads to a slight curvature in the $k_{\text {aerosol }}$ plot, adding a quadratic term (King \& Byrne 1976)

$$
\log \left(k_{\text {aerosol }}\right)=\log (\beta)-\alpha \log (\lambda)+\gamma[\log (\lambda)]^{2}
$$

The second class is an approximately Gaussian size distribution, which was found for $~ 30 \%$ of King's observations. In the $k_{\text {aeros ol }}$ diagram it is characterized by an line with some curvature and a positive slope, giving a negative $\alpha$.

The third class is actually a combination of the two previous size distributions and is char- 
(o) Junge distribution

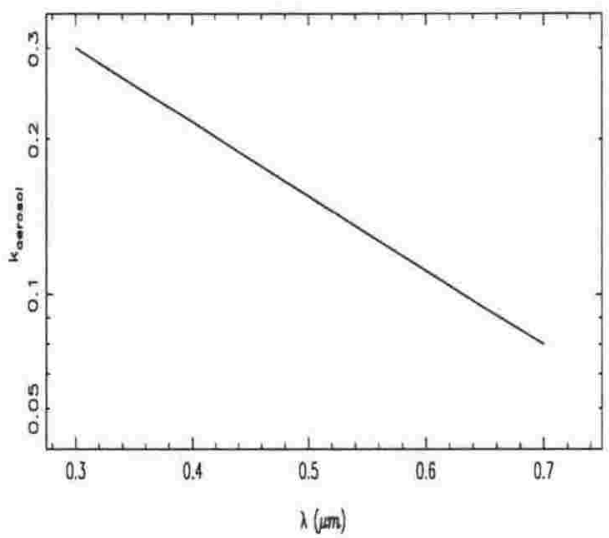

(b) Goussion distribution

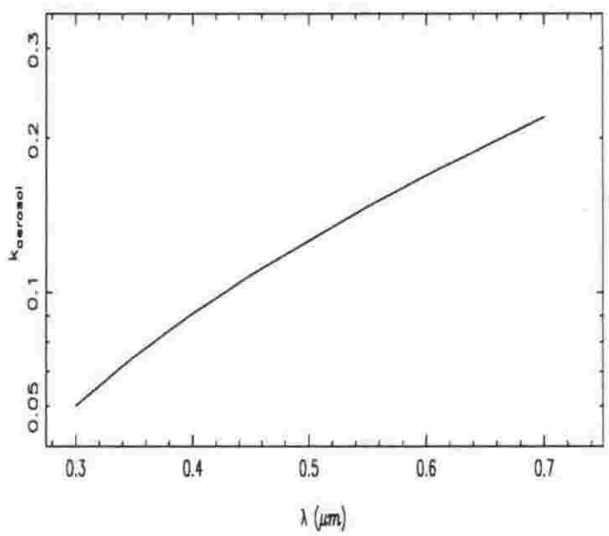

(c) combination distribution

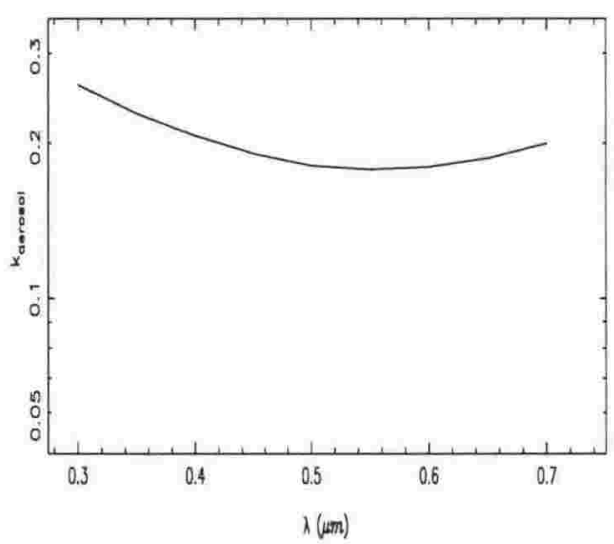

(0) Junge distribution

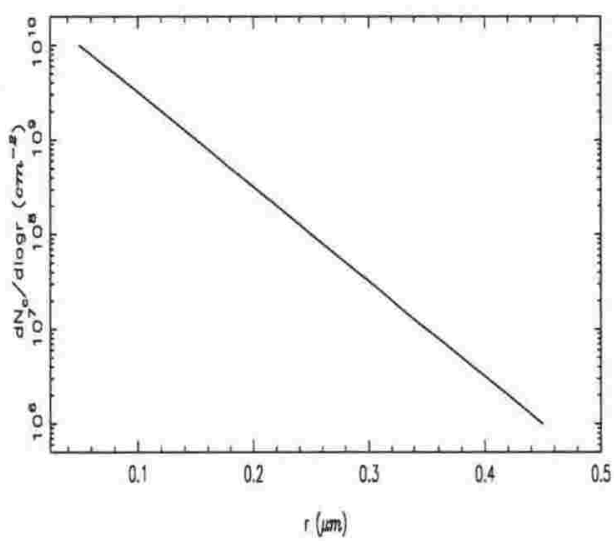

(b) Goussion distribution

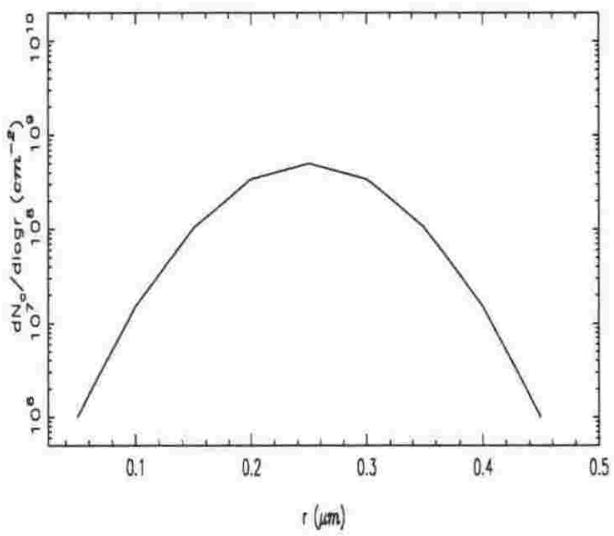

(c) combination distribution

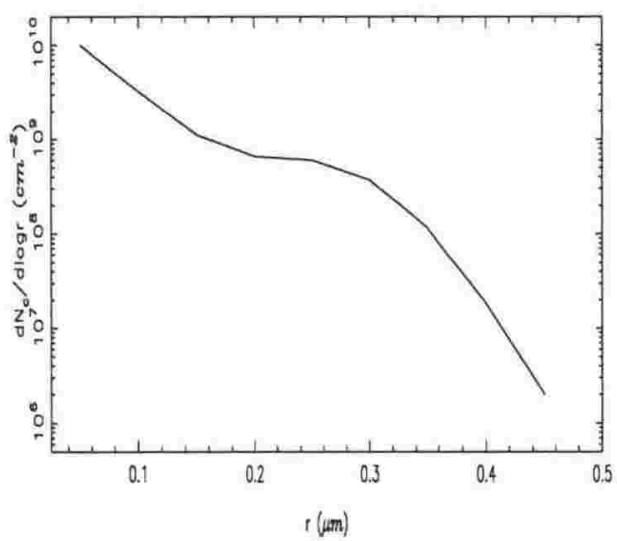

Figure H.1: The three different aerosol size distributions and their corresponding extinction spectrum. 
acterized by a small $\alpha$. It was the most common distribution, being found for $\sim 50 \%$ of the observations. This two component distribution is thought to arise from two aerosol production mechanisms (King et al. 1978), namely

a) nucleation from a gas phase and later coagulation, predominately with $r \leqslant 0.5 \mu \mathrm{m}$

b) particles created by mechanical or wind stress from the Earth's surface, predominately with $r \widetilde{>} 1 \mu \mathrm{m}$.

Thus even when Ångström's empirical formula (equation H.9) is not an appropriate model for the aerosol size distribution, the sign and magnitude of the parameter $\alpha$ appears to indicate the type of distribution (Junge, Gaussian or combination).

\section{H.5 Fitting procedures.}

The Rayleigh extinction coefficients at STP were calculated using equations H.2 and H.3 and convolved with the response functions of the standard Vilnius system (see Table 1.3) to produce the effective extinction coefficients of each filter, given in Table H.1.

Similarly, the absorption coef-

ficients of ozone were taken from

Gast (1960) and convolved with

the response functions of the stan-

dard Vilnius system to produce

the effective absorption coefficients $\left(\mathrm{cm}^{-1}\right)$ of each filter. For comparison with the Rayleigh coefficients, the ozone absorption coefficients were then converted to

Table H.1: Effective Rayleigh and Ozone extinction coefficients for the standard Vilnius photometric system.

\begin{tabular}{|cccll|}
\hline filter & $\begin{array}{c}\lambda_{0} \\
(\mu \mathrm{m})\end{array}$ & $\begin{array}{l}\text { Rayleigh } \\
\text { extinction }\end{array}$ & $\begin{array}{l}\text { Ozone } \\
\text { absorption }\end{array}$ & $\begin{array}{l}\text { Ozone } \\
\text { extinction }\end{array}$ \\
\hline $\mathrm{U}$ & $0 \cdot 345$ & $0 \cdot 661$ & $6 \cdot 7 \times 10^{-2}$ & $2 \cdot 0 \times 10^{-2}$ \\
$\mathrm{P}$ & 0.374 & $0 \cdot 464$ & $2 \cdot 5 \times 10^{-4}$ & $7 \cdot 4 \times 10^{-5}$ \\
$\mathrm{X}$ & 0.405 & $0 \cdot 333$ & $5 \cdot 4 \times 10^{-5}$ & $1 \cdot 6 \times 10^{-5}$ \\
$\mathrm{Y}$ & 0.466 & $0 \cdot 186$ & $3 \cdot 9 \times 10^{-3}$ & $1 \cdot 2 \times 10^{-3}$ \\
$\mathrm{Z}$ & 0.516 & $0 \cdot 122$ & $1.9 \times 10^{-2}$ & $5 \cdot 6 \times 10^{-3}$ \\
$\mathrm{~V}$ & 0.544 & $0 \cdot 099$ & $3 \cdot 3 \times 10^{-2}$ & $9 \cdot 8 \times 10^{-3}$ \\
$\mathrm{~S}$ & $0 \cdot 656$ & 0.047 & $2 \cdot 7 \times 10^{-2}$ & $8 \cdot 0 \times 10^{-3}$ \\
\hline
\end{tabular}

mean extinction coefficients using

equation H.5 and the total ozone (averaged over a year) at latitude $40^{\circ} \mathrm{S}$ from Allen (1963).

The aerosol extinction coefficients (equation H.9) were calculated at the effective wavelength $\left(\lambda_{0}\right)$ of each filter as the wavelength dependence in the optical region was found to be nearly flat - also, it would have been very difficult to calculate the effective extinction as it depends on $\alpha$, one of the parameters we wish to fit.

On 13 April 1992, the monochromatic extinction was determined approximately hourly using Nikonov's method. The extinction on this night showed a gradual, linear, decrease. Fitting the extinction components may allow Young's(1974) explanation that such decreases in extinction are due to a slow fallout of aerosols (when the solar driven convection which stirs up the aerosols 
Fitted Extinction Components on 13 April 1992

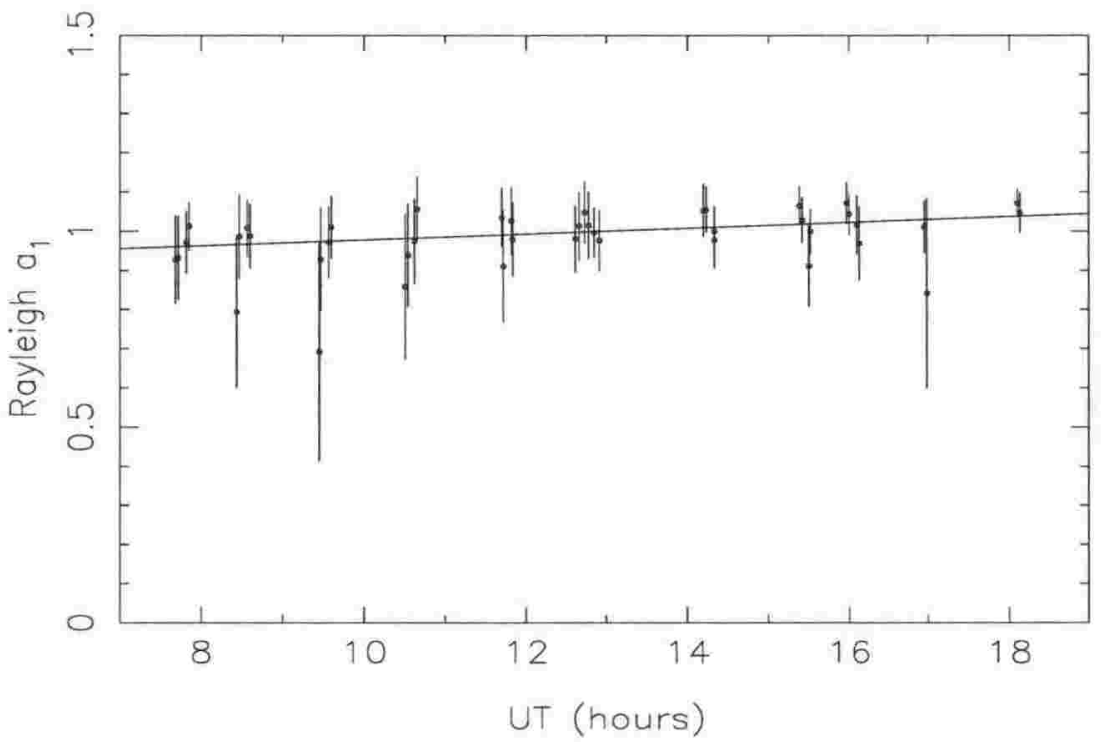

Figure H.2: Behaviour of the Rayleigh extinction during 13 April 1992.

ceases) to be checked. Hence, the extinction was fitted as a trial using a Levenberg-Marquardt non-linear least squares subroutine (Press et al. 1992) on

$$
k_{\text {total }}=a_{1} k_{\text {Rayleigh }_{S T P}}+a_{2} k_{\text {ozone }} \text { mean }+a_{3} \lambda^{-a_{4}}
$$

where $a_{1}$ measures the fractional change from STP and $a_{2}$ measures the fractional change from the mean ozone content.

\section{H.6 Results.}

The total extinction was fitted for each extinction star measurement with all four parameters free. The Rayleigh component $\left(a_{1}\right)$ shows a gradual increase during the night (see Figure H.2) while the other parameters have such large scatter that no trends can be seen. This large scatter is due to strong correlations between the parameters (especially the Rayleigh component which is essentially proportional to $\lambda^{-4}$ and the aerosol component, which is proportional to $\lambda^{-\alpha}$ ), which makes the fits very sensitive to measurement errors.

To try to reduce this scatter, a straight line was fitted to the Rayleigh coefficients and $a_{1}$ was fixed at the appropriate value from this line for each measurement during the night, while the remaining three parameters were refitted. This succeeded in reducing the scatter sufficiently that trends could be seen in all the coefficients, which will be tentatively interpreted next. 
Fitted Extinction Components on 13 April 1992

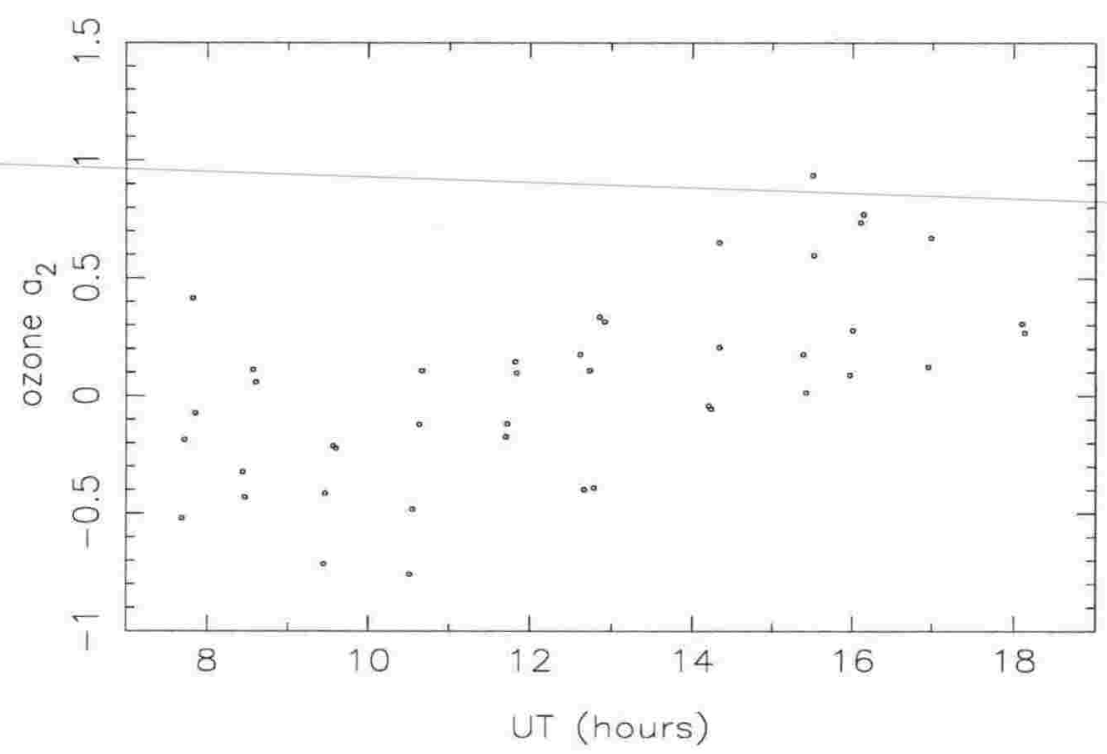

Figure H.3: Behaviour of the ozone extinction during 13 April 1992.

At the beginning of the night, the Rayleigh coefficient was

$$
a_{1}=\frac{T_{S T P}}{T} \frac{P}{P_{S T P}} \approx 0.96
$$

Assuming $P=P_{S T P}$, this implies the air temperature $T \approx 11^{\circ} C$, which shows good agreement with the recorded dome temperature of $12^{\circ} \mathrm{C}$. A similar calculation for the end of the night gives $T \approx-10^{\circ} \mathrm{C}$. However the dome (interior) temperature showed no significant change from $12^{\circ} \mathrm{C}$ (i.e. within $\pm 1^{\circ}$ ) during the night. The insulating properties of the dome walls and roof together with the heat generated by the electronics and telescope \& dome motors would tend to hold the dome interior at a higher temperature than the outside air. Thus it seems more likely that the air temperature did decrease during the night, to $0^{\circ} \mathrm{C}$ say, and the air pressure also increased slightly.

The ozone content shows a decreasing trend until $\sim 8-9^{h}$ UT, an increase until $\sim 15^{h}$ UT, when it decreased again (see Figure H.3). As astronomical twilight ended and began at $\sim 7^{h}$ UT and $17^{h}$ UT respectively at sealevel on 13 April 1992(The Astronomical Almanac 1992), and the ozone layer is $\sim 21 \mathrm{~km}$ above sealevel (Allen 1963), a simple calculation shows that the twilight for the ozone layer ended/began at $\sim 8^{h} / 16^{h}$ UT. Thus, it seems likely that the decrease in ozone content is due to the Sun's ultraviolet light breaking up the ozone during the day and the increase is the layer reforming during the night.

The negative values for the ozone coefficients around $9^{h}$ UT are not realistic. As the ozone 
is the weakest component of the total extinction (in the wavelength region measured), slightly too large values in the other components would have a significant effect on the derived ozone content, forcing it too far down.

To reduce this problem, another filter would be needed to measure a wavelength region with stronger ozone absorption. The $6000 \AA$ region is ruled out, as the $\mathrm{H}_{2} \mathrm{O}$ absorption band is of approximately equal size to the ozone absorption there. This only leaves a region at $\sim 3000 \AA$. The Rayleigh extinction is also large at that wavelength, but fortunately it can be accurately predicted from measurements of the air temperature and pressure. A blue star (i.e. O or B stars) would probably be needed as the extinction star to get a good signal at these wavelengths.

The aerosol content ( $a_{3}$, see Figure H.4) shows an overall decrease during the night, with slight increases at the beginning of the night and between $12-14^{h}$ UT. These increases seem to be correlated with a slight breeze present at those times. However the aerosol size distribution parameter $\left(a_{4}\right.$, see Figure H.5) shows a slight rise until $\sim 11^{h}$ UT, then a decrease for the rest of the night. The small, positive value of $a_{4}$ at the beginning of the night indicates a mixture of Junge and Gaussian particle size distributions. The increasingly negative value after $11^{h} \mathrm{UT}$ indicates the distribution is becoming more Gaussian as the night progresses, i.e. there are less small sized particles. It is possible that these small particles (which are caused by nucleation from a gas phase) coalesce together, forming larger particles while decreasing the total number of particles (and hence reducing $a_{3}$ ). Hence, the results are consistent with Young's contention that the decrease in extinction is due to a decreasing number of aerosols during the night, but indicates a different mechanism is removing these particles.

The rapid changes in the extinction components (i.e. on a time scale of hours) means fitting the components to the average extinction coefficients for a night would only be valid for nights with constant extinction. However, over a long enough time scale (i.e. months or years), the average extinction might be accurately fitted by the average amount of the components. To test this, the mean extinction coefficients for all photometric nights were fitted - see Figure H.6.

It was necessary to split the data into two sets; before and after the aerosols from the Mount Pinatubo eruption arrived at MJUO - see Table H.2. As the aerosols ejected into the stratosphere may also deplete the ozone layer (Stowe et al. 1992), it was necessary to fit the ozone and aerosol components to both data sets. However, the Rayleigh component was not expected to be changed by the eruption so the value derived in the Pre-Pinatubo fit was used (as a fixed parameter) in the Post-Pinatubo fit. The results are given in Table H.3, where $\chi^{2}$ is the chi squared goodness-of-fit parameter.

From Allen (1963), the mean temperature (at sea-level) for latitude $-45^{\circ}$ is $\sim 8^{\circ} \mathrm{C}$ while 
Fitted Extinction Components on 13 April 1992

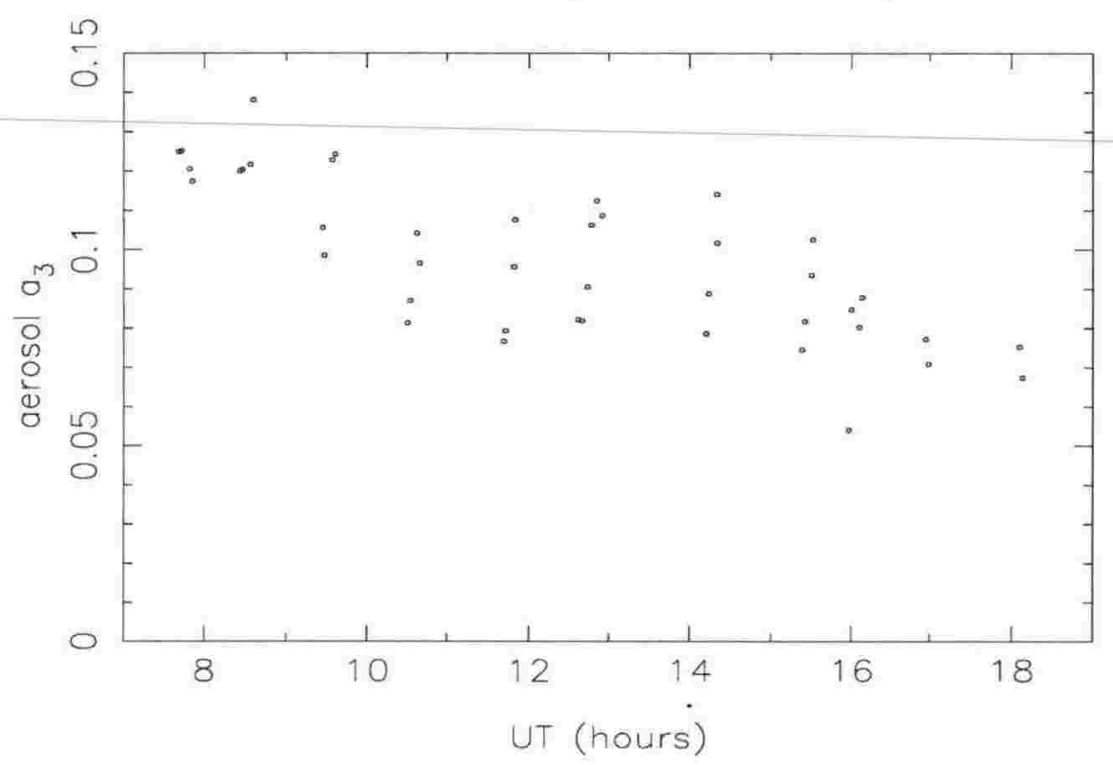

Figure H.4: Behaviour of the aerosol extinction during 13 April 1992.

Fitted Extinction Components on 13 April 1992

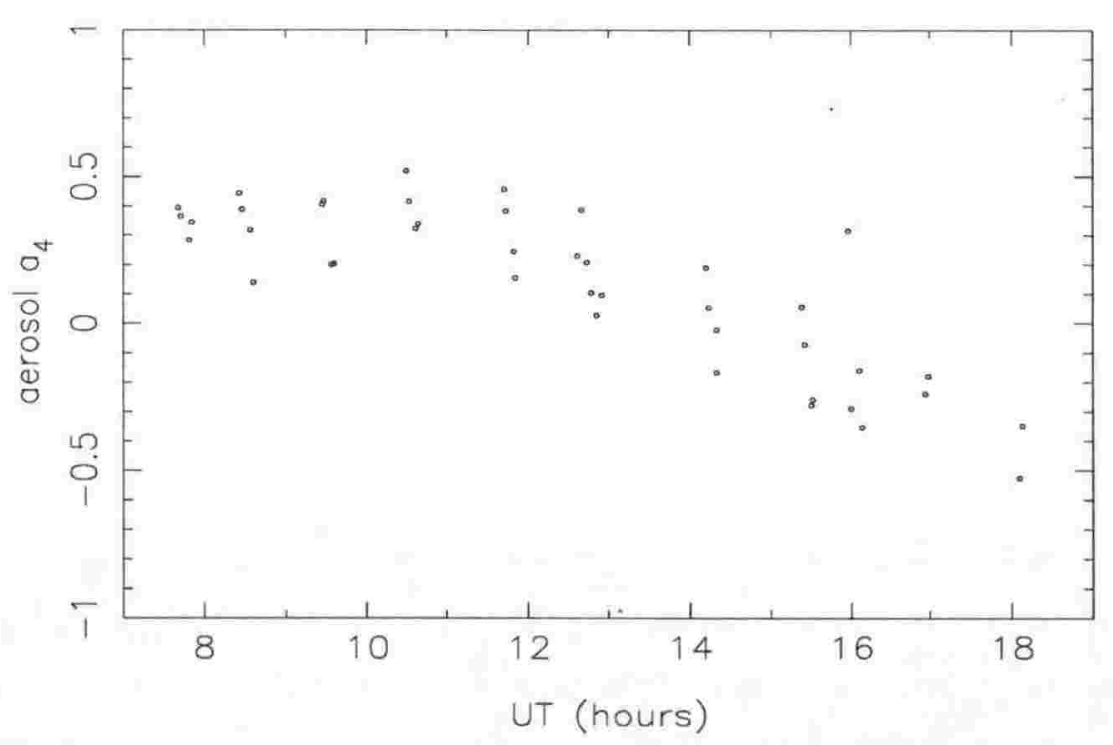

Figure H.5: Behaviour of the aerosol size distribution parameter during 13 April 1992. 


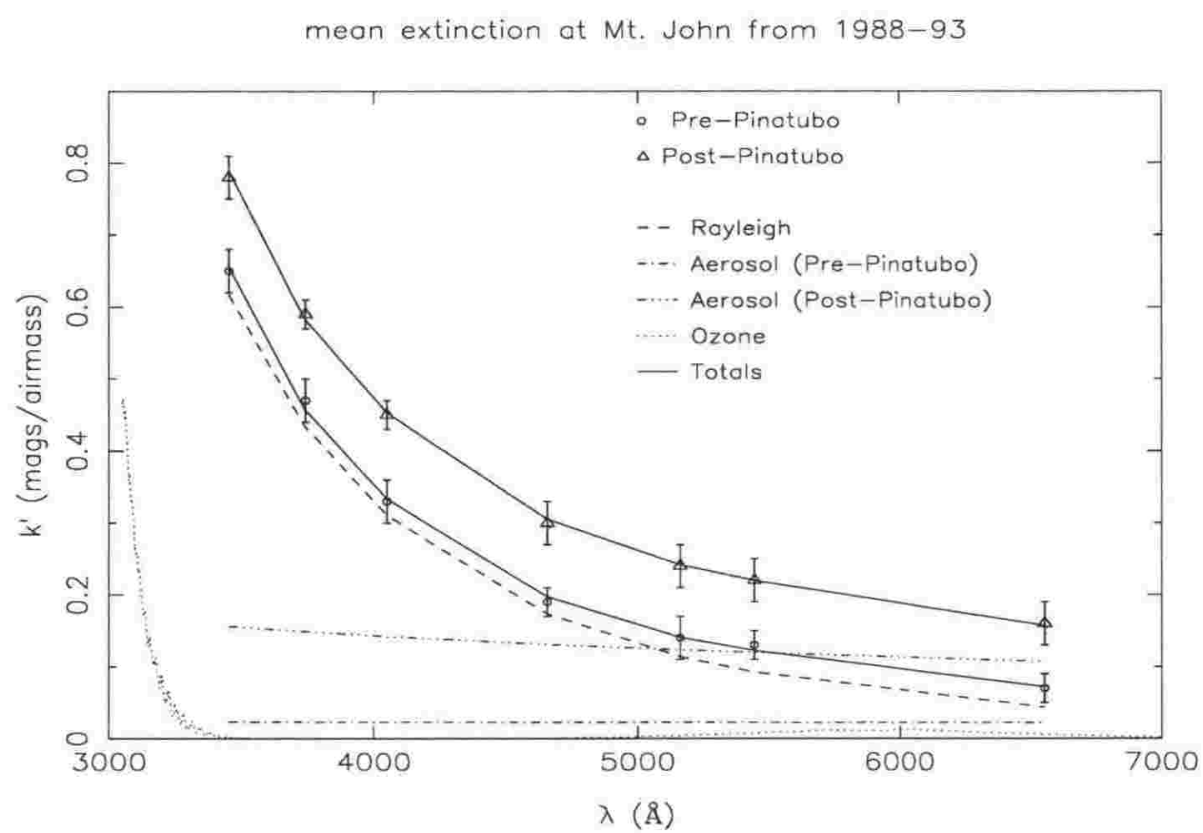

Figure H.6: The mean extinction at MJUO fitted with Rayleigh, Ozone and aerosol components. The extinction has been divided into two groups; before and after the Mount Pinatubo eruption.

Table H.2: The mean Vilnius extinction coefficients at MJUO — before, after, and the changes due to, the Mount Pinatubo eruption. All uncertainties are between $0.02-0.03$.

\begin{tabular}{|lccccccc|}
\hline date & \multicolumn{8}{c|}{ monochromatic extinction coefficients } \\
(Pinatubo) & $\mathrm{U}$ & $\mathrm{P}$ & $\mathrm{X}$ & $\mathrm{Y}$ & $\mathrm{Z}$ & $\mathrm{V}$ & $\mathrm{S}$ \\
\hline Pre- (Jun 88 - May 91) & $0 \cdot 65$ & 0.47 & 0.33 & $0 \cdot 19$ & $0 \cdot 14$ & 0.13 & $0 \cdot 07$ \\
Post- (Nov 91 - Jul 93) & 0.78 & 0.59 & 0.45 & 0.30 & 0.24 & 0.22 & 0.16 \\
$\Delta$ Pinatubo & 0.13 & 0.12 & 0.12 & $0 \cdot 11$ & $0 \cdot 12$ & 0.09 & 0.09 \\
\hline
\end{tabular}

the mean air pressure at $h=1 \mathrm{~km}$ is $899 \mathrm{mb}$ (at sea-level, $P_{S T P}=1013 \mathrm{mb}$ ), so

$$
a_{1} \approx \frac{8+273 \cdot 15}{273 \cdot 15} \frac{899}{1013} \approx 0 \cdot 91
$$

which agrees (within the uncertainties) with the derived value. The ozone content is approximately halved following the eruption, but in view of the large uncertainties in the coefficients, this decrease may not be real but a result of correlations in the fitting procedure. The aerosol content has increased by a factor of four, with the particle size distribution becoming more Gaussian (from the Pre-Pinatubo Junge and Gaussian mixture). The aerosol extinction spectrum looks nearly flat (i.e. grey - see $\Delta$ Pinatubo in Table H.3), as was also found by GutiérrezMoreno et al. (1982), Sterken \& Manfroid (1992b) and Grothues \& Gochermann (1992). 
Table H.3: The fitted Rayleigh, Ozone and aerosol coefficients to the mean extinction.

\begin{tabular}{|l|c|c|c|c|c|}
\hline date & Rayleigh & Ozone & \multicolumn{2}{|c|}{ aerosol } & \\
(Pinatubo) & $a_{1}$ & $a_{2}$ & $a_{3}$ & $a_{4}$ & $\chi^{2}$ \\
\hline Pre- & $0.93 \pm 0 \cdot 19$ & $0.76 \pm 2.3$ & $0.022 \pm 0 \cdot 087$ & $0.0077 \pm 7.4$ & 0.55 \\
Post- & 0.93 (fixed) & $0.38 \pm 1.5$ & $0.086 \pm 0.030$ & $0.57 \pm 0.39$ & 0.24 \\
\hline
\end{tabular}

\section{H.7 Conclusions.}

The extinction data from the Vilnius filter set can be used to derive the contributions from the Rayleigh, ozone and aerosol components. However the major component, Rayleigh scattering, should be predicted (from equation H.2 and measuring the air temperature and pressure) rather than fitted, to reduce the large uncertainties due to the correlations between the parameters. The rapid changes in all three components mean Nikonov's method (or other similar method) must be used to measure the extinction hourly — only a night with constant extinction may be fitted from the night's average extinction. The Mount Pinatubo eruption significantly reduced the ozone content and increased the aerosol content (in the stratosphere). 


\section{Formulation of the Vilnius Filters using Schott glasses.}

Here the possibility of using Schott glasses to make a Vilnius filter set was investigated, ${ }^{1}$ as an alternative to those produced by the Vilnius Observatory. The transmittance response curves were taken from the Schott catalog up to $\lambda=10000 \AA$, assuming the detector used does not response to longer wavelengths - this assumption must be checked as many Schott glasses show a second (and larger) red leak for $\lambda>10000 \AA$. Changes in transmittance due to differing thickness for any particular glass are calculated according to

$$
R_{d_{1}}(\lambda)=\left[R_{d_{2}}(\lambda)\right]^{d_{1} / d_{2}}
$$

where $R_{d_{i}}$ is the transmittance at $\lambda$ for a glass thickness $d_{i}$.

It has been found that the transmittance curves in the catalog are only average values and may not be accurate for any particular sample of a glass (Young 1994). In particular, the long-pass glasses (for example WG, GG, OG, and RG glasses) typically vary by $60 \AA$, which corresponds to approximately a factor of four in thickness. Similarly, the band-pass glasses (for example UG and BG) have typical errors corresponding to $\pm 10 \%$ thickness variations. This effect can be seen in Figure I.1 where the catalog transmittance curve of BG39 is compared with a sample measured at VUW - the red side differs by $\sim 90 \AA$ from the catalog value. This is a significant fraction of the FWHM of the Vilnius System filters (typically $250 \AA$ ), hence orders to manufacturers should be specified by the required transmittance curves rather than the glass thickness and the glass combinations given here should be taken only as a guide.

Many different Schott glass combinations were investigated for each Vilnius filter, and initially compared visually from plots of the transmittance curves. To quantify the closer matches, the following formula was used

$$
\text { rating }=\frac{\left|\Delta \lambda_{\text {short }}-\Delta \lambda_{\text {long }}\right|\left|\Delta \lambda_{\text {peak }}\right|(\text { Red Leak } \%)}{\left(R_{\text {peak }} \%\right)}
$$

\footnotetext{
${ }^{1}$ A prescription using Schott glasses has already been given by Kučinskas (1990) but four of the filters require glasses which are no longer available and the $\mathrm{S}$ filter used the detector as its long-wavelength cutoff.
} 
BG039 $1 \mathrm{~mm}$ Schott Glasses

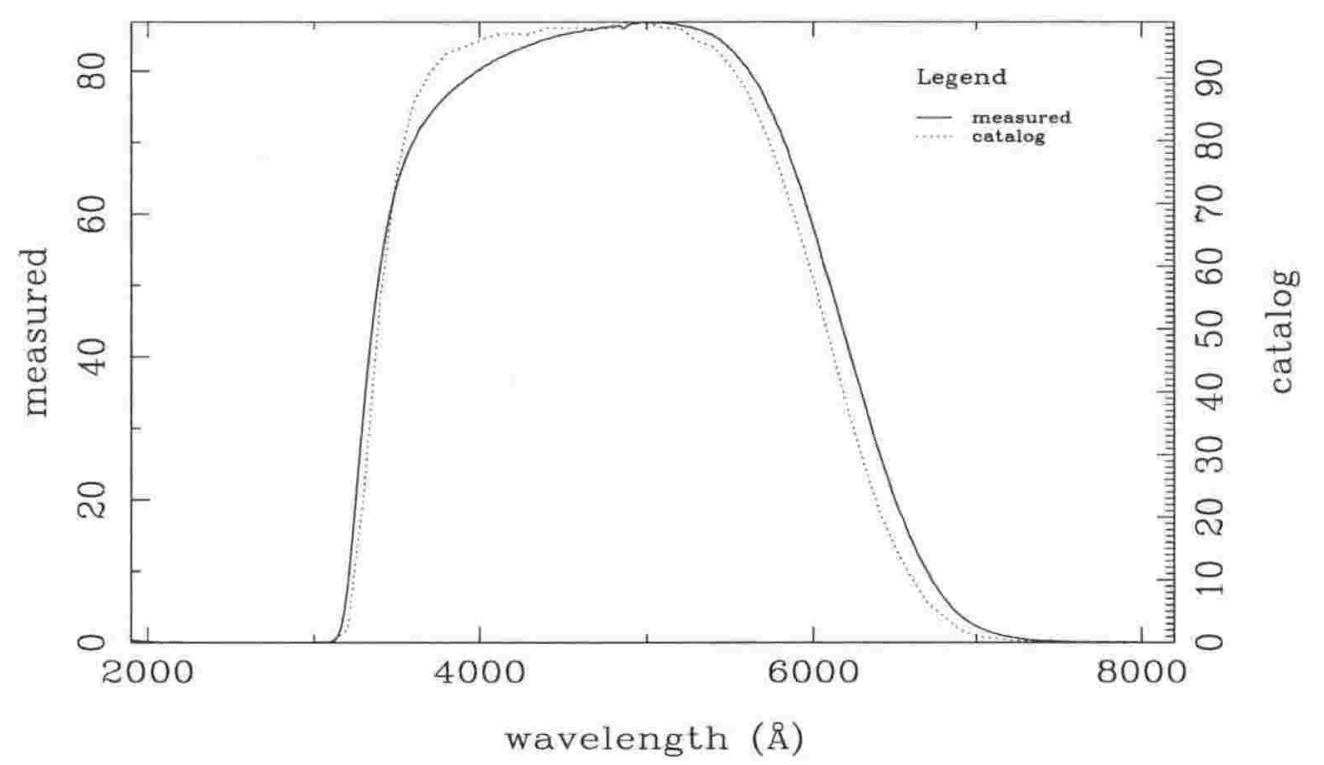

Figure I.1: Comparison of Measured and Catalog responses of Schott glasses, here BG39 is shown spline fitted.

where $\Delta \lambda_{\text {short }}, \Delta \lambda_{\text {long }}$, and $\Delta \lambda_{\text {peak }}$ are the required shifts (in wavelength) of the half-maximum (of the short and long wavelength sides) and the peak transmittance to match the standard filter. The $R_{\text {peak }} \%$ is the maximum (peak) transmittance as a percentage and the Red Leak\% $=\frac{\text { peak transmittance of the red leak }}{\text { peak transmittance of the Schott filter }}$. Using this crude, but easy to calculate formula, the combination of glasses with the smallest rating gives the best match to the standard filter.

Young (1994) gives a more accurate formula for comparing filters

$$
\cos (\phi)=\frac{\int R_{A} \cdot R_{B} d \lambda}{\left|R_{A}\right|\left|R_{B}\right|}
$$

where the transmittance of the filters are $R_{A}$ and $R_{B}$, and the magnitude of a filter is

$$
|R|=\sqrt{\int R^{2} d \lambda} .
$$

The angle $\phi$ measures the difference between the filters, $\phi=0^{\circ}$ means the filters are identical (apart from a scaling factor in the transmittance) while $\phi=90^{\circ}$ corresponds to completely non-overlapping filters.

This formula is derived by treating each filter as a vector in a multi-dimensional space and 
Table I.1: The Vilnius Filter System from Schott glasses.

\begin{tabular}{|c|c|c|c|c|c|}
\hline Filter & Schott glass combination & $\begin{array}{l}R_{\text {peak }} \\
(\%)\end{array}$ & Rating & $\begin{array}{c}\phi_{\text {Schott }} \\
\text { (degrees) }\end{array}$ & $\begin{array}{c}\phi_{\text {local }} \\
\text { (degrees) }\end{array}$ \\
\hline $\mathrm{U}$ & WG355 $(1 \mathrm{~mm})+\mathrm{UG} 11(7 \mathrm{~mm})$ & 45 & 0.003 & $5 \cdot 7$ & $7 \cdot 3$ \\
\hline $\mathrm{P}$ & $\mathrm{BG} 23(6 \mathrm{~mm})+\mathrm{UG} 1(2 \mathrm{~mm})$ & 17 & 0.01 & $6 \cdot 5$ & $5 \cdot 0$ \\
\hline $\mathrm{X}$ & GG400 $(1 \mathrm{~mm})+\mathrm{BG} 3(9 \mathrm{~mm})+\mathrm{BG} 39(1 \mathrm{~mm})$ & 53 & $0 \cdot 9$ & $18 \cdot 8$ & $2 \cdot 8$ \\
\hline $\mathrm{Y}$ & GG455 $(2 \mathrm{~mm})+\mathrm{BG} 3(1 \mathrm{~mm})+\mathrm{BG} 39(2 \mathrm{~mm})$ & 42 & 0.01 & $6 \cdot 6$ & $9 \cdot 5$ \\
\hline Z & GG495 $(6 \mathrm{~mm})+\mathrm{BG} 18(9 \mathrm{~mm})$ & 38 & 4.0 & $35 \cdot 6$ & $6 \cdot 0$ \\
\hline V & OG530 $(3 \mathrm{~mm})+\mathrm{BG} 39(12 \mathrm{~mm})$ & 44 & $2 \cdot 0$ & $9 \cdot 7$ & $3 \cdot 1$ \\
\hline $\mathrm{S}$ & $\mathrm{RG} 645(2 \mathrm{~mm})+\mathrm{BG} 40(2 \mathrm{~mm})$ & 13 & $12 \cdot 0$ & $30 \cdot 5$ & $30 \cdot 8$ \\
\hline
\end{tabular}

taking the dot product between the vectors. The components of each vector are

$$
\tilde{R}=\left\{R\left(\lambda_{1}\right), R\left(\lambda_{2}\right), \ldots, R\left(\lambda_{N}\right)\right\}
$$

where $N$ is the number of transmittance measurements made of the filter. To ensure each vector has measurements at the same wavelengths, the transmittance measurements are interpolated (using a cubic spline) to all the wavelengths measured in both filters.

The results are shown in Table I.1 and Figure I.2. The table also includes the angle between the Standard Vilnius filters (Straižys \& Zdanavičius 1970) and the local Vilnius filter set (\#1, as measured in 1990) for comparison.

The Schott U filter shows a slightly better agreement with the standard filter (as measured by $\phi$ ) than does the local filter. The most significant difference between the Schott filter and the standard filter is a reduced blue response, which would be more strongly seen for early stars. However the atmospheric extinction is large at these wavelengths so the effect is expected to be small. Both glasses (WG335 and UG11) are sensitive to staining by weak acids (for example from finger-prints or breath) and need either a protective coating (the Schott catalog states a coating is available but gives no further details) or be sandwiched between other glasses with stronger resistance to weak acids and which will not change the transmittance curve, for example WG280. This extra glass can also be used to pad the filters out to a common optical depth, required for imaging detectors (such as CCD's) where all filters should have the same focal point, and optional for non-imaging detectors (such as PMT's) where this is a second order source of error.

The Schott P filter is a slightly worse match to the standard filter (as measured by $\phi$ ) than the local filter. It also has a low peak transmittance, being approximately half that of the local P filter. Like the Schott U filter, it also needs protection against stains, with WG280 again being suitable.

No Schott glass combination could be found to give a good match to the standard X filter. The best combination has too strong a red response and a 'blue' leak. The 'blue' leak (at 
Spectral Transmittance (\%)

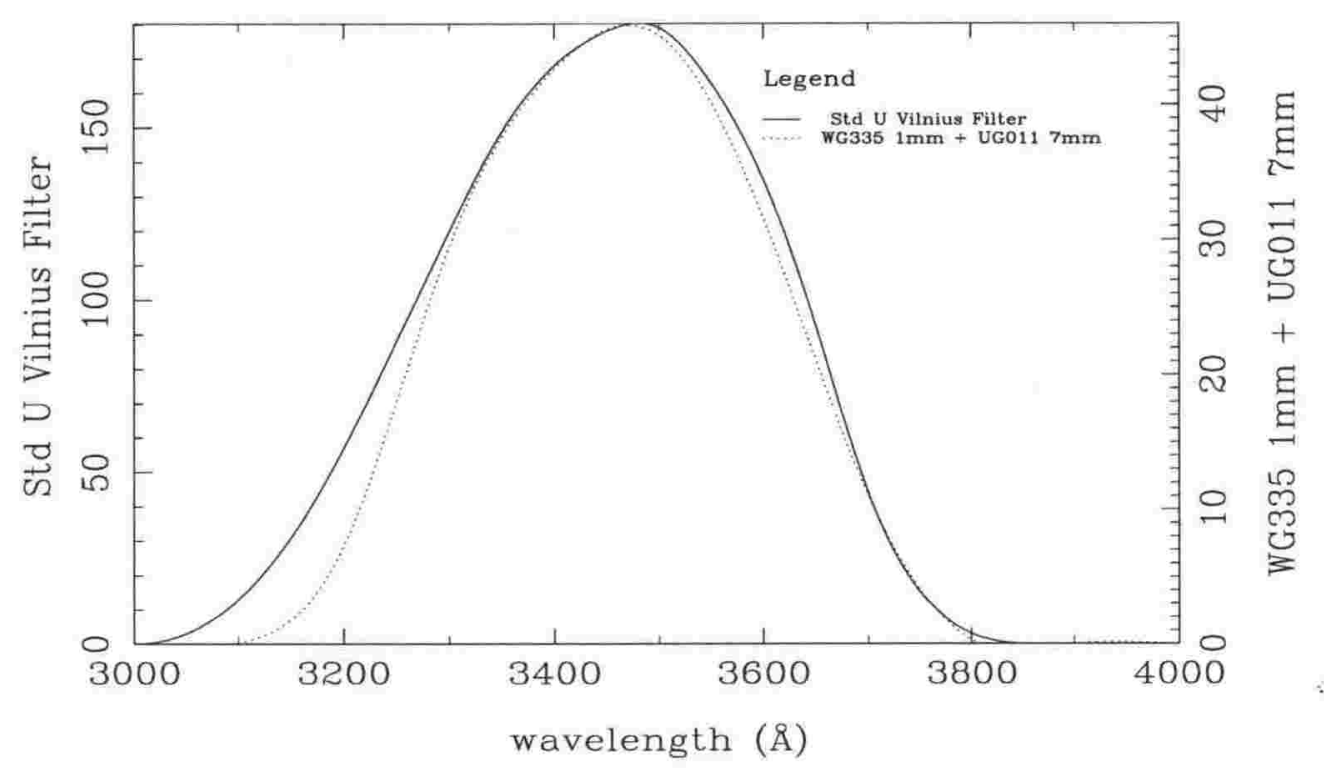

Figure I.2: Realization of the Vilnius Photometric System using Schott glasses, with the curves drawn using a cubic spline. The Standard filter responses are in arbitary units while the Schott glass combinations are a percentage of full transmittance. See following pages for the remaining filters.

Spectral Transmittance (\%)

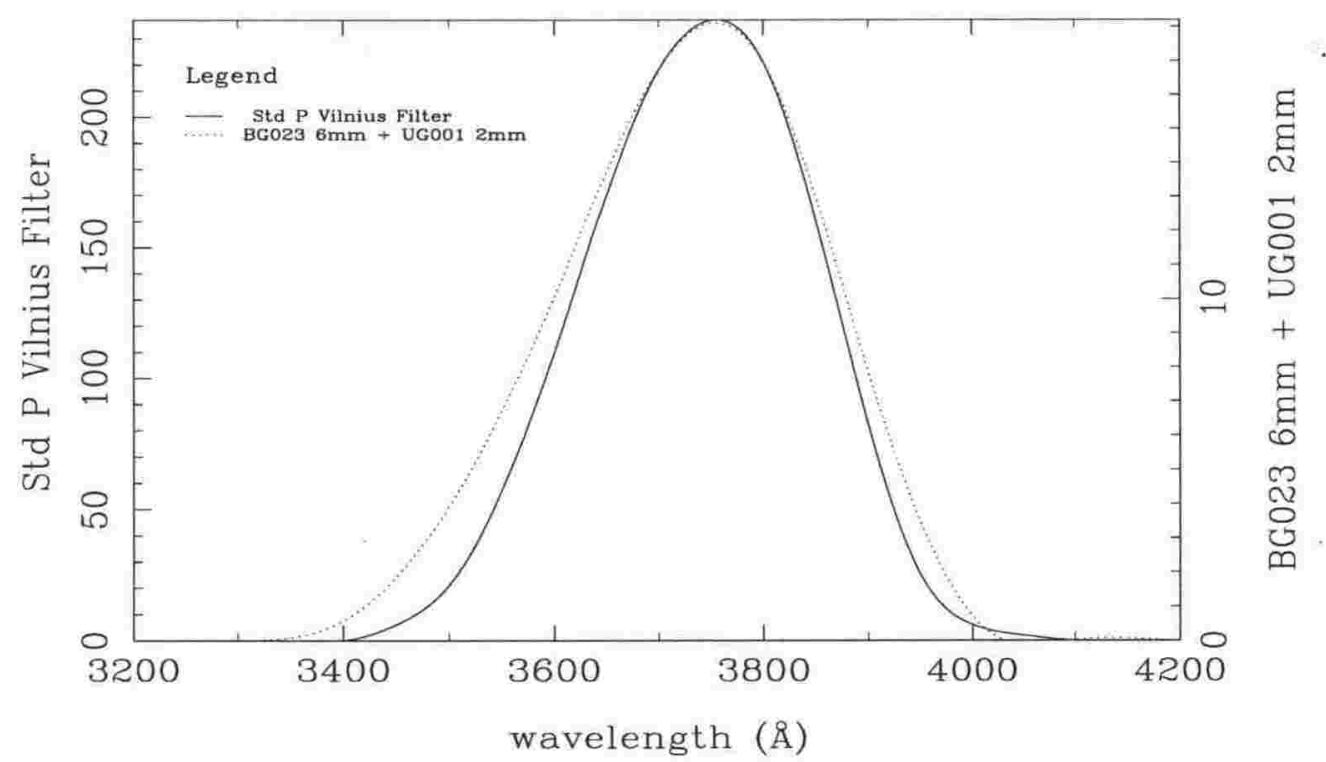

Figure I.2 continued. 


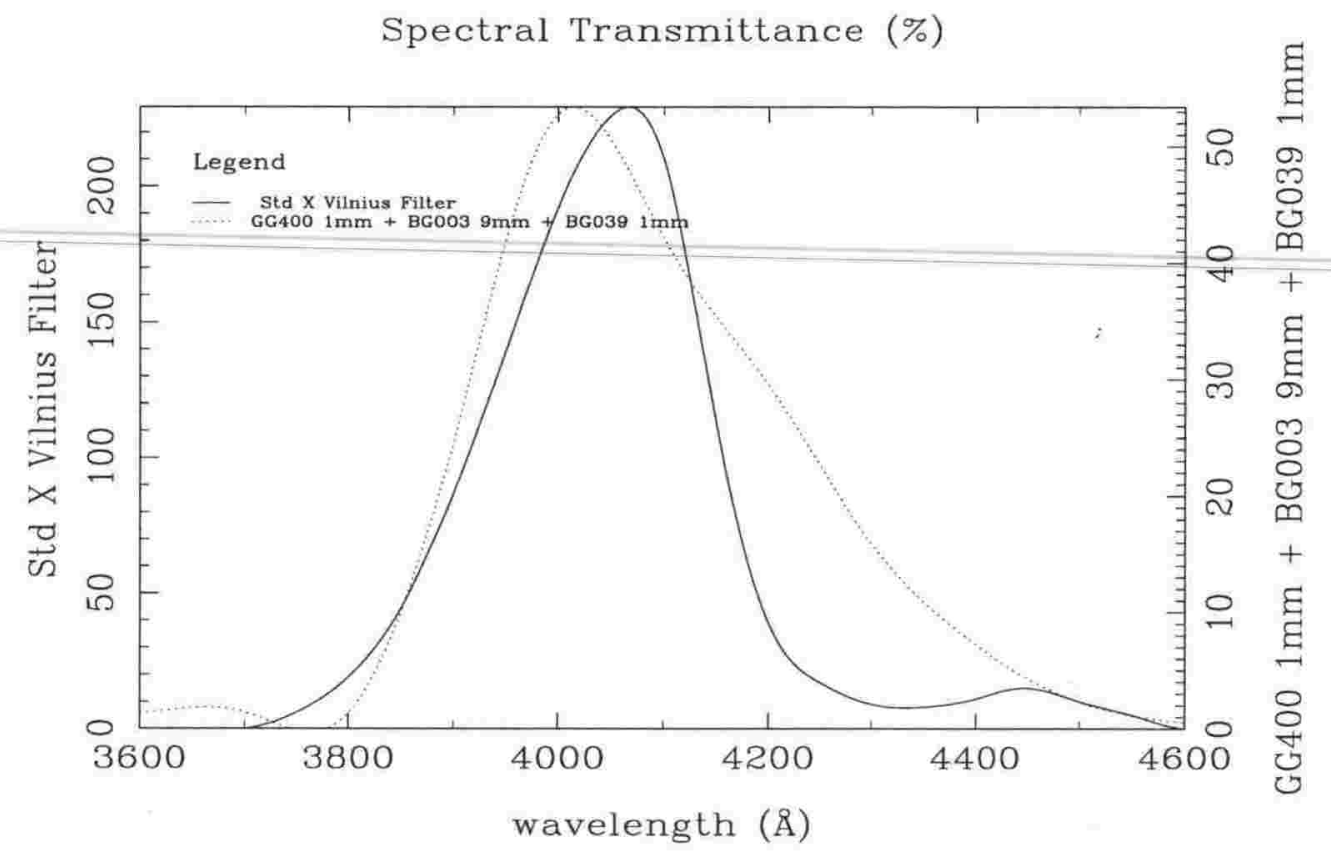

Figure I.2 continued.

$\sim 3650 \AA$ ) arises from changing the thickness of the GG400 glass from $3 \mathrm{~mm}$ (as given in the catalog) to $1 \mathrm{~mm}$, and is probably an artifact of the thickness scaling formula (equation I.1) and measuring uncertainties of the small transmittance in the cut-on of the glass's response. If it is a real leak, then it can be removed by adding $1 \mathrm{~mm}$ of GG375 glass. The BG39 glass is used to remove a red leak at $\sim 8000 \AA$, and can be increased in thickness if any red leak remains. Both BG3 and BG39 are sensitive to staining and either GG375 or WG280 can be used to protect them.

The Schott Y filter is a better match to the standard filter than the local filter, with a large peak transmittance but a slightly longer effective wavelength. Again, the BG3 and BG39 glasses need protection.

The large difference on the red side between the Schott $\mathrm{Z}$ filter and the standard filter occurs as there are no short-pass glasses with a steep enough cut-off at the right wavelength. A more symmetric combination with nearly the right effective wavelength (GG495(1mm) + BG18 $(9 \mathrm{~mm})$ ) was found but the blue side of the Schott filter would include an absorption line at $\sim 4850 \AA$ (for normal, early stars) so this combination was rejected. The effect of the larger red side of the Schott filter can be estimated as (for normal stars at least) a stellar spectra is approximately straight between $5000-5800 \AA$, with a slope proportional to Y-V. The BG18 glass requires protection against staining - use either the Schott protective coating or sandwich the BG18 glass between two GG495 glasses ( $3 \mathrm{~mm}$ thick each). The usual WG280 glass should not be used as the Schott $\mathrm{Z}$ filter already has a total thickness of $15 \mathrm{~mm}$. 


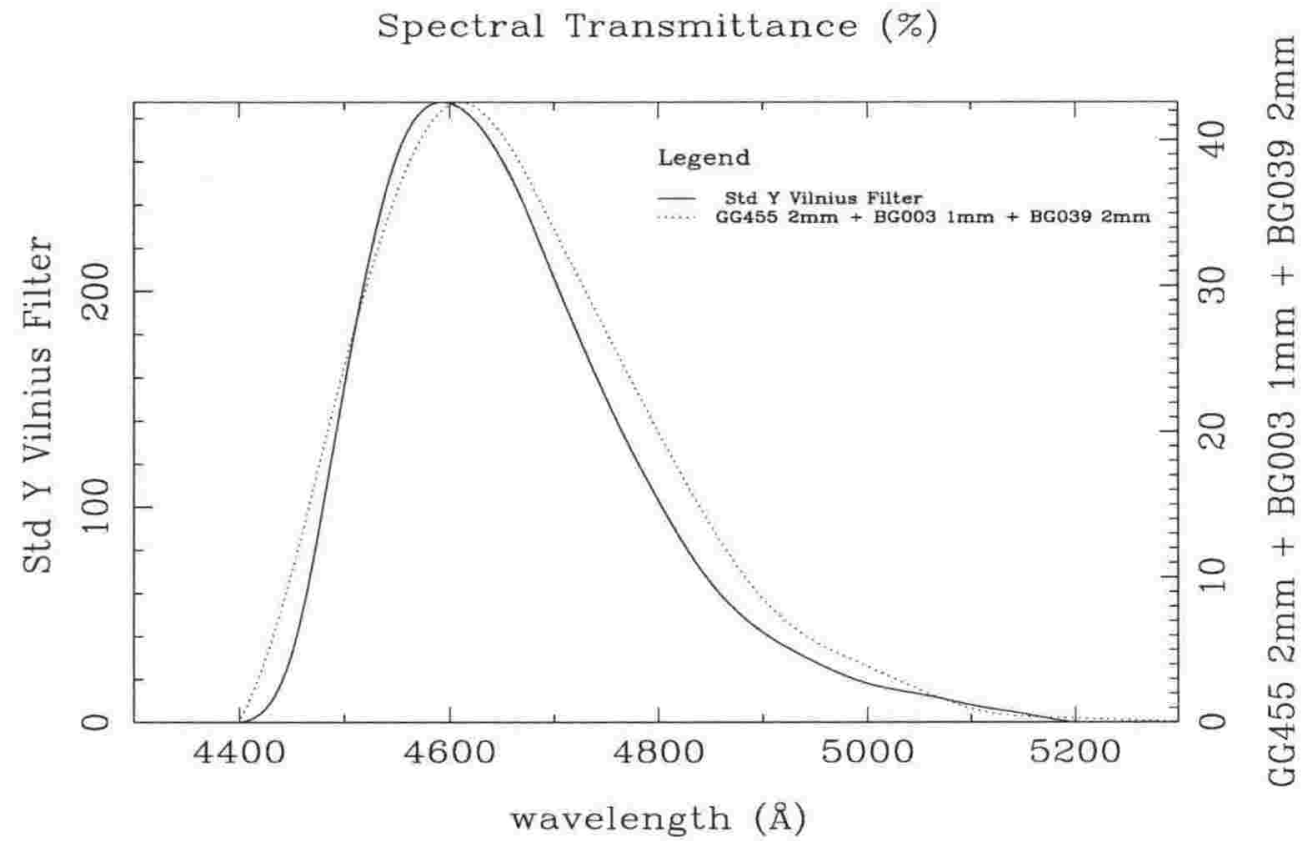

Figure I.2 continued.

Spectral Transmittance (\%)

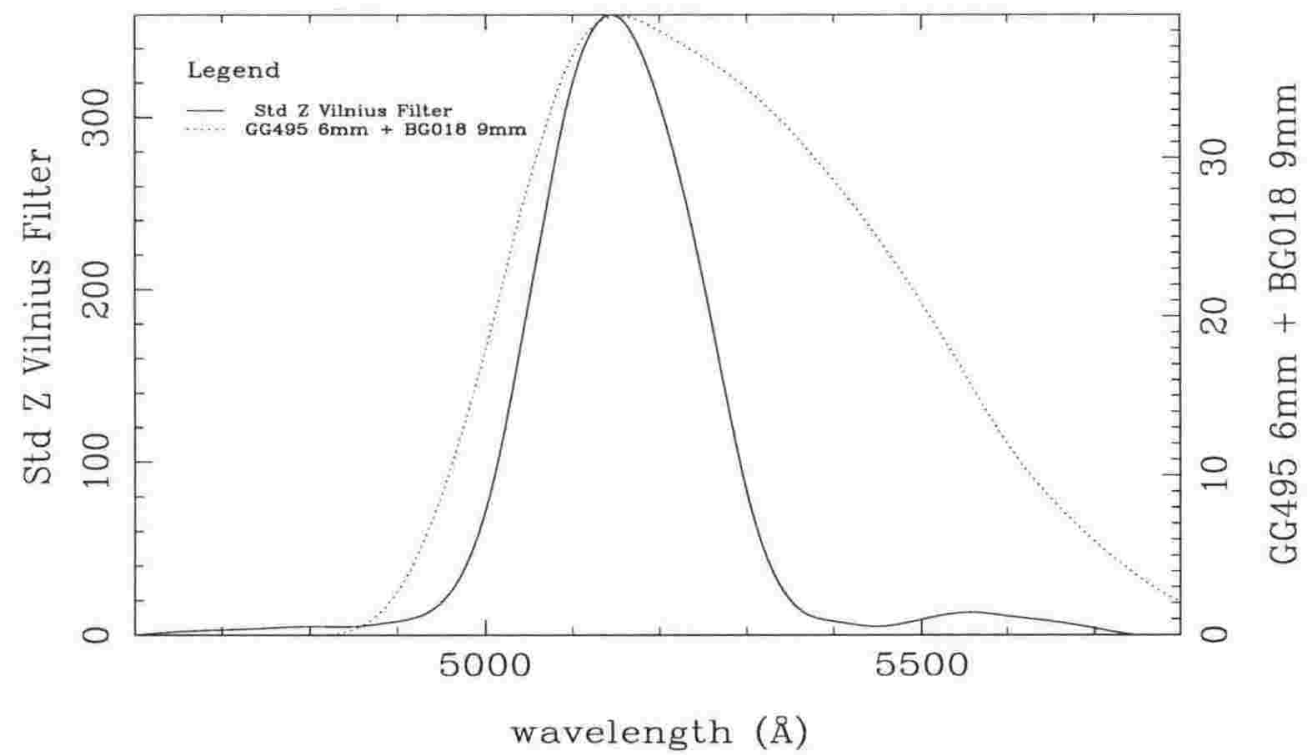

Figure I.2 continued. 
Spectral Transmittance (\%)

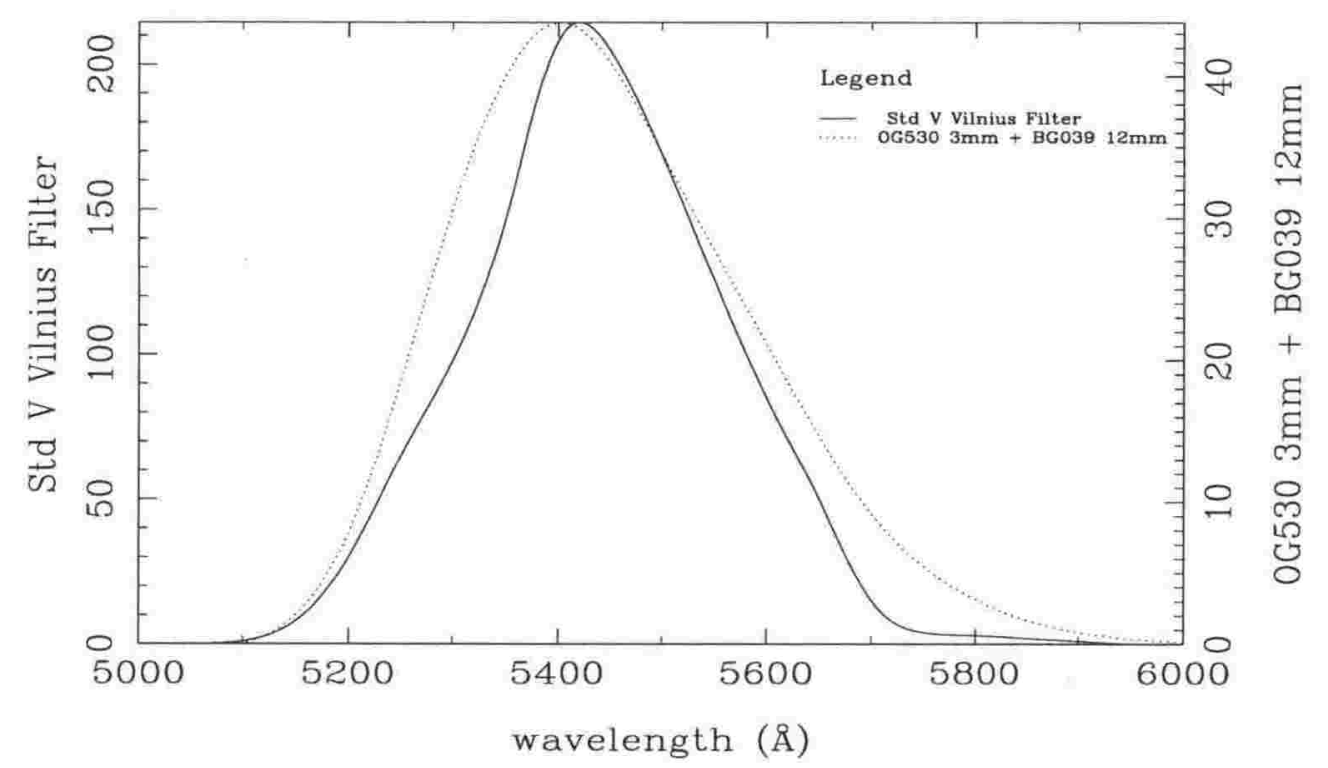

Figure I.2 continued.

The shape of the standard V filter is difficult to match with Schott glasses. However, for normal stars, the stellar spectra between $5000-6000 \AA$ is approximately straight (whose slope can be estimated from $\mathrm{Y}-\mathrm{V}$ ), with larger absorption features in late stars. Fortunately the two largest absorption features occur at the blue and red tails of the filters and so are not expected to cause major transformation errors. The BG39 glass should be sandwiched between two OG530 glasses (each $1.5 \mathrm{~mm}$ thick) for stain protection - the WG280 glass can not be used as the Schott V filter already has a total thickness of $15 \mathrm{~mm}$. The more stain resistant class of OG530 glass should be used. Either the $\mathrm{Z}$ or V filter would define the optical depth to which the other filters should be padded.

As the standard $\mathrm{S}$ filter is an interference filter with a narrow passband, it was not possible to duplicate it accurately using glass filters. The Schott filter and local filter show similar angles $(\phi)$ from the standard filters, however for the local (interference) filter this is due to its passband being shifted to a longer wavelength. Stellar spectra on the red side of the Schott filter imply that late stars will not transform well (using Y-V - another filter centred at 6700-6800 should provide the required information).

As only four of the Vilnius filters may be matched by Schott filters to an acceptable accuracy $\left(\phi<10^{\circ}\right)$, it was concluded that the filters provided by the Vilnius Observatory will, in general, give better transformations to the standard system. However, adding extra filters at wavelengths between the standard filters (to give Young's critical sampling at $\frac{1}{2}$ FWHM spacing (Young 1974, Young 1988, Young 1992a, Young 1992b)) may give better transformations in combination with 
Spectral Transmittance (\%)

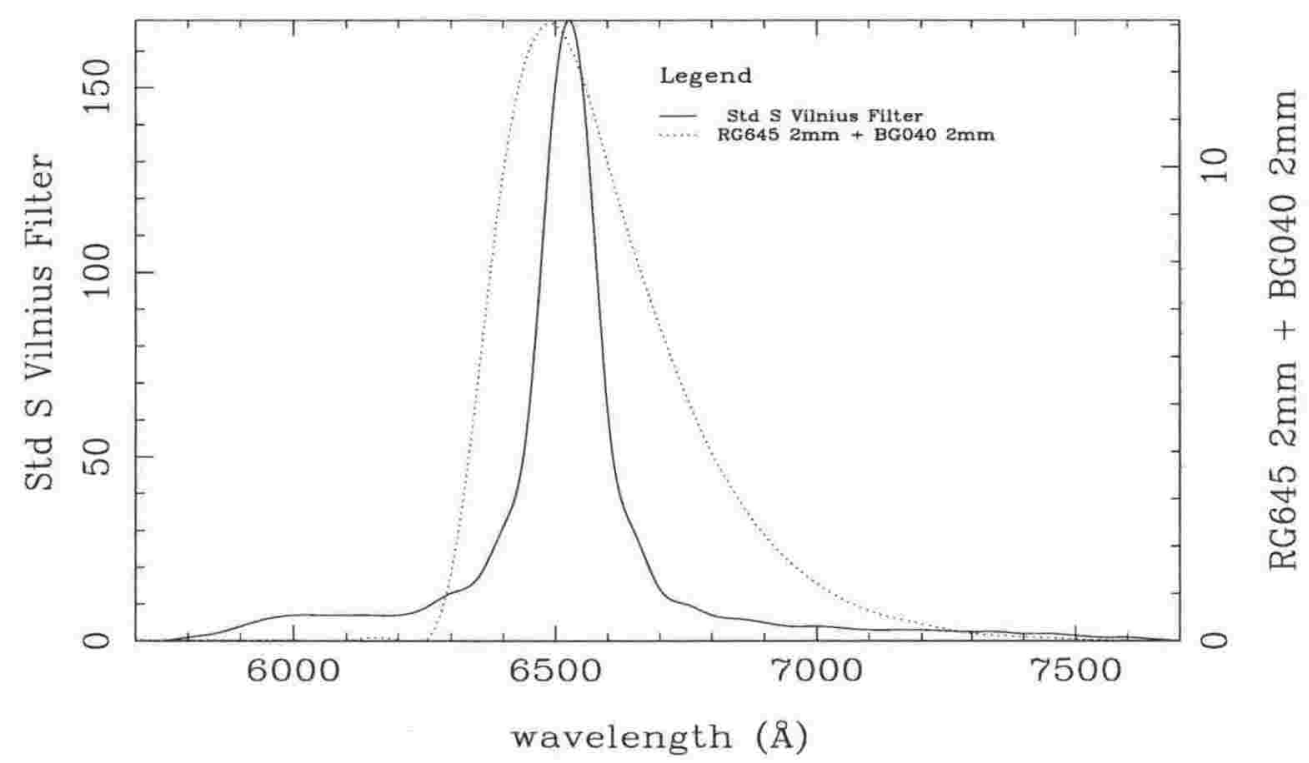

Figure I.2 continued.

Table I.2: Filters between the wavelengths of the Vilnius Filter System from Schott glasses.

\begin{tabular}{|c|c|c|c|}
\hline Filter & Schott glass combination & $\begin{array}{c}R_{\text {peak }} \\
(\%)\end{array}$ & $\begin{array}{c}\lambda_{0} \\
(\AA)\end{array}$ \\
\hline $\mathrm{U}_{\text {short }}$ & WG320 $(2 \mathrm{~mm})+\mathrm{UG} 11(7 \mathrm{~mm})$ & 49 & 3420 \\
\hline $\mathrm{U}_{\text {long }}\left(=\mathrm{P}_{\text {short }}\right)$ & WG345 $(2 \mathrm{~mm})+\mathrm{UG} 11(5 \mathrm{~mm})+\mathrm{BG} 39(1 \mathrm{~mm})$ & 36 & 3570 \\
\hline $\mathrm{P}_{\text {long }}\left(=\mathrm{X}_{\text {short }}\right)$ & $\mathrm{GG} 375(3 \mathrm{~mm})+\mathrm{UG} 1(1 \mathrm{~mm})+\mathrm{BG} 39(1 \mathrm{~mm})$ & 43 & 3840 \\
\hline $\mathrm{X}_{\text {long }}\left(=\mathrm{Y}_{\text {short }}\right)$ & $\mathrm{GG} 400 \dagger(1 \mathrm{~mm})+\mathrm{GG} 420(1 \mathrm{~mm})+\mathrm{BG} 3(3 \mathrm{~mm})+\mathrm{BG} 39(2 \mathrm{~mm})$ & 52 & 4340 \\
\hline Ylong & GG455† $(1 \mathrm{~mm})+\mathrm{GG} 475(1 \mathrm{~mm})+\mathrm{BG} 3(1 \mathrm{~mm})+\mathrm{BG} 39(2 \mathrm{~mm})$ & 24 & 4760 \\
\hline $\mathrm{Z}_{\text {long }}\left(=\mathrm{V}_{\text {short }}\right)$ & OG515(3mm $)+$ BG $18(9 \mathrm{~mm})$ & 31 & 5370 \\
\hline $\mathrm{V}_{\text {long }}$ & $\mathrm{OG} 550(3 \mathrm{~mm})+\mathrm{BG} 18(4 \mathrm{~mm})+\mathrm{BG} 39(4 \mathrm{~mm})$ & 20 & 5610 \\
\hline $\mathrm{S}_{\text {short }}$ & $\mathrm{RG} 610 \dagger(1 \mathrm{~mm})+\mathrm{RG} 630(1 \mathrm{~mm})+\mathrm{BG} 40(2 \mathrm{~mm})$ & 24 & 6420 \\
\hline
\end{tabular}

either the Schott filters or the local filters. To investigate this, the 'intermediate wavelength' Schott filters were calculated from the Schott catalog response curves, with the results given in Table I.2 and in Figure I.3. The daggers $(\dagger)$ in the table mean the indicated glass was added to the combination to remove the 'blue' leak discussed earlier (arising from thinning long-pass glasses). Two filters $\left(Z_{\text {short }}, S_{\text {long }}\right)$ are missing from the table as they could not be made using Schott glasses.

One way of investigating the usefulness of these extra filters is to add the corresponding short and long wavelength filters in a linear combination with the local filter (or the Schott glass version) to see if this improves the match to the standard filter. Using the angle $\phi$, the linear combinations were found to be an improvement in approximately half the cases. This is a surprisingly good result, considering the extra filters were chosen to achieve $\frac{1}{2}$ FWHM spacing 


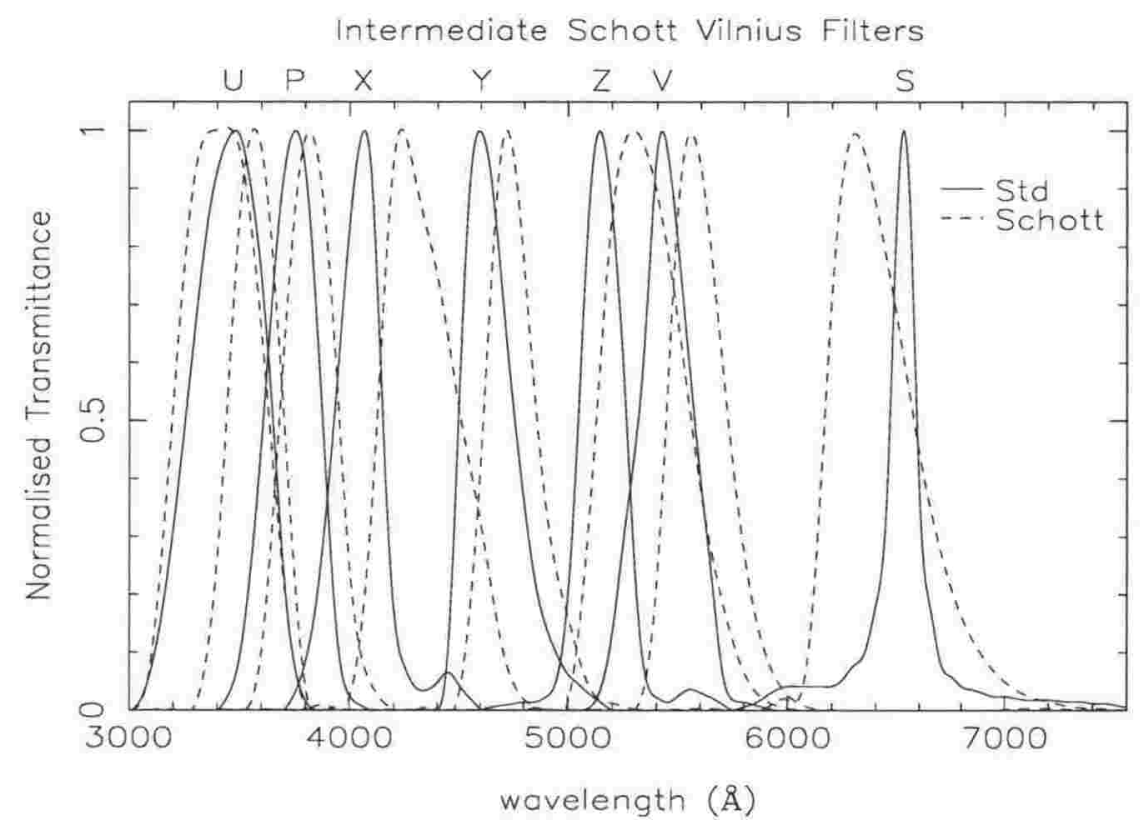

Figure I.3: Comparison of intermediate Schott filters with the Vilnius System, normalised and spline fitted.

rather than give better matches to the standard filters via linear combinations.

Another way to evaluate the usefulness of the extra filters is to fit the transformations, in this case using synthesized magnitudes derived by convolving stellar spectra (Gunn \& Stryker 1983) with the passbands. Even using transformations to only first order, for example,

$$
\mathrm{V}-\mathrm{V}_{0}=a_{0}+a_{1}\left(\mathrm{~V}_{\text {short }}-\mathrm{V}\right)+a_{2}\left(\mathrm{~V}-\mathrm{V}_{\text {long }}\right)
$$

gives results with a precision better (or at least the same) as the usual transformations, for example,

$$
\mathrm{V}-\mathrm{V}_{0}=a_{0}+a_{1}(\mathrm{Y}-\mathrm{V})_{0}
$$

and should give more accurate transformations for stars with unusual spectra.

The most important test is to make some actual observations of Vilnius standard stars using the existing local filters and the 'intermediate' filters. As up to ten extra filters would be required to achieve $\frac{1}{2}$ FWHM sampling for the Vilnius system, it is recommended that only two extra filters be made for the initial trial, for example, $V_{\text {short }}$ and $V_{\text {long }}$. It is expected that a significant improvement could be made using only four extra filters; $Y_{\text {short }}, Y_{\text {long }}, V_{\text {short }}$ and $\mathrm{V}_{\text {long }}$, to give more accurate transformations to $(\mathrm{Y}-\mathrm{V})_{0}$, which is then used to transform all the other magnitudes and colours (see equation 2.29). 


\section{Appendix $\mathbf{J}$}

\section{Tables and Appendices from Straižys book.}

Some of the tables from the literature (mainly from Straižys (1992)) have been reproduced in this appendix for the reader's convenience.

Table J.1: Ratio of extinction (in the UBV system for a B0 V star) to colour excess (in the Vilnius system), $\mathrm{R}=\mathrm{A}_{\mathrm{V}}(\mathrm{B} 0 \mathrm{~V}) / E_{\mathrm{Y}-\mathrm{V}}$. Taken from Straižys (1992, Table 74).

\begin{tabular}{|cc||cc|}
\hline Sp & $\mathrm{R}$ & $\mathrm{Sp}$ & $\mathrm{R}$ \\
\hline B0-B5 V-III & 4.48 & G5-K5 III & 4.50 \\
A0-A5 V-III & 4.46 & M0-M2 III & 4.52 \\
F0-G5 V-III & 4.48 & M3 III & 4.54 \\
K0 V & 4.50 & M4-M5 III & 4.55 \\
K3 V & 4.48 & B0-F5 I & 4.48 \\
K5-M0 V & 4.44 & F8-G0 I & 4.50 \\
M2-M4 V & 4.48 & G2-G8 I & 4.52 \\
M5 V & 4.50 & K2-K3 I & 4.54 \\
& & M2 I & 4.60 \\
\hline
\end{tabular}


Table J.2: Vilnius colour excess ratios for the normal interstellar extinction law. Taken from Straižys (1992, Table 64).

\begin{tabular}{|llllll|}
\hline & & & & & \\
Sp & $\frac{E_{\mathrm{U}-\mathrm{Y}}}{E_{\mathrm{Y}-\mathrm{V}}}$ & $\frac{E_{\mathrm{P}-\mathrm{Y}}}{E_{\mathrm{Y}-\mathrm{V}}}$ & $\frac{E_{\mathrm{X}-\mathrm{Y}}}{E_{\mathrm{Y}-\mathrm{V}}}$ & $\frac{E_{\mathrm{Y}-\mathrm{Z}}}{E_{\mathrm{Y}-\mathrm{V}}}$ & $\frac{E_{\mathrm{Y}-\mathrm{S}}}{E_{\mathrm{Y}-\mathrm{V}}}$ \\
\hline B0 V-III & 1.62 & 1.14 & 0.76 & 0.64 & 1.84 \\
B5 V-III & 1.60 & 1.12 & 0.75 & 0.64 & 1.84 \\
A0 V-III & 1.58 & 1.09 & 0.73 & 0.64 & 1.84 \\
A5 V-III & 1.58 & 1.09 & 0.73 & 0.64 & 1.84 \\
F0 V-III & 1.58 & 1.11 & 0.74 & 0.64 & 1.84 \\
F5 V-III & 1.58 & 1.12 & 0.74 & 0.64 & 1.84 \\
G0 V-III & 1.58 & 1.14 & 0.73 & 0.64 & 1.84 \\
G5 V & 1.58 & 1.14 & 0.73 & 0.64 & 1.84 \\
K0 V & 1.58 & 1.15 & 0.72 & 0.64 & 1.84 \\
K5 V & 1.56 & 1.13 & 0.69 & 0.65 & 1.83 \\
M0 V & 1.55 & 1.12 & 0.69 & 0.65 & 1.83 \\
M2 V & 1.54 & 1.14 & 0.70 & 0.66 & 1.85 \\
M5 V & 1.55 & 1.15 & 0.72 & 0.66 & 1.87 \\
G5 III & 1.59 & 1.15 & 0.72 & 0.64 & 1.85 \\
K0 III & 1.57 & 1.15 & 0.71 & 0.64 & 1.85 \\
K5 III & 1.57 & 1.15 & 0.69 & 0.64 & 1.85 \\
M0 III & 1.56 & 1.15 & 0.68 & 0.65 & 1.85 \\
M2 III & 1.55 & 1.15 & 0.68 & 0.65 & 1.86 \\
M4 III & 1.53 & 1.15 & 0.71 & 0.65 & 1.89 \\
M6 III & 1.51 & 1.15 & 0.74 & 0.64 & 1.90 \\
B0 I & 1.62 & 1.14 & 0.75 & 0.64 & 1.84 \\
B5 I & 1.60 & 1.12 & 0.75 & 0.64 & 1.84 \\
A0 I & 1.59 & 1.11 & 0.75 & 0.64 & 1.84 \\
A5 I & 1.57 & 1.10 & 0.75 & 0.64 & 1.84 \\
F0 I & 1.57 & 1.08 & 0.74 & 0.64 & 1.84 \\
F5 I & 1.57 & 1.10 & 0.74 & 0.64 & 1.84 \\
G0 I & 1.57 & 1.14 & 0.73 & 0.64 & 1.84 \\
G5 I & 1.57 & 1.16 & 0.72 & 0.64 & 1.84 \\
K0 I & 1.57 & 1.17 & 0.71 & 0.64 & 1.85 \\
K3 I & 1.56 & 1.18 & 0.71 & 0.64 & 1.85 \\
M2 I & 1.57 & 1.17 & 0.71 & 0.65 & 1.88 \\
\hline & & & & & \\
\hline
\end{tabular}


Table J.3: Intrinsic Vilnius colours for the luminosity V and the ZAMS stars. The O6.5-B2 stars are taken from Sūdžius \& Bobinas (1994), with the remainder from Straižys (1992, Table 66).

\begin{tabular}{|c|c|c|c|c|c|c|c|c|}
\hline \multirow[t]{2}{*}{ Sp } & \multicolumn{2}{|c|}{ U-P } & \multicolumn{2}{|c|}{$\mathrm{P}-\mathrm{X}$} & $X-Y$ & $\mathrm{Y}-\mathrm{Z}$ & $\mathrm{Z}-\mathrm{V}$ & V-S \\
\hline & ZAMS & V & ZAMS & V & V & V & V & V \\
\hline $\mathrm{O} 6.5$ & & 0.00 & & 0.00 & -0.02 & -0.01 & 0.00 & 0.00 \\
\hline O7 & & 0.00 & & 0.00 & -0.02 & -0.01 & $0 \cdot 00$ & 0.00 \\
\hline O 7.5 & & 0.00 & & $0 \cdot 00$ & -0.02 & 0.00 & $0 \cdot 00$ & 0.00 \\
\hline O8 & & $0 \cdot 00$ & & 0.00 & -0.02 & $0 \cdot 00$ & 0.00 & 0.00 \\
\hline O8.5 & & 0.00 & & 0.01 & -0.01 & 0.00 & $0 \cdot 00$ & 0.00 \\
\hline O9 & & 0.00 & & 0.01 & $-0 \cdot 01$ & 0.01 & 0.00 & 0.00 \\
\hline O9.5 & & 0.00 & & 0.02 & -0.01 & 0.01 & 0.00 & 0.01 \\
\hline B0 & & 0.01 & & 0.05 & 0.00 & 0.01 & 0.00 & 0.01 \\
\hline B0.5 & & 0.04 & & 0.07 & 0.02 & 0.02 & $0 \cdot 00$ & 0.01 \\
\hline B1 & & 0.06 & & $0 \cdot 10$ & 0.04 & 0.02 & 0.00 & 0.02 \\
\hline B1.5 & & 0.09 & & $0 \cdot 15$ & 0.05 & 0.03 & 0.01 & 0.02 \\
\hline B2 & & 0.13 & & 0.22 & 0.06 & 0.03 & 0.02 & 0.03 \\
\hline B0 & & 0.01 & & 0.04 & 0.02 & 0.00 & 0.00 & 0.00 \\
\hline B1 & 0.07 & 0.06 & $0 \cdot 13$ & 0.13 & 0.04 & 0.01 & 0.01 & 0.01 \\
\hline B2 & $\cdot 11$ & $0 \cdot 15$ & & & 0.06 & 0.02 & 0.02 & 0.03 \\
\hline B3 & & $0 \cdot 20$ & & & 0.08 & 0.04 & 0.03 & 0.05 \\
\hline B5 & & $0.2 ?$ & & & $0 \cdot 14$ & 0.06 & 0.04 & 0.08 \\
\hline B6 & 27 & $0 \cdot 34$ & & & $0 \cdot 15$ & 0.06 & 0.04 & 0.09 \\
\hline B7 & 0.34 & 0.43 & 0.54 & 0.52 & 0.17 & 0.06 & 0.04 & $0 \cdot 10$ \\
\hline B8 & 0.43 & $0 \cdot 50$ & 0.64 & 0.58 & $0 \cdot 19$ & 0.07 & 0.05 & 0.11 \\
\hline B9 & & 0.57 & 71 & 0.67 & 0.23 & 0.08 & 0.06 & 0.12 \\
\hline A0 & & 0.64 & & 0.7 & 0.28 & $0 \cdot 10$ & 0.07 & 0.14 \\
\hline $\mathrm{A} 1$ & 55 & $0 \cdot 6$ & & & $0 \cdot 32$ & $0 \cdot 11$ & .08 & $0 \cdot 15$ \\
\hline A2 & & $0 \cdot t$ & & & 0 . & $0 \cdot 12$ & 0.08 & $0 \cdot 17$ \\
\hline A3 & 57 & 0.68 & & 0.8 & 0.39 & $0 \cdot 14$ & 0.08 & $0 \cdot 19$ \\
\hline A5 & 0.57 & $0 \cdot 66$ & 0.78 & $0.8:$ & $0 \cdot 44$ & $0 \cdot 16$ & $0 \cdot 10$ & 0.22 \\
\hline A7 & 0.56 & 0.62 & 0.74 & 0.77 & $0 \cdot 48$ & $0 \cdot 17$ & $0 \cdot 10$ & 0.27 \\
\hline F0 & 0.54 & 0.55 & 0.65 & 0.68 & 0.53 & $0 \cdot 21$ & $0 \cdot 13$ & $0 \cdot 34$ \\
\hline F2 & 0. & 0.52 & 61 & 0.62 & 0.57 & 0.25 & 0.15 & 0.40 \\
\hline F5 & 50 & 0.49 & $0 \cdot 60$ & 0.5 & 0.64 & $0 \cdot 29$ & $0 \cdot 17$ & 0.45 \\
\hline F8 & 48 & 0.47 & 62 & 0 & 0.7 & $0 \cdot 32$ & $0 \cdot 19$ & 0.50 \\
\hline G0 & & & & & 77 & $0 \cdot 33$ & $0 \cdot 20$ & $0 \cdot 54$ \\
\hline G2 & 0 . & & & & & $0 \cdot 34$ & 0.22 & 0.56 \\
\hline G5 & 0.46 & 0 . & & & & $0 \cdot 34$ & $0 \cdot 23$ & 0.58 \\
\hline G8 & 0.46 & 0.4 & & & 96 & $0 \cdot 34$ & 0.27 & 0.62 \\
\hline K0 & 0.46 & $0 \cdot 45$ & & & 1.06 & $0 \cdot 34$ & $0 \cdot 30$ & $0 \cdot 65$ \\
\hline K1 & & 0.44 & 0.91 & & 1.12 & 0.33 & $0 \cdot 32$ & 0.68 \\
\hline K2 & & 0.43 & 0.96 & 0.91 & 1.19 & 0.32 & $0 \cdot 36$ & 0.72 \\
\hline K3 & & 0.42 & 1.03 & 0.97 & $1 \cdot 30$ & $0 \cdot 30$ & $0 \cdot 40$ & 0.77 \\
\hline K4 & & 0.42 & $1 \cdot 12$ & 1.05 & 1.47 & 0.27 & 0.47 & 0.83 \\
\hline K5 & & 0.45 & & $1 \cdot 10$ & 1.61 & 0.25 & 0.53 & 0.91 \\
\hline K7 & & 0.55 & & 1.12 & 1.77 & 0.31 & $0 \cdot 61$ & 1.08 \\
\hline M0 & & 0.56 & & 1.12 & 1.74 & 0.31 & $0 \cdot 61$ & 1.10 \\
\hline M2 & & 0.72 & & 1.06 & 1.68 & 0.50 & 0.62 & 1.23 \\
\hline
\end{tabular}


Table J.4: Intrinsic Vilnius colours for the luminosity IV stars. Taken from Straižys (1992, Table 67).

\begin{tabular}{|lllllll|}
\hline Sp & U-P & P-X & X-Y & Y-Z & Z-V & V-S \\
\hline B0 & & $0.02:$ & 0.00 & 0.00 & 0.00 & 0.00 \\
B1 & & $0 \cdot 11:$ & 0.03 & 0.01 & 0.01 & 0.03 \\
B2 & $0.15:$ & $0.20:$ & 0.07 & 0.02 & 0.02 & 0.04 \\
B3 & $0.21:$ & $0.29:$ & 0.10 & 0.04 & 0.02 & 0.06 \\
B5 & 0.32 & $0.41:$ & 0.15 & 0.06 & 0.03 & 0.08 \\
B6 & $0.37:$ & $0.47:$ & 0.17 & 0.07 & 0.04 & 0.10 \\
B7 & $0.44:$ & $0.52:$ & 0.19 & 0.07 & 0.05 & 0.11 \\
B8 & $0.50:$ & $0.58:$ & 0.21 & 0.08 & 0.05 & 0.12 \\
B9 & $0.59:$ & $0.67:$ & 0.24 & 0.09 & 0.06 & 0.13 \\
A0 & $0.65:$ & $0.78:$ & 0.27 & 0.10 & 0.06 & 0.14 \\
A1 & $0.68:$ & $0.82:$ & 0.32 & 0.11 & 0.06 & 0.15 \\
A2 & $0.68:$ & $0.84:$ & 0.36 & 0.12 & 0.07 & 0.17 \\
A3 & $0.68:$ & $0.85:$ & 0.39 & 0.14 & 0.08 & 0.20 \\
A5 & $0.67:$ & 0.82 & 0.43 & 0.17 & 0.09 & 0.26 \\
A7 & $0.65:$ & 0.77 & 0.47 & 0.19 & 0.10 & 0.32 \\
F0 & $0.62:$ & 0.70 & 0.54 & 0.22 & 0.11 & 0.35 \\
F2 & $0.57:$ & 0.65 & 0.58 & 0.25 & 0.15 & 0.39 \\
F5 & $0.53:$ & 0.59 & 0.63 & 0.29 & 0.17 & 0.43 \\
F8 & $0.50:$ & 0.61 & 0.72 & 0.31 & 0.19 & 0.48 \\
G0 & $0.48:$ & 0.67 & 0.79 & 0.32 & 0.21 & 0.52 \\
G2 & 0.47 & 0.71 & 0.86 & 0.33 & 0.22 & 0.55 \\
G5 & 0.47 & 0.75 & 0.93 & 0.35 & 0.24 & 0.59 \\
G8 & 0.49 & 0.81 & 1.05 & 0.38 & 0.27 & 0.64 \\
K0 & 0.52 & 0.88 & 1.16 & 0.40 & 0.30 & 0.69 \\
K1 & 0.57 & 1.10 & 1.40 & 0.41 & 0.36 & 0.74 \\
\hline & & & & & & \\
\hline
\end{tabular}


Table J.5: Intrinsic Vilnius colours for the luminosity III stars. The O6.5-B2 stars are taken from Sūdžius \& Bobinas (1994), with the remainder from Straižys (1992, Table 68).

\begin{tabular}{|c|c|c|c|c|c|c|}
\hline Sp & U-P & $\mathrm{P}-\mathrm{X}$ & $\mathrm{X}-\mathrm{Y}$ & $\mathrm{Y}-\mathrm{Z}$ & $\mathrm{Z}-\mathrm{V}$ & $\mathrm{V}-\mathrm{S}$ \\
\hline $\mathrm{O} 7$ & $-0 \cdot 01$ & +0.00 & $+0 \cdot 00$ & +0.00 & $+0 \cdot 00$ & +0.01 \\
\hline $07 \cdot 5$ & +0.00 & $+0 \cdot 00$ & +0.00 & +0.00 & +0.00 & +0.01 \\
\hline $\mathrm{O} 8$ & +0.00 & +0.01 & +0.00 & +0.00 & +0.00 & $+0 \cdot 01$ \\
\hline O8.5 & +0.01 & +0.02 & +0.00 & +0.01 & $+0 \cdot 00$ & $+0 \cdot 01$ \\
\hline O9 & +0.01 & +0.03 & +0.01 & +0.01 & +0.00 & $+0 \cdot 01$ \\
\hline O9.5 & +0.02 & +0.04 & +0.01 & +0.01 & +0.00 & $+0 \cdot 01$ \\
\hline B0 & +0.03 & +0.05 & +0.02 & +0.01 & $+0 \cdot 00$ & $+0 \cdot 01$ \\
\hline B0.5 & +0.04 & $+0 \cdot 06$ & +0.02 & +0.02 & +0.00 & $+0 \cdot 01$ \\
\hline B1 & +0.07 & +0.12 & +0.03 & +0.03 & $+0 \cdot 00$ & $+0 \cdot 01$ \\
\hline B1.5 & $+0 \cdot 10$ & $+0 \cdot 14$ & +0.04 & +0.04 & +0.01 & $+0 \cdot 02$ \\
\hline B2 & $+0 \cdot 12$ & $+0 \cdot 17$ & +0.06 & +0.04 & +0.02 & +0.03 \\
\hline B0 & & +0.00 & +0.00 & $+0 \cdot 00$ & $+0 \cdot 00$ & +0.00 \\
\hline B1 & +0.07 & $+0 \cdot 09$ & +0.03 & +0.01 & +0.01 & +0.02 \\
\hline B2 & $+0 \cdot 15$ & +0.18 & +0.07 & +0.02 & +0.03 & $+0 \cdot 05$ \\
\hline B3 & +0.21 & +0.27 & +0.08 & +0.03 & +0.04 & +0.07 \\
\hline B5 & $+0 \cdot 35$ & +0.45 & $+0 \cdot 14$ & +0.05 & +0.04 & +0.09 \\
\hline B6 & $+0 \cdot 40$ & +0.47 & $+0 \cdot 16$ & +0.06 & $+0 \cdot 04$ & $+0 \cdot 11$ \\
\hline B7 & $+0.44:$ & +0.50 & +0.18 & +0.07 & $+0 \cdot 05$ & $+0 \cdot 12$ \\
\hline B8 & $+0.50=$ & +0.54 & +0.20 & +0.08 & $+0 \cdot 05$ & $+0 \cdot 13$ \\
\hline B9 & $+0 \cdot 60:$ & $+0 \cdot 64$ & +0.23 & +0.09 & $+0 \cdot 05$ & $+0 \cdot 14$ \\
\hline A0 & $+0.67:$ & +0.75 & +0.26 & $+0 \cdot 10$ & +0.06 & $+0 \cdot 15$ \\
\hline A1 & $+0.70:$ & +0.82 & +0.30 & $+0 \cdot 11$ & $+0 \cdot 06$ & $+0 \cdot 16$ \\
\hline A2 & $+0.71:$ & +0.84 & +0.34 & $+0 \cdot 12$ & +0.07 & +0.18 \\
\hline A3 & $+0.71:$ & +0.85 & +0.35 & $+0 \cdot 13$ & +0.08 & +0.20 \\
\hline A5 & $+0 \cdot 71:$ & +0.82 & +0.43 & $+0 \cdot 15$ & +0.09 & +0.22 \\
\hline A7 & $+0.71:$ & +0.81 & +0.48 & $+0.19:$ & $+0 \cdot 10$ & $+0 \cdot 26$ \\
\hline F0 & $+0.71:$ & +0.77 & +0.54 & $+0 \cdot 23:$ & +0.12 & $+0 \cdot 37$ \\
\hline $\mathrm{F} 2$ & $+0 \cdot 70:$ & +0.73 & +0.59 & $+0 \cdot 25:$ & +0.15 & $+0 \cdot 41$ \\
\hline F5 & $+0.66:$ & +0.68 & +0.65 & $+0.28:$ & $+0 \cdot 17$ & $+0 \cdot 47$ \\
\hline F8 & $+0.61:$ & +0.65 & +0.73 & +0.32 & $+0 \cdot 20$ & +0.53 \\
\hline G0 & +0.58 & +0.65 & +0.86 & +0.35 & +0.22 & +0.58 \\
\hline G2 & +0.54 & +0.69 & +0.95 & +0.38 & +0.23 & $+0 \cdot 61$ \\
\hline G5 & +0.53 & +0.82 & +1.08 & +0.42 & +0.25 & +0.66 \\
\hline G8 & +0.55 & +0.92 & $+1 \cdot 19$ & $+0 \cdot 45$ & +0.27 & +0.69 \\
\hline K0 & +0.57 & +1.01 & $+1 \cdot 30$ & +0.46 & +0.31 & +0.73 \\
\hline K1 & +0.61 & $+1 \cdot 10$ & $+1 \cdot 40$ & $+0 \cdot 47$ & +0.34 & $+0 \cdot 77$ \\
\hline K2 & +0.63 & $+1 \cdot 18$ & +1.53 & $+0 \cdot 48$ & $+0 \cdot 37$ & +0.81 \\
\hline K3 & +0.65 & +1.27 & $+1 \cdot 70$ & +0.50 & +0.41 & +0.87 \\
\hline K4 & $+0 \cdot 71$ & $+1 \cdot 35$ & +1.90 & +0.52 & +0.47 & +0.95 \\
\hline K5 & +0.79 & $+1 \cdot 38$ & $+2 \cdot 05$ & +0.54 & +0.53 & $+1 \cdot 00$ \\
\hline M0 & $+0.81:$ & +1.42 & $+2 \cdot 10$ & +0.57 & +0.54 & +1.06 \\
\hline M1 & $+0 \cdot 84:$ & $+1 \cdot 43$ & $+2 \cdot 13$ & +0.59 & +0.54 & $+1 \cdot 10$ \\
\hline M2 & $+0 \cdot 87:$ & $+1 \cdot 42$ & +2.08 & $+0 \cdot 62$ & +0.53 & $+1 \cdot 14$ \\
\hline M3 & $+0.93:$ & $+1 \cdot 38$ & $+1.90=$ & $+0 \cdot 70:$ & +0.50 & $+1 \cdot 20$ \\
\hline M4 & $+1 \cdot 00:$ & +1.34 & $+1 \cdot 65:$ & $+0.85:$ & +0.45 & $+1 \cdot 27$ \\
\hline M5 & $+1 \cdot 10:$ & +1.35 & $+1 \cdot 47:$ & $+0.95:$ & +0.40 & $+1 \cdot 36:$ \\
\hline M6 & $+1 \cdot 30=$ & $+1 \cdot 39$ & $+0.96:$ & $+1 \cdot 15:$ & +0.35 & $+1.56:$ \\
\hline
\end{tabular}


Table J.6: Intrinsic Vilnius colours for the luminosity I stars. The O6.5-B2 stars are taken from Südžius \& Bobinas (1994), with the remainder from Straižys (1992, Table 69).

\begin{tabular}{|c|c|c|c|c|c|c|c|c|c|c|}
\hline \multirow[t]{2}{*}{ Sp } & \multicolumn{3}{|c|}{$\mathrm{U}-\mathrm{P}$} & \multicolumn{3}{|c|}{$\mathrm{P}-\mathrm{X}$} & $X-Y$ & $\mathrm{Y}-\mathrm{Z}$ & $\mathrm{Z}-\mathrm{V}$ & V-S \\
\hline & Ia & I & $\mathrm{Ib}$ & Ia & I & Ib & I & I & I & I \\
\hline O7.5 & & +0.00 & & & -0.03 & & -0.01 & +0.02 & +0.00 & +0.03 \\
\hline O8 & & +0.01 & & & -0.03 & & -0.01 & +0.02 & +0.00 & $+0 \cdot 03$ \\
\hline O 8.5 & & +0.01 & & & -0.03 & & $+0 \cdot 00$ & +0.02 & +0.00 & +0.03 \\
\hline O9 & & +0.01 & & & -0.01 & & +0.01 & +0.02 & +0.00 & +0.03 \\
\hline O9.5 & & +0.02 & & & +0.01 & & +0.02 & $+0 \cdot 02$ & +0.00 & +0.03 \\
\hline B0 & & +0.03 & & & +0.02 & & +0.03 & +0.03 & $+0 \cdot 00$ & +0.03 \\
\hline B 0.5 & & +0.05 & & & +0.03 & & +0.04 & +0.04 & +0.01 & +0.05 \\
\hline B1 & & +0.08 & & & +0.06 & & +0.06 & +0.05 & +0.02 & +0.08 \\
\hline B1.5 & & +0.10 & & & +0.08 & & +0.08 & +0.06 & +0.03 & +0.09 \\
\hline B2 & & +0.11 & & & +0.09 & & +0.09 & +0.06 & +0.03 & +0.09 \\
\hline B0 & $+0.03:$ & & & $+0.04:$ & & & +0.06 & +0.04 & +0.02 & +0.06 \\
\hline B1 & $+0.06:$ & & & $+0.06:$ & & & +0.08 & +0.06 & +0.03 & +0.09 \\
\hline B2 & $+0 \cdot 10:$ & & & $+0 \cdot 10$ & & & $+0 \cdot 10$ & +0.08 & +0.04 & +0.11 \\
\hline B3 & $+0 \cdot 17:$ & & & $+0 \cdot 13:$ & & & +0.12 & $+0 \cdot 09$ & +0.05 & +0.13 \\
\hline B5 & $+0 \cdot 26:$ & & & $+0 \cdot 18:$ & & & $+0 \cdot 14$ & $+0 \cdot 10$ & +0.06 & +0.14 \\
\hline B6 & $+0.31:$ & & & $+0.22:$ & & & $+0 \cdot 16$ & $+0 \cdot 11$ & +0.06 & $+0 \cdot 16$ \\
\hline B7 & $+0.35:$ & & & $+0 \cdot 25:$ & & & +0.18 & $+0 \cdot 12$ & +0.06 & +0.17 \\
\hline B8 & $+0 \cdot 40:$ & & & $+0 \cdot 28:$ & & & +0.19 & +0.13 & +0.07 & +0.18 \\
\hline B9 & $+0 \cdot 47:$ & & & $+0.32:$ & & & $+0 \cdot 20$ & $+0 \cdot 13$ & +0.07 & +0.19 \\
\hline A 0 & $+0.56:$ & & & $+0.38:$ & & & +0.20 & $+0 \cdot 13$ & +0.07 & +0.20 \\
\hline A1 & $+0.65:$ & & & $+0.44:$ & & & +0.20 & $+0 \cdot 14$ & +0.08 & +0.22 \\
\hline A2 & $+0.75:$ & & & $+0.50:$ & & & +0.22 & +0.15 & +0.08 & +0.23 \\
\hline A3 & $+1 \cdot 00:$ & & & $+0.56:$ & & & +0.23 & +0.15 & +0.09 & +0.23 \\
\hline A5 & $+1 \cdot 12:$ & & & $+0 \cdot 64:$ & & & +0.26 & +0.16 & +0.09 & +0.23 \\
\hline A7 & $+1 \cdot 14:$ & & & $+0 \cdot 69:$ & & & +0.31 & +0.17 & +0.09 & +0.25 \\
\hline F0 & $+1 \cdot 15:$ & & & $+0.73:$ & & & +0.36 & $+0 \cdot 18$ & $+0 \cdot 10$ & +0.27 \\
\hline F2 & & & +1.07 & & & +0.80 & +0.48 & +0.20 & +0.13 & +0.33 \\
\hline F5 & & & +0.92 & & & +0.74 & +0.64 & $+0 \cdot 23$ & $+0 \cdot 16$ & +0.39 \\
\hline F8 & & & +0.75 & & & +0.74 & +0.84 & +0.32 & +0.19 & +0.50 \\
\hline G0 & & & +0.68 & & & +0.77 & +1.00 & $+0 \cdot 38$ & +0.22 & +0.58 \\
\hline G2 & & & +0.62 & & & +0.82 & $+1 \cdot 15$ & +0.44 & +0.25 & +0.65 \\
\hline G5 & & & +0.63 & & & +0.95 & $+1 \cdot 37$ & +0.49 & +0.29 & +0.70 \\
\hline G8 & & & $+0.66:$ & & & $+1 \cdot 09:$ & $+1.57:$ & +0.53 & +0.33 & +0.77 \\
\hline K0 & & & $+0 \cdot 69:$ & & & $+1 \cdot 20$ & $+1 \cdot 74:$ & +0.57 & +0.39 & +0.84 \\
\hline K1 & & & $+0 \cdot 70:$ & & & $+1 \cdot 24:$ & +1.90 & +0.61 & +0.43 & +0.90 \\
\hline K2 & & & $+0 \cdot 72:$ & & & $+1 \cdot 26=$ & $+2 \cdot 00$ : & +0.64 & +0.47 & +0.96 \\
\hline K3 & & & $+0.74:$ & & & $+1 \cdot 26$; & $+2 \cdot 06:$ & +0.67 & +0.50 & +1.01 \\
\hline K4 & & & $+0 \cdot 77:$ & & & $+1 \cdot 26=$ & +2.08: & +0.67 & +0.50 & +1.02 \\
\hline K5 & & & $+0 \cdot 81:$ & & & $+1 \cdot 26:$ & $+2 \cdot 09:$ & +0.67 & +0.51 & +1.03 \\
\hline M0 & & & $+0.85:$ & & & $+1 \cdot 27:$ & $+2 \cdot 12:$ & +0.68 & +0.51 & +1.02 \\
\hline M1 & & & $+0 \cdot 89:$ & & & $+1 \cdot 30:$ & $+2 \cdot 12$ : & +0.73 & $+0 \cdot 50$ & +1.02 \\
\hline M2 & & & $+0.94:$ & & & $+1 \cdot 35:$ & $+2 \cdot 09:$ & +0.82 & +0.48 & $+1 \cdot 10$ \\
\hline M3 & & & & & & & $+1 \cdot 85$ & +0.93 & +0.44 & +1.22 \\
\hline M4 & & & & & & & $+1.45:$ & $+1.05:$ & +0.37 & +1.35 \\
\hline
\end{tabular}


Table J.7: Intrinsic color indices $(\mathrm{Y}-\mathrm{V})_{0}$ as a function of $\mathrm{Sp}$ and $\mathrm{M}_{\mathrm{V}}$, Taken from Straižys (1992, Table 73).

\begin{tabular}{|c|c|c|c|c|c|c|c|}
\hline $\mathrm{M}_{\mathrm{V}}$ & -7 & -6 & -5 & -4 & -3 & -2 & -1 \\
\hline B1 & 0.08 & 0.08 & 0.06 & 0.03 & & & \\
\hline B2 & $0 \cdot 105$ & 0.11 & $0 \cdot 10$ & 0.08 & 0.045 & 0.03 & \\
\hline B3 & 0.14 & 0.14 & 0.13 & 0.11 & 0.08 & 0.07 & \\
\hline B4 & $0 \cdot 16$ & 0.16 & 0.15 & 0.13 & 0.105 & 0.09 & 0.09 \\
\hline B5 & $0 \cdot 16$ & 0.16 & 0.15 & 0.14 & $0 \cdot 12$ & $0 \cdot 10$ & $0 \cdot 10$ \\
\hline B6 & $0 \cdot 18$ & 0.18 & 0.17 & 0.155 & 0.13 & $0 \cdot 11$ & $0 \cdot 10$ \\
\hline B7 & 0.195 & 0.19 & $0 \cdot 19$ & 0.18 & 0.17 & 0.145 & 0.12 \\
\hline B8 & 0.20 & $0 \cdot 20$ & 0.20 & $0 \cdot 195$ & $0 \cdot 18$ & $0 \cdot 16$ & 0.13 \\
\hline B9 & $0 \cdot 20$ & 0.20 & 0.20 & $0 \cdot 20$ & 0.195 & 0.18 & 0.16 \\
\hline A0 & 0.20 & 0.20 & 0.20 & 0.20 & 0.20 & 0.19 & $0 \cdot 18$ \\
\hline A1 & 0.22 & 0.22 & 0.22 & $0 \cdot 21$ & 0.205 & $0 \cdot 20$ & 0.195 \\
\hline A2 & 0.23 & 0.23 & $0 \cdot 23$ & $0 \cdot 225$ & 0.22 & 0.215 & 0.21 \\
\hline A3 & 0.24 & 0.24 & 0.24 & $0 \cdot 24$ & 0.24 & 0.23 & 0.23 \\
\hline A4 & 0.245 & 0.24 & 0.245 & $0 \cdot 245$ & $0 \cdot 245$ & $0 \cdot 24$ & 0.24 \\
\hline A5 & 0.25 & 0.25 & 0.25 & $0 \cdot 25$ & $0 \cdot 25$ & 0.25 & 0.245 \\
\hline A6 & 0.25 & 0.25 & $0 \cdot 25$ & 0.25 & 0.25 & 0.25 & 0.25 \\
\hline A7 & 0.26 & 0.26 & 0.26 & 0.26 & 0.26 & 0.26 & 0.26 \\
\hline A 8 & 0.26 & 0.26 & 0.26 & 0.26 & 0.26 & 0.26 & 0.27 \\
\hline A9 & 0.27 & 0.27 & $0 \cdot 27$ & 0.27 & 0.27 & 0.27 & 0.28 \\
\hline F0 & 0.28 & 0.28 & 0.28 & $0 \cdot 28$ & 0.28 & 0.28 & 0.295 \\
\hline F1 & & & $0 \cdot 30$ & $0 \cdot 30$ & $0 \cdot 30$ & $0 \cdot 305$ & 0.31 \\
\hline F2 & & & 0.33 & $0 \cdot 33$ & 0.33 & 0.335 & 0.34 \\
\hline F3 & & & 0.35 & $0 \cdot 35$ & $0 \cdot 35$ & 0.35 & 0.36 \\
\hline F4 & & & 0.36 & $0 \cdot 36$ & 0.36 & 0.365 & 0.375 \\
\hline F5 & & & 0.38 & 0.38 & 0.38 & 0.38 & 0.39 \\
\hline $\mathrm{F} 6$ & & & 0.41 & 0.41 & 0.41 & 0.41 & 0.41 \\
\hline F7 & & & 0.45 & 0.45 & 0.45 & 0.45 & 0.45 \\
\hline F8 & & & 0.485 & 0.49 & 0.49 & 0.49 & 0.49 \\
\hline F9 & & & 0.54 & $0 \cdot 54$ & 0.535 & 0.53 & 0.52 \\
\hline G0 & & & $0 \cdot 60$ & 0.59 & 0.585 & 0.57 & 0.55 \\
\hline G1 & & & $0 \cdot 65$ & 0.65 & 0.64 & 0.62 & 0.595 \\
\hline G2 & & & 0.70 & 0.69 & 0.68 & 0.665 & 0.64 \\
\hline G3 & & & $0 \cdot 73$ & 0.725 & 0.71 & 0.69 & 0.66 \\
\hline G4 & & & 0.76 & 0.75 & 0.73 & 0.71 & 0.68 \\
\hline G5 & & & 0.78 & 0.77 & 0.75 & 0.73 & 0.71 \\
\hline G6 & & & 0.80 & $0 \cdot 795$ & 0.78 & 0.75 & 0.73 \\
\hline G7 & & & 0.82 & $0 \cdot 82$ & 0.80 & 0.775 & 0.75 \\
\hline G8 & & & 0.86 & 0.85 & 0.83 & 0.81 & 0.78 \\
\hline G9 & & & 0.895 & 0.885 & 0.86 & 0.835 & 0.805 \\
\hline K0 & & & 0.96 & 0.95 & 0.91 & 0.875 & 0.84 \\
\hline K1 & & & 1.025 & 1.01 & 0.965 & 0.92 & 0.885 \\
\hline K2 & & & 1.11 & 1.095 & 1.04 & 0.99 & 0.945 \\
\hline K3 & & & 1.14 & 1.12 & 1.07 & 1.02 & 0.98 \\
\hline K4 & & & $1 \cdot 16$ & 1.15 & $1 \cdot 12$ & 1.08 & 1.04 \\
\hline K5 & & & 1.18 & 1.17 & 1.15 & 1.13 & $1 \cdot 10$ \\
\hline
\end{tabular}


Table $\mathbf{J} .7$ continued from previous page.

\begin{tabular}{|c|c|c|c|c|c|c|c|c|}
\hline $\mathrm{M}_{\mathrm{V}}$ & 0 & +1 & +2 & +3 & +4 & +5 & +6 & +7 \\
\hline \multicolumn{9}{|l|}{ B1 } \\
\hline \multicolumn{9}{|l|}{ B2 } \\
\hline \multicolumn{9}{|l|}{ B3 } \\
\hline \multicolumn{9}{|l|}{ B4 } \\
\hline \multicolumn{9}{|l|}{ B5 } \\
\hline \multicolumn{9}{|l|}{ B6 } \\
\hline B7 & $0 \cdot 105$ & & & & & & & \\
\hline B8 & 0.12 & & & & & & & \\
\hline B9 & 0.14 & $0 \cdot 14$ & & & & & & \\
\hline $\mathrm{A} 0$ & $0 \cdot 16$ & 0.15 & & & & & & \\
\hline Al & $0 \cdot 19$ & 0.175 & & & & & & \\
\hline A2 & 0.20 & $0 \cdot 19$ & 0.21 & & & & & \\
\hline A3 & $0 \cdot 225$ & 0.205 & 0.225 & & & & & \\
\hline A4 & $0 \cdot 23$ & 0.22 & 0.23 & & & & & \\
\hline A5 & 0.24 & 0.24 & 0.25 & & & & & \\
\hline A6 & 0.25 & $0 \cdot 255$ & $0 \cdot 26$ & 0.28 & & & & \\
\hline A7 & 0.26 & 0.28 & 0.28 & 0.28 & & & & \\
\hline A8 & $0 \cdot 28$ & 0.29 & 0.30 & 0.30 & & & & \\
\hline A9 & 0.30 & 0.32 & 0.33 & 0.33 & & & & \\
\hline F0 & 0.315 & 0.33 & 0.35 & 0.365 & & & & \\
\hline F1 & $0 \cdot 33$ & 0.35 & 0.37 & $0.39:$ & & & & \\
\hline F2 & 0.35 & 0.37 & 0.39 & 0.42 & & & & \\
\hline F3 & 0.37 & 0.39 & 0.41 & 0.43 & & & & \\
\hline F4 & 0.39 & 0.41 & 0.43 & 0.44 & 0.45 & & & \\
\hline F5 & 0.40 & 0.43 & 0.45 & 0.45 & 0.45 & & & \\
\hline F6 & 0.42 & 0.44 & 0.46 & 0.46 & 0.46 & & & \\
\hline F7 & 0.45 & 0.46 & 0.475 & 0.475 & 0.475 & & & \\
\hline F8 & 0.49 & 0.49 & 0.49 & 0.50 & 0.50 & & & \\
\hline F9 & 0.51 & 0.51 & 0.15 & 0.51 & 0.52 & 0.52 & & \\
\hline G0 & 0.535 & 0.52 & 0.51 & 0.52 & 0.53 & 0.55 & & \\
\hline G1 & 0.57 & 0.55 & 0.54 & 0.54 & 0.55 & 0.55 & & \\
\hline G2 & 0.615 & 0.59 & 0.585 & 0.58 & 0.57 & 0.56 & & \\
\hline G3 & 0.635 & 0.62 & $0 \cdot 61$ & 0.595 & 0.58 & 0.56 & & \\
\hline G4 & 0.66 & 0.64 & 0.63 & 0.61 & 0.58 & 0.56 & & \\
\hline G5 & 0.69 & 0.67 & 0.66 & 0.63 & 0.61 & 0.575 & & \\
\hline G6 & 0.71 & 0.69 & 0.67 & 0.64 & 0.615 & 0.59 & & \\
\hline G7 & 0.73 & 0.71 & 0.68 & 0.65 & 0.625 & $0 \cdot 60$ & & \\
\hline G8 & 0.755 & 0.73 & 0.70 & 0.67 & 0.64 & $0 \cdot 61$ & 0.58 & \\
\hline G9 & $0 \cdot 775$ & 0.745 & 0.715 & $0 \cdot 685$ & 0.655 & 0.625 & 0.595 & \\
\hline K0 & $0 \cdot 81$ & 0.775 & 0.74 & 0.715 & 0.68 & 0.65 & 0.62 & \\
\hline K1 & $0 \cdot 85$ & 0.81 & 0.775 & 0.74 & 0.71 & 0.68 & $0 \cdot 65$ & \\
\hline K2 & 0.895 & 0.85 & 0.82 & 0.78 & 0.75 & 0.72 & $0 \cdot 68$ & \\
\hline K3 & 0.935 & $0 \cdot 895$ & 0.86 & 0.83 & 0.80 & 0.77 & 0.74 & $0 \cdot 70$ \\
\hline K4 & 1.01 & 0.97 & 0.93 & 0.89 & 0.85 & 0.815 & 0.78 & 0.74 \\
\hline K5 & 1.08 & 1.04 & 1.00 & 0.96 & 0.92 & 0.88 & 0.84 & 0.80 \\
\hline
\end{tabular}


Table J.8: Calibration of spectral classes MK in absolute magnitude $\mathrm{M}_{\mathrm{V}}$. Taken from Straižys (1992, Appendix 1), originally from Straižys \& Kurilienè (1981).

\begin{tabular}{|c|c|c|c|c|c|c|c|c|}
\hline Sp & ZAMS & V & IV & III & II & $\mathrm{Ib}$ & Iab & Ia \\
\hline O5 & -4.6 & $-5 \cdot 6$ & $-5 \cdot 8$ & $-6 \cdot 0$ & $-6 \cdot 3$ & $-6 \cdot 6$ & $-6 \cdot 9$ & $-7 \cdot 2$ \\
\hline O6 & $-4 \cdot 0$ & $-5 \cdot 4$ & $-5 \cdot 7$ & $-5 \cdot 9$ & $-6 \cdot 3$ & $-6 \cdot 6$ & $-6 \cdot 9$ & -7.2 \\
\hline O7 & $-3 \cdot 9$ & $-5 \cdot 2$ & $-5 \cdot 5$ & $-5 \cdot 8$ & $-6 \cdot 2$ & $-6 \cdot 5$ & $-6 \cdot 8$ & $-7 \cdot 2$ \\
\hline O8 & $-3 \cdot 7$ & $-4 \cdot 9$ & $-5 \cdot 2$ & $-5 \cdot 6$ & $-6 \cdot 1$ & $-6 \cdot 4$ & $-6 \cdot 7$ & $-7 \cdot 2$ \\
\hline O9 & $-3 \cdot 5$ & $-4 \cdot 5$ & -4.9 & $-5 \cdot 3$ & -5.9 & $-6 \cdot 3$ & $-6 \cdot 6$ & $-7 \cdot 2$ \\
\hline B0 & $-3 \cdot 1$ & $-4 \cdot 0$ & $-4 \cdot 4$ & -4.9 & $-5 \cdot 6$ & $-6 \cdot 1$ & $-6 \cdot 5$ & $-7 \cdot 2$ \\
\hline B1 & $-2 \cdot 3$ & $-3 \cdot 3$ & $-3 \cdot 9$ & $-4 \cdot 5$ & $-5 \cdot 2$ & $-5 \cdot 9$ & $-6 \cdot 4$ & $-7 \cdot 2$ \\
\hline B2 & $-1 \cdot 6$ & $-2 \cdot 5$ & $-3 \cdot 1$ & $-3 \cdot 7$ & $-5 \cdot 0$ & $-5 \cdot 9$ & $-6 \cdot 4$ & $-7 \cdot 2$ \\
\hline B3 & $-1 \cdot 0$ & $-1 \cdot 7$ & $-2 \cdot 3$ & -3.0 & -4.8 & $-5 \cdot 9$ & $-6 \cdot 4$ & $-7 \cdot 2$ \\
\hline B5 & -0.1 & -0.8 & $-1 \cdot 2$ & -1.7 & $-4 \cdot 6$ & $-5 \cdot 9$ & $-6 \cdot 4$ & $-7 \cdot 2$ \\
\hline B6 & $+0 \cdot 3$ & -0.5 & -0.9 & $-1 \cdot 3$ & $-4 \cdot 4$ & -5.8 & $-6 \cdot 4$ & $-7 \cdot 2$ \\
\hline B7 & +0.6 & $-0 \cdot 2$ & -0.6 & $-1 \cdot 0$ & $-4 \cdot 2$ & $-5 \cdot 8$ & $-6 \cdot 4$ & $-7 \cdot 2$ \\
\hline B8 & $+1 \cdot 0$ & $+0 \cdot 1$ & -0.3 & -0.7 & $-3 \cdot 9$ & $-5 \cdot 8$ & $-6 \cdot 4$ & $-7 \cdot 2$ \\
\hline B9 & $+1 \cdot 4$ & +0.5 & +0.1 & -0.4 & $-3 \cdot 6$ & $-5 \cdot 7$ & $-6 \cdot 4$ & $-7 \cdot 2$ \\
\hline $\mathrm{A} 0$ & +1.6 & +0.8 & +0.4 & $-0 \cdot 1$ & $-3 \cdot 4$ & $-5 \cdot 5$ & $-6 \cdot 4$ & $-7 \cdot 2$ \\
\hline A1 & $+1 \cdot 7$ & $+1 \cdot 1$ & +0.7 & +0.2 & $-3 \cdot 2$ & $-5 \cdot 3$ & $-6 \cdot 4$ & $-7 \cdot 2$ \\
\hline A2 & +1.8 & $+1 \cdot 3$ & +0.9 & +0.4 & $-3 \cdot 1$ & $-5 \cdot 2$ & $-6 \cdot 4$ & $-7 \cdot 3$ \\
\hline A3 & +1.9 & +1.5 & +1.0 & +0.5 & $-3 \cdot 0$ & $-5 \cdot 1$ & -6.4 & $-7 \cdot 3$ \\
\hline A5 & $+2 \cdot 3$ & +1.9 & $+1 \cdot 4$ & +0.8 & -2.9 & $-5 \cdot 0$ & -6.5 & -7.5 \\
\hline A7 & $+2 \cdot 6$ & $+2 \cdot 3$ & $+1 \cdot 7$ & $+1 \cdot 1$ & $-2 \cdot 8$ & -5.0 & $-6 \cdot 7$ & $-7 \cdot 7$ \\
\hline F0 & $+3 \cdot 0$ & $+2 \cdot 8$ & $+2 \cdot 2$ & +1.5 & $-2 \cdot 7$ & -5.0 & -6.9 & -7.9 \\
\hline $\mathrm{F} 2$ & $+3 \cdot 2$ & $+3 \cdot 1$ & $+2 \cdot 4$ & +1.8 & $-2 \cdot 6$ & -4.9 & -7.0 & $-8 \cdot 0$ \\
\hline F5 & $+3 \cdot 7$ & $+3 \cdot 6$ & $+2 \cdot 6$ & $+2 \cdot 0$ & $-2 \cdot 6$ & -4.8 & $-7 \cdot 1$ & $-8 \cdot 0$ \\
\hline F8 & $+4 \cdot 2$ & $+4 \cdot 1$ & $+2 \cdot 8$ & & -2.5 & $-4 \cdot 7$ & $-7 \cdot 2$ & $-8 \cdot 1$ \\
\hline G0 & +4.5 & $+4 \cdot 4$ & $+2 \cdot 9$ & & $-2 \cdot 4$ & $-4 \cdot 6$ & $-7 \cdot 2$ & $-8 \cdot 2$ \\
\hline G2 & & $+4 \cdot 7$ & +3.0 & $+1 \cdot 1$ & $-2 \cdot 4$ & -4.5 & $-7 \cdot 2$ & $-8 \cdot 2$ \\
\hline G5 & & $+5 \cdot 1$ & $+3 \cdot 1$ & +1.0 & $-2 \cdot 4$ & $-4 \cdot 4$ & $-7 \cdot 2$ & $-8 \cdot 2$ \\
\hline G8 & & $+5 \cdot 6$ & $+3 \cdot 2$ & +0.9 & -2.5 & $-4 \cdot 3$ & -7.0 & $-8 \cdot 1$ \\
\hline K0 & & $+6 \cdot 0$ & $+3 \cdot 2$ & +0.8 & -2.5 & $-4 \cdot 3$ & $-6 \cdot 8$ & -7.9 \\
\hline K1 & & $+6 \cdot 2$ & $+3 \cdot 2$ & +0.8 & $-2 \cdot 5$ & $-4 \cdot 3$ & $-6 \cdot 7$ & $-7 \cdot 7$ \\
\hline K2 & & +6.5 & & +0.7 & $-2 \cdot 5$ & $-4 \cdot 3$ & $-6 \cdot 6$ & -7.6 \\
\hline K3 & & +6.7 & & +0.6 & -2.5 & $-4 \cdot 3$ & $-6 \cdot 5$ & -7.5 \\
\hline K4 & & $+7 \cdot 0$ & & +0.5 & $-2 \cdot 6$ & $-4 \cdot 4$ & $-6 \cdot 4$ & -7.4 \\
\hline K5 & & $+7 \cdot 3$ & & +0.3 & $-2 \cdot 6$ & $-4 \cdot 4$ & $-6 \cdot 2$ & $-7 \cdot 2$ \\
\hline K7 & & $+8 \cdot 1$ & & +0.0 & $-2 \cdot 7$ & -4.5 & $-6 \cdot 0$ & $-7 \cdot 0$ \\
\hline M0 & & +8.9 & & -0.6 & $-2 \cdot 8$ & $-4 \cdot 6$ & $-5 \cdot 8$ & -6.9 \\
\hline M1 & & +9.4 & & -0.8 & -2.9 & $-4 \cdot 6$ & $-5 \cdot 8$ & $-6 \cdot 8$ \\
\hline M2 & & $+10 \cdot 0$ & & -0.9 & $-3 \cdot 0$ & $-4 \cdot 7$ & -5.8 & $-6 \cdot 7$ \\
\hline M3 & & +10.5 & & -1.0 & $-3 \cdot 0$ & $-4 \cdot 7$ & $-5 \cdot 8$ & $-6 \cdot 7$ \\
\hline M4 & & +11.5 & & -0.6 & $-3 \cdot 1$ & $-4 \cdot 7$ & $-5 \cdot 8$ & $-6 \cdot 7$ \\
\hline M5 & & $+13 \cdot 5$ & & $-0 \cdot 1$ & $-3 \cdot 1$ & $-4 \cdot 7$ & $-5 \cdot 8$ & $-6 \cdot 7$ \\
\hline
\end{tabular}


Table J.9: Logarithms of stellar effective temperatures and bolometric corrections. Taken from Straižys (1992, Appendix 3), originally from Straižys \& Kuriliené (1981).

\begin{tabular}{|c|c|c|c|c|c|c|c|c|}
\hline \multirow[t]{2}{*}{ Sp } & \multicolumn{4}{|c|}{$\log T_{e}$} & \multicolumn{4}{|c|}{$\mathrm{BC}$} \\
\hline & V & V-III & III & I-II & V & V-III & III & I-II \\
\hline O5 & & 4.626 & & $4 \cdot 618$ & & $-4 \cdot 15$ & & $-3 \cdot 80$ \\
\hline $\mathrm{O} 6$ & & 4.593 & & 4.585 & & -3.90 & & -3.55 \\
\hline O7 & & 4.568 & & 4.556 & & -3.65 & & $-3 \cdot 30$ \\
\hline O8 & & $4 \cdot 550$ & & 4.535 & & $-3 \cdot 40$ & & $-3 \cdot 15$ \\
\hline O9 & & 4.525 & & 4.512 & & $-3 \cdot 15$ & & -2.95 \\
\hline B0 & & 4.498 & & $4 \cdot 431$ & & -2.95 & & -2.50 \\
\hline B1 & & $4 \cdot 423$ & & 4.371 & & $-2 \cdot 60$ & & -2.15 \\
\hline B2 & & $4 \cdot 362$ & & $4 \cdot 307$ & & $-2 \cdot 20$ & & -1.75 \\
\hline B3 & & $4 \cdot 286$ & & $4 \cdot 243$ & & -1.85 & & -1.40 \\
\hline B5 & & $4 \cdot 188$ & & $4 \cdot 137$ & & $-1 \cdot 30$ & & -0.90 \\
\hline B6 & & $4 \cdot 152$ & & $4 \cdot 100$ & & $-1 \cdot 05$ & & -0.75 \\
\hline B7 & & $4 \cdot 107$ & & 4.068 & & -0.80 & & -0.60 \\
\hline B8 & & $4 \cdot 061$ & & $4 \cdot 041$ & & -0.55 & & -0.45 \\
\hline B9 & & 4.017 & & 4.013 & & -0.35 & & -0.35 \\
\hline A0 & & $3 \cdot 982$ & & 3.991 & & $-0 \cdot 25$ & & -0.25 \\
\hline A1 & & 3.973 & & 3.978 & & -0.16 & & -0.16 \\
\hline A2 & & $3 \cdot 961$ & & $3 \cdot 964$ & & -0.10 & & $-0 \cdot 10$ \\
\hline A3 & & 3.949 & & 3.949 & & -0.03 & & -0.03 \\
\hline A5 & & 3.924 & & 3.919 & & +0.02 & & +0.05 \\
\hline A7 & & $3 \cdot 903$ & & 3.897 & & +0.02 & & +0.09 \\
\hline F0 & & 3.863 & & 3.869 & & +0.02 & & +0.13 \\
\hline $\mathrm{F} 2$ & & $3 \cdot 845$ & & 3.851 & & $+0 \cdot 01$ & & $+0 \cdot 11$ \\
\hline F5 & & $3 \cdot 813$ & & 3.813 & & -0.02 & & +0.08 \\
\hline F8 & 3.789 & & $3 \cdot 782$ & 3.778 & & -0.03 & & +0.03 \\
\hline G0 & $3 \cdot 774$ & & $3 \cdot 763$ & 3.756 & & -0.05 & & +0.00 \\
\hline G2 & $3 \cdot 763$ & & $3 \cdot 740$ & 3.732 & & -0.07 & & -0.05 \\
\hline G5 & $3 \cdot 740$ & & $3 \cdot 712$ & $3 \cdot 699$ & -0.09 & & -0.22 & -0.13 \\
\hline G8 & $3 \cdot 720$ & & $3 \cdot 695$ & 3.663 & $-0 \cdot 13$ & & -0.28 & -0.22 \\
\hline K0 & $3 \cdot 703$ & & $3 \cdot 681$ & 3.643 & $-0 \cdot 19$ & & -0.37 & -0.29 \\
\hline K1 & $3 \cdot 695$ & & $3 \cdot 663$ & 3.633 & & & -0.43 & -0.35 \\
\hline K2 & $3 \cdot 686$ & & $3 \cdot 648$ & $3 \cdot 623$ & -0.30 & & -0.49 & -0.42 \\
\hline K3 & 3.672 & & 3.628 & 3.613 & & & -0.66 & -0.57 \\
\hline K4 & 3.663 & & $3 \cdot 613$ & & & & -0.86 & -0.75 \\
\hline K5 & $3 \cdot 643$ & & $3 \cdot 602$ & 3.585 & -0.62 & & $-1 \cdot 15$ & -1.17 \\
\hline K7 & $3 \cdot 602$ & & & & -0.89 & & & \\
\hline M0 & 3.591 & & 3.591 & 3.568 & $-1 \cdot 17$ & & -1.25 & -1.25 \\
\hline M1 & 3.574 & & $3 \cdot 580$ & 3.556 & -1.45 & & -1.45 & -1.40 \\
\hline M2 & $3 \cdot 550$ & & 3.574 & $3 \cdot 544$ & -1.71 & & $-1 \cdot 65$ & -1.60 \\
\hline M3 & 3.531 & & 3.562 & 3.518 & -1.92 & & -1.95 & $-2 \cdot 0$ \\
\hline M4 & $3 \cdot 512$ & & $3 \cdot 550$ & 3.491 & $-2 \cdot 24$ & & $-2 \cdot 4$ & $-2 \cdot 6$ \\
\hline M5 & 3.491 & & 3.531 & 3.470 & -2.55 & & $-3 \cdot 1$ & $-3 \cdot 3$ \\
\hline M6 & & & 3.512 & & $-4 \cdot 4$ & & $-4 \cdot 0$ & \\
\hline
\end{tabular}


Table J.10: Logarithms of stellar surface gravities $(\log g)$. Taken from Straižys (1992, Appendix 4), originally from Straižys \& Kurilienè (1981).

\begin{tabular}{|c|c|c|c|c|c|c|c|c|}
\hline Sp & ZAMS & V & IV & III & II & $\mathrm{Ib}$ & Iab & Ia \\
\hline O5 & $+4 \cdot 13$ & +3.90 & +3.86 & +3.82 & $+3 \cdot 76$ & +3.74 & $+3 \cdot 69$ & \\
\hline O6 & $+4 \cdot 16$ & $+3 \cdot 86$ & +3.80 & $+3 \cdot 76$ & $+3 \cdot 69$ & $+3 \cdot 64$ & $+3 \cdot 60$ & +3.53 \\
\hline O7 & $+4 \cdot 18$ & +3.85 & +3.80 & $+3 \cdot 74$ & $+3 \cdot 64$ & $+3 \cdot 57$ & +3.52 & $+3 \cdot 45$ \\
\hline 08 & $+4 \cdot 17$ & $+3 \cdot 87$ & +3.81 & $+3 \cdot 75$ & $+3 \cdot 62$ & $+3 \cdot 53$ & $+3 \cdot 49$ & $+3 \cdot 39$ \\
\hline 09 & $+4 \cdot 21$ & +3.95 & +3.82 & $+3 \cdot 74$ & +3.58 & $+3 \cdot 50$ & $+3 \cdot 44$ & $+3 \cdot 31$ \\
\hline B0 & $+4 \cdot 22$ & +4.00 & +3.88 & $+3 \cdot 74$ & $+3 \cdot 39$ & $+3 \cdot 27$ & $+3 \cdot 19$ & +3.05 \\
\hline B1 & $+4 \cdot 28$ & $+4 \cdot 00$ & +3.86 & $+3 \cdot 71$ & $+3 \cdot 31$ & $+3 \cdot 17$ & +3.01 & +2.87 \\
\hline B2 & $+4 \cdot 28$ & +4.06 & +3.88 & $+3 \cdot 68$ & $+3 \cdot 19$ & $+3 \cdot 00$ & +2.84 & +2.68 \\
\hline B3 & $+4 \cdot 31$ & +4.06 & +3.89 & $+3 \cdot 71$ & $+3 \cdot 12$ & +2.79 & $+2 \cdot 68$ & +2.49 \\
\hline B5 & $+4 \cdot 32$ & $+4 \cdot 10$ & +3.98 & $+3 \cdot 81$ & +2.90 & +2.52 & $+2 \cdot 40$ & +2.22 \\
\hline B6 & $+4 \cdot 32$ & +4.09 & +3.96 & $+3 \cdot 84$ & +2.77 & $+2 \cdot 42$ & $+2 \cdot 29$ & +2.13 \\
\hline B7 & $+4 \cdot 35$ & +4.07 & +3.95 & $+3 \cdot 82$ & +2.77 & $+2 \cdot 33$ & $+2 \cdot 21$ & +2.02 \\
\hline B8 & $+4 \cdot 34$ & +4.07 & +3.92 & $+3 \cdot 79$ & +2.79 & $+2 \cdot 27$ & $+2 \cdot 11$ & +1.97 \\
\hline B9 & $+4 \cdot 34$ & +4.03 & +3.94 & $+3 \cdot 75$ & $+2 \cdot 81$ & $+2 \cdot 20$ & +2.04 & +1.88 \\
\hline $\mathrm{A} 0$ & $+4 \cdot 32$ & +4.07 & +3.91 & $+3 \cdot 75$ & +2.85 & $+2 \cdot 23$ & $+2 \cdot 01$ & +1.81 \\
\hline A1 & $+4 \cdot 35$ & $+4 \cdot 10$ & +3.96 & $+3 \cdot 78$ & +2.88 & $+2 \cdot 22$ & +1.96 & $+1 \cdot 76$ \\
\hline A2 & $+4 \cdot 32$ & $+4 \cdot 16$ & +3.98 & +3.78 & +2.87 & $+2 \cdot 23$ & +1.92 & +1.71 \\
\hline A3 & $+4 \cdot 34$ & $+4 \cdot 20$ & +4.03 & $+3 \cdot 83$ & $+2 \cdot 85$ & $+2 \cdot 20$ & +1.86 & $+1 \cdot 65$ \\
\hline A5 & $+4 \cdot 36$ & $+4 \cdot 22$ & +4.06 & $+3 \cdot 86$ & +2.81 & $+2 \cdot 14$ & +1.74 & +1.53 \\
\hline A7 & $+4 \cdot 36$ & $+4 \cdot 26$ & $+4 \cdot 10$ & $+3 \cdot 86$ & +2.75 & +2.08 & $+1 \cdot 65$ & $+1 \cdot 38$ \\
\hline F0 & $+4 \cdot 32$ & +4.28 & +4.05 & +3.83 & +2.67 & $+2 \cdot 00$ & +1.51 & +1.25 \\
\hline F2 & $+4 \cdot 30$ & +4.26 & +4.01 & $+3 \cdot 81$ & $+2 \cdot 63$ & +1.92 & +1.39 & $+1 \cdot 15$ \\
\hline F5 & $+4 \cdot 32$ & $+4 \cdot 28$ & +3.93 & $+3 \cdot 74$ & +2.48 & +1.81 & +1.22 & +1.00 \\
\hline F8 & $+4 \cdot 39$ & $+4 \cdot 35$ & +3.89 & & $+2 \cdot 38$ & $+1 \cdot 71$ & $+1 \cdot 06$ & +0.83 \\
\hline G0 & $+4 \cdot 39$ & +4.39 & +3.84 & & $+2 \cdot 29$ & +1.62 & +0.95 & +0.72 \\
\hline G2 & $+4 \cdot 40$ & $+4 \cdot 40$ & $+3 \cdot 77$ & $+3 \cdot 20$ & $+2 \cdot 20$ & +1.53 & +0.86 & +0.61 \\
\hline G5 & & +4.49 & +3.71 & $+3 \cdot 07$ & +2.04 & +1.45 & +0.71 & +0.45 \\
\hline G8 & & +4.55 & +3.64 & +2.95 & +1.84 & $+1 \cdot 30$ & +0.60 & +0.30 \\
\hline K0 & & +4.57 & +3.57 & +2.89 & +1.74 & $+1 \cdot 20$ & +0.54 & +0.25 \\
\hline K1 & & +4.55 & +3.55 & +2.78 & +1.66 & $+1 \cdot 16$ & +0.54 & +0.25 \\
\hline K2 & & +4.55 & & $+2 \cdot 63$ & +1.59 & $+1 \cdot 10$ & +0.48 & +0.23 \\
\hline K3 & & +4.56 & & $+2 \cdot 36$ & +1.52 & +1.00 & +0.46 & $+0 \cdot 19$ \\
\hline K4 & & +4.57 & & $+2 \cdot 16$ & & & & \\
\hline K5 & & +4.57 & & +1.93 & $+1 \cdot 20$ & +0.77 & +0.35 & $+0 \cdot 10$ \\
\hline K7 & & $+4 \cdot 62$ & & & & & & \\
\hline M0 & & +4.61 & & $+1 \cdot 63$ & +1.01 & +0.61 & +0.30 & +0.00 \\
\hline M1 & & +4.67 & & $+1 \cdot 41$ & +0.84 & +0.51 & $+0 \cdot 19$ & -0.07 \\
\hline M2 & & +4.69 & & $+1 \cdot 31$ & $+0 \cdot 70$ & +0.39 & +0.09 & -0.13 \\
\hline M3 & & +4.71 & & $+1 \cdot 12$ & +0.38 & $+0 \cdot 10$ & $-0 \cdot 16$ & -0.34 \\
\hline M4 & & +4.77 & & +0.98 & & & & \\
\hline M5 & & $+5 \cdot 06$ & & $(+0 \cdot 76)$ & & & & \\
\hline M6 & & & & $(+0.52)$ & & & & \\
\hline
\end{tabular}


Table J.11: Logarithms of stellar masses in solar units $\left(\log \mathcal{M} / \mathcal{M}_{\odot}\right)$. Taken from Straižys (1992, Appendix 5), originally from Straižys \& Kurilienẻ (1981).

\begin{tabular}{|c|c|c|c|c|c|c|c|c|}
\hline Sp & ZAMS & V & IV & III & II & $\mathrm{Ib}$ & $\mathrm{Iab}$ & Ia \\
\hline O5 & $+1 \cdot 60$ & +1.81 & +1.85 & +1.89 & +1.90 & +1.92 & +1.99 & \\
\hline O6 & +1.48 & +1.70 & +1.76 & +1.80 & +1.80 & +1.87 & +1.91 & $+2 \cdot 00$ \\
\hline O7 & +1.40 & +1.59 & +1.65 & +1.68 & $+1 \cdot 71$ & $+1 \cdot 76$ & +1.83 & +1.92 \\
\hline O8 & $+1 \cdot 34$ & +1.48 & +1.54 & $+1 \cdot 60$ & +1.65 & +1.72 & +1.76 & +1.90 \\
\hline O9 & +1.28 & $+1 \cdot 38$ & +1.45 & +1.49 & +1.58 & $+1 \cdot 66$ & +1.72 & +1.83 \\
\hline B0 & $+1 \cdot 20$ & $+1 \cdot 30$ & $+1 \cdot 34$ & +1.40 & +1.40 & +1.48 & +1.56 & +1.70 \\
\hline B1 & +1.04 & $+1 \cdot 11$ & $+1 \cdot 18$ & +1.23 & +1.28 & $+1 \cdot 38$ & +1.46 & +1.64 \\
\hline B2 & +0.92 & +0.99 & +1.04 & +1.08 & $+1 \cdot 18$ & $+1 \cdot 30$ & +1.38 & +1.54 \\
\hline B3 & +0.78 & +0.84 & +0.88 & +0.94 & $+1 \cdot 11$ & +1.23 & +1.32 & +1.45 \\
\hline B5 & +0.62 & +0.68 & +0.72 & +0.75 & +1.00 & $+1 \cdot 18$ & +1.26 & +1.40 \\
\hline B6 & +0.56 & +0.61 & +0.64 & +0.68 & +0.94 & $+1 \cdot 15$ & +1.26 & +1.38 \\
\hline B7 & +0.49 & +0.53 & +0.57 & +0.60 & +0.91 & $+1 \cdot 11$ & +1.23 & $+1 \cdot 36$ \\
\hline B8 & $+0 \cdot 43$ & +0.48 & +0.49 & +0.52 & +0.88 & +1.08 & +1.20 & $+1 \cdot 34$ \\
\hline B9 & +0.36 & +0.41 & +0.45 & +0.49 & +0.85 & +1.04 & +1.20 & $+1 \cdot 32$ \\
\hline A 0 & $+0 \cdot 32$ & +0.35 & +0.39 & +0.43 & +0.81 & +1.04 & $+1 \cdot 18$ & $+1 \cdot 30$ \\
\hline A1 & +0.31 & +0.34 & +0.36 & +0.41 & +0.78 & +1.00 & $+1 \cdot 18$ & $+1 \cdot 30$ \\
\hline A2 & $+0 \cdot 29$ & +0.32 & $+0 \cdot 34$ & +0.39 & +0.75 & +0.98 & $+1 \cdot 15$ & $+1 \cdot 30$ \\
\hline A 3 & +0.27 & +0.30 & $+0 \cdot 32$ & +0.36 & +0.75 & +0.97 & $+1 \cdot 11$ & $+1 \cdot 30$ \\
\hline A5 & $+0 \cdot 23$ & +0.26 & +0.29 & +0.33 & +0.74 & +0.95 & $+1 \cdot 11$ & $+1 \cdot 30$ \\
\hline A7 & $+0 \cdot 20$ & +0.22 & +0.26 & +0.30 & +0.73 & +0.94 & $+1 \cdot 15$ & $+1 \cdot 32$ \\
\hline F0 & $+0 \cdot 16$ & +0.16 & +0.20 & +0.23 & +0.72 & +0.93 & $+1 \cdot 20$ & $+1 \cdot 38$ \\
\hline F2 & +0.13 & +0.13 & $+0 \cdot 16$ & $+0 \cdot 20$ & +0.72 & +0.93 & +1.20 & +1.40 \\
\hline F5 & +0.08 & +0.08 & $+0 \cdot 13$ & $+0 \cdot 18$ & +0.72 & +0.93 & +1.26 & +1.40 \\
\hline F8 & +0.04 & +0.04 & +0.11 & & +0.72 & +0.93 & +1.28 & +1.41 \\
\hline G0 & +0.02 & +0.02 & $+0 \cdot 10$ & & +0.72 & +0.93 & $+1 \cdot 30$ & +1.43 \\
\hline G2 & $+0 \cdot 00$ & +0.00 & +0.10 & +0.33 & +0.72 & +0.93 & $+1 \cdot 30$ & +1.45 \\
\hline G5 & & -0.02 & +0.08 & +0.39 & +0.73 & +0.94 & +1.32 & +1.46 \\
\hline G8 & & -0.04 & +0.08 & +0.42 & +0.76 & +0.94 & +1.32 & +1.46 \\
\hline K0 & & -0.07 & +0.11 & +0.46 & +0.78 & +0.96 & $+1 \cdot 30$ & +1.45 \\
\hline K1 & & $-0 \cdot 10$ & +0.13 & +0.46 & +0.78 & +0.96 & $+1 \cdot 30$ & +1.45 \\
\hline K2 & & $-0 \cdot 10$ & & +0.45 & +0.79 & +0.98 & $+1 \cdot 28$ & +1.43 \\
\hline K3 & & -0.12 & & +0.38 & +0.80 & +1.00 & $+1 \cdot 30$ & +1.43 \\
\hline K4 & & -0.15 & & +0.36 & & & & \\
\hline K5 & & $-0 \cdot 19$ & & +0.37 & +0.83 & +1.08 & $+1 \cdot 30$ & +1.45 \\
\hline K7 & & -0.22 & & & & & & \\
\hline M0 & & -0.26 & & +0.48 & +0.83 & $+1 \cdot 15$ & $+1 \cdot 32$ & +1.46 \\
\hline M1 & & -0.30 & & +0.54 & +0.83 & $+1 \cdot 18$ & $+1 \cdot 34$ & +1.48 \\
\hline M2 & & -0.35 & & +0.54 & +0.81 & $+1 \cdot 18$ & +1.36 & +1.50 \\
\hline M3 & & -0.40 & & +0.52 & +0.84 & +1.20 & $+1 \cdot 38$ & +1.56 \\
\hline M4 & & -0.52 & & +0.51 & & & & \\
\hline M5 & & $(-0.82)$ & & $(+0.41)$ & & & & \\
\hline M6 & & & & $(+0.40)$ & & & & \\
\hline
\end{tabular}


Table J.12: Logarithms of stellar radii in solar units $\left(\log R / R_{\odot}\right)$. Taken from Straižys $(1992$, Appendix 6), originally from Straižys \& Kurilienè (1981).

\begin{tabular}{|c|c|c|c|c|c|c|c|c|}
\hline Sp & ZAMS & V & IV & III & II & $\mathrm{Ib}$ & Iab & Ia \\
\hline O5 & +0.95 & $+1 \cdot 17$ & +1.21 & $+1 \cdot 25$ & +1.28 & $+1 \cdot 30$ & +1.36 & \\
\hline O6 & +0.87 & $+1 \cdot 13$ & $+1 \cdot 19$ & +1.23 & +1.27 & $+1 \cdot 33$ & $+1 \cdot 37$ & +1.45 \\
\hline O7 & +0.82 & +1.08 & $+1 \cdot 14$ & $+1 \cdot 18$ & +1.25 & $+1 \cdot 31$ & $+1 \cdot 37$ & +1.45 \\
\hline O8 & +0.80 & +1.02 & $+1 \cdot 08$ & $+1 \cdot 14$ & +1.23 & $+1 \cdot 31$ & $+1 \cdot 35$ & +1.47 \\
\hline O9 & +0.75 & +0.93 & +1.03 & +1.09 & $+1 \cdot 22$ & $+1 \cdot 30$ & $+1 \cdot 36$ & +1.48 \\
\hline B0 & $+0 \cdot 70$ & +0.86 & +0.94 & +1.04 & +1.20 & $+1 \cdot 32$ & $+1 \cdot 40$ & +1.54 \\
\hline B1 & +0.59 & +0.77 & +0.87 & +0.97 & +1.20 & $+1 \cdot 32$ & +1.44 & +1.60 \\
\hline B2 & +0.54 & +0.68 & +0.80 & +0.92 & $+1 \cdot 21$ & $+1 \cdot 37$ & +1.49 & +1.65 \\
\hline B3 & +0.45 & +0.61 & +0.71 & +0.83 & $+1 \cdot 21$ & +1.43 & +1.53 & +1.69 \\
\hline B5 & $+0 \cdot 36$ & +0.50 & +0.58 & +0.68 & $+1 \cdot 27$ & +1.55 & +1.65 & +1.81 \\
\hline B6 & +0.34 & +0.48 & +0.56 & $+0 \cdot 64$ & $+1 \cdot 30$ & +1.58 & $+1 \cdot 70$ & +1.84 \\
\hline B7 & +0.29 & $+0 \cdot 45$ & +0.53 & +0.61 & $+1 \cdot 28$ & $+1 \cdot 60$ & +1.72 & +1.88 \\
\hline B8 & +0.26 & $+0 \cdot 42$ & +0.50 & +0.58 & +1.26 & $+1 \cdot 62$ & $+1 \cdot 76$ & +1.90 \\
\hline B9 & +0.23 & +0.41 & +0.47 & +0.59 & $+1 \cdot 23$ & $+1 \cdot 63$ & +1.79 & +1.93 \\
\hline A0 & +0.22 & +0.36 & +0.46 & +0.56 & $+1 \cdot 20$ & $+1 \cdot 62$ & +1.80 & +1.96 \\
\hline $\mathrm{A} 1$ & $+0 \cdot 19$ & +0.33 & +0.41 & +0.53 & $+1 \cdot 16$ & $+1 \cdot 60$ & +1.82 & +1.98 \\
\hline A2 & +0.20 & $+0 \cdot 30$ & +0.40 & +0.52 & $+1 \cdot 15$ & +1.59 & +1.83 & $+2 \cdot 01$ \\
\hline A3 & $+0 \cdot 18$ & +0.26 & +0.36 & +0.48 & $+1 \cdot 16$ & $+1 \cdot 60$ & +1.84 & $+2 \cdot 04$ \\
\hline A5 & $+0 \cdot 15$ & $+0 \cdot 23$ & +0.33 & +0.45 & $+1 \cdot 18$ & $+1 \cdot 62$ & +1.90 & $+2 \cdot 10$ \\
\hline A 7 & +0.13 & +0.19 & +0.29 & +0.43 & +1.21 & $+1 \cdot 65$ & +1.97 & $+2 \cdot 19$ \\
\hline F0 & $+0 \cdot 13$ & $+0 \cdot 15$ & +0.29 & $+0 \cdot 41$ & $+1 \cdot 24$ & +1.68 & $+2 \cdot 06$ & $+2 \cdot 28$ \\
\hline $\mathrm{F} 2$ & +0.13 & +0.15 & +0.29 & +0.41 & $+1 \cdot 26$ & +1.72 & $+2 \cdot 12$ & $+2 \cdot 34$ \\
\hline F5 & +0.09 & +0.11 & +0.31 & +0.43 & $+1 \cdot 30$ & +1.77 & $+2 \cdot 23$ & +2.41 \\
\hline F8 & +0.04 & +0.06 & +0.33 & & $+1 \cdot 38$ & +1.82 & $+2 \cdot 32$ & +2.50 \\
\hline G0 & +0.03 & +0.03 & +0.34 & & +1.43 & +1.87 & $+2 \cdot 39$ & +2.57 \\
\hline G2 & +0.01 & +0.01 & +0.38 & +0.78 & +1.48 & +1.92 & +2.44 & +2.64 \\
\hline G5 & & -0.04 & +0.41 & +0.88 & $+1 \cdot 56$ & +1.96 & +2.52 & +2.72 \\
\hline G8 & & -0.08 & +0.43 & +0.95 & +1.67 & +2.03 & +2.57 & +2.79 \\
\hline K0 & & -0.11 & +0.48 & +1.00 & $+1 \cdot 73$ & +2.09 & +2.59 & +2.81 \\
\hline K1 & & -0.11 & +0.50 & +1.05 & +1.77 & $+2 \cdot 11$ & $+2 \cdot 61$ & +2.81 \\
\hline K2 & & -0.11 & & $+1 \cdot 12$ & +1.81 & $+2 \cdot 15$ & $+2 \cdot 61$ & +2.81 \\
\hline K3 & & -0.12 & & +1.22 & +1.85 & $+2 \cdot 21$ & $+2 \cdot 63$ & +2.83 \\
\hline K4 & & -0.15 & & $+1 \cdot 31$ & & & & \\
\hline K5 & & -0.17 & & +1.44 & +2.03 & $+2 \cdot 37$ & $+2 \cdot 69$ & +2.89 \\
\hline K7 & & -0.20 & & & & & & \\
\hline M0 & & -0.22 & & +1.64 & $+2 \cdot 12$ & +2.48 & +2.72 & +2.92 \\
\hline M1 & & -0.27 & & $+1 \cdot 78$ & $+2 \cdot 21$ & +2.55 & +2.78 & +2.99 \\
\hline M2 & & -0.30 & & +1.83 & $+2 \cdot 27$ & $+2 \cdot 61$ & +2.85 & +3.03 \\
\hline M3 & & $-0 \cdot 36$ & & +1.92 & +2.44 & $+2 \cdot 76$ & +2.98 & $+3 \cdot 16$ \\
\hline M4 & & -0.42 & & +1.98 & & & & \\
\hline M5 & & -0.72 & & $(+2 \cdot 04)$ & & & & \\
\hline M6 & & & & $(+2 \cdot 16)$ & & & & \\
\hline
\end{tabular}




\section{Appendix K}

\section{Glossary of Acronyms.}

$\begin{array}{ll}\text { B\&C } & \text { Boller and Chivens } \\ \text { BHB } & \text { Blue Horizontal Branch } \\ \text { BS } & \text { Bright Star catalog } \\ \text { CCD } & \text { Charge Coupled Device } \\ \text { CDROM } & \text { Compact Disk Read Only Mem- } \\ & \text { ory }\end{array}$

CDS Centre de Données Astronomiques de Strasbourg

CMD Colour-Magnitude Diagram

CPC Cape Photographic Catalogue

DC Direct Current

Dec Declination

FRST Foundation for Research, Science and Technology (NZ)

FWHM Full Width at Half Maximum

GCVS General Catalog of Variable Stars

HA Hour Angle

HD Henry Draper catalog

HJD Heliocentric Julian Date

HR Harvard Revised catalog - synonym for the Bright Star catalog

HST Hubble Space Telescope

IAU International Astronomical Union

IGC Internal Grants Committee

IRAF Image Reduction and Analysis Facility

ITS Information Technology Services

JD Julian Date
KPO Kitt Peak Observatory

LED Light Emitting Diode

LMC Large Magellanic Cloud

LST Local Sidereal Time

MJUO Mount John University Observatory

NGC New General Catalog

NPS North Polar Sequence

O/C Optical Craftsman

PC Pulse Counting or Personal Computer

PMT PhotoMultiplier Tube

QF Quality Factor

RA Right Ascension

SAO Smithsonian Astrophysical Observatory

SB Spectroscopic Binary

SIMBAD Set of Identifications, Measurements and Bibliography for Astronomical Data

SPS South Polar Sequence

STP Standard Temperature and Pressure

UoC University of Canterbury

UT Universal Time

UV ultra-violet

VUW Victoria University of Wellington

ZAMS Zero Age Main Sequence 


\section{Appendix L}

\section{List of Publications.}

Below is a list of relevant papers published during this project.

- "Vilnius CCD photometry of southern clusters - some preliminary results" Banks, T., Forbes, M. C., Sullivan, D. J. \& Dodd, R .J.

Southern Stars 36(1 \& 2), 69-73, (1994)

- "Vilnius photometry in the southern hemisphere" Dodd, R. J., Forbes, M. C. \& Sullivan, D. J.

'IAU Colloquium 136 on Stellar Photometry - Current Techniques and Future Developments', Dublin, August 1992

Cambridge University Press.

- "Determining time-dependant atmospheric extinction"

Forbes, M. C.

Southern Stars 35(1), 1-11, (1992)

- "Progress report on the establishment of the southern hemisphere Vilnius standard photometric system"

Forbes, M. C.

Southern Stars 35(3), 69-79, (1993)

- "The southern Vilnius photometric system. I.

Transformation to the standard Vilnius system"

Forbes, M. C., Dodd, R. J. \& Sullivan, D. J.

Baltic Astronomy 2(2), 246-255, (1993)

- "The southern Vilnius photometric system. II.

The bright, southern standard stars"

Forbes, M. C., Dodd, R. J. \& Sullivan, D. J.

Baltic Astronomy 3(3) 227-231, (1994)

- "The Southern Vilnius Photometric System. III.

Observations of $\mathrm{E}$ and $\mathrm{F}$ Harvard standard regions, 47 Tuc and some metal-deficient stars" Zdanavičius, K., Dodd, R. J., Forbes, M. C. \& Sullivan, D. J.

Baltic Astronomy 4, 25-38, (1995)

- "The southern Vilnius photometric system. IV.

The E region standard stars"

Forbes, M. C., Dodd, R. J. \& Sullivan, D. J.

submitted to Baltic Astronomy. 
- "Mount Pinatubo and atmospheric extinction at Mount John University Observatory 1987-94"

Forbes, M. C., Banks, T., Sullivan, D. J., Dodd , R. J., Gilmore, A. C. \& Kilmartin, P. M. The Observatory 115, 29-30, (1995)

- "A Southern Hemisphere Network of the Secondary Standard Stars in the Vilnius Photometric System."

Dodd, R. J., Forbes, M. C., Sullivan, D. J. \& Zdanavičius, K.

Baltic Astronomy 5, 271-275, (1996)

- "A detailed investigation of atmospheric extinction via Vilnius photometry" Forbes, M. C., Dodd, R. J. \& Sullivan, D. J.

Baltic Astronomy 5, 281-295, (1996) 


\section{References}

Acker, A. \& Jaschek, C. (1986), Astronomical Methods and Calculations, John Wiley and Sons, Brisbane. Translated by Chris Kitchin.

Allen, C. W. (1963), Astrophysical Quantities, The Athlone Press, University of London, 4 Gower Street, London WC1. 2nd edition.

Ångström, A. (1929), Geogr. Ann. 11, 156-166.

Ardeberg, A. \& Virdefors, B. (1975), Astron. Astrophys. 39, 26. Cited by Jaschek \& Jaschek (1987).

Arp, H. (1961), Astrophys. J. 133, 874. Cited by Straižys (1992).

Arp, H. C. \& Van Sant, C. T. (1958), Astron. J. 63(1262), 341-346.

Ažusienis, A. \& Straižys, V. (1969), Astron. Zurnal 46, 402. alternatively Soviet Astron. 13, 316. Cited by Straižys (1992).

Balona, L. A. (1977), Mem. R. Astr. Soc. 84, 101-117.

Balona, L. A. (1982), Inf. Bull. Variable Stars.

Balona, L. A. \& Koen, C. (1994), Mon. Not. R. Astr. Soc. 267, 1071-1080.

Balona, L. A. \& Shobbrook, R. R. (1984), Mon. Not. R. Astr. Soc. 211, 375-390.

Banks, T. (1994). pers. comm.

Banks, T. (1995), PhD thesis, Victoria University of Wellington, New Zealand.

Banks, T. et al. (1994), Southern Stars 36(1-2), 69-73.

Bartkevičius, A. \& Lazauskaitè, R. (1994), Baltic Astronomy 3, 58-66.

Bartkevičius, A. \& Metik, L. P. (1969), Bull. Vilnius Obs. 26, 13-22.

Bartkevičius, A. \& Straižys, V. (1970a), Bull. Vilnius Obs. 30, 3-15.

Bartkevičius, A. \& Straižys, V. (1970b), Bull. Vilnius Obs, 30, 33-39.

Bartkevičius, A. \& Straižys, V. (1970c), Bull. Vilnius Obs, 30, 16-32.

Bartkevičius, A. et al. (1973), Bull. Vilnius Obs. 36(3), 17-24.

Bartkevičius, A. et al. (1992), Baltic Astronomy 1, 216-238.

Becker, W. (1961), Z. Astrophys. 51, 151. Cited by Perry et al. (1976).

Becker, W. (1963), Z. Astrophys. 57, 117. Cited by Perry et al. (1976).

Becker, W. \& Fenkart, R. (1970), in W. Becker \& G. Contopoulos, eds, 'The Spiral Structure of our Galaxy', D. Reidel Publishing Company, Dordrecht-Holland/Boston-USA, p. 205. 
Bemporad, A. (1904), Grossh. Sternwarte Heidelberg. Cited by Hardie (1962), Young (1974), Sterken \& Manfroid (1992a).

Bessell, M. S. (1986), Publ. Astron. Soc. Pac. 98, 354. Cited by Sterken \& Manfroid (1992a).

Bessell, M. S. (1990), Publ. Astron. Soc. Pac. 102, 1181-1199.

Bessell, M. S. (1993), Photometric systems, in C. J. Butler \& I. Elliot, eds, 'IAU Colloquium 136 on Stellar Photometry - Current Techniques and Future Developments', Dublin, August 1992, Cambridge University Press.

Bidelman, W. P. (1954), Publ. Astron. Soc. Pac. 66, 249-251.

Brown, P. J. F. et al. (1986), Mon. Not. R. Astr. Soc. 220, 1003-1020.

Budding, E. (1993), An introduction to Astronomical Photometry, Cambridge University Press.

Buscombe, W. (1963), Mem. Mt. Stromlo Obs. Cited by Perry et al. (1976).

Buscombe, W. (1965), Mon. Not. R. Astr. Soc. 129, 411-417.

C̆ernis, K. \& Jasevičius, V. (1992), Baltic Astronomy 1, 83-95.

C̆ernis, K. et al. (1989), Bull. Vilnius Obs. 84, 9.

Cooper \& Walker (1989), Getting the measure of the stars, Adam Hilger Publ. Cited by Poretti (1992).

Cousins, A. W. J. (1987), South African Astron. Obs. Circ. 11, 93-120.

Cousins, A. W. J. (1989), South African Astron. Obs. Circ. 13, 15-25.

Crawford, D. L. (1958), Astrophys. J. 128, 185-206.

Crawford, D. L. (1966), in K. Loden et al., eds, 'Spectral Classification and Multicolour Photometry', IAU Symp. No. 24, Academic Press. Cited by Straižys (1992).

Crawford, D. L. (1975), Astron. J. 80, 955. Cited by Sterken \& Manfroid (1992a).

Crawford, D. L. \& Barnes, J. V. (1970), Astron. J. 75, 978. Cited by Golay (1974), Straižys (1992).

Crawford, D. L. \& Mander, J. (1966), Astron. J. 71(2), 114-118.

Crawford, D. L., Barnes, J. V. \& Golson, J. C. (1971), Astron. J. 76(7), 621-630.

Dachs, J. \& Kaiser, D. (1984), Astron. Astrophys. Suppl. Ser. 58, 411-429.

de Waard, G. J. et al. (1984), Astron. Astrophys. Suppl. Ser. 56, 373-380.

Dodd, R. J. (1994). pers. comm.

Duffett-Smith, P. (1985), Astronomy with your Personal Computer, Cambridge University Press, London.

Dzérvítis, U. \& Paupers, O. (1994), Baltic Astronomy 3, 335-347.

Eggen, O. J. (1972), Astrophys. J. 173, 63-86.

Eggen, O. J. (1976), Publ. Astron. Soc. Pac. 88, 732. Cited by Straižys (1992), Bessell (1993).

Eggen, O. J. (1982), Astrophys. J., Suppl. Ser. 50, 221. Cited by Straižys (1992).

Eggen, O. J. (1983), Astron. J. 88(2), 209-214. 
Evans, R. D. (1955), The Atomic Nucleus, McGraw Hill, New York. Cited by Fernie (1976), Poretti (1992).

Feast, M. W. (1963), Mon. Not. R. Astr. Soc. 126, 11-28.

Feinstein, A. (1961), Publ. Astron. Soc. Pac. 73, 410-417.

Fernie, J. D. (1976), Publ. Astron. Soc. Pac. 88, 969-970.

Forbes, J. D. (1842), Phil. Trans. 132, 225. Cited by Young (1974), Sterken \& Manfroid $(1992 a)$.

Forbes, M. C. (1992), Southern Stars 35(1), 1-11.

Gast, P. R. (1960), Absorption coefficients of ozone, in 'Handbook of Geophysics', U.S. Air Forces, Cambridge Research Center, Geophysics Research Directorate, rev. ed.;MacMillan, New York, p. 16.21.

Gieseking, F. (1981), Astron. Astrophys. Suppl. Ser. 43, 33-74.

Gilmore, A. C. \& Kilmartin, P. M. (1994). pers. comm.

Golay, M. (1974), Introduction to Astronomical Photometry, Vol. 41 of Astrophysics and Space Science Library, D. Reidel Publishing Company, Dordrecht-Holland/Boston-USA.

Graham, J. A. (1967), Mon. Not. R. Astr. Soc. 135, 377-390.

Grønbech, B. \& Olsen, E. H. (1976), Astron. Astrophys. Suppl. Ser. 25, 213-270.

Grønbech, B. \& Olsen, E. H. (1977), Astron. Astrophys. Suppl. Ser. 27, 433-462.

Grønbech, B. et al. (1976), Astron. Astrophys. Suppl. Ser. 26, 155. Cited by Straižys (1992), Sterken \& Manfroid (1992a).

Grothues, H.-G. \& Gochermann, J. (1992), The ESO Messenger 68, 43-44.

Gunn, J. E. \& Stryker, L. L. (1983), Astrophys. J., Suppl. Ser. 52, 121-153.

Gutiérrez-Moreno, A. et al. (1982), Publ. Astron. Soc. Pac. 94, 722-728.

Hall, D. S. \& Genet, R. M. (1982), Photoelectric Photometry of Variable Stars, International Amateur-Professional Photoelectric Photometry. Printed by William L. Meyers, MinutemanPress.

Hardie, R. H. (1962), Photoelectric reductions, in W. A. Hiltner, ed., 'Astronomical Techniques', Vol. 2 of Stars and Stellar Systems, The University of Chicago Press, Chicago, Illinois, U.S.A.

Harris, W. E., FitzGerald, M. P. \& Reed, B. C. (1981), Publ. Astron. Soc. Pac. 93, 507.

Hauck, B. \& Mermilliod, M. (1980), Astron. Astrophys. Suppl. Ser. 40, 1. Cited by Straižys (1992).

Hayes, D. S. \& Latham, D. W. (1975), Astrophys. J. 197, 593-601.

Hendon, A. A. \& Kaitchuck, R. H. (1989), Astronomical Software for the IBM-PC, WillmannBell Inc., P.O Box 35025, Richmond, Virginia 23235, U.S.A.

Hendon, A. A. \& Kaitchuck, R. H. (1990), Astronomical Photometry, Willmann-Bell Inc., P.O Box 35025, Richmond, Virginia 23235, U.S.A.

Hernández, C. (1959), Bol. Astron. Assn. Argentina 2, 1. Cited by de Waard et al. (1984). 
Hernández, C. (1960), Publ. Astron. Soc. Pac. 72, 416-418.

Hiltner, W. A. et al. (1969), Astrophys. J. 157, 313. Cited by Crawford et al. (1971).

Hoffleit, D. (1964), Yale University Observatory. Cited by Crawford et al. (1971).

Hogg, A. R. (1958), Mt. Stromlo Min. Cited by Marraco \& Orsatti (1982).

Hogg, A. R. (1960), Publ. Astron. Soc. Pac. 72, 85-93.

Houk, N. (1978), University of Michigan, Ann Arbor. Cited by Crawford et al. (1971).

Jakate, S. M. (1978), Astron. J. 83(10), 1179-1183.

Jakate, S. M. (1979), Astron. J. 84(7), 1042-1045.

Jaschek, C. \& Frankel, S. (1985), Astron. Astrophys. 158, 174. Cited by Jaschek \& Jaschek (1987).

Jaschek, C. \& Jaschek, M. (1987), The Classification of Stars, Cambridge University Press, London. Reprinted with corrections 1990.

Jasevičius, V. (1986), Bull. Vilnius Obs. 74, 40-55.

Johnson, H. L. (1955), Ann. d'Astrophys. 18, 292. Cited by Straižys (1992).

Johnson, H. L. (1962), Astrophys. J. 135, 975. Cited by Straižys (1992).

Johnson, H. L. (1963), in K. A. Strand, ed., 'Basic Astronomical Data', The University of Chicago Press, Chicago, Illinois, U.S.A., p. 204. Cited by Straižys (1992).

Johnson, H. L. (1965a), Astrophys. J. 141, 923. Cited by Straižys (1992).

Johnson, H. L. (1965b), Comm. Lunar. \& Planet. Lab., Uni. Arizona 3, 73. Cited by Jaschek \& Jaschek (1987).

Johnson, H. L. \& Harris, W. E. (1954), Astrophys. J. 120, 196. Cited by Straižys (1992).

Johnson, H. L. \& Morgan, W. W. (1951), Astrophys. J. 114, 522. Cited by Straižys (1992), Sterken \& Manfroid (1992a).

Johnson, H. L. \& Morgan, W. W. (1953), Astrophys. J. 117, 313. Cited by (Jaschek \& Jaschek1987 Straižys1992 Sterken \& Manfroid1992a).

Junge, C. E. (1963), in J. van Mieghem, ed., 'Air Chemistry and Radioactivity', Vol. 4 of International Geophysics Series, Academic Press, New York and London.

Kaiser, D. (1980), preprint, Cited by de Waard et al. (1984).

Kakaras, G. \& Straižys, V. (1969), Bull. Vilnius Obs. 23, 3-35.

Kakaras, G. et al. (1968), Bull. Vilnius Obs. 22, 3-24.

Kennedy, P. (1966), Mem. Mt. Stromlo Obs. Cited by Perry et al. (1976).

Kilkenny, D. \& Laing, J. D. (1992), Mon. Not. R. Astr. Soc. 255, 308-318.

King, D. S. (1980), Sydney observatory papers. Reprinted from the Journal and Proceedings, Royal Society of New South Wales, Vol 113, pp. 65-68, 1980.

King, I. R. (1952), Astron. J. 57, 253. Cited by Straižys (1992), Sterken \& Manfroid (1992a).

King, M. D. \& Byrne, D. M. (1976), J. Atmos. Sci. 33, 2242-2251. 
King, M. D. et al. (1978), J. Atmos. Sci. 35, 2153-2167.

Koen, C. (1993), Mon. Not. R. Astr. Soc. 264, 165-170.

Koester, D. \& Reimers, D. (1985), Astron. Astrophys. 153, 260-264.

Kučinskas, A. (1990), J. Br. Astron. Assoc. 100(4), 191-194.

Kurilienè, G. \& Sūdžius, J. (1974), Bull. Vilnius Obs. 40, 10-25.

Kurucz, R. L. (1995). pers. comm.

Lamla, E. (1982), Vol. 2b of Numerical data and functional relationships in Science and Technology, p. 35. New Series, Group 6. Cited by Jaschek \& Jaschek (1987).

Lesh, J. R. (1969), Astrophys. J., Suppl. Ser. 17(151), 371. Cited by Crawford et al. (1971).

Lesh, J. R. (1972), Astron. Astrophys. Suppl. Ser. 5, 129. Cited by Eggen (1983).

Levato, H. \& Malaroda, S. (1984), Astrophys. Lett. 24, 37-41.

Levato, H. et al. (1988), Astrophys. Space. Sci. 146, 361-373.

Lyngå, G. (1959), Arkiv för Astronomi 2(34), 379-390.

Lyngå, G. (1961), Arkiv för Astronomi 3(8), 65.

Lyngå, G. \& Wramdemark, S. (1984), Astron. Astrophys. 132, 58-74.

Maitzen, H. M. \& Catalano, F. A. (1986), Astron. Astrophys. Suppl. Ser. 66, 37-44.

Manfroid, J. (1985), in D. S. Hayes et al., eds, 'Calibration of Fundamental Stellar Quantites', IAU Colloquium No. 111, D. Reidel Publishing Company, Dordrecht-Holland/Boston-USA, p. 505. Cited by Sterken \& Manfroid (1992a).

Manfroid, J. (1992), Astron. Astrophys. 260, 517-524.

Manfroid, J. \& Sterken, C. (1987), Astron. Astrophys. Suppl. Ser. 71, 539. Cited by Sterken \& Manfroid (1992a).

Marraco, H. G. \& Orsatti, A. M. (1982), Rev. Mexicana Astron. Astrof. 5, 183-208.

Matsushima, S. (1969), Astrophys. J. 158, 1137. Cited by Golay (1974), Straižys (1992).

Matthews, T. A. \& Sandage, A. R. (1963), Astrophys. J. 138, 49. Cited by Straižys (1992), Sterken \& Manfroid (1992a).

McGregor, P. J. \& Hyland, A. R. (1984), Astrophys. J. 277, 149-163.

Melbourne, W. G. (1960), Astrophys. J. 132, 101. Cited by Straižys (1992).

Menzies, J. W. \& Laing, J. D. (1988), Mon. Not. R. Astr. Soc. 231, 1047-1053.

Menzies, J. W. et al. (1989), South African Astron. Obs. Circ. 13, 1-13.

Menzies, J. W. et al. (1991), Mon. Not. R. Astr. Soc. 248, 642-652.

Mermilliod, J. C. (1981), Astron. Astrophys. Suppl. Ser. 44, 467-500.

Mermilliod, J. C. (1987a), Astron. Astrophys. Suppl. Ser. 71, 119 and 413. Cited by Straižys (1992).

Mermilliod, J. C. (1987b), Bull. Inf. CDS 32, 37. Cited by Straižys (1992).

Mermilliod, J. C. \& Maeder, A. (1986), Astron. Astrophys. 158, 45-49. 
Meynet, G. et al. (1993), Astron. Astrophys. Suppl. Ser. 98, 477-504.

Morris, S. L. \& Naftilan, S. A. (1994), Astron. Astrophys. Suppl. Ser. 107, 71-75.

Nicolet, B. (1978), Astron. Astrophys. Suppl. Ser. 34, 1. Cited by Straižys (1992).

Nikonov, V. B. (1953), Bull. Abastumani Obs.

North, P. (1978), Etude du systeme GENVIL, Technical report, Lausanne. Cited by Straižys (1992).

North, P. (1980), Astron. Astrophys. Suppl. Ser. 41, 395-396.

North, P. et al. (1982), Astron. Astrophys. 108, 373. Cited by Straižys (1992).

Olsen, E. H. (1983), Astron. Astrophys. Suppl. Ser. 54, 55-134.

Olson, E. C. (1974), Publ. Astron. Soc. Pac. 86, 80. Cited by Golay (1974), Straižys (1992).

Optical Glass Filters (1990). SCHOTT Glaswerke.

Penndorf, R. (1957), J. Opt. Soc. Am. 47(2), 176-182.

Perry, C. L. \& Bond, H. E. (1969), Publ. Astron. Soc. Pac. 81, 629-636.

Perry, C. L. \& Hill, G. (1969), Astron. J. 74(7), 899-907.

Perry, C. L. et al. (1976), Astron. J. 81(8), 632-637.

Photomultipliers and Accessories (1990). Thorn EMI Electron Tubes.

Pogson, N. (1856), Mon. Not. R. Astr. Soc. 17, 12. Cited by Sterken \& Manfroid (1992a).

Poretti, E. (1992), The ESO Messenger 68, 52-53. See also correction in No. 71, 1993.

Poretti, E. \& Zerbi, F. (1993), Astron. Astrophys. 268, 369-373.

Press, W. H. et al. (1992), Numerical Recipes in FORTRAN, 2 edn, Cambridge University Press.

Reimann, H.-G. et al. (1992), Astron. Astrophys. 265, 360-369.

Renson, P. (1988), Astron. Astrophys. Suppl. Ser. 76, 127-135.

Rufener, F. (1981), Astron. Astrophys. Suppl. Ser. 45, 207-366.

Rufener, F. (1986), Astron. Astrophys. 165, 275-286.

Sagar, R. (1987), Mon. Not. R. Astr. Soc. 228, 483-499.

Schaerer, D. et al. (1993), Astron. Astrophys. Suppl. Ser. 98, 523-527.

Schaller, G. et al. (1992), Astron. Astrophys. Suppl. Ser. 96, 269-331.

Schild, R. E. (1970), Astrophys. J. 161, 855-866.

Sharov, A. S. (1962), Bull. Abastumani Obs. 27, 133. Cited by Straižys (1992).

Shobbrook, R. R. (1984), Mon. Not. R. Astr. Soc. 206, 273-284.

S̆leivytè, J. (1992), Baltic Astronomy 1, 83-95.

Slettebak, A. (1985), Astrophys. J., Suppl. Ser. 59, 769-784.

Smriglio, F. et al. (1986), Astron. Astrophys. Suppl. Ser. 66, 181-190. 
Smriglio, F. et al. (1988), Astron. Astrophys. Suppl. Ser. 75, 185-190.

Smriglio, F. et al. (1991), Astron. Astrophys. Suppl. Ser. 88, 87-93.

Sowell, J. R. (1987), Astrophys. J., Suppl. Ser. 64, 241-267.

Stauffer, J. et al. (1989), Astrophys. J. 342, 285-294.

Stebbins, J. et al. (1950), Astrophys. J. 112, 469. Cited by Straižys (1992).

Sterken, C. \& Manfroid, J. (1992a), Astronomical Photometry - A Guide, Vol. 175 of Astrophysics and Space Science Library, Kluwer Academic Publishers, London.

Sterken, C. \& Manfroid, J. (1992b), Astron. Astrophys. 266, 619-627.

Stokes, N. R. (1972), Mon. Not. R. Astr. Soc. 159, 165. Cited by Straižys (1992).

Stowe, L. L. et al. (1992), Geophys. Res. Lett. 19(2), 159-162.

Straižys, V. (1963), Bull. Vilnius Obs. 6, 1-10.

Straižys, V. (1964), Soviet Astron. 8(4), 596-597.

Straižys, V. (1965), Bull. Vilnius Obs. 15, 3-27.

Straižys, V. (1966), Trans. IAU 12b, 261-265. Commission 25.

Straižys, V. (1973a), Astron. Astrophys. 28, 349-354.

Straižys, V. (1973b), Bull. Vilnius Obs. 36(3), 3-16.

Straižys, V. (1974), Astron. Astrophys. 36, 435-440.

Straižys, V. (1977), Multicolor Stellar Photometry, Mokslas Publishers, Vilnius. In Russian. Cited by Straižys (1992).

Straižys, V. (1978), From multicolor photometric indices to the HR diagram, in A. G. D. Philip \& D. S. Hayes, eds, 'The HR Diagram', IAU Symp. No. 80, pp. 293-300.

Straižys, V. (1992), Multicolor Stellar Photometry, Vol. 15 of Astronomy and Astrophysics Series, Pachart Publishing House, 1130 San Lucas Circle, P.O. Box 35549, Tucson, Arizona 85740 .

Straižys, V. \& Kazlauskas, A. (1993), Baltic Astronomy 2, 1-169.

Straižys, V. \& Kurilienè, G. (1981), Astrophys. Space. Sci. 80, 353. Cited by Straižys (1992).

Straižys, V. \& Philip, A. G. D. (1994), Baltic Astronomy 3, 146-157.

Straižys, V. \& Sviderskienè, Z. (1972), Astron. Astrophys. 17, 312-322.

Straižys, V. \& Zdanavičius, K. (1965), Bull. Vilnius Obs. 14, 3-23.

Straižys, V. \& Zdanavičius, K. (1970), Bull. Vilnius Obs. 29, 15-24.

Straižys, V. et al. (1970), Bull. Vilnius Obs. 29, 10-14.

Straižys, V. et al. (1982), Bull. Vilnius Obs. 60, 50. Cited by Straižys (1992).

Straižys, V. et al. (1993), Baltic Astronomy 2, 326-347.

Strömgren, B. (1937), Handbuch der Experimentalphysik (Astrophysik), Vol. 26, Akad. Verlagsgesellschaft, Leipzig. Cited by Golay (1974), Straižys (1992), Sterken \& Manfroid (1992a). 
Strömgren, B. (1963), in K. A. Strand, ed., 'Basic Astronomical Data', The University of Chicago Press, Chicago, Illinois, U.S.A., p. 123. Cited by (Golay1974 Jaschek \& Jaschek1987 Straižys1992 Sterken \& Manfroid1992a).

Strömgren, B. (1966), Ann. Rev. Astron. Astrophys. 4, 433. Cited by (Golay1974 Jaschek \& Jaschek1987 Hendon \& Kaitchuck1990 Straižys1992 Sterken \& Manfroid1992a).

Sūdžius, J. \& Bobinas, V. (1994), Baltic Astronomy 3, 158-167.

Sūdžius, J. et al. (1970), Bull. Vilnius Obs. 29, 3-9.

Sullivan, D. J. (1989), A fast two-star photometer, in E. Budding \& J. Richard, eds, 'Third New Zealand Conference on Photoelectric Photometry', Special issue of Southern Stars, Fairborn Press.

Sullivan, D. J. (1994). pers. comm.

Sviderskienè, Z. \& Straižys, V. (1970), Bull. Vilnius Obs. 28, 55-77.

The Astronomical Almanac (1992).

Tobin, W. (1991), Southern Stars 34, 219.

Tody, D. C. (1986), in D. L. Crawford, ed., 'Instrumentation in Astronomy IV', Vol. 627, p. 733.

van de Hulst, H. C. (1981), Light Scattering by Small Particles, Dover Publications, Inc., 180 Varick Street, New York, N.Y. 10014. un-abridged and corrected edition of original 1957 edition.

Van Hoof, A. (1972), Astron. Astrophys. 18, 51-54.

Van Hoof, A. (1973), Inf. Bull. Variable Stars.

Wadsworth, A. D. (1988), The dead-time correction of the Mount John University Observatory EMI 9558 B photomultiplier tube used with photon-amplifier No. 2 in the No. 2 photometer, Stage III physics project, University of Canterbury.

Wadsworth, A. D. \& Cole, C. S. (1994), Southern Stars pp. 163-166. in press.

Waelkens, C. \& Rufener, F. (1983), Astron. Astrophys. Suppl. Ser. 52, 21-25.

Wallenquist, A. (1959), Ann. Uppsala Aston. Obs. Cited by Perry et al. (1976).

Wilson, R. E. (1953), Carnegie Inst. Washington Publ. No. 601. Cited by Eggen (1983).

Young, A. T. (1974), Observational technique and data reduction, in N. Carleton, ed., 'Astrophysics. Part A - Optical and Infrared', Vol, 12 of Methods of Experimental Physics, Academic Press, New York and London.

Young, A. T. (1988), Improvements to photometry. 1. better estimation of derivatives in extinction and transformation equations, in W. J. Borucki, ed., 'Proc. Second Workshop Improvements to Photometry', Vol. 10015, p. 215. Cited by Sterken \& Manfroid (1992a).

Young, A. T. (1992a), High-precision photometry, in S. J. Adelman, R. J. Dukes, Jr \& C. J. Adelman, eds, 'Automated Telescopes for Photometry and Imaging', Vol. 28 of ASP Conference Series.

Young, A. T. (1992b), Astron. Astrophys. 257, 366-388.

Young, A. T. (1994), Astron. Astrophys. 288, 683-696.

Young, A. T. \& Irvine, W. M. (1967), Astron. J. 72, 945. Cited by Young (1974). 
Zdanavičius, K. (1970), Bull. Vilnius Obs. 28, 24-32.

Zdanavičius, K. \& Cerniene, E. (1985), Bull. Vilnius Obs. 69, 3.

Zdanavičius, K. \& Nikonov, V. B. (1967), Bull. Vilnius Obs. 19, 3-27.

Zdanavičius, K. \& Straižys, V. (1964), Bull. Vilnius Obs. 11, 1-10.

Zdanavičius, K. et al. (1969), Bull. Vilnius Obs. 26, 3-12. 